DEPARTAMENTO DE INGENIERÍA ENERGÉTICA

ESCUELA TÉCNICA SUPERIOR DE INGENIEROS INDUSTRIALES

UNIVERSIDAD POLITÉCNICA DE MADRID

\title{
NUEVA GENERACION DE CENTRALES \\ TERMOSOLARES CON COLECTORES SOLARES \\ LINEALES ACOPLADOS A CICLOS SUPERCRITICOS DE POTENCIA
}

TESIS DOCTORAL

\author{
Por \\ Luis Coco Enriquez \\ Ingeniero Industrial por la E. T. S. de Ingenieros \\ Industriales de la Universidad de Navarra
}

\begin{abstract}
Director de la Tesis:
Dr. D. Javier Muñoz Antón

Doctor Ingeniero Industrial por la E. T. S. de Ingenieros Industriales de la Universidad Politécnica de Madrid
\end{abstract}


Tribunal nombrado por el Magfco. y Excmo. Sr. Rector de la Universidad Politécnica de Madrid, el día XX de xxxxxxxxx de 2017.

Presidente:

Vocal:

Vocal:

Vocal:

Secretario:

Suplente:

Suplente:

Realizado el acto de defensa y lectura de la tesis el día xxx de xxxx de 2017 en la E.T.S. Ingenieros Industriales.

CALIFICACIÓN:

EL PRESIDENTE

LOS VOCALES

EL SECRETARIO 


\section{Agradecimientos}

En primer lugar agradezco a mi esposa, a mi familia y amigos, por su apoyo afectivo y económico incondicional, que me ha permitido realizar un trabajo de investigación sobre energías renovables para mejora del bienestar de la Humanidad.

Mi estudio se enmarca en el programa de Doctorado de Energía Sostenible, Nuclear y Renovable liderado por el Catedrático Dr. Don Eduardo Gallego Díaz. Ha sido codesarrollado por el Dr. Don Javier Muñoz-Antón, Director de la Tesis, en el seno del Grupo de Investigación de Modelización de Sistemas Termoenergéticos de la Universidad Politécnica de Madrid (UPM), donde también encontré el apoyo incondicional de Dr. Don Alberto Abanades Velasco.

Esta Tesis nació gracias al patrocinio de Dr. Don José Luis de la Fuente O'Connor profesor titular de la UPM y Responsable de Inteligencia Estratégico-Tecnológica en Iberdrola, quién me puso en contacto con el Catedrático y Académico de Número de la Sección de Ingeniería de la Real Academia de Doctores de España Dr. Don José María Martínez-Val Peñalosa, mi Codirector en mis primeros años y Tutor de Honor desde el comienzo.

Especial agradecimiento a los trabajos de investigación de los investigadores: Dr. Don John J. Dyreby (University of Wisconsin-Madison), Dr. D. Eduardo Zarza (investigador del CIEMAT y responsable del Programa SolarPaces de la AIE), Dr. Don Antonio Rovira (profesor de la UNED), Dr. Doña María José Montes Pita (profesor de la UNED), Dr. D. Rubén Abbas (profesor de la UPM) y Dr. Don Luca Moretti (Politecnico di Milano), y otros autores citados en las referencias de esta tesis.

Finalmente, doy las gracias a todos los que me han permitió realizar un primer estudio de diseño conceptual de la nueva generación de plantas termosolares lineales acopladas a los ciclos de potencia supercríticos. 


\section{Publicaciones y conferencias}

Los contenidos principales de esta memoria así como otros derivados de forma directa de los trabajos realizados en la misma han sido sometidos a un proceso de revisión por pares cuyo resultado hasta la fecha es el siguiente:

L.Coco-Enriquez, J. Muñoz-Antón, J.M. Martínez-Val. Comparing SAM and Thermoflow for Linear Fresnel Plants. SAM Virtual Conference: July 23, 2013.

L.Coco-Enriquez, J. Muñoz-Antón, J.M. Martínez-Val. Innovations on Direct Steam Generation in Linear Fresnel Collectors. SOLARPACES 2013 International Conference.

L.Coco-Enriquez, J. Muñoz-Antón, J.M. Martínez-Val. New generation Line-Focusing Solar Power Plants with Molten Salts and Supercritical Carbon Dioxide Joule-Brayton power cycles.NURER 2014 International Conference.

L.Coco-Enriquez, J. Muñoz-Antón, J.M. Martínez-Val. Supercritical Steam power cycle for LineFocus Solar Power Plants.Journal of Polytechnic, 2015 18(4), Gazi University Turquia.

L.Coco-Enriquez, J. Muñoz-Antón, J.M. Martínez-Val. Integration between Direct Steam Generation in linear solar collectors and Supercritical Carbon Dioxide Brayton Power cycles. International Journal of Hydrogen Energy 40(2015).

L.Coco-Enriquez, J. Muñoz-Antón, J.M. Martínez-Val. Supercritical Ethane Brayton Power conversion systems for concentrated solar power plant. ASME POWER 2015, Journal of Solar Energy and Engineering.

L.Coco-Enriquez, J. Muñoz-Antón, J.M. Martínez-Val. Dual Loop Line-Focusing Solar Power Plants with Supercritical Brayton Power cycles. ECRES 2016 European Conference Renewable Energies (selected to be published on 'International Journal on Hydrogen Energy')

L.Coco-Enriquez, J. Muñoz-Antón, J.M. Martínez-Val. Comparison between s-CO2 and other working Fluids (s-Ethane, s-SFG, s-Xe, s-CH4, s-N2) in Line-Focusing Solar Power Plants with supercritical Brayton power cycles. ECRES 2016 European Conference Renewable Energies (selected to be published on 'International Journal on Hydrogen Energy').

L.Coco-Enriquez, J. Muñoz-Antón, J.M. Martínez-Val, Thermodynamic optimisation of supercritical $\mathrm{CO}_{2}$ brayton power cycles coupled to direct steam generation line-focusing solar fields, International Energy \& Engineering Conference 2016, 13-14 October 2016, Gaziantep, Turkey. Proceedings ISBN 978-975-7375-39-5.

L.Coco-Enriquez, J. Muñoz-Antón, J.M. Martínez-Val, Thermodynamic optimization of supercritical CO2 Brayton power cycles coupled to Line-Focusing Solar Fields. Proceedings of the ASME 2017 Power and Energy Conference PowerEnergy 2017 June 25-30, 2017, Charlotte, North Carolina, USA. Article number: PowerEnergy2017-3082. 


\section{Resumen}

El cambio climático representa uno de los principales desafíos de la humanidad hacia un futuro de paz, prosperidad y sostenibilidad del desarrollo de la sociedad. El Mundo necesita un nuevo modelo de crecimiento sostenible, seguro, duradero y beneficioso para todos. Reconociendo que el desarrollo sostenible, el acceso universal a la energía, y la seguridad energética son críticas para compartir la prosperidad y el futuro de nuestro planeta. Para garantizar y fomentar las energías sin impacto ambiental, sostenibles y de acceso universal, y beneficiarse del Sol como fuente energética. La energía termosolar jugará un papel importante para acelerar la transición entre los combustibles fósiles y nucleares hacia fuentes energéticas renovables.

Esta tesis trata de ahondar en este contexto buscando la integración de sinergias entre colectores solares lineales (cilindro parabólicos y Fresnel), los diferentes fluidos de trabajo disponibles en el estado de la técnica (aceite térmico, sales fundidas, generación directa de vapor) y ciclos de potencia (Joule-Brayton, Rankine).

Para abordar este planteamiento, se parte del estado de la técnica de plantas termosolares, fluidos de trabajo y ciclos de potencia (capítulo 1) que será analizado con la metodología descrita en el capítulo 2. Con esta base, se comienza por evaluar posibles mejoras al rendimiento de las plantas termosolares de colectores lineales y generación directa de vapor (capítulo 3), la utilización de ciclos Rankine supercríticos (capítulo 4), el uso de sales fundidas en el campo solar y ciclos Brayton con Dióxido de Carbono como fluido de trabajo (capítulo 5). A la vista de los resultados se introduce la necesidad de optimizar los parámetros de operación de plantas termosolares, siguiendo pautas recomendadas por otros autores (capítulo 6), lo que conduce a la realización de una herramienta de cálculo propia, SCSP, que minimiza el área de captación solar mediante algoritmos de optimización multivariable SUBPLEX, UOBYQA y NEWUOA. Con esta herramienta se estudian campos solares con aceite térmico acoplados a ciclos Brayton con Dióxido de Carbono (capítulo 7), campos solares con generación directa de vapor y ciclos Brayton con Dióxido de Carbono (capítulo 8), campos solares Dual-Loop con ciclos Brayton con Dióxido de Carbono (capítulo 9) y campos solares con sales fundidas o generación directa de vapor con ciclos Brayton de etano supercrítico (capítulo 10). En el capítulo 11 se compara el comportamiento del Dióxido de Carbono con otros posibles fluidos de trabajo en ciclos Brayton. Por último en el capítulo 12 se describen las generalidades del software desarrollado, SCSP y en el capítulo 13 se indican los principales resultados, conclusiones y trabajos futuros que derivan de esta tesis.

El trabajo desarrollado constituye un completo catálogo de configuraciones y prestaciones, habiéndose puesto énfasis en varias configuraciones novedosas, que en su conjunto orientan al diseñador sobre las capacidades de cada posible combinación de campo solar / bloque de potencia en función de sus fluidos de trabajo. 
Resumen 


\begin{abstract}
The climate change is one of the main challenges for the humanity towards a peace future, prosperity and sustainability of society development. The world needs a new sustainable growth model, secure, durable and beneficial for all. Recognizing the sustainable development, the universal access to energy, and the secure access to energy are critical for sharing the prosperity and the future of our planet. For assuring and fostering the energy sources without any environmental impact, sustainable and with universal access, and getting the benefits from the Sun as energy resource. The thermosolar energy will play an important role for accelerating the transition between the fossil and the nuclear fuels towards renewable energy resources.
\end{abstract}

This thesis deal with the integration and searching the synergies between the linear solar collectors (parabolic troughs and Fresnel), with the different state of the art heat transfer fluids (thermal oils, molten salts, direct steam generation) and power cycles (Joule-Brayton, Rankine).

For developing these issues, the concentrated solar plants arrangements, heat transfer fluids, and power cycles state of the art are considered the starting points in this thesis (chapter 1 ), and will be analysed with the methodology described in (chapter 2). This is the reference, for assessing further improvements as the line-focusing solar plants with direct reheating (chapter 3), supercritical water Rankine cycles in line-focusing solar plants (chapter 4), and solar fields with molten salts as heat transfer fluid coupled to supercritical Carbon Dioxide Brayton power cycles (chapter 5).

Reviewing the results obtained arise the requirement of optimizing the thermosolar power plants operation parameters, following the guidelines recommended by other authors (chapter 6), driving to the development of a new software, the Surpercritical Concentrated Solar Plants software (SCSP), for minimizing the solar field efficient aperture area by means of the multivariable optimization algorithms SUBPLEX, UOBYQA, and NEWOUA. With this tool the solar fields with thermal oil coupled to supercritical Carbon Dioxide Brayton cycles are studied (chapter 7), solar fields with direct steam generation coupled to supercritical Brayton cycles (chapter 8), and Dual-Loop solar fields also coupled to Brayton cycles (chapter 9), and the solar fields with molten salt or direct steam generation coupled to supercritical Ethane Bratyon cycles. In the chapter 11 is compared the behaviour of Carbon Dioxide with other supercritical working fluids in the Brayton cycles. Finally, in the chapter 12, are described the SCSP software capabilities, and in the chapter 13 are summarized and discussed the main results and conclusions and the further works derived from this these are proposed.

This researching work constitutes a complete catalogue about the configurations and benefits, highlighting the innovative arrangements, advising the designer about the capabilities deducted from the combination of the solar fields and power cycles with different working fluids. 
Abstract 


\section{Índice}

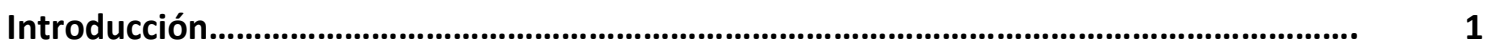

1. Contexto Energético 1

2. Contexto Tecnológico

3. Contexto Histórico $\quad 8$

4. La Iniciativa SUNSHOT 13

5. Objetivos de la tesis 15

6. Referencias 16

Capítulo 1. Estado de la técnica en las centrales termosolares con colectores solares lineales.

1.1. Introducción $\quad 21$

1.2. El Sol y la radiación solar como fuente energética 21

1.3. Colectores Cilindro Parabólicos (PTC) 24

1.3.1. Introducción $\quad 24$

1.3.2. Balance energético y pérdidas energéticas en un colector cilindro-parabólico PTC

1.3.3. Otros elementos de los PTC

1.4. Colectores Lineales Fresnel (LF) 36

1.4.1. Introducción 36

1.4.2. Caracterización del colector LF 40

1.5. Sistemas de Receptor Central o Torre Solar $\quad 46$

$\begin{array}{ll}\text { 1.6. Sistemas de Disco Parabólico } & 47\end{array}$

1.7. Tecnologías de almacenamiento térmico en plantas termosolares 48

1.7.1. Almacenamiento indirecto con sales fundidas $\quad 49$

1.7.2. Almacenamiento directo de vapor $\quad 49$

1.7.3. Almacenamiento indirecto con hormigón $\quad 49$

1.7.4 Almacenamiento indirecto en un medio de cambio de fase 50

1.8. Centrales termosolares con colectores lineales, con aceites térmicos como fluido 50 caloportador, y ciclos de potencia Rankine (Oil + Water Rankine)

1.9. Centrales termosolares con colectores lineales, con sales fundidas como fluido caloportador, ciclos de potencia Rankine (MS + Water Rankine)

1.10. Conclusiones 53

1.11. Referencias 53

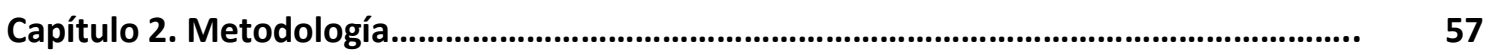

2.1. Modelo energético de los colectores solares lineales

2.2. Modelo energético y rendimiento del ciclo Rankine 62

2.3. Modelo energético y rendimiento del ciclo Brayton 63

2.4. Propiedades de los fluidos de trabajo del ciclo de potencia 63

2.5. Turbomáquinas (turbinas y compresores) 63

2.5.1. Turbinas (Diseño Básico) 63

2.5.2. Compresores (Diseño Básico) 64 
2.5.4. Compresores (Diseño Detallado) 71

2.6. Intercambiadores de calor del ciclo Brayton (Diseño Básico) 78

2.7. Intercambiadores de calor del ciclo Brayton (Diseño Detallado) 81

2.8. Intercambiadores de calor del ciclo Brayton. Operación fuera de su punto de diseño. Off-Design performance

2.9. Algoritmos de optimización multivariable 85

2.9.1. Algoritmo SUBPLEX $\quad 85$

2.9.2.Algoritmo UOBYQA - Unconstrained Optimization BY Quadratic Approximation $\quad 87$

2.9.3. Algoritmo NEWOUA-NEW Unconstrained Optimization Algorithm 89

2.10. Referencias $\quad 89$

Capítulo 3. Plantas Termosolares con colectores lineales Fresnel con Generación de Vapor y ciclos de potencia Subcríticos de Rankine con Recalentamiento Directo.............

3.1. Introducción $\quad 92$

3.2. Selección del programa informático para diseño de las plantas con colectores LF y DSG

3.3. Configuraciones de Campo Solar con colectores LF 95

3.3.1. Configuración de Referencia 96

3.3.2. Configuración de Referencia con Recalentamiento Directo o Direct ReHeating $\quad 99$

$\begin{array}{ll}\text { 3.3.3. Configuración propuesta } & 101\end{array}$

$\begin{array}{ll}\text { 3.4. Conclusiones } & 104\end{array}$

3.5. Referencias 105

Capítulo 4. Plantas Termosolares con Colectores Lineales con DSG y sales fundidas como HTF acoplados a ciclos Supercríticos Rankine...

4.1. Introducción $\quad 110$

4.2. Hipótesis de cálculo $\quad 112$

4.3. Planta Termosolar con Colectores Lineales con DSG como HTF acoplado a ciclo subcrítico Rankine (configuración de referencia)

4.4. Planta Termosolar con Colectores Lineales con DSG como HTF acoplado a ciclo supercrítico Rankine

4.5. Planta Termosolar con Colectores Lineales con sales fundidas como HTF acoplado a ciclo supercrítico Rankine

4.6. Conclusiones

Capítulo 5. Plantas Termosolares con Colectores Lineales con Sales Fundidas como HTF y ciclos de potencia Brayton con $\mathrm{CO}_{2}$ supercrítico como fluido de trabajo.................

$\begin{array}{ll}\text { 5.1. Introducción } & 125\end{array}$

5.2. Hipótesis de cálculo 130

5.3. Resultados 132

5.4. Diseño de detalle de los Intercambiadores de Calor Primario y de Recalentamiento 134

5.5. Conclusiones 136

$\begin{array}{ll}\text { 5.6. Referencias } & 137\end{array}$ 
Capítulo 6. Optimización de las plantas termosolares con colectores lineales con sales fundidas como HTF acopladas a ciclos de potencia Brayton con $\mathrm{CO}_{2}$ supercrítico como fluido de trabajo.

6.1. Introducción

6.2. Hipótesis de cálculo

6.3. Resultados

6.3.1. Caracterización de la Eficiencia Bruta del Ciclo de Potencia Brayton

6.3.2. Parámetros termodinámicos para la optimización de la eficiencia del ciclo Brayton

6.3.3. Tamaño Térmico de los recuperadores del ciclo Brayton

6.3.4. Área de Apertura Efectiva del Campo Solar

6.3.5. Estimación de Costes del Campo Solar

6.4. Conclusiones

6.5. Referencias

Capítulo 7. Optimización de las plantas termosolares con colectores lineales con aceites térmicos como HTF acopladas a ciclos Brayton de $\mathrm{CO}_{2}$ supercrítico

7.1. Introducción

7.2. Hipótesis de cálculo

7.3. Resultados

7.3.1. Eficiencia de la planta termosolar

7.3.2. Parámetros termodinámicos para la optimización de la eficiencia del ciclo termodinámico Brayton

7.3.3. Área apertura efectiva del campo solar

7.3.4. Estimación de costes del campo solar

7.3.5. Diseño de los Recuperadores de Baja y Alta presión

7.4. Conclusiones

Capítulo 8. Plantas termosolares de Colectores Lineales con DSG acopladas a ciclos Brayton con $\mathrm{CO}_{2}$

8.1. Introducción

8.2. Planta termosolar con colectores lineales con generación directa de vapor acopladas a ciclo Rankine sin Recalentamiento (configuración de referencia)

8.4. Plantas termosolares con colectores lineales con generaciones directas de vapor acopladas a ciclos de potencia s- $\mathrm{CO}_{2}$ Brayton

8.4.1. Configuración 1

8.4.2. Configuración 2

8.4.3. Configuración 3 
8.5.3. Tamaño térmico (UA) de los intercambiadores de calor

8.5.4. Estimación de costes de los Intercambiadores de calor del ciclo s- $\mathrm{CO}_{2}$ Brayton

8.6. Optimización de los parámetros de operación de plantas termosolares con colectores lineales con DSG, acopladas a ciclos de potencia s- $\mathrm{CO}_{2}$ Brayton

8.6.1. Caracterización de la eficiencia energética del ciclo de potencia s- $\mathrm{CO}_{2}$ Brayton

8.6.2. Caracterización del campo solar

8.6.3. Intercambiadores de calor del campo solar y del sumidero final de calor

8.7. Conclusiones

8.8. Referencias

Capítulo 9. Plantas Termosolares con Colectores Lineales, configuración del campo solar Dual- Loop acopladas a ciclos de potencia s-CO2 Brayton.

9.1. Introducción

9.2. Hipótesis de cálculo

9.3. Caracterización del ciclo de potencia Brayton en el punto de diseño

9.4. Caracterización de la producción de energia de la planta termosolar en el punto de diseño

9.5. Estimación de Coste de Inversión

9.6. Integración de otras configuraciones del ciclo s-CO2 Brayton (RC, PCRC y RCMCl) con los campos solares de tipología Dual-Loop

9.6.1. Caracterización del ciclo de potencia

9.6.2. Área de apertura efectiva y estimación de costes del campo solar

9.7. Conclusiones

9.8. Referencias

Capítulo 10. Plantas Termosolares con Colectores Lineales con Sales Fundidas o DSG como fluido caloportador acopladas a ciclos de pontencia supercritcos Brayton con $\mathrm{C}_{2} \mathrm{H}_{6}$ como fluido de trabajo

10.1. Introducción

10.2. Estabilidad del Etano en los ciclos de potencia supercríticos Brayton

10.3. Planta termosolar con colectores lineales con DSG y ciclo subcrítico de potencia Rankine (configuración de referencia)

10.4. El Campo Solar con DSG o Sales Fundidas

10.5. Hipótesis de cálculo

10.6. Eficiencia Neta en el Punto de Diseño

10.7. Fracción de Caudal Óptimo

10.8. Análisis de Sensibilidad del tamaño térmico (UA) en los Recuperadores

10.9. Análisis de Sensibilidad de las pérdidas de presión en los Intercambiadores de Calor

10.10. Análisis de Sensibilidad de la Temperatura de Entrada en el Compresor (CIT)

10.11. Efecto combinado de pérdida de presión en los cambiadores de calor y temperatura de entrada al compresor

10.12. Área de Apertura y Coste de Inversión del Campo Solar 270

10.13. Conclusiones $\quad 271$

10.14. Referencias $\quad 274$ 
Capítulo 11. Comparación entre el s- $\mathrm{CO}_{2}$ y otros fluidos de trabajo $\left(\mathrm{s}-\mathrm{C}_{2} \mathrm{H}_{6}, \mathrm{~s}-\mathrm{SF}{ }_{6}, \mathrm{~s}-\mathrm{Xe}\right.$, $\mathrm{s}-\mathrm{CH}_{4}, \mathrm{~s}-\mathrm{N}_{2}$ ) en las plantas termosolares acopladas a ciclos supercríticos Brayton...........

11.1. Introducción

11.2. Hipótesis de cálculo

11.3. Configuraciones ciclos de potencia Brayton

11.4. Caracterización del ciclo de potencia Brayton

11.5. Área de Apertura Efectiva del Campo Solar

11.6. Caracterización del coeficiente de transferencia de calor (HTC) de los fluidos de trabajo

11.7. Caracterización de las pérdidas de presión de los fluidos de trabajo

11.8. Conclusiones

11.9. Referencias

Capítulo 12. Programas Informático para diseño y optimización de los ciclos Brayton en las plantas termosolares con colectores lineales $y$ diferentes fluidos caloportadores.

12.1. Introducción

12.2. Configuraciones de las plantas CSP acopladas ciclos Brayton 306

12.3. Cálculo del punto de diseño (Design-Point)

12.4. Cálculo de las condiciones de operación óptimas

12.5. Diseño de los equipos de la planta CSP acoplada al ciclo supercrítico Brayton

12.5.1. Diseño de los Compresores

12.5.2. Diseño de las Turbinas

12.5.3. Diseño de intercambiadores del ciclo Brayton

12.5.4. Diseño del campo solar.

12.5.5. Diseño del Generador.

12.6. Validación de resultados con Thermoflow

12.7. Conclusiones

12.8. Referencias

Capítulo 13. Conclusiones.

ANEXO. Resultados simulaciones capítulo 9. Plantas termosolares con colectores lineales, configuración del campo solar "Dual-Loop" acopladas a ciclos de potencia s$\mathrm{CO}_{2}$ Brayton

Lista de acrónimos 


\section{Introducción}

\section{Contexto Energético}

De acuerdo a los últimos informes de la Agencia Internacional de la Energía International Energy Agency (IEA), si se continúa con la tendencia actual de consumo de combustibles fósiles para la generación energética, figuras 1 y 2 , se prevé que las emisiones de gases de efecto invernadero den lugar a un cambio climático sustancial produciendo un calentamiento medio global de $6^{\circ} \mathrm{C}$ [1]. Se debe cambiar esta tendencia hacia una economía sustentada por tecnologías de generación eléctrica sostenibles con baja emisión de gases de efecto invernadero, figuras 3 y 4 , si se quiere cumplir con el objetivo de reducir un $50 \%$ los niveles de $\mathrm{CO}_{2}$ en 2050 por debajo de los niveles actuales y limitar el calentamiento global en 2050 a $2{ }^{\circ} \mathrm{C}$ por encima de la era pre-industrial [2]. Estos objetivos requieren un esfuerzo inversor sustancial en energías renovables.

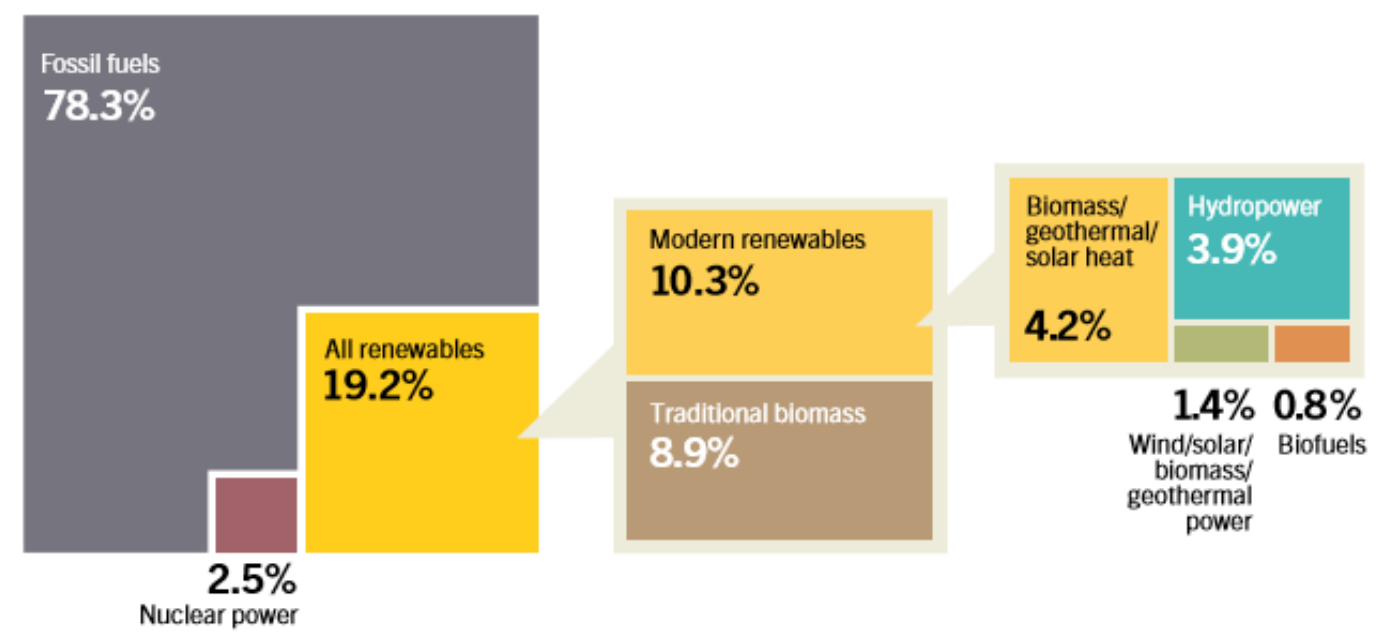

Figura 1. Estimación de la aportación de las Energías Renovables al Consumo Global de Energía en 2014 [3].

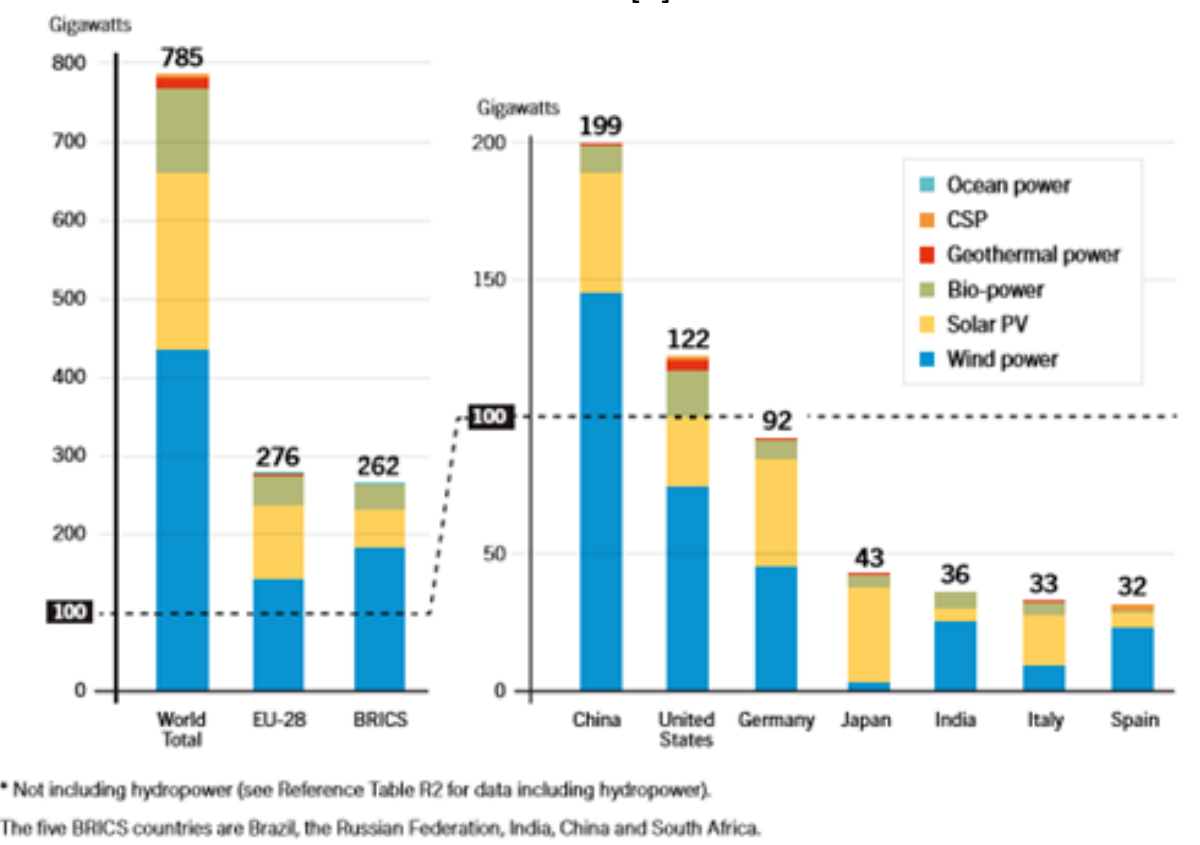

Figura 2. Potencia instalada en el mundo de energías renovables 2014 [3]. 
La energía termosolar o Solar Termal Electricity (STE) generada mediante concentración solar Concentrating Solar Power (CSP), es una de las fuentes de energías renovables con más perspectivas de crecimiento en los próximo años. La IEA ha previsto para la STE una cuota del $11 \%$ para el 2050 [4], figura 4. Las plantas CSP no producen emisiones contaminantes que incrementen el efecto invernadero, constituyéndose en una tecnología clave para mitigar el cambio climático. Además, la flexibilidad de las plantas CSP debido a su capacidad de almacenamiento térmico incrementan la seguridad energética del sistema. Esta es una de las principales características en que la CSP aventaja actualmente a las plantas fotovoltaicas photovoltaic (PV), el almacenamiento térmico o Thermal Energy Storage System (TES). Además, muchas plantas CSP también están equipadas con una caldera de combustible fósil de suficiente potencia como para ayudar a respaldar térmicamente las necesidades de demanda de la red eléctrica. Gracias al TES con capacidad para operar a carga completa durante varias horas, las plantas CSP pueden operar durante períodos nublados y después de la caída del Sol cuando se produce un incremento de la demanda eléctrica [5].

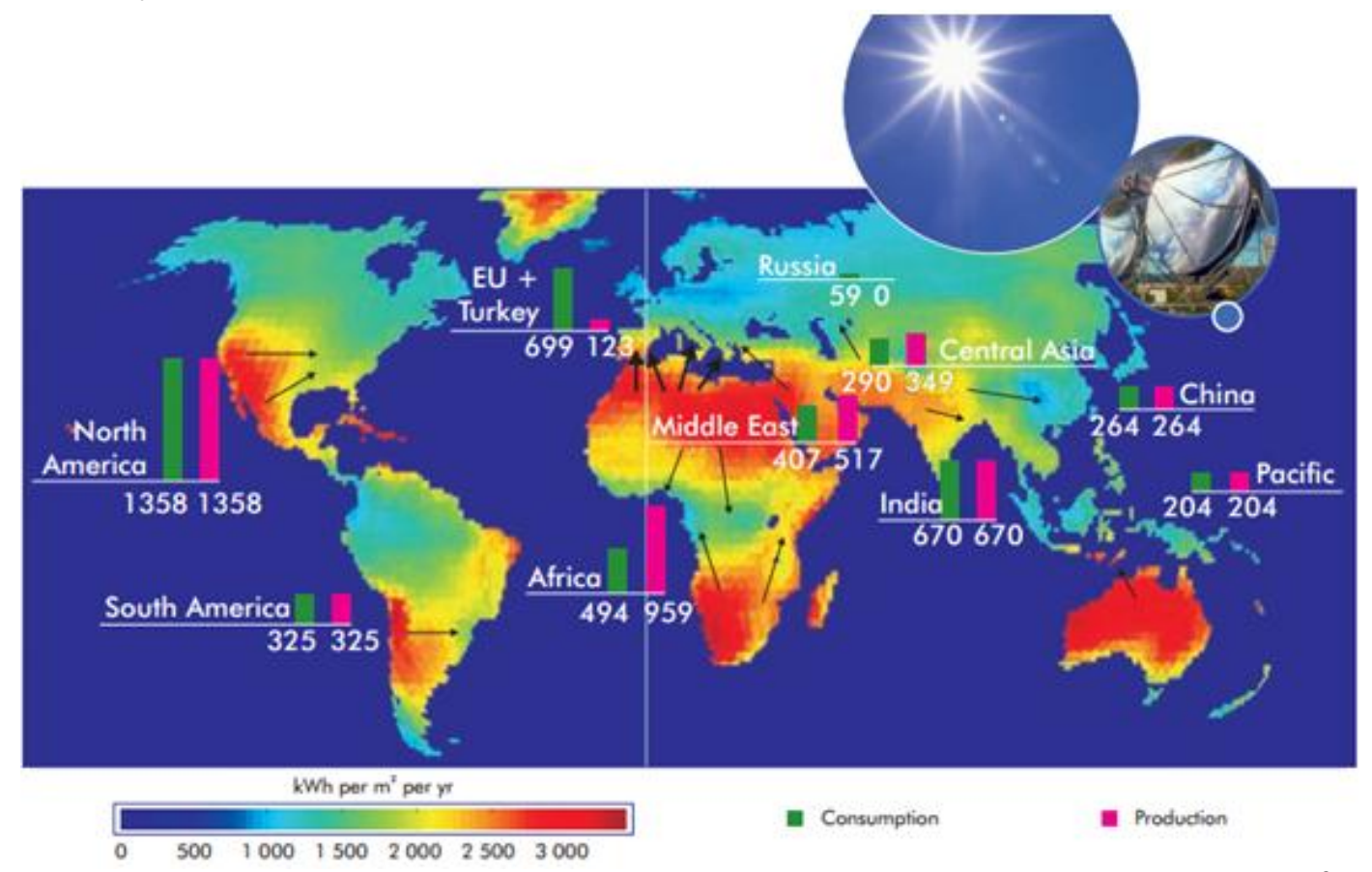

Figura 3. Mapa mundial de la distribución de Irradiación solar directa (DNI) anual $\mathrm{kWh} / \mathrm{m}^{2} / \mathrm{yr}$, y previsiones de Producción y Consumo de energía termosolar en el año 2050 en TWh [6]. Las flechas indican la transferencia de electricidad generadas por CSP desde las zonas con mayor irradiación solar hacia las zonas con mayor consumo eléctrico.

Entre las principales conclusiones obtenidas a la hora de elaborar las previsiones de energía termosolar en el 2014 se citan las siguientes:

- En 2050, con un fomento adecuado de la energía termosolar, la cuota global de CSP sera del $11.3 \%$, desglosado en un $9.6 \%$ cuyo origen será la radiación solar, y un $1.7 \%$ producido por las calderas de respaldo con combustibles fósiles o de biomasa.

- En los países con mayor irradiación solar, la energía termosolar serán una fuente de generación eléctrica competitiva para los períodos picos e intermedios en 2020, y constituirán una fuente de energía eléctrica base del sistema entre 2025 y 2030.

- La posibilidad de integrar almacenamiento térmico, como se ha mencionado previamente, es una característica diferenciadora de las plantas termosolares, 
proporcionando una capacidad de generación eléctrica de respaldo alternativa a las calderas de combustible fósil. Por tanto, las plantas CSP ofrecen, una flexibilidad de producción a los operadores de la red eléctrica que permite una mayor disponibilidad y adaptación a la demanda que otras fuentes renovables como son la energía eólica y la fotovoltaica.

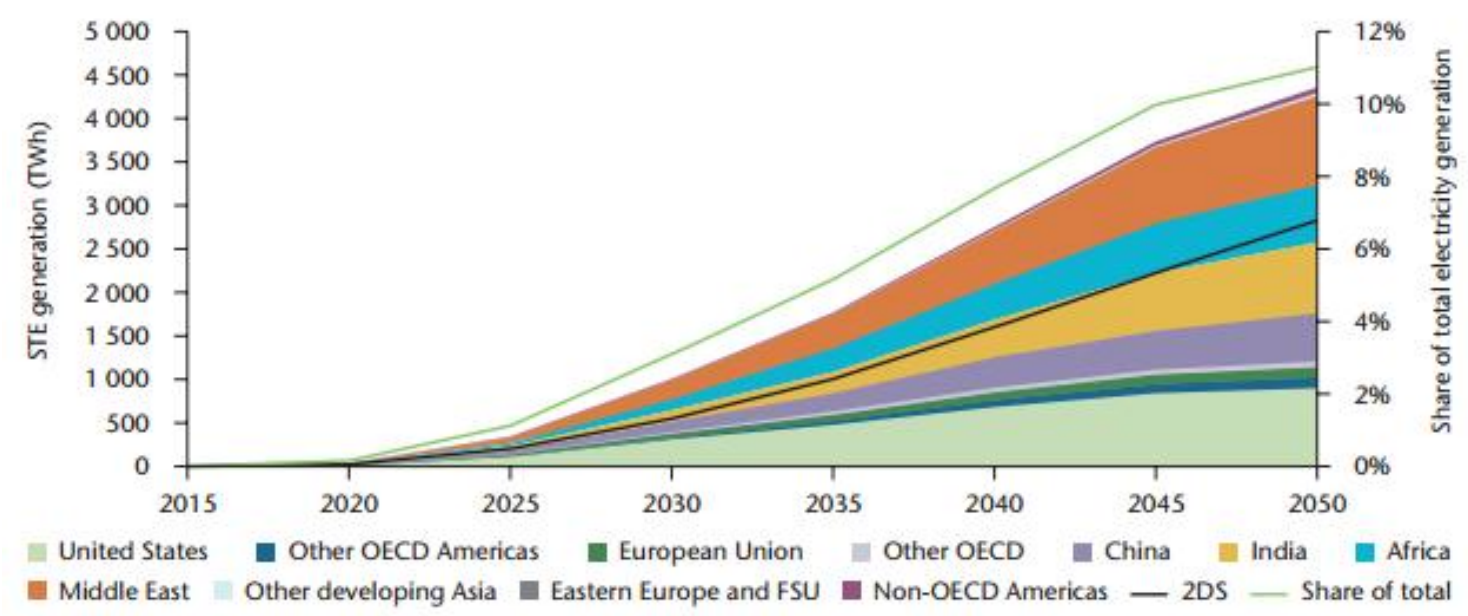

Figura 4. Previsión de incremento de la producción en CSP [4].

- Se prevé que Estados Unidos sea el mayor productor y consumidor de energía termosolar, seguido por África, India y Oriente Medio. El Norte de África tiene el potencial de constituirse en un gran exportador de energía eléctrica a Europa, y la gran irradiación solar recibida compensa el coste adicional de las líneas eléctricas de transmisión de potencia.

- La energía termosolar puede producir calor a altas temperaturas para procesos industriales, y en particular puede ayudar a cubrir la demanda de agua desalada en los países áridos.

- Dado el clima árido o semiárido de la locación donde se ubican las plantas termosolares, un desafío importante es acceder a los recursos hídricos en esas zonas que permitan una refrigeración óptima de las plantas CSP. En este sentido se han desarrollado los sistemas híbridos de refrigeración con aire/agua para las zonas con mayor escasez de agua.

- La principal limitación para incrementar la implantación de plantas termosolares, no es la inexistencia de áreas para su localización, sino la distancia de esas áreas a los principales centros de consumo de electricidad. Por este motivo se están desarrollando en paralelo las tecnologías de transmisión de electricidad a largas distancias.

Las plantas CSP pueden comenzar suministrando energía eléctrica competitiva o también suministrando combustibles [7]: líquidos (Metanol, Hidrocarbonos sintéticos, etc.) o gaseosos (Hidrógeno, Metano, Syngas) en 2030. Para el 2050, se prevé que las plantas CSP podrían producir hidrógeno para desplazar el 3\% del consumo global de Gas Natural, y cerca del 3\% del consumo de combustibles líquidos. 
Se resumen en base a escala temporal los hitos tecnológicos esperados en el campo de la energía termosolar:

a) En 2020 la potencia de CSP mundial será de 148 GW y su factor de operación del 32\%. Se cumplirán las siguientes metas:

- Primera torre central con generación directa de vapor [8].

- Primera torre central con sales fundidas [9-10].

- Primera planta a gran escala con colectores lineales Fresnel [11-12].

- Primera planta con 100 colectores de discos Stirling [13].

- Generación directa de vapor en las plantas con colectores lineales [14-15].

- Almacenamiento térmico en tres fases para las plantas con generación directa de vapor [16-17].

- Almacenamiento térmico y sistemas de respaldo para grandes colectores de discos [18].

- Demostración de la utilización de las sales fundidas como fluido caloportador Heat Transfer Fluid (HTF) en los colectores lineales Parabolic Trough Collector (PTC) o Linear Fresnel Collector (LF) a gran escala. En los últimos años ya se han construido y experimentado en instalaciones piloto para este fin [19-21].

- Desarrollo de colectores solares con bajo peso, y bajo coste de fabricación para garantizar su capacidad reflectante [22].

- Optimización del tamaño de los heliostatos, el diseño de los campos solares, el diseño de las torres centrales, y el número de torres por turbina para un almacenamiento de 6 a 18 horas [23].

- Incremento de la energía recibida en los tubos absorbedores con una óptica innovadora en los colectores lineales [24].

- Introducción de las turbinas de vapor supercrítico en las plantas CSP [25].

b) En 2030 la potencia de CSP mundial será de 337 GW y su factor de operación del 39\%. Se cumplirán las siguientes metas:

- Desarrollo de sistemas híbridos con paneles fotovoltaicos y energía termosolar mediante el aprovechamiento selectivo del espectro solar [26].

- Todas las plantas tendrán un sistema de enfriamiento seco sin agua. Operarán a $540^{\circ} \mathrm{C}$ y dispondrán de grandes capacidades de almacenamiento térmico [27].

- El proceso de desalación será un proceso de cogeneración en las plantas CSP [28].

- Primera planta con torre central con fluido HTF aire y turbinas de gas [29].

- Primera planta CSP supercrítica.

- Desarrollo e introducción de ciclos de potencia con Dióxido de carbono supercrítico. Introducción turbinas Brayton con multietapas de recalentamiento [30-31].

- Introducción de innovadores fluidos caloportadores HTF: aire [32], gases [33-34], nanofluidos [35], metales líquidos Liquid metals [36-37], aire y partículas en las torres centrales [38-39].

c) En 2050 la potencia de CSP mundial será de 1089 GW y su factor de operación del 50\%. Intensificación en la investigación y desarrollo de combustibles de origen solar (gaseoso, líquido y sólido) [40-42].

- Biogás y combustibles de origen solar sustituirán al gas natural como combustible de respaldo en las centrales CSP [43].

- La producción de Hidrógeno en las plantas CSP en torres centrales y colectores de discos se introducirán en la red de gas natural [44]. 
- Producción de hidrógeno de origen solamente solar para producir combustibles líquidos [45].

\section{Contexto Tecnológico}

Hasta la fecha se han desarrollado cuatro tipos diferentes de tecnologías en las plantas termosolares dependiendo del sistema de captación y concentración solar utilizado: PTC, LF, torre central y discos parabólicos. Cada una de ellas se encuentra en distintas fases de desarrollo e implantación. El tipo de tecnología más extendido es la de captación solar mediante canales/espejos parabólicos (figura 5). Otras tecnologías de captación/concentración solar son el receptor central o de torre con campo de helióstatos (figura 5), está emergiendo con fuerza y representará un porcentaje significativo de las futuras plantas. Las tecnologías de reflectores lineales LF (figura 5), y la de discos parabólicos con motores Stirling (figura 5), están empezando a entrar en la fase comercial con argumentos diferenciados frente a las otras dos tecnologías más extendidas mencionadas anteriormente.
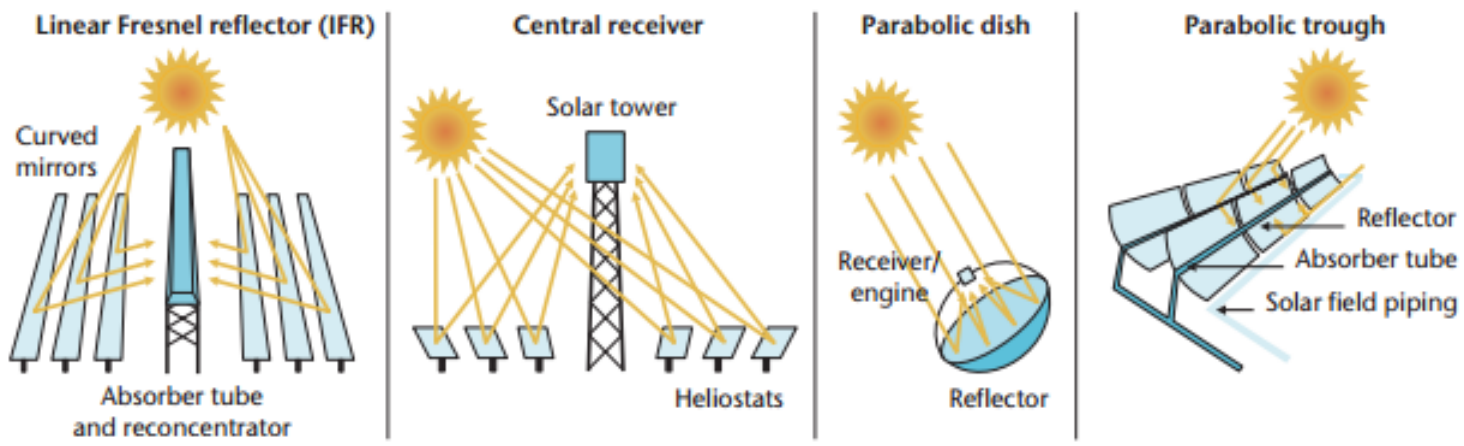

Figura 5. Representación esquemática de las diferentes tecnologías de concentración solar en las plantas termosolares de generación de energía eléctrica [4].

La concentración de la radiación solar permite unas altas temperaturas de operación y por tanto una buena eficiencia a nivel del colector solar. Estos altos niveles térmicos permiten una alta eficiencia térmica, y una alta conversión de la energía calorífica en trabajo, como consecuencia del teorema de Carnot, ver ecuación 1 [46].

$$
\eta=1-\frac{\mathrm{T}_{2}}{\mathrm{~T}_{1}}
$$

Donde:

- $\quad \mathrm{T}_{1}$ : temperatura del foco caliente.

- $\quad T_{2}$ : temperatura del foco frío.

Las eficiencias de los colectores solares, la eficiencia de Carnot, y la eficiencia total de conversión de energía solar en electricidad, se muestran en la figura 6, en función de varias temperaturas de fluido caloportador HTF, para diferentes factores de concentración solar.

En la parte izquierda de la figura 6, las relaciones de concentración entre 40 a 100 están representadas para los colectores "lineales" tanto parabólicos como Fresnel. En la parte derecha de la figura 6, las relaciones de concentración entre 100 y 2000 representan a los colectores "puntuales" de torres centrales y discos. 
La eficiencia de los colectores depende del estado del arte de la tecnología, mientras que el principio termodinámico de Carnot representa una ley física y expresa la máxima eficiencia posible de conversión. La eficiencia global, es el producto de la eficiencia del colector por la eficiencia de Carnot y por un coeficiente fijado en 0.7, expresando las imperfecciones de un motor termodinámico imperfecto [46-47]. Gracias a su mayor ratio de concentración solar, los colectores "puntuales" pueden convertir en electricidad mayores fracciones de energía que los colectores "lineales". Para cada nivel de concentración solar, hay una temperatura óptima de operación, para la tecnología del colector solar, que maximiza la eficiencia global de conversión solar a electricidad. La temperatura óptima de operación para los colectores lineales con un factor de concentración de 100 es alrededor de $400^{\circ} \mathrm{C}$, y para los colectores puntuales con concentración de 1000 la temperatura de operación óptima es de alrededor de $750^{\circ} \mathrm{C}[48]$.
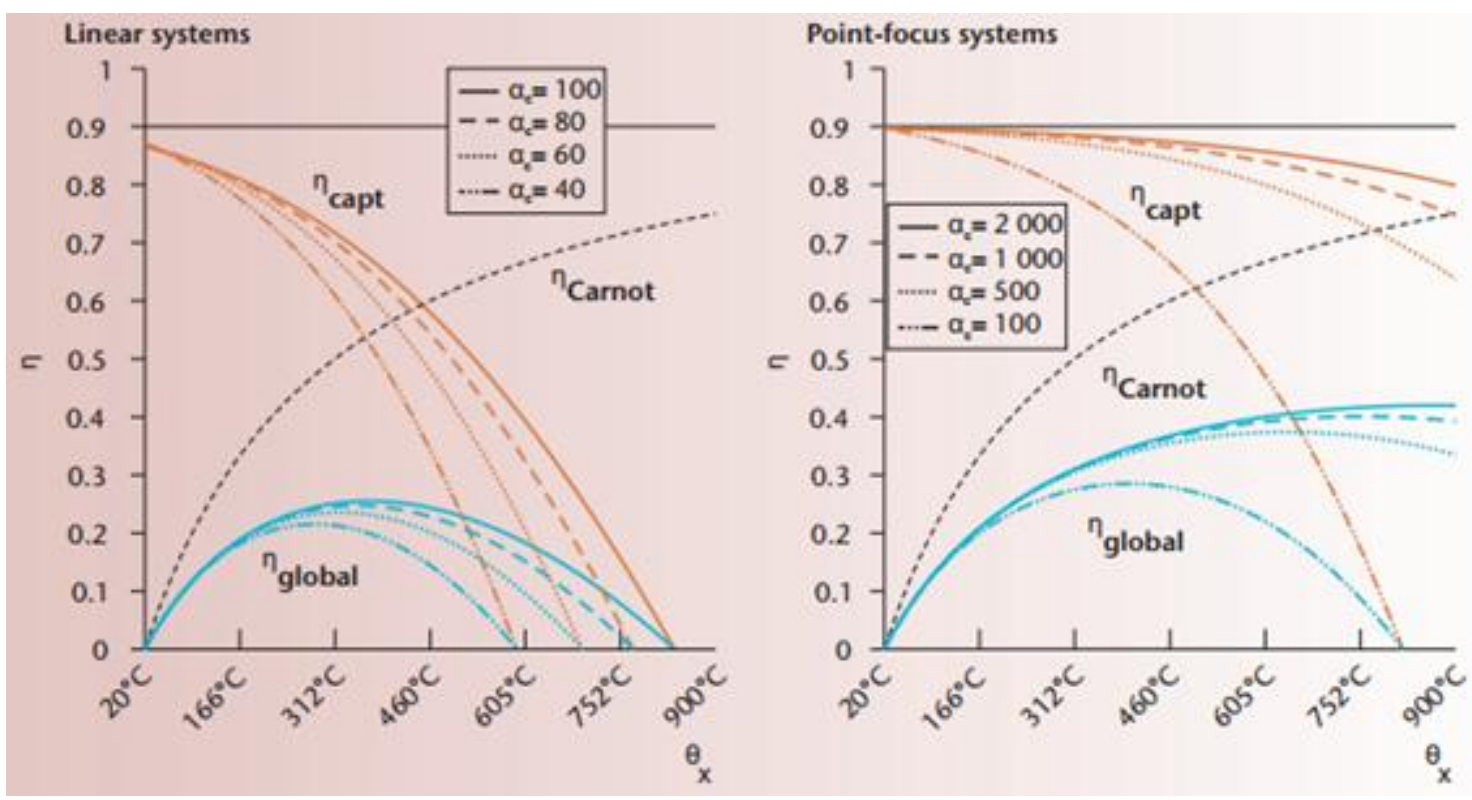

Figura 6. Eficiencia en función de la temperatura de trabajo para varias relaciones de concentración solar [47].

Los colectores parabólicos lineales (PTC) con aceite térmico como fluido caloportador es la tecnología más madura, pero todavía permite mejoras sustanciales:

- Las dimensiones de los colectores solares pueden ser incrementadas.

- Las láminas reflectoras pueden reemplazar el cristal para reducir el peso y coste de los colectores solares.

- Los tubos absorbedores están siendo constantemente optimizados.

- Nuevos fluidos caloportadores están siendo investigados. La generación directa de vapor (o Direct Steam Generation- DSG) es una opción viable (figura 9).

Mediante este avance tecnológico, DSG, se reduce el número de intercambiadores de calor y los equipos relacionados con la estabilidad química del fluido caloportador HTF, pero su alta presión de operación hace que otros componentes sean más caros. Mediante la tecnología DSG se pueden obtener mayores presiones y temperaturas de trabajo a la entrada de la turbina de vapor, si el proceso de generación de vapor es adecuadamente controlado, lo cual está resultando una tarea compleja en las instalaciones piloto. El motivo de la complejidad de 
control de la tecnología DSG, radica en el fenómeno físico de la ebullición, cambio de fase del agua de la fase líquida a vapor. Los procesos de cambio de fase en la naturaleza son muy complejos desde el punto de vista de su simulación numérica. La mayor planta termosolar con colectores PTC y DSG tiene una capacidad de 5 MWe (TSE 1 en Tailandia) [48], sin embargo, ya se han desarrollado plantas con la tecnología de torre central con más de $100 \mathrm{MWe}$ (proyecto Ivanpah 392 MWe brutos, en Estados unidos [8].

El almacenamiento térmico en las plantas DSG también están resultando un desafío tecnológico, ya que la evaporación del agua es un proceso isotermo, a lo contrario de la adición de calor en las sales fundidas, las pérdidas de presión en estos sistemas de almacenamiento reducen la eficiencia termodinámica del sistema. Por otra parte el almacenamiento de vapor en vasijas a presión es caro y no garantiza una optimización de coste a gran escala. Una opción es la utilización de un sistema de almacenamiento en tres fases, para precalentar el agua, evaporarla y sobrecalentar el vapor. En las fases 1 y 3 sería un almacenamiento de calor en el que cambia la temperatura de almacenamiento del medio (calor sensible). En la fase 2, sería un almacenamiento de calor de cambio de fase a temperatura constante (calor latente), utilizando un material de almacenamiento térmico de cambio de fase.

Como alternativa, actualmente se utilizan principalmente sales fundidas para el sistema de almacenamiento térmico principalmente, y a menor escala como fluido de trabajo en el receptor (a esta tecnología se denomina almacenamiento directo). La utilización de sales en el receptor permite un incremento de la temperatura de trabajo desde unos $380^{\circ} \mathrm{C}$ en los aceites térmicos hasta un rango $530^{\circ} \mathrm{C}$ a $550^{\circ} \mathrm{C}$, incrementando la eficiencia bruta del ciclo de potencia desde $39 \%$ hasta $42 \%$ según autores como Lenzen [49]. Gracias a las mayores diferencias de temperatura entre los tanques de almacenamiento térmico caliente y frío (actualmente se utiliza una mezcla de sales fundidas que solidifica por debajo de $238^{\circ} \mathrm{C}$ ), las plantas que utilizan sales fundidas como HTF necesitan tres veces menos cantidad de sales que las plantas que utilizan aceite térmico como fluido caloportador para la misma capacidad de almacenamiento. Este decremento se debe al beneficio que supone el aumento en la temperatura, que para una misma cantidad de energía almacenada reduce la masa de sales requeridas proporcionalmente, ver ecuación 2:

$$
Q=\dot{m} \cdot C p \cdot \Delta T
$$

Esta consideración lleva a que el ahorro en masa de sales fundidas pueda llegar a reducir el coste del sistema de almacenamiento térmico, lo que representa alrededor del $24 \%$ del coste total de la planta para un almacenamiento de 7.5 horas en una planta con colectores lineales [50]. Otra ventaja de las sales fundidas como fluido HTF sobre el vapor, es que este fluido precisa menores presiones de operación, y por tanto los requerimientos asociados a la resistencia mecánica de los materiales de los tubos absorbedores pueden ser aliviados, lo cual reduce el coste de inversión del campo solar.

Los colectores Fresnel están siendo investigados actualmente tanto con DSG, un ejemplo representativo es la planta de Puerto Errado, Murcia [11], como con sales fundidas en los 
mediante desarrollos de AREVA instalados en un lazo piloto en Sandia National Laboratories en Estados Unidos (SNL) [51]. Por otro lado, la tecnología de torre central, a pesar de requerir mayores inversiones iniciales por sus características intrínsecas, está constituyéndose en una alternativa competitiva, entre otros motivos por dar lugar a una solución muy compacta en superficie del campo solar, y por la posibilidad de un drenaje natural de las sales fundidas hacia los tanques de almacenamiento por gravedad. En España y Estados Unidos se han construido recientemente plantas termosolares integrando sales fundidas para refrigerar los receptores como son Gemasolar $20 \mathrm{MW}$ de Torresol Energy en Sevilla, España [9] y Crescent Dunes - SolarReserve (110 MWe), localizada en Nevada, Estados Unidos [10], entre otras.

\section{Contexto Histórico}

La crisis del petróleo en 1973 provocó un fuerte efecto inflacionista y una reducción de la actividad económica de Estados Unidos y Europa Occidental, como consecuencia directa de la decisión de la Organización de Países Árabes Exportadores de Petróleo (OPEP) de no exportar más petróleo a los países que habían apoyado a Israel durante la guerra de Yom Kipur. Europa y Estados Unidos respondieron con una serie de medidas permanentes para frenar su dependencia energética del exterior. Como consecuencia directa de la crisis del petróleo, hay que remontarse a mediados de 1970 cuando en Estados Unidos comenzó el impulso de las renovables, destacando el apoyo del Energy Research and Development Administration (ERDA) en Estados Unidos, y que dio lugar al actual U.S. Department of Energy (DOE) en 1978.

Realizando una breve recapitulación histórica de las plantas termosolares con colectores PTC, la mayor parte del desarrollo estaba patrocinado por SNL. Numerosas aplicaciones para generación de calor se pusieron en servicio, ocupando hasta $5000 \mathrm{~m}^{2}$ de área de colectores. Acurex, SunTex y Solar Kinetics fueron los fabricantes de colectores PTC en Estados Unidos durante este período. Los colectores PTC son capaces de generar temperaturas de $500^{\circ} \mathrm{C}$ $\left(932^{\circ} \mathrm{F}\right)$ fueron inicialmente desarrollados para aplicaciones de calor industriales Industrial Process Heat (IPH).

En España también como consecuencia directa de la citada crisis del petróleo en 1973, se puso en marcha la investigación en Energía Solar, con la construcción de la Plataforma Solar de Almería (PSA) a finales de los 70 en el desierto de Tabernas. En 1980 se firmó un acuerdo para la construcción de la PSA, entre Alemania, Austria, Bélgica, Estados Unidos, Grecia, España, Italia, Suiza y Suecia. Su objetivo principal era demostrar la viabilidad técnica de la energía termosolar de concentración como fuente de energía eléctrica. Con la bajada del precio del petróleo, siete países se retiraron del negocio, quedando solamente España y Alemania, que también se retiró en 1999. En estas instalaciones de la PSA también se desarrollaron los colectores parabólicos PTC en Europa, con la construcción del Small Solar Power Systems Project, Distributed Collector System (SSPS/DCS) en Tabernas, España, en 1981. Esta instalación consistía en dos campos de colectores parabólicos con un área total de apertura de $7602 \mathrm{~m}^{2}$. Estos campos utilizaban el sistema de seguimiento solar de eje único Acurex y los colectores de doble eje de seguimiento solar desarrollados por M.A.N en Munich, Alemania. En 1982, Luz International Limited (LUZ) desarrolló los colectores parabólicos para aplicación de generación de calor industrial (IPH) que estaban basados en la gran experiencia que había aportado SNL. Aunque varios de los desarrolladores de los colectores PTC vendieron sistemas IPH en $1970 \mathrm{y}$ 
1980, ellos normalmente encontraron dos barreras para difundir sus tecnologías. La primera de ellas era la necesidad de desarrollo de ingeniería y de publicidad para realizar incluso los proyectos más pequeños. La segunda, la mayoría de los potenciales clientes industriales disponían de complejos mecanismos de toma de decisión lo cual daba lugar a rechazar los proyectos después del gran coste y esfuerzo que se había gastado en ellos.

En 1983, Southern California Edison (SCE) firmó un acuerdo con Acurex Corporation para la compra de la electricidad producida en los campos solares integrados por colectores PTC. Acurex fue incapaz de financiar dicho proyecto. LUZ negoció un contrato con términos similares con SCE para la construcción de las plantas solares Solar Electric Generating System SEGS I y II, tablas 1 a 3 y figura 7.

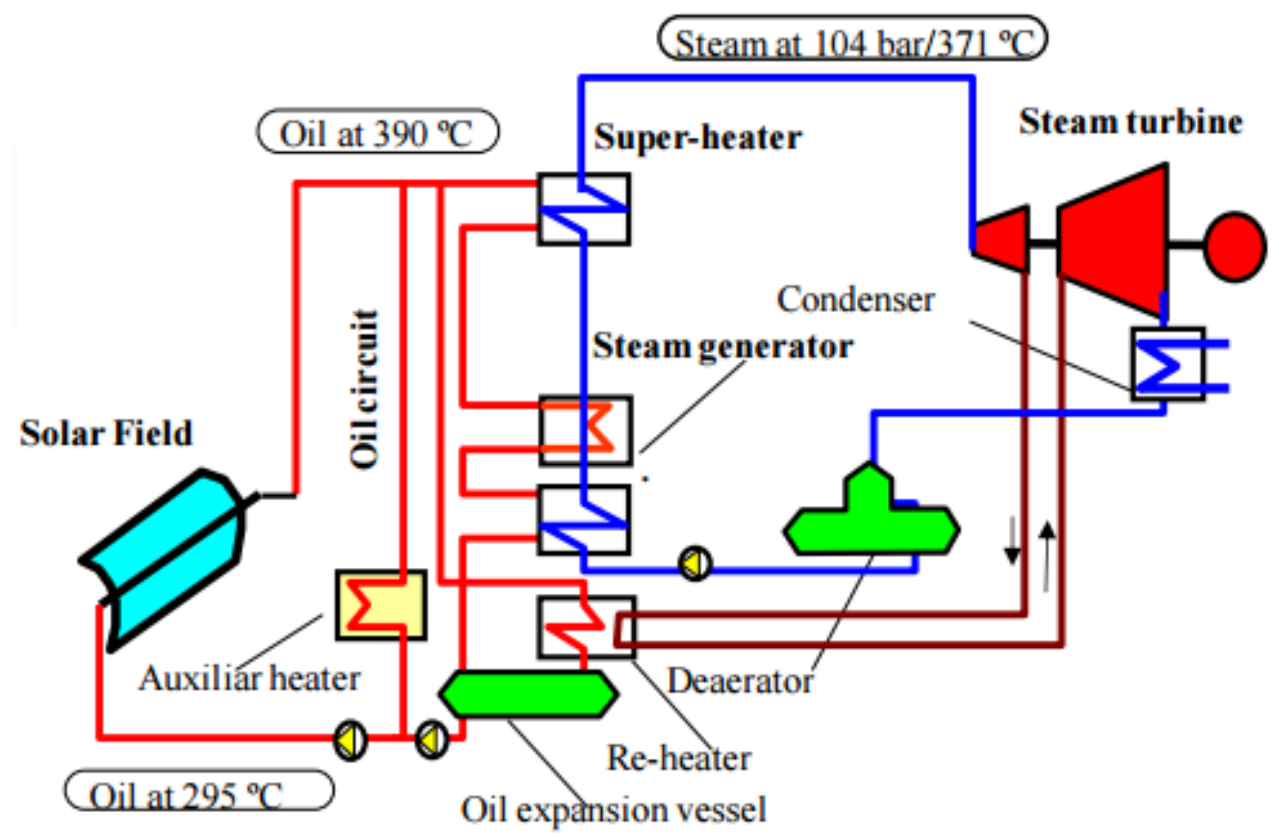

Figura 7. Planta termosolar de tipología SEGS-I a SEGS-IX, construida por la compañía Luz en Estados Unidos, entre 1985 y 1991. Ciclo de potencia Rankine con recalentamiento. Eficiencia del ciclo 37.6\%. Potencia nominal eléctrica 80 MWe. Eficiencia eléctrica anual 13.6\%.

Más tarde con la aparición del California Standard Offer (SO) LUZ consiguió la firma de numerosos contratos con el SCE que darían lugar al desarrollo de la plantas SEGS III hasta SEGS IX. Inicialmente, estas plantas estaban limitadas por la legislación de USA hasta $30 \mathrm{MWe}$; más tarde esta limitación fue elevada hasta 80 MWe. En 1991 la empresa LUZ quebró siendo incapaz de financiar la construcción de su planta décima SEGS X. Aunque muchos factores contribuyeron a la quiebra de LUZ, el problema principal de base fue que el coste de la tecnología era muy alto para competir en el mercado. Lotker [52] describió los acontecimientos que permitieron a la empresa LUZ tener éxito en el mercado eléctrico entre los años 1984 y 1990, y las numerosas barreras administrativas e institucionales que contribuyeron a su quiebra. Es importante hacer hincapié en que todas las plantas de LUZ, es decir, las plantas SEGS, tablas 1 a 3, fueron vendidas a inversores y actualmente continúan en operación. En la figura 7 se muestran sus características técnicas principales, equipos y componentes. 
Las plantas termosolares continúan madurando tecnológicamente para reducir el coste unitario de la energía producida y poder competir con las tradicionales centrales de generación con combustibles fósiles o nucleares. El objetivo es disponer de una fuente de energía renovable sin coste alguno de su combustible, sin impacto ambiental durante su explotación, y minimizar su coste ambiental durante su fabricación. La configuración de planta termosolar que mayor implantación industrial y comercial a nivel mundial está integrada por colectores solares PTC, con aceite térmico como HTF, ciclos de potencia Rankine con agua en estado subcrítico y opcionalmente con almacenamiento térmico mediante dos tanques de sales fundidas [53]. Como alternativa para optimización energética de la citada planta termosolar, actualmente se está sustituyendo los aceites térmicos por sales fundidas como HTF (figura 8).

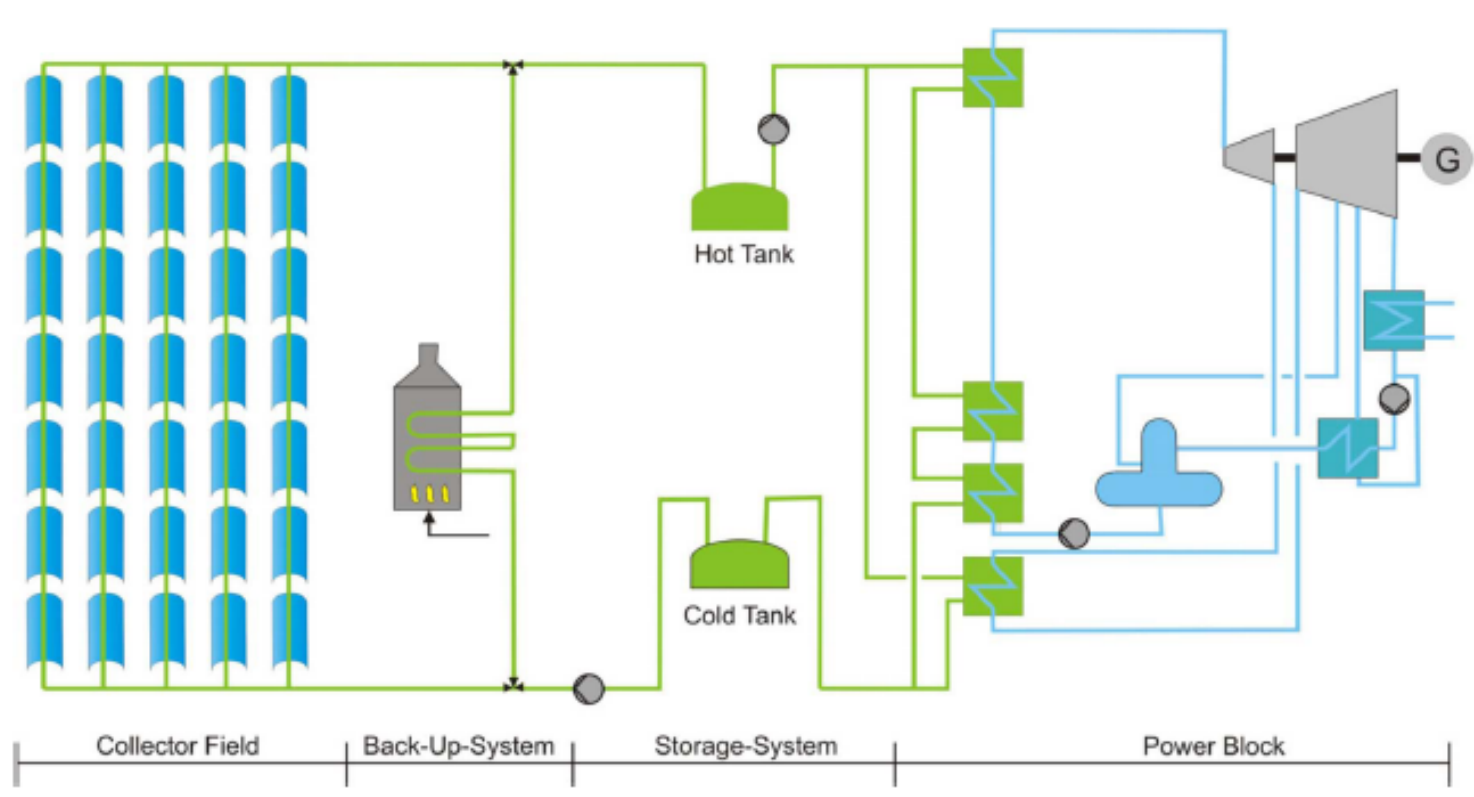

Figura 8. Planta termosolar con colectores lineales (PTC), sales fundidas (MS) como fluido caloportador (HTF), sistema de almacenamiento térmico (TES) con sales fundidas, y caldera de fuel de apoyo [53].

La generación directa de vapor Direct Steam Generation (DSG) en los colectores lineales como fluido HTF, en las plantas termosolares con colectores lineales PTC o LF, está constituyendo otro desarrollo tecnológico que permite la sustitución tanto de aceites como de sales fundidas por vapor de agua en los colectores lineales de concentración solar (figura 9). Lo cual tiene un beneficio directo desde el punto de vista medioambiental en esta tipología de instalaciones. 


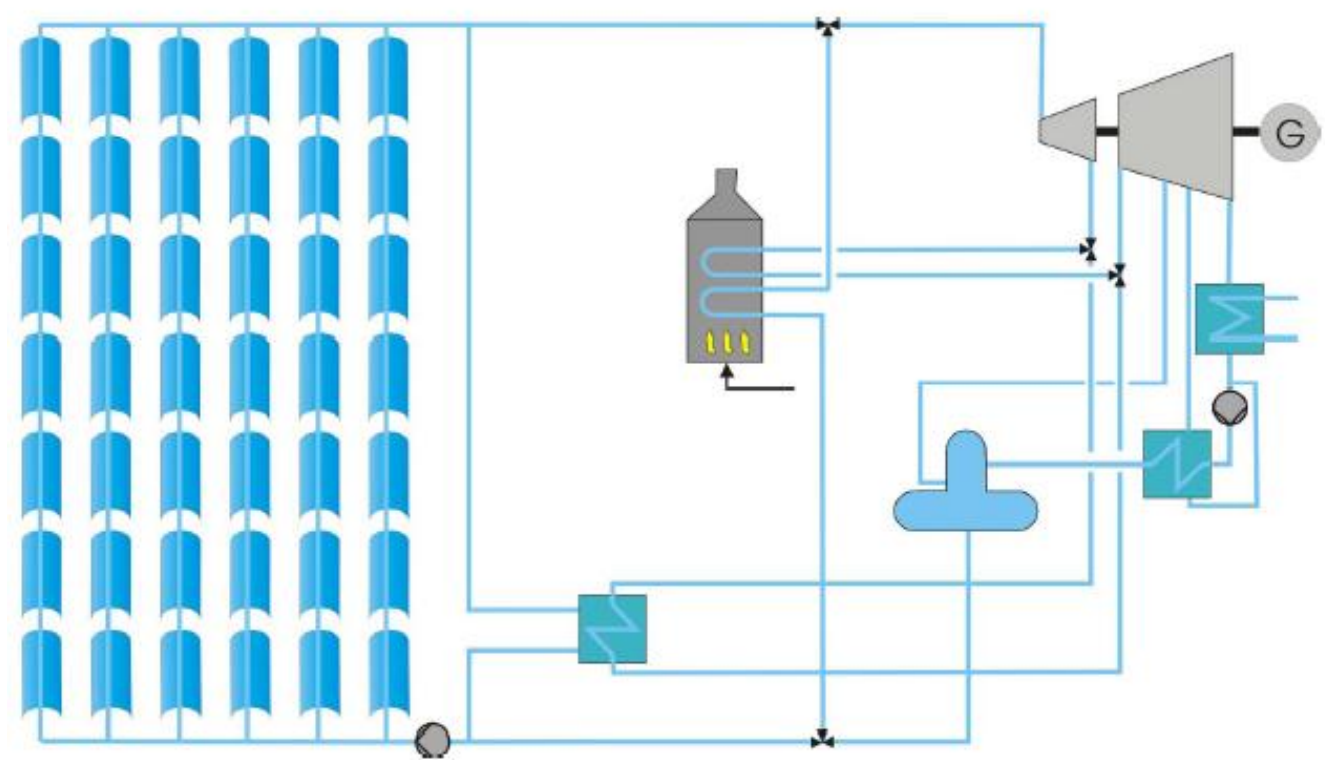

Figura 9. Planta termosolar con colectores lineales PTC, DSG como HTF, y caldera de biomasa/biogas de apoyo [53].

Como principal exponente a nivel internacional, de las plantas termosolares con colectores lineales PTC o LF y ciclos de potencia Rankine, sirva como ejemplo el complejo termosolar Solar Energy Generating Systems (SEGS) en California, Estados Unidos, es el segundo con mayor potencia instalada, después de Ivanpah [8]. Integrado por nueve plantas termosolares con colectores cilindroparabólicos y aceite como fluido caloportador. Su potencia es de 354 MWe. En las tablas 1 a 3 se puede apreciar en detalle la potencia instalada y el histórico de potencia producida en el complejo SEGS [54].

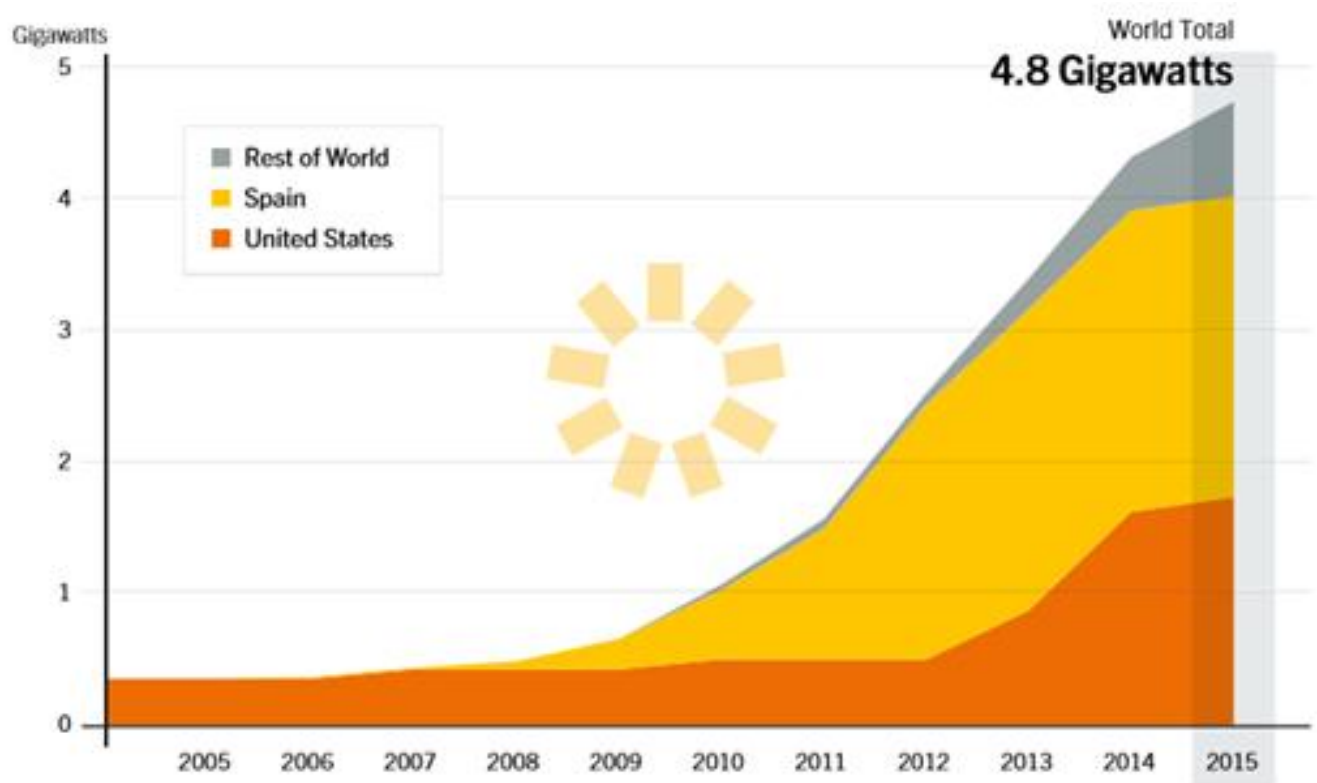

Figura 10. Capacidad Global de potencia termosolar por países en el Mundo (GW) [3].

Como se indica en la tabla 1, la primera planta termosolar con colectores PTC fue construida en el desierto de Mojave en California U.S., en 1984. Desde entonces la potencia se ha incrementado considerablemente como muestra la figura 10. En relación a las plantas con 
colectores lineales, se están construyendo plantas con mayor potencia (250MWe): proyecto Genesis [55] y el proyecto Mojave [56], ambas en Estados Unidos y sin almacenamiento térmico.

Tabla 1. Principales características técnicas de las plantas termosolares SEGS [54].

\begin{tabular}{lllcccc}
\hline \multirow{2}{*}{ Planta } & \multirow{2}{*}{$\begin{array}{c}\text { Año } \\
\text { construcción }\end{array}$} & Localización & $\begin{array}{c}\text { Capacidad } \\
\text { Neta }\end{array}$ & $\begin{array}{c}\text { Capacidad } \\
\text { Bruta }\end{array}$ & Superficie & $\begin{array}{c}\text { Temperatura } \\
\text { Aceite }\end{array}$ \\
\cline { 4 - 7 } & & $(\mathrm{MW})$ & $(\mathrm{MW})$ & $\left(\mathrm{m}^{2}\right)$ & $\left({ }^{\circ} \mathrm{C}\right)$ \\
\hline SEGS I & 1984 & Dagget & 14 & 14 & 82960 & 307 \\
\hline SEGS II & 1985 & Dagget & 30 & 33 & 190338 & 316 \\
\hline SEGS III & 1986 & Kramer Jct. & 30 & 33 & 230300 & 349 \\
\hline SEGS IV & 1986 & Kramer Jct. & 30 & 33 & 230300 & 349 \\
\hline SEGS V & 1987 & Kramer Jct. & 30 & 33 & 250500 & 349 \\
\hline SEGS VI & 1988 & Kramer Jct. & 30 & 35 & 188000 & 390 \\
\hline SEGS VII & 1988 & Kramer Jct. & 30 & 35 & 194280 & 390 \\
\hline SEGS VIII & 1989 & Harper Lake & 80 & 89 & 464340 & 390 \\
\hline SEGS IX & 1990 & Harper Lake & 80 & 89 & 483960 & 390 \\
\hline Total & & & 354 & 394 & 2314978 & \\
\hline
\end{tabular}

Tabla 2. Producción Anual de las plantas termosolares SEGS [54].

\begin{tabular}{llllllr}
\hline \multirow{2}{*}{ Planta } & \multicolumn{5}{c}{ Producción Anual Bruta (MWh) } \\
\cline { 2 - 7 } & 1985 & 1986 & 1987 & 1988 & 1989 & 1990 \\
\hline SEGS I & 19261 & 22510 & 25055 & 16927 & 23527 & 21491 \\
\hline SEGS II & & 25085 & 23431 & 38914 & 43862 & 39156 \\
\hline SEGS III & & 49444 & 61475 & 63096 & 69410 \\
\hline SEGS IV & & 52181 & 64762 & 70552 & 74661 \\
\hline SEGS V & & & 62858 & 65280 & 72449 \\
\hline SEGS VI & & & & 48045 & 62690 \\
\hline SEGS VII & & & & 38868 & 57661 \\
\hline SEGS VIII & & & & & 114996 \\
\hline SEGS IX & & & & & 5974 \\
\hline Total & 19261 & 47595 & 150111 & 244936 & 353230 & 518488 \\
\hline
\end{tabular}

Además de los proyectos más representativos ya citados, otras instalaciones termosolares con colectores lineales de interés se resumen en la tabla 4. Actualmente en España se cuenta con 50 centrales en operación que suman $2.300 \mathrm{MW}$ de potencia, siendo nuestro país el mercado con mayor capacidad operativa del mundo [57].

Tabla 3. Potencia eléctrica Neta producida (MWh) en las plantas termosolares SEGS [54].

\begin{tabular}{lll}
\hline Planta & $\begin{array}{l}\text { Año } \\
\mathbf{2 0 1 5} \\
\text { (MWh) }\end{array}$ & $\begin{array}{l}\text { Total } \\
\mathbf{1 9 8 5 - 2 0 1 5} \\
\text { (MWh) }\end{array}$ \\
\hline SEGS I & 12562 & 442543 \\
\hline SEGS II & & 752862 \\
\hline SEGS III & 52073 & 1760550 \\
\hline SEGS IV & 53117 & 1787814 \\
\hline SEGS V & 52646 & 2747851 \\
\hline
\end{tabular}




\begin{tabular}{lll}
\hline Planta & $\begin{array}{l}\text { Año } \\
\mathbf{2 0 1 5} \\
(\mathrm{MWh})\end{array}$ & $\begin{array}{l}\text { Total } \\
\mathbf{1 9 8 5 - 2 0 1 5} \\
\text { (MWh) }\end{array}$ \\
\hline SEGS VI & 46937 & 1651282 \\
\hline SEGS VII & 37771 & 1551591 \\
\hline SEGS VIII & 138149 & 2465981 \\
\hline SEGS IX & 145863 & 3352060 \\
\hline Total & 539118 & 16512534 \\
\hline
\end{tabular}

Tabla 4. Plantas termosolares con colectores lineales PTC o LF y ciclo de potencia Rankine.

\begin{tabular}{llll}
\hline Localización & Nombre & $\begin{array}{l}\text { Potencia } \\
\text { (Mwe) }\end{array}$ & TES \\
\hline $\begin{array}{llll}\text { Emiratos } \\
\text { Árabes Unidos }\end{array}$ & Shams 1 [58] & 100 & No \\
\hline India & Godawari [59] & 50 & No \\
\hline India & Diwakar [60] & 100 & Si \\
\hline India & Megha [61] & 50 & No \\
\hline India & Gujurat Solar One [62] & 28 & $\mathrm{Si}$ \\
\hline India & KVK Energy Solar [63] & 100 & $\mathrm{Si}$ \\
\hline Marruecos & Noor I [64] & 160 & $\mathrm{Si}$ \\
\hline Sudáfrica & KaXu [65] & 100 & $\mathrm{Si}$ \\
\hline Sudáfrica & Bokpoort [66] & 54.5 & $\mathrm{Si}$ \\
\hline
\end{tabular}

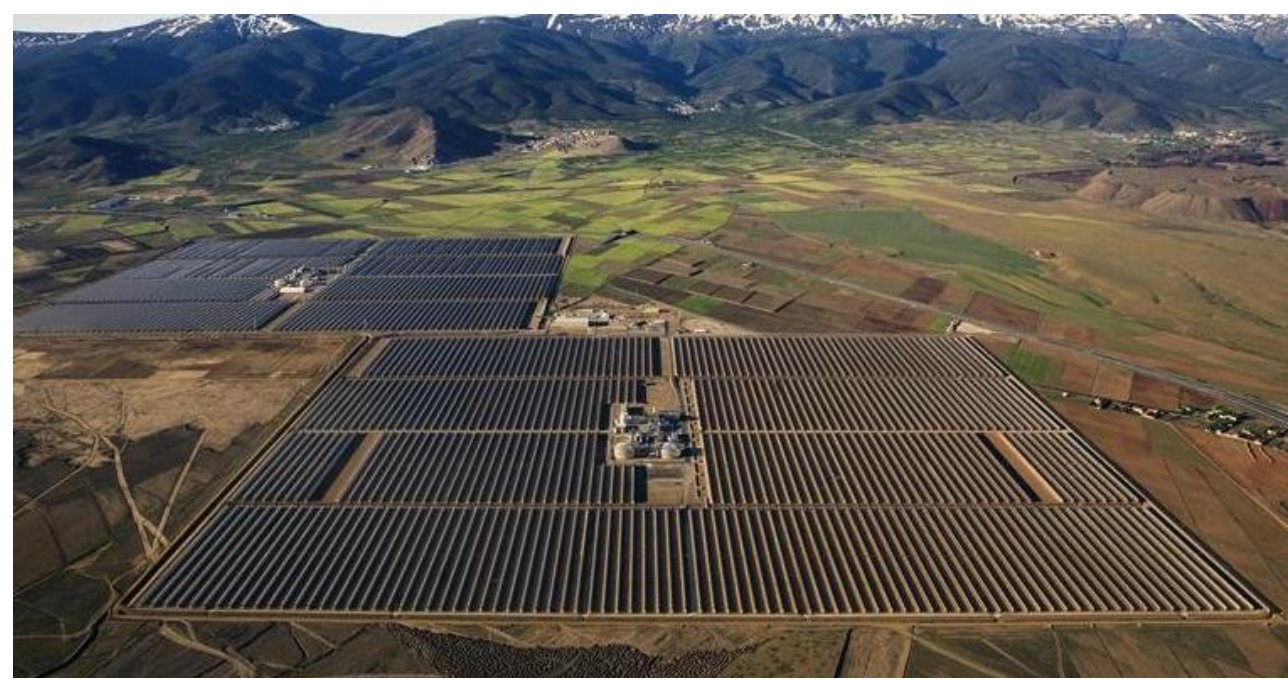

Figura 11. Vista aérea de las plantas termosolares Andasol 1 y 2, con colectores PTC [50].

\section{La Iniciativa SUNSHOT}

El programa SunShot está liderado por la Oficina de Eficiencia Energética y Energía Renovable, Office of Energy Efficiency and Renewable Energy, en el seno del Ministerio de Energía de los Estados Unidos de América, U.S. Department of Energy. El programa SunShot fue anunciado en Febrero del 2011, y se desarrolla con un equipo de trabajo que comprende cinco áreas: energía fotovoltaica (PV), energía termosolar (CSP), soft costs (coste cadena proveedores, coste instalación, impuestos, permisos, etc), integración de sistemas, y difusión tecnológica hacia el mercado. El objetivo de la iniciativa SunShot [67] es conseguir que la energía solar sea 
económicamente competitiva con las fuentes tradicionales de energía, antes del final de esta década (año 2020). Su principal meta es reducir el coste de la energía solar a $0.06 \$$ por kWh o $\$ 1$ por watio (no incluyendo los incentivos), figuras 12 y 13.

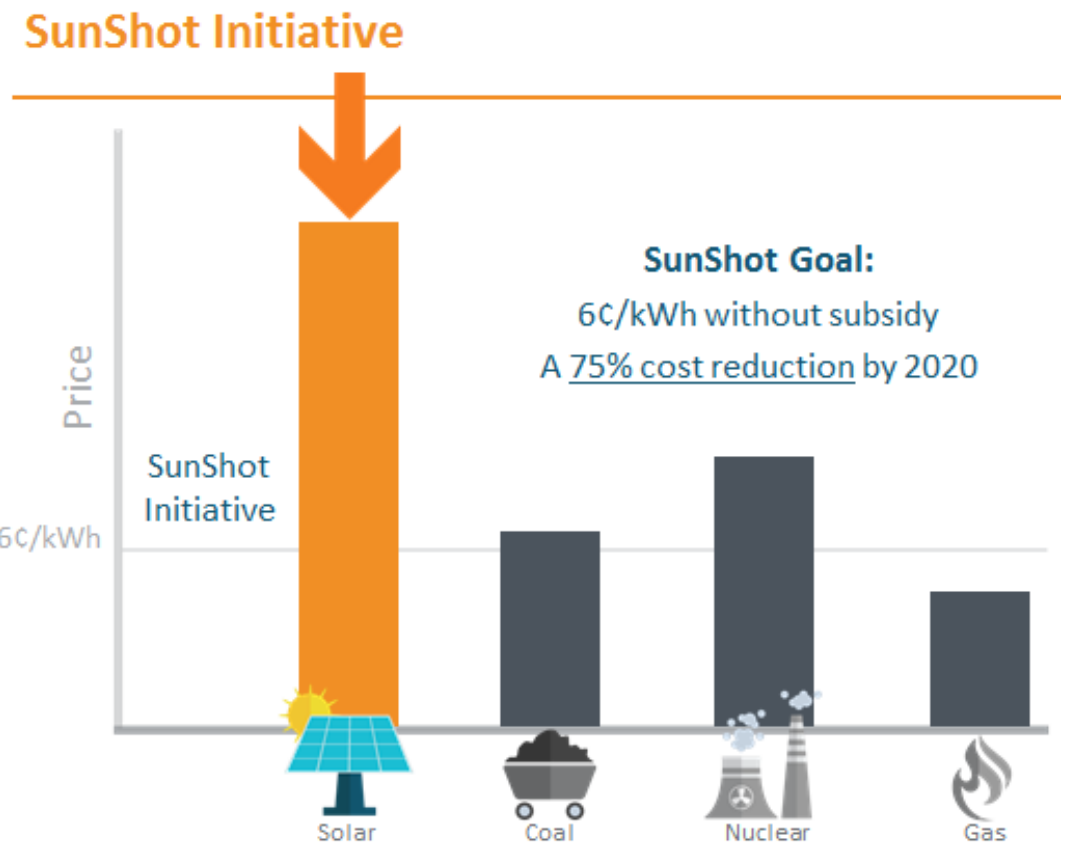

Figura 12. Representación gráfica del principal objetivo de la iniciativa SunShot, reducir el coste unitario de le energía generada en la planta termosolar a $6 c \$ / k W h$ [67].

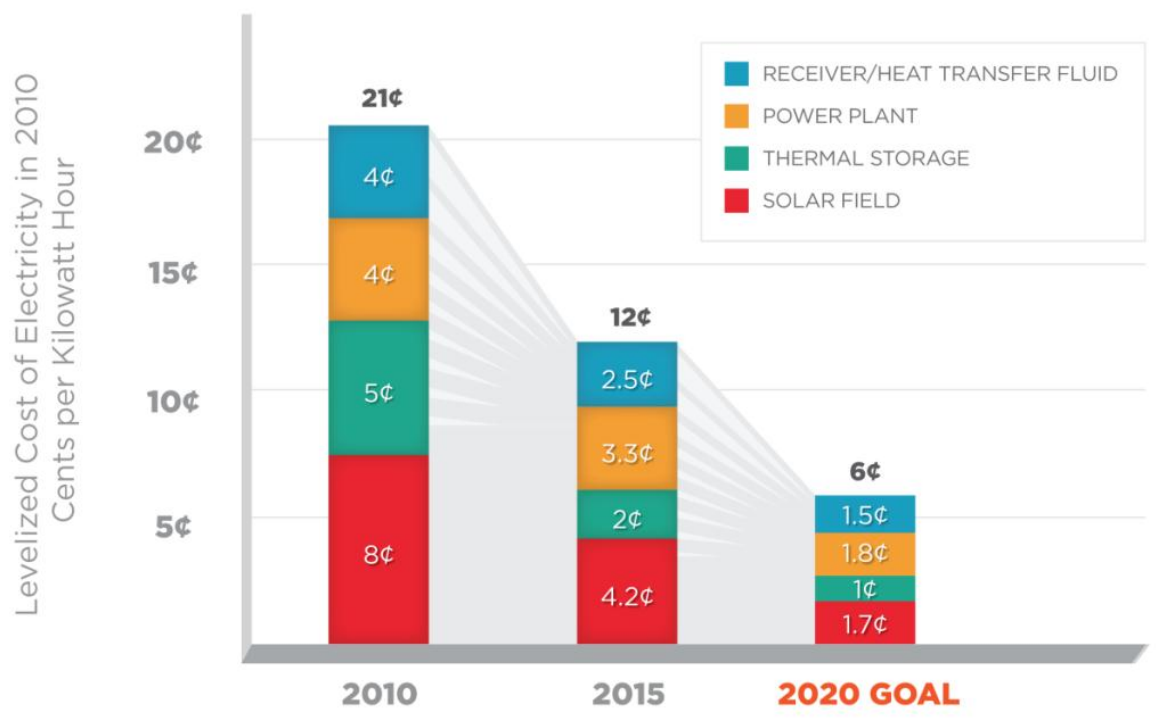

Figura 13. Desglose de los objetivos de coste en las plantas termosolares, SunShot [67].

La iniciativa Sunshot impartió recientemente en Abril 19-21 2016 una cumbre [68] en la ciudad de San Diego para informar, compartir e inspirar a los participantes sobre los avances tecnológicos, mercados e innovaciones de energía solar. Las principales líneas de investigación de la cumbre SunShot 2016 son:

- Desarrollo de los ciclos de potencia supercríticos s- $\mathrm{CO}_{2}$ Brayton para su integración en las plantas termosolares [69-78]. 
- Investigación en innovadores fluidos caloportadores HTFs (metales líquidos LeadBismuth-Eutectic (LBE), Oxy-halide Eutectic, etc) para operar a más altas temperaturas e incrementar el rendimiento energético de la planta termosolar [79-80].

- Desarrollo de innovadores sistemas de almacenamiento térmico TES en las plantas CSP, y evaluación de sus beneficios frente a otras tecnologías alternativas como la solar fotovoltaica con baterías [81-85].

- Reducción del coste de los colectores solare PTC y LF. Desarrollo de tubos absorbedores con mayore temperaturas de operación y optimización de las pérdidas térmicas [86-92].

- Recopilación de las lecciones aprendidas de los principales proyectos de plantas termosolares implantadas en Estados Unidos [93]: Solana (280 MWe) desarrollado por Abengoa, Ivanpah desarrollado por BrightSource (392 MWe), Genesis (250 MWe) desarrollado por NextEra, Crescent Dunes (110 MWe) desarrollado por SolarReserve, Mojave (280 MWe) desarrollado por Abengoa, en total $1312 \mathrm{MWe}$ instalados de CSP en Estados Unidos en los últimos 3 años [94-95].

- Nuevos materiales y recubrimientos resistentes a mayores temperaturas de operación [96-97].

- Desarrollo de innovadores diseños de torres centrales para CSP [98-100].

\section{Objetivos de la tesis}

Atendiendo a los párrafos previos, se ha detectado la necesidad del estudio de la integración de plantas termosolares con colectores lineales, tanto PTC como LF, con las tecnologías pioneras en el campo de la generación de potencia, es decir, con ciclos termodinámicos de potencia con fluidos en estado supercrítico, tanto de tipología Rankine, con agua como fluido caloportador, como con ciclos Brayton con Dióxido de Carbono como fluido caloportador, entre otros. Para llevar a la práctica el objetivo anteriormente propuesto se han desarrollado un trabajo que puede ser organizado en los siguientes capítulos:

- El capítulo primero incluye una recapitulación del estado del arte de las principales tecnologías en el campo de la energía termosolar con colectores solares lineales, tanto PTC como LF.

- El capítulo segundo describe la metodología seguida para el desarrollo de la presente tesis doctoral. Se incluye la definción de los principales fenómenos físicos y ecuaciones que rigen el balance energético de las plantas termosolares. Se incluye los diagramas de flujo de los algoritmos de cálculo de los principales equipos de las CSP.

- En capítulo tercero se realiza una definición del sistema de recalentamiento directo Direct ReHeating (DRH) en las plantas termosolares con colectores lineales con DSG y ciclos de potencia Rankine.

- En el cuarto capítulo integración de los campos solares con colectores lineales, con MS o DSG como fluidos caloportadores (HTF), y los ciclos de potencia Supercríticos Rankine. Y su consiguiente comparación con ciclos Subcríticos de Agua con Recalentamiento Directo y DSG en los colectores solares.

- Los capítulos quinto, sexto y séptimo, tienen como principal objetivo la integración de los campo solares con colectores lineales y MS o aceites térmicos como fluidos caloportadores, con los ciclos de potencia Supercríticos con Dióxido de Carbono (s$\mathrm{CO}_{2}$ ) de tipología Brayton, mediante intercambiadores de calor tipo Carcasa y Tubos. 
- En el capítulo octavo se definen cuatro configuraciones de plantas termosolares para la integración entre los colectores lineales con DSG y los ciclos Supercríticos Brayton.

- El noveno capítulo estudia la integración de los campos solares de tipología Dual-Loop, con combinación de dos fluidos calorportadores HTF en una misma planta termosolar, y los ciclos de potencia s- $\mathrm{CO}_{2}$ Brayton.

- El décimo capítulo define los nuevos sistemas de generación de potencia con Etano en estado supercrítico como fluido de trabajo en el ciclo Brayton para plantas termosolares con colectores lineales y su comparación con ciclos Brayton con Dióxido de Carbono $\left(\mathrm{s}-\mathrm{CO}_{2}\right)$ y con plantas con colectores con DSG y ciclos Rankine.

- En el undécimo, se realiza una comparación de la eficiencia y producción energéticas de las plantas termosolares con colectores lineales, y ciclos supercríticos Brayton, con diferentes fluidos de trabajo: Etano, Xenón, Hexafluoruro de Azufre, Nitrógeno, Metano y Dióxido de Carbono.

- El duodécimo capítulo describe el interface gráfico, Graphical User Interface (GUI), y las capacidades de programa informático desarrollado ad hoc en esta tesis doctoral, para el diseño y optimización de los parámetros de operación, de las plantas termosolares con colectores lineales (PTC o LF), y ciclos Brayton con el fluido de trabajo en estado supercrítico. El software fue desarrollado con los lengujes C\#, Fortran, y es también una herramienta informática para diseño y dimensionamiento de los equipos y componentes que integran la instalación termosolar. La verificación de dicha herramienta informática se ha realizado con el programa comercial Thermoflow.

- Finalmente el capítulo de conclusiones y trabajos futuros incluye una recopilación de los principales resultados obtenidos durante el desarrollo de la tesis. Se incluye en este capítulo las principales soluciones tecnológicas propuestas para integrar los ciclos supercríticos de potencia en las plantas termosolares con colectores lineales. Se proponen diferentes trabajos de análisis e investigación para poder materializar a escala industrial las plantas termosolares de nueva generación con ciclos de potencia con fluidos de trabajo en estado supercrítico.

\section{Referencias}

[1] Six degrees could change the world. National Geo. Channel. http://www.natgeotv.com

[2] NASA, Global Climate Change, Vital Signs of the Planet. http://climate.nasa.gov/

[3] Renewables 2016 Global Status Report. http://www.ren21.net

[4] Technology Roadmap. Solar Thermal Electricity. 2014 Edition. https://www.iea.org

[5] Red Eléctrica de España. http://www.ree.es/es/

[6] Concentrating Solar Power Roadmap. OECD/IEA, 2010. https://www.iea.org

[7] Gilles Flamant, CNRS-PROMES. Introduction to solar reactors. SFERA II 2014-2017, Summer School, June 25-27 2014.

[8] Ivanpah project, BrigthSource. http://www.brightsourceenergy.com

[9] Gemasolar project.Torresol Energy, SENER. http://www.torresolenergy.com

[10] Crescent Dunes project. SolarReserve. http://www.solarreserve.com

[11] Puerto Errado 2. Novatec Solar. http://www.novatecsolar.com/56-1-PE-2.html

[12] Reliance Solar Thermal Power Plant Project, Dhursar. Areva Solar. http://india.areva.com

[13] Maricopa Solar Project and Tooele Army Depot Solar Project. http://www.nrel.gov

[14] J.F. Feldhoff, Analysis and Potential of Once-Through Steam Generators in Line Focus Systems - Final Results of the DUKE Project. 
[15] A. Khenissi, D. Krüger, T. Hirsch, K. Hennecke. Return of Experience on Transient Behavior at the DSG Solar Thermal Power Plant in Kanchanaburi, Thailand. Energy Procedia, Volume 69, May 2015, Pages 1603-1612.

[16] P.Garcia, V.Vuillerme, M.Olcese, N.El Mourchid. Design and modelling of an innovative three-stage termal storage system for direct steam generation CSP plants. AIP Conference Proceedings 1734, 050015 (2016). SolarPACES 2015.

[17] M. Seitz, S.Hübner, M.Johnson. Detailed partial load investigation of a thermal energy storage concept for solar thermal power plants with direct steam generation. AIP Conference Proceedings 1734, 050042 (2016). SolarPACES 2015.

[18] Monne C., Bravo Y., Moreno F., Muñoz M.. Analysis of a solar dish-stirling system with hybridization and thermal storage. Int. J. of En. and Env. Eng., 2014, 5:2, pp. 1 to 5.

[19] M.Eickhoff, M. Grünefeldt, L. Keller. New operating strategies for molten salt in line focusing solar fields - daily drainage and solar receiver preheating. AIP Conference Proceedings 1734, 070007 (2016). SolarPACES 2015.

[20] W.Gaggioli, P.Ascenzi, L.Rinaldi, P.Tarquini, F.Fabrizi. Effects assesment of 10 functioning years on the main componentes of the molten salt PCS experimental facility of ENEA. AIP Conference Proceedings 1734, 070009 (2016). SolarPACES 2015.

[21] E.Hakkarainen, M.Tähtinen. Dynamic modelling and simulation of linear Fresnel solar field model based on molten salt heat transfer fluid. AIP Conference Proceedings 1734, 070014 (2016). SolarPACES 2015.

[22] F.Sallaberry et al. Standards for components in CSP - status of the Spanish working group. AIP Conference Proceedings 1734, 110003 (2016). SolarPACES 2015.

[23] F.von Reeken, G.Weinrebe, T.Keck, M.Balz. Heliostat cost optimization study. AIP Conference Proceedings 1734, 160018 (2016). SolarPACES 2015.

[24] A. Stollo, T. Chiarappa, A. D'Angelo, A. Maccari, F. Matino. LCOE reduction for parabolic trough CSP: Innovative solar receiver with improved performance at medium temperatura. AIP Conference Proceedings 1734, 030034 (2016). SolarPACES 2015.

[25] J.E.Pacheco, T.Wolf, N.Muley. Incorporating Supercritical Steam Turbines into Advanced Molten-Salt Power Tower Plants: Feasibility and Performance. SAND2013-1960. Unlimited release, printed March 2013.

[26] A. Green, C. Diep, R. Dunn, J. Dent. High capacity factor CSP-PV hybrid systems. Energy Procedia 69 ( 2015 ) 2049 - 2059. SolarPACES 2014.

[27] Akesai Solar Thermal power plant (ASTPP). Achimede Solar Energy (ASE).

[28] S. Dieckmann et al. Integration of Solar Process Heat into an Existing Thermal Desalination Plant in Qatar. SolarPACES 2015.

[29] A. del Río et al. Soltrec-Pressurized Volumetric Solar Air Receiver Technology. Energy Procedia, Volume 69, May 2015, Pages 360-368. SolarPACES 2014.

[30] L.Coco Enríquez, J. Muñoz-Antón, J.M. Martínez-Val. Integration between direct steam generation in linear solar collectors and supercritical carbon dioxide Brayton power cycles. International Journal of Hydrogen Energy, 40:44, 2015, pp.15284, 15300.

[31] L.Coco-Enríquez, J.Muñoz-Antón, J.M.Martínez-Val Peñalosa. S-Ethane Brayton Power Conversion Systems for CSP. ASME J.Sol.En.Eng. 138(1), 011012 (December 29, 2015).

[32] S.A. Zavattoni et al. A Novel CSP Receiver Based on Airlight Energy Technology Optimization of the Thermal Insulation System by Means of CFD Analysis. Energy Procedia, Volume 49, 2014, Pages 589-598. SolarPACES 2013.

[33] M.Biencinto, L.González, E.Zarza, L.E. Díez, J.Muñoz-Antón, Performance model and annual yield comparison of parabolic-trough solar thermal power plants with either nitrogen or synthetic oil as heat transfer fluid, En.Conversion and Management, 87, 2014, pp.238-249.

[34] J. Muñoz-Anton, M. Biencinto, E. Zarza, L.E. Díez, Theoretical basis and experimental facility for parabolic trough collectors at high temperature using gas as heat transfer fluid, Applied Energy, Volume 135, 15 December 2014, Pages 373-381. 
[35] S.M. Sohel Murshed, C.A. Nieto de Castro. Superior thermal features of carbon nanotubesbased nanofluids - A review. Renewable and Sustainable Energy Reviews, Volume 37, September 2014, Pages 155-167.

[36] A. Fritsch, J.Flesch, V.Geza, Cs.Singer, R.Uhlig, B. Hoffschmidt. Conceptual Study of Central Receiver Systems with Liquid Metals as Efficient Heat Transfer Fluids. Energy Procedia, Volume 69, May 2015, Pages 644-653. SolarPACES 2014.

[37] Nikola Lorenzin, Alberto Abánades. A review on the application of liquid metals as heat transfer fluid on Concentrated Solar Power technologies. International Journal of Hydrogen Energy, Volume 41, Issue 17, May 2016, pages 6990-6995.

[38] Kenneth M. Armijo, Clifford Ho, Ryan Anderson, Joshua Chirstian, Sean Babiniec, Jesus Ortega. Magnetic field flow phenomena in a falling particle receiver. AIP Conference Proceedings 1734, 030004 (2016). SolarPACES 2015.

[39] Joshua Christian, Clifford Ho. Design requirements, challenges, and solutions for hightemperature falling particle receivers. AIP Conference Proceedings 1734, 030004 (2016). SolarPACES 2015.

[40] Ivan Ermanoski, Johannes Grobbel, Abhishek Singh, Justing Lapp, Stefan Brendelberger, Martin Roeb, Christian Sattler, Josh Whaley, Anthony McDaniel, Nathan P. Siegel. Design and Construction of a Cascading Pressure Reactor Prototype for Solar-Thermochemical Hydrogen Production. AIP Conference Proceedings 1734, 120001 (2016). SolarPACES 2015.

[41] Raffaele Liberatore, Michela Lanchi, Luca Turchetti. Hydrogen Production by the Solarpowered Hybrid Sulfur Process: Analysis of the Integration of the CSP and Chemical Plants in Selected Scenarios. AIP Conference Proceedings. SolarPACES 2015.

[42] Martin Roeb, Aldo Steinfeld, Günter Borchardt, Claus Feldmann, Martin Schmücker, Christian Sattler, Robert Pitz-Paal. SolarSyngas: Results from a virtual institute developing materials and key components for solar thermochemical fuel production. AIP Conference Proceedings 1734, 120007 (2016). SolarPACES 2015.

[43] Antonio Colmenar-Santos, José-Luis Bonilla-Gómez, David Borge-Diez, Manuel Castro-Gil. Hybridization of concentrated solar power plants with biogas production systems as an alternative to premiums: The case of Spain. Renewable and Sustainable Energy Reviews 47 (2015) 186-197.

[44] Abdullah A., AlZahrani, Ibrahim Dincer. Design and analysis of a solar tower based integrated system using high temperatura electrolyzer for hydrogen production. International Journal of Hydrogen Energy, Volume 41, Issue 19, 25 May 2016, Pages 8042-8056. Special Issue on Progress in Hydrogen Production and Applications (ICH2P-2015), 3-6 May 2015, Oshawa, Ontario, Canada.

[45] Javier Sanz-Bermejo, Javier Muñoz-Antón, José González-Aguilar, Manuel Romero. Part Load operation of a solid oxide electrolysis system for integration with renewable energy source. International Journal of Hydrogen Energy, Volume 40, Issue 26, 13 July 2015, pages 8291-8303.

[46] Sadi Carnot. Réflexions sur la puissance motrice du feu et sur les machines propes à developer ate puissance. https://books.google.es/books?id=QX9ilWF3yOMC\&redir esc=y

[47] Tardieu Alaphilippe, M. (2007), Recherche d'un Nouveau Procede de Conversion Thermodynamique de l'Energie Solaire, en Vue de son Application a la Cogeneration de Petite Puissance, dissertation presented to the I'Universite de Pau et des Pays de l'Adour, Pau, France.

[48] "TSE 1" Kanchanaburi solar power plant, Tailandia. Solarlite Project.

http://solarlite-csp.com/en/reference/tse-1-kanchanaburi-provinz-thailand/

[49] Frank Lenzen. SCHOTT Solar CSP. IEA CSP Workshop. 03-03-2014.

https://www.iea.org

[50] System Advisor Model (SAM) Case Study: Andasol-1. NREL https://sam.nrel.gov

[51] AREVA Compact Linear Fresnel Reflector (CLFR) in SNL. http://us.areva.com 
[52] M.Lotker. Barriers to commercialization of large-scale solar electricity: lessons learned from the LUZ experience. Sandia National Laboraoty, USA. SAND91-1974. November 1991.

http://www.osti.gov/scitech/servlets/purl/10108287

[53] (Jan) Fabian Feldhoff. Direct Steam Generation (DSG). Technology Overview. SFERA Summer School 2012, June 28, 2012, Almería, Spain.

[54] Solar Energy Generating Systems (SEGS). http://www.energy.ca.gov/sitingcases/solar/

[55] Genesis Solar Energy Project. http://www.energy.ca.gov/sitingcases/genesis solar/

[56] Mojave Solar power plant, Abengoa Solar. http://www.abengoasolar.com

[57] Protermosolar www.protermosolar.com

[58] Shams 1 solar power plant. http://shamspower.ae/en/

[59] Godawari solar power plant . http://www.nrel.gov

[60] Diwakar solar power plant. http://www.nrel.gov

[61] Megha solar power plant. http://www.meil.in/index.html

[62] Gujurat Solar One power plant. http://www.nrel.gov

[63] KVK Energy Solar. http://www.kvkenergy.com/business ct.html

[64] Noor I solar power plant. http://acwapower.com/project/noor-1-csp-ipp/

[65] KaXu Solar One solar power plant, Abengoa Solar.

http://www.abengoasolar.com/web/es/plantas solares/plantas propias/sudafrica/

[66] Bokpoort solar power plant.

http://www.acciona.com/emblematic-projects/bokpoort-concentrating-solar-power-plant/

[67] SunShot Initiative. http://energy.gov/eere/sunshot/sunshot-initiative

[68] SunShot CSP Summit and Integration Workshop 2016.

http://energy.gov/eere/sunshot/2016-sunshot-csp-summit-and-integration-workshop

[69] Craig Turchi. Supercritical CO2 Power Cycles: Next-Gen Power for CSP?. 2016 SunShot CSP Summit and Integration Workshop. U.S. Department of Energy.

[70] Shaun Sullivan, Ragaiy Zidan. Brayton Energy and Savannah River National Lab. HighEfficiency low-cost solar receiver for use in a supercritical $\mathrm{CO} 2$ recompression cycle. Low-cost metal hydride thermal energy storage system for CSP systems. Solar receiver with integrated thermal storage for a supercritical CO2 power cycle. 2016 SunShot CSP Summit. U.S. DOE.

[71] J.Jeffrey Moore. Development of a High Efficiency Hot Gas Turbo-Expander and Low Cost Heat Exchanger for Optimized CSP s-CO2 Operation. 2016 SunShot CSP Summit and Integration Workshop. U.S. Department of Energy.

[72] Mark Anderson. University of Wisconsin Madison. Advanced s-CO2 cycles. 2016 SunShot CSP Summit and Integration Workshop. U.S. Department of Energy.

[73] Craig Turchi. CSP Systems Analysis. 2016 SunShot CSP Summit and Integration Workshop. U.S. Department of Energy.

[74] A.Thatte. General Electric. An Integrated Coupled-Physics Framework for Performance and Life Prediction of Supercritical CO2 Turbomachines. 2016 SunShot CSP Workshop. U.S. DOE.

[75] J.Wilkes et al. Application of an Integrally Geared Compander (integrally-geared compressor-expander) to an s-CO2. 2016 SunShot CSP Summit. U.S. DOE.

[76] Jeff Moore, Azam Thatte. Apollo High-Efficiency s-CO2 Centrifugal Compressor Development. 2016 SunShot CSP Summit and Integration Workshop. U.S. DOE.

[77] K.H. Sandhage. Robust, Cost-Effective Heat Exchangers for $8000 \mathrm{C}$ Operation with supercritical CO2. 2016 SunShot CSP Summit Workshop. U.S. Department of Energy.

[78] Bruce Pint. Lifetime Model Development for Supercritical CO2, CSP Systems. . 2016 SunShot CSP Summit and Integration Workshop. U.S. Department of Energy.

[79] P.W.Li et al. Halide and Oxy-halide Eutectic Systems for high performance High Temperatura Heat Transfer Fluids. 2016 SunShot CSP Summit Workshop. U.S. DOE.

[80] M.Asta et al. High-operating temperature heat-transfer fluids for solar thermal power generation (Liquid Metals). . 2016 SunShot CSP Summit and Integration Workshop. U.S. DOE.

[81] Mark S. Mehos. Beyond LCOE: The Value of CSP with Thermal Energy Storage. 2016 SunShot CSP Summit and Integration Workshop. U.S. Department of Energy. 
Introducción

[82] B.Gould. SolarReserve, The Value of CSP with Storage and Cost Reduction opportunities. 2016 SunShot CSP Summit and Integration Workshop. U.S. Department of Energy.

[83] A.Lavine. Thermochemical Storage with Anhydrous Ammonia. 2016 SunShot CSP Summit and Integration Workshop. U.S. Department of Energy.

[84] A.Ambrosini. High Performance Reduction/Oxidation Metal Oxides for Thermochemical Energy Storage (PROMOTES). 2016 SunShot CSP Summit and Integration Workshop. U.S. DOE.

[85] S.Gangwal, A.Muto. Demonstration of High-Temperature Calcium-Based Thermochemical TES for Use with Concentrating Solar Power Facilities. 2016 SunShot CSP Workshop. U.S. DOE.

[86] J.Stettenheim. Norwich Technologies. Advanced low cost receiver for parabolic trough solar power. Design for manufacturing. 2016 SunShot CSP Workshop. U.S. DOE.

[87] S.Obrey et al. High Temperature Heat Pipe Receiver for Parabolic Trough Collectors. 2016 SunShot CSP Summit and Integration Workshop. U.S. Department of Energy.

[88] K.C.Toussaint, L.J. Guo, G.Zhu. Development of a plannar focusing collector for CSP. 2016 SunShot CSP Summit and Integration Workshop. U.S. Department of Energy.

[89] G.Mungas. Hyperlight Energy. Low Cost Concentrated Solar Power (CSP) Collector. 2016 SunShot CSP Summit and Integration Workshop. U.S. Department of Energy.

[90] P.Gleckman, Sunvapor Inc. Green Parabolic Trough Collector (GPTC). 2016 SunShot CSP Summit and Integration Workshop. U.S. Department of Energy.

[91] G.Mungas, Hyperlight Energy. Low Cost Concentrated Solar Power (CSP) Collector. 2016 SunShot CSP Summit and Integration Workshop. U.S. Department of Energy.

[92] G.Glatzmaier. Hydrogen Mitigation Project. 2016 SunShot CSP Summit and Integration Workshop. U.S. Department of Energy.

[93] Concentrating Solar Power Projects by Technology. http://www.nrel.gov

[94] Joseph Desmond. BrigthSource Limitless. 2016 SunShot CSP Summit and Integration Workshop. U.S. Department of Energy.

[95] H.Price, Agengoa Solar. Lessons Learned from Solana: The Worlds largest parabolic trough solar power plant. 280 MWe with 6 hours of TES. 2016 SunShot CSP Workshop. U.S. DOE.

[96] J.Gomez, M.Anderson. Degradation Mechanisms and Development of Protective Coatings for TES and HTF Containment Materials. 2016 SunShot CSP Workshop. U.S. DOE.

[97] B.L. Garcia-Diaz et al. High Temperature Molten Salt Corrosion in CSP Systems. 2016 SunShot CSP Summit and Integration Workshop. U.S. Department of Energy.

[98] Michael Wagner. Direct s-CO2 Receiver Development. 2016 SunShot CSP Summit and Integration Workshop. U.S. Department of Energy.

[99] Clifford K. Ho. High-Temperature Falling Particle Receiver. 2016 SunShot CSP Summit and Integration Workshop. U.S. Department of Energy.

[100] Clifford K. Ho. High-Temperature Particle Heat Exchanger for s-CO2 Power Cycles. 2016 SunShot CSP Summit and Integration Workshop. U.S. Department of Energy. 
Capítulo 1

\title{
Estado de la técnica en las centrales termosolares con colectores solares lineales
}

\author{
1.1. Introducción \\ El sol constituye la principal fuente de energía renovable en la Tierra. Para captación y \\ concentración de la radiación solar se han ido diseñando con el paso de los años diferentes \\ tipologías de colectores solares, entre ellos los colectores solares lineales, tanto el colector \\ cilindro-parabólico Parabolic Trough Collector (PTC), como el colector Lineal Fresnel (LF), son \\ especialmente tratados en este trabajo, definiendo sus principales parámetros de operación, \\ sus características geométricas principales y su balance energético que condiciona el \\ funcionamiento como sistema de aprovechamiento energético.
}

En este capítulo se hace un recorrido bibliográfico centrado en las tecnologías PTC y LF, sin olvidar los sistemas de torre central y los discos parabólicos, atendiendo a los aspectos definitorios de cada una de estas tecnologías como son los diferentes fluidos caloportadores con los que trabajan las plantas comerciales actuales (principalmente aceites térmicos y sales fundidas) y los ciclos de potencia que utilizan para producir energía eléctrica.

En las primeras secciones de este capítulo se incluye una descripción de los dos tipos principales de colectores lineales, PTC y LF. En los siguientes apartados se incluye una caracterización tanto de la eficiencia energética como de la potencia generada y de la superficie de apertura efectiva de los colectores de planta termosolar con colectores lineales acoplada a ciclo Rankine con aceite térmico o MS como HTF. La tecnología Direct Steam Generation (DSG), con menor incorporación en el mercado, se explica con mayor grado de detalle en el capítulo 8.

\subsection{El Sol y la radiación solar como fuente energética}

La radiación emitida por el Sol que alcanza al planeta Tierra (radiación solar) constituye su principal fuente de energía. En cierto lapso de la evolución en el planeta Tierra, el Sol proporcionaba energía para todas las criaturas de la Tierra gracias al proceso de fotosíntesis, mediante el cual las plantas absorben la radiación solar y la convierten en energía para su crecimiento y desarrollo. El Sol se puede describir de forma somera como un gran reactor de fusión nuclear respecto a los proyectos que el hombre trata de desarrollar ( $7 \times 10^{5} \mathrm{~km}$ de radio) que emite una potencia radiante de alrededor de $3.86 \times 10^{23} \mathrm{~kW}$ a una temperatura equivalente de cuerpo negro de $5250^{\circ} \mathrm{C}$ [1] (figura 1.1). 
1. Estado de la técnica en las centrales termosolares con colectores solares lineales.

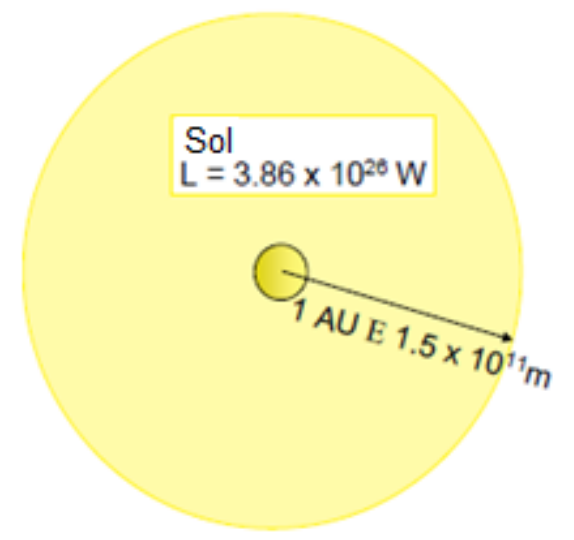

Figura 1.1. Ilustración del cálculo de la constante solar.

El principio de conservación de la energía requiere que la totalidad de la energía emitida por el Sol lo haga a través de la esfera de radio 1 Astronomical Unit (AU). La densidad del flujo energía a una distancia de 1 AU es:

$\frac{L}{4 \pi r^{2}}=1367 \frac{W}{m^{2}}$ (Definición de Constante Solar)

Al valor del flujo de energía solar radiante sobre una superficie situada en el exterior de la atmósfera y perpendicular al vector solar, cuando la Tierra se encuentra a su distancia media del Sol, se denomina Constante Solar. Normalmente se toma como valor de esta Constante Solar $1367 \mathrm{~W} / \mathrm{m}^{2}$, ver ecuación 1.1 , compuesta por un $50 \%$ de luz infrarroja, el $40 \%$ luz visible y el $10 \%$ por luz ultravioleta como puede deducirse de su espectro de radiación (figura 1.2).

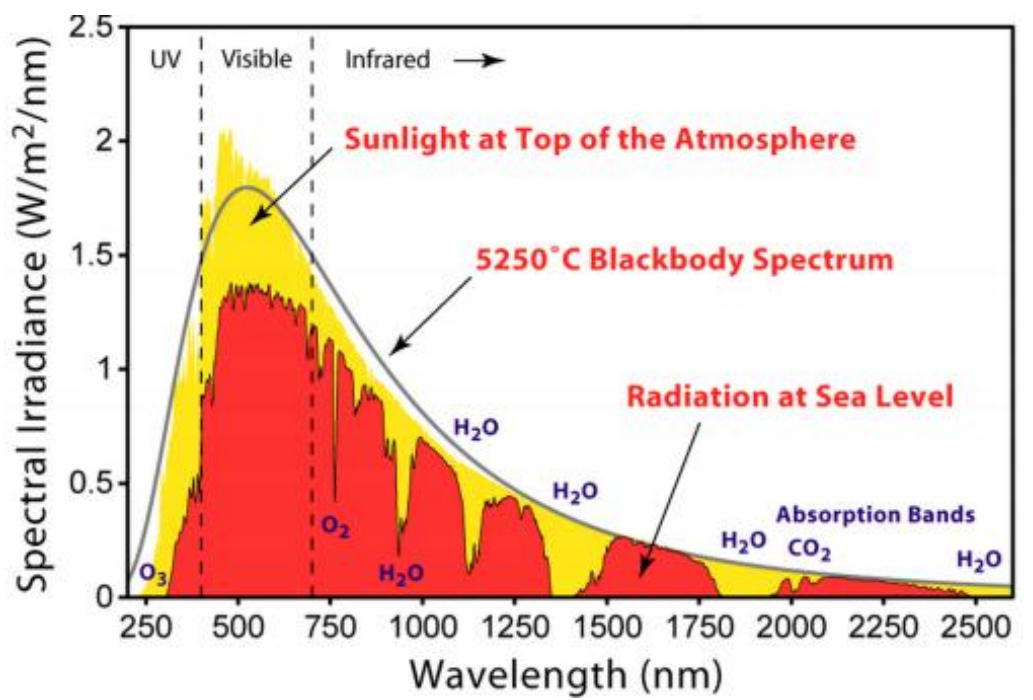

Figura 1.2. Representación del espectro de la radiación solar a nivel extraterrestres y a nivel de la superficie terrestre. También se compara dichos espectros con el espectro de emisión de un cuerpo negro a una temperatura de $5250^{\circ} \mathrm{C}$ (temperatura del Sol) [2].

La Tierra recibe 174000 TW de radiación solar incidente en la capa superior de la atmósfera terrestre. Aproximadamente el $30 \%$ de esta radiación es reflejada al espacio, mientras que el resto es absorbida por la nubes, los océanos y la tierra. 84000 TW llegan hasta los océanos y la 
tierra. El espectro de la luz solar en la superficie de la tierra se extiende entre diferentes longitudes de onda como se muestra en la figura 1.2.

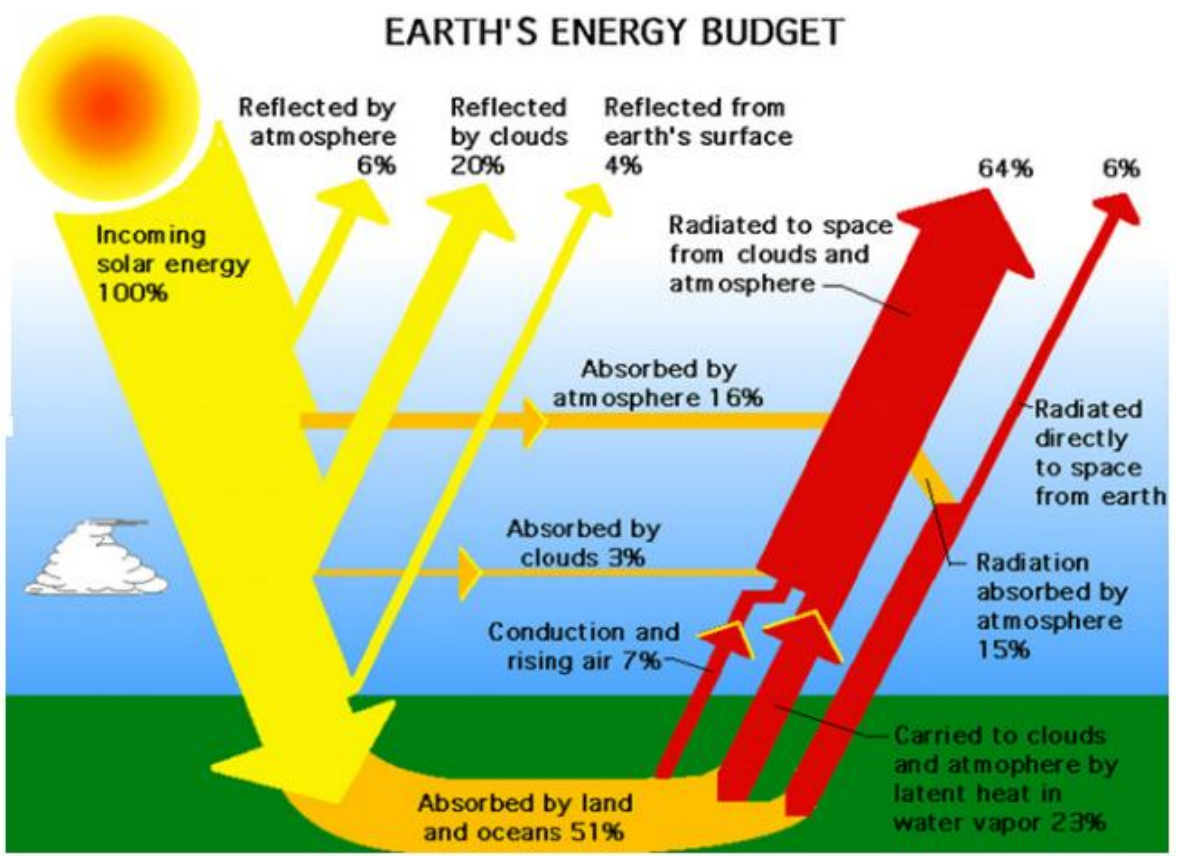

Figura 1.3. Balance Energético de la Tierra. Se representa gráficamente los flujos energéticos, tanto de la energía incidente sobre la Tierra procedente del Sol, la energía absorbida por la Tierra, y la energía que la Tierra emite al Espacio [3].

La radiación solar en su camino hacia la tierra interacción con la atmósfera sufriendo un proceso de absorción y otro de difusión (figura 1.3). A nivel del suelo la cantidad de energía decrece hasta alrededor de $1120-1000 \mathrm{~W} / \mathrm{m}^{2}$, estando compuesta por un $44 \%$ de luz visible, el $3 \%$ de luz ultravioleta y el resto de radiación dentro del espectro de infrarrojo. La radiación solar tiene dos componentes la radiación directa y la radiación difusa. Sólo la radiación directa puede ser concentrada mediante los colectores de las plantas termosolares. En la figura 1.4 se ilustra la distribución de la radiación solar directa incidente (DNI) en la superficie terrestre. Este parámetro es la variable de referencia a la hora de diseñar las plantas termosolares para generación de energía eléctrica.

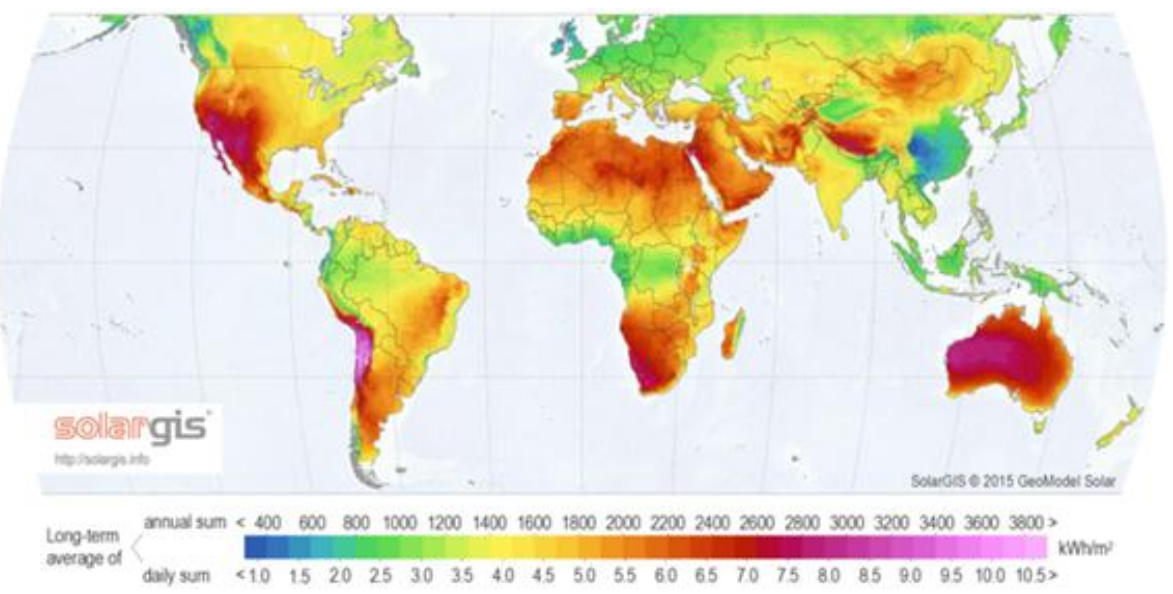

Figura 1.4. Mapa de la distribución de Irradiación Solar Directa DNI $\left(\mathrm{kWh} / \mathrm{m}^{2}\right)$ [3]. 
1. Estado de la técnica en las centrales termosolares con colectores solares lineales.

\subsection{Colectores Cilindro Parabólicos (PTC)}

\subsubsection{Introducción}

El colector solar cilindro parabólico consiste en un concentrador de sección parabólica (superficie especular) que refleja la radiación solar directa sobre la línea focal de la parábola, en la que se sitúa el receptor, que habitualmente se denomina tubo absorbedor. En la figura 1.5 se muestra la localización del tubo absorbedor en el Focal Point de la parábola del colector cilindro-parabólico. En la misma figura 1.5 se ilustra la sección transversal de un colector constituido por espejos con forma parabólica. Esta forma geométrica de los espejos permite focalizar la radiación solar hacia el punto focal de la parábola donde se localiza el tubo absorbedor. El eje de la parábola es la línea que une el punto focal con el vértice de la parábola. Se designa como distancia focal a la distancia entre el punto focal y el vértice de la parábola "A". La altura de la parábola es la distancia "B", y la distancia "C" es la longitud de apertura de la parábola. El área de apertura es el resultado de multiplicar la longitud " $\mathrm{C}$ " por la longitud del colector.

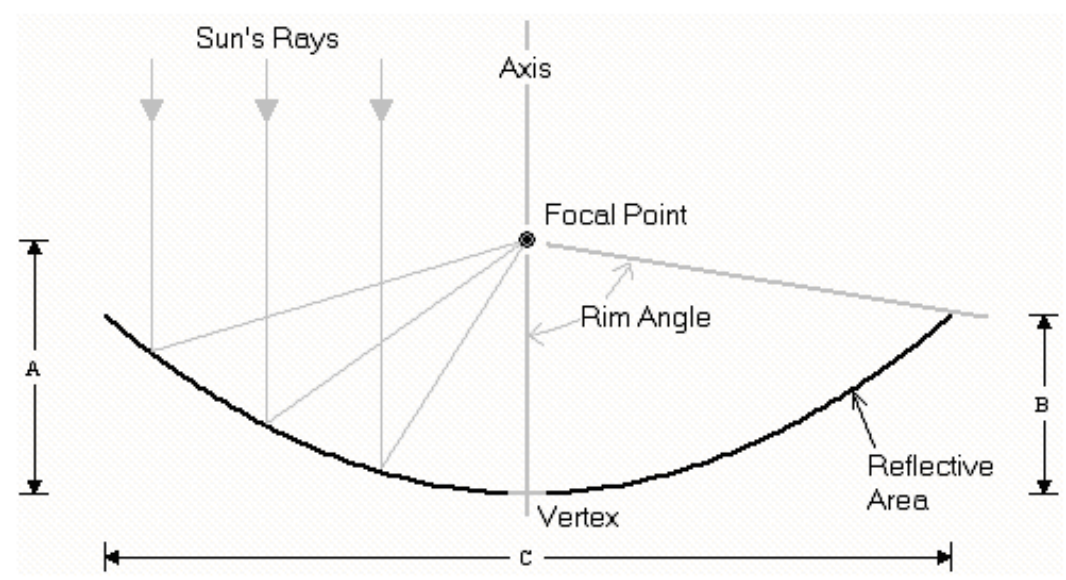

Figura 1.5. Sección transversal de un colector solar tipo cilindro parabólico (PTC) [5].

La forma geométrica de este colector figura 1.6, permite focalizar la radiación incidente en la parábola que es paralela a su eje y dirigirla hacia su foco donde se localiza el tubo absorbedor. Definimos en las figuras 1.7 y 1.8 , y en la tabla 1.1 los ángulos solares para caracterizar el comportamiento de los colectores solares parabólicos.

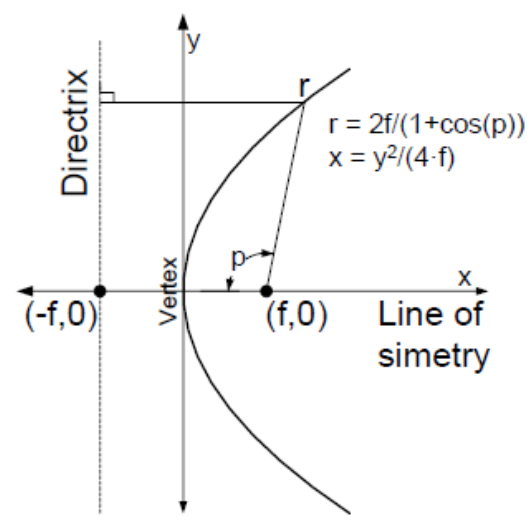

Figura 1.6. Relaciones geométricas que definen una parábola. Esta forma geométrica ha sido utilizada para el diseño de los espejos de los PTC [6]. 
1. Estado de la técnica en las centrales termosolares con colectores solares lineales.

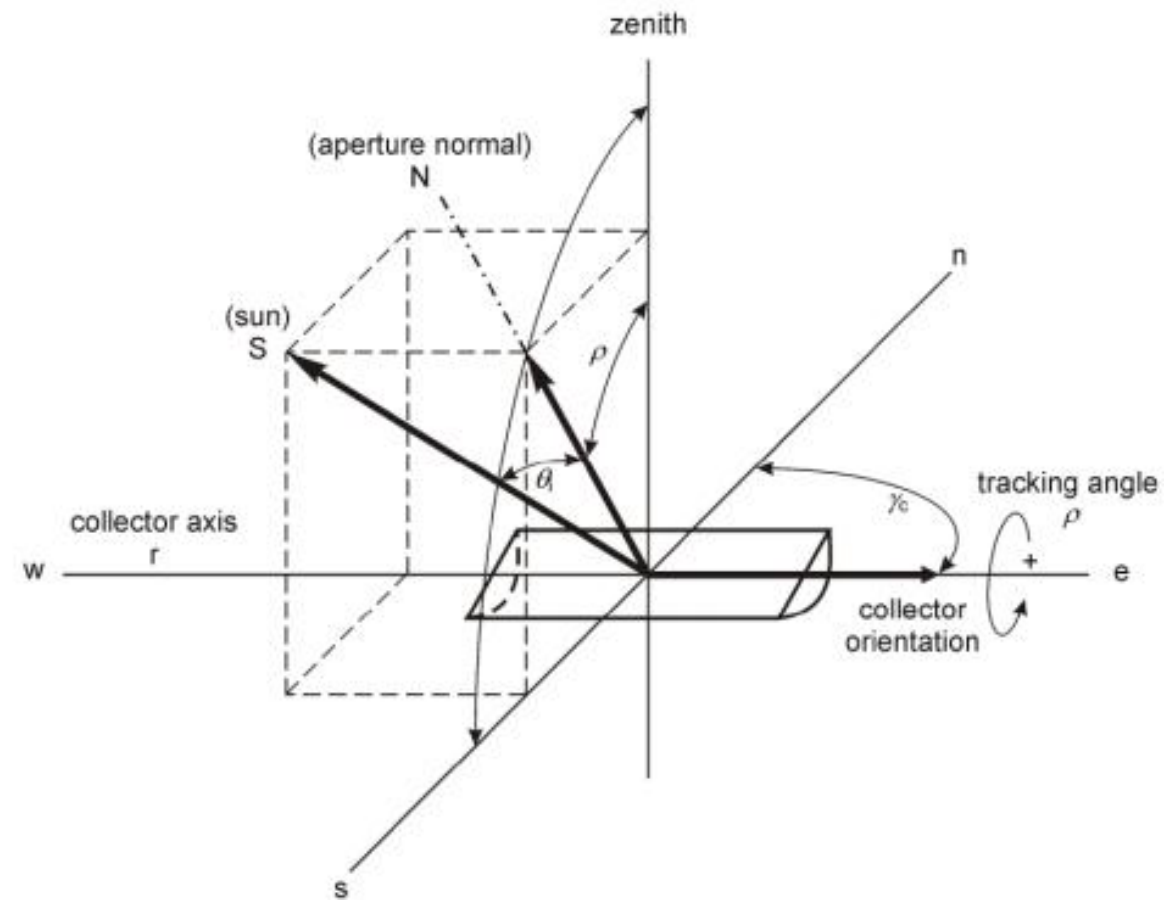

Figura 1.7. Representación gráfica de los ángulos solares y orientación de un colector PTC [7].

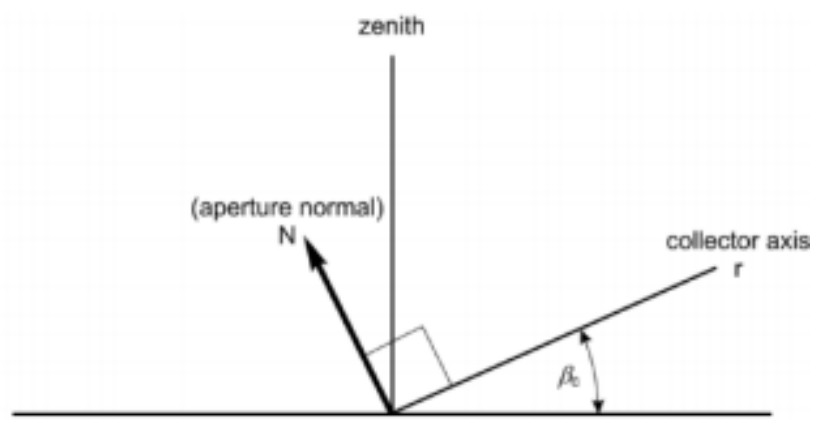

Figura 1.8. Definición de la dirección normal al área de apertura del colector solar (PTC) [7].

Tabla 1.1. Definición de los ángulos solares y de orientación de un colector PTC.

\begin{tabular}{ll}
\hline Nombre & Explicación \\
\hline Ángulo Azimuth $\left(\gamma_{a}\right):$ & el ángulo entre el Norte y la proyección \\
& del Sol sobre el plano horizontal. \\
\hline Ángulo Elevación solar $\left(\alpha_{s}\right):$ & el ángulo entre una línea recta hasta el \\
& Sol y el plano horizontal. \\
\hline Ángulo Zenith solar $\left(\theta_{z}\right):$ & ángulo complementario a $\alpha_{s} ; \theta_{z}=90^{\circ}-\alpha_{s}$ \\
\hline Azimuth del colector $\left(\gamma_{c}\right):$ & ángulo entre el Norte y la orientación de \\
& apertura del colector solar. \\
\hline
\end{tabular}


1. Estado de la técnica en las centrales termosolares con colectores solares lineales.

\begin{tabular}{ll}
\hline Ángulo inclinación tilt & ángulo de inclinación entre la superficie del \\
del colector $\left(\beta_{c}\right):$ & colector solar y el plano horizontal. \\
\hline Ángulo de incidencia $\left(\theta_{i}\right):$ & ángulo entre una línea recta hasta el Sol y \\
\end{tabular}

Para maximizar la energía capturada, el colector PTC es rotado mediante un sistema mecánico (figura 1.9), para realizar un seguimiento de la radiación solar. Este sistema permite mantener la orientación del cilindro-parabólico de forma que los rayos solares incidan paralelamente al eje de la parábola y normalmente a su área de apertura. Los colectores parabólicos están posicionados normalmente a lo largo de los meridianos, y rotan para seguir el desplazamiento del sol de Este a Oeste. Sin embargo, también se utilizan otras orientaciones, como la perpendicular a los meridianos para obtener un mejor balance de la irradiación capturada a lo largo del año.

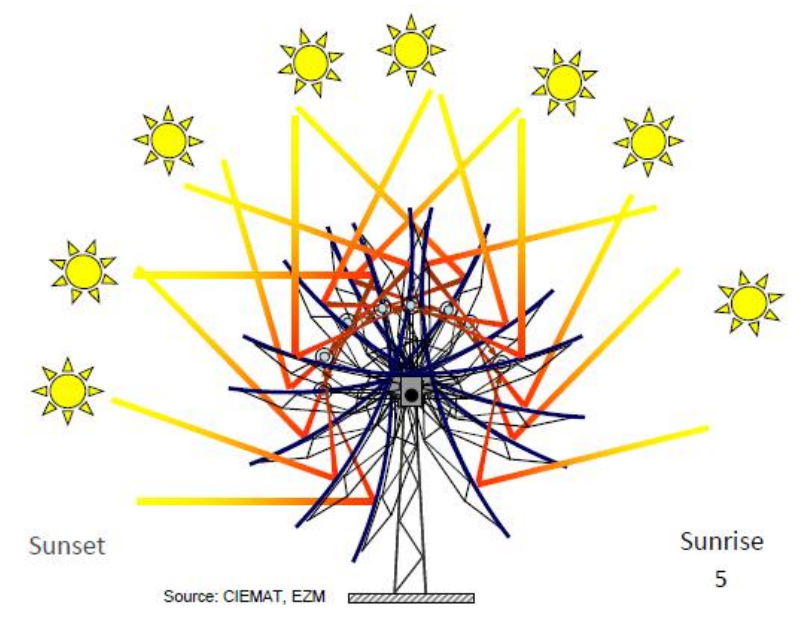

Figura 1.9. Sistema de seguimiento solar, mediante la rotación del colector PTC [6].

Merece la pena destacar las principales marcas comerciales de colectores PTC actualmente en el mercado:

- $\quad$ Luz: LS-1 (SEGS I+II), LS-2 (SEGS II-VII) en 1985 [8] y LS-3 (SEGS IX) en 1989 [9].

- Eurotrough: ET-I en 2000, ET-II en 2005 [10].

- Colectores solares SENER: SENERtrough ${ }^{\circledR}-2$ [11].

- Colectores solares Abengoa: ASTR0 150 en 2007, Phoenix en 2009, E2 (Eucumsa) en 2011, ST8 Spacetube en 2013 [12].

- Colectores solares SkyFuel: SkyTrough [13].

- Helios Trough [14].

- Ultimate Trough - Flabeg [15]. 
1. Estado de la técnica en las centrales termosolares con colectores solares lineales.

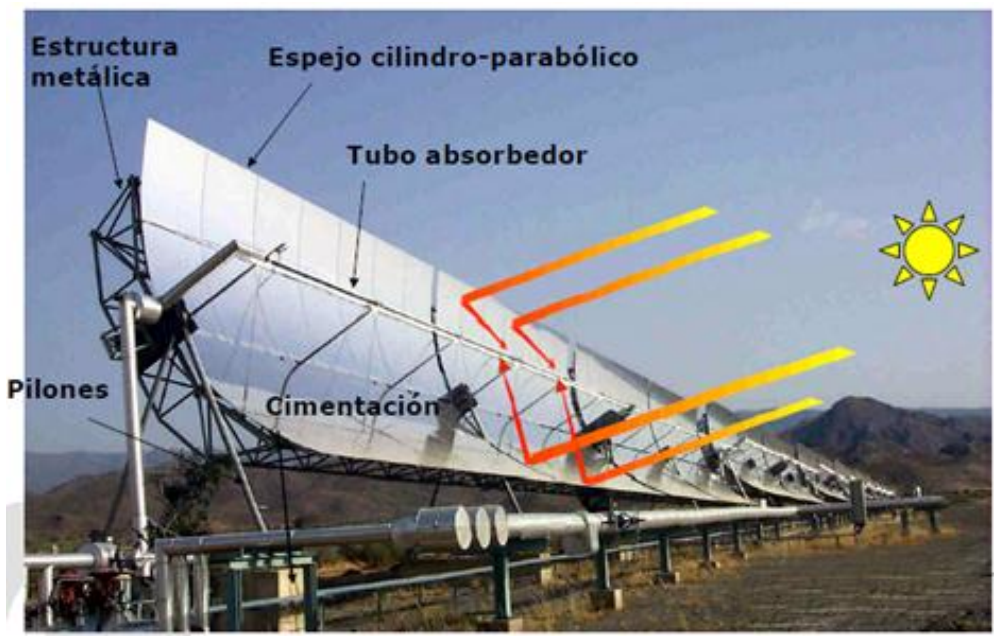

Figura 1.10. Ilustración de un captador solar PTC [1].

\subsubsection{Balance Energético y pérdidas energéticas en un colector cilindro-parabólico PTC}

La radiación solar incidente en el colector PTC, ecuación 1-2, no es la radiación finalmente absorbida por el HTF, figura 1.11. Existen tres grupos de pérdidas de energía en los colectores solares: las pérdidas ópticas, las pérdidas térmicas y las pérdidas geométricas. El calor transmitido al fluido viene definido mediante la ecuación 1-3.

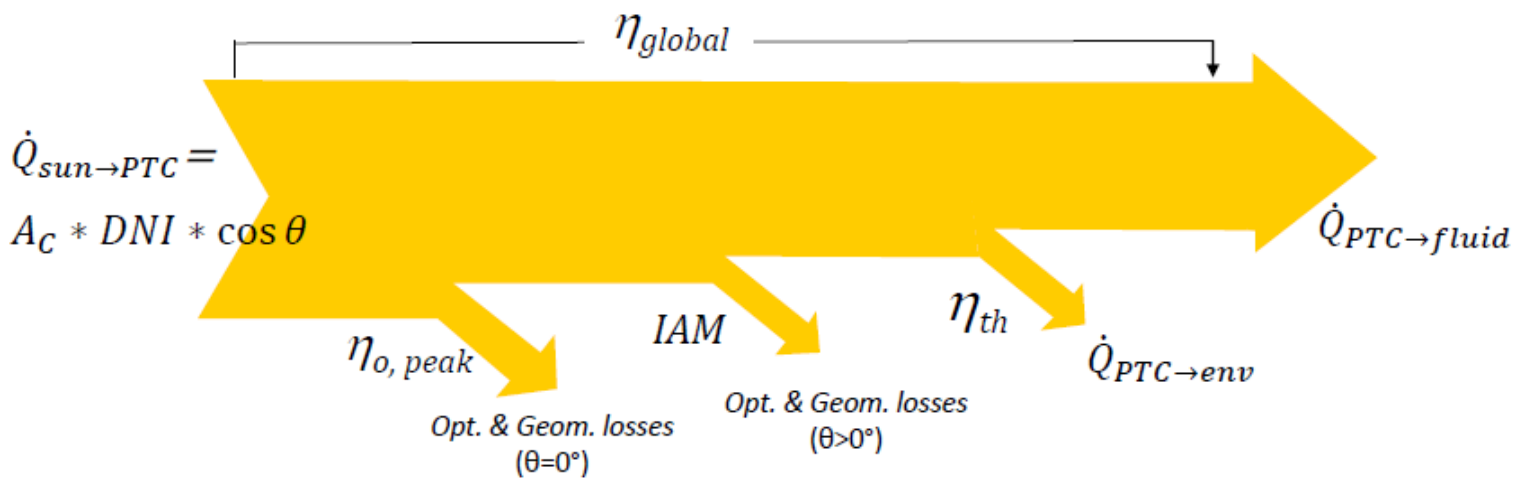

Figura 1.11. Balance energético de un colector solar PTC [6].

$Q_{\text {Sun } \rightarrow P T C}=A_{C} \cdot D N I \cdot \cos \theta$

$Q_{P T C \rightarrow \text { fluid }}=A_{C} \cdot D N I \cdot \cos \theta \cdot \eta_{o, p e a k} \cdot I A M-Q_{P T C \rightarrow e n v}$

Ac : área de apertura del colector solar $\left(\mathrm{m}^{2}\right)$.

DNI : radiación normal incidente $\left(\mathrm{W} / \mathrm{m}^{2}\right)$.

$\cos \theta$ : coseno del ángulo de incidencia.

$\eta_{o, p e a k}$ : eficiencia óptica pico.

IAM : modificación del ángulo de incidencia Incidence Angle Modifier (IAM).

$Q_{P T C \rightarrow \text { env }}$ : pérdidas térmicas $(\mathrm{W})$.

\section{a) Pérdidas ópticas}

Los colectores parabólicos incluyen diferentes imperfecciones en la superficie de los espejos, en la posición de los tubos absorbedores y otras imperfecciones geométricas. Así mismo, los materiales que constituyen los espejos y el tubo absorbedor constituyen otro foco de pérdidas 
energéticas. Para medir la relación entre la radiación solar absorbida por el colector en relación con radiación solar incidente definimos la Eficiencia Óptica $\left(\eta_{o}\right)$, ecuación 1-4. Esta eficiencia depende de los siguientes factores: del factor de interceptación $(\curlyvee)$, la transmitancia del tubo de vidrio $(\tau)$, la absortancia del tubo absorbedor $(\alpha)$, y la reflectancia del espejo parabólico $(\rho)$, figura 1.12 .

$\eta_{o}=\gamma \cdot \tau \cdot \alpha \cdot \rho$

- El Factor de Interceptación ( $\curlyvee$ ): agrupa los errores superficiales del espejo y del sistema de seguimiento solar hace que no todos los rayos incidentes en el espejo parabólico vayan a interceptar sobre el tubo absorbedor. Los valores típicos en los espejos comerciales es superior al $98 \%$ [6].

- La Transmitancia del tubo de vidrio $(\tau)$ : se define como la relación entre la radiación solar incidente en la cubierta del cristal del tubo absorbedor, y la radiación que realmente pasa a través del tubo de vidrio >97\% [21].

- La Absortancia del tubo absorbedor $(\alpha)$ : cuantifica la cantidad de radiación solar absorbida por el material selectivo del tubo absorbedor. El valor máximo de este parámetro es de $>95.5 \%$ (normativa ISO), >96\% (normativa ASTM) [21].

- Emisividad (emitancia) térmica del tubo absorbedor $(\varepsilon)$ : es la proporción de radiación térmica emitida por una superficie u objeto debido a su temperatura. $\varepsilon<9.5 \%$ [21].

- La Reflectividad del espejo parabólico ( $\rho$ ): fracción de energía incidente solar que es reflejada por la superficie (espejos) del colector solar. Depende de la longitud de onda, la dirección y la naturaleza de la radiación incidente, el acabado de la superficie reflectante y la temperatura de dicha superficie. Este factor disminuye conforme se incrementa la suciedad de los espejos del colector solar. Su valor típico para reflectores parabólicos es 93\% [6].

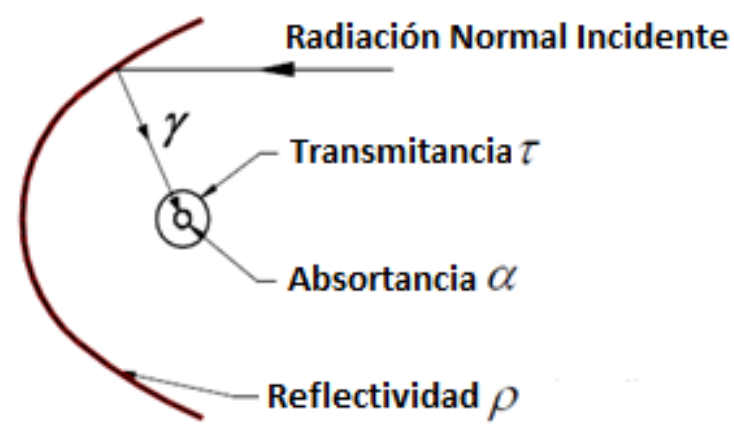

Figura 1.12. Sección transversal de un colector cilindro-parabólico representando los parámetros ópticos principales de los componentes que lo integran: transmitancia, absortancia y reflectancia [6]. 
1. Estado de la técnica en las centrales termosolares con colectores solares lineales.

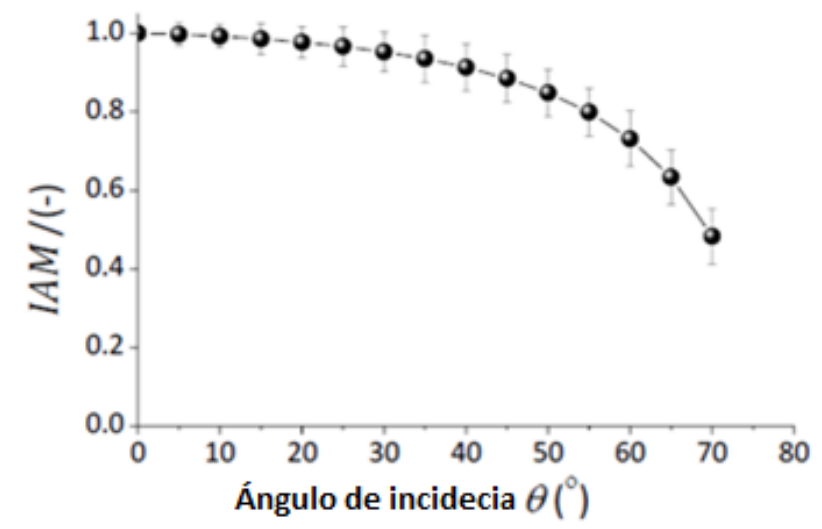

Figura 1.13. Representación del valor de los factores de modificación del ángulo de incidencia IAM para diferentes valores del ángulo de incidencia [6].

La Eficiencia óptica-pico $\left(\eta_{o \text {,peak }}\right)$ es la eficiencia óptica máxima cuando el ángulo de incidencia $(\theta)$ es cero, y la radiación solar es perpendicular al plano de apertura del colector solar. Cuando el ángulo de incidencia varía la eficiencia óptica pico se ve modificada mediante el factor de modificación del ángulo de incidencia Incidence Angle Modifier (IAM), ecuación 1-5 y figura 1.13. Este factor evalúa el funcionamiento del colector incluyendo los siguientes efectos:

- Incluye los cambios de los parámetros geométricos y ópticos cuando el ángulo de incidencia no es $0^{\circ}$.

- No incluye el efecto del coseno.

- Varia de 0 a 1 (el valor de 1 lo obtenemos cuando $\theta=0^{\circ}$ ).

$\eta_{o}\left(\theta \neq 0^{\circ}\right)=\eta_{o, \text { peak }} \cdot \mathrm{IAM}$

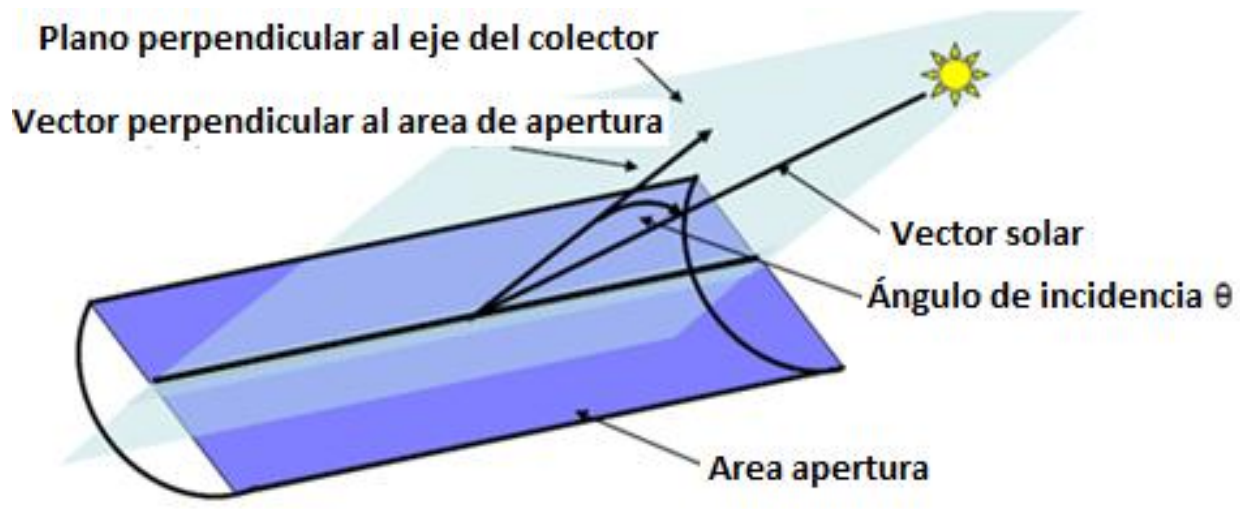

Figura 1.14. En esta figura se ilustran los parámetros: área de apertura, ángulo de incidencia en un colector PTC [6].

El ángulo de incidencia $(\theta)$ es función de la posición del Sol y del ángulo de seguimiento solar (figura 1.14). Para averiguar la posición del Sol, para una posición particular de la planta termosolar, altitud $(\phi)$ y longitud $(\psi)$, primero se calcula el tiempo "hora" solar $\mathrm{t}_{\text {sol }}$ ecuación 19. La ecuación horaria Equation Of Time (EOT), ecuación 1-6 y 1-7, que determina la desviación entre hora local y hora solar en función del día del año. Otra desviación medida es el shift, 
ecuación 1-8, que representa un valor fijo en minutos debido a la diferencia entre la longitud para una zona horaria determinada y la longitud de la localización estudiada (la zona horaria se considera con valores negativos al Oeste y con valores positivos al Este del GMT+0). El día del año y la hora del día (medido en 0 a 24 horas) también son parámetros de entrada en los cálculos de los ángulos solares. El tiempo solar es convertido en ángulo horario $(\omega)$ mediante la siguiente ecuación 1-10, que se define con la ayuda de las ecuaciones 1-6 a 1-9:

$B=($ day -1$) \cdot \frac{360}{365}$

$\mathrm{EOT}=9.2(0.000075+0.001868 \cdot \cos \mathrm{B}-0.032077 \cdot \sin \mathrm{B}-0.014615 \cdot \cos (2 \mathrm{~B})-$ $0.04089 \cdot \sin (2 \mathrm{~B}))$

shift $=$ zone $\cdot 15-\Psi$

$\mathrm{t}_{\text {sol }}=$ hour $+\frac{\text { shift }}{15}+\frac{\text { EOT }}{60}$

$\omega=\left(t_{\text {sol }}-12\right) \cdot 15^{\circ}$

Ya que la posición del Sol está influenciada por la posición del eje de la Tierra tilt, el ángulo de declinación $\delta$ se determina con la siguiente ecuación 1-11:

$\delta=23.45^{\circ} \cdot \sin \left(360 \cdot \frac{(284+\text { day })}{365}\right)$

Los ángulos de elevación solar $\left(\theta_{\mathrm{e}}\right)$, ángulo cenital solar $\left(\theta_{\mathrm{z}}\right)$ y ángulo azimut solar $\left(\gamma_{\mathrm{sol}}\right)$, son calculados respectivamente con las ecuaciones 1.12 a 1.14 :

$$
\begin{aligned}
& \theta_{\mathrm{e}}=\sin ^{-1}(\sin (\delta) \cdot \sin (\phi)+\cos (\phi) \cdot \cos (\delta) \cdot \cos (\omega)) \\
& \theta_{\mathrm{z}}=90^{\circ}-\theta_{\mathrm{e}} \\
& \gamma_{\text {sol }}=\operatorname{sign}(\omega) \cdot\left[\cos ^{-1} \cdot\left(\frac{\cos \left(\theta_{\mathrm{z}}\right) \cdot \sin (\phi)-\sin (\delta)}{\sin \left(\theta_{\mathrm{z}}\right) \cdot \cos (\phi)}\right)\right]
\end{aligned}
$$

Los colectores solares parabólicos (PTC) siguen la trayectoria solar según un solo eje al que normalmente se le refiere con el tracking angle. Éste ángulo puede ser orientado en cualquier dirección, normalmente se suele orientar con los puntos cardinales Norte-Sur o Este-Oeste. El colector realiza un seguimiento de la posición solar de tal forma que el ángulo entre la normal a su plano de apertura y la irradiación solar incidente es mínimo. El objetivo es captar la radiación normal incidente en la superficie de apertura del colector solar. El ángulo de seguimiento solar $\left(\omega_{\text {col }}\right)$ es calculado con la siguiente ecuación 1-15, donde la orientación del colector solar viene definida por dos ángulos de orientación, el ángulo azimuth $\left(\gamma_{\text {col }}\right)$, y el ángulo de inclinación tilt angle $\left(\theta_{\text {col }}\right)$.

$$
\omega_{\text {col }}=\tan ^{-1} \cdot\left[\frac{\cos \left(\theta_{\mathrm{e}}\right) \cdot \sin \left(\gamma_{\mathrm{sol}}-\gamma_{\mathrm{col}}\right)}{\sin \left(\theta_{\mathrm{e}}-\theta_{\mathrm{col}}\right)+\sin \left(\theta_{\mathrm{col}}\right) \cdot \cos \left(\theta_{\mathrm{e}}\right) \cdot\left(1-\cos \left(\gamma_{\mathrm{sol}}-\gamma_{\mathrm{col}}\right)\right)}\right]
$$


1. Estado de la técnica en las centrales termosolares con colectores solares lineales.

Toda la información necesaria para calcular el ángulo de incidencia $(\theta)$ ha sido determinada en las ecuaciones anteriormente descritas, dando lugar a la ecuación 1-16:

$\theta=\cos ^{-1} \sqrt{1-\left[\cos \left(\theta_{\mathrm{e}}-\theta_{\mathrm{col}}\right)-\cos \left(\theta_{\mathrm{col}}\right) \cdot \cos \left(\theta_{\mathrm{e}}\right) \cdot\left(1-\cos \left(\gamma_{\mathrm{sol}}-\gamma_{\mathrm{col}}\right)\right)\right]^{2}}(1-16)$

De esta forma quedan definidos todos los parámetros asociados al cálculo de la energía que llega del Sol al PTC.

\section{b) Pérdidas geométricas:}

Tanto la sombra entre dos filas de colectores adyacentes, como las pérdidas por concentración solar en el extremo del colector, son explicadas con mayor detalle en el siguiente capítulo, Metodología. A continuación, se incluyen las figuras 1-14 a 1-16 de ambos fenómenos físicos, y las principales ecuaciones para su cálculo 1-17 a 1-20.

- Sombra de una fila de colectores PTC: Las ecuaciones 1-17 y 1-18 definen el rendimiento $\eta$ shadow

$$
\begin{aligned}
& \omega_{\mathrm{a}}=\cos \left(\omega_{\text {col }}\right) \cdot \mathrm{L}_{\text {spacing }} \\
& \eta_{\text {shadow }}=\frac{\mathrm{w}_{\mathrm{a}}}{\mathrm{w}}=\left[\cos \left(\omega_{\text {col }}\right)\right] \cdot \frac{\mathrm{L}_{\text {spacing }}}{\mathrm{w}}
\end{aligned}
$$

Los parámetros $w, w_{a}, L_{\text {spacing }} y w_{c o l}$ quedan definidos en la figura 1.15. El rendimiento $\eta$ shadow se multiplica por $\mathrm{A}_{\mathrm{C}} \cdot \mathrm{DNI} \cdot \cos \theta$, ecuación 1.2 , afectando directamente al valor de $\mathrm{Q}_{\text {Sun } \rightarrow \text { PTC }}$.

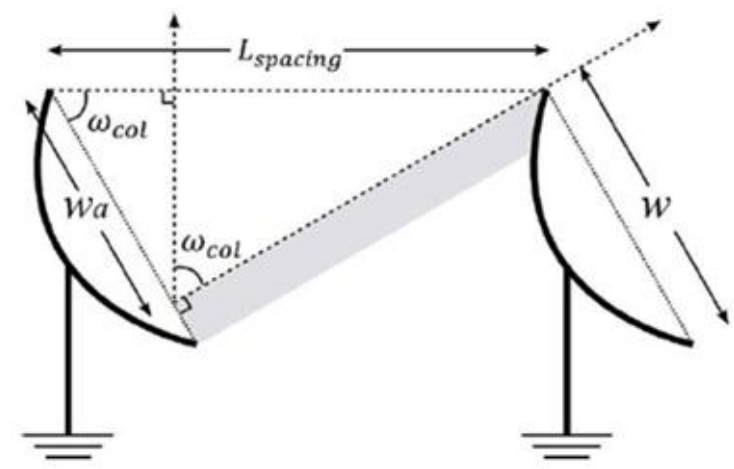

Figura 1.15. Dos filas de colectores adyacentes pueden darse sombra una fila sobre la otra si el ángulo de seguimiento solar llega a unos valores extremos. La sombra depende de la anchura de apertura de los colectores, de la distancia entre las filas (entre los ejes centrales de las dos filas), y del ángulo de seguimiento solar de los colectores [20]. 
1. Estado de la técnica en las centrales termosolares con colectores solares lineales.

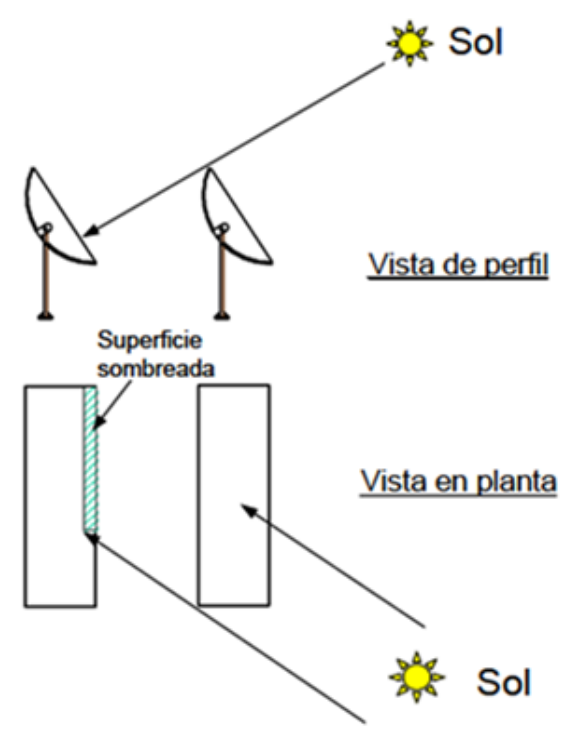

Figura 1.16. Sombra entre dos filas adyacentes de los colectores solares PTC [6].

- Cálculo de las pérdidas por concentración solar en los extremos de los colectores de cada lazo. Las ecuaciones 1-19 y 1-20 definen el cálculo del rendimiento de pérdidas en el extremo $\eta_{\text {end Loss, } i}$ de una fila de PTC. Los parámetros de dichas ecuaciones están explicados en la figura 1.17. El rendimiento $\eta_{\text {end Loss }, i}$ se multiplica por $A_{C} \cdot D N I \cdot \cos \theta$, en la ecuación 1.2, afectando directamente al valor de $Q_{S u n \rightarrow P T C}$.

$$
\begin{gathered}
\eta \text { end Loss,i }=1-\frac{\mathrm{L}_{\mathrm{f}, \mathrm{ave}, \mathrm{i}} \cdot \tan (\theta)}{\mathrm{L}_{\mathrm{i}}} \\
\mathrm{L}_{\mathrm{f}, \mathrm{ave}}=\sqrt{\frac{\left[4 \mathrm{a}^{2}+\left(\frac{\mathrm{w}}{2}\right)^{2}\right]^{2}}{\mathrm{a}^{2}}} \cdot \frac{12 \mathrm{a}^{2}+\left(\frac{\mathrm{w}}{2}\right)^{2}}{12\left(4 \mathrm{a}^{2}+\left(\frac{\mathrm{w}}{2}\right)^{2}\right)}
\end{gathered}
$$

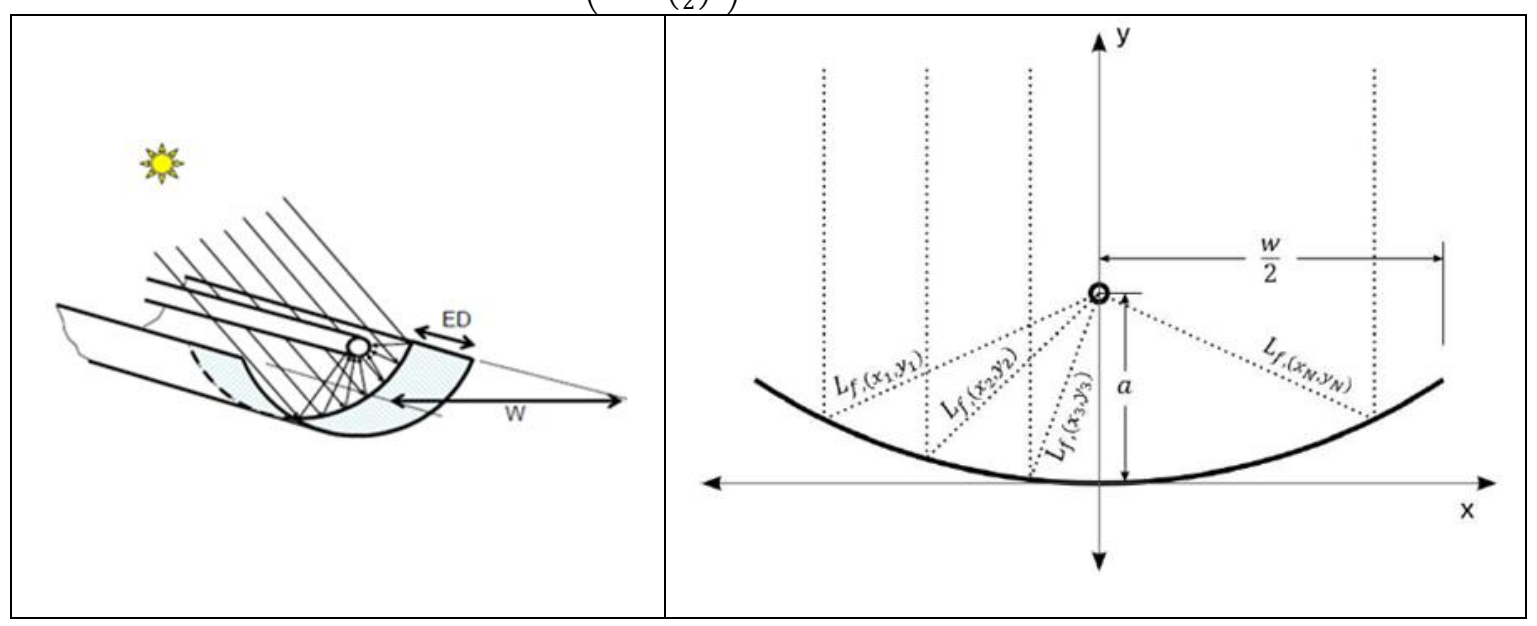

Figura 1.17. Pérdidas geométricas del extremo de un colector CCP (izq.) [6]. Sección transversal del colector parabólico para definición de su la longitud focal media $\mathrm{L}_{\text {f,ave }}$ (der.) [20].

c) Pérdidas Térmicas:

El tubo absorbedor es el elemento donde se producen la mayor parte de las pérdidas térmicas de un PTC. En la figura 1.18 se muestran los elementos integrantes de los tubos absorbedores. Principalmente están constituidos por dos tubos concéntricos, el tubo exterior de vidrio y el 
interior de acero al carbono o acero inoxidable. Por el interior del tubo metálico fluye el fluido caloportador. En la zona entre el tubo metálico y el tubo de vidrio se realiza el vacio para reducir las pérdidas térmicas por convección y para evitar el deterioro del recubrimiento selectivo que se aplica en la superficie de los tubos para mejorar las propiedades ópticas del mismo.

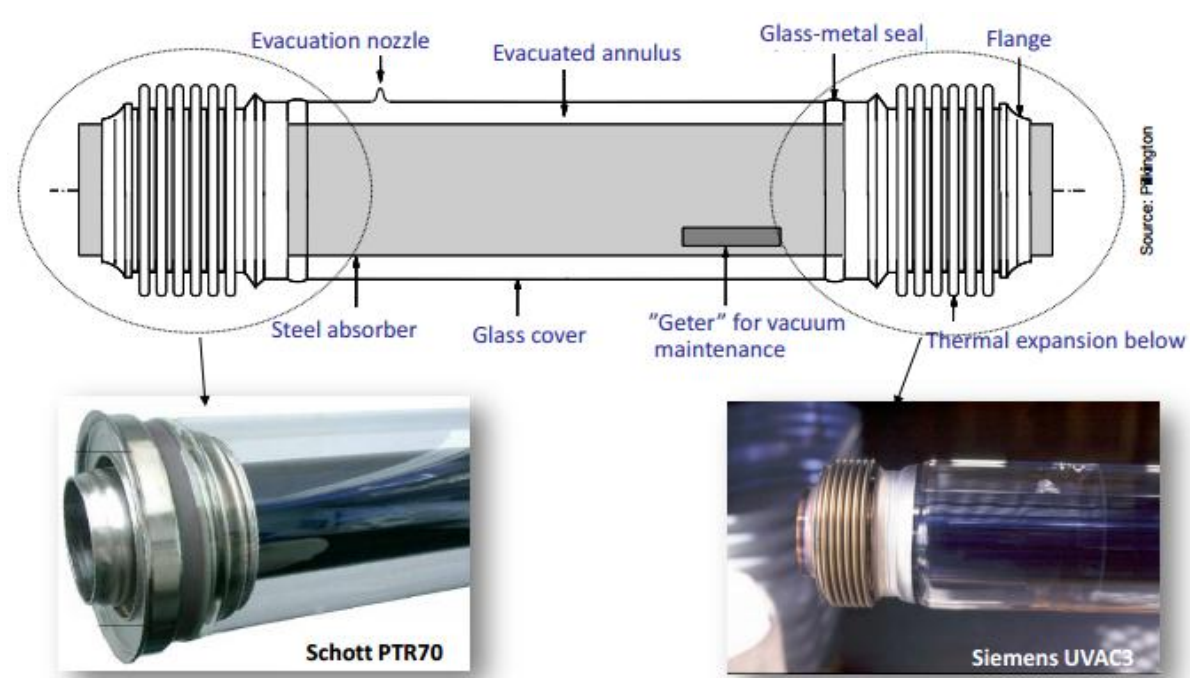

Figura 1.18. Sección longitudinal de un tubo absorbedor de un colector solar PTC [6].

Resulta de interés señalar algunas de las principales denominaciones comerciales de fabricantes de tubos absorbedores:

- SCHOTT Solar, con tres productos en el mercado: SCHOTT PTR70 para uso con aceites térmicos, SCHOTT PTR70 Premium incluye además una cápsula con gas noble para incrementar la vida del colector, y SCHOTT PTR70 Advance para utilización con sales fundidas como HTF hasta $550^{\circ} \mathrm{C}$ [21].

- Archimede Solar Energy (ASE), con tres productos comerciales: HCEMS-11 para sales fundidas, HCEOI-12 para aceites térmicos y HCESHS-12 para generación directa de Vapor (DSG) [22].

El modelo analítico detallado de las pérdidas térmicas de un tubo absorbedor queda explicado en el documento [23]. En las figuras 1.19 y 1.20 se representan los flujos energéticos y resistencias térmicas asociadas a dicho modelo. 
1. Estado de la técnica en las centrales termosolares con colectores solares lineales.

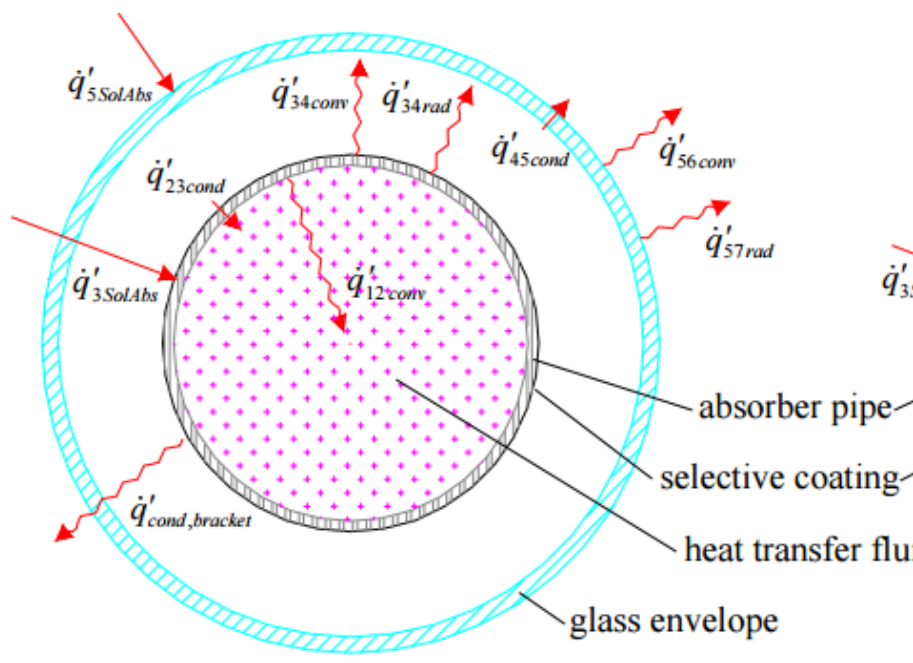

With Glass Envelope

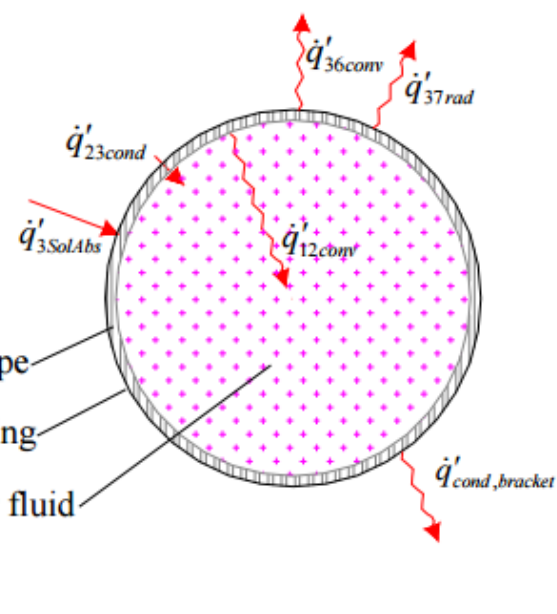

$\underline{\text { Without Glass Envelope }}$

Figura 1.19. Balance energético de una sección transversal de tubo absorbedor [23].

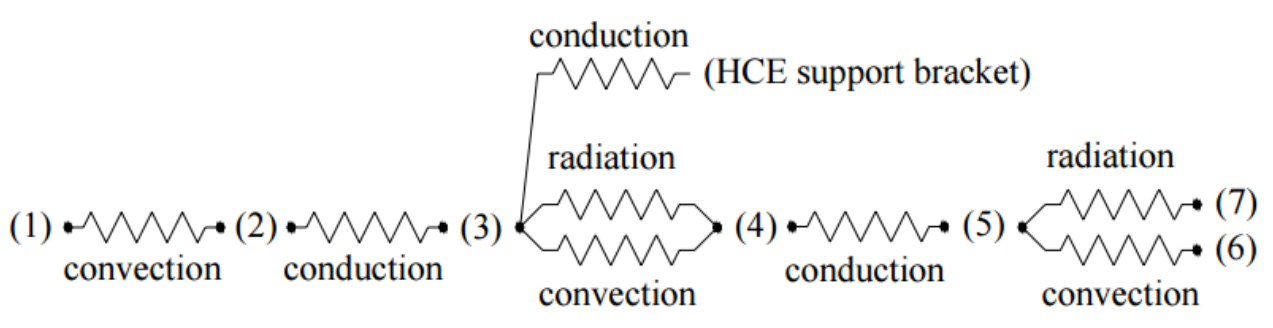
(1) heat transfer fluid
(5) glass envelope outer surface
(2) absorber inner surface
(6) surrounding air
(3) absorber outer surface
(7) sky
(4) glass envelope inner surface

Figura 1.20. Representación gráfica de las resistencias térmicas correspondientes al modelo unidimensional del tubo absorbedor representado en la figura 1.19 [23].

Los modelos analíticos han sido validados por diferentes ensayos experimentales proporcionando correlaciones el cálculo de las pérdidas térmicas en los tubos absorbedores. Cabe destacar los siguientes trabajos a este respecto [24-28].

\subsubsection{Otros elementos de los CCP}

Desde el punto de vista estructural, este tipo de colectores se compone de cuatro elementos principales: cimentación y la estructura soporte, el reflector cilindro parabólico, el tubo absorbedor o receptor y el sistema de seguimiento solar, figura 1.21. 
1. Estado de la técnica en las centrales termosolares con colectores solares lineales.

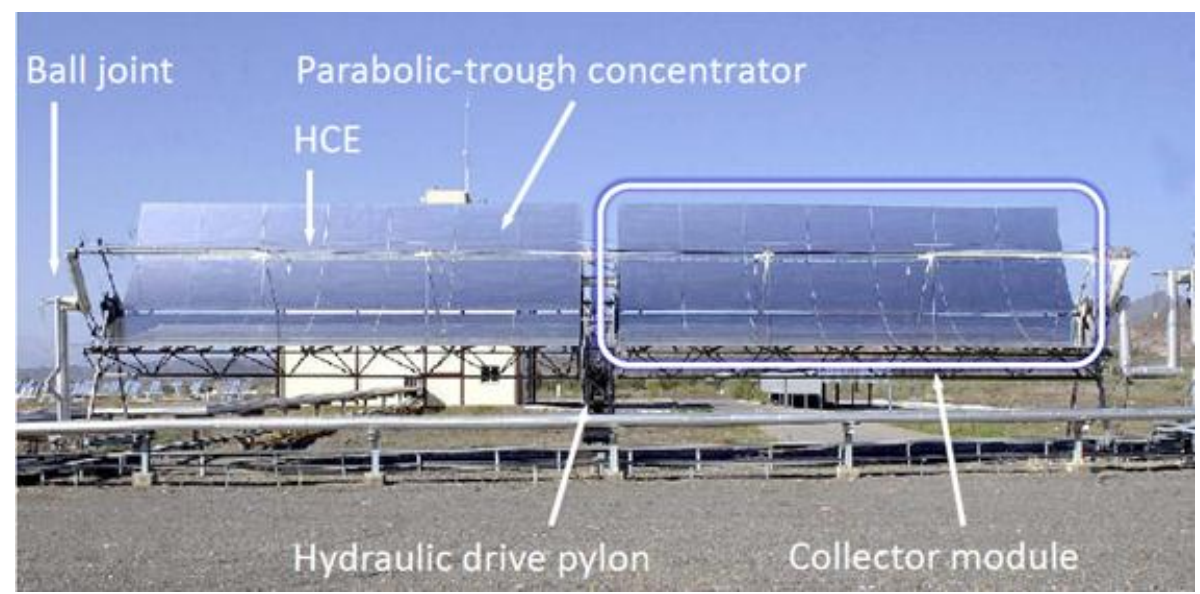

Figura 1.21. Principales componentes que integran un colector solar PTC [6].

Como puede verse en la figura 1.22 el sistema de seguimiento solar está constituido por dos pistones olehidráulicos, que giran el colector PTC alrededor de su eje central para enfocarlo al Sol. La radiación solar incidente sobre el colector debe ser lo más perpendicular posible a su área de apertura.

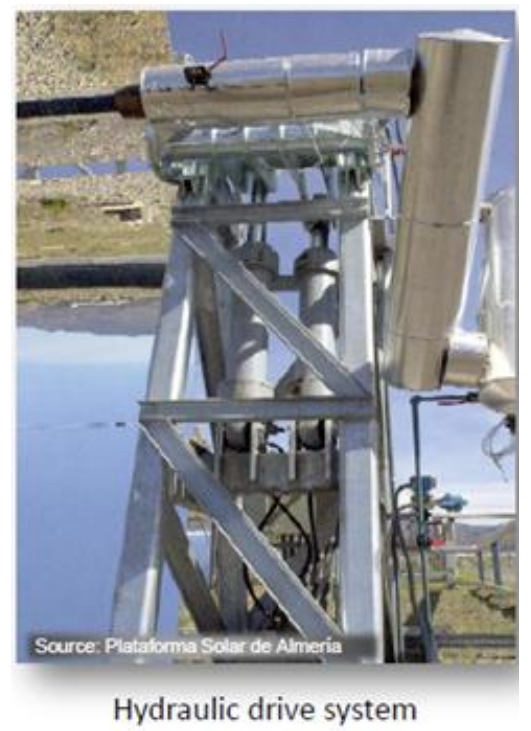

Figura 1.22. Sistema de seguimiento solar mediante actuadores de dos pistones [6].

El sistema de seguimiento solar también está integrado por unos sensores que detectan el movimiento del Sol (figura 1.23). 
1. Estado de la técnica en las centrales termosolares con colectores solares lineales.

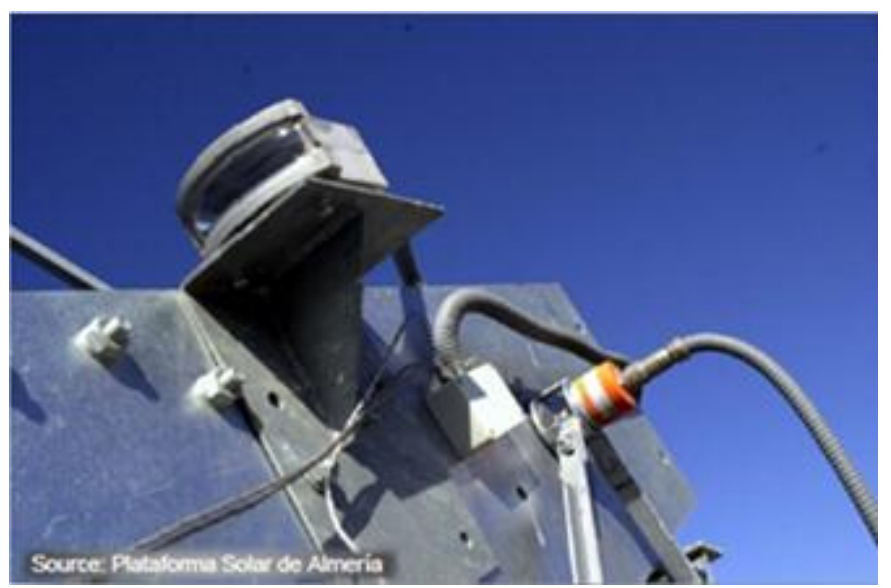

Figura 1.23. Sistema de seguimiento solar. Sensor de seguimiento de la luz [6].

Para conectar los colectores parabólicos PTC a las tuberías de distribución del fluido caloportador en el campo solar headers o para conexión con otro colector PTC se utilizan los siguientes componentes (figura 1.24):

a) Mangueras flexibles: grandes pérdidas de carga, juntas de expansión de acero inoxidable, y limitada flexibilidad a alta temperatura.

b) Juntas de bola Ball joints: provista de un sello interno de grafito para reducir la fricción y las posibles fugas.
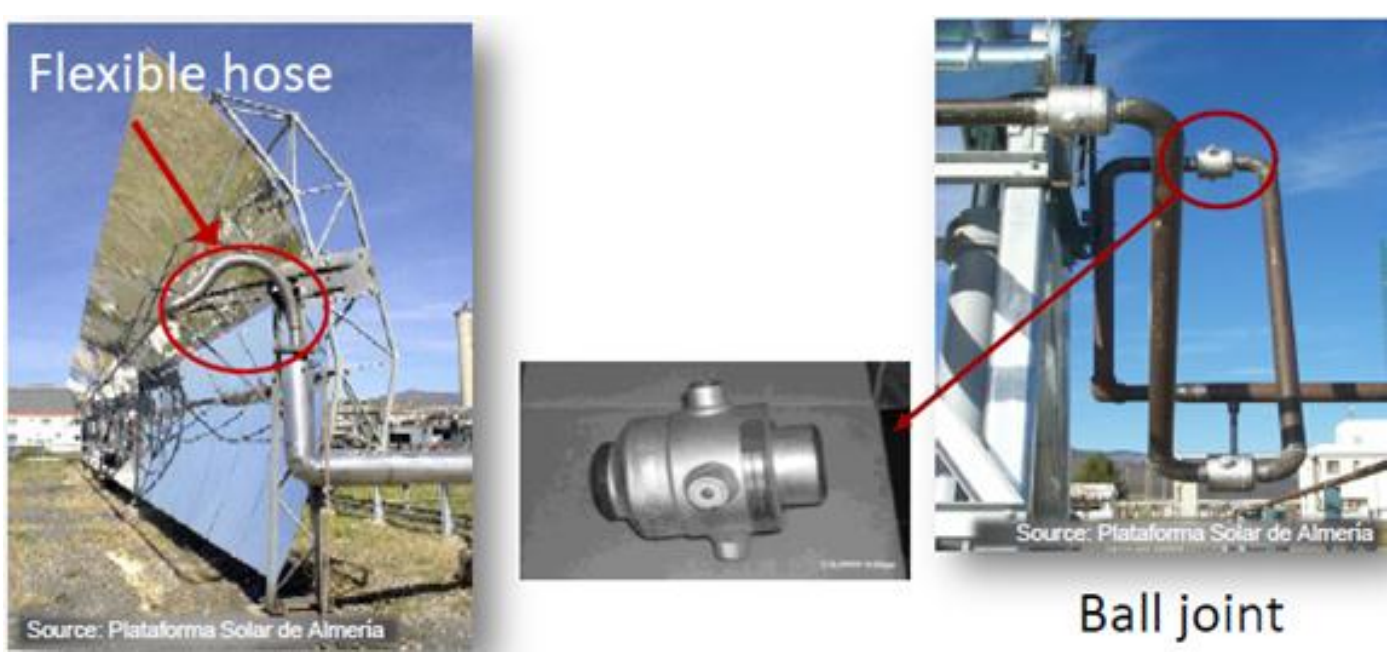

Figura 1.24. Manguera flexibe y junta de bola, dos elementos que permiten el acoplamiento entre los tubos absorbedores y las tuberías principales de distribución del campo solar [6].

\subsection{Colectores Lineales Fresnel (LF)}

\subsubsection{Introducción}

Un conjunto de reflectores planos o casi planos concentran la radiación solar en receptores lineales invertidos elevados haciendo que el fluido que fluye por los receptores incrementa su entalpía. Este sistema es de concentración lineal, similar a un canal parabólico, con la ventaja de su bajo coste en soporte estructural y reflectores, juntas fijas del fluido, un receptor separado del sistema de reflectores, y grandes longitudes focales que permiten utilizar espejos planos o casi planos. Esta tecnología es contemplada como una alternativa potencial de menor coste a la tecnología de PTC para la el aprovechamiento solar, figuras 1.25 y 1.26. 


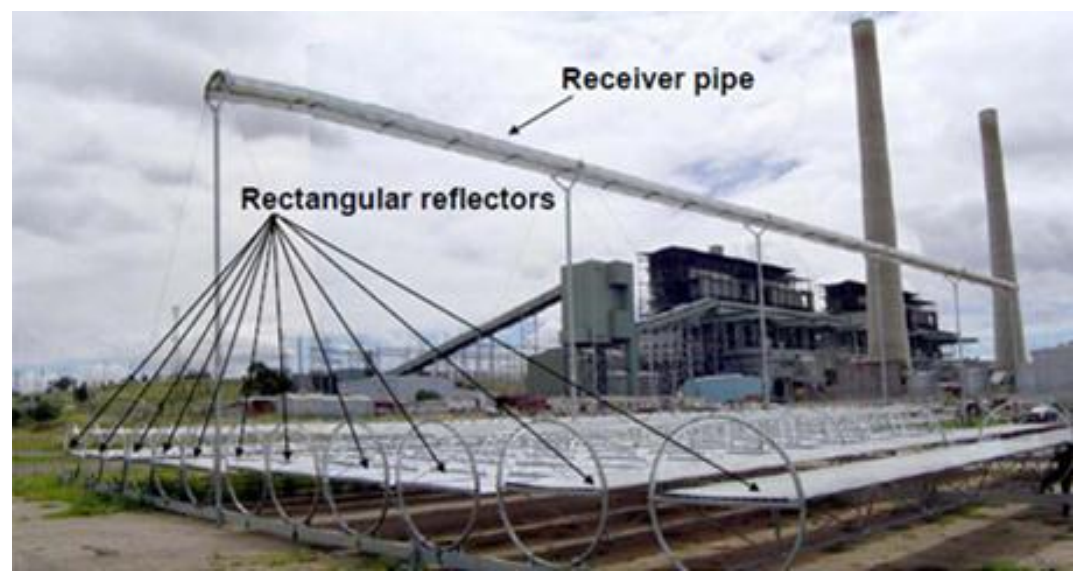

Figura 1.25. Sección transversal de un colector solar tipo LF [2].

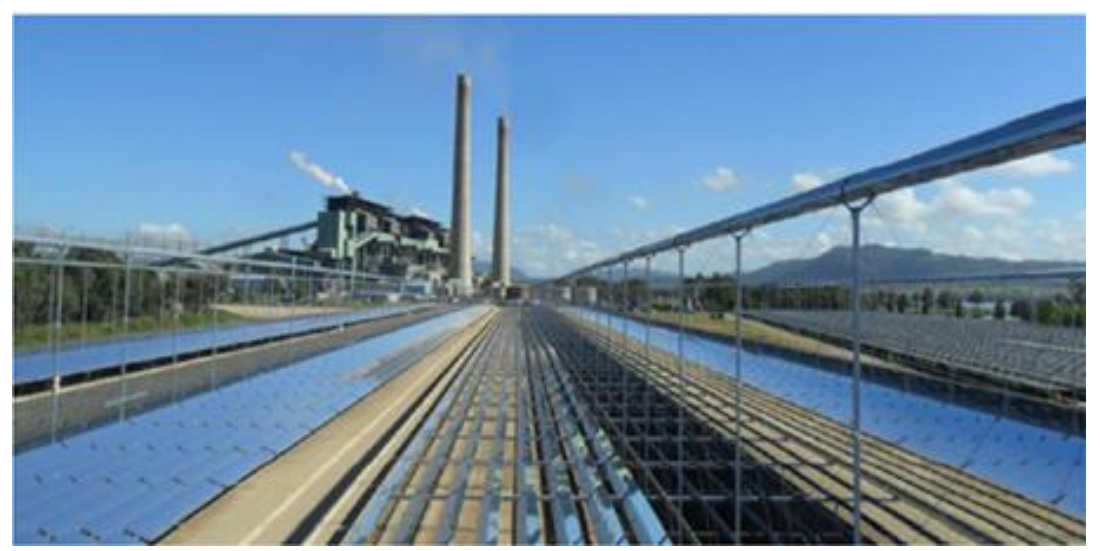

Figura 1.26. Vista de los colectores solares tipo LF en la Central Térmica de Liddell en Australia [27].

La lente de Fresnel, llamada así por su inventor Augustin Fresnel (1788-1828), Físico francés, es un diseño de lentes que permite la construcción de lentes de gran apertura y una corta distancia focal sin el peso y volumen de material que debería usar en una lente de diseño convencional aprovechando el principio de refracción. La primera aplicación de este tipo de lentes fue para focalizar la luz en las casas horizontalmente para llevarla a mayor distancia, figura $1.27[8]$.

Cuando las lentes son grandes, su grosor puede hacerse excesivo, haciendo la lente muy pesada y cara. En vez de ello, se puede mantener los radios de curvatura de las lentes separándolas en anillos circulares. El grosor de la lente en cada anillo es diferente, eliminando el enorme espesor que tendría la lente de ser sus superficies continúas, mientras que la superficie presenta un aspecto escalonado. Se emplean en lupas planas con formato de tarjeta de crédito, linternas de los faros, faros de los automóviles, indicadores de dirección, etc.

En su versión para aprovechamiento solar que se describe en este documento, el sistema Fresnel no hace uso de la refracción si no de la reflexión, un concepto que destaca por la sencillez de su construcción y por su bajo coste [8]. 
1. Estado de la técnica en las centrales termosolares con colectores solares lineales.
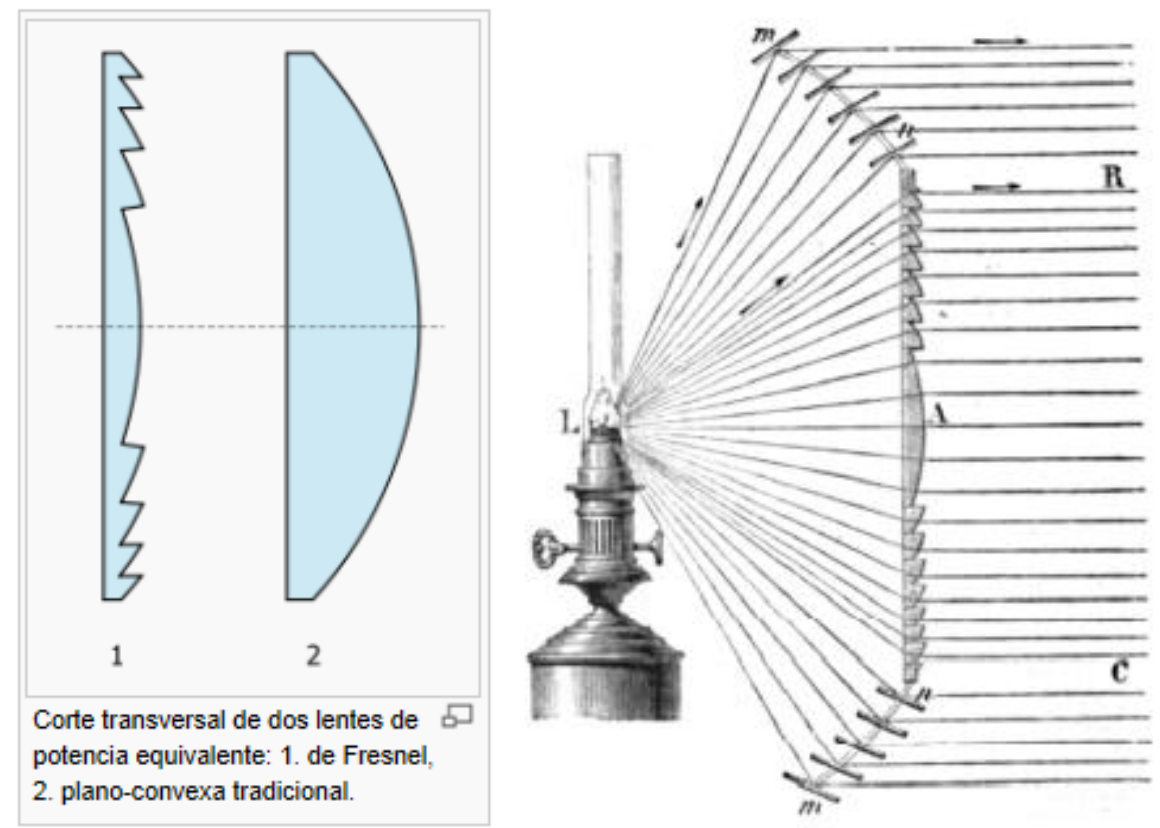

Figura 1.27. Las lentes patentadas por Augustin Fresnel permiten reducir el espesor de las lentes utilizadas hasta entonces para focalizar la luz en luminarias doméstricas [8].

Los reflectores utilizados en este sistema son espejos de vidrio convencionales, lo que abarata esta partida. La forma curvada de los espejos cilindro parabólicos hace que sean más eficientes que los espejos Fresnel, pero con el ahorro de costes de construcción y diseño se compensa el precio [29].

En la figura 1.28 se ilustra la sección transversal de un colector solar tipo LF. Los espejos son orientados de forma individual conforme el sol va desarrollando su trayectoria. Se han diseñado diferentes tipos de colectores de estas características: unos con espejos secundarios enfocando el sol sobre un tubo absorbedor, figura 1.30, y otros con una fila de tubos absorbedores formando una pared a la radiación solar, figuras 1.31 y 1.32. La longitud focal es definida por el parámetro " $\mathrm{A}$ " y se define como la distancia normal entre el plano horizontal que forman todos los espejos y el eje del tubo absorbedor. El área de apertura es la suma de todas las áreas de reflexión de cada uno de los espejos. La anchura unitaria " $\mathrm{C}$ " es la suma de las anchuras de cada uno de los espejos que integran el colector.

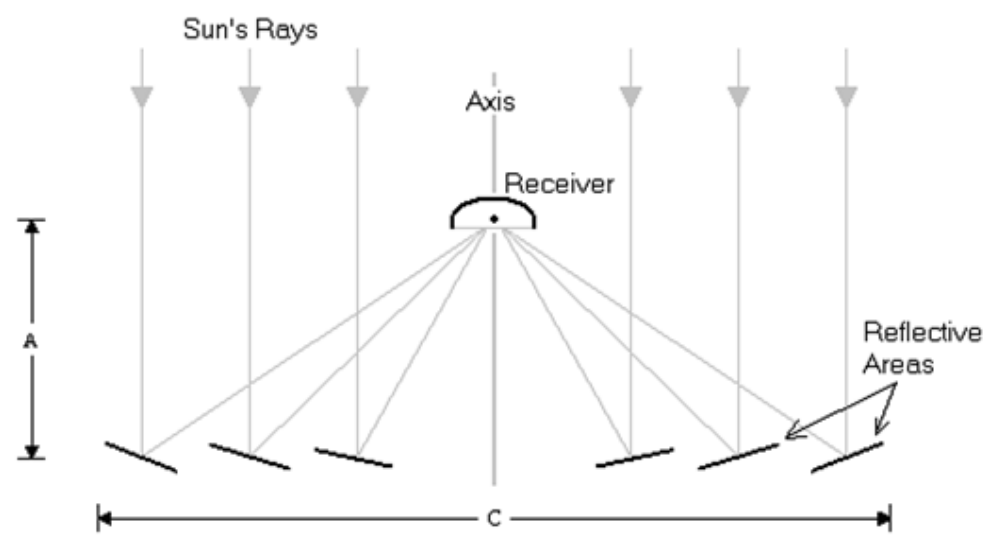

Figura 1.28. Sección transversal de un colector solar lineal tipo LF [5]. 
1. Estado de la técnica en las centrales termosolares con colectores solares lineales.

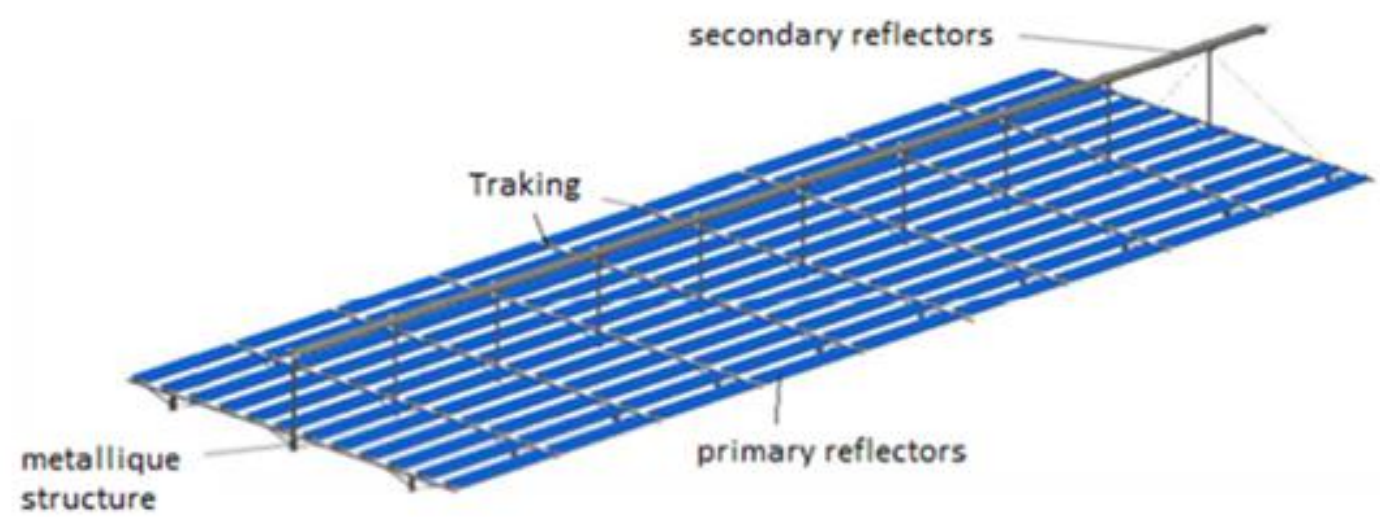

Figura 1.29. Vista general de un colector solar tipo LF.

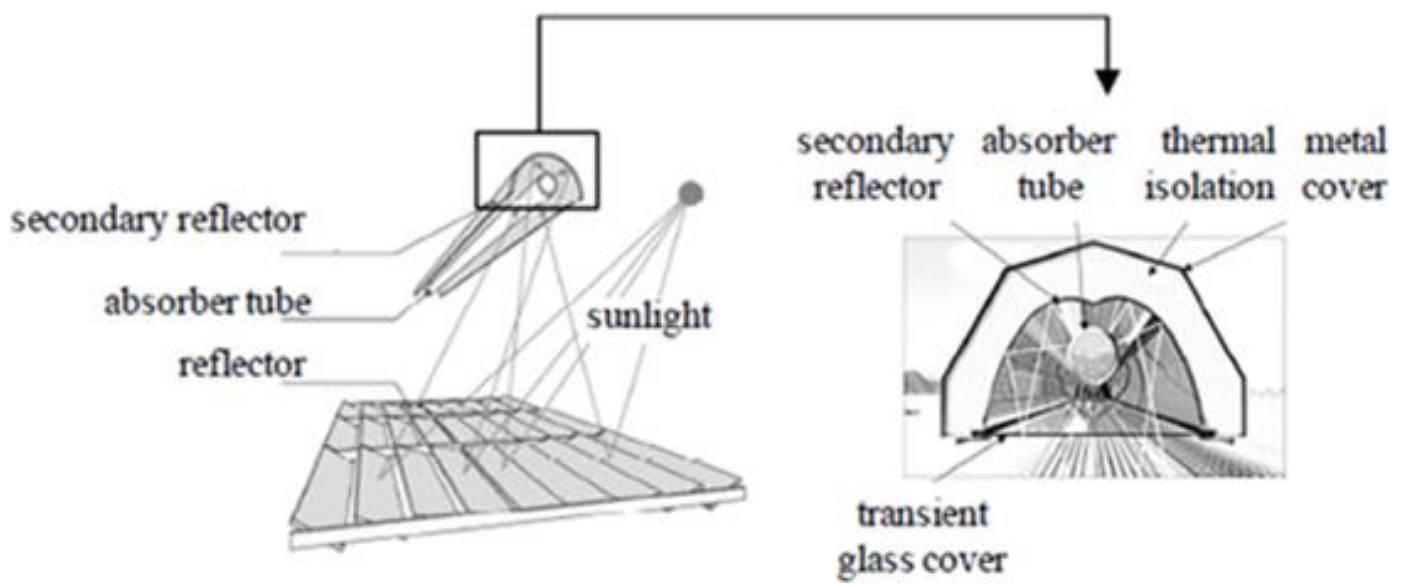

Figura 1.30. Vista detallada de los elementos que integran el colector LF con un solo tubo absorbedor y su reflector secundario.
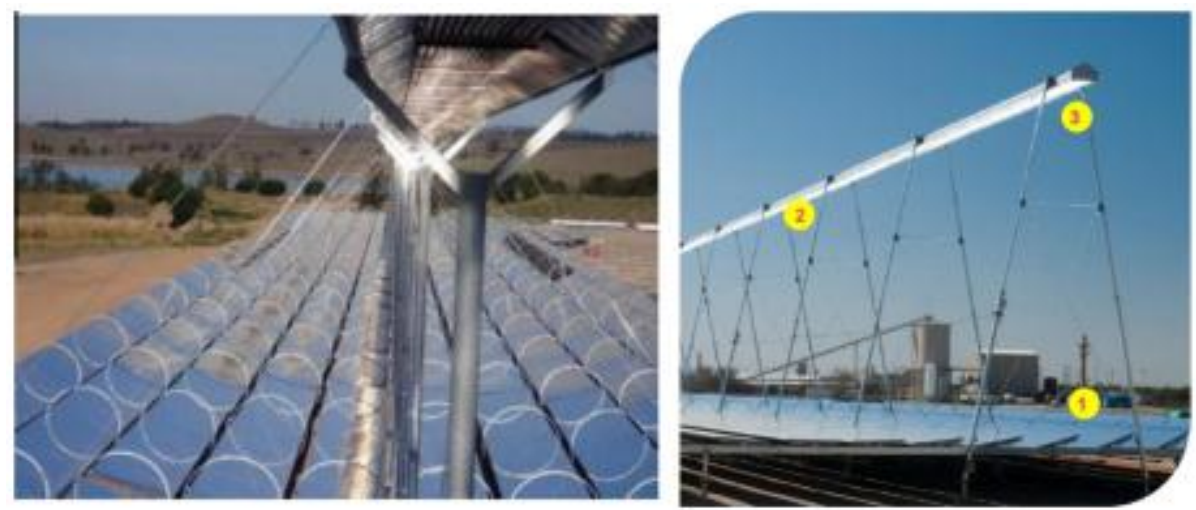

Figura 1.31. Esquema de funcionamiento de un colector solar tipo LF multitubos, 1) el agua circula a través del interior del tubo absorbedor, 2) los espejos reflectores enfocan los rayos del Sol hacia la superficie del tubo absorbedor y hacia el reflector secundario, 3) el calor de la superficie del tubo absorbedor, debido a la incidencia de los rayos solares, calienta el agua del interior del tubo aborbedor y la convierte en vapor sobrecalentado [17]. 
1. Estado de la técnica en las centrales termosolares con colectores solares lineales.
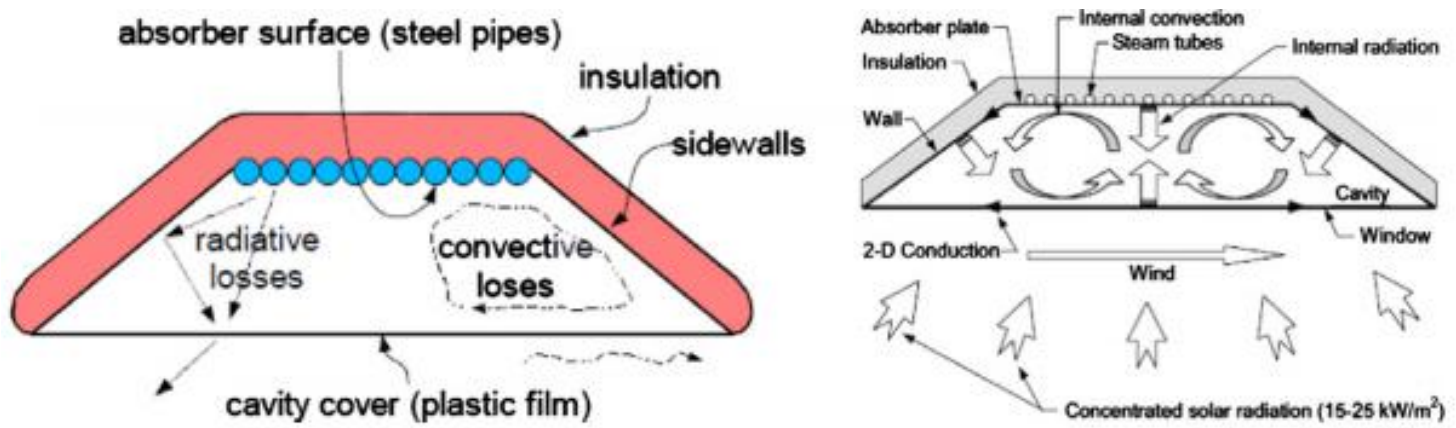

Figura 1.32. Sección transversal del diseño de dos receptores (cavidades trapezoidales) en los colectores LF, figura izquierda [18], figura derecha [19].
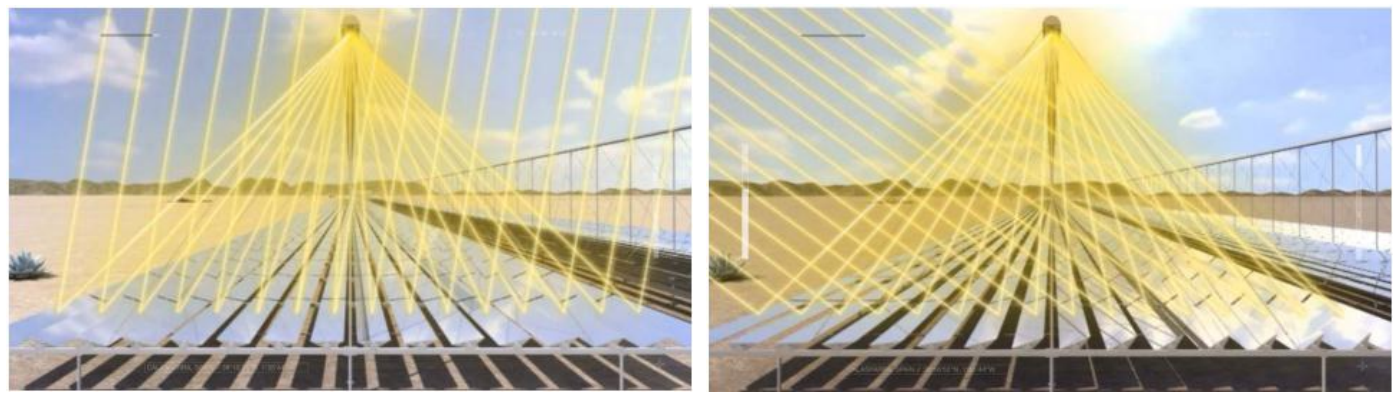

Figura 1.33. Sistema de seguimiento solar de un colector LF [7].

\subsubsection{Caracterización del colector LF.}

La modelización de un colector LF se realiza mediante las siguientes ecuaciones, comenzando por aquella por la que se determina la potencia térmica, 1-21.

$P_{\text {th }}=A \cdot\left(D N I \cdot f_{\text {clean }} \cdot \eta_{\text {opt }}-q_{\text {loss }}\right)$

Donde:

- $\quad P_{\text {th }}=$ potencia térmica $(W)$.

- $\quad A$ = área de apertura del módulo $\left(\mathrm{m}^{2}\right)$

- $\quad D N I=$ irradiación solar directa $\left(\mathrm{W} / \mathrm{m}^{2}\right)$

- $\quad \mathrm{f}_{\text {clean }}=$ factor de limpieza

- $\quad \eta_{\text {opt }}=$ eficiencia óptica

- $q_{\text {loss }}=$ pérdidas térmicas $\left(\mathrm{W} / \mathrm{m}^{2}\right)$

La eficiencia óptica es calculada con la ecuación 1-22.

$\eta_{\text {opt }}=\eta_{o} \cdot I_{A M_{\text {Transversal }}} \cdot I A M_{\text {Longitudinal }}$

Los valores de los modificadores del ángulo de incidencia Incidence Angle Modifier (IAM) son facilitados por el fabricante del colector y de forma similar a como se indican en la figura 136.

Las pérdidas térmicas son calculadas con la correlación proporcionada por el fabricante del colector según la ecuación 1-23 y los valore indicados en la tabla 1.2. 
1. Estado de la técnica en las centrales termosolares con colectores solares lineales.

$\mathrm{q}_{\text {loss }}=\mathrm{u}_{\mathrm{o}} \cdot \Delta \mathrm{T}+\mathrm{u}_{1} \cdot \Delta \mathrm{T}^{2}+\mathrm{u}_{2} \cdot \Delta \mathrm{T}^{3}+\mathrm{u}_{3} \cdot \Delta \mathrm{T}^{4}$

Tabla 1.2. Coeficientes de pérdidas térmicas y eficiencia óptica de los colectores LF marca comercial Novatec Solar [18].

\begin{tabular}{cccc}
\hline Tipo de colector & No vacío & Vacío & Unidades \\
\hline Eficiencia óptica nominal & $67 \%$ & $64.7 \%$ & - \\
\hline Coeficiente $u_{o}$ & 0.671 & 0.15 & $\mathrm{~W} / \mathrm{m} \cdot \mathrm{K}$ \\
\hline Coeficiente $\mathrm{u}_{1}$ & 0.00256 & 0 & $\mathrm{~W} / \mathrm{m} \cdot \mathrm{K}^{2}$ \\
\hline Coeficiente $\mathrm{u}_{2}$ & 0 & 0 & $\mathrm{~W} / \mathrm{m} \cdot \mathrm{K}^{3}$ \\
\hline Coeficiente $\mathrm{u}_{3}$ & 0 & $7.5 \mathrm{e}-9$ & $\mathrm{~W} / \mathrm{m} \cdot \mathrm{K}^{4}$ \\
\hline
\end{tabular}

La definición de la sección transversal del colector LF de la marca comercial Novatec [18] Solar se define en la figura 1.34 y tabla.1.3.

Los modificadores de las eficiencias ópticas para los diferentes ángulos de incidencia IAM en función de los ángulos de incidencia $\left(\theta_{\mathrm{i}}\right)$, y una formulación empírica que representa las pérdidas térmicas del colector. Normalmente los fabricantes de colectores solares utilizan simulaciones de irradiación solar para caracterizan la capacidad de sus colectores para focalizar la irradiación solar normal que reciben. Los resultados de estos análisis son expresados mediante tablas de IAM en dos dimensiones, función de los ángulos de incidencia longitudinal $\left(\theta_{\text {long }}\right)$ y transversal $\left(\theta_{\text {trans }}\right)$, figuras 1.35 y 1.36 . Así mismo utilizan métodos experimentales para determinar las pérdidas térmicas características de los tubos absorbedores.

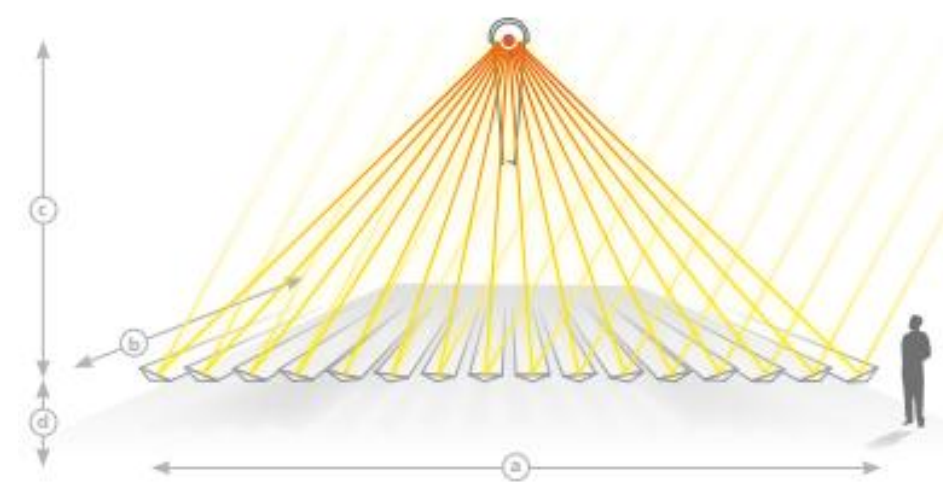

Figura 1.34. Definición de la sección transversal de un módulo de los colectores LF de la marca comercial Novatec Solar [18].

Tabla 1.3. Principales parámetros de diseño del colector LF de la marca comercial Novatec [18].

\begin{tabular}{ll}
\hline Geometría & Valor \\
\hline Anchura & $16.56 \mathrm{~m}$ \\
\hline Longitud & $44.8 \mathrm{~m}$ \\
\hline $\begin{array}{l}\text { Altitud del tubo absorbedor en relación al } \\
\text { nivel de los reflectores }\end{array}$ & $7.4 \mathrm{~m}$ \\
\hline
\end{tabular}


1. Estado de la técnica en las centrales termosolares con colectores solares lineales.

\begin{tabular}{ll}
\hline Geometría & Valor \\
\hline Altitud de los reflectores respecto al suelo & $0.75-1.05 \mathrm{~m}$ \\
\hline $\begin{array}{l}\text { Espacio recomendado entre filas de } \\
\text { colectores }\end{array}$ & $4.5 \mathrm{~m}$ \\
\hline Área de Apertura & $513.6 \mathrm{~m}^{2}$ \\
\hline Longitud mínima de la fila de colectores & 5 unidades de \\
& control, $224 \mathrm{~m}$ de \\
& longitud \\
\hline Longitud máxima de fila de colectores & 22 unidades de \\
& control, $985.6 \mathrm{~m}$ de \\
& longitud \\
\hline
\end{tabular}

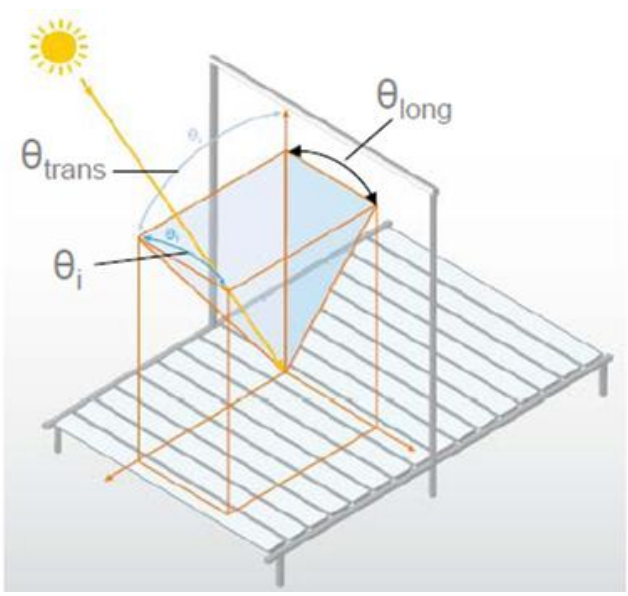

Figura 1.35. Ilustración de los ángulos de incidencia $\left(\theta_{i}\right)$, ángulo de incidencia longitudinal $\left(\theta_{\text {long }}\right)$ y transversal $\left(\theta_{\text {trans }}\right)$ en un colector LF de la empresa Novatec [18].

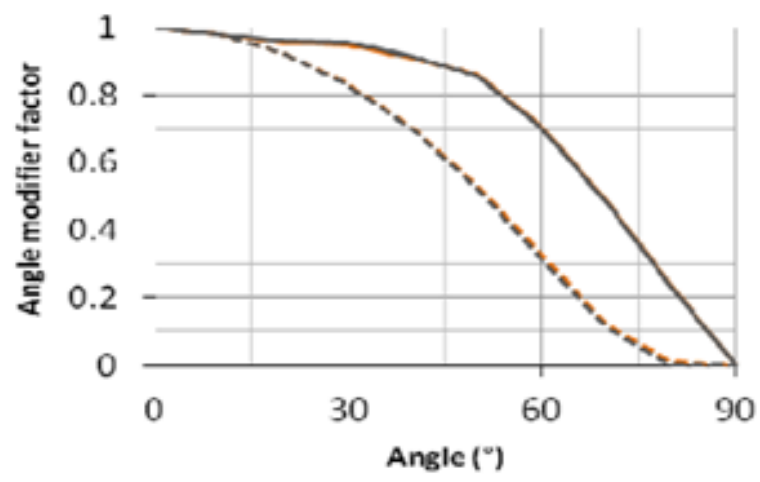

Figura 1.36. Modificadores del ángulo de incidencia en función del ángulo de incidencia solar. En línea continua los valores del IAM transversales y en línea discontinua los valores del IAM longitudinales [7]. 
1. Estado de la técnica en las centrales termosolares con colectores solares lineales.

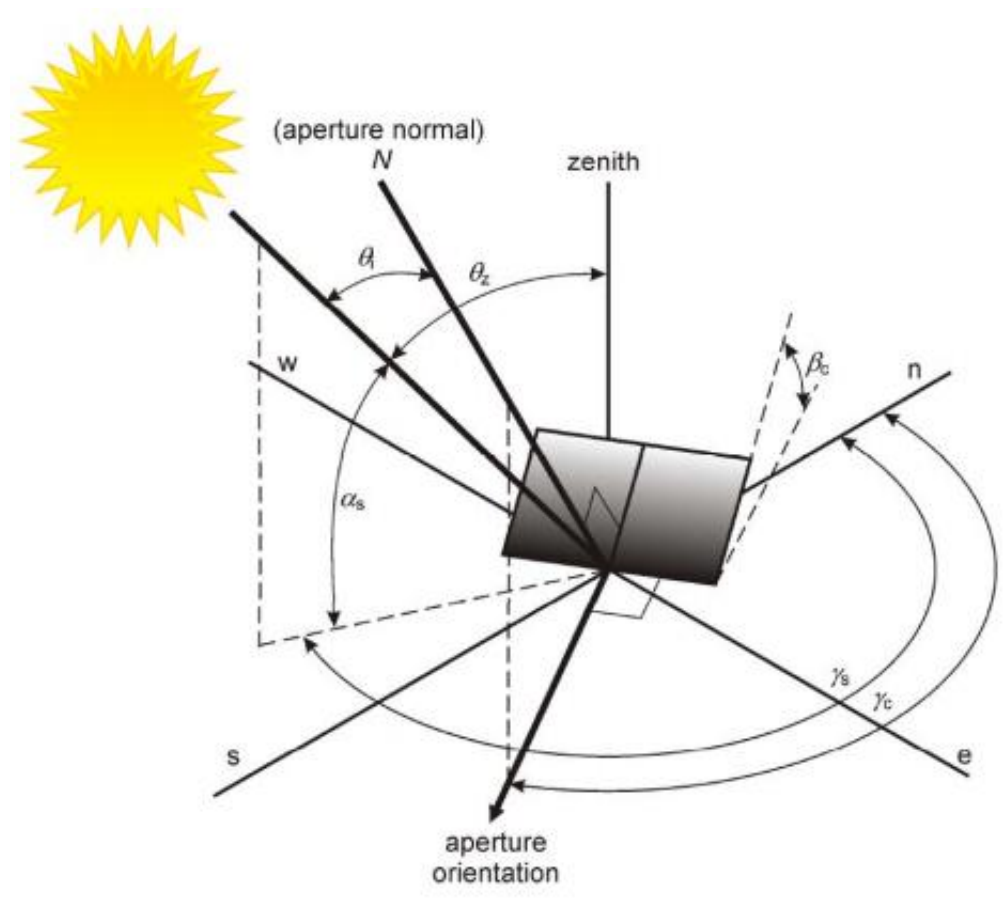

Figura 1.37. Representación gráfica de los ángulos solares sobre un espejo plano de un colector lineal de tipología LF [7].

Tabla 1.4. Definición de los ángulos solares de un colector LF [7].

\begin{tabular}{|c|c|c|}
\hline Nombre & Símbol & Explicación \\
\hline Ángulo Azimut solar & $\gamma_{s}$ & $\begin{array}{l}\text { El ángulo entre el Norte y la posición del Sol } \\
\text { proyectada sobre el plano horizontal. }\end{array}$ \\
\hline $\begin{array}{l}\text { Ángulo Elevación } \\
\text { solar }\end{array}$ & $\alpha_{s}$ & $\begin{array}{l}\text { Ángulo vertical entre una línea recta hasta el } \\
\text { Sol y el plano horizontal. }\end{array}$ \\
\hline Ángulo Zenit & $\theta_{z}$ & Ángulo complementario de $\alpha_{s} ; \theta_{z}=90^{\circ}-\alpha_{s}$ \\
\hline $\begin{array}{l}\text { Ángulo orientación } \\
\text { colector azimut }\end{array}$ & $\gamma_{c}$ & $\begin{array}{l}\text { Ángulo entre el Norte y la orientación del área } \\
\text { de apertura del colector. }\end{array}$ \\
\hline $\begin{array}{l}\text { Ángulo orientación } \\
\text { colector inclinación }\end{array}$ & $\beta_{c}$ & $\begin{array}{l}\text { Ángulo de elevación entre la superficie del } \\
\text { colector y el plano horizontal. }\end{array}$ \\
\hline Ángulo de incidencia & $\theta_{i}$ & $\begin{array}{l}\text { Ángulo entre una línea recta hasta el Sol y la } \\
\text { normal al colector. }\end{array}$ \\
\hline Declinación & $\Delta$ & $\begin{array}{l}\text { Ángulo entre los rayos solares y el plano } \\
\text { ecuatorial de la Tierra; Positivo en Verano } \\
\text { (entre finales de Marzo y finales de } \\
\text { Septiembre); }-23.45^{\circ}<\delta<23.45^{\circ}\end{array}$ \\
\hline Ángulo Horario & $\Omega$ & $\begin{array}{l}\text { Ángulo entre el meridiano del observador y el } \\
\text { meridiano paralelo a los rayos solares; } \\
\qquad-180^{\circ}<\omega<180^{\circ}\end{array}$ \\
\hline Latitud Geográfica & $\Phi$ & Positivo en el hemisferio norte; $-90^{\circ}<\phi<90^{\circ}$ \\
\hline Longitud Geográfica & $\Lambda$ & Positivo al este de Greenwich; $-180^{\circ}<\lambda<180^{\circ}$ \\
\hline
\end{tabular}


1. Estado de la técnica en las centrales termosolares con colectores solares lineales.

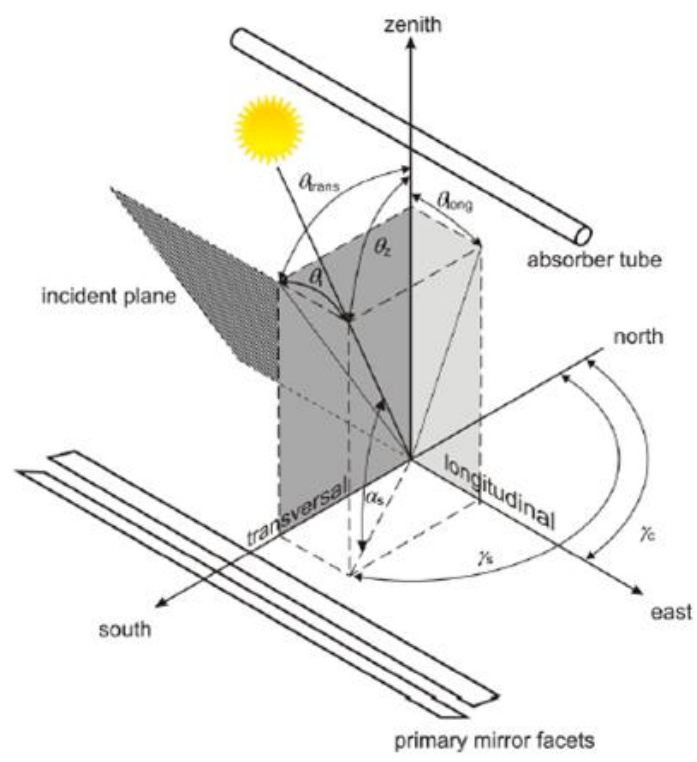

Figura 1.38. Definición de los parámetro trigonométricos que definen el ángulo de incidencia tanto longitudinal como transversal dependiendo de la orientación del colector LF en relación al Sol (orientación arbitraria, orientación Norte-Sur, orientación Este-Oeste) [7].

Tabla 1.5. Ecuaciones de ángulos de incidencia longitudinal $\left(\theta_{1}\right)$ y transversal $\left(\theta_{\text {trans }}\right)$ en LF [6].

\begin{tabular}{|c|c|c|}
\hline Orientación & Ángulo & Ecuación \\
\hline \multirow{2}{*}{ Arbitraria } & $\cos \theta_{l}$ & $=\sqrt{1-\left(\cos \left(\alpha_{\mathrm{s}}-\beta_{\mathrm{c}}\right)-\cos \beta_{\mathrm{c}} \cos \alpha_{\mathrm{s}}\left[1-\cos \left(\gamma_{\mathrm{s}}-\gamma_{\mathrm{c}}\right)\right]\right)^{2}}$ \\
\hline & $\tan \theta_{\text {trans }}$ & $\begin{aligned}=\cos \alpha_{s} \sin \left(\gamma_{s}-\right. & \left.\gamma_{c}\right) /\left(\sin \left(\alpha_{s}-\beta_{c}\right)\right. \\
& \left.+\sin \beta \cos \alpha_{s}\left[1-\cos \left(\gamma_{s}-\gamma_{c}\right)\right]\right)\end{aligned}$ \\
\hline \multirow{2}{*}{ Norte-Sur } & $\cos \theta_{l}$ & $=\sqrt{1-\cos ^{2} \alpha_{s} \cos ^{2} \gamma_{s}}$ \\
\hline & $\tan \theta_{\text {trans }}$ & $=\sin \gamma_{s} / \tan \alpha_{s}$ \\
\hline \multirow{2}{*}{ Este-Oeste } & $\cos \theta_{l}$ & $=\sqrt{1-\cos ^{2} \alpha_{s} \sin ^{2} \gamma_{s}}$ \\
\hline & $\tan \theta_{\text {trans }}$ & $=\cos \gamma_{s} / \tan \alpha_{s}$ \\
\hline
\end{tabular}

La eficiencia de pico es menor en los colectores LF que en los PTC, debido a los siguientes factores (figura 1.39): astigmatismo (los diferentes espejos que integran los colectores LF no pueden comportarse igual que un espejo parabólico continuo, la sombra proyectada por el tubo absorbedor y resto del reflector secundario que integran el receptor, la sombra que proyecta un espejo sobre los adyacentes.
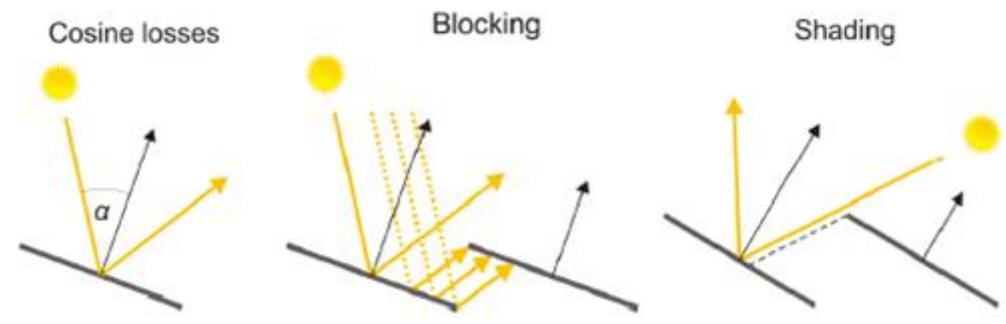

Figura 1.39. Explicación de los tres efectos físicos que disminuyen la radiación solar reflejada por los espejos de los colectores LF cuando el Sol está en posición bajas [7]. 
Los modificadores del ángulo de incidencia IAM, introducidos en base a la documentación aportada por el fabricante del colector, tienen en cuenta tanto los efectos ópticos y geométricos. Los efectos ópticos son las pérdidas por reflexión debido al choque y absorción de la irradiación solar al incidir sobre los espejos. Por su parte, los efectos geométricos incluyen la disposición de los colectores, la forma geométrica de los espejos y su propiedades de alineamiento, el sistema de seguimiento solar, y las características de cómo redirecciona la estructura del colector los rayos del sol sobre el tubo absorbedor.

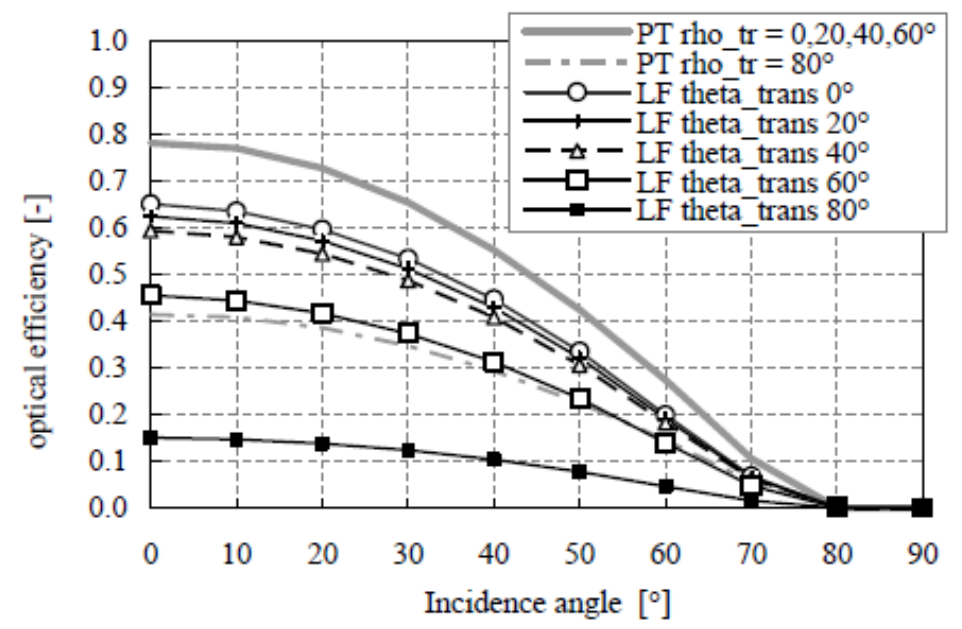

Figura 1.40. Estudio de la variación de la eficiencia óptica, de los colectores solares PTC y LF con tubos absorbedores al vacío, en relación al ángulo de incidencia de la radiación solar [7].

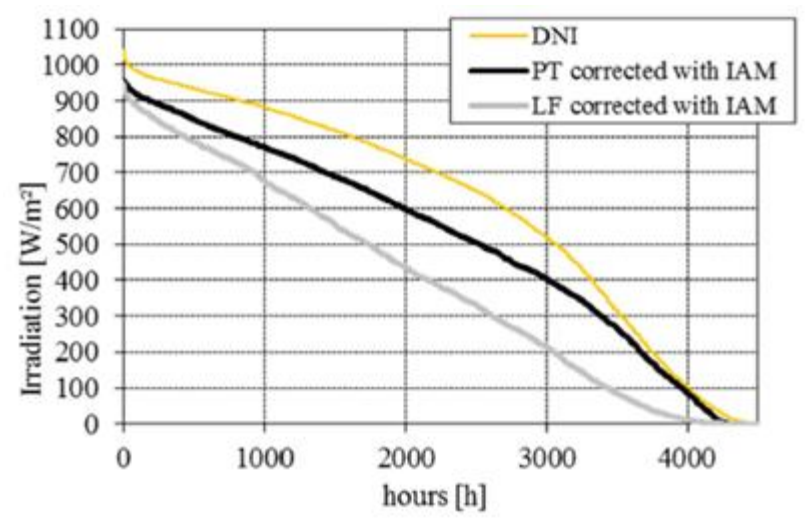

Figura 1.41. Se ilustra la radiación solar $\left(\mathrm{W} / \mathrm{m}^{2}\right)$ sobre los colectores lineales, LF y PTC, corregidas por el IAM, y su comparación con la radiación solar directa incidente DNI [7]. 
1. Estado de la técnica en las centrales termosolares con colectores solares lineales.

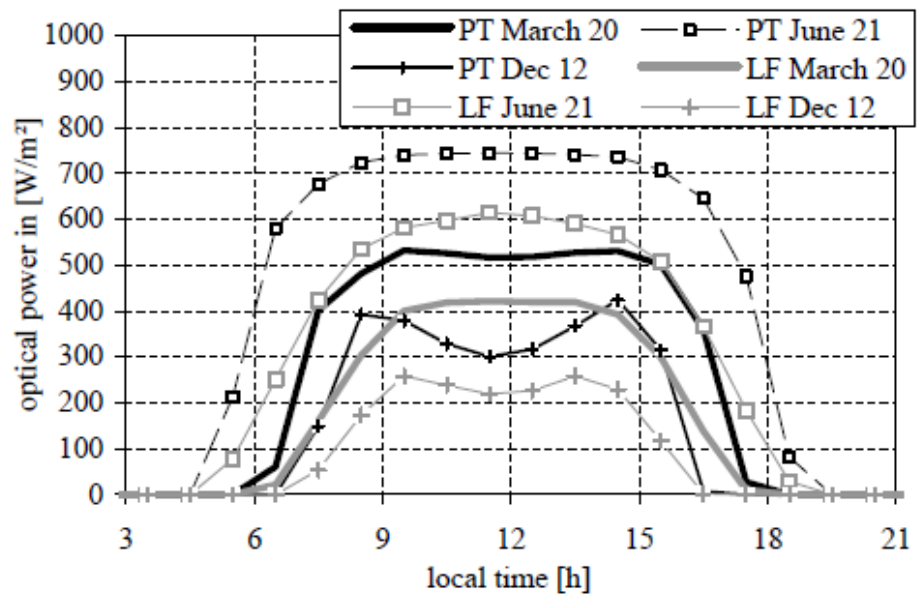

Figura 1.42. La potencia por metro cuadrado en LF muestra un pico en Verano, mientras que en PTC muestra una meseta más amplia. Localización considerada Daggett USA [7].

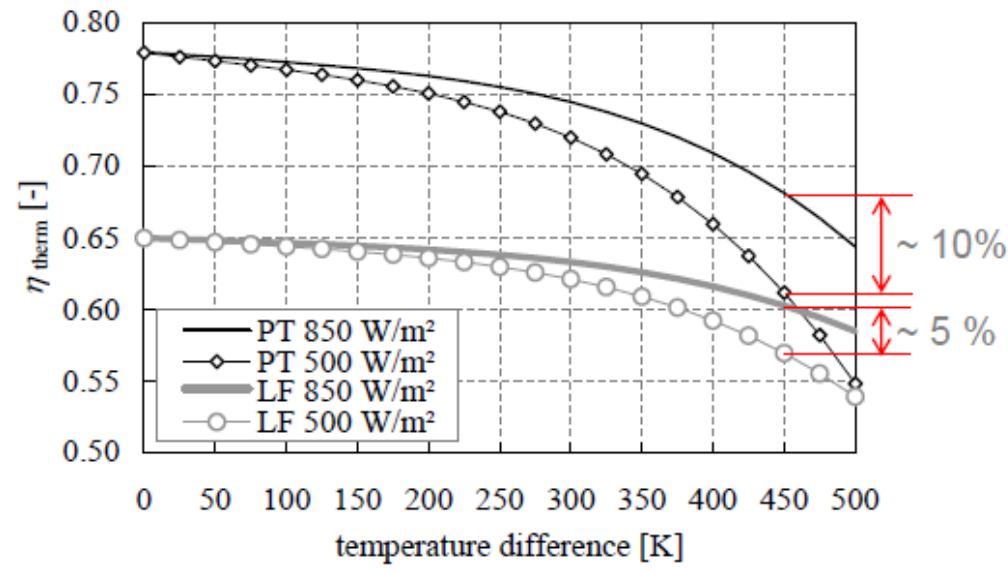

Figura 1.43. Comparación de la variación de la Eficiencia Térmica de PTC y LF respecto a la temperatura de los colectores. Se han estudiado dos escenarios radiación solar incidente de $850 \mathrm{~W} / \mathrm{m}^{2}$ y de $500 \mathrm{~W} / \mathrm{m}^{2}[7]$

Sin embargo, con bajos niveles de radiación solar directa DNI, los colectores solares LF absorben mayores cantidades de energía en relación a los colectores PTC. El motivo fundamental es el siguiente: el colector LF debido a su mayor anchura de apertura con el mismo tubo absorbedor consigue mayores factores de concentración solar, por tanto las pérdidas energéticas son relativamente menores. Parte del calor absorbido por el tubo es perdido hacia el exterior. Los mecanismos de transmisión de calor son similares a los descritos en colectores cilindro parabólicos (PTC) en el apartado anterior.

\subsection{Sistemas de Receptor Central o Torre Solar}

Un conjunto circular de heliostatos (grandes espejos con sistemas de seguimiento solar) concentra la luz solar en un receptor central montado sobre una torre, figura 1.44. Un medio de transferencia térmica en este receptor central absorbe la radiación altamente concentrada reflejada por los heliostatos, figura 1.45, y la convierte en energía térmica que se utiliza para generar el vapor sobrecalentado para la turbina. 
1. Estado de la técnica en las centrales termosolares con colectores solares lineales.

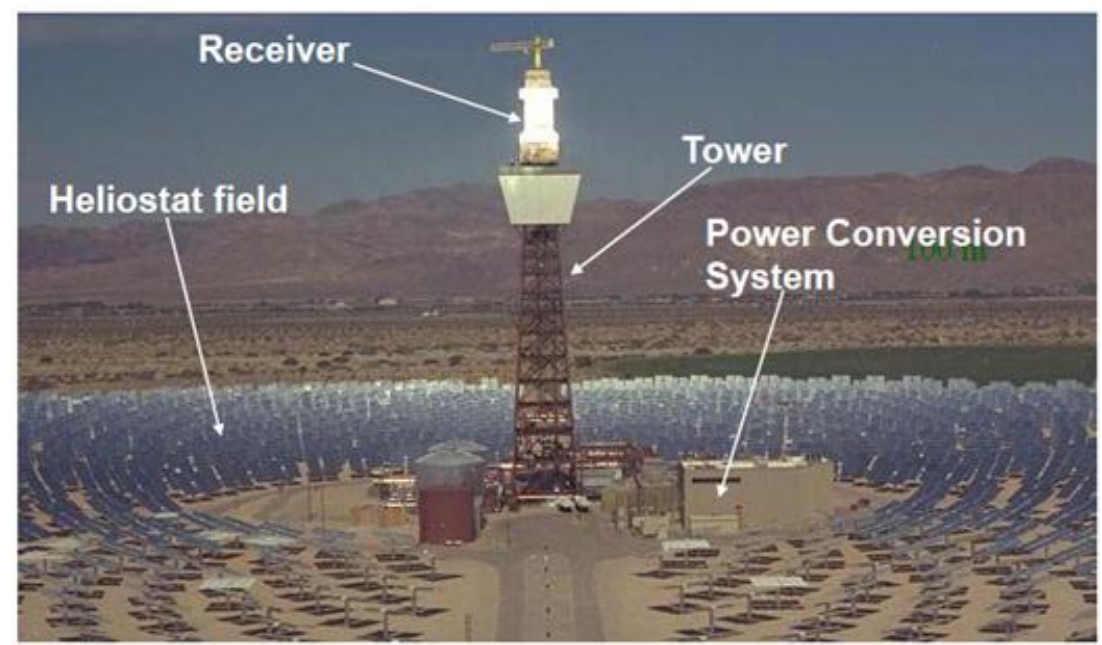

Figura 1.44. Planta Termosolar con tecnología de receptor central y campo de heliostatos [1].
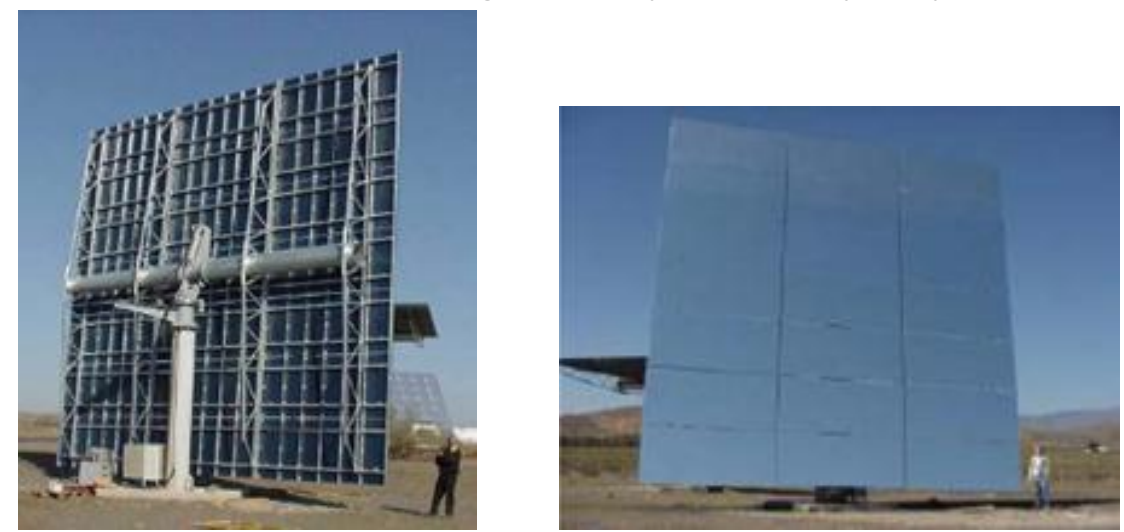

Figura 1.45. Vista de los colectores solares tipo Heliostatos en una Central Termosolar con tecnología de Torre Central [1].

Hasta la fecha, los fluidos de transferencia térmica con que trabajan los sistemas de receptor central incluyen agua/vapor (Ivanpah project [30]), sales fundidas (Gemasolar project [31], Crescent Dunes project [32]) y aire (Soltrec [33]). Si se utiliza aire o gas a presión a temperaturas muy elevadas de unos $1000^{\circ} \mathrm{C}$ o más como medio de transferencia térmica, puede también utilizarse en hibridación en los ciclos combinados (Solugas Project [34]), lo que saca el máximo rendimiento del ciclo ( $60 \%$ y más).

\subsection{Sistemas de Disco Parabólico}

En estos sistemas, un reflector parabólico en forma de disco concentra los rayos solares en un receptor situado en el punto focal de un disco (figura 1.46). La radiación concentrada se absorbe en el receptor para calentar un fluido o gas (aire) a unos $750^{\circ} \mathrm{C}$ [35]. Este fluido o gas se utiliza para generar electricidad en un pequeño pistón o motor Stirling o incluso en una micro-urbina, conectada al receptor. En Enero de 2010, Stirling Energy Systems y Tessera Solar pusierón en funcionamiento la primera planta de demostración con $1.5 \mathrm{MWe}$, la planta termosolar Maricopa Solar, utilizando tecnología Stirling, en Peoria, Arizona [36]. Al principio de 2011, Tessera Solar, vendío sus dos principales proyectos, el proyecto Imperial con $709 \mathrm{MW}$ y el proyecto Calico con 850 MW a AES Solar y K.Road, respectivamente [37-38] (figura 1.47). En 2015 una empresa Sueca, Rispasso Energy probó su sistema de generación de electricidad 
mediante discos Sterling en el Desierto Kalahari en Sudafrica [39] proporcionando una eficiencia energética del $34 \%$.

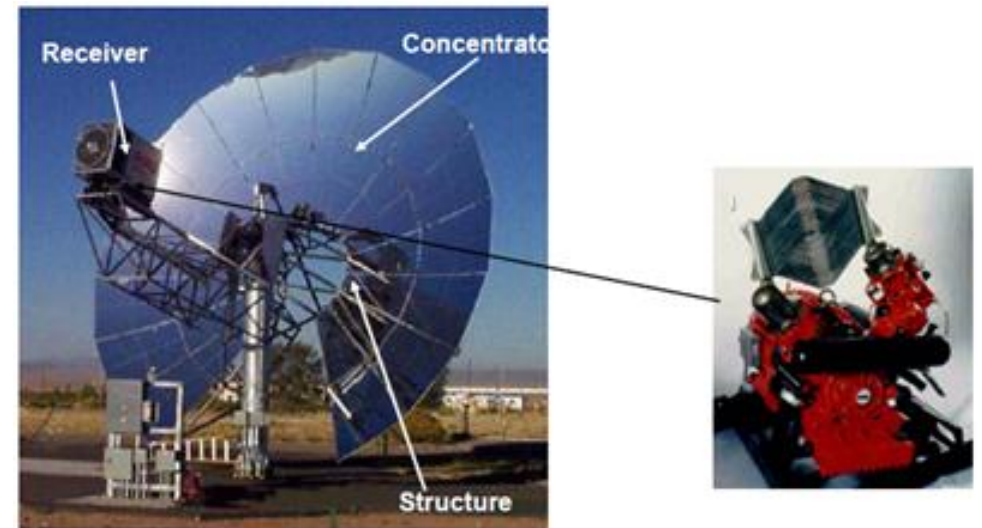

Figura 1.46. Captador Solar Tipo Disco de Stirling [36].

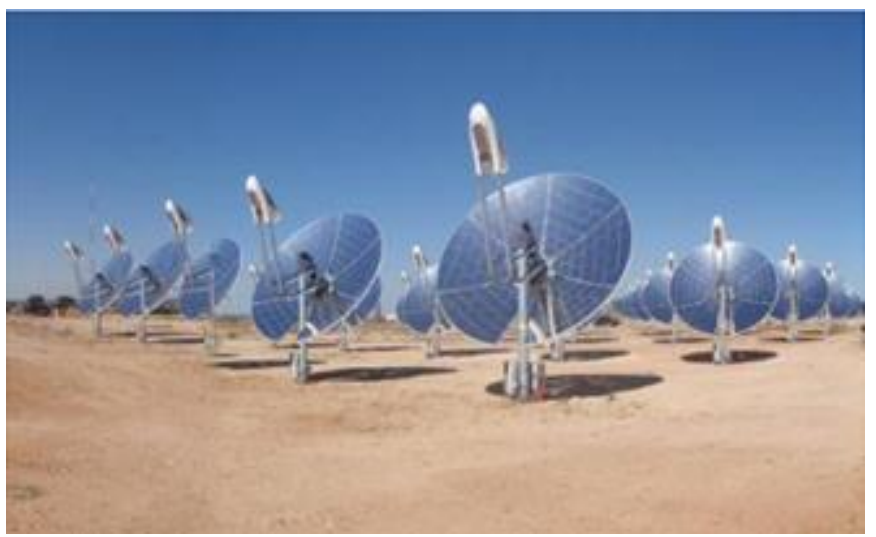

Figura 1.47. Captadores/concentradores solares tipo discos Stirling [1].

\subsection{Tecnologías de almacenamiento térmico en plantas termosolares}

La energía solar térmica de concentración puede llegar a ser más "gestionable" con la incorporación del almacenamiento térmico. Esto significa que la central termosolar puede funcionar de forma más flexible, no sólo bajo condiciones de irradiación óptimas. A los sistemas de almacenamiento térmico se les suele denominar Thermal Energy Storage (TES), esta tecnología almacena parte de la energía térmica recogida por el campo solar para convertirla más adelante en electricidad [40]. El almacenamiento puede adaptar el perfil de demanda de producción a lo largo del día y puede aumentar el rendimiento energético total de una planta para una potencia máxima dada de la turbina. Esto se logra almacenando el exceso de energía de un campo solar mayor antes de su uso en la turbina [41-42]. Eventualmente las plantas con almacenamiento pueden llegar a operarse con un factor de capacidad elevado, lo que significa que concentrar la energía solar puede proporcionar electricidad en base en ubicaciones apropiadas. Las diferentes configuraciones de plantas termosolares requieren soluciones de almacenamiento de energía térmica a la medida adecuadas para la mezcla de tecnologías de cada caso en particular, como, por ejemplo, el fluido de trabajo primario, la temperatura y la presión de funcionamiento, la capacidad y el nivel de potencia. Con el fin de lograr una mayor eficiencia y economía, los sistemas TES 
1. Estado de la técnica en las centrales termosolares con colectores solares lineales.

requerirán una variedad de tecnologías de almacenamiento, materiales y métodos para alcanzar todas las especificaciones de las centrales.

Las tecnologías de almacenamiento pueden ser directas o indirectas:

- En las indirectas el medio de almacenamiento no es calentado directamente por los concentradores. Los sistemas indirectos utilizan un fluido de transferencia térmica, generalmente un aceite sintético en el caso de los PTC, que pasa por un intercambiador térmico con el medio de almacenamiento, generalmente sales fundidas, calentándolo indirectamente. Generalmente el fluido de transferencia es aceite sintético.

- En las directas se utiliza el mismo fluido como fluido caloportador HTF en los colectores solares, y para almacenamiento térmico en los tanques del sistema TES. La principal ventaja de estos sistemas es que no utilizan intercambiador de calor intermedio entre el sistema TES y el campo solar. La utilización de dos fluidos diferentes, uno en el campo solar y otro para el sistema TES, permite optimizar diferentes propiedades físicas por separado en las dos instalaciones. En el campo solar el objetivo principal es obtener un alto coeficiente de transferencia de calor, para reducir la longitud de los colectores solares, y minimizar su viscosidad para minimizar las pérdidas de presión y consumo eléctrico de las bombas del campo solar. El coste del fluido de almacenamiento y el calor específico a presión constante $\mathrm{Cp}$ son las dos principales variables a optimizar en el fluido para almacenamiento térmico TES.

\subsubsection{Almacenamiento indirecto con sales fundidas}

Un ejemplo de este tipo de tecnología se observa en las centrales de Andasol 3 de Granada (España), que emplea un tanque de frío (alrededor de $290^{\circ} \mathrm{C}$ ) y un tanque caliente (alrededor de $390^{\circ} \mathrm{C}$ ) de sales fundidas, con 7.5 horas de almacenamiento, con unas 28.500 toneladas en cada tanque [43]. Las sales frías se pasan por un intercambiador térmico con el aceite que es calentado por el concentrador, y se almacenan después en el tanque caliente para uso futuro. Para extraer el calor se invierte el proceso por el intercambiador, con el fin de transferir el calor de nuevo al aceite y producir vapor para el generador. Una ventaja de este proceso es que los aceites para la transferencia térmica son tecnología probada y testada, y un inconveniente es el alto precio de los intercambiadores térmicos, que añaden costes a la inversión.

\subsubsection{Almacenamiento directo de vapor}

Su capacidad de almacenamiento es limitada debido a los costes elevados de los recipientes a presión para grandes capacidades de almacenamiento y volúmenes de vapor. En principio esto es una tecnología convencional, conocida también como almacenamiento de Ruth. El uso más idóneo de esta tecnología es como almacenamiento intermedio para potencia punta. Esta técnica se utiliza comercialmente en la planta PS10 y ofreciendo entre 30 minutos y una hora de operación extra [44]. 
1. Estado de la técnica en las centrales termosolares con colectores solares lineales.

\subsubsection{Almacenamiento indirecto con hormigón}

El uso de hormigón para almacenar calor se encuentra en diferentes etapas de desarrollo en instalaciones prototipo con buenas perspectivas de desarrollo. El hormigón opera a temperaturas entre 400 y $500^{\circ} \mathrm{C}$, y es un diseño modular y escalable para capacidades de 500 kWh a 1000 MWh. Actualmente los costes de inversión son del orden de 30 euros/KWh, pero el objetivo es menos de 20 euros/kWh. Los módulos de primera generación tienen una capacidad de $300 \mathrm{kWh}$ y llevan dos años operativos [45]. Los de segunda generación tienen 400 kWh y están ya listos para una demostración [46].

\subsubsection{Almacenamiento indirecto en un medio de cambio de fase}

Esta tecnología se encuentra en proceso de desarrollo y utiliza el punto de fusión/congelación de sales como los nitratos de sodio o de potasio para almacenar y obtener calor para la condensación y evaporación de vapor en plantas de vapor directas [47]. Sólo ha sido testada en algunos prototipos [48], y no hay de momento aplicaciones comerciales. En este sistema fluye líquido de transferencia térmica caliente por un colector incrustado en los materiales de fase cambiante, y transfiere su calor al material de almacenamiento. La ventaja principal de esta tecnología es su densidad volumétrica y el bajo coste de los materiales de almacenamiento. Existen algunos retos de desarrollo de este método que deben superarse antes de hacer comercial esta solución.

\subsection{Centrales termosolares con colectores lineales, con aceites térmicos como fluido caloportador, y ciclos de potencia Rankine (Oil + Rankine)}

En este apartado se realiza una comparativa de diferentes aceites térmicos: Dowtherm A [49], Syltherm 800 [50], Therminol VP1 [51] y Therminol 75 [52]. Cada uno de estos fluidos caloportadores tiene diferente temperatura máxima de operación, y por tanto, la eficiencia de la planta termosolar es diferente. Los resultados obtenidos de la simulación con Thermoflow [5] (figuras 1.48 y 1.49), de los correspondientes balances energéticos están resumidos en las tablas 1.6 a 1.9 para colectores PTC y LF respectivamente. Como hemos comentado podemos apreciar existe una correlación entre la eficiencia neta de la planta termosolar y la superficie efectiva de los colectores solares. En las tablas 1.6 a 1.9 se caracterizan en términos de área de apertura efectiva y de coste de los colectores para diferentes temperaturas de entrada en turbina TIT para cada uno de los aceites térmicos estudiados.

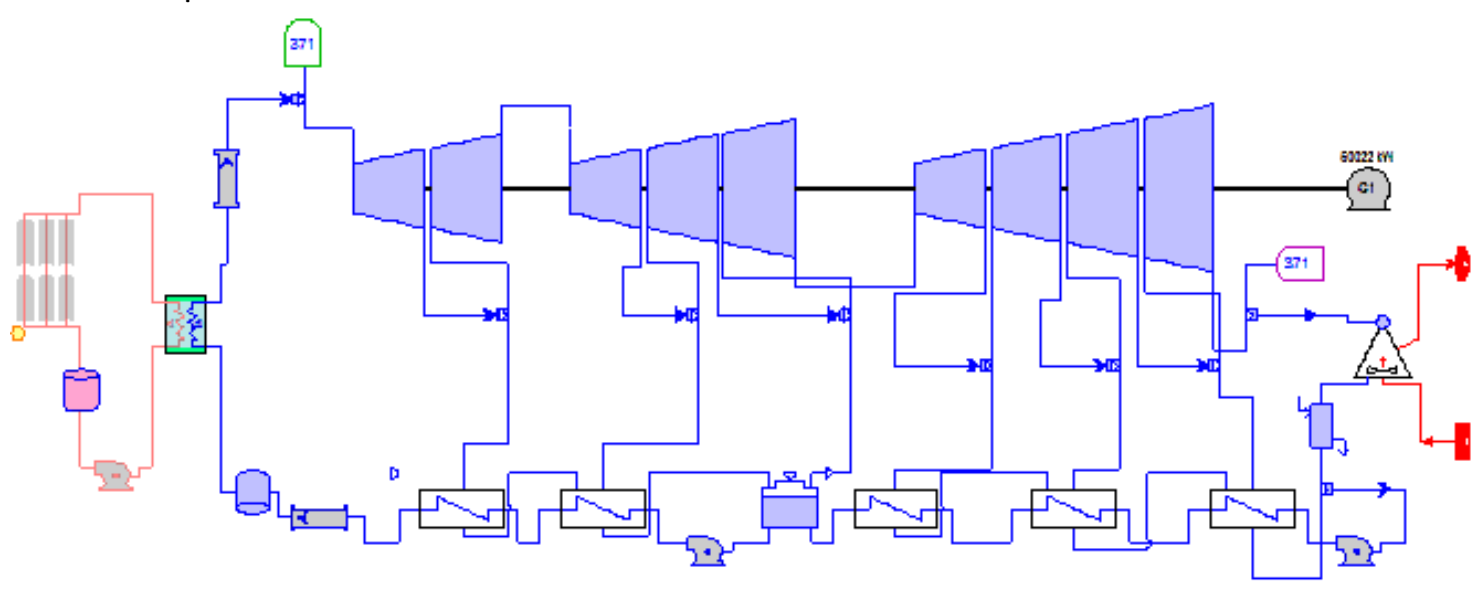

Figura 1.48. Esquema de planta solar con campo solar con aceite térmico o sales fundidas como HTF, y ciclo de potencia Rankine sin Recalentamiento. 
1. Estado de la técnica en las centrales termosolares con colectores solares lineales.

Tabla 1.6. Eficiencia y potencia de las centrales termosolares con colectores lineales PTC y ciclo de potencia Rankine sin Recalentamiento. Valores en punto de diseño.

\begin{tabular}{|c|c|c|c|c|c|c|c|}
\hline $\begin{array}{l}\text { TIT } \\
\left({ }^{\circ} \mathrm{C}\right)\end{array}$ & $\begin{array}{l}\text { SF } \\
\text { Config. }\end{array}$ & $\begin{array}{l}\text { PTC } \\
\text { Area } \\
\left(\mathrm{m}^{2}\right) \\
\end{array}$ & $\begin{array}{l}\text { PTC } \\
\text { Coste } \\
\text { (M\$) }\end{array}$ & $\begin{array}{l}\text { Eficiencia } \\
\text { Neta } \\
(\%)\end{array}$ & $\begin{array}{l}\text { Eficiencia } \\
\text { Bruta } \\
(\%)\end{array}$ & $\begin{array}{l}\text { Potencia } \\
\text { Bruta } \\
\text { (Mwe) }\end{array}$ & $\begin{array}{l}\text { Potencia } \\
\text { Neta } \\
\text { (MWe) }\end{array}$ \\
\hline 415 & Dowtherm A & 201319 & 68.2 & 35.40 & 37.52 & 50006 & 47175 \\
\hline 390 & Syltherm 800 & 203879 & 69.1 & 34.69 & 36.94 & 50025 & 46978 \\
\hline 390 & Therminol VP1 & 203693 & 68.9 & 34.74 & 36.92 & 50025 & 47083 \\
\hline 375 & Therminol 75 & 205506 & 69.6 & 34.32 & 36.53 & 50015 & 46987 \\
\hline
\end{tabular}

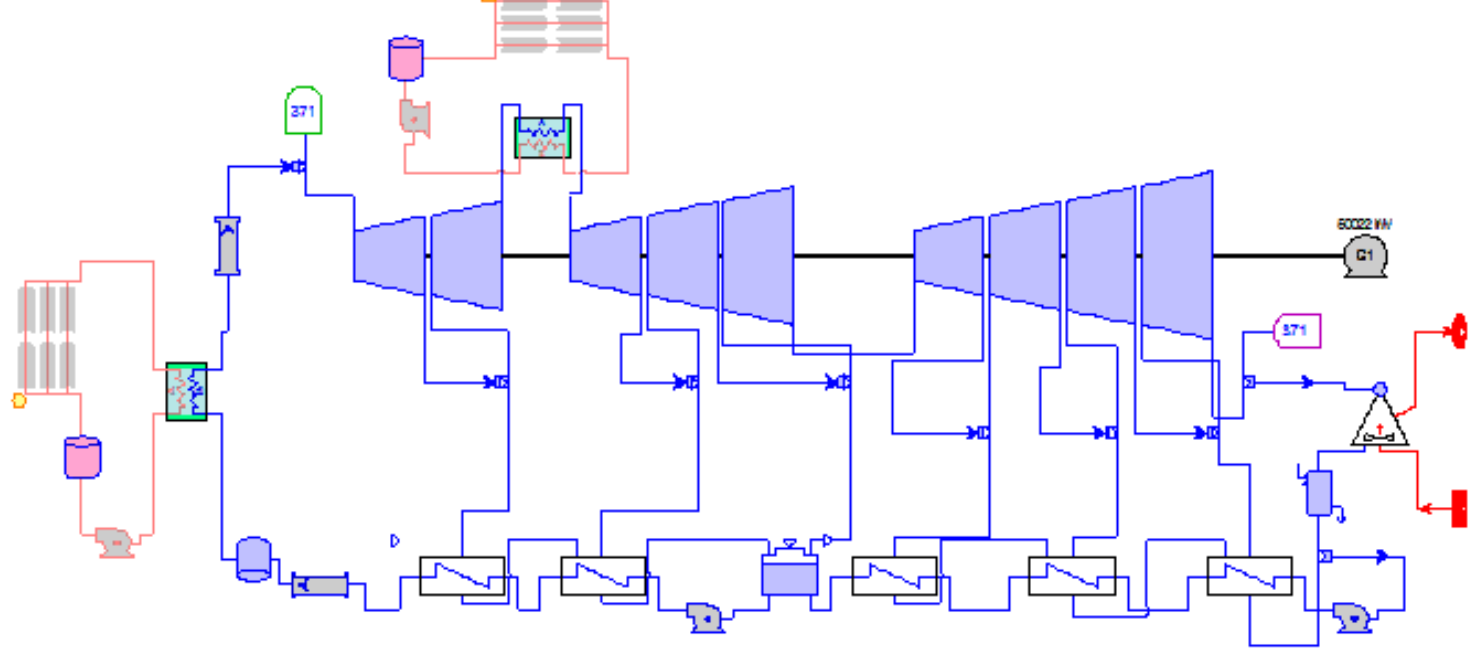

Figura 1.49. Esquema de planta solar con campo solar con aceite térmico o sales fundidas como HTF, y ciclo de potencia Rankine con Recalentamiento.

Tabla 1.7. Eficiencia y potencia de las centrales termosolares con colectores lineales LF y ciclo de potencia Rankine sin Recalentamiento. Valores en punto de diseño.

\begin{tabular}{|c|c|c|c|c|c|c|c|}
\hline $\begin{array}{l}\text { TIT } \\
\left({ }^{\circ} \mathrm{C}\right)\end{array}$ & $\begin{array}{l}\text { SF } \\
\text { Config. }\end{array}$ & $\begin{array}{l}\mathrm{LF} \\
\text { Area } \\
\left(\mathrm{m}^{2}\right)\end{array}$ & $\begin{array}{l}\text { LF } \\
\text { Coste } \\
\text { (M\$) }\end{array}$ & $\begin{array}{l}\text { Eficiencia } \\
\text { Neta } \\
(\%)\end{array}$ & $\begin{array}{l}\text { Eficiencia } \\
\text { Bruta } \\
(\%)\end{array}$ & $\begin{array}{l}\text { Potencia } \\
\text { Bruta } \\
\text { (Mwe) }\end{array}$ & $\begin{array}{l}\text { Potencia } \\
\text { Neta } \\
\text { (MWe) }\end{array}$ \\
\hline 415 & Dowtherm A & 234944 & 61.3 & 35.41 & 37.51 & 50006 & 47200 \\
\hline 390 & Syltherm 800 & 239310 & 62.5 & 34.71 & 36.93 & 50025 & 47013 \\
\hline 390 & Therminol VP1 & 238651 & 62.3 & 34.76 & 36.91 & 50025 & 47105 \\
\hline 375 & Therminol 75 & 241776 & 63.1 & 34.33 & 36.53 & 50038 & 47028 \\
\hline
\end{tabular}

Tabla 1.8. Eficiencia y potencia de las centrales termosolares con colectores lineales PTC y ciclo de potencia Rankine con Recalentamiento. Valores en punto de diseño.

\begin{tabular}{|c|c|c|c|c|c|c|c|}
\hline $\begin{array}{l}\text { TIT } \\
\left({ }^{\circ} \mathrm{C}\right)\end{array}$ & $\begin{array}{l}\text { SF } \\
\text { Config. }\end{array}$ & $\begin{array}{l}\text { PTC } \\
\text { Area } \\
\left(\mathrm{m}^{2}\right)\end{array}$ & $\begin{array}{l}\text { PTC } \\
\text { Coste } \\
\text { (M\$) }\end{array}$ & $\begin{array}{l}\text { Eficiencia } \\
\text { Neta } \\
(\%)\end{array}$ & $\begin{array}{l}\text { Eficiencia } \\
\text { Bruta } \\
(\%)\end{array}$ & $\begin{array}{l}\text { Potencia } \\
\text { Bruta } \\
\text { (Mwe) }\end{array}$ & $\begin{array}{l}\text { Potencia } \\
\text { Neta } \\
\text { (MWe) }\end{array}$ \\
\hline 415 & Dowtherm & 193910 & 65.7 & 37.00 & 39.2 & 50017 & 47211 \\
\hline 390 & Syltherm & 195622 & 66.3 & 36.25 & 38.56 & 50022 & 47035 \\
\hline 390 & Therminol & 195584 & 66.3 & 36.33 & 38.51 & 50022 & 47182 \\
\hline 375 & Therminol & 197115 & 66.8 & 35.92 & 38.1 & 50010 & 47140 \\
\hline
\end{tabular}

Otro valor a destacar son las pérdidas energéticas debido al consumo de equipos auxiliares. Los principales consumos eléctricos son debido a los ventiladores de los condensadores de 
agua en el ciclo Rankine $\sim 1.5 \mathrm{MWe}$, la bomba principal de agua de alimentación del ciclo Rankine $\sim 0.5 \mathrm{MWe}$, las bombas de los campos solares con aceite térmico como HTF consumen alrededor de 0.5 MWe entre las dos (campo solar principal y campo solar de recalentamiento). En total se cuantifican unos 2.5-3 MWe de consumos auxiliares.

En las tablas 1.6 a 1.9 también se incluye el coste de los colectores solares para los diferentes aceites térmicos utilizados. Como se puede apreciar no existe una diferencia de costes significativas entre las diferentes alternativa de aceites térmicos estudiadas. En todos los casos se considera que los tubos absorbedores están constituidos por acero al carbono, y su coste unitario es: PTC $\left(292 \$ / \mathrm{m}^{2}\right)$ y LF $\left(225 \$ / \mathrm{m}^{2}\right)$ [53].

Tabla 1.9. Eficiencia y potencia de las centrales termosolares con colectores lineales LF y ciclo de potencia Rankine con Recalentamiento. Valores en punto de diseño.

\begin{tabular}{|c|c|c|c|c|c|c|c|}
\hline TIT & SF & LF & LF & Eficiencia & Eficiencia & Potencia & Potencia \\
\hline$\left({ }^{\circ} \mathrm{C}\right)$ & Config. & $\begin{array}{l}\text { Area } \\
\left(\mathrm{m}^{2}\right)\end{array}$ & $\begin{array}{l}\text { Coste } \\
\text { (M\$) }\end{array}$ & $\begin{array}{l}\text { Neta } \\
(\%)\end{array}$ & $\begin{array}{l}\text { Bruta } \\
\text { (\%) }\end{array}$ & $\begin{array}{l}\text { Bruta } \\
\text { (Mwe) }\end{array}$ & $\begin{array}{l}\text { Neta } \\
\text { (MWe) }\end{array}$ \\
\hline 415 & Dowtherm A & 227119 & 59.3 & 37.02 & 39.19 & 50037 & 47266 \\
\hline 390 & Syltherm 800 & 230048 & 60.1 & 36.28 & 38.54 & 50022 & 47086 \\
\hline 390 & Therminol VP1 & 229652 & 59.9 & 36.34 & 38.51 & 50022 & 47215 \\
\hline 375 & Therminol 75 & 232124 & 60.6 & 35.93 & 38.10 & 50019 & 47176 \\
\hline
\end{tabular}

1.9. Centrales termosolares con colectores lineales (PTC o LF), con sales fundidas como fluido caloportador, ciclos de potencia Rankine (MS + Rankine)

El incremento de la eficiencia neta proporcionado por las sales fundidas (Solar Salt [54], Hitec $X L$ [55]) como fluido caloportador es cuantificado en las tablas 1.10 a 1.13 . Como puede apreciarse el rendimiento se incrementa desde un $35.4 \%$ hasta un $38.4 \%$, es decir, alrededor del $3 \%$ para la tipología de ciclo de potencia Rankine sin recalentamiento. Para el ciclo de potencia con recalentamiento su incremento de eficiencia va desde el $37 \%$ hasta el $40.5 \%$.

Tabla 1.10. Eficiencia y potencia de las centrales termosolares con colectores lineales PTC y ciclo de potencia Rankine sin Recalentamiento. Valores en punto de diseño.

\begin{tabular}{|c|c|c|c|c|c|c|c|}
\hline TIT & SF & PTC & PTC & Eficiencia & Eficiencia & Potencia & Potencia \\
\hline$\left({ }^{\circ} \mathrm{C}\right)$ & Config. & $\begin{array}{l}\text { Area } \\
\left(\mathrm{m}^{2}\right)\end{array}$ & $\begin{array}{l}\text { Coste } \\
\text { (M\$) }\end{array}$ & $\begin{array}{l}\text { Neta } \\
(\%)\end{array}$ & $\begin{array}{l}\text { Bruta } \\
\text { (\%) }\end{array}$ & $\begin{array}{l}\text { Bruta } \\
\text { (MWe) }\end{array}$ & $\begin{array}{l}\text { Neta } \\
\text { (MWe) }\end{array}$ \\
\hline 550 & Solar Salt & 198092 & 89.6 & 38.46 & 40.35 & 50018 & 47679 \\
\hline 530 & Hitec XL & 196380 & 88.8 & 38.07 & 39.96 & 50017 & 47643 \\
\hline
\end{tabular}

Tabla 1.11. Eficiencia y potencia de las centrales termosolares con colectores lineales LF y ciclo de potencia Rankine sin Recalentamiento. Valores en punto de diseño.

\begin{tabular}{|c|c|c|c|c|c|c|c|}
\hline TIT & SF & $\mathrm{LF}$ & $\mathrm{LF}$ & Eficiencia & Eficiencia & Potencia & Potencia \\
\hline$\left({ }^{\circ} \mathrm{C}\right)$ & Config. & $\begin{array}{l}\text { Area } \\
\left(\mathrm{m}^{2}\right)\end{array}$ & $\begin{array}{l}\text { Coste } \\
\text { (M\$) }\end{array}$ & $\begin{array}{l}\text { Neta } \\
\text { (\%) }\end{array}$ & $\begin{array}{l}\text { Bruta } \\
\text { (\%) }\end{array}$ & $\begin{array}{l}\text { Bruta } \\
\text { (Mwe) }\end{array}$ & $\begin{array}{l}\text { Neta } \\
\text { (MWe) }\end{array}$ \\
\hline 550 & Solar Salt & 227083 & 79.1 & 38.46 & 40.34 & 50018 & 47686 \\
\hline 530 & Hitec XL & 226578 & 78.8 & 38.07 & 39.96 & 50017 & 47649 \\
\hline
\end{tabular}

Tabla 1.12. Eficiencia y potencia de las centrales termosolares con colectores lineales PTC y ciclo de potencia Rankine con Recalentamiento. Valores en punto de diseño. 
1. Estado de la técnica en las centrales termosolares con colectores solares lineales.

\begin{tabular}{|c|c|c|c|c|c|c|c|}
\hline TIT & SF & PTC & PTC & Eficiencia & Eficiencia & Potencia & Potencia \\
\hline$\left({ }^{\circ} \mathrm{C}\right)$ & Config. & $\begin{array}{l}\text { Area } \\
\left(\mathrm{m}^{2}\right)\end{array}$ & $\begin{array}{l}\text { Coste } \\
\text { (M\$) }\end{array}$ & $\begin{array}{l}\text { Neta } \\
(\%)\end{array}$ & $\begin{array}{l}\text { Bruta } \\
(\%)\end{array}$ & $\begin{array}{l}\text { Bruta } \\
\text { (Mwe) }\end{array}$ & $\begin{array}{l}\text { Neta } \\
\text { (MWe) }\end{array}$ \\
\hline 550 & Solar Salt & 192494 & 87.1 & 40.50 & 42.32 & 50028 & 47880 \\
\hline 530 & Hitec XL & 190616 & 86.2 & 40.06 & 41.88 & 50025 & 47846 \\
\hline
\end{tabular}

Sin embargo, si se cuantifica en términos monetarios este incremento de eficiencia neta del ciclo no se traduce linealmente en una reducción de costes de inversión en el campo solar a pesar de que su área de apertura efectiva es menor. La principal razón se encuentra en el coste de los materiales de los tubos absorbedores. Debida a la utilización de sales fundidas es necesario utilizar aceros inoxidables austeníticos con aleación de Titanio (Ti) o Niobio ( $\mathrm{Nb}$ ) para su estabilización. El coste unitario de los colectores lineales utilizando sales fundidas como fluido caloportador es: PTC (390 $\left.\$ / \mathrm{m}^{2}\right)$ y LF $\left(300 \$ / \mathrm{m}^{2}\right)$ [53].

Tabla 1.13. Eficiencia y potencia de las centrales termosolares con colectores lineales LF y ciclo de potencia Rankine con Recalentamiento. Valores en punto de diseño.

\begin{tabular}{|c|c|c|c|c|c|c|c|}
\hline $\begin{array}{l}\mathrm{TIT} \\
\left({ }^{\circ} \mathrm{C}\right)\end{array}$ & $\begin{array}{l}\text { SF } \\
\text { Config. }\end{array}$ & $\begin{array}{l}\text { LF } \\
\text { Area } \\
\left(\mathrm{m}^{2}\right)\end{array}$ & $\begin{array}{l}\text { LF } \\
\text { Coste } \\
\text { (M\$) }\end{array}$ & $\begin{array}{l}\text { Eficiencia } \\
\text { Neta } \\
(\%)\end{array}$ & $\begin{array}{l}\text { Eficiencia } \\
\text { Bruta } \\
\text { (\%) }\end{array}$ & $\begin{array}{l}\text { Potencia Bruta } \\
\text { (Mwe) }\end{array}$ & $\begin{array}{l}\text { Potencia } \\
\text { Neta } \\
\text { (MWe) }\end{array}$ \\
\hline 550 & Solar Salt & 220498 & 76.7 & 40.50 & 42.31 & 50053 & 47912 \\
\hline 530 & Hitec XL & 219906 & 76.5 & 40.06 & 41.88 & 50050 & 47877 \\
\hline
\end{tabular}

\subsection{Conclusiones}

Se han comparado los resultados de eficiencia neta obtenidos en los apartados 1.8 y 1.9 con los de Giostri [56], y se concluye que los resultados calculados son similares. Las hipótesis de cálculo de las pérdidas auxiliares del ciclo de potencia del presente trabajo son algo menos conservadoras, y la localización y temperatura ambiente es levemente diferente.

Se concluye que con el aceite térmico Therminol VP-1 en la configuración de ciclo Rankine sin recalentamiento en turbinas, la eficiencia del ciclo de potencia es del $34.7 \%$ tablas 1.6 y 1.7 . Con la configuración con recalentamiento se incrementa hasta el $36.4 \%$ la eficiencia del ciclo (tablas 1.8 y 1.9). En ambos casos la TIT es de $390^{\circ} \mathrm{C}$. En el caso del Dowtherm A las eficiencias netas se mejoran en un $0.7 \%$ en términos absolutos, con una $\mathrm{TIT}=415^{\circ} \mathrm{C}$, tablas 1.6 a 1.9.

Con sales fundidas (Solar Salt o Hitec XL) como fluidos caloportadores (HTF) los rendimientos energéticos se incrementan hasta un $40.5 \%$ con Solar Salt, y $40 \%$ con Hitec XL, en ambos casos con recalentamiento en el ciclo Rankine (tablas 1.12 y 1.13 ). Estos valores se ven reducidos en la configuración sin recalentamiento hasta $38.4 \%$ y $38 \%$ respectivamente, tablas 1.10 y 1.11 .

Los valores máximos de rendimiento entorno al $41 \%$ para una $\mathrm{TIT}=565^{\circ} \mathrm{C}$ con ciclos Rankine quedan confirmado por Turchi [57].

\subsection{Referencias}

[1] Eduardo Zarza. Basic principles of solar radiation and STE plants. CIEMAT, Plataforma Solar de Almería. $3^{\text {rd }}$ SFERA Summer School. 
1. Estado de la técnica en las centrales termosolares con colectores solares lineales.

[2] Geoffrey Toon Jet Propulsion Laboratory, California Institute of Technology. The Solar Spectrum: an Atmospheric Remote Sensing Perspective. Noble Seminar, University of Toronto, Oct 21, 2013

[3] The University of Tennessee Institute of Agriculture. https://ag.tennessee.edu

[4] SolarGis, Direct Normal Irradiation (DNI). http://solargis.info

[5] Thermoflow Software manual. https://www.thermoflow.com/

[6] L. Valenzuela. STE plants with parabolic trough collectors. CIEMAT, Plataforma Solar de Almería. http://sfera.sollab.eu/downloads/Schools/Loreto Valenzuela Presentation.pdf

[7] Jan Fabian Feldhoff. Linear Fresnel Collectors. SFERA Summer School 2012, June 28, 2012, Almería, Spain.

[8] Dudley, E.V. et al. SEGS LS-2 Solar Collector (Test Results), Report No. SAND94-1884, Sandia National Laboratories, California (EEUU), 1994.

[9] Solar Electric Generating System IX Technical Description, LUZ International Limited: 1990. http://www.nrel.gov/csp/solarpaces/project detail.cfm/projectlD=36

[10] Eurotrough solar collectors: ET-I en 2000, ET-II en 2005.

http://www.fika.org/jb/resources/EuroTrough.pdf

[11] Sener solar collector: SENERtrough ${ }^{\circledR}-2$.

http://www.sener-power-process.com

[12] Abengoa solar collectors: ASTRO 150 en 2007, Phoenix en 2009, E2 (Eucumsa) en 2011, ST8 Spacetube en 2013.

http://energy.gov/sites/prod/files/2014/01/f7/csp review meeting 042513 price.pdf

[13 ]SkyFuel solar collector: SkyTrough http://www.skyfuel.com/skytrough.shtml

[14] Flagsol solar collectors: HelioTrough. http://www.heliotrough.com/publications.html

[15] Ultimate Trough, Flabeg. www.flabeg-fe.com/en/engineering/ultimate-trough.html

[16] Fresnel lens. https://www.bibnum.education.fr

[17] Areva solar Fresnel solar collector technology. http://www.areva.com

[18]Pye, J.D., Morrison, G., Behnia, M., 2003. Transient modelling of cavity receiver heat transfer for the compact linear Fresnel reflector. Manufacturing Engineering, 1-9.

[19]Reynolds, D.J., Jance, M.J., Behnia, M., Morrison, G.L., 2004. An experimental and computational study of the heat loss characteristics of a trapezoidal cavity absorber. Solar Energy 2004, 229-234

[20] Novatec Solar. http://www.novatecsolar.com/

[21] Camille Bachelier. SAM Linear Fresnel solar boiler model. SAM Virtual Conference 2013. Novatec Solar 23-07-2013. https://sam.nrel.gov/content/sam-virtual-conference-july-23-2013

[22] System Advisor Model (SAM) software technical Manual. https://sam.nrel.gov

[23] SCHOOT receivers. http://www.schott.com/csp/english/schott-solar-receivers.html

[24] Archimede Solarenergy receivers. http://www.archimedesolarenergy.it

[25] R.Forristal. Heat Transfer Analysis and Modeling of a Parabolic Trough Solar Receiver Implemented in Engineering Equation Solver. NREL/TP-550-34169, October 2003.

[26] A.Maccari, Archimede Solar Energy. Archimede Solar Energy Molten Salt Parabolic Trough Demo Plant: A Step Ahead Towards the New Frontiers of CSP. SolarPaces 2014.

[27] F.Matino, Archimede Solar Energy Molten Salt Receivers Operated on Parabolic Trough Demo Plant and in Laboratory Conditions. SolarPaces 2014.

[28] F.Burkholder and C.Kutscher. Heat Loss Testing of Schott's 2008 PTR70 Parabolic Trough Receiver. Report NREL/TP-550-45633, May 2009. 
1. Estado de la técnica en las centrales termosolares con colectores solares lineales.

[29] R. Abbas, M.J. Montes, A. Rovira, J.M. Martínez-Val. Parabolic trough collector or linear Fresnel collector?. A comparison of optical features including thermal quality based on commercial solutions. Solar Energy, Volume 124, February 2016, Pages 198-215.

[30] Ivanpah solar power plant. A BrightSource Project. http://www.brightsourceenergy.com

[31] Gemasolar solar power plant. SENER Project. http://www.torresolenergy.com

[32] Crescent Dunes solar power plant. A SolarReserve Project. http://www.solarreserve.com

[33] A. del Rio, R. Korzynietz, J.A. Brioso, et al. Soltrec-Pressurized Volumetric Solar Air Receiver Technology. Energy Procedia, Volume 69, May 2015, Pages 360-368.

[34] M.Quero et al. Solugas-Operation Experience of the First Solar Hybrid Turbine System at MW Scale. Energy Procedia, Volume 49, 2014, Pages 1820-1830.

[35] Mohammad Hossein Ahmadi, Mohammad Ali Ahmadi, Adel Mellit, Fathollah Pourfayaz, Michel Feidt. Thermodynamic analysis and multi objective optimization of performance of solar dish Stirling engine by the centrality of entransy and entropy generation. Energy Systems, Volume 78, June 2016, Pages 88-95.

[36] O'Grady, Patrick (23 January 2010). SES, Tessera debut new solar plant in Peoria. Phoenix Business Journal. Retrieved June 17, 2010.

[37] Solar buys Tessera Solar's Imperial Valley project with intent to turn CSP into PV. PVtech.org. Retrieved 2013-08-20.

[38] Wang, Ucilia (2010-12-29). Tessera Solar Sells Troubled 850 MW Project. Gigaom.com. Retrieved 2013-08-20.

[39] Jeffrey Barbee (May 13, 2015). Could this be the world's most efficient solar electricity system? Using military technology and a zero-emission engine invented by a 19th-century Scot, Swedish firm seeks to revolutionise solar energy production. The Guardian. Retrieved May 13, 2015. 34\% of the sun's energy hitting the mirrors is converted directly to grid-available electric power. http://www.theguardian.com

[40] Ming Liu, N.H. Steven Tay, Stuart Bell, Martin Belusko, et al. Review on concentrating solar power plants and new developments in high temperature thermal energy storage technologies. Renewable and Sustainable Energy Reviews, Volume 53, January 2016, Pages 1411-1432.

[41] J. Stekli, L. Irwin, R. Pitchumani. Technical challenges and opportunities for concentrating solar power with thermal energy storage. J Therm Sci Eng Appl, 5 (2013), p. 021011

[42] S. Kuravi, J. Trahan, D.Y. Goswami, M.M. Rahman, E.K. Stefanakos. Thermal energy storage technologies and systems for concentrating solar power plants. Prog Energy Combust Sci, 39 (2013), pp. 285-319

[43] F.Dinter, D. Mayorga González. Operability, reliability and economic benefits of CSP with thermal energy storage: first year of operation of ANDASOL 3. SolarPACES 2013, Elesevier, Energy Procedia 49 (2014) 2472-2481.

[44] R. Osuna, R. Olavarría, R. Morillo, M.A. Silva. PS10, Construction of 11 MWe Solar Thermal Tower Plant in Seville, Spain. SolarPACES 2006, Seville, Spain.

[45] D. Laing, W. D. Steinmann, P. Viebahn, F. Gräter and C. Bahl. Economic Analysis and Life Cycle Assessment of Concrete Thermal Energy Storage for Parabolic Trough Power Plants. J. Sol. Energy Eng 132(4), 041013 (Oct 12, 2010).

[46] Laing D, Bahl C, Bauer T, Fiss M, Breidenbach N, Hempel M. High-temperature solid-media thermal energy storage for solar thermal power plants. IEEE Proceedings 2012; PP(99):1-9. 
1. Estado de la técnica en las centrales termosolares con colectores solares lineales.

[47] S. Hübner, M. Eck, C. Stiller, M. Seitz. Techno-economic heat transfer optimization of large scale latent heat energy storage systems in solar thermal power plants. Applied Thermal Engineering, Volume 98, April 2016, Pages 483-491.

[48] A. Caron-Soupart, J.F. Fourmigué, P. Marty. Performance analysis of thermal energy storage systems using phase change material. Applied Thermal Engineering, Volume 98, 5 April 2016, Pages 1286-1296.

[49] Dowtherm A heat transfer fluid. http://msdssearch.dow.com

[50] Syltherm 800 heat transfer fluid. http://www.dow.com

[51] Therminol VP1 heat transfer fluid. https://www.therminol.com

[52] Therminol 75 heat transfer fluid. https://www.therminol.com

[53] Thermoflow Inc. http://www.thermoflow.com/

[54] Archimede. Solar Salt heat transfer fluid. http://www.archimedesolarenergy.it

[55] D.Grogan. Development of Molten-Salt Heat Transfer Fluid Technology for Parabolic Trough Solar Power Plants. Abengoa Solar Sunshot Conference, 24-03-2013.

[56] A.Giostri, M.Binotti, M.Astolfi, P.Silva, E.Macchi, G.Manzolini. Comparison of different solar plants based on parabolic trough technology. Solar Energy 86 (2012) 1208-1221.

[57] C.Turchi, C.Bing, M.Lausten. $10 \mathrm{MW}$ Supercritical $\mathrm{CO}_{2}$ Turbine Test. University of Wisconsin at Madison and Sandia National Laboratories. DE-EE0001589 NREL. 


\section{Capítulo 2 \\ Metodología}

\section{Resumen}

El principal objetivo de este trabajo es diseñar nuevas configuraciones de plantas termosolares con colectores lineales (PTC o LF), y ciclos de potencia Supercríticos Rankine o Brayton, con el fin de optimizar su eficiencia energética y reducir su coste de inversión. Para ello, en los siguientes capítulos de esta tesis se han simulado los balances energéticos de diferentes configuraciones de plantas termosolares con ciclos supercríticos, y calculado el área de apertura efectiva de los colectores solares para cuantificar su coste de inversión. Se han utilizado principalmente dos programas informáticos: el programa comercial Thermoflow [1], y una herramienta informática desarrollada ad hoc para la presente tesis en los lenguajes $\mathrm{C \#} \mathrm{y}$ Fortran, capítulo 12.

En este capítulo de la tesis se explican los procedimientos y principios físicos, que rigen el cálculo de los balances térmicos y dimensionamiento de los equipos, de las plantas termosolares con ciclos de potencia supercriticos Brayton. Se estudian dos posible soluciones de colectores solares lineales, PTC y LF. En el apartado 2.2 de este capítulo, se realiza un modelo energético común para ambas tipologías de colectores. En los apartados 2.3 y 2.4 se definen las principales ecuaciones que gobiernan el cálculo del rendimiento energético de los ciclos de potencia Rankine y Brayton. Posteriormente, en el apartado 2.5 se explica el cálculo de las propiedades físicas del fluido de trabajo en estado supercrítico. En los apartados 2.6 a 2.8 se han definido las ecuaciones que gobiernan el dimensionamiento y diseño de los equipos que integran el ciclo Brayton, y se finaliza este capítulo explicando los algoritmos de optimización multivariable SUBPLEX [22], UOBYQA [23-25], NEWUOA [26-27], para maximizar la eficiencia energética de los ciclos Brayton.

\subsection{Modelo Energético de los colectores solares lineales}

Cálculo del balance energético entre la energía solar incidente en el tubo absorbedor y las pérdidas térmicas, con la ecuación (2-1), obteniendo como resultado la energía neta térmica absorbida en el campo solar ( $Q_{S F}$ net):

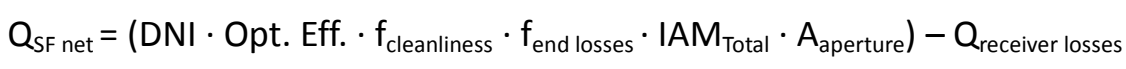

Donde

- DNI : Irradiación solar normal incidente $\left(\mathrm{W} / \mathrm{m}^{2}\right)$

- Opt. Eff.: Eficiencia óptica nominal (a 0 grados de incidencia)

- $\quad f_{\text {cleanliness: }}$ factor de limpieza de los espejos de los colectores solares

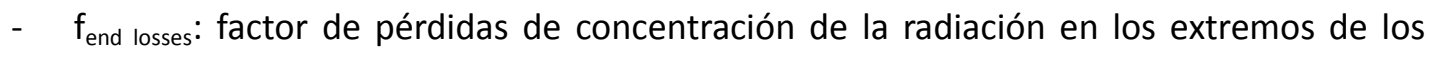
colectores.

- $\quad \mid A M_{\text {Total }}$ : factores de modificador del ángulo de incidencia

- $\quad A_{\text {aperture: área de apertura efectiva del colector solar }\left(\mathrm{m}^{2}\right)}$

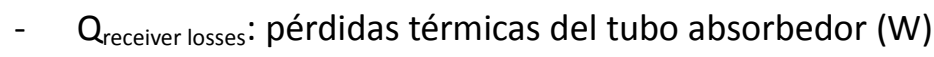


Las configuraciones de planta termosolar estudiadas en este trabajo están integradas por dos campos solares, un campo solar principal ( $Q_{\mathrm{SF} 1}$ net) y un campo solar para recalentemiento ( $\mathrm{Q}_{\mathrm{SF} 2}$ net).

El calor total transferido desde los campos solares hacia el ciclo de potencia viene dado por la ecuación (2-2):

$Q_{\text {Total SF net }}=Q_{S F 1 \text { net }}+Q_{S F 2 \text { net }}$

$\mathrm{Q}_{\mathrm{SF} 1 \text { net }}$ : calor neto absorbido en el campo solar principal (W)

$\mathrm{Q}_{\mathrm{SF2} \text { net }}$ : calor neto absorbido en el campo solar de recalentamiento (W)

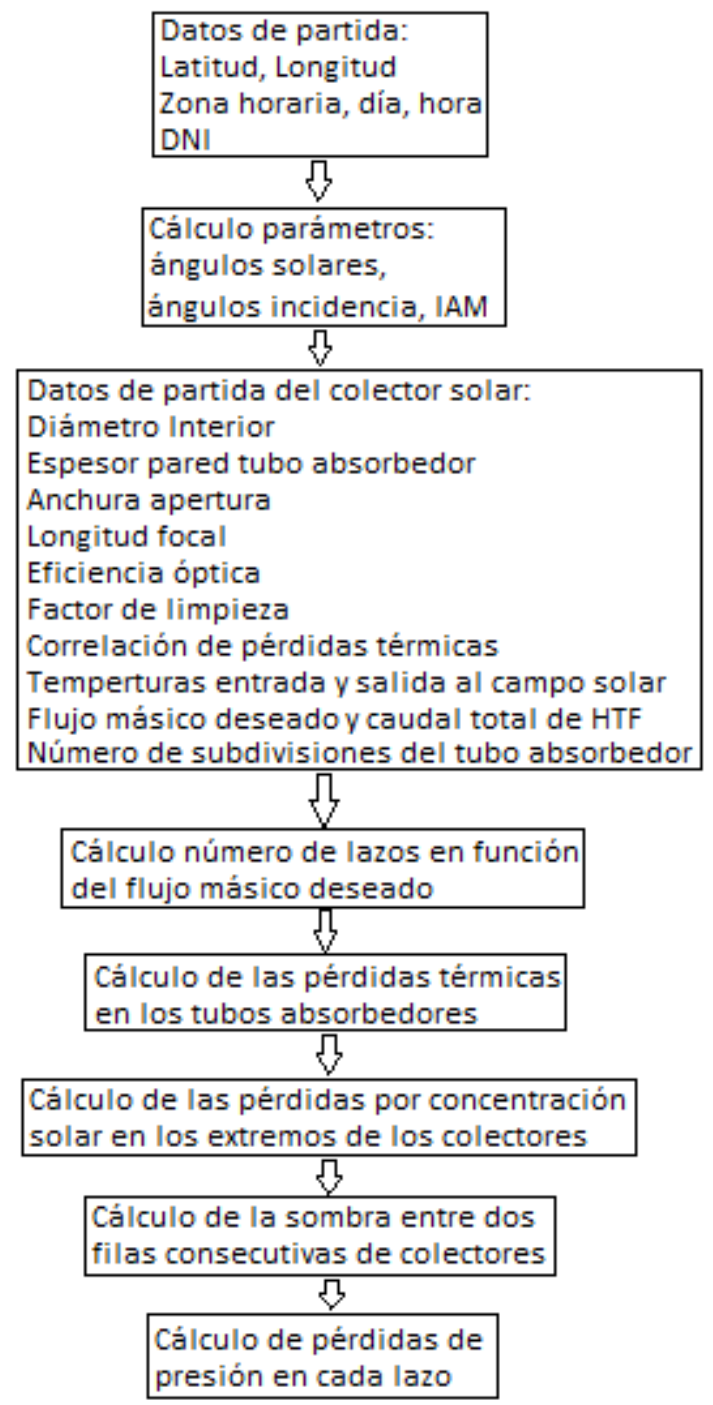

Figura 2.1. Algoritmo de cálculo del área de apertura efectiva y pérdidas de presión en los campos solares con colectores lineales.

En la figura 2.1 se resume el algoritmo de cálculo desarrollado ad hoc en esta tesis para calcular el área de apertura efectiva de los colectores lineales. Su explicación y código detallado se definen a continuación. Como datos de partida se consideran las temperaturas de entrada y salida del campo solar, definidas por el intercambiador de calor entre el campo solar y el ciclo de potencia. 


\section{Metodología}

Proceso:

1. Cálculo del ángulo de incidencia solar $(\theta)$.

1.1. Datos de partida: la Longitud, la Latitud, la zona horaria, el DNI, el día y la hora.

1.2. Cálculo del shift con la ecuación (2-3), que representa el desfase horario debido a la diferencia entre la longitud de la zona horaria considerada (la zona horaria tiene valores negativos hacia el Oeste y valores positivos hacia el Este), y la longitud de la localización estudiada.

$$
\text { MerSD }=15^{*}(\text {-zone })
$$

1.3. Cálculo del parámetro " $B$ " definido mediante las ecuaciones (2-4 y 2-5):

$$
\begin{aligned}
& B=(D A Y-1) \\
& B=(B * 360) / 365
\end{aligned}
$$

1.4. Cálculo de la ecuación horaria Equation Of Time (EOT), con la ecuación (2-6):

$$
\begin{aligned}
& \text { EOT }=229.18 *(0.000075+0.001868 * \operatorname{Cos}(\operatorname{rad}(B))-0.032077 * \operatorname{Sin}(\operatorname{rad}(B))-0.014615 \\
& * \operatorname{Cos}(\operatorname{rad}(2 * B))-0.04089 * \operatorname{Sin}(\operatorname{rad}(2 * B))) ;
\end{aligned}
$$

1.5. Cálculo de la hora solar solar time Tsun con la ecuación (2-7):

$$
\text { Tsun }=(\text { HOUR })+(\text { MerSD }+ \text { Lon }) / 15+\text { EOT } / 60
$$

1.6. Cálculo de los diferentes ángulos solares: ángulo horario, ecuación (2-8), ángulo declinación, ecuación (2-9), ángulo zenit, ecuación (2-10), ángulo incidencia, ecuación (2-11), altura solar, ecuación (2-12) y azimuth, ecuación (2-13).

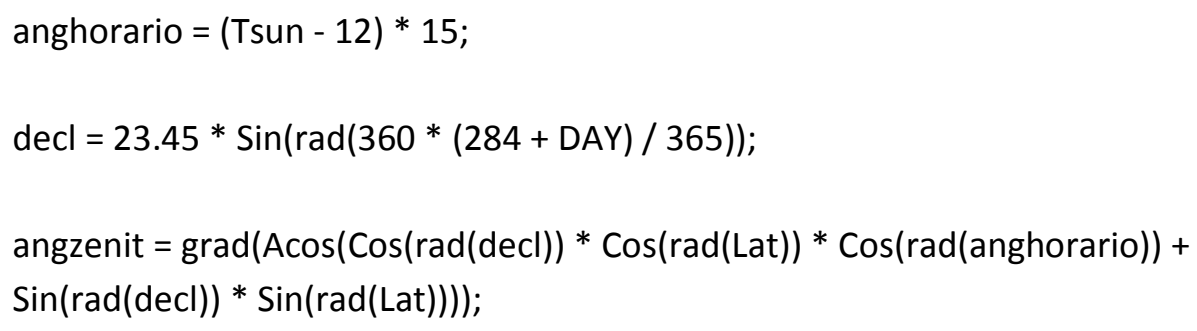

alt_solar = 90 - angzenit;

azimuth $=180-(\operatorname{grad}($ Asin $(-\operatorname{Cos}(\operatorname{rad}(\operatorname{dec} l)) * \operatorname{Sin}(\operatorname{rad}($ anghorario $)) /$

$\operatorname{Cos}(\operatorname{rad}($ alt_solar) $))))$; 


\section{Metodología}

2. Tomando como dato de partida el ángulo de incidencia calculado con la ecuación (2-11), se interpola en las tablas de IAM proporcionadas por el fabricante del colector solar (PTC o LF).

3. Cálculo del valor del IAMOverall, con la ecuación (2-14), que es el producto del IAM longitudinal multiplicado por el IAM transversal.

$$
\text { IAMOverall = IAMLongitudinal * IAMTransversal; }
$$

4. Datos de partida para realizar el dimensionamiento del campo solar en base a su balance térmico: DNI, eficiencia óptica nominal, factor limpieza, factor pérdidas en los extremos, anchura apertura, energía térmica del campo solar, temperatura entrada en campo solar, temperatura salida del campo solar, coeficiente de pérdidas térmicas $A 1$, coeficiente de pérdidas térmicas $A 2$, número de subdivisiones del tubo absorbedor en cada lazo, flujo másico deseado, distancia focal y diámetro interior del tubo absorbedor.

5. Cálculo del número de lazos en función del flujo másico máximo $\left(\mathrm{kg} / \mathrm{m}^{2} \cdot \mathrm{s}\right)$ Desired_Mass_Flux, valor fijado por usuario para limitar las pérdidas de presión para una longitud excesiva de los colectores solares.

6. Cálculo de las pérdidas térmicas de los tubos absorbedores. Con el siguiente código se subdivide el colector solar en 10 zonas, y en cada una de ellas se calculan las pérdidas térmicas en función de una correlación matemática proporcionada por el fabricante del colector solar, por ejemplo: $0.141 \Delta T+6.48 \mathrm{e}-9 \Delta \mathrm{T}^{4}$.

7. Cálculo de las pérdidas por concentración solar en los extremos de los colectores de cada lazo.

Durante las horas del día durante las cuales la radiación solar no incide directamente en el área de apertura, parte de la radiación es reflejado hacia el exterior del colector y no incide directamente en el tubo absorberdor. Las pérdidas de radiación en el extremo del colector puede incidir en el colector adyacente, sin embargo, en este estudio no se ha considerado esta ganancia de radiación. La ecuación (2-15) calcula las pérdidas de radiación en el extremo del colector solar:

$$
\eta_{\text {end Loss }, \mathrm{i}}=1-\frac{\mathrm{L}_{\mathrm{f}, \mathrm{ave}, \mathrm{i}} \cdot \tan (\theta)}{\mathrm{L}_{\mathrm{i}}}
$$

La ecuación está elaborada en base a la longitud focal media del colector cilindro parabólico. En la figura 2.2 se representa gráficamente las longitudes focales en diferentes puntos de la superficie del colector parabólico.

Mediante la ecuación (2.16) se calcula la longitud focal media $\mathrm{L}_{\mathrm{f}, \text { ave }}$ :

$$
\mathrm{L}_{\mathrm{f}, \mathrm{ave}}=\sqrt{\frac{\left[4 \mathrm{a}^{2}+\left(\frac{\mathrm{w}}{2}\right)^{2}\right]^{2}}{\mathrm{a}^{2}}} \cdot \frac{12 \mathrm{a}^{2}+\left(\frac{\mathrm{w}}{2}\right)^{2}}{12\left(4 \mathrm{a}^{2}+\left(\frac{\mathrm{w}}{2}\right)^{2}\right)}
$$




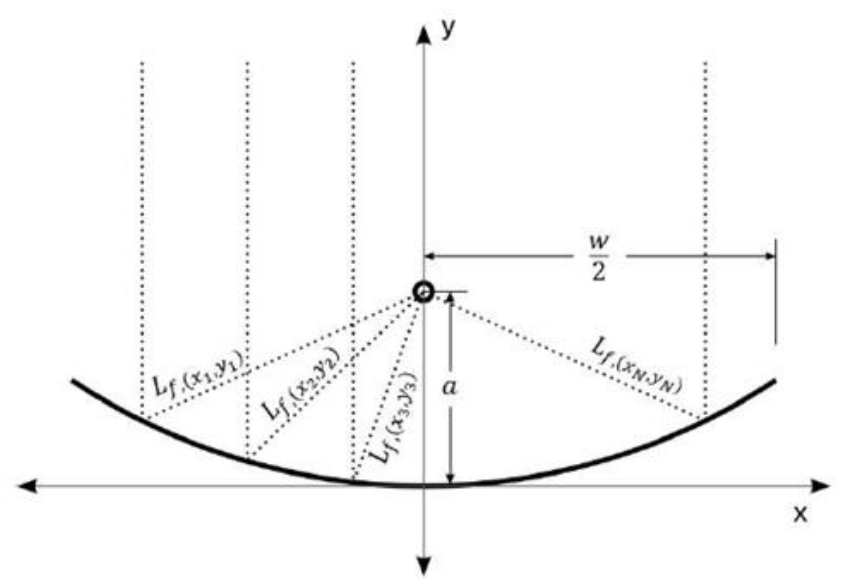

Figura 2.2. Representación gráfica de las longitudes focales para el cálculo de la longitud focal media [2].

8. Cálculo de la sombra entre dos filas consecutivas de colectores cilindro-parabólcos: las zonas de sombra entre dos colectores en paralelo queda representada en la figura 2.2.

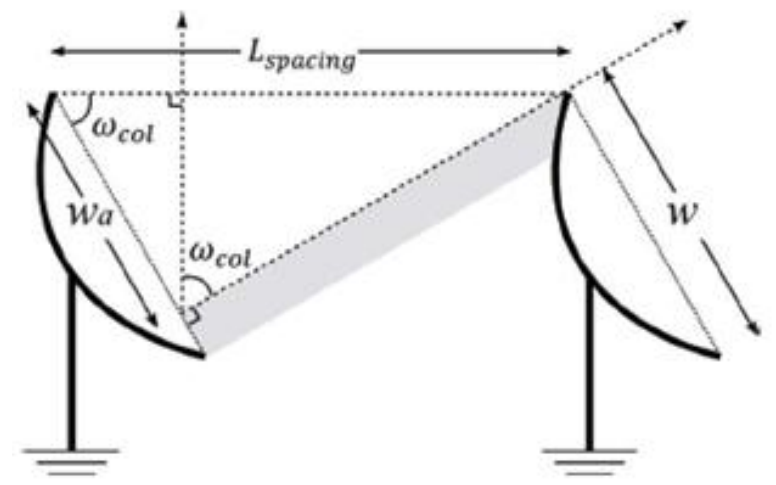

Figura 2.3. Sombra entre dos filas paralelas de colectores parabólicos. La geometría de la sombra depende de la distancia entre las dos filas de colectores $\left(L_{\text {spacing }}\right)$, de su ángulo de seguimiento solar $\left(\mathrm{W}_{\text {col }}\right)$, y de la longitud de apertura de los colectores (W) [2].

Tal y como se ha mostrado en la figura 2.3, el efecto de sombra entre las dos filas de colectores afecta al area de apertura efectiva de una de las fila de colectores. Para determinar la anchura de apertura disponible no afectada por la sombra, se ha definido un triángulo rectángulo cuya hipotenusa es igual a la distancia entre las dos filas de colectores y cateto menor es la anchura de apertura no afectada por la sombra $W_{a}$. Mediante la ecuación (2-17) y teniendo en cuenta el ángulo de seguimiento solar de los colectores $W_{\text {col }}$ tracking angle se obtiene el resultado del problema la anchura de apertura no afectada por la sombra, $\mathrm{w}_{\mathrm{a}}$.

$\mathrm{w}_{\mathrm{a}}=\cos \left(\mathrm{w}_{\mathrm{col}}\right) \cdot \mathrm{L}_{\mathrm{spacing}}$

Se define en la ecuación (2-18) la eficiencia debida al efecto de sombra entre los colectores, como la relación entre la anchura de apertura disponible después de deducir el efecto de sombra, y el area de apertura total del colector. La eficiencia por el efecto sombra está limitada entre dos valores 0.5 y 1.0. Si la eficiencia es menor de 0.5 el campo solar es probable que no opere correctamente y se considera cero la eficiencia óptica global del campo solar. 


\section{Metodología}

$\eta_{\text {shadow }}=\frac{w_{a}}{w}=\left[\cos \left(w_{\text {col }}\right)\right] \cdot \frac{L_{\text {spacing }}}{w}$

Donde:

- RS: Row spacing separación entre filas paralelas de colectores

- W: anchura de apertura

9. Cálculo de las pérdidas de presión en cada lazo.

Se subdivide el lazo en diferentes tramos y se calcula la caída de presión en cada uno de los tramos mediante la ecuación 2-19:

$\Delta P_{i}=f_{f d, i} \cdot \Delta x_{i} \cdot \rho_{i} \cdot\left(\frac{v_{i}^{2}}{2}\right)$

Donde:

- $\Delta P_{i}=$ caida de presión $(\mathrm{Pa})$.

- $f_{f d, i}=$ coeficiente de Darcy en cada tramo de la tubería.

- $\Delta x_{i}=$ longitud de cada tramo de tubería $(\mathrm{m})$.

- $\quad \rho_{i}=$ densidad en cada tramo de tubería $\left(\mathrm{kg} / \mathrm{m}^{3}\right)$.

- $v_{\mathrm{i}}=$ velocidad media en cada tramo de tubería $(\mathrm{m} / \mathrm{s})$.

Para el cálculo de la citada ecuación es necesario calcular el coeficiente de fricción de Darcy en cada uno de los tramos. El cálculo del coeficiente de Darcy se realiza mediante dos ecuaciones, dependiendo si el flujo es turbulento se utiliza la ecuación de Colebrook, cuando el número de Reynolds mayor de 4000, ecuación 2-20, o en caso de que el flujo sea laminar, cuando el número de Reynold menor de 2300, se aplica la ecuaicón 2-21:

$\frac{1}{\lambda^{0.5}}=-2 \log \left[\frac{2.51}{\left(R e \cdot \lambda^{0.5}\right)}+\frac{\left(\frac{k}{d_{h}}\right)}{3.72}\right]$

Donde:

- $\lambda=$ coeficiente de fricción Darcy-Weisbach.

- $\quad \operatorname{Re}=$ número de Reynolds.

- $\quad k$ = rugosidad de la pared interna de la tubería $(\mathrm{mm})$.

- $\quad d_{h}=$ diámetro hidráulico $(m)$.

El coeficiente de fricción para regimen de flujo laminar se cálcula con la ecuación 2-21:

$\lambda=\frac{64}{R e}$

Donde:

- $\lambda=$ coeficiente de fricción Darcy-Weisbach.

- $\quad \operatorname{Re}=$ número de Reynolds. 


\subsection{Modelo energético y rendimiento del ciclo Rankine}

$\eta_{\text {neto }}=\left(\mathrm{W}_{\text {turbinas }}-\mathrm{W}_{\text {bombas }}-\mathrm{E}_{\text {generador }}-\mathrm{E}_{\text {auxiliares }}\right) / \mathrm{Q}_{\text {Total SF neta }}$

Donde:

- $\quad W_{\text {turbinas }}$ : Balance Of Plant (BOP) potencia de las turbinas (W)

- $\quad W_{\text {bombas: }}:$ BOP potencia de las bombas (W)

- $E_{\text {generador: }}$ BOP pérdidas en el generador (W)

- $\quad E_{\text {auxiliares: }}$ consumes auxiliaries del ciclo de potencia (ventiladores del condensador, etc.) (W)

- $\quad \eta_{\text {neto }}$ : Eficiencia neta de la planta (\%)

\subsection{Modelo energético y rendimiento del ciclo Brayton}

$\eta_{\text {neto }}=\left(\mathrm{W}_{\text {turbinas }}-\mathrm{W}_{\text {compresores }}-\mathrm{E}_{\text {generator }}-\mathrm{E}_{\text {auxiliares }}\right) / \mathrm{Q}_{\text {Total SF neta }}$

Donde:

- $\quad W_{\text {turbinas }}$ : potencia de las tubinas (W)

- $\quad W_{\text {compresores: }}$ potencia de los compresores (W)

- $E_{\text {generator: }}$ pérdidas en el generador (W)

- Eauxiliares: consumes auxiliaries de la planta (ventiladores y bombas del foco frío) (W)

- $\quad \eta_{\text {neto: }}$ Eficiencia neta de la planta (\%)

\subsection{Propiedades de los fluidos de trabajo del ciclo de potencia}

Se han estudiado diferentes fluidos como posibles candidatos para integrar el bloque de potencia Brayton: Dióxido de Carbono $\left(\mathrm{CO}_{2}\right)$, Etano $\left(\mathrm{C}_{2} \mathrm{H}_{6}\right)$, Metano $\left(\mathrm{CH}_{4}\right)$, Nitrógeno $\left(\mathrm{N}_{2}\right)$, Xenón (Xe) y Hexafluoruro de Azufre $\left(\mathrm{SF}_{6}\right)$. Las propiedades de estos fluidos en condiciones supercríticas se han calculado con la base de datos REFPROP: Reference Fluid Thermodynamic and Transport Properties Database (REFPROP): Version 9.0., desarrollada por E.W. Lemmon, M.L. Huber, and M.O. McLinden en el National Institute of Standards and Technology (NIST) de Estados Unidos. Esta base de datos ha sido desarrollada en Fortran, y se encuentra integrada en el programa informático Thermoflow. Así mismo, en esta tesis doctoral se ha creado un interface en lenguaje C\# para acceder a las propiedades de los fluidos supercríticos y permitir el cálculo termodinámicos de los ciclos Brayton integrados en las plantas termosolares con colectores PTC o LF. Para los fluidos indicados anteriormente las ecuaciones de estado Equation Of State (EOS) que definen sus propiedades han sido publicadas en las siguientes referencias: $\mathrm{CO}_{2}$ [3], Etano $\left(\mathrm{C}_{2} \mathrm{H}_{6}\right)$ [4], Metano $\left(\mathrm{CH}_{4}\right)$ [5], Nitrógeno $\left(\mathrm{N}_{2}\right)$ [6], Xenon (Xe) [7], Hexafluoruro de Azufre $\left(\mathrm{SF}_{6}\right)[8]$.

En relación al agua, como fluido de trabajo en los ciclos de potencia Rankine, sus propiedades en el programa informático Thermoflow han sido obtenidas de las siguientes fuentes: IAPWSIF97 [9] y del ASME IFC-67 [10]. 


\section{Metodología}

\subsection{Tubomáquinas (turbinas y compresores)}

\subsubsection{Turbinas (Diseño Básico)}

Los valores de partida para la modelización de las turbinas son la temperatura y presión a la entrada, la presión a la salida, y la eficiencia isoentrópica de la turbina. Una vez conocidas las condiciones termodinámicas a la entrada de la turbina (presión y temperatura) se calculan la entropía y la entalpía de entrada. Se considera como hipótesis inicial que se trata de una turbina isoentrópica, la entropía a la salida de la turbina es igual a la entropía a la entrada ecuación (2-21). Se calcula la entalpía a la salida de la turbina en condiciones isoentrópicas ecuación (2-22). Con el rendimiento isoentrópico se calcula la entalpía real a la salida de la turbina ecuación (2-23 y 2-24).

$$
\begin{aligned}
& \mathrm{S}_{\text {out }}=\mathrm{S}_{\text {in }} \\
& \mathrm{w}_{\mathrm{s}}=\mathrm{i}_{\text {in }}-\mathrm{i}_{\text {outs }} \\
& \mathrm{w}=\mathrm{w}_{\mathrm{s}} \cdot \eta \\
& \mathrm{i}_{\text {out }}=\mathrm{i}_{\text {in }}-\mathrm{w}
\end{aligned}
$$

Con las condiciones de presión y entalpía a la salida de la turbina, se obtienen aplicando las ecuaciones (2-21 a 2-24) y con la ayuda de las tablas de propiedades del fluido de trabajo.

\subsubsection{Compresores (Diseño Básico)}

Los compresores son calculados siguiendo idéntica metodología a la indicada con las turbinas, con una sola diferencia, la relación entre el trabajo ideal y el trabajo real en el compersor están definidas por la ecuación (2-25):

$$
\mathrm{w}=\frac{\mathrm{w}_{\mathrm{s}}}{\eta}
$$

Donde $\eta$, es la eficiencia del compresor.

Mediante el siguiente código informático se ha desarrollado la metodología de cálculo antes explicada para definir las condiciones termodinámicas a la salida de las turbomáquinas una vez conocidas sus condiciones de entrada, la presión requerida a la salida y su rendimiento isoentrópico.

\subsubsection{Turbinas (Diseño Detallado)}

Para el diseño de las turbinas y compresores se han tomado como referencia fundamental los datos experimentales obtendios en el lazo experimental de ciclo s- $\mathrm{CO}_{2}$ Brayton localizado en el SNL (figuras 2.4-2.5), e integrado por dos turbo-alternadores-compresores (figuras 2.6 y 2.7). El código informático utilizado ha sido obtenido de la tesis de Dyreby [12] y ha sido adaptado a lenguaje C\# para su integración en las plantas termosolares con colectores lineales. 


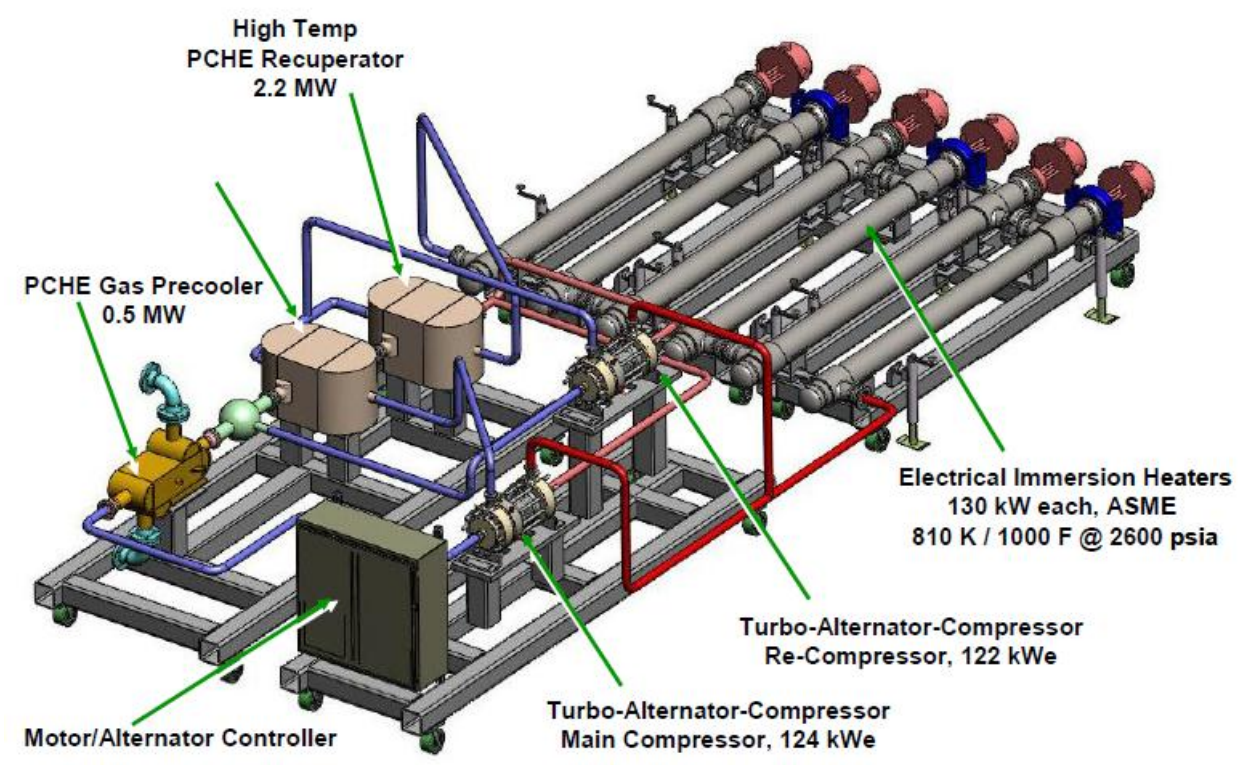

Figura 2.4. Representación gráfica $3 D$ de los principales componentes y equipos que integran el lazo de pruebas de ciclo supercritical Carbon Dioxide $\left(\mathrm{s}-\mathrm{CO}_{2}\right)$ Brayton en SNL [11].

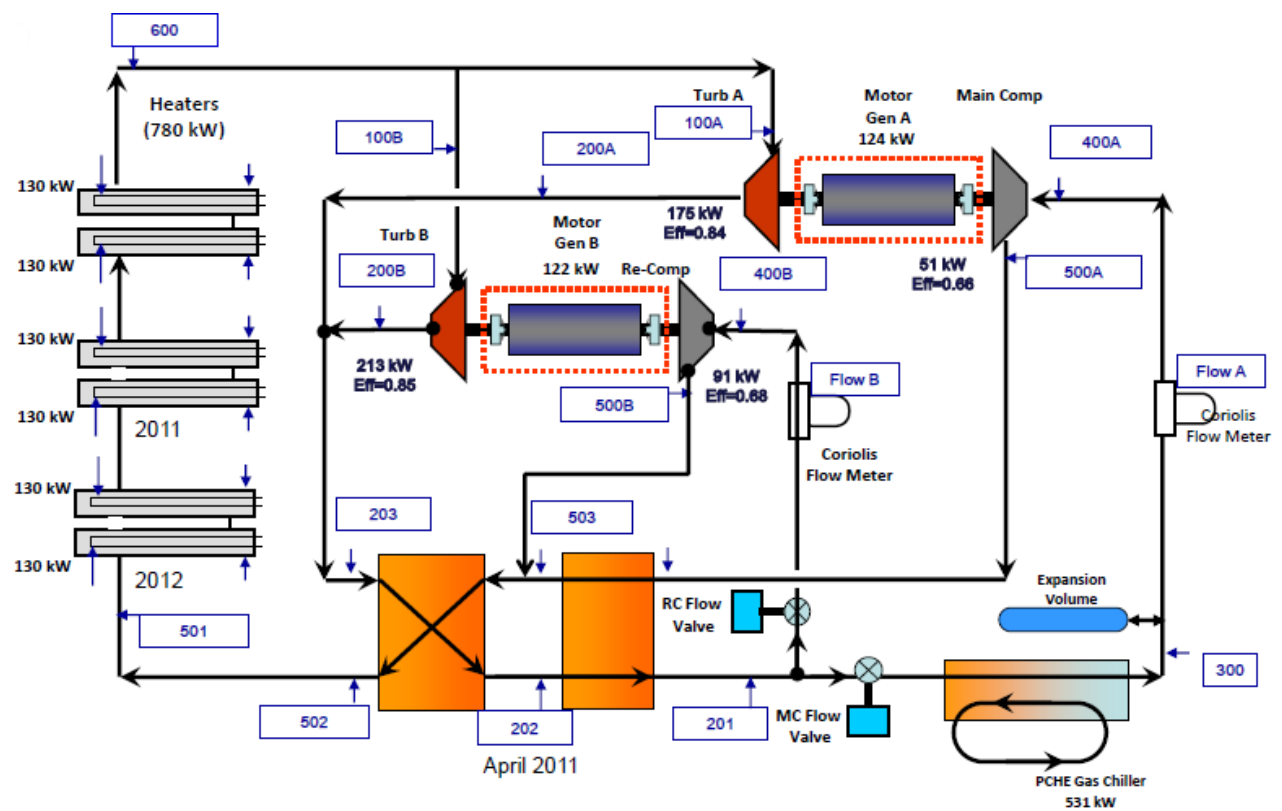

Figura 2.5. Esquema del ciclo de potencia s- $\mathrm{CO}_{2}$ Brayton del lazo de pruebas de SNL [11]. 


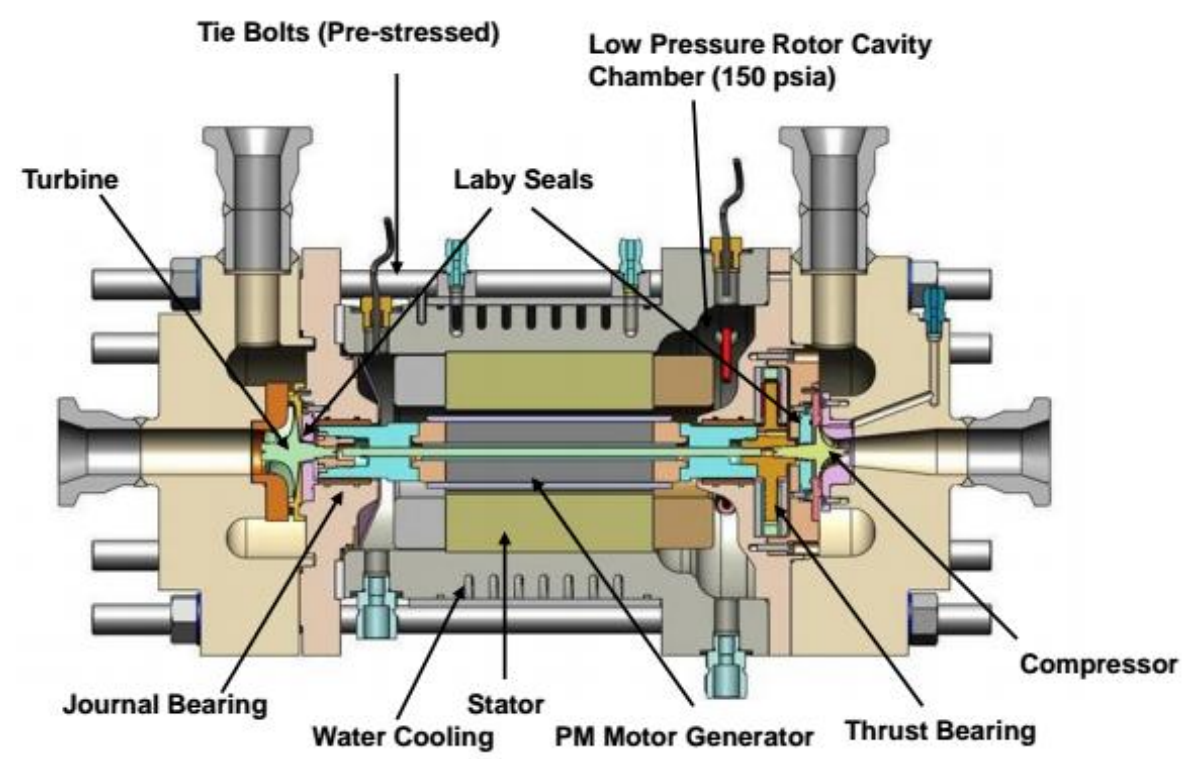

Figura 2.6. Tecnología principal o key technology, Turbo-Alternador-Compresor diseñado con rodamientos tipología gas foil bearings. Longitud 24 pulgadas y diámetro 12 pulgadas [11].

Las turbomáquinas diseñadas ad hoc para el experimento en SNL son de tipología "Radial". En la figura 2.7 se muestra una fotografía las dos turbinas, la turbina que comparte eje con el compresor principal, y la turbina que comparte eje con el recompresor. Cabe destacar su reducido tamaño, principal ventaja de la tecnología analizada, debido a la utilización de un fluido de trabajo en el ciclo de potencia con alta densidad, el s- $\mathrm{CO}_{2}$.
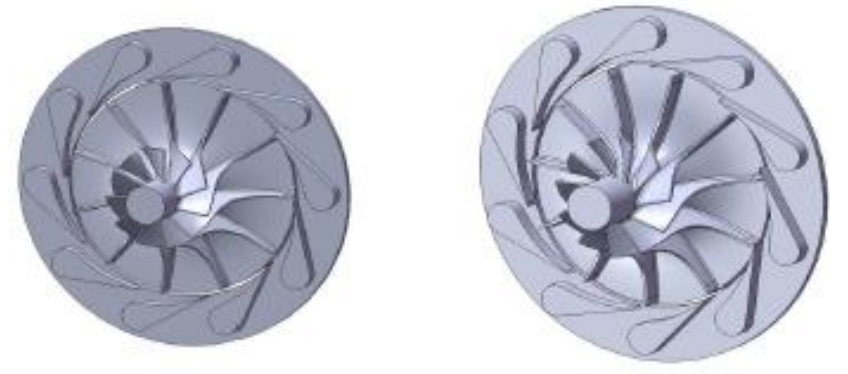

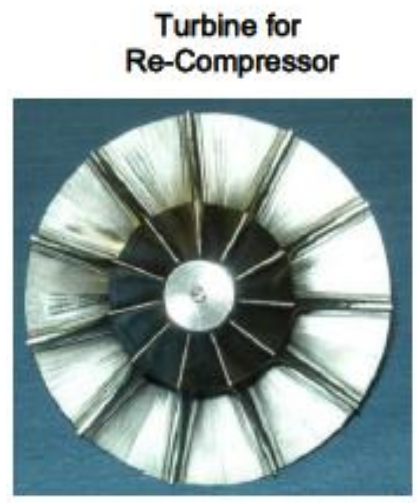

$\mathrm{OD}=68.3 \mathrm{~mm}$ $2.69^{\prime \prime}$

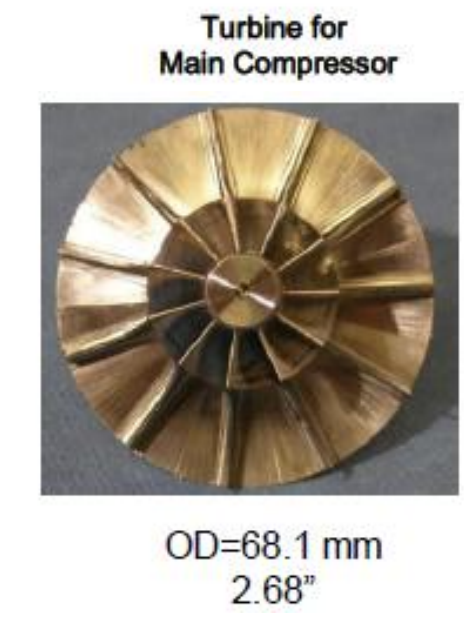

Turbine for Main Compressor

Figura 2.7. Fotografía de las dos turbinas radiales fabricadas por la empresa Baber -Nichols [13], que integran el ciclo de pruebas de ciclo de potencia s- $\mathrm{CO}_{2}$ Brayton en SNL [11]. 
Las turbinas radiales son adecuadas para potencias inferiores a 50MWe según lo indicado en la referencia Gibbs 2006 [14]. El caudal a través de la turbina viene definido por la ecuación (226):

$m^{\prime}=C_{S} \cdot A_{\text {nozzle }} \cdot \rho$

Donde:

- Anozzle: area

- $\quad \rho:$ densidad de salida

La velocidad de chorro spouting velocity $C_{s}$ es la velocidad que se alcanzaría si el fluido fuera expandido en condiciones isoentrópicas y viene definido por la ecuación (2-27):

$C_{s}=\sqrt{2 \cdot \Delta h_{i}}$

Para el dimensionamiento de las turbinas radiales se ha seguido el método de seleccionar un valor nominal de la velocidad del tip del rotor. Se utiliza la gráfica de la figura 2.8 para optimizar su rendimiento aerodinámico. En la citada figura se utilizan dos parámetros, $\mathrm{U}_{\text {tip }}$ es la velocidad del alabe inducido, y $\mathrm{C}_{\mathrm{s}}$ spouting velocity la velocidad de flujo que corresponde a la expansión ideal desde la entrada hacia la salida en condiciones estáticas atravesando la turbina. La variación de la eficiencia aerodinámica de la turbina, obtenida en su totalidad por análisis, alcanza el máximo en la $\mathrm{U}_{\text {tip }} / \mathrm{C}_{\mathrm{s}}$ cercano a 0.7 Japikse y Baines [15].

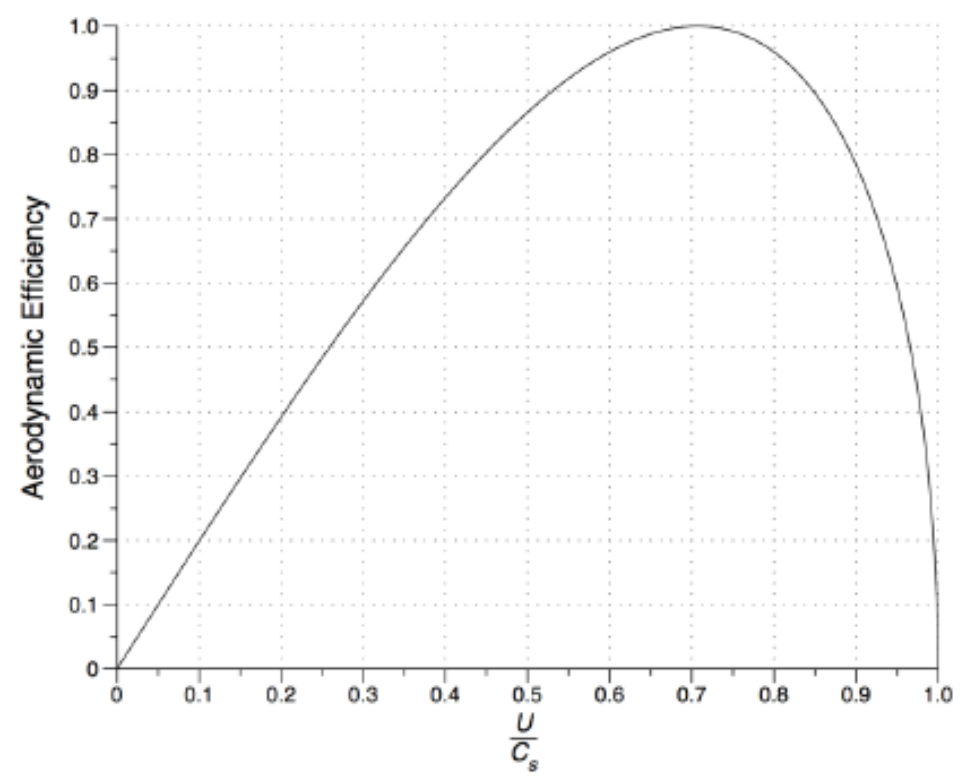

Figura 2.8. Eficiencia de una turbina radial ideal en función de la relación de velocidades $\left(v=U_{\text {tip }} / C_{s}\right)$. El valor normalmente elegido es de $v=0.707$ para obtener una eficiencia aerodinámica máxima (Japikse y Baines [15]).

Japikse y Baines [16] propusieron una ecuación 2-28 que relaciona la eficiencia ideal $\eta_{\text {ideal, }} 0$ también llamado rendimiento aerodinámico, y la relación de velocidades tip velocity/spouting velocity $\left(\mathrm{v}=\mathrm{U}_{\mathrm{tip}} / \mathrm{C}_{\mathrm{s}}\right)$. 


\section{Metodología}

$\eta_{\text {idel }}=2 \cdot v \cdot \sqrt{1-v^{2}}$

La figura 2.8 viene definida por la ecuación (2-29), y por el siguiente polinomio de aproximación de cuarto grado: $\eta_{\text {ideal }}=0.179921180+1.3567 \cdot v+1.3668 \cdot v^{2}-3.0874 \cdot v^{3}+$ $1.0626 \cdot v^{4}$

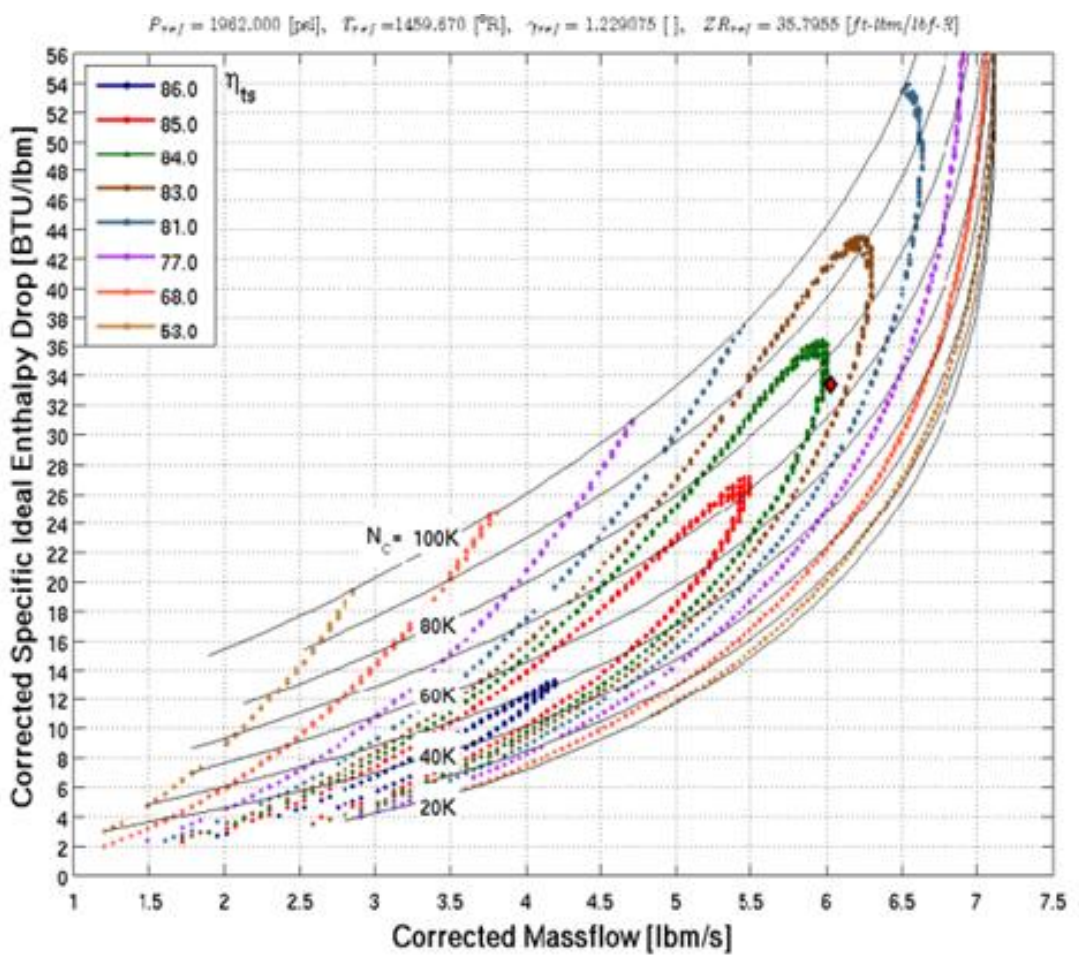

Figura 2.9. Mapa de funcionamiento de la turbina acoplada al compresor principal [11].

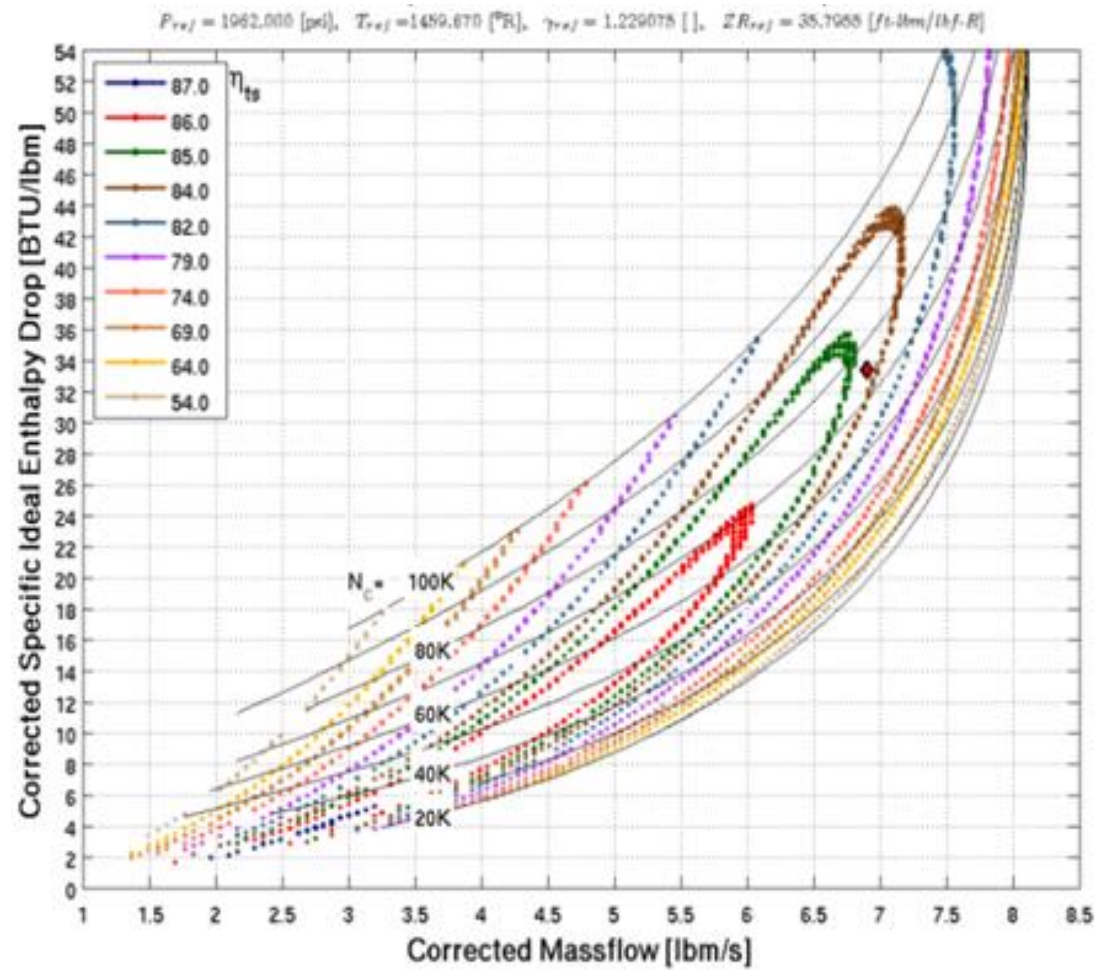

Figura 2.10. Mapa de funcionamiento de la turbina acoplada al recompresor [11]. 
Una vez diseñadas y fabricadas las turbinas radiales se someten a diferentes pruebas experimentales para obtener el mapa de funcionamiento, que relaciona la caída de entalpía en el equipo con el caudal que atraviesa la turbina. En las figuras 2.9 y 2.10 se representan gráficamente los mapas de operación de las turbinas utilizadas en el lazo experimental de ciclo $\mathrm{SCO}_{2}$ en SNL. Cabe destacar que los rendimientos obtenidos en las turbinas están entre un 84$85 \%$. Estos mapas de operación solo son válidos para la geometría de las turbinas consideradas y para las condiciones de referencia de presión y temperatura indicadas en los mismos. Una vez obtenidos los mapas de operación por parte del fabricante de la turbina, se pueden utilizar para obtener los valores de $A_{\text {nozzle }}$ aplicando las ecuaciones (2-27 y 2-28). De esta manera se puede estudiar el comportamiento de la turbina bajo los diferentes rangos de velocidades de operación. Así mismo, si se utiliza la información del mapa de operación de las turbinas para obtener su eficiencia aerodinámica, se comprueba que su comportamiento bajo los diferentes rangos de velocidades no es el mismo que el indicado en la figura 2.8 .

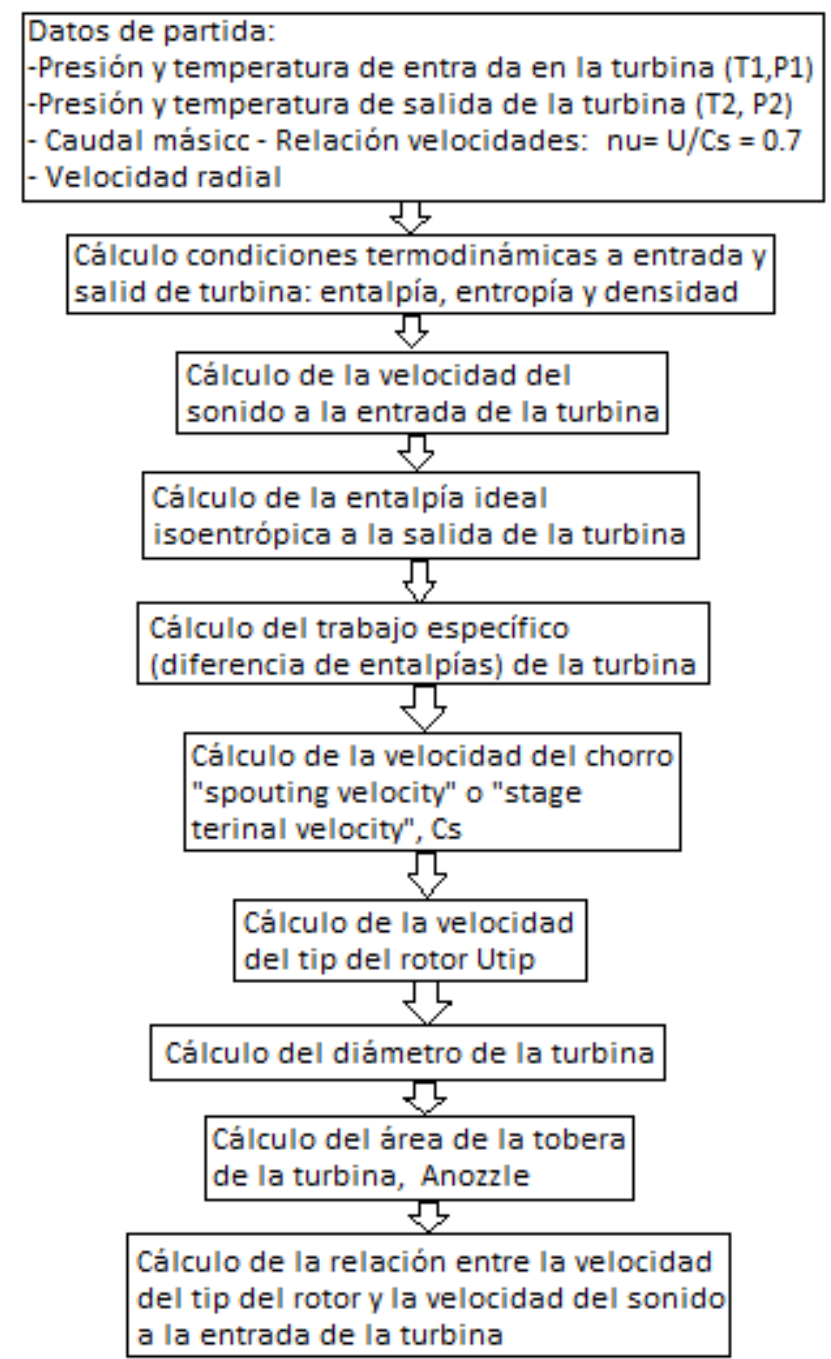

Figura 2.11. Algoritmo para el diseño Design-Point de las turbinas radiales del ciclo de potencia s-CO2 Brayton [12].

En el programa informático desarrollado ad hoc para esta tesis doctoral, se han simulado dos tipologías de turbinas, la turbina utilizada en SNL para los experimentos en el lazo de $\mathrm{s}-\mathrm{CO}_{2}$ 
(SNL_Turbine), y la turbina de tipología Low-reaction Radial_Trubine. Los únicos parámetros que diferencian ambos diseños son:

- La relación ( $v$, nu_design) entre velocidades del rotor $\left(U_{\text {tip }}\right)$ y velocidad de chorro $\left(C_{s}\right.$, spouting velocity). Los valores de $v$ considerados son: $v=0.7476$ para el diseño SNL_Turbine, y v $=0.707$ para el diseño Radial_Turbine.

- El valor de la densidad considerada a la hora de dimensionar el área de la boquilla de la turbina $\left(\mathrm{A}_{\text {nozzle }}\right)$. En el diseño $S N L$ Turbine se ha considerado la densidad de entrada en la turbina, mientras que el diseño Radial_Turbine se elige la densidad de salida de la turbina para el dimensionamiento del parámetro $A_{\text {nozzle }}$.

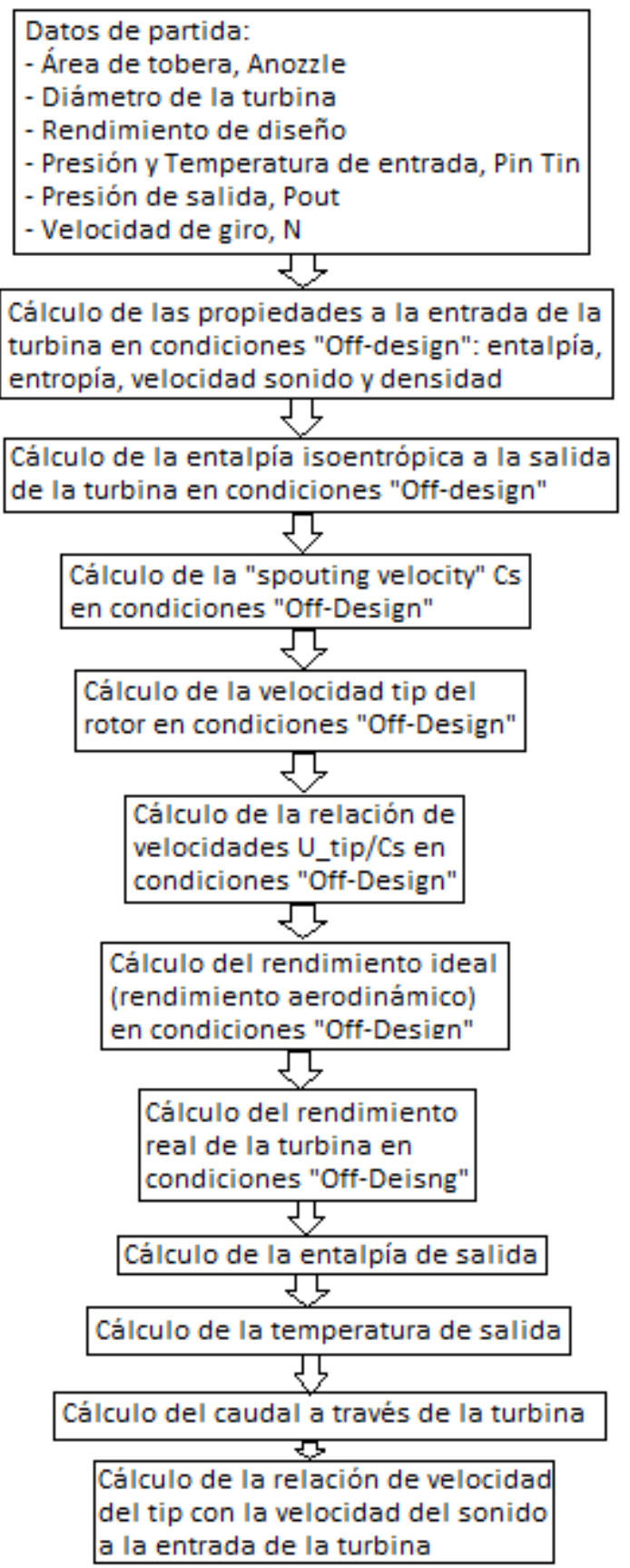

Figura 2.12. Algoritmo de cálculo para determinar las condiciones de operación

Off-design de la turbina [12]. 
En los cálculos Off-design se parte de los valores de diseño: $A_{\text {nozzle, }} \mathrm{D}$ y $\eta$, y se fijan los datos de partida: presión a la entrada $\mathrm{P}_{\mathrm{in}}$, temperatura a la entrada $\mathrm{T}_{\mathrm{in}}$, velocidad de giro $\mathrm{N}$ y presión a la salida $\mathrm{P}_{\text {out }}$. Con estos datos se realizan los cálculos del algoritmo de la figura 2.12 y se obtienen: la relación de velocidades $v$, el caudal másico $\mathrm{m}^{\prime}$, y la eficiencia real de operación $\eta$.

Respecto a la condiciones de operación de la turbina fuera del punto de diseño Off-Design, en la figura 2.12 se ha resumido su algoritmo de cálculo. Se aplica la ecuación (2-29) para calcular su rendimiento.

$\eta=\eta_{\text {design }} \cdot \eta_{\text {ideal }}=\eta_{\text {design }} \cdot 2 \cdot v \cdot \sqrt{1-v^{2}}$

\subsubsection{Compresores (Diseño Detallado)}

En la etapa de diseño del compresor radial se parte de los valores siguientes: temperatura de entrada $T_{\text {in }}$, presión de entrada $P_{\text {in, }}$ temperatura de salida $T_{\text {out }}$, presión de salida $P_{\text {out }}$, caudal a través del compresor $\mathrm{m}^{\prime}$. Se fijan los parámetros: coeficiente de altura manométrica $\Psi$ y coeficiente de flujo $\phi$. Tras el diseño básico del compresor se obtienen sus principales características: el diámetro del compresor $\mathrm{D}_{c}$, la velocidad de tip del rotor $\mathrm{U}_{\mathrm{c}}$, y la velocidad de rotación del compresor $\mathrm{N}_{\mathrm{c}}$.

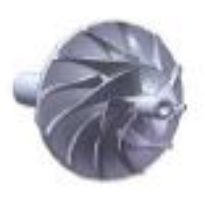

Main Compressor

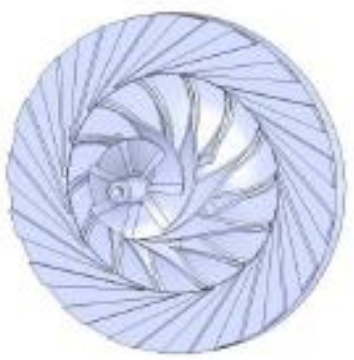

ReCompressor

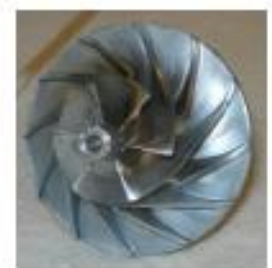

$\mathrm{OD}=37.3 \mathrm{~mm}$ $1.47^{\prime \prime}$

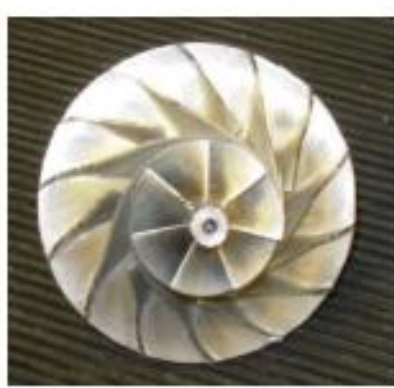

$\mathrm{OD}=57.9 \mathrm{~mm}$ $2.27^{\prime \prime}$

Figura 2.13. Representación 3D y fotografía de los compresores radiales de la marca comercial Baber-Nichols [13] que integran el lazo experimental de ciclo de potencia s-CO2 en SNL [11].

Una vez definidas las dimensiones del compresor por el fabricante, mediante los dos parámetros no dimensionales, que rigen el diseño de los compresores radiales, el coeficiente de altura manométrica $(\Psi)$ y el coeficiente de flujo $(\phi)$, definidos en las ecuaciones (2-30 y 2$31)$, se transforman los mapas de operación de las figuras 2.14 y 2.15 en las curvas no dimensionales de la eficiencia y el coeficiente de altura manométrica, ambas en función del coeficiente de flujo (figura 2.16). 


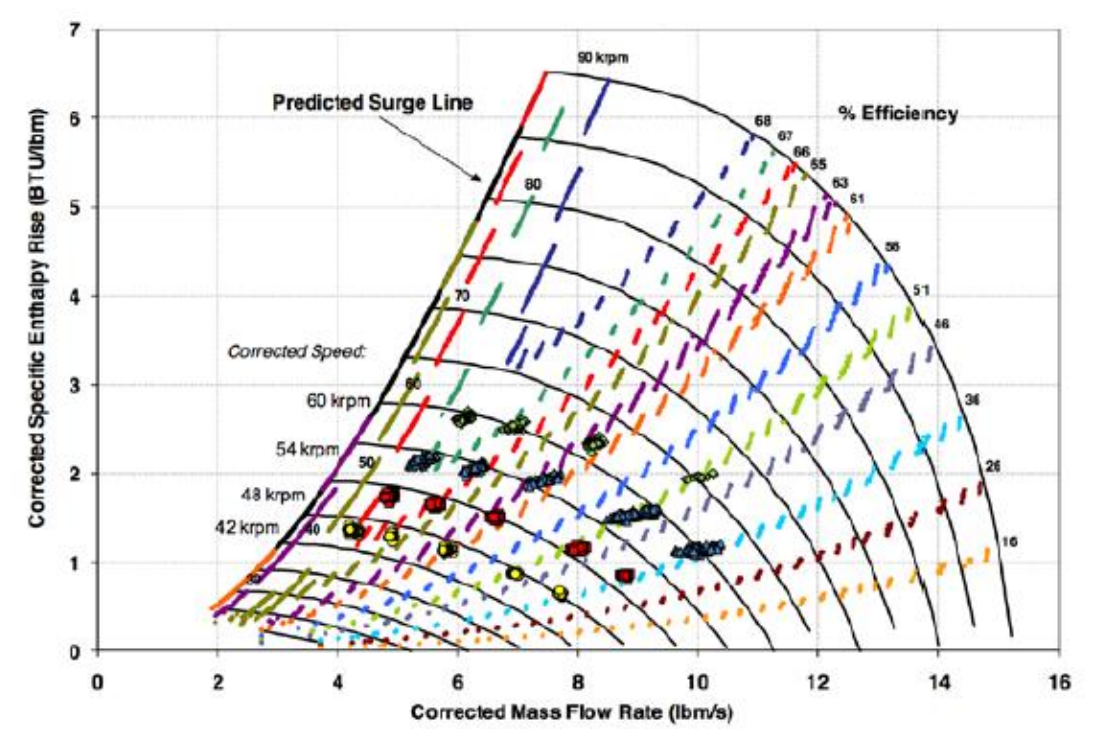

Figura 2.14. El mapa de operación del compresor radial es representado para un rango de velocidades de 35,40 y $45 \mathrm{krpm}[11]$.

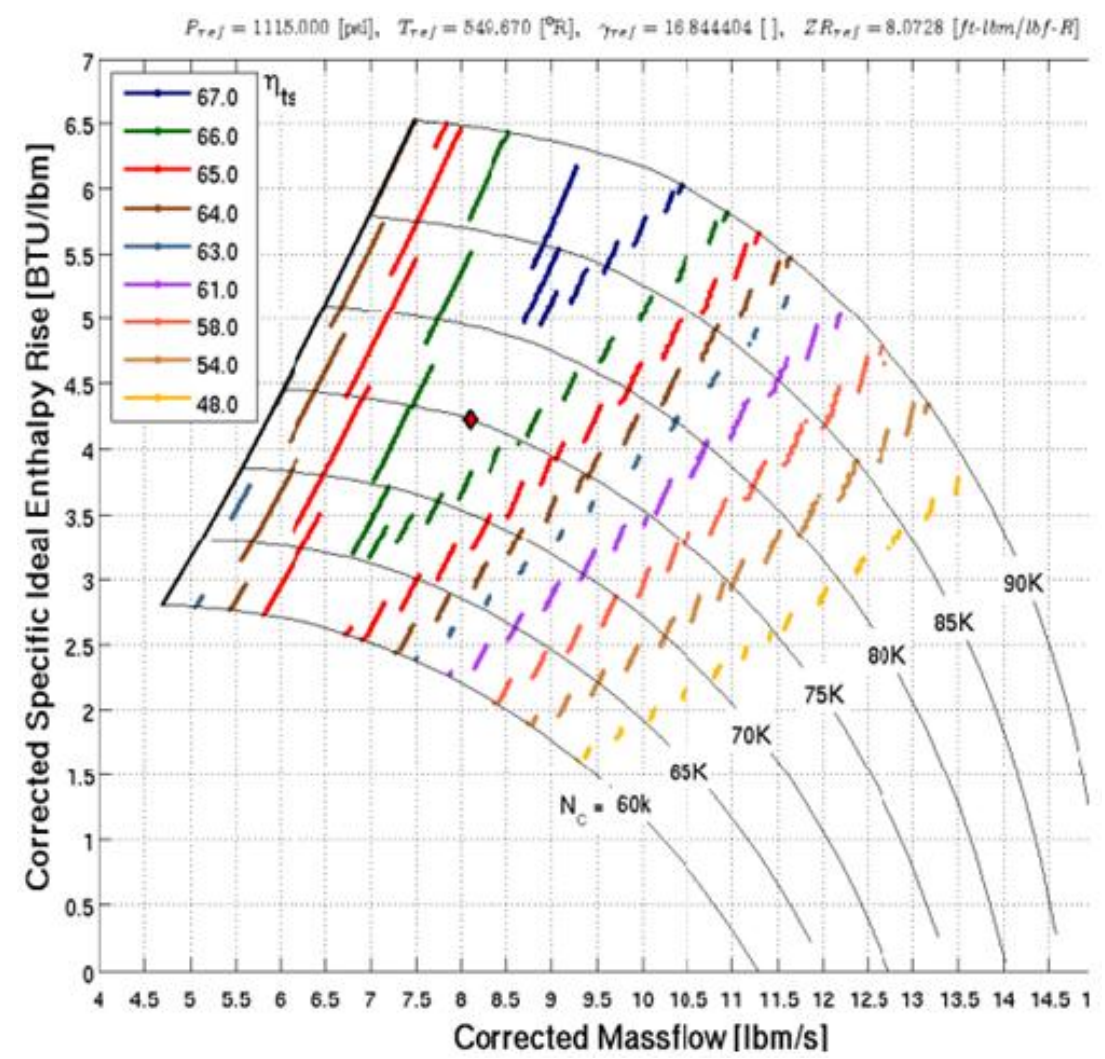

Figura 2.15. Gráfica de detalle del mapa de operación del compresor radial, en lazo de pruebas del ciclo s-CO2 Brayton, en SNL [11].

$$
\begin{gathered}
\Psi=\frac{\Delta \mathrm{h}}{\mathrm{U}_{\mathrm{c}}^{2}} \\
\phi=\frac{\mathrm{m}^{\prime}}{\rho \cdot \mathrm{U}_{\mathrm{c}} \cdot \mathrm{D}_{\mathrm{c}}^{2}}
\end{gathered}
$$




\section{Metodología}

Donde:

- $\quad \Psi$ : coeficiente de altura manométrica

- $\phi$ : coeficiente de flujo

- $\quad \Delta$ h: cambio idel (isoentrópico)de entalpía

- $\quad \mathrm{U}_{\mathrm{c}}$ : velocidad del tip del rotor

- m': caudal a través del compresor

- $\quad \rho:$ densidad

- $\quad D_{c}$ : diámetro del rotor
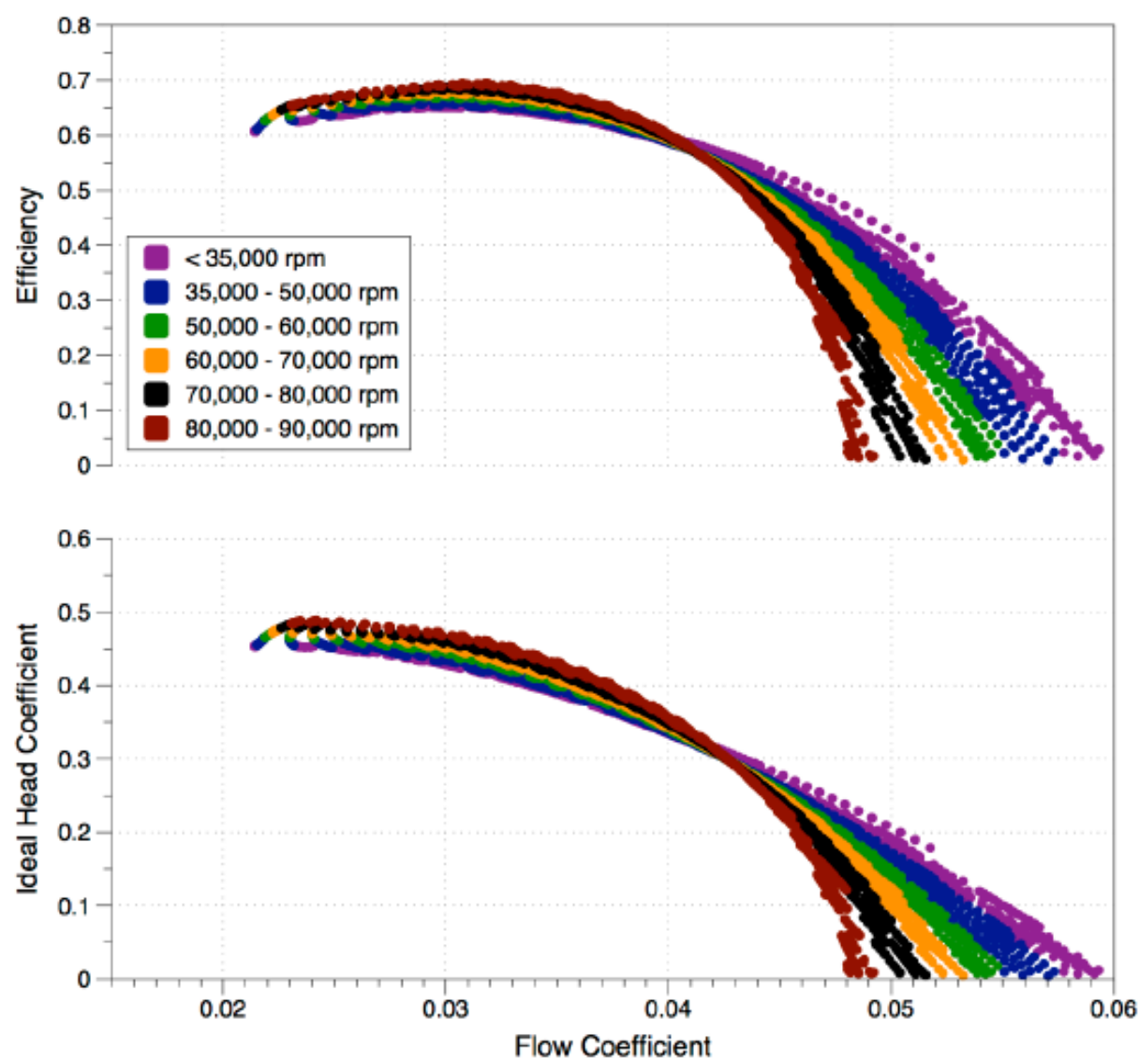

Figura 2.16. Curvas adimensionales que relacionan el coeficiente de flujo $(\phi)$, con la eficiencia $(\eta)$ y el coeficiente de altura manométrica $(\Psi)$, para el compresor del lazo de pruebas de SNL

[12].

Las curvas adimensionales de la figura 2.16 han sido modificadas y condensadas mediante las ecuaciones (2-32, 2-33 y 2-34), obteniendo como resultado las curvas de la figura 2.17 , que permite definir polinomios aproximativos del factor de flujo $\phi$, factor de altura manométrica $\Psi$, y eficiencia $\eta$, todos estos parámetros se consideran modificados y adimensionales. Las ecuaciones (2-32 a 2-34) son empíricas y específicas para el compresor del lazo de pruebas del ciclo SCO2 en SNL [11].

$\phi^{*}=\frac{\mathrm{m}^{\prime}}{\rho \cdot \mathrm{U} \cdot \mathrm{D}^{2}}\left(\frac{\mathrm{N}}{\mathrm{N}_{\text {design }}}\right)^{\frac{1}{5}}$ 


\section{Metodología}

$$
\begin{aligned}
& \Psi^{*}=\frac{\Delta h}{U^{2}}\left(\frac{N_{\text {design }}}{N}\right)^{\left(20 \phi^{*}\right)^{3}} \\
& \eta^{*}=\eta\left(\frac{N_{\text {design }}}{\mathrm{N}}\right)^{\left(20 \phi^{*}\right)^{5}}
\end{aligned}
$$

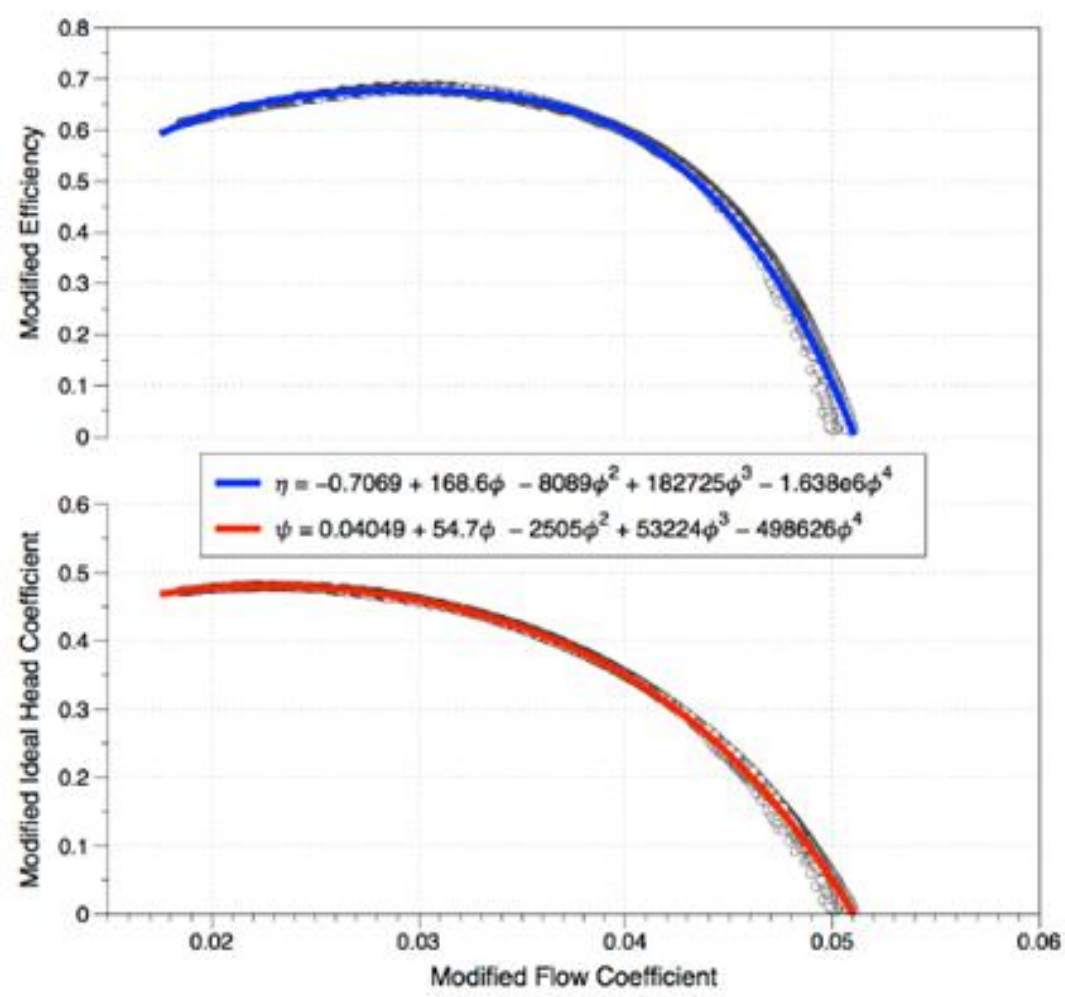

Figura 2.17. Polinomios aproximativos de la altura manométrica y eficiencia no dimensionales para el compresor de lazo de pruebas en SNL [12].

Para evitar que el diámetro del compresor sea excesivo, para el diseño del recompresor se han considerado dos etapas de compresión. El algoritmo de diseño de un compresor radial con dos etapas de compresión queda explicado en las figuras $2.19,2.20$ y 2.21 . 


\section{Metodología}

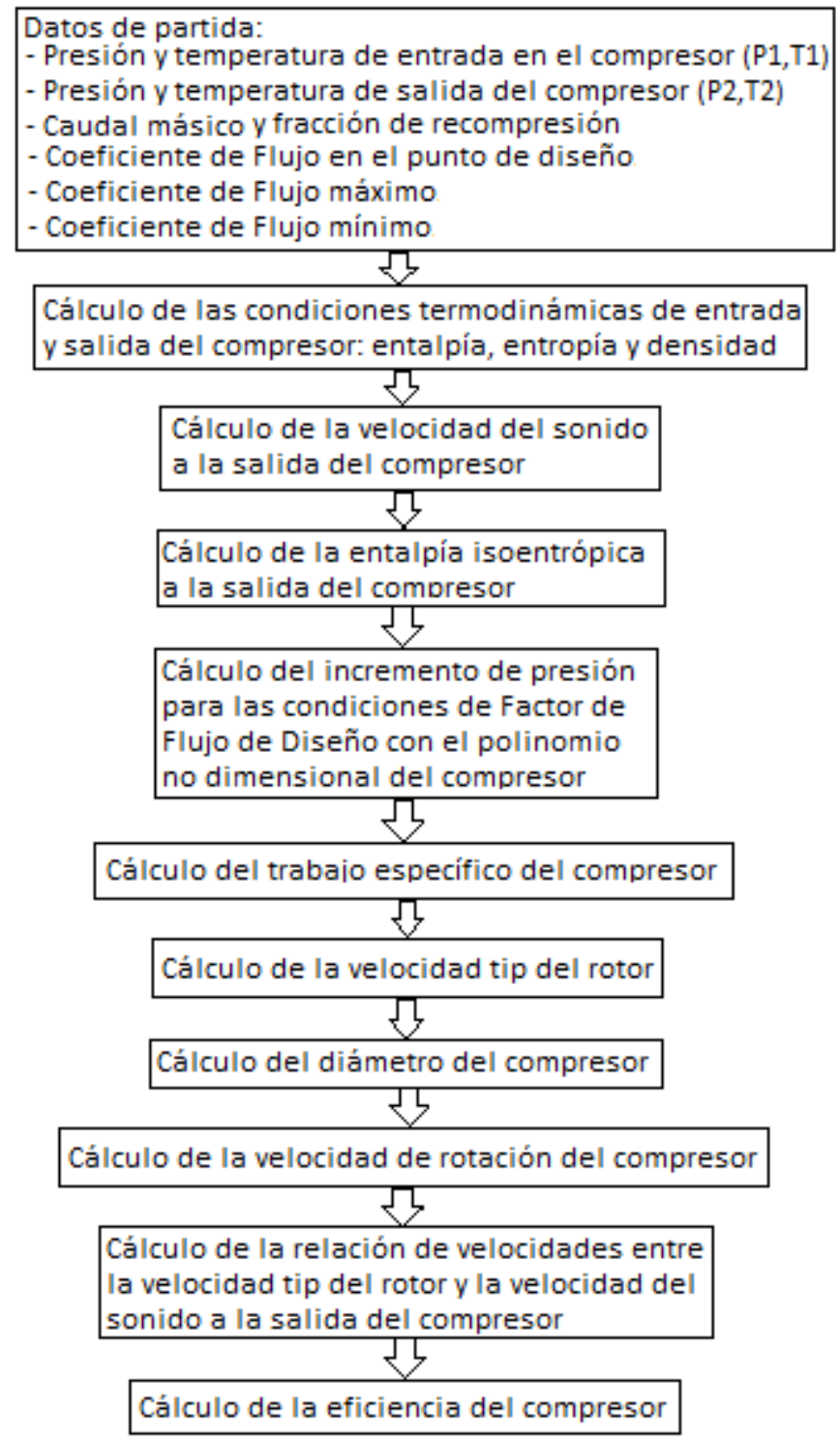

Figura 2.18. Algoritmo de diseño de los compresores radiales del ciclo Brayton con una etapa de compresión [12]. 


\section{Metodología}

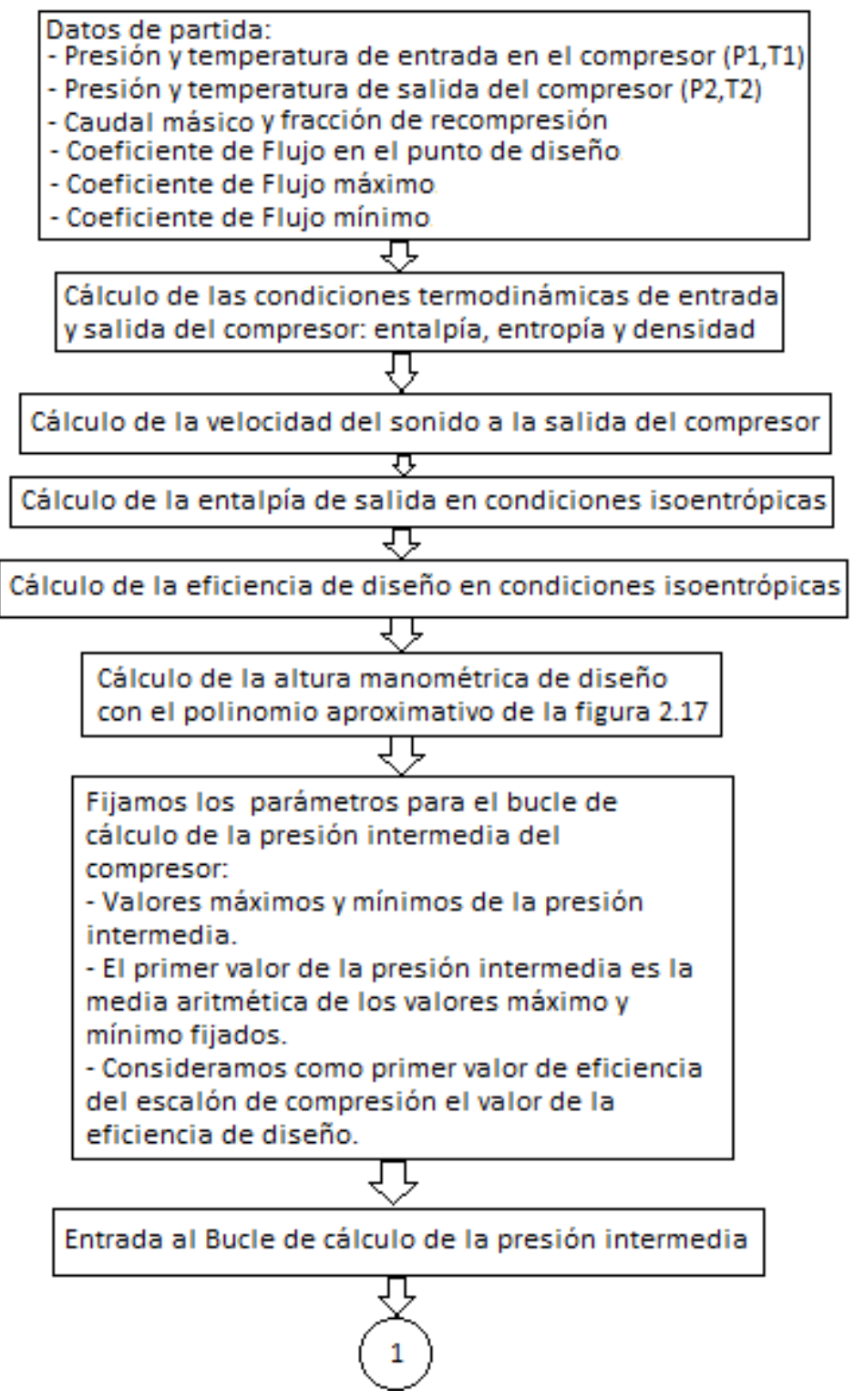

Figura 2.19. Algoritmo de diseño del Recompresor radial de dos etapas de compresión del ciclo Brayton [12]. 


\section{Metodología}

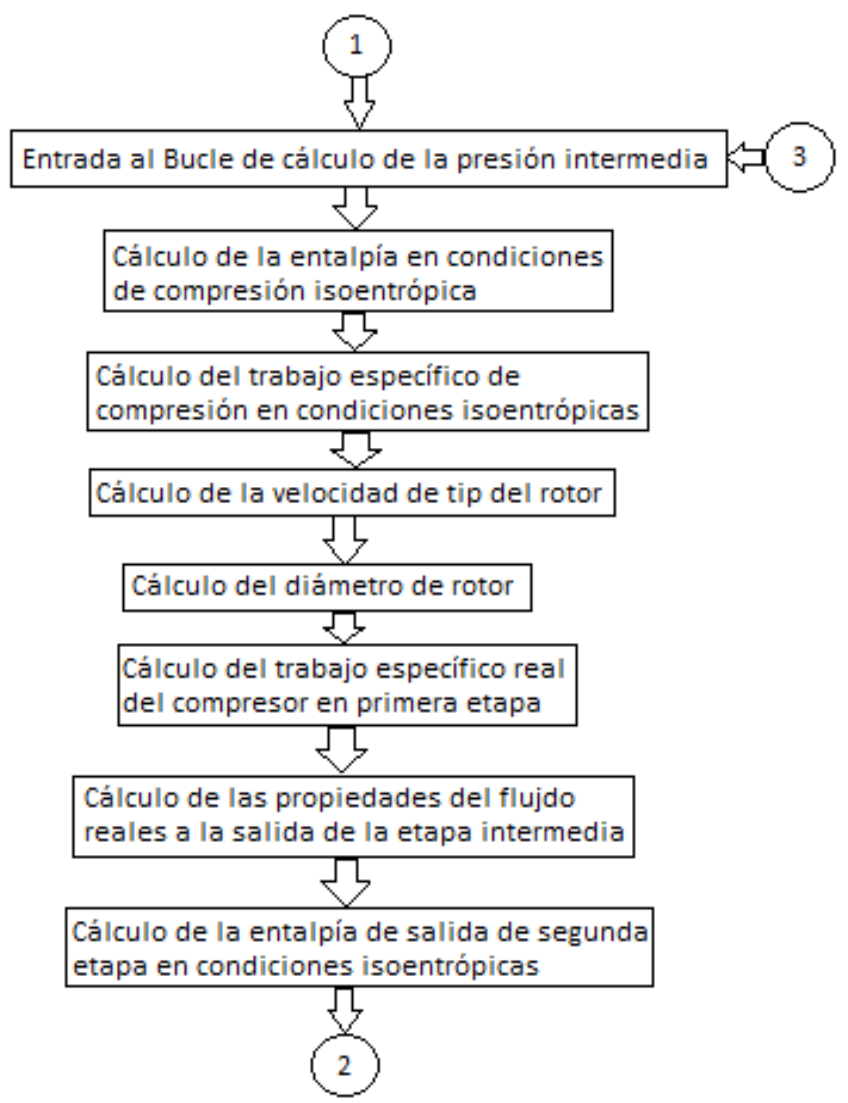

Figura 2.20. Algoritmo de diseño del Recompresor radial de dos etapas de compresión del ciclo Brayton [12].

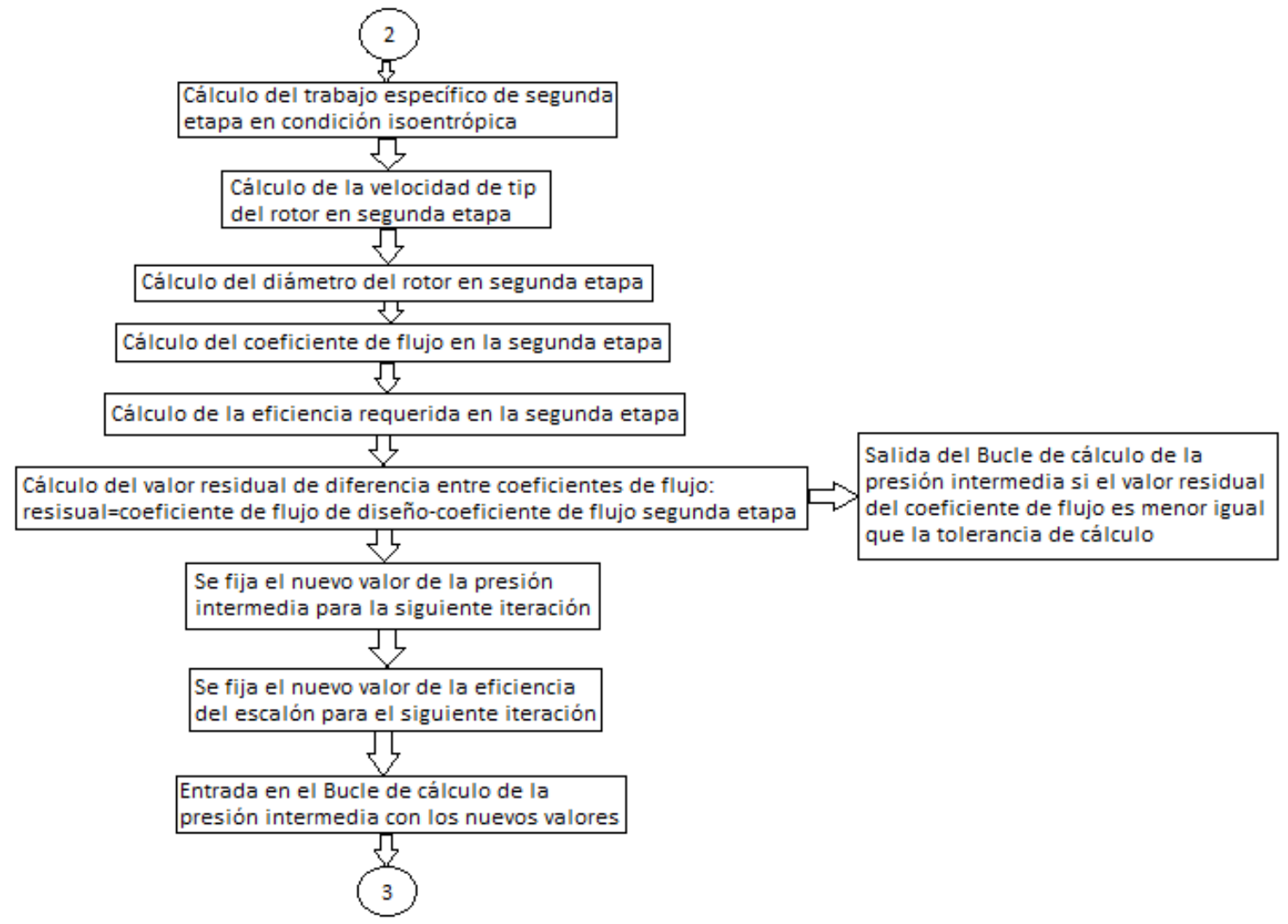

Figura 2.21. Algoritmo de diseño del Recompresor radial de dos etapas de compresión del ciclo Brayton [12]. 
En la simulación de los compresores en condiciones de operación fuera de su punto de diseño Off-Design, se parte de los siguientes datos: diámetro de diseño del compresor Dc, presión de entrada Pin, Temperatura de entrada Tin, velocidad de rotación Nc y caudal másico a través del compresor. Y se obtienen como soluciones: el factor de flujo y de altura manométrica, la presión de salida y la eficiencia real en condiciones Off-Design. En la figura 2.22 se resume el algoritmo de cálculo utilizado para definir los parámetros de operación fuera del punto de diseño en los compresores radiales con una etapa de compresión.

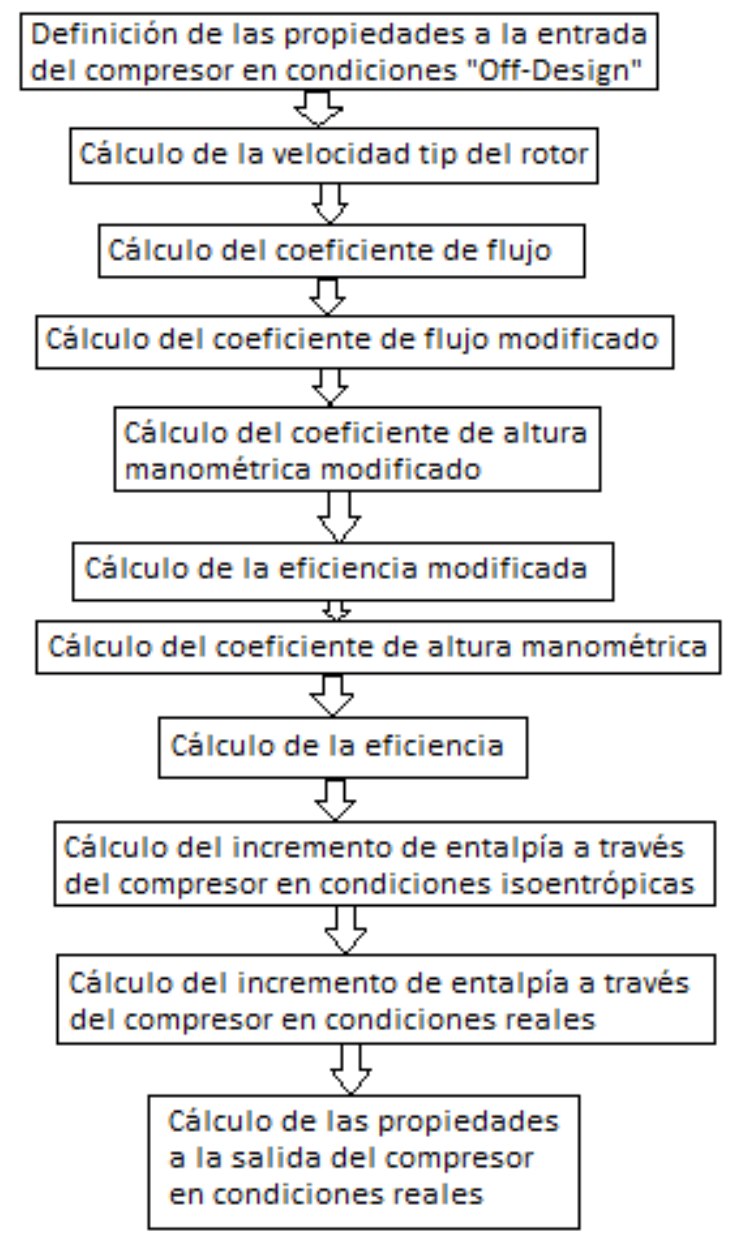

Figura 2.22. Algoritmo de cálculo para simulación de un Compresor radial de una sola etapa de compresión fuera de sus condiciones del punto de diseño Off-Design [12].

\subsection{Intercambiadores de calor del ciclo Brayton (Diseño Básico)}

Cuanto se trabaja con fluidos en estado supercrítico se confirma que existe una gran variación de sus propiedades físicas alrededor de su punto de criticidad. Debido a este motivo, para el dimensionamiento y diseño de los intercambiadores de calor, es necesario realizar su subdivisión y discretización en $\mathrm{N}$ nodos (figura 2.23), para estudiar como varían las propiedades de los fluidos caloportadores en cada uno de los nodos. 


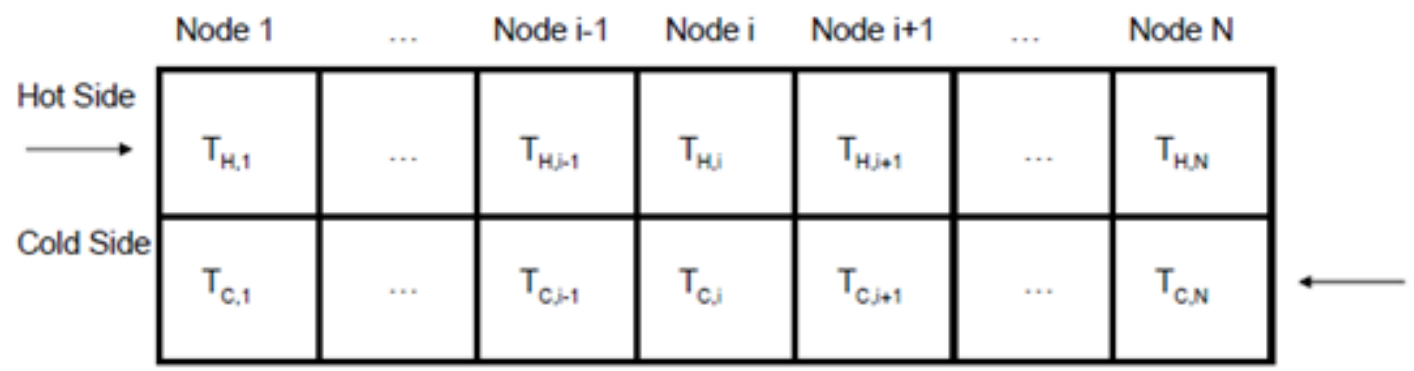

Figura 2.23. Subdivisión del intercambiador de calor con fluidos supercriticos [17, 18].

Cuanto mayor sea el número de nodos de nuestra subdivisión del intercambiador de calor, mayor será la precisión de los resultados obtenidos en su diseño y dimensionamiento. El tamaño del intercambiador de calor puede ser definido especificando su eficiencia y calculando los valores de tamaño térmico (UA) en cada uno de los nodos, o especificando su tamaño térmico total y calculando su eficiencia, o simplemente especificando dos temperaturas de entrada y una temperatura de salida de los fluidos caloportadores.

La eficiencia global del intercambiador de calor $(\varepsilon)$ es definida en la ecuación (2-35) como la relación entre el calor real intercambiado y el calor máximo que podría ser transferido en el equipo si este fuera "infinitamente" largo.

$\varepsilon=\frac{q^{\prime}}{q_{\max }}$

donde $\varepsilon$ representa la eficiencia, $\mathrm{q}$ el calor real intercambiado, y $\mathrm{q}_{\max }$ el calor máximo intercambiado con un equipo "infinitamente" largo.

A continuación, se explica en detalle el algoritmo de cálculo de la figura 2.24 para el diseño de un intercambiador conociendo las dos entalpías (presión y temperatura) de entrada (lado caliente) y una entalpía (presión y temperatura) de salida (lado frío).

1. Como primer paso se tienen que estimar las presiones a la entrada y salida de los dos fluidos (lado caliente y lado frío). Tomando como base esta hipótesis se considera una distribución lineal de caídas de presiones a lo largo de cada de las corrientes caliente y fría.

2. Con la entalpía de entrada y salida en una de las corrientes (en este caso caliente), se calcula el calor real intercambiado en el equipo mediante la ecuación (2-36).

$q^{\prime}=m_{H}^{\prime} \cdot\left(i_{H, 1}-i_{H, N}\right)$

3. Una vez conocido el calor real intercambiado se calcula la entalpía de entrada incógnita con la ecuación (2-37).

$i_{C, 1}=i_{C, N}+\frac{q^{\prime}}{m_{C}^{\prime}}$ 


\section{Metodología}

4. Siguiendo la metodología establecida por Klein and Nellis [18], se calculan las entalpías a la entrada y salida de cada nodo con las ecuaciones (2-38 y 2-39):

$$
\begin{aligned}
& i_{H, i}=i_{H, i-1}+\frac{q^{\prime}}{N \cdot m_{H}^{\prime}} \\
& i_{C, i}=i_{C, i-1}+\frac{q^{\prime}}{N \cdot m_{C}^{\prime}}
\end{aligned}
$$

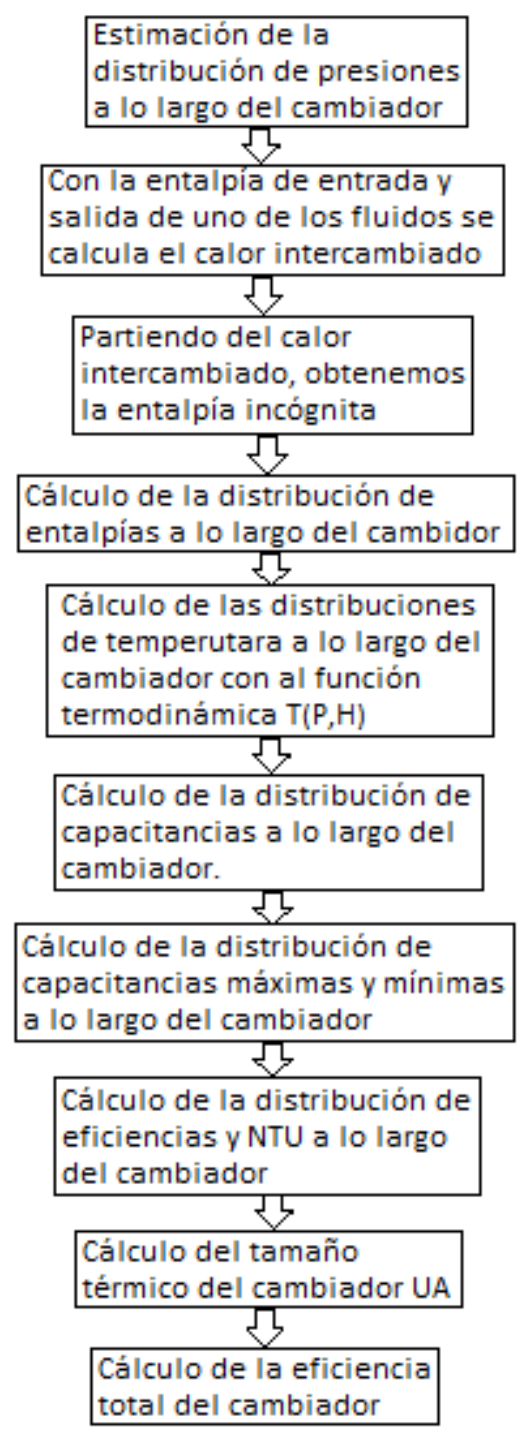

Figura 2.24. Algoritmo para el cálculo de los intercambiadores de calor en un ciclo de potencia Brayton con fluido de trabajo en estado supercrítico [17-18].

5. Una vez calculadas las distribuciones de entalpía en cada uno de los nodos, con las tablas de propiedades de los fluidos se calculan las distribuciones de temperaturas en los diferentes nodos, con la función termodinámica de cálculo de temperatura en función de la presión y entalpía $T(P, H)$, ver ecuaciones (2-40, 2-41, 2-42, 2-43):

$\Delta i_{H, i}=i_{H, i}-i_{H, i+1}$ 
$\Delta i_{C, i}=i_{C, i}-i_{C, i+1}$

$\Delta T_{H, i}=T_{H, i}-T_{H, i+1}$

$\Delta T_{C, i}=T_{C, i}-T_{C, i+1}$

6. La capacitancia del lado frio y caliente en cada uno de los nodos se calcula mediante la ecuación (2-44):

$C_{H, i}^{\prime}=m_{H}^{\prime} \cdot \frac{\Delta i_{H, i}}{\Delta T_{H, i}}$

7. La eficiencia de cada uno de los intercambiadores en los que hemos subdividido el equipo principal es calculada con la siguiente ecuación (2-45):

$q^{\prime}=\Sigma \varepsilon_{\mathrm{i}} \cdot C_{\min , \mathrm{i}}^{\prime} \cdot\left(T_{H, i}-T_{C, i+1}\right)$

8. Respecto al tamaño térmico de cada nodo es calculado con la siguiente ecuación (2-46):

$U A_{i}=N T U_{i} \cdot \min \left(C_{H, i}^{\prime}, C_{C, i}^{\prime}\right)$

EI NTU (Number of Transfer Units) viene definido por la ecuación (2-47), como la relación de las capacitancias $C_{R}$, ver ecuación (2-48).

$N T U_{i}=\frac{1-\exp \left[-N T U \cdot\left(1-C_{R}\right)\right]}{1-C_{R} \cdot \exp \left[-N T U \cdot\left(1-C_{R}\right)\right]}$

Donde: $C_{R}=\frac{C_{\min }^{\prime}}{C_{\max }^{\prime}}$

9. El cálculo de la eficiencia del intercambiador $\varepsilon$ cuantifica la relación entre el calor real intercambiado y el calor que sería posible transferir en el caso de disponer de un cambiador infinitamente largo.

\subsection{Intercambiadores de calor del ciclo Brayton (Diseño Detallado)}

Los ciclos de potencia Brayton requieren intercambiadores de calor que soporten grandes presiones de trabajo. A la vez los mencionados cambiadores requieren gran área de intercambio de calor, y que el equipo no ocupe un gran volumen. Para cumplir con todos estos requisitos se ha considerado como solución técnica más apropiada los intercambiadores de calor de circuito impreso compactos o Printed Circuit Heat Exchangers (PCHE). Los canales semihemisféricos que integran estos equipos son fabricados mediante agresión química y tienen un diámetro de 1 a $2 \mathrm{~mm}$. Su fabricación se realiza mediante placas unidas por un proceso metalúrgico de calentamiento y presión. Para el diseño en detalle de estos equipos se ha considerado la correlación de transferencia de calor de Gnielinsky, ver ecuación (2-49): 


\section{Metodología}

$\mathrm{Nu}_{\mathrm{D}_{\mathrm{h}}}=\frac{\left(\frac{\mathrm{f}}{8}\right) \cdot\left(\mathrm{Re}_{\mathrm{D}_{\mathrm{h}}}-1000\right) \cdot \operatorname{Pr}}{1+12.7 \cdot\left(\operatorname{Pr}^{\frac{2}{3}}-1\right) \cdot \sqrt{\frac{\mathrm{f}}{8}}}$

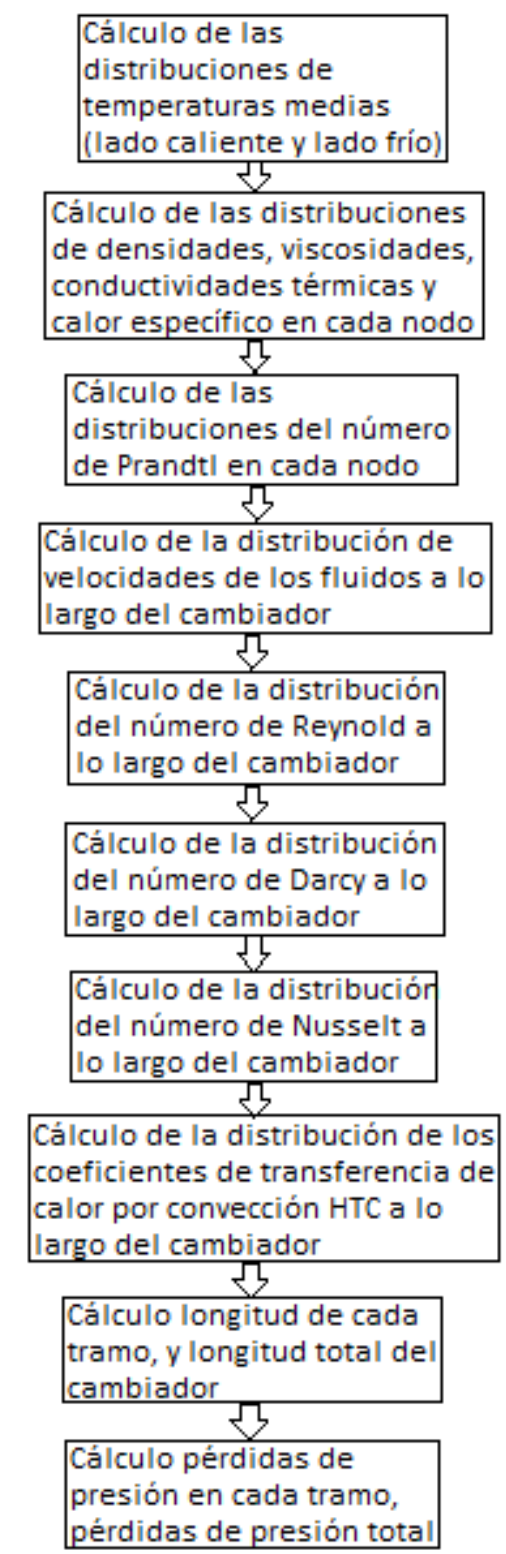

Figura 2.25. Algoritmo de cálculo para cálculo de la longitud total y pérdidas de presiones de un cambiador de calor en un ciclo Brayton con fluido de trabajo en estado supercrítico [17].

Tomando como punto de partida la distribución de temperaturas calculadas en base al procedimiento explicado en el apartado anterior, se calcula la distribución de coeficientes de transferencia de calor o Heat Transfer Coefficients (HTC) siguiendo la metodología descrita a continuación (figura 2.25):

1. Cálculo de la distribución de temperaturas medias (Tave_h y Tave_c) en cada uno de los nodos.

2. Cálculo de densidades en cada nodo. 


\section{Metodología}

3. Cálculo de viscosidades en cada nodo.

4. Cálculo de la relación entre densidad y viscosidad en cada nodo.

5. Cálculo de la conductividad térmica en cada nodo.

6. Cálculo del calor específico a presión constante $C_{P}$ en cada nodo.

7. Cálculo del número de Prandtl en cada nodo.

8. Cálculo de caudales, y distribución de velocidades.

9. Cálculo del número de Reynold en cada nodo.

10. Cálculo del número de Darcy en cada nodo, ecuación (2-50):

$f_{f d}=\frac{1}{\left[0.79 \cdot \ln \left(R e_{D_{h}}\right)-1.64\right]^{2}}$

11. Cálculo del número de Nusselt en cada nodo de acuerdo a la correlación de Gnielinsky.

12. Resolución de los coeficientes de transferencia de calor en cada nodo, ecuación (2-51):

$h=\frac{N u_{D_{h}} \cdot k}{D_{h}}$

13. Cálculo de la longitud de cada tramo del intercambiador de calor y de su longitud total, ecuación (2-52):

$\Delta x=\frac{U A_{i} \cdot\left(\frac{1}{h_{H, i}}+\frac{1}{h_{C, i}}+\frac{t h}{k_{m}}+f f\right)}{W_{c h} \cdot N_{c h}}$

14. Cálculo de las pérdidas de presión en el lado caliente y lado frío del intercambiador, ecuación (2-53).

$\Delta P_{i}=f_{f d, i} \cdot\left(\frac{\Delta x_{i}}{W_{c h}}\right) \cdot \rho_{i} \cdot\left(\frac{v_{i}^{2}}{2}\right)$

2.8. Intercambiadores de calor del ciclo Brayton. Operación fuera de su punto de diseño OffDesign performance

Se han desarrollado recientemente dos modelos para simular las condiciones de operación de los intercambiadores de calor del ciclo Brayton cuando operan fuera de su punto de diseño, es decir, cuando operan en condiciones Off-Design. Los modelos están explicados en las 


\section{Metodología}

referencias $[12,19,20]$. A continuación se realiza una breve descripción y explicación de los dos modelos:

1. Modelo desarrollado por J.Dyreby [12], L.Tse y T.Neises [19].

- Las pérdidas de presión a través del intercambiador son simuladas con la ecuación (2-54) [20]:

$\Delta \mathrm{P}=\Delta \mathrm{P}_{\text {design }} \cdot\left(\frac{\mathrm{m}^{\prime}}{\mathrm{m}_{\text {design }}^{\prime}}\right)^{\frac{7}{4}}$

- El coeficiente de transferencia de calor U en condiciones Off-design se calcula con la ecuación (2-55) [20]:

$U=U_{\text {design }} \cdot\left(\frac{1}{2} \cdot\left(\frac{m_{\mathrm{CO}_{2}}^{\prime}}{m_{\mathrm{CO}_{2, \text { design }}}^{\prime}}+\frac{m_{\mathrm{HTF}}^{\prime}}{m_{H T F, \text { design }}^{\prime}}\right)\right)^{0.8}$

2. Modelo desarrollado Kevin Hoopes, D.Sánchez, Francesco Crespi [21]. Para tener un conocimiento del comportamiento bajo condiciones de carga parcial de los intercambiadores de calor del ciclo Brayton, antes de especificar la geometría del equipo, se define la relación de la ecuación (2-56):

$\mathrm{h} \cdot \mathrm{A}_{\text {ratio }}=\frac{\mathrm{h} \cdot \mathrm{A}_{\text {hot }}}{\mathrm{h} \cdot \mathrm{A}_{\text {cold }}}$

$\frac{1}{\mathrm{UA}}=\frac{1}{\mathrm{hA}_{\mathrm{hot}}}+\frac{1}{\mathrm{hA}_{\text {cold }}}$

Las ecuaciones (2-56 y 2-57) se resuelven obteniendo la ecuación (2-58).

$\frac{1}{U A}=\frac{1}{h A_{\text {hot }}}+\frac{1}{\frac{h A_{\text {hot }}}{h A_{\text {ratio }}}}$

Para comenzar con los cálculos de las condiciones Off-design se necesitan los siguientes parámetros: el caudal de ambas corrientes, la temperatura y presión de ambos lados del intercambiador. Se cálcula el intercambio de calor en cada una de las divisiones del intercambiador con la ecuación (2-59):

$Q_{o f f-d e s i g n}=U A_{o f f-d e s i g n} * \Delta T_{o f f-d e s i g n}$

Se estima el valor $U A_{\text {off-design }}$ mediante las ecuaciones (2-60 y 2-61), tanto para la corriente fría como para la corriente caliente:

$h A_{o f f-\text { design }}=h A_{o n-\text { design }} *\left[\frac{\lambda_{o f f-\text { design }}}{\lambda_{\text {on-design }}}\right] *\left[\frac{R e_{o f f-\text { design }}}{R e_{\text {on-design }}}\right]^{x} *\left[\frac{P r_{o f f-d e s i g n}}{P r_{o n-d e s i g n}}\right]^{y}$ 


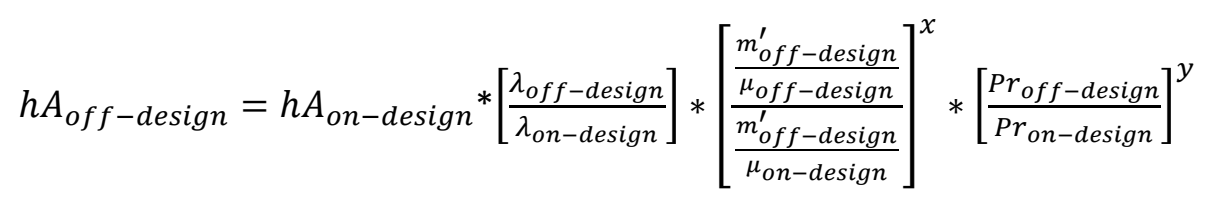

Donde:

- $\quad \lambda$ : conductividad térmica.

- $\quad \mu$ : viscosidad dinámica.

- Pr: número de Prandtl.

Los valores de $\mathrm{x}$ e y, pueden ser especificados separadamente para las corrientes fría y caliente tal y como se indica en la correlación de Dittus-Boelter. Finalmente mediante la aplicación de la ecuación (2-57) se obtiene el valor de $U \mathrm{~A}_{\text {off-design. }}$.

\subsection{Algoritmos de optimización multivariable}

Se han utilizado tres algoritmos matemáticos para realizar una optimización multivariable de los parámetros termodinámicos del ciclo de potencia Brayton en las plantas termosolares con colectores lineales (PTC o LF). Los algoritmos empleados son: SUBPLEX [22], UOBYQA [23-25], NEWUOA [26-27].

\subsubsection{Algoritmo SUPBLEX}

Fue desarrollado por Tom H. Rowan (Laboratorio Central Oak Ridge en Estados Unidos) para la optimización de una función multivariable sin condiciones de contorno alguna. El método Subplex está basado en el método Simplex detallado a continuación:

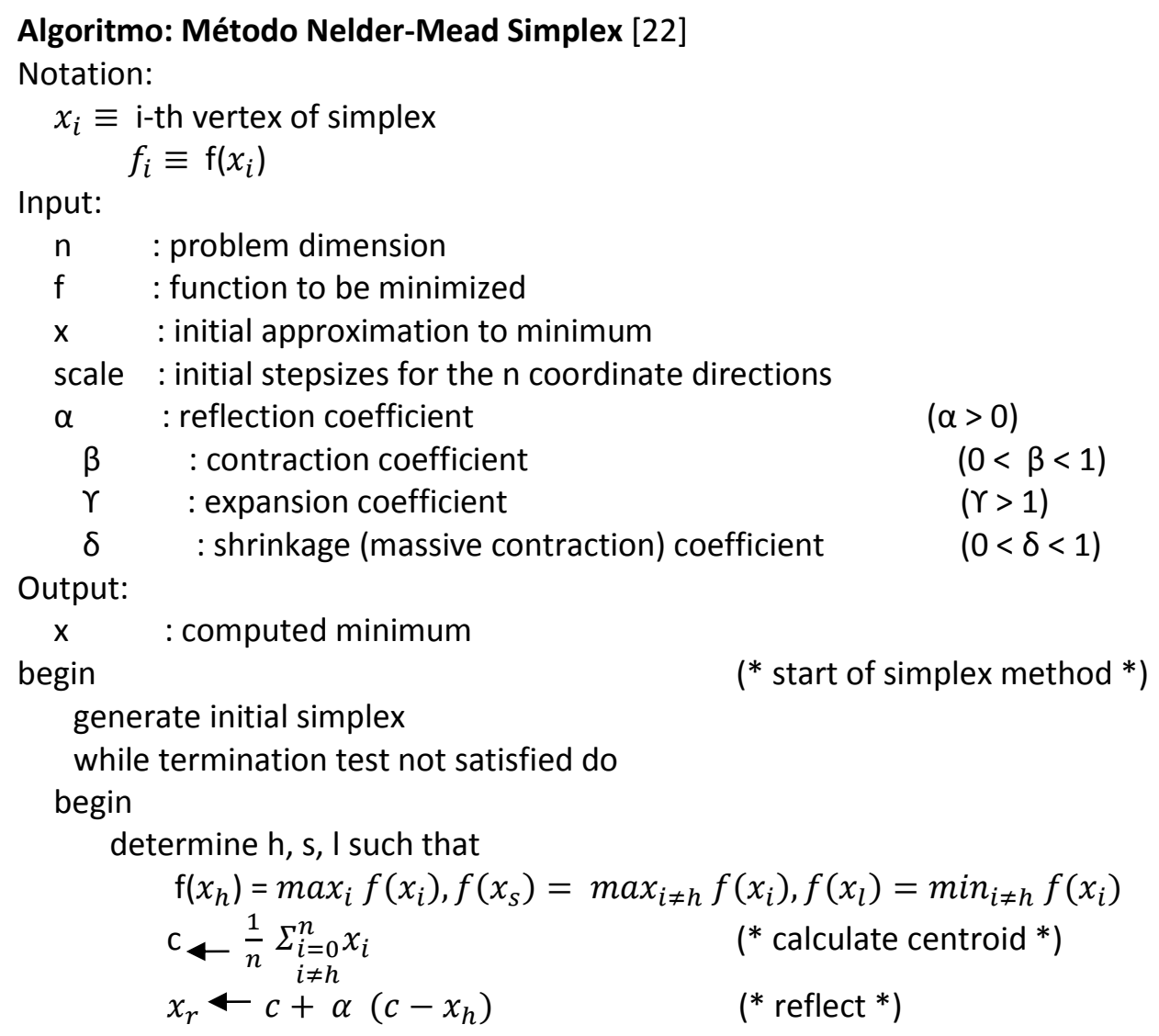




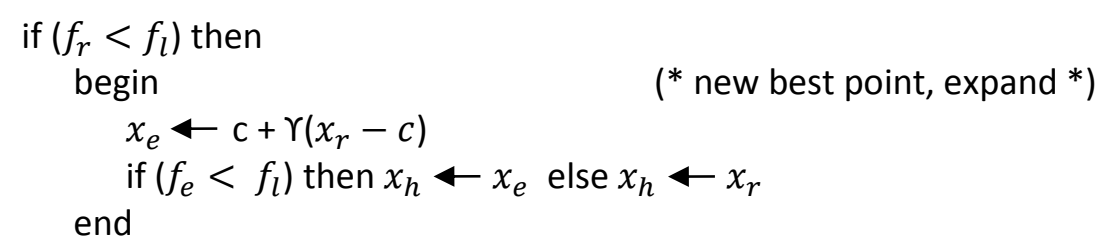

else if $\left(f_{r}<f_{s}\right)$ then $x_{h} \leftarrow x_{r} \quad\left({ }^{*}\right.$ neither best nor worst point, accept *) else

end

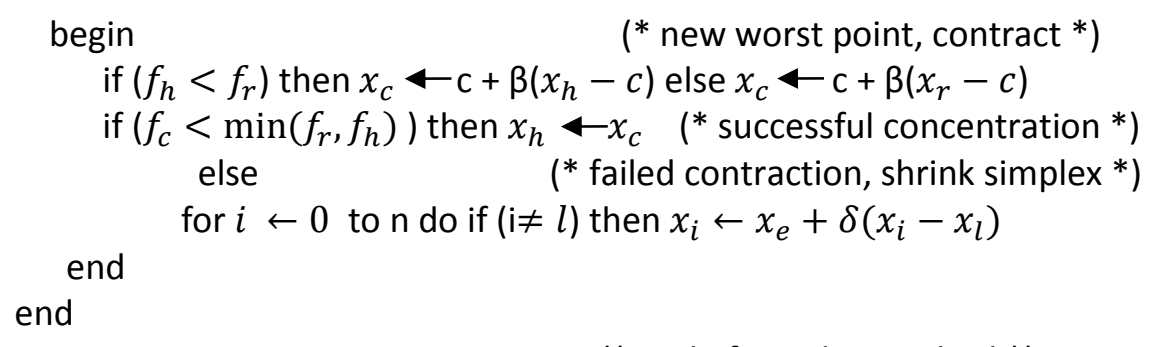

El Método Simplex es un método analítico de solución de problemas de programación lineal capaz de resolver modelos más complejos que los resueltos mediante el método gráfico sin restricción en el número de variables. El Método Simplex es un método iterativo que permite ir mejorando la solución en cada paso.
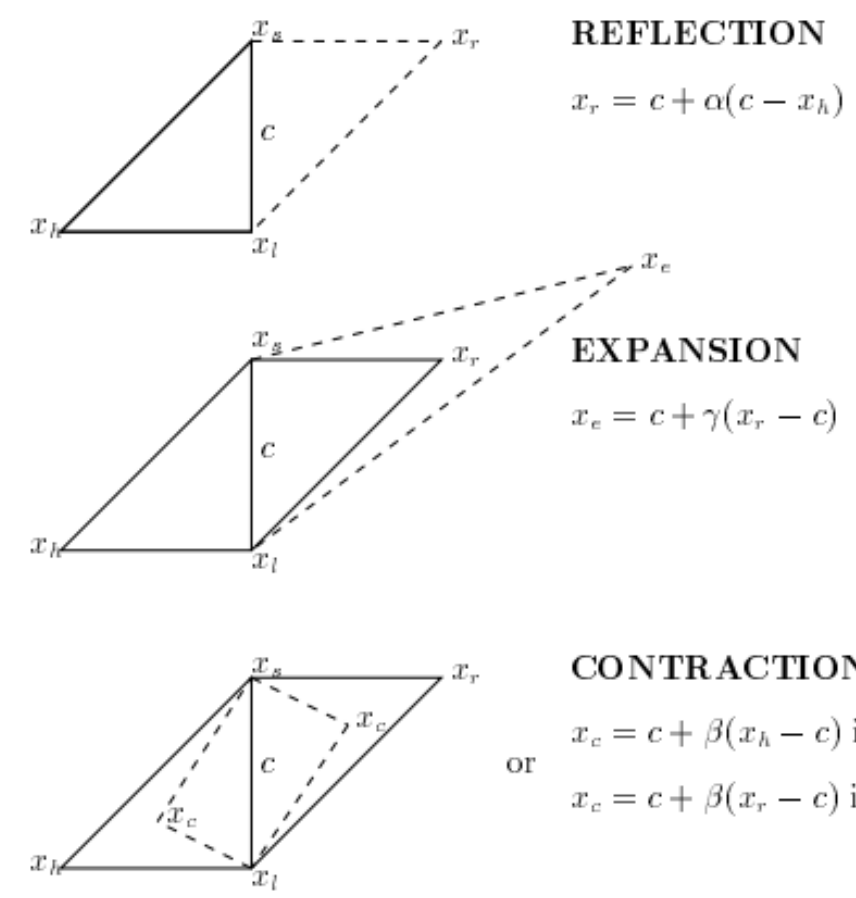

CONTRACTION
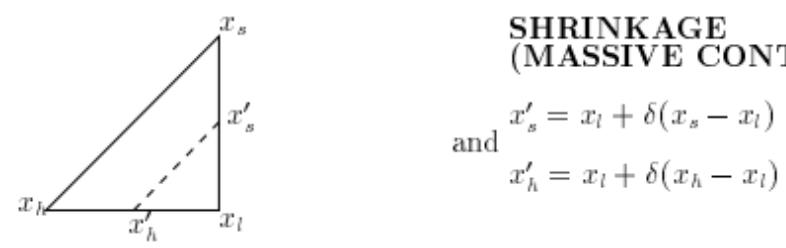

Figura 2.26. Movimientos básicos del método Nelder-Mead para buscar la solución [22].

La razón matemática de esta mejora radica en que el método consiste en caminar del vértice de un poliedro a un vértice vecino de manera que aumente o disminuya (según el contexto 
de la función objetivo, sea maximizar o minimizar), dado que el número de vértices que presenta un poliedro solución es finito siempre se hallará solución (figura 2.26). Este famosísimo método fue creado en el año de 1947 por el estadounidense George Bernard Dantzig y el ruso Leonid Vitalievich Kantorovich, con el ánimo de crear un algoritmo capaz de solucionar problemas de $\mathrm{m}$ restricciones y $\mathrm{n}$ variables. En el código Subplex el número de llamadas a la función de evaluación se incrementa linealmente con el tamaño del problema, por tanto para la mayor parte de aplicaciones el método Subplex es más eficiente que el método Simplex. La idea principal del algoritmo Subplex es: Search the input space attempting to maximize the ratio of the forward error to the condition number.

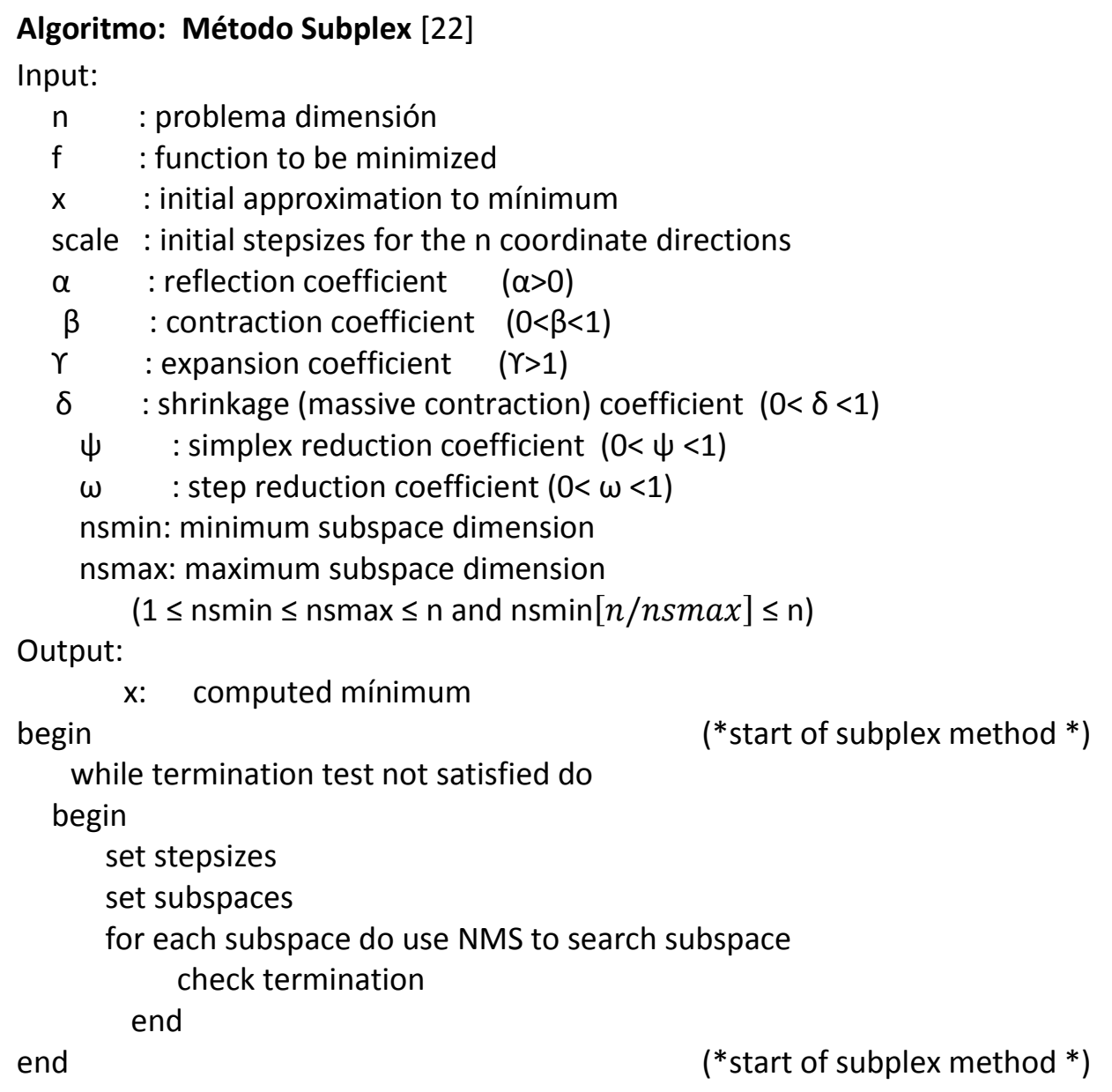

El algoritmo Subplex tiene similar estructura a los métodos, en que en cada iteración, determinan un conjunto de direcciones mejoradas para la búsqueda de la solución (figura 2.26), y realizan la búsqueda de la solución de forma lineal a lo largo de cada dirección. El algoritmo Subplex determina una serie de subespacios optimizados, donde aplica posteriormente el método Nelder-Mead Simplex (NMS).

\subsubsection{Algoritmo UOBYQA - Unconstrained Optimization BY Quadratic Approximation}

UOBYQA es un nuevo algoritmo para la optimización de funciones multivariable sin condiciones de contorno, que tienen en cuenta la curvatura de la función objetivo, F, generando modelos cuadráticos mediante interpolación [23-25]. Por ello, como no se requieren primeras derivadas, cada modelo es definido por $1 / 2(n+1)(n+2)$ valores de $F$, donde $\mathrm{n}$ es el número de variables, y los puntos de interpolación deben de tener la propiedad que 
los polinomios cuadráticos se hacen nulos para todos estos puntos. Una iteración típica del algoritmo genera una nueva colección de variables $\mathrm{X}_{\mathrm{t}}$, es decir, mediante la minimización del modelo cuadrático restringido a la región de confianza, o mediante un procedimiento que debería mejorar la exactitud del modelo. Entonces $F\left(X_{t}\right)$ es obtenida y uno de los puntos de interpolación es sustituido por $X_{t}$. Por ello el algoritmo explica cómo se consideran inicialmente la posición inicial de los puntos de interpolación, el ajuste posterior del radio de la región de confianza, el cálculo de Xt en los dos casos mencionados, y la selección del punto a reemplazar. UOBYQA trabaja con funciones de Lagrange de las ecuaciones de interpolación explícitamente, de esta forma sus coeficientes son actualizados cada vez que uno de los puntos de interpolación es movido. Las funciones de Lagrange ayudan al procedimiento para mejora del modelo, y también proporcionan una estimación del error de la aproximación cuadrática de la función $F$, lo cual permite al algoritmo un mayor ratio de convergencia.

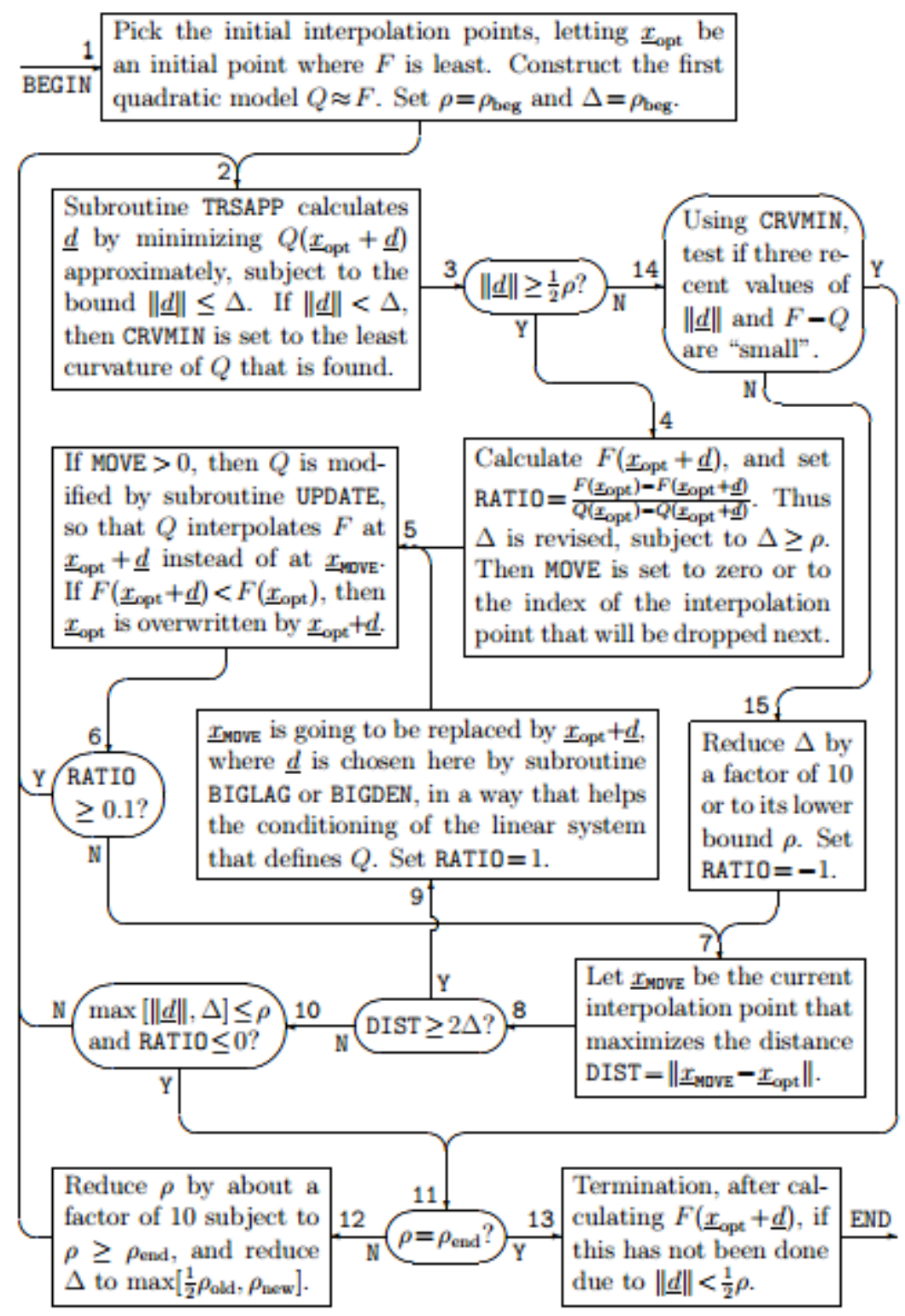

Figura 2.27. Definición del algoritmo NEWUOA [26-27]. 


\subsubsection{Algoritmo NEWUOA - NEW Unconstrained Optimization Algorithm}

El programa informático NEWUOA (figura 2.27) realiza una búsqueda de los valores que minimizan la función $\mathrm{F}(\mathrm{x})$. El algoritmo es iterativo, al inicio de cada iteración se realiza un modelo cuadrático $Q$ que se ajusta lo más posible a la función $F$. Este modelo cuadrático $Q$ es utilizado dentro de la región de confianza para ajustar el valor de las variables. Cuando el modelo cuadrático es revisado, la nueva función $\mathrm{Q}$ interpola $\mathrm{F}$ en $\mathrm{m}$ puntos, recomendando un valor de $m=2 n+1$. La nueva $Q$ es calculada en base a la minimización de la norma de Frobenius de la variación de la segunda derivada de la función $Q$.

Únicamente un solo punto es modificado en cada iteración. Por tanto, excepto para casos ocasionales donde el origen se varia, el coste de cálculo de cada iteración es del orden $(m+n)^{2}$, lo cual permite que $\mathrm{n}$ sea suficientemente grande. En el desarrollo de NEWUOA se han tenido en cuenta diferentes soluciones para conseguir una buena exactitud y solidez del algoritmo.

Se incluye la elección del modelo cuadrático inicial, la necesidad mantener una independencia lineal en las condiciones de interpolación en la presencia de errores de redondeo del ordenador, y la estabilidad de la actualización de ciertas matrices que permiten una rápida revisión de la función cuadrática $Q$.

\subsection{Referencias}

[1] Thermoflow Software. https://www.thermoflow.com/

[2] System Advisor Model (SAM) software technical Manual. https://sam.nrel.gov

[3] Span, R. and Wagner, W., A New Equation of State for Carbon Dioxide Covering the Fluid Region from the Triple-Point Temperature to $1100 \mathrm{~K}$ at Pressures up to $800 \mathrm{MPa}$. J. Phys. Chem. Ref. Data, 25(6):1509-1596, 1996.

[4] Buecker, D. and Wagner, W. A Reference Equation of State for the Thermodynamic Properties of Ethane for Temperatures from the Melting Line to $675 \mathrm{~K}$ and Pressures up to 900 MPa. J. Phys. Chem. Ref. Data, 35(1):205-266, 2006.

[5] Setzmann, U. and Wagner, W. A New Equation of State and Tables of Thermodynamic Properties for Methane Covering the Range from the Melting Line to $625 \mathrm{~K}$ at Pressures up to 1000 MPa. J. Phys. Chem. Ref. Data, 20(6):1061-1151, 1991.

[6] Span, R., Lemmon, E.W., Jacobsen, R.T, Wagner, W., and Yokozeki, A. A Reference Equation of State for the Thermodynamic Properties of Nitrogen for Temperatures from 63.151 to 1000 K and Pressures to 2200 MPa. J. Phys. Chem. Ref. Data, 29(6):1361-1433, 2000.

[7] Lemmon, E.W. and Span, R. Short Fundamental Equations of State for 20 Industrial Fluids. J. Chem. Eng. Data, 51:785-850, 2006.

[8] Guder, C. and Wagner, W. A Reference Equation of State for the Thermodynamic Properties of Sulfur Hexafluoride (SF6) for Temperatures from the Melting Line to $625 \mathrm{~K}$ and Pressures up to 150 MPa. J. Phys. Chem. Ref. Data, 38(1):33-94, 2009.

[9] International Association for the Properties of Water and Steam. http://www.iapws.org/ [10] C.A. Meyer, R.B. McClintock, G.J.Silvestri, and R.C.Spencer. ASME Steam Tables: Thermodynamic and Transport Properties of Steam. New York, 1967.

[11] S.A. Wright, R.F. Radel, T.M. Conboy, G.E. Rochau. Modeling and Experimental Results for Condensing Supercritical CO2 Power cycles. Sandia National Laboratories in USA. SAN 20108840, unlimited release, printed January 2011. 
[12] J. Dyreby. Thesis: Modeling the Supercritical Carbon Dioxide Brayton Cycle with Recompression. University of Wisconsin-Madison, 2014.

[13] Baber-Nichols Companny. http://www.barber-nichols.com/products/turbines

[14] Gibbs, J. P., Hejzlar, P., \& Driscoll, M. J., Applicability of Supercritical CO2 Power Conversion Systems to GEN IV React. MIT, Topical Report No. MIT-GFR-037, (2006).

[15] Japikse, D., \& Baines, N. C., Introduction to Turbomachinery, Concepts ETI, (1997).

[16] Chen, H., \& Baines, N. C. The aerodynamic loading of radial and mixed-flow turbines. International Journal of Mechanical Sciences, Vol. 36, No. 1, pp. 63-79, (1994).

[17] Seidel W. Master Thesis: Model Development and Annual Simulation of the Supercritical Carbon Dioxide Brayton Cycle for Concentrating Solar Power Applications. University of Wisconsin, Madison, 2010. http://sel.me.wisc.edu/publications/theses/seidel10.zip

[18] Klein, Nellis. 2009. Heat Transfer. Cambridge University Press.

[19] Louis A. Tse, Ty Neises. Analysis and Optimization for Off-Design performance of the recompression sCO2 cycles for high temperature CSP applications. The 5th International Symposium- Supercritical CO2 Power Cycles, March 29-31, 2016, San Antonio, Texas.

[20] Patnode, A. M., Simulation and Performance Evaluation of Parabolic Trough Solar Power Plants, MS Thesis, University of Wisconsin-Madison, Madison, WI, (2006).

[21] K.Hoopes, D.Sánchez. A new method for modeling Off-Design performance of sCO2 Heat Exchangers without specifying detailed geometry. The $5^{\text {th }}$ International Symposium Supercritical CO2 Power Cycles, March 29-31, 2016, San Antonio, Texas, Estados Unidos.

[22] T. Rowan. Functional Stability Analysis of Numerical Algorithms. Ph.D. thesis, Department of Computer Sciences, University of Texas at Austin, 1990.

[23] Powell, M.J.D. (December 2000). UOBYQA: unconstrained optimization by quadratic approximation (Report). Department of Applied Mathematics and Theoretical Physics, Cambridge University. DAMTP 2000/NA14. Retrieved 2015-04-06.

[24] Powell, M.J.D. (2002). UOBYQA: unconstrained optimization by quadratic approximation. Mathematical Programming, Series B (Springer) 92: 555-582.

[25] Source code of UOBYQA software. Retrieved 2015-04-06.

[26] Source code of NEWUOA software. Retrieved 2014-01-14.

[27] Powell, M.J.D. (November 2004). The NEWUOA software for unconstrained optimization without derivatives (PDF) (Report). Department of Applied Mathematics and Theoretical Physics, Cambridge University. DAMTP 2004/NA05. Retrieved 2014-01-14. 


\section{Capítulo 3 \\ Plantas Termosolares con colectores lineales Fresnel con Generación de Vapor y ciclos de potencia Subcríticos Rankine con Recalentamiento Directo}

\section{Resumen.}

La generación directa de vapor (DSG) en los colectores solares lineales PTC o LF se está consolidando como una tecnología viable para su implantación a escala industrial en plantas termsolares [1-2], al igual que ocurrió con el aceite térmico en los colectores solares PTC [3].

Las principales ventajas de la DSG respecto a la tecnología de sales fundidas o de aceite térmico son:

- fluido caloportador agua a través de los tubos receptores, presiones y temperaturas de vapor vivo elevadas $\left(500^{\circ} \mathrm{C}\right.$ y $90 \mathrm{bar}$ ) a la entrada de la turbina de vapor

- no es necesario la utilización de sistema de calentamiento auxiliar para evitar solidificación del fluido caloportador

- no hay riesgo de degradación del fluido caloportador por alta temperatura o por el número de ciclos de operación

- no es necesaria la instalación de intercambiador de calor intermedio entre el ciclo de potencia y el campo solar

- bajo coste de mantenimiento y operación.

Los colectores solares LF han sido desarrollados como alternativa a los colectores PTC. La optimización de su diseño continúa en fase de desarrollo [4-6]. Las principales ventajas de los colectores LF son:

- el coste reducido de fabricación y mantenimiento

- los espejos son más sencillos, sin la complejidad de la fabricación espejos parabólicos

- las tuberías de los tubos receptores son fijas sin juntas rotativas o móviles, únicamente incluyen juntas térmicas de expansión o bellows, con lo que el riesgo de fugas del fluido caloportador se reduce

- baja susceptibilidad a daños por cargas de viento,

- estructura soporte más liviano que reduciendo el peso general del conjunto.

Las compañías Novatec [7], Areva [8], Solar Euromed [9], etc, están invirtiendo en la tecnología de colectores LF y construyendo diferentes plantas pilotos para demostrar sus ventajas competitivas y viabilidad técnica. Sirvan como ejemplo las plantas piloto Puerto Errado 1 and 2 in Murcia Spain [10], Lidell in New South Wales Australia [11], Kogan Creek in Brigalow, Queensland Australia [12], Kimberlina in Bakersfield California USA [13], Llo Solar in Pyrénées France [14], Dhursar in India [15], etc.

Hay numerosas decisiones críticas que deben tenerse en cuenta para optimizar el diseño de una planta termosolar en general, y de colectores lineales y DSG en particular. Se tiene que obtener un óptimo balance entre la producción de electricidad neta de la planta, su coste de 
3. Plantas Termosolares con colectores lineales Fresnel con Generación de Vapor y ciclos de potencia Subcríticos Rankine con Recalentamiento Directo

capital inicial, su durabilidad. Algunas de estas decisiones pasan por realizar un diseño optimizado del campo solar: se deben elegir los parámetros termodinámicos de operación óptimos, seleccionar el material adecuado de los tubos receptores para soportar las presiones de operación y evitar la corrosión del material en contacto con el HTF (en el caso de las plantas con DSG la selección de tanques de separación de fases y sus dimensiones constituyen una pieza clave para garantizar la operación correcta de la planta frente a transitorios y evitar puntos calientes en los tubos receptores), configuración del sistema de control y bombas de recirculación de fase líquida, minimizar la longitud y perdidas de presión de las tuberías de distribución entre colectores, etc.

Atendiendo a estos aspectos, el diseño y selección de los parámetros termodinámicos de operación y su correcta evaluación son un objetivo prioritario para el diseño de las plantas termosolares con colectores LF y DSG. Para este propósito en los últimos años se han desarrollado programas informáticos para simulación de este tipo de instalaciones industriales. Entre los programas más especializados en este materia se pueden citar Thermoflow [16] y System Advisor Model (SAM) [17], entre otros. Una vez seleccionada y validada la herramienta de simulación, se realiza un estudio con objeto de proponer una configuración de campo solar óptima. Este es el principal objetivo de este capítulo de la tesis, que se llevará a cabo con la obligada comparación respecto a la tipología de planta que indica el estado de la técnica. Las fases de transitorios de operación (puesta en marcha, parada, etc.) también es simulado con SAM de forma detallada para optimizar la producción anual de la planta termosolar. SAM incluye como opción la simulación de las inercias térmicas de los equipos del campo solar. Con Thermoflow también se ha realizado la simulación anual de la planta termosolar, pero con menos grado de detalle. Este programa comercial no incluye como opción la simulación de inercias térmicas en condiciones transitorias de operación.

En este capítulo se ha propuesto una configuración de campo solar innovadora que ha sido analizada para optimizar la producción de las plantas termosolares con DSG y colectores LF. Finalmente, se ha evaluado como la inercia térmica y el modo de regulación del ciclo de potencia son puntos críticos cuando se opera con baja irradiación solar, siendo parámetros decisivos a la hora de optimizar la producción neta anual de la plantas termosolar.

\subsection{Introducción}

Las plantas termosolares con DSG en los colectores solares lineales tipo PTC y LF están madurando como una alternativa a las plantas termosolares tradicionales con aceites térmicos como HTF. Las grandes compañías apuestan por dos tecnologías a nivel industrial con colectores LF y DSG, la integración de los colectores LF en las plantas de ciclos combinados, Integrated Solar Combined cycle (ISCC) [18], y la integración de los colectores LF con un sistema de almacenamiento térmico mediante tanques de sales fundidas o Thermal Energy Storage system (TES) [19-20] y caldera de biomas auxiliar [21] para garantizar una generación de electricidad adecuada a los requisitos de la red eléctrica dispatchable generation y eliminando los transitorios inherentes de la radiación solar. Así mismo, el objetivo del desarrollo futuro de las instalaciones termosolares con colectores LF está alineado con la reducción del coste de la electricidad generada. 
3. Plantas Termosolares con colectores lineales Fresnel con Generación de Vapor y ciclos de potencia Subcríticos Rankine con Recalentamiento Directo

Hay numerosos obstáculos técnicos que están siendo superados progresivamente como son:

- nuevas configuraciones de los espejos y de la estructura del colector para obtener mayores eficiencias ópticas

- el sistema de control de la generación directa de vapor en la configuración OnceThrough (OT) [22-25]

- la integración con un sistema TES

- el material de recubrimiento selectivo de los tubos absorbedores,

- su integración con nuevos ciclos de potencia, etc.

La automatización del diseño de las plantas termosolares con colectores LF está limitada por el desarrollo de programas informáticos que permitan una optimización de la potencia neta generada y de la eficiencia del ciclo de potencia. En este capítulo de la tesis se ha comparado, Thermoflow [16] y el SAM [17], las dos herramientas informáticas más avanzadas para el diseño de instalaciones termosolares con LF y DSG. Las principales ventajas y desventajas de estas herramientas informáticas están resumidas en la tabla 3.1.

Mediante este tipo de software se pueden cuantificar los flujos energéticos entre los equipos y componentes que integran la instalación termosolar para las diferentes configuraciones definidas (figuras 3.1 a 3.6). Y se cuantifican los principales parámetros termodinámicos de caudal, presión, temperatura y entalpía para maximizar la eficiencia del ciclo y optimizar la potencia generada.

Como hipótesis fundamental se ha fijado la temperatura de entrada a turbina, TIT, en $500^{\circ} \mathrm{C} \mathrm{y}$ la presión TIP en 90 bar, tal y como aconseja el proveedor de los receptores Fresnel Novatec [7]. Se ha demostrado que temperaturas superiores a los $500^{\circ} \mathrm{C}$ no reducen el coste unitario de la electricidad generada debido a un incremento de las pérdidas térmicas por radiación en los tubos absorbedores, pese a que el rendimiento de la conversión térmo-eléctrica sí que se ve mejorado por el aumento de TIT.

En este estudio se ha realizado un análisis de incremento de TIP, manteniendo fija TIT y el área de apertura efectiva del campo solar. Se ha concluido que para presiones mayores de 90 bar, se incrementa la eficiencia del ciclo, pero se reduce la potencia generada.

La configuración de campo solar elegida como configuración de Referencia está integrada por receptores Fresnel con DSG en modo recirculación (RC), figuras 3.2 y 3.4, ampliamente validada y desarrollada desde el proyecto de investigación DISS [26], hasta su implantación a nivel industrial [1]. La configuración propuesta por la Universidad Politécnica de Madrid [27] también ha sido evaluada (figuras 3.5 y 3.6). Esta nueva solución de diseño está basada en la recirculación de la fase líquida desde los tanques de separación de fases hacia un grupo de lazos con colector común, permitiendo un escenario de operación del campo solar más estable, y reduciendo las fluctuaciones de TIT. Los resultados obtenidos están resumidos en las tablas 3.13 y 3.14. Finalmente, se ha modelado también la configuración OT [22- 25], con inyecciones de agua líquida para reducir el efecto de punto caliente o dry-out [28-30], y los resultados obtenidos resumidos en la conclusión de este capítulo. 
3. Plantas Termosolares con colectores lineales Fresnel con Generación de Vapor y ciclos de potencia Subcríticos Rankine con Recalentamiento Directo

La inercia térmica en los tubos absorbedores o cabezales de distribución también ayuda a la estabilización de los parámetros operacionales de la instalación durante los transitorios de la radiación solar, y durante las fases de puesta en marcha y parada. Estos parámetros de inercia térmica también son considerados por el programa informático SAM, pero no por Thermoflow.

Finalmente, indicar que la tecnología de Recalentamiento Directo o Direct ReHeating (DRH) con módulos solares LF y DSG, (figuras 3.3b y 3.4), permitiría incrementar la potencia unitaria generada en los colectores $\left(\mathrm{kW} / \mathrm{m}^{2}\right)$ alrededor de un $4 \%$ [16], y reducir el coste unitario de la electricidad producida según las estimaciones realizadas.

\subsection{Selección del programa informático para diseño de las plantas con colectores LF y DSG}

El programa informático System Advisory Model (SAM) [17], desarrollado National Renewable Energy Laboratory (NREL) de Estados Unidos, ha sido elegido un primer cálculo de diseño, fiable y rápido a tenor de las referencias disponibles [31-35], proporcionando incluso el cálculo de la producción de energía anual de la instalación termosolar. Dicho software integra los módulos necesarios para el cálculo de los principales transitorios de operación (puesta en marcha, paradas, transitorios de radiación solar debido a nubes, etc.). Sin embargo, la principal desventaja del SAM es que no permite un diseño flexible de la configuración de los equipos y componentes que integran el ciclo de potencia ni del campo solar. Por esta razón con SAM no es posible simular la tecnología DRH. Como alternativa a SAM se ha elegido Thermoflow [16], la comparativa entre ambos programas informáticos se resume en la tabla 3.1. Thermoflow posee un interface gráfico flexible que permite la definición de las corrientes energéticas entre equipos y componentes. Thermoflow está basado en la resolución de un sistema de ecuaciones no lineales mediante el método de Newthon Raphson y está bien considerado en el sector de la producción de energía eléctrica [36-40].

Tabla 3.1. Comparación entre las capacidades de los programas informáticos Thermoflow y SAM para el diseño de plantas termosolares con colectores LF y DSG.

\begin{tabular}{lll}
\hline & Thermoflow & SAM \\
\hline Entorno de simulación gráfica mostrando flujos energéticos entre equipos. & $\mathrm{SI}$ & $\mathrm{No}$ \\
Flexibilidad de modelización de las configuraciones del campo solar & $\mathrm{SI}$ & $\mathrm{No}$ \\
Datos meteorológicos desde archivos tipo: TMY2, TMY3, EPW & $\mathrm{No}$ & $\mathrm{SI}$ \\
Modelización de inercias térmicas en el campo solar & $\mathrm{No}$ & $\mathrm{SI}$ \\
Modelos de pérdidas de presión para flujos bifásicos y compresibles & $\mathrm{SI}$ & $\mathrm{No}$ \\
Parámetros de control: límite de flujo, limite congelación o viento, etc. & $\mathrm{No}$ & $\mathrm{SI}$ \\
Modelos de coeficientes de película HTC: Kandlikar, Ditus-Boelter, etc. & $\mathrm{SI}$ & $\mathrm{No}$ \\
Cálculo de espesor de tubos y límite de tensiones mecánicas. & $\mathrm{SI}$ & $\mathrm{No}$ \\
Modelo de regresión para cálculo en condiciones off-design & $\mathrm{No}$ & $\mathrm{SI}$ \\
Modos de operación del BOP (arranque, paradas, stand by, etc.) & No & $\mathrm{SI}$ \\
Simulación de pérdidas energéticas auxiliares en campo solar y BOP & No & $\mathrm{SI}$ \\
Simulación de ciclo de potencia supercrítico Rankine & $\mathrm{SI}$ & $\mathrm{No}$ \\
Simulación de Recalentamiento en el BOP & $\mathrm{SI}$ & $\mathrm{No}$ \\
Simulación operación off-design del condensador & No & $\mathrm{SI}$ \\
Modelos financieros & No & $\mathrm{SI}$ \\
\hline
\end{tabular}


3. Plantas Termosolares con colectores lineales Fresnel con Generación de Vapor y ciclos de potencia Subcríticos Rankine con Recalentamiento Directo

\subsection{Configuraciones de Campo Solar con colectores LF}

Tomando como referencia las plantas termosolares con colectores LF implantadas a escala industrial: Puerto Errado 1 y 2 en Murcia, España [10], Lidell en New South Wales, Australia [11], Kogan Creek en Brigalow, Queensland, Australia [12], Kimberlina en Bakersfield, California, USA [13], Llo Solar en Pyrénées, France [14], Dhursar en India [15], etc., y las plantas con colectores PTC y DSG también en vía de implantación comercial: $[1,30,41]$, se han definido las configuraciones de campo solar descritas en los siguientes epígrafes. En las tablas 3.2 a 3.5 se incluyen las principales hipótesis de cálculo y datos de partida comunes a todas las simulaciones realizadas.

Tabla 3.2. Parámetros de localización de la planta termosolar con colectores LF y DSG.

\begin{tabular}{ll}
\hline Ciudad & Dagget \\
País & USA \\
Zona horaria & GMT-8 \\
Elevación & $558 \mathrm{~m}$ \\
Latitud & $34.86^{\circ}$ \\
Longitud & -116.8 \\
Datos Meteorológicos & $\mathrm{TMY3}$ \\
Radiación Anual Incidente & $2723.5 \mathrm{kWh} / \mathrm{m}^{2}$ \\
\hline
\end{tabular}

Tabla 3.3a. Parámetros de los colectores LF con DSG y del campo solar.

\begin{tabular}{ll}
\hline Tipo de colector solar & SuperNova [7] \\
Longitud (por unidad) & $44.8 \mathrm{~m}$ \\
Anchura (por unidad) & $16.5 \mathrm{~m}$ \\
Anchura Efectiva (por unidad) & $11.48 \mathrm{~m}$ \\
Área Apertura Efectiva (por unidad) & $513.6 \mathrm{~m}^{2}$ \\
Longitud Focal & $7.4 \mathrm{~m}$ \\
Eficiencia óptica nominal (ebullición) & 0.67 \\
Eficiencia óptica nominal (sobrecalentamiento) & 0.65 \\
Factor de Limpieza & 0.96 \\
Múltiplo solar & 1 \\
Área apertura efectiva (Thermoflow) & $227251 \mathrm{~m}^{2}$ \\
Área apertura efectiva (SAM) & $227011 \mathrm{~m}^{2}$ \\
Área apertura módulos de ebullición & $161373 \mathrm{~m}^{2}$ \\
Área apertura módulos de sobrecalentamiento & $65878 \mathrm{~m}^{2}$ \\
\hline
\end{tabular}

Tabla 3.3b. Parámetros de los colectores LF con DSG y del campo solar.

\begin{tabular}{|c|c|}
\hline Número de lazos en el campo solar & 26 \\
\hline $\begin{array}{l}\text { Número de módulos de ebullición y } \\
\text { recalentamiento en cada lazo }\end{array}$ & 12 \\
\hline Módulos de sobrecalentamiento en cada lazo & 5 \\
\hline Distancia entre dos lazos & $4 \mathrm{~m}$ \\
\hline Temperatura de entrada del campo solar (diseño) & $235^{\circ} \mathrm{C}$ \\
\hline
\end{tabular}


3. Plantas Termosolares con colectores lineales Fresnel con Generación de Vapor y ciclos de potencia Subcríticos Rankine con Recalentamiento Directo

\begin{tabular}{ll}
\hline Temperatura de salida del campo solar (diseño) & $500^{\circ} \mathrm{C}$ \\
Calidad de salida del campo solar (diseño) & 0.8 \\
Caída de presión a lo largo de cada lazo & $12 \mathrm{bar}$ \\
Potencia Térmica del campo solar & 132.4 \\
& $\mathrm{MWth}$ \\
Inercia Térmica por unidad de área & 2.7 \\
& $\mathrm{~kJ} / \mathrm{Km}{ }^{2}$ \\
Calidad del vapor a la salida de los módulos de & 0.8 \\
ebullición & \\
Longitud cabezales calientes & 1000 \\
& $\mathrm{~m}$ \\
& 1000 \\
Longitud cabezales fríos & $\mathrm{m}$ \\
\end{tabular}

Tabla 3.4. BOP principales parámetros termodinámicos.

\begin{tabular}{ll}
\hline Potencia Bruta & $50 \mathrm{MWe}$ \\
Potencia Neta & $47.5 \mathrm{MWe}$ \\
Potencia Bruta/Potencia Neta & 0.94 \\
Presión Entrada Turbina TIP (diseño) & $90 \mathrm{bar}$ \\
Presión mínima a entrada de Turbina & $45 \mathrm{bar}$ \\
Temperatura Entrada Turbina TIT (diseño) & $500^{\circ} \mathrm{C}$ \\
Caudal másico a la entrada de Turbina & $53.46 \mathrm{~kg} / \mathrm{s}$ \\
\hline
\end{tabular}

Tabla 3.5. Datos de entrada en el punto de Diseño 21 Junio.

\begin{tabular}{ll}
\hline Hora & $11.5 \mathrm{hr}$ \\
DNI & $986 \mathrm{~W} / \mathrm{m}^{2}$ \\
Humedad Relativa & $18 \%$ \\
Temperatura bulbo seco & $31.9^{\circ} \mathrm{C}$ \\
Temperatura bulbo húmedo & $16^{\circ} \mathrm{C}$ \\
Altitud sobre nivel del mar & $588 \mathrm{~m}$ \\
\hline
\end{tabular}

\subsubsection{Configuración de Referencia}

En las figuras 3.1 y 3.2 se ilustra la configuración de referencia integrada por lazos de colectores LF conectados en paralelo con GDV en modo recirculación. Los resultados obtenidos del cálculo de la producción de electricidad en el punto de diseño se resumen en las tablas 3.6 a 3.11. Se concluye que tanto con SAM [17] como con Thermoflow [16] los resultados obtenidos son muy similares, confirmando la coherencia de los modelos elaborados en ambos programas informáticos. En las tablas 3.6, 3.7 y 3.8 se incluyen los parámetros y cálculos ópticos del campo solar, y en las tablas 3.9, 3.10 y 3.11 los parámetros termodinámicos. Respecto a la producción anual de electricidad con esta configuración de Referencia se detallan en la tabla 3.12 . 
3. Plantas Termosolares con colectores lineales Fresnel con Generación de Vapor y ciclos de potencia Subcríticos Rankine con Recalentamiento Directo

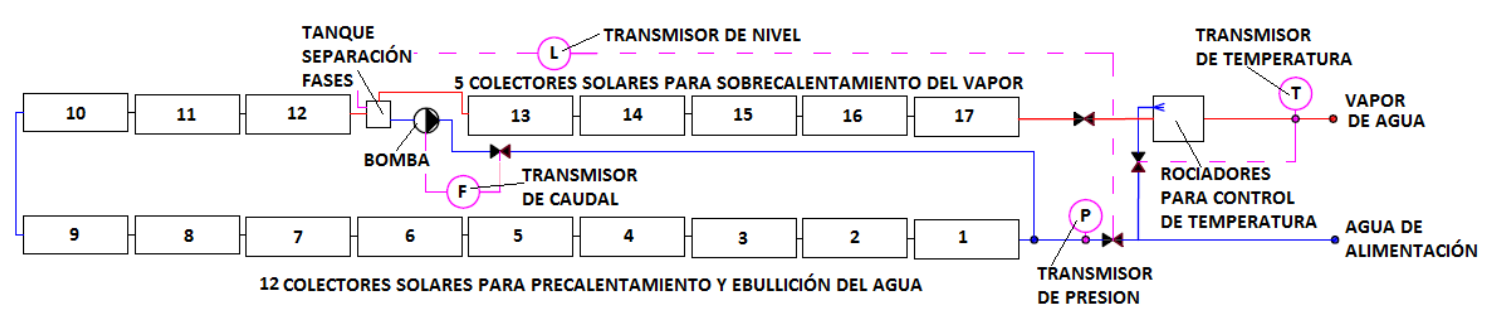

Figura 3.1. Lazo integrado por colectores solares LF y DSG en modo recirculación.

En la figura 3.1 se ilustra detalladamente uno de los lazos que integran la configuración de Referencia. Cada lazo incluye 18 módulos de colectores LF: en los 12 primeros módulos se precalienta el agua y vaporiza hasta obtener a la salida del módulo 12 el vapor con título de vapor 0.8 y en el resto de módulos se sobrecalienta el vapor para obtener orden de $500^{\circ} \mathrm{C}$ en la entrada de la turbina principal.

La configuración del lazo representado en la figura 3.1 es similar al utilizado en el proyecto DISS con PTC [26].
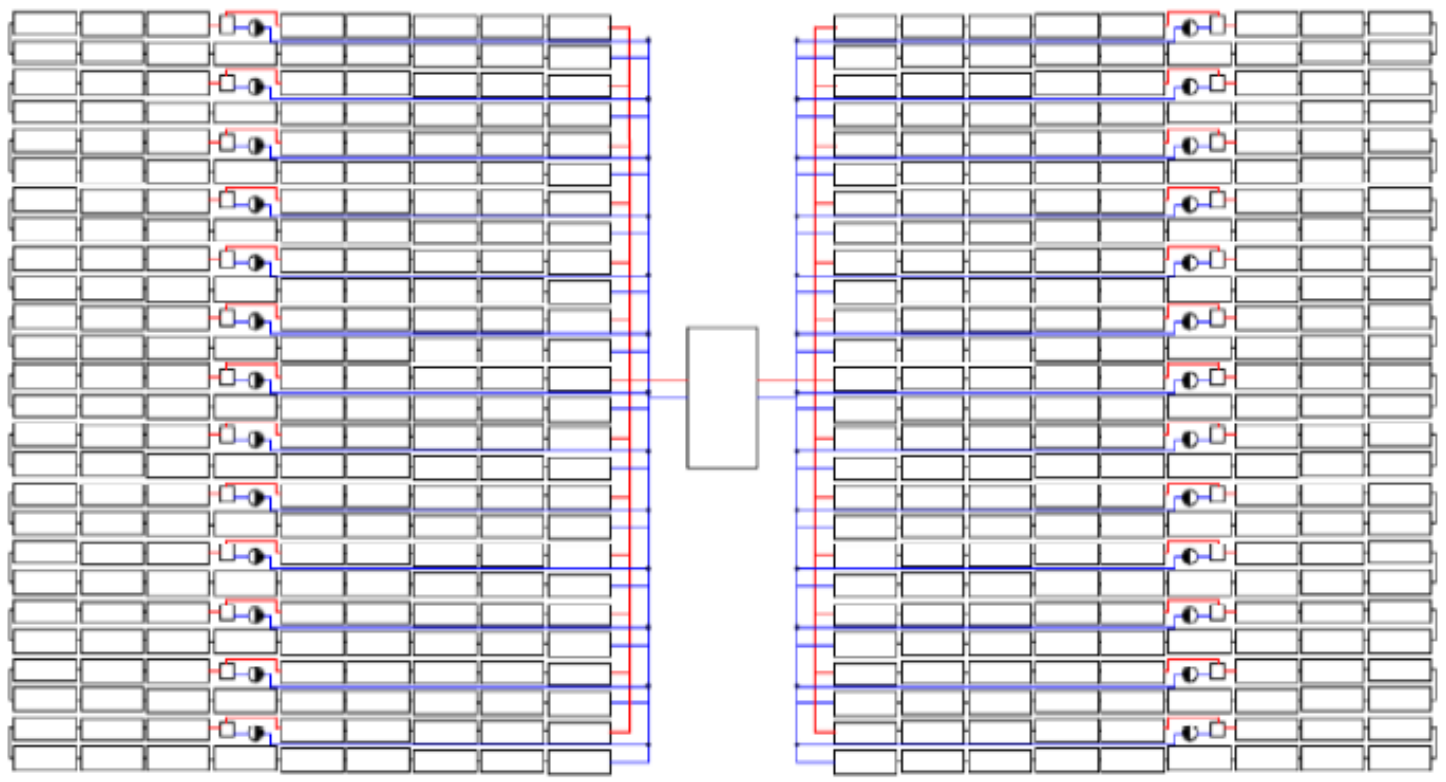

Figura 3.2. Configuración de referencia integrada por lazos de colectores solares LF con DSG en modo RC conectados en paralelo [26].

Tabla 3.6. Comparación parámetros del campo solar LF (punto de diseño 21 Junio).

\begin{tabular}{lll}
\hline & Thermoflow & SAM \\
Elevación & $77.8^{\circ}$ & $77.9^{\circ}$ \\
Ángulo Zenit & $12.1^{\circ}$ & $12.1^{\circ}$ \\
Ángulo Azimut & $159.1^{\circ}$ & $159.1^{\circ}$ \\
Ángulo incidencia long. & $11.31^{\circ}$ & $11.3^{\circ}$ \\
Ángulo incidencia transv. & $4.372^{\circ}$ & $4.4^{\circ}$ \\
\hline
\end{tabular}


3. Plantas Termosolares con colectores lineales Fresnel con Generación de Vapor y ciclos de potencia Subcríticos Rankine con Recalentamiento Directo

Tabla 3.7. Comparación parámetros del campo solar LF (punto de diseño 21 Junio).

\begin{tabular}{lll}
\hline & Thermoflow & SAM \\
Eficiencia óptica & $67.00 \%$ & $67.00 \%$ \\
Factor Limpieza & $96.00 \%$ & $96.00 \%$ \\
Factor end losses & $99.45 \%$ & $\mathrm{~N} / \mathrm{A}$ \\
IAM & $94.45 \%$ & $95.28 \%$ (deducida) \\
Eficiencia óptica real (\%) & $60.42 \%$ & $61.29 \%$ \\
\hline
\end{tabular}

Tabla 3.8. Parámetros óptimos del modulo de recalentamiento (punto de diseño 21 Junio).

\begin{tabular}{lll}
\hline & Thermoflow & SAM \\
Eficiencia óptica & $65.00 \%$ & $65.00 \%$ \\
Factor Limpieza & $96.00 \%$ & $96.00 \%$ \\
Factor end losses & $98.71 \%$ & $\mathrm{~N} / \mathrm{A}$ \\
IAM & $94.45 \%$ & $98.22 \%$ (deducida) \\
Eficiencia óptica real (\%) & $58.18 \%$ & $61.29 \%$
\end{tabular}

Tabla 3.9. Comparación parámetros termodinámicos SF LF (punto de Diseño 21 Junio).

\begin{tabular}{lll}
\hline & Thermoflow & SAM \\
SF energía incidente & $224.0 \mathrm{MWth}$ & $223.8 \mathrm{MWth}$ \\
SF energía recibida & $133.9 \mathrm{MW}$ th & $137.1 \mathrm{MWth}$ \\
SF pérdidas térmicas & $6.6 \mathrm{MWth}$ & $6.7 \mathrm{MWth}$ \\
SF potencia térmica & $127.3 \mathrm{MWth}$ & $129.7 \mathrm{MWth}$ \\
\hline
\end{tabular}

Tabla 3.10. Comparación resultados BOP (punto de Diseño 21 Junio).

\begin{tabular}{lll}
\hline & Thermoflow & SAM \\
Potencia Bruta & $50284 \mathrm{kWe}$ & $51564 \mathrm{kWe}$ \\
Eficiencia Bruta & $39.50 \%$ & $39.72 \%$ \\
Potencia Neta & $47387 \mathrm{kWe}$ & $48395 \mathrm{kWe}$ \\
Eficiencia Neta & $37.23 \%$ & $37.30 \%$ \\
Potencia Ventiladores & $1599.7 \mathrm{kWe}$ & $2708.3 \mathrm{kWe}$ \\
Bomba condensado & $61.7 \mathrm{kWe}$ & $131.9 \mathrm{kWe}$ \\
Bomba agua alimentación & $718.1 \mathrm{kWe}$ & \\
Pérdidas energéticas SF & $15.1 \mathrm{kWe}$ & $45.4 \mathrm{kWe}$ \\
Pérdidas energéticas BOP & $502.8 \mathrm{kWe}$ & $283.2 \mathrm{kWe}$ \\
\hline
\end{tabular}

Tabla 3.11. Principales propiedades termodinámicas de las Corrientes energéticas entre equipos en la configuración de Referencia (punto de Diseño 21 Junio).

\begin{tabular}{llllll}
\hline BOP & $\begin{array}{l}\text { Caudal } \\
(\mathrm{kg} / \mathrm{s})\end{array}$ & $\begin{array}{l}\text { Presión } \\
(\mathrm{bar})\end{array}$ & $\begin{array}{l}\text { Temperatura } \\
\left({ }^{\circ} \mathrm{C}\right)\end{array}$ & $\begin{array}{l}\text { Entalpía } \\
(\mathrm{kJ} / \mathrm{kg})\end{array}$ & Calidad del Vapor \\
\hline 1 & 53.51 & 101.5 & 239 & 1033 & Subenfriado Líquido \\
2 & 66.89 & 101.5 & 254 & 1104 & Subenfriado Líquido \\
3 & 13.37 & 101.5 & 308 & 1389 & Subenfriado Líquido \\
4 & 66.89 & 95.6 & 307.7 & 2464 & 0.8 \\
5 & 13.37 & 95.6 & 307.7 & 1388 & Saturado Líquido \\
6 & 53.51 & 95.6 & 307.7 & 2733 & Saturado Vapor \\
7 & 53.51 & 90.3 & 510.1 & 3412 & Sobrecalentado. Vapor \\
8 & 53.46 & 87.7 & 508.9 & 3412 & Sobrecalentado. Vapor \\
9 & 5.047 & 37.0 & 387.3 & 3190 & Sobrecalentado. Vapor \\
10 & 2.622 & 14.4 & 271.8 & 2976 & Sobrecalentado. Vapor \\
11 & 2.594 & 6.4 & 185.6 & 2816 & Sobrecalentado. Vapor \\
\hline
\end{tabular}


3. Plantas Termosolares con colectores lineales Fresnel con Generación de Vapor y ciclos de potencia Subcríticos Rankine con Recalentamiento Directo

\begin{tabular}{llllll}
\hline 12 & 2.423 & 3.1 & 135 & 2698 & 0.9 \\
13 & 2.272 & 1.2 & 104.9 & 2563 & 0.9 \\
14 & 1.903 & 0.4 & 74.8 & 2417 & 0.9 \\
15 & 36.6 & 0.08 & 41.8 & 2265 & 0.8 \\
16 & 43.25 & 9.7 & 42.8 & 180 & Subenfriado Líquido \\
17 & 43.25 & 9.3 & 69.0 & 289 & Subenfriado Líquido \\
18 & 43.25 & 8.9 & 98.9 & 415 & Subenfriado Líquido \\
19 & 43.25 & 7.3 & 128.9 & 541 & \\
20 & 53.51 & 104.7 & 161.6 & 688 & \\
21 & 53.51 & 103.6 & 189.9 & 811 & \\
22 & 53.51 & 101.5 & 239 & 1033 & \\
\hline
\end{tabular}

Tabla 3.12. Configuración de Referencia. Potencia energética anual (resultados mensuales).

\begin{tabular}{lllllll}
\hline & $\begin{array}{l}\text { Thermoflow } \\
\text { Potencia } \\
\text { Bruta } \\
\text { (MWh) }\end{array}$ & $\begin{array}{l}\text { SAM } \\
\text { Potencia } \\
\text { Bruta } \\
\text { (MWh) }\end{array}$ & $\begin{array}{l}\text { Thermoflow } \\
\text { Potencia } \\
\text { Bruta } \\
\text { (MWh) }\end{array}$ & $\begin{array}{l}\text { Thermoflow } \\
\text { Potencia } \\
\text { Neta } \\
\text { (MWh) }\end{array}$ & $\begin{array}{l}\text { SAM } \\
\text { Potencia } \\
\text { Neta } \\
\text { (MWh) }\end{array}$ & $\begin{array}{l}\text { Thermoflow } \\
\text { Potencia } \\
\text { Neta } \\
\text { (MWh) * }\end{array}$ \\
\hline Enero & 2438 & 2336 & 2294 & 2330 & 2205 & 2189 \\
Febrero & 3649 & 3577 & 3498 & 3482 & 3389 & 3334 \\
Marzo & 6053 & 5871 & 5881 & 5769 & 5546 & 5600 \\
Abril & 8132 & 8009 & 7982 & 7721 & 7495 & 7573 \\
Mayo & 10396 & 10282 & 10263 & 9838 & 9531 & 9705 \\
Junio & 11650 & 11539 & 11529 & 10930 & 10613 & 10807 \\
Julio & 11001 & 10772 & 10848 & 10273 & 9903 & 10119 \\
Agosto & 10973 & 10848 & 10858 & 10282 & 9985 & 10166 \\
Septiembre & 8420 & 8324 & 8272 & 7933 & 7651 & 7786 \\
Octubre & 5651 & 5404 & 5481 & 5363 & 4969 & 5197 \\
Noviembre & 3324 & 3024 & 3193 & 3173 & 2850 & 3045 \\
Diciembre & 2290 & 2275 & 2164 & 2187 & 2124 & 2063 \\
TOTAL & 83977 & 82260 & 82263 & 79281 & 76262 & 77584 \\
\hline
\end{tabular}

\subsubsection{Configuración de referencia con Recalentamiento Directo o Direct ReHeating (DRH)}

El Recalentamiento Directo [42-43] considerado,figura 3.3b, utiliza colectores lineales LF o PTC, para el sobrecalentamiento del vapor proveniente de las turbinas en el campo solar. De este modo se prescinden del intercambiador de calor intermedio, que serían necesario si el HTF en el campo solar fuera aceite térmico o sales fundidas.

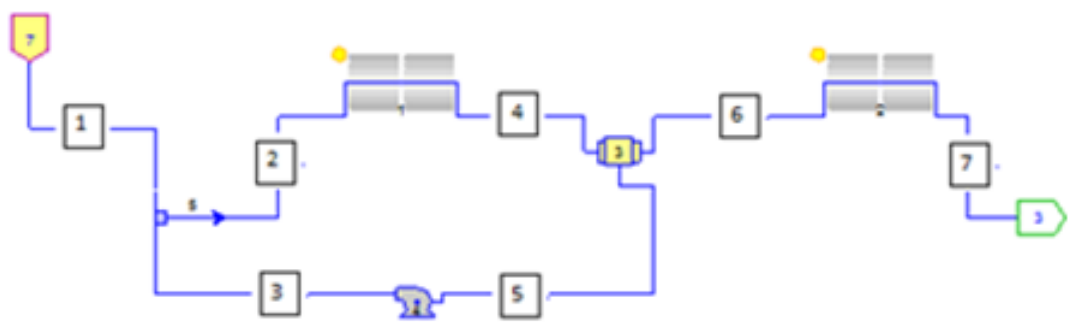

Figura 3.3. (a) Modelo del campo solar con colectores LF y DSG con modo recirculación RC.

Realizado con el programa Informático Thermoflow [26].

A la hora de diseñar el campo solar para DRH, es muy importante limitar las pérdidas de presión en los colectores solares, ya que una caída de presión impacta de forma directa en la presión de entrada en las turbinas de media y baja presión, y por tanto, reduce considerablemente tanto la potencia generada en el ciclo de potencia como su eficiencia neta. Para limitar las pérdidas de presión en el campo solar (figuras 3.3 y 3.4), se ha limitado el flujo 
3. Plantas Termosolares con colectores lineales Fresnel con Generación de Vapor y ciclos de potencia Subcríticos Rankine con Recalentamiento Directo

másico $\left(\mathrm{kg} / \mathrm{m}^{2} \cdot \mathrm{s}\right)$ a través de los tubos absorbedores y de la longitud de los colectores solares en cada lazo.

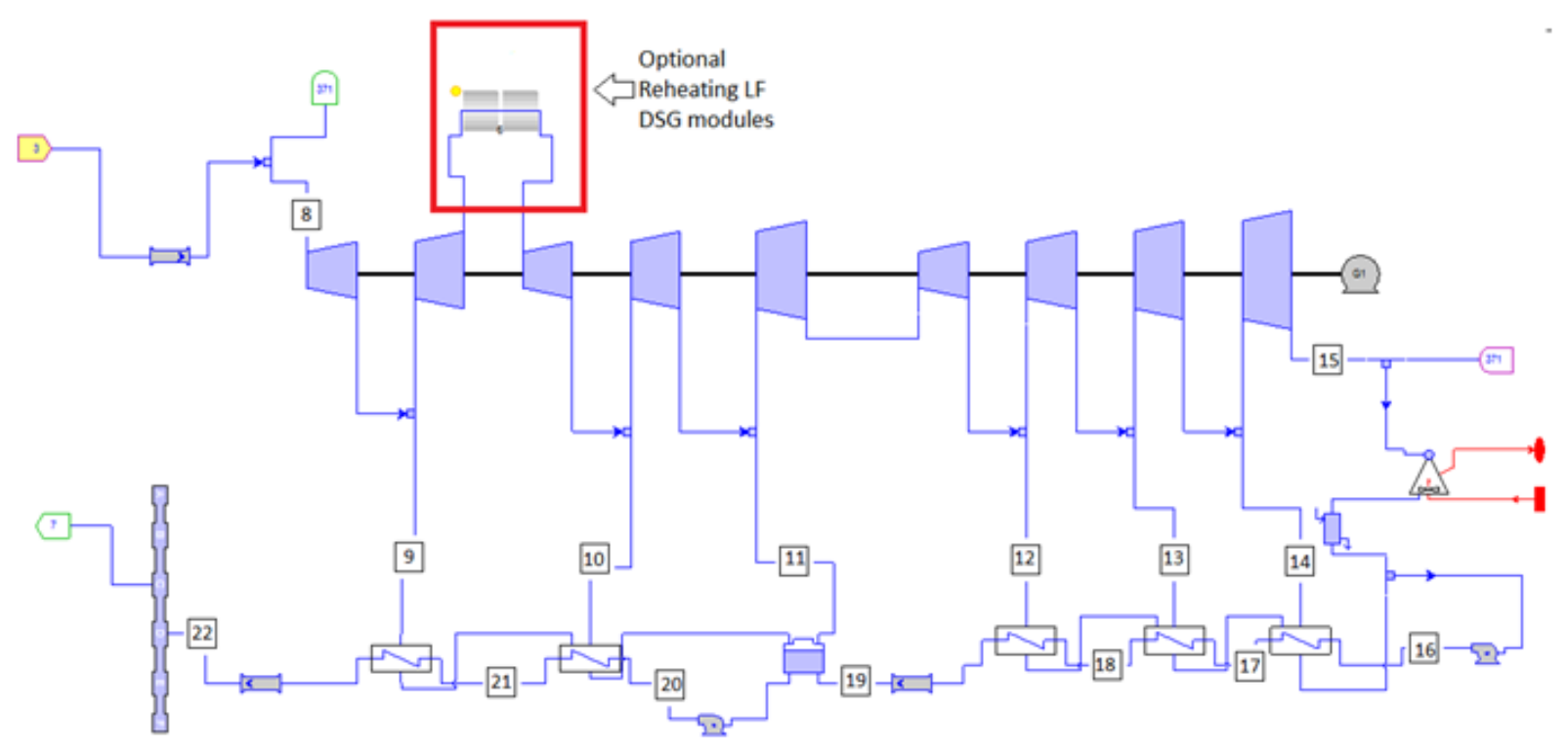

Figura 3.3. (b) Modelo Thermoflow del BOP con DRH con colectores LF.

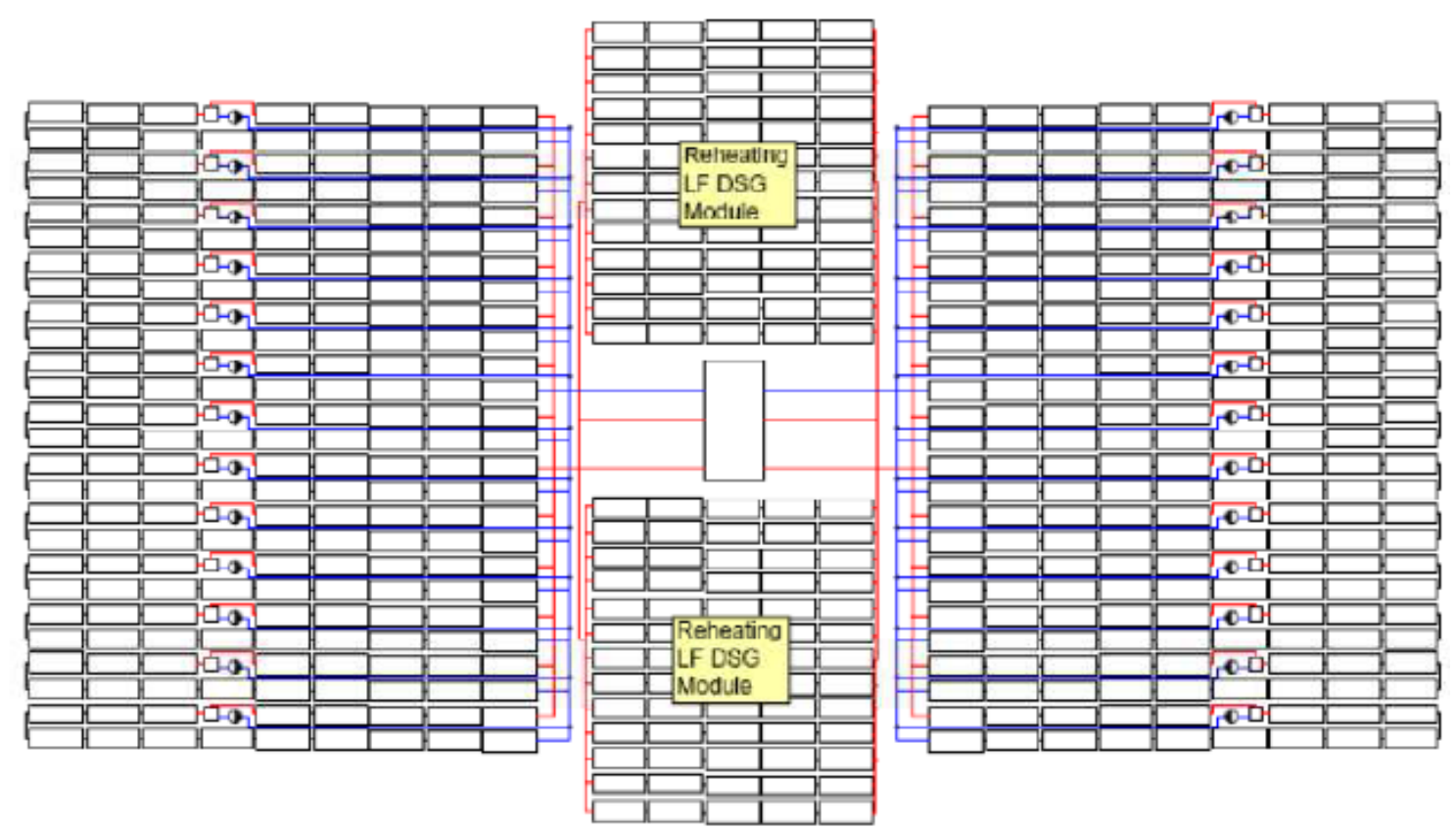

Figura 3.4. Planta termosolar de $50 \mathrm{MWe}$ con dos grupos de 13 lazos conectados en paralelo. Cada lazo dispone de 12 módulos para ebullición del agua y 5 módulos para sobrecalentamiento del vapor. Todos los módulos están integrados por colectores LF tipo Novatec [7]. El bloque de potencia BOP está localizado en el centro del campo solar.

En la tabla 3.13 se resume la potencia eléctrica generada en el punto de diseño, y la eficiencia bruta y neta del ciclo de potencia con la configuración con DRH para diferentes temperaturas de recalentamiento. Conforme vamos incrementando la temperatura de recalentamiento vamos incrementando la eficiencia neta del ciclo de potencia y maximizando la potencia unitaria generada en sus colectores solares. El incremento de la potencia unitaria desde una 
3. Plantas Termosolares con colectores lineales Fresnel con Generación de Vapor y ciclos de potencia Subcríticos Rankine con Recalentamiento Directo

temperatura de recalentamiento de $373^{\circ} \mathrm{C}$ hasta la temperatura de recalentamiento de $517^{\circ} \mathrm{C}$ es del $\sim 7 \%$. La producción anual de electricidad también fue simulada, y los resultados obtenidos están resumidos en la tabla 3.14 .

Tabla 3.13. Potencia y eficiencia de la configuración de Referencia con Recalentamiento Directa DRH (punto de diseño 21 Junio).

\begin{tabular}{llllllll}
\hline $\begin{array}{l}\text { Temperatura } \\
\text { Recalentamiento } \\
\left({ }^{\circ} \mathrm{C}\right)\end{array}$ & $\begin{array}{l}\text { Potencia } \\
\text { Bruta } \\
(\mathrm{MWe})\end{array}$ & $\begin{array}{l}\text { Eficiencia } \\
\text { Bruta } \\
(\%)\end{array}$ & $\begin{array}{l}\text { Potencia } \\
\text { Neta } \\
(\mathrm{MWe})\end{array}$ & $\begin{array}{l}\text { Eficiencia } \\
\text { Neta } \\
(\%)\end{array}$ & $\begin{array}{l}\text { Área } \\
\text { Recalentamiento } \\
\left(\mathrm{m}^{2}\right)\end{array}$ & $\begin{array}{l}\text { Área } \\
\text { Total } \\
\left(\mathrm{m}^{2}\right)\end{array}$ & $\begin{array}{l}\text { Potencia } \\
\text { Unitaria } \\
\left(\mathrm{MWe} / \mathrm{m}^{2}\right)\end{array}$ \\
\hline 373 & 50460 & 38.3 & 47465 & 36.03 & 8139 & 235457 & 0.20158 \\
400 & 52799 & 39.02 & 49755 & 36.77 & 14469 & 241787 & 0.20578 \\
450 & 56963 & 40.17 & 53830 & 37.96 & 26282 & 253600 & 0.21226 \\
500 & 59553 & 40.63 & 56349 & 38.44 & 34639 & 261957 & 0.21510 \\
517 & 60508 & 40.82 & 57279 & 38.64 & 37524 & 264842 & 0.21627 \\
\hline
\end{tabular}

Tabla 3.14. Configuración de Referencia, potencia anual (mensual).

\begin{tabular}{lllll}
\hline & $\begin{array}{l}\text { Thermoflow sin } \\
\text { Recalentamiento } \\
\text { Potencia } \\
\text { Bruta(MWh) }\end{array}$ & $\begin{array}{l}\text { Thermoflow con } \\
\text { Recalentamiento } \\
\text { Potencia } \\
\text { Bruta(MWh) }\end{array}$ & $\begin{array}{l}\text { Thermoflow sin } \\
\text { Recalentamiento } \\
\text { Potencia } \\
\text { Neta(MWh) }\end{array}$ & $\begin{array}{l}\text { Thermoflow con } \\
\text { Recalentamiento } \\
\text { Potencia Neta } \\
\text { (MWh) }\end{array}$ \\
\hline Enero & 2294 & 2770 & 2189 & 2651 \\
Febrero & 3498 & 4143 & 3334 & 3960 \\
Marzo & 5881 & 7081 & 5600 & 6767 \\
Abril & 7982 & 9563 & 7573 & 9108 \\
Mayo & 10263 & 12393 & 9705 & 11764 \\
Junio & 11529 & 13826 & 10807 & 13019 \\
Julio & 10848 & 13099 & 10119 & 12279 \\
Agosto & 10858 & 13027 & 10166 & 12250 \\
Septiembre & 8272 & 9924 & 7786 & 9379 \\
Octubre & 5481 & 6555 & 5197 & 6237 \\
Noviembre & 3193 & 3767 & 3045 & 3603 \\
Diciembre & 2164 & 2602 & 2063 & 2487 \\
TOTAL & 82263 & 98750 & 77584 & 93504 \\
\hline
\end{tabular}

\subsubsection{Configuración propuesta}

La configuración de campo solar propuesta [27], está fundamentada en la recirculación de la fase líquida desde el tanque de separación de fases de un grupo de lazos hacia el grupo de lazos adyacente, figuras 3.5 y 3.6. Los equipos auxiliares están agrupados (bombas de recirculación, tanques de separación de fases, sistema de control, etc.), para ser compartido por un grupo de lazos conectados en paralelo. El sistema de recalentamiento y sobrecalentamiento del vapor está constituido por grupos de lazos situados en las inmediaciones del bloque de potencia para minimizar las pérdidas de presión del vapor como fluido compresible en su camino entre el campo solar hacia la entrada de las turbinas. Las principales ventajas de la configuración propuesta [27] se citan a continuación:

- Gracias a la acumulación de vapor y almacenamiento de fluido caloportador (agua) en los tanques de separación de fases, las fluctuaciones de temperatura en el campo solar son minimizadas durante los desequilibrios de radiación solar incidente en los colectores LF local shadowing. 
3. Plantas Termosolares con colectores lineales Fresnel con Generación de Vapor y ciclos de potencia Subcríticos Rankine con Recalentamiento Directo

Así mismo, este almacenamiento temporal en los tanques de separación de fases favorece a la estabilización de los parámetros de operación durante los períodos de arranque y parada, u otros transitorios de operación.

- El sistema de control de caudal de flujo de agua de alimentación es optimizado.

- El sistema de control para la configuración propuesta [27] puede ser muy similar al desarrollado durante el proyecto DISS [26].

- La configuración propuesta [27], figuras 3.5 y 3.6, con recirculación de la fase líquida hacia el grupo de lazos adyacentes, puede ser intercambiada por la configuración tradicional (figuras 3.2 y 3.4), mediante la instalación de un sistema de by-passes y válvulas adecuados. Los parámetros termodinámicos de su simulación con Thermoflow (figura 3.6) en su punto de diseño, están resumidos en la tabla 3.15; y la simulación de la producción anual de potencia está detallada en la tabla 3.16.

- La recirculación de la fase líquida entre grupos de lazos adyacentes, es una innovación tecnológica cuyas ventajas se incrementan conforme se aumenta la potencia neta de la planta termosolar; la mayor parte de las plantas solares en España son de $50 \mathrm{MWe}$ con almacenamiento térmico TES.

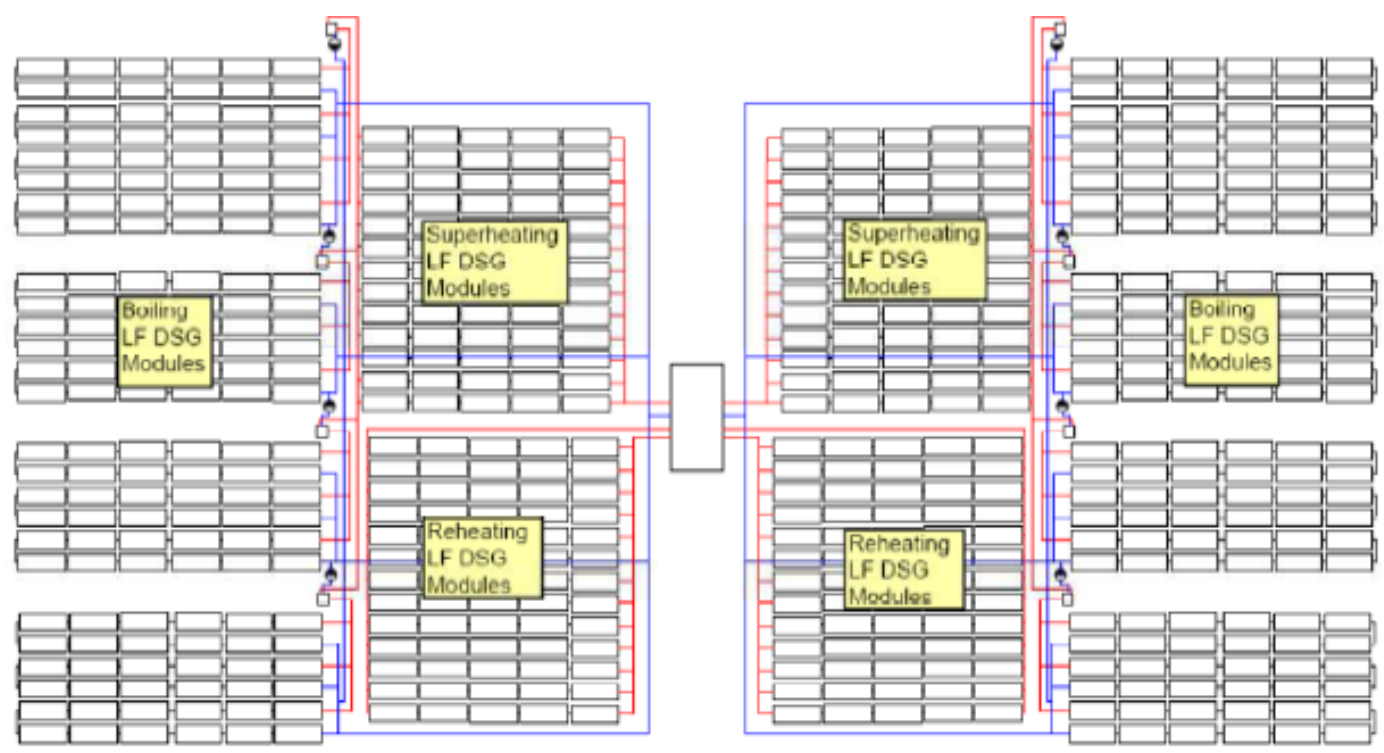

Figura 3.5. Configuración propuesta [27]. 
3. Plantas Termosolares con colectores lineales Fresnel con Generación de Vapor y ciclos de potencia Subcríticos Rankine con Recalentamiento Directo

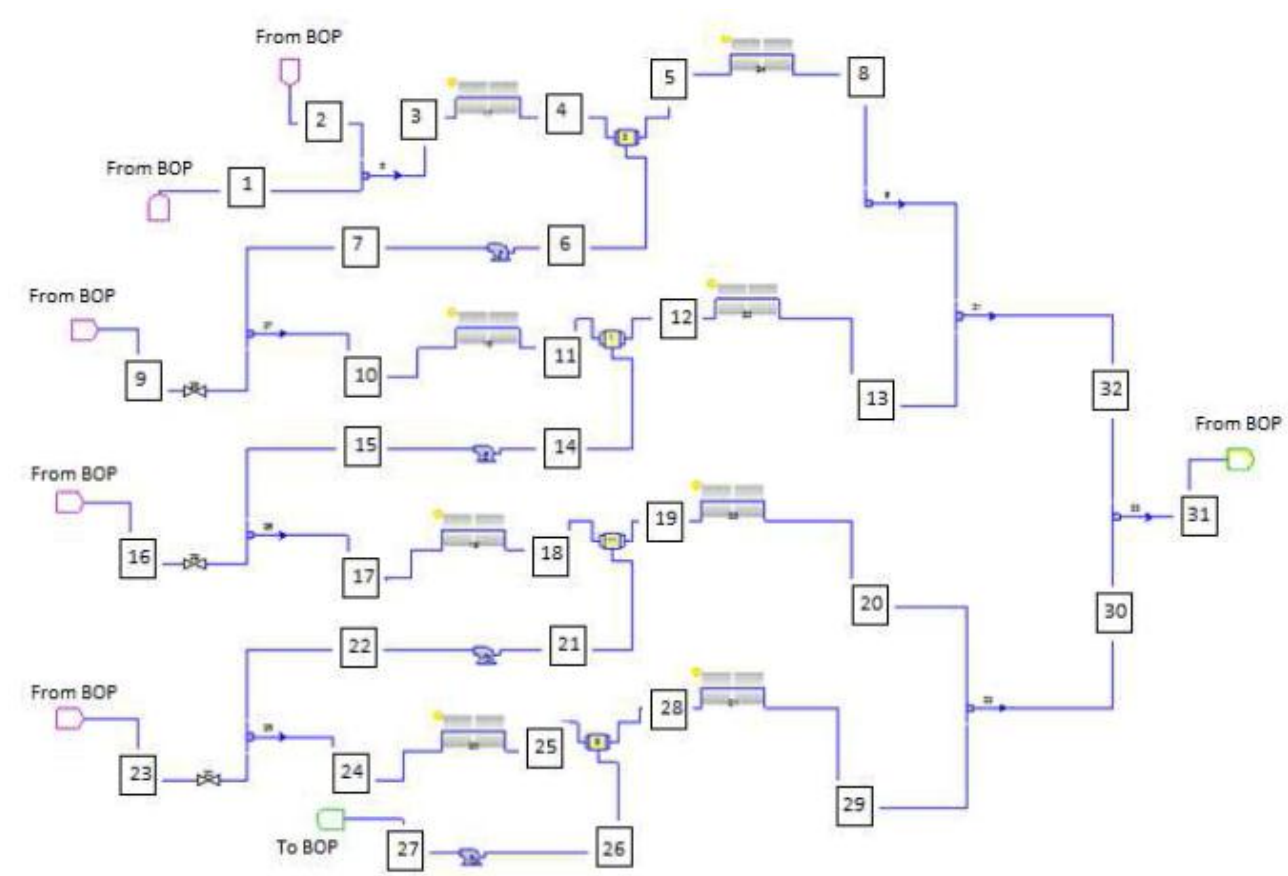

Figura 3.6. Configuración propuesta [27].

Tabla 3.15. Parámetros termodinámicos de operación de la configuración propuesta [27]

(Punto de diseño 21 de Junio).

\begin{tabular}{|c|c|c|c|c|c|}
\hline BOP & $\begin{array}{l}\text { Flujo Másico } \\
(\mathrm{kg} / \mathrm{s})\end{array}$ & $\begin{array}{l}\text { Presión } \\
\text { (bar) }\end{array}$ & $\begin{array}{l}\text { Temperatura } \\
\left({ }^{\circ} \mathrm{C}\right)\end{array}$ & $\begin{array}{l}\text { Entalpía } \\
\text { (kJ/kg) }\end{array}$ & $\begin{array}{l}\text { Calidad } \\
\text { del Vapor }\end{array}$ \\
\hline 1 & 3.3 & 101 & 239 & 1033 & Subenfriado \\
\hline 2 & 13.3 & 101 & 308 & 1389 & Subenfriado \\
\hline 3 & 16.7 & 101 & 254 & 1104 & Subenfriado \\
\hline 4 & 16.7 & 96 & 307 & 2464 & 0.8 \\
\hline 5 & 13.3 & 96 & 307 & 2733 & 1 \\
\hline 6 & 3.3 & 96 & 307 & 1388 & 0 \\
\hline 7 & 3.3 & 101 & 308 & 1389 & Subenfriado \\
\hline 8 & 13.3 & 91 & 514 & 3422 & Sobrecalentado \\
\hline 9 & 13.3 & 101 & 239 & 1033 & Subenfriado \\
\hline 10 & 16.7 & 101 & 254 & 1104 & Subenfriado \\
\hline 11 & 16.7 & 96 & 307 & 2464 & 0.8 \\
\hline 12 & 13.3 & 96 & 307 & 2733 & 1 \\
\hline 13 & 13.3 & 91 & 514 & 3422 & \\
\hline 14 & 3.3 & 96 & 307 & 1388 & 0 \\
\hline 15 & 3.3 & 10 & 308 & 1389 & Subenfriado \\
\hline 16 & 13.3 & 101 & 239 & 1033 & Subenfriado \\
\hline 17 & 16.7 & 101 & 254 & 1104 & Subenfriado \\
\hline 18 & 16.7 & 96 & 307 & 2464 & 0.8 \\
\hline 19 & 13.3 & 96 & 307 & 2733 & 1 \\
\hline 20 & 13.3 & 91 & 514 & 3422 & Sobrecalentado \\
\hline 21 & 3.3 & 96 & 307 & 1388 & 0 \\
\hline 22 & 3.3 & 101 & 308 & 1389 & Subenfriado \\
\hline 23 & 13.3 & 101 & 239 & 1033 & Subenfriado \\
\hline 24 & 16.7 & 101 & 254 & 1104 & Subenfriado \\
\hline 25 & 16.7 & 96 & 307 & 2464 & 0.8 \\
\hline 26 & 3.3 & 96 & 307 & 1388 & 0 \\
\hline 27 & 3.3 & 101 & 308 & 1389 & Subenfriado \\
\hline 28 & 13.3 & 96 & 307 & 2733 & 1 \\
\hline
\end{tabular}


3. Plantas Termosolares con colectores lineales Fresnel con Generación de Vapor y ciclos de potencia Subcríticos Rankine con Recalentamiento Directo

\begin{tabular}{llllll}
\hline 29 & 13.3 & 91 & 514 & 3422 & Sobrecalentado \\
30 & 26.7 & 91 & 514 & 3422 & Sobrecalentado \\
31 & 53.4 & 91 & 514 & 3422 & Sobrecalentado \\
32 & 26.7 & 91 & 514 & 3422 & Sobrecalentado \\
\hline
\end{tabular}

Tabla 3.16. Configuración propuesta [27], potencia anual (mensual).

\begin{tabular}{lllllll}
\hline & $\begin{array}{l}\text { Thermoflow } \\
\text { sin recalent. } \\
\text { Potencia } \\
\text { Bruta } \\
\text { (MWh) }\end{array}$ & $\begin{array}{l}\text { Thermoflow } \\
\text { Sin recalent. } \\
\text { Potencia } \\
\text { Bruta* } \\
\text { (MWh) }\end{array}$ & $\begin{array}{l}\text { Thermoflow } \\
\text { con } \\
\text { recalent. } \\
\text { Potencia } \\
\text { Bruta } \\
\text { (MWh) }\end{array}$ & $\begin{array}{l}\text { Thermoflow } \\
\text { sin recalent. } \\
\text { Potencia } \\
\text { Neta } \\
\text { (MWh) }\end{array}$ & $\begin{array}{l}\text { Thermoflow } \\
\text { Sin recalent. } \\
\text { Potencia } \\
\text { Neta* } \\
\text { (MWh) }\end{array}$ & $\begin{array}{l}\text { Thermoflow } \\
\text { con } \\
\text { recalent. } \\
\text { Potencia } \\
\text { Neta } \\
\text { (MWh) }\end{array}$ \\
\hline Enero & 2222 & 2251 & 2726 & 2120 & 2148 & 2611 \\
Febrero & 3369 & 3420 & 4127 & 3211 & 3261 & 3949 \\
Marzo & 5764 & 5864 & 6996 & 5489 & 5584 & 6687 \\
Abril & 7833 & 7894 & 9433 & 7431 & 7490 & 8986 \\
Mayo & 10070 & 10110 & 12421 & 9522 & 9559 & 11792 \\
Junio & 11314 & 11389 & 13810 & 10603 & 10671 & 13007 \\
Julio & 10674 & 10740 & 12999 & 9951 & 10013 & 12187 \\
Agosto & 10652 & 10710 & 13010 & 9975 & 10026 & 12246 \\
Septiembre & 8096 & 8201 & 9862 & 7617 & 7715 & 9323 \\
Octubre & 5359 & 5404 & 6529 & 5080 & 5123 & 6215 \\
Noviembre & 3088 & 3133 & 3836 & 2943 & 2988 & 3670 \\
Deciembre & 2074 & 2102 & 2606 & 1977 & 2005 & 2495 \\
TOTAL & 80520 & 81221 & 98357 & 75921 & 76586 & 93170 \\
\hline
\end{tabular}

\subsection{Conclusiones}

La selección del programa informático para la simulación y modelización de las plantas termosolares con colectores LF y DSG, es una pieza clave en el proceso de diseño de este tipo de plantas de generación de energía eléctrica. En este capítulo se han evaluado y comparado las capacidades de dos de herramientas informáticas muy bien consideradas en el sector: Thermoflow [16] y SAM [17].

Thermoflow proporciona un entorno flexible de modelización, adaptable a diferentes configuraciones de campo solar, permitiendo definir los grados de libertad de localización de los diferentes equipos y componentes en el campo solar y las corrientes de interconexión entre ellos.

La principal ventaja del SAM es un entorno integrando de simulación de plantas termosolares con gran velocidad de cálculo en la estimación de la producción anual, los transitorios de irradiación solar y sus inercias térmicas.

Así mismo, SAM incluye las bases de datos de radiación solar dependiendo de la ubicación de la instalación industrial. En este estudio se trabaja de forma simultánea con ambas herramientas informáticas combinando sus capacidades para obtener una optimización final del diseño de la planta termosolar.

El incremento de la producción neta de las plantas termosolares con colectores LF y DSG requiere la optimización del diseño de la disposición de localización de los colectores LF en el 
3. Plantas Termosolares con colectores lineales Fresnel con Generación de Vapor y ciclos de potencia Subcríticos Rankine con Recalentamiento Directo

campo solar. Las plantas termosolares de grandes dimensiones requieren un equilibrio y estabilización de los parámetros termodinámicos de diseño en todos los bucles que integran el campo solar. Los transitorios de operación en este tipo de instalaciones durante su puesta en marcha, paradas, $u$ otros transitorios de operación generados por irregularidades en la radiación solar incidente, produce un impacto directo en los gradientes térmicos que debe soportar las tuberías integrantes del campo solar. La nueva configuración [27], ilustrada en las figuras 3.5 y 3.6, proporciona muchas ventajas para mantener la estabilidad de los parámetros de operación del planta termosolar. Esta configuración requiere cambios menores en relación al lazo diseñado en la instalación piloto del proyecto DISS [26] (figuras 3.1, 3.2 y 3.4). También permite la intercambiabilidad con la configuración de referencia mediante la operación de apertura y cierre de válvulas.

La configuración OT fue desarrollada en el seno del proyecto de investigación DUKE [22- 25] para colectores solares PTC. Esta configuración OT, constituye otra oportunidad para reducir el coste unitario de la electricidad producida en la planta termosolar, y podría diseñarse una planta termosolar que permitiera el intercambio entre las configuraciones presentadas en este trabajo para aprovechar las sinergias generadas por cada una de ellas. El desarrollo de nuevos materiales para las plantas con combustibles fósiles [44-45] favorece al desarrollo de la configuración OT, ya que las propiedades optimizadas de los materiales más innovadores mejorará el desempeño de los sistemas de control, permitiendo una mayor tenacidad del material a altas temperaturas y una mayor resistencia mecánica en las zonas críticas hot-spots susceptibles de potenciales fracturas o acumulación de tensiones en el material.

La configuración OT también fue simulada con Thermoflow [16] y SAM [17]. La producción bruta anual de electricidad obtenida ha sido detallada en la tabla 3.17.

Tabla 3.17. Configuración propuesta [27], potencia anual (mensual) comparada para el cálculo con SAM y Thermoflow.

\begin{tabular}{lcc}
\hline & $\begin{array}{c}\text { Potencia Bruta } \\
\text { (MWh) }\end{array}$ & $\begin{array}{c}\text { Potencia Neta } \\
(\text { MWh })\end{array}$ \\
\hline Thermoflow & 80779 & 76158 \\
SAM & 79799 & 73592 \\
\hline
\end{tabular}

\subsection{Referencias}

[1] D. Krüger, J. Krüger, J. Pandian, K. Hennecke. Kanchanaburi Solar Thermal Power Plant with Direct Steam Generation. SolarPACES 2010.

[2] M.Seling, Novatec Solar GmbH. Two years experience in operating the largest commercial Fresnel CSP Plant. SolarPaces 2014, Beijing, China.

[3] Solar Energy Generating Systems (SEGS). http://www.energy.ca.gov/sitingcases/solar/

[4] Hani H. Sait, José M. Martínez-Val, Rubén Abbas, Javier Muñoz-Antón. Fresnel-based modular solar fields for performance/cost optimization in solar thermal power plants: A comparison with parabolic trough collectors. Applied Energy 141 (2015) 175-189. 
3. Plantas Termosolares con colectores lineales Fresnel con Generación de Vapor y ciclos de potencia Subcríticos Rankine con Recalentamiento Directo

[5] María J. Montes, Carlo Rubbia, Rubén Abbas, José M. Martínez-Val . A comparative analysis of configurations of linear Fresnel collectors for concentrating solar power. Energy 73 (2014) 192-203.

[6] José M. Martínez-Val, Carlo Rubbia, Hani H. Sait, Rubén Abbas, Javier Muñoz-Antón. A coherent integration of design choices for advancing in solar thermal power. Solar Energy 119 (2015) 474-485.

[7] Novatec Solar. http://www.novatecsolar.com/

[8] Areva Solar. http://www.areva.com/EN/solar-220/areva-solar.html

[9] Euromed Solar. http://www.solareuromed.com/

[10] Puerto Errado 1 y 2, Murcia, Spain. http://www.novatecsolar.com/56-1-PE-2.html

[11] Liddell, New South Wales, Australia . http://www.austela.com.au

[12] Kogan Creek in Brigalow, Queensland Australia.http://arena.gov.au

[13] Kimberlina, Bakersfield California USA. http://www.areva.com/EN/solar-164/areva-solar

[14] Llo Solar, Pyrénées Orientales, Francia. http://www.nrel.gov

[15] Dhursar, Rajasthan India. http://india.areva.com

[16] Copyright Thermoflow, Inc. 1987-2012, USA. Thermoflow Fully-Flexible Heat Balance Engineering Software. https://www.thermoflow.com

[17] NREL, DOE, USA. SAM System Advisor Model. https://sam.nrel.gov/

[18] A.Rovira, R.Barbero, M.J.Montes , R.Abbas , F.Varela. Analysis and comparison of Integrated Solar Combined Cycles using parabolic troughs and linear Fresnel reflectors as concentrating systems. Elsevier Applied Energy 162 (2016) 990-1000.

[19] C.Prieto, P.Coope, A.I.Fernández, L.F. Cabeza. Review of technology: Thermochemical energy storage for concentrated solar power plants. Renewable and Sustainable Energy Reviews 60 (2016) 909-929.

[20] C. Parrado, A. Marzo, E. Fuentealba, A.G. Fernández. 2050 LCOE improvement using new molten salts for thermal energy storage in CSP plants. Renewable and Sustainable Energy Reviews 57 (2016) 505-514.

[21] Y.Tanaka, S.Mesfun, K.Umeki, A.Toffolo, Y.Tamaura, K.Yoshikawa. Thermodynamic performance of a hybrid power generation system using biomass gasification and concentrated solar thermal processes. Applied Energy 160 (2015) 664-672.

[22] J.B.FabianFeldhoff, M.Eck, DLR, Germany. Pub. No.: US 2012/0144831 A1. Method of generating superheated steam in solar thermal power plant and solar thermal power plant. Unites States, Patent Application Publication.

[23] J.B.Feldhoff, et al., SolarPACES 2012. Concept comparison and test facility design for the analysis of Direct Steam Generation in Once-Through mode.

[24] J.B.Feldhoff, T.Hirsch, R.Pitz-Paal, L.Valenzuela. Transient models and characteristics of once-through line focus systems. International Conference on Concentrating Solar Power and Chemical Energy Systems, SolarPACES 2014. Energy Procedia 69 ( 2015 ) 626-637.

[25] A.Aurousseau, V.Vuillerme, J.J.Bezian. Control systems for direct steam generation in linear concentrating solar power plants - A review. Renewable and Sustainable Energy Reviews 56 (2016) 611-630.

[26] E.Zarza, L.Valenzuela, Javier León, K.Hennecke, M.Eck, H.D. Weyers, Martin Eickhoff. Direct steam generation in parabolic troughs: Final results and conclusions of the DISS project. Energy Volume 29, Issues 5-6, April-May 2004, Pages 635-644 SolarPACES 2002. 
3. Plantas Termosolares con colectores lineales Fresnel con Generación de Vapor y ciclos de potencia Subcríticos Rankine con Recalentamiento Directo

[27] J.M.Martinez-Val Peñalosa, M.Valdés Del Fresno, A.Abánades Velasco, R.R. Amengual Matas, M.Piera Carrete, M.J.Montes Pita, A.Rovira de Antonio, A.Ramos Millán, J.Muñoz Antón, R.Abbas Cámara. Universidad Politécnica de Madrid, Spain. International Patent, Publication Number: WO 2013/045721/ A1. Solar Power Plant for Direct Steam Generation.

[28] T.G. Theofanous, T.N. Dinh, J.P. Tu, A.T. Dinh. The boiling crisis phenomenon: Part II: dryout dynamics and burnout. Experimental Thermal and Fluid Science, Volume 26, Issues 6-7, August 2002, Pages 793-810.

[29] S.D. Odeh, G.L. Morrison, M.Behnia. Modelling of parabolic trough direct steam generation solar collectors. Solar Energy, VoL. 62, Issue 6, Jun 1998, Pages 395-406.

[30] J.F.Feldhoff, T.Hirsch, R.Pitz-Paal, L.Valenzuela. Analysis and potential of Once-Through steam generators in line focus systems-Final results of the DUKE project. SolarPACES 2015. AIP Conference Proceedings 1734, 100006(2016).

[31] Price H, Parabolic Trough Solar Power Plant Simulation Model. Proceedings of the ISEC 2003: International Solar Energy Conference, 15-18 March 2003, Kohala Coast, Hawaii. New York: American Society of Mechanical Engineers. 665-673 pp.; NREL Report No. CP-550-34742. [32] Wagner, M. J.; Gilman, P. (2011). Technical Manual for the SAM Physical Trough Model. 124 pp.; NREL Report No. TP-5500-51825. http://www.nrel.gov/docs/fy11osti/51825.pdf

[33] Turchi, C.; Neises, T. (2015). Parabolic Trough Solar-Thermal Output Model Decoupled from SAM Power Block Assumptions. Milestone report prepared for the U.S. Department of Energy. https://sam.nrel.gov

[34] Wagner, M.; Zhu, G. (2012). A Direct-steam Linear Fresnel Performance Model for NREL's System Advisor Model. NREL Conference Paper CP-5500-55044.

[35] Wagner, M. (2012). Results and Comparison from the SAM Linear Fresnel Technology Performance Model: Preprint. NREL Conference Paper CP-5500-54758.

[36] P.Griffin, K.Huschka, G.Morin. Sofware for design, simulation, and cost estimation of solar thermal power and heat cycles. SolarPACES 2009. https://www.thermoflow.com

[37] A. Giostri, M. Binotti, P. Silva, E. Macchi and G. Manzolini. Comparison of Two Linear Collectors in Solar Thermal Plants: Parabolic Trough Versus Fresnel. ASME Journal of Solar Energy Engineering, Volume 135, Issue 1.

[38] G.Manente, S.Rech, A.Lazzaretto. Optimum choice and placement of concentrating solar power technologies in integrated solar combined cycle systems. Renewable Energy 96 (2016) 172-189.

[39] G.Manente. High performance integrated solar combined cycles with minimum modifications to the combined cycle power plant design. Energy Conversion and Management 111 (2016) 186-197.

[40] F.Rinaldi, M.Binotti, A.Giostri, G.Manzolini. Comparison of linear and point focus collectors in solar power plants. SolarPACES 2013. Energy Procedia 49 (2014) 1491-1500.

[41] A.Khenissi. Return of Experience on Transient Behaviour at the DSG Solar Thermal Power Plant in Kanchanaburi Thailand. SolarPaces 2014, Beijing, China.

[42] T.Hirsch, A.Khenissi, A systematic comparison on power block efficiencies for CSP plants with direct steam generation, Institute of Solar Research, German Aerospace Center (DLR), SolarPaces 2013.

[43] L.Coco-Enríquez, J.Muñoz-Antón, J.M. Martínez-Val. Innovations on direct steam generation in linear Fresnel collectors. SolarPaces 2013, Las Vegas, U.S. 
3. Plantas Termosolares con colectores lineales Fresnel con Generación de Vapor y ciclos de potencia Subcríticos Rankine con Recalentamiento Directo

[44]Electric Power Research Institute. G8 Cleaner Fossil Fuels Workshop. IEA Secretariat, Paris, 17-18 January, 2008. Boiler material for USC pulverized coal (PC) Plants.

[45] John A. Siefert, Cara Libby, John Shingledecker. Concentrating solar power (CSP) power cycle improvements through application of advanced materials. SolarPACES 2015. AIP Conference Proceedings 1734, 070030 (2016). 
Capítulo 4

\title{
Plantas Termosolares con Colectores Lineales con DSG y sales fundidas como HTF acoplados a ciclos Supercriticos Rankine
}

\author{
Resumen \\ Entre las principales limitaciones de las plantas termosolares con ciclos Rankine están la \\ temperatura máxima y la presión máxima a la entrada a turbina, lo que limita la eficiencia de la \\ planta solar debido a la limitación impuesta por el principio de Carnot. Estos dos parámetros \\ son muy importantes para incrementar la potencia generada y optimizar la eficiencia del \\ sistema.
}

Aumentar la presión a la entrada de la turbina permite aumentar el número de calentadores de agua de alimentación del ciclo de potencia Rankine, lo cual reduce las pérdidas energéticas y proporciona un incremento exergético neto de la instalación termosolar, debido al aumento de la energía intercambiada entre los equipos que integran el Balance Of Plant (BOP). Sin embargo, con presión y temperaturas superiores a ciertos valores, el agua se presenta en la naturaleza en su estado crítico, no en estado de vapor: El punto supercrítico del agua viene definido por los siguientes parámetros termodinámicos: presión 220 bar y temperatura $374^{\circ} \mathrm{C}$.

Las turbinas de vapor de agua han sido la tecnología más difundida e implantada en los ciclos de potencia Rankine de las plantas de generación de energía eléctrica. Sin embargo, recientemente han comenzado a desarrollarse e implantarse a escala industrial las turbinas de agua en estado supercrítico. Las plantas con combustibles fósiles (carbón principalmente) han sido las primeras centrales termoeléctricas en integrar estas innovadores turbinas en sus ciclos de potencia. Las turbinas supercríticas comerciales tienen una potencia mínima de $\mathbf{4 0 0}$ MWe [1], fruto del diseño optimizado y de factores como el sellado con los ejes. Por tanto, un primer desafío tecnológico con el estado de la técnica es la adaptación del tamaño de las turbinas supercríticas para su integración en plantas termosolares con capacidades de generación inferiores, del orden de $50 \mathrm{MWe}$.

En este sentido un estudio reciente de SNL y de la empresa Siemens Energy, publicado en Marzo de 2013 [2], confirma la viabilidad de adaptación de las turbinas comerciales Siemens modelo SST-900 para la generación de electricidad en ciclos Rankine con agua en estado supercrítico. El estudio ha sido realizado para condiciones de vapor vivo de 230 a 260 bar, y un rango de potencias entre 140 y $200 \mathrm{MWe}$. Este estudio fue realizado para plantas termosolares con torre central y campo de heliostatos. En la presente tesis se ha continuado la misma línea de investigación, realizando un estudio de integración de un campo solar con colectores lineales PTC o LF, con un ciclo de potencia Rankine con agua en estado supercrítico. Los dos fluidos caloportadores estudiados en los colectores solares lineales han sido: sal fundida marca comercial HITEC XL, y DSG. Las condiciones de referencia fijadas a la entrada de la turbina han sido de $550^{\circ} \mathrm{C}$ y 260 bar, y de $165 \mathrm{MWe}$ de potencia bruta generada.

Una vez simulado el comportamiento termodinámico y los flujos energéticos de los equipos de la planta termosolar, se concluye que la eficiencia de la planta con ciclo Rankine supercrítico es 
4. Plantas Termosolares con Colectores Lineales con DSG y sales fundidas como HTF acoplados a ciclos Supercriticos Rankine

de $43 \%-45 \%$, dependiendo de la configuración de los calentadores, y del número de extracciones de turbina, como se detallará en este capítulo. Sin embargo, con el ciclo de potencia Rankine subcrítico, la eficiencia de la planta termosolar está limitada entre un $40 \%$ a $41 \%$, dependiendo principalmente del número de etapas de recalentamiento directo de vapor en el campo termosolar.

\subsection{Introducción}

Las plantas termosolares de generación eléctrica no emiten durante su funcionamiento gases de efecto invernadero, como $\mathrm{CO}_{2}$. Mediante la energía renovable de origen termosolar se produce una transformación limpia de la radiación solar en energía eléctrica. En las plantas termosolares el objetivo no es reducir la cantidad de combustible fósil consumido, sino reducir el coste de inversión en su fabricación y construcción. Se puede considerar que el coste de la totalidad del combustible "teórico" consumido durante la vida de la instalación se integra en el coste inicial de la instalación termosolar, ya que la radiación solar es gratuita. El coste variable de las instalaciones termosolares es predominantemente derivado de su operación y mantenimiento. El parámetro de referencia seleccionado para realizar una comparativa del diseñó más óptimo es la eficiencia energética neta de la planta, que tiene relación directa con el tamaño del campo solar.

La tecnología más difundida en las plantas termosolares españolas es la de colectores solares PTC y aceite térmico como HTF, como es el caso de la planta termosolar Andasol 1. Esta tipología de plantas tiene limitada su temperatura de operación a $390^{\circ} \mathrm{C}$ para evitar la degradación del aceite térmico. Por esta razón los parámetros termodinámicos del vapor vivo a la entrada de la turbina son de $380^{\circ} \mathrm{C}$ y 100 bar. Con estas condiciones y el estado de la técnica en los ciclos Rankine, se obtienen unas eficiencias energéticas netas del orden de 37.5\% [3]. Los ciclos subcríticos Rankine más modernos tecnológicamente hablando disponen de 7 calentadores de agua, 4 de baja presión, 3 de alta presión y un desaireador, como en California U.S.A. en los balances preliminares de la empresa Alstom para los proyectos: Rio Mesa, Hidden Hills, Blythe, Palen [4].

Otra tecnología recientemente desarrollada, en vías de implantación a escala industrial, es la combinación entre la tecnología DSG en los colectores lineales PTC o LF, y los ciclos subcríticos de potencia Rankine [5-8]. Esta tecnología estudiada en el capítulo 3, proporciona rendimientos netos del orden de $40-41 \%$. Para un mayor grado de detalle respecto a estos valores de eficiencia ver los resultados tabulados en las tablas 4.7 a 4.10 en el presente capítulo.

Las sales fundidas se presentan como la principal alternativa a la tecnología DSG en las plantas termosolares con colectores lineales como HTF [9-11]. Gracias a las sales fundidas se dispone de mayor temperatura de operación en el vapor vivo a la entrada de la turbina, mejorando considerablemente el rendimiento termodinámico del ciclo Rankine. Las sales fundidas permiten operar a presiones menores que los aceites térmicos del orden de 15 bar, reduciéndose las pérdidas parásitas en los campos solares por bombeo. Sin embargo, presentan el gran inconveniente de requerir sistemas Heat-Tracing, para evitar la solidificación de las sales en el interior de los tubos receptores. 
4. Plantas Termosolares con Colectores Lineales con DSG y sales fundidas como HTF acoplados a ciclos Supercriticos Rankine

En las centrales térmicas con combustibles fósiles, carbón principalmente, se está desarrollando la tecnología de vapor en estado supercrítico en ciclos Rankine [12-14]. En el presente capítulo de esta tesis doctoral, se demuestra como los ciclos supercríticos Rankine combinados con colectores lineales, PTC o LF, consiguen incrementar la eficiencia neta de las plantas termosolares hasta un $43-45 \%$, para unas condiciones de diseño del vapor vivo a la entrada de turbina principal de $550^{\circ} \mathrm{C}$ y de 260 bar de presión [15]. Los resultados detallados se han resumido en las tablas 4.7 a 4.10 . El incremento de la presión del vapor vivo a la entrada de la turbina principal a valores superiores a su punto crítico, permite incrementar el número de extracciones de vapor para precalentamiento del agua en fase líquida a la salida del ciclo Rankine. De esta forma se consigue una mayor temperatura del agua de alimentación a la entrada del campo solar, y la energía solar necesaria para su calentamiento se ve disminuida. El ciclo supercrítico Rankine propuesto en este capítulo considera siete calentadores de agua de alimentación, frente a los cinco calentadores en los ciclos subcriticos Rankine, como se propone en la referencia [16].

El agua en condiciones supercríticas, proporciona unas propiedades físicas ventajosas, como es por ejemplo su alta densidad en comparación con otros estados del agua, lo que permitedisminuir las dimensiones de sus turbinas. Si se consigue un diseño apropiado de los caminos del vapor y de los álabes en las turbinas supercríticas, se puede optimizar su eficiencia y optimizar las perdidas termo-hidráulicas secundarias. El estudio ya referido de SNL y de Siemens [2], concluye que la turbina de Siemens SST-900 puede ser adaptada para altos valores de velocidad y presión del vapor, permitiendo su utilización con vapor en estado supercrítico, y proporcionando una potencia generada entre 140 y 200 MWe. Esta turbina también puede ser adaptada a rápidos transitorios derivados de la puesta en marcha de la instalación y de su operación diaria, realizando un seguimiento adecuado de la variación de la radiación solar. La tecnología de estas turbinas de vapor supercríticas está sustentada por la utilización de materiales más sofisticados con mejores propiedades mecánicas (aceros inoxidables AISI 347, Inconel, etc.), materiales ya ampliamente validados, y utilizados en las centrales térmicas con ciclos Rankine supercríticos o ultrasupercríticos [17-18].

De acuerdo al principio de Carnot, la eficiencia del ciclo termodinámico de potencia mejora para mayores valores de presión y temperatura del vapor vivo a la entrada de la turbina principal. Pero la temperatura de operación de la central termosolar con colectores lineales también está limitada por la máxima temperatura soportada por el material selectivo de recubrimiento de los tubos absorbedores, y para minimizar las pérdidas térmicas por radiación derivadas a grandes temperaturas. Por este motivo se ha fijado como temperatura máxima de operación $550^{\circ} \mathrm{C}$.

Como HTF en el campo solar se ha elegido la sal fundida comercial HITEC XL, o la tecnología DSG. En ambos casos las temperaturas de entrada a turbina son del orden de $550^{\circ} \mathrm{C}$.

Respecto a los colectores solares, se han estudiado los colectores PTC y LF. La eficiencia óptica nominal de los colectores PTC es del orden del $75 \%$ mayor que la eficiencia de los LF $65 \%$ [19]. 
4. Plantas Termosolares con Colectores Lineales con DSG y sales fundidas como HTF acoplados a ciclos Supercriticos Rankine

La optimización del campo solar se ha cuantificado en términos de la potencia unitaria nominal generada en los colectores solares o Unitary Power Output, definida como la relación entre la potencia neta eléctrica generada por la planta termosolar y la superficie de apertura efectiva del campo solar. El objetivo es maximizar este parámetro para reducir la superficie de los colectores solares para una potencia neta considerada.

Respecto al flujo másico $\left(\mathrm{kg} / \mathrm{m}^{2} \cdot \mathrm{s}\right)$ del HTF en los tubos absorbedores, se ha limitado a un valor máximo. Los objetivos perseguidos son: minimizar las pérdidas de presión, obtener una velocidad del HTF que no produzca una degradación por erosión-corrosión, y reducir la probabilidad de fugas o vibraciones en los elementos y componentes que integran el campo solar.

Otra variable de diseño que impacta directamente en la eficiencia neta de la planta es el número de etapas de recalentamiento de vapor en las turbinas de alta y media presión. El recalentamiento del vapor proporciona otro medio para incrementar la eficiencia energética en los ciclos Rankine, permitiendo un mayor intercambio energético entre los equipos que integran el ciclo termodinámico. No obstante, el número de etapas de recalentamiento está limitado por las pérdidas de presión en cada una de las etapas y por el diseño de la turbina. El recalentamiento directo DRH [20-21] constituye una solución innovadora para evitar los intercambiadores intermedios en las etapas de recalentamiento entre el ciclo de potencia y el campo solar. El diseño de estos equipos sería de grandes dimensiones por los bajos coeficientes de transferencia de calor de agua en estado vapor. La presión del orden de 260 bar a la entrada de la turbina de alta presión permitirían la integración en el ciclo de hasta tres etapas de recalentamiento (figuras 4.3 y 4.4). El número de etapas de recalentamiento tiene que ser confirmada con el fabricante de la turbina, que debe adaptar los diferentes escalones de presión a las extracciones realizadas para recalentamiento. En las turbinas de vapor se debe garantizar que las pérdidas de carga intermedias en cada una de las etapas de turbinado no reduzca el título de vapor por debajo de valores de título 0.9. De lo contrario la condensación del vapor en gotitas de líquido produciría la erosión de los álabes de la turbina.

\subsection{Hipótesis de cálculo}

Los principales datos de partida de las simulaciones termodinámicas se han resumido en las tablas 4.1 a la 4.6 .

Tabla 4.1. Localización y condiciones ambientales.

\begin{tabular}{ll}
\hline Localización: & Dagget, CA, USA. \\
Latitud: & $34.86^{\circ}$ \\
Longitud: & $-116.8^{\circ}$ \\
Zona horaria: & -8 \\
Hora: & $11: 30 \mathrm{hr}$ \\
DNI: & $986 \mathrm{~W} / \mathrm{m}^{2}$ \\
Temperatura ambiente: & $25^{\circ} \mathrm{C}$ \\
Altitud: & $588 \mathrm{~m}$ \\
\hline
\end{tabular}


4. Plantas Termosolares con Colectores Lineales con DSG y sales fundidas como HTF acoplados a ciclos Supercriticos Rankine

Tabla 4.2. Parámetros tubo absorbedor.

\begin{tabular}{ll}
\hline Material tubo: & Acero inoxidable \\
Diámetro exterior: & $70 \mathrm{~mm}$ \\
Espesor tubo: & $4-8 \mathrm{~mm}$ \\
Rugosidad interna: & $\mathrm{Ra}=0.0457 \mathrm{~mm}$ \\
Max. DSG velocidad: $(\mathrm{m} / \mathrm{s})$ & $40-50$ \\
Max. MS velocidad: $(\mathrm{m} / \mathrm{s}):$ & $2-4$ \\
\hline
\end{tabular}

Tabla 4.3. Parámetros colector PTC.

\begin{tabular}{ll}
\hline Tipo colector: & EuroTrough II \\
Anchura Apertura: & $5.77 \mathrm{~m}$ \\
Longitud focal: & $1.71 \mathrm{~m}$ \\
Factor limpieza: & 0.96 \\
Eficiencia óptica: & 0.75 \\
Pérdidas térmicas: & $0.141 \Delta \mathrm{T}+6.48 \mathrm{e}-9 \Delta \mathrm{T}^{4}[22]$ \\
\hline
\end{tabular}

Tabla 4.4. Parámetros colector LF.

\begin{tabular}{ll}
\hline Tipo colector: & SuperNova1 (Novatec) \\
Dimensiones: & $16.56 \mathrm{~m} \times 44.8 \mathrm{~m}$ \\
Apertura area: & $513.6 \mathrm{~m}^{2} /$ per module \\
Eficiencia óptica: & $0.67 ; 0.647$ \\
Pérdias térmicas: & $1.06 \Delta \mathrm{T}+1.2 \mathrm{e}-8 \Delta \mathrm{T}^{4}$ \\
Pérdidas térmicas: & $0.15 \Delta \mathrm{T}+7.15 \mathrm{e}-9 \Delta \mathrm{T}^{4}[19]$ \\
\hline
\end{tabular}

Tabla 4.5. Ciclo Rankine (supercritico).

\begin{tabular}{ll}
\hline HP entrada turbina (bar): & 87.7 \\
HP entrada turbina $\left({ }^{\circ} \mathrm{C}\right):$ & 550 \\
Eficiencia isentrópica (\%): & 0.85 \\
№ escalones HP: & 2 \\
№ escalones IP: & 3 \\
№ escalones LP: & 4 \\
Ta recalentamieto $\left({ }^{\circ} \mathrm{C}\right):$ & 550 \\
Calidad salida turbina LP: & $>0.9$ \\
Condensador (bar): & 0.08 \\
№ calentadores: & 5 \\
Desaireador (bar): & 6.2 \\
Calentadores TTD ( $\left.{ }^{\circ} \mathrm{C}\right):$ & 5 \\
Calentadores DCA $\left({ }^{\circ} \mathrm{C}\right):$ & 5 \\
\hline & \\
Tabla 4.6. Ciclo Rankine (supercrítico). \\
\hline HP entrada turbina (bar): & 260 \\
HP entrada turbina $\left({ }^{\circ} \mathrm{C}\right):$ & 550 \\
Eficiencia isentrópica turbina & 0.85 \\
№ escalones HP: & 2 \\
№ escalones IP: & 3 \\
\hline
\end{tabular}


4. Plantas Termosolares con Colectores Lineales con DSG y sales fundidas como HTF acoplados a ciclos Supercriticos Rankine

\begin{tabular}{ll}
\hline № escalones LP: & 4 \\
Ta recalentamiento $\left({ }^{\circ} \mathrm{C}\right):$ & 550 \\
Calidad salida turbina LP: & $>0.9$ \\
Condensador (bar): & 0.08 \\
№ calentadores: & 7 \\
Desaireador (bar): & 8.5 \\
Calentadores TTD $\left({ }^{\circ} \mathrm{C}\right):$ & 5 \\
Calentadores DCA $\left({ }^{\circ} \mathrm{C}\right):$ & 5 \\
\hline
\end{tabular}

4.3. Plantas Termosolares con Colectores Lineales con DSG como HTF acoplado a ciclo Subcrítico Rankine (configuración de referencia)

Siguiendo las últimas tendencias en las plantas termosolares colectores lineales y ciclos Rankine, para el presente estudio se ha considerado como configuración de referencia la representada en las figuras 4.1 y 4.2 , con colectores solares lineales con DSG en modo de recirculación de la fase líquida, y un ciclo subcritico Rankine. Los resultados de eficiencia neta de esta tipología de plantas termosolares se han detallado en las tablas 4.7 a 4.10 .

El principal proyecto relacionado con la tecnología DSG en colectores PTC es el proyecto DISS [5-6]. En este proyecto se ha construido una planta piloto para demostrar la viabilidad tecnológica de la DSG como HTF. Recientemente, se han implantado las primeras centrales termosolares con colectores lineales con DSG. Los principales proyectos son: planta termosolar Kanchanaburi Thailand [8] con colectores PTC, y planta de Puerto Errado [7] con colectores LF.

En una primera fase de desarrollo se ha diseñado el modo de generación de vapor con recirculación de la fase líquida (figura 4.1). En este modo de operación, el agua es precalentada y evaporada en el campo solar, obteniéndose un título de vapor típico a la salida de los colectores del $80 \%$; la fase líquida es separada en los tanques de separación de fases, y el vapor vivo es recalentado en colectores solares diseñados para tal efecto (figura 4.1). Las principales ventajas de la tecnología DSG son:

- no hay impacto ambiental alguno ni solidificación del HTF.

- la corrosión de las tuberías es reducida.

- no hay límite de presión ni de temperatura, etc.

Entre sus principales desventajas se puede indicar el complejo sistema de control para evitar que la ebullición en los tubos absorbedores genere puntos calientes que dañen a la pared del tubo, por gradientes térmicos excesivos entre la fase líquida y vapor.

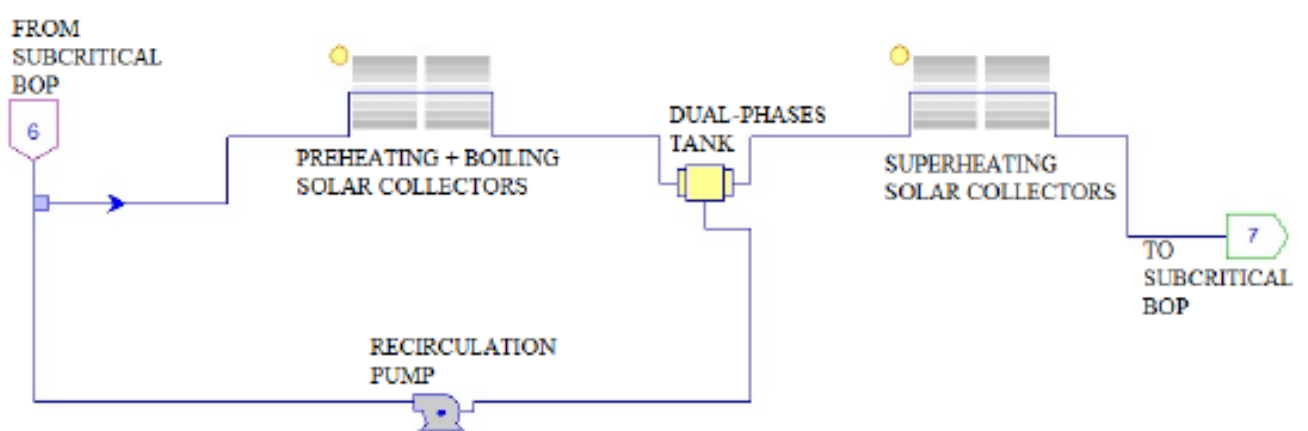

Figura 4.1. DSG en los colectores lineales PTC o LF en modo Recirculación de la fase líquida. 


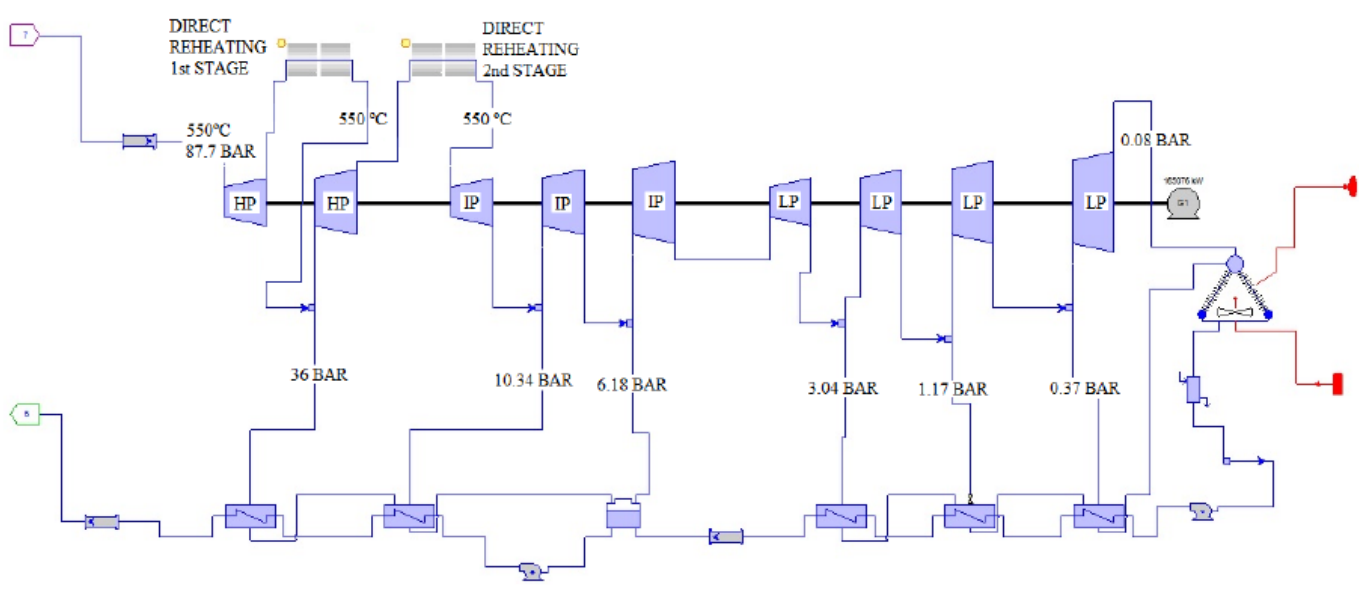

Figura 4.2. Ciclo subcrítico Rankine con 5 calentadores del agua de alimentación y un desaireador.

En relación al ciclo de potencia, las principales características del ciclo representado en la figura 4.2, es la integración de dos etapas de Recalentamiento Directo DRH, en el ciclo Rankine, sin intercambiador intermedio entre los campos solares y el ciclo de potencia [2021]. Con una etapa de recalentamiento directo $D R H$, la eficiencia neta de la planta termosolar es de un $40 \%$ (tablas 4.7 a 4.10), en comparación con la solución sin recalentamiento con eficiencia neta $38.4 \%$. Si incorporamos en el ciclo de potencia dos etapas de recalentamiento la eficiencia neta del ciclo se incrementa hasta un $41 \%$.

\subsection{Planta Termosolar con Colectores Lineales con DSG como HTF ay ciclo supercrítico Rankine}

En lugar de la tecnología DSG con recirculación de la fase líquida, ya validada a escala industria, explicada en detalle en el apartado anterior (figura 4.1); en este apartado se propone otra técnica para producción del vapor y su recirculación en los campos solares con colectores lineales. El vapor saturado puede ser producido durante la puesta en marcha de la planta termosolar mediante una caldera con combustible fósil, o en un campo solar con DSG en modo recirculación de la fase líquida. Durante las condiciones normales de operación, el vapor sobrecalentado no condensa en los tubos absorbedores, y sus pérdidas de carga serían contrarrestadas mediante la compresión del vapor. La principal ventaja de este sistema es la simplicidad de la instalación, reduciendo el coste de la planta termosolar. El principal desafío se materializa en el desarrollo industrial de compresores de vapor para las condiciones termodinámicas consideradas en el presente estudio. Sería necesario garantizar la eficiencia de los compresores operando en rangos de presión superiores a 150 bar. El objetivo es minimizar su consumo eléctrico, ya que impacta muy negativamente en la eficiencia neta de la instalación termosolar.

La selección del material de los tubos absorbedores con DSG, constituye otra ventaja comparativa con la utilización de sales fundidas como HTF. El vapor de agua presenta requerimientos menores a corrosión que los solicitados por las sales fundidas. Sin embargo, la elevada presión de operación, del orden 150 bar, obliga a confirmar que los espesores de pared de los tubos absorbedores garantizan el nivel de transferencia de calor característico de 
4. Plantas Termosolares con Colectores Lineales con DSG y sales fundidas como HTF acoplados a ciclos Supercriticos Rankine

este tipo de instalaciones. En este trabajo se ha confirmado de acuerdo a la normativa ASME 31.3 , que el espesor de los tubos es inferior a $9 \mathrm{~mm}$. Los cálculos han sido realizados con la siguiente ecuación (4-1):

$\mathrm{T}=\frac{\mathrm{P} \cdot(\mathrm{d}+2 \cdot \mathrm{c})}{2 \cdot(\mathrm{S} \cdot \mathrm{E}-\mathrm{P} \cdot(1-\mathrm{Y}))}$

Donde:

- T: Espesor de la tubería.

- P: Presión de diseño.

- d: Diámetro nominal de la tubería.

- c: espesor por corrosión.

- S: Tensión admisible.

- E: Factor de calidad.

- $\quad$ Y: Factor de material.

El diseño del campo solar, la disposición en planta de los colectores, y la longitud de cada lazo, son variables muy importantes a la hora de realizar el diseño de detalle de estas plantas temosolares. El objetivo es minimizar las pérdidas de presión y reducir, por tanto, la potencia y consumo de los compresores de vapor. Para ello, es necesario limitar la longitud de los colectores y de los cabezales de distribución de vapor. Con este fin se ha fijado el flujo másico $\left(\mathrm{kg} / \mathrm{m}^{2} \cdot \mathrm{s}\right)$ por debajo de ciertos límites. Otra posible solución de diseño para reducir las pérdidas de presión, estaría relacionada con el incremento del diámetro de los tubos absorbedores desde $70 \mathrm{~mm}$ hasta $90 \mathrm{~mm}$. Este incremento de diámetro favorecería la transferencia de calor debido al incremento de área de transferencia de calor por unidad de longitud de los tubos absorbedores. Los valores límite de flujo másico considerados en este estudio son de: $750 \mathrm{~kg} / \mathrm{m}^{2} \cdot \mathrm{s}$ para tubos con $70 \mathrm{~mm}$ de diámetro y $650 \mathrm{~kg} / \mathrm{m}^{2} \cdot \mathrm{s}$ para tubos absorbedores con $90 \mathrm{~mm}$ de diámetro. Para minimizar también las pérdidas de carga en las etapas con DRH, el flujo másico máximo en los colectores también se limitó a $600 \mathrm{~kg} / \mathrm{m}^{2} \mathrm{~s}$, en la primera y segunda etapa de recalentamiento, y a $300 \mathrm{~kg} / \mathrm{m}^{2} \mathrm{~s}$ en la tercera etapa de recalentamiento. Se explica mediante un ejemplo el cálculo del límite de flujo másico:

Caso de primera etapa de recalentamiento DRH.

- Caudal másico en el campo solar para recalentamiento: $134.6 \mathrm{~kg} / \mathrm{s}$

- Número de lazos: 81

- Caudal másico en un lazo: $134.6 / 81=1.662 \mathrm{~kg} / \mathrm{s}$

- Diámetro exterior del tubo absorbedor: $70 \mathrm{~mm}$

- Espesor de pared del tubo absorbedor: $5 \mathrm{~mm}$

- Diámetro interior del tubo absorbedor: $60 \mathrm{~mm}$

- Área interior del tubo absorbedor: $0.00282744 \mathrm{~m}^{2}$

- Flujo másico en cada lazo: 1.662/0.0028744 = $587.7 \mathrm{~kg} / \mathrm{m}^{2} \cdot \mathrm{s}<600 \mathrm{~kg} / \mathrm{m}^{2} \cdot \mathrm{s}$ (flujo límite)

- Velocidad del vapor a la entrada del lazo: $16.04 \mathrm{~m} / \mathrm{s}$

- Velocidad del vapor a la salida del lazo: $23.02 \mathrm{~m} / \mathrm{s}$ 
4. Plantas Termosolares con Colectores Lineales con DSG y sales fundidas como HTF acoplados a ciclos Supercriticos Rankine

Los resultados de las simulaciones y modelos de la planta termosolar se han resumido en las tablas 4.7 y 4.8. Se confirmó que los mayores valores de eficiencia energética de la instalación solar se obtienen con la instalación de 7 calentadores. La configuración con tres etapas de recalentamiento directo, en las turbinas de alta y media presión, optimiza el aprovechamiento energético, y maximiza el rendimiento energético en la planta termosolar. Sin embargo, esta configuración no se ha implantado en ninguna instalación industrial, y su viabilidad está sujeta a la aprobación de los fabricantes de turbinas. Se deben diseñar las etapas de turbinado correspondientes, ajustándose los valores de presión, temperatura y caudal óptimos. La configuración del ciclo supercrítico Rankine, con una etapa de recalentamiento en la turbina de alta presión, es la solución técnica más adoptada, y mejora el rendimiento de la instalación desde el $40 \%$ hasta un $42.7 \%$. Esta mejora también se ve reflejada en una disminución del área de apertura efectiva de los colectores, reduciéndose la potencia unitaria desde 0.22 hasta 0.23 (incremento del 5\%).

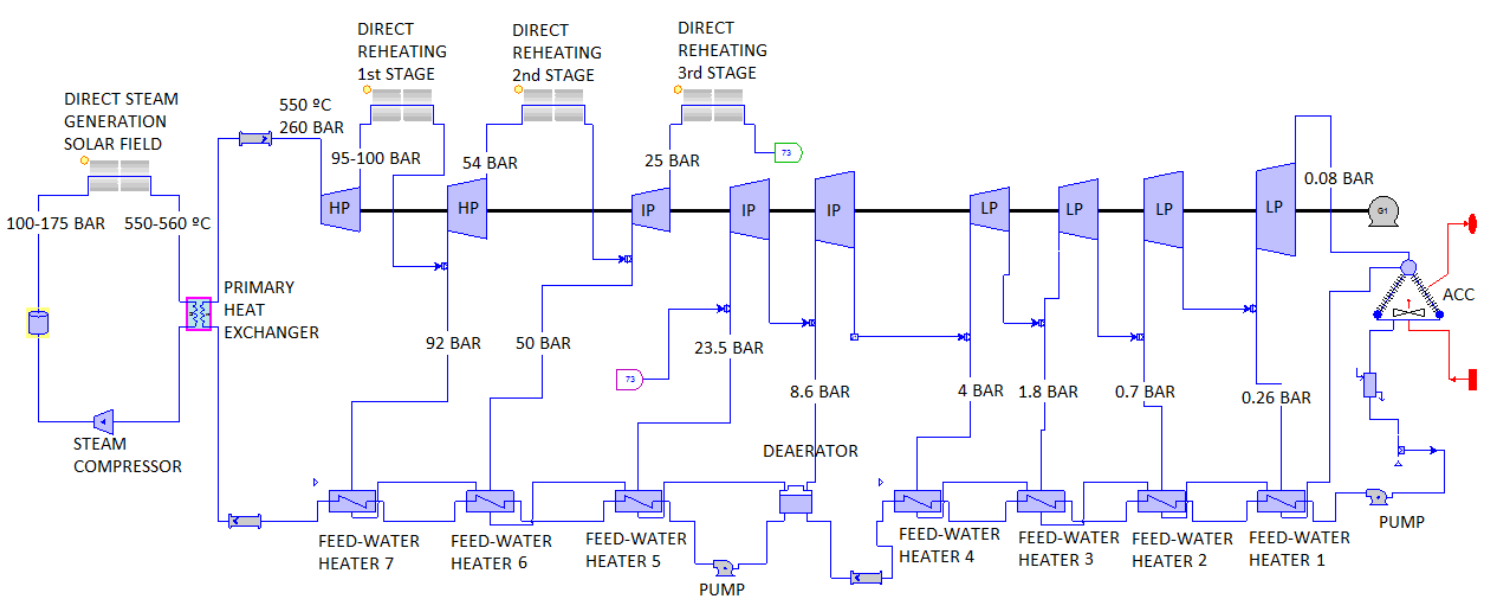

Figura 4.3. Planta Termosolar con colectores lineales (PTC o LF) con generación directa de vapor como HTF acoplado con ciclo de potencia supercrítico Rankine.

Tabla 4.7. Planta Termosolar con colectores LF con DSG y ciclos de potencia Rankine. Comparación de la eficiencia neta de los ciclos subcríticos y los supercríticos (TIT $=550^{\circ} \mathrm{C}$ ).

\begin{tabular}{lllllll}
\hline Ciclo de Potencia & $\begin{array}{l}\text { Subcrítico } \\
\text { Referencia }\end{array}$ & $\begin{array}{l}\text { Subcrítico } \\
\text { Referencia }\end{array}$ & Superc. & Superc. & Superc. & Superc. \\
\hline Figura & 4.2 & 4.2 & 4.3 & 4.3 & 4.3 & 4.3 \\
Etapas de Recalentamiento & $2^{\text {nd }}$ & $1^{\text {st }}$ & $2^{\text {nd }}$ & $1^{\text {st }}, 2^{\text {nd }}, 3^{\text {rd }}$ & $1^{\text {st }}, 2^{\text {nd }}$ & $2^{\text {nd }}, 3^{\text {rd }}$ \\
Presión Campo Solar (bar) & 104 & 104.5 & 150 & 150 & 150 & 150 \\
Presión Entrada Turbina (bar) & 87.7 & 87.7 & 260 & 260 & 260 & 260 \\
Eficiencia Neta (\%) & 40.44 & 40.16 & 42.66 & 44.32 & 43.90 & 43.56 \\
Potencia Unitaria $\left(\mathrm{W} / \mathrm{m}^{2}\right)$ & 226.5 & & 222.1 & 227 & 235 & 234.2 \\
\hline
\end{tabular}

En la tabla 4.8 se han resumido los resultados obtenidos para colectores lineales PTC. Con los colectores PTC se obtienen valores de potencia unitaria superiores ( 16\%) a los obtenidos con colectores LF. El motivo es la mayor eficiencia óptica nominal $~ 75 \%$ con colectores PTC y $\sim 65 \%$ con colectores LF. 
4. Plantas Termosolares con Colectores Lineales con DSG y sales fundidas como HTF acoplados a ciclos Supercriticos Rankine

Tabla 4.8. Planta Termosolar con colectores PTC con DSG, y ciclos de potencia Rankine. Comparación de la eficiencia neta de los ciclos subcríticos y los supercríticos (TIT $=550^{\circ} \mathrm{C}$ ).

\begin{tabular}{lllllll}
\hline Ciclo de Potencia & $\begin{array}{l}\text { Subcrítico } \\
\text { Referencia }\end{array}$ & $\begin{array}{l}\text { Subcrítico } \\
\text { Referencia }\end{array}$ & Superc. & Superc. & Superc. & Superc. \\
\hline Figura Número & 4.2 & 4.2 & 4.3 & 4.3 & 4.3 & 4.3 \\
Etapas de Recalentamiento & $2^{\text {nd }}$ & $1^{\text {st }}$ & $2^{\text {nd }}$ & $1^{\text {st }}, 2^{\text {nd }}, 3^{\text {rd }}$ & $1^{\text {st }}, 2^{\text {nd }}$ & $2^{\text {nd }}, 3^{\text {rd }}$ \\
Presión Campo Solar (bar) & 104.6 & 104.7 & 150 & 150 & 150 & 150 \\
Presión Entrada Turbina (bar) & 87.7 & 87.7 & 260 & 260 & 260 & 260 \\
Eficiencia Neta (\%) & 40.41 & 40.09 & 42.90 & 44.52 & 43.81 & 43.78 \\
Potencia Unitaria (W/m ${ }^{2}$ ) & 265.8 & 263.7 & 268.8 & 276.8 & 273.2 & 274.4 \\
\hline
\end{tabular}

\subsection{Planta Termosolar con Colectores Lineales con sales fundidas como HTF acoplado a ciclo Supercritico Rankine.}

La configuración de planta termosolar de la figura 4.4, con sales fundidas como HTF, la tecnología DMS, y colectores PTC o LF, es una alternativa a la utilización de aceites térmicos, o utilización de DSG como HTF. Una de las principales ventajas DMS es la reducción de las pérdidas parásitas del ciclo, debido al reducido consumo eléctrico de las bombas del campo solar. Gracias a la menor viscosidad de las sales fundidas en relación con el aceite térmico, y la mayor densidad de las sales en relación con la baja densidad del vapor de agua, permite obtener una mayor eficiencia en su bombeo a lo largo de los tubos absorbedores, y de las tuberías de distribución del campo solar. Se considera buena práctica la limitación de la velocidad de flujo de la sales entre 2 y $4 \mathrm{~m} / \mathrm{s}$, de esta forma se garantiza bajas pérdidas de presión en el circuito hidráulico, garantizando una baja corrosión y erosión de las tuberías [23]. Optimizándose a su vez los diámetros y longitud los tubos para garantizar un correcto funcionamiento hidráulico del campo solar.

La modificación de la composición de las tradicionales de sales fundidas Solar Salt $\left(60 \% \mathrm{NaNO}_{3}\right.$, $40 \% \mathrm{KNO}_{3}$ ) ha permitido obtener otras sales para su uso como HTF, en los campo solares con colectores lineales, y con propiedades físicas optimizadas para este fin. Es el caso de las sal fundida HITEC XL $\left(48 \% \mathrm{Ca}\left(\mathrm{NO}_{3}\right) \quad 2.7 \% \mathrm{NaNO}_{3}, 45 \% \mathrm{KNO}_{3}\right)$. El precio unitario de las sales tradicionales $(0.49 \$ / \mathrm{kg})$, es inferior a la del HITEC XL $(1.43 \$ / \mathrm{kg})$ sin embargo, el punto de solidificación de las sales solares es de $200^{\circ} \mathrm{C}$ y este valor se ve reducido para el HITEC XL hasta $142^{\circ} \mathrm{C}[24-25]$. 
4. Plantas Termosolares con Colectores Lineales con DSG y sales fundidas como HTF acoplados a ciclos Supercriticos Rankine

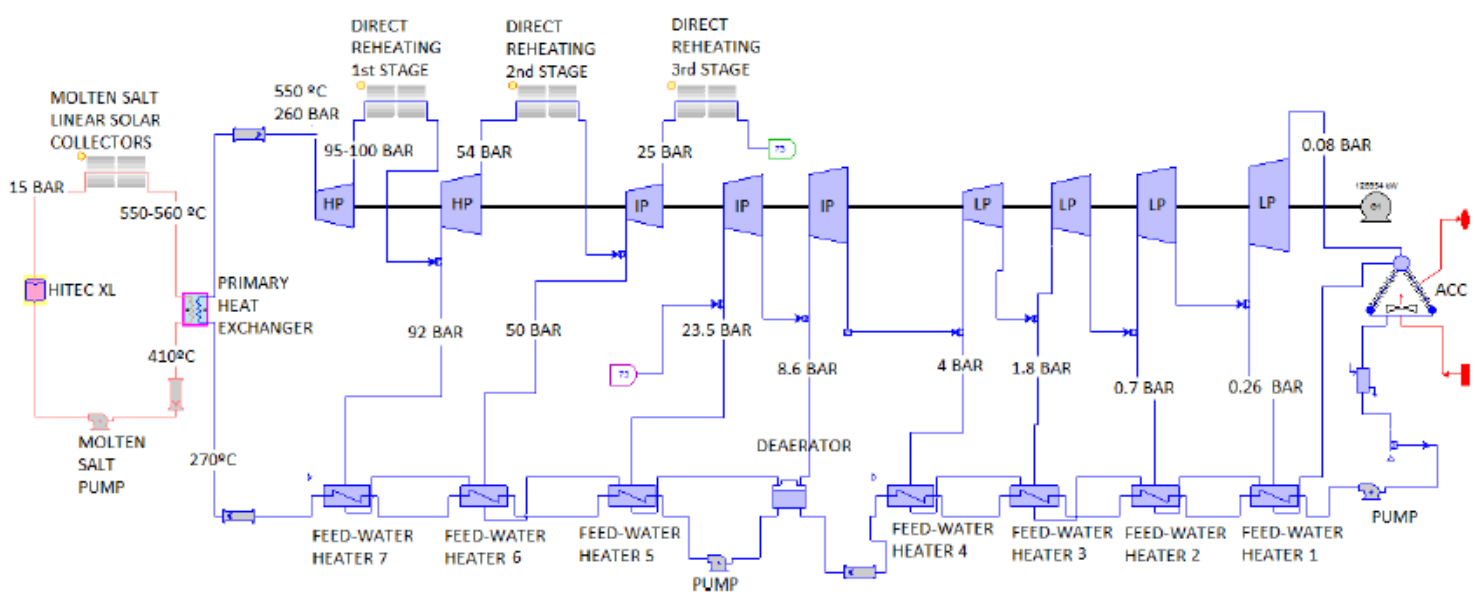

Figura 4.4. Planta Termosolar con colectores lineales con sales fundidas como HTF acoplado a ciclo de potencia supercrítico Rankine.

Otra ventaja comparativa de la utilización de las sales fundidas respecto a la utilización tanto de aceite térmico como de DSG como HTF, se materializa en el diseño de los cambiadores de calor entre el campo solar y el ciclo de potencia. Gracias a las propiedades físicas de las sales, su coeficiente de transferencia de calor es más alto que el del aceite térmico y el del vapor de agua.

Tabla 4.9. Planta Termosolar con colectores LF, con sales fundidas como HTF, y ciclos de potencia de Rankine. Comparación de la eficiencia neta de los ciclos subcríticos y los supercríticos $\left(\mathrm{TIT}=550^{\circ} \mathrm{C}\right)$.

\begin{tabular}{lllllll}
\hline Ciclo de Potencia & $\begin{array}{l}\text { Subcrítico } \\
\text { Referencia }\end{array}$ & $\begin{array}{l}\text { Subcrítico } \\
\text { Referencia }\end{array}$ & Superc. & Superc. & Superc. & Superc. \\
\hline Número de Figura & 4.2 & 4.2 & 4.4 & 4.4 & 4.4 & 4.4 \\
Etapas Recalentamiento & $2^{\text {nd }}$ & $1^{\text {st }}$ & $2^{\text {nd }}$ & $1^{\text {st }}, 2^{\text {nd }}, 3^{\text {rd }}$ & $1^{\text {st }}, 2^{\text {nd }}$ & $2^{\text {nd }}, 3^{\text {rd }}$ \\
Campo Solar (bar) & 104 & 104.5 & 15 & 15 & 15 & 15 \\
Entrada Turbina (bar) & 87.7 & 87.7 & 260 & 260 & 260 & 260 \\
Eficiencia Neta (\%) & 40.44 & 40.16 & 43.77 & 45.06 & 44.62 & 44.27 \\
Potencia Unitaria (W/m & 226.5 & 222.1 & 235.6 & 240 & 238.9 & 236.9 \\
\hline
\end{tabular}

Como resultado, se obtiene un diseño final de los cambiadores de calor con dimensiones menores, lo cual impacta directamente en un menor coste de la planta termosolar. Como contrapartida los requerimientos de corrosión del material del lado tubos, cuando utilizamos sales fundidas, es mayor que cuando se utiliza vapor de agua o aceite térmico. Las sales fundidas hacen necesario la selección de aceros inoxidables austeníticos con altos grados de aleación con Níquel, para garantizar la resistencia a corrosión del material en contacto con las sales con grandes temperaturas de operación $\left(300^{\circ} \mathrm{C}-550^{\circ} \mathrm{C}\right)$. El coste del Níquel es una variable importante a la hora de caracterizar el coste final de los cambiadores.

En las tablas 4.9 y 4.10 se ha confirmado que la tecnología DMS, y ciclo de potencia supercrítico Rankine, proporciona valores de eficiencia neta superiores en un $3 \%$ a $5 \%$ a la de los ciclos de Rankine subcríticos (tablas 4.9 y 4.10). Si comparamos los resultados obtenidos con DSG y ciclos de Rankine supercríticos, se confirma que las solución técnica de sales fundidas en los colectores lineales proporciona valores superiores de eficiencia neta entre un 
4. Plantas Termosolares con Colectores Lineales con DSG y sales fundidas como HTF acoplados a ciclos Supercriticos Rankine

$0.5 \%$ y $1 \%$. Se puede confirmar este hecho comparando los resultados de las tablas 4.7 y 4.8 para colectores lineales con DSG, y los de las tablas 4.9 y 4.10 , con sales fundidas como HTF.

Tabla 4.10. Planta Termosolar con colectores PTC, con sales fundidas como HTF, y ciclos de potencia de Rankine. Comparación de la eficiencia neta de los ciclos subcríticos y los

supercríticos $\left(\mathrm{TIT}=550^{\circ} \mathrm{C}\right)$.

\begin{tabular}{lllllll}
\hline Ciclo de Potencia & $\begin{array}{l}\text { Subcrítico } \\
\text { Referencia }\end{array}$ & $\begin{array}{l}\text { Subcrítico } \\
\text { Referencia }\end{array}$ & Superc. & Superc. & Superc. & Superc. \\
\hline Número de Figura & 4.2 & 4.2 & 4.4 & 4.4 & 4.4 & 4.4 \\
Etapas Recalentamiento & $2^{\text {nd }}$ & $1^{\text {st }}$ & $2^{\text {nd }}$ & $1^{\text {st }}, 2^{\text {nd }}, 3^{\text {rd }}$ & $1^{\text {st }}, 2^{\text {nd }}$ & $2^{\text {nd }}, 3^{\text {rd }}$ \\
Campo Solar (bar) & 104.6 & 104.7 & 15 & 15 & 15 & 15 \\
Entrada Turbina (bar) & 87.7 & 87.7 & 260 & 260 & 260 & 260 \\
Eficiencia Neta (\%) & 40.41 & 40.09 & 43.82 & 44.72 & 44.39 & 43.82 \\
Potencia Unitaria $\left(\mathrm{W} / \mathrm{m}^{2}\right)$ & 265.8 & 263.7 & 274.7 & 277 & 276.2 & 273.4 \\
\hline
\end{tabular}

\subsection{Conclusiones}

Las plantas termosolares con colectores lineales con ciclos supercríticos Rankine, proporcionan mayores niveles de eficiencia energética del orden del $43 \%$ a $45 \%$, en comparación con las plantas termosolares con colectores lineales con DSG y ciclos subcríticos Rankine, con una eficiencia del $40 \%$ con una etapa de recalentamiento, y de $41 \%$ con dos etapas de recalentamiento. Los resultados que apoyan esta afirmación se recogen en las tablas 4.7 a 4.10.

El incremento de rendimiento de las plantas termosolares con ciclos supercríticos Rankine se debe principalmente a la utilización de siete calentadores de agua de alimentación [3], y dos o tres etapas de DRH integradas en las turbinas de vapor. Los ciclos subcríticos Rankine operaban a presiones inferiores y no permitían incrementar el número de extracciones de vapor para recalentar el agua de alimentación. El incremento de la potencia de entrada a la turbina principal proporciona mayores rangos de presión a la hora de recalentar el vapor en los campos solares, y permiten evitar la utilización de intercambiadores de vapor intermedios en las etapas de recalentamiento. Sin embargo, como se ha indicado a lo largo de este capítulo, el estado de la tecnología de diseño de las turbinas supercríticas no ha sido aún desarrollada para turbinas con potencias inferiores a $400 \mathrm{MWe}$ [1]. Otro importante inconveniente de la tecnología supercrítica es la necesidad de materiales sofisticados resistentes a la corrosión y erosión a altas temperaturas y presiones. Principalmente su desarrollo está acompañado a la utilización de aleaciones con grandes contenidos de Níquel [17-18], material con un elevado coste.

Se ha demostrado en este capítulo como la utilización de sales fundidas como HTF en los colectores lineales proporciona mayores valores de eficiencia de la planta termosolar. En el caso comparado de la tecnología DSG, las pérdidas energéticas derivadas del consumo de los compresores de vapor impactan negativamente en la potencia neta generada. Sin embargo, la DSG como HTF en los colectores lineales evita la utilización de Heat-tracing, para evitar la solidificación de las sales, cuyo consumo eléctrico también impacta negativamente en la potencia neta generada. 
4. Plantas Termosolares con Colectores Lineales con DSG y sales fundidas como HTF acoplados a ciclos Supercriticos Rankine

Los requerimientos de corrosión y tensiones mecánicas en los ciclos supercríticos Rankine tienen que ser también evaluados en detalle, y constituyen otra desventaja de esta innovadora tecnología en relación a la operación con agua con sus propiedades en estado subcrítico. La necesidad del Níquel, como principal elemento aleante, introduce un inconveniente a la hora de disminuir el coste de equipos y componentes en las plantas supercríticas. El desarrollo de nuevos materiales con menor coste, y similares resistencia química y mecánicas, constituirán uno de los pilares principales a la hora garantizar la viabilidad industrial de esta solución tecnológica [18].

Así mismo, este trabajo constituye una referencia de la viabilidad energética y termodinámica del sistema estudiado. Es necesario el desarrollo en detalle de los equipos, garantizando que su eficiencia permite obtener valores de rendimiento similar a los obtenidos de las simulaciones.

Como trabajos futuros, sería conveniente realizar la evaluación de la producción anual de la planta termosolar, teniendo en cuenta su localización, poniendo en especial consideración las condiciones ambientales de temperatura y humedad del foco frío, y de irradiación solar. La integración de la planta termosolar con un sistema de almacenamiento térmico y/o con calderas de biomasa, constituyen las últimas tendencias para garantizar la flexibilidad de suministro eléctrico, adaptadas a la variación de energía solar incidente característica de lugar donde se ubique a instalación industrial.

\subsection{Referencias}

[1] Bruce Kelly. Advanced Thermal Storage for Central Receivers with Supercritical Coolants. Abengoa Solar Inc, Grant DE-FG36-08G018149.

[2] J.E.Pacheco, T.Wolf, N.Muley Incorporating Supercritical Steam Turbines into Advanced Molten-Salt Power Tower Plants: Feasibility and Performance. Sandia report, SAND2013-1960, March 2013.

[3] Solar Energy Generating Systems (SEGS). http://www.energy.ca.gov/sitingcases/solar/

[4] California Solar Power Plants project status. http://www.energy.ca.gov

[5] Zarza E, Valenzuela L, Leon J, Weyers HD, Eickhoff M, Eck M, Hennecke K, The DISS Project: Direct Steam Generation in Parabolic Trough Systems. Operation and Maintenance Experience and Update on Project Status, J. of Solar Energy Engineering, 124 (2), 126-133, 2002

[6] Eck M., Zarza E., Eickhoff M., Rheinländer J., and Valenzuela L.Applied research concerning the direct steam generation in parabolic troughs, Solar Energy, 74 (4), 341-351, (2003)

[7] M.Seling, Novatec Solar GmbH. Two years experience in operating the largest commercial Fresnel CSP Plant. SolarPaces 2014, Beijing, China.

[8] A.Khenissi. Return of Experience on Transient Behaviour at the DSG Solar Thermal Power Plant in Kanchanaburi Thailand. SolarPaces 2014, Beijing, China.

[9] G.Morin, Novatec Solar GmbH. Molten Salt as Heat Transfer Fluid in a Linear Fresnel Collector Comercial Application Backed by Demonstration. SolarPaces 2014.

[10] A.Maccari, Archimede Solar Energy. Archimede Solar Energy Molten Salt Parabolic Trough Demo Plant: A Step Ahead Towards the New Frontiers of CSP. SolarPaces 2014.

[11] F. Matino, Archimede Solar Energy Molten Salt Receivers Operated on Parabolic Trough Demo Plant and in Laboratory Conditions. SolarPaces 2014. 
4. Plantas Termosolares con Colectores Lineales con DSG y sales fundidas como HTF acoplados a ciclos Supercriticos Rankine

[12] Technology Roadmap. High-Efficiency, Low-Emissions, Coal-Fired Power Generation. International Energy Agency, IEA. http://www.iea.org

[13] Alstom. (2014). Major milestone achieved in the development of advanced ultrasupercritical steam power plants. www.alstom.com

[14] Siemens. (2013). Siemens commissions record-high-effi-ciency 750MW steam power plant Lünen in Germany. www.siemens.com

[15] L.Coco-Enriquez, J.Muñoz-Anton, J.M.Martinez-Val. Supercritical Steam Power cycle for Line-Focusing Solar Power Plants. Journal of Polytechnic, 2015; 18 (4) : 219-225.

[16] B.Kelly. Advanced Thermal Storage for Central Receivers with Supercritical Coolants. Abengoa Solar Inc. DE-FG36-08G018149, June 2010.

[17] Electric Power Research Institute. G8 Cleaner Fossil Fuels Workshop.IEA Secretariat, Paris France, 17-18 January, 2008. Boiler material for USC pulverized coal (PC) Plants.

[18] J.A. Siefert, C.Libby, John J.Shingledecker. Concentrating solar power power cycle improvements through application of advanced materials. SolarPACES 2015. AIP Conference Proceedings 1734, 070030 (2016).

[19] C.Bachelier. SAM Linear Fresnel solar boiler model, SAM Webinar. Novatec Solar. NREL SAM Conference 2013.

[20] T.Hirsch, A.Khenissi. A systematic comparison on power block efficiencies for CSP plants with direct steam generation. Institute of Solar Research, German Aerospace Center (DLR), SolarPaces 2013.

[21] L.Coco-Enríquez, J.Muñoz-Antón, J.M. Martínez-Val. Innovations on direct steam generation in linear Fresnel collectors. SolarPaces 2013, Las Vegas, U.S.

[22] F.Burkholder and C.Kutscher Heat Loss Testing of Schott's 2008 PTR70 Parabolic Trough Receiver, report NREL/TP-550-45633, May 2009.

[23] M.Wagner. Modeling Parabolic Trough Systems. National Renewable Energy Laboratory (NREL). SAM Webinar, June 18, 2014. https://sam.nrel.gov

[24] HITEC $^{\circledR}$, Technical Bulletin, Coastal Chemical Co., L.L.C. http://www.coastalchem.com/

[25] T.Wang, D.Mantha, R.Reddy. Novel low melting point quaternary eutectic system for solar thermal energy storage. Applied Energy 102(Feb 2013): 1422-1429. 


\title{
Capítulo 5 \\ Plantas Termosolares con Colectores Lineales con Sales Fundidas como HTF y ciclos de potencia Brayton con $\mathrm{CO}_{2}$ supercrítico como fluido de trabajo
}

\begin{abstract}
Resumen
Continuando con la reciente tendencia [1-3] de sustituir el aceite térmico en los colectores lineales por sales fundidas, en el presente capítulo se propone un nuevo concepto de planta termosolar con colectores lineales con sales fundidas como HTF, y generación de potencia mediante ciclos $\mathrm{s}-\mathrm{CO}_{2}$ Brayton [4-5]. En este estudio se demuestra como esta solución tecnológica es un medio viable para incrementar la eficiencia neta de la planta termosolar, y permite una reducción asociada de los costes de inversión en el campo solar y en su área de apertura efectiva. Los ciclos de potencia Brayton con $\mathrm{CO}_{2}$ en estado supercrítico, gracias a la alta densidad del fluido de trabajo en comparación con el vapor de agua en los ciclos Rankine, reducen las dimensiones tanto de las turbinas como de los compresores y de la obra civil asociada.
\end{abstract}

En el presente trabajo se han analizado cuatro configuraciones de ciclos supercríticos [6]:

- ciclo simple con recuperación de calor o Simple with heat recuperation (SB), figura 5.4.

- ciclo con recompresión o with Recompression (RC), figura 5.5.

- ciclo con enfriamiento parcial y recompresión o Partial Cooling with Recompresion (PCRC), figura 5.6.

- ciclo con recompresión y enfriamiento intermedia en el compresor principal o Recompression with Main Compression Intercooling ( $\mathrm{RCMCl}$ ), figura 5.7.

En todas estas configuraciones se ha incluido una etapa intermedia de recalentamiento en las turbinas de generación de potencia, para incrementar la eficiencia energética del ciclo de potencia como había propuesto y analizado Dostal [7]. Los resultados de las simulaciones de las configuraciones propuestas en este capítulo, se han comparado con otra tecnología del estado del arte con DSG en los colectores solares y ciclo subcrítico Rankine.

De acuerdo al principio de Carnot, la temperatura máxima de operación del ciclo termodinámico tiene un impacto directo en la eficiencia neta del mismo, por tanto, como parámetro de operación principal se destaca la TIT, su valor ha sido fijado a un máximo de $550^{\circ} \mathrm{C}$. El principal motivo para limitar el valor del TIT es que los fabricantes de tubos absorbedores, Archimede Solar (HCEMS-11) [8] y SCHOTT [9], han desarrollado unos materiales especiales de recubrimiento de los tubos absorbedores, para minimizar las perdidas térmicas por radiación, y maximizar la radiación solar absorbida. La temperatura máxima de operación del material de recubrimiento de los tubos absorbedores es cercana a los $560^{\circ} \mathrm{C}$.

Sin embargo, se ha realizado un estudio aproximativo con una temperatura de entrada de turbina de $650^{\circ} \mathrm{C}$, extrapolando los valores de las pérdidas por radiación en los colectores solares, con las correlaciones definidas en la tabla 5.4 del presente capítulo. El principal 
5. Plantas Termosolares con Colectores Lineales con Sales Fundidas como HTF y ciclos de potencia Brayton con $\mathrm{CO}_{2}$ supercrítico como fluido de trabajo

inconveniente de este estudio es que a partir de ciertas temperaturas de operación las perdidas por radiación no son linealmente extrapolables, por eso se hace hincapié en que se debe considerar como un estudio estimativo. Sin embargo, las últimas tecnologías de tubos receptores en colectores lineales están desarrollando tecnologías para minimizar las perdidas térmicas por radiación para temperaturas superiores a $550^{\circ} \mathrm{C}$, como es el caso de la empresa comercial Norwich [10].

Respecto a la máxima presión de operación de los ciclos Brayton, se ha fijado su máximo valor a 250 bar: Dyreby [11] en su tesis ha demostrado que un incremento de presión por encima de estos valores no proporciona un incremento significativo en el rendimiento termodinámico del ciclo de potencia.

La eficiencia neta de las plantas termosolares propuestas en este capítulo asciende a $46.84 \%$ $\left(550^{\circ} \mathrm{C}\right.$ TIT) y $50.85 \%\left(650^{\circ} \mathrm{C}\right.$ TIT). En ambos casos, con una configuración de ciclo Brayton con recompresión y recalentamiento intermedio entre turbinas. El incremento de la eficiencia neta en las plantas termosolares reduce y optimiza la superficie efectiva de captación solar necesaria, y la correspondiente superficie del terreno para implantación de los colectores solares para una potencia generada fija de referencia.

Los intercambiadores de calor en los ciclos Brayton son otra pieza clave para conseguir una solución técnica económicamente competitiva con los ciclos Rankine. Las grandes necesidades de intercambio térmico entre los equipos que integran el ciclo Brayton, hacen necesarias grandes áreas de intercambio de calor que ocupen el menor volumen posible. Para ello se han desarrollado los intercambiadores de calor de circuito impreso o Printed Circuit Heat Exchangers (PCHE), de acero inoxidable austenítico AISI 347. La principal marca comercial que fabrica esta tipología de cambiadores es Heatric [12]. Los primeros diseños de los cambiadores $\mathrm{PCHE}$, estaban integrados por canales semicirculares o rectangulares, por los que fluyen los fluidos caloríficos con unas dimensiones del orden de $1 \mathrm{a} 2 \mathrm{~mm}$. Sin embargo, con el objetivo de minimizar las pérdidas de presión, se han definido otras geometrías de canales más complejas [13], como fin profiles, s-fins, etc.

En este capítulo se demuestra cómo estos intercambiadores son más apropiados cuando los dos fluidos son $\mathrm{CO}_{2}$, como es el caso de los recuperadores de baja presión Low Temperature Recuperator (LTR) y de alta presión High Temperature Recuperator (HTR). Como innovación se ha propuesto utilizar intercambiadores de tipo carcasa y tubos para diseño de los cambiadores de calor entre el campo solar principal o Primary Heat Exchanger (PHX), y para el campo solar de recalentamiento ReHeating Heat Exchanger (RHX) y el ciclo de potencia.

Otra pieza fundamental en la optimización de los ciclos Brayton es el diseño apropiado del sumidero final de calor o Ultimate Heat Sink (UHS). Uno de los estudios principales en este sentido ha sido realizado por Gavic [14]. El citado autor concluye que la solución óptima es la utilización de un sistema híbrido de enfriamiento integrado por un intercambiador PCHE con agua para refrigeración del $\mathrm{CO}_{2}$ y un enfriador con aire del agua de refrigeración o Air Cooling Heat Exchanger (ACHE) interconectados en un ciclo cerrado. Mediante este sistema se minimiza las dimensiones del $\mathrm{ACHE}$, y se evita un consumo de agua debido a la utilización 
5. Plantas Termosolares con Colectores Lineales con Sales Fundidas como HTF y ciclos de potencia Brayton con $\mathrm{CO}_{2}$ supercrítico como fluido de trabajo

convencional de torres de refrigeración. A lo largo de esta tesis se desarrollará la relación directa entre las dimensiones de los recuperadores LTR, HTR, y las dimensiones del ACHE, PHX, RHX, y sus costes asociados.

\subsection{Introducción}

El diseño de las plantas termosolares de nueva generación vendrá condicionado por el desarrollo de los fluidos supercríticos con una composición óptima para maximizar el rendimiento de los ciclos de potencia de Bratyon, y a su vez que reduzcan los costes necesarios por los requisitos de presión y corrosión del material de los equipos y componentes.

Tal y como se ha destacado a lo largo de este trabajo, la tendencia es ir alcanzando materiales y tecnologías compatibles con mayores valores de presión y temperatura, para maximizar el rendimiento termodinámico de los ciclos de potencia. Si en el capítulo anterior se trataba la integración de los ciclos de agua en estado supercrítico en las plantas termosolares con colectores lineales, este capítulo se centra en la integración de las plantas con colectores lineales y ciclos de potencia Brayton con fluido de trabajo $\mathrm{CO}_{2}$ en estado supercrítico. En subsiguientes capítulos se estudiarán otras posibles alternativas de fluidos de trabajo en los ciclos Brayton, como el Etano, $\mathrm{SF}_{6}$, Xenon, $\mathrm{CH}_{4}, \mathrm{~N}_{2}$, y el proceso de optimización de sus parámetros termodinámicos de operación.

El objetivo de los ciclos de potencia supercríticos es la reducción del volumen y dimensiones de las turbinas, reducir la obra civil de implantación del ciclo de potencia, y optimizar el coste de la electricidad producida en la planta termosolar. Los ciclos supercríticos Brayton además evitan trabajar con cambios de fases en el fluido de trabajo, lo cual conlleva a la modelización compleja de los dos estados de la materia y sus interfases a la hora de diseñar los equipos. Sin embargo, los ciclos supercríticos presentan también una desventaja competitiva con los ciclos subcríticos Rankine, en lo relativo a los requisitos de corrosión de los materiales.

Los ciclos supercríticos Brayton hasta ahora diseñados confirman la necesidad de aceros inoxidable austeníticos estabilizados con Niobio ( $\mathrm{Nb}$ ), Titanio (Ti), etc. para garantizar su resistencia a altas presiones y temperaturas. El material más utilizado es el acero inoxidable austenítico AISI 347. Por esta razón principal y debido al alto coste del Níquel, Niobio, Titanio y otros elementos aleantes en los aceros inoxidables austeníticos compatibles con el s- $\mathrm{CO}_{2}$, el desarrollo de ciclos de potencia Rankine con agua en estado supercrítico también jugaran un papel importante a medio y largo plazo, hasta que se desarrollen materiales alternativos de bajo coste e igual resistencia mecánica y química como cerámicos, etc.

Los ciclos s- $\mathrm{CO}_{2}$ Brayton fueron inicialmente diseñados en Estados Unidos por E.G. Feher, 1967. Angelino en 1967 también realizó un diseño detallado de los ciclos de s- $\mathrm{CO}_{2}$, enfocado en los ciclos con condensación. El renacimiento de la investigación con los ciclos s- $\mathrm{CO}_{2}$ fue en 1997, liderado por la Czech Technical University, República Checa. El ciclo con recompresión y recalentamiento presenta valores de eficiencia energética neta óptimos. En 2004 se publica la tesis doctoral referente en este tema, titulada: A supercritical Carbon Dioxide Cycle for Next Generation Nuclear Reactors, by Dostal et al [7]. Un análisis termodinámico y comparativo de los ciclos de $\mathrm{s}-\mathrm{CO}_{2}$ es realizado comparando la eficiencia de los ciclos: simple con recuperación, 
5. Plantas Termosolares con Colectores Lineales con Sales Fundidas como HTF y ciclos de potencia Brayton con $\mathrm{CO}_{2}$ supercrítico como fluido de trabajo

con pre-compresión, con recompresión, división de la etapa de expansión, ciclo con enfriamiento parcial.

La respuesta dinámica de los ciclos $\mathrm{s}-\mathrm{CO}_{2}$ Brayton con recompresión ante transitorios del sistema fue inicialmente estudiada en la tesis publicada en el MIT 2009 por Pavel Hejzlar, et al. Posteriormente, en el Laboratorio Central de Argonne se desarrolló un programa informático Plant Dynamic Code (PDC) [15]. La empresa Bechtel Marine Propulsion Corporation adaptó el programa informático TRACE para simulación de los transitorios en los ciclos s- $\mathrm{CO}_{2}$ [16], y validado en la instalación piloto de pruebas Integrated System Test (IST), con aceite caliente como fuente térmica a una temperatura de $282.8^{\circ} \mathrm{C}$. El primer bucle de experimentación para garantizar la viabilidad de la tecnología de generación de potencia con ciclos Brayton, fue construido en SNL [17], en base a la patente internacional no WO 2013/022664 A2.

Resumiendo: existen dos prometedoras tecnologías en vía de implantación industrial para mejorar la eficiencia de las centrales termosolares con colectores lineales: las sales fundidas como fluidos caloportadores en los colectores lineales DMS, y los ciclos s- $\mathrm{CO}_{2}$ de Bratyon. Para aumentar las sinergias entre ambas tecnologías se han integrado en una misma planta termosolar y se ha conseguido mejoras del rendimiento y potencia generada neta.

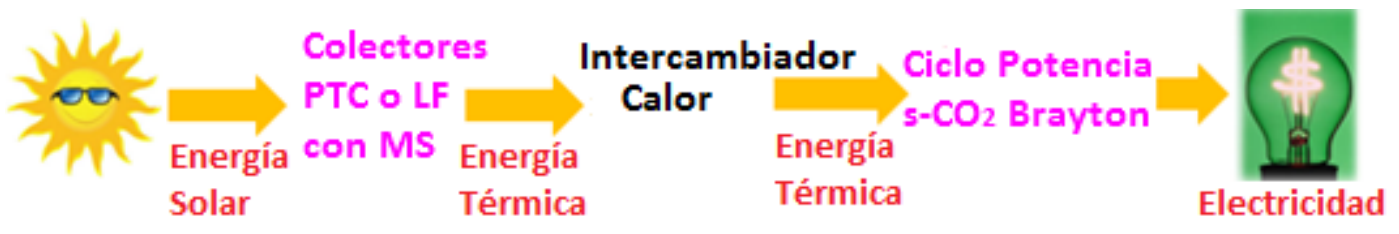

\section{Sales Fundidas + Ciclo de Potencia s- $\mathrm{CO}_{2}$ Brayton}

Figura 5.1. Adaptación de los ciclos supercriticos $\mathrm{s}-\mathrm{CO}_{2}$ Brayton a las plantas termosolares con colectores lineales.

En este capítulo se continúa con el estudio de la implantación de las sales fundidas como HTF en los colectores lineales como alternativa a los aceites térmicos y a la DSG. Como se ha explicado, las principales ventajas de la DSG son: bajo impacto medioambiental, temperaturas de operación mayores a $400^{\circ} \mathrm{C}$ (compatibles con los aceites sintéticos), sin necesidad de intercambiadores de calor intermedios entre el campo solar y el ciclo de potencia, permite obtener presiones elevadas a entrada de turbina, baja corrosión de las tuberías del campo solar y de los tubos absorbedores, permite utilización de materiales poco aleados de bajo coste, etc. Como principal competidor a la DSG, se pueden citar las sales fundidas. Las principales ventajas de las sales fundidas son: la simplicidad en el sistema de control ante transitorios de la radiación solar, el HTF siempre presenta una fase líquida evitando su evaporación, se reduce por tanto el número de equipos y su coste asociado. La presión de operación de las sales fundidas ( 15 bar) es menor que la del vapor (70-150 bar) y la del aceite térmico (20 bar), por tanto las tensiones en las tuberías son menores, no existe degradación alguna de las sales a altas temperaturas.

Como principales desventajas de las sales fundidas, ya mencionadas en el capítulo anterior, están la necesidad de materiales aleados para soportar la corrosión de las sales y el consumo 
5. Plantas Termosolares con Colectores Lineales con Sales Fundidas como HTF y ciclos de potencia Brayton con $\mathrm{CO}_{2}$ supercrítico como fluido de trabajo

eléctrico de las resistencias eléctricas, tecnología heat-tracing, para evitar su solidificación. Las sales fundidas como HTF, es una tecnología muy investigada en otras ramas de la ciencia como en la generación nuclear [18-20], siendo la principal tecnología para transferir calor a altas temperaturas.

En la figura 5.2 se representa la disposición de equipos en las plantas tradicionales con aceite térmico como HTF. La madurez de las tecnologías de los equipos que las componen, y su amplia experiencia operativa en numerosas plantas en el mundo, junto con un gran número de proveedores, se constituyen como las principales ventajas competitivas en relación a las alternativas estudiadas en el presente trabajo. A medio plazo, esta tipología de plantas termosolares continuarán proporcionando una fuente de energía renovable fiable y a su vez con costes inversión muy competitivos [21].

\section{OIL}

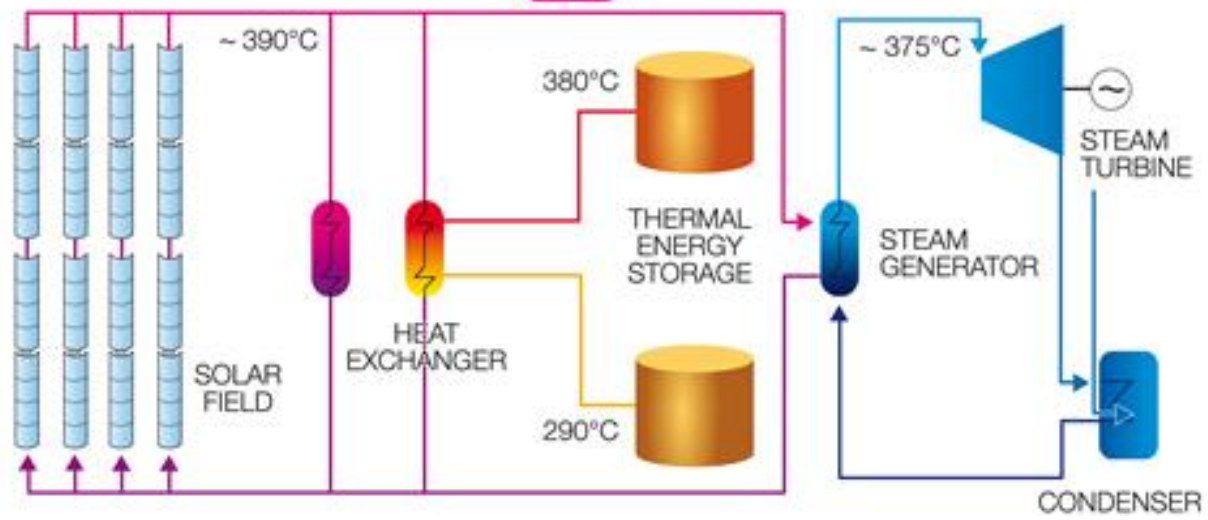

Figura 5.2. Planta Termosolar con colectores lineales y aceite como HTF [22].

Como se ha mencionado, las primeras plantas piloto están siendo implantadas para demostrar la viabilidad de utilización de las sales fundidas como HTF [1-3] (figura 5.3). Su configuración permite eliminar el intercambiador intermedio entre el campo solar y el ciclo de potencia Rankine, garantizando una alta TIT $550^{\circ} \mathrm{C}$ y su integración directa con el sistema de almacenamiento térmico.

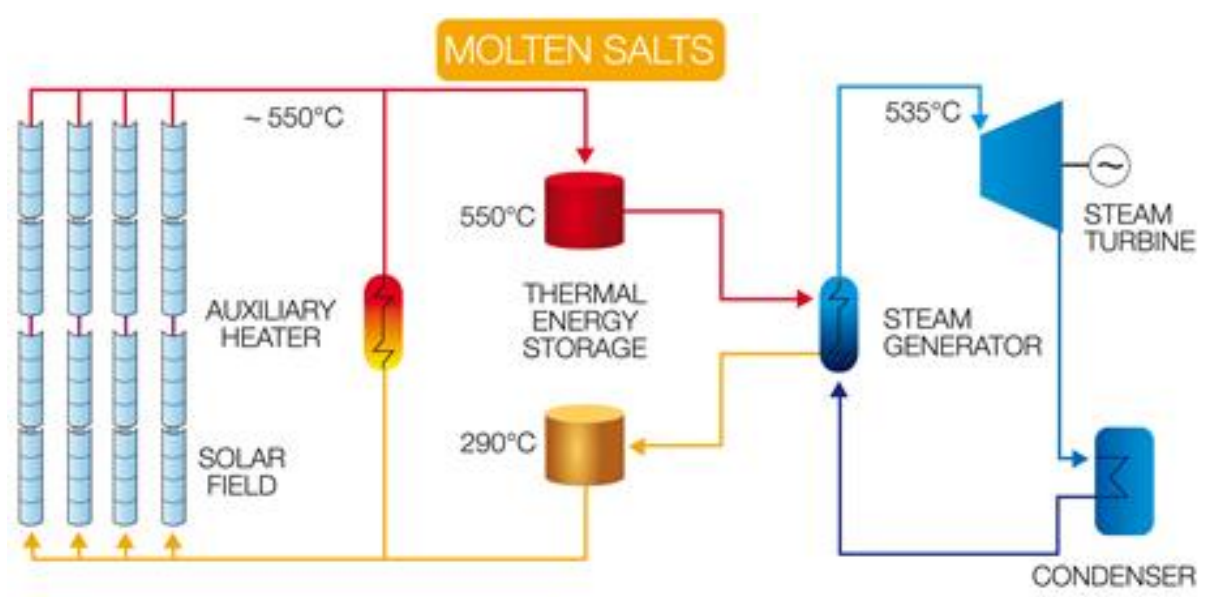

Figura 5.3. Planta Termosolar con colectores lineales (PTC o LF) y sales fundidas como HTF [22] 
5. Plantas Termosolares con Colectores Lineales con Sales Fundidas como HTF y ciclos de potencia Brayton con $\mathrm{CO}_{2}$ supercrítico como fluido de trabajo

En la figura 5.4 se ha ilustrado una primera representación gráfica de la disposición en planta de los equipos en las plantas termosolares de nueva generación con colectores lineales y ciclos Brayton. Como se ha explicado, los intercambiadores PHX y RHX permiten la transferencia del calor desde el campo solar para calentamiento del fluido de trabajo en el BOP.

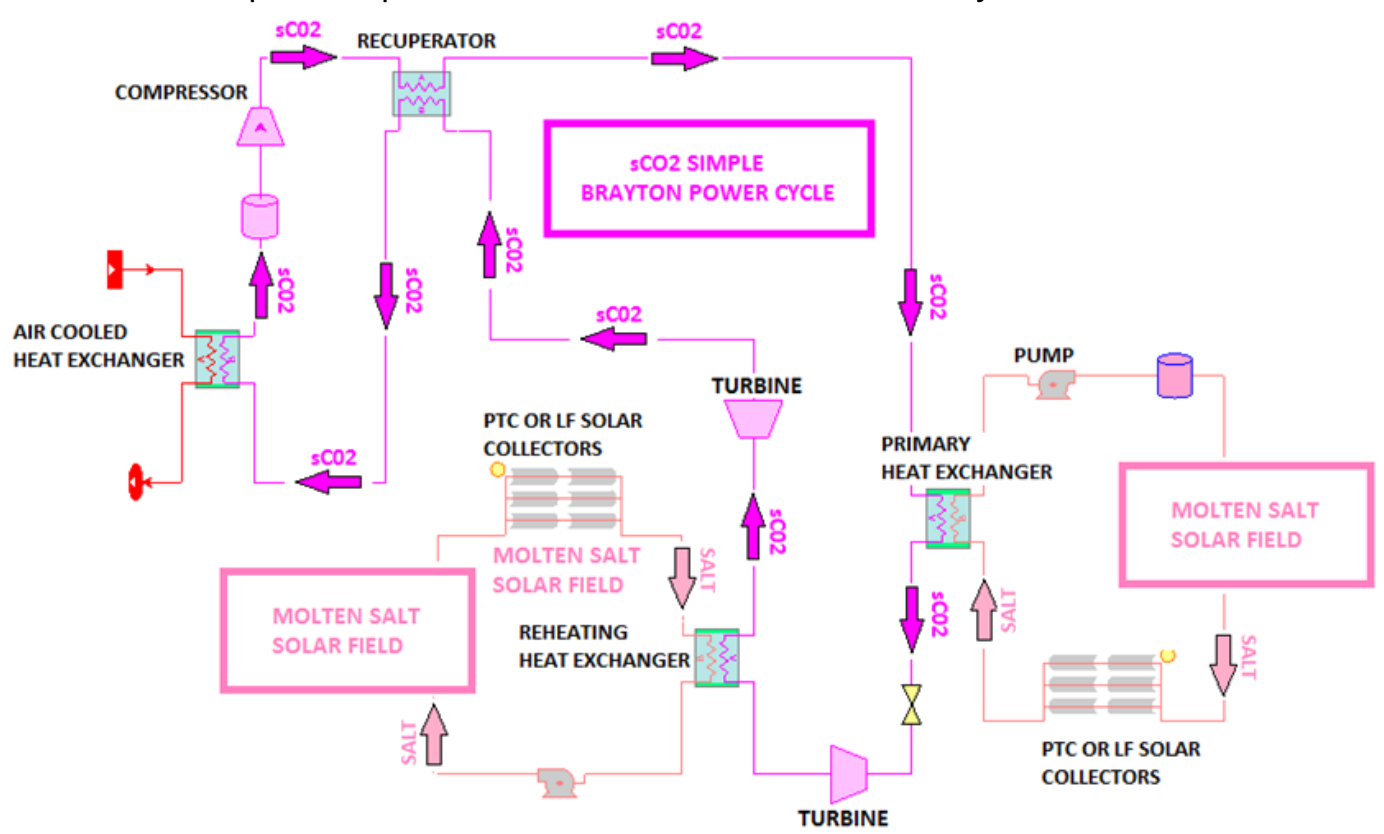

Figura 5.4. Planta termosolar con colectores lineales y ciclo de potencia Brayton con s- $\mathrm{CO}_{2}$ y configuración básica con recuperación SB.

La adaptación de los ciclos supercríticos Brayton a los parámetros termodinámicos de las plantas termosolares ha sido motivo de recientes investigaciones. En el presente estudio se ha tomado como referencia las configuraciones de ciclos Brayton desarrolladas por el NREL [6] y ya mencionados en el resumen de este capítulo: el ciclo simple SB (figura 5.4.), el ciclo con recompresión RC (figura 5.5.), el ciclo con enfriamiento parcial y recompressión PCRC (figura 5.6.), y el ciclo con recompresión y enfriamiento intermedia en el compresor principal RCMCI (figura 5.7).

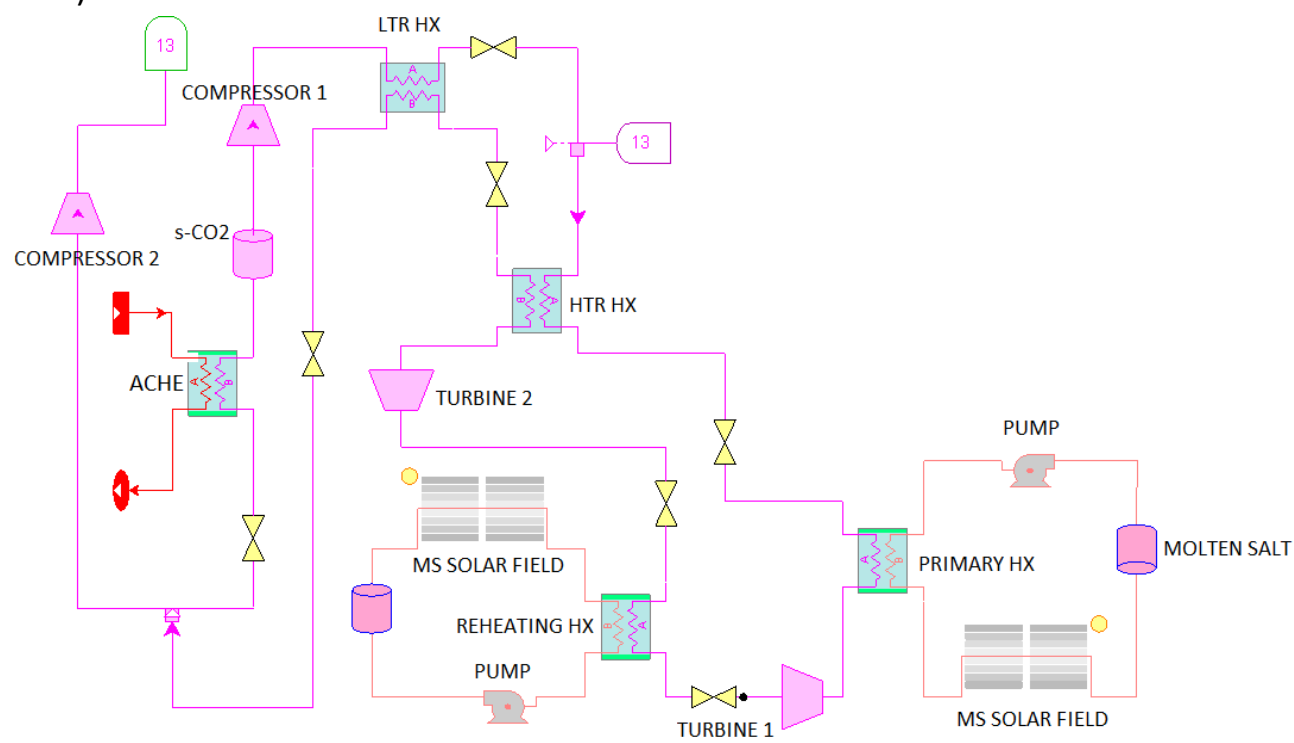

Figura 5.5. Planta termosolar con colectores lineales y ciclo de potencia Brayton con s- $\mathrm{CO}_{2}$ y configuración con recompresión RC. 
5. Plantas Termosolares con Colectores Lineales con Sales Fundidas como HTF y ciclos de potencia Brayton con $\mathrm{CO}_{2}$ supercrítico como fluido de trabajo

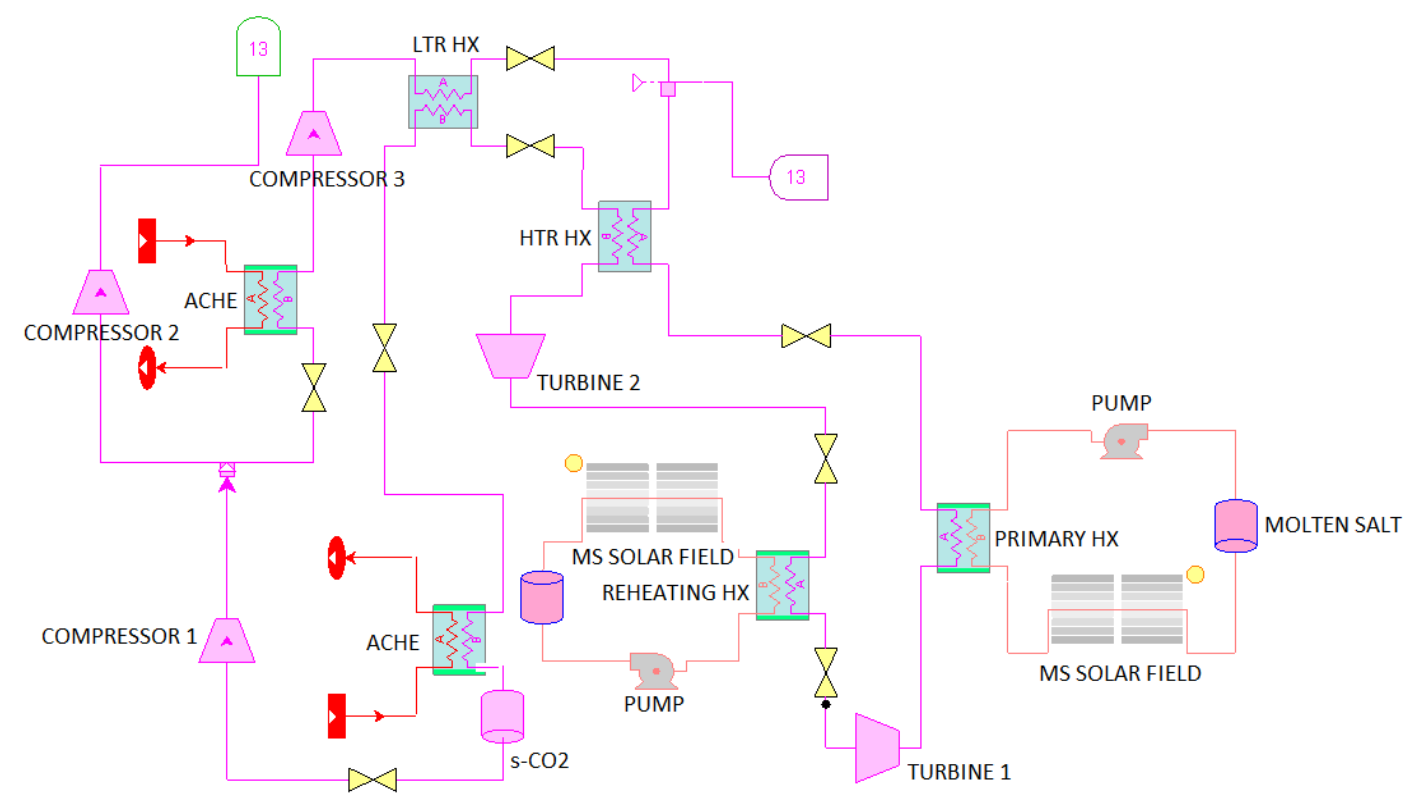

Figura 5.6. Planta termosolar con colectores lineales y ciclo de potencia Brayton con s- $\mathrm{CO}_{2}$ y configuración con enfriamiento parcial y recompresión PCRC.

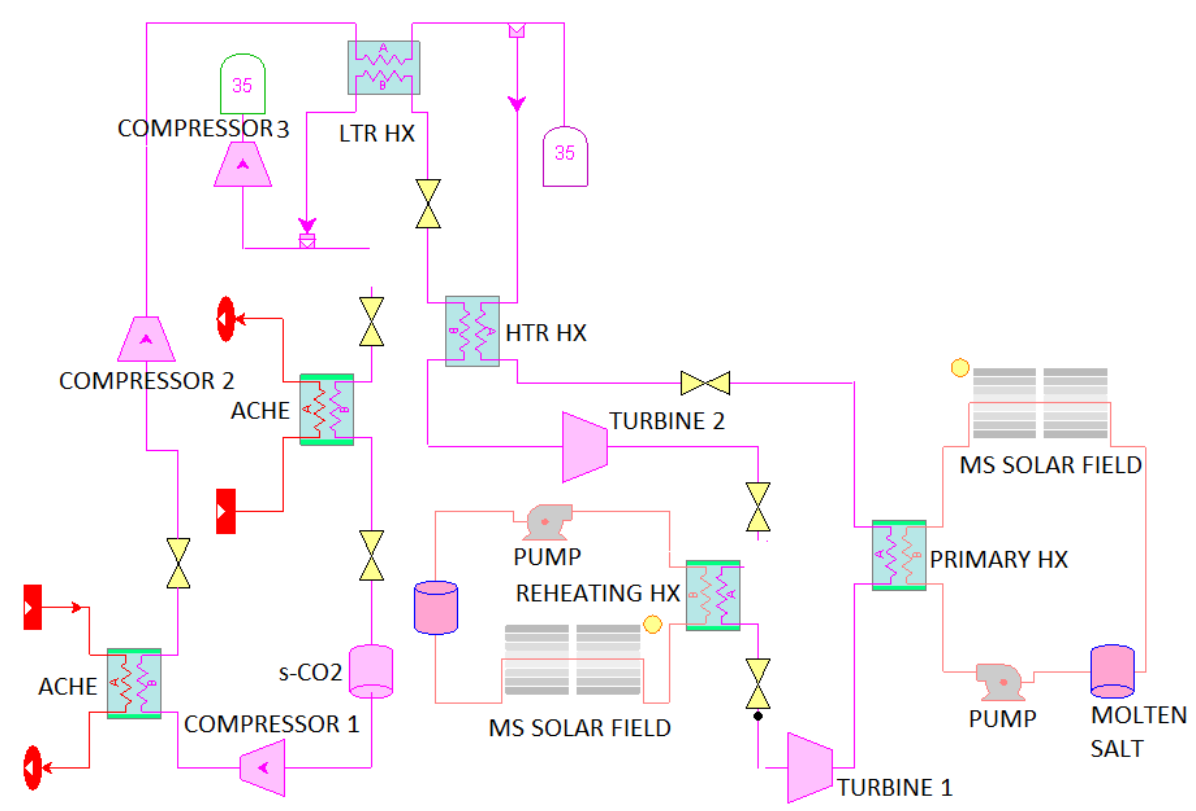

Figura 5.7. Planta termosolar con colectores lineales y ciclo de potencia Brayton con s- $\mathrm{CO}_{2}$ y configuración con recompresión y enfriamiento intermedia en el compresor principal RCMCI.

La configuración Simple con Recuperación de calor (SB) es la más compacta (figura 5.4), con el menor número de equipos, pero su eficiencia es menor que el resto de configuraciones. La viabilidad de inversión en este tipo de configuración está muy influenciada por la simplicidad de su sistema de control frente a condiciones de operación transitorias. En las instalaciones solares se constituye como una posible alternativa debido a la variación intrínseca de la radiación solar a lo largo del día y sujeta al impacto de fenómenos meteorológicos cambiantes como la nubosidad. 
5. Plantas Termosolares con Colectores Lineales con Sales Fundidas como HTF y ciclos de potencia Brayton con $\mathrm{CO}_{2}$ supercrítico como fluido de trabajo

La configuración de ciclo de potencia con Recompresión (RC), figura 5.5, es la alternativa más difundidas y estudiada ya que presenta un balance óptimo entre el número de equipos y su eficiencia energética. En esta configuración una parte del caudal de $\mathrm{CO}_{2}$ es enfriada antes de su entrada en el compresor, el resto del caudal será comprimido a la temperatura proveniente de la salida de turbina de recalentamiento sin enfriamiento previo alguno. En la presente tesis, en capítulos subsiguientes, se ha definido con el algoritmo de cálculo SUBPLEX los valores de los parámetros óptimos de operación de esta configuración, eligiendo la fracción óptima de caudal que va a ser enfriado antes de su compresión.

Respecto a la solución RCMCl (figura 5.7), su principal ventaja es la reducción del tamaño térmico (UA) en relación la configuración RC para valores similares de eficiencia, los resultados detallados están incluidos en las figuras 5.8 a 5.11. La configuración $\mathrm{RCMCl}$ estudiada en este trabajo incluye dos etapas de enfriamiento, sin embargo, el número óptimo de etapas de enfriamiento debería ser evaluado cuantificando si son rentables las pérdidas de presión y costes en los enfriadores suplementarios, en relación al ahorro en coste que supone una reducción del área de apertura efectiva en el campo solar.

Finalmente la configuración PCRC proporciona rendimiento energéticos intermedios entre los proporcionados por las configuraciones SB y RC, pero se consigue una optimización del tamaño térmico UA en los recuperadores.

\subsection{Hipótesis de cálculo}

Todas las simulaciones realizadas fueron calculadas en su punto de diseño, tomando como hipótesis los siguientes valores de temperatura a entrada en la turbina TIT: $400^{\circ} \mathrm{C}, 450^{\circ} \mathrm{C}$, $500^{\circ} \mathrm{C}$ y $550^{\circ} \mathrm{C}$. El resto de hipótesis consideradas en los cálculos se resumen en las tablas $5.1 \mathrm{a}$ 5.6 .

Tabla 5.1. Localización y condiciones ambientales.

\begin{tabular}{lc}
\hline Localización: & Dagget,CA, USA. \\
Latitud: & $34.86^{\circ}$ \\
Longitud: & $-116.8^{\circ}$ \\
Zona horaria: & -8 \\
Hora: & $11: 30 \mathrm{hr}$ \\
DNI: & $986 \mathrm{~W} / \mathrm{m}^{2}$ \\
Temperatura: & $25^{\circ} \mathrm{C}$ \\
Altitud: & $588 \mathrm{~m}$ \\
\hline
\end{tabular}

Tabla 5.2. Parámetros tubo absorbedor.

\begin{tabular}{ll}
\hline Diámetro exterior: & $70 \mathrm{~mm}$ \\
Espesor de pared: & $4.191 \mathrm{~mm}$ \\
Material: & Acero Inoxidable \\
Rugosidad: & $0.0457 \mathrm{~mm}$ \\
\hline
\end{tabular}


5. Plantas Termosolares con Colectores Lineales con Sales Fundidas como HTF y ciclos de potencia Brayton con $\mathrm{CO}_{2}$ supercrítico como fluido de trabajo

Tabla 5.3 Parámetros colectores PTC.

\begin{tabular}{ll}
\hline Tipo de colector: & EuroTrough II \\
Anchura apertura: & $5.77 \mathrm{~m}$ \\
Longitud focal: & $1.71 \mathrm{~m}$ \\
Separación entre filas/anchura colector: & 2.5 \\
Factor Limpieza: & 0.96 \\
Eficiencia óptica: & 0.75 \\
Pérdidas térmicas: & $0.141 \Delta \mathrm{T}+6.48 \cdot 10^{-9} \Delta \mathrm{T}^{4}$ Schott PTR70 NREL Ref.[23] \\
\hline
\end{tabular}

Tabla 5.4. Parámetros colectores LF.

\begin{tabular}{ll}
\hline Tipo colector: & SuperNova 1 (Novatec) \\
Dimensiones: & $16.56 \mathrm{~m} \times 44.8 \mathrm{~m}$ \\
Área apertura: & $513.6 \mathrm{~m}^{2} /$ per Módulo \\
Eficiencia óptica: & $0.67 ; 0.647$ \\
Pérdidas térmicas: & $1.06 \Delta \mathrm{T}+1.2 \cdot 10^{-8} \Delta \mathrm{T}^{4}[24]$ \\
Pérdidas térmicas: & $0.15 \Delta \mathrm{T}+7.1510^{-9} \Delta \mathrm{T}^{4}[24]$ \\
\hline
\end{tabular}

Tabla 5.5. Ciclo de potencia Rankine.

\begin{tabular}{ll}
\hline Turbina HP: & 2 etapas (87.7 bar; 36 bar) \\
Turbina IP: & $\begin{array}{l}3 \text { etapas (16.5 bar; } 10.34 \\
\text { bar; } 6.18 \text { bar) }\end{array}$ \\
& 4 etapas (5.17 bar; 3.04 bar; \\
Turbina LP: & 1.17 bar; 0.37 bar) \\
& $85 \%$ \\
Eficiencia Turbina: & 0.08 bar \\
Presión condensador: & $98.23 \%$ (en Punto-Diseño) \\
Eficiencia Generador: & $0.01 \%$ (Potencia Bruta) \\
Pérdidas auxiliares BOP: & $5^{\circ} \mathrm{C}$ \\
TTD calentadores agua: & $5^{\circ} \mathrm{C}$ \\
DCA calentadores agua: & 6.17 bar \\
Presión desaireador: & \\
\hline
\end{tabular}

Tabla 5.6. Ciclo de potencia s-CO2 Brayton.

\begin{tabular}{ll}
\hline Eficiencia Turbina: & $93 \%$ \\
Eficiencia Compresor: & $89 \%$ \\
Eficiencia recuperadores: & $95 \%$ \\
No pérdidas de presión en intercambiadores & \\
Temperatura entrada Turbina: & $550^{\circ} \mathrm{C}$ \\
Presión entrada Turbina: & $250 \mathrm{bar}$ \\
Presión recalentamiento: & $173 \mathrm{bar}$ \\
\hline
\end{tabular}


5. Plantas Termosolares con Colectores Lineales con Sales Fundidas como HTF y ciclos de potencia Brayton con $\mathrm{CO}_{2}$ supercrítico como fluido de trabajo

\begin{tabular}{ll}
\hline Temperatura entrada compresor: & $32^{\circ} \mathrm{C}$ \\
Presión entrada compresor: & $74 \mathrm{bar}$ \\
Fracción flujo: & $71 \% ; 29 \%$ \\
Pérdidas auxiliares BOP: & $0.01 \%$ \\
Eficiencia Generador: & 98.23 \\
\hline
\end{tabular}

\subsection{Resultados}

En el presente capítulo de la Tesis se ha desmostrado que las plantas termosolares con colectores lineales, sales fundidas como HTF y ciclo supercrítico Brayton tienen rendimientos mayores que los diseños tradicionales integrando ciclos subcríticos Rankine. La principal razón es la mayor densidad de $\mathrm{s}-\mathrm{CO}_{2}$, mayores eficiencias en las turbinas, y menores trabajos de compresión del fluido de trabajo del ciclo BOP.

Los resultados detallados se han resumido en las figuras 5.8 a 5.11. En este capítulo se ha propuesto un primer diseño con las hipótesis de cálculo detalladas en el apartado 5.2, para la integración de ambas tecnologías pioneras, Direct Molten Salt in line-focusing solar collectors y supercritical Brayton power cycles. En subsiguientes capítulos se utilizarán algoritmos de cálculo SUBPLEX para obtener los parámetros termodinámicos óptimos de operación en el punto de diseño de los ciclos Brayton, y conseguir valores superiores de eficiencia energética.

La configuración de ciclo Brayton óptima, por encima de $\mathrm{TIT}=500^{\circ} \mathrm{C}$, es la solución con recompresión RC. Proporcionando mayores eficiencias la configuración $\mathrm{RCMCl}$ para valores inferiores de TIT. Ambas configuraciones en su punto de diseño $550^{\circ} \mathrm{C}$, proporcionan una eficiencia superior al 45\%, sin embargo, las configuraciones de planta termosolar con DSG y ciclo subcrítico Rankine con recalentamiento, proporcionaban eficiencias del orden del $40 \%$.

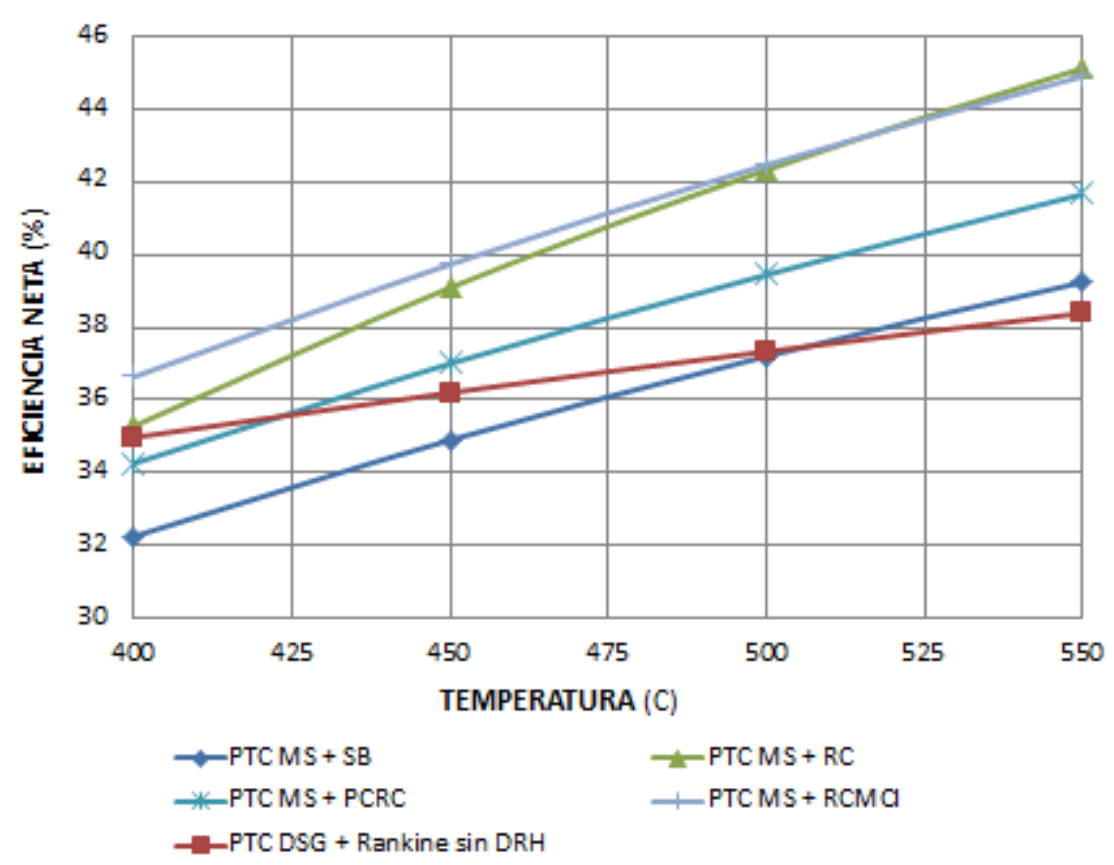

Figura 5.8. Eficiencia Neta frente a TIT. Ciclos Brayton sin Recalentamiento. 
5. Plantas Termosolares con Colectores Lineales con Sales Fundidas como HTF y ciclos de potencia Brayton con $\mathrm{CO}_{2}$ supercrítico como fluido de trabajo

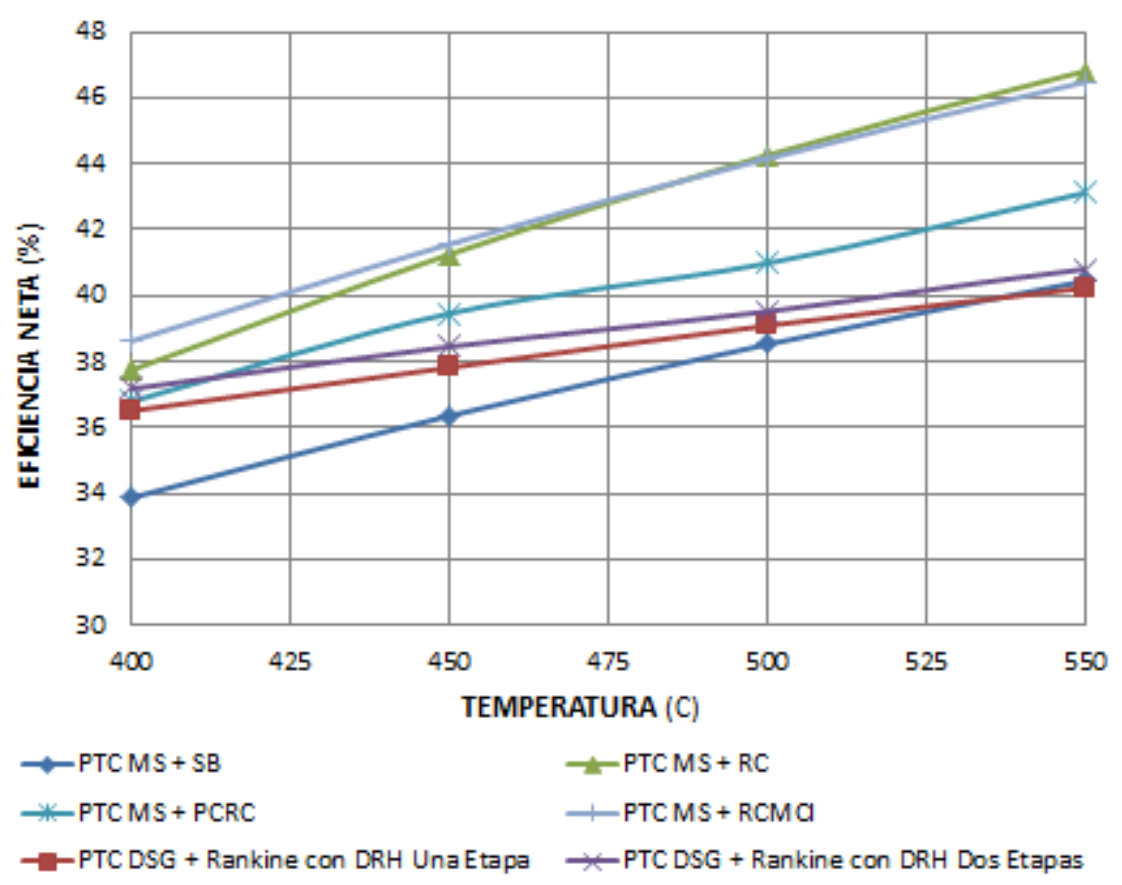

Figura 5.9. Eficiencia Neta frente a TIT. Ciclos Brayton con Recalentamiento.

En las plantas termosolares la mayor eficiencia neta del ciclo termodinámico se traduce directamente en un ahorro de la superficie de apertura efectiva en los colectores solares, lo cual supone un ahorro sustancial en los costes de inversión en la instalación (figuras $5.10 \mathrm{y}$ 5.11). Para cuantificar este ahorro se define el parámetro "potencia unitaria", que es la relación entre la potencia neta de la planta termosolar y el área de apertura efectiva de los colectores solares que la integran. Los resultados de potencia unitaria obtenidos para las diferentes configuraciones se detallan en las figuras 5.10 y 5.11. La configuración PTC MS+RC s$\mathrm{CO}_{2}$, permite un ahorro del $6 \%$ en área de apertura en relación a la configuración de referencia PTC DSG+Rankine, y un ahorro de la superficie del solar para instalar los colectores del $13.5 \%$. Con colectores LF, el ahorro de área de apertura efectiva es alrededor del $10 \%$ con la configuración LF MS+RC s- $\mathrm{CO}_{2}$, en comparación con la configuración de referencia LF DSG+Rankine, y la reducción del solar es del 23.76\%.

Así mismo, el incremento de las temperaturas de entrada en la turbina principal TIT, desde $400^{\circ} \mathrm{C}$ hasta $550^{\circ} \mathrm{C}$, permite un incremento de las potencias unitarias, desde $0.249 \mathrm{~kW} / \mathrm{m}^{2}$ hasta $0.282 \mathrm{~kW} / \mathrm{m}^{2}$ para la configuración PTC MS+RC s- $\mathrm{CO}_{2}$, y para la configuración con colectores Fresnel LF MS+RC s- $\mathrm{CO}_{2}$ desde $0.209 \mathrm{~kW} / \mathrm{m}^{2}$ hasta $0.249 \mathrm{~W} / \mathrm{m}^{2}$, figuras 5.10 y 5.11 . 
5. Plantas Termosolares con Colectores Lineales con Sales Fundidas como HTF y ciclos de potencia Brayton con $\mathrm{CO}_{2}$ supercrítico como fluido de trabajo

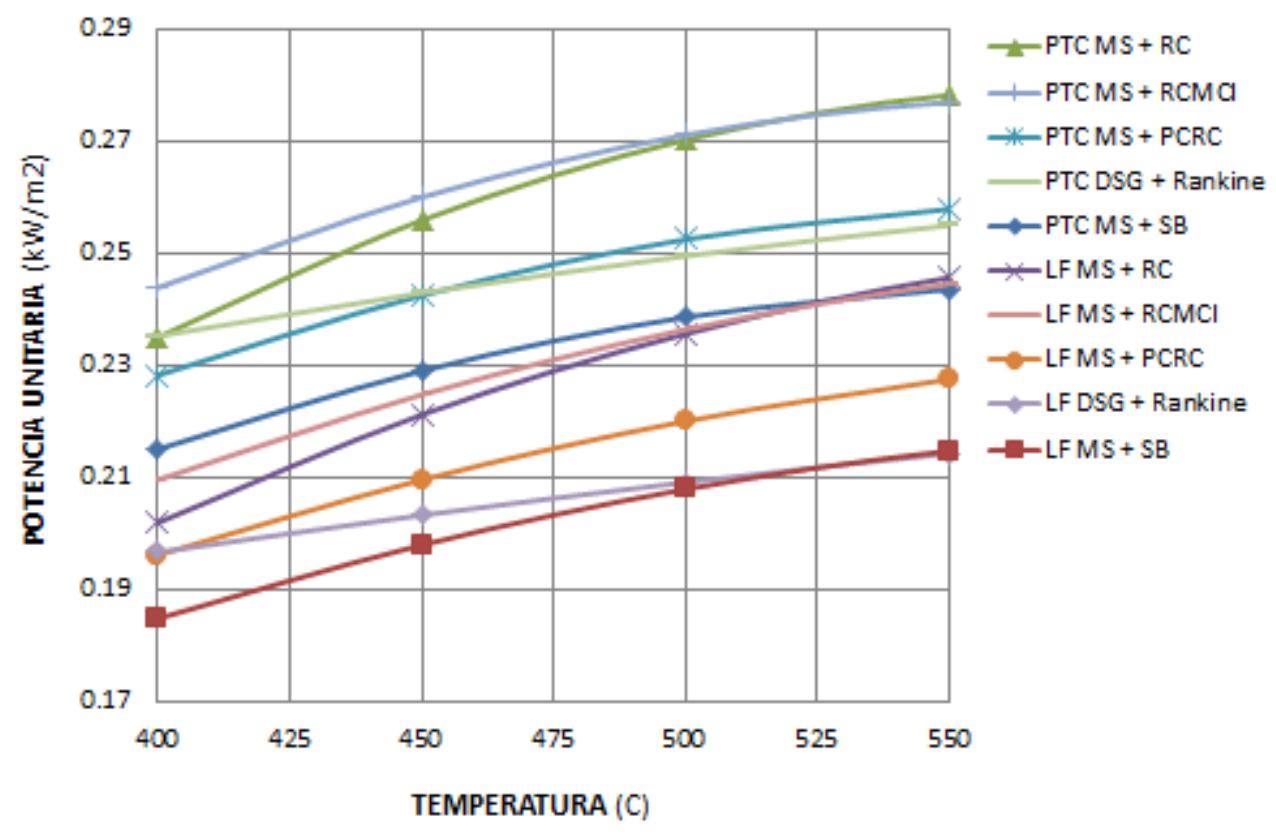

Figura 5.10. Potencia Unitaria (Potencia Neta / Área Apertura Efectiva SF) frente a TIT, Sin Recalentamiento.

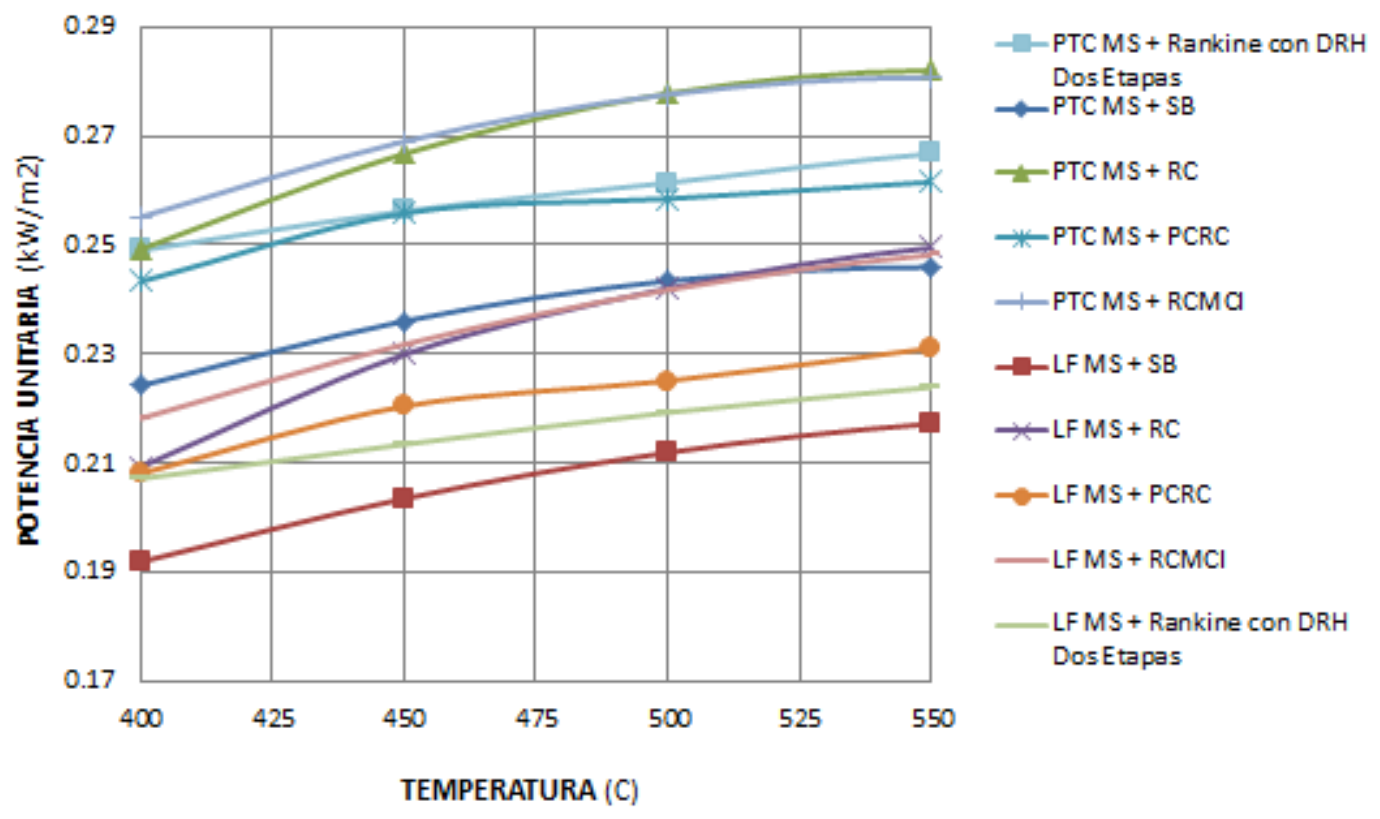

Figura 5.11. Potencia Unitaria (Potencia Neta / Área Apertura Efectiva SF) frente a TIT, Con Recalentamiento.

\subsection{Diseño de detalle de los Intercambiadores de Calor Primario y de Recalentamiento}

Otra característica destacable de los ciclos de potencia Brayton son las grandes tasas de intercambio de energía entre los equipos que integran el ciclo de potencia. Esto hace necesaria la elección de intercambiadores de calor de última tecnología PCHE, para maximizar la superficie de intercambio de calor y reducir al máximo el volumen de estos equipos. Sin embargo, debido a las características de transferencia de calor del $\mathrm{CO}_{2}$, estos intercambiadores son aconsejables para los recuperadores de baja y alta presión. 
5. Plantas Termosolares con Colectores Lineales con Sales Fundidas como HTF y ciclos de potencia Brayton con $\mathrm{CO}_{2}$ supercrítico como fluido de trabajo

Tabla 5.7. Planta Termosolar con colectores lineales con sales fundidas como fluido caloportador y ciclos de potencia Brayton. Principales parámetros del diseño básico de los intercambiadores de calor ( $\mathrm{PHX}$ y $\mathrm{RHX}$ ) entre los campos solars y el ciclo de potencia s- $\mathrm{CO}_{2}$ Brayton. Para la comparativa de resultados se ha fijado la potencia neta generada $55 \mathrm{MWe}$ y la $\mathrm{TIT}=550^{\circ} \mathrm{C}$.

\begin{tabular}{lccccccccc}
\hline & & NTU & $\begin{array}{c}\text { Eficiencia } \\
\text { Recuperadores }\end{array}$ & Uds & $\begin{array}{c}\text { UA } \\
(\mathrm{kW} / \mathrm{K})\end{array}$ & $\begin{array}{c}\mathbf{Q} \\
(\mathrm{kW})\end{array}$ & $\begin{array}{c}\text { UA } \\
\text { Total } \\
(\mathrm{kW} / \mathrm{K})\end{array}$ & $\begin{array}{c}\text { Q Total } \\
(\mathrm{kW})\end{array}$ & $\begin{array}{c}\text { Pinch- } \\
\text { Point } \\
\left({ }^{\circ} \mathrm{C}\right)\end{array}$ \\
\hline RC & PHX & 5.4 & 0.93 & 6 & 588.7 & 14529 & 3532.2 & 87174 & 10.1 \\
RC & RHX & 2.7 & 0.81 & 6 & 303.1 & 5070 & 1818.6 & 30420 & 10.8 \\
PCRC & PHX & 5.2 & 0.95 & 6 & 452.7 & 17203 & 2716.4 & 103218 & 10.1 \\
PCRC & RHX & 2.4 & 0.81 & 6 & 212.2 & 4069 & 1273.2 & 24414 & 10.8 \\
RCMCl & PHX & 5.1 & 0.94 & 6 & 478.1 & 15374 & 2868.4 & 92244 & 9.9 \\
RCMCl & RXH & 2.5 & 0.81 & 6 & 235.5 & 4355 & 1413 & 26130 & 10.8 \\
\hline
\end{tabular}

Tabla 5.8. Planta Termosolar con colectores lineales con sales fundidas como fluido caloportador y ciclos de potencia Brayton. Diseño de detalle de los intercambiadores de calor ( $\mathrm{PHX}$ y $\mathrm{RHX}$ ) entre los campos solares y el ciclo de potencia s- $\mathrm{CO}_{2}$ Brayton. Para la comparativa de resultados se ha fijado la potencia neta generada $55 \mathrm{MWe}$ y la $\mathrm{TIT}=550^{\circ} \mathrm{C}$.

\begin{tabular}{|c|c|c|c|c|c|c|c|c|c|c|}
\hline & & Longitud & Diámetro & & & Coste & & Coste & AP & AP \\
\hline & & $\begin{array}{l}\text { carcasa } \\
(\mathrm{m})\end{array}$ & $\begin{array}{l}\text { carcasa } \\
(\mathrm{m})\end{array}$ & (\$) & Uds & $\begin{array}{l}\text { Total } \\
\text { (M\$) }\end{array}$ & $(\mathrm{MW})$ & $\begin{array}{l}\text { Unitario } \\
(\$ / k W)\end{array}$ & $\begin{array}{l}\text { Tubos } \\
\text { (bar) }\end{array}$ & $\begin{array}{l}\text { Carcasa } \\
\text { (bar) }\end{array}$ \\
\hline $\mathbf{R C}$ & PHX & 12.86 & 1.41 & 1.37 & 6 & 8.2 & 87.2 & 94.2 & 0.1 & 0.5 \\
\hline RC & $\mathrm{RHX}$ & 8.76 & 1.35 & 1.15 & 6 & 6.9 & 30.4 & 226.6 & 0.1 & 0.1 \\
\hline RC & & & & 2.50 & & 15.1 & 117.6 & 128.5 & & \\
\hline PCRC & PHX & 12.17 & 1.05 & 1.05 & 6 & 6.3 & 103.2 & 61.3 & 0.1 & 0.7 \\
\hline PCRC & $\mathrm{RHX}$ & 7.68 & 1.22 & 0.87 & 6 & 5.3 & 24.4 & 215.3 & 0.1 & 0.2 \\
\hline PCRC & & & & 1.92 & & 11.6 & 127.6 & 90.8 & & \\
\hline $\mathrm{RCMCl}$ & PHX & 12.04 & 1.06 & 1.13 & 6 & 6.7 & 92.2 & 73.3 & 0.1 & 0.6 \\
\hline $\mathrm{RCMCl}$ & $\mathrm{RHX}$ & 7.98 & 1.25 & 0.95 & 6 & 5.7 & 26.1 & 217.3 & 0.1 & 0.1 \\
\hline $\mathrm{RCMCl}$ & & & & 2.08 & & 12.4 & 118.4 & 105.1 & & \\
\hline
\end{tabular}

En este trabajo se ha realizado el diseño de detalle de los intercambiadores, tipo carcasatubos, con acero inoxidable austenítico AISI 347, para transferencia de calor entre los campos solares y el ciclo de potencia. En las tablas 5.7 y 5.8 se detallan los resultados obtenidos del dimensionamiento de estos intercambiadores tipología carcasa-tubos. El coste unitario calculado $\left(90-130 \$ / \mathrm{kW}_{\text {th }}\right)$ es asimilable a los valores indicados en la referencia [25] para los 
5. Plantas Termosolares con Colectores Lineales con Sales Fundidas como HTF y ciclos de potencia Brayton con $\mathrm{CO}_{2}$ supercrítico como fluido de trabajo

intercambiadores de tipología PCHE. La principal ventaja de este diseño de intercambiador de calor es que su tecnología de fabricación está ampliamente difundida y que los canales por los que fluyen las sales no sufren de posible solidificación por bajas temperaturas.

Se puede decir, por tanto, que para la difusión e implantación de los ciclos de potencia a escala industrial, sería necesaria una reducción del precio de los cambiadores PCHE y una difusión de su tecnología de fabricación.

\subsection{Conclusiones}

Como configuración de referencia se ha definido una planta termosolar con colectores lineales con DSG y un ciclo subcrítico de Rankine con dos etapas DRH, figura 4.2 en el capítulo 4. Esta tipología de planta termosolar tiene una eficiencia energética de $40.4 \%$ en su punto de diseño, y con condiciones de vapor vivo a la entrada de turbina de $550^{\circ} \mathrm{C}$ y 87.7 bar. Los diseños de las plantas SEGS, con colectores PTC y aceite como HTF, tienen una eficiencia neta de $35 \%$ sin recalentamiento en el ciclo Rankine, y del $37 \%$ con recalentamiento en el ciclo Rankine, para unas condiciones de vapor vivo de $385^{\circ} \mathrm{C}$ y 100 bar a la entrada de la turbina principal. En este capítulo se ha definido una primera solución de diseño para mejorar la eficiencia de las plantas termosolares, mediante la integración de un ciclo de potencia Brayton en lugar de un ciclo de potencia Rankine.

Por tanto, se considera que se ha puesto la "primera piedra" para la definición de la plantas termosolares de "Nueva Generación" incluyendo los ciclos de potencia supercríticos del estado del arte en la generación de potencia. Las configuraciones de plantas termosolares con colectores lineales, y con ciclos de potencia Brayton están representadas en las figuras 5.4 a 5.7 De los resultados de simulación de las plantas termosolares: MS+SB, MS+RC, MS+PCRC y $\mathrm{MS}+\mathrm{RCMCl}$, resumidos en las figuras 5.8 a 5.11 , se concluye que la configuración MS+RCMCl es la que tiene mayor eficiencia neta $46 \%$ (para unas condiciones de entrada en turbina de $550^{\circ} \mathrm{C}$ y 250 bar). Se confirma también que los ciclos de potencia Brayton permiten reducir el área de apertura efectiva de los colectores solares. Para cuantificar este ahorro se ha definido en este estudio un parámetro, la potencia unitaria (relación entre la potencia neta producida en la instalación solar dividida por el área de apertura efectiva de los colectores solares). En base a este parámetro, la potencia unitaria, se han comparado las diferentes configuraciones y los resultados quedan cuantificados y resumidos en las figuras 5.10 y 5.11 .

Es muy importante recalcar que este estudio se ha realizado tomando como base hipótesis de eficiencia de los componentes del ciclo Brayton similares a las consideradas en por NREL en la publicación considerada de referencia [6]. Los principales componentes de los ciclos de potencia Brayton (turbinas, compresores, recuperadores, intercambidadores de calor, enfriadores) están siendo desarrollados industrialmente y su eficiencia de funcionamiento tendrán que ser confirmada con los suministradores finales de los equipos.

Otra importante conclusión deducida en este capítulo, se centra en la elección de los intercambiadores de calor de tipo carcasa y tubos, como alternativa óptima para sustituir a los intercambiadores tipo PCHE, para la integración entre el ciclo de potencia, el campo solar principal (PHX) y el campo solar de recalentamiento (RHX). La tecnología de los cambiadores 
5. Plantas Termosolares con Colectores Lineales con Sales Fundidas como HTF y ciclos de potencia Brayton con $\mathrm{CO}_{2}$ supercrítico como fluido de trabajo

de carcasa y tubos es común entre los suministradores de esta tipología de equipamiento, permitiendo obtener un precio competitivo. Los intercambiadores de carcasa y tubostambién son un elemento óptimo para intercambio de calor entre el ciclo de potencia Brayton y el sistema de almacenamiento energético TES.

Se está realizando un gran esfuerzo para reducir el coste actual tanto de los materiales de fabricación de los equipos que integran los ciclos de potencia Brayton como Rankine. A corto y medio plazo se continuarán construyendo plantas termosolares con colectores lineales y ciclos de potencia Rankine con agua en estado subcrítico ya que es una tecnología muy madura y ampliamente validada en numerosas centrales termosolares en operación. El esfuerzo inversor en investigación a nivel mundial y liderado por Estados Unidos está enfocado a la implantación a nivel industrial de los ciclos supercríticos Brayton [4-5] como alternativa más eficiente y económica que los ciclos Rankine. En este capítulo se ha mostrado un primer estudio conceptual de la integración de los colectores solares lineales con los ciclos Brayton como solución técnica para la "Nueva Generación" de plantas termosolares.

\subsection{Referencias}

[1] A.Maccari, Archimede Solar Energy. Archimede Solar Energy Molten Salt Parabolic Trough Demo Plant: A Step Ahead Towards the New Frontiers of CSP. SolarPaces 2014.

[2] F.Matino, Archimede Solar Energy. Molten Salt Receivers Operated on Parabolic Trough Demo Plant and in Laboratory Conditions. SolarPaces 2014.

[3] G.Morin, Novatec Solar GmbH. Molten Salt as Heat Transfer Fluid in a Linear Fresnel Collector Comercial Application Backed by Demonstration. SolarPaces 2014.

[4] Supercritical CO2 Power Cycles Symposium. http://www.swri.org/4org/d18/sco2/

[5] Y.Ahn et al. Review of supercritical $\mathrm{CO}_{2}$ power cycle technology and current status of research and development. Nuclear Engineering and Technology. 47-6, 2015, Pages 647-661.

[6] Z.Ma, C.S. Turchi. Advanced Supercritical Carbon Dioxide Power Cycle Configurations for Use in Concentrating Solar Power Systems. National Renewable Energy Laboratory.

[7] V.Dostal, M.J. Driscoll, P.Hejzlar. A Supercritical Carbon Dioxide Cycle for Next Generation Nuclear Reactors. Advanced Nuclear Power Technology Program. MIT-ANP-TR-100.

[8] HCEMS-11, Archimede Solarenergy. http://www.archimedesolarenergy.it

[9] SCHOTT PTR 70 Premium Receivers. http://www.schott.com

[10] Cavity receivers for parabolic solar troughs, Norwich. http://patents.com

[11] Dyreby J. Thesis: Modeling the Supercritical Carbon Dioxide Brayton Cycle with Recompression. University of Wisconsin, Madison. http://sel.me.wisc.edu

[12] Heatric, compact diffusion bonded heat exchangers. www.heatric.com/

[13] Jin Gyu Kwon, Tae Ho Kim, Hyun Sun Park, Moo Hwan Kim. Numerical Analysis of a fin arrangement for an optimal design of airfoil fin PCHE. The $4^{\text {th }}$ International SymposiumSupercritical $\mathrm{CO}_{2}$ Power Cycles, September 9-10, 2014, Pittsburgh, Pennsylvania.

[14] Gavic D., Investigation Of Water, Air, and Hybrid Cooling for Supercritical Carbon Dioxide Brayton Cycles. University of Wisconsin, Madison. http://sel.me.wisc.edu

[15] A.Moisseytsev, J.J. Sienicki. Development of the ANL Plant Dynamics Code and Control Strategies for the Supercritical Carbon Dioxide Brayton Cycle and Code Validation with Data from the Sandia Small-Scale Supercritical CO2 Brayton Cycle Test Loop. Argonne National Laboratory. ANL-ARC-218. 
5. Plantas Termosolares con Colectores Lineales con Sales Fundidas como HTF y ciclos de potencia Brayton con $\mathrm{CO}_{2}$ supercrítico como fluido de trabajo

[16] E.M. Clementoni, T.L. Cox. Practical Aspects of Supercritical Carbon Dioxide Brayton System Testing. The $4^{\text {th }}$ International Symposium-Supercritcial $\mathrm{CO}_{2}$ Power Cycles. September 910, 2014, Pittsburgh, Pennsylvania. http://www.swri.org

[17] S.A. Wright, R.F. Radel, M.E. Vernon, G.E. Rochau, P.S. Pickard. Operation and Analysis of a Supercritical CO2 Brayton Cycle. Sandia Report, SAND2010-0171, printed September 2010.

[18] Molten salt reactor. http://www.world-nuclear.org

[19] Transatomic nuclear reactor. http://www.transatomicpower.com

[20] Terrestrial Energy, Integral molten salt reactor. http://terrestrialenergy.com

[21] F. De Luca, V.Ferraro, V.Marinelli. On the performance of CSP oil-cooled plants, with and without heat storage in tanks of molten salts. Energy 83 (2015) 230-239.

[22] Archimede Solar Energy. http://www.archimedesolarenergy.it/molten salt vs oil.htm

[23] F.Burkholder and C.Kutscher Heat Loss Testing of Schott's 2008 PTR70 Parabolic Trough Receiver. Report NREL/TP-550-45633, May 2009.

[24] Novatec Solar. SAM Linear Fresnel solar boiler model. NREL SAM Conference 2013.

[25] S.A. Wright, T. M. Conboy, G.E. Rochau. Overview of supercritical CO2 power cycle development at Sandia National Laboratories. October 25-27, 2011 Columbus, Ohio, SNL. 


\title{
Capítulo 6 \\ Optimización de las plantas termosolares con colectores lineales con sales fundidas como HTF acopladas a ciclos de potencia Brayton con $\mathrm{CO}_{2}$ supercrítico como fluido de trabajo
}

\begin{abstract}
Resumen
En el capítulo 5 del presente trabajo se han definido diseños preliminares para integrar las plantas termosolares con colectores lineales y ciclos de potencia supercríticos $\mathrm{s}-\mathrm{CO}_{2}$ Brayton. En el presente capítulo, el objetivo principal es maximizar la eficiencia energética de los ciclos Brayton mediante algoritmos matemáticos de optimización multiparámetro. Como consecuencia directa se optimiza el área de apertura efectiva de los colectores solares, y se minimiza su coste de inversión.
\end{abstract}

Se han utilizado tres algoritmos matemáticos para la optimización de los parámetros de operación de los ciclos s- $\mathrm{CO}_{2}$ Brayton: SUBPLEX [1], UOBYQA [2-4] y NEWUOA [5-6]. Estos algoritmos han sido descritos y explicados en detalle en el capítulo 2 "Metodología".

Como punto de partida de este trabajo parte de la tesis doctoral desarrollada por Dyreby [7]. El código informático en lenguaje Fortran y Python desarrollado por Dyreby estaba concebido únicamente para una configuración de ciclo Brayton con recompresión, pero sin etapa de recalentamiento intermedio después del primer escalón de expansión en la turbina. En esta tesis doctoral se ha desarrollado el código Fortran para un mayor número de configuraciones de ciclos Brayton [8]: configuración con enfriamiento parcial antes de recompresión (PCRC) y configuración con enfriamiento intermedio en el compresor principal (RCMCl). También se ha incluido como innovación, la opción de recalentamiento del fluido de trabajo en las etapas intermedias de expansión en turbina. Se ha incluido el dimensionamiento en detalle de los recuperadores y resto de intercambiadores de calor del ciclo, ver capítulo 2 "Metodología". Mediante el diseño detallado de los cambiadores se ha realizado también un cálculo más aproximado de sus pérdidas de presión. Otra mejora significativa es el acoplamiento del ciclo Brayton a los modelos de campo solar con colectores lineales PTC o LF, y con diferentes HTF (sales fundidas, aceites térmicos, DSG). Permitiendo el cálculo de su área de apertura efectiva y estimación de su coste. Se ha optimizado también el interface de usuario Graphical User Interface. Se ha desarrollado el programa bajo el entorno Windows, con el lenguaje de programación C\#, en el entorno de desarrollo Visual Studio 2010 [9]. Permitiendo interactuar de forma más rápida y sencilla con las variables del ciclo de potencia y realizar análisis de sensibilidad.

\subsection{Introducción}

Como se ha mencionado en el capítulo anterior hay dos tecnologías que se encuentran en fase de desarrollo industrial para mejora del rendimiento energético de las plantas termosolares con colectores lineales y ciclos de potencia Brayton. Por un lado se están desarrollando nuevas composiciones químicas de sales fundidas como HTF, y de almacenamiento directo en el campo solar, para minimizar el coste de los materiales de los equipos y componentes en los 
6. Optimización de las plantas termosolares con colectores lineales con sales fundidas como

HTF acopladas a ciclos de potencia Brayton con $\mathrm{CO}_{2}$ supercrítico como fluido de trabajo

campo solares. Estos fluidos deberán tener un bajo punto de fusión para evitar su solidificación a bajas temperaturas, no deberían ser nocivos al medio ambiente en caso de fugas y no sufrir degradación alguna de sus propiedades a lo largo de la vida de la instalación [10-13] (tabla 6.1).

Tabla 6.1. Propiedades físicas de las sales fundidas como HTF.

\begin{tabular}{ccccccc}
\hline $\begin{array}{c}\text { Sal } \\
\text { fundida }\end{array}$ & $\begin{array}{c}\text { Viscosidad } \\
\text { Dinámica } \\
(\mathrm{MPa} \cdot \mathrm{s}) \\
\left(300^{\circ} \mathrm{C}\right)\end{array}$ & $\begin{array}{c}\text { Densidad } \\
\left(\mathrm{kg} / \mathrm{m}^{3}\right) \\
\left(300^{\circ} \mathrm{C}\right)\end{array}$ & $\begin{array}{c}\mathrm{C}_{\mathrm{p}} \\
(\mathrm{kJ} / \mathrm{kg} \cdot \mathrm{K})\end{array}$ & $\begin{array}{c}\text { Composición } \\
\text { (Porcentaje } \\
\text { en peso })\end{array}$ & $\begin{array}{c}\text { Temperatura } \\
\text { Máxima } \\
\text { Operación } \\
\left({ }^{\circ} \mathrm{C}\right)\end{array}$ & $\begin{array}{c}\text { Temperatura } \\
\text { Mínima } \\
\text { Operación } \\
\left({ }^{\circ} \mathrm{C}\right)\end{array}$ \\
\hline Solar Salt & 3.26 & 1899 & 1.49 & $\begin{array}{c}60 \%\left(\mathrm{NaNO}_{3}\right) \\
40 \%\left(\mathrm{KNO}_{3}\right)\end{array}$ & 593.3 & 260 \\
HITEC XL & 6.37 & 1992 & 1.45 & $\begin{array}{c}42 \% \mathrm{Ca}\left(\mathrm{NO}_{3}\right)_{2} \\
15 \%\left(\mathrm{NaNO}_{3}\right) \\
43 \%\left(\mathrm{KNO}_{3}\right)\end{array}$ & 540 & 120 \\
\hline
\end{tabular}

En lo que respecta a los ciclos de potencia Brayton, se están desarrollando a escala industrial [14-15], y los equipos que lo integran (turbinas [16-17], compresores [18-19], intercambiadores de calor [20-21]). Se están definiendo los materiales necesarios para los niveles tan altos de presión y temperatura que estos ciclos de potencia requieren. Se intenta optimizar los procesos de fabricación para obtener unos intercambiadores de calor con altos tamaños térmicos UA, que sean compactos y ocupen el menor volumen posible. El sistema de control de estos ciclos en condiciones de transitorios de radiación solar también se está desarrollando [22-23].

Tal y como se ha explicado en detalle a lo largo de este trabajo en relación a las tecnologías que impactan directamente en el diseño de los campos solares, se tiende a la implantación de las sales fundidas como HTF [24-25] y como sistema de almacenamiento térmico [26-27].

Este capítulo se centrará en la caracterización y optimización de los parámetros de operación de los ciclos de potencia Brayton con diferentes configuraciones [8]:

- Brayton simple (SB)

- con recompersión (RC)

- con enfriamiento parcial antes de la recompresión (PCRC)

- con enfriamiento intermedio en el compresor principal (RCMCI)

Estas soluciones de diseño ya han sido definidas y explicadas en el capítulo 5, sin embargo, en este capítulo se integran diferentes algoritmos matemáticos: SUBPLEX [1], UOBYQA [2-4] y NEWUOA [5, 6], en el cálculo de los balances térmicos de los ciclos Brayton con objeto de optimizar la eficiencia energética de las plantas termosolares, y optimizar tanto el tamaño de sus campos solares como el coste de inversión en los colectores lineales.

En relación al procedimiento de cálculo seguido, se han fijado diferentes valores del tamaño térmico de los recuperadores, UA, y se han fijado diferentes TIT. Ambos parámetros tienen gran impacto en la eficiencia del ciclo de potencia Brayton. El UA está limitado por el volumen y coste de fabricación de los intercambiadores de calor con $\mathrm{s}-\mathrm{CO}_{2}$ en ambas corrientes. 
6. Optimización de las plantas termosolares con colectores lineales con sales fundidas como

$\mathrm{HTF}$ acopladas a ciclos de potencia Brayton con $\mathrm{CO}_{2}$ supercrítico como fluido de trabajo

Actualmente, en este ámbito, se están desarrollando diferentes tipologías de canales para incrementar el área de intercambio y reducir las pérdidas de presión [20-21]. Recientemente ha comenzado a experimentarse con materiales cerámicos para obtener mayores temperaturas de operación [28-29]. Sin embargo, el UA está limitado por la mínima diferencia de temperatura entre corrientes o pinch-point [30]. En el capítulo 11 de este trabajo se proponen también otros fluidos de trabajo como alternativa al $\mathrm{CO}_{2}$ (Etano, Xenón, $\mathrm{SF}_{6}$, Nitrógeno y Metano), que permiten un mayor recorrido en el incremento del UA de los cambiadores. La corrosión de los materiales de los cambiadores también juega un papel fundamental en los ciclos Brayton, dado que supone aumentar espesores lo que implica un aumento de material, y el excesivo coste de los materiales de cambiadores de gran tamaño repercute en gran medida el coste de la planta [31-32].

\subsection{Hipótesis de cálculo}

Todas las simulaciones reproducen las condiciones de operación de la planta termosolar en su punto de diseño para diferentes TIT en la turbina principal y de recalentamiento: $400^{\circ} \mathrm{C}, 450^{\circ} \mathrm{C}$, $500^{\circ} \mathrm{C}$ y $550^{\circ} \mathrm{C}$. El resto de hipótesis de cálculo quedan resumidas en las tablas 6.2 a 6.7. En todas las simulaciones se ha fijado una potencia bruta generada de $50 \mathrm{MWe}$.

Tabla 6.2. Localización, condiciones ambientales.

\begin{tabular}{lc}
\hline Localización: & Dagget, CA, USA. \\
Latitud: & $34.86^{\circ}$ \\
Longitud: & $-116.8^{\circ}$ \\
Zona horaria: & -8 \\
Hora: & $11: 30 \mathrm{hr}$ \\
DNI: & $986 \mathrm{~W} / \mathrm{m}^{2}$ \\
Temperatura: & $25^{\circ} \mathrm{C}$ \\
Altitud: & $588 \mathrm{~m}$ \\
\hline
\end{tabular}

Tabla 6.3. Parámetros tubo absorbedor.

\begin{tabular}{ll}
\hline Diámetro exterior: & $70 \mathrm{~mm}$ \\
Espesor pared: & $4.191 \mathrm{~mm}$ \\
Material: & Acero Inoxidable \\
Rugosidad: & $0.0457 \mathrm{~mm}$ \\
\hline
\end{tabular}

Tabla 6.4. Parámetros colectores PTC.

\begin{tabular}{ll}
\hline Tipo colector: & EuroTrough II \\
Anchura apertura: & $5.77 \mathrm{~m}$ \\
Longitud focal: & $1.71 \mathrm{~m}$ \\
Distancia/anchura (lazos): & 2.5 \\
Factor Limpieza: & 0.96 \\
Eficiencia óptica: & 0.75 \\
Pérdidas térmicas: & $0.141 \Delta \mathrm{T}+6.48 \cdot 10^{-9} \Delta \mathrm{T}^{4}$ \\
\hline
\end{tabular}


6. Optimización de las plantas termosolares con colectores lineales con sales fundidas como $\mathrm{HTF}$ acopladas a ciclos de potencia Brayton con $\mathrm{CO}_{2}$ supercrítico como fluido de trabajo

Tabla 6.5. Parámetros colectores Fresnel.

\begin{tabular}{ll}
\hline Tipo colector: & SuperNova 1 (Novatec) \\
Dimensiones: & $16.56 \mathrm{~m} \times 44.8 \mathrm{~m}$ \\
Área & $513.6 \mathrm{~m}^{2} /$ por Modulo \\
Eficiencia & 0.647 \\
Pérdidas: & $1.06 \Delta \mathrm{T}+1.2110^{-8} \Delta \mathrm{T}^{4}$ \\
Pérdidas: & $0.15 \Delta \mathrm{T}+7.1510^{-9} \Delta \mathrm{T}^{4}$ \\
\hline
\end{tabular}

Tabla 6.6. Ciclo potencia Rankine.

\begin{tabular}{ll}
\hline Turbina HP: & 2 etapas (87.7 bar; 36 bar) \\
Turbina IP: & 3 etapas \\
& (16.5 bar;10.34 bar; 6.18 bar) \\
Turbina LP: & 4 etapas \\
& $(5.17$ bar; 3.04bar; 1.17 bar; 0.37 bar) \\
Eficiencia Turbina: & $85 \%$ \\
Presión condensador: & 0.08 bar \\
Eficiencia Generador: & $98.23 \%$ (punto de diseño) \\
Auxiliary BOP: & $0.01 \%$ (potencia bruta) \\
TTD calentadores agua: & $5^{\circ} \mathrm{C}$ \\
DCA calentadores agua : & $5^{\circ} \mathrm{C}$ \\
Presión desaireador: & 6.17 bar \\
\hline
\end{tabular}

Tabla 6.7. Ciclo potencia s- $\mathrm{CO}_{2}$ Brayton.

\begin{tabular}{ll}
\hline Eficiencia Turbina: & $93 \%$ \\
Eficiencia Compresor: & $89 \%$ \\
Tamaño térmico cambiadores fijado (UA) \\
No pérdidas de presión en cambiadores \\
Temperatura entrada turbina: & $550^{\circ} \mathrm{C}$ \\
Presión entrada turbina: & $250 \mathrm{bar}$ \\
Presión recalentamiento: & optimizado \\
Temperatura compresor: & $32^{\circ} \mathrm{C}$ \\
Presión entrada compresor: & Optimizado \\
Fracción de Flujo: & optimizado \\
Pérdidas auxiliares BOP: & $0.01 \%$ \\
Eficiencia Generador: & 98.23 \\
\hline
\end{tabular}

\subsection{Resultados}

\subsubsection{Caracterización de la Eficiencia Bruta del Ciclo de Potencia Brayton}

En este apartado se ha realizado una recopilación de la evolución de la eficiencia Bruta de los ciclos Brayton una vez realizada la optimización de sus parámetros termodinámicos de operación mediante los algoritmos matemáticos: SUBPLEX [1], UOBYQA [2-4], NEWUOA [5-6].

Como se ha indicado anteriormente, se han fijado los tamaños térmicos, UA, de los recuperadores que integran el ciclo Brayton y se ha graficado la evolución de la eficiencia bruta 
6. Optimización de las plantas termosolares con colectores lineales con sales fundidas como HTF acopladas a ciclos de potencia Brayton con $\mathrm{CO}_{2}$ supercrítico como fluido de trabajo

para diferentes temperaturas de entrada en la turbina principal TIT. Se ha realizado el modelado de tres tipologías de ciclos Brayton [8] (con Recompresión, con enfriamiento parcial antes de la Recompresión, y con Recompresión y enfriamiento intermedio en el compresor principal. En los tres casos se han simulado los ciclos de potencia con recalentamiento en las turbinas de generación de potencia y sin recalentamiento. Los resultados obtenidos están resumidos en las figuras 6.1 a 6.6. Como se puede apreciar en dichas gráficas hay una serie de valores umbrales del tamaño térmico de los recuperadores del ciclo (UA $=10000-15000$ $\mathrm{kW} / \mathrm{K}$ ) a partir de los cuales no obtenemos un incremento significativo en la eficiencia bruta del ciclo. Por tanto, hay que elegir unos valores de tamaño térmico en los recuperadores que permita reducir la superficie de los colectores solares.

Como dato indicativo el coste de incrementar el tamaño térmico en los recuperadores es menor que el coste de incrementar la superficie de los colectores solares. Los intercambiadores de calor de los recuperadores tienden a ser compactos y ocupar poco volumen, reduciendo el coste de sus materiales y coste de su fabricación. Sin embargo, el coste de los colectores solares lleva asociado diversos componentes: los espejos reflectantes, su estructura soporte, su obra civil y su mantenimiento para mantenerlos limpios. Compensa, por tanto, incrementar el tamaño térmico de los recuperadores e intentar ahorrar en el área de apertura efectiva y coste de capital de los colectores solares. En subsiguientes capítulos se han desglosado ambos costes de inversión.

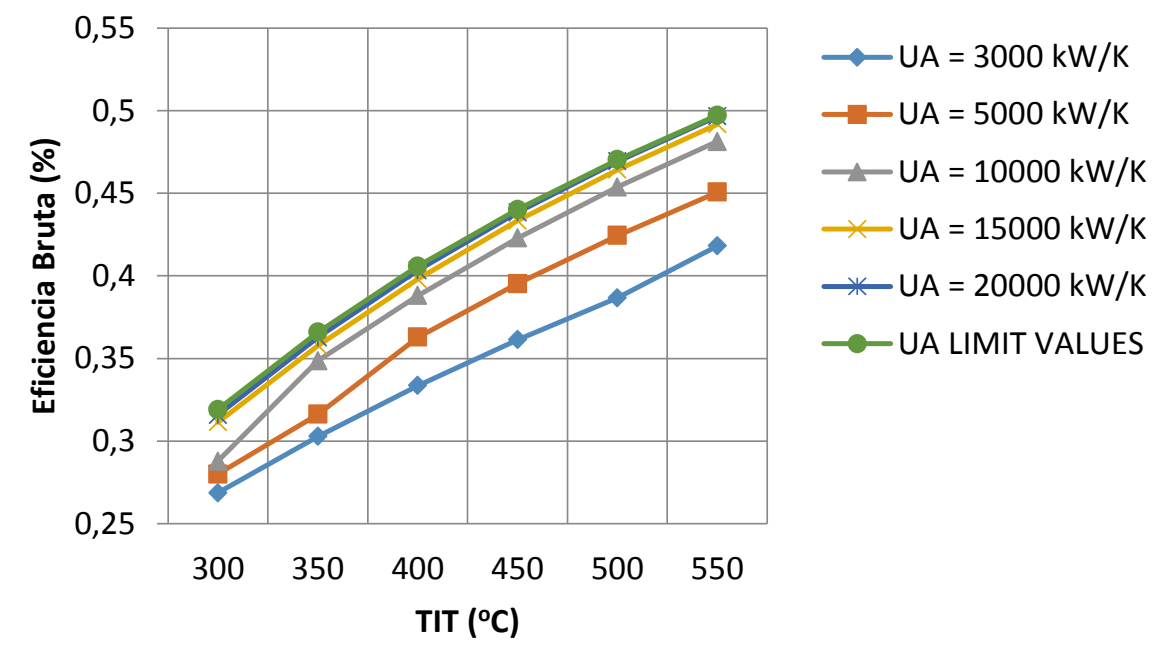

Figura 6.1. Eficiencia Bruta \& $\mathrm{TIT}$, ciclo $\mathrm{RC} \mathrm{s}-\mathrm{CO}_{2}$ sin recalentamiento. 
6. Optimización de las plantas termosolares con colectores lineales con sales fundidas como $\mathrm{HTF}$ acopladas a ciclos de potencia Brayton con $\mathrm{CO}_{2}$ supercrítico como fluido de trabajo

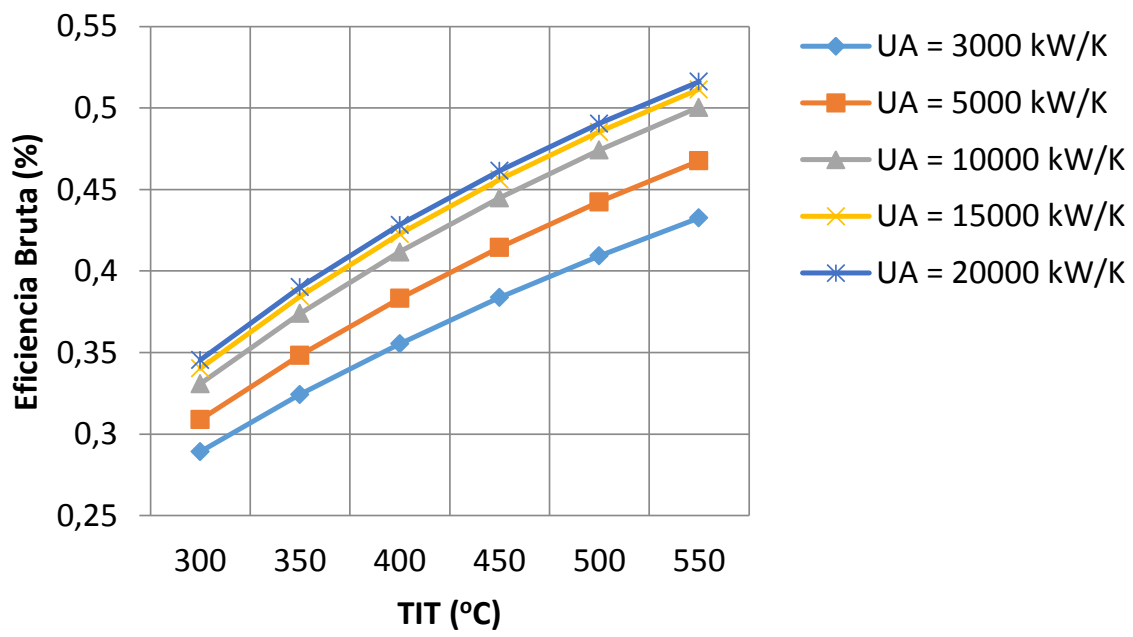

Figura 6.2. Eficiencia Bruta \& $\mathrm{TIT}$, ciclo $\mathrm{RC} \mathrm{s}-\mathrm{CO}_{2}$ con recalentamiento.

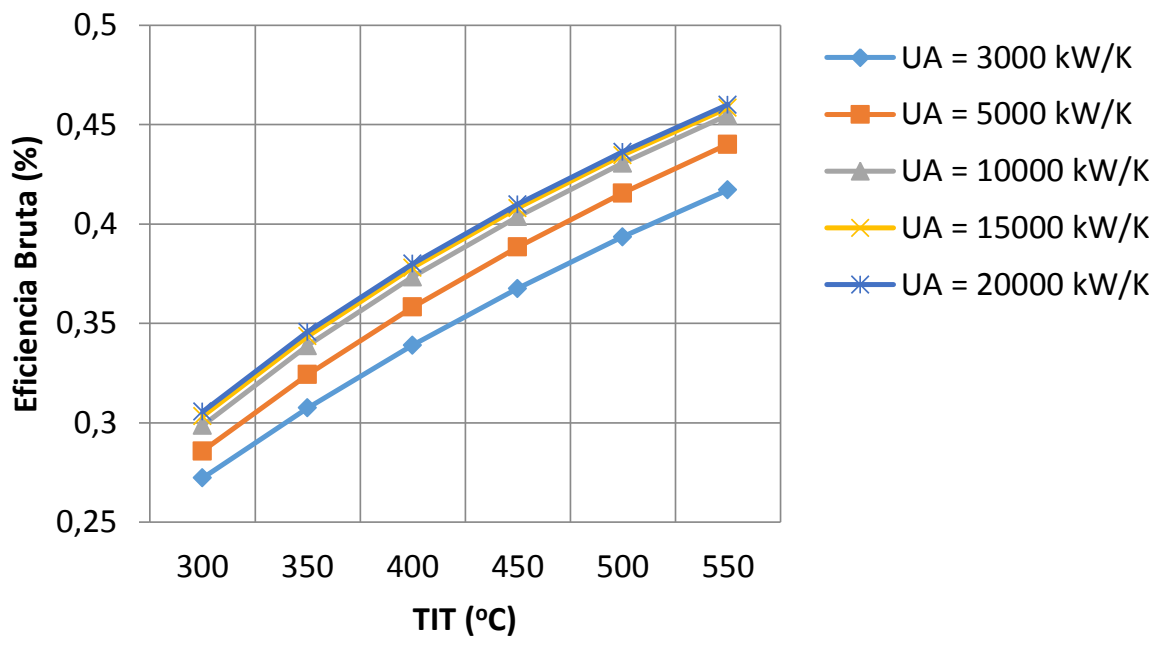

Figura 6.3. Eficiencia Bruta \& $\mathrm{TIT}$, ciclo $\mathrm{PCRC} s-\mathrm{CO}_{2} \sin$ recalentamiento.

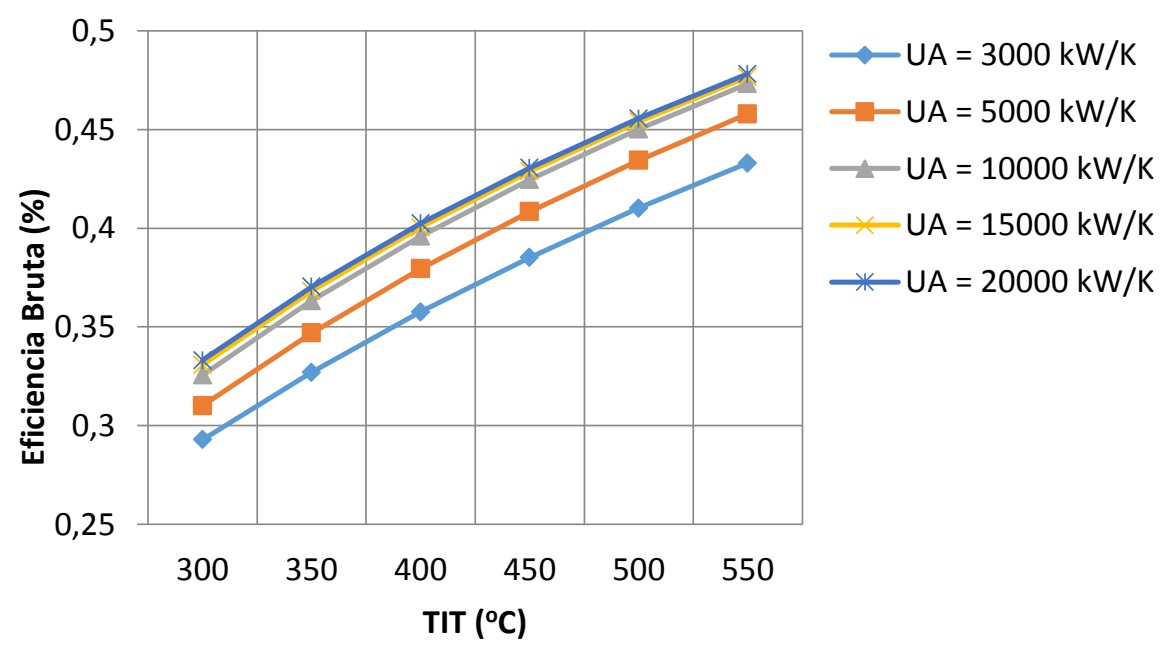

Figura 6.4. Eficiencia Bruta \& TIT, ciclo PCRC s- $\mathrm{CO}_{2}$ con recalentamiento. 
6. Optimización de las plantas termosolares con colectores lineales con sales fundidas como $\mathrm{HTF}$ acopladas a ciclos de potencia Brayton con $\mathrm{CO}_{2}$ supercrítico como fluido de trabajo

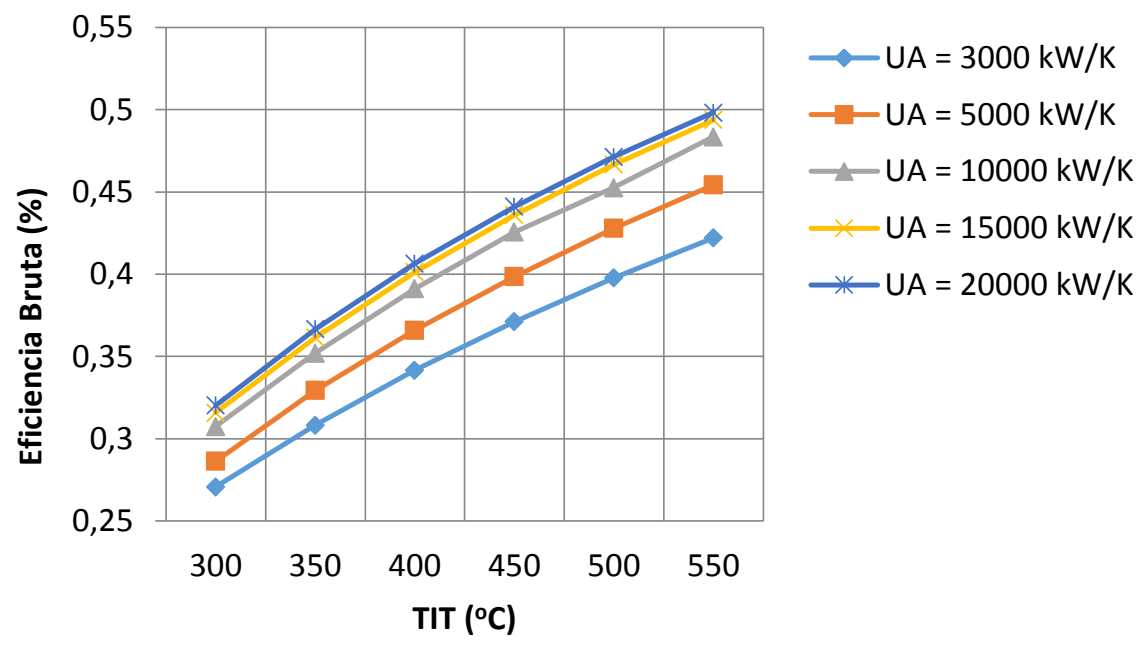

Figura 6.5. Eficiencia Bruta \& TIT, ciclo $\mathrm{RCMCl} \mathrm{s}-\mathrm{CO}_{2}$ sin recalentamiento.

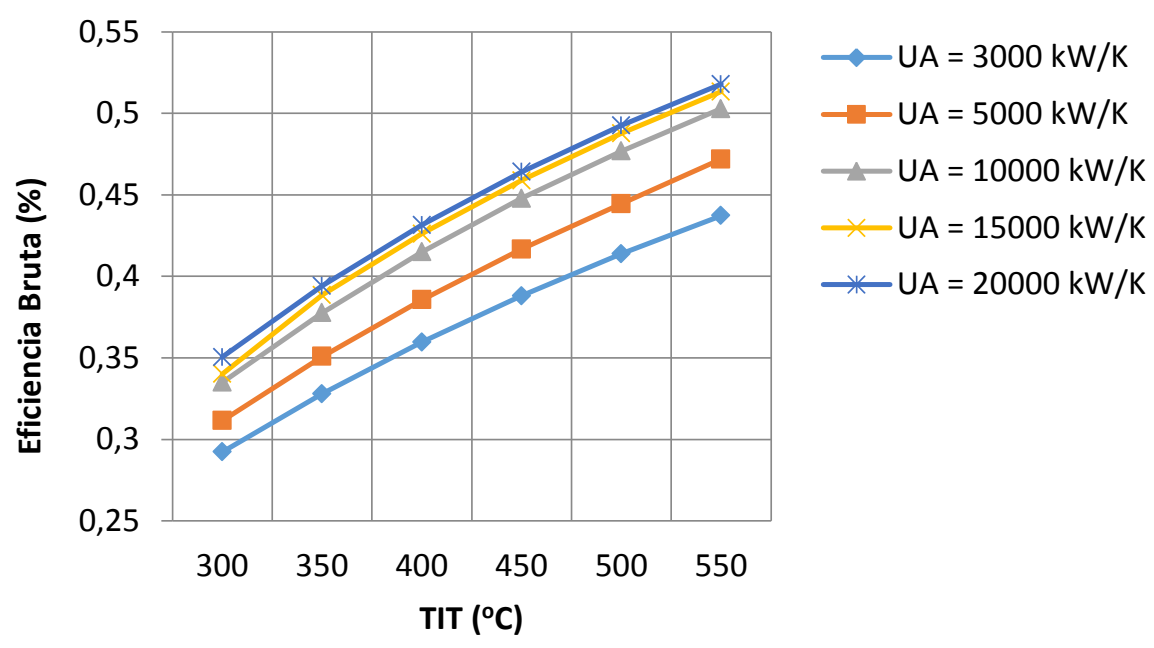

Figura 6.6. Eficiencia Bruta \& $\mathrm{TIT}$, ciclo $\mathrm{RCMCl} \mathrm{s-} \mathrm{CO}_{2}$ con recalentamiento.

\subsubsection{Parámetros termodinámicos para la optimización de la eficiencia del ciclo Brayton}

En los siguientes apartados se han identificado los parámetros termodinámicos que presentan un impacto directo en la eficiencia bruta de los ciclos Brayton.

Como regla general la presión optima de entrada a los compresores no presenta una variación significativa, y es lo más cercana posible a las condiciones de criticidad para aprovechar la alta densidad del fluido de trabajo bajo estas condiciones. La fracción de recompresión de flujo óptima si tiene un especial impacto en la eficiencia energética del ciclo, y se incrementa su valor conforme se aumenta el tamaño térmico de los recuperadores. Y el caudal másico óptimo para una potencia fija, en este estudio $50 \mathrm{MWe}$, es menor conforme se incrementa el tamaño térmico de los recuperadores. La presión de recalentamiento no tiene gran impacto en la eficiencia neta del ciclo.

La presión de entrada en la turbina principal, en este estudio se ha fijado en $250 \mathrm{MPa}$, mayores valores requieren materiales muy sofisticados de alto coste [33]. La temperatura ambiental también se ha fijado en $25^{\circ} \mathrm{C}$ para permitir una temperatura fija en todas las configuraciones 
6. Optimización de las plantas termosolares con colectores lineales con sales fundidas como

$\mathrm{HTF}$ acopladas a ciclos de potencia Brayton con $\mathrm{CO}_{2}$ supercrítico como fluido de trabajo

del fluido de trabajo de $32^{\circ} \mathrm{C}$ a la entrada de los compresores CIT. Este parámetro tiene un gran impacto en el rendimiento del ciclo, ya que pequeñas variaciones de la temperatura a la entrada del compresor dan lugar a variaciones excesivas en la densidad del fluido de trabajo, incrementando el trabajo de los compresores e impactando negativamente en el rendimiento energético de la planta termosolar [33].

\subsubsection{Ciclo con recompresión ( $\mathrm{RC}$ ) sin Recalentamiento}

El parámetro termodinámico que más influye en esta tipología de ciclos es la fracción de caudal de recompresión, con una variación entre 0.2 y 0.4 . Para los valores de tamaño térmico de los recuperadores, $U A=3000 \mathrm{~kW} / \mathrm{K}$, la fracción de recompresión nula, y por tanto, el ciclo de potencia presentaría una tipología SB. El caudal másico también varía significativamente cuando se incrementa la TIT, sufriendo un decremento significativo en sus valores. Si reducimos el caudal másico también se reduce el trabajo necesario para su compresión, incrementando el rendimiento del ciclo. Los resultados de la optimización de las variables de operación del ciclo para la configuración del ciclo Brayton RC, quedan resumidos en las tablas 6.8 a 6.10 .

Tabla 6.8. Variables termodinámicas para optimizar el rendimiento energético del ciclo de potencia Brayton ( $\mathrm{RC}$ sin recalentamiento) en centrales termosolares con colectores lineales.

\begin{tabular}{|c|c|c|c|c|c|c|c|}
\hline \multicolumn{4}{|c|}{$U A=3000 \mathrm{~kW} / \mathrm{K}$} & \multicolumn{4}{|c|}{$U A=5000 \mathrm{~kW} / \mathrm{K}$} \\
\hline $\begin{array}{l}\text { TIT } \\
\left({ }^{\circ} \mathrm{C}\right)\end{array}$ & $\begin{array}{l}\text { CIP } \\
\text { (bar) }\end{array}$ & $\begin{array}{l}\text { Flow } \\
\text { Fraction } \\
\text { (\%) }\end{array}$ & $\begin{array}{l}\text { Mass } \\
\text { Flow } \\
(\mathrm{kg} / \mathrm{s})\end{array}$ & $\begin{array}{l}\text { TIT } \\
\left({ }^{\circ} \mathrm{C}\right)\end{array}$ & $\begin{array}{l}\text { CIP } \\
\text { (bar) }\end{array}$ & $\begin{array}{l}\text { Flow } \\
\text { Fraction (\%) }\end{array}$ & $\begin{array}{l}\text { Mass } \\
\text { Flow } \\
(\mathrm{kg} / \mathrm{s})\end{array}$ \\
\hline 300 & 75.8 & 0.00 & 724.9 & 300 & 75.9 & 0.00 & 720.3 \\
\hline 350 & 75.6 & 0.00 & 615.6 & 350 & 75.7 & 0.00 & 612.1 \\
\hline 400 & 75.6 & 0.00 & 539.0 & 400 & 76.1 & 0.28 & 631.3 \\
\hline 450 & 75.5 & 0.00 & 482.0 & 450 & 76.1 & 0.28 & 553.5 \\
\hline 500 & 75.5 & 0.00 & 437.1 & 500 & 76.0 & 0.29 & 493.8 \\
\hline 550 & 75.7 & 0.21 & 433.2 & 550 & 76.0 & 0.29 & 446.5 \\
\hline
\end{tabular}

Tabla 6.9. Variables termodinámicas para optimizar el rendimiento energético del ciclo de potencia Brayton ( $\mathrm{RC}$ sin Recalentamiento) en centrales termosolares con colectores lineales.

\begin{tabular}{|c|c|c|c|c|c|c|c|}
\hline \multicolumn{4}{|c|}{$\mathrm{UA}=10000 \mathrm{~kW} / \mathrm{K}$} & \multicolumn{4}{|c|}{$\mathrm{UA}=15000 \mathrm{~kW} / \mathrm{K}$} \\
\hline $\begin{array}{l}\text { TIT } \\
\left({ }^{\circ} \mathrm{C}\right)\end{array}$ & $\begin{array}{l}\text { CIP } \\
\text { (bar) }\end{array}$ & $\begin{array}{l}\text { Flow } \\
\text { Fraction } \\
(\%)\end{array}$ & $\begin{array}{l}\text { Mass } \\
\text { Flow } \\
(\mathrm{kg} / \mathrm{s})\end{array}$ & $\begin{array}{l}\mathrm{TIT} \\
\left({ }^{\circ} \mathrm{C}\right)\end{array}$ & $\begin{array}{l}\text { CIP } \\
\text { (bar) }\end{array}$ & $\begin{array}{l}\text { Flow } \\
\text { Fraction (\%) }\end{array}$ & $\begin{array}{l}\text { Mass } \\
\text { Flow } \\
(\mathrm{kg} / \mathrm{s})\end{array}$ \\
\hline 300 & 76.6 & 0.00 & 715.5 & 300 & 78.4 & 0.34 & 932.8 \\
\hline 350 & 77.0 & 0.33 & 755.5 & 350 & 77.6 & 0.35 & 759.1 \\
\hline 400 & 76.8 & 0.34 & 644.6 & 400 & 77.1 & 0.36 & 647.4 \\
\hline 450 & 76.6 & 0.35 & 564.4 & 450 & 76.8 & 0.36 & 565.3 \\
\hline 500 & 76.4 & 0.35 & 503.0 & 500 & 76.6 & 0.37 & 503.8 \\
\hline 550 & 76.3 & 0.36 & 454.2 & 550 & 76.4 & 0.37 & 454.4 \\
\hline
\end{tabular}


6. Optimización de las plantas termosolares con colectores lineales con sales fundidas como HTF acopladas a ciclos de potencia Brayton con $\mathrm{CO}_{2}$ supercrítico como fluido de trabajo

Tabla 6.10. Variables termodinámicas para optimizar el rendimiento energético del ciclo de potencia Brayton ( $\mathrm{RC}$ sin Recalentamiento) en centrales termosolares con colectores lineales.

\begin{tabular}{|c|c|c|c|}
\hline \multicolumn{4}{|c|}{$U A=20000 \mathrm{~kW} / \mathrm{K}$} \\
\hline $\begin{array}{l}\text { TIT } \\
\left({ }^{\circ} \mathrm{C}\right)\end{array}$ & $\begin{array}{l}\text { CIP } \\
\text { (bar) }\end{array}$ & $\begin{array}{l}\text { Flow } \\
\text { Fraction } \\
(\%)\end{array}$ & $\begin{array}{l}\text { Mass } \\
\text { Flow } \\
(\mathrm{kg} / \mathrm{s})\end{array}$ \\
\hline 300 & 78.7 & 0.35 & 930.6 \\
\hline 350 & 78.0 & 0.35 & 760.3 \\
\hline 400 & 77.3 & 0.36 & 647.8 \\
\hline 450 & 76.9 & 0.37 & 565.7 \\
\hline 500 & 76.6 & 0.37 & 502.9 \\
\hline 550 & 76.4 & 0.37 & 453.3 \\
\hline
\end{tabular}

\subsubsection{Ciclo con recompresión (RC) con recalentamiento}

Al igual que en el ciclo RC sin recalentamiento, la fracción de flujo de recompresión optima es una variable muy importante, impactando directamente en el rendimiento neto del ciclo y sus valores varían entre 0.1-0.37. La presión de recompresión óptima también presenta una variación entre 152-141 bar. Respecto a la presión de entrada en el compresor principal su valor óptimo varía ligeramente alrededor de 76 bar.

Los valores de fracción de recompresión se incrementan conforme se incrementa el tamaño térmico de los recuperadores UA y la presión de recalentamiento se reduce conforme se incrementa UA.

El caudal de flujo másico óptimo se reduce sustancialmente conforme se aumenta la temperatura de entrada a la turbina de alta presión TIT, y también sufre un pequeño incremento cuando se aumenta la UA de los recuperadores.

Los resultados de la optimización multivariable están resumidos en las tablas 6.11 a 6.13.

Tabla 6.11. Variables termodinámicas para optimizar el rendimiento energético del ciclo de potencia Brayton (RC con Recalentamiento) en centrales termosolares con colectores lineales.

\begin{tabular}{|c|c|c|c|c|c|c|c|c|c|}
\hline \multicolumn{5}{|c|}{$\mathrm{UA}=3000 \mathrm{~kW} / \mathrm{K}$} & \multicolumn{5}{|c|}{$\mathrm{UA}=5000 \mathrm{~kW} / \mathrm{K}$} \\
\hline $\begin{array}{l}\text { TIT } \\
\left({ }^{\circ} \mathrm{C}\right)\end{array}$ & $\begin{array}{l}\text { CIP } \\
\text { (bar) }\end{array}$ & $\begin{array}{l}\text { ReHeating } \\
\text { Pressure } \\
\text { (bar) }\end{array}$ & $\begin{array}{l}\text { Flow } \\
\text { Fraction } \\
(\%)\end{array}$ & $\begin{array}{l}\text { Mass } \\
\text { Flow } \\
(\mathrm{kg} / \mathrm{s})\end{array}$ & $\operatorname{TIT}\left({ }^{\circ} \mathrm{C}\right)$ & $\begin{array}{l}\text { CIP } \\
\text { (bar) }\end{array}$ & $\begin{array}{l}\text { ReHeating } \\
\text { Pressure } \\
\text { (bar) }\end{array}$ & $\begin{array}{l}\text { Flow } \\
\text { Fraction } \\
(\%)\end{array}$ & $\begin{array}{l}\text { Mass } \\
\text { Flow } \\
(\mathrm{kg} / \mathrm{s})\end{array}$ \\
\hline 300 & 75.8 & 153.9 & 0.08 & 686.2 & 300 & 76.2 & 152.6 & 0.23 & 760.9 \\
\hline 350 & 75.8 & 156.8 & 0.16 & 618.8 & 350 & 76.1 & 152.9 & 0.25 & 650.6 \\
\hline 400 & 75.7 & 158.1 & 0.16 & 541.8 & 400 & 76.0 & 152.6 & 0.26 & 567.8 \\
\hline 450 & 75.7 & 157.8 & 0.18 & 486.4 & 450 & 76.0 & 152.5 & 0.27 & 504.3 \\
\hline 500 & 75.7 & 158.2 & 0.19 & 439.2 & 500 & 75.9 & 152.5 & 0.28 & 454.0 \\
\hline 550 & 75.7 & 158.7 & 0.19 & 400.5 & 550 & 75.9 & 152.7 & 0.29 & 413.1 \\
\hline
\end{tabular}


6. Optimización de las plantas termosolares con colectores lineales con sales fundidas como HTF acopladas a ciclos de potencia Brayton con $\mathrm{CO}_{2}$ supercrítico como fluido de trabajo

Tabla 6.12. Variables termodinámicas para optimizar el rendimiento energético del ciclo de potencia Brayton ( $\mathrm{RC}$ con Recalentamiento) en centrales termosolares con colectores lineales lineales.

\begin{tabular}{|c|c|c|c|c|c|c|c|c|c|}
\hline \multicolumn{5}{|c|}{$\mathrm{UA}=10000 \mathrm{~kW} / \mathrm{K}$} & \multicolumn{5}{|c|}{$U A=15000 \mathrm{~kW} / \mathrm{K}$} \\
\hline $\begin{array}{l}\text { TIT } \\
\left({ }^{\circ} \mathrm{C}\right)\end{array}$ & $\begin{array}{l}\text { CIP } \\
\text { (bar) }\end{array}$ & $\begin{array}{l}\text { ReHeating } \\
\text { Pressure } \\
\text { (bar) }\end{array}$ & $\begin{array}{l}\text { Flow } \\
\text { Fraction } \\
\text { (\%) }\end{array}$ & $\begin{array}{l}\text { Mass } \\
\text { Flow } \\
(\mathrm{kg} / \mathrm{s})\end{array}$ & $\begin{array}{l}\text { TIT } \\
\left({ }^{\circ} \mathrm{C}\right)\end{array}$ & $\begin{array}{l}\text { CIP } \\
\text { (bar) }\end{array}$ & $\begin{array}{l}\text { ReHeating } \\
\text { Pressure } \\
\text { (bar) }\end{array}$ & $\begin{array}{l}\text { Flow } \\
\text { Fraction } \\
\text { (\%) }\end{array}$ & $\begin{array}{l}\text { Mass } \\
\text { Flow } \\
(\mathrm{kg} / \mathrm{s})\end{array}$ \\
\hline 300 & 76.9 & 148.5 & 0.31 & 794.8 & 300 & 77.4 & 146.3 & 0.34 & 804.5 \\
\hline 350 & 76.6 & 147.3 & 0.32 & 671.7 & 350 & 76.9 & 144.8 & 0.35 & 677.7 \\
\hline 400 & 76.5 & 146.4 & 0.34 & 583.1 & 400 & 76.7 & 143.9 & 0.36 & 586.3 \\
\hline 450 & 76.3 & 145.9 & 0.34 & 515.9 & 450 & 76.5 & 143.1 & 0.36 & 517.7 \\
\hline 500 & 76.2 & 145.6 & 0.35 & 463.1 & 500 & 76.3 & 142.6 & 0.37 & 464.1 \\
\hline 550 & 76.1 & 145.1 & 0.36 & 420.7 & 550 & 76.2 & 142.1 & 0.37 & 420.9 \\
\hline
\end{tabular}

Tabla 6.13. Variables termodinámicas para optimizar el rendimiento energético del ciclo de potencia Brayton ( $R C$ con Recalentamiento) en centrales termosolares con colectores lineales.

\begin{tabular}{|c|c|c|c|c|}
\hline \multicolumn{5}{|c|}{$U A=20000 \mathrm{~kW} / \mathrm{K}$} \\
\hline $\begin{array}{l}\text { TIT } \\
\left({ }^{\circ} \mathrm{C}\right)\end{array}$ & $\begin{array}{l}\text { CIP } \\
\text { (bar) }\end{array}$ & $\begin{array}{l}\text { ReHeating } \\
\text { Pressure } \\
\text { (bar) }\end{array}$ & $\begin{array}{l}\text { Flow } \\
\text { Fraction } \\
(\%)\end{array}$ & $\begin{array}{l}\text { Mass } \\
\text { Flow } \\
(\mathrm{kg} / \mathrm{s})\end{array}$ \\
\hline 300 & 77.7 & 145.0 & 0.35 & 808.8 \\
\hline 350 & 77.3 & 143.7 & 0.36 & 679.2 \\
\hline 400 & 76.8 & 142.6 & 0.36 & 586.9 \\
\hline 450 & 76.5 & 141.9 & 0.37 & 517.3 \\
\hline 500 & 76.3 & 141.3 & 0.37 & 463.3 \\
\hline 550 & 76.2 & 140.8 & 0.37 & 420.0 \\
\hline
\end{tabular}

\subsubsection{Ciclo con enfriamiento parcial y recompresión (PCRC), sin y con recalentamiento}

La presión a la salida del precompresor es el principal parámetro diferenciador, si se comparan las tipologías de ciclos RC y PCRC. Cabe destacar que el valor óptimo de esta presión varía entre 80-82 bar. El resto de parámetros termodinámicos presentan un comportamiento similar al de los ciclos RC. La fracción de recompresión óptima también se ve incrementada desde 0.3 hasta 0.45 . Respecto a la presión de recalentamiento optima, varía desde 154-141 bar. Cabe destacar que se ha fijado la presión de entrada en precompresor al valor de 73.77 bar, la presión del punto de criticidad del $\mathrm{CO}_{2}$. Conforme se incrementa la TIT, y el tamaño térmico de los recuperadores UA, también se incrementa la fracción de caudal óptima. Lo mismo ocurre con la presión de descarga del precompresor, es mayor conforme se aumentan los valores de TIT y UA. Comportamiento opuesto presenta la presión de entrada a la turbina de recalentamiento, un aumento de los valores de TIT y UA da como resultado una menor presión de entrada a la turbina de recalentamiento. Los resultados detallados de la optimización multiparamétrica de los ciclos (PCRC) están resumidos en las tablas 6.14 a 6.19. 
6. Optimización de las plantas termosolares con colectores lineales con sales fundidas como HTF acopladas a ciclos de potencia Brayton con $\mathrm{CO}_{2}$ supercrítico como fluido de trabajo

Tabla 6.14. Variables termodinámicas para optimizar el rendimiento energético del ciclo de potencia Brayton (PCRC sin Recalentamiento) en centrales termosolares con colectores lineales.

\begin{tabular}{|c|c|c|c|c|c|c|c|c|c|}
\hline \multicolumn{5}{|c|}{$U A=3000 \mathrm{~kW} / \mathrm{K}$} & \multicolumn{5}{|c|}{$\mathrm{UA}=5000 \mathrm{~kW} / \mathrm{K}$} \\
\hline $\begin{array}{l}\text { TIT } \\
\left({ }^{\circ} \mathrm{C}\right)\end{array}$ & $\begin{array}{l}\text { CIP } \\
\text { (bar) }\end{array}$ & $\begin{array}{l}\text { PreComp. } \\
\text { Inlet (bar) }\end{array}$ & $\begin{array}{l}\text { Flow } \\
\text { Fraction } \\
(\%)\end{array}$ & $\begin{array}{l}\text { Mass } \\
\text { Flow } \\
(\mathrm{kg} / \mathrm{s})\end{array}$ & $\begin{array}{l}\mathrm{TIT} \\
\left({ }^{\circ} \mathrm{C}\right)\end{array}$ & $\begin{array}{l}\text { CIP } \\
\text { (bar) }\end{array}$ & $\begin{array}{l}\text { PreComp. } \\
\text { Inlet (bar) }\end{array}$ & $\begin{array}{l}\text { Flow } \\
\text { Fraction } \\
(\%)\end{array}$ & $\begin{array}{l}\text { Mass } \\
\text { Flow } \\
(\mathrm{kg} / \mathrm{s})\end{array}$ \\
\hline 300 & 73.7 & 76.9 & 0.28 & 739.9 & 300 & 73.7 & 78.1 & 0.34 & 746.2 \\
\hline 350 & 73.7 & 76.9 & 0.32 & 625.4 & 350 & 73.7 & 78.2 & 0.39 & 630.1 \\
\hline 400 & 73.7 & 76.9 & 0.35 & 544.0 & 400 & 73.7 & 78.3 & 0.42 & 548.1 \\
\hline 450 & 73.7 & 76.9 & 0.39 & 484.3 & 450 & 73.7 & 78.5 & 0.45 & 486.5 \\
\hline 500 & 73.7 & 77.0 & 0.42 & 436.8 & 500 & 73.7 & 78.6 & 0.46 & 438.1 \\
\hline 550 & 73.7 & 77.0 & 0.44 & 398.3 & 550 & 73.7 & 78.8 & 0.48 & 399.1 \\
\hline
\end{tabular}

Tabla 6.15. Variables termodinámicas para optimizar el rendimiento energético del ciclo de potencia Brayton (PCRC sin Recalentamiento) en centrales termosolares con colectores lineales.

\begin{tabular}{|c|c|c|c|c|c|c|c|c|c|}
\hline \multicolumn{5}{|c|}{$U A=10000 \mathrm{~kW} / \mathrm{K}$} & \multicolumn{5}{|c|}{$\mathrm{UA}=15000 \mathrm{~kW} / \mathrm{K}$} \\
\hline $\begin{array}{l}\text { TIT } \\
\left({ }^{\circ} \mathrm{C}\right)\end{array}$ & $\begin{array}{l}\text { CIP } \\
\text { (bar) }\end{array}$ & $\begin{array}{l}\text { PreComp. } \\
\text { Inlet (bar) }\end{array}$ & $\begin{array}{l}\text { Flow } \\
\text { Fraction } \\
\text { (\%) }\end{array}$ & $\begin{array}{l}\text { Mass } \\
\text { Flow } \\
(\mathrm{kg} / \mathrm{s})\end{array}$ & $\begin{array}{l}\text { TIT } \\
\left({ }^{\circ} \mathrm{C}\right)\end{array}$ & $\begin{array}{l}\text { CIP } \\
\text { (bar) }\end{array}$ & $\begin{array}{l}\text { PreComp. } \\
\text { Inlet (bar) }\end{array}$ & $\begin{array}{l}\text { Flow } \\
\text { Fraction } \\
\text { (\%) }\end{array}$ & $\begin{array}{l}\text { Mass } \\
\text { Flow } \\
(\mathrm{kg} / \mathrm{s})\end{array}$ \\
\hline 300 & 73.7 & 79.8 & 0.38 & 751.1 & 300 & 73.7 & 80.6 & 0.39 & 752.0 \\
\hline 350 & 73.7 & 80.0 & 0.41 & 631.7 & 350 & 73.7 & 80.8 & 0.41 & 631.4 \\
\hline 400 & 73.7 & 80.2 & 0.43 & 547.9 & 400 & 73.7 & 81.0 & 0.42 & 547.0 \\
\hline 450 & 73.7 & 80.4 & 0.44 & 485.3 & 450 & 73.7 & 81.1 & 0.42 & 484.3 \\
\hline 500 & 73.7 & 80.6 & 0.44 & 436.6 & 500 & 73.7 & 81.2 & 0.43 & 435.6 \\
\hline 550 & 73.7 & 80.7 & 0.45 & 397.4 & 550 & 73.7 & 81.3 & 0.43 & 396.5 \\
\hline
\end{tabular}

Tabla 6.16. Variables termodinámicas para optimizar el rendimiento energético del ciclo de potencia Brayton (PCRC sin Recalentamiento) en centrales termosolares con colectores

\begin{tabular}{|c|c|c|c|c|}
\hline \multicolumn{5}{|c|}{$U A=20000 \mathrm{~kW} / \mathrm{K}$} \\
\hline $\begin{array}{l}\text { TIT } \\
\left({ }^{\circ} \mathrm{C}\right)\end{array}$ & $\begin{array}{l}\text { CIP } \\
\text { (bar) }\end{array}$ & $\begin{array}{l}\text { PreComp. } \\
\text { Inlet (bar) }\end{array}$ & $\begin{array}{l}\text { Flow } \\
\text { Fraction } \\
\text { (\%) }\end{array}$ & $\begin{array}{l}\text { Mass } \\
\text { Flow } \\
(\mathrm{kg} / \mathrm{s})\end{array}$ \\
\hline 300 & 73.7 & 81.0 & 0.39 & 752.2 \\
\hline 350 & 73.7 & 81.2 & 0.40 & 631.0 \\
\hline 400 & 73.7 & 81.3 & 0.41 & 546.5 \\
\hline 450 & 73.7 & 81.4 & 0.42 & 483.8 \\
\hline 500 & 73.7 & 81.5 & 0.42 & 435.1 \\
\hline 550 & 73.7 & 81.7 & 0.42 & 396.1 \\
\hline
\end{tabular}


6. Optimización de las plantas termosolares con colectores lineales con sales fundidas como HTF acopladas a ciclos de potencia Brayton con $\mathrm{CO}_{2}$ supercrítico como fluido de trabajo

Tabla 6.17. Variables termodinámicas para optimizar el rendimiento energético del ciclo de potencia Brayton (PCRC con Recalentamiento) en centrales termosolares con colectores lineales.

\begin{tabular}{|c|c|c|c|c|c|c|c|c|c|}
\hline \multicolumn{5}{|c|}{$\mathrm{UA}=3000 \mathrm{~kW} / \mathrm{K}$} & \multicolumn{5}{|c|}{$\mathrm{UA}=5000 \mathrm{~kW} / \mathrm{K}$} \\
\hline $\begin{array}{l}\text { TIT } \\
\left({ }^{\circ} \mathrm{C}\right)\end{array}$ & $\begin{array}{l}\text { CIP } \\
\text { (bar) }\end{array}$ & $\begin{array}{l}\text { PreCom. } \\
\text { (bar) }\end{array}$ & $\begin{array}{l}\text { ReHeat } \\
\text { Pressure } \\
\text { (bar) }\end{array}$ & $\begin{array}{l}\text { Flow } \\
\text { Fraction } \\
(\%)\end{array}$ & $\begin{array}{l}\mathrm{TIT} \\
\left({ }^{\circ} \mathrm{C}\right)\end{array}$ & $\begin{array}{l}\text { CIP } \\
\text { (bar) }\end{array}$ & $\begin{array}{l}\text { PreCom. } \\
\text { (bar) }\end{array}$ & $\begin{array}{l}\text { ReHeat } \\
\text { Pressure } \\
\text { (bar) }\end{array}$ & $\begin{array}{l}\text { Flow } \\
\text { Fraction } \\
(\%)\end{array}$ \\
\hline 300 & 73.7 & 76.7 & 151.6 & 0.26 & 300 & 73.7 & 78.0 & 145.6 & 0.35 \\
\hline 350 & 73.7 & 76.7 & 151.0 & 0.32 & 350 & 73.7 & 78.1 & 144.8 & 0.40 \\
\hline 400 & 73.7 & 76.8 & 150.7 & 0.36 & 400 & 73.7 & 78.3 & 144.2 & 0.43 \\
\hline 450 & 73.7 & 76.8 & 150.7 & 0.39 & 450 & 73.7 & 78.5 & 143.7 & 0.45 \\
\hline 500 & 73.7 & 76.9 & 150.9 & 0.42 & 500 & 73.7 & 78.6 & 143.4 & 0.47 \\
\hline 550 & 73.7 & 77.0 & 151.1 & 0.44 & 550 & 73.7 & 78.8 & 143.2 & 0.48 \\
\hline
\end{tabular}

Tabla 6.18. Variables termodinámicas para optimizar el rendimiento energético del ciclo de potencia Brayton (PCRC con Recalentamiento) en centrales termosolares con colectores lineales.

\begin{tabular}{|c|c|c|c|c|c|c|c|c|c|}
\hline \multicolumn{5}{|c|}{$U A=10000 \mathrm{~kW} / \mathrm{K}$} & \multicolumn{5}{|c|}{$U A=15000 \mathrm{~kW} / \mathrm{K}$} \\
\hline $\begin{array}{l}\text { TIT } \\
\left({ }^{\circ} \mathrm{C}\right)\end{array}$ & $\begin{array}{l}\text { CIP } \\
\text { (bar) }\end{array}$ & $\begin{array}{l}\text { PreCom. } \\
\text { (bar) }\end{array}$ & $\begin{array}{l}\text { ReHeat } \\
\text { Pressure } \\
\text { (bar) }\end{array}$ & $\begin{array}{l}\text { Flow } \\
\text { Fraction } \\
(\%)\end{array}$ & $\begin{array}{l}\text { TIT } \\
\left({ }^{\circ} \mathrm{C}\right)\end{array}$ & $\begin{array}{l}\text { CIP } \\
\text { (bar) }\end{array}$ & $\begin{array}{l}\text { PreCom. } \\
\text { (bar) }\end{array}$ & $\begin{array}{l}\text { ReHeat } \\
\text { Pressure } \\
\text { (bar) }\end{array}$ & $\begin{array}{l}\text { Flow } \\
\text { Fraction } \\
(\%)\end{array}$ \\
\hline 300 & 73.7 & 79.8 & 140.9 & 0.40 & 300 & 73.7 & 80.7 & 139.6 & 0.40 \\
\hline 350 & 73.7 & 80.1 & 140.1 & 0.42 & 350 & 73.7 & 80.9 & 139.0 & 0.42 \\
\hline 400 & 73.7 & 80.3 & 139.5 & 0.43 & 400 & 73.7 & 81.0 & 138.6 & 0.42 \\
\hline 450 & 73.7 & 80.5 & 139.1 & 0.44 & 450 & 73.7 & 81.2 & 138.2 & 0.43 \\
\hline 500 & 73.7 & 80.6 & 138.7 & 0.45 & 500 & 73.7 & 81.3 & 137.9 & 0.43 \\
\hline 550 & 73.7 & 80.7 & 138.4 & 0.45 & 550 & 73.7 & 81.3 & 137.7 & 0.43 \\
\hline
\end{tabular}

Tabla 6.19. Variables termodinámicas para optimizar el rendimiento energético del ciclo de potencia Brayton (PCRC con Recalentamiento) en centrales termosolares con colectores lineales.

\begin{tabular}{lllll}
\hline UA $=20000 \mathrm{~kW} / \mathrm{K}$ \\
\hline
\end{tabular}


6. Optimización de las plantas termosolares con colectores lineales con sales fundidas como $\mathrm{HTF}$ acopladas a ciclos de potencia Brayton con $\mathrm{CO}_{2}$ supercrítico como fluido de trabajo

6.3.2.4. Ciclo con recompresión y enfriamiento intermedio en el compresor principal ( $\mathrm{RCMCl})$, sin y con recalentamiento

Los resultados de la optimización de los parámetros de operación de la configuración (RCMCl) están resumidos en las tablas 6.20 a 6.25. Al igual que ocurría en las configuraciones anteriormente estudiadas, RC y PCRC, un incremento de la UA de los recuperadores, o de la TIT, da lugar a un incremento de las fracción de caudal óptimas, y un decremento del flujo másico del ciclo para la potencia bruta fija de $50 \mathrm{MWe}$. La presión optima de entra en turbina de recalentamiento tiene unos valores comprendidos entre 140 bar y 157 bar. Su valor se ve reducido conforme se incrementa el TIT o el UA de los recuperadores. También se han optimizado las presiones de entrada en los dos compresores principales. Las presiones óptimas de entrada a los compresores (CIP 1 y CIP 2) se incrementan cuando se aumenta el valor de UA de los recuperadores, y disminuyen conforme se aumenta la TIT.

Tabla 6.20. Variables termodinámicas para optimizar el rendimiento energético del ciclo de potencia Brayton ( $\mathrm{RCMCl} \sin$ Recalentamiento) en centrales termosolares con colectores lineales.

\begin{tabular}{|c|c|c|c|c|c|c|c|c|c|}
\hline \multicolumn{5}{|c|}{$U A=3000 \mathrm{~kW} / \mathrm{K}$} & \multicolumn{5}{|c|}{$\mathrm{UA}=5000 \mathrm{~kW} / \mathrm{K}$} \\
\hline $\begin{array}{l}\text { TIT } \\
\left({ }^{\circ} \mathrm{C}\right)\end{array}$ & $\begin{array}{l}\text { CIP } 1 \\
\text { (bar) }\end{array}$ & $\begin{array}{l}\text { CIP } 2 \\
\text { (bar) }\end{array}$ & $\begin{array}{l}\text { Flow } \\
\text { Fraction } \\
(\%)\end{array}$ & $\begin{array}{l}\text { Mass } \\
\text { Flow } \\
(\mathrm{kg} / \mathrm{s})\end{array}$ & $\begin{array}{l}\text { TIT } \\
\left({ }^{\circ} \mathrm{C}\right)\end{array}$ & $\begin{array}{l}\text { CIP } \\
\text { (bar) }\end{array}$ & $\begin{array}{l}\text { CIP } 2 \\
\text { (bar) }\end{array}$ & $\begin{array}{l}\text { Flow } \\
\text { Fraction } \\
\text { (\%) }\end{array}$ & $\begin{array}{l}\text { Mass } \\
\text { Flow } \\
(\mathrm{kg} / \mathrm{s})\end{array}$ \\
\hline 300 & 73.7 & 76.1 & 0.07 & 745.4 & 300 & 73.7 & 77.5 & 0.26 & 863.0 \\
\hline 350 & 73.7 & 76.4 & 0.21 & 694.5 & 350 & 73.7 & 77.2 & 0.30 & 730.1 \\
\hline 400 & 73.7 & 76.3 & 0.23 & 602.8 & 400 & 73.7 & 77.1 & 0.29 & 619.3 \\
\hline 450 & 73.7 & 76.2 & 0.23 & 527.7 & 450 & 73.7 & 77.0 & 0.30 & 542.2 \\
\hline 500 & 73.7 & 76.2 & 0.23 & 471.0 & 500 & 73.7 & 77.0 & 0.31 & 483.6 \\
\hline 550 & 73.7 & 76.2 & 0.23 & 426.1 & 550 & 73.7 & 76.9 & 0.31 & 437.2 \\
\hline
\end{tabular}

Tabla 6.21. Variables termodinámicas para optimizar el rendimiento energético del ciclo de potencia Brayton ( $\mathrm{RCMCl}$ sin Recalentamiento) en centrales termosolares con colectores lineales.

\begin{tabular}{|c|c|c|c|c|c|c|c|c|c|}
\hline \multicolumn{5}{|c|}{$U A=10000 \mathrm{~kW} / \mathrm{K}$} & \multicolumn{5}{|c|}{$U A=15000 \mathrm{~kW} / \mathrm{K}$} \\
\hline $\begin{array}{l}\text { TIT } \\
\left({ }^{\circ} \mathrm{C}\right)\end{array}$ & $\begin{array}{l}\text { CIP } \\
\text { (bar) }\end{array}$ & $\begin{array}{l}\text { CIP } 2 \\
\text { (bar) }\end{array}$ & $\begin{array}{l}\text { Flow } \\
\text { Fraction } \\
\text { (\%) }\end{array}$ & $\begin{array}{l}\text { Mass } \\
\text { Flow } \\
(\mathrm{kg} / \mathrm{s})\end{array}$ & $\begin{array}{l}\text { TIT } \\
\left({ }^{\circ} \mathrm{C}\right)\end{array}$ & $\begin{array}{l}\text { CIP } \\
\text { (bar) }\end{array}$ & $\begin{array}{l}\text { CIP } 2 \\
\text { (bar) }\end{array}$ & $\begin{array}{l}\text { Flow } \\
\text { Fraction } \\
\text { (\%) }\end{array}$ & $\begin{array}{l}\text { Mass } \\
\text { Flow } \\
\text { (kg/s) }\end{array}$ \\
\hline 300 & 76.1 & 90.2 & 0.35 & 888.6 & 300 & 76.3 & 92.7 & 0.36 & 884.1 \\
\hline 350 & 75.9 & 86.8 & 0.35 & 729.3 & 350 & 76.1 & 89.6 & 0.36 & 728.8 \\
\hline 400 & 75.8 & 85.1 & 0.36 & 625.8 & 400 & 75.9 & 86.6 & 0.37 & 625.8 \\
\hline 450 & 75.7 & 83.7 & 0.37 & 550.1 & 450 & 75.8 & 84.2 & 0.38 & 549.9 \\
\hline 500 & 75.7 & 82.6 & 0.37 & 491.7 & 500 & 75.7 & 82.4 & 0.38 & 491.3 \\
\hline 550 & 75.6 & 81.6 & 0.38 & 445.1 & 550 & 75.6 & 80.9 & 0.39 & 444.6 \\
\hline
\end{tabular}


6. Optimización de las plantas termosolares con colectores lineales con sales fundidas como HTF acopladas a ciclos de potencia Brayton con $\mathrm{CO}_{2}$ supercrítico como fluido de trabajo

Tabla 6.22. Variables termodinámicas para optimizar el rendimiento energético del ciclo de potencia Brayton ( $\mathrm{RCMCl}$ sin Recalentamiento) en centrales termosolares con colectores lineales.

\begin{tabular}{|c|c|c|c|c|}
\hline \multicolumn{5}{|c|}{$U A=20000 \mathrm{~kW} / \mathrm{K}$} \\
\hline $\begin{array}{l}\text { TIT } \\
\left({ }^{\circ} \mathrm{C}\right)\end{array}$ & $\begin{array}{l}\text { CIP } \\
\text { (bar) }\end{array}$ & $\begin{array}{l}\text { CIP } 2 \\
\text { (bar) }\end{array}$ & $\begin{array}{l}\text { Flow } \\
\text { Fraction } \\
(\%)\end{array}$ & $\begin{array}{l}\text { Mass } \\
\text { Flow } \\
(\mathrm{kg} / \mathrm{s})\end{array}$ \\
\hline 300 & 76.5 & 95.2 & 0.36 & 880.1 \\
\hline 350 & 76.2 & 90.8 & 0.37 & 728.1 \\
\hline 400 & 75.9 & 86.8 & 0.38 & 625.0 \\
\hline 450 & 75.8 & 83.9 & 0.38 & 548.9 \\
\hline 500 & 75.7 & 81.8 & 0.38 & 490.3 \\
\hline 550 & 75.6 & 80.2 & 0.38 & 443.5 \\
\hline
\end{tabular}

Tabla 6.23. Variables termodinámicas para optimizar el rendimiento energético del ciclo de potencia Brayton ( $\mathrm{RCMCl}$ con Recalentamiento) en centrales termosolares con colectores lineales.

\begin{tabular}{|c|c|c|c|c|c|c|c|c|c|}
\hline \multicolumn{5}{|c|}{$\mathrm{UA}=3000 \mathrm{~kW} / \mathrm{K}$} & \multicolumn{5}{|c|}{$\mathrm{UA}=5000 \mathrm{~kW} / \mathrm{K}$} \\
\hline $\begin{array}{l}\text { TIT } \\
\left({ }^{\circ} \mathrm{C}\right)\end{array}$ & $\begin{array}{l}\text { CIP } 1 \\
\text { (bar) }\end{array}$ & $\begin{array}{l}\text { CIP } 2 \\
\text { (bar) }\end{array}$ & $\begin{array}{l}\text { ReHeating } \\
\text { Pressure } \\
\text { (bar) }\end{array}$ & $\begin{array}{l}\text { Flow } \\
\text { Fraction } \\
(\%)\end{array}$ & $\begin{array}{l}\text { TIT } \\
\left({ }^{\circ} \mathrm{C}\right)\end{array}$ & $\begin{array}{l}\text { CIP } 1 \\
\text { (bar) }\end{array}$ & $\begin{array}{l}\text { CIP } 2 \\
\text { (bar) }\end{array}$ & $\begin{array}{l}\text { ReHeating } \\
\text { Pressure } \\
\text { (bar) }\end{array}$ & $\begin{array}{l}\text { Flow } \\
\text { Fraction } \\
(\%)\end{array}$ \\
\hline 300 & 73.7 & 76.1 & 152.3 & 0.11 & 300 & 75.6 & 80.7 & 153.2 & 0.26 \\
\hline 350 & 73.7 & 76.2 & 155.3 & 0.18 & 350 & 75.6 & 80.0 & 152.6 & 0.28 \\
\hline 400 & 73.7 & 76.2 & 155.7 & 0.19 & 400 & 75.6 & 79.7 & 152.2 & 0.29 \\
\hline 450 & 73.7 & 76.2 & 156.0 & 0.20 & 450 & 75.5 & 79.3 & 152.1 & 0.30 \\
\hline 500 & 73.7 & 76.2 & 156.4 & 0.21 & 500 & 75.4 & 78.7 & 152.1 & 0.31 \\
\hline 550 & 73.7 & 76.2 & 156.8 & 0.21 & 550 & 73.7 & 76.9 & 150.4 & 0.31 \\
\hline
\end{tabular}

Tabla 6.24. Variables termodinámicas para optimizar el rendimiento energético del ciclo de potencia Brayton ( $\mathrm{RCMCl}$ con Recalentamiento) en centrales termosolares con colectores lineales.

\begin{tabular}{|c|c|c|c|c|c|c|c|c|c|}
\hline \multicolumn{5}{|c|}{$U A=10000 \mathrm{~kW} / \mathrm{K}$} & \multicolumn{5}{|c|}{$U A=15000 \mathrm{~kW} / \mathrm{K}$} \\
\hline $\begin{array}{l}\text { TIT } \\
\left({ }^{\circ} \mathrm{C}\right)\end{array}$ & $\begin{array}{l}\text { CIP } 1 \\
\text { (bar) }\end{array}$ & $\begin{array}{l}\text { CIP } 2 \\
\text { (bar) }\end{array}$ & $\begin{array}{l}\text { ReHeating } \\
\text { Pressure } \\
\text { (bar) }\end{array}$ & $\begin{array}{l}\text { Flow } \\
\text { Fraction } \\
\text { (\%) }\end{array}$ & $\begin{array}{l}\text { TIT } \\
\left({ }^{\circ} \mathrm{C}\right)\end{array}$ & $\begin{array}{l}\text { CIP } 1 \\
\text { (bar) }\end{array}$ & $\begin{array}{l}\text { CIP } 2 \\
\text { (bar) }\end{array}$ & $\begin{array}{l}\text { ReHeating } \\
\text { Pressure } \\
\text { (bar) }\end{array}$ & $\begin{array}{l}\text { Flow } \\
\text { Fraction } \\
\text { (\%) }\end{array}$ \\
\hline 300 & 75.9 & 87.4 & 147.4 & 0.33 & 300 & 76.0 & 90.6 & 144.7 & 0.35 \\
\hline 350 & 75.8 & 85.6 & 146.2 & 0.35 & 350 & 75.8 & 87.3 & 143.5 & 0.37 \\
\hline 400 & 75.7 & 84.1 & 145.5 & 0.36 & 400 & 75.7 & 84.8 & 142.7 & 0.38 \\
\hline 450 & 75.7 & 82.9 & 145.0 & 0.37 & 450 & 75.7 & 82.8 & 142.1 & 0.38 \\
\hline 500 & 75.6 & 81.9 & 144.6 & 0.37 & 500 & 75.6 & 81.3 & 141.7 & 0.38 \\
\hline 550 & 75.6 & 81.1 & 144.3 & 0.38 & 550 & 75.6 & 79.9 & 141.3 & 0.39 \\
\hline
\end{tabular}


6. Optimización de las plantas termosolares con colectores lineales con sales fundidas como HTF acopladas a ciclos de potencia Brayton con $\mathrm{CO}_{2}$ supercrítico como fluido de trabajo

Tabla 6.25. Variables termodinámicas para optimizar el rendimiento energético del ciclo de potencia Brayton ( $\mathrm{RCMCl}$ con Recalentamiento) en centrales termosolares con colectores lineales (PTC o LF).

\begin{tabular}{|c|c|c|c|c|}
\hline \multicolumn{5}{|c|}{$U A=20000 \mathrm{~kW} / \mathrm{K}$} \\
\hline $\begin{array}{l}\text { TIT } \\
\left({ }^{\circ} \mathrm{C}\right)\end{array}$ & $\begin{array}{l}\text { CIP } \\
\text { (bar) }\end{array}$ & $\begin{array}{l}\text { CIP } 2 \\
\text { (bar) }\end{array}$ & $\begin{array}{l}\text { ReHeating } \\
\text { Pressure } \\
\text { (bar) }\end{array}$ & $\begin{array}{l}\text { Flow } \\
\text { Fraction } \\
\text { (\%) }\end{array}$ \\
\hline 300 & 76.1 & 92.0 & 143.3 & 0.36 \\
\hline 350 & 75.9 & 87.7 & 142.2 & 0.37 \\
\hline 400 & 75.8 & 84.5 & 141.5 & 0.38 \\
\hline 450 & 75.7 & 82.2 & 140.9 & 0.38 \\
\hline 500 & 75.6 & 80.5 & 140.5 & 0.38 \\
\hline 550 & 75.6 & 79.1 & 140.1 & 0.38 \\
\hline
\end{tabular}

\subsubsection{Tamaño Térmico de los recuperadores del ciclo Brayton}

En las figuras 6.7 a 6.18 se representan gráficamente las variaciones de los tamaños térmicos óptimos de los recuperadores de calor de baja presión LTR, y de alta presión HTR, para diferentes valores de la TIT, y fijando el valor total de tamaño térmico de los recuperadores.
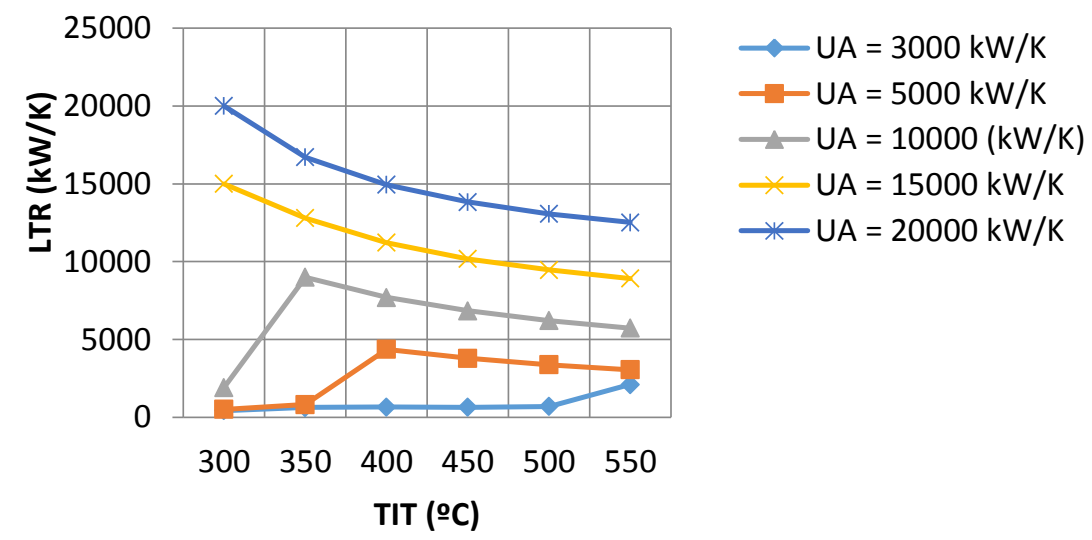

Figura 6.7. Tamaño térmico óptimo del Recuperador de Baja Presión (LTR), frente a TIT, ciclo termodinámico $(\mathrm{RC}) \mathrm{s}-\mathrm{CO}_{2} \sin$ Recalentamiento.

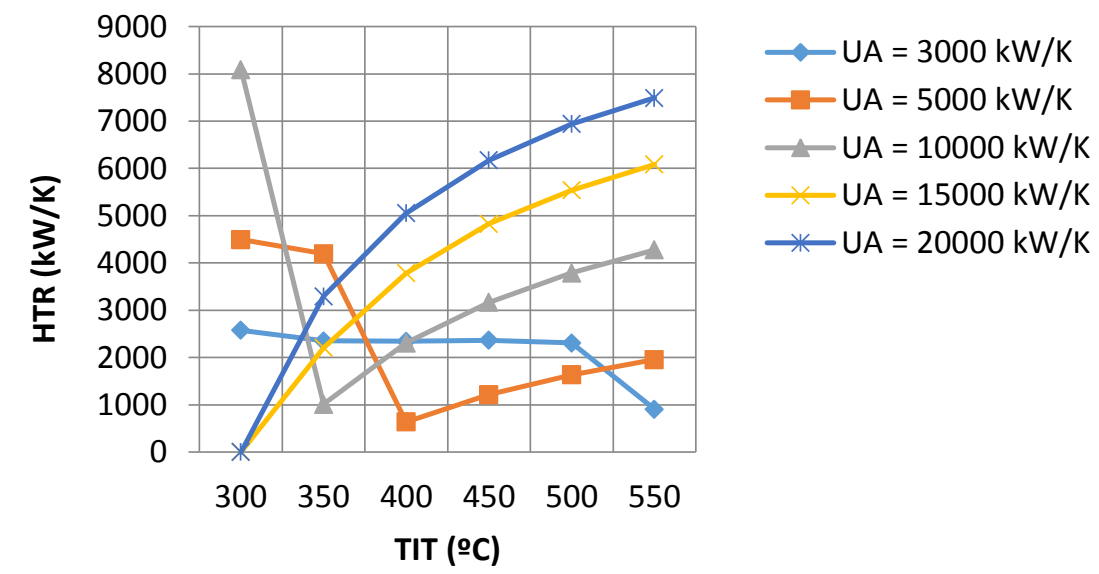

Figura 6.8. Tamaño térmico óptimo del Recuperador de Alta Presión (HTR), frente a TIT. Ciclo termodinámico $(\mathrm{RC}) \mathrm{s}-\mathrm{CO}_{2} \sin$ Recalentamiento. 
6. Optimización de las plantas termosolares con colectores lineales con sales fundidas como $\mathrm{HTF}$ acopladas a ciclos de potencia Brayton con $\mathrm{CO}_{2}$ supercrítico como fluido de trabajo

Es decir, mediante las citadas gráficas, figuras 6.7 a 6.18 , se ha cuantificado la fracción de tamaño térmico óptimo, tanto de los recuperadores LTR, como de los recuperadores HTR, para diferentes valores del tamaño térmico total UA de los recuperadores del ciclo Brayton.

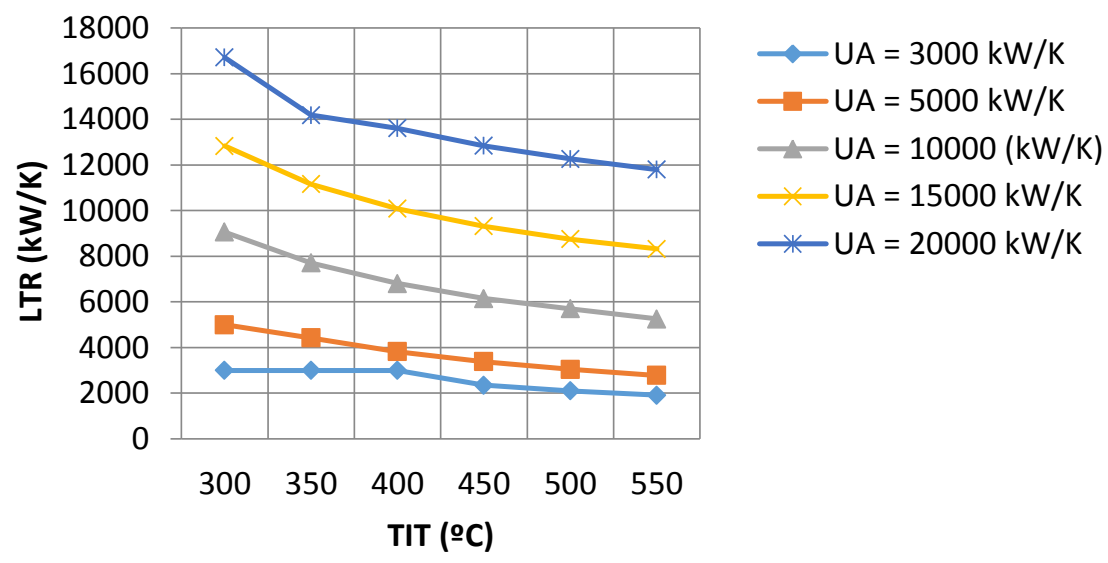

Figura 6.9. Tamaño térmico óptimo del Recuperador de Baja Presión (LTR), frente a TIT. Ciclo termodinámico $(\mathrm{RC}) \mathrm{s}-\mathrm{CO}_{2}$ con Recalentamiento.
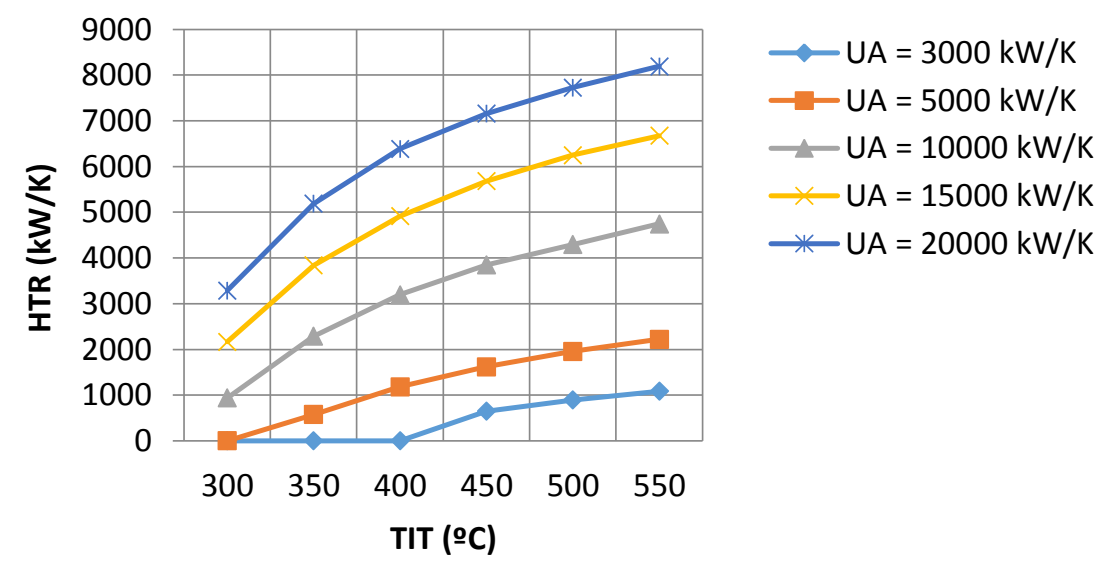

Figura 6.10. Tamaño térmico óptimo del Recuperador de Baja Presión (HTR), frente a TIT. Ciclo termodinámico $(\mathrm{RC}) \mathrm{s}-\mathrm{CO}_{2}$ con Recalentamiento.

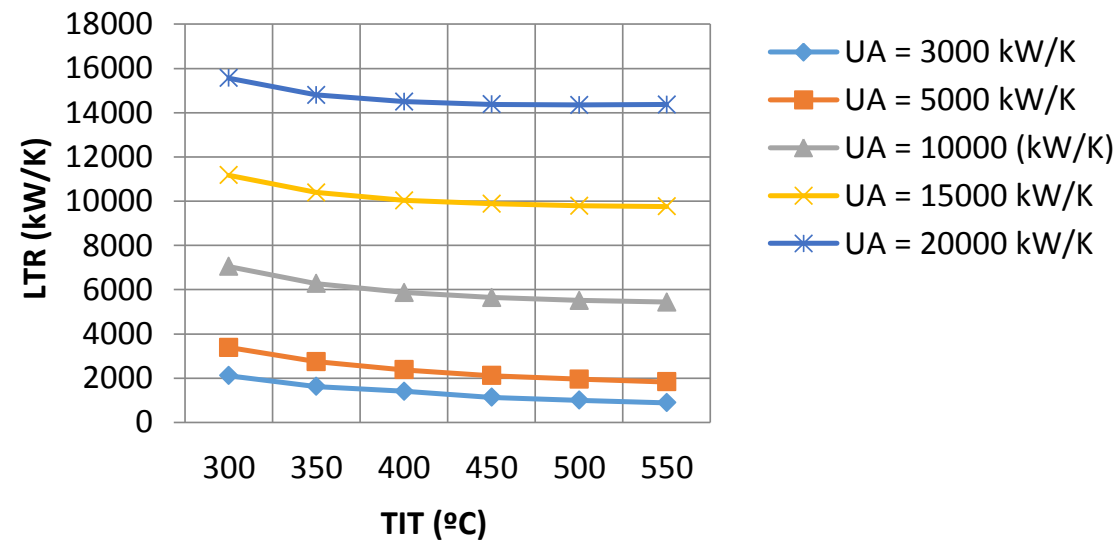

Figura 6.11. Tamaño térmico óptimo del Recuperador de Baja Presión (LTR), frente a TIT. Ciclo termodinámico ( $\mathrm{PCRC}$ ) $\mathrm{s}-\mathrm{CO}_{2} \sin$ Recalentamiento. 
6. Optimización de las plantas termosolares con colectores lineales con sales fundidas como HTF acopladas a ciclos de potencia Brayton con $\mathrm{CO}_{2}$ supercrítico como fluido de trabajo

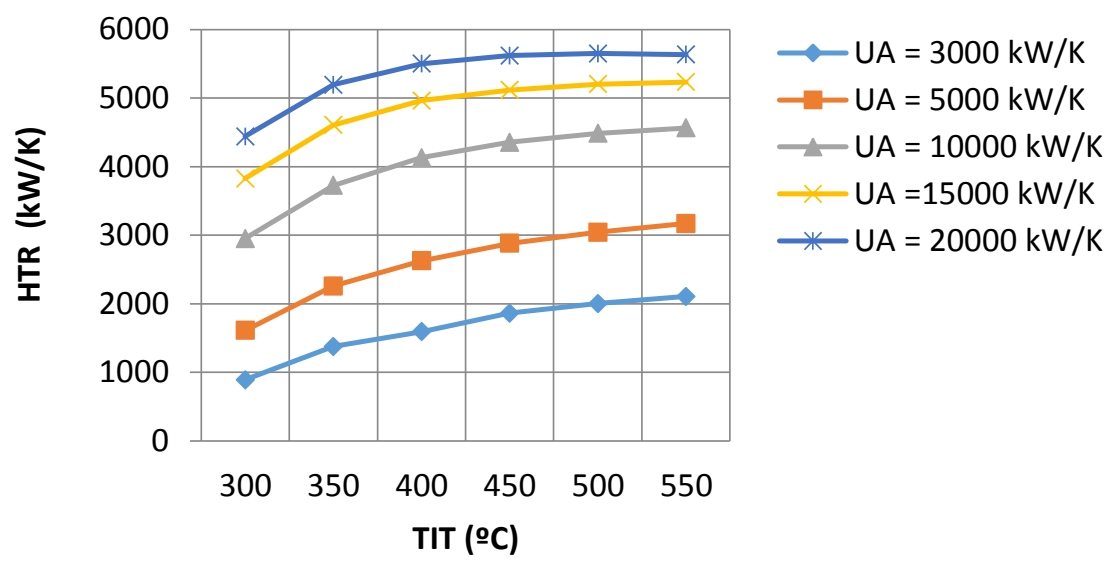

Figura 6.12. Tamaño térmico óptimo del Recuperador de Baja Presión (HTR), frente a la TIT. Ciclo termodinámico (PCRC) s- $\mathrm{CO}_{2} \sin$ Recalentamiento.

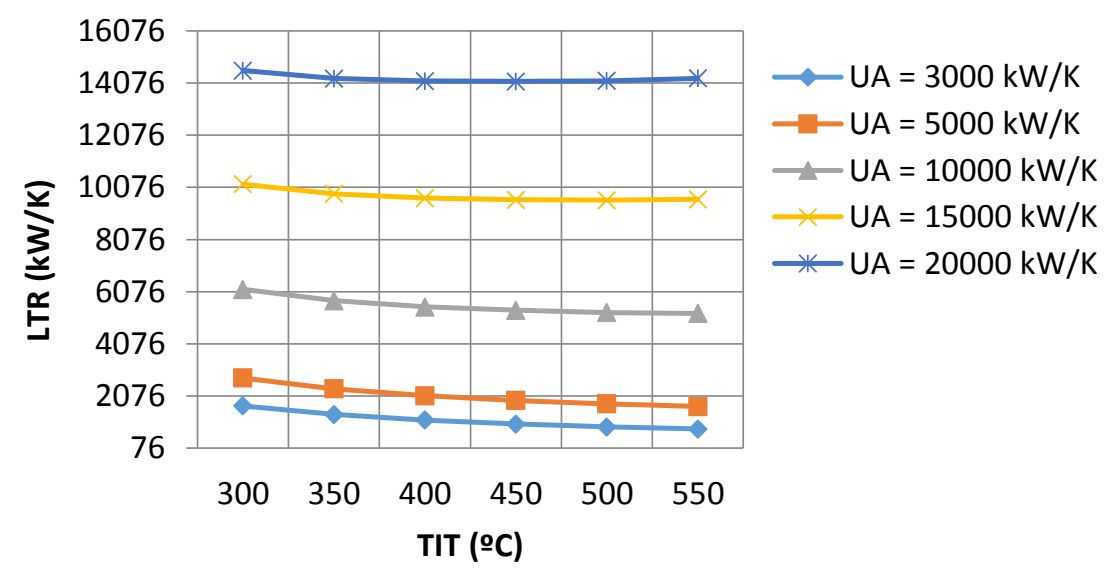

Figura 6.13. Tamaño térmico óptimo del Recuperador de Baja Presión (LTR), frente a TIT. Ciclo termodinámico (PCRC) $\mathrm{s}-\mathrm{CO}_{2}$ con Recalentamiento.

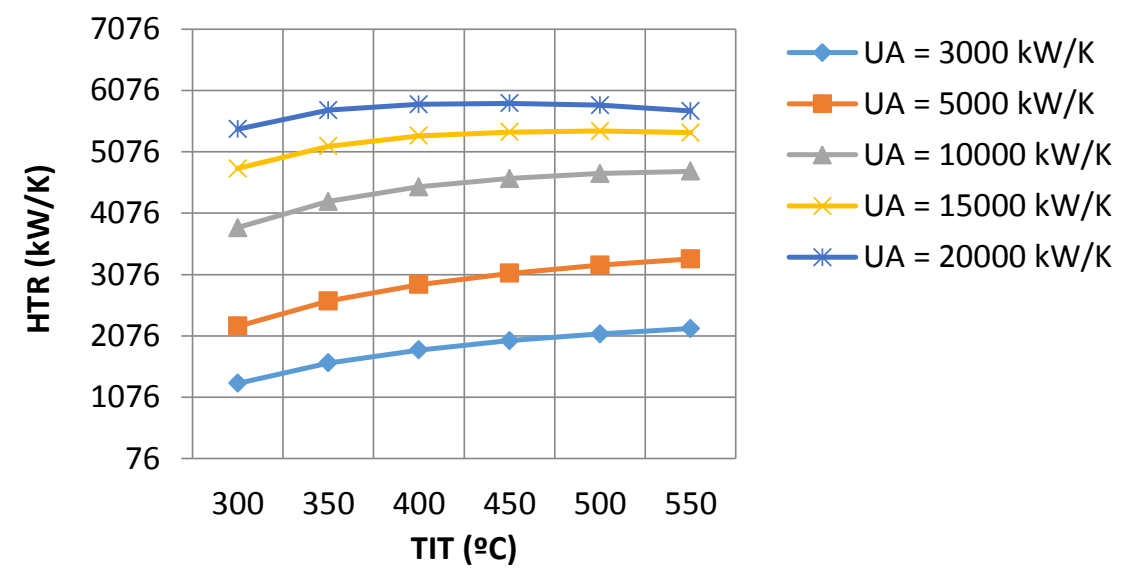

Figura 6.14. Tamaño térmico óptimo del Recuperador de Baja Presión (HTR), frente a TIT. Ciclo termodinámico (PCRC) s- $\mathrm{CO}_{2}$ con Recalentamiento. 
6. Optimización de las plantas termosolares con colectores lineales con sales fundidas como HTF acopladas a ciclos de potencia Brayton con $\mathrm{CO}_{2}$ supercrítico como fluido de trabajo

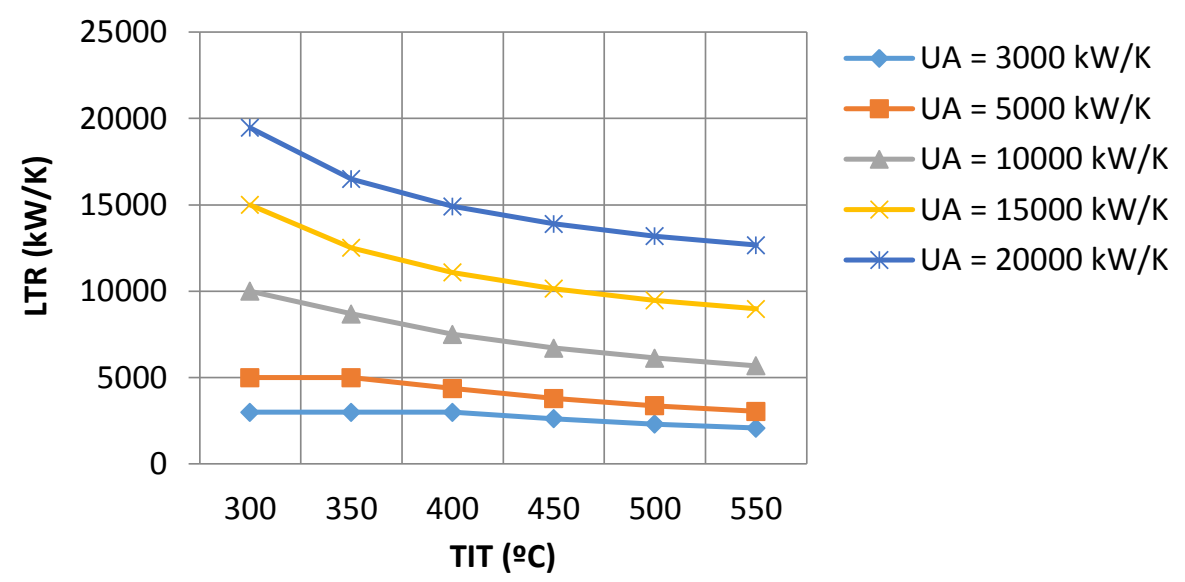

Figura 6.15. Tamaño térmico óptimo del Recuperador de Baja Presión (LTR), frente a TIT. Ciclo termodinámico $(\mathrm{RCMCl}) \mathrm{s}-\mathrm{CO}_{2} \sin$ Recalentamiento.

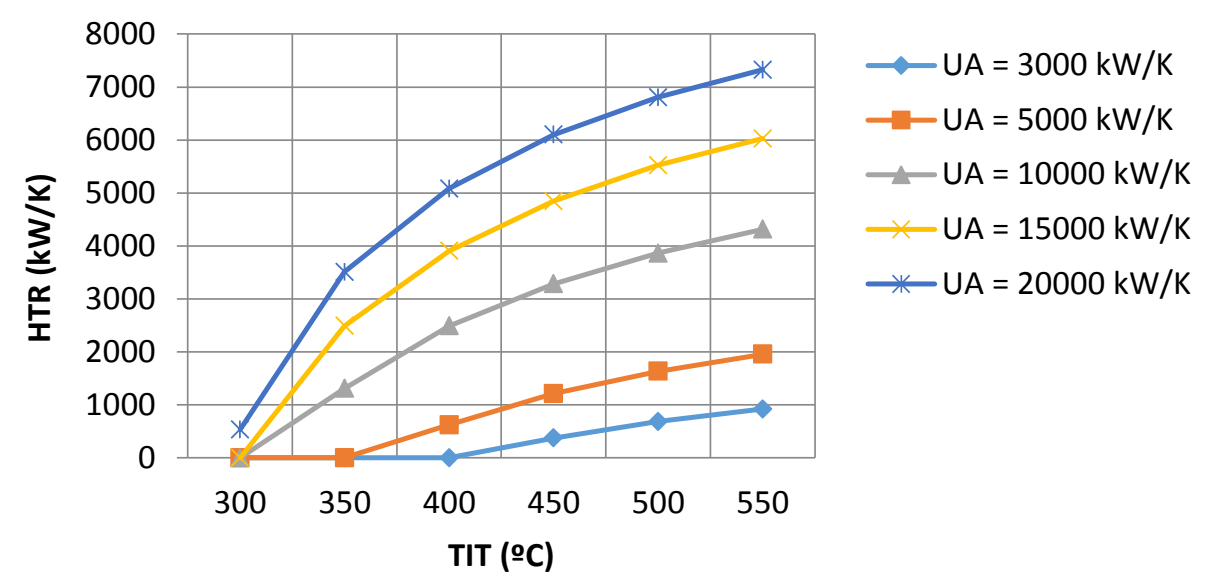

Figura 6.16. Tamaño térmico óptimo del Recuperador de Baja Presión (HTR), frente a TIT. Ciclo termodinámico ( $\mathrm{RCMCl}$ ) $\mathrm{s}-\mathrm{CO}_{2} \sin$ Recalentamiento.

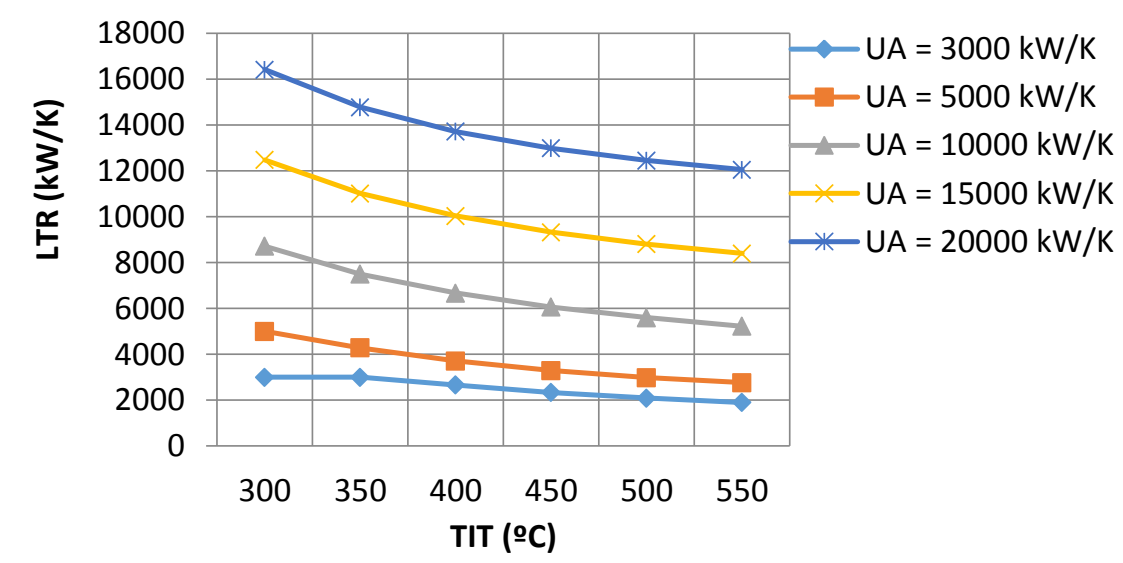

Figura 6.17. Tamaño térmico óptimo del Recuperador de Baja Presión (LTR), frente a TIT. Ciclo termodinámico $(\mathrm{RCMCl}) \mathrm{s}-\mathrm{CO}_{2}$ con Recalentamiento. 
6. Optimización de las plantas termosolares con colectores lineales con sales fundidas como $\mathrm{HTF}$ acopladas a ciclos de potencia Brayton con $\mathrm{CO}_{2}$ supercrítico como fluido de trabajo

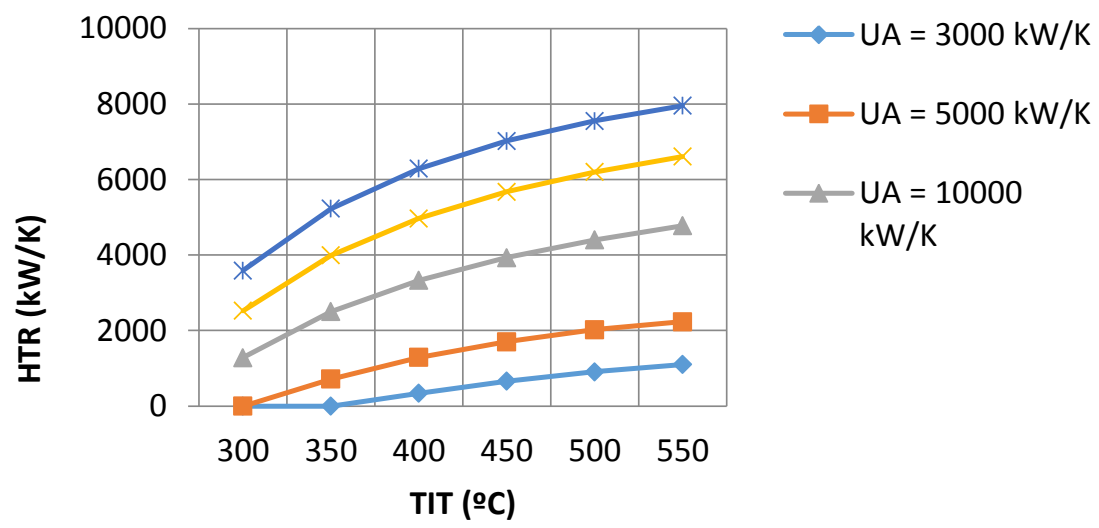

Figura 6.18. Tamaño térmico óptimo del Recuperador de Baja Presión (HTR), frente a TIT. Ciclo termodinámico ( $\mathrm{RCMCl}) \mathrm{s}-\mathrm{CO}_{2}$ con Recalentamiento.

\subsection{4. Área de Apertura Efectiva del Campo Solar}

En este capítulo se ha demostrado como un incremento tanto de la TIT, como del tamaño térmico total de los recuperadores en el ciclo Brayton, dan lugar a un incremento de la eficiencia neta del ciclo Brayton, y como consecuencia directa, una reducción del área de apertura efectiva de los colectores solares. En las figuras 6.19 a 6.24 se representan gráficamente las diferentes áreas de apertura efectiva conforme se incrementan TIT y UA de los recuperadores del ciclo Brayton. Cabe destacar que en todas la figuras indicadas, hay un valor de tamaño térmico umbral (UA=10000-15000 kW/K), a partir del cual la reducción del área de apertura efectiva de los colectores solares no es significativa. El motivo también ha sido comentado en los apartados anteriores: un incremento de UA de los recuperadores da lugar a una reducción de la diferencia mínima de temperaturas o pinch-point entre las corrientes fría y caliente de los recuperadores. Se considera como valores característicos de operación de los recuperadores una temperaturas de pinch-point entre $5-10^{\circ} \mathrm{C}$.

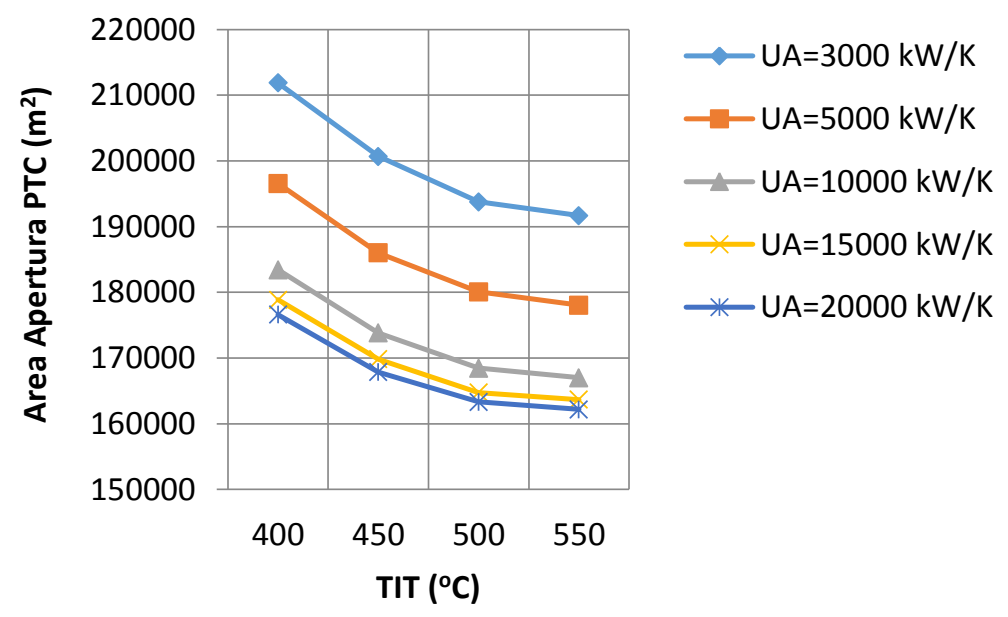

Figura 6.19. Área de Apertura Efectiva con colectores solares MS PTC y ciclo de potencia $\mathrm{RC} \mathrm{s}-\mathrm{CO}_{2}$ con recalentamiento. 
6. Optimización de las plantas termosolares con colectores lineales con sales fundidas como $\mathrm{HTF}$ acopladas a ciclos de potencia Brayton con $\mathrm{CO}_{2}$ supercrítico como fluido de trabajo

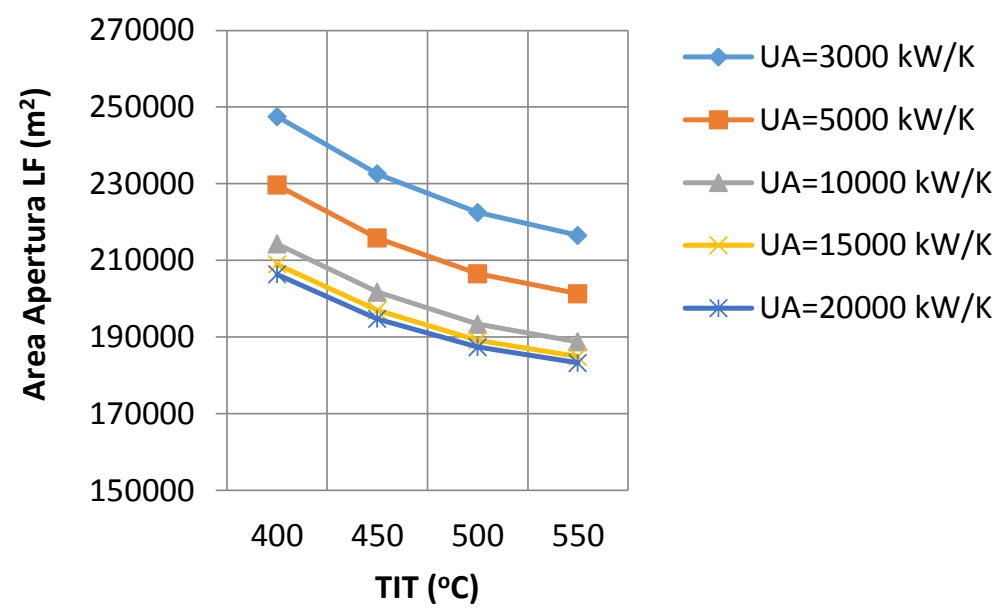

Figura 6.20. Área de Apertura Efectiva con colectores solares MS LF y ciclo de potencia $\mathrm{RC}$ s- $\mathrm{CO}_{2}$ con recalentamiento.

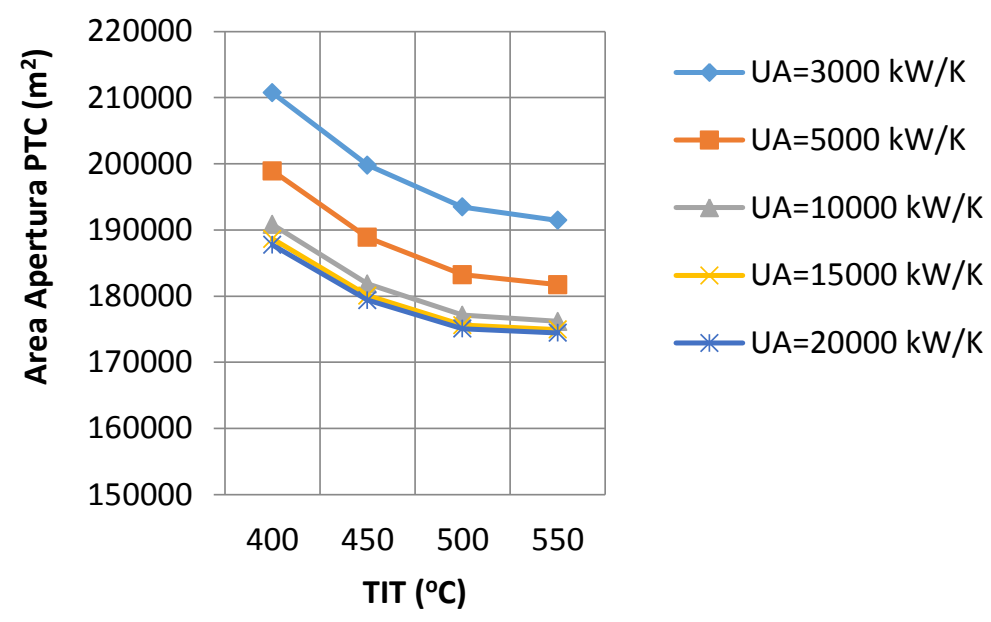

Figura 6.21. Área de Apertura Efectiva con colectores solares MS PTC y ciclo de potencia $\mathrm{PCRC}$ s- $\mathrm{CO}_{2}$ con recalentamiento.

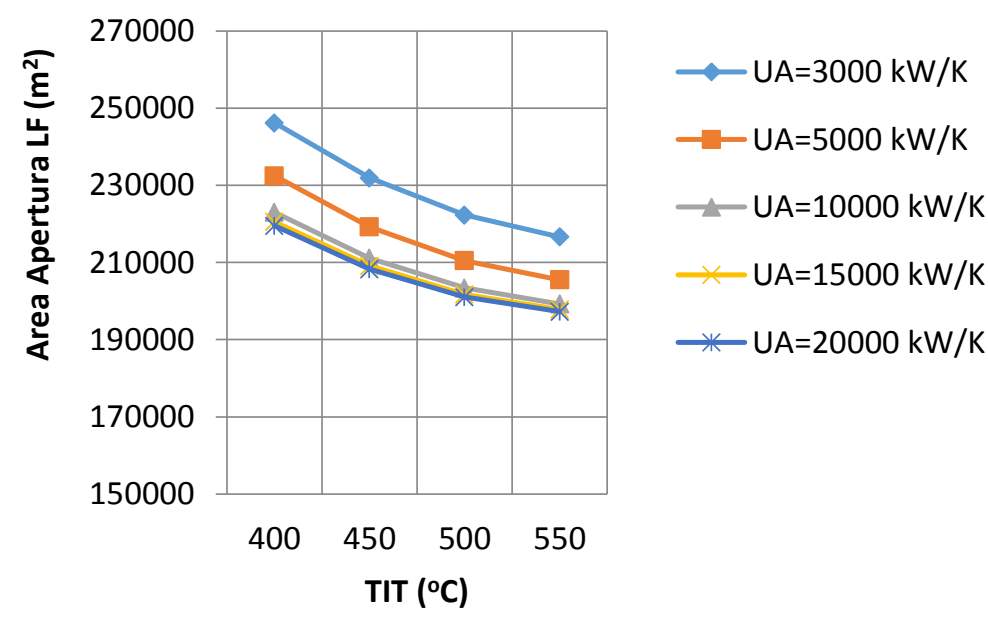

Figura 6.22. Área de Apertura Efectiva con colectores solares MS LF y ciclo de potencia $\mathrm{PCRC}$ s- $\mathrm{CO}_{2}$ con recalentamiento. 
6. Optimización de las plantas termosolares con colectores lineales con sales fundidas como $\mathrm{HTF}$ acopladas a ciclos de potencia Brayton con $\mathrm{CO}_{2}$ supercrítico como fluido de trabajo

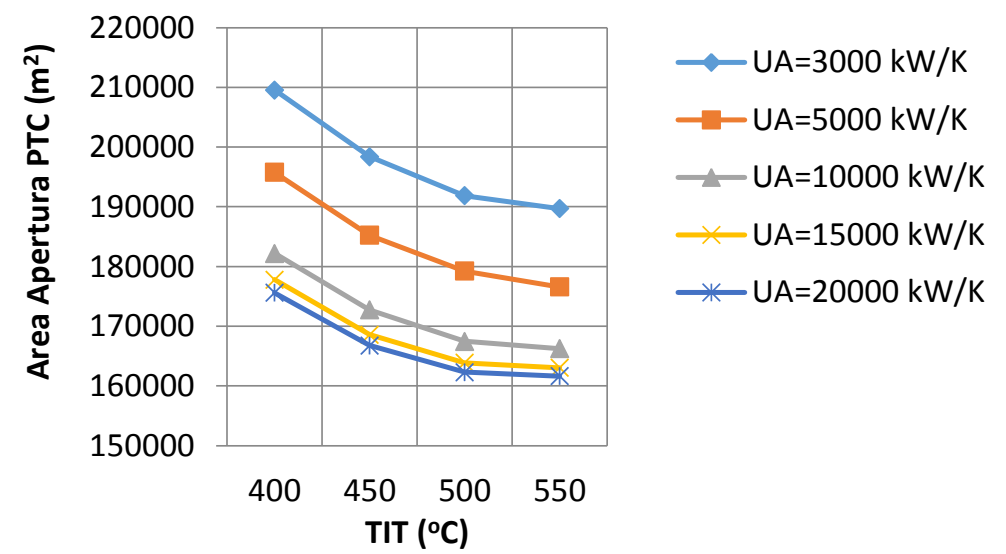

Figura 6.23. Área de Apertura Efectiva con colectores solares MS PTC y ciclo de potencia $\mathrm{RCMCl}$ s- $\mathrm{CO}_{2}$ con recalentamiento.

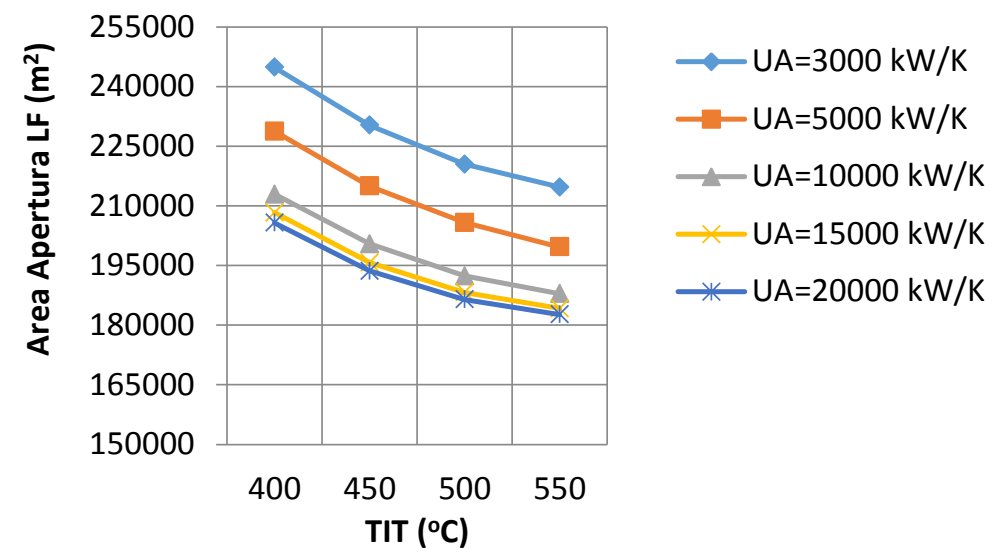

Figura 6.24. Área de Apertura Efectiva con colectores solares MS LF y ciclo de potencia $\mathrm{RCMCl} \mathrm{s-} \mathrm{CO}_{2}$ con recalentamiento.

\subsubsection{Estimación de Costes del Campo Solar}

Los resultados del apartado anterior, áreas de apertura efectiva total de los campos solares de las diferentes configuraciones de planta termosolar estudiadas, han sido traducidas en términos monetarios para facilitar la comparación entre ellas. En este apartado se ha cuantificado económicamente el coste de los campos solares para diferentes valores de tamaño térmico de los recuperadores del ciclo Brayton. Los resultados detallados del coste de inversión de los campos solares están resumidos en las figuras 6.25 a 6.30. Se han estudiado las dos posibles tipologías de colectores lineales PTC o LF. Los costes unitarios considerados en dichas estimaciones económicas se detallan a continuación:

- Colectores solares PTC, tipología Eurotrough II:

HTF sales fundidas, material Acero Inoxidable AISI 347.

Precio unitario: $432 \$ / \mathrm{m}^{2}$ [34]

Factor multiplicativo para cubrir costes de instalación: 1.16 [34]

- Colectores solares LF, marca comercial Novatec:

HTF sales fundidas, material Acero Inoxidable AISI 347.

Precio unitario: $300 \$ / \mathrm{m}^{2}$ [34]

Factor multiplicativo para cubrir costes de instalación: 1.16 [34] 
6. Optimización de las plantas termosolares con colectores lineales con sales fundidas como $\mathrm{HTF}$ acopladas a ciclos de potencia Brayton con $\mathrm{CO}_{2}$ supercrítico como fluido de trabajo

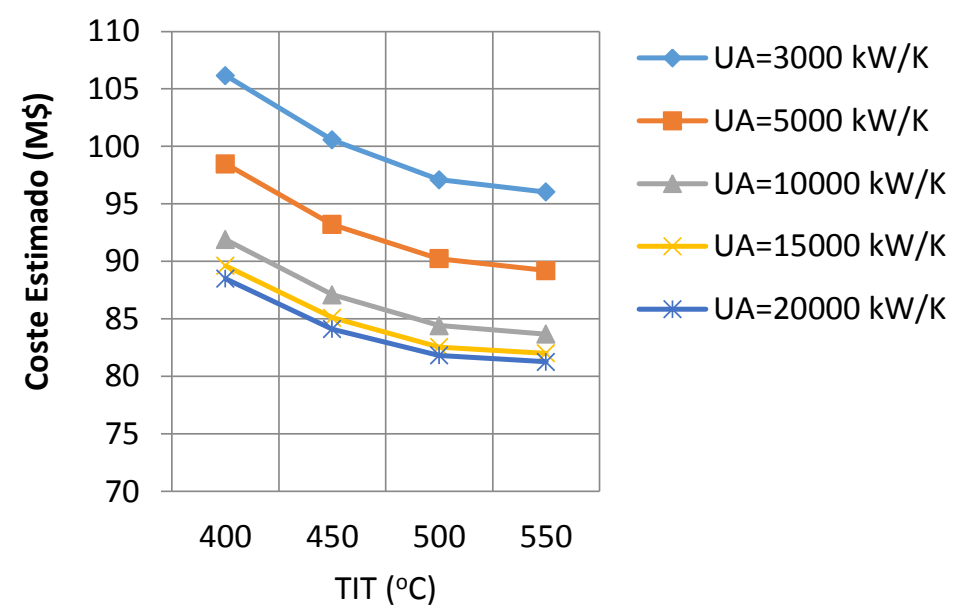

Figura 6.25. Estimación de Costes de los colectores solares PTC y ciclo de potencia $\mathrm{RC}$ s- $\mathrm{CO}_{2}$ con recalentamiento.

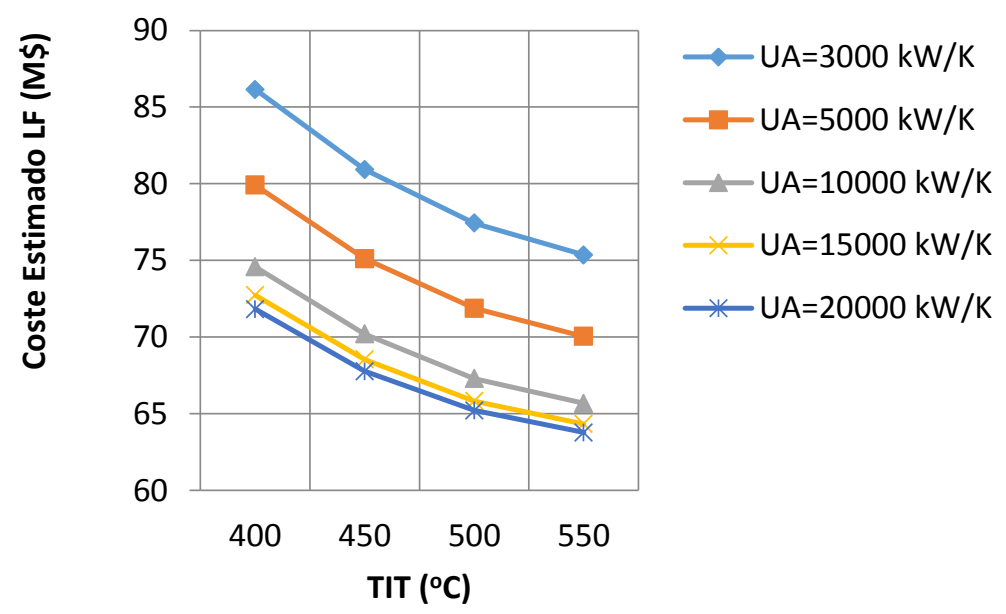

Figura 6.26. Estimación de Costes de los colectores solares LF y ciclo de potencia $\mathrm{RC} \mathrm{s}-\mathrm{CO}_{2}$ con recalentamiento.

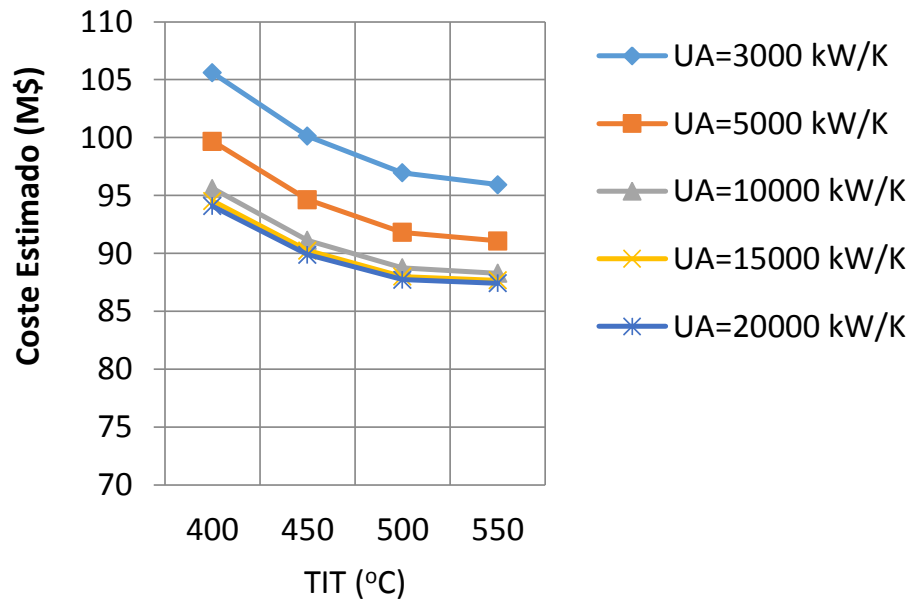

Figura 6.27. Estimación de Costes de los colectores solares PTC y ciclo de potencia $\mathrm{PCRC}$ s- $-\mathrm{CO}_{2}$ con recalentamiento. 
6. Optimización de las plantas termosolares con colectores lineales con sales fundidas como HTF acopladas a ciclos de potencia Brayton con $\mathrm{CO}_{2}$ supercrítico como fluido de trabajo

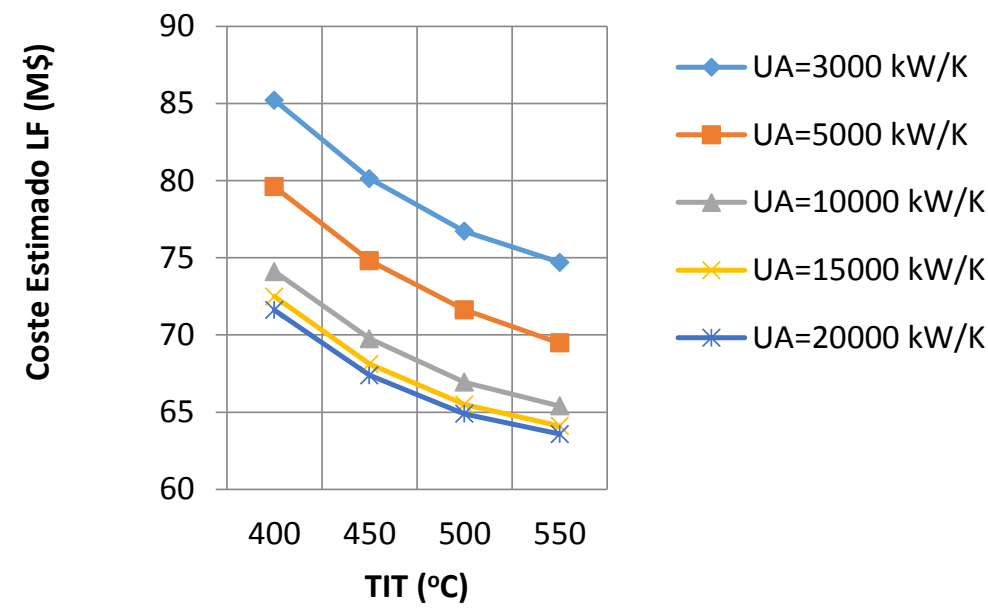

Figura 6.28. Estimación de Costes de los colectores solares LF y ciclo de potencia $\mathrm{PCRC} s-\mathrm{CO}_{2}$ con recalentamiento.

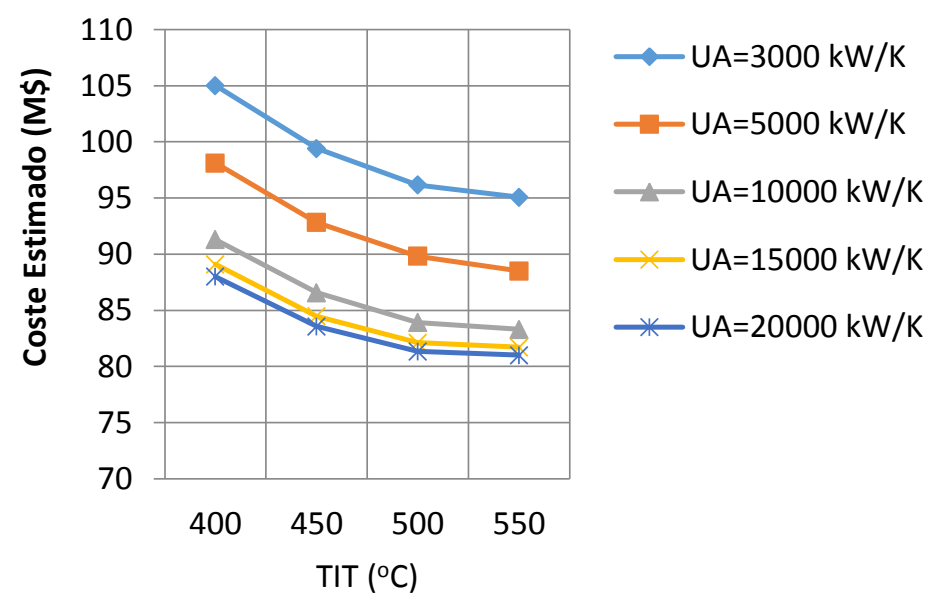

Figura 6.29. Estimación de Costes de los colectores solares PTC y ciclo de potencia $\mathrm{RCMCl} \mathrm{s}-\mathrm{CO}_{2}$ con recalentamiento.

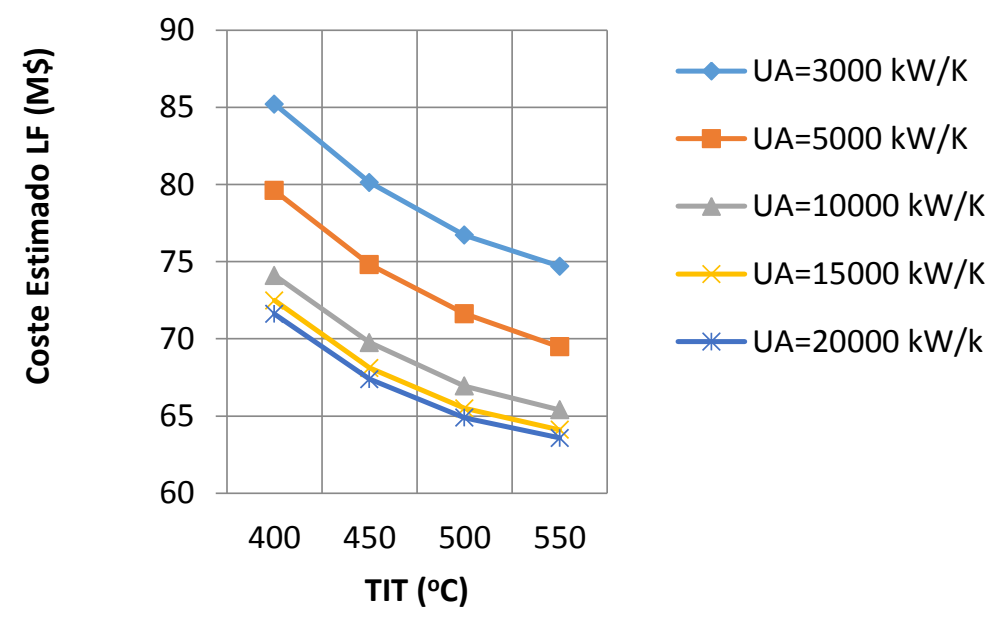

Figura 6.30. Estimación de Costes de los colectores solares LF y ciclo de potencia $\mathrm{RCMCl} \mathrm{s}-\mathrm{CO}_{2}$ con recalentamiento. 
6. Optimización de las plantas termosolares con colectores lineales con sales fundidas como

$\mathrm{HTF}$ acopladas a ciclos de potencia Brayton con $\mathrm{CO}_{2}$ supercrítico como fluido de trabajo

\subsection{Conclusiones}

Gracias a la integración de los algoritmos de optimización multiparámetro SUBPLEX [1], UOBYQA [2-4] y NEWUOA [5-6], en los métodos de cálculo de los balances energéticos de las plantas termosolares con colectores lineales, sales fundidas como HTF y ciclos de potencia Brayton, se han conseguido obtener unos valores de eficiencia energética del $50 \%$ con las configuraciones de los ciclos $\mathrm{RC}$ y $\mathrm{RCMCl}$, muy superiores a los resultados obtenidos en la presente tesis, en el capítulo 5, sin algoritmos de optimización. Similares trabajos de optimización multiparámetro de las variables de operación de los ciclos de potencia supercríticos Brayton se han realizado recientemente en las referencias [7, 35-37].

Como principal innovación en esta tesis en relación a las publicaciones citadas es la integración de los algoritmos UOBYQA [2-4] y NEWUOA [5-6], para validar los resultados proporcionados por SUBPLEX [1]. Se ha continuado con el trabajo utilizado como referencia [7], integrando los ciclos Brayton en las plantas termosolares con colectores lineales. Se ha realizado el dimensionamiento en detalle de los recuperadores y resto de intercambiadores del ciclo de potencia. En lo referente a las configuraciones de dichos ciclos, se han incrementado el número de configuraciones simuladas, incluyendo también PCRC y $\mathrm{RCMCl}$, además de las $\mathrm{SB}$ y $\mathrm{RC}$, en todas ellas se ha incluido como nueva opción el recalentamiento en turbinas.

Se ha demostrado en el apartado 6.4.1 como el incremento de eficiencia de los ciclos Brayton está íntimamente relacionado con el incremento del tamaño térmico de sus recuperadores UA, con la temperatura de entrada a turbina TIT, y con la optimización de sus parámetros operacionales. En este sentido, en este trabajo se ha definido en los apartado 6.4 .2 y 6.4.3, los parámetros operacionales óptimos: fracción de caudal, presión de recalentamiento, presión de entrada en los compresores, tamaño térmico de los recuperadores de alta y baja presión, etc., que proporcionan el mencionado valor máximo de eficiencia neta.

Finalmente en los apartados 6.4 .4 y 6.4 .5 , se ha traducido a términos de área de apertura efectiva y coste de inversión de los colectores solares, los valores de eficiencia bruta del ciclo de potencia Brayton. Se confirma como valores umbrales del tamaño térmico de los recuperadores, $\mathrm{UA}=10000-15000 \mathrm{~kW} / \mathrm{K}$, para optimizar el coste de los colectores solares.

\subsection{Referencias}

[1] T. Rowan. Functional Stability Analysis of Numerical Algorithms. Ph.D. thesis, Department of Computer Sciences, University of Texas at Austin, 1990.

[2] Powell M.J.D. (December 2000). UOBYQA: unconstrained optimization by quadratic approximation (Report). Department of Applied Mathematics and Theoretical Physics, Cambridge University. DAMTP 2000/NA14. Retrieved 2015-04-06.

[3] Powell M.J.D. (2002). UOBYQA: Unconstrained Optimization BY Quadratic Approximation. Mathematical Programming, Series B (Springer) 92: 555-582.

[4] Powell M.J.D. Source code of UOBYQA software: Unconstrained Optimization BY Quadratic Approximation. Department of Applied Mathematics and Theoretical Physics, Cambridge University. http://mat.uc.pt/ zhang/software.html

[5] Powell M.J.D. Source code of NEWUOA software: NEW Unconstrained Optimization Algorithm. Department of Applied Mathematics and Theoretical Physics, Cambridge University. 
6. Optimización de las plantas termosolares con colectores lineales con sales fundidas como HTF acopladas a ciclos de potencia Brayton con $\mathrm{CO}_{2}$ supercrítico como fluido de trabajo

[6] Powell M.J.D. (November 2004). The NEWUOA software for unconstrained optimization without derivatives. Department of Applied Mathematics and Theoretical Physics, Cambridge University. DAMTP 2004/NA05. Retrieved 2014-01-14.

[7] John J. Dyreby, Gregory F.Nellis, Sanford A. Klein, et al. Thesis: Modeling the Supercritical Carbon Dioxide Brayton Cycle with Recompression. University of Wisconsin-Madison, 2014.

[8] T.Neises, C.Turchi. A comparison of supercritical carbon dioxide power cycle configurations with an emphasis on csp applications. Energy Procedia, 49:1187-1196, 2014.

[9] Visual Studio 2010, developed by Microsoft.

[10] A.G. Fernandez, F.J. Pérez. Improvement of the corrosión properties in ternary molten nitrate salts for direct energy storage in CSP plants. Solar Energy 134 (2016) 468-478.

[11] Glatzmaier, Gomez, J.C., 2013. $\mathrm{Ca}(\mathrm{NO} 3) 2-\mathrm{NaNO3}-\mathrm{KNO}$ molten salt mixtures for direct thermal energy storage systems in parabolic trough plants. J. Sol. Energy Eng. 135.

[12] Wang, T., Mantha, D., Reddy, R.G., 2013. Thermodynamic properties of LiNO3- NaNO3KNO3-2KNO3Mg(NO3)2 system. Thermochim. Acta 551, 92-98.

[13] Mantha, D., Wang, T., Reddy, R.G., 2013. Thermodynamic modeling of eutectic point in the LiNO3-NaNO3-KNO3-NaNO2 quaternary system. Sol. Energy Mater. Sol. Cells 118, 18-21.

[14] Douglas Hofer. Phased Approach to Development of a High Temperature sCO2 Power Cycle Pilot Test Facility. General Electric Company. GE Global Research, One Research Circle, Niskayuna, NY 12309 USA. The $5^{\text {th }}$ International Symposium - Supercritical $\mathrm{CO}_{2}$ Power Cycles, March 28-31, 2016, San Antonio, Texas.

[15] Lon Dawson and Matthew Carlson. Demonstration testing and facility requirements for sCO2 Brayton Commercialization. The $5^{\text {th }}$ International Symposium - Supercritical $\mathrm{CO}_{2}$ Power Cycles, March 28-31, 2016, San Antonio, Texas.

[16] C.Turchi, Golden CO, Christine Bing, Mark Lausten. 10 MW Supercritical CO2 Turbine Test. DE-EE0001589 NREL. University of Wisconsin, Fall 2014-Summer 2015.

[17] M.Anderson. WISCO2. Madison, sCO2 Advanced Power Cycle Development. 3-26-2016.

[18] F.Behafarid, M.Z. Podowski. Modeling and Computer Simulation of Centrifugal $\mathrm{CO}_{2}$ Compressors at Supercritical Pressures. ASME, Journal of Fluids Engineering, 138-6.

[19] B.Monje. Thesis: Design of supercritical carbon dioxide centrifugal compressors. Universidad de Sevilla, June 2014.

[20] X.Zhang, X.Sun, R.N. Christensen, M.Anderson, M.Carlson. Optimization of S-Shaped Fin Channels in a Printed Circuit Heat Exchanger for Supercritical $\mathrm{CO}_{2}$ Test Loop. The $5^{\text {th }}$ International Symposium - Supercritical $\mathrm{CO}_{2}$ Power Cycles, March 28-31, 2016, San Antonio.

[21] J.G.Kwon, T.Ho Kim, H.Sun Park, M.Hwan Kim. Numerical analysis of a fin arrangement for an optimal design of airfoil fin PCHE. The $4^{\text {th }}$ International Symposium - Supercritical CO2 Power Cycles September 9-10, 2014, Pittsburgh, Pennsylvania.

[22] C.Nathan. Thesis: Control strategies for supercritical carbon dioxide power conversion systems. MIT. Dept. of Nuclear Science and Engineering, 2007.

[23] L.A. Tse, T.Neises. Analysis and Optimization for Off-Design Performance of the Recompression s-CO2 cycles for high temperature CSP Applications. The $5^{\text {th }}$ International Symposium - Supercritical $\mathrm{CO}_{2}$ Power Cycles, March 28-31, 2016, San Antonio, Texas.

[24] W. Gaggioli, ENEA - Casaccia .Effects Assessment of 10 Functioning Years on Main Components of ENEA's Molten Salt Experimental PCS Facility. SolarPaces 2015, Cape Town, South Africa, October 13-16 2015. 
6. Optimización de las plantas termosolares con colectores lineales con sales fundidas como HTF acopladas a ciclos de potencia Brayton con $\mathrm{CO}_{2}$ supercrítico como fluido de trabajo

[25] A. Maccari, Archimede Solar Energy. Archimede Solar Energy Molten Salt Parabolic Trough Demo Plant: Improvements and Second Year of Operation. SolarPaces 2015, Cape Town, South Africa, October 13-16 2015.

[26] S.Kuravi, J.Trahan, D.Y.Goswami, M.M.Rahman, E.K. Stefanakos. Thermal energy storage technologies and systems for concentrating solar power plants. Progress in Energy and Combustion Science, 39(4):285-319, 2013.

[27] K Nithyanandam, R Pitchumani. Cost and performance analysis of concentrating solar power systems with integrated latent thermal energy storage. Energy, 64:793-810, 2014.

[28] M.Mohagheghi, H.Zawati, T.Pinol, J.Gou, C.Xu, J.Kapat. Use of 1-D Finite Enthalpy Method for a High-Temperature Recuperator made of Polymer Derived Ceramic Composite for a Supercritical Carbon Dioxide Power System. The $5^{\text {th }}$ International Symposium - Supercritical $\mathrm{CO}_{2}$ Power Cycles, March 28-31, 2016, San Antonio, Texas.

[29] C.A. Lewinsohn, J.Fellows (Ceramatec, Inc.), N.Sullivan, R.J. Kee, R. Braun (The Colorado School of Mines). Ceramic, Microchannel Heat Exchangers for Supercritical Carbon Dioxide Power Cycles. The $5^{\text {th }}$ International Symposium - Supercritical $\mathrm{CO}_{2}$ Power Cycles, March 28-31, 2016, San Antonio, Texas.

[30] V.Ladislav, V.Dostál, B.Ondrej, V.Novotny. Pinch Point Analysis of Heat Exchangers for Supercritical CO2 with Gaseous Admixtures in CCS Systems. Energy Proc. 86: 489-499, 2016.

[31] D.Shiferaw, J.Montero Carrero, R.Le Pierres. Economic analysis of SCO2 cycles with PCHE Recuperator deseign optimisation. The $5^{\text {th }}$ International Symposium - Supercritical $\mathrm{CO}_{2}$ Power Cycles, March 28-31, 2016, San Antonio, Texas.

[32] V.T. Cheang, R.A. Hedderwick, C.McGregor. Benchmarking supercritical carbon dioxide cycles against steam Rankine cycles for Concentrated Solar Power. Solar Energy, Volume 113, March 2015, Pages 199-211.

[33] J.Dyreby, S.Klein, G.Nellis, D.Reindl. Design considerations for supercritical carbon dioxide brayton cycles with recompression. Journal of Engineering for Gas Turbines and Power, 136(10):101701, 2014.

[34] Thermoflow, Inc. https://www.thermoflow.com/

[35] Hang Zhao; Qinghua Deng; Wenting Huang; Dian Wang; Zhenping Feng. Thermodynamic and Economic Analysis and Multi-objective Optimization of Supercritical CO 2Brayton Cycles.

ASME, J. Eng. Gas Turbines Power. 2016; 138(8):081602-081602-9.GTP-15-1585.

[36] Mahmood Mohagheghi, Jayanta Kapat. Thermodynamic Optimization of recuperated s$\mathrm{CO} 2$ Brayton cycles for waste heat recovery applications. The $4^{\text {th }}$ International SymposiumSupercritical CO2 Power cycles, September 9-10, 2014, Pittsburgh, Pennsylvania.

[37] Ricardo Vasquez Padilla, Yen Chean, Regano Benito, Wes Stein. Multi-objective thermodynamic optimisation of supercritical $\mathrm{CO} 2$ Brayton cycles integrated with solar central receivers. International Journal of Sustainable Energy, April 2016. 


\title{
Capítulo 7 \\ Optimización de plantas termosolares de colectores lineales con aceites térmicos como HTF acopladas a ciclos Brayton de $\mathrm{CO}_{2}$ supercrítico
}

\begin{abstract}
Resumen
En el presente capítulo se propone la integración de la tecnología de aceites térmicos como $\mathrm{HTF}$, en colectores solares lineales, y los ciclos de potencia $\mathrm{s}-\mathrm{CO}_{2}$ Brayton. El objetivo es combinar las dos mencionadas tecnologías y optimizar las sinergias obtenidas. Los aceites térmicos constituyen una solución tecnológica muy madura a nivel industrial, ampliamente validada e implantada en numerosas plantas comerciales, y que proporciona una fiabilidad muy alta en su diseño y operación [1]. La segunda de estas tecnologías está en fase de desarrollo industrial de los equipos y componentes que integran los nuevos ciclos de potencia Brayton, pero en la cual se está realizando un importante esfuerzo inversor para su implantación comercial en Estados Unidos desde principios del año 2000 [2]. El resultado de la integración de ambas tecnologías no persigue la obtención de ciclos de potencia con una eficiencia neta muy alta, sino que su principal objetivo es la reducción del coste total de la instalación termosolar.
\end{abstract}

Los aceites térmicos presentan unas características químicas que no requieren materiales altamente aleados, como aceros inoxidables, en las tuberías de la planta termosolar, lo cual reduce el coste de la instalación notablemente. Se ha indicado en los primeros capítulos de este trabajo que dada la relación de concentración solar de los colectores parabólicos para minimizar la eficiencia del sistema solar que lo integran, su temperatura optima de operación es de $400^{\circ} \mathrm{C}$, con ello se consigue minimizar las pérdidas térmicas por radiación en los tubos absorbedores. Estas temperaturas son soportadas por los últimos aceites térmicos desarrollados comercialmente: Dowtherm A (mezcla de Óxido de difenilo 73\% y Bifenilo 27\%), [3], Syltherm 800 [4], Therminol VP1 [5], Therminol 75 [6].

Por otra parte, los ciclos $\mathrm{s}-\mathrm{CO}_{2}$ Brayton presentan las siguientes ventajas, explicadas en detalle en capítulos anteriores, resumidas ahora: la alta densidad del fluido de trabajo en el compresor y en la turbina, mejora el rendimiento de las tubomáquinas, mejorando su eficiencia isentrópica. Gracias también a la densidad del $\mathrm{s}-\mathrm{CO}_{2}$ en estado supercrítico, las dimensiones y volumen de las turbomáquinas es más reducido. Como consecuencia directa se reduce considerablemente el trabajo de la obra civil asociada. Pequeñas fugas de los ciclos de potencia Brayton $\mathrm{s}-\mathrm{CO}_{2}$ no producen un efecto muy adverso en el medio ambiente. La toxicidad del s$\mathrm{CO}_{2}$ es casi nula. No se produce descomposición química del $\mathrm{s}-\mathrm{CO}_{2}$ a altas presiones y temperaturas. Ni hay riesgo de explosiones por incursión de aire. El coste del s- $\mathrm{CO}_{2}$ es bajo. Es un fluido muy abundante en la naturaleza. El tiempo de respuesta frente a transitorios en turbinas y compresores es más rápido que en el caso del vapor de agua; esta ventaja tiene gran impacto en las plantas termosolares sometidas a transitorios medioambientales de radiación solar y de temperatura ambiente. 
7. Optimización de plantas termosolares de colectores lineales con aceites térmicos como HTF acopladas a ciclos Brayton de $\mathrm{CO}_{2}$ supercrítico

Los principales inconvenientes de los ciclos s- $\mathrm{CO}_{2}$ Brayton son:

- el alto coste de los materiales de los equipos: debido a las altas presiones y temperaturas se requieren aceros inoxidables muy aleados, con Niobio, Titanio, Níquel, y otros elementos con alto coste.

- la tecnología de compresores y turbinas están siendo desarrolladas para altos niveles de potencia del ciclo.

- el tamaño térmico de los intercambiadores de calor es una pieza clave para incrementar la eficiencia energética neta de la instalación de potencia. Conseguir intercambiadores lo más compactos posible, a un coste reducido, es uno de los principales objetivos de investigación en este campo.

- el sumidero final de calor del ciclo en zonas con escasez de agua, incrementa sustancialmente las pérdidas eléctricas de los ventiladores de los intercambiadores de calor refrigerados por aire en convección forzada. Este aspecto constituye una pieza clave, para optimizar el rendimiento energético de las plantas termosolares acopladas a ciclos s- $\mathrm{CO}_{2}$ Brayton.

Respecto a los tradicionales ciclos de Rankine, se destacan sus principales ventajas frente a los ciclos Brayton: la tecnología tanto de turbinas como del resto de equipos y componentes que integran el ciclo, están ampliamente validadas a escala industrial, y disponibles comercialmente. Se están desarrollando turbinas con materiales sofisticados para condiciones de operación extremas con agua en estado supercrítico, pero para potencias superiores a 400 MWe. El coste de los materiales de los equipos y componentes que operan con agua como fluido de trabajo, tiene menores requisitos de corrosión que cuando se utiliza s- $\mathrm{CO}_{2}$. Como principal inconveniente de los ciclos de Rankine destaca su menor eficiencia energética neta en relación a los ciclos Brayton, lo cual incrementa sustancialmente las dimensiones y coste de inversión del campo solar. Respecto al BOP, en los ciclos de Rankine, el tamaño de los equipos, y la obra civil asociada, incrementa sustancialmente el coste de la instalación termosolar.

En este capítulo el fluido de trabajo seleccionado para el ciclo Brayton ha sido el $\mathrm{CO}_{2}$, proporcionando unos valores de eficiencia neta del ciclo Brayton entre 42-43\%, para un tamaño térmico de los recuperadores UA=10000-15000 kW/K, Dowtherm A como HTF, y una temperatura de entrada en turbina $\mathrm{TIT}=415^{\circ} \mathrm{C}$. Comparando estos resultados con la eficiencia del ciclo Rankine con recalentamiento, con un rendimiento neto del $37 \%$ con $\mathrm{TIT}=415^{\circ} \mathrm{C}$ y Dowtherm A como HTF. También puede compararse con el rendimiento de un ciclo Rankine con recalentamiento, con $\mathrm{TIT}=550^{\circ} \mathrm{C}$, con sales fundidas Solar Salt como HTF, tiene un eficiencia neta del $40.5 \%$, y con $\mathrm{HITEC} \mathrm{XL}$ con $\mathrm{TIT}=530^{\circ} \mathrm{C}$ presenta una eficiencia del $40 \%$.

Se concluye que la solución tecnológica de los ciclos Brayton garantiza un incremento de la eficiencia del 2-3\%. La mayor eficiencia de los ciclos Brayton en relación a la eficiencia de los ciclos Rankine para similares TIT, permite optimizar y minimizar el área de apertura efectiva de los colectores solares y reducir su coste de inversión. Por tanto, en estas plantas termosolares con colectores lineales, con aceites térmicos como HTF acopladas a ciclos de potencia Brayton, disponemos de numerosas ventajas para reducir el coste de la instalación: acero al carbono o con baja aleación en los tubos absorbedores, una reducción significativa del área de apertura 
7. Optimización de plantas termosolares de colectores lineales con aceites térmicos como HTF acopladas a ciclos Brayton de $\mathrm{CO}_{2}$ supercrítico

de sus colectores solares debido al alto rendimiento de los ciclos Brayton, simplicidad del sistema de control, no requieren Heat-Tracing, las pérdidas térmicas en los tubos absorbedores son menores debidas a la menor TIT, una amplia validación y disponibilidad comercial de los equipos auxiliares para bombeo y operación del HTF. Como principales inconvenientes de los aceites térmicos en relación a las sales fundidas caben destacar: la menor TIT y el mayor consumo de potencia de las bombas de recirculación del HTF en el campo solar debidas a la mayor viscosidad de los aceites.

Los intercambiadores de calor entre los campos solares y el ciclo de potencia Brayton son piezas clave para la integración entre las dos tecnologías. En la actualidad se están desarrollando diferentes tipologías de intercambiadores de calor: tubos-carcasa [7], PCHE [8], de placas [9], etc. Recientemente se han experimentado en una instalación piloto de la empresa Bechtel $[7,10]$, el intercambiador de calor tipo tubo-carcasa con aceite en la zona de la carcasa, y $\mathrm{CO}_{2}$ por el interior de los tubos. Dada las propiedades físicas de los aceites térmicos esta tipología de cambiadores es la más adecuada para minimizar las pérdidas de presión en el cambiador debidas a la alta viscosidad de los aceites térmicos. Así mismo, se consigue reducir su coste de fabricación y de materiales de la carcasa. Por ello en este trabajo se propone como solución tecnológica la utilización de intercambiadores de calor de tipología carcasa-tubo.

\subsection{Introducción}

La tecnología de transferencia de calor mediante aceite térmico es la más utilizada en las plantas termoslares con colectores PTC y ciclos de Rankine, desde la construcción de la primera instalación termosolar en el desierto de Mojave en California en 1984 [11]. Posteriormente se han implantado a nivel comercial en numerosas plantas [12]. Una de las primeras referencias que estima y optimiza el tamaño del campo solar como función de la irradiación solar en plantas sin almacenamiento térmico, fue el trabajo de Quashing et al [13], y un modelo simplificado por Rolim [14]. El NREL publicó en 2009 una primera revisión del programa informático System Advisor Model (SAM), para simular tanto el comportamiento transitorio, como la producción anual de plantas termosolares con colectores lineales y aceites térmicos [15].

Uno de los trabajos de referencia en la evaluación de plantas termosolares con aceite térmico como HTF y sin almacenamiento térmico es Montes et al. [16], en la cual se incluye información para el cálculo de la producción anual de electricidad, y la optimización económica de la instalación. Kumaresan et al. [17], investigó los parámetros de operación incluyendo la temperatura de trabajo, la ganancia de energía y la eficiencia térmica de las plantas con colectores PTC. Consideró Therminol 55 como fluido caloportador HTF, e incluyó un sistema de almacenamiento térmico TES. Manzoline et al [18-19] presentó un código informático, denominado PATTO, para el diseño en condiciones nominales de diferentes tipos de plantas solares sin almacenamiento térmico, y refrigeradas mediante aceites, sales fundidas o agua. Hermann et al. [20] estimó una reducción en los costes de la energía eléctrica en un 10\%, mediante la utilización de almacenamiento térmico 12 horas con sales fundidas, en las plantas termosolares con aceites térmicos. Llorente Garcia et al. [21] presenta un modelo detallado para calcular la producción de esta tipología de plantas con colectores lineales y 
7. Optimización de plantas termosolares de colectores lineales con aceites térmicos como HTF acopladas a ciclos Brayton de $\mathrm{CO}_{2}$ supercrítico

almacenamiento térmico. Un modelo económico comparativo de plantas termosolares con aceites térmicos con almacenamiento térmico entre 0 a 12 horas fue publicado por Wagner y Rubin [22]. Fabrizio De Luca et al [23] estudió la producción y costes de plantas termosolares en función del múltiplo solar (entre 1 y 3), y su capacidad de almacenamiento térmico (entre 0 y 24 horas), en términos de producción anual de la planta, eficiencia media anual, factor de carga, inversión inicial, y coste unitario de la energía producida Levelized Cost Of Energy (LCOE). También incluyó un método para la optimización económica, basado en la evaluación del valor mínimo del LCOE. El valor mínimo del LCOE, en el caso de incluir almacenamiento térmico, es obtenido para el múltiplo solar 2.2 y una capacidad de almacenamiento de 16 horas.

En las plantas sin almacenamiento el valor óptimo del múltiplo solar es de 1.2 para minimizar el LCOE. T.E. Boukelia et al [24] incluye un análisis $4 E$ (Energético, Exergético, Medioambiental y Económico) comparando 8 configuraciones diferentes de plantas termosolares con colectores PTC, y dos fluidos caloportadores diferentes HTF: aceite térmico Therminol VP-1 y sales fundidas. Se consideran las opciones de almacenamiento térmico y la instalación de una caldera con combustible fósil para respaldar la producción energética de la instalación. La configuración de sales fundidas con almacenamiento térmico, presentó los mayores valores de eficiencia energética (18.48\%). Mientras que los valores mayores de eficiencia exergética (21.77\%), factor de disponibilidad (38.2\%) y producción anual de electricidad (114 GWh) son obtenidos en la configuración con aceites térmicos incluyendo el almacenamiento térmico y la caldera de respaldo con combustibles fósiles. Los resultados también confirmaron que la configuración con sales fundidas es mejor en términos de minimizar el impacto ambiental, y optimizar desde el punto de vista económico la instalación industrial. Las configuraciones que integran el sistema de almacenamiento térmico y la caldera de respaldo, son las mejores soluciones tecno-económicas, pero presentan un importante inconveniente medioambiental.

Tabla 7.1.Principales aceites térmicos como HTF en las plantas termosolaresy sus principales propiedades físicas.

\begin{tabular}{|c|c|c|c|c|c|c|}
\hline $\begin{array}{l}\text { Aceite } \\
\text { térmico }\end{array}$ & $\begin{array}{l}\text { Viscosidad } \\
\text { Dinámica } \\
\text { (Mpa.s) } \\
\left(25^{\circ} \mathrm{C}\right)\end{array}$ & $\begin{array}{l}\text { Densidad } \\
\left(\mathrm{kg} / \mathrm{m}^{3}\right) \\
\left(25^{\circ} \mathrm{C}\right)\end{array}$ & $\begin{array}{l}\mathrm{Cp} \\
(\mathrm{kJ} / \mathrm{kg} \mathrm{K})\end{array}$ & $\begin{array}{l}\mathrm{K} \\
(\mathrm{W} / \mathrm{m} \mathrm{K})\end{array}$ & $\begin{array}{l}\text { Temperatura } \\
\text { Máxima } \\
\text { Operación } \\
\left({ }^{\circ} \mathrm{C}\right)\end{array}$ & $\begin{array}{l}\text { Temperatura } \\
\text { Mínima } \\
\text { Operación } \\
\left({ }^{\circ} \mathrm{C}\right)\end{array}$ \\
\hline Dowtherm A & 4.31 & 1056 & 1.58 & 0.13 & 426.7 & 12.8 \\
\hline Dowtherm RP & 33.41 & 1025.8 & 1.63 & 0.13 & 343.3 & 10.0 \\
\hline Syltherm 800 & 9.1 & 936 & 1.61 & 0.13 & 398.9 & -40.0 \\
\hline Therminol 66 & 89.53 & 1005.1 & 1.57 & 0.11 & 343.3 & -1.1 \\
\hline Therminol 72 & 11.11 & 1074.5 & 1.56 & 0.13 & 379.4 & -9.4 \\
\hline Therminol 75 & --- & --- & --- & --- & 385.0 & 79.4 \\
\hline Therminol VP-1 & 3.78 & 1060 & 1.56 & 0.13 & 398.9 & 12.8 \\
\hline
\end{tabular}

A pesar de sus inconvenientes, el aceite térmico como fluido caloportadore presenta numerosas ventajas sobre el resto de HTFs recientemente desarrollados: es una tecnología muy madura, ampliamente validada a escala industrial, existencia de equipos comerciales para su implantación en instalaciones solares, se continúan desarrollando formulas químicas 
7. Optimización de plantas termosolares de colectores lineales con aceites térmicos como HTF acopladas a ciclos Brayton de $\mathrm{CO}_{2}$ supercrítico

avanzadas para soportar temperaturas superiores Dowtherm A [3] (mezcla de Oxido de difenilo $73 \%$ y Bifenilo $27 \%$ ), su sistema de control no es tan complejo como el de otras tecnologías como DSG. Existen numerosas marcas comerciales de aceites térmicos, las principales se han resumido en la tabla 7.1 junto con sus principales propiedades físicas.

En la tabla 7.2 se han incluido las dos principales alternativas de sales fundidas para su combinación o sustitución con los aceites térmicos. Actualmente, se han implantado principalmente en tres instalaciones piloto: planta piloto de Novatec en Puerto Errado con colectores fresnel y sales fundidas [25], planta piloto Archimede en Italia [26], planta piloto en SNL con colectores Fresnel de la marca comercial Areva [27]. Archimede solar ha firmado recientemente un contrato en China para la construcción de la primera planta termosolares con colectores parabólicos y sales fundidas como HTF, con una potencia de 55MWe y 15 horas de almacenamiento térmico [28]. El proyecto se conoce con el nombre de AKESAI Solar Thermal Power Plant (ASTPP).

Tabla 7.2. Principales sales fundidas como HTF en las plantas termosolares y sus principales propiedades físicas.

\begin{tabular}{lllllll}
\hline $\begin{array}{l}\text { Sal } \\
\text { fundida }\end{array}$ & $\begin{array}{l}\text { Viscosidad } \\
\text { Dinámica } \\
(\mathrm{MPa} \cdot \mathrm{s}) \\
\left(300^{\circ} \mathrm{C}\right)\end{array}$ & $\begin{array}{l}\text { Densidad } \\
\left(\mathrm{kg} / \mathrm{m}^{3}\right) \\
\left(300^{\circ} \mathrm{C}\right)\end{array}$ & $\begin{array}{l}\mathrm{Cp} \\
(\mathrm{kJ} / \mathrm{kg} \mathrm{K})\end{array}$ & $\begin{array}{l}\text { Composición } \\
\text { porcentaje } \\
\text { en peso })\end{array}$ & $\begin{array}{l}\text { Temperatura } \\
\text { Máxima } \\
\text { Operación } \\
\left({ }^{\circ} \mathrm{C}\right)\end{array}$ & $\begin{array}{l}\text { Temperatura } \\
\text { Mínima } \\
\text { Operación } \\
\left({ }^{\circ} \mathrm{C}\right)\end{array}$ \\
\hline Solar Salt & 3.26 & 1899 & 1.49 & $\begin{array}{l}60 \%\left(\mathrm{NaNO}_{3}\right) \\
40 \%\left(\mathrm{KNO}_{3}\right) \\
42 \%\left(\mathrm{Ca}\left(\mathrm{NO}_{3}\right)_{2}\right.\end{array}$ & 593.3 & 260 \\
HITEC XL & 6.37 & 1992 & 1.45 & $\begin{array}{l}15 \%\left(\mathrm{NaNO}_{3}\right) \\
43 \%\left(\mathrm{KNO}_{3}\right)\end{array}$ & 540 & 120 \\
\hline
\end{tabular}

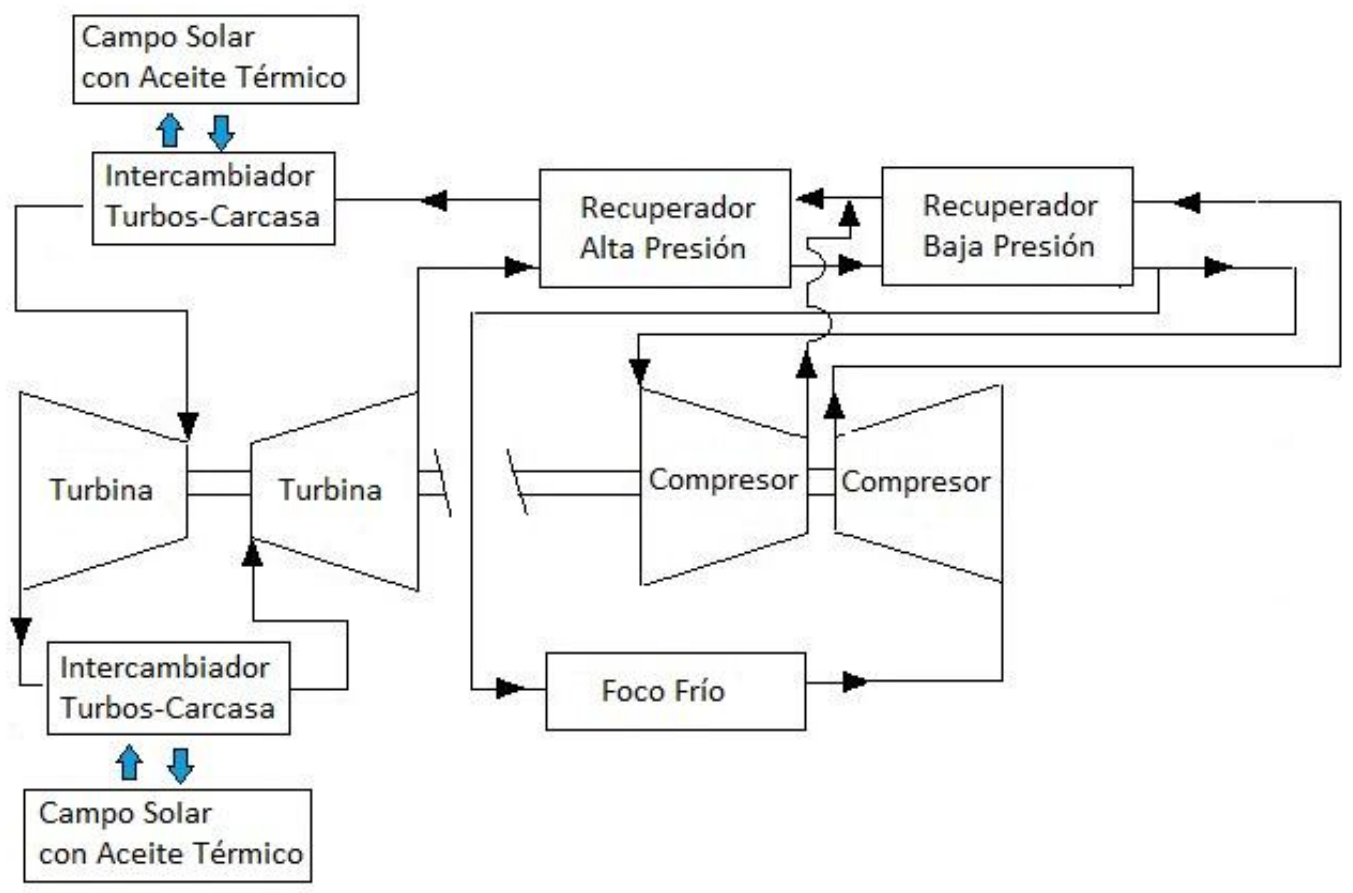

Figura 7.1. Planta termosolar de colectores lineales con aceite térmico como HTF acoplada a ciclo de potencia s- $\mathrm{CO}_{2}$ Brayton RC. 
7. Optimización de plantas termosolares de colectores lineales con aceites térmicos como HTF acopladas a ciclos Brayton de $\mathrm{CO}_{2}$ supercrítico

En este capítulo se comparar la producción y eficiencia neta de plantas termosolares integradas por colectores lineales, con los siguientes HTF: Solar Salt, Hitec XL, Dowtherm A, Syltherm 800, Therminol VP1 y Therminol 75, y ciclos de potencia s- $\mathrm{CO}_{2}$ Brayton con diferentes disposición de sus equipos, tal y como se aconsejaba en trabajos de relevancia [29, 30] para las plantas termosolares: ciclo con recompresión (RC), ciclo con enfriamiento parcial antes de la recompresión (PCRC), y finalmente el ciclo con recompresión y enfriamiento intermedio en el compresor principal (RCMCl). Todas las configuraciones de ciclos de potencia Brayton definidas anteriormente incluyen una etapa de recalentamiento en las turbinas para incrementar la eficiencia del ciclo. Como resultado obtenemos las configuraciones de plantas termosolares ilustradas en las figuras $7.1,7.2$ y 7.3 .

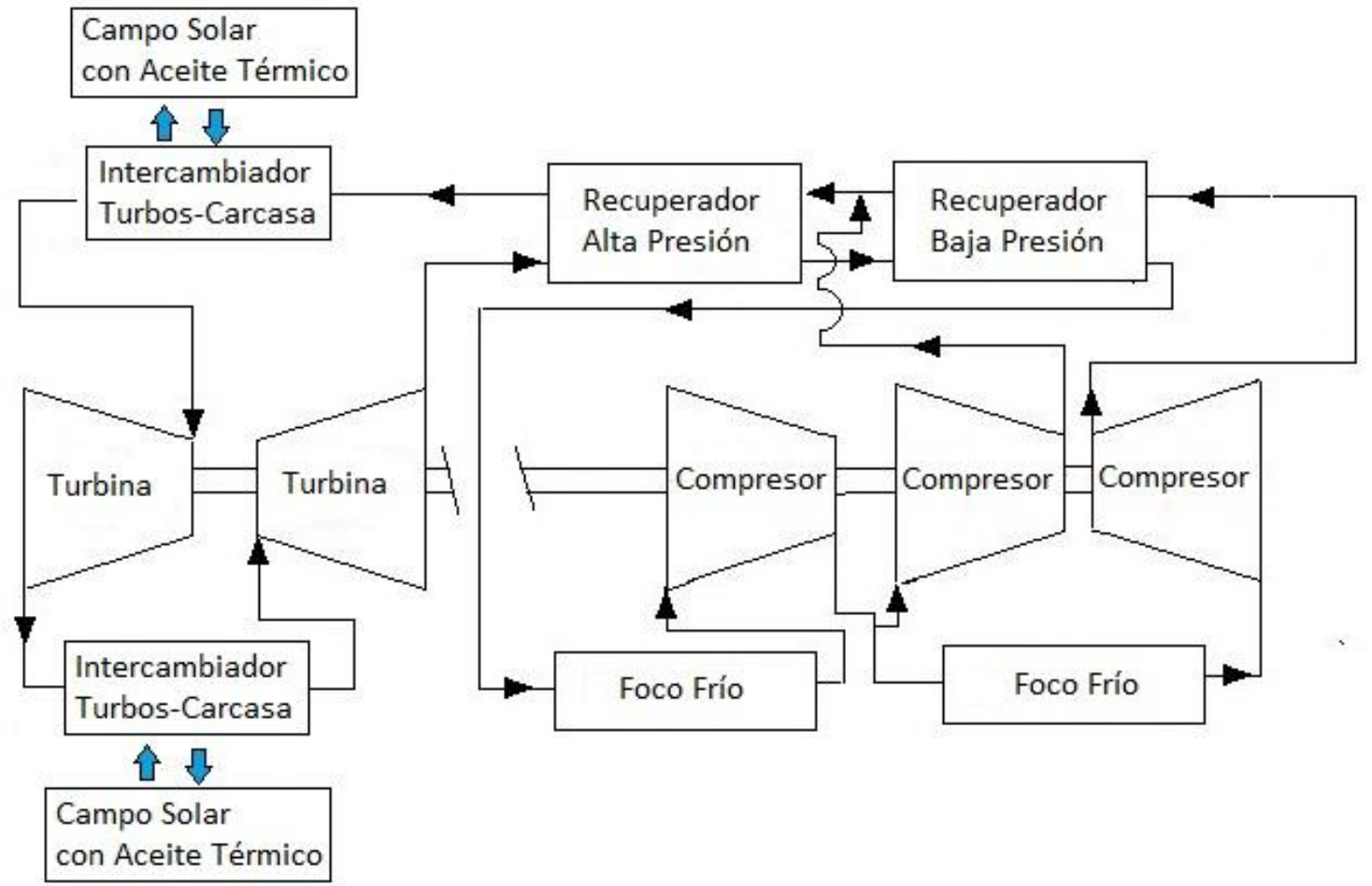

Figura 7.2. Planta termosolar con colectores lineales con aceite térmico como HTF acoplada a ciclo de potencia s- $\mathrm{CO}_{2}$ Brayton PCRC.

\subsection{Hipótesis del modelo}

Todas las simulaciones fueron calculadas en su punto de diseño, fijando una potencia bruta de $50 \mathrm{MWe}$. Se consideraron diferentes valores de temperatura de entrada en turbina $\operatorname{TIT}\left(400^{\circ} \mathrm{C}\right.$, $450^{\circ} \mathrm{C}, 500^{\circ} \mathrm{C}$ y $550^{\circ} \mathrm{C}$ ). El resto de hipótesis de cálculo están resumidas en las tablas 7.3 a 7.8.

Tabla 7.3. Localización, condiciones ambientales.

\begin{tabular}{lc}
\hline Localización: & Dagget,CA, USA. \\
Latitud: & $34.86^{\circ}$ \\
Longitud: & $-116.8^{\circ}$ \\
Zona horaria: & -8 \\
Hora: & $11: 30 \mathrm{hr}$ \\
DNI: & $986 \mathrm{~W} / \mathrm{m}^{2}$ \\
Temperatura: & $25^{\circ} \mathrm{C}$ \\
Altitud: & $588 \mathrm{~m}$ \\
\hline
\end{tabular}


7. Optimización de plantas termosolares de colectores lineales con aceites térmicos como HTF acopladas a ciclos Brayton de $\mathrm{CO}_{2}$ supercrítico

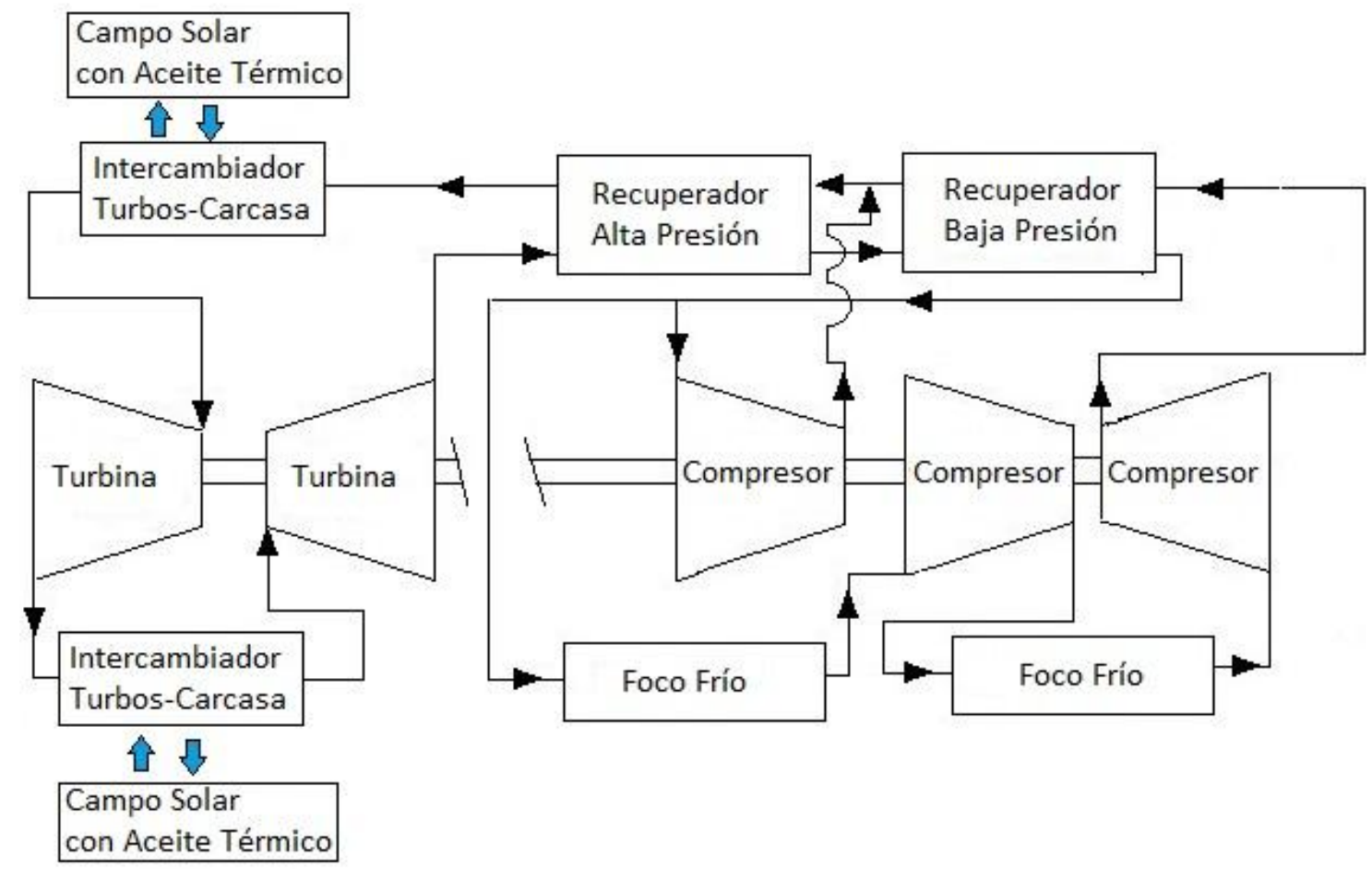

Figura 7.3. Planta termosolar con colectores lineales con aceite térmico como HTF acoplada a ciclo de potencia s- $\mathrm{CO}_{2}$ Brayton $\mathrm{RCMCl}$.

Tabla 7.4. Parámetros tubo absorbedor.

\begin{tabular}{ll}
\hline Diámetro exterior: & $70 \mathrm{~mm}$ \\
Espesor pared: & $4.191 \mathrm{~mm}$ \\
Material: & Acero Inoxidable \\
Rugosidad: & $0.0457 \mathrm{~mm}$ \\
\hline
\end{tabular}

Tabla 7.5. Parámetros colectores PTC.

\begin{tabular}{ll}
\hline Tipo colector: & EuroTrough II \\
Anchura apertura: & $5.77 \mathrm{~m}$ \\
Longitud focal: & $1.71 \mathrm{~m}$ \\
Distancia/anchura (lazos): & 2.5 \\
Factor Limpieza: & 0.96 \\
Eficiencia óptica: & 0.75 \\
Pérdidas térmicas: & $0.141 \Delta \mathrm{T}+6.48 \cdot 10^{-9} \Delta \mathrm{T}^{4}$ \\
\hline
\end{tabular}

Tabla 7.6. Parámetros colector LF.

\begin{tabular}{ll}
\hline Tipo & SuperNova 1 (Novatec) \\
Dimensiones & $16.56 \mathrm{~m} \times 44.8 \mathrm{~m}$ \\
Área & $513.6 \mathrm{~m}^{2} /$ por Modulo \\
Eficiencia & 0.647 \\
Pérdidas: & $1.06 \Delta \mathrm{T}+1.2 \cdot 10^{-8} \Delta \mathrm{T}^{4}$ \\
Pérdidas: & $0.15 \Delta \mathrm{T}+7.1510^{-9} \Delta \mathrm{T}^{4}$ \\
\hline
\end{tabular}


7. Optimización de plantas termosolares de colectores lineales con aceites térmicos como HTF acopladas a ciclos Brayton de $\mathrm{CO}_{2}$ supercrítico

Tabla 7.7. Ciclo potencia Rankine.

\begin{tabular}{ll}
\hline Turbina HP: & 2 etapas (87.7 bar; 36 bar) \\
Turbina IP: & 3 etapas (16.5 bar;10.34 bar; 6.18 bar) \\
Turbina LP: & 4 etapas (5.17 bar; 3.04bar; 1.17 bar; 0.37 bar) \\
Eficiencia Turbina: & $85 \%$ \\
Presión condensador: & 0.08 bar \\
Eficiencia Generador: & $98.23 \%$ (punto de diseño) \\
Auxiliary BOP: & $0.01 \%$ (potencia bruta) \\
TTD calentadores agua: & $5^{\circ} \mathrm{C}$ \\
DCA calentadores agua : & $5^{\circ} \mathrm{C}$ \\
Presión desaireador: & 6.17 bar \\
\hline
\end{tabular}

Tabla 7.8. Ciclo potencia s- $\mathrm{CO}_{2}$ Brayton.

\begin{tabular}{lc}
\hline Eficiencia Turbina: & $93 \%$ \\
Eficiencia Compresor: & $89 \%$ \\
Tamaño térmico cambiadores fijado (UA) & No pérdidas de presión en cambiadores \\
Temperatura entrada turbina: & $550^{\circ} \mathrm{C}$ \\
Presión entrada turbina: & $250 \mathrm{bar}$ \\
Presión recalentamiento: & optimizado \\
Temperatura compresor: & $32^{\circ} \mathrm{C}$ \\
Presión entrada compresor: & Optimizado \\
Fracción de Flujo: & optimizado \\
Pérdidas auxiliares BOP: & $0.01 \%$ \\
Eficiencia Generador: & 98.23 \\
\hline
\end{tabular}

\subsection{Resultados}

En un primer apartado 7.4.1 se ha realizado una comparación detallada de las eficiencias netas con las diferentes soluciones de ciclos de potencia Rankine o Brayton. Y se ha cuantificado en detalle el incremento de eficiencia energética como consecuencia directa de sustituir los ciclos Rankine por ciclos supercríticos Brayton. En este mismo apartado se realiza una comparativa de los valores de diferencia de temperatura mínima pinch-point en los recuperadores. Se confirma el hecho explicado en capítulos anteriores, como un incremento del tamaño térmico de los recuperadores da lugar a una reducción del pinch-point en los recuperadores y un incremento de la eficiencia del ciclo.

En el segundo apartado 7.4.2, se han definidos los parámetros termodinámicos óptimos para garantizar un óptimo funcionamiento de los ciclos Brayton. Se han establecidos los valores óptimos de fracción de flujo, presión de entrada en la turbina de recalentamiento, tamaño térmico de los recuperadores, presión de entrada y descarga de los compresores, etc.

En el apartado 7.4.3 y 7.4.4 se traduce a términos de área de apertura efectiva de los colectores, y a térmicos monetarios de inversión de capital, los resultados de eficiencia energética del ciclo de potencia Brayton obtenidos en el apartado 7.4.1. A pesar de proporcionar menores temperaturas de entrada en turbina, el coste final de los campos 
7. Optimización de plantas termosolares de colectores lineales con aceites térmicos como HTF acopladas a ciclos Brayton de $\mathrm{CO}_{2}$ supercrítico

solares considerando las configuraciones con aceites térmicos, es mucho menor que el proporcionado por la solución de sales fundidas. El coste de los materiales de los tubos absorbedores, y resto de componentes auxiliares del campo solar (tuberías de distribución, válvulas, etc.) es el principal motivo. Los aceites térmicos, como se ha recalcado en este capítulo, no son corrosivos en contacto con el metal. Sin embargo, las sales fundidas requieren aceros inoxidables austeníticos estabilizados con Niobio ( $\mathrm{Nb}$ ), Titanio (Ti), u otros metales, que encarecen el coste de la instalación termosolar. No se ha cuantificado en este apartado por falta de datos concretos de los equipos, el coste de inversión y consumo eléctrico del sistema auxiliar de calefacción heat-tracing para evitar la solidificación de las sales fundidas. El sistema heat-tracing también constituye una importante ventaja comparativa de los aceites térmicos en relación a las sales fundidas.

\subsubsection{Eficiencia de la Planta Termosolar}

\subsubsection{Ciclo de Potencia Rankine}

Los resultados resumidos en las tablas 7.9 y 7.10, son similares a los incluidos en el capítulo 1 de esta tesis doctoral para las plantas termosolares con colectores lineales y ciclos de potencia Rankine. Para las configuraciones sin recalentamineto, la mayor eficiencia de la planta termosolar es $38.4 \%$, y la proporcionan las sales fundidas Solar Salt como HTF, con una temperatura máxima de entrada en turbina $\mathrm{TIT}=550^{\circ} \mathrm{C}$, tabla 7.9. La solución tecnológica con recalentamiento del vapor, proporciona un incremento de la eficiencia neta del ciclo de potencia del $2 \%$, en términos absolutos, dando como resultado una eficiencia neta del $40.5 \%$, tabla 7.10. Traducido a términos económicos el ahorro de inversión en los colectores solares es de 2-3 millones de euros.

Tabla 7.9. Eficiencia Neta de planta termosolar con colectores lineales acoplada a ciclo de potencia Rankine sin Recalentamiento.

\begin{tabular}{lll}
\hline TIT $\left({ }^{\circ} \mathrm{C}\right)$ & SF HTF & Eficiencia Neta (\%) \\
\hline 550 & Solar Salt & 38.47 \\
530 & Hitec XL & 38.07 \\
415 & Dowtherm A & 35.37 \\
390 & Syltherm 800 & 34.65 \\
390 & Therminol VP1 & 34.70 \\
375 & Therminol 75 & 34.26 \\
\hline
\end{tabular}

Tabla 7.10. Eficiencia Neta de planta termosolar con colectores lineales acoplada a ciclo de potencia Rankine con Recalentamiento.

\begin{tabular}{lll}
\hline TIT $\left({ }^{\circ} \mathrm{C}\right)$ & SF HTF & Eficiencia \\
\hline 550 & Solar Salt & 40.54 \\
530 & Hitec XL & 40.09 \\
415 & Dowtherm A & 37.02 \\
390 & Syltherm 800 & 36.27 \\
390 & Therminol VP1 & 36.34 \\
375 & Therminol 75 & 35.92 \\
\hline
\end{tabular}


7. Optimización de plantas termosolares de colectores lineales con aceites térmicos como HTF acopladas a ciclos Brayton de $\mathrm{CO}_{2}$ supercrítico

\subsubsection{Ciclo de potencia con recompresión}

Cabe destacar como a partir de un valor umbral del tamaño térmico de los recuperadores $\mathrm{UA}=20000 \mathrm{~kW} / \mathrm{K}$, la eficiencia neta de la planta termosolar no se incrementa en gran medida, y el pinch-point en los recuperadores de calor ya es del orden de $2-5^{\circ} \mathrm{C}$. La evolución de incremento de eficiencia y reducción de pinch-point conforme se incrementa el tamaño térmico de los recuperadores UA está detallado en las tablas 7.11 a 7.15.

Para los valores menores de $U A=3000 \mathrm{~kW} / \mathrm{K}$, la eficiencia de los ciclos de potencia Brayton es superior a la proporcionada por los ciclos Rankine para el rango de $\mathrm{TIT}>500^{\circ} \mathrm{C}$, comparar resultados en tabla 7.10 con tabla 7.11. En el caso de $\mathrm{UA}=5000 \mathrm{~kW} / \mathrm{K}$, para los valores de temperatura de entrada en turbina, $390 \leq \mathrm{TIT} \leq 550$, proporcionan rendimientos energéticos de los ciclos Brayton superiores a los de los ciclos Rankine con similares TIT, como puede verse al comparar los resultados en las tablas 7.10 y tabla 7.12 .

Tabla 7.11. Eficiencia Neta de planta termosolar con colectores lineales acoplada a ciclo de potencia s- $\mathrm{CO}_{2}$ Brayton $\mathrm{RC}$ con recalentamiento, $\mathrm{UA}=3000 \mathrm{~kW} / \mathrm{K}$.

\begin{tabular}{|c|c|c|c|c|c|}
\hline $\operatorname{TIT}\left({ }^{\circ} \mathrm{C}\right)$ & SF Configuración & Eficiencia Bruta (\%) & EficienciaNeta (\%) & LTR Pinch $\left({ }^{\circ} \mathrm{C}\right)$ & HTR Pinch $\left({ }^{\circ} \mathrm{C}\right)$ \\
\hline 550 & Solar Salt & 43.37 & 41.97 & 23.6 & 61.9 \\
\hline 520 & Hitec XL & 41.93 & 40.59 & 23.8 & 62.6 \\
\hline 415 & Dowtherm A & 36.49 & 35.27 & 22.5 & 64.6 \\
\hline 390 & Syltherm 800 & 35.05 & 33.80 & 22.1 & 65.4 \\
\hline 390 & Therminol VP1 & 35.02 & 33.85 & 22.1 & 65.4 \\
\hline 375 & Therminol 75 & 34.09 & 32.96 & 21.5 & 0 \\
\hline
\end{tabular}

Tabla 7.12. Eficiencia Neta de planta termosolar con colectores lineales acoplada a ciclo de potencia s- $\mathrm{CO}_{2}$ Brayton $\mathrm{RC}$ con recalentamiento, $\mathrm{UA}=5000 \mathrm{~kW} / \mathrm{K}$.

\begin{tabular}{cccccc}
\hline TIT $\left({ }^{\circ} \mathrm{C}\right)$ & SF Configuración & Eficiencia Bruta $(\%)$ & Eficiencia Neta $(\%)$ & LTR Pinch $\left({ }^{\circ} \mathrm{C}\right)$ & HTR Pinch $\left({ }^{\circ} \mathrm{C}\right)$ \\
\hline 550 & Solar Salt & 46.87 & 45.37 & 15.8 & 32.1 \\
520 & Hitec XL & 45.32 & 43.88 & 15.7 & 33.1 \\
415 & Dowtherm A & 39.36 & 38.04 & 15.1 & 36.1 \\
390 & Syltherm 800 & 37.75 & 36.4 & 14.8 & 37.1 \\
390 & Therminol VP1 & 37.72 & 36.45 & 14.8 & 37.1 \\
375 & Therminol 75 & 36.70 & 35.47 & 14.6 & 37.7 \\
\hline
\end{tabular}

Tabla 7.13. Eficiencia Neta de planta termosolar con colectores lineales acoplada a ciclo de potencia s- $\mathrm{CO}_{2}$ Brayton $\mathrm{RC}$ con recalentamiento, $\mathrm{UA}=10000 \mathrm{~kW} / \mathrm{K}$.

\begin{tabular}{cccccc}
\hline TIT $\left({ }^{\circ} \mathrm{C}\right)$ & SF Configuración & Eficiencia Bruta $(\%)$ & Eficiencia Neta $(\%)$ & LTR Pinch $\left({ }^{\circ} \mathrm{C}\right)$ & HTR Pinch $\left({ }^{\circ} \mathrm{C}\right)$ \\
\hline 550 & Solar Salt & 50.15 & 48.55 & 8.6 & 10.4 \\
520 & Hitec XL & 48.58 & 47.05 & 9.1 & 10.9 \\
415 & Dowtherm A & 42.28 & 40.86 & 8.8 & 13.9 \\
390 & Syltherm 800 & 40.56 & 39.11 & 8.6 & 14.9 \\
390 & Therminol VP1 & 40.53 & 39.18 & 8.6 & 14.9 \\
375 & Therminol 75 & 39.41 & 38.09 & 8.5 & 15.5 \\
\hline
\end{tabular}


7. Optimización de plantas termosolares de colectores lineales con aceites térmicos como HTF acopladas a ciclos Brayton de $\mathrm{CO}_{2}$ supercrítico

Tabla 7.14. Eficiencia Neta de planta termosolar con colectores lineales acoplada a ciclo de potencia s- $\mathrm{CO}_{2}$ Brayton $\mathrm{RC}$ con recalentamiento, UA=15000 kW/K.

\begin{tabular}{cccccc}
\hline TIT $\left({ }^{\circ} \mathrm{C}\right)$ & SF Configuración & Eficiencia Bruta (\%) & Eficiencia Neta $(\%)$ & LTR Pinch $\left({ }^{\circ} \mathrm{C}\right)$ & HTR Pinch $\left({ }^{\circ} \mathrm{C}\right)$ \\
\hline 550 & Solar Salt & 51.24 & 49.61 & 4.3 & 4.3 \\
520 & Hitec XL & 49.71 & 48.15 & 4.7 & 4.7 \\
415 & Dowtherm A & 43.42 & 41.96 & 6.2 & 7.1 \\
390 & Syltherm 800 & 41.66 & 40.17 & 6.2 & 7.8 \\
390 & Therminol VP1 & 41.63 & 40.22 & 6.2 & 7.8 \\
375 & Therminol 75 & 40.50 & 39.14 & 5.8 & 8.7 \\
\hline
\end{tabular}

Tabla 7.15. Eficiencia Neta de planta termosolar con colectores lineales acoplada a ciclo de potencia s- $\mathrm{CO}_{2}$ Brayton $\mathrm{RC}$ con recalentamiento, $\mathrm{UA}=20000 \mathrm{~kW} / \mathrm{K}$.

\begin{tabular}{llllll}
\hline TIT $\left({ }^{\circ} \mathrm{C}\right)$ & SF Configuración & Eficiencia Bruta $(\%)$ & Eficiencia Neta $(\%)$ & LTR Pinch $\left({ }^{\circ} \mathrm{C}\right)$ & HTR Pinch $\left({ }^{\circ} \mathrm{C}\right)$ \\
\hline 550 & Solar Salt & 51.73 & 50.09 & 2.1 & 2.1 \\
520 & Hitec XL & 50.21 & 48.63 & 2.4 & 2.5 \\
415 & Dowtherm A & 43.99 & 42.51 & 4.1 & 4.1 \\
390 & Syltherm 800 & 42.25 & 40.74 & 4.5 & 4.8 \\
390 & Therminol VP1 & 42.21 & 40.79 & 4.5 & 4.8 \\
375 & Therminol 75 & 41.07 & 39.69 & 4.6 & 5.1 \\
\hline
\end{tabular}

\subsubsection{Ciclo de potencia con enfriamiento previo a la recompresión}

La configuración PCRC de los ciclos s- $\mathrm{CO}_{2}$ Brayton proporciona menores valores de eficiencia energética que los ciclos RC, comparar los resultados de las tablas 7.11 a 7.15 y las tablas 7.16 a 7.20. Para los ciclos de tipología PCRC, el valor umbral del tamaño térmico de los recuperadores $U A=10000 \mathrm{~kW} / \mathrm{K}$, reduce las temperaturas de pinch-point en los recuperadores a un rango entre $2-5^{\circ} \mathrm{C}$. Lo cual marca una clara diferencia con los ciclos RC. A partir del mismo valor UA $=10000 \mathrm{~kW} / \mathrm{K}$, la eficiencia de los ciclos PCRC Brayton es superior a los valores de rendimiento energéticos proporcionados por los ciclos de potencia Rankine, comparando los resultados de las tablas 7.18 a 7.20, con la tabla 7.10.

Tabla 7.16. Eficiencia Neta de planta termosolar con colectores lineales acoplada a ciclo de potencia s- $\mathrm{CO}_{2}$ Brayton PCRC con recalentamiento, $\mathrm{UA}=3000 \mathrm{~kW} / \mathrm{K}$.

\begin{tabular}{llllll}
\hline TIT $\left({ }^{\circ} \mathrm{C}\right)$ & SF Configuración & Eficiencia Bruta (\%) & Eficiencia Neta (\%) & LTR Pinch $\left({ }^{\circ} \mathrm{C}\right)$ & HTR Pinch $\left({ }^{\circ} \mathrm{C}\right)$ \\
\hline 550 & Solar Salt & 43.37 & 41.97 & 23.3 & 23.9 \\
520 & Hitec XL & 42.01 & 40.67 & 23.3 & 24.6 \\
415 & Dowtherm A & 36.68 & 35.45 & 22.4 & 27.7 \\
390 & Syltherm 800 & 35.25 & 34.02 & 22.1 & 28.6 \\
390 & Therminol VP1 & 35.23 & 34.05 & 22.1 & 28.6 \\
375 & Therminol 75 & 34.35 & 33.13 & 22.8 & 28.2 \\
\hline
\end{tabular}

Tabla 7.17. Eficiencia Neta de planta termosolar con colectores lineales acoplada a ciclo de potencia s- $\mathrm{CO}_{2}$ Brayton PCRC con recalentamiento, UA=5000 kW/K.

\begin{tabular}{llllll}
\hline TIT $\left({ }^{\circ} \mathrm{C}\right)$ & SF Configuración & Eficiencia Bruta $(\%)$ & Eficiencia Neta $(\%)$ & LTR Pinch $\left({ }^{\circ} \mathrm{C}\right)$ & HTR Pinch $\left({ }^{\circ} \mathrm{C}\right)$ \\
\hline 550 & Solar Salt & 45.86 & 44.39 & 9.3 & 9.3 \\
520 & Hitec XL & 44.47 & 43.05 & 9.9 & 9.9 \\
415 & Dowtherm A & 38.87 & 37.56 & 12.9 & 12.8 \\
390 & Syltherm 800 & 37.41 & 36.1 & 13.2 & 13.7 \\
390 & Therminol VP1 & 37.39 & 36.14 & 13.2 & 13.7 \\
375 & Therminol 75 & 36.45 & 35.17 & 13.3 & 14.3 \\
\hline
\end{tabular}


7. Optimización de plantas termosolares de colectores lineales con aceites térmicos como HTF acopladas a ciclos Brayton de $\mathrm{CO}_{2}$ supercrítico

Tabla 7.18. Eficiencia Neta de planta termosolar con colectores lineales acoplada a ciclo de potencia s- $\mathrm{CO}_{2}$ Brayton $\mathrm{PCRC}$ con recalentamiento, $\mathrm{UA}=10000 \mathrm{~kW} / \mathrm{K}$.

\begin{tabular}{llllll}
\hline TIT $\left({ }^{\circ} \mathrm{C}\right)$ & SF Configuración & Eficiencia Bruta (\%) & Eficiencia Neta (\%) & LTR Pinch $\left({ }^{\circ} \mathrm{C}\right)$ & HTR Pinch $\left({ }^{\circ} \mathrm{C}\right)$ \\
\hline 550 & Solar Salt & 47.40 & 45.88 & 2.7 & 2.7 \\
520 & Hitec XL & 46.04 & 44.58 & 2.9 & 2.9 \\
415 & Dowtherm A & 40.57 & 39.2 & 4.3 & 4.3 \\
390 & Syltherm 800 & 39.07 & 37.7 & 4.7 & 4.7 \\
390 & Therminol VP1 & 39.04 & 37.74 & 4.7 & 4.7 \\
375 & Therminol 75 & 38.08 & 36.82 & 5.1 & 5.1 \\
\hline
\end{tabular}

Tabla 7.19. Eficiencia Neta de planta termosolar con colectores lineales acopladas a ciclo de potencia s- $\mathrm{CO}_{2}$ Brayton $\mathrm{PCRC}$ con recalentamiento, $\mathrm{UA}=15000 \mathrm{~kW} / \mathrm{K}$.

\begin{tabular}{llllll}
\hline TIT $\left({ }^{\circ} \mathrm{C}\right)$ & SF Configuración & Eficiencia Bruta (\%) & Eficiencia Neta $(\%)$ & LTR Pinch $\left({ }^{\circ} \mathrm{C}\right)$ & HTR Pinch $\left({ }^{\circ} \mathrm{C}\right)$ \\
\hline 550 & Solar Salt & 47.75 & 46.22 & 1.5 & 1.5 \\
520 & Hitec XL & 46.41 & 44.94 & 1.7 & 1.6 \\
415 & Dowtherm A & 41.03 & 39.64 & 2.4 & 2.4 \\
390 & Syltherm 800 & 39.54 & 38.16 & 2.7 & 2.7 \\
390 & Therminol VP1 & 38.56 & 38.20 & 2.7 & 2.7 \\
375 & Therminol 75 & 39.52 & 37.28 & 2.9 & 2.9 \\
\hline
\end{tabular}

Tabla 7.20. Eficiencia Neta de planta termosolar con colectores lineales acoplada a ciclo de potencia s- $\mathrm{CO}_{2}$ Brayton PCRC con Recalentamiento, UA=20000 kW/K.

\begin{tabular}{llllll}
\hline TIT $\left({ }^{\circ} \mathrm{C}\right)$ & SF Configuración & Eficiencia Bruta $(\%)$ & Eficiencia Neta $(\%)$ & LTR Pinch $\left({ }^{\circ} \mathrm{C}\right)$ & HTR Pinch $\left({ }^{\circ} \mathrm{C}\right)$ \\
\hline 550 & Solar Salt & 47.89 & 46.37 & 1.1 & 1.1 \\
520 & Hitec XL & 46.56 & 45.09 & 1.2 & 1.1 \\
415 & Dowtherm A & 41.22 & 39.83 & 1.7 & 1.7 \\
390 & Syltherm 800 & 39.74 & 38.35 & 1.8 & 1.9 \\
390 & Therminol VP1 & 39.72 & 38.40 & 1.8 & 1.9 \\
375 & Therminol 75 & 38.79 & 37.44 & 1.9 & 2.0 \\
\hline
\end{tabular}

\subsubsection{Ciclo de potencia con recompresión y enfriamiento intermedio en el compresor principal}

La configuración $\mathrm{RCMCl}$ es similar a la configuración de ciclo RC, pero con incrementando el número de enfriamientos intermedios en el compresor principal, figuras 7.1 y 7.3. Gracias a incluir una etapa más de enfriamiento, se obtiene un incremento de la eficiencia bruta del ciclo de potencia, comparar los resultados en las tablas 7.11 a 7.15 con las tablas 7.21 a 7.25. El comportamiento de los ciclos RCMCl es muy similar a los ciclos RC. Para un tamaño térmico de los recuperaros $U A=20000 \mathrm{~kW} / \mathrm{K}$, el incremento de eficiencia energética no es significativo, al igual que ocurría con la tipología de ciclos de potencia RC. Así mismo, a partir de UA=10000 $\mathrm{kW} / \mathrm{K}$ el rendimiento de esta tipología de ciclos Brayton proporcionan un rendimiento energético superior a los ciclos Rankine para todo el rango de temperaturas de entrada en turbina TIT estudiados en este capítulo, para más detalle comparar los resultados de la tabla 7.10 con las tablas 7.23 a 7.25 . 
7. Optimización de plantas termosolares de colectores lineales con aceites térmicos como HTF acopladas a ciclos Brayton de $\mathrm{CO}_{2}$ supercrítico

Tabla 7.21. Eficiencia Neta de planta termosolar con colectores lineales acoplada a ciclo de potencia s- $\mathrm{CO}_{2}$ Brayton $\mathrm{RCMCl}$ con Recalentamiento, $\mathrm{UA}=3000 \mathrm{~kW} / \mathrm{K}$.

\begin{tabular}{llllll}
\hline TIT $\left({ }^{\circ} \mathrm{C}\right)$ & SF Configuración & Eficiencia Bruta (\%) & Eficiencia Neta $(\%)$ & LTR Pinch $\left({ }^{\circ} \mathrm{C}\right)$ & HTR Pinch $\left({ }^{\circ} \mathrm{C}\right)$ \\
\hline 550 & Solar Salt & 43.78 & 42.36 & 24.3 & 60.3 \\
520 & Hitec XL & 42.39 & 41.03 & 24.2 & 60.7 \\
415 & Dowtherm A & 36.93 & 35.62 & 22.9 & 62.6 \\
390 & Syltherm 800 & 35.42 & 34.08 & 22.4 & 63.3 \\
390 & Therminol VP1 & 35.43 & 34.19 & 22.4 & 63.3 \\
375 & Therminol 75 & 34.49 & 33.30 & 21.9 & 63.9 \\
\hline
\end{tabular}

Tabla 7.22. Eficiencia Neta de planta termosolar con colectores lineales acoplada a ciclo de potencia s- $\mathrm{CO}_{2}$ Brayton $\mathrm{RCMCl}$ con Recalentamiento, $\mathrm{UA}=5000 \mathrm{~kW} / \mathrm{K}$.

\begin{tabular}{llllll}
\hline TIT $\left({ }^{\circ} \mathrm{C}\right)$ & SF Configuración & Eficiencia Bruta (\%) & Eficiencia Neta $(\%)$ & LTR Pinch $\left({ }^{\circ} \mathrm{C}\right)$ & HTR Pinch $\left({ }^{\circ} \mathrm{C}\right)$ \\
\hline 550 & Solar Salt & 47.26 & 45.74 & 16.3 & 30.9 \\
520 & Hitec XL & 45.74 & 44.28 & 16.3 & 31.6 \\
415 & Dowtherm A & 39.66 & 38.25 & 16.9 & 34.7 \\
390 & Syltherm 800 & 38.01 & 36.56 & 16.8 & 35.9 \\
390 & Therminol VP1 & 37.97 & 36.64 & 16.8 & 35.9 \\
375 & Therminol 75 & 36.94 & 35.65 & 16.7 & 36.6 \\
\hline
\end{tabular}

Tabla 7.23. Eficiencia Neta de planta termosolar con colectores lineales acoplada a ciclo de potencia s- $\mathrm{CO}_{2}$ Brayton $\mathrm{RCMCl}$ con Recalentamiento), UA=10000 kW/K.

\begin{tabular}{llllll}
\hline TIT $\left({ }^{\circ} \mathrm{C}\right)$ & SF Configuración & Eficiencia Bruta $(\%)$ & Eficiencia Neta $(\%)$ & LTR Pinch $\left({ }^{\circ} \mathrm{C}\right)$ & HTR Pinch $\left({ }^{\circ} \mathrm{C}\right)$ \\
\hline 550 & Solar Salt & 50.38 & 48.78 & 9.2 & 9.3 \\
520 & Hitec XL & 48.82 & 47.27 & 9.4 & 10.1 \\
415 & Dowtherm A & 42.63 & 41.12 & 10.4 & 12.9 \\
390 & Syltherm 800 & 40.93 & 39.37 & 10.3 & 13.9 \\
390 & Therminol VP1 & 40.88 & 39.46 & 10.3 & 13.9 \\
375 & Therminol 75 & 39.78 & 38.40 & 10.3 & 14.6 \\
\hline
\end{tabular}

Tabla 7.24. Eficiencia Neta de planta termosolar con colectores lineales acoplada a ciclo de potencia s- $\mathrm{CO}_{2}$ Brayton $\mathrm{RCMCl}$ con Recalentamiento, $\mathrm{UA}=15000 \mathrm{~kW} / \mathrm{K}$.

\begin{tabular}{llllll}
\hline TIT $\left({ }^{\circ} \mathrm{C}\right)$ & SF Configuración & Eficiencia Bruta (\%) & Eficiencia Neta $(\%)$ & LTR Pinch $\left({ }^{\circ} \mathrm{C}\right)$ & HTR Pinch $\left({ }^{\circ} \mathrm{C}\right)$ \\
\hline 550 & Solar Salt & 51.43 & 49.79 & 3.8 & 3.8 \\
520 & Hitec XL & 49.90 & 48.32 & 3.6 & 4.4 \\
415 & Dowtherm A & 43.78 & 42.23 & 6.4 & 6.4 \\
390 & Syltherm 800 & 42.05 & 40.45 & 6.9 & 7.1 \\
390 & Therminol VP1 & 42.00 & 40.54 & 6.9 & 7.1 \\
375 & Therminol 75 & 40.88 & 39.46 & 6.9 & 7.6 \\
\hline
\end{tabular}

Tabla 7.25. Eficiencia Neta de planta termosolar con colectores lineales acoplada a ciclo de potencia s- $\mathrm{CO}_{2}$ Brayton $\mathrm{RCMCl}$ con Recalentamiento, $\mathrm{UA}=20000 \mathrm{~kW} / \mathrm{K}$.

\begin{tabular}{llllll}
\hline TIT $\left({ }^{\circ} \mathrm{C}\right)$ & SF Configuración & Eficiencia Bruta (\%) & Eficiencia Neta (\%) & LTR Pinch $\left({ }^{\circ} \mathrm{C}\right)$ & HTR Pinch $\left({ }^{\circ} \mathrm{C}\right)$ \\
\hline 550 & Solar Salt & 51.89 & 50.24 & 1.8 & 1.9 \\
520 & Hitec XL & 50.44 & 48.85 & 1.9 & 2.3 \\
415 & Dowtherm A & 44.32 & 42.75 & 3.6 & 3.7 \\
390 & Syltherm 800 & 42.56 & 40.94 & 4.4 & 4.6 \\
390 & Therminol VP1 & 42.51 & 41.00 & 4.4 & 4.6 \\
375 & Therminol 75 & 41.46 & 40.02 & 4.6 & 4.6 \\
\hline
\end{tabular}


7. Optimización de plantas termosolares de colectores lineales con aceites térmicos como HTF acopladas a ciclos Brayton de $\mathrm{CO}_{2}$ supercrítico

\subsubsection{Parámetros termodinámicos para la optimización de la eficiencia del ciclo termodinámico Brayton.}

De las tablas 7.26 a 7.34 se deducen las siguientes conclusiones comunes a las tres tipologías de ciclos Brayton: RC, PCRC y RCMCl.

- Un incremento del tamaño térmico UA Total de los recuperadores de calor, da lugar a una reducción significativa de la presión de entrada en la turbina de recalentamiento RHP. Comparando las tres configuraciones, la tipología de ciclo RC es la que requiere mayores valores de RHP, seguida por la configuración RCMCl y la PCRC.

- En relación a la fracción de flujo óptima, su valor se ve incrementado con el incremento del tamaño térmico de los recuperadores. Si se comparan las tres tipologías de ciclos, la configuración PCRC es la requiere mayores valores de fracción de flujo óptima, y la que menor la configuración RC.

- En las tipología de ciclo $\mathrm{RC}$ y $\mathrm{RCMCl}$, un incremento del tamaño térmico de los recuperadores UA da lugar a un pequeño incremento de la presión óptima de entrada al compresor principal. En la configuración PCRC el aumento del UA de los recuperadores da lugar a un incremento de la presión de descarga óptima del precompresor.

Las conclusiones anteriores son similares a las obtenidas en el apartado 6.4.2. en el capítulo 6 de este trabajo.

Tabla 7.26. Variables en la optimización del rendimiento energético de planta termosolar con colectores lineales, Dowtherm A como HTF, acopladas a ciclos de potencia s- $\mathrm{CO}_{2}$ Brayton RC

con Recalentamiento.

\begin{tabular}{llllll}
\hline UA (kW/K) & TIT $\left({ }^{\circ} \mathrm{C}\right)$ & CIP (bar) & RHP (bar) & FracciónFlujo (\%) & Caudal (kg/s) \\
\hline 3000 & 415 & 75.7 & 157.5 & 0.18 & 526.1 \\
5000 & 415 & 76.0 & 152.5 & 0.27 & 547.0 \\
10000 & 415 & 76.4 & 146.3 & 0.34 & 561.1 \\
15000 & 415 & 76.6 & 143.6 & 0.36 & 563.7 \\
20000 & 415 & 76.7 & 142.3 & 0.37 & 563.8 \\
\hline
\end{tabular}

Tabla 7.27. Variables en la optimización del rendimiento energético de planta termosolar con colectores lineales, Therminol VP/Syltherm 800 como HTF, acoplada a ciclos de potencia s- $\mathrm{CO}_{2}$ Brayton RC con Recalentamiento.

\begin{tabular}{llllll}
\hline UA $(\mathrm{kW} / \mathrm{K})$ & TIT $\left({ }^{\circ} \mathrm{C}\right)$ & CIP (bar) & RHP $($ bar $)$ & FracciónFlujo $(\%)$ & Caudal $(\mathrm{kg} / \mathrm{s})$ \\
\hline 3000 & 390 & 75.8 & 157.4 & 0.17 & 558.5 \\
5000 & 390 & 76.0 & 152.6 & 0.26 & 582.6 \\
10000 & 390 & 76.5 & 146.6 & 0.33 & 598.8 \\
15000 & 390 & 76.7 & 144.0 & 0.36 & 602.4 \\
20000 & 390 & 76.8 & 142.8 & 0.36 & 602.1 \\
\hline
\end{tabular}


7. Optimización de plantas termosolares de colectores lineales con aceites térmicos como HTF acopladas a ciclos Brayton de $\mathrm{CO}_{2}$ supercrítico

Tabla 7.28. Variables en la optimización del rendimiento energético de planta termosolar con colectores lineales, Therminol 75 como HTF, acopladas a ciclos de potencia s- $\mathrm{CO}_{2}$ Brayton RC con Recalentamiento.

\begin{tabular}{llllll}
\hline UA $(\mathrm{kW} / \mathrm{K})$ & TIT $\left({ }^{\circ} \mathrm{C}\right)$ & CIP (bar) & RHP (bar) & Fracción Flujo $(\%)$ & Caudal $(\mathrm{kg} / \mathrm{s})$ \\
\hline 3000 & 375 & 75.8 & 157.6 & 0.16 & 579.3 \\
5000 & 375 & 76.0 & 152.7 & 0.26 & 606.3 \\
10000 & 375 & 76.5 & 146.9 & 0.33 & 624.0 \\
15000 & 375 & 76.8 & 144.3 & 0.35 & 626.0 \\
20000 & 375 & 76.9 & 143.1 & 0.36 & 629.2 \\
\hline
\end{tabular}

Tabla 7.29. Variables en la optimización del rendimiento energético de planta termosolar con colectores lineales, Dowtherm A como HTF, acopladas a ciclos de potencia s- $\mathrm{CO}_{2}$ Brayton PCRC con Recalentamiento.

\begin{tabular}{lllllll}
\hline UA (kW/K) & TIT $\left({ }^{\circ} \mathrm{C}\right)$ & $\begin{array}{l}\text { Precomp.Inlet } \\
\text { (bar) }\end{array}$ & $\begin{array}{l}\text { Precomp. } \\
\text { Outlet (bar) }\end{array}$ & RHP (bar) & FracciónFlujo (\%) & Caudal (kg/s) \\
\hline 3000 & 415 & 73.7 & 76.8 & 150.7 & 0.37 & 481.1 \\
5000 & 415 & 73.7 & 78.4 & 144.0 & 0.44 & 483.6 \\
10000 & 415 & 73.7 & 80.4 & 139.4 & 0.44 & 482.6 \\
15000 & 415 & 73.7 & 81.1 & 138.4 & 0.42 & 481.7 \\
20000 & 415 & 73.7 & 81.4 & 138.2 & 0.42 & 481.1 \\
\hline
\end{tabular}

Tabla 7.30. Variables en la optimización del rendimiento energético de planta termosolar con colectores lineales, Therminol VP/Syltherm 800 como HTF, acopladas a ciclos de potencia s$\mathrm{CO}_{2}$ Brayton PCRC con Recalentamiento).

\begin{tabular}{lllllll}
\hline UA $(\mathrm{kW} / \mathrm{K})$ & TIT $\left({ }^{\circ} \mathrm{C}\right)$ & $\begin{array}{l}\text { Precomp. } \\
\text { Inlet (bar) }\end{array}$ & $\begin{array}{l}\text { Precomp. Outlet } \\
\text { (bar) }\end{array}$ & RHP (bar) & $\begin{array}{l}\text { Fracción Flujo } \\
(\%)\end{array}$ & Caudal (kg/s) \\
\hline 3000 & 390 & 73.7 & 76.8 & 150.8 & 0.35 & 509.9 \\
5000 & 390 & 73.7 & 78.3 & 144.3 & 0.43 & 512.9 \\
10000 & 390 & 73.7 & 80.3 & 139.6 & 0.43 & 512.5 \\
15000 & 390 & 73.7 & 81.0 & 138.7 & 0.42 & 511.5 \\
20000 & 390 & 73.7 & 81.4 & 138.3 & 0.42 & 511.0 \\
\hline
\end{tabular}

Tabla 7.31. Variables en la optimización del rendimiento energético de planta termosolar con colectores lineales, Therminol 75 como HTF, acopladas a ciclos de potencia s- $\mathrm{CO}_{2}$ Brayton PCRC con Recalentamiento.

\begin{tabular}{lllllll}
\hline UA $(\mathrm{kW} / \mathrm{K})$ & TIT $\left({ }^{\circ} \mathrm{C}\right)$ & $\begin{array}{l}\text { Precomp.Inlet } \\
\text { (bar) }\end{array}$ & $\begin{array}{l}\text { Precomp.Outlet } \\
\text { (bar) }\end{array}$ & RHP (bar) & $\begin{array}{l}\text { Fracción Flujo } \\
(\%)\end{array}$ & Caudal $(\mathrm{kg} / \mathrm{s})$ \\
\hline 3000 & 375 & 73.7 & 77.2 & 150.9 & 0.37 & 530.1 \\
5000 & 375 & 73.7 & 78.2 & 144.4 & 0.42 & 532.5 \\
10000 & 375 & 73.7 & 80.2 & 139.8 & 0.43 & 532.5 \\
15000 & 375 & 73.7 & 81.0 & 138.8 & 0.42 & 531.6 \\
20000 & 375 & 73.7 & 81.3 & 138.5 & 0.41 & 531.0 \\
\hline
\end{tabular}


7. Optimización de plantas termosolares de colectores lineales con aceites térmicos como HTF acopladas a ciclos Brayton de $\mathrm{CO}_{2}$ supercrítico

Tabla 7.32. Variables en la optimización del rendimiento energético de planta termosolar con colectores lineales, Dowtherm A como HTF, acopladas a ciclos de potencia s- $\mathrm{CO}_{2}$ Brayton

$\mathrm{RCMCl}$ con Recalentamiento.

\begin{tabular}{lllllll}
\hline UA (kW/K) & TIT ( $\left.{ }^{\circ} \mathrm{C}\right)$ & CIP1(bar) & $\begin{array}{l}\text { CIP2 } \\
\text { (bar) }\end{array}$ & RHP (bar) & Fracción Flujo (\%) & Caudal (kg/s) \\
\hline 3000 & 415 & 73.7 & 76.2 & 155.8 & 0.20 & 517.4 \\
5000 & 415 & 75.5 & 79.6 & 152.2 & 0.29 & 538.8 \\
10000 & 415 & 75.7 & 83.7 & 145.3 & 0.36 & 546.6 \\
15000 & 415 & 75.7 & 84.1 & 142.5 & 0.38 & 547.2 \\
20000 & 415 & 75.7 & 83.7 & 141.3 & 0.38 & 546.6 \\
\hline
\end{tabular}

Tabla 7.33. Variables en la optimización del rendimiento energético de planta termosolar con colectores lineales, Therminol VP/Syltherm 800 como HTF, acopladas a ciclos de potencia s$\mathrm{CO}_{2}$ Brayton $\mathrm{RCMCl}$ con Recalentamiento.

\begin{tabular}{lllllll}
\hline UA $(\mathrm{kW} / \mathrm{K})$ & TIT $\left({ }^{\circ} \mathrm{C}\right)$ & CIP1(bar) & CIP2(bar) & RHP (bar) & Fracción Flujo (\%) & Caudal $(\mathrm{kg} / \mathrm{s})$ \\
\hline 3000 & 390 & 73.7 & 76.2 & 155.7 & 0.19 & 549.8 \\
5000 & 390 & 75.6 & 79.7 & 152.3 & 0.29 & 573.4 \\
10000 & 390 & 75.7 & 84.4 & 145.6 & 0.36 & 582.3 \\
15000 & 390 & 75.8 & 85.3 & 142.9 & 0.37 & 583.3 \\
20000 & 390 & 75.8 & 85.1 & 141.6 & 0.38 & 582.8 \\
\hline
\end{tabular}

Tabla 7.34. Variables en la optimización del rendimiento energético de planta termosolar con colectores lineales, Therminol 75 como HTF, acopladas a ciclos de potencias- $\mathrm{CO}_{2}$ Brayton $\mathrm{RCMCl}$ con Recalentamiento.

\begin{tabular}{lllllll}
\hline UA $(\mathrm{kW} / \mathrm{K})$ & TIT $\left({ }^{\circ} \mathrm{C}\right)$ & CIP1(bar) & CIP2(bar) & RHP (bar) & Fracción Flujo (\%) & Caudal (kg/s) \\
\hline 3000 & 375 & 73.7 & 76.2 & 155.7 & 0.19 & 571.2 \\
5000 & 375 & 75.6 & 79.8 & 152.4 & 0.29 & 596.5 \\
10000 & 375 & 75.7 & 84.8 & 145.8 & 0.35 & 606.1 \\
15000 & 375 & 75.8 & 86.0 & 143.1 & 0.37 & 607.4 \\
20000 & 375 & 75.8 & 86.0 & 141.8 & 0.38 & 607.1 \\
\hline
\end{tabular}

\subsection{3. Área apertura efectiva del campo solar}

En las figuras 7.4 a 7.9 se han representado gráficamente el área de apertura efectiva, tanto con colectores PTC como con colectores LF, de las diferentes configuraciones de plantas termosolares con ciclos Brayton estudiadas en este capítulo de la tesis. Como primera conclusión deducida se confirma que un incremento de la temperatura de entrada en turbina TIT desde $400^{\circ} \mathrm{C}$ hasta $550^{\circ} \mathrm{C}$, proporciona una reducción del área de apertura efectiva superior al $10 \%$. Como segunda conclusión se confirma que un incremento del tamaño térmico de los recuperadores proporciona una reducción del área de apertura efectiva de los colectores solares. Se fija en UA=10000 kW/K, el valor umbral a partir del cual un incremento adicional de UA no proporciona reducción significativas del área de apertura efectiva de los colectores solares. 
7. Optimización de plantas termosolares de colectores lineales con aceites térmicos como HTF acopladas a ciclos Brayton de $\mathrm{CO}_{2}$ supercrítico

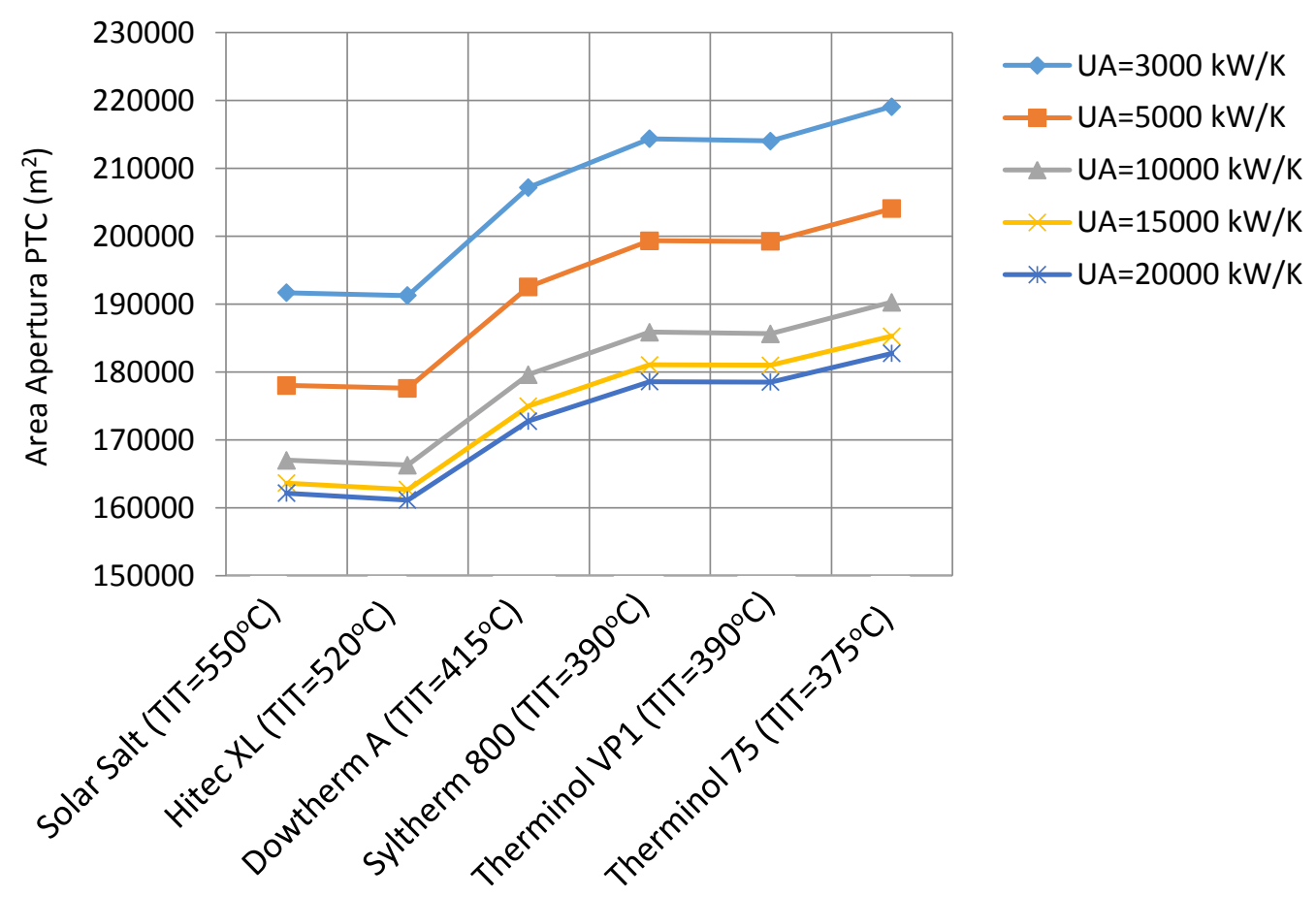

Figura 7.4. Área de Apertura Efectiva con colectores solares lineales PTC acoplada a ciclo de potencia $\mathrm{s}-\mathrm{CO}_{2}$ Brayton $\mathrm{RC}$ con recalentamiento.
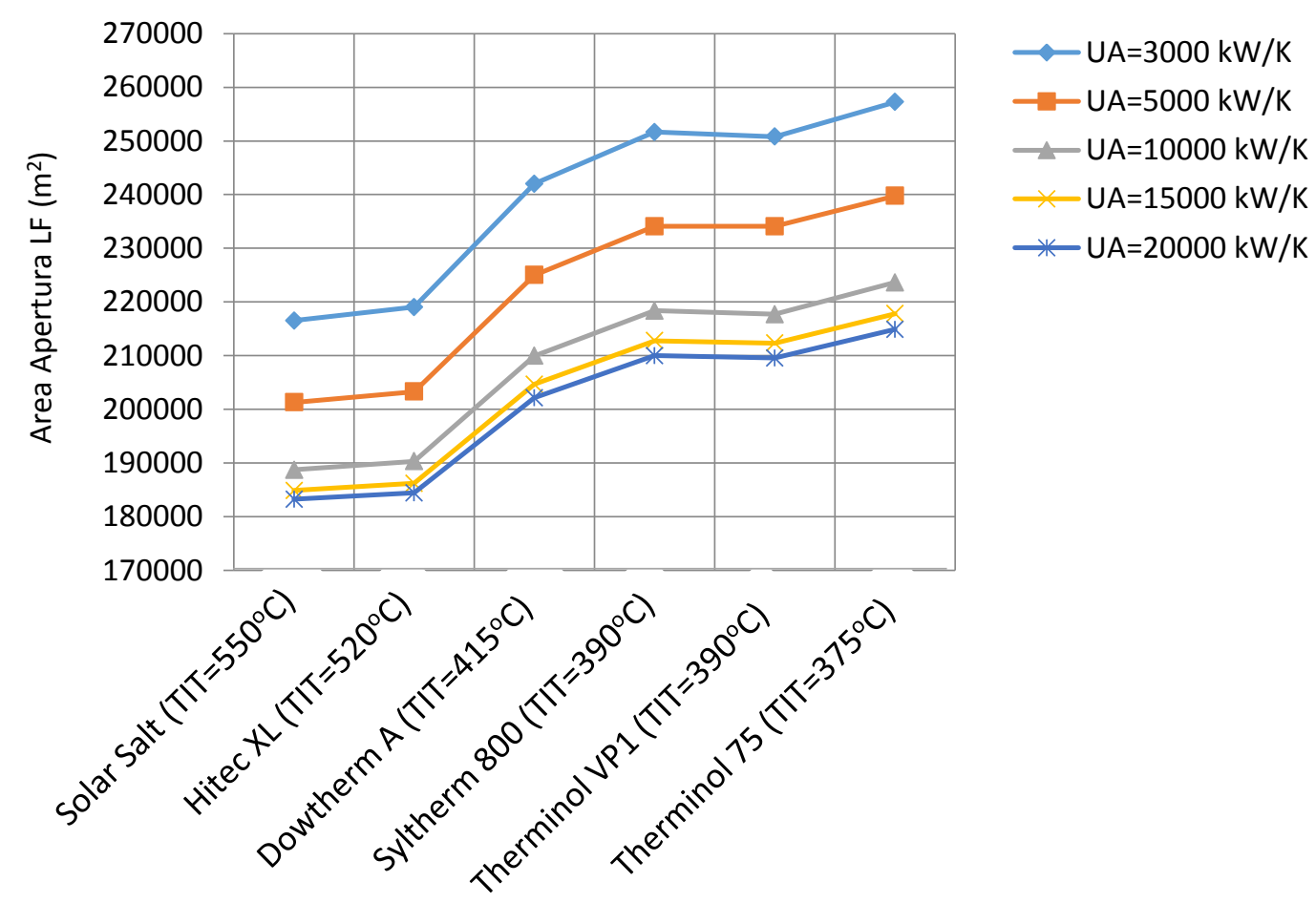

Figura 7.5. Área de Apertura Efectiva con colectores solares lineales LF acoplada a ciclo de potencia s- $\mathrm{CO}_{2}$ Brayton $\mathrm{RC}$ con recalentamiento. 
7. Optimización de plantas termosolares de colectores lineales con aceites térmicos como HTF acopladas a ciclos Brayton de $\mathrm{CO}_{2}$ supercrítico

7.3.3.2. Ciclo de potencia con enfriamiento previo a la recompresión
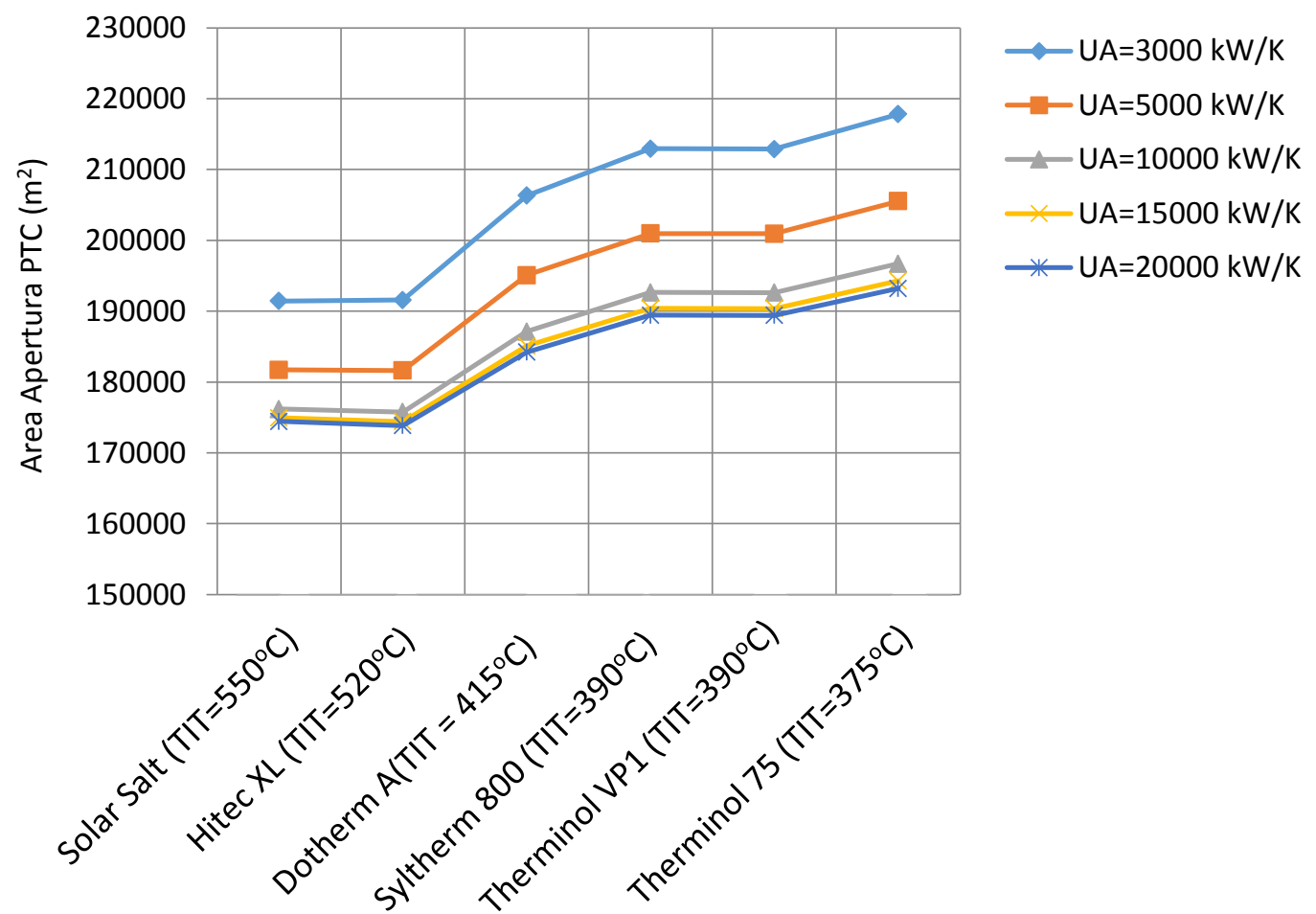

Figura 7.6. Área de Apertura Efectiva con colectores solares lineales PTC acoplada a ciclo de potencia s- $\mathrm{CO}_{2}$ Brayton PCRC con recalentamiento.

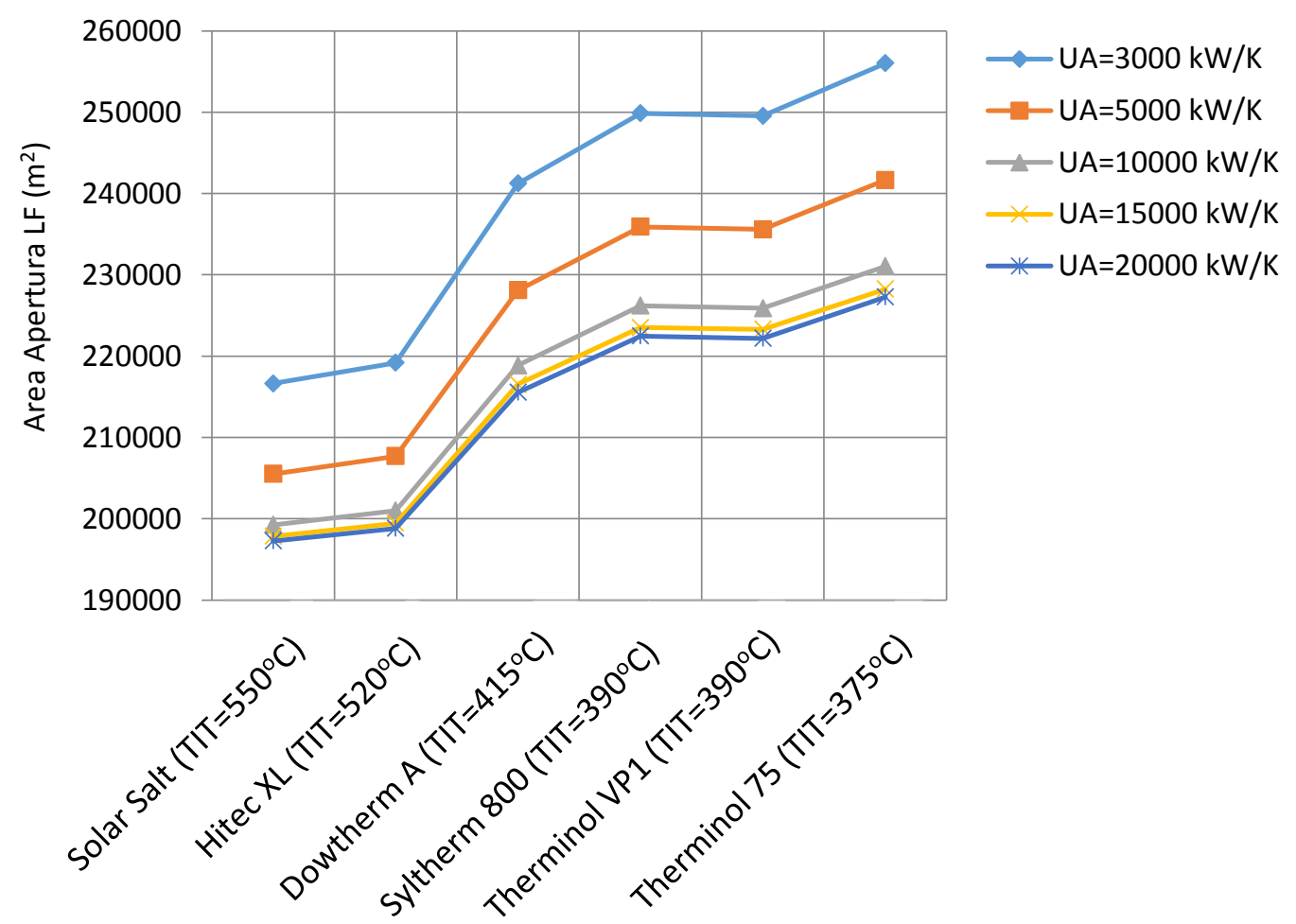

Figura 7.7. Área de Apertura Efectiva con colectores solares lineales $L F$ acoplada a ciclo de potencia s- $\mathrm{CO}_{2}$ Brayton PCRC con recalentamiento. 
7. Optimización de plantas termosolares de colectores lineales con aceites térmicos como HTF acopladas a ciclos Brayton de $\mathrm{CO}_{2}$ supercrítico

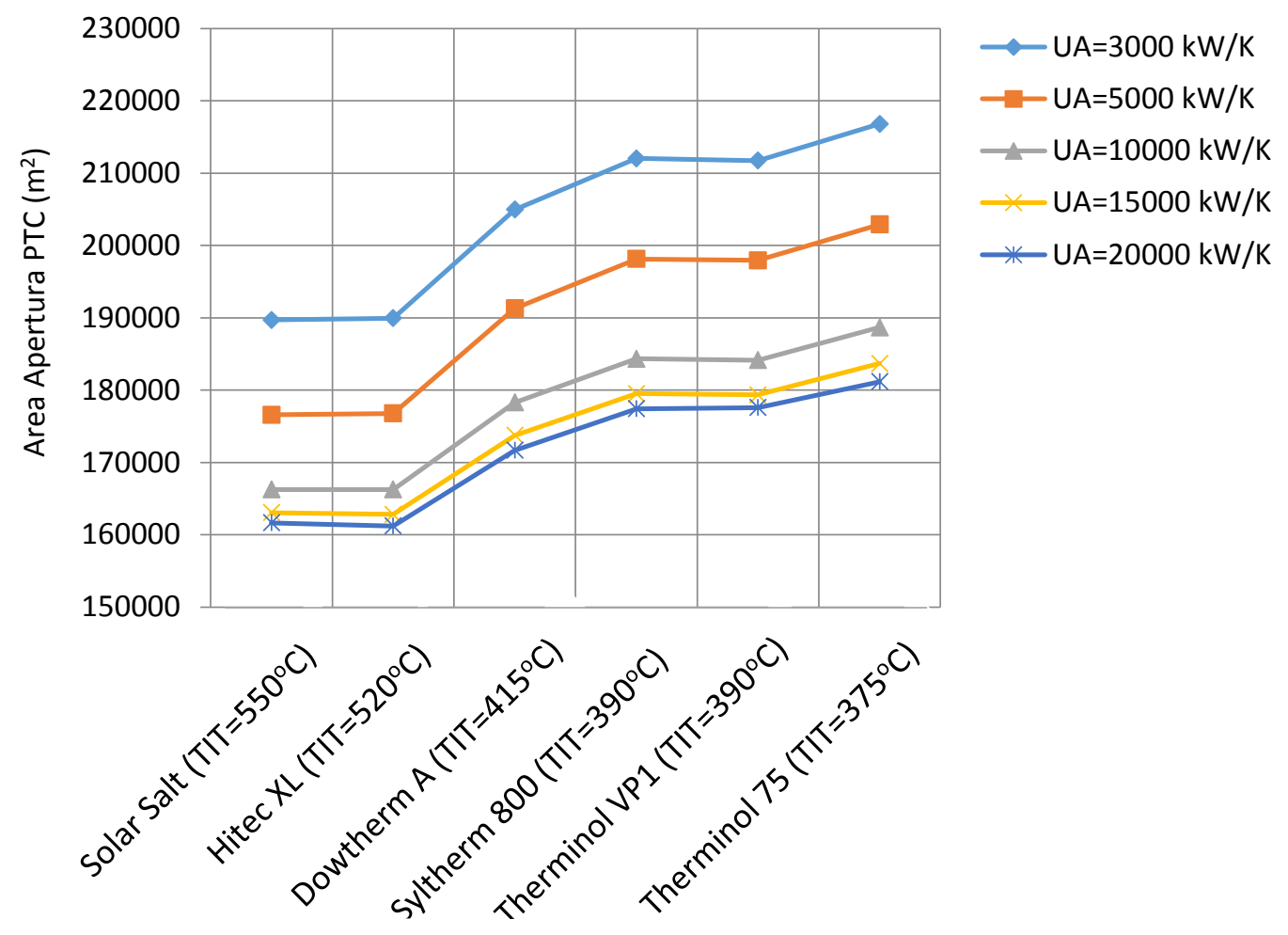

Figura 7.8. Área de Apertura Efectiva con colectores solares lineales PTC acoplada a ciclo de potencia s- $\mathrm{CO}_{2}$ Brayton $\mathrm{RCMCl}$ con recalentamiento.

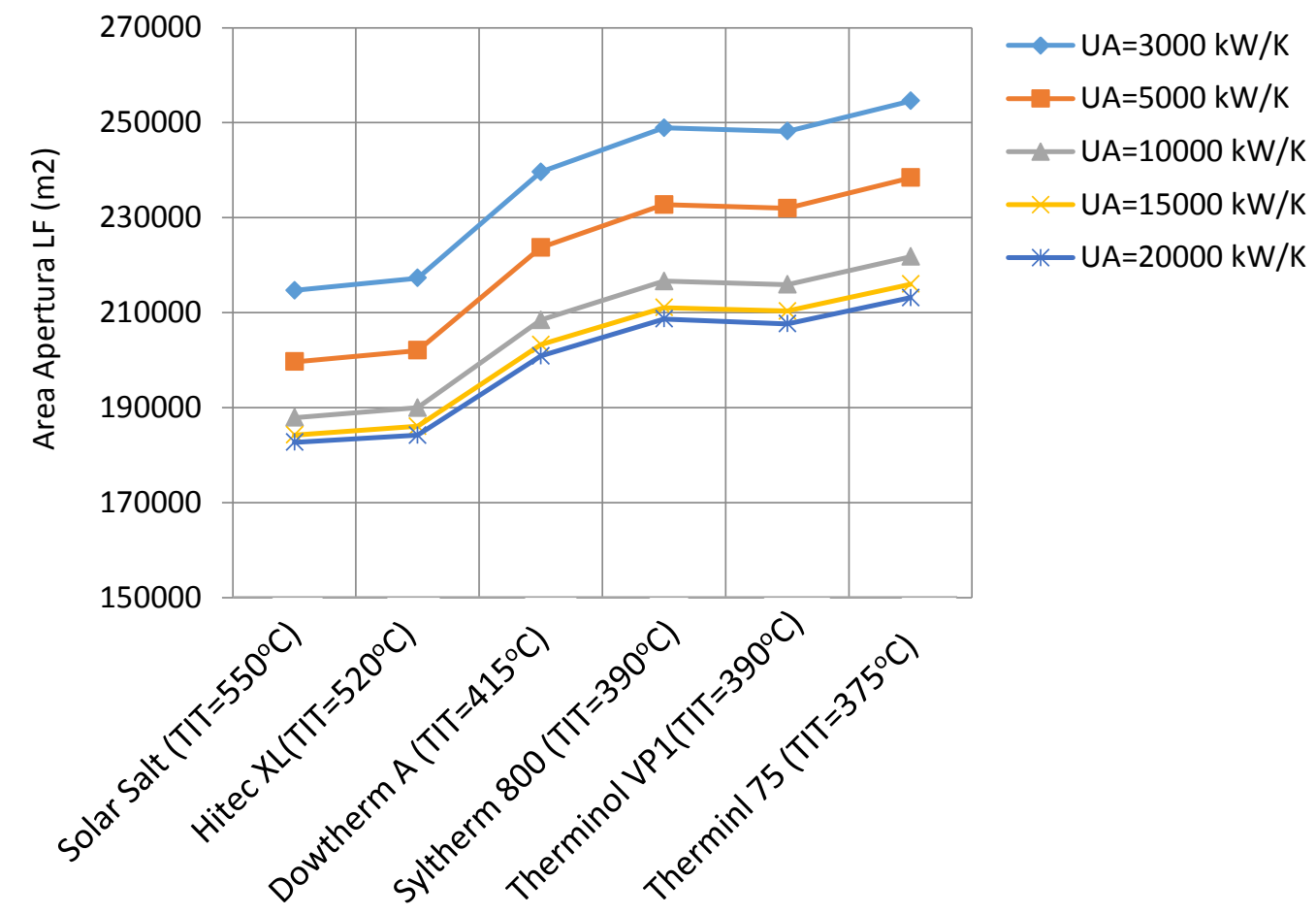

Figura 7.9. Área de Apertura Efectiva con colectores solares lineales LF acoplada a ciclo de potencia s- $\mathrm{CO}_{2}$ Brayton $\mathrm{RCMCl}$ con recalentamiento. 
7. Optimización de plantas termosolares de colectores lineales con aceites térmicos como HTF acopladas a ciclos Brayton de $\mathrm{CO}_{2}$ supercrítico

\subsubsection{Estimación de costes del campo solar}

De acuerdo a los costes unitarios aplicados, el ahorro económico consecuencia directa de la elección de la tecnología de aceites térmicos con ciclos $\mathrm{s}-\mathrm{CO}_{2}$ Brayton, en relación a la alternativa de las sales fundidas, se materializa en un ahorro de costes de inversión en los campos solares entre el $15-20 \%$. Como se ha explicado, a pesar de que las sales fundidas proporcionan mayores valores de temperatura de entrada en turbina TIT, el ahorro en los costes de los materiales con menor grado de aleación del acero al carbono, la eliminación de la tecnología heat-tracing para evitar la solidificación de las sales, y las menores pérdidas térmicas por radiación en los tubos absorbedores, marcan la ventaja competitiva de los aceites térmicos frente a las sales fundidas en los plantas termosolares con colectores lineales y ciclos s- $\mathrm{CO}_{2}$ Brayton.
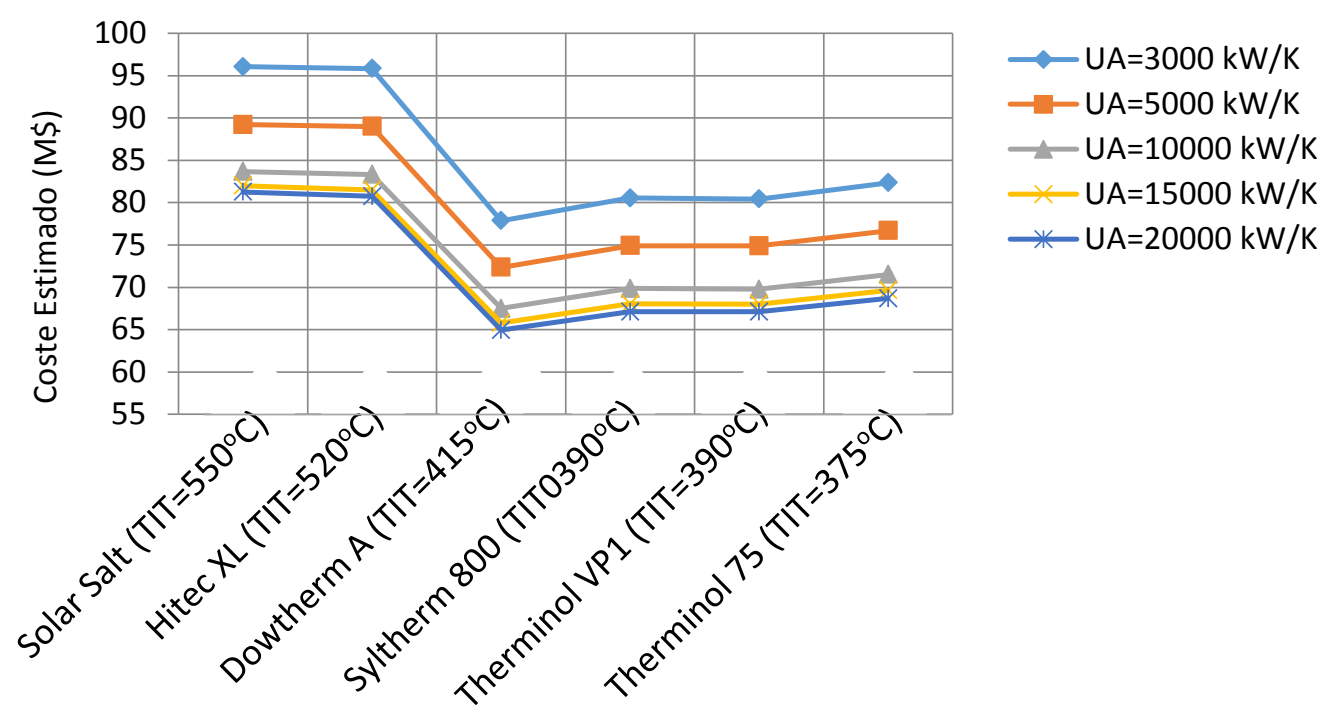

Figura 7.10. Estimación de Costes de campo solar con colectores lineales PTC acoplada a ciclo de potencia $\mathrm{s}-\mathrm{CO}_{2}$ Brayton $\mathrm{RC}$ con recalentamiento.
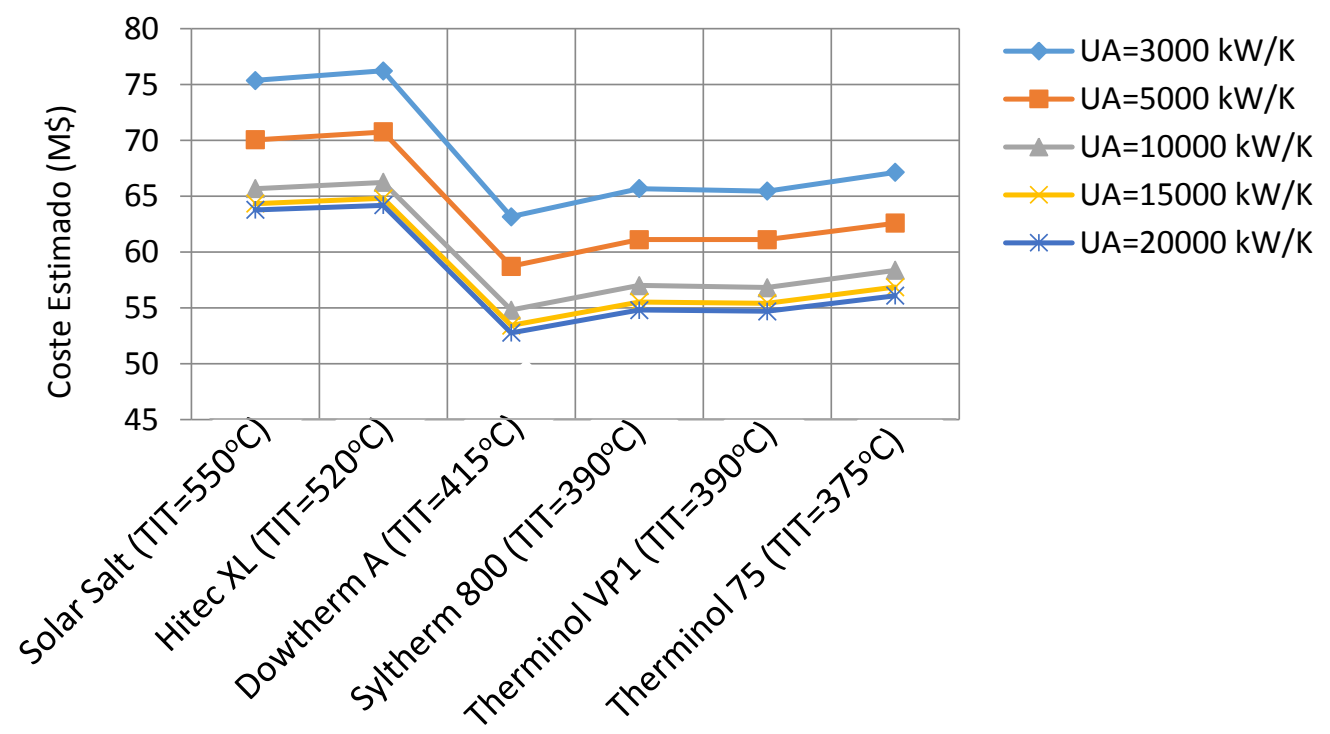

Figura 7.11. Estimación de Costes de campo solar con colectores lineales LF acoplada a ciclo de potencia s- $\mathrm{CO}_{2}$ Brayton $\mathrm{RC}$ con recalentamiento. 
7. Optimización de plantas termosolares de colectores lineales con aceites térmicos como HTF acopladas a ciclos Brayton de $\mathrm{CO}_{2}$ supercrítico

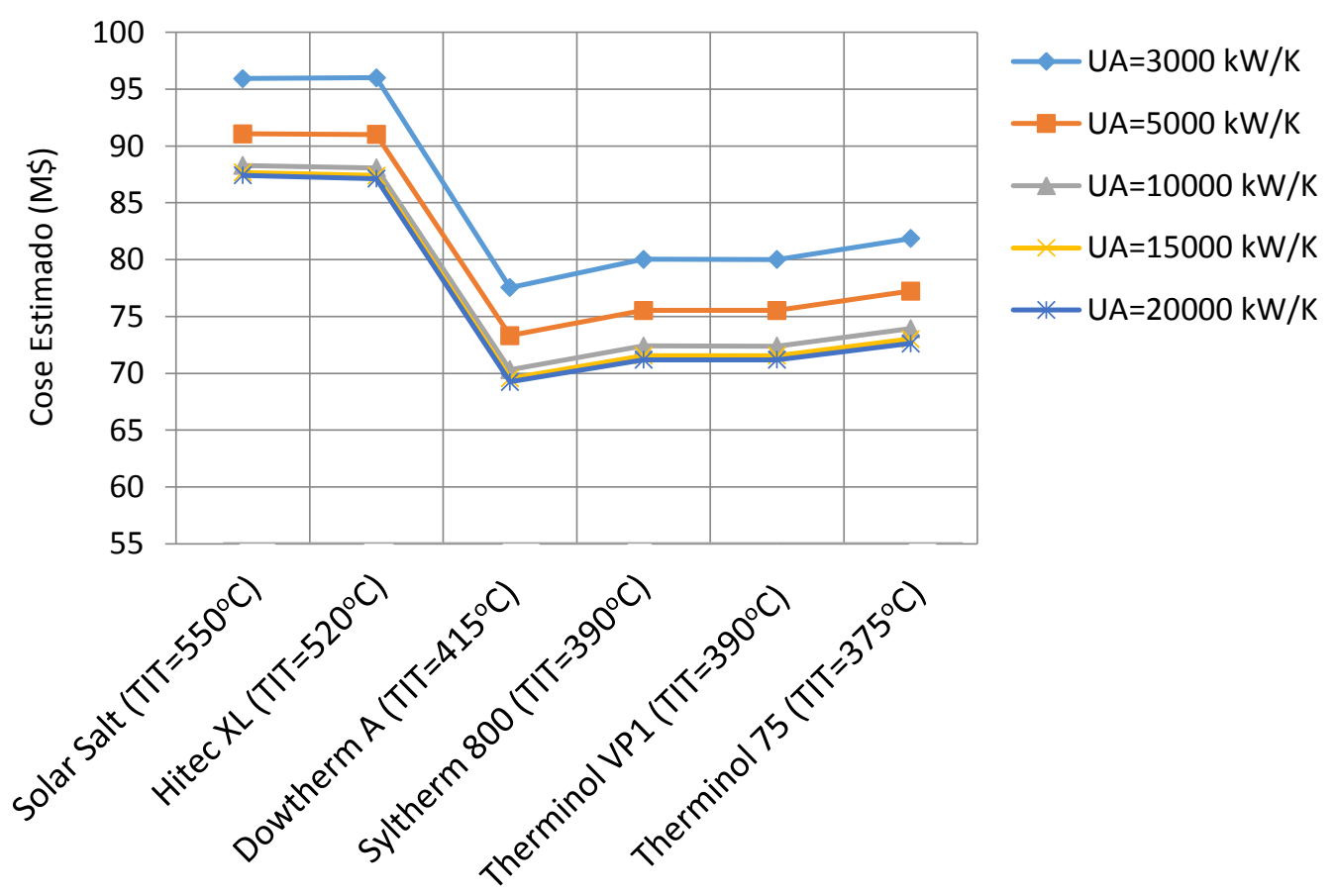

Figura 7.12. Estimación de Costes de campo solar con colectores lineales PTC acoplada a ciclo de potencia s- $\mathrm{CO}_{2}$ Brayton PCRC con recalentamiento.

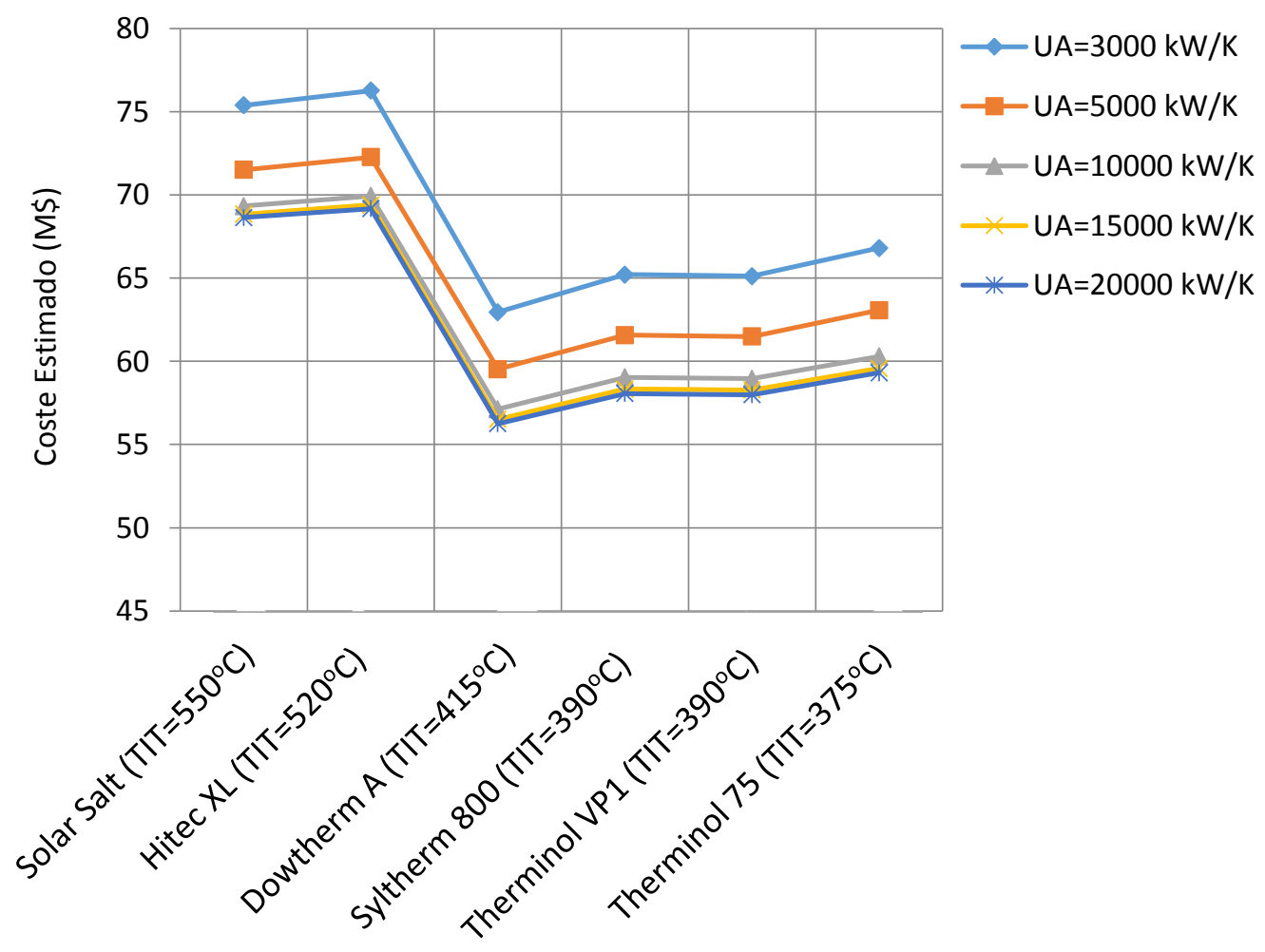

Figura 7.13. Estimación de Costes de campo solar con colectores lineales LF acoplada a ciclo de potencia s- $\mathrm{CO}_{2}$ Brayton PCRC con recalentamiento. 
7. Optimización de plantas termosolares de colectores lineales con aceites térmicos como HTF acopladas a ciclos Brayton de $\mathrm{CO}_{2}$ supercrítico

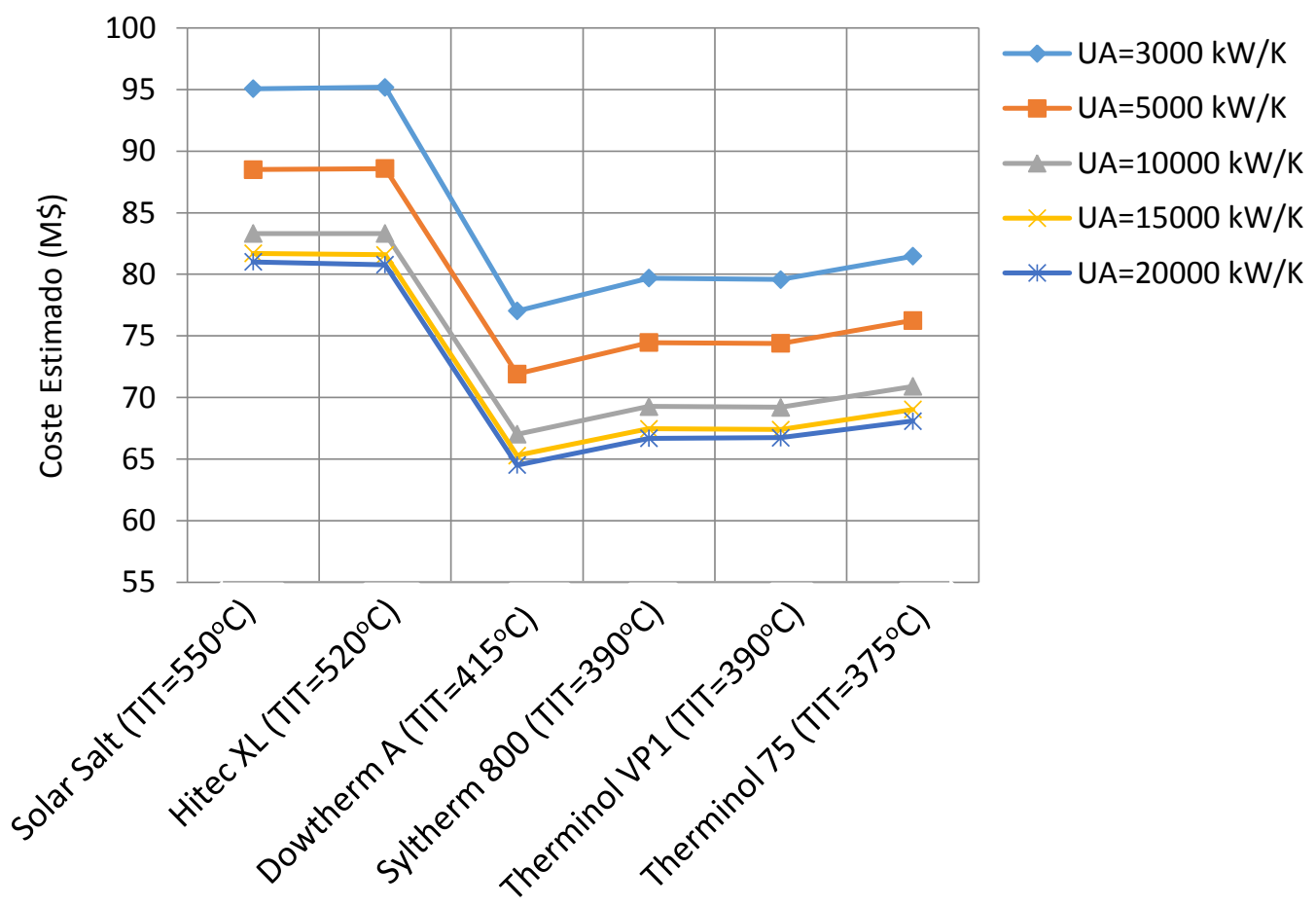

Figura 7.14. Estimación de Costes de campo solar con colectores lineales PTC acoplada a ciclo de potencia s- $\mathrm{CO}_{2}$ Brayton $\mathrm{RCMCl}$ con recalentamiento.

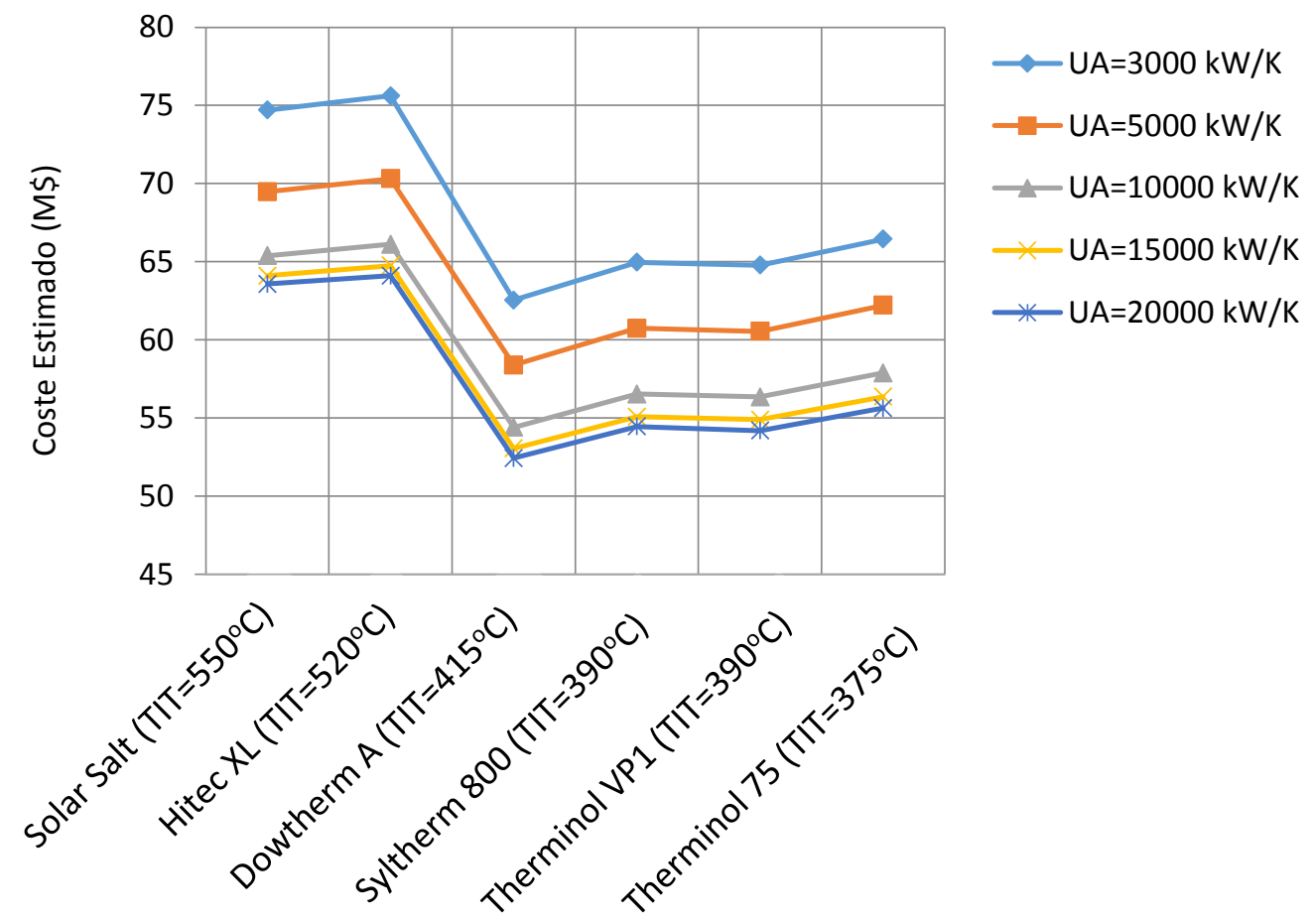

Figura 7.15. Estimación de Costes de campo solar con colectores lineales LF acoplada a ciclo de potencia s- $\mathrm{CO}_{2}$ Brayton $\mathrm{RCMCl}$ con recalentamiento. 
7. Optimización de plantas termosolares de colectores lineales con aceites térmicos como HTF acopladas a ciclos Brayton de $\mathrm{CO}_{2}$ supercrítico

\subsubsection{Diseño de los Recuperadores de Baja y Alta presión}

En este apartado se ha realizado una estimación del coste de inversión de los diferentes tamaños térmicos UA de los recuperadores del ciclo Brayton, ver resultados detalladas en las tablas 7.35 a 7.49. Se ha fijado como precio unitario de los recuperadores entre $90-100$ $\$ / \mathrm{kWth}$, considerando recuperadores de tipología PCHE.

Como primera conclusión se confirma que debido al menor precio unitario de los recuperadores en relación con los colectores solares lineales, un incremento del tamaño térmico de los recuperadores UA proporciona una reducción significativa del coste de la instalación industrial. Se han fijado en los apartados anteriores los valores umbrales del tamaño térmico de los recuperadores a partir de los cuales el incremento de rendimiento energéticos significativo debido a la limitación de temperaturas pinch-point en los recuperadores.

Tabla 7.35. Diseño y parámetros de operación de los recuperadores. Tamaño térmico total de los recuperadores UA=3000 kW/K. Ciclo de potencia s- $\mathrm{CO}_{2}$ Brayton RC.

\begin{tabular}{lllllllll}
\hline $\begin{array}{l}\text { TIT } \\
\left({ }^{\circ} \mathrm{C}\right)\end{array}$ & $\begin{array}{l}\text { SF } \\
\text { Config. }\end{array}$ & $\begin{array}{l}\text { LTR Pinch } \\
\left({ }^{\circ} \mathrm{C}\right)\end{array}$ & $\begin{array}{l}\text { HTR Pinch } \\
\left({ }^{\circ} \mathrm{C}\right)\end{array}$ & $\begin{array}{l}\text { LTR } \\
(\mathrm{kW})\end{array}$ & $\begin{array}{l}\text { HTR } \\
(\mathrm{kW})\end{array}$ & $\begin{array}{l}\text { Total Cost } \\
(\mathrm{M} \$)\end{array}$ & $\begin{array}{l}\text { LTR UA } \\
(\mathrm{kW} / \mathrm{K})\end{array}$ & $\begin{array}{l}\text { HTR UA } \\
(\mathrm{kW} / \mathrm{K})\end{array}$ \\
\hline 550 & Solar Salt & 23.7 & 61.9 & 85062 & 80723 & 16.58 & 1928.8 & 1097 \\
520 & Hitec XL & 23.8 & 62.6 & 89892 & 71268 & 16.12 & 2023.7 & 976.3 \\
415 & Dowtherm A & 22.6 & 64.6 & 112960 & 29767 & 14.27 & 2568.8 & 431.3 \\
390 & Syltherm 800 & 22.1 & 65.4 & 120367 & 16960 & 13.73 & 2751 & 249.6 \\
390 & Therminol VP1 & 22.1 & 65.4 & 120342 & 16957 & 13.73 & 2750.6 & 249.6 \\
375 & Therminol 75 & 21.5 & 0 & 133523 & 0 & 13.35 & 3000 & 0 \\
\hline
\end{tabular}

Tabla 7.36. Diseño y parámetros de operación de los recuperadores. Tamaño térmico total de los recuperadores UA=5000 kW/K. Ciclo de potencia s- $\mathrm{CO}_{2}$ Brayton RC.

\begin{tabular}{lllllllll}
\hline $\begin{array}{l}\text { TIT } \\
\left({ }^{\circ} \mathrm{C}\right)\end{array}$ & $\begin{array}{l}\text { SF } \\
\text { Config. }\end{array}$ & $\begin{array}{l}\text { LTR Pinch } \\
\left({ }^{\circ} \mathrm{C}\right)\end{array}$ & $\begin{array}{l}\text { HTR Pinch } \\
\left({ }^{\circ} \mathrm{C}\right)\end{array}$ & $\begin{array}{l}\text { LTR } \\
(\mathrm{kW})\end{array}$ & $\begin{array}{l}\text { HTR } \\
(\mathrm{kW})\end{array}$ & $\begin{array}{l}\text { Total Cost } \\
(\mathrm{M} \$)^{2}\end{array}$ & $\begin{array}{l}\text { LTR UA } \\
(\mathrm{kW} / \mathrm{K})\end{array}$ & $\begin{array}{l}\text { HTR UA } \\
(\mathrm{kW} / \mathrm{K})\end{array}$ \\
\hline 550 & Solar Salt & 15.8 & 32.1 & 73088 & 105974 & 17.91 & 2777 & 2241.3 \\
520 & Hitec XL & 15.7 & 33.1 & 77780 & 97182 & 17.50 & 2930 & 2069.9 \\
415 & Dowtherm A & 15.1 & 36.2 & 99202 & 59118 & 15.83 & 3673 & 1326.6 \\
390 & Syltherm 800 & 14.8 & 37.1 & 106336 & 47145 & 15.35 & 3924 & 1075.8 \\
390 & Therminol VP1 & 14.8 & 37.1 & 106336 & 47145 & 15.35 & 3924 & 1075.8 \\
375 & Therminol 75 & 14.6 & 37.7 & 111106 & 39203 & 15.03 & 4096 & 905.3 \\
\hline
\end{tabular}

Tabla 7.37. Diseño y parámetros de operación de los recuperadores. Tamaño térmico total de los recuperadores UA=10000 kW/K. Ciclo de potencia s- $\mathrm{CO}_{2}$ Brayton RC.

\begin{tabular}{lllllllll}
\hline $\begin{array}{l}\text { TIT } \\
\left({ }^{\circ} \mathrm{C}\right)\end{array}$ & $\begin{array}{l}\text { SF } \\
\text { Config. }\end{array}$ & $\begin{array}{l}\text { LTR Pinch } \\
\left({ }^{\circ} \mathrm{C}\right)\end{array}$ & $\begin{array}{l}\text { HTR Pinch } \\
\left({ }^{\circ} \mathrm{C}\right)\end{array}$ & $\begin{array}{l}\text { LTR } \\
(\mathrm{kW})\end{array}$ & $\begin{array}{l}\text { HTR } \\
(\mathrm{kW})\end{array}$ & $\begin{array}{l}\text { Total Cost } \\
(\mathrm{M} \$)\end{array}$ & $\begin{array}{l}\text { LTR UA } \\
(\mathrm{kW} / \mathrm{K})\end{array}$ & $\begin{array}{l}\text { HTR UA } \\
(\mathrm{kW} / \mathrm{K})\end{array}$ \\
\hline 550 & Solar Salt & 8.6 & 10.4 & 63808 & 126568 & 19.04 & 5306 & 4695 \\
520 & Hitec XL & 9.1 & 10.9 & 67972 & 119181 & 18.72 & 5485 & 4514 \\
415 & Dowtherm A & 8.8 & 13.9 & 87286 & 85495 & 17.28 & 6587 & 3414 \\
390 & Syltherm 800 & 8.7 & 14.9 & 93787 & 74853 & 16.86 & 6962 & 3038 \\
390 & Therminol VP1 & 8.7 & 14.9 & 93859 & 74787 & 16.86 & 6961 & 3038 \\
375 & Therminol 75 & 8.6 & 15.6 & 98133 & 67769 & 16.59 & 7217 & 2782.9 \\
\hline
\end{tabular}


7. Optimización de plantas termosolares de colectores lineales con aceites térmicos como HTF acopladas a ciclos Brayton de $\mathrm{CO}_{2}$ supercrítico

Tabla 7.38. Diseño y parámetros de operación de los recuperadores. Tamaño térmico total de los recuperadores UA=15000 kW/K. Ciclo de potencia s- $\mathrm{CO}_{2}$ Brayton RC.

\begin{tabular}{lllllllll}
\hline $\begin{array}{l}\text { TIT } \\
\left({ }^{\circ} \mathrm{C}\right)\end{array}$ & $\begin{array}{l}\text { SF } \\
\text { Config. }\end{array}$ & $\begin{array}{l}\text { LTR Pinch } \\
\left({ }^{\circ} \mathrm{C}\right)\end{array}$ & $\begin{array}{l}\text { HTR Pinch } \\
\left({ }^{\circ} \mathrm{C}\right)\end{array}$ & $\begin{array}{l}\text { LTR } \\
(\mathrm{kW})\end{array}$ & $\begin{array}{l}\text { HTR } \\
(\mathrm{kW})\end{array}$ & $\begin{array}{l}\text { Total Cost } \\
(\mathrm{M} \$)\end{array}$ & $\begin{array}{l}\text { LTR UA } \\
(\mathrm{kW} / \mathrm{K})\end{array}$ & $\begin{array}{l}\text { HTR UA } \\
(\mathrm{kW} / \mathrm{K})\end{array}$ \\
\hline 550 & Solar Salt & 4.3 & 4.3 & 61154 & 132814 & 19.40 & 8329 & 6673 \\
520 & Hitec XL & 4.7 & 4.7 & 65022 & 125789 & 19.08 & 8564 & 6437 \\
415 & Dowtherm A & 6.2 & 7.1 & 83441 & 94285 & 17.77 & 9851 & 5149 \\
390 & Syltherm 800 & 6.2 & 7.8 & 89256 & 84865 & 17.41 & 10265 & 4735 \\
390 & Therminol VP1 1 & 6.2 & 7.8 & 89256 & 84865 & 17.41 & 10265 & 4735 \\
375 & Therminol 75 & 5.8 & 8.7 & 93196 & 78124 & 17.13 & 10710 & 4292 \\
\hline
\end{tabular}

Tabla 7.39. Diseño y parámetros de operación de los recuperadores. Tamaño térmico total de los recuperadores UA=20000 kW/K. Ciclo de potencia s- $\mathrm{CO}_{2}$ Brayton RC.

\begin{tabular}{|c|c|c|c|c|c|c|c|c|}
\hline $\begin{array}{l}\text { TIT } \\
\left({ }^{\circ} \mathrm{C}\right) \\
\end{array}$ & $\begin{array}{l}\text { SF } \\
\text { Config. }\end{array}$ & $\begin{array}{l}\text { LTR Pinch } \\
\left({ }^{\circ} \mathrm{C}\right) \\
\end{array}$ & $\begin{array}{l}\text { HTR Pinch } \\
\left({ }^{\circ} \mathrm{C}\right) \\
\end{array}$ & $\begin{array}{l}\text { LTR } \\
(\mathrm{kW}) \\
\end{array}$ & $\begin{array}{l}\text { HTR } \\
(\mathrm{kW}) \\
\end{array}$ & $\begin{array}{l}\text { Total Cost } \\
\text { (M\$) }\end{array}$ & $\begin{array}{l}\text { LTR UA } \\
(\mathrm{kW} / \mathrm{K}) \\
\end{array}$ & $\begin{array}{l}\text { HTR UA } \\
(\mathrm{kW} / \mathrm{K}) \\
\end{array}$ \\
\hline 550 & Solar Salt & 2.1 & 2.1 & 60024 & 135080 & 19.51 & 11806 & 8188 \\
\hline 520 & Hitec XL & 2.4 & 2.5 & 63738 & 128323 & 19.21 & 12107 & 7892 \\
\hline 415 & Dowtherm A & 4.1 & 4.1 & 81452 & 98558 & 18.00 & 13378 & 6623 \\
\hline 390 & Syltherm 800 & 4.5 & 4.8 & 87336 & 89282 & 17.66 & 13959 & 6041 \\
\hline 390 & Therminol VP1 & 4.5 & 4.8 & 87336 & 89282 & 17.66 & 13959 & 6041 \\
\hline 375 & Therminol 75 & 4.6 & 5.1 & 91262 & 83280 & 17.45 & 14187 & 5813 \\
\hline
\end{tabular}

Tabla 7.40. Diseño y parámetros de operación de los recuperadores. Tamaño térmico total de los recuperadores $U A=3000 \mathrm{~kW} / \mathrm{K}$. Ciclo de potencia s- $\mathrm{CO}_{2}$ Brayton PCRC.

\begin{tabular}{lllllllll}
\hline $\begin{array}{l}\text { TIT } \\
\left({ }^{\circ} \mathrm{C}\right)\end{array}$ & $\begin{array}{l}\text { SF } \\
\text { Config. }\end{array}$ & $\begin{array}{l}\text { LTR Pinch } \\
\left({ }^{\circ} \mathrm{C}\right)\end{array}$ & $\begin{array}{l}\text { HTR Pinch } \\
\left({ }^{\circ} \mathrm{C}\right)\end{array}$ & $\begin{array}{l}\text { LTR } \\
(\mathrm{kW})\end{array}$ & $\begin{array}{l}\text { HTR } \\
(\mathrm{kW})\end{array}$ & $\begin{array}{l}\text { Total Cost } \\
(\mathrm{M} \$)^{2}\end{array}$ & $\begin{array}{l}\text { LTR UA } \\
(\mathrm{kW} / \mathrm{K})\end{array}$ & $\begin{array}{l}\text { HTR UA } \\
(\mathrm{kW} / \mathrm{K})\end{array}$ \\
\hline 550 & Solar Salt & 23.3 & 23.9 & 18975 & 138926 & 15.79 & 806.4 & 2193.5 \\
520 & Hitec XL & 23.3 & 24.6 & 20315 & 133210 & 15.35 & 855.2 & 2144.7 \\
415 & Dowtherm A & 22.4 & 27.7 & 27239 & 107971 & 13.52 & 1105.3 & 1894.7 \\
390 & Syltherm 800 & 22.1 & 28.6 & 29542 & 100386 & 12.99 & 1189.5 & 1810.5 \\
390 & Therminol VP1 & 22.1 & 28.6 & 29542 & 100386 & 12.99 & 1189.5 & 1810.5 \\
375 & Therminol 75 & 22.8 & 28.2 & 30326 & 96498 & 12.68 & 1201.4 & 1799 \\
\hline
\end{tabular}

Tabla 7.41. Diseño y parámetros de operación de los recuperadores. Tamaño térmico total de los recuperadores $U A=5000 \mathrm{~kW} / \mathrm{K}$. Ciclo de potencia s- $\mathrm{CO}_{2}$ Brayton PCRC.

\begin{tabular}{lllllllll}
\hline $\begin{array}{l}\text { TIT } \\
\left({ }^{\circ} \mathrm{C}\right)\end{array}$ & $\begin{array}{l}\text { SF } \\
\text { Config. }\end{array}$ & $\begin{array}{l}\text { LTR Pinch } \\
\left({ }^{\circ} \mathrm{C}\right)\end{array}$ & $\begin{array}{l}\text { HTR Pinch } \\
\left({ }^{\circ} \mathrm{C}\right)\end{array}$ & $\begin{array}{l}\text { LTR } \\
(\mathrm{kW})\end{array}$ & $\begin{array}{l}\text { HTR } \\
(\mathrm{kW})\end{array}$ & $\begin{array}{l}\text { Total Cost } \\
(\mathrm{M} \$)\end{array}$ & $\begin{array}{l}\text { LTR UA } \\
(\mathrm{kW} / \mathrm{K})\end{array}$ & $\begin{array}{l}\text { HTR UA } \\
(\mathrm{kW} / \mathrm{K})\end{array}$ \\
\hline 550 & Solar Salt & 9.3 & 9.3 & 18988 & 148542 & 16.75 & 1672 & 3328 \\
520 & Hitec XL & 9.9 & 9.9 & 20220 & 143213 & 16.34 & 1729 & 3271 \\
415 & Dowtherm A & 12.9 & 12.7 & 26133 & 120138 & 14.63 & 1967 & 2972.9 \\
390 & Syltherm 800 & 13.2 & 13.7 & 28672 & 112832 & 14.15 & 2135 & 2864.9 \\
390 & Therminol VP1 & 13.2 & 13.7 & 28672 & 112832 & 14.15 & 2135 & 2865 \\
375 & Therminol 75 & 13.3 & 14.3 & 30172 & 108192 & 13.84 & 2208.2 & 2791.9 \\
\hline
\end{tabular}


7. Optimización de plantas termosolares de colectores lineales con aceites térmicos como HTF acopladas a ciclos Brayton de $\mathrm{CO}_{2}$ supercrítico

Tabla 7.42. Diseño y parámetros de operación de los recuperadores. Tamaño térmico total de los recuperadores UA=10000 kW/K. Ciclo de potencia s- $\mathrm{CO}_{2}$ Brayton PCRC.

\begin{tabular}{lllllllll}
\hline $\begin{array}{l}\text { TIT } \\
\left({ }^{\circ} \mathrm{C}\right)\end{array}$ & $\begin{array}{l}\text { SF } \\
\text { Config. }\end{array}$ & $\begin{array}{l}\text { LTR Pinch } \\
\left({ }^{\circ} \mathrm{C}\right)\end{array}$ & $\begin{array}{l}\text { HTR Pinch } \\
\left({ }^{\circ} \mathrm{C}\right)\end{array}$ & $\begin{array}{l}\text { LTR } \\
(\mathrm{kW})\end{array}$ & $\begin{array}{l}\text { HTR } \\
(\mathrm{kW})\end{array}$ & $\begin{array}{l}\text { Total Cost } \\
\left.(\mathrm{M} \$)^{2}\right)\end{array}$ & $\begin{array}{l}\text { LTR UA } \\
(\mathrm{kW} / \mathrm{K})\end{array}$ & $\begin{array}{l}\text { HTR UA } \\
(\mathrm{kW} / \mathrm{K})\end{array}$ \\
\hline 550 & Solar Salt & 2.7 & 2.7 & 21218 & 152563 & 17.38 & 5242 & 4760 \\
520 & Hitec XL & 2.9 & 2.9 & 22387 & 147666 & 17.01 & 5265 & 4736 \\
415 & Dowtherm A & 4.3 & 4.3 & 28082 & 126649 & 15.47 & 5447 & 4553 \\
390 & Syltherm 800 & 4.7 & 4.7 & 30015 & 120360 & 15.04 & 5532 & 4469 \\
390 & Therminol VP1 & 4.7 & 4.7 & 30015 & 120360 & 15.04 & 5532 & 4469 \\
375 & Therminol 75 & 5.1 & 5.1 & 31335 & 116246 & 14.76 & 5594 & 4406 \\
\hline
\end{tabular}

Tabla 7.43. Diseño y parámetros de operación de los recuperadores. Tamaño térmico total de los recuperadores UA=15000 kW/K. Ciclo de potencia s- $\mathrm{CO}_{2}$ Brayton PCRC.

\begin{tabular}{lllllllll}
\hline $\begin{array}{l}\text { TIT } \\
\left({ }^{\circ} \mathrm{C}\right)\end{array}$ & $\begin{array}{l}\text { SF } \\
\text { Config. }\end{array}$ & $\begin{array}{l}\text { LTR Pinch } \\
\left({ }^{\circ} \mathrm{C}\right)\end{array}$ & $\begin{array}{l}\text { HTR Pinch } \\
\left({ }^{\circ} \mathrm{C}\right)\end{array}$ & $\begin{array}{l}\text { LTR } \\
(\mathrm{kW})\end{array}$ & $\begin{array}{l}\text { HTR } \\
(\mathrm{kW})\end{array}$ & $\begin{array}{l}\text { Total Cost } \\
(\mathrm{M} \$)\end{array}$ & $\begin{array}{l}\text { LTR UA } \\
(\mathrm{kW} / \mathrm{K})\end{array}$ & $\begin{array}{l}\text { HTR UA } \\
(\mathrm{kW} / \mathrm{K})\end{array}$ \\
\hline 550 & Solar Salt & 1.5 & 1.5 & 22185 & 153014 & 17.52 & 9614 & 5385 \\
520 & Hitec XL & 1.6 & 1.6 & 23348 & 148256 & 17.16 & 9587 & 5413 \\
415 & Dowtherm A & 2.4 & 2.4 & 29102 & 127877 & 15.70 & 9635 & 5365 \\
390 & Syltherm 800 & 2.7 & 2.7 & 31010 & 121831 & 15.28 & 9688 & 5314 \\
390 & Therminol VP1 & 2.7 & 2.7 & 31011 & 121829 & 15.28 & 9688 & 5313 \\
375 & Therminol 75 & 2.9 & 2.9 & 32313 & 117900 & 15.02 & 9729 & 5270 \\
\hline
\end{tabular}

Tabla 7.44. Diseño y parámetros de operación de los recuperadores. Tamaño térmico total de los recuperadores UA=20000 kW/K. Ciclo de potencia s- $\mathrm{CO}_{2}$ Brayton PCRC.

\begin{tabular}{|c|c|c|c|c|c|c|c|c|}
\hline $\begin{array}{l}\text { TIT } \\
\left({ }^{\circ} \mathrm{C}\right)\end{array}$ & $\begin{array}{l}\text { SF } \\
\text { Config. }\end{array}$ & $\begin{array}{l}\text { LTR Pinch } \\
\left({ }^{\circ} \mathrm{C}\right)\end{array}$ & $\begin{array}{l}\text { HTR Pinch } \\
\left({ }^{\circ} \mathrm{C}\right)\end{array}$ & $\begin{array}{l}\text { LTR } \\
(\mathrm{kW})\end{array}$ & $\begin{array}{l}\text { HTR } \\
(\mathrm{kW})\end{array}$ & $\begin{array}{l}\text { Total Cost } \\
\text { (M\$) }\end{array}$ & $\begin{array}{l}\text { LTR UA } \\
(\mathrm{kW} / \mathrm{K})\end{array}$ & $\begin{array}{l}\text { HTR UA } \\
(\mathrm{kW} / \mathrm{K})\end{array}$ \\
\hline 550 & Solar Salt & 1.1 & 1.1 & 22639 & 153107 & 17.57 & 14259 & 5740 \\
\hline 520 & Hitec XL & 1.2 & 1.2 & 23806 & 148419 & 17.22 & 14188 & 5819 \\
\hline 415 & Dowtherm A & 1.7 & 1.7 & 29640 & 128264 & 15.79 & 14143 & 5859 \\
\hline 390 & Syltherm 800 & 1.8 & 1.9 & 31549 & 122349 & 15.39 & 14160 & 5842 \\
\hline 390 & Therminol VP1 & 1.8 & 1.9 & 31549 & 122349 & 15.39 & 14160 & 5842 \\
\hline 375 & Therminol 75 & 1.9 & 2.1 & 32842 & 118494 & 15.13 & 14184 & 5816 \\
\hline
\end{tabular}

Tabla 7.45. Diseño y parámetros de operación de los recuperadores. Tamaño térmico total de los recuperadores UA=3000 kW/K. Ciclo de potencia s- $\mathrm{CO}_{2}$ Brayton $\mathrm{RCMCI}$.

\begin{tabular}{lllllllll}
\hline $\begin{array}{l}\text { TIT } \\
\left({ }^{\circ} \mathrm{C}\right)\end{array}$ & $\begin{array}{l}\text { SF } \\
\text { Config. }\end{array}$ & $\begin{array}{l}\text { LTR } \\
\text { Pinch } \\
\left({ }^{\circ} \mathrm{C}\right)\end{array}$ & $\begin{array}{l}\text { HTR } \\
\text { Pinch } \\
\left({ }^{\circ} \mathrm{C}\right)\end{array}$ & LTR $(\mathrm{kW})$ & $\begin{array}{l}\text { HTR } \\
(\mathrm{kW})\end{array}$ & $\begin{array}{l}\text { Total Cost } \\
(\mathrm{M} \$)\end{array}$ & $\begin{array}{l}\text { LTR UA } \\
(\mathrm{kW} / \mathrm{K})\end{array}$ & $\begin{array}{l}\text { HTR UA } \\
(\mathrm{kW} / \mathrm{K})\end{array}$ \\
\hline 550 & Solar Salt & 24.3 & 60.3 & 83479 & 79783 & 16.33 & 1897.9 & 1103.1 \\
520 & Hitec XL & 24.1 & 60.7 & 88399 & 70683 & 15.91 & 2010.2 & 990.5 \\
415 & Dowtherm A & 22.8 & 62.6 & 111035 & 30164 & 14.12 & 2551.6 & 449 \\
390 & Syltherm 800 & 22.4 & 63.3 & 118586 & 17611 & 13.62 & 2737.4 & 266.7 \\
390 & Therminol VP1 & 22.4 & 63.3 & 118380 & 17585 & 13.60 & 2734 & 266.3 \\
375 & Therminol 75 & 21.9 & 63.8 & 123361 & 9219 & 13.26 & 2859.6 & 141.2 \\
\hline
\end{tabular}


7. Optimización de plantas termosolares de colectores lineales con aceites térmicos como HTF acopladas a ciclos Brayton de $\mathrm{CO}_{2}$ supercrítico

Tabla 7.46. Diseño y parámetros de operación de los recuperadores. Tamaño térmico total de los recuperadores UA=5000 kW/K. Ciclo de potencia s- $\mathrm{CO}_{2}$ Brayton $\mathrm{RCMCl}$.

\begin{tabular}{lllllllll}
\hline $\begin{array}{l}\text { TIT } \\
\left({ }^{\circ} \mathrm{C}\right)\end{array}$ & $\begin{array}{l}\text { SF } \\
\text { Config. }\end{array}$ & $\begin{array}{l}\text { LTR } \\
\text { Pinch } \\
\left({ }^{\circ} \mathrm{C}\right)\end{array}$ & $\begin{array}{l}\text { HTR Pinch } \\
\left({ }^{\circ} \mathrm{C}\right)\end{array}$ & $\begin{array}{l}\text { LTR } \\
(\mathrm{kW})\end{array}$ & $\begin{array}{l}\text { HTR } \\
(\mathrm{kW})\end{array}$ & $\begin{array}{l}\text { Total Cost } \\
(\mathrm{M} \$)\end{array}$ & $\begin{array}{l}\text { LTR UA } \\
(\mathrm{kW} / \mathrm{K})\end{array}$ & $\begin{array}{l}\text { HTR UA } \\
(\mathrm{kW} / \mathrm{K})\end{array}$ \\
\hline 550 & Solar Salt & 16.3 & 30.9 & 71987 & 104002 & 17.60 & 2766 & 2234.8 \\
520 & Hitec XL & 16.3 & 31.6 & 76439 & 95763 & 17.22 & 2916 & 2084 \\
415 & Dowtherm A & 16.9 & 34.7 & 96930 & 63961 & 16.09 & 3588 & 1442.5 \\
390 & Syltherm 800 & 16.8 & 35.9 & 104000 & 52215 & 15.62 & 3807 & 1192.4 \\
390 & Therminol VP1 & 16.8 & 35.9 & 103999 & 52215 & 15.62 & 3807 & 1192.4 \\
375 & Therminol 75 & 16.7 & 36.6 & 108665 & 44579 & 15.32 & 3971 & 1029.3 \\
\hline
\end{tabular}

Tabla 7.47. Diseño y parámetros de operación de los recuperadores. Tamaño térmico total de los recuperadores UA=10000 kW/K. Ciclo de potencia s- $\mathrm{CO}_{2}$ Brayton $\mathrm{RCMCI}$.

\begin{tabular}{lllllllll}
\hline $\begin{array}{l}\text { TIT } \\
\left({ }^{\circ} \mathrm{C}\right)\end{array}$ & $\begin{array}{l}\text { SF } \\
\text { Config. }\end{array}$ & $\begin{array}{l}\text { LTR } \\
\text { Pinch } \\
\left({ }^{\circ} \mathrm{C}\right)\end{array}$ & $\begin{array}{l}\text { HTR } \\
\text { Pinch } \\
\left({ }^{\circ} \mathrm{C}\right)\end{array}$ & $\begin{array}{l}\text { LTR } \\
(\mathrm{kW})\end{array}$ & $\begin{array}{l}\text { HTR } \\
(\mathrm{kW})\end{array}$ & $\begin{array}{l}\text { Total Cost } \\
(\mathrm{M} \$)\end{array}$ & $\begin{array}{l}\text { LTR UA } \\
(\mathrm{kW} / \mathrm{K})\end{array}$ & $\begin{array}{l}\text { HTR UA } \\
(\mathrm{kW} / \mathrm{K})\end{array}$ \\
\hline 550 & Solar Salt & 9.2 & 9.3 & 62610 & 128761 & 19.14 & 5225 & 4776 \\
520 & Hitec XL & 9.3 & 10.1 & 67172 & 121099 & 18.83 & 5431 & 4550 \\
415 & Dowtherm A & 10.4 & 12.9 & 84817 & 89782 & 17.46 & 6469 & 3531 \\
390 & Syltherm 800 & 10.3 & 13.9 & 90994 & 79798 & 17.08 & 6813 & 3187 \\
390 & Therminol VP1 & 10.3 & 13.9 & 90994 & 79798 & 17.08 & 6813 & 3187 \\
375 & Therminol 75 & 10.3 & 14.6 & 95185 & 73172 & 16.84 & 7047 & 2952.6 \\
\hline
\end{tabular}

Tabla 7.48. Diseño y parámetros de operación de los recuperadores. Tamaño térmico total de los recuperadores UA=15000 kW/K. Ciclo de potencia s- $\mathrm{CO}_{2}$ Brayton $\mathrm{RCMCl}$.

\begin{tabular}{lllllllll}
\hline $\begin{array}{l}\text { TIT } \\
\left({ }^{\circ} \mathrm{C}\right)\end{array}$ & $\begin{array}{l}\text { SF } \\
\text { Config. }\end{array}$ & $\begin{array}{l}\text { LTR } \\
\text { Pinch } \\
\left({ }^{\circ} \mathrm{C}\right)\end{array}$ & $\begin{array}{l}\text { HTR } \\
\text { Pinch } \\
\left({ }^{\circ} \mathrm{C}\right)\end{array}$ & $\begin{array}{l}\text { LTR } \\
(\mathrm{kW})\end{array}$ & $\begin{array}{l}\text { HTR } \\
(\mathrm{kW})\end{array}$ & $\begin{array}{l}\text { Total Cost } \\
(\mathrm{M} \$)\end{array}$ & $\begin{array}{l}\text { LTR UA } \\
(\mathrm{kW} / \mathrm{K})\end{array}$ & $\begin{array}{l}\text { HTR UA } \\
(\mathrm{kW} / \mathrm{K})\end{array}$ \\
\hline 550 & Solar Salt & 3.8 & 3.8 & 59945 & 134011 & 19.40 & 8393 & 6607 \\
520 & Hitec XL & 3.6 & 4.4 & 64473 & 126714 & 19.12 & 8601 & 6358 \\
415 & Dowtherm A & 6.4 & 6.4 & 81067 & 97696 & 17.88 & 9794 & 5206 \\
390 & Syltherm 800 & 6.9 & 7.1 & 86730 & 88670 & 17.54 & 10199 & 4803 \\
390 & Therminol VP1 & 6.9 & 7.1 & 86730 & 88669 & 17.54 & 10196 & 4803 \\
375 & Therminol 75 & 6.9 & 7.6 & 90600 & 82661 & 17.33 & 10472 & 4527 \\
\hline
\end{tabular}

Tabla 7.49. Diseño y parámetros de operación de los recuperadores. Tamaño térmico total de los recuperadores UA=20000 kW/K. Ciclo de potencia s- $\mathrm{CO}_{2}$ Brayton $\mathrm{RCMCl}$.

\begin{tabular}{lllllllll}
\hline TIT & SF & LTR & HTR & LTR \\
$\left({ }^{\circ} \mathrm{C}\right)$ & Config. & $\begin{array}{l}\text { Pinch } \\
\left({ }^{\circ} \mathrm{C}\right)\end{array}$ & $\begin{array}{l}\text { Pinch } \\
\left({ }^{\circ} \mathrm{C}\right)\end{array}$ & $\begin{array}{l}\text { HTR } \\
(\mathrm{kW})\end{array}$ & $\begin{array}{l}\text { Total Cost } \\
(\mathrm{M} \$)\end{array}$ & $\begin{array}{l}\text { LTR UA } \\
(\mathrm{kW} / \mathrm{K})\end{array}$ & $\begin{array}{l}\text { HTR UA } \\
(\mathrm{kW} / \mathrm{K})\end{array}$ \\
\hline 550 & Solar Salt & 1.8 & 1.9 & 59014 & 135683 & 19.47 & 12043 & 7950 \\
520 & Hitec XL & 1.9 & 2.3 & 63314 & 124042 & 18.74 & 12245 & 7743 \\
415 & Dowtherm A & 3.6 & 3.7 & 79315 & 101044 & 18.04 & 13465 & 6535 \\
390 & Syltherm 800 & 4.4 & 4.6 & 85275 & 91889 & 17.72 & 13448 & 5915 \\
390 & Therminol VP1 & 4.4 & 4.6 & 85243 & 91920 & 17.72 & 13446 & 5915 \\
375 & Therminol 75 & 4.6 & 4.6 & 88638 & 86689 & 17.53 & 14185 & 5813 \\
\hline
\end{tabular}


7. Optimización de plantas termosolares de colectores lineales con aceites térmicos como HTF acopladas a ciclos Brayton de $\mathrm{CO}_{2}$ supercrítico

\subsection{Conclusiones}

En este capítulo se proponen diferentes diseños innovadores de plantas termosolares con colectores solares lineales, con aceites térmicos (Dowtherm A, Syltherm 800, Therminol VP1 y Therminol 75) como HTF acopladas a ciclos de potencia $\mathrm{s}-\mathrm{CO}_{2}$ Brayton, con diferentes tipologías (RC, PCRC y $\mathrm{RCMCl}$ ) con una etapa de recalentamiento en las turbinas. Es la tecnología conocida con el acrónico Oil+s- $\mathrm{CO}_{2}$.

Se ha comparado la tecnología Oil+s- $\mathrm{CO}_{2}$ con la solución con sales fundidas, $\mathrm{MS}+\mathrm{s}-\mathrm{CO}_{2}$, desarrollada en los capítulos 5 y 6 , y con la opción de colectores lineales acoplados a ciclos Rankine, analizada en el capítulo 1 . Se concluye que la opción $\mathrm{MS}+\mathrm{s}-\mathrm{CO}_{2}$ es la que proporciona valores mayores de eficiencia energética combinado los mayores valores de temperatura de entrada en turbina $\mathrm{TIT}=550^{\circ} \mathrm{C}$, con los ciclos Brayton (RC, PCRC y RCMCI).

Sin embargo, la principal conclusión de este capítulo es que la solución de Oil+s- $\mathrm{CO}_{2}$, a pesar de su menor eficiencia neta en relación a la solución $\mathrm{MS}+\mathrm{s}-\mathrm{CO}_{2}$, el coste de inversión de la planta termosolar para una potencia fija, es más reducido gracias a la combinación de diferentes factores como: el menor coste de los materiales de los equipos y componentes no afectados por el fenómeno de corrosión, no requieren la tecnología heat-tracing, los equipos son comerciales y ampliamente validados en numerosas instalaciones en operación actualmente, las pérdidas térmicas y materiales selectivos en los tubos absorbedores son menos restrictivas.

En este capítulo también se ha demostrado como un incremento del tamaño térmico de los recuperadores UA proporciona un aumento de la eficiencia neta del ciclo, y una reducción del coste de inversión de sus campos solares. Se han identificado los valores umbrales de UA a partir de los cuales, la limitación de pinch-point en los recuperadores, no proporciona una mejora significativa ni de la eficiencia energética ni del coste de los campos solares.

\subsection{Referencias}

[1] Solar Energy Generating Systems (SEGS). http://www.energy.ca.gov/sitingcases/solar/

[2] Supercritical $\mathrm{CO}_{2}$ Power Cycles Symposium. http://www.swri.org/4org/d18/sco2/

[3] Dowtherm A heat transfer fluid. http://msdssearch.dow.com

[4] Syltherm 800 heat transfer fluid. http://www.dow.com

[5] Therminol VP1 heat transfer fluid. https://www.therminol.com

[6] Therminol 75 heat transfer fluid. https://www.therminol.com

[7] T.L. Cox, P.M. Fourspring. Comparison of Meassured and Analytical Performance of ShellTube Heat Exchangers cooling and heating supercritical CO2. The $4^{\text {th }}$ International Symposium - Supercritical CO2 Power Cycles, September 9-10, 2014, Pittsburgh, Pennsylvania.

[8] D. Shiferaw, J. Montero Carrero, R. Le Pierres. Economic analysis of sCO2 cycles with PCHE Recuperator design optimization. The $5^{\text {th }}$ Int. Sym. sCO2 March 28-31, 2016, San Antonio.

[9] Carlson M.D., Kruizenga A.K., Schalansky C., Fleming D.F. Sandia progress on advanced heat exchangers for $\mathrm{SCO} 2$ Brayton cycles. The $4^{\text {th }}$ International Symposium - Supercritical $\mathrm{CO} 2$ Power Cycles, September 9-10, 2014, Pittsburgh, Pennsylvania.

[10] E.M. Clementoni, T.L.Cox. Steady-state power operation of a sCO2 Brayton cycle. The $4^{\text {th }}$ Int.Symp. sCO2 Power Cycles, September 9-10, 2014, Pittsburgh, Pennsylvania. 
7. Optimización de plantas termosolares de colectores lineales con aceites térmicos como HTF acopladas a ciclos Brayton de $\mathrm{CO}_{2}$ supercrítico

[11] SEGS I en Mojave 1984. U.S. Energy Informacion Administration. Electricity Data Browser.

[12] CSP Projects. National Renewable Laboratory NREL. http://www.nrel.gov

[13] Quasching V et al. Influence of DNI on the optimal parabolic trough field size: a problem solved with technical and economical simulations. Trans ASME 2002; 124(5):160-4.

[14] Rolim MM, Fraidenraich N, Tiba C. Analytic modeling of a solar power plant with parabolic linear collectors. Sol Energy 2009; 83:126-33.

[15] SAM version 3.0, solar advisor model reference manual for CSP trough systems. Golden, Colorado, USA: National Renewable Energy Laboratory; July 2009.

[16] Montes MJ, Abanades A, Martinez-Val JM, Valdes M. Solar multiple optimization for a solar-only thermal power plant, using oil as heat transfer fluid in the parabolic trough collectors. Sol Energy 2009; 83:2165-76.

[17] Kumaresan G, Sridhar R, Velraj R. Performance studies of a solar parabolic trough collector with a thermal energy storage system. Energy 2012; 47(1): 395-402.

[18] Manzolini G, Giostri A, Saccillotto C, Silva P, Macchi E. Development of an innovative code for the design of thermodynamic solar power plants part A: code description and test case. Renew Energy 2011; 36:1993-2003.

[19] Manzolini G, Giostri A, Saccillotto C, Silva P, Macchi E. Development of an innovative code for the design of thermodynamic solar power plants part B: performance assessment of commercial and innovative technologies. Renew Energy 2011; 36:2465-73.

[20] Hermann U, Kelly B, Price H. Two-tank molten salts for parabolic trough solar power plants. Energy 2004; 29:883-93.

[21] Llorente Garcia I, Alvarez JL, Blanco D. Performance mode for parabolic solar thermal power plants with TES: comparison to operating plant data. Sol Energy 2011; 85:2443-60.

[22] Wagner SJ, Rubin ES. Economic implications of thermal energy storage for concentrated solar thermal power. Renew Energy 2014; 61:81-95.

[23] Fabrizio De Luca, Vittorio Ferraro, Valerio Marinelli. On the performance of CSP oil-cooled plants, with and without heat storage in tanks of molten salts. Energy 83 (2015) 230-239.

[24] T.E. Boukelia et al. Investigation of solar parabolic trough power plants with and without integrated TES and FBS using thermic oil and solar salt. Energy 88 (2015) 292-303.

[25] G.Morin et al. Molten Salt as a Heat Transfer Fluid in a Linear Fresnel Collector Commercial Application Backed by Demonstration. En. Proc. 69, 2015, pp 689-698.

[26] A.Maccari, Archimede Solar Energy. Archimede Solar Energy Molten Salt Parabolic Trough Demo Plant: A Step Ahead Towards the New Frontiers of CSP. SolarPaces 2014.

[27] Reliance Solar Thermal Power Plant Project, Areva Solar. http://india.areva.com

[28] Archimede Solarenergy receivers. http://www.archimedesolarenergy.it/en prodotti.htm

[29] Zhiwen Ma, Craig S. Turchi. Advanced Supercritical CO2 Power cycle Configurations for Use in Concentrating Solar Power Systems. Supercritical CO2 Power Cycle Symposium Boulder, Colorado, May 24-25, 2011. Conference Paper NREL/CP-5500-50787, March 2011.

[30] T.Neises, C.Turchi. A comparison of Supercritical Carbon Dioxide Power Cycle Configurations with an Emphasis on CSP Applications. En.Proc., 49, 2014, pp. 1187-1196. 


\title{
Capítulo 8 \\ Plantas Termosolares de Colectores Lineales con DSG acopladas a ciclos Brayton con $\mathrm{CO}_{2}$
}

\begin{abstract}
Resumen
La generación de vapor en los colectores solares lineales (DSG), está en desarrollo industrial desde principios de 1990, para sustituir el aceite térmico y las sales fundidas como HTF en plantas termosolares. Se destaca como su principal ventaja, la eliminación de los impactos ambientales, al tratarse el agua como fluido inocuo al medio ambiente, pero con el inconveniente de que donde hay elevada irradiación solar suele escasear el recurso hídrico.
\end{abstract}

En paralelo con el desarrollo tecnológico de DSG, se está invirtiendo un gran esfuerzo de investigación en la tecnología de generación de potencia con ciclos s- $\mathrm{CO}_{2}$ Brayton, como alternativa a los ciclos de potencia Rankine. El principal objetivo de los ciclos de potencia s- $\mathrm{CO}_{2}$ Brayton es la reducción de las dimensiones de los equipos que integran el ciclo y de la obra civil asociada, y el incremento de la eficiencia de la planta termosolar.

Tal y como se ha definido en capítulos anteriores (capítulo 5 y siguientes), para realizar la integración de los campos solares con colectores lineales con los ciclos s- $\mathrm{CO}_{2}$ Brayton, se han elegido cuatro tipologías de ciclos para adaptarse a las características de las instalaciones termosolares [1]. Dichas tipologías se han representado en las figuras 8.1 a 8.4: ciclo simple con recompresión y con recuperación de calor (SB), ciclo con recompresión (RC), ciclo con enfriamiento parcial antes de recompresión (PCRC), y ciclo con enfriamiento intermedio en el compresor principal (RCMCI).

Las plantas termosolares diseñadas en el presente capítulo son integradas por colectores lineales con DSG, y los ciclos supercríticos de potencia s- $\mathrm{CO}_{2}$ Brayton. Se han definido cuatro configuraciones de plantas termosolares compartiendo todas ellas las características antes citadas:

- La Configuración 1, explicada en el apartado 8.5.1, figura 8.8, consiste en la instalación de un condensador entre el campo solar y el ciclo de potencia. El vapor generado en los colectores solares es condensado transmitiendo su energía al fluido de trabajo del ciclo de potencia. El intercambiador elegido para realizar esta función es carcasa y tubos. Por los tubos fluye el $\mathrm{CO}_{2}$, y por el lado carcasa condensa el agua del campo solar. La principal ventaja del condensador es su alto coeficiente de transferencia de calor en el lado de agua condensada, minimizando las dimensiones y pesos finales del equipo. Respecto a las desventajas de esta Configuración 1, la alta presión requerida en el campo solar para condensación del agua vapor en agua líquida. La presión tiene que estar entre 150 bar y 175 bar para obtener una condensación completa del agua en fase vapor y conseguir valores de entrada de turbina de $400^{\circ} \mathrm{C}$.

- En la Configuración 2, explicada en el apartado 8.5.2, figura 8.9, el vapor recalentado suministrado en el campo solar transfiere su energía calorífica al fluido de trabajo del ciclo de potencia a través de un intercambiador de calor Primary Heat Exchanger (PHX). En esta configuración el vapor no es condensado en la fase líquida del agua, y 
solamente es sometido a una reducción de temperatura desde $550^{\circ} \mathrm{C}$ a $420^{\circ} \mathrm{C}$. La principal ventaja de esta configuración es por tanto evitar el cambio de fase en del agua, lo cual reduce considerablemente la complejidad del sistema de control, ya que no hace falta controlar la aparición de puntos calientes o hot-spots en los tubos absorbedores. Se reduce también el número de equipos, se evita la bomba de recirculación de condensado, los tanques de separación de fases y los separadores de humedad; y se obtiene una configuración similar a la obtenida en el modo de generación de vapor en modo OT, con inyecciones puntuales de agua líquida para controlar la temperatura de sobrecalentamiento del vapor a la salida del campo solar. En esta configuración las caídas de presión del vapor en el campo solar, por el rozamiento del fluido caloportador con las paredes de los tubos absorbedores y con las paredes de las tuberías de distribución de vapor entre colectores solares, es compensada mediante compresores de vapor. Esta segunda configuración es compatible con temperaturas de entrada en turbina del orden de $550^{\circ} \mathrm{C}$. Las claves fundamentales de esta configuración son las condiciones de presión y temperatura (175 bar y $420^{\circ} \mathrm{C}$ ) a la entrada de los compresores de vapor para minimizar un consumo energético y garantizar su eficiencia.

- La Configuración 3, descrita en el apartado 8.5.3 y representada en la figura 8.11, incluye un campo solar con DSG en modo de operación de recirculación de la fase líquida, solución desarrollada y validada experimentalmente en el proyecto de investigación DISS, con dos ciclos s- $\mathrm{CO}_{2}$ Brayton en cascada. El primer ciclo de potencia operando a $\mathrm{TIT}=550^{\circ} \mathrm{C}$, y el segundo ciclo con una $\mathrm{TIT}=410^{\circ} \mathrm{C}$. La principal ventaja de esta configuración es la utilización de un modo de recirculación, sistema de generación de vapor en el campo solar ya implantado a escala industrial en algunas instalaciones pioneras [2]. Se evita por tanto la utilización de compresores, equipos que no están comercialmente disponibles para su aplicación en plantas termosolares. El principal inconveniente de este diseño es el incremento del número de equipos al duplicar los ciclos s- $\mathrm{CO}_{2}$ Brayton.

- La configuración 4, definida en el apartado 8.5.4, es similar a la configuración 2, figura 8.12, con el mismo campos solar con generación de vapor con recalentamiento sin condensación y con compresores de vapor para contrarrestar las pérdidas de presión en los tubos absorbedores. Para recalentamiento del vapor se dispone de un campo solar con colectores lineales y sales fundidas como HTF.

Tabla 8.1. Eficiencia energética de las plantas termosolares con colectores lineales con DSG acopladas a ciclos de potencia s- $\mathrm{CO}_{2}$ Brayton.

\begin{tabular}{lllllll}
\hline Configuración & 1 & & 2 & & 3 & 4 \\
\hline $\mathrm{HTF}$ & $\mathrm{DSG}$ & $\mathrm{MS}$ & $\mathrm{DSG}$ & $\mathrm{MS}$ & $\mathrm{DSG}$ & $\mathrm{DSG}, \mathrm{MS}$ \\
Ciclo & $\mathrm{SCO}_{2}$ & $\mathrm{sCO}_{2}$ & $\mathrm{sCO}_{2}$ & $\mathrm{sCO}_{2}$ & $\mathrm{sCO}_{2}$ & $\mathrm{sCO}_{2}$ \\
potencia & $\mathrm{RCMCl}$ & $\mathrm{RCMCl}$ & $\mathrm{RC}$ & $\mathrm{RC}$ & $\mathrm{RCMCl}$ & $\mathrm{RC}$ \\
$\mathrm{TIT}\left({ }^{\circ} \mathrm{C}\right)$ & 400 & 400 & 550 & 550 & $\begin{array}{l}\text { Ciclo1:550 } \\
\text { Ciclo2:400 }\end{array}$ & 550 \\
$\begin{array}{l}\text { Eficiencia } \\
\text { Neta (\%) }\end{array}$ & 36.6 & 36.7 & 43.6 & 45.1 & 39.7 & 45.8 \\
\hline
\end{tabular}


En la tabla 8.1 se realiza una comparativa de la eficiencia energética de las configuraciones con DSG propuestas en el presente capítulo. La configuración 1, proporciona valores de eficiencia y potencia neta de $36.6 \%$, similares a los obtenidos con una configuración de planta termosolar integrada por colectores solares con sal fundida como fluido caloportador y ciclo de potencia s$\mathrm{CO}_{2}$ Brayton $\mathrm{RCMCl}$, tablas 8.1, 8.9 y 8.10 .

La eficiencia neta de la segunda solución de diseño, Configuración 2 , sufre el impacto de la potencia eléctrica consumida por los compresores de vapor. La eficiencia neta del ciclo es de $43.6 \%$ con colectores solares con generación de vapor, frente al $45.16 \%$ con un campo solar con colectores con sal fundida como HTF, tablas 8.1, 8.11 y 8.12. En ambos casos la temperatura de entrada en turbina es de $550^{\circ} \mathrm{C}$. La eficiencia neta de la configuración 3 es de $39.7 \%$, con dos ciclos s- $\mathrm{CO}_{2}$ Brayton en cascada tipología $\mathrm{RCMCl}$, tablas 8.1 y 8.13. Finalmente, la configuración 4 es la que proporciona valores mayores de eficiencia neta $\sim 45.77 \%$, pero limitado por las desventajas de la utilización de sales fundidas en el campo solar para recalentamiento (corrosión del material, coste del material en los tubos receptores, impacto ambiental, etc.), tablas 8.1, 8.14.

\subsection{Introducción}

La mayor parte de las calderas de generación de vapor en las centrales de generación de electricidad con combustibles fósiles son de la tipología de recirculación de agua o water recirculation $(\mathrm{RC})$ con tanques para separación del vapor de la fase líquida. Otra tipología son las calderas de un solo paso sin separación de fases, Once-Through (OT) ó también llamadas Benson boilers.

La tecnología DSG en los colectores solares lineales nace como resultado de adopción de las tecnologías desarrolladas en las calderas de las centrales térmicas con combustible fósiles a las plantas termosolares con colectores lineales. El objetivo es sustituir los tubos de las calderas en las centrales térmicas con combustibles fósiles por tubos absorbedores calentados por la radiación solar concentrada mediante espejos. La primera planta con generación directa de vapor construida fue en Estados Unidos en 1870 por John Ericsson [3]. Posteriormente, una planta solar con una superficie de $1250 \mathrm{~m}^{2}$ fue construida por Frank Shumman en Egipto [4]. Esta planta producía vapor saturado a 10 bar de presión para alimentar estaciones de bombeo. Un intensivo esfuerzo en investigación en esta tecnología fue llevado a cabo desde principios de los '90 en las instalaciones de la Plataforma Solar de Almeria (PSA) en España, en una instalación experimental enmarcada en el proyecto de investigación DISS [5]. Durante los ensayos se consiguió diseñar y experimentar la generación de vapor en PTC con recirculación de la fase líquida. La instalación estaba constituida por un lazo de $500 \mathrm{~m}$ con colectores parabólicos tipo LS-3. El principal resultado de este proyecto fue la demostración de la viabilidad de los diferentes modos de generación de vapor y evaluación de las pérdidas de presión en los tubos abosorbedores [6]. Con posterioridad, dentro del proyecto marco europeo INDITEP, se instalaron dos PTC Eurotrough-100 para incrementar la potencia térmica de la instalación experimental y se realiza la prueba de separadores de humedad [7]. En subsiguientes proyectos de investigación y experimentación se han probado componentes para el desarrollo a escala industrial de la tecnología de generación de vapor, tabla 8.2. 
8. Plantas Termosolares de Colectores Lineales con DSG acopladas a ciclos Brayton con $\mathrm{CO}_{2}$

Tabla 8.2. Listado de proyectos de investigación más significativos para el desarrollo de la DSG en los colectores lineales.

\begin{tabular}{|c|c|c|}
\hline Año & Referencia & Descripción del Proyecto \\
\hline 2008 & [8] & $\begin{array}{l}\text { El potencial de la generación directa de vapor en colectores lineales. } \\
\text { Resultados del proyecto Alemán DIVA. }\end{array}$ \\
\hline 2008 & [9] & $\begin{array}{l}\text { Generación de vapor en colectores lineales Fresnel. Planta termosolar } \\
\text { PuertoErrado } 1 .\end{array}$ \\
\hline 2009 & [10] & $\begin{array}{l}\text { Areva Solar Generación directa de vapor en colectores lineales Fresnel } \\
\text { CLFR. }\end{array}$ \\
\hline 2011 & [11] & $\begin{array}{l}\text { La generación directa de vapor en los colectores parabólicos a } 500^{\circ} \mathrm{C} \text {. } \\
\text { Resultados del proyecto REAL-DISS. }\end{array}$ \\
\hline 2011 & [12] & $\begin{array}{l}\text { El almacenamiento de energía térmica para la generación directa de } \\
\text { vapor. }\end{array}$ \\
\hline 2012 & [13] & $\begin{array}{l}\text { Comienza la operación de la planta termosolar Puerto Errado 2, de } 30 \\
\text { Mwe, basada en la tecnología de colectores Fresnel. }\end{array}$ \\
\hline 2012 & [14] & $\begin{array}{l}\text { Diseño y construcción de una planta piloto con colectores Fresnel } \\
\text { localizada en los Pirineos Franceses. Proyecto Augusting Fresnel } 1 . \\
\text { Empresa SolarEuromed. }\end{array}$ \\
\hline 2012 & [15] & $\begin{array}{l}\text { Proyecto de integración de la tecnología de colectores solares Fresnel en } \\
\text { una planta de generación térmica de carbón, localizada en Liddell. } \\
\text { Empresa Novatec Solar. }\end{array}$ \\
\hline 2012 & [16] & $\begin{array}{l}\text { Finalización del proyecto TRESERT: cogeneración de electricidad, calor y } \\
\text { refrigeración, a partir de la energía solar y de biomasa. }\end{array}$ \\
\hline 2012 & [17] & $\begin{array}{l}\text { Resultados operacionales y simulación de un colectores lineal Fresnel } \\
\text { para sobrecalentamiento del vapor. }\end{array}$ \\
\hline 2012 & [18] & $\begin{array}{l}\text { Comparación conceptual y diseño de una instalación de pruebas para el } \\
\text { análisis de la generación directa de vapor en modo OT. }\end{array}$ \\
\hline 2012 & [19] & $\begin{array}{l}\text { Desarrollo de un siste de almacenamiento térmico de alta temperatura } \\
\text { mediante materiales de cambio de fase o Phase Change Material (PCM), } \\
\text { para su integración en plantas termosolares con DSG. Resultados con el } \\
\text { primer prototipo. }\end{array}$ \\
\hline 2013 & [20] & $\begin{array}{l}\text { Estado y primeros resultados del proyecto DUKE, para demostrar la } \\
\text { viabilidad de la generación directa de vapor en modo OT. }\end{array}$ \\
\hline 2014 & [21] & $\begin{array}{l}\text { Dos años de experiencia operacional en la planta termoslar Puerto } \\
\text { Errado } 2 \text { con colectores lineales Fresnel. }\end{array}$ \\
\hline 2014 & [22] & $\begin{array}{l}\text { Una guía práctica para el control de la generación de vapor en modo OT } \\
\text { en colectores lineales parabólicos. }\end{array}$ \\
\hline 2014 & [23] & $\begin{array}{l}\text { Modelos de los transitorios en las instalaciones termosolares con } \\
\text { colectores lineales parabólicos y generación directa de vapor en modo } \\
\text { OT. }\end{array}$ \\
\hline 2015 & [24] & $\begin{array}{l}\text { Análisis y potencial de la generación directa de vapor en modo OT en } \\
\text { colectores lineales. Resultados finales del proyecto DUKE. }\end{array}$ \\
\hline
\end{tabular}


La primera planta comercial para generación de electricidad con colectores PTC y DSG, entró en operación en 2012 en Kanchanburi, Tahilandia [2, 26]. La planta produce vapor a 30 bar y $330^{\circ} \mathrm{C}$ a una turbina de vapor de $5 \mathrm{MWe}$. Las primeras experiencias de operación confirmaron que el sistema opera correctamente bajo condiciones transitorias de irradiación solar.

Otro desarrollo reciente relacionado con los colectores lineales con DSG es el Recalentamiento Directo sin intercambiador intermedio DRH [27-28], ya explicado en el capítulo 3 y todavía no implantado en ninguna instalación industrial.

En relación a los tubos absorbedores para DSG, se pueden mencionar dos empresas suministradoras Archimede SolarEnergy y SCHOTT. El objetivo del desarrollo de este tipo de componentes persigue el desarrollo de recubrimientos para los tubos absorbedores que resistan temperaturas superiores a $550^{\circ} \mathrm{C}$ y minimizar las pérdidas por radiación térmica para permitir incrementar las temperaturas a la entrada de turbina hasta $650^{\circ} \mathrm{C}$, como ya es posible con otras tecnologías termosolares como torre central. En este contexto, la empresa SCHOTT ha desarrollado recientemente los tubos absorbedores de 4ạ Generación [29] y la empresa Archimede SolarEnergy ha desarrollado los tubos HCESHS-12 [30] especializados en DSG. EI tubo del modelo HCESHS-12 tiene una geometría y una resistencia mecánica tales que permiten la optimización del funcionamiento y durabilidad para generación de vapor a altas presiones y temperaturas (superiores a 100 bar y $550^{\circ} \mathrm{C}$ ). Estos tubos HCESHS- 12 disponen en el espacio anular de condiciones de vacío y de un material para absorción de los gases residuales generados por el metal del tubo evitando la degradación del funcionamiento térmico del tubo absorbedor.

Como se ha citado en capítulos anteriores, la DSG es una tecnología en la que se han invertido muchos recursos ya que dispone de muchas ventajas en comparación con el resto de fluidos de trabajo: el fluido no sufre degradación alguna, es una tecnología limpia, sin impacto ambiental alguno, el coste del HTF es prácticamente nulo, es industrialmente escalable, ya se ha validado en plantas industriales, la corrosión del material de las tuberías no es tan grande como en el caso de las sales, su coste de mantenimiento no es alto, no requiere sistema alguno de calentamiento para evitar solidificaciones.

El estado del arte de los ciclos de potencia s- $\mathrm{CO}_{2}$ Brayton ya ha sido tratado en capítulos anteriores. Es una tecnología que está madurando a escala industrial. Constituye uno de los objetivos en las políticas de investigación y desarrollo de países tan potentes como Estados Unidos, Corea del Sur, China, etc. Por tanto, está garantizado el desarrollo de los equipos a escala industrial que permita su adaptación a las centrales termosolares. En particular, en el presente capítulo se realiza un primer estudio para la integración de los parámetros termodinámicos de DSG con ciclos $\mathrm{s}-\mathrm{CO}_{2}$ Brayton. Con este propósito se han definido diferentes configuraciones de planta termosolar con colectores lineales.

- La configuración 1, figura 8.8, con la instalación de un condensador de vapor entre el ciclo de potencia y el campo solar con DSG en modo de recirculación de la fase líquida. El condensador es de tipología Carcasa-Tubos con condensación del vapor en el lado carcasa y fluyendo el s- $\mathrm{CO}_{2}$ por el interior de los tubos. Las dimensiones de este equipo están muy beneficiadas por el buen coeficiente de transferencia de calor en la zona de 
8. Plantas Termosolares de Colectores Lineales con DSG acopladas a ciclos Brayton con $\mathrm{CO}_{2}$

cambio de fase de vapor a líquido. Esta primera configuración es tecnológicamente factible con los equipos y materiales disponibles en el mercado, su principal hándicap pasaría por confirmar el espesor de los tubos absorbedores. En un primer estudio aproximativo se ha fijado en $9 \mathrm{~mm}$, con tubos de acero inoxidable.

- La configuración 2, figura 8.9, tiene por objetivo evitar la condensación del vapor y permitir la recirculación del vapor sobrecalentado en el campo solar por medio de compresores. No se ha demostrado que esta solución técnica sea viable desde el punto de vista del diseño de los equipos que la constituyen, principalmente de los compresores de vapor. En este trabajo se ha demostrado su viabilidad termodinámica. Estas dos configuraciones 1 y 2 puede ser integradas en una sola planta termosolar como se representa en la figura 8.7.

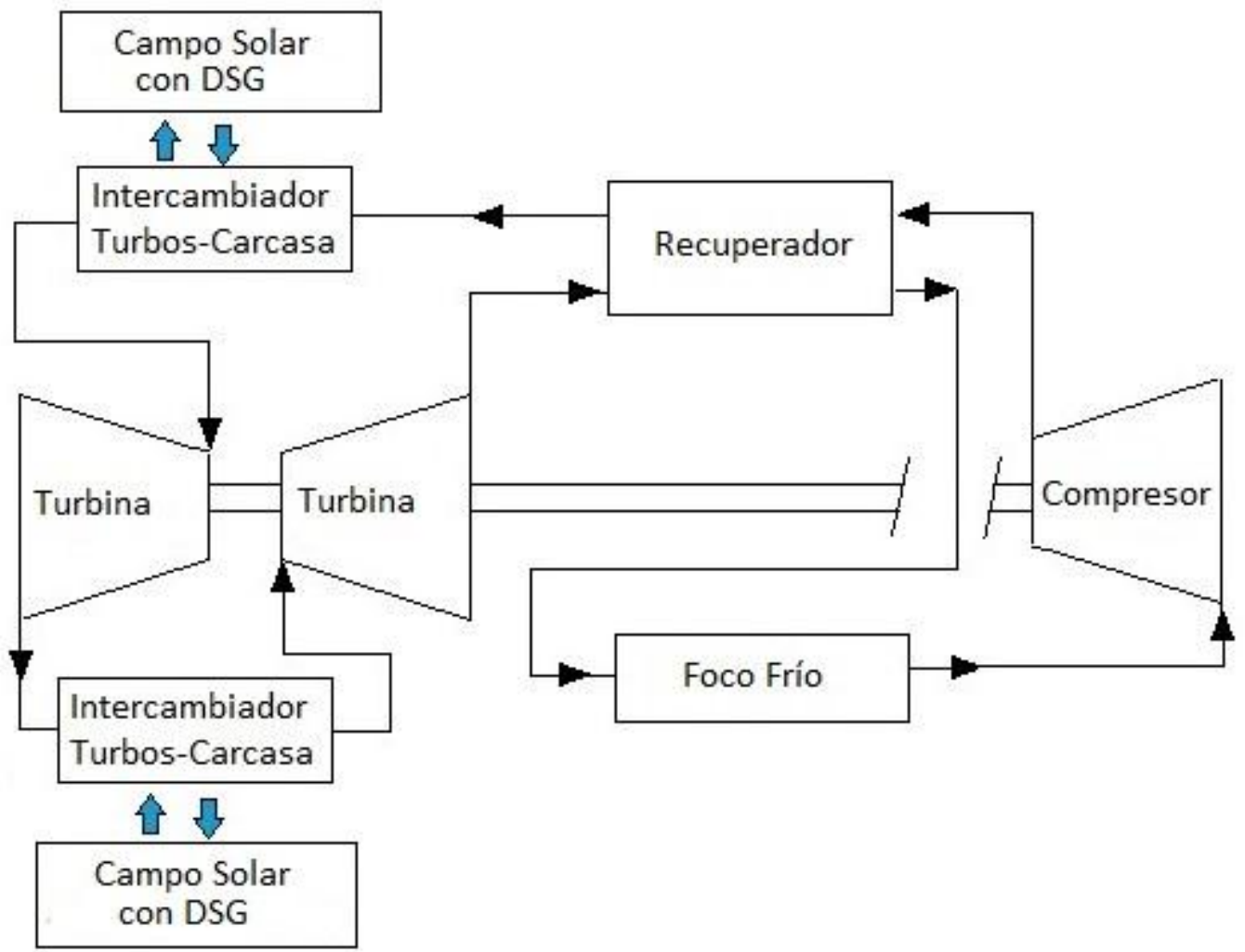

Figura 8.1. Configuración de central termosolar con colectores lineales acoplada a ciclo de potencia s- $\mathrm{CO}_{2}$ Brayton SB. 
8. Plantas Termosolares de Colectores Lineales con DSG acopladas a ciclos Brayton con $\mathrm{CO}_{2}$

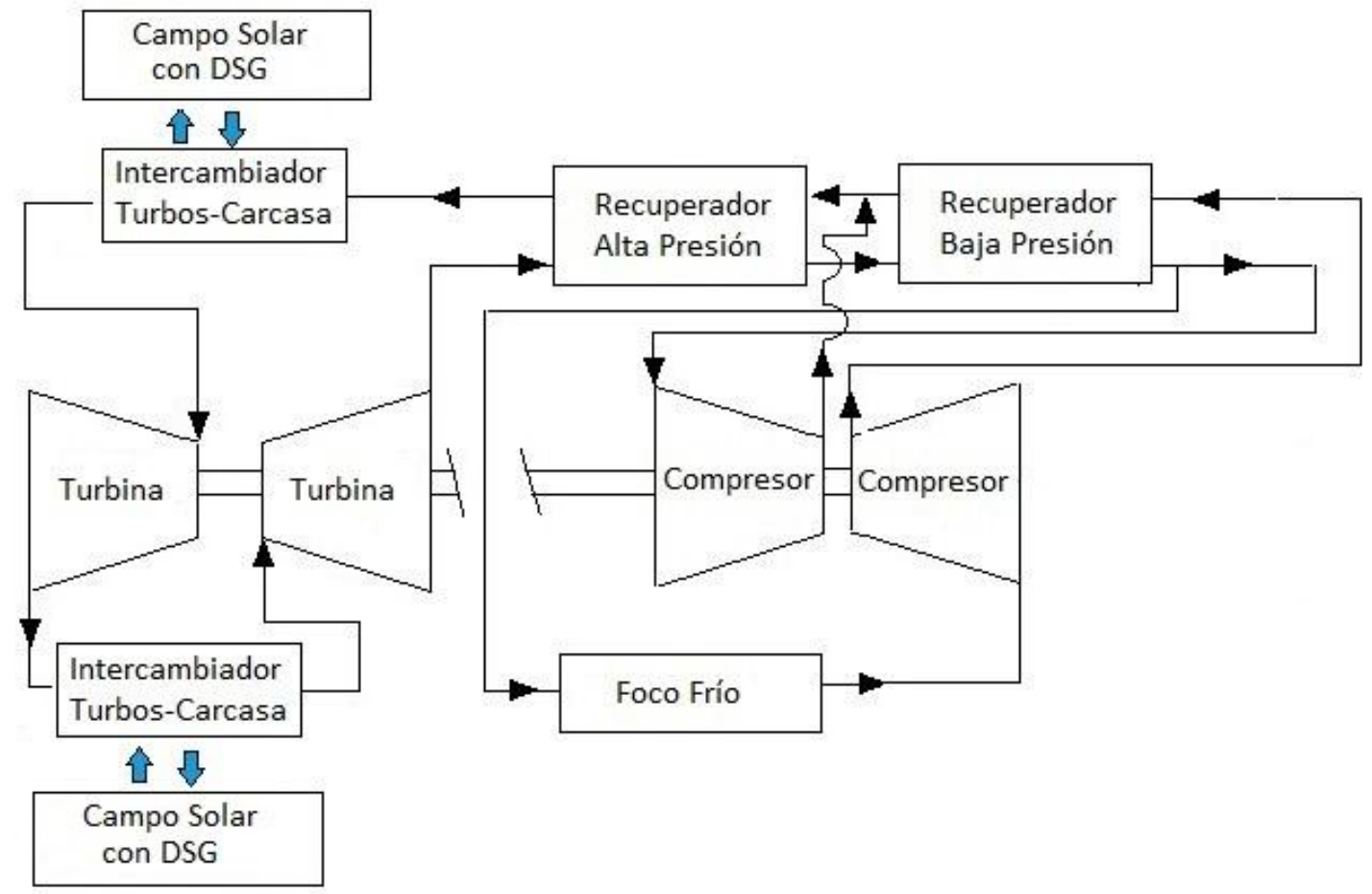

Figura 8.2. Configuración de central termosolar con colectores lineales acoplada a ciclo de potencia s- $\mathrm{CO}_{2}$ Brayton RC.

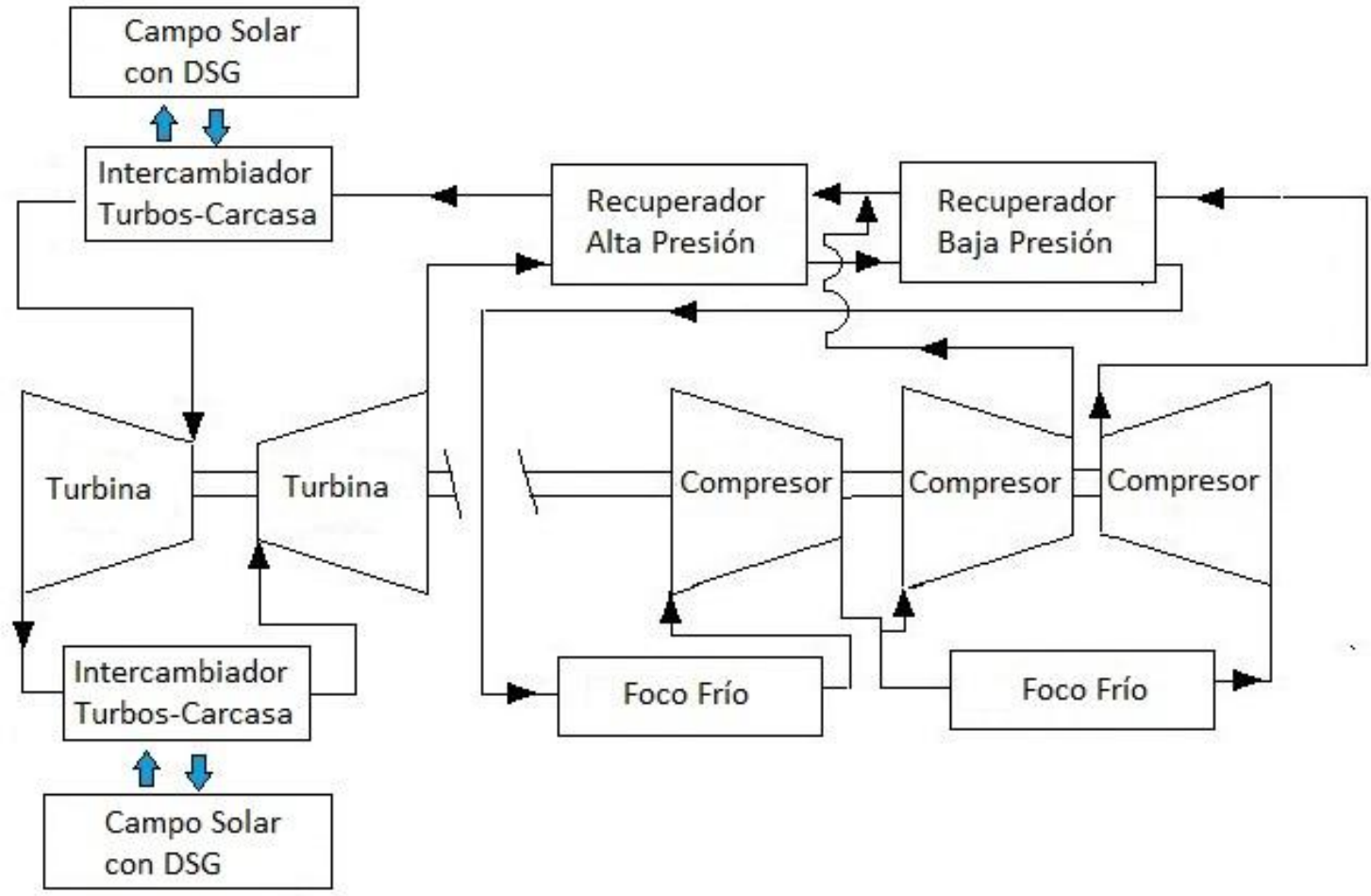

Figura 8.3. Configuración de central termosolar con colectores lineales acoplada a ciclo de potencia s- $\mathrm{CO}_{2}$ Brayton PCRC. 


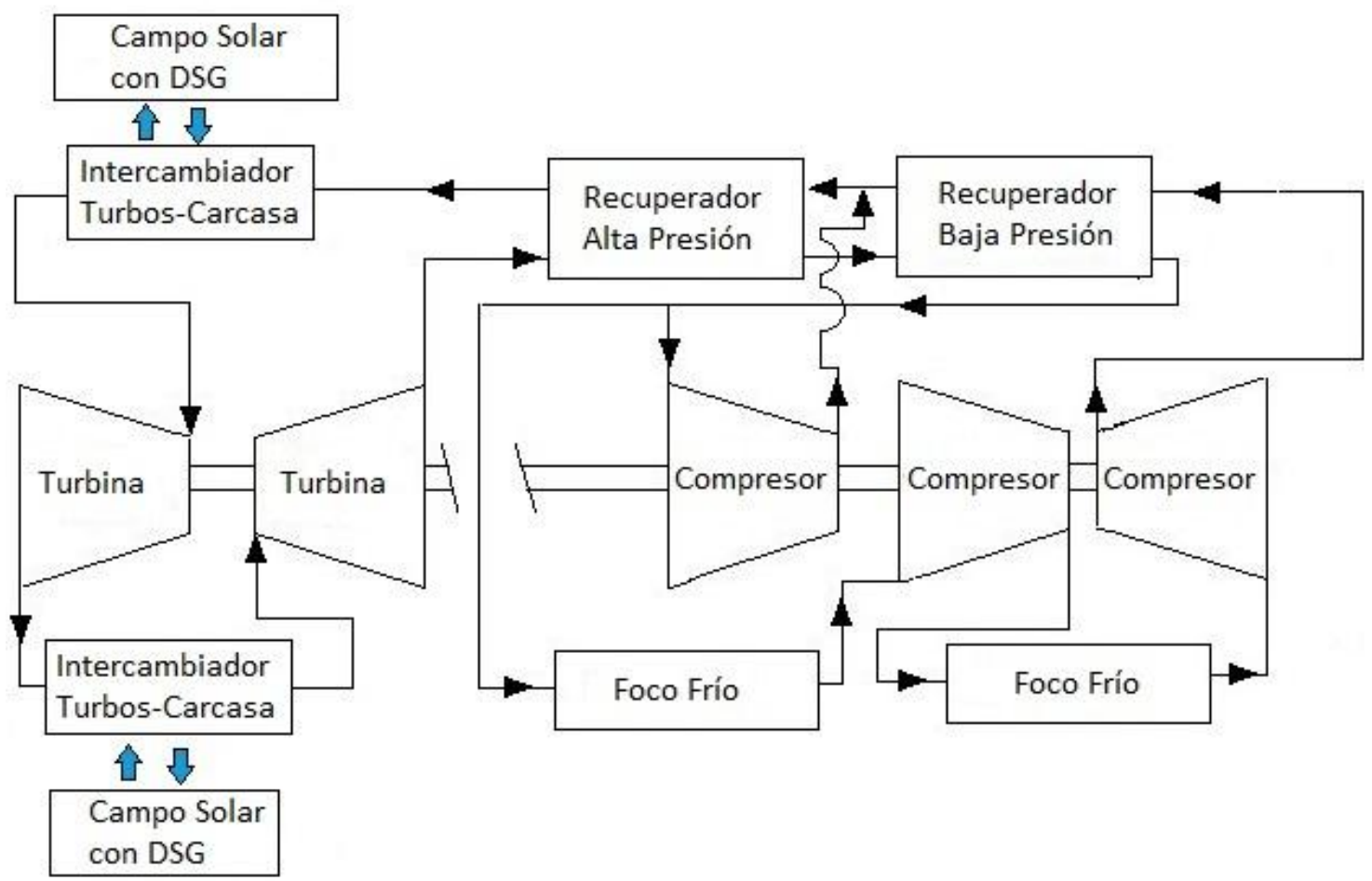

Figura 8.4. Configuración de central termosolar con colectores lineales acoplada a ciclo de potencia s- $\mathrm{CO}_{2}$ Brayton $\mathrm{RCMCl}$.

- La configuración 3, figura 8.11, es similar a la configuración 1 con DSG en modo de recirculación de la fase líquida pero con dos ciclos $\mathrm{s}-\mathrm{CO}_{2}$ Brayton interconectados en cascada. Mediante esta alternativa se consiguen temperaturas de entrada en turbina de $550^{\circ} \mathrm{C}$ incrementando la eficiencia neta del ciclo de potencia. Como contrapartida se duplica el número de equipos con dos ciclos de potencia.

- La configuración 4, figura 8.12 es una combinación de la configuración 2 con una etapa de recalentamiento en turbina mediante campo solar con colectores con sales fundidas como fluido caloportador. Estas plantas termosolares $\left(\mathrm{DSG}+\mathrm{sCO}_{2}\right.$ ) han sido comparadas con la otra tecnología de referencia ya validada a nivel industrial en plantas piloto, la generación directa de vapor en colectores lineales (PTC o LF) y ciclo de potencia Rankine con agua en estado subcrítico, figuras. 8.5 y 8.6 .

\subsection{Planta termosolar con colectores lineales con generación directa de vapor acopladas a} ciclo Rankine sin Recalentamiento (configuración de referencia).

Como base comparativa se ha simulado la producción de energía de una planta termosolar con colectores lineales con DSG acoplada a ciclo de potencia Rankine. El plano de disposición de los equipos y componentes de esta tipología de instalaciones termosolares queda representado en las figuras 8.5 y 8.6 . 
8. Plantas Termosolares de Colectores Lineales con DSG acopladas a ciclos Brayton con $\mathrm{CO}_{2}$

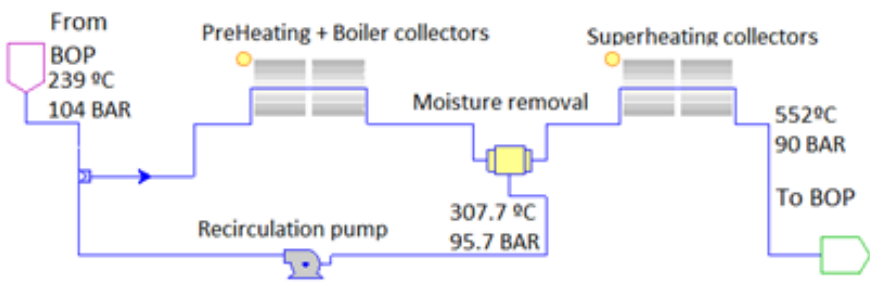

Figura 8.5. Campo solar integrado por colectores lineales con generación directa de vapor en modo recirculación (configuración de referencia).

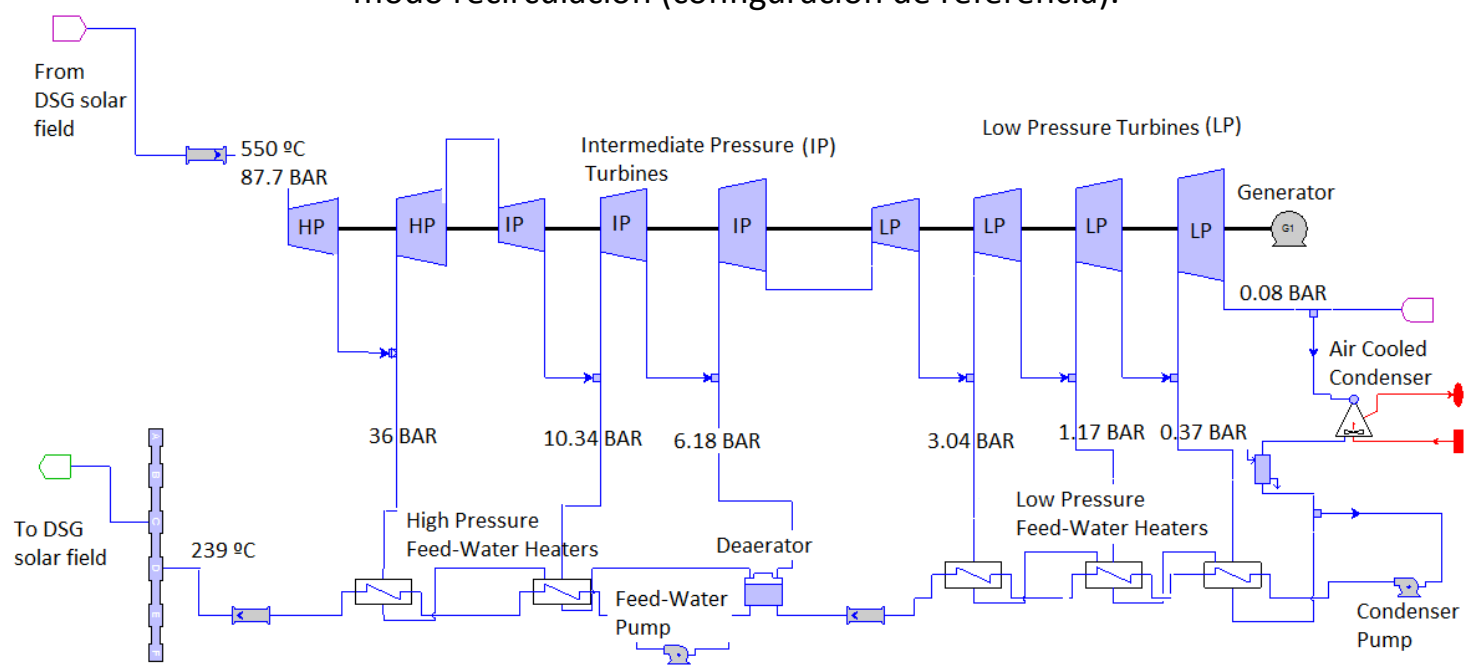

Figura 8.6. Ciclo de potencia Rankine sin recalentamiento en turbinas (configuración de referencia).

\subsection{Hipótesis de cálculo}

Todos los modelos de plantas termosolares estudiados en este capítulo han considerado como hipótesis de diseño los valores incluidos en las tabla 8.3 a 8.7 .

Tabla 8.3. Localización y condiciones ambientales.

\begin{tabular}{ll}
\hline Localización: & Dagget, CA, USA. \\
Latitud: & $34.86^{\circ}$ \\
Longitud: & $-116.8^{\circ}$ \\
Zona Horaria: & -8 \\
Hora: & $11: 30 \mathrm{hr}$ \\
DNI: & $986 \mathrm{~W} / \mathrm{m}^{2}$ \\
Temperatura ambiente: & $25^{\circ} \mathrm{C}$ \\
Altitud: & $588 \mathrm{~m}$ \\
\hline
\end{tabular}

Tabla 8.4. Parámetros tubos receptores.

\begin{tabular}{ll}
\hline Material tuberías: & Acero Inoxidable \\
Diámetro exterior: & $70 \mathrm{~mm}$ \\
Espesor: & $4-8 \mathrm{~mm}$ \\
Rugosidad interna: & $\mathrm{Ra}=0.0457 \mathrm{~mm}$ \\
\hline
\end{tabular}


8. Plantas Termosolares de Colectores Lineales con DSG acopladas a ciclos Brayton con $\mathrm{CO}_{2}$

Tabla 8.5. Parámetros de los colectores PTC.

\begin{tabular}{ll}
\hline Tipo de colector: & SuperNova1 (Novatec) \\
Dimensiones del & $16.56 \mathrm{~m} \times 44.8 \mathrm{~m}$ \\
Módulo: & $513.6 \mathrm{~m}^{2} /$ por módulo \\
Área de apertura: & 0.67 (evaporación); \\
Eficiencia óptica & 0.647 (sobrecalentamiento) \\
nominal: & $1.06 \Delta \mathrm{T}+1.2 \cdot 10^{-8} \Delta \mathrm{T}^{4}[31]$ \\
Pérdidas térmicas: & $0.15 \Delta \mathrm{T}+7.15 \cdot 10^{-9} \Delta \mathrm{T}^{4}[31]$ \\
\hline
\end{tabular}

Tabla 8.6. Parámetros de los colectores LF.

\begin{tabular}{ll}
\hline Tipo de colector: & EuroTrough II \\
Anchura de apertura: & $5.77 \mathrm{~m}$ \\
Longitud Focal: & $1.71 \mathrm{~m}$ \\
Factor de limpieza: & 0.96 \\
Eficiencia óptica & 0.75 \\
nominal: & $0.141 \Delta \mathrm{T}+6.4810^{-9} \Delta \mathrm{T}^{4}[32]$ \\
\hline
\end{tabular}

Tabla 8.7. Parámetros del ciclo s- $\mathrm{CO}_{2}$ Brayton.

\begin{tabular}{ll}
\hline Eficiencia de Turbina: & $93 \%$ \\
Eficiencia de Compresor: & $89 \%$ \\
Eficiencia de Intercambiador: & $95 \%$ \\
No perdidas de presión en $\mathrm{HX}$ & \\
Temperatura entrada Turbina: & $550^{\circ} \mathrm{C}$ \\
Presión entrada Turbina: & $250 \mathrm{bar}$ \\
Presión recalentamiento: & $173 \mathrm{bar}$ \\
Entrada al Compresor: & $32^{\circ} \mathrm{C}$ and 74 bar \\
Pérdidas parásitas: & $0.01 \%$ (Potencia Bruta) \\
Eficiencia del Generador: & 98.23 (Punto de Diseño) \\
\hline
\end{tabular}

\subsection{Plantas termosolares con colectores lineales con generaciones directas de vapor acopladas a ciclos de potencia s- $\mathrm{CO}_{2}$ Brayton}

\subsubsection{Configuración 1}

Esta solución de diseño para la integración de la tecnología de generación de vapor con modo de recirculación de la fase líquida con los ciclos de potencia s- $\mathrm{CO}_{2}$ Brayton está representada gráficamente en las figuras 8.7 y 8.8. El campo solar está subdividido en tres zonas: los colectores lineales para evaporación del agua líquida, los colectores solares lineales para el sobrecalentamiento del vapor saturado, y una caldera con combustible fósil de respaldo del campo solar para la generación de vapor de agua. 


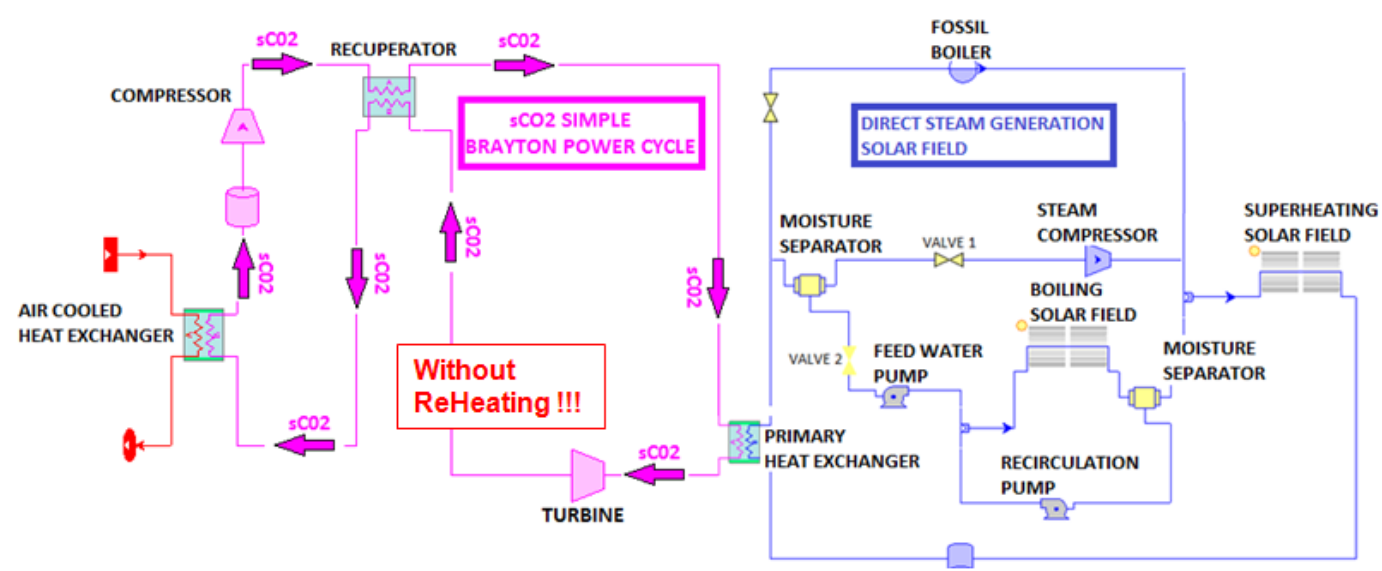

Figura 8.7. Integración de las Configuraciones 1 y 2 en la misma planta termosolar.

En esta configuración es necesaria la instalación de dos intercambiadores de calor entre el campo solar y el ciclo de potencia $\mathrm{s}-\mathrm{CO}_{2}$ Brayton. Un primer intercambiador reduce la temperatura del vapor sobrecalentado $\mathrm{y}$ en un segundo cambiador operando como condensador, figura 8.8, se produce la condensación del vapor de agua en su fase líquida. En el primer intercambiador se transfiere al $\mathrm{s}-\mathrm{CO}_{2}$ el calor sensible del vapor y en el segundo intercambiador se transfiere al s- $\mathrm{CO}_{2}$ el calor latente de cambio de fase del vapor de agua a la fase líquida. El almacenamiento de la energía térmica mediante el calor latente de cambio de fase es un mecanismo óptimo de transferencia de calor, y debido a esta razón las dimensiones del condensador serán menores que las del otro intercambiador de calor. Para condensar el vapor generado en los colectores solares lineales, es necesario incrementar la presión de operación del campo a valores entre 150 bar a 175 bar. Por esta razón el material de los tubos absorbedores y su espesor tiene que ser diseñado para soportar la presión de 175 bar. Las tuberías de acero inoxidable con $8 \mathrm{~mm}$ de espesor son una selección óptima. Sin embargo, también es posible evaluar la solución de seleccionar acero al carbono como material de los tubos e incrementar su espesor de pared para permitir un margen por corrosión ya que el agua no es tan corrosiva como las sales fundidas.

Esta configuración de planta termosolar tiene dos modos de operación, en el Modo 1 de operación el vapor saturado es generado en el campo solar y en Modo 2 de operación el vapor es generado en la caldera con combustibles fósiles y posteriormente es sobrecalentado en el campo solar. El Modo 2 de operación es adecuado para la puesta en marcha de la instalación y para compensar los transitorios de operación provocados por fenómenos meteorológicos (como la nubosidad). La configuración 1 puede ser integrada e intercambiarse con la configuración 2 que se explicará posteriormente, en una misma planta termosolar mediante la configuración ilustrada en la figura 8.7. La temperatura de entrada en turbina de la configuración 1 es de $400^{\circ} \mathrm{C}$, por tanto, es una solución de diseño apropiada para bajos valores de radiación solar incidente. El caudal del fluido caloportador, tanto en el campo solar como en el ciclo de potencia, debe ser regulado durante el funcionamiento de la planta para garantizar la totalidad de la condensación del vapor de agua y evitar daños de cavitación por inclusiones de vapor en la bomba de agua de alimentación en el campo solar. Con este fin también se ha instalado un separador de humedad, figura 8.7, y un tanque de separación de fases para garantizar el NPHS y la fase líquida a la entrada de la citada bomba del campo solar. 


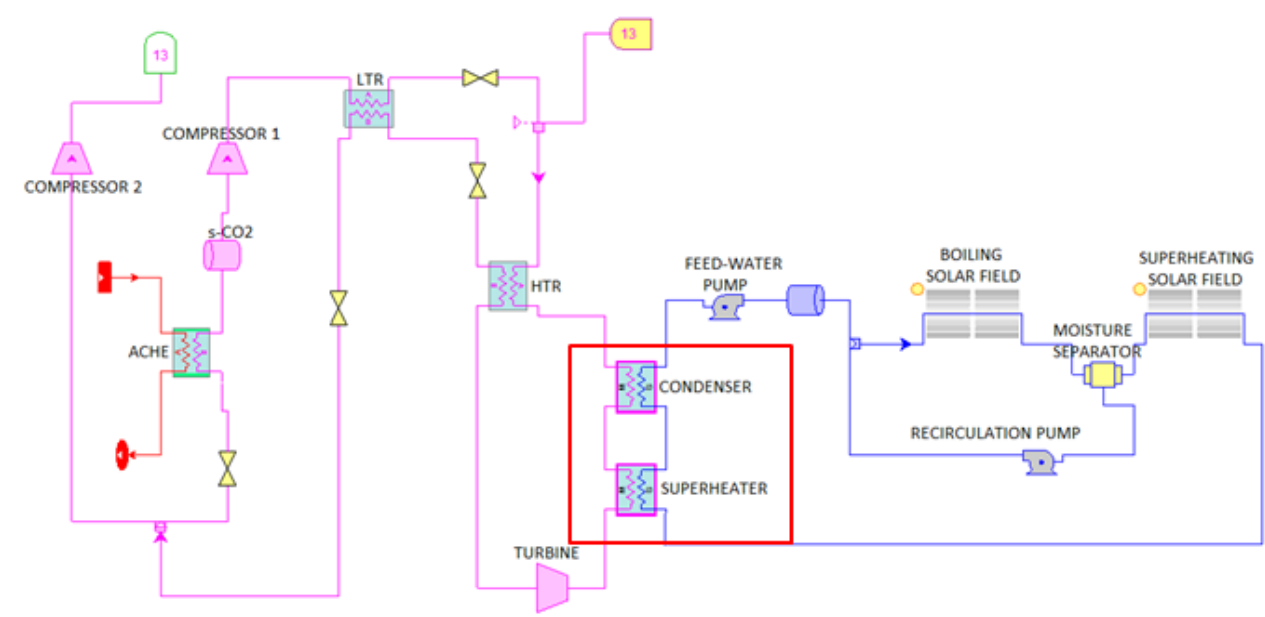

Figura 8.8. Planta termosolar con colectores lineales con generación directa de vapor acoplada a ciclo de potencia s- $\mathrm{CO}_{2}$ Brayton sin recalentamiento (configuración 1).

\subsubsection{Configuración 2}

En esta configuración 2 el vapor generado en los colectores lineales es también sobrecalentado en un campo solar, pero no es condensado en su fase líquida, figura 8.9. El compresor de vapor compensa las caídas de presión del vapor por su rozamiento con las tuberías tanto de los tubos absorbedores como de las tuberías de distribución del campo solar. Para compensar os transitorios de radiación solar y para generar el vapor durante la puesta en marcha de la instalación, en paralelo con los colectores para sobrecalentamiento del vapor se ha instalado una caldera con combustible fósil, figura 8.7. La configuración 2 está diseñada para temperaturas de entrada en turbina superiores a $400^{\circ} \mathrm{C}$ y hasta $550^{\circ} \mathrm{C}$. Con esta solución, el vapor sobrecalentado generado en los colectores solares transfiere su calor al s- $\mathrm{CO}_{2}$ en un intercambiador de calor tipo carcasa-tubo PHX, y sin condensar en su fase líquida regresa al campo solar para su calentamiento.

Otro parámetro importante en el funcionamiento de estas instalaciones es garantizar que la temperatura y presión $\left(427^{\circ} \mathrm{C}\right.$ y 175 bar) a la entrada del campo solar son los adecuados para optimizar el funcionamiento del compresor de vapor y minimizar su consumo eléctrico, y a la vez se consiga una optimización del diseño del intercambiador PHX para calentamiento de fluido de trabajo en el ciclo $\mathrm{s}-\mathrm{CO}_{2}$ Brayton. El compromiso se explica a continuación: si la temperatura del vapor a la salida del cambiador PHX es muy baja lo cual favorece a los compresores de vapor, no se optimizará el diseño de este equipo. Por el contrario, si la temperatura a la salida del PHX es muy alta el consumo de los compresores de vapor se incrementará, ya que la densidad del vapor aumentará lo cual impacta negativamente en el rendimiento de los compresores de vapor.

Respecto al material de las tuberías de distribución del campo solar y de los tubos absorbedores, se considera que el acero inoxidable ferrítico T91 o el acero inoxidable austenítico con Molibdeno y Titanio, es adecuado para soportar la susceptibilidad de corrosión a altas presiones y temperaturas $560^{\circ} \mathrm{C}$ and 175 bar. Sin embargo, para minimizar los costes del material también sería conveniente evaluar la selección de acero al carbono incrementando el espesor de pared de los tubos garantizando que se mantienen sus características de transferencia de calor. 
8. Plantas Termosolares de Colectores Lineales con DSG acopladas a ciclos Brayton con $\mathrm{CO}_{2}$

Como se ha mencionado anteriormente, el principal hándicap de la configuración 2 es el consumo eléctrico de los compresores de vapor, para minimizar su impacto directo en la eficiencia neta de la planta termosolar. En este sentido, otro parámetro a optimizar en el diseño del campo solar es la pérdida de carga del vapor de agua a lo largo de los tubos absorbedores y de los tubos de distribución de vapor. Por ello se limita el flujo másico $\left(\mathrm{kg} / \mathrm{m}^{2}\right.$ s) en los tubos absorbedores, este parámetro está limitado a $1000 \mathrm{~kg} / \mathrm{m}^{2} \mathrm{~s}$, en los colectores LF, y a $850 \mathrm{~kg} / \mathrm{m}^{2} \mathrm{~s}$ en los colectores PTC. La limitación del flujo másico garantiza la optimización de las pérdidas de carga en el campo solar, y por tanto, proporciona un diseño óptimo de la longitud de los colectores solares.

Otra variable decisiva en para garantizar un consumo mínimo de los compresores de vapor es la presión de operación del campo solar. Los altos valores de presión en el campo solar incremental la densidad del vapor y reduce el trabajo en los compresores. Sin embargo, los altos valores de presión en el campo solar requieren un material con alta resistencia mecánica y altos espesores en los tubos absorbedores, tuberías de distribución del campo solar, y juntas rotativas móviles en el caso de colectores parabólicos.

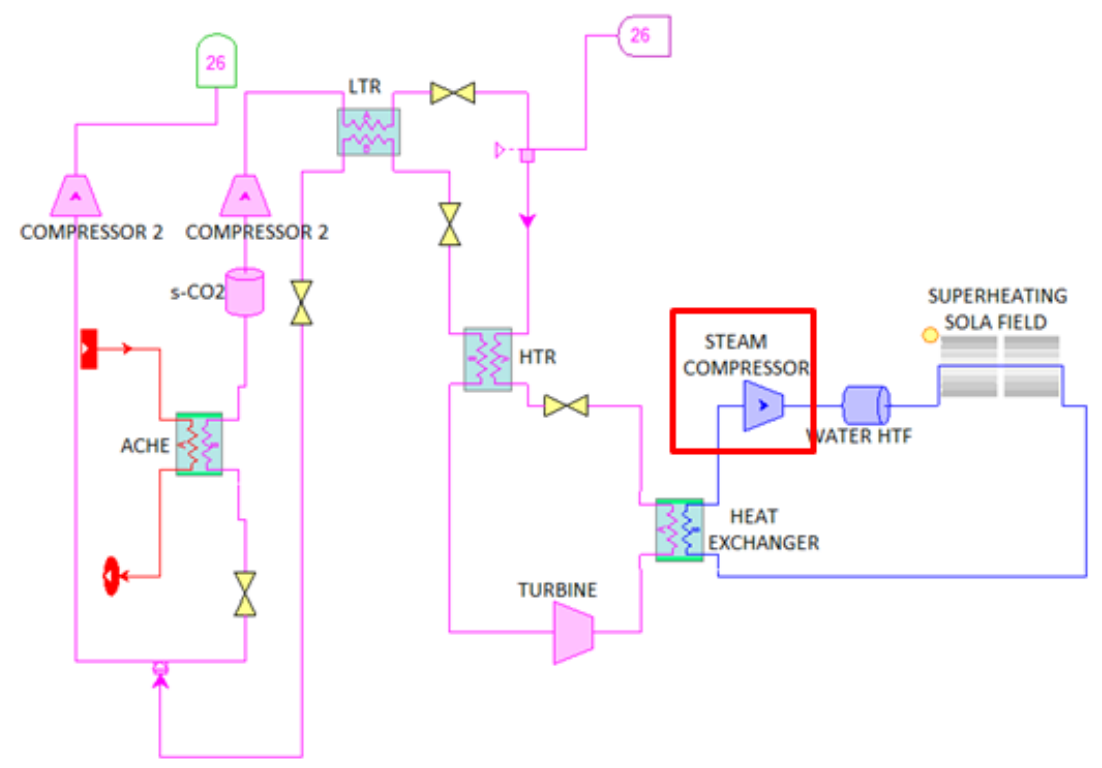

Figura 8.9. Planta termosolar con colectores lineales para sobrecalentamineto del vapor acoplada a ciclo de potencia s- $\mathrm{CO}_{2}$ Brayton sin recalentamiento (configuración 2).

Resumiendo la viabilidad de la configuración 2 está condicionada principalmente a minimizar el consumo eléctrico de los compresores de vapor. Para optimizar las pérdidas de presión en el campo solar y en el intercambiador PHX, se han propuesto las siguientes medidas:

- Limitación de la longitud de los colectores y los lazos que constituyen a longitud<150 $\mathrm{m}$ para colectores LF y a longitud $<220 \mathrm{~m}$ para colectores parabólicos.

- Limitar el flujo másico en los tubos absorbedores: $1000 \mathrm{~kg} / \mathrm{m}^{2}$ s en colectores LF y 850 $\mathrm{kg} / \mathrm{m}^{2}$ s en colectores parabólicos.

- Fijar la presión (175 bar) y temperatura $\left(420^{\circ} \mathrm{C}\right)$ a la entrada de los compresores de vapor.

- Obtener un diseño óptimo de los tubos absorbedores. Se han simulado dos opciones: 
8. Plantas Termosolares de Colectores Lineales con DSG acopladas a ciclos Brayton con $\mathrm{CO}_{2}$

a) Tubo absorbedor con diámetro exterior de $70 \mathrm{~mm}$ de Acero al Carbono y espesor de pared $8.5 \mathrm{~mm}$.

b) Tubo absorbedor con diámetro exterior de $90 \mathrm{~mm}$ de Acero al Carbono y espesor de pared $10.5 \mathrm{~mm}$.

- Optimizar las pérdidas de presión en el intercambiador de calor para acoplamiento entre campo solar y ciclo de potencia, adoptando las tecnologías más innovadoras del diseño de los canales (Airfoil fin, $\mathrm{S}$ fin, etc.).

Para confirmar que las hipótesis de pérdida de presión consideradas con Thermoflow en el campo solar de la configuración 2 son correctas, se ha realizado un Balance Hidráulico con el programa informático AFT Arrow [33], simulando el diseño de un campo solar como el mostrado en la figura 8.10.

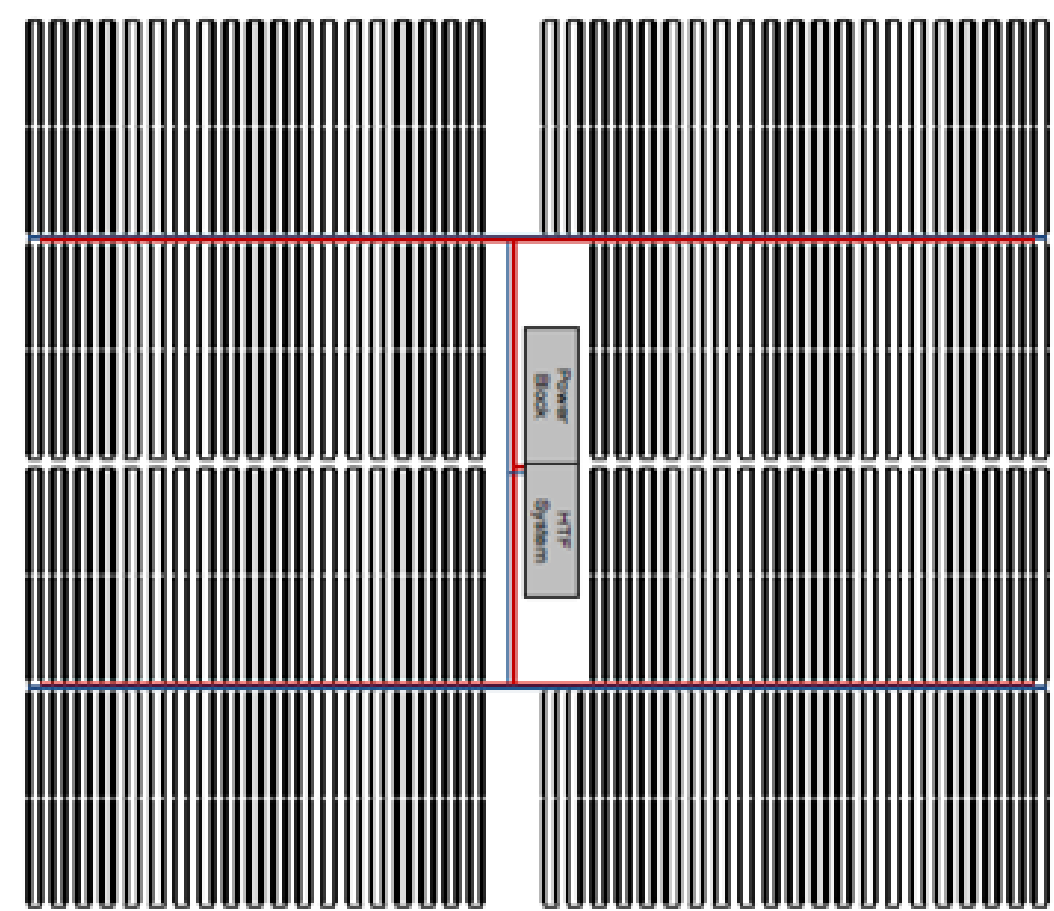

Figura 8.10. Plano de planta del Campo Solar diseñado para la configuración 2.

Las hipótesis de cálculo consideradas en el Balance Hidráulico del campo solar de la configuración 2 y sus resultados se citan en la tabla 8.8. Como puede apreciarse el programa informático AFT Arrow proporciona una pérdida de carga en los tubos absorbedores de 3.7 bar, y en Thermoflow se ha considerado 5.2 bar.

Tabla 8.8. Hipótesis y resultados del Balance Hidráulico del campo solar de la configuración 2.

\begin{tabular}{llll}
\hline & AFT ARROW & AFT ARROW & Thermoflow \\
\hline Fluido & ASME 97 & ASME 97 & IFC-67 \\
Tipo de colector & Fresnel & Fresnel & Fresnel \\
Material & Carbon steel & Carbon steel & Carbon steel \\
Rugosidad $(\mathrm{mm})$ & 0.045 & 0.045 & 0.0457 \\
Diámetro exterior $(\mathrm{mm})$ & 70 & 70 & 70 \\
Diámetro interior $(\mathrm{mm})$ & 53.5 & 53.5 & 53.5
\end{tabular}


8. Plantas Termosolares de Colectores Lineales con DSG acopladas a ciclos Brayton con $\mathrm{CO}_{2}$

\begin{tabular}{llll}
\hline & AFT ARROW & AFT ARROW & Thermoflow \\
\hline Longitud $(\mathrm{m})$ & 143 & 143 & 143 \\
Caudal $(\mathrm{kg} / \mathrm{s})$ & 2.244 & 2.24 & 2.24 \\
Presión entrada (bar) & 175 & 175 & 175 \\
Temperatura entrada $\left({ }^{\circ} \mathrm{C}\right)$ & 564 & 433.6 & 433.6 \\
Caída Presión (bar) & $\mathbf{5 . 1 0 1}$ & $\mathbf{3 . 6 8 2}$ & $\mathbf{5 . 2 1 7}$ \\
\hline
\end{tabular}

En relación a las pérdidas de carga de las tuberías de distribución de vapor en el campo solar de la configuración 2, es necesario realizar su dimensionamiento para minimizar las pérdidas de presión para ello se ha fijado su diámetro entre 8 pulgadas y 10 pulgadas, dependiendo de las restricciones en la velocidad del vapor para evitar condensaciones significativas. Como resultado se estima una pérdida de carga entre 0.25 bar y 0.6 bar. Finalmente, se evalúan las pérdidas de carga totales de la configuración 2 , suma de las pérdidas de presión en los tubos absorbedores, tuberías de distribución de vapor e intercambiador de calor:

6-6.75 bar (configuración 2) = 5 bar (Tubos absorbedores) + 0.25-0.6 bar (10"-12" tuberías distribución)+ 0.75 bar (Intercambiador de Calor PHX).

\subsubsection{Configuración 3}

El propósito de la configuración 3 es la integración de la tecnología de generación de vapor en modo recirculación, ya validad en instalaciones industriales, con los ciclos supercríticos de potencia s- $\mathrm{CO}_{2}$ Brayton, pero con unas temperaturas de $550^{\circ} \mathrm{C}$ a la entrada de la turbina. La disposición de los equipos en la configuración 3 está representada en la figura 8.11, y combina dos ciclos s- $\mathrm{CO}_{2}$ Bayton de tipología $\mathrm{RCMCl}$ operando en cascada. El objetivo es conseguir una condensación del vapor generador en el campo solar en su fase líquida una vez transferido todo su calor latente al fluido de trabajo del ciclo $\mathrm{s}-\mathrm{CO}_{2}$ Brayton. Para ello se instalan dos cambiadores designados como PHX1 y PHX2, figura 8.11.

El intercambiador de calor PHX2 es un condensador tipo Carcasa-Tubos. Por el lado tubos fluye el s- $\mathrm{CO}_{2}$ y por el lado carcasa se produce la condensación del vapor de agua en su fase líquida. En el I intercambiador PHX1 el vapor reduce su temperatura desde alrededor de $550^{\circ} \mathrm{C}$ hasta $410^{\circ} \mathrm{C}$, temperatura a la que entra en el condensador PHX2. Otro punto significativo en esta configuración es la etapa de recalentamiento del $\mathrm{s}-\mathrm{CO}_{2}$ en el ciclo Brayton inferior de la cascada. El recalentamiento del $\mathrm{s}-\mathrm{CO}_{2}$ se realiza mediante un intercambiador, $\mathrm{RHX}$ (figura 8.11), con una extracción de vapor proveniente directamente del campo solar de un $55 \%$ del caudal de vapor sobrecalentado. Una vez que esta corriente ha cedido su calor reduce su temperatura desde alrededor de $550^{\circ} \mathrm{C}$ hasta unos $500^{\circ} \mathrm{C}$ y pasa a mezclarse con el vapor proveniente del intercambiador PHX1. Por tanto al ciclo s- $\mathrm{CO}_{2}$ Brayton inferior es calentado en su intercambiador principal PHX1 con el $45 \%$ del caudal de vapor sobrecalentado por el campo solar, el resto, es decir, el $55 \%$ es utilizado en su etapa de recalentamiento.

Hay otros parámetros de diseño de la configuración 3 a destacar para obtener su rendimiento óptimo cercano al $40 \%$. El campo solar opera a una alta presión 175 bar (con una temperatura de saturación de $354.63^{\circ} \mathrm{C}$ ) para permitir la condensación del vapor de agua en el cambiador $\mathrm{PHX} 2$ a $350^{\circ} \mathrm{C}$. 
8. Plantas Termosolares de Colectores Lineales con DSG acopladas a ciclos Brayton con $\mathrm{CO}_{2}$

Otro parámetro importante es la relación entre el caudal de $\mathrm{s}-\mathrm{CO}_{2}$ en el ciclo 1 y el caudal de s$\mathrm{CO}_{2}$ en el ciclo 2, esta relación es cercana a 6.2, para permitir la condensación completa del vapor de agua antes de su entrada de nuevo en el campo solar. Finamente, la fracción óptima de caudal de compresión en los ciclos s- $\mathrm{CO}_{2}$ Brayton es de $60 \%$ y $40 \%$.

En los dos ciclos $\mathrm{s}-\mathrm{CO}_{2}$ Brayton en cascada es posible incrementar su tamaño térmico para reducir el pinch-point a la salida de los recuperadores, esta metodología establecida en la referencia [34], permite un incremento de eficiencia neta del ciclo de alrededor del $1 \%$.

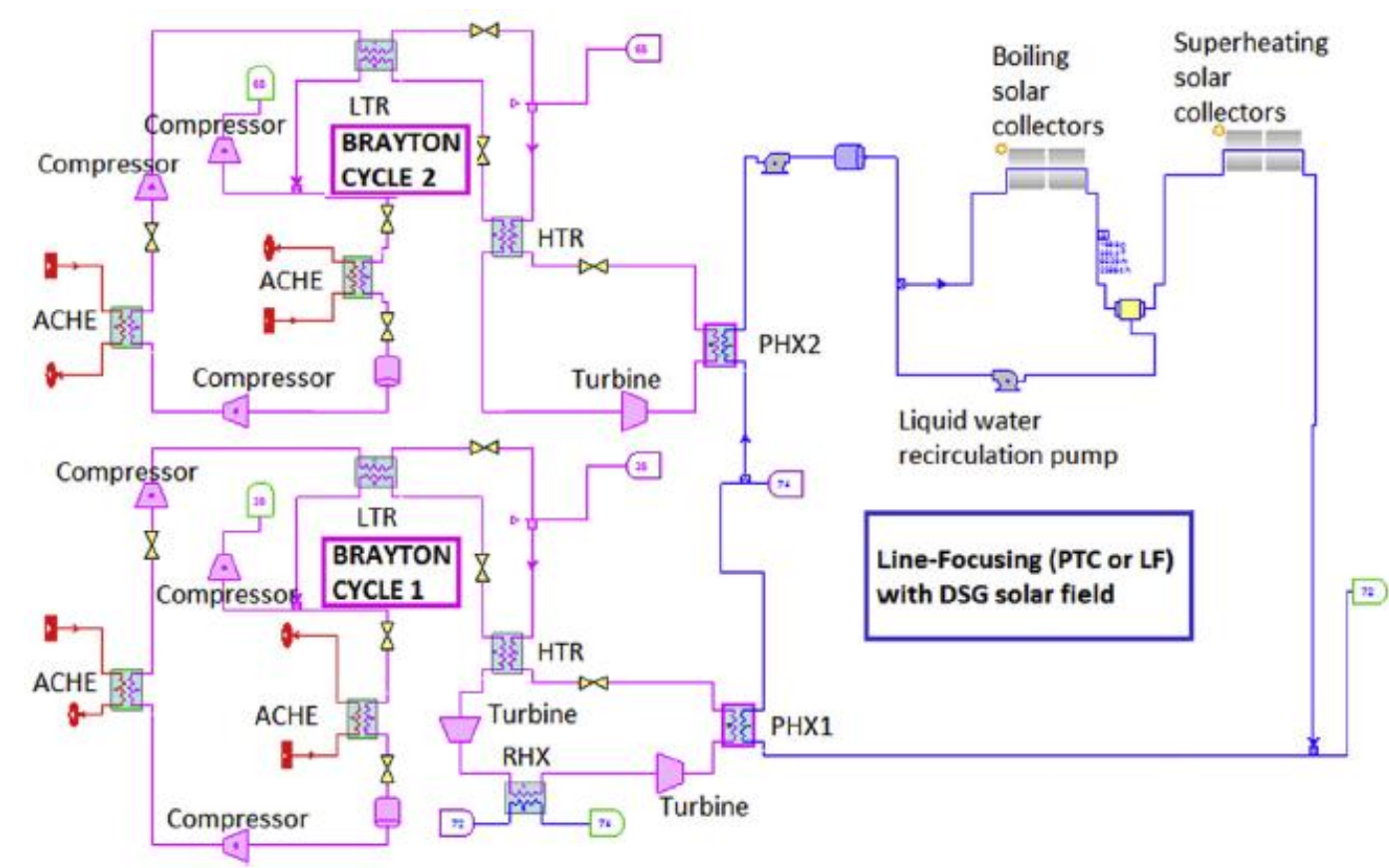

Figura 8.11. Planta Termosolar con colectores lineales con DSG, acoplada a dos ciclos de potencia s- $\mathrm{CO}_{2}$ Bratyon $\mathrm{RCMCl}$ en cascada con recalentamiento en el ciclo inferior (configuración 3).

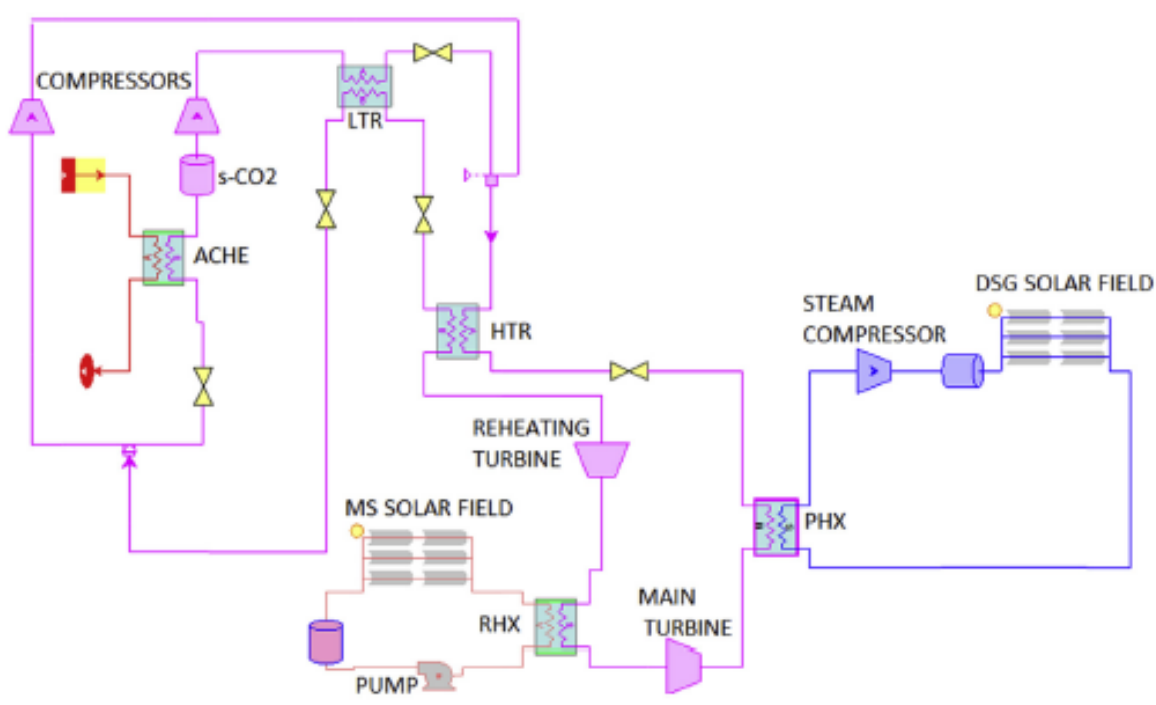

Figura 8.12. Planta termosolar con colectores lineales con DSG en campo solar principal, y con sales fundidas en el campo solar para recalentamiento, acoplada a ciclo de potencia s- $\mathrm{CO}_{2}$ Brayton (configuración 4). 


\subsubsection{Configuración 4}

El objetivo de esta configuración es incrementar la eficiencia energética de la configuración 2 incluyendo una etapa de recalentamiento del vapor en turbina mediante campo solar. Mediante esta mejora se conseguirá reducir las dimensiones de los recuperador y del campo solar proporcionando una ahorro económico sustancial de inversión en la planta termosolar. Los beneficios de las etapas de recalentamiento en turbina ya fueron cuantificados por MIT Dostal [35]. En la configuración 4, figura 8.12, el campo solar principal está integrado por colectores con generación directa de vapor y el campo de recalentamiento del $\mathrm{CO}_{2}$ por colectores solares con sales fundidas como HTF. Esta configuración tipo Dual-Loop integrada por un ciclo s- $\mathrm{CO}_{2}$ Brayton se considera innovadora respecto al estado del arte, y combina los beneficios de ambas tecnologías, minimizando el coste de los materiales, reduciendo su susceptibilidad a la corrosión, y reduciendo las dimensiones del campo con sales fundidas, y minimizando los costes de los equipos de Heat-tracing .

\subsection{Resultados}

\subsubsection{Eficiencia neta en el punto de diseño}

En plantas termosolares con colectores parabólicos como Andasol-1 (España), el fluido caloportador era aceite térmico, cuya limitación de temperatura de entrada en turbina era de $390^{\circ} \mathrm{C}$, y con un ciclo de potencia Rankine con tres calentadores de agua de alimentación de baja presión, un desaireador, y un calentador de agua de alimentación de alta presión, la eficiencia neta del ciclo era cercana al 35\%, ver configuraciones con aceites térmicos sin recalentamiento en el capítulo 1 de esta tesis doctoral. Las configuraciones recientes de ciclo Rankine con recalentamiento en las turbinas de vapor, y con cuatro calentadores del agua de alimentación de baja presión, un desaireador, tres calentadores del agua de alimentación de alta presión, y con las mismas condiciones de temperatura $\left(380^{\circ} \mathrm{C}\right)$ y presión (100 bar) a la entrada de turbina, presentan un rendimiento de la instalación del orden del 37\%, ver el capítulo 1.

El estado del arte de plantas tipo DSG+Rankine con recalentamiento directo o Direct ReHeating (DRH) también puede alcanzar eficiencias del orden del $40.4 \%$ para temperaturas de entrada en turbina de $550^{\circ} \mathrm{C}$, con dos etapas de recalentamiento, tal y como se explica en el capítulo 4. Esta configuración a corto y medio plazo puede jugar un papel muy importante en el desarrollo de las centrales termosolares de nueva generación, ya que a pesar de que las plantas con ciclos $\mathrm{s}-\mathrm{CO}_{2}$ Brayton proporcionan eficiencias netas mayores, el coste de los materiales y equipos requeridos por los fluidos supercríticos, tiene que ser todavía optimizado en relación a los valores de referencias ofertados en plantas con ciclos Rankine.

En este capítulo, se ha considerado como referencia una planta con configuración DSG+Rankine sin recalentamiento de vapor en turbinas. En las figuras 8.5 y 8.6 está representada la configuración con sus equipos y componentes. Las condiciones termodinámicas de entrada en turbina se han fijado en $400^{\circ} \mathrm{C}$ y 90 bar. Los resultados de su simulación en el punto de diseño junto con los de la configuración 1 están resumidos en las tablas 8.9 y 8.10 . Se demuestra por tanto, que la configuración 1 , innovación propuesta en este estudio, con un ciclo s- $\mathrm{CO}_{2}$ Brayton $\mathrm{RC}$ como el representado en la figura 8.8, tiene una eficiencia neta en su punto de diseño de $35.5 \%$ y con una configuración del ciclo $\mathrm{RCMCl}$ es 
8. Plantas Termosolares de Colectores Lineales con DSG acopladas a ciclos Brayton con $\mathrm{CO}_{2}$

incrementado hasta $36.65 \%$, permitiendo reducir las dimensiones del ciclo de potencia de vapor y a su vez disminuir el área de apertura efectiva de los colectores solares, tal y como indica en las tablas 8.17 y 8.18 .

Tabla 8.9. Eficiencia neta de plantas termosolares con colectores LF. Comparación de resultados entre la configuración 1 y la de referencia.

\begin{tabular}{llllllllll}
\hline Ciclo de potencia & Rankine & SB & SB & RC & RC & PCRC & PCRC & RCMCl & RCMCl \\
\hline HTF & DSG & DSG & MS & DSG & MS & DSG & MS & DSG & MS \\
Eficiencia (\%) & 35.01 & 32.25 & 32.3 & 35.58 & 35.34 & 34.25 & 34.31 & 36.65 & 36.69 \\
\hline
\end{tabular}

Nota: Todos los ciclos de potencia han sido simulados sin recalentamiento, TIT $=400^{\circ} \mathrm{C}$.

Tabla 8.10. Eficiencia neta de plantas termosolares con colectores PTC. Comparación resultados entre la configuración 1 y la de referencia.

\begin{tabular}{llllllllll}
\hline Ciclo de potencia & Rankine & SB & SB & RC & RC & PCRC & PCRC & RCMCl & RCMCl \\
\hline HTF & DSG & DSG & MS & DSG & MS & DSG & MS & DSG & MS \\
Eficiencia (\%) & 35.18 & 32.24 & 32.26 & 35.53 & 35.3 & 34.2 & 34.27 & 36.65 & 36.65 \\
\hline
\end{tabular}

Nota: Todos los ciclos de potencia han sido simulados sin recalentamiento, TIT $=400^{\circ} \mathrm{C}$.

Cabe destacar que para la configuración 1, al tener consumos eléctricos similares en los equipos que integran el campo solar tanto con colectores con generación de vapor como con colectores con sales fundidas, los rendimientos energéticos de ambas configuraciones (MS+s$\mathrm{CO}_{2}$ y DSG+s- $-\mathrm{CO}_{2}$ ) es muy aproximado, tablas 8.9 y 8.10 .

Si se incrementa la temperatura de entrada en turbina de la configuración de referencia (DSG+Rankine sin recalentamiento) hasta $550^{\circ} \mathrm{C}$, obtenemos un incremento de la eficiencia neta hasta el $38.4 \%$, tablas 8.11 y 8.12 . La configuración 2 integrando un ciclo s- $\mathrm{CO}_{2}$ Brayton tipo RC, como el representado en la figura 8.2, proporciona una eficiencia de $43.6 \%$, tabla 8.9 y 8.10. La misma configuración 2 acoplada a un campo solar con sales fundidas incrementa su eficiencia neta en un $1.5 \%$ hasta $45.16 \%$, tablas 8.9 y 8.10 , debido al consumo eléctrico de los compresores de vapor en la solución con generación directa de vapor en los colectores lineales.

Tabla 8.11. Eficiencia neta de plantas termosolares con colectores LF. Comparación de resultados entre la configuración 2 y la de referencia.

\begin{tabular}{llllllllll}
\hline Ciclo de & Rankine & SB & SB & RC & RC & PCRC & PCRC & RCMCl & RCMCl \\
\hline HTF & DSG & DSG & MS & DSG & MS & DSG & MS & DSG & MS \\
Eficiencia (\%) & 38.39 & 37.9 & 39.28 & 43.68 & 45.16 & 40.23 & 41.71 & 43.45 & 44.94 \\
\hline
\end{tabular}

Nota: Todos los ciclos de potencia han sido simulados sin recalentamiento, TIT $=550^{\circ} \mathrm{C}$.

Tabla 8.12. Eficiencia neta de plantas termosolares con colectores PTC. Comparación de resultados entre la configuración 2 y la de referencia.

\begin{tabular}{llllllllll}
\hline Ciclo de potencia & Rankine & SB & SB & RC & RC & PCRC & PCRC & RCMCl & RCMCl \\
\hline HTF & DSG & DSG & MS & DSG & MS & DSG & MS & DSG & MS \\
Eficiencia (\%) & 38.36 & 37.85 & 39.24 & 43.66 & 45.12 & 40.19 & 41.66 & 43.43 & 44.9 \\
\hline
\end{tabular}

Nota: Todos los ciclos de potencia han sido simulados sin recalentamiento, TIT $=550^{\circ} \mathrm{C}$. 
Es muy importante hacer hincapié en que las configuraciones 1 y 2 no incluyen ninguna etapa de recalentamiento en los ciclos de potencia, tal y como se ha remarcado en los notas al pie de las tablas 8.9 a 8.12. El objetivo de la configuración 3 fue incrementar la temperatura de entrada en turbina de la configuración 1 desde $400^{\circ} \mathrm{C}$ hasta $500^{\circ} \mathrm{C}$, y mantener el mismo campo solar con generación de vapor en modo de recirculación. Para ellos, se han acoplado dos ciclos $\mathrm{s}-\mathrm{CO}_{2}$ Brayton en cascada, figura 8.11. Como resultado se ha incrementado la eficiencia neta hasta un $39.7 \%$, con la configuración $\mathrm{RCMCl}$ de ambos ciclos s- $\mathrm{CO}_{2}$ Brayton en cascada. El detalle de la simulación de la configuración 3 y de la configuración de referencia, DSG+Rankine con una etapa de recalentamiento, se encuentran resumidos en la tabla 8.13.

Tabla 8.13. Eficiencia neta de plantas termosolares con colectores LF. Comparación de resultados entre la configuración 3 y la de referencia.

\begin{tabular}{llll}
\hline Ciclo de potencia & Rankine One DRH & Rankine Two DRH & RCMCI \\
\hline HTF & DSG & DSG & DSG \\
Eficiencia (\%) & 40.23 & 40.79 & 39.7 \\
\hline
\end{tabular}

Nota: En la comparativa se incluye una etapa de recalentamiento en los ciclos, $\mathrm{TIT}=550^{\circ} \mathrm{C}$.

Como ya se ha explicado en otras secciones anteriores, la configuración 4 tiene por objetivo la integración de una etapa de recalentamiento en turbina en la configuración 2. Con esta nueva solución de diseño representada en la figura 8.12, obtenemos una eficiencia neta de $45.76 \%$, ver el resto de resultados de simulaciones de la configuración 4 en la tabla 8.14.

Tabla 8.14. Eficiencia neta de plantas termosolares con colectores lineales. Comparación de resultados entre la configuración 4 y diferentes tipologías de ciclos s- $\mathrm{CO}_{2}$ Brayton.

\begin{tabular}{lllll}
\hline Tipo colector & SB & RC & PCRC & RCMCl \\
\hline LF & 39.39 & 45.76 & 42.01 & 45.40 \\
PTC & 39.38 & 45.77 & 41.97 & 45.37 \\
\hline
\end{tabular}

Nota: En la comparativa se incluye una etapa de recalentamiento en los ciclos, TIT $=550^{\circ} \mathrm{C}$.

\subsection{2. Área de apertura efectiva en el campo solar.}

La eficiencia neta de la planta termosolar es traducida en el presente capítulo en términos de ahorro del área de apertura efectiva de los colectores solares. Para la comparación de las diferentes tecnologías, se ha definido un parámetro llamado potencia unitaria, que se define como la potencia eléctrica producida por cada metro cuadrado de área de apertura efectiva de los colectores solares. Su expresión matemática se define en la ecuación (8-1):

$P_{\text {unitaria }}\left(W / m^{2}\right)=$ Potencia $_{e, \text { neta }}(W) / A_{\text {apertura }}\left(m^{2}\right)$

Para la configuración 1 , como se ha mencionado en el capítulo anterior, la configuración óptima es la de ciclo de potencia s- $\mathrm{CO}_{2}$ Brayton con tipología $\mathrm{RCMCl}$. La diferencia promedio de potencia unitaria entre las configuraciones DSG+Rankine y $\mathrm{DSG}+\mathrm{SCO}_{2}$ es alrededor del $4.5 \%$. Similar a los valores obtenidos comparando las eficiencias netas de la planta termosolar. Los resultados de potencia unitaria están resumidos en las tablas 8.15 y 8.16. Para la configuración 1 y 2, la tipología de ciclo s- $\mathrm{CO}_{2}$ Brayton óptima es la $\mathrm{RCMCl}$. En la configuración 1 la potencia unitaria es similar con las tecnologías de DSG y MS para los ciclos s- $\mathrm{CO}_{2}$ Brayton. En el caso de la configuración 2, el consumo del compresor de vapor en el campo solar hace que la potencia 
unitaria con la tecnología $\mathrm{DSG}+\mathrm{sCO}_{2}$ sea algo menor que con la tecnología $\mathrm{MS}+\mathrm{sCO}_{2}$. Sin embargo, no se ha tenido en cuenta los consumos eléctricos de las resistencias para calefacción de las sales fundidas y evitar su solidificación.

Otro asunto importante a considerar es la similitud entre la eficiencia neta con colectores PTC o LF, como se detalla en las tablas 8.9 a 8.12. La principal diferencia se debe a la eficiencia óptica nominal de ambas tipologías de colectores solares: $75 \%$ con colectores parabólicos PTC, y entre $64 \%$ y $67 \%$ para colectores LF. Existe una correlación entre la diferencia de potencia unitaria y las diferencias de eficiencia óptica para las dos tipologías de campos solares PTC y LF. En la configuración 1, si se considera una diferencia de eficiencia óptica de entorno al 14\%, similar diferencia es obtenida al comparar los valores de potencia unitaria de la tabla 8.15. En el caso de la configuración 2, la diferencia de eficiencia óptica es del $12 \%$, y también se obtienen similares valores comparando los resultados de potencia unitaria de la tabla 8.15.

Para la configuración 3, con dos ciclos $\mathrm{s}-\mathrm{CO}_{2}$ Brayton con tipología $\mathrm{RCMCl}$ conectados en cascada, el área de apertura efectiva es de $247764 \mathrm{~m}^{2}$, y la correspondiente potencia unitaria de $221.98 \mathrm{~W} / \mathrm{m}^{2}$, con colectores solares LF, y con colectores PTC el área de apertura efectiva es de $212845 \mathrm{~m}^{2}$ y una potencia unitaria de $258.5 \mathrm{~W} / \mathrm{m}^{2}$.

En relación a la configuración 4, la configuración RC es la óptima para minimizar el área de apertura efectiva en el campo solar. Para esta configuración 4, las diferencias relativas en el área de apertura son del 3.5\% para LF y de 4\% para colectores PTC. El resto de los resultados detallados de potencia unitaria para la configuración 4 están resumidos en la tabla 8.16.

Tabla 8.15. Potencia Unitaria de plantas termosolares con colectores LF. Comparación de resultados entre la configuración 1 y la de referencia, $\mathrm{TIT}=400^{\circ} \mathrm{C}$.

\begin{tabular}{|c|c|c|c|c|c|c|c|c|c|c|c|}
\hline & TIT (으) & Colector & Rankine & SB & SB & RC & RC & PCRC & PCRC & $\mathrm{RCMCl}$ & $\mathrm{RCMCl}$ \\
\hline HTF & 400 & LF & DSG & DSG & MS & DSG & MS & DSG & MS & DSG & MS \\
\hline $\begin{array}{l}\text { Potencia } \\
\left(\mathrm{W} / \mathrm{m}^{2}\right)\end{array}$ & & & 196.5 & 182 & 185 & 198.8 & 201.7 & 193.4 & 196.2 & 206.4 & 209.6 \\
\hline HTF & 400 & PTC & DSG & DSG & MS & DSG & MS & DSG & MS & DSG & MS \\
\hline $\begin{array}{l}\text { Potencia } \\
\left(\mathrm{W} / \mathrm{m}^{2}\right)\end{array}$ & & & 236.4 & 212 & 215 & 231.4 & 234.6 & 225.9 & 228 & 240.8 & 243.8 \\
\hline HTF & 550 & LF & DSG & DSG & MS & DSG & MS & DSG & MS & DSG & MS \\
\hline $\begin{array}{l}\text { Potencia } \\
\left(\mathrm{W} / \mathrm{m}^{2}\right)\end{array}$ & & & 214.2 & 206 & 215 & 235.7 & 245.4 & 217.5 & 227.4 & 234.2 & 244.4 \\
\hline & 550 & PTC & DSG & DSG & MS & DSG & MS & DSG & MS & DSG & MS \\
\hline $\begin{array}{l}\text { Potencia } \\
\left(\mathrm{W} / \mathrm{m}^{2}\right)\end{array}$ & & & 254.8 & 234 & 244 & 267.7 & 278.2 & 247.2 & 257.9 & 266.1 & 276.7 \\
\hline
\end{tabular}

Tabla 8.16. Potencia Unitaria de plantas termosolares con colectores LF. Comparación de resultados entre la configuración 4 y la de referencia, $\mathrm{T}=550^{\circ} \mathrm{C}$.

\begin{tabular}{rrrrrrr}
\hline & TIT $($ oC $)$ & Colector & SB & RC & PCRC & RCMCI \\
\hline Potencia $\left(\mathrm{W} / \mathrm{m}^{2}\right)$ & 550 & LF & 212 & 245 & 209 & 242.2 \\
Potencia $\left(\mathrm{W} / \mathrm{m}^{2}\right)$ & 550 & PTC & 241 & 278 & 256 & 274.2 \\
\hline
\end{tabular}


Las principales variables que tienen un impacto directo en el coste de los campos solares, es el tipo de colector (PTC o LF) y el material de los tubos absorbedores. Con la tecnología DSG se puede utilizar un acero inoxidable de baja aleación o incluso un acero al carbono teniendo en cuenta el espesor por corrosión en el diseño de los tubos. Los tubos absorbedores con generación directa de vapor no requieren un alto grado de materiales de aleación porque el agua no tiene un alto grado de corrosión, y es incluso menor cuando se trata previamente el agua químicamente.

En las tablas 8.17 a 8.22, están resumidas las diferencias entre el coste de inversión de capital para campos solares con DSG y con sales fundidas MS como fluidos caloportadores HTF. La diferencia de costes de inversión entre ambas tipologías de campos solares se debe principalmente a los materiales utilizados para evitar el alto grado de corrosión de las sales fundidas. La configuración de planta termosolar con menor coste de inversión de capital es la configuración 1 con ciclo Brayton tipo RCMCl y con TIT $400^{\circ} \mathrm{C}$. Para las Configuraciones 2 y 4 los costes de inversión son similares para las tipologías de ciclos $\mathrm{RC}$ y $\mathrm{RCMCl}$.

Tabla 8.17. Área de apertura y coste del campo solar en plantas con LF. Comparación de resultados entre la configuración 1 y la de referencia. Potencia neta $40 \mathrm{MWe}, \mathrm{TIT}=400$ ㅇ․

\begin{tabular}{llllllllll}
\hline Ciclo & Rankine & SB & SB & RC & RC & PCRC & PCRC & RCMCI & RCMCI \\
\hline HTF & DSG & DSG & MS & DSG & MS & DSG & MS & DSG & MS \\
SF Área Apertura $\left(\mathrm{m}^{2}\right) \times 10^{3}$ & 203.5 & 220.2 & 216.5 & 201.2 & 198.2 & 207.3 & 204.1 & 194.2 & 190.9 \\
SF Coste (M\$) & 48.75 & 51.08 & 75.35 & 47.50 & 68.99 & 47.95 & 70.99 & 45.36 & 66.45 \\
\hline
\end{tabular}

Tabla 8.18. Área de apertura y coste del campo solar en plantas con PTC. Comparación de resultados entre la configuración 1 y la de referencia. Potencia neta $40 \mathrm{MWe}, \mathrm{TIT}=400 \circ \mathrm{C}$.

\begin{tabular}{llllllllll}
\hline Ciclo & Rankine & SB & SB & RC & RC & PCRC & PCRC & RCMCI & RCMCI \\
\hline HTF & DSG & DSG & MS & DSG & MS & DSG & MS & DSG & MS \\
SF Área Apertura $\left(\mathrm{m}^{2}\right) \times 10^{3}$ & 169.2 & 188.5 & 186.1 & 172.9 & 170.5 & 177.5 & 175.4 & 166.1 & 164.1 \\
SF Coste (M\$) & 63.59 & 70.83 & 93.21 & 64.97 & 85.42 & 66.69 & 87.91 & 62.42 & 82.21 \\
\hline
\end{tabular}

Tabla 8.19. Área de apertura y coste del campo solar en plantas con LF. Comparación de resultados entre la configuración 2 y la de referencia. Potencia neta $55 \mathrm{MWe}, \mathrm{TIT}=550^{\circ} \mathrm{C}$.

\begin{tabular}{llllllllll}
\hline Ciclo & Rankine & SB & SB & RC & RC & PCRC & PCRC & RCMCI & RCMCI \\
\hline HTF & DSG & DSG & MS & DSG & MS & DSG & MS & DSG & MS \\
SF Área Apertura $\left(\mathrm{m}^{2}\right) \times 10^{3}$ & 256.8 & 266.9 & 256.4 & 233.3 & 224.1 & 252.9 & 241.8 & 234.8 & 225.1 \\
SF Coste (M\$) & 61.52 & 69.66 & 89.21 & 60.9 & 77.99 & 66 & 84.16 & 61.28 & 78.3 \\
\hline
\end{tabular}

Tabla 8.20. Área de apertura y coste del campo solar en plantas con PTC. Comparación de resultados entre la configuración 2 y la de referencia. Potencia neta $55 \mathrm{MWe}, \mathrm{TIT}=550^{\circ} \mathrm{C}$.

\begin{tabular}{llllllllll}
\hline Ciclo & Rankine & SB & SB & RC & RC & PCRC & PCRC & RCMCI & RCMCI \\
\hline HTF & DSG & DSG & MS & DSG & MS & DSG & MS & DSG & MS \\
SF Área Apertura $\left(\mathrm{m}^{2}\right) \times 10^{3}$ & 215.8 & 243.8 & 225.9 & 205.3 & 197.8 & 222.4 & 213.3 & 206.7 & 198.8 \\
SF Coste (M\$) & 81.12 & 91.63 & & 77.15 & 99.14 & 83.57 & 106.92 & 77.7 & 99.62 \\
\hline
\end{tabular}


8. Plantas Termosolares de Colectores Lineales con DSG acopladas a ciclos Brayton con $\mathrm{CO}_{2}$

Tabla 8.21. Área de apertura y coste del campo solar en plantas con LF. Comparación de resultados entre la configuración 4 y la de referencia. Potencia neta $55 \mathrm{MWe}, \mathrm{TIT}=550^{\circ} \mathrm{C}$.

\begin{tabular}{lllll}
\hline Ciclo de potencia & SB & RC & PCRC & RCMCI \\
\hline SF Area Apertura $\left(\mathrm{m}^{2}\right) \times 10^{3}$ & 259.4 & 224.8 & 263.3 & 227.1 \\
SF Coste (M\$) & 72.37 & 63.94 & 73.34 & 63.87 \\
\hline
\end{tabular}

Tabla 8.22. Área de apertura y coste del campo solar en plantas con PTC. Comparación de resultados entre la configuración 4 y la de referencia. Potencia neta $55 \mathrm{MWe}, \mathrm{TIT}=550^{\circ} \mathrm{C}$.

\begin{tabular}{lllll}
\hline Ciclo de potencia & SB & RC & PCRC & RCMCI \\
\hline SF Area Apertura $\left(\mathrm{m}^{2}\right) \times 10^{3}$ & 228.3 & 198.1 & 215.1 & 200.6 \\
SF Coste (M\$) & 91.71 & 81.22 & 86.33 & 81.28 \\
\hline
\end{tabular}

\footnotetext{
Notas: Coste Unitario de acuerdo a Thermoflow [36]:

LF DSG Rankine: $206.55 \$ / \mathrm{m}^{2}$

LF DSG Brayton (8.5 mm espesor pared tubo de absorbedor): $225 \$ / \mathrm{m}^{2}$

LF MS Brayton : $300 \$ / \mathrm{m}^{2}$

PTC DSG Rankine: $324 \$ / \mathrm{m}^{2}$

PTC DSG Brayton (8.5 mm espesor pared tubo de absorbedor): $324 \$ / \mathrm{m}^{2}$

PTC MS Brayton: $432 \$ / \mathrm{m}^{2}$

Los precios unitarios indicados anteriormente deben ser multiplicados por un factor de 1.16

para incluir los costes de instalación. Esta metodología ha sido establecida por el programa informático Thermoflow [36].
}

\subsubsection{Tamaño térmico (UA) de los intercambiadores de calor}

En este epígrafe se realiza una comparativa de la tamaño térmico UA, definida como el producto entre el coeficiente general de transferencia de calor $(U)$ y el área de total de transferencia de calor (A), de los intercambiadores de calor que integran los ciclos de potencia en las diferentes configuraciones de plantas termosolares.

En las Configuraciones 1 y 2, el tamaño térmico de los recuperadores de calor de alta y baja presión (LTR y HTR) son similares. Los resultados detallados están resumidos en las tablas 8.23 y 8.26 .

La tipología de ciclo Brayton PCRC requiere unos valores menores de tamaño térmico en los recuperadores para una potencia fija, en relación con el resto de configuraciones $\mathrm{RC}$ y $\mathrm{RCMCl}$. Sin embargo, su eficiencia neta es menor y el área de los colectores solares mayor.

En relación con el tamaño térmico (UA) del intercambiador principal PHX en la configuración 1 ( $\mathrm{TIT}<=400^{\circ} \mathrm{C}$ ), se consigue un ahorro sustancial en la configuración $\mathrm{DSG}+\mathrm{SCO}_{2}$ en relación con la configuraciones $\mathrm{MS}+\mathrm{sCO}_{2}$ gracias a los altos valores de coeficiente de transferencia de calor proporcionados en el fenómeno de condensación de vapor de agua. Sirva como ejemplo ilustrativo, que para la tipología de ciclo RC en la configuración 1, la tamaño térmico del PHX es de $2337 \mathrm{~kW} / \mathrm{K}$ para la tecnología $\mathrm{DSG}+\mathrm{sCO}_{2}$, y para la tecnología $\mathrm{MS}+\mathrm{sCO}_{2}$ el valor de tamaño térmico para el PHX es de $6275 \mathrm{~kW} / \mathrm{K}$, proporcionando un ahorro de alrededor del $60 \%$, ver valores detallados en las tablas 8.23 y 8.24 . Otro asunto importante en relación a los PHX es la tipología de intercambiador a utilizar íntimamente relacionado con su coste y sus coeficientes de transferencia de calor HTC. La tipología de cambiador de calor tubos-carcasa puede ser adoptada en ambas tecnologías $\mathrm{DSG}+\mathrm{sCO}_{2}$ y $\mathrm{MS}+\mathrm{sCO}_{2}$, fluyendo el $\mathrm{sCO}_{2}$ por el lado tubos y el HTF por el lado carcasa. Esto permite un ahorro sustancial en relación a la utilización de 
8. Plantas Termosolares de Colectores Lineales con DSG acopladas a ciclos Brayton con $\mathrm{CO}_{2}$

cambiadores compactos PCHE cuyo coste y tecnologías de fabricación es sustancialmente superior.

El sumidero final de calor o Ultimate Heat Sink (UHS) de los ciclos de potencia Brayton en plantas termosolares, en la mayoría de los casos están constituidos por intercambiadores de calor refrigerados por aire ACHE cuyos valores de tamaño térmico, UA, también varían sensiblemente dependiendo de la tipología de ciclo elegida RC, PCRC o RCMCl. En las tablas 8.23 a 8.29 se resumen los valores de tamaño térmico, UA, de las diferentes configuraciones. La tipología de ciclo RC es la que requiere menores valores de UA en los ACHE. Los ahorros respecto al resto de configuraciones son sustanciales entre un $15 \%$ y un $25 \%$.

Tabla 8.23. Tamaño térmico de los intercambiadores en plantas termosolares con LF. Comparación entre la configuración 1 y la referencia. Potencia neta $40 \mathrm{MWe}, \mathrm{TIT}=400^{\circ} \mathrm{C}$.

\begin{tabular}{lllllllll}
\hline Ciclo de potencia & SB & SB & RC & RC & PCRC & PCRC & RCMCl & RCMCl \\
\hline HTF & DSG & MS & DSG & MS & DSG & MS & DSG & MS \\
LTR UA (kW $\left./{ }^{\circ} \mathrm{C}\right)$ & 3253 & 3247 & 7606 & 7692 & 4230 & 4219 & 7228 & 7227 \\
HTR UA (kW $\left./{ }^{\circ} \mathrm{C}\right)$ & - & - & 3322 & 3196 & 2882.6 & 2875.5 & 3419 & 3413 \\
PHX UA (kW $\left./{ }^{\circ} \mathrm{C}\right)$ & 1829.3 & 4057 & 2337 & 6275 & 1706.8 & 3208 & 1948.7 & 3661 \\
ACHE UA $\left(\mathrm{kW} /{ }^{\circ} \mathrm{C}\right)$ & 4615 & 4604 & 3913 & 3965 & 5942.1 & 5927.2 & 5158.5 & 5150 \\
\hline
\end{tabular}

Tabla 8.24. Tamaño térmico de los intercambiadores en plantas termosolares con PTC. Comparación entre la configuración 1 y la referencia. Potencia neta $40 \mathrm{MWe}, \mathrm{TIT}=400^{\circ} \mathrm{C}$.

\begin{tabular}{lllllllll}
\hline Ciclo de potencia & SB & SB & RC & RC & PCRC & PCRC & RCMCl & RCMCl \\
\hline HTF & DSG & MS & DSG & MS & DSG & MS & DSG & MS \\
LTR UA $\left(\mathrm{kW} /{ }^{\circ} \mathrm{C}\right)$ & 3253 & 3251 & 7623 & 7715 & 4239 & 4232 & 7227 & 7228 \\
HTR UA $\left(\mathrm{kW} /{ }^{\circ} \mathrm{C}\right)$ & - & - & 3330 & 3206 & 2891.7 & 2883.2 & 3419 & 3417 \\
PHX UA $\left(\mathrm{kW} /{ }^{\circ} \mathrm{C}\right)$ & 1856.9 & 4069 & 2061.5 & 6326 & 1889.3 & 3213 & 1653.8 & 3662 \\
ACHE UA $\left(\mathrm{kW} /{ }^{\circ} \mathrm{C}\right)$ & 4615 & 4610 & 3923 & 3975 & 5965.5 & 5942.1 & 5158.5 & 5158.5 \\
\hline
\end{tabular}

Tabla 8.25. Tamaño térmico de los intercambiadores en plantas termosolares con LF. Comparación entre la configuración 2 y la de referencia. Potencia neta $55 \mathrm{MWe}, \mathrm{TIT}=550^{\circ} \mathrm{C}$.

\begin{tabular}{lllllllll}
\hline Ciclo de potencia & SB & SB & RC & RC & PCRC & PCRC & RCMCl & RCMCl \\
\hline HTF & DSG & MS & DSG & MS & DSG & MS & DSG & MS \\
LTR UA $\left(\mathrm{kW} /{ }^{\circ} \mathrm{C}\right)$ & 3491 & 3297 & 7797 & 7355 & 5049 & 4757 & 7986 & 7532 \\
HTR UA $\left(\mathrm{kW} /{ }^{\circ} \mathrm{C}\right)$ & - & - & 6123 & 5779 & 3161 & 2976 & 3944 & 3717 \\
PHX UA $\left(\mathrm{kW} /{ }^{\circ} \mathrm{C}\right)$ & 4228 & 3864 & 5130 & 4812 & 3297 & 3253 & 3564 & 3542 \\
ACHE UA $\left(\mathrm{kW} /{ }^{\circ} \mathrm{C}\right)$ & 4769 & 4460 & 3766 & 3524 & 6347.9 & 5919.8 & 5325.7 & 4980.2 \\
\hline
\end{tabular}

Tabla 8.26. Tamaño térmico de los intercambiadores en plantas termosolares con PTC. Comparación entre la configuración 2 y la de referencia. Potencia neta $55 \mathrm{MWe}, \mathrm{TIT}=550^{\circ} \mathrm{C}$.

\begin{tabular}{lllllllll}
\hline Ciclo de potencia & SB & SB & RC & RC & PCRC & PCRC & RCMCl & RCMCl \\
\hline HTF & DSG & MS & DSG & MS & DSG & MS & DSG & MS \\
LTR UA (kW $\left./{ }^{\circ} \mathrm{C}\right)$ & 3634 & 3302 & 7809 & 7367 & 5060 & 4765 & 8000 & 7540 \\
HTR UA (kW $\left./{ }^{\circ} \mathrm{C}\right)$ & - & - & 6131 & 5787 & 3167 & 2980.8 & 3949 & 3724 \\
PHX UA (kW $\left./{ }^{\circ} \mathrm{C}\right)$ & 4411 & 3876 & 5138 & 4828 & 3308 & 3251 & 3572 & 3544 \\
ACHE UA (kW $\left./{ }^{\circ} \mathrm{C}\right)$ & 5003 & 4468 & 3770 & 3529 & 6362.3 & 5930.6 & 5334.1 & 4992 \\
\hline
\end{tabular}




\subsubsection{Estimación de costes de los Intercambiadores de calor del ciclo s- $\mathrm{CO}_{2}$ Brayton}

Para realizar una primera estimación de coste de los recuperadores en las centrales termosolares con colectores lineales y ciclos s- $\mathrm{CO}_{2}$ Brayton, se ha considerado la tipología de intercambiadores de calor compactos de circuito impreso tipología Heatric. Su coste unitario estimado está íntimamente ligado con el coste de los materiales requeridos para las altas temperaturas y presiones de operación, tabla 8.27. Se ha considerado un coste unitario general para los recuperadores de tipología PCHE de $92 \$ / \mathrm{kW}_{\text {th }}$ [37-38]. Como resultado se obtiene el coste total de los recuperadores de calor para las diferentes configuraciones de planta termosolar estudiadas en este capítulo de la tesis, ver los resultados detallados en las tablas 8.28 a 8.37 .

El primer comentario a estos resultados es en relación a la configuración PCRC. A pesar de que el tamaño térmico total, UA, en la tipología PCRC es la menor en comparación con la SB, RC y $\mathrm{RCMCl}$, el coste total de los cambiadores en la configuración $\mathrm{RC}$ es al menor de todos. La razón principal es la diferencia de temperatura en los intercambiadores, ya que el calor transferido es directamente proporcional al producto entre el tamaño térmico y la diferencia de temperaturas.

Tabla 8.27. Caracterización de los materiales del ciclo de potencia s- $\mathrm{CO}_{2}$ Brayton [39].

\begin{tabular}{|c|c|c|c|}
\hline $\begin{array}{l}\text { Temperatura } \\
(\stackrel{\circ}{C})\end{array}$ & $\begin{array}{l}\text { Presión } \\
\text { (bar) }\end{array}$ & $\begin{array}{l}\text { Aleación } \\
\text { recomendada }\end{array}$ & Comentarios \\
\hline$T<200$ & 100 & 304ss, P91, T22 & $\begin{array}{l}\text { Para temperaturas inferiores a } 200 C \text { aleaciones } \\
\text { de acero ferrítico. }\end{array}$ \\
\hline$T<250$ & 240 & 304ss, P91 & $\begin{array}{l}\text { Aleaciones de acero austenítico de bajo coste } \\
\text { o acero ferrítico. }\end{array}$ \\
\hline $\mathrm{T}<400$ & 240 & $\begin{array}{l}347 s s, 310 s s, 304 s s \\
\text { o } 316 s s\end{array}$ & $\begin{array}{l}\text { Aleaciones de acero austenítico son recomendables } \\
\text { o de P91 para bajos períoidos de tiempo. }\end{array}$ \\
\hline$T<550$ & 240 & $347 s s, 310 s s$ & $\begin{array}{l}\text { Aceros austeníticos con altos contenidos de } \mathrm{Ni}, \mathrm{Cr} \text {, } \\
\text { Co } \\
\text { ( } 310 \text { ss, } 347 \text { ss) } 304 \text { ss o } 316 \text { ss es adecuado para } \\
\text { bajos períodos de tiempo. }\end{array}$ \\
\hline$T<650$ & 240 & $\begin{array}{l}\text { Haynes 230, IN617, } \\
800 \mathrm{H}, 347 \text { ss o } 310 \text { ss }\end{array}$ & $\begin{array}{l}\text { Aleaciones con altos contenidos de Ni y Cr. Los } \\
\text { aceros inoxidables } 347 \mathrm{ss} \text { y } 310 \text { ss son suficientes para } \\
\text { bajos períodos de tiempo. }\end{array}$ \\
\hline $\mathrm{T}>650$ & 240 & Haynes 282 o IN740 & $\begin{array}{l}\text { Haynes } 230 \text { y IN617 son suficientes para bajos } \\
\text { períodos de tiempo. }\end{array}$ \\
\hline
\end{tabular}

Otro asunto importante es el coste del material en contacto con el fluido de trabajo $\mathrm{sCO}_{2}$, y las condiciones de presión y temperatura en los diferentes cambiadores del ciclo de potencia. Conforme incrementemos la presión y temperatura del $\mathrm{sCO}_{2}$ mayor degradación produce el fenómeno de corrosión en los cambiadores de calor. En la referencia [39] se realiza un estudio detallado de los materiales necesarios para cada condición termodinámica y resumida en la tabla 8.27.

Los ciclos de potencia Rankine disponen de intercambiadores de calor de acero al carbono, debido a la baja susceptibilidad del agua a producir fenómenos de corrosión, y aun menor 
cuando se trata el agua del ciclo de potencia para controlar su composición química y sus impurezas reduciendo los fenómenos de corrosión. El ahorro de coste de material comparando los ciclos Rankine y los ciclos s- $\mathrm{CO}_{2}$ Brayton está resumido en las tablas 8.28 a 8.35. Se obtiene un ahorro entorno al 50\%. La tendencia en el futuro será fabricar intercambiadores en los ciclos s- $\mathrm{CO}_{2}$ Brayton con mayor resistencia a la corrosión y similares resistencias mecánicas a alta temperatura, y alto tamaño térmico (UA) ocupando el mínimo volumen posible. Se están estudiando diferentes materiales cerámicos para este propósito [40-41].

El estado del arte de los intercambiadores de calor de los ciclos s- $\mathrm{CO}_{2}$ Brayton tiene como objetivo reducir las pérdidas de carga en los cambiadores compactos. Las pérdidas de carga tienen un impacto directo en la eficiencia neta del ciclo y por tanto en el coste de inversión del campo solar. Los últimos diseños introducen diferentes geometrías en los canales con una sección de perfil de ala de avión air foil [42] o con secciones s-shaped fins [43].

Tabla 8.28. Coste de inversión de los intercambiadores en plantas termosolares con LF. Comparación entre la configuración 1 y la de referencia. Potencia Neta $40 \mathrm{MWe}, \mathrm{TIT}=400^{\circ} \mathrm{C}$.

\begin{tabular}{llllll}
\hline Ciclo de potencia & Rankine & SB & RC & PCRC & RCMCI \\
\hline HTF & & DSG & DSG & DSG & DSG \\
LTR (MWth) & 87.3 & 83.5 & 28.1 & 56.8 \\
HTR (MWth) & - & 26.6 & 86.1 & 68.7 \\
PHX (MWth) & & 124.3 & 112.5 & 117.1 & 109.3 \\
ACHE (MWth) & & 82.8 & 71.2 & 75.5 & 67.9 \\
Coste Total HXs (M\$) & 15.2 & 27.1 & 27.1 & 28.2 & 27.8 \\
\hline
\end{tabular}

Tabla 8.29. Coste de inversión de los intercambiadores del ciclo de potencia Rankine. Planta termosolar con LF. Potencia Neta $40 \mathrm{MWe}, \mathrm{TIT}=400^{\circ} \mathrm{C}$.

\begin{tabular}{ll}
\hline Ciclo de potencia & Rankine (sin Recalentamiento) \\
\hline Calentadores agua alimentación 1 al 5 (M\$) & 1.18 \\
Deaireador (M\$) & 0.38 \\
ACC (M\$) & 13.61 \\
Coste Total HXs (M\$) & 15.18 \\
\hline
\end{tabular}

Tabla 8.30. Coste de inversión de los intercambiadores en plantas termosolares con PTC. Comparación entre la configuración 1 y la de referencia. Potencia Neta $40 \mathrm{MWe}, \mathrm{TIT}=400^{\circ} \mathrm{C}$.

\begin{tabular}{llllll}
\hline Ciclo de potencia & Rankine & SB & RC & PCRC & RCMCI \\
\hline HTF & & DSG & DSG & DSG & DSG \\
LTR (MWth) & & 87.3 & 83.7 & 28.1 & 56.8 \\
HTR (MWth) & - & 26.6 & 86.3 & 68.7 \\
PHX (MWth) & & 124.3 & 112.8 & 117.4 & 109.3 \\
ACHE (MWth) & & 82.8 & 71.3 & 75.8 & 67.9 \\
Coste Total HXs (M\$) & 15.2 & 27.1 & 27.1 & 28.3 & 27.8 \\
\hline
\end{tabular}


8. Plantas Termosolares de Colectores Lineales con DSG acopladas a ciclos Brayton con $\mathrm{CO}_{2}$

Tabla 8.31. Coste de inversión de los intercambiadores del ciclo de potencia Rankine. Planta termosolar con PTC. Potencia Neta $40 \mathrm{MWe}, \mathrm{TITT}=400^{\circ} \mathrm{C}$.

\begin{tabular}{ll}
\hline Ciclo de potencia & Rankine (sin Recalentamiento) \\
\hline Calentadores agua alimentación 1 al 5 (M\$) & 1.19 \\
Deaireador (M\$) & 0.39 \\
ACC (M\$) & 13.63 \\
Coste Total HXs (M\$) & 15.21 \\
\hline
\end{tabular}

Tabla 8.32. Coste de inversión de los intercambiadores en plantas termosolares con LF. Comparación entre la configuración 2 y la de referencia. Potencia Neta $55 \mathrm{MWe}, \mathrm{TIT}=550^{\circ} \mathrm{C}$.

\begin{tabular}{llllll}
\hline Ciclo de potencia & Rankine & SB & RC & PCRC & RCMCI \\
\hline HTF & & DSG & DSG & DSG & DSG \\
LTR (MWth) & 163.1 & 90.4 & 37.8 & 68.5 \\
HTR (MWth) & - & 104.1 & 154.7 & 137.6 \\
PHX (MWth) & & 148.4 & 129.3 & 140.3 & 130.1 \\
ACHE (MWth) & & 88.3 & 69.1 & 80.1 & 69.8 \\
Coste Total HXs (M\$) & 17.4 & 36.8 & 36.1 & 37.9 & 37.3 \\
\hline
\end{tabular}

Tabla 8.33. Coste de inversión de los intercambiadores del ciclo de potencia Rankine. Planta termosolar con LF. Potencia Neta $55 \mathrm{MWe}, \mathrm{TIT}=550^{\circ} \mathrm{C}$.

\begin{tabular}{ll}
\hline Ciclo de potencia & Rankine \\
\hline Calentadores agua alimentación 1 al 5 (M\$) & 1.18 \\
Deaireador (M\$) & 0.39 \\
ACC (M\$) & 15.87 \\
Coste Total HXs (M\$) & 17.46 \\
\hline
\end{tabular}

Tabla 8.34. Coste de inversión de los intercambiadores en plantas termosolares con PTC. Comparación entre la configuración 2 y la de referencia. Potencia Neta $55 \mathrm{MWe}, \mathrm{TIT}=550^{\circ} \mathrm{C}$.

\begin{tabular}{llllll}
\hline Ciclo de potencia & Rankine & SB & RC & PCRC & RCMCI \\
\hline HTF & & DSG & DSG & DSG & DSG \\
LTR (MWth) & 169.8 & 90.5 & 37.8 & 68.6 \\
HTR (MWth) & - & 104.2 & 154.8 & 137.8 \\
PHX (MWth) & & 154.5 & 129.4 & 140.4 & 130.2 \\
ACHE (MWth) & & 92.1 & 69.2 & 80.1 & 69.9 \\
Coste Total(M\$) & 17.47 & 38.3 & 36.2 & 38.1 & 37.4 \\
\hline
\end{tabular}

Tabla 8.35. Coste de inversión de los intercambiadores del ciclo de potencia Rankine. Planta termosolar con PTC. Potencia Neta $55 \mathrm{MWe}, \mathrm{TIT}=550^{\circ} \mathrm{C}$.

\begin{tabular}{ll}
\hline Ciclo de potencia & Rankine \\
\hline Calentadores agua alimentación 1 al 5 (M\$) & 1.19 \\
Deaireador (M\$) & 0.39 \\
ACC (M\$) & 15.88 \\
Coste Total HXs (M\$) & 17.47 \\
\hline
\end{tabular}


8. Plantas Termosolares de Colectores Lineales con DSG acopladas a ciclos Brayton con $\mathrm{CO}_{2}$

Tabla 8.36. Coste de inversión de los intercambiadores en plantas termosolares con LF. Comparación entre la configuración 4 y la de referencia. Potencia Neta $55 \mathrm{MWe}, \mathrm{TIT}=550^{\circ} \mathrm{C}$

\begin{tabular}{lllll}
\hline Ciclo de potencia & SB & RC & PCRC & RCMCI \\
\hline LTR (MWth) & 173.5 & 87.4 & 40.5 & 66.5 \\
HTR (MWth) & - & 117.2 & 175.8 & 147.4 \\
PHX (MWth) & 114.5 & 90.9 & 116.5 & 96.5 \\
RHX (MWth) & 27.6 & 31.7 & 27.5 & 27.3 \\
ACE (MWth) & 82.9 & 63.5 & 80.1 & 64.5 \\
Total Coste (M\$) & 36.6 & 35.9 & 40.5 & 37.0 \\
\hline
\end{tabular}

Tabla 8.37. Coste de inversión de los intercambiadores en plantas termosolares con PTC. Comparación entre la configuración 4 y la de referencia. Potencia Neta $55 \mathrm{MWe}, \mathrm{TIT}=550^{\circ} \mathrm{C}$.

\begin{tabular}{lllll}
\hline Ciclo de potencia & SB & RC & PCRC & RCMCI \\
\hline LTR (MWth) & 173.7 & 87.3 & 37.6 & 66.5 \\
HTR (MWth) & - & 117.2 & 163.4 & 147.6 \\
PHX (MWth) & 114.7 & 90.9 & 108.3 & 96.6 \\
RHX (MWth) & 27.7 & 31.7 & 25.6 & 27.3 \\
ACE (MWth) & 83.1 & 63.5 & 74.4 & 64.6 \\
Total Coste (M\$) & 36.74 & 35.9 & 37.6 & 61.5 \\
\hline
\end{tabular}

En las tablas 8.38 a 8.41 se ha resumido el coste de los colectores solares y el coste de los intercambiadores del ciclo de potencia. El objetivo es realizar una comparativa entre las diferentes soluciones de diseño propuestas. Se concluye que los ciclos Rankine continúan siendo una solución muy atractiva a corte y medio plazo, desde el punto de vista del coste de los materiales que integran sus equipos. El agua es un fluido caloportador que no provoca una alta corrosión, y permite reducir el coste de los materiales. Así mismo, la disponibilidad comercial de los equipos del ciclo Rankine reduce también el precio de los equipos y componentes.

Si bien existe una amplia experiencia operativa y de validación de todos los equipos del ciclo Rankine la solución de ciclo de potencia $\mathrm{s}-\mathrm{CO}_{2}$ Brayton está en pleno desarrollo industrial sustentada por las subvenciones de los principales gobiernos mundiales, ya que proporciona mayores niveles de eficiencia energética, y requiere menores costes de la obra civil para albergar el ciclo de potencia. En un futuro proporcionará menores costes unitarios de la electricidad producida en plantas termosolares con colectores lineales.

Tabla 8.38. Coste de inversión total. Planta termosolar con LF. Suma del coste de los colectores solares y de los intercambiadores de calor del ciclo de potencia s- $\mathrm{CO}_{2}$ Brayton. Comparación entre la configuración 1 y la de referencia. Potencia Neta $40 \mathrm{MWe}, \mathrm{TIT}=400^{\circ} \mathrm{C}$.

\begin{tabular}{llllll}
\hline Ciclo de potencia & Rankine & SB & RC & PCRC & RCMCI \\
\hline HTF & DSG & DSG & DSG & DSG & DSG \\
Coste SF (Millo. \$) & 48.75 & 51.08 & 47.51 & 47.95 & 45.36 \\
Coste HXs (Millo. \$) & 15.19 & 27.09 & 27.04 & 28.22 & 27.85 \\
Coste Total (Millo. \$) & 63.94 & 78.18 & 74.54 & 76.17 & 73.22 \\
\hline
\end{tabular}


8. Plantas Termosolares de Colectores Lineales con DSG acopladas a ciclos Brayton con $\mathrm{CO}_{2}$

Tabla 8.39. Coste de inversión total. Planta termosolar con PTC. Suma del coste de los colectores solares y de los intercambiadores de calor del ciclo de potencia s- $\mathrm{CO}_{2}$ Brayton. Comparación entre la configuración 1 y la de referencia. Potencia Neta $40 \mathrm{MWe}, \mathrm{TIT}=400^{\circ} \mathrm{C}$.

\begin{tabular}{llllll}
\hline Ciclo de potencia & Rankine & SB & RC & PCRC & RCMCI \\
\hline HTF & DSG & DSG & DSG & DSG & DSG \\
Coste SF (Millo. \$) & 63.59 & 70.83 & 64.97 & 66.69 & 62.42 \\
Coste HXs (Millo. \$) & 15.21 & 27.09 & 27.1 & 28.31 & 27.85 \\
Coste Total (Millo. \$) & 78.81 & 97.93 & 92.07 & 95.01 & 90.27 \\
\hline
\end{tabular}

Tabla 8.40. Coste de inversión total. Planta termosolar con LF. Suma del coste de los colectores solares y de los intercambiadores de calor del ciclo de potencia s- $\mathrm{CO}_{2}$ Brayton. Comparación entre la configuración 2 y la de referencia. Potencia Neta $55 \mathrm{MWe}, \mathrm{TIT}=550^{\circ} \mathrm{C}$.

\begin{tabular}{llllll}
\hline Ciclo de potencia & Rankine & SB & RC & PCRC & RCMCI \\
\hline HTF & DSG & DSG & DSG & DSG & DSG \\
Coste SF (M Millo. \$) & 61.52 & 69.66 & 60.9 & 66 & 61.28 \\
Coste HXs (Millo. \$) & 17.46 & 36.8 & 36.16 & 37.98 & 37.35 \\
Coste Total (Millo. \$) & 78.98 & 106.46 & 97.06 & 103.98 & 98.63 \\
\hline
\end{tabular}

Tabla 8.41. Coste de inversión total. Planta termosolar con PTC. Suma del coste de los colectores solares y de los intercambiadores de calor del ciclo de potencia s- $\mathrm{CO}_{2}$ Brayton. Comparación entre la configuración 2 y la de referencia. Potencia Neta $55 \mathrm{MWe}, \mathrm{TIT}=550^{\circ} \mathrm{C}$.

\begin{tabular}{llllll}
\hline Ciclo de potencia & Rankine & SB & RC & PCRC & RCMCI \\
\hline HTF & DSG & DSG & DSG & DSG & DSG \\
Coste SF (Millo. \$) & 81.12 & 91.63 & 77.15 & 83.57 & 77.7 \\
Coste HXs (Millo. \$) & 17.47 & 38.31 & 36.2 & 38.03 & 37.4 \\
Coste Total (Millo. \$) & 98.56 & 129.94 & 113.35 & 121.61 & 115.1 \\
\hline
\end{tabular}

8.6. Optimización de los parámetros de operación de plantas termosolares con colectores lineales con DSG, acopladas a ciclos de potencia s- $\mathrm{CO}_{2}$ Brayton

\subsubsection{Caracterización de la eficiencia energética del ciclo de potencia s- $\mathrm{CO}_{2}$ Brayton}

Como se ha explicado previamente, la elección óptima de los parámetros de operación de los ciclos supercríticos Brayton (fracción flujo de recompresión, presión de entrada a turbina de recalentamiento, presión a la entrada de los compresores, etc.) permite incrementar la potencia generada y la eficiencia del ciclo. Para este fin se han integrado en los programas de simulación energética los algoritmos matemáticos de optimización multivariable SUBPLEX [44], UOBYQA [45], NEWUOA [46]. Como resultado directo de su aplicación se han obtenido los valores de eficiencia energética resumidos en las tablas 8.42 a 8.44 . Las configuraciones de campo solar estudiadas son la configuración 1 y 2, ilustradas en las figuras 8.7 a 8.9. Se confirma que el tamaño térmico UA de los recuperadores tiene un impacto directo en el incremento de la eficiencia neta del ciclo de potencia. Su incremento está limitado por la reducción de la diferencia mínima de temperatura entre corrientes en los recuperadores, es la llamada temperatura pinch-point. 
8. Plantas Termosolares de Colectores Lineales con DSG acopladas a ciclos Brayton con $\mathrm{CO}_{2}$

Tabla 8.42. Eficiencia neta del ciclo de potencia s- $\mathrm{CO}_{2}$ Brayton $\mathrm{RC}$, y diferencia mínimas de temperaturas en los recuperadores pinch-point.

\begin{tabular}{llllllll}
\hline $\begin{array}{l}\text { TIT } \\
\left({ }^{\circ} \mathrm{C}\right)\end{array}$ & $\begin{array}{l}\text { UA } \\
(\mathrm{kW} / \mathrm{K})\end{array}$ & $\begin{array}{l}\text { SF } \\
\text { Config. }\end{array}$ & $\begin{array}{l}\text { Eficiencia } \\
\text { Bruta } \\
(\%)\end{array}$ & $\begin{array}{l}\text { Eficiencia } \\
\text { Neta } \\
(\%)\end{array}$ & $\begin{array}{l}\text { Potencia } \\
\text { Neta } \\
(\mathrm{MWe})\end{array}$ & $\begin{array}{l}\text { LTR } \\
\text { Pinch } \\
\left({ }^{\circ} \mathrm{C}\right)\end{array}$ & $\begin{array}{l}\text { HTR } \\
\text { Pinch } \\
\left({ }^{\circ} \mathrm{C}\right)\end{array}$ \\
\hline 400 & 3000 & Configuración 1 & 33.39 & 32.34 & 48434 & 12.2 & 17.9 \\
550 & 3000 & Configuración 2 & 42.94 & 38.91 & 45306 & 24.1 & 58.7 \\
400 & 5000 & Configuración 1 & 36.32 & 35.19 & 48446 & 14.8 & 33.6 \\
550 & 5000 & Configuración 2 & 46.26 & 42.16 & 45572 & 15.8 & 30.9 \\
400 & 10000 & Configuración 1 & 38.84 & 37.63 & 48451 & 8.7 & 14.2 \\
550 & 10000 & Configuración 2 & 49.45 & 45.25 & 45750 & 9.1 & 10.2 \\
400 & 15000 & Configuración 1 & 39.84 & 38.61 & 48457 & 6.1 & 7.8 \\
550 & 15000 & Configuración 2 & 50.54 & 46.28 & 45795 & 4.5 & 4.5 \\
400 & 20000 & Configuración 1 & 40.36 & 39.11 & 48458 & 4.6 & 4.9 \\
550 & 20000 & Configuración 2 & 51.01 & 46.75 & 45830 & 2.4 & 2.4 \\
\hline
\end{tabular}

Tabla 8.43. Eficiencia neta del ciclo de potencia s- $\mathrm{CO}_{2}$ Brayton PCRC, y diferencia mínimas de temperaturas en los recuperadores pinch-point.

\begin{tabular}{llllllll}
\hline $\begin{array}{l}\text { TIT } \\
\left({ }^{\circ} \mathrm{C}\right)\end{array}$ & $\begin{array}{l}\text { UA } \\
(\mathrm{kW} / \mathrm{K})\end{array}$ & $\begin{array}{l}\text { SF } \\
\text { Config. }\end{array}$ & $\begin{array}{l}\text { Eficiencia } \\
\text { Bruta } \\
(\%)\end{array}$ & $\begin{array}{l}\text { Eficiencia } \\
\text { Neta } \\
(\%)\end{array}$ & $\begin{array}{l}\text { Potencia } \\
\text { Neta } \\
(\mathrm{MWe})\end{array}$ & $\begin{array}{l}\text { LTR } \\
\text { Pinch } \\
\left({ }^{\circ} \mathrm{C}\right)\end{array}$ & $\begin{array}{l}\text { HTR } \\
\text { Pinch } \\
\left({ }^{\circ} \mathrm{C}\right)\end{array}$ \\
\hline 400 & 3000 & Configuración 1 & 33.94 & 32.87 & 48430 & 20.8 & 28.4 \\
550 & 3000 & Configuración 2 & 42.74 & 38.87 & 45490 & 22.3 & 23.1 \\
400 & 5000 & Configuración 1 & 35.86 & 34.73 & 48438 & 12.8 & 13.6 \\
550 & 5000 & Configuración 2 & 45.15 & 41.19 & 45614 & 9.4 & 9.5 \\
400 & 10000 & Configuración 1 & 37.38 & 36.21 & 48444 & 5.1 & 5.1 \\
550 & 10000 & Configuración 2 & 46.57 & 42.57 & 45727 & 2.8 & 2.9 \\
400 & 15000 & Configuración 1 & 37.83 & 36.65 & 48451 & 2.9 & 3.1 \\
550 & 15000 & Configuración 2 & 46.94 & 42.92 & 45744 & 1.6 & 1.7 \\
400 & 20000 & Configuración 1 & 38.04 & 36.85 & 48450 & 1.9 & 2.1 \\
550 & 20000 & Configuración 2 & 47.085 & 43.09 & 45778 & 1.1 & 1.2 \\
\hline
\end{tabular}

Tabla 8.44. Eficiencia neta del ciclo de potencia s- $\mathrm{CO}_{2}$ Brayton $\mathrm{RCMCl}$, y diferencia mínimas de temperaturas en los recuperadores pinch-point.

\begin{tabular}{llllllll}
\hline $\begin{array}{l}\text { TIT } \\
\left({ }^{\circ} \mathrm{C}\right)\end{array}$ & $\begin{array}{l}\text { UA } \\
(\mathrm{kW} / \mathrm{K})\end{array}$ & SF Config. & $\begin{array}{l}\text { Eficiencia } \\
\text { Bruta }(\%)\end{array}$ & $\begin{array}{l}\text { Eficiencia } \\
\text { Neta }(\%)\end{array}$ & $\begin{array}{l}\text { Potencia Neta } \\
(\mathrm{MWe})\end{array}$ & $\begin{array}{l}\text { LTR } \\
\text { Pinch }\left({ }^{\circ} \mathrm{C}\right)\end{array}$ & $\begin{array}{l}\text { HTR Pinch } \\
\left({ }^{\circ} \mathrm{C}\right)\end{array}$ \\
\hline 400 & 3000 & Configuración 1 & 34.20 & 33.13 & 48443 & 22.6 & 0 \\
550 & 3000 & Configuración 2 & 43.38 & 39.28 & 45280 & 24.3 & 56.7 \\
400 & 5000 & Configuración 1 & 36.63 & 35.49 & 48457 & 15.1 & 32.3 \\
550 & 5000 & Configuración 2 & 46.68 & 42.51 & 45548 & 16.2 & 29.5 \\
400 & 10000 & Configuración 1 & 39.16 & 37.95 & 48460 & 10.1 & 13.4 \\
550 & 10000 & Configuración 2 & 49.70 & 45.46 & 45737 & 9.3 & 9.3 \\
400 & 15000 & Configuración 1 & 40.17 & 38.93 & 48460 & 6.8 & 7.3 \\
550 & 15000 & Configuración 2 & 50.75 & 46.47 & 45782 & 4.1 & 4.1 \\
400 & 20000 & Configuración 1 & 40.69 & 39.43 & 48460 & 4.5 & 4.5 \\
550 & 20000 & Configuración 2 & 51.21 & 46.92 & 45814 & 2.1 & 2.1 \\
\hline
\end{tabular}

\subsubsection{Caracterización del campo solar}

El área de apertura efectiva de los campos solares con colectores lineales y DSG, está íntimamente relacionada con el tamaño térmico de los recuperadores del ciclo s- $\mathrm{CO}_{2}$ Brayton. Conforme se aumenta de tamaño térmico UA (producto del área de intercambio, A, por el 
8. Plantas Termosolares de Colectores Lineales con DSG acopladas a ciclos Brayton con $\mathrm{CO}_{2}$

coeficiente global de transmisión de calor, $U$ ) de los recuperadores se reduce el tamaño del campo solar para una potencia fija debido al aumento del rendimiento del ciclo de potencia.

En las figuras 8.13 a 8.18 se ha realizado un estudio de sensibilidad cuantificando el área de apertura efectiva de los campos solares para diferentes tamaños térmicos de los recuperadores del ciclo s- $\mathrm{CO}_{2}$ Brayton. El análisis se ha realizado para las Configuraciones 1 y 2 representadas gráficamente en las figuras 8.7 a 8.9. Se confirma que el tamaño térmico $U A=20000 \mathrm{~kW} / \mathrm{K}$ es un valor umbral, a partir del cual un incremento de UA no proporciona una reducción significativa del área de apertura efectiva del campo solar.

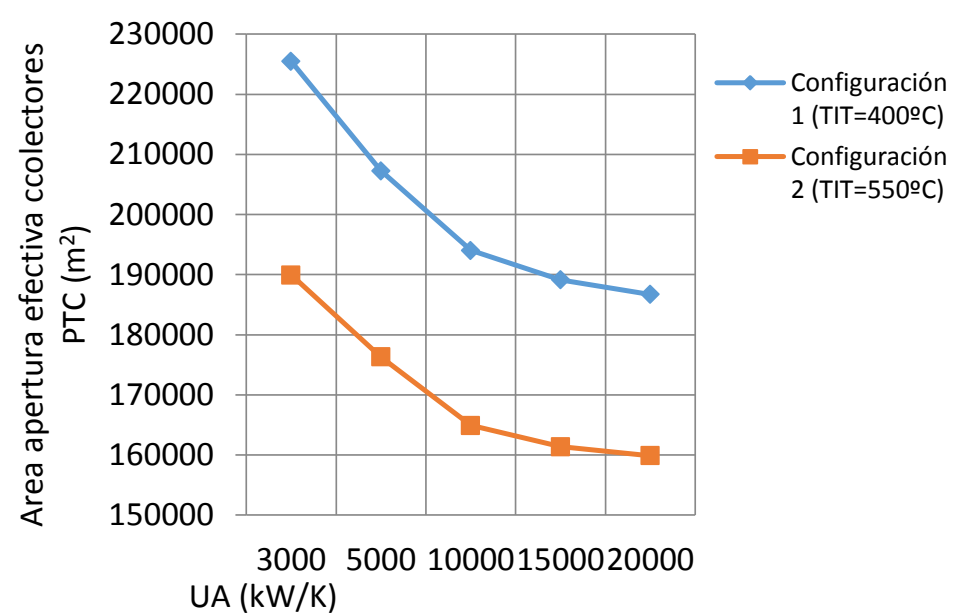

Figura 8.13. Área de apertura efectiva del campo solar con colectores PTC Vs. UA de los recuperadores del ciclo $\mathrm{s}-\mathrm{CO}_{2}$ Brayton $\mathrm{RC}$ sin recalentamiento.

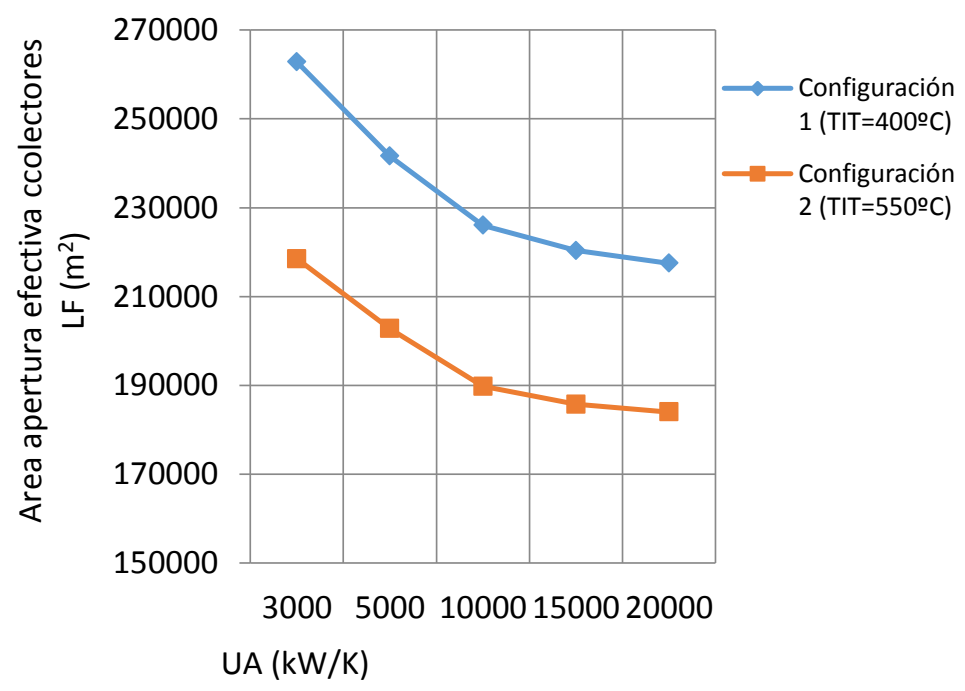

Figura 8.14. Área de apertura efectiva del campo solar con colectores LF Vs. UA de los recuperadores del ciclo $\mathrm{s}-\mathrm{CO}_{2}$ Brayton $\mathrm{RC}$ sin recalentamiento. 
8. Plantas Termosolares de Colectores Lineales con DSG acopladas a ciclos Brayton con $\mathrm{CO}_{2}$

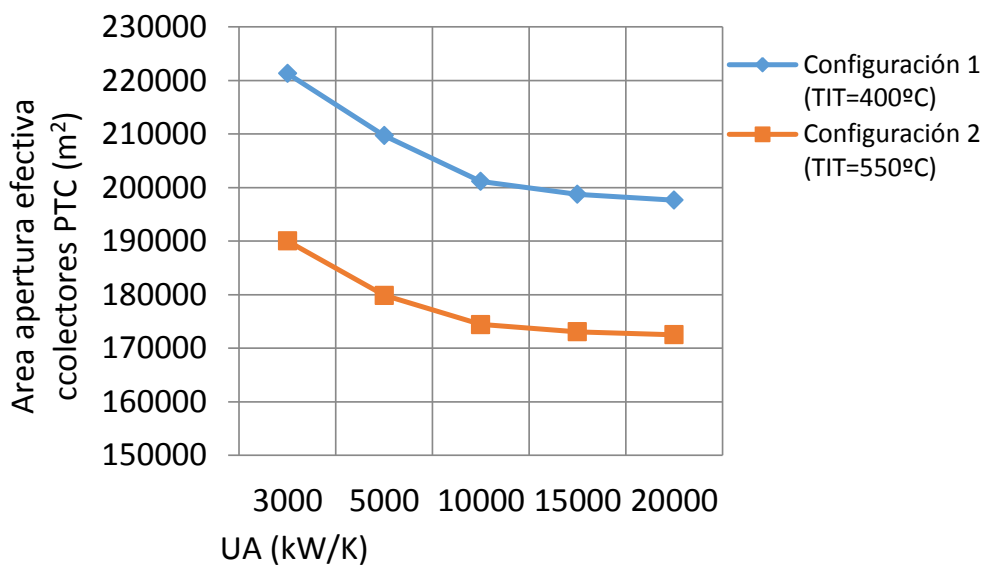

Figura 8.15. Área de apertura efectiva del campo solar con colectores PTC Vs. UA de los recuperadores del ciclo $\mathrm{s}-\mathrm{CO}_{2}$ Brayton $\mathrm{PCRC}$ sin recalentamiento.

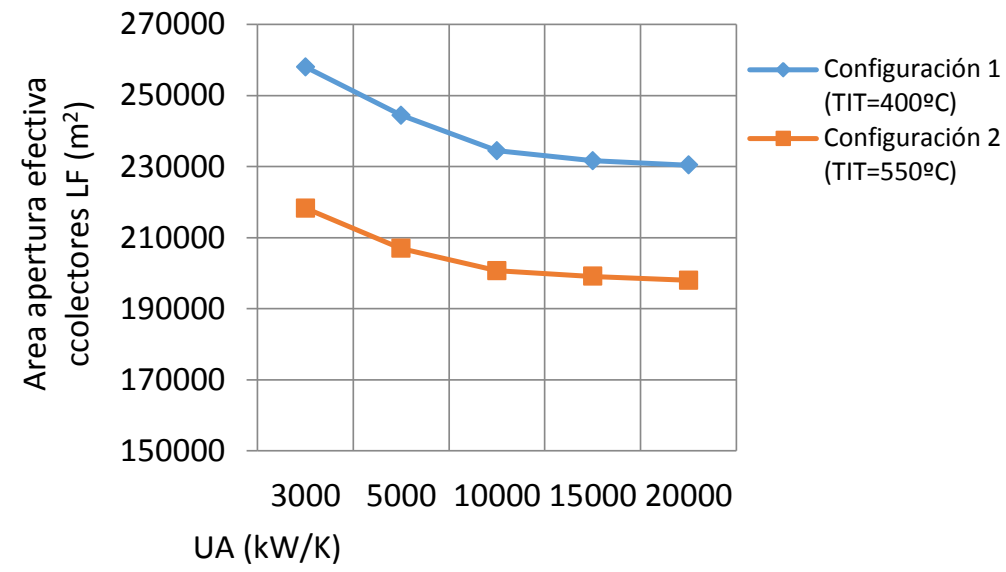

Figura 8.16. Área de apertura efectiva del campo solar con colectores LF Vs. el UA de los recuperadores del ciclo $\mathrm{s}-\mathrm{CO}_{2}$ Brayton $\mathrm{PCRC}$ sin recalentamiento.

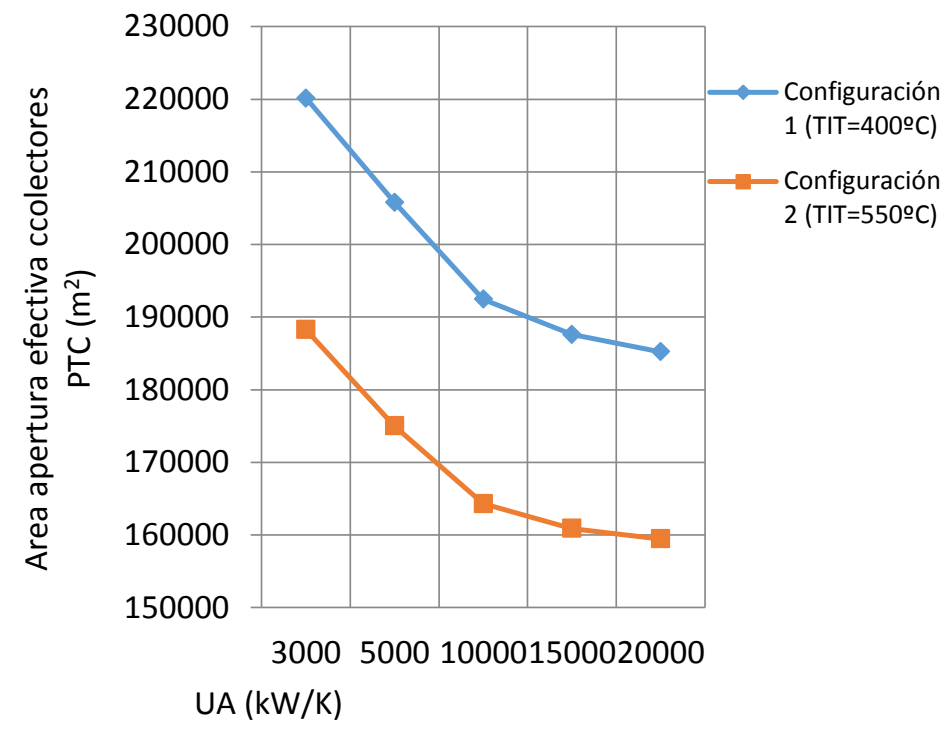

Figura 8.17. Área de apertura efectiva del campo solar con colectores PTC Vs. UA de los recuperadores del ciclo $\mathrm{s}-\mathrm{CO}_{2}$ Brayton $\mathrm{RCMCl}$ sin recalentamiento. 
8. Plantas Termosolares de Colectores Lineales con DSG acopladas a ciclos Brayton con $\mathrm{CO}_{2}$

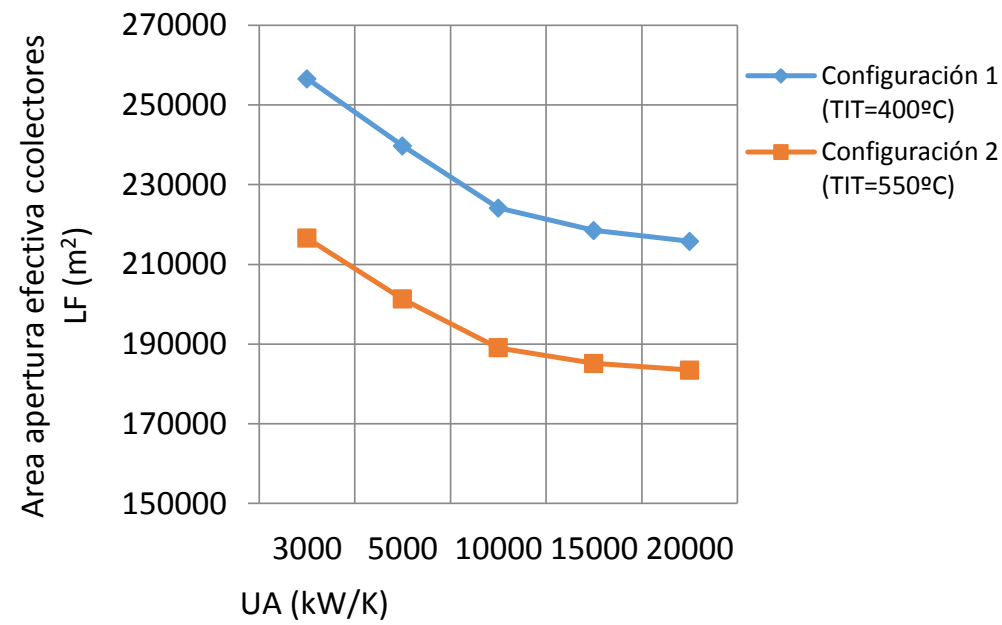

Figura 8.18. Área de apertura efectiva del campo solar con colectores LF Vs. UA de los recuperadores del ciclo $\mathrm{s}-\mathrm{CO}_{2}$ Brayton $\mathrm{RCMCl}$ sin recalentamiento.

\subsubsection{Intercambiadores de calor del campo solar y del sumidero final de calor}

En este apartado se ha cuantificado el tamaño térmico UA, y el coste de los intercambiadores de calor entre el ciclo s- $\mathrm{CO}_{2}$ Brayton y el campo solar ( $\mathrm{PHX}$ y $\mathrm{RHX}$ ), y entre el ciclo s- $\mathrm{CO}_{2}$ Brayton y el sumidero final de calor ACHE. Los resultados obtenidos se han resumido en las tablas 8.45 a 8.47 . Se confirma que el incremento del tamaño térmico de los recuperadores UA permite reducir el coste de los cambiadores PHX, RHX y ACHE. El incremento de la temperatura de entrada en turbina, desde $400^{\circ} \mathrm{C}$ hasta $500^{\circ} \mathrm{C}$, reduce también significativamente el UA de los intercambiadores, $\mathrm{PHX}$ y RHX, entre el campo solar y el ciclo de potencia. Como consecuencia directa se reduce su coste unitario, y se incrementa su eficiencia térmica.

Tabla 8.45. Tamaño térmico UA y estimación del coste de los intercambiadores de plantas termosolares con colectores lineales con DSG, acopladas a ciclos s- $\mathrm{CO}_{2}$ Brayton RC.

\begin{tabular}{lllllllll}
\hline $\begin{array}{l}\text { TIT } \\
\left({ }^{\circ} \mathrm{C}\right)\end{array}$ & $\begin{array}{l}\text { UA } \\
(\mathrm{kW} / \mathrm{K})\end{array}$ & $\begin{array}{l}\text { SF } \\
\text { Config. }\end{array}$ & $\begin{array}{l}\text { PHX+RHX } \\
\text { UA }(\mathrm{kW} / \mathrm{K})\end{array}$ & $\begin{array}{l}\text { ACHE UA } \\
(\mathrm{kW} / \mathrm{K})\end{array}$ & $\begin{array}{l}\text { PHX+RHX } \\
(\mathrm{kW})\end{array}$ & $\begin{array}{l}\text { ACHE } \\
(\mathrm{kW})\end{array}$ & $\begin{array}{l}\text { PHX+RHX } \\
(\mathrm{M} \$)\end{array}$ & $\begin{array}{l}\text { ACHE } \\
(\mathrm{M} \$)\end{array}$ \\
\hline 400 & 3000 & Configuración 1 & 4414 & 11618 & 149908 & 99904 & 14.99 & 24.98 \\
550 & 3000 & Configuración 2 & 3010 & 6654 & 119622 & 69606 & 11.96 & 17.41 \\
400 & 5000 & Configuración 1 & 7797 & 10603 & 137806 & 87804 & 13.78 & 21.95 \\
550 & 5000 & Configuración 2 & 3344 & 6144 & 111007 & 60951 & 11.11 & 15.24 \\
400 & 10000 & Configuración 1 & 6113 & 9611 & 128864 & 78861 & 12.88 & 19.72 \\
550 & 10000 & Configuración 2 & 3698 & 5607 & 103855 & 53907 & 10.38 & 13.47 \\
400 & 15000 & Configuración 1 & 6634 & 9162 & 125626 & 75618 & 12.56 & 18.90 \\
550 & 15000 & Configuración 2 & 3799 & 5451 & 101646 & 51695 & 10.16 & 12.92 \\
400 & 20000 & Configuración 1 & 6907 & 8937 & 124003 & 73997 & 12.40 & 18.50 \\
550 & 20000 & Configuración 2 & 3822 & 5403 & 100706 & 50720 & 10.07 & 12.68 \\
\hline
\end{tabular}

Tabla 8.46. Tamaño térmico UA y estimación del coste de los intercambiadores de plantas termosolares con colectores lineales con DSG, acopladas a ciclos s- $\mathrm{CO}_{2}$ Brayton PCRC.

\begin{tabular}{|c|c|c|c|c|c|c|c|c|}
\hline $\begin{array}{l}\text { TIT } \\
\left({ }^{\circ} \mathrm{C}\right)\end{array}$ & $\begin{array}{l}\text { UA } \\
(\mathrm{kW} / \mathrm{K})\end{array}$ & $\begin{array}{l}\text { SF } \\
\text { Config. }\end{array}$ & $\begin{array}{l}\text { PHX+RHX } \\
\text { UA (kW/K) }\end{array}$ & $\begin{array}{l}\text { ACHE UA } \\
(\mathrm{kW} / \mathrm{K})\end{array}$ & $\begin{array}{l}\text { PHX+RHX } \\
(\mathrm{kW})\end{array}$ & $\begin{array}{l}\text { ACHE } \\
(k W)\end{array}$ & $\begin{array}{l}\text { PHX+RHX } \\
\text { (M\$) }\end{array}$ & $\begin{array}{l}\text { ACHE } \\
(\mathrm{M} \$)\end{array}$ \\
\hline 400 & 3000 & Configuración 1 & 5044 & 8975 & 147497 & 97490 & 14.75 & 24.37 \\
\hline 550 & 3000 & Configuración 2 & 2780.3 & 5743 & 120036 & 70017 & 12.01 & 17.50 \\
\hline 400 & 5000 & Configuración 1 & 4032 & 8298 & 139621 & 89610 & 13.96 & 22.40 \\
\hline
\end{tabular}




\begin{tabular}{lllllllll}
\hline $\begin{array}{l}\text { TIT } \\
\left({ }^{\circ} \mathrm{C}\right)\end{array}$ & $\begin{array}{l}\text { UA } \\
(\mathrm{kW} / \mathrm{K})\end{array}$ & $\begin{array}{l}\text { SF } \\
\text { Config. }\end{array}$ & $\begin{array}{l}\text { PHX+RHX } \\
\text { UA }(\mathrm{kW} / \mathrm{K})\end{array}$ & $\begin{array}{l}\text { ACHE UA } \\
(\mathrm{kW} / \mathrm{K})\end{array}$ & $\begin{array}{l}\text { PHX+RHX } \\
(\mathrm{kW})\end{array}$ & $\begin{array}{l}\text { ACHE } \\
(\mathrm{kW})\end{array}$ & $\begin{array}{l}\text { PHX+RHX } \\
(\mathrm{M} \$)\end{array}$ & $\begin{array}{l}\text { ACHE } \\
(\mathrm{M} \$)\end{array}$ \\
\hline 550 & 5000 & Configuración 2 & 2888.3 & 5381.2 & 113635 & 63628 & 11.36 & 15.91 \\
400 & 10000 & Configuración 1 & 4320 & 7905 & 133931 & 83920 & 13.39 & 20.98 \\
550 & 10000 & Configuración 2 & 2929.5 & 5327.9 & 110212 & 60185 & 11.02 & 15.04 \\
400 & 15000 & Configuración 1 & 4368 & 7815 & 132317 & 82304 & 13.23 & 20.58 \\
550 & 15000 & Configuración 2 & 2935.4 & 5320.6 & 109345 & 59323 & 10.93 & 14.83 \\
400 & 20000 & Configuración 1 & 4420 & 7780 & 131604 & 81593 & 13.16 & 20.40 \\
550 & 20000 & Configuración 2 & 2935.9 & 5309.5 & 108979 & 58968 & 10.89 & 14.74 \\
\hline
\end{tabular}

Tabla 8.47. Tamaño térmico UA y estimación del coste de los intercambiadores de plantas

termosolares con colectores lineales con DSG, acopladas a ciclos s- $\mathrm{CO}_{2}$ Brayton $\mathrm{RCMCl}$.

\begin{tabular}{lllllllll}
\hline $\begin{array}{l}\text { TIT } \\
\left({ }^{\circ} \mathrm{C}\right)\end{array}$ & $\begin{array}{l}\text { UA } \\
(\mathrm{kW} / \mathrm{K})\end{array}$ & $\begin{array}{l}\text { SF } \\
\text { Config. }\end{array}$ & $\begin{array}{l}\text { PHX+RHX } \\
\text { UA }(\mathrm{kW} / \mathrm{K})\end{array}$ & $\begin{array}{l}\text { ACHE UA } \\
(\mathrm{kW} / \mathrm{K})\end{array}$ & $\begin{array}{l}\text { PHX+RHX } \\
(\mathrm{kW})\end{array}$ & $\begin{array}{l}\text { ACHE } \\
(\mathrm{kW})\end{array}$ & $\begin{array}{l}\text { PHX+RHX } \\
(\mathrm{M} \$)\end{array}$ & $\begin{array}{l}\text { ACHE } \\
(\mathrm{M} \$)\end{array}$ \\
\hline 400 & 3000 & Configuración 1 & 5889 & 10164 & 146354 & 96344 & 14.63 & 24.09 \\
550 & 3000 & Configuración 2 & 2874.9 & 6444.2 & 118489 & 68474 & 11.85 & 17.11 \\
400 & 5000 & Configuración 1 & 4860 & 9395 & 136679 & 86661 & 13.67 & 21.67 \\
550 & 5000 & Configuración 2 & 3144 & 5866.3 & 110124 & 60096 & 11.01 & 15.02 \\
400 & 10000 & Configuración 1 & 5621 & 9610 & 127813 & 77805 & 12.78 & 19.45 \\
550 & 10000 & Configuración 2 & 3426 & 5751.6 & 103378 & 53413 & 10.34 & 13.35 \\
400 & 15000 & Configuración 1 & 5945 & 9353.3 & 124584 & 74579 & 12.46 & 18.64 \\
550 & 15000 & Configuración 2 & 3494 & 5612.6 & 101236 & 51287 & 10.12 & 12.82 \\
400 & 20000 & Configuración 1 & 6154 & 9235.5 & 123017 & 73010 & 12.31 & 18.25 \\
550 & 20000 & Configuración 2 & 3510 & 5562 & 100324 & 50375 & 10.03 & 12.59 \\
\hline
\end{tabular}

\subsection{Conclusiones}

Las plantas termosolares de nueva generación tienen que integrar las últimas tecnologías de generación de potencia para garantizar la competitividad con el resto de plantas de generación eléctrica. En este sentido, una de las principales innovaciones en el campo de generación de electricidad lo constituye los ciclos s- $\mathrm{CO}_{2}$ Brayton, que vienen siendo objeto de investigación desde el año 2000 [31]. La primera solución comercial integrando un ciclo de potencia s- $\mathrm{CO}_{2}$ Brayton para aprovechamiento del calor residual en las instalaciones industriales, ha sido fabricada recientemente por la empresa Echogen [47]. Esta tecnología constituye una revolución en el campo de la generación de potencia, como una alternativa a los ciclos Rankine. No obstante su implantación generalizada está pendiente de la fabricación a gran escala y reducción de coste de los equipos que los integran. Para ello, se hace necesario continuar con el esfuerzo de investigación para conseguir optimizar el sistema completo, atendiendo a pérdidas de carga en los PCHE, reducir los costes de fabricación de los equipos, diseñar materiales más resistentes a corrosión y tensiones, turbomáquinas adaptables a los fluidos supercríticos y sus mezclas, sistemas de control de operación, etc.

El objetivo de este estudio ha sido la integración entre dos tecnologías novedosas, los campos solares con generación directa de vapor, y los ciclos de potencia s- $\mathrm{CO}_{2}$ Brayton. Para ello se han propuesto cuatro configuraciones de planta termosolar: La configuración 1, figura 8.8, es más apropiada para temperaturas de operación de entrada a turbina hasta $400^{\circ} \mathrm{C}$, y las configuraciones 2 (figura 8.9), 3 (figura 8.11) y 4 (figura 8.12) para temperaturas de entrada en turbina hasta $550^{\circ} \mathrm{C}$. Todas estas configuraciones han sido comparadas con las configuración de referencia, planta termosolar con colectores lineales con DSG y ciclo de potencia subcrítico Rankine, figuras 8.5 y 8.6 . 
8. Plantas Termosolares de Colectores Lineales con DSG acopladas a ciclos Brayton con $\mathrm{CO}_{2}$

La eficiencia neta proporcionada por la configuración 1 (DSG+RCMCl s- $\mathrm{CO}_{2}$ ) es de $36.6 \%$, mejorando el valor de 35\%, eficiencia de la configuración de Referencia (DSG+Rankine). Así mismo, se consigue una reducción del área de apertura del campo solar, cuantificada en un $2 \%$ (con colectores PTC) y en un 5\% (con colectores LF). Un mayor grado de detalle de los resultados obtenidos de la simulación de la configuración 1 se encuentran resumidos en las tablas 8.9 y 8.10 .

Respecto a la configuración 2 ( $\mathrm{DSG}+\mathrm{RC} \mathrm{s}-\mathrm{CO}_{2}$ ) la eficiencia neta proporcionada por esta solución de diseño es de un 43.6\%, mejorando el valor de la configuración de Referencia (DSG+Rankine) con un 38.39\%. Respecto al ahorro en el área de apertura efectiva de los colectores solares, con la configuración 2 se ha reducido un 5\% (con colectores PTC) y un $9 \%$ (con colectores LF). Los resultados de la simulación de la configuración 2 están reflejados en las tablas 8.11 y 8.12 .

En relación a la configuración 3, la eficiencia neta de este diseño es de un 39.7\%, tabla 8.13, valor muy cercano al proporcionado por la solución de referencia (DSG+Rankine) con un rendimiento entre el $40 \%$ y el $41 \%$, dependiendo del número de etapas de recalentamiento intermedio en turbinas.

Las configuraciones 1, 2, y 3 no incluyen ninguna etapa de recalentamiento en turbina lo cual impacta negativamente en la eficiencia del ciclo. Sin embargo la configuración 4, gracias la integración de una etapa de recalentamiento en turbina mediante un campo solar con sales fundidas, proporciona unos valores de eficiencia neta cercanos al $45.7 \%$, con una temperatura de entrada a turbina de $550^{\circ} \mathrm{C}$. En esta solución de diseño se combinan los campos solares con generación directa de vapor y campos solares con sales fundidas como HTF, figura 8.12. Las principales desventajas de los campos con sales fundidas son la necesidad de calefacción de los tubos receptores y resto de componentes del campo solar para evitar la solidificación de las sales, y la corrosión de los equipos debido a la composición química de las sales fundidas y sus altas temperaturas. Los resultados detallados de la simulación de la configuración 4 están resumidos en la tabla 8.14 .

Los valores de eficiencia que se han detallado anteriormente tienen su traducción directa en un ahorro de la superficie del campo solar asociado y de los colectores que lo integran, tablas 8.17 a 8.22. Comparando las configuraciones de plantas termosolares con ciclos de potencia Brayton con las plantas con ciclos Rankine se concluye que para una potencia fija generada, gracias a los innovadores ciclos supercríticos se reduce considerablemente la inversión en los colectores solares, en el terreno que ocupan y en la obra civil asociada (así como los gastos de operación y mantenimiento respectivos).

Los tubos absorbedores de acero inoxidable de baja aleación con un espesor de pared mínimo de $8.5 \mathrm{~mm}$ fueron elegidos como los más apropiados para la tecnología DSG+s-CO2. Lo cual permite un ahorro sustancial respecto a los tubos absorbedores utilizados en la tecnología Direct Molten Salt, que requiere acero inoxidables austeníticos con alto grado de aleantes especialmente de Níquel y otros elementos estabilizantes como el Molibdeno y Titanio o 
Niobio (AISI 347). Los resultados de la estimación de costes de los campos solares están resumidos en las tablas 8.17 a 8.22 . La configuración óptima es la correspondiente a un ciclo Brayton $\mathrm{s}-\mathrm{CO}_{2}$ de tipología $\mathrm{RCMCl}$. Esta es la configuración que presenta menores costes unitarios y mejor eficiencia energética.

Se ha realizado una estimación de los costes de los intercambiadores que integran el ciclo Brayton y los resultados obtenidos se encuentran en las tablas 8.28 a 8.37. La configuración de ciclo Brayton RC es la que proporciona unos mayores valores de eficiencia energética en comparación con el tamaño térmico y coste de los cambiadores del ciclo Brayton. Los intercambiadores de calor de los ciclos Rankine tienen un menor coste económico, gracias al menor coste de sus materiales, y al tratarse de una tecnología más difundida comercialmente, ampliamente implantada a escala industrial. Para realizar una comparativa entre las plantas termosolares con ciclos Rankine y las plantas con ciclos Brayton se ha resumido en las tablas 8.38 a 8.41 la suma del coste de los colectores solares y de los intercambiadores de calor de los ciclos de potencia. Se confirma que la reducción de coste de los campos solares en plantas acopladas a ciclos Brayton, no compensa la reducción de costes obtenida en los ciclos Rankine gracias al menor coste de sus intercambiadores. Otro aspecto importante a considerar en la reducción de costes de los cambiadores de plantas acopladas a ciclos Brayton, es la elección de cambiadores de tipología Tubos-Carcasa entre los campos solares y los ciclos de potencia. Los cambiadores Tubos-Carcasa acoplados a colectores solares con DSG permiten reducir el coste de los materiales en el lado por el que fluye el agua como HTF, y garantiza un alto coeficiente de transferencia de calor.

Como complemento final de este trabajo se ha realizado la optimización multivariable con los algoritmos de cálculo: SUBPLEX [44], UOBYQA [45] y NEWUOA [46] de los parámetros de operación de los ciclos Brayton acoplados a los colectores solares lineales con DSG. La metodología seguida para realizar este estudio de optimización consiste en fijar los valores de tamaño térmico UA de los recuperadores. Se confirma que un incremento del tamaño térmico de los recuperadores beneficia el rendimiento energético de la planta termosolar. Gracias al aumento del UA se reduce el área de apertura efectiva de sus colectores solares para una potencia fija. El UA también impacta de forma beneficiosa en el tamaño térmico de los intercambiadores de calor entre el ciclo Brayton y los campos solares (PHX y RHX), y en el tamaño térmico del intercambiador acoplado al sumidero final de calor (ACHE).Los resultados detallados de la eficiencia energética obtenidos están incluidos en las tablas 8.42 a 8.44 . Se ha realizado una estimación del área de apertura efectiva de los colectores solares en función del UA de los recuperadores, los resultados se han representado en las figuras 8.13 a 8.18. Así mismo, se ha incluido en las tablas 8.45 a 8.47 la estimación de costes de los intercambiadores de calor: PHX, RHX y ACHE.

Los últimos avances tecnológicos de colectores solares lineales (PTC O LF) con DSG, están encaminados con el modo de generación de vapor OT en colectores PTC [26]. La línea de investigación estará marcada por la integración de la tecnología OT acoplada a ciclos de potencia supercríticos Brayton, tal y como se ha mostrado en este trabajo para el modo de recirculación de la fase líquida para producción de vapor. 
8. Plantas Termosolares de Colectores Lineales con DSG acopladas a ciclos Brayton con $\mathrm{CO}_{2}$

La integración con un sistema de almacenamiento térmico está siendo una de las prioridades de las instalaciones con DSG en los colectores lineales. Los últimos avances en este sentido están explicados en la referencia [48].

El diseño de detalle de los cambiadores tubos carcasa para acoplamiento de los campos solares con los ciclos supercríticos Brayton es otro trabajo de investigación pendiente. En estos cambiadores, el agua como HTF se encontrará en estado líquido, bifásico o vapor. Dependiendo de la tipología de configuración considerada, se tiene que realizar la ingeniería de detalle de estos cambiadores tipo carcasa y tubo.

\subsection{Referencias}

[1] Z.Ma, C.S. Turchi. Advanced Supercritical Carbon Dioxide Power Cycle Configurations for Use in Concentrating Solar Power Systems. National Renewable Energy Laboratory.

[2] Khenissi A. Return of experience on transient behaviour at the DSG Solar Thermal Power Plant in Kanchanaburi Thailand. Beijing, China: SolarPaces 2014.

[3] Ericson J. The sun motor and the sun's temperature. Nature 1884;29:217-23.

[4] Pytlinski JT. Solar energy installations for pumping irrigation water. Sol Energy 1978; 21:255-8.

[5] Zarza E, Valenzuela L, Leon J, Weyers HD, Eickhoff M, Eck M, Hennecke K. The DISS project: direct steam generation in parabolic trough systems. Operation and maintenance experience and update on project status. J Sol Energy Eng 2002;124(2):126-33.

[6] Eck M, Zarza E, Eickhoff M, Rheinl€ander J, Valenzuela L. Applied research concerning the direct steam generation in parabolic troughs. Sol Energy 2003;74(4):341-51.

[7] Eck $M$, Schmidt $H$, Eickhoff $M$, Hirsch T. Field test of water steam separators for the DSG process. In: 13th International Symposium on Concentrated Solar Power and Chemical Energy Technologies, Seville; 2006.

[8] Eck M, Benz N, Feldhoff JF, Gilon Y, Hacker Z, Mu“ Iler T. The potential of direct steam generation in parabolic troughs results of the German project DIVA. 14th Biennial CSP. Las

Vegas, USA: SolarPACES Symposium; 2008.

[9] PuertoErrado 1 Thermosolar power plant: Novatec linear Fresenel technology.

http://www.nrel.gov/csp/solarpaces/project detail.cfm/projectlD $=46$

[10] Conlon WM. Direct steam form CLFR solar steam generators. 17th SolarPaces conference, Granada, Spain; 2011.

[11] Eck M, Eickhoff M, Feldhoff JF, Fontela P, Gathmann N, Meyer-Gru“ nefeldt M, et al. Direct steam generation in parabolic troughs at $500^{\circ} \mathrm{C}$ e first results of the REAL-DISS project. SolarPACES, Granada, Spain; 2011.

[12] Laing D, Bahl C, Bauer T, Lehmann D, Wolf-Dieter. Thermal energy storage for direct steam generation. Sol Energy 2011;85(4):627-33.

[13] Mertins M, Selig M, Ternedde A, Glanzel J. Experiences of operation of $30 \mathrm{MWe}$ solarthermal power station based on Fresnel collector technology. SolarPACES 2012 Conference, Marrakech, Morocco 2012.

[14] PuertoErrado 2 Thermosolar power plant: Novatec linear Fresenel technology. http://www.nrel.gov/csp/solarpaces/project detail.cfm/projectlD=46

[15] Itskhokine D, Lecuillier P, Benmarraze S, Guillier L, Rabut Q. Augustin Fresnel 1 project eDesign, construction and testing of a linear Fresnel pilot plant in the Pyrenees. SolarPaces 
8. Plantas Termosolares de Colectores Lineales con DSG acopladas a ciclos Brayton con $\mathrm{CO}_{2}$

2012 conference, Marrakech, Morocco; 2012.

http://www.solareuromed.com/augustin-fresnel-1

[16] Paul C, Teichrew O, Ternedde A. Operation experience of the integration of a solar boiler based on Fresnel collector technology into a coal-fired power station. SolarPaces 2012 conference, Marrakech, Morocco; 2012.

[17] Krüger D, Krüger J, Sukchai S, Breitzke $P$, Rahbani M, Schenk $H$, et al. Solar cogeneration with parabolic trough collectors in TRESERT. 18th SolarPaces Conference, Marrakech, Morocco 2012.

[18] Morin G, Kirchberger J, Lemmertz N, Mertins M. Operational results and simulation of a superheating Fresnel collector. SolarPaces 2012 conference,Marrakech, Morocco; 2012.

[19] Feldhoff JF, Eickhoff M, Karthikeyan R, Kruger J, Le_on Alonso J, Meyer-Gru“ nefeldt M, et al. Concept comparison and test facility design for the analysis of direct steam generation in once-through mode. SolarPACES 2012 Conference, Marrakech, Morocco; 2012.

[20] Zipf V, Neuh€auser A, Willert D, Gschwander S, Platzer W. Development of a high temperature PCM storage for DSG: first test results with a prototype. SolarPACES 2012 Conference, Marrakech, Morocco; 2012.

[21] DUKE project Feldhoff Jan Fabian, Eickhoff Martin, Keller Lothar, Alonso Javier Leon, Meyer-Grunefeldt Mirko, Valenzuela Loreto, et al. Status and first results of the DUKE project e component qualification of new receivers and collectors. SolarPaces; 2013.

[22] Seling M, Novatec Solar GmbH. Two years experience in operating the largest commercial Fresnel CSP Plant. Beijing, China: SolarPaces 2014.

[23] Feldhoff JF. A practical guide to solar once-through boiler control. Beijing, China: SolarPaces; 2014.

[24] Feldhoff JF. Transient models and characteristics of one through line focus systems. Beijing, China: SolarPaces; 2014.

[25] Jan Fabian Feldhoff, Tobias Hirsch, Robert Pitz-Paal and Loreto Valenzuela. Analysis and potential of once-through steam generators in line focus systems - Final results of the DUKE project. SolarPaces 2015.AIP Conf. Proc. 1734, 100006 (2016).

[26] Krüger D, Krüger J, Pandian Y, O'Connell B, Feldhoff JF, Karthikeyan R, et al. Experience with direct steam generation at the Kanchanaburi Solar Thermal Power Plant. In: $18^{\text {th }}$ SolarPACES Conference, Marrakech, Morocco 2012.

[27] Hirsch T, Khenissi A. A systematic comparison on power block efficiencies for CSP plants with direct steam generation. Las Vegas, U.S: SolarPaces 2013.

[28] Coco-Enríquez L, Muñoz-Antón J, Martínez-Val JM. Innovations on direct steam generation in linear Fresnel collectors. Las Vegas, U.S. SolarPaces 2013.

[29] Schoot PTR 70 4th Generation: http://www.schott.com.

[30] Maccari A, Archimede Solar Energy. Performance of direct steam generation solar receiver: laboratory vs real plant. Beijing, China: SolarPaces; 2014.

https://solarpaces2014.pse.de

[31] Burkholder F, Kutscher C. Heat loss testing of Schott's 2008 PTR70 parabolic trough receiver. NREL/TP-550-45633. May 2009.

[32] Solar Novatec. SAM lineal Fresnel solar boiler model, SAM Webinar. NREL SAM

Conference 2013.

[33] AFT Arrow Software. www.aft.com/products/arrow 
8. Plantas Termosolares de Colectores Lineales con DSG acopladas a ciclos Brayton con $\mathrm{CO}_{2}$

[34] Dyreby J, Klein S, Nellis G, Reindl D. Design considerations for supercritical carbon dioxide Brayton cycles with recompression. J Eng Gas Turbines Power Jul.22, 2014; 136(10):101710.

[35] V.Dostal, M.J. Driscoll, P.Hejzlar. A Supercritical Carbon Dioxide Cycle for Next Generation Nuclear Reactors. Advanced Nuclear Power Technology Program. MIT-ANP-TR-100.

[36] Thermoflow Software. https://www.thermoflow.com/

[37] S.A. Wright, T.M. Conboy, G.E. Rochau, Overview of supercritical CO2 power cycle development at Sandia National Laboratories, October 25-27, 2011 Columbus, Ohio, SNL.

[38] D. Shiferaw, J. Montero Carrero, R. Le Pierres. Economic analysis of SCO2 cycles with PCHE Recuperator design optimization. The 5th International Symposium - Supercritical CO2 Power Cycles March 28-31, 2016, San Antonio, Texas.

[39] C.Turchi, et al. 10 MW Supercritical CO2 Turbine Test. NREL, DE-EE0001589, 2014.

[40] M.Mohagheghi, H.Zawati, T.Pinol, J.Gou, C.Xu, J.Kapat. Use of 1-D Finite Enthalpy Method for a High-Temperature Recuperator Made of Polymer Derived Ceramic Composite for a Supercritical Carbon Dioxide Power System. The 5th International Symposium - Supercritical CO2 Power Cycles March 28-31, 2016, San Antonio, Texas.

[41] N. Sullivan, R.J. Kee, R. Braun. Ceramic, Microchannel Heat Exchangers for Supercritical Carbon Dioxide Power Cycles. The 5th International Symposium - Supercritical CO2 Power Cycles March 28-31, 2016, San Antonio, Texas.

[42] Jin Gyu Kwon, Tae Ho Kim, Hyun Sun Park, Moo Hwan Kim. Numerical analysis of a fin arrangement for an optimal design of Airfoil Fin PCHE. The $4^{\text {th }}$ International Symposium Supercritical CO2 Power Cycles, September 9-10, 2014, Pittsburgh, Pennsylvania.

[43] X. Zhang, X.Sun, R.N. Christensen. Optimization of S-Shaped Fin Channels in a Printed Circuit Heat Exchanger for Supercritical CO2 Test Loop. The 5th International Symposium Supercritical CO2 Power Cycles March 28-31, 2016, San Antonio, Texas.

[44] T. Rowan, Functional Stability Analysis of Numerical Algorithms, Ph.D. thesis, Department of Computer Sciences, University of Texas at Austin, 1990.

[45] Powell, M. J. D. (2002). UOBYQA: unconstrained optimization by quadratic approximation. Mathematical Programming, Series B (Springer) 92: 555-582. doi: 10.1007/s101070100290.

[46] Powell, M. J. D. (November 2004). The NEWUOA software for unconstrained optimization without derivatives (PDF) (Report). Department of Applied Mathematics and Theoretical Physics, Cambridge University. DAMTP 2004/NA05. Retrieved 2014-01-14.

[47] Echogen power systems. http://www.echogen.com

[48] M. Seitz, S. Hübner, M. Johnson. Detailed partial load investigation of a thermal energy storage concept for solar thermal power plants with direct steam generation.

AIP Conf. Proc. 1734, 050042 (2016). 


\title{
Capítulo 9 \\ Plantas Termosolares con Colectores Lineales, configuración del campo solar Dual Loop acopladas a ciclos de potencia s- $\mathrm{CO}_{2}$ \\ Brayton
}

\begin{abstract}
Resumen
La mayor parte de las plantas termosolares comerciales en operación están integradas por colectores PTC, y aceites térmicos como HTF. Sirvan como ejemplo las instalaciones de tipología Solar Energy Generating Systems (SEGS) en Estados Unidos [1]. En España también se han construido numerosas planta termosolares con esta configuración [2], sirva como ejemplo más significativo la planta Andasol 1 [3]. Como se ha explicado en capítulos anteriores, la máxima temperatura de operación de los aceites sintéticos debe ser menor de $400^{\circ} \mathrm{C}$, para garantizar que no se produce su degradación térmica, por tanto, de acuerdo al principio termodinámico de Carnot, se limita la eficiencia bruta del ciclo a un máximo del 37.5\% y neta del 35.4\%. En el capítulo 1 se ha realizado una comparativa de la eficiencia neta proporcionada por diferentes aceites térmicos. Estas instalaciones continúan en fase de estudio y mejora continua, cabe destacar el trabajo de Fabrizio De Luca [4] publicado recientemente. En este trabajo se analiza la producción y coste de inversión, en función del múltiplo solar (valores entre 1 y 3) y la capacidad de almacenamiento térmico (de 0 a 24 horas), en términos de la producción anual de electricidad, la eficiencia media anual, el factor de carga, el coste de capital y el Levelized Cost Of Energy (LCOE). En esta publicación se concluye que el mínimo valor de LCOE corresponde al escenario con almacenamiento térmico (16 horas), y un múltiplo solar de 2.2. Y para la configuración sin almacenamiento térmico, el mínimo valor de LCOE tiene correspondencia directa con un valor del múltiplo solar de 1.2.
\end{abstract}

Para solucionar la limitación de los aceites térmicos de máxima temperatura de entrada en turbina $\left(\mathrm{TIT}<400^{\circ} \mathrm{C}\right)$, se están adoptando las sales fundidas (MS) como HTF en colectores lineales [5-7]. En un estudio reciente del NREL [8], se confirma que el inventario de materiales de una planta convencional termosolar con colectores parabólicos y sales fundidas como HTF es un $25 \%$ inferior que el de una instalación solar con aceite térmico como HTF, ver detalle en tabla $9.1[8]$.

La mayor desventaja de las sales fundidas como HTF es la corrosión de los equipos y componentes del campo solar [9-10], así como el consumo eléctrico de los equipos auxiliares necesarios para evitar la solidificación de la sal dentro de los tubos absorbedores, Heat-Tracing [11-12]. Las temperaturas de solidificación de las MS más comunes se indican a continuación:

- Solar Salt $\left(60 \% \mathrm{NaNO}_{3}, 40 \% \mathrm{KNO}_{3}\right) 260^{\circ} \mathrm{C}$

- Hitec XL $\left(42 \% \mathrm{Ca}\left(\mathrm{NO}_{3}\right)_{2}, 15 \% \mathrm{NaNO}_{3}, 43 \% \mathrm{KNO}_{3}\right) 120^{\circ} \mathrm{C}$.

Los campos solares con configuración Dual-Loop están siendo estudiados desde el año 2012 [13], para combinar las sinergias proporcionadas por la combinación de dos fluidos caloportadores en una misma planta termosolar, el aceite sintético y las sales fundidas. En la configuración Dual-Loop de las configuraciones 2 y 3, figuras 9.3 y 9.4 respectivamente, se 
9. Plantas termosolares con Colectores Lineales, configuración del campo solar Dual Loop acopladas a ciclos de potencia s- $\mathrm{CO}_{2}$ Brayton

dispone de dos campos solares separados físicamente, en el primer campo solar con Dowtherm A [14], se realiza el calentamiento del fluido de trabajo del ciclo de potencia desde $300^{\circ} \mathrm{C}$ hasta $400^{\circ} \mathrm{C}$. En el segundo campo solar con sales fundidas $\left(60 \% \mathrm{NaNO}_{3}, 40 \% \mathrm{KNO}_{3}\right)$, se calienta el fluido de trabajo del bloque de potencia, $\mathrm{CO}_{2}$, desde $400^{\circ} \mathrm{C}$ hasta $550^{\circ} \mathrm{C}$.

Tabla 9.1. Comparación del contenido de materiales (en Toneladas) de tres plantas termosolares con diferente tecnología. Potencia 103 MWe y 6 horas de almacenamiento térmico [8].

\begin{tabular}{llll}
\hline & PTC Oil & PTC Sales & Torre Sales \\
\hline Acero al carbono, hierro v Zinc & 30804 & 26367 & 28107 \\
Acero Inoxidable & 1918 & 2283 & 1010 \\
Acero aleado & 1 & 261 & 335 \\
Cobre & 140 & 334 & 427 \\
Plata & 1 & 1 & 1 \\
FerroNiquel & 11 & 10 & \\
Aluminio & 441 & 333 & 287 \\
Aislamiento & 2755 & 2169 & 1277 \\
Vidrio & 12211 & 11261 & 10055 \\
Plástico & 508 & 400 & 617 \\
Adhesivos & 12 & 11 & \\
Pintura & 233 & 215 & \\
Aceites y lubricantes & 4600 & 95 & 95 \\
Nitrato de Sodio & 40100 & 16301 & 10451 \\
Nitrato de Potasio & 26700 & 10867 & 6967 \\
Nitrógeno & 18 & & \\
Hormigón y ladrillos & 66661 & 59088 & 78829 \\
Cemento & 49 & & \\
Asfalto & 7960 & 7347 & 3879 \\
Grava y piedras molidas & 53081 & 49087 & 46889 \\
Totales (Toneladas) & $\mathbf{2 4 8 2 0 4}$ & $\mathbf{1 8 6 4 3 0}$ & $\mathbf{1 8 9 2 2 6}$ \\
& & & \\
\hline
\end{tabular}

El estado de la técnica en relación a la configuración de campo solar Dual-Loop incluye a los ciclos de potencia Rankine [15-17]. El principal objetivo de este capítulo es el análisis de la integración de la configuración de campo solar con la tipología Dual-Loop acoplada al ciclo de potencia s- $\mathrm{CO}_{2}$ Brayton con recalentamiento [18] en las configuraciones indicadas.

La principal conclusión que se deduce de este estudio es el incremento de la eficiencia bruta hasta $44.4 \%$, con $\mathrm{TIT}=550^{\circ} \mathrm{C}$, proporcionada por las configuraciones indicadas, y ciclo de potencia simple Brayton con recuperación de calor y recalentemiento en turbina frente al 41.8\% eficiencia bruta obtenida con la configuración 1, figura 9.1, con disposición de campo solar Dual-Loop y ciclo de potencia Rankine.

\subsection{Introducción}

La mayor parte de las plantas comerciales de energía termosolar están integradas por colectores PTC con aceite sintético como HTF; como es el caso de las plantas SEGS [1] y de Andasol [3]. España es el país líder en el mundo en número de instalaciones termosolares, con 43 plantas de $50 \mathrm{MWe}$ cada una de ellas. Veintitrés de estas instalaciones con almacenamiento térmico con sales fundidas, y otras 20 sin almacenamiento térmico. 
9. Plantas termosolares con Colectores Lineales, configuración del campo solar Dual Loop acopladas a ciclos de potencia s- $\mathrm{CO}_{2}$ Brayton

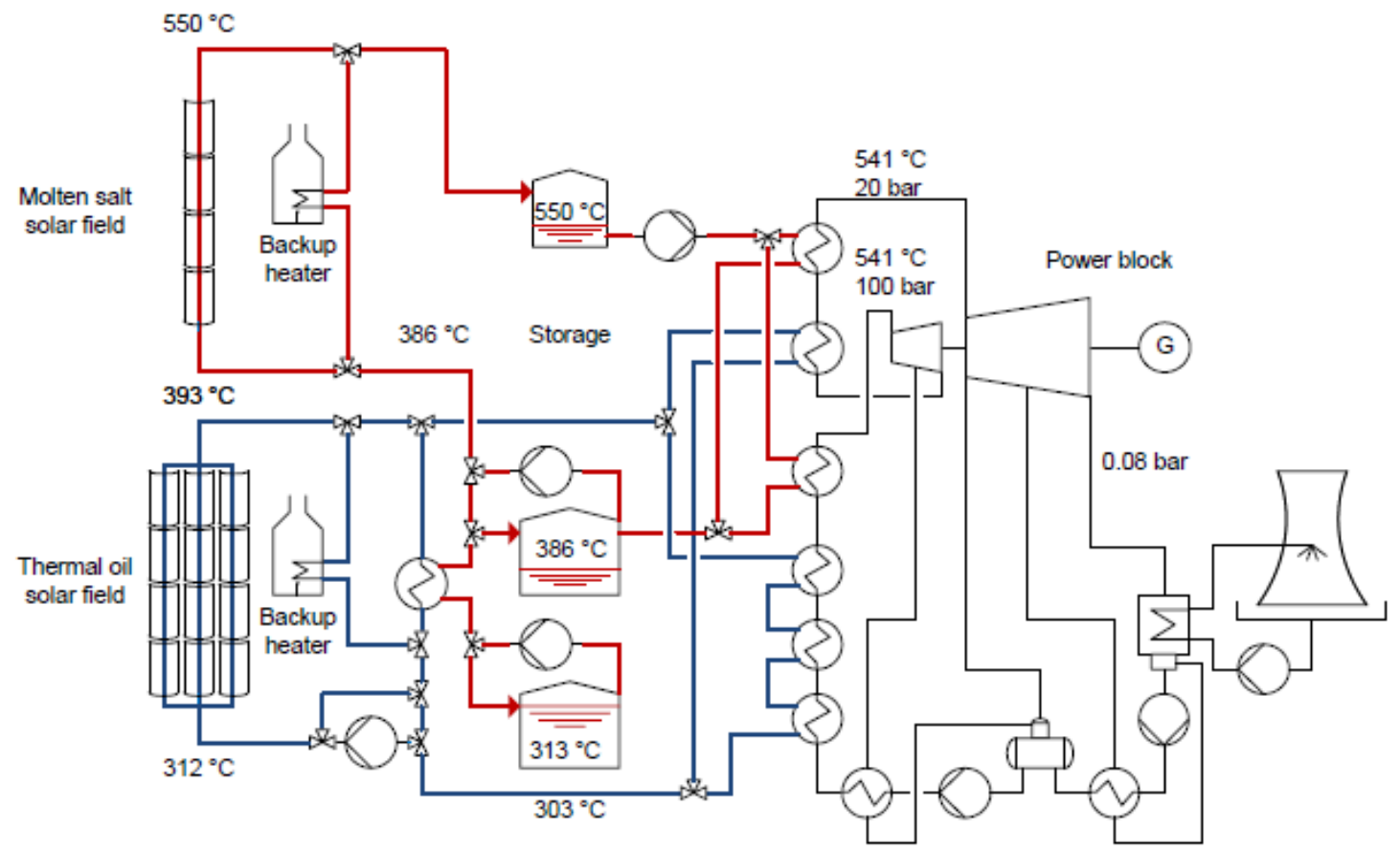

Figura 9.1. Diagrama de flujo del sistema Dual-Loop propuesto por Vogel 2014 [10].

La temperatura máxima de operación del aceite sintético es de $400^{\circ} \mathrm{C}$ para evitar su degradación. Esta limitación de TIT limita negativamente la potencia neta generada, como consecuencia directa de la aplicación del principio de Carnot. La utilización de sales fundidas como HTF en los colectores solares lineales es propuesto inicialmente por Rubbia et al. [19], para incrementar la TIT y mejorar la eficiencia energética del ciclo de potencia.

La mayor desventaja de las sales fundidas es su alta temperatura de solidificación, cercana a $220^{\circ} \mathrm{C}$ para la Solar Salt: $60 \% \mathrm{NaNO}_{3}-40 \% \mathrm{KNO}_{3}$, haciendo necesaria la utilización de resistencia eléctricas para evitar la solidificación de la sal durante la noche o con temperaturas ambientales frías. Shinners [15] ha realizado la comparación de tres configuraciones acopladas a ciclo de potencia Rankine: campo solar Single-Loop con Dowtherm A como HTF, campo solar Single-Loop con Solar Salt: $60 \% \mathrm{NaNO}_{3}-40 \% \mathrm{KNO}_{3}$, y campo solar Dual-Loop combinando los dos HTF citados. Los resultados obtenidos están resumidos en la tabla 9.2. Cabe destacar que ENEA [21] ha verificado experimentalmente la viabilidad técnica de las sales fundidas como HTF en los colectores parabólicos PTC, con una temperatura de operación alrededor de los $550^{\circ} \mathrm{C}$. Wang et al. [22] han estimado que la eficiencia térmica de los colectores PTC con sales fundidas, operando a $500^{\circ} \mathrm{C}$, es solamente $7.9 \%$ menor que con los aceites térmicos, operando a $300^{\circ} \mathrm{C}$. Giostri et al. [23] han documentado que la eficiencia de la planta termosolar puede ser mejorada un $6 \%$ si se utiliza sales como HTF en lugar de aceites térmicos. Zaversky et al. [24] desarrollaron un modelo para simulación de los transitorios de los colectores PTC utilizando sales fundidas. Este modelo ha sido satisfactoriamente validado con las medidas obtenidas en la instalación industrial SOLTERM en Italia. Adicionalmente, Raade et al. [25] investigaron experimentalmente las propiedades térmicas de las mezcla de sales (Nitrato de Litio, Nitrato de Sodio, Nitrato de Potasio, Nitrato de Cesio y Nitrato de Calcio), y obtuvieron como resultado una composición química que reduce la temperatura de fusión de las sales 
9. Plantas termosolares con Colectores Lineales, configuración del campo solar Dual Loop acopladas a ciclos de potencia s- $\mathrm{CO}_{2}$ Brayton

hasta $65^{\circ} \mathrm{C}$ y con una estabilidad térmica hasta $500^{\circ} \mathrm{C}$. Recientemente la compañía Archimede Solar Energy [26] ha continuado con su campaña experimental para confirmar la viabilidad técnica de las sales fundidas como HTF. Dicha compañía ha desarrollado especialmente para las sales fundidas Solar Salt, los tubos absorbedores HCEMS-11 con acero inoxidable AISI 316Ti, de alta resistencia a la corrosión.

Tabla 9.2. Comparación resultados configuraciones acopladas a ciclo de potencia Rankine [15].

\begin{tabular}{lcccccc}
\hline & $\begin{array}{c}\text { Area } \\
\text { colectores } \\
\left(\mathrm{m}^{2}\right)\end{array}$ & $\begin{array}{c}\text { Volumen } \\
\text { TES }\end{array}$ & $\begin{array}{c}\text { Total UA (kW/K) } \\
\text { no incluye el }\end{array}$ & $\begin{array}{c}\text { Potencia } \\
\text { Neta }\end{array}$ & $\begin{array}{c}\text { Eficiencia } \\
\text { Neta } \\
\text { condensador }\end{array}$ & $\begin{array}{c}\text { Eficiencia } \\
\text { Total Sistema } \\
(\%)\end{array}$ \\
\hline Dowtherm A & 200000 & 63500 & 2888 & $1.26 \mathrm{E}+05$ & 36.8 & 26.5 \\
Solar Salt & 200000 & 30000 & 2746 & $1.35 \mathrm{E}+05$ & 42.6 & 28.9 \\
Dual-Loop & 200000 & 45000 & 3156 & $1.32 \mathrm{E}+05$ & 42.7 & 28.1 \\
\hline
\end{tabular}

La solución técnica de campo solar Dual-Loop, combinando dos campos solares con aceite térmico y sales fundidas, fue propuesta inicialmente por Lang y Cuthbert [13], para maximizar las sinergias que proporcionan los dos HTF citados. La configuración de planta termosolar propuesta utiliza sales fundidas para almacenamiento térmico de ambos HTF. El gas natural es utilizado como fuente térmica de respaldo, suplementando el aporte térmico del campo solar. En condiciones nominales la turbina Andasol alcanza una eficiencia bruta del $38.2 \%$ son una configuración en el campo solar Single-Loop, con aceite térmico como HTF. Con la configuración de campo solar Dual-Loop, de acuerdo a la simulación realizada por A.K. Vogel et al. [16], la eficiencia bruta de la turbina se incrementa hasta un $41.8 \%$. La planta termosolar integra tres tanques de almacenamiento térmico con sales fundidas, y una caldera de gas natural para calentamiento auxiliar. La eficiencia bruta del $42 \%$ es corroborada por Shinners [15].

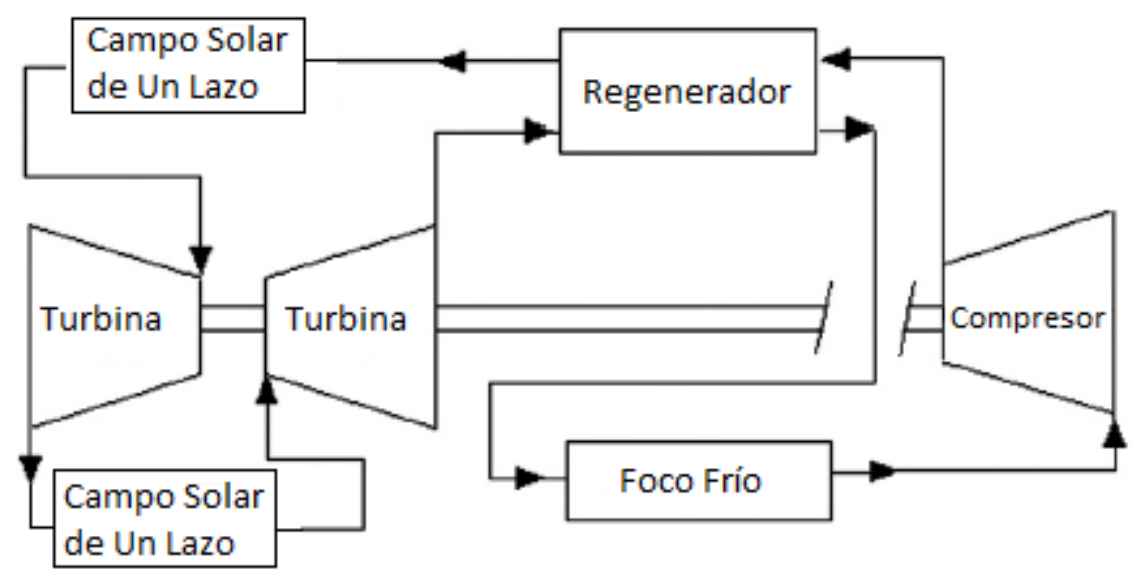

Figura 9.2. Configuración 1. Planta termosolar con campos solares con aceites térmicos o sales fundidas como HTF acopladas a ciclo de potencia s- $\mathrm{CO}_{2}$ Brayton SB con recuperación de calor y recalentamiento.

El sistema Dual-Loop proporciona diferentes ventajas: se alcanzan mayores TIT que con aceites térmicos. A bajas temperaturas la utilización de aceites térmicos permite reducir el consumo eléctrico del sistema Heat Tracing para evitar la solidificación de las sales fundidas. Los aceites térmicos permiten operar a bajos niveles de radiación solar, lo cual permite operar a la planta 
9. Plantas termosolares con Colectores Lineales, configuración del campo solar Dual Loop acopladas a ciclos de potencia s- $\mathrm{CO}_{2}$ Brayton

termosolar durante más días a lo largo del año. El coste del campo solar con aceites térmicos no requiere materiales resistentes a la corrosión.

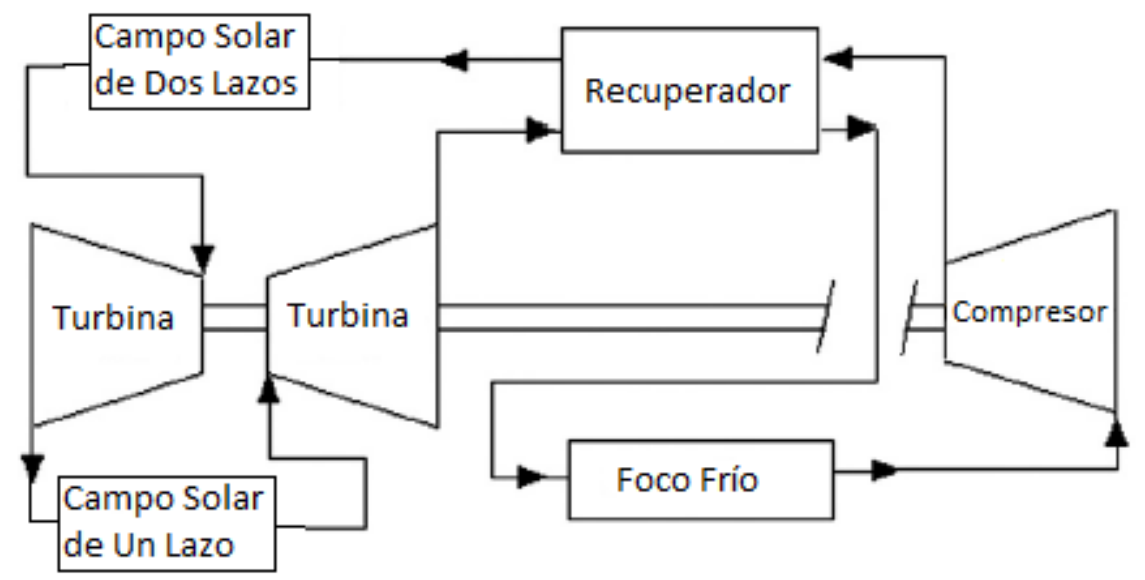

Figura 9.3. Configuración 2. Planta termosolar con campos solar principal de dos lazos o DualLoop, y campo solar de recalentamiento Single-Loop, acoplada a ciclo de potencia s- $\mathrm{CO}_{2}$ Brayton SB con recuperación de calor y recalentamiento.

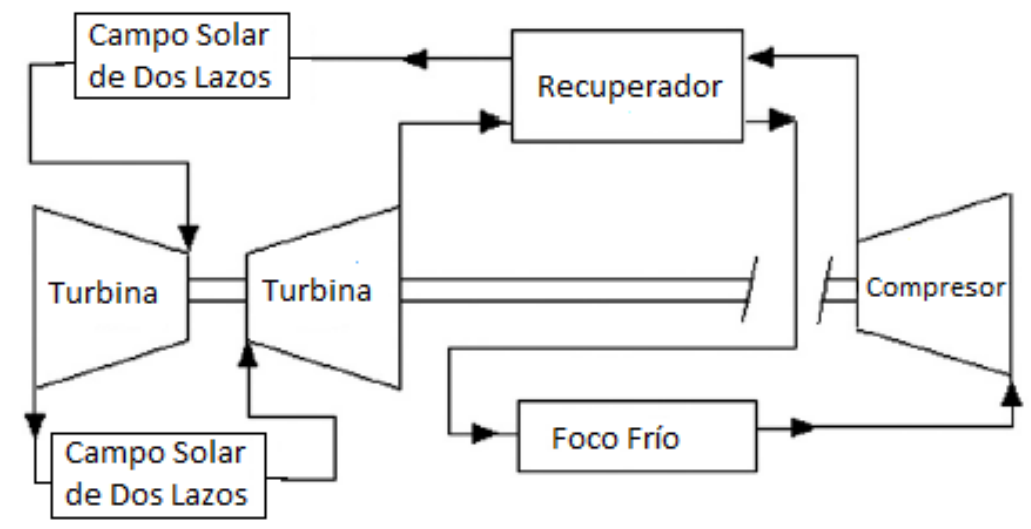

Figura 9.4. Configuración 3. Planta termosolar con campo solar principal y de recalentamiento de tipo Dual-Loop, acoplada a ciclo de potencia s- $\mathrm{CO}_{2}$ Brayton SB con recuperación de calor y recalentamiento.

En el presente capítulo se parte de la solución tecnológica de campo solar Dual-Loop, figura 9.1, y se integra con los ciclos de potencia $\mathrm{s}-\mathrm{CO}_{2}$ Brayton. Para ello, se diseñan las configuraciones 2 y 3 de campo solar con tipología Dual-Loop, figuras 9.3 y 9.4, la primera considera Dual-Loop en el campo principal y la segunda tanto en el campo solar principal como en de recalentamiento. Dichas configuraciones se comparan con la configuración 1 de referencia Single-Loop, figura 9.2. La primera solución de diseño, la configuración 1, considera Dowtherm A [14] o solar salt $\left(60 \% \mathrm{NaNO}_{3}, 40 \% \mathrm{KNO}_{3}\right)$ tanto en el campo solar principal como en el campo solar de recalentamiento. El campo solar Dual-Loop está integrado por dos campos solares:

- un primer campo con fluido caloportador Dowtherm A, que calienta el fluido de trabajo del ciclo hasta $400^{\circ} \mathrm{C}-415^{\circ} \mathrm{C}$.

- un segundo campo solar donde la temperatura es incrementada hasta $550^{\circ} \mathrm{C}$. 
9. Plantas termosolares con Colectores Lineales, configuración del campo solar Dual Loop acopladas a ciclos de potencia s- $\mathrm{CO}_{2}$ Brayton

\subsection{Hipótesis de cálculo.}

En las tablas 9.3 a 9.6 se resumen los principales parámetros de operación e hipótesis de diseño consideradas en la simulación de las configuraciones de planta termosolar propuestas en este capítulo. En todas las simulaciones se ha fijado una potencia bruta de $50 \mathrm{MWe}$ para poder realizar una comparativa entre las diferentes alternativas analizadas.

Tabla 9.3. Localización y condiciones ambientales.

\begin{tabular}{lc}
\hline Localización: & Dagget,CA, USA \\
Latitud: & $34.86^{\circ}$ \\
Longitud: & $-116.8^{\circ}$ \\
Zona horaria: & -8 \\
Hora: & $11: 30 \mathrm{hr}$ \\
DNI: & $986 \mathrm{~W} / \mathrm{m}^{2}$ \\
Temperatura ambiente: & $25^{\circ} \mathrm{C}$ \\
Altitud: & $588 \mathrm{~m}$ \\
\hline
\end{tabular}

Tabla 9.4. Parámetros colectores PTC y LF.

\begin{tabular}{ll}
\hline Tipo de colector PTC: & EuroTrough II \\
Anchura aperture PTC: & $5.77 \mathrm{~m}$ \\
Longitud Focal PTC: & $1.71 \mathrm{~m}$ \\
Factor Limpieza: & 0.96 \\
Eficiencia óptica PTC: & 0.75 \\
Pérdidas térmicas PTC: & $0.141 \Delta \mathrm{T}+6.48 \mathrm{e}-9 \Delta \mathrm{T}^{4}[27]$ \\
Tipo colector LF: & Novatec \\
Dimensiones LF: & $16.56 \mathrm{~m} \times 44.8 \mathrm{~m}$ \\
Eficiencia óptica LF: & 0.647 \\
Pérdidas térmicas LF: & $0.15 \Delta \mathrm{T}+7.15 .10-9[28]$ \\
\hline
\end{tabular}

Tabla 9.5. Parámetros tubo absorbedor [29]

\begin{tabular}{lc}
\hline Diámetro exterior: & $70 \mathrm{~mm}$ \\
Espesor tubo: & $4.191 \mathrm{~mm}$ \\
Material: Acero Carbono (para Dowtherm A) & \multicolumn{1}{c}{ Acero Inoxidable (para Solar Salt) } \\
Vacio entre el tubo de acero y el tubo de vidrio \\
Rugosidad: & $0.0457 \mathrm{~mm}$ \\
\hline
\end{tabular}

Tabla 9.6. Parámetros ciclo s- $\mathrm{CO}_{2}$ Brayton.

\begin{tabular}{lc}
\hline Eficiencia Turbina: & $93 \%$ \\
Eficiencia Compresor: & $89 \%$ \\
Efectividad Intercambiadores: & $95 \%$ \\
Pérdidas presión: & $1 \%$ lado caliente \\
Pérdidas presión en foco frio: & $1 \%$ lado caliente \\
TIT: & $400^{\circ} \mathrm{C}-550^{\circ} \mathrm{C}$ \\
Presión entrada Turbina: & 250 bar \\
Presión recalentamiento: & optimizado \\
CIT: & $32^{\circ} \mathrm{C}$ \\
Presión entrada Compresor: & optimizado \\
Eficiencia Generador: & 98.23 \\
\hline
\end{tabular}


9. Plantas termosolares con Colectores Lineales, configuración del campo solar Dual Loop acopladas a ciclos de potencia s- $\mathrm{CO}_{2}$ Brayton

\subsection{Caracterización del ciclo de potencia Brayton en el punto de diseño}

El ciclo de potencia s- $\mathrm{CO}_{2}$ Brayton analizado en este estudio para su integración en plantas termosolares de colectores lineales, ha sido caracterizado previamente siguiendo la metodología establecida por Dyreby [30]. Para ello se han calculado los parámetros termodinámicos de operación óptimos mediante los algoritmos matemáticos: SUBPLEX [31], UOBYQA [32] y NEWUOA [33]. Se ha caracterizado la eficiencia neta del ciclo para diferentes valores de del tamaño térmico de los recuperadores, y diferentes temperaturas de entrada en la turbina principal. Los resultados obtenidos se han resumido en la figura 9.5.

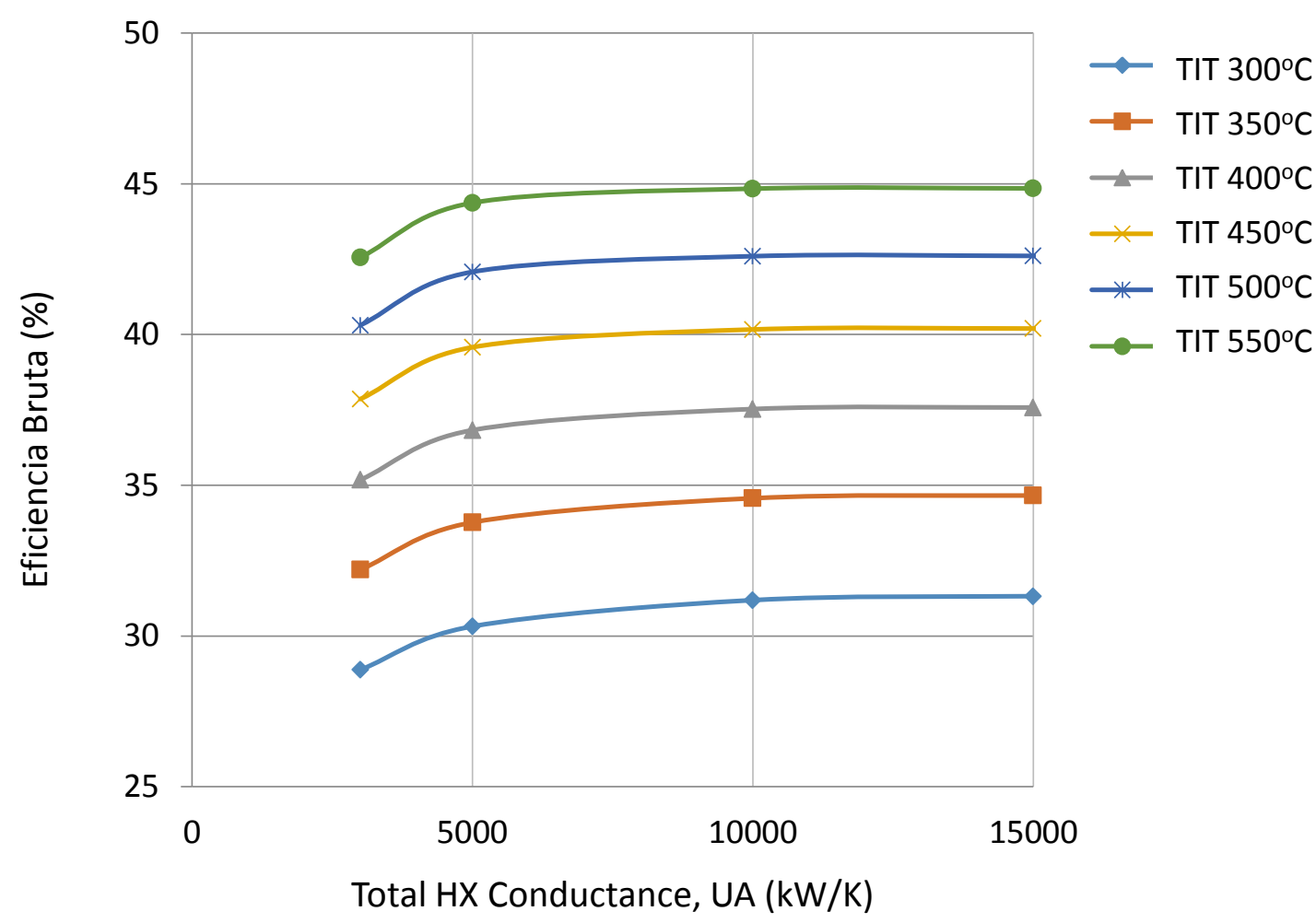

Figura 9.5. Eficiencia Neta de los ciclos de potencia s- $\mathrm{CO}_{2}$ Brayton $\mathrm{SB}$ con recuperación de calor y recalentamiento en función del UA de los recuperadores de calor y de TIT.

\subsection{Caracterización de la producción de energía de la planta termosolar en el punto de diseño}

La mínima diferencia de temperatura entre corrientes pinch-point en los recuperadores está muy relacionada con su tamaño térmico UA. Si se incrementan los valores de UA de los recuperadores el pinch-point se reduce, como se detalla en las tablas 9.7 y 9.8. La eficiencia neta máxima está limitada por el menor pinch-point en los recuperadores. Por esta razón se ha limitado el tamaño térmico de los recuperadores a un valor máximo de $U A=5000 \mathrm{~kW} / \mathrm{K}$, obteniendo un pinch-point entre $2.6^{\circ} \mathrm{C}$ y $4^{\circ} \mathrm{C}$.

Las eficiencias energéticas netas, de las plantas termosolares con campo solar Dual-Loop acopladas a ciclo s- $\mathrm{CO}_{2}$ Brayton $\mathrm{SB}$, se resumen en las tablas 9.7 y 9.8. Estas simulaciones incluyen las pérdidas en el Generador, y el consumo eléctrico de las bombas auxiliares en el 
9. Plantas termosolares con Colectores Lineales, configuración del campo solar Dual Loop acopladas a ciclos de potencia s- $\mathrm{CO}_{2}$ Brayton

campo solar. Sin embargo, no se ha considerado en nuestros cálculos el consumo de los ventiladores y resto de equipos del sumidero de calor, Air Cooler Heat Exchanger (ACHE). De acuerdo a las estimaciones de T. Neises [34], el consumo de los ventiladores de los ACHE, debería estar limitado a un $1 \%$ de la potencia neta generada. Sin embargo, con los diseños comerciales actuales de los $\mathrm{ACHE}$, se ha confirmado con los cálculos realizados durante el desarrollo de la presente tesis, que el consumo de los ventiladores de los ACHE es $\geq 4 \%$ de la potencia neta de la planta [34]. Es decir, para una planta de $50 \mathrm{MWe}$, sería del orden de $\geq 2$ MWe. Como conclusión se hace hincapié que las eficiencias energéticas netas resumidas en las tablas 9.6 y 9.7 , deberían ser reducidas en un $4 \%$, para tener en cuenta el consumo de los ventiladores de los ACHE.

Tabla 9.7. Planta termosolar con colectores lineales acoplada a ciclo de potencia s- $\mathrm{CO}_{2}$ Brayton SB con recalentamiento. Potencia Bruta fija 50MWe. Tamaño térmico de los recuperadores

$$
U A=3000 \mathrm{~kW} / \mathrm{K} \text {. }
$$

\begin{tabular}{llllll}
\hline & \multicolumn{3}{c}{ Sin $\Delta \mathrm{P}$ en los $\mathrm{HX}$} & \multicolumn{2}{l}{ Con $\Delta \mathrm{P}$ en los $\mathrm{HX}$} \\
\hline $\begin{array}{l}\text { Config. } \\
\text { SF }\end{array}$ & $\begin{array}{l}\text { TIT } \\
\left({ }^{\circ} \mathrm{C}\right)\end{array}$ & $\begin{array}{l}\text { Effic. } \\
\text { Neta } \\
(\%)\end{array}$ & $\begin{array}{l}\text { Recuperador } \\
\text { pinch point } \\
\left({ }^{\circ} \mathrm{C}\right)\end{array}$ & $\begin{array}{l}\text { Effic. } \\
\text { Neta } \\
(\%)\end{array}$ & $\begin{array}{l}\text { Recuperador } \\
\text { pinch point } \\
\left({ }^{\circ} \mathrm{C}\right)\end{array}$ \\
\hline 1 & 400 & 34.41 & 13.2 & 33.79 & 13.8 \\
1 & 400 & 34.41 & 13.2 & 33.66 & 13.9 \\
3 (ver Nota) & 450 & 36.95 & 12.7 & 36.19 & 13.4 \\
2 & 450 & 36.99 & 12.7 & 36.39 & 13.3 \\
1 & 450 & 37.04 & 12.7 & 36.44 & 13.2 \\
2 & 500 & 39.4 & 12.5 & 38.76 & 12.8 \\
1 & 500 & 39.42 & 12.4 & 38.85 & 12.7 \\
2 & 550 & 41.57 & 12.5 & 40.96 & 12.4 \\
1 & 550 & 41.59 & 12.5 & 41.06 & 12.3 \\
\hline
\end{tabular}

Nota: La configuración 3 incluye campo solar Dual-Loop, tanto en el campo solar principal como en el campo solar de recalentamiento, figura 9.4. El campo con aceite térmico Dowtherm $A$, caliente al fluido de trabajo $\mathrm{s}-\mathrm{CO}_{2}$ hasta $380^{\circ} \mathrm{C}$, y el campo solar con Solar Salt, sobrecalienta el fluido de trabajo s- $\mathrm{CO}_{2}$ hasta $450^{\circ} \mathrm{C}$.

El consumo eléctrico del Heat Tracing para evitar la solidificación de las sales fundidas no ha sido cuantificado, pero su impacto en el ciclo depende de la ubicación de la instalación, y de la temperatura ambiente y humedad relativa de cada localización.

La máxima eficiencia bruta del ciclo de potencia es de un $44.4 \%$, para la mayor $\mathrm{TIT}=550^{\circ} \mathrm{C}$, sin considerar las pérdidas energéticas auxiliares, sino únicamente cuantificando la potencia generada en las turbinas y el calor transferido en los intercambiadores de los campos solares. Otro parámetro importante cuantificado en este apartado son las pérdidas de presión y su impacto final en la eficiencia de la planta termosolar. Se han simulado en detalle los intercambiadores de calor que integran el ciclo de potencia como se ha explicado en la sección 2. Como puede deducirse de las tablas 9.7 y 9.8 , la eficiencia neta del ciclo disminuye entre un $0.5 \%-0.7 \%$, debido a las pérdidas de carga en los intercambiadores de calor. 
9. Plantas termosolares con Colectores Lineales, configuración del campo solar Dual Loop acopladas a ciclos de potencia s- $\mathrm{CO}_{2}$ Brayton

Tabla 9.8. Planta termosolar con colectores lineales acoplada a ciclo de potencia s- $\mathrm{CO}_{2}$ Brayton SB con recalentamiento. Potencia Bruta fija 50MWe. Tamaño térmico de los recuperadores

\begin{tabular}{|c|c|c|c|c|c|}
\hline \multirow[b]{2}{*}{$\begin{array}{l}\text { Config. } \\
\text { SF }\end{array}$} & \multirow[b]{2}{*}{$\begin{array}{l}\text { TIT } \\
\left({ }^{\circ} \mathrm{C}\right)\end{array}$} & \multicolumn{2}{|c|}{ Sin $\Delta \mathrm{P}$ en los $\mathrm{HX}$} & \multicolumn{2}{|c|}{ Con $\Delta \mathrm{P}$ en los $\mathrm{HX}$} \\
\hline & & $\begin{array}{l}\text { Effic. } \\
\text { Neta } \\
(\%) \\
\end{array}$ & $\begin{array}{l}\text { Recuperador } \\
\text { pinch point } \\
\left({ }^{\circ} \mathrm{C}\right)\end{array}$ & $\begin{array}{l}\text { Effic. } \\
\text { Neta } \\
(\%) \\
\end{array}$ & $\begin{array}{l}\text { Recuperador } \\
\text { pinch point } \\
\left({ }^{\circ} \mathrm{C}\right)\end{array}$ \\
\hline 1 & 400 & 36.05 & 3.7 & 35.48 & 4.1 \\
\hline 1 & 400 & 36.05 & 3.7 & 35.35 & 4.1 \\
\hline 3 (ver Nota) & 450 & 38.67 & 3.1 & 37.94 & 3.6 \\
\hline 2 & 450 & 38.70 & 3.1 & 38.15 & 3.4 \\
\hline 1 & 450 & 38.75 & 3.1 & 38.22 & 3.4 \\
\hline 2 & 500 & 41.17 & 2.6 & 40.59 & 3.1 \\
\hline 1 & 500 & 41.20 & 2.6 & 40.67 & 2.9 \\
\hline 2 & 550 & 43.42 & 2.3 & 42.84 & 2.7 \\
\hline 1 & 550 & 43.44 & 2.3 & 42.93 & 2.6 \\
\hline
\end{tabular}

Nota: La configuración 3 incluye campo solar Dual-Loop, tanto en el campo solar principal como en el campo solar de recalentamiento, figura 9.4. El campo con aceite térmico Dowtherm $A$, caliente al fluido de trabajo s- $\mathrm{CO}_{2}$ hasta $380^{\circ} \mathrm{C}$, y el campo solar con Solar Salt, sobrecalienta el fluido de trabajo s- $\mathrm{CO}_{2}$ hasta $450^{\circ} \mathrm{C}$.

\subsection{Estimación de Coste de Inversión}

Una de las principales razonas para optar por el diseño de campos solares Dual-Loop es la optimización del coste de los materiales gracias a los menores requerimientos de resistencia a la corrosión de los equipos y componentes en contacto con el Dowtherm A en relación a los elementos que están en contacto con las sales fundidas. Para recalcar esta ventaja, en este trabajo se ha considerado acero al carbono como material en los tubos receptores en contacto con el Dowtherm A y acero inoxidable austenítico AISI 347 en las tuberías en contacto en las sales fundidas.

El coste de los materiales están muy influenciada por el elemento aleante Níquel en los aceros inoxidable austeníticos y en otras aleaciones de Níquel con gran resistencia a la corrosión. Las estimaciones del coste de inversión de las diferentes configuraciones de campo solar analizadas en el presente estudio se han resumido en las tablas 9.9 y 9.10 . Tal y como se ha explicado a lo largo de esta Tesis, otro equipo decisivo para garantizar la eficiencia de los ciclos Brayton es el sumidero de calor de la planta termosolar Ultimate Heat Sink (UHS). Las dimensiones del sistema de sumidero frio están muy relacionadas con la eficiencia neta de la instalación y por tanto con la temperatura de entrada a turbina del fluido de trabajo en el ciclo de potencia, tablas 9.9 y 9.10$)$.

La optimización del diseño y el coste final del UHS en ciclos Brayton ha sido ampliamente estudiado por Gavic [35], concluyendo que la configuración óptima de UHS para minimizar el consumo de agua y las dimensiones de los equipos auxiliares de ventilación, es la solución híbrida compuesta por un circuito cerrado de agua de refrigeración integrado por un enfriador del agua con aire en convección forzada y un intercambiador de calor tipo PCHE para enfriamiento del $\mathrm{CO}_{2}$. El coste unitario de este sistema hibrido de enfriamiento se ha estimado entre 200-300 $\$ / \mathrm{kWe}$, compuesto por el intercambiador de calor PCHE (100 $\$ / \mathrm{kWe}$ ) y el ACHE (150\$/kWe). 
9. Plantas termosolares con Colectores Lineales, configuración del campo solar Dual Loop acopladas a ciclos de potencia s- $\mathrm{CO}_{2}$ Brayton

Tabla 9.9. Estimación central termosolar con colectores PTC acoplada a ciclo Potencia s- $\mathrm{CO}_{2}$ Brayton SB. Potencia bruta fija 50 MWe.

\begin{tabular}{|c|c|c|c|c|c|c|c|c|c|}
\hline & \multirow{2}{*}{$\begin{array}{l}\text { TIT } \\
\left({ }^{\circ} \mathrm{C}\right)\end{array}$} & \multicolumn{4}{|c|}{$\begin{array}{c}\text { Recuperador } \\
\text { UA }=3000 \mathrm{~kW} / \mathrm{K}\end{array}$} & \multicolumn{4}{|c|}{$\begin{array}{c}\text { Recuperador } \\
U A=5000 \mathrm{~kW} / \mathrm{K}\end{array}$} \\
\hline & & $\begin{array}{l}\mathrm{SF} \\
(\mathrm{M} \$)\end{array}$ & $\begin{array}{l}\mathrm{HX} \\
(\mathrm{M} \$)\end{array}$ & $\begin{array}{l}\text { Foco } \\
\text { Frío } \\
(\mathrm{M} \$)\end{array}$ & $\begin{array}{l}\text { Total } \\
\text { (M\$) }\end{array}$ & $\begin{array}{l}\mathrm{SF} \\
(\mathrm{M} \$)\end{array}$ & $\begin{array}{l}\mathrm{HX} \\
(\mathrm{M} \$)\end{array}$ & $\begin{array}{l}\text { Foco } \\
\text { Frío } \\
(\mathrm{M} \$)\end{array}$ & $\begin{array}{l}\text { Total } \\
\text { (M\$) }\end{array}$ \\
\hline 1 & 400 & 110.31 & 22.06 & 23.68 & 156.05 & 105.12 & 23.08 & 21.94 & 150.14 \\
\hline 1 & 400 & 83.05 & 21.94 & 23.82 & 128.81 & 79.16 & 22.96 & 22.08 & 124.21 \\
\hline 3 & 450 & 90.83 & 28.94 & 21.25 & 141.02 & 88.66 & 30.25 & 19.73 & 138.64 \\
\hline 2 & 450 & 92.72 & 25.47 & 21.10 & 139.29 & 90.00 & 26.24 & 19.54 & 135.78 \\
\hline 1 & 450 & 104.97 & 22.17 & 21.07 & 148.21 & 100.24 & 23.19 & 19.50 & 142.94 \\
\hline 2 & 500 & 93.04 & 26.48 & 19.04 & 138.56 & 91.02 & 27.18 & 17.67 & 135.87 \\
\hline 1 & 500 & 102.01 & 22.53 & 18.97 & 143.52 & 97.89 & 23.58 & 17.60 & 139.07 \\
\hline 2 & 550 & 96.69 & 26.27 & 17.36 & 140.32 & 95.12 & 26.90 & 16.06 & 138.08 \\
\hline 1 & 550 & 101.70 & 22.97 & 17.29 & 141.96 & 97.57 & 23.94 & 15.98 & 137.48 \\
\hline
\end{tabular}

Como puede apreciarse en la estimación de costes de inversión de la planta termosolar en las tablas 9.9 y 9.10 , el coste del UHS es similar el coste del resto de cambiadores de la planta termosolar. Por tanto, la elección del la solución técnica optima de diseño del UHS es una pieza clave para elegir la configuración de planta más competitiva. Se concluye que la configuración 1 con Dowtherm A como HTF y TIT $=400^{\circ} \mathrm{C}$, es la solución técnica cuyo coste de inversión es menor. El incremento del tamaño térmico del recuperador hasta $5000 \mathrm{~kW} / \mathrm{K}$ a pesar de incrementar el coste de recuperador permite una mayor eficiencia neta en la instalación y por tanto un ahorro del coste de inversión en el campo solar y en el sumidero de calor.

El ahorro neto de incremento del tamaño térmico del recuperador desde $3000 \mathrm{~kW} / \mathrm{K}$ hasta $5000 \mathrm{~kW} / \mathrm{K}$ es del $4 \%$. Es muy importante la conclusión a la que se ha llegado ya que se ha demostrado que un incremento del tamaño térmico de los recuperadores del ciclo Brayton tiene un efecto directo en la reducción de coste final de la totalidad de la instalación termosolar. A pesar de incrementar las dimensiones y coste de inversión en los recuperadores, el ahorro asociado en el campo solar y en el sumidero de calor es mayor. El incremento de las dimensiones del recuperador está limitado a su vez por el mínimo pinch-point a la salida de este equipo. Es decir, cuando se incrementan las dimensiones del recuperador se disminuye la diferencia de temperatura pinch-point entre la corriente fría y la corriente caliente de s- $\mathrm{CO}_{2}$. El pinch-point de los cambiadores está normalmente limitado entre $3^{\circ} \mathrm{C}$ y $10^{\circ} \mathrm{C}$.

Los resultados de la estimación de costes están resumidos en las tablas 9.9 y 9.10 , mostrando la solución de diseño más competitiva de la configuración $1\left(\mathrm{TIT}=400^{\circ} \mathrm{C}\right)$, con aceite térmico Dowtherm A como HTF tanto en el campo solar principal, como en el campo solar para recalentamiento. La segunda configuración con un menor coste de capital, sería la configuración $2\left(\mathrm{TIT}=500^{\circ} \mathrm{C}\right)$, con un campo solar principal Dual-Loop, y un campo solar para recalentamiento tipo Single-Loop con sales fundidas, en ambos casos con colectores solares LF. La misma conclusión es obtenida con recuperadores con tamaño térmico UA=3000 kW/K y $\mathrm{UA}=5000 \mathrm{~kW} / \mathrm{K}$. En el segundo caso la diferencia de coste es de un $5 \%$ entre la solución más competitiva y el resto de alternativas. 
9. Plantas termosolares con Colectores Lineales, configuración del campo solar Dual Loop acopladas a ciclos de potencia s- $\mathrm{CO}_{2}$ Brayton

Tabla 9.10. Estimación central termosolar con colectores LF acoplada a ciclo Potencia s- $\mathrm{CO}_{2}$ Brayton SB. Potencia bruta fija 50 MWe.

\begin{tabular}{|c|c|c|c|c|c|c|c|c|c|}
\hline & \multirow{2}{*}{$\begin{array}{l}\text { TIT } \\
\left({ }^{\circ} \mathrm{C}\right)\end{array}$} & \multicolumn{4}{|c|}{$\begin{array}{c}\text { Recuperador } \\
\text { UA }=3000 \mathrm{~kW} / \mathrm{K}\end{array}$} & \multicolumn{4}{|c|}{$\begin{array}{c}\text { Recuperador } \\
\text { UA }=5000 \mathrm{~kW} / \mathrm{K}\end{array}$} \\
\hline & & $\begin{array}{l}\mathrm{SF} \\
(\mathrm{M} \$)\end{array}$ & $\begin{array}{l}\mathrm{HX} \\
(\mathrm{M} \$)\end{array}$ & $\begin{array}{l}\text { Foco } \\
\text { Frío } \\
(\mathrm{M} \$)\end{array}$ & $\begin{array}{l}\text { Total } \\
(\mathrm{M} \$)\end{array}$ & $\begin{array}{l}\mathrm{SF} \\
(\mathrm{M} \$)\end{array}$ & $\begin{array}{l}\mathrm{HX} \\
(\mathrm{M} \$)\end{array}$ & $\begin{array}{l}\text { Foco } \\
\text { Frío } \\
(\mathrm{M} \$)\end{array}$ & $\begin{array}{l}\text { Total } \\
(\mathrm{M} \$)\end{array}$ \\
\hline 1 & 400 & 83.05 & 22.54 & 23.68 & 129.27 & 79.84 & 23.12 & 21.93 & 124.8 \\
\hline 1 & 400 & 62.59 & 21.94 & 23.82 & 108.35 & 59.68 & 22.98 & 22.08 & 104.7 \\
\hline 3 & 450 & 68.99 & 28.92 & 21.25 & 119.16 & 66.58 & 30.28 & 19.72 & 116.5 \\
\hline 2 & 450 & 69.25 & 25.45 & 21.10 & 115.80 & 67.09 & 26.22 & 19.54 & 112.8 \\
\hline 1 & 450 & 78.29 & 22.17 & 21.07 & 121.52 & 74.74 & 23.19 & 19.50 & 117.4 \\
\hline 2 & 500 & 78.33 & 26.50 & 19.04 & 123.87 & 66.77 & 27.18 & 17.66 & 111.6 \\
\hline 1 & 500 & 74.76 & 22.53 & 18.97 & 116.26 & 72.22 & 23.58 & 17.60 & 113.4 \\
\hline 2 & 550 & 69.90 & 26.27 & 17.36 & 113.53 & 68.29 & 26.90 & 16.05 & 111.2 \\
\hline 1 & 550 & 72.97 & 22.93 & 17.29 & 113.19 & 69.91 & 23.94 & 15.98 & 109.8 \\
\hline
\end{tabular}

Nota: PTC tubo de acero para el Dowtherm A: $324 \$ / \mathrm{m}^{2}$. PTC tubo de acero inoxidable AISI 347 para la Solar Salt: $432 \$ / \mathrm{m}^{2}$. LF tubo de acero para el Dowtherm A: $207 \$ / \mathrm{m}^{2}$. LF tubo de acero inoxidable AISI 347 para la Solar Salt: $275 \$ / \mathrm{m}^{2}$.Factor por costes de construcción: 1.16 x coste área de apertura. PCHE: $92 \$ / \mathrm{kWe}$. ACHE $250 \$ / \mathrm{kWe}$.

\subsection{Integración de otras configuraciones del ciclo s- $\mathrm{CO}_{2}$ Brayton (RC, PCRC y $\mathrm{RCMCl}$ ) con los campos solares de tipología Dual-Loop}

El estudio realizado hasta ahora con las configuraciones mostradas en las figuras $9.2,9.3$ y 9.4 con un ciclo de potencia Brayton simple con recuperación de calor, también se han realizado con otras tipologías de ciclos Brayton con diferentes disposiciones de los equipos para maximizar la eficiencia energéticas mediante la instalación de etapas adicionales de enfriamiento en compresores, figuras $9.6,9.7$ y 9.8 .

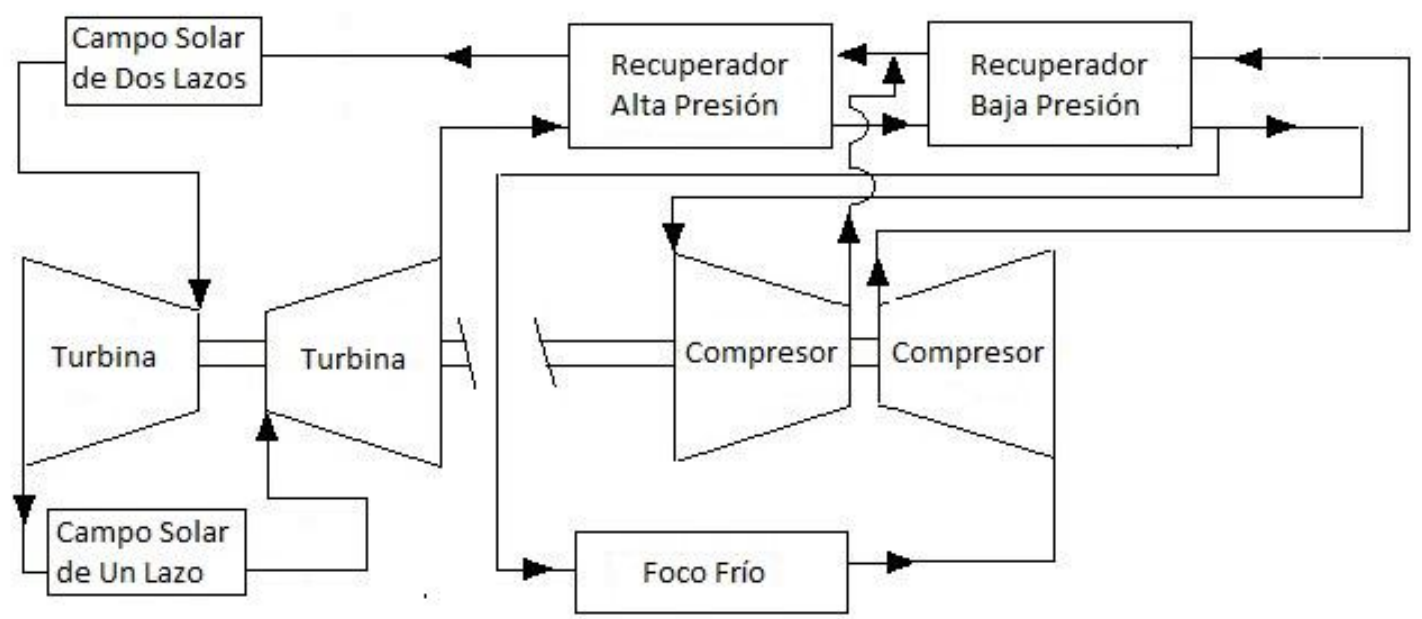

Figura 9.6. Planta CSP con colectores solares lineales (PTC o LF), con dos campos solares: el campo principal de tipología 'Dual-Loop' y el campo de recalentamiento acoplados a ciclo s$\mathrm{CO}_{2}$ Brayton RC con recalentamiento. 
9. Plantas termosolares con Colectores Lineales, configuración del campo solar Dual Loop acopladas a ciclos de potencia s- $\mathrm{CO}_{2}$ Brayton

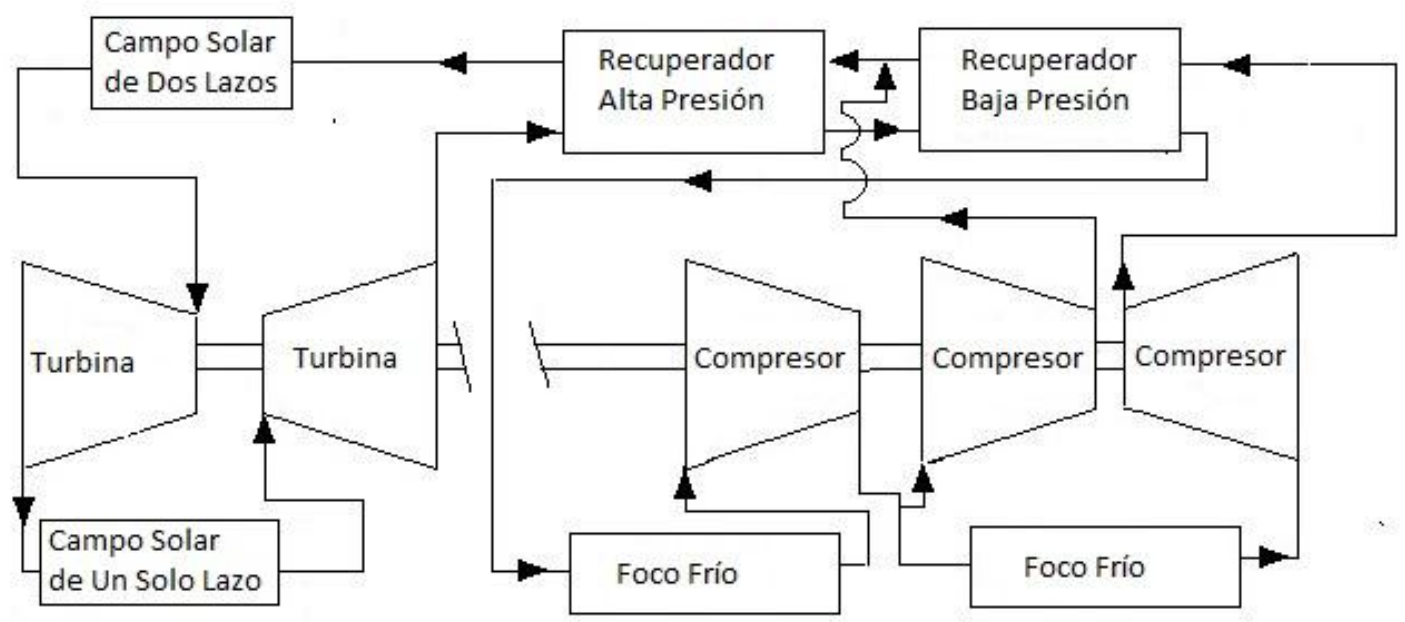

Figura 9.7. Planta CSP con colectores solares lineales (PTC o LF), con dos campos solares: el campo principal de tipología 'Dual-Loop' y el campo de recalentamiento acoplados a ciclo s$\mathrm{CO}_{2}$ Brayton PCRC con recalentamiento.

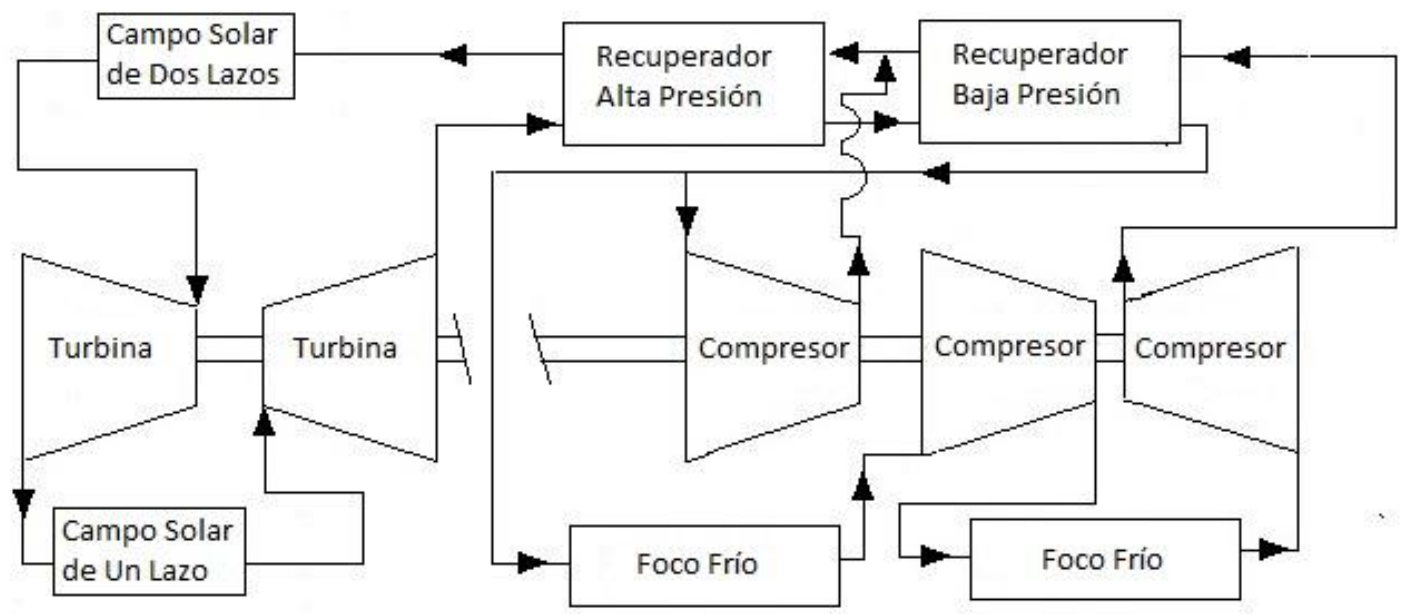

Figura 9.8. Planta CSP con colectores solares lineales (PTC o LF), con dos campos solares: el campo principal de tipología 'Dual-Loop' y el campo de recalentamiento acoplados a ciclo s$\mathrm{CO}_{2}$ Brayton RCMCI con recalentamiento. 
9. Plantas termosolares con Colectores Lineales, configuración del campo solar Dual Loop acopladas a ciclos de potencia s- $\mathrm{CO}_{2}$ Brayton

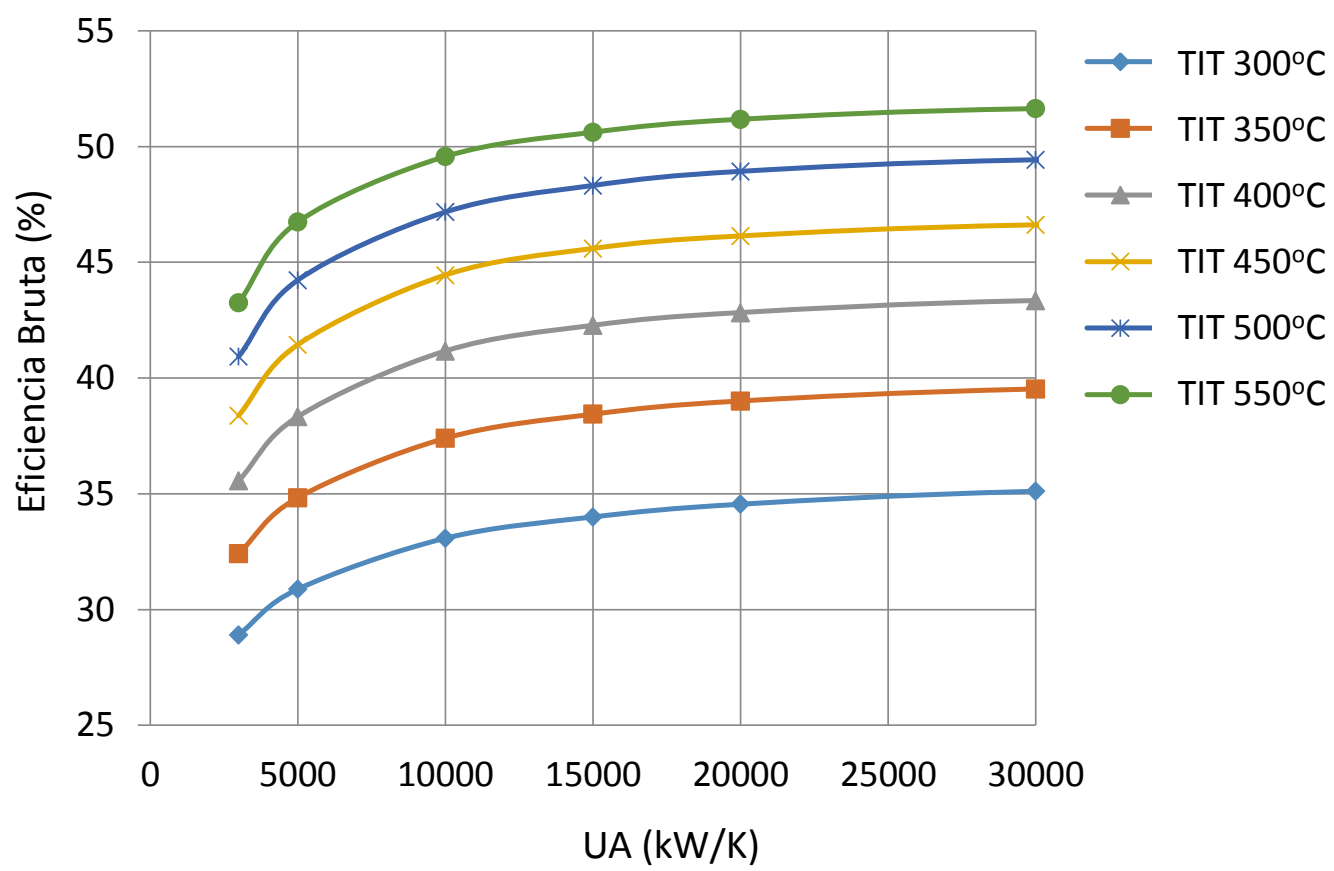

Figura 9.9. Eficiencia Neta de los ciclos de potencia s- $\mathrm{CO}_{2}$ Brayton $\mathrm{RC}$ con recuperación de calor y recalentamiento en función del UA de los recuperadores de calor y de TIT.

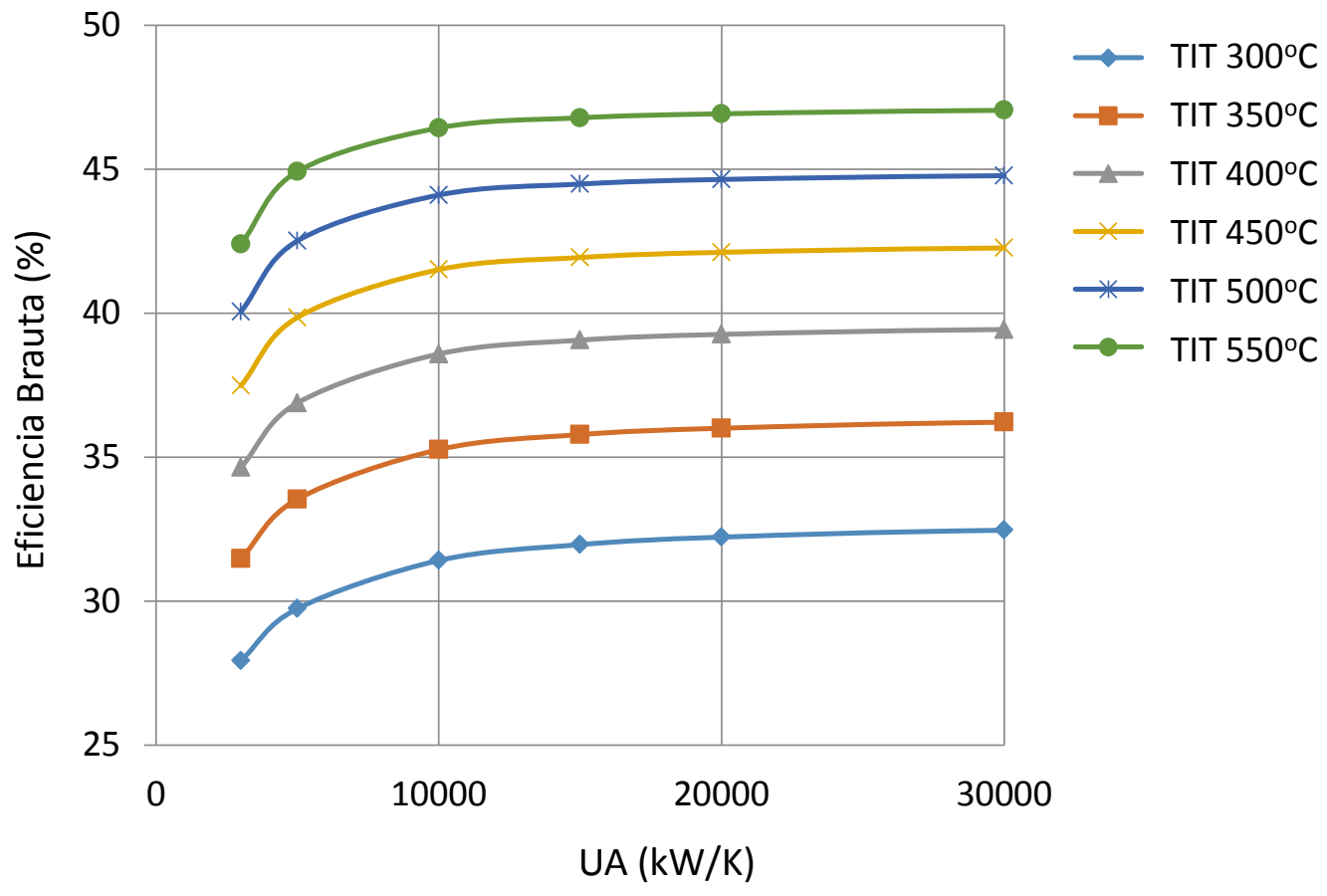

Figura 9.10. Eficiencia Neta de los ciclos de potencia s- $\mathrm{CO}_{2}$ Brayton PCRC con recuperación de calor y recalentamiento en función del UA de los recuperadores de calor y de TIT.

En este apartado se han simulado diferentes configuraciones de campo solar Dual-Loop, integrado por diferentes fluidos caloportadores (aceites térmicos, DSG y sales fundidas). A continuación, se definen las alternativas consideradas: 
9. Plantas termosolares con Colectores Lineales, configuración del campo solar Dual Loop acopladas a ciclos de potencia s- $\mathrm{CO}_{2}$ Brayton

- DSG en combinación con Hitec XL, designación "2: Dual (DSG+Hitec XL)", tablas 9.11 a 9.16 .

- DSG en combinación con aceites térmicos, designación "3: Dual (DSG+Thermal Oil)", tablas 9.11 a 9.16 .

- DSG en combinación con sales fundidas "Solar Salt", designación "4: Dual (DSG+Solar Salt)", tablas 9.11 a 9.16 .

- Aceites Térmicos en combinación con sales fundidas Solar Salt, designación "8: Dual", tablas 9.11 a 9.16 .

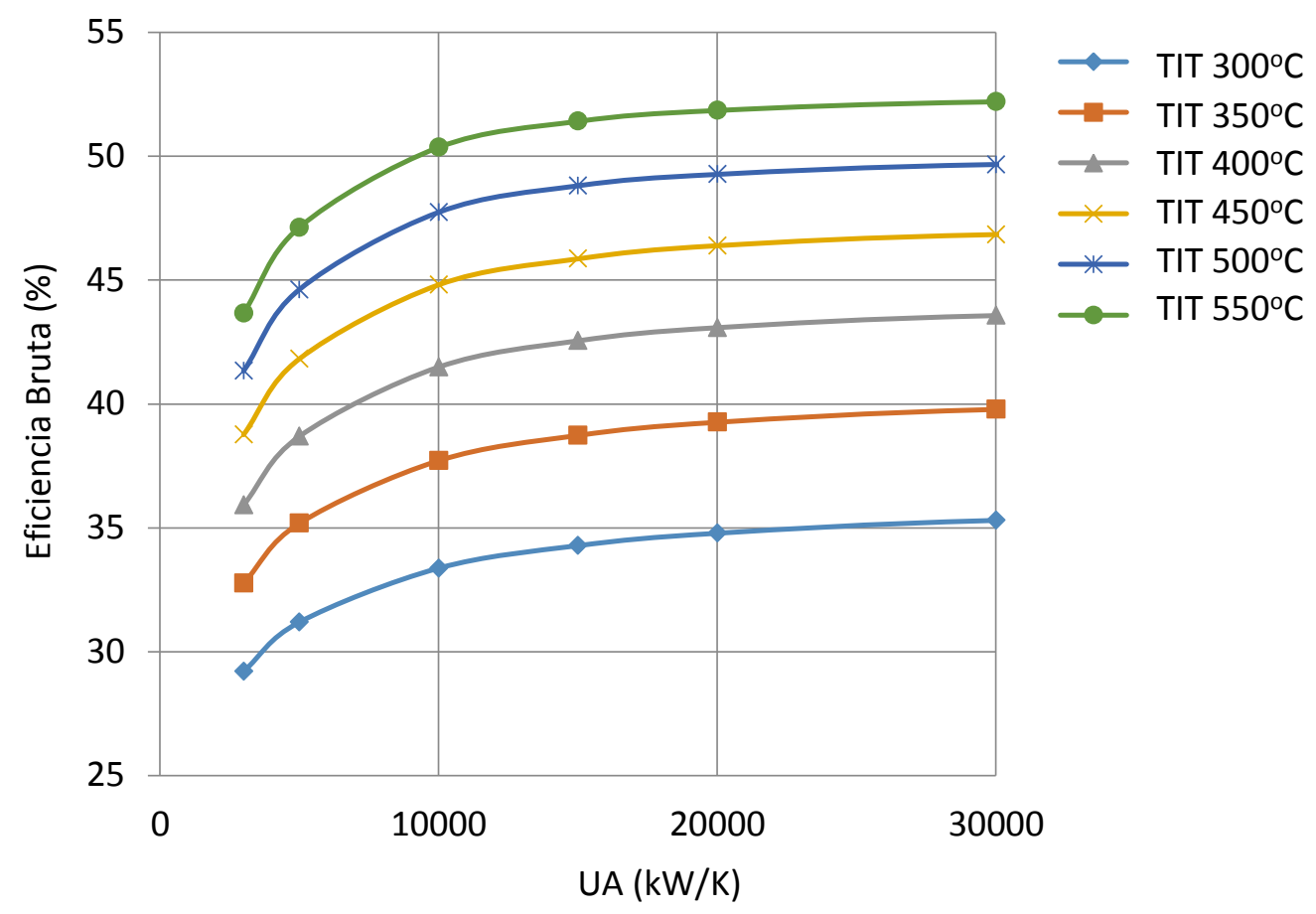

Figura 9.11. Eficiencia Neta de los ciclos de potencia s- $\mathrm{CO}_{2}$ Brayton $\mathrm{RCMCl}$ con recuperación de calor y recalentamiento en función del UA de los recuperadores de calor y de TIT.

\subsubsection{Caracterización del ciclo de potencia}

Los resultados de eficiencia bruta y neta, para diferentes tamaños térmicos de los recuperadores se detallan en las tablas 1 a 6 del anexo. Los resultados más significativos se resumen en las tablas 9.11 a 9.13. En las citadas tablas también se resumen la potencia bruta, fijada en todas las simulaciones en $50 \mathrm{MWe}$, y la potencia neta. Se puede apreciar como los valores de potencia neta son diferentes para las diferentes configuraciones simuladas. Ponemos especial atención en los consumos auxiliares debidos a los compresores en los campo solares en la configuración con DSG.

Tabla 9.11. Centrales Termosolares con campo Dual-Loop acopladas a ciclo de potencia s- $\mathrm{CO}_{2}$ Brayton RC. Eficiencia y producción de energía.

\begin{tabular}{llllll}
\hline $\begin{array}{l}\text { TIT } \\
\left({ }^{\circ} \mathrm{C}\right)\end{array}$ & UA $(\mathrm{kW} / \mathrm{K})$ & $\begin{array}{l}\text { Eficiencia } \\
\text { Bruta }(\%)\end{array}$ & $\begin{array}{l}\text { Potencia } \\
\text { Bruta }(\mathrm{kwe})\end{array}$ & $\begin{array}{l}\text { Eficiencia } \\
\text { Neta }(\%)\end{array}$ & $\begin{array}{l}\text { Potencia } \\
\text { Neta }(\mathrm{kWe})\end{array}$ \\
\hline 450 & 3000 & 38.43 & 50002 & 37.17 & 48361
\end{tabular}


9. Plantas termosolares con Colectores Lineales, configuración del campo solar Dual Loop acopladas a ciclos de potencia s- $\mathrm{CO}_{2}$ Brayton

\begin{tabular}{llllll}
500 & 3000 & 40.98 & 50000 & 39.65 & 48371 \\
450 & 5000 & 41.49 & 50005 & 40.15 & 48389 \\
500 & 5000 & 44.29 & 50001 & 42.86 & 48389 \\
450 & 10000 & 44.54 & 50006 & 43.11 & 48402 \\
500 & 10000 & 47.50 & 50004 & 45.98 & 48405 \\
450 & 15000 & 45.70 & 50000 & 44.24 & 48401 \\
500 & 15000 & 48.67 & 50004 & 47.05 & 48339 \\
450 & 20000 & 46.22 & 50002 & 44.75 & 48405 \\
500 & 20000 & 49.21 & 50004 & 47.5 & 48271 \\
\hline
\end{tabular}

Tabla 9.12. Centrales Termosolares con campo Dual-Loop acopladas a ciclo de potencia s- $\mathrm{CO}_{2}$ Brayton PCRC. Eficiencia y producción de energía.

\begin{tabular}{llllll}
\hline TIT & $\begin{array}{l}\text { UA } \\
\left({ }^{\circ} \mathrm{C}\right)\end{array}$ & $\begin{array}{l}\text { Eficiencia } \\
(\mathrm{kW} / \mathrm{K})\end{array}$ & $\begin{array}{l}\text { Potencia } \\
\text { Bruta }(\%)\end{array}$ & $\begin{array}{l}\text { Eficiencia } \\
\text { Bruta }(\mathrm{kwe})\end{array}$ & $\begin{array}{l}\text { Potencia } \\
\text { Neta }(\%)\end{array}$ \\
\hline 450 & 3000 & 38.57 & 50003 & 37.32 & 48372 \\
500 & 3000 & 41.08 & 50003 & 39.74 & 48375 \\
450 & 5000 & 40.89 & 50007 & 39.57 & 48389 \\
500 & 5000 & 43.50 & 50005 & 42.10 & 48390 \\
450 & 10000 & 42.53 & 50007 & 41.16 & 48396 \\
500 & 10000 & 45.09 & 50004 & 43.64 & 48395 \\
450 & 15000 & 42.96 & 50006 & 41.57 & 48396 \\
500 & 15000 & 45.48 & 50004 & 44.02 & 48397 \\
450 & 20000 & 43.13 & 50005 & 41.74 & 48397 \\
500 & 20000 & 45.64 & 50003 & 44.17 & 48397 \\
\hline
\end{tabular}

Tabla 9.13. Centrales Termosolares con campo Dual-Loop acopladas a ciclo de potencia s- $\mathrm{CO}_{2}$ Brayton RCMCl. Eficiencia y producción de energía.

\begin{tabular}{llllll}
\hline $\begin{array}{l}\text { TIT } \\
\left({ }^{\circ} \mathrm{C}\right)\end{array}$ & $\begin{array}{l}\text { UA } \\
(\mathrm{kW} / \mathrm{K})\end{array}$ & $\begin{array}{l}\text { Eficiencia } \\
\text { Bruta }(\%)\end{array}$ & $\begin{array}{l}\text { Potencia } \\
\text { Bruta }(\mathrm{kwe})\end{array}$ & $\begin{array}{l}\text { Eficiencia } \\
\text { Neta }(\%)\end{array}$ & $\begin{array}{l}\text { Potencia } \\
\text { Neta }(\mathrm{kWe})\end{array}$ \\
\hline 450 & 3000 & 38.86 & 50004 & 37.58 & 48357 \\
500 & 3000 & 41.44 & 50000 & 40.09 & 48365 \\
450 & 5000 & 41.73 & 50000 & 40.37 & 48374 \\
500 & 5000 & 44.50 & 50006 & 43.06 & 48389 \\
450 & 10000 & 44.84 & 50004 & 43.39 & 48393 \\
500 & 10000 & 47.78 & 50004 & 46.25 & 48402 \\
450 & 15000 & 45.97 & 50000 & 44.50 & 48400 \\
500 & 15000 & 48.91 & 50004 & 47.26 & 48326 \\
450 & 20000 & 46.49 & 50001 & 45.00 & 48403 \\
500 & 20000 & 49.40 & 50001 & 47.72 & 48292 \\
\hline
\end{tabular}

\subsection{2. Área de apertura efectiva y estimación de costes del campo solar}

Los resultados de la simulación y diseño de los correspondientes colectores solares PTC están detallados en las tablas 7 a 12 del anexo. Los resultados más significativos se incluyen en las tablas 9.14 a 9.16. Los valores de coste unitarios menores corresponden a las soluciones de diseño con campos solares con configuración Dual-Loop. 
9. Plantas termosolares con Colectores Lineales, configuración del campo solar Dual Loop acopladas a ciclos de potencia s- $\mathrm{CO}_{2}$ Brayton

Tabla 9.14. Centrales Termosolares con campo Dual-Loop (oil+sal) acopladas a ciclo de potencia s- $\mathrm{CO}_{2}$ Brayton RC. Área de apertura efectiva y estimación de costes de los colectores PTC.

\begin{tabular}{llllll}
\hline TIT $\left({ }^{\circ} \mathrm{C}\right)$ & UA $(\mathrm{kW} / \mathrm{K})$ & $\begin{array}{l}\text { PTC SF }\left(\mathrm{m}^{2}\right) \\
\text { Acerolnoxidable }\end{array}$ & $\begin{array}{l}\text { PTC SF } \\
\text { Acero }\left(\mathrm{m}^{2}\right)\end{array}$ & PTC SF (M\$) & $\begin{array}{l}\text { SF Coste Unitario } \\
(\mathrm{M} \$ / \mathrm{MWe})\end{array}$ \\
\hline 450 & 3000 & 97661 & 102528 & 87.47 & 1.81 \\
500 & 3000 & 136428 & 56628 & 89.65 & 1.85 \\
450 & 5000 & 104769 & 81226 & 83.03 & 1.72 \\
500 & 5000 & 144630 & 34970 & 85.62 & 1.77 \\
450 & 10000 & 111783 & 61956 & 79.30 & 1.64 \\
500 & 10000 & 152239 & 16028 & 82.31 & 1.70 \\
450 & 15000 & 114128 & 55323 & 77.98 & 1.61 \\
500 & 15000 & 154664 & 9756 & 81.17 & 1.68 \\
450 & 20000 & 114970 & 52622 & 77.39 & 1.60 \\
500 & 20000 & 155312 & 7381 & 80.60 & 1.67 \\
\hline
\end{tabular}

Tabla 9.15. Centrales Termosolares con campo Dual-Loop acopladas a ciclo de potencia PCRC s- $\mathrm{CO}_{2}$ Brayton. Área de apertura efectiva y estimación de costes de los colectores PTC.

\begin{tabular}{llllll}
\hline TIT $\left({ }^{\circ} \mathrm{C}\right)$ & UA $(\mathrm{kW} / \mathrm{K})$ & $\begin{array}{l}\text { PTC SF }\left(\mathrm{m}^{2}\right) \\
\text { Acero Inoxidable }\end{array}$ & $\begin{array}{l}\text { PTC SF } \\
\text { Acero } \\
\left(\mathrm{m}^{2}\right)\end{array}$ & $\begin{array}{l}\text { PTC SF Coste } \\
(\mathrm{M} \$)\end{array}$ & $\begin{array}{l}\text { SF Coste Unitario } \\
(\mathrm{M} / \mathrm{MWe})\end{array}$ \\
\hline 450 & 3000 & 93918 & 105424 & 86.68 & 1.79 \\
500 & 3000 & 130308 & 62092 & 88.63 & 1.83 \\
450 & 5000 & 98582 & 89888 & 83.18 & 1.72 \\
500 & 5000 & 135162 & 47248 & 85.49 & 1.77 \\
450 & 10000 & 101246 & 80241 & 80.89 & 1.67 \\
500 & 10000 & 137544 & 38824 & 83.51 & 1.73 \\
450 & 15000 & 101572 & 78150 & 80.27 & 1.66 \\
500 & 15000 & 137731 & 37217 & 83.00 & 1.72 \\
450 & 20000 & 101626 & 77379 & 80.00 & 1.65 \\
500 & 20000 & 137727 & 36633 & 82.78 & 1.71 \\
\hline
\end{tabular}

Tabla 9.16. Centrales Termosolares con campo Dual-Loop acopladas a ciclo de potencia s- $\mathrm{CO}_{2}$ Brayton $\mathrm{RCMCl}$. Área de apertura efectiva y estimación de costes de los colectores PTC.

\begin{tabular}{llllll}
\hline TIT $\left({ }^{\circ} \mathrm{C}\right)$ & UA $(\mathrm{kW} / \mathrm{K})$ & $\begin{array}{l}\text { PTC SF }\left(\mathrm{m}^{2}\right) \\
\text { Acero Inoxidable }\end{array}$ & $\begin{array}{l}\text { PTC SF } \\
\text { Acero } \\
\left(\mathrm{m}^{2}\right)\end{array}$ & $\begin{array}{l}\text { PTC SF Coste } \\
(\mathrm{M} \$ \text { ) }\end{array}$ & $\begin{array}{l}\text { SF Coste Unitario } \\
(\mathrm{M} \$ \mathrm{MWe})\end{array}$ \\
\hline 450 & 3000 & 97133 & 100863 & 86.58 & 1.79 \\
500 & 3000 & 135190 & 55778 & 88.71 & 1.83 \\
450 & 5000 & 103473 & 81443 & 82.46 & 1.70 \\
500 & 5000 & 143064 & 35678 & 85.10 & 1.76 \\
450 & 10000 & 109813 & 62808 & 78.63 & 1.62 \\
500 & 10000 & 149809 & 17468 & 81.63 & 1.69 \\
450 & 15000 & 111865 & 56543 & 77.30 & 1.60 \\
500 & 15000 & 151799 & 11785 & 80.49 & 1.67 \\
450 & 20000 & 112554 & 54046 & 76.71 & 1.58 \\
500 & 20000 & 152338 & 9671 & 79.97 & 1.66 \\
\hline
\end{tabular}


9. Plantas termosolares con Colectores Lineales, configuración del campo solar Dual Loop

acopladas a ciclos de potencia s- $\mathrm{CO}_{2}$ Brayton

\subsection{Conclusiones}

En este capítulo se han estudiado plantas termosolares con colectores lineales y la combinación de dos HTF, sales fundidas y aceite térmico en una misma planta termosolar. Esta tecnología de campo solar conocida como Dual-Loop, ya adaptada en numerosos trabajos a ciclos de potencia Rankine [9-11], en este capítulo se han acoplado con ciclos de potencia s$\mathrm{CO}_{2}$ Brayton, figuras 9.2-9.8. Las configuraciones Dual-Loop, permiten aprovechar las sinergias resultantes de la combinación de dos fluidos caloportadores diferentes, en los receptores solares. En el campo solar con Dowtherm A como HTF, se calienta el fluido del trabajo del BOP desde $300^{\circ} \mathrm{C}$ hasta alrededor de $400^{\circ} \mathrm{C}$. Y un segundo campo solar, con sales fluidas como HTF, se incrementa la temperatura del fluido de trabajo del BOP desde $400^{\circ} \mathrm{C}$ hasta $550^{\circ} \mathrm{C}$.

La potencia bruta generada en todas las configuraciones estudiadas se ha fijado en $50 \mathrm{MWe}$. Respecto a la metodología de trabajo, se ha realizado una primera simulación para optimización multiparámetro de las variables de operación de los ciclos s- $\mathrm{CO}_{2}$ Brayton con los algoritmos SUBPLEX [31], UOBYQA [32], NEWUOA [33], explicados en el capítulo 2, y posteriormente con Thermoflow [36] se ha realizado la simulación en detalle de los campos solares y dimensionamiento de los equipos del ciclo de potencia.

Las configuraciones con campo solar Dual-Loop y ciclo de potencia SB s- $\mathrm{CO}_{2}$ Brayton, proporcionan un rendimiento bruto de hasta un $45 \%$ con una $\mathrm{TIT}=550^{\circ} \mathrm{C}$, figura 9.3 y 9.4 , que teniendo en cuenta las pérdidas del ciclo se reduce al $\approx 43 \%$, tabla 9.8 . Se concluye que los ciclos de potencia s- $\mathrm{CO}_{2}$ Brayton constituyen una mejora sustancial de la eficiencia energética de la planta termosolar, ya que la configuración Dual-Loop con ciclo Rankine proporcionaba una eficiencia bruta del $41.8 \%$, valor similar al obtenido en las tablas 1.11 y 1.12 en el capítulo 1, con Hitec XL como HTF.

El incremento de eficiencia energética de las configuraciones Dual-Loop+s- $\mathrm{CO}_{2}$, es aun mayor con el resto de tipologías de ciclos s- $\mathrm{CO}_{2}$ Brayton estudiadas en el apartado 9.8 (RC, PCRC y $\mathrm{RCMCl}$ ), figuras $9.6,9.7$ y 9.8. En estos casos se obtienen valores superiores de eficiencia energética de la planta termosolar, apartado 9.8 .1 y tablas 1 a 6 del anexo.

Desde el punto de vista económico, el coste de inversión de campo solar presenta un valor predominante a la hora de evaluar el coste total de la planta termosolar con colectores lineales. Las soluciones de diseño propuestas en este capítulo con campos solares de tipología Dual-Loop permiten aprovechar las sinergias proporcionadas por las diferentes tecnologías de HTF estudiadas: aceites térmicos, sales fundidas y DSG. En las tablas 7 a 12 del anexo, se confirma que los campos solares Dual-Loop son los que presentan menores costes de inversión unitarios. Como hemos reiterado a lo largo de este capítulo el coste de los materiales cuando se utilizan aceites térmicos (acero al carbono) o DSG (acero inoxidable ferrítico A335 P22) [3738], se reduce sensiblemente en comparación con la opción de sales fundidas que requieren materiales muy resistentes a la corrosión (acero inoxidables AISI 316, 316 Ti, 347) [39-40].

\subsection{Referencias}

[1] Solar Energy Generating Systems (SEGS). http://www.energy.ca.gov/sitingcases/solar/

[2] Concentrated Solar Power Project, NREL. http://www.nrel.gov 
9. Plantas termosolares con Colectores Lineales, configuración del campo solar Dual Loop acopladas a ciclos de potencia s- $\mathrm{CO}_{2}$ Brayton

[3] Relloso S. Experience with molten salt thermal storage in a commercial parabolic trough plant: Andasol-1 commissioning and operation. 15th SolarPaces Conference, 15. - 18. September, Berlin, Germany; 2009.

[4] F. De Luca, V.Ferraro, V.Marinelli. On the performance of CSP oil-cooled plants, with and without heat storage in tanks of molten salts. Energy 83 (2015) 230-239.

[5] F.Matino, A. Maccari. Molten salt receivers operated on parabolic trough demo plant and in laboratory conditions. SolarPACES 2014. Energy Procedia 69 (2015) 481-486.

[6] A. Maccaria, D. Bissib, G. Casuboloc, F. Guerrinid, L. Lucatelloe, G. Lunaf, A. Rivabeng, E. Savoldih, S. Tamanoi, M. Zuanellaj. Archimede Solar Energy Molten Salt Parabolic Trough Demo Plant: A Step Ahead towards the New Frontiers of CSP. Energy Procedia. Volume 69, May 2015, Pages 1643-165.

[7] G. Morin, M. Karl, M. Mertins, M. Seling. Molten Salt as a Heat Transfer Fluid in a Linear Fresnel Collector - Commercial Application Backed by Demonstration. Energy Procedia.

Volume 69, May 2015, Pages 689-698. International Conference on Concentrating Solar Power and Chemical Energy Systems, SolarPaces 2014.

[8] C. Turchi, P.Kurup, S.Akar, F.Flores. Domestic Material Content in Molten-Salt

Concentrating Solar Power Plants. NREL. NREL/TP-5500-64429, August 2015.

[9] W. Gaggioli. Effects Assessment of 10 Functioning Years on Main Components of ENEA's Molten Salt Experimental PCS Facility. SolarPACES 2015.

[10] A. Maccari. Archimede Solar Energy Molten Salt Parabolic Trough Demo Plant: Improvements and Second Year of Operation.SolarPACES 2015.

[11] A. Vogel. Freeze Protection in Line-Focusing Systems: Comparison of Different Collector Types, Molten Salt Mixtures and Heat Sources. SolarPACES 2015.

[12] M. Eickhoff. New Operating Strategies for Molten Salt in Line Focusing Solar Fields - Daily Drainage and Solar Preheating. SolarPACES 2015.

[13] Lang C, Cuthbert J. Evaluation of dual loop design for parabolic trough. 18th SolarPaces Conference, 11. - 14. September, Marrakech, Morocco; 2012.

[14] Christoph Lang. Dow Chemical Companny Europe GmbH. http://www.dow.com

[15] Joseph R. Shinners. Analysis of Dual Loop Parabolic Trough Concentrating Solar Power Plants. University of Wisconsin-Madison (2014). http://sel.me.wisc.edu

[16] A.K. Vogel, H. Reilinga, T.P. Fluria W.J. Platzera. High temperatures in line focusing systems: Dual loop cycle efficiency and heat losses. Energy Procedia 69 (2015) $1461-1470$.

[17] Q.Liu, Z.Bai, J.Sun, Y.Yan, Z.Gao, H.Jin. Thermodynamics investigation of a solar power system integrated oil and molten salt as heat transfer fluids. App. Th. Eng. 93 (2016) 967-977.

[18] Gary E. Rochau, Thomas Drennen, James Pasch, Darryn Fleming, Matthew Carlson, Lon Dawson, Alan Kruizenga. Commercialization of SCO2 Brayton power cycles. $2016 \mathrm{CO} 25^{\text {th }}$ International Symposium, San Antonio, Texas, USA.

[19] Rubbia C, Antonaia A, Arabito C, Avitabile M, Boccardi G, Braccio G, et al. Solar thermal energy production: guidelines and future programs of ENEA. June 2001. ENEA/TM/PRES/2001_07. Available at: http://www.enea.it/com/ing.

[20] D. Kearney, U. Herrmann, P. Nava, B. Kelly, R. Mahoney, J. Pacheco, R. Cable, N.

Potrovitza, D. Blakeand H. Price. Assessment of a Molten Salt Heat Transfer Fluid in a Parabolic Trough Solar Field. J. Sol. Energy Eng 125(2), 170-176 (May 08, 2003).

[21] A. Maccari ENEA activities on CSP (concentrating solar power) technologies, Technologies Parabolic TroughWorkshop, 2006.

[22] Y. Wang, Q. Liu, J. Lei, H. Jin. A three-dimensional simulation of a parabolic trough solar collector system using molten salt as heat transfer fluid. Appl. Therm. Eng. 70 (2014) 462-476. 
9. Plantas termosolares con Colectores Lineales, configuración del campo solar Dual Loop acopladas a ciclos de potencia s- $\mathrm{CO}_{2}$ Brayton

[23] A. Giostri, M. Binotti, M. Astolfi, P. Silva, E. Macchi, G. Manzolini, Comparison of different solar plants based on parabolic trough technology, Sol. Energy 86 (2012) 1208-1221.

[24] F. Zaversky, R. Medina, J. Garcia-Barberena, M. Sanchez, D. Astrain, Objectoriented modeling for the transient performance simulation of parabolic trough collectors using molten salt as heat transfer fluid, Sol. Energy 95 (2013) 192-215.

[25] J.W. Raade, D. Padowitz. Development of molten salt heat transfer fluid with low melting point and high thermal stability. J. Sol. Energy Eng. 133 (2011) 310131-310136.

[26] A.Maccari, Archimede Solar Energy. Archimede Solar Energy Molten Salt Parabolic Trough Demo Plant: A Step Ahead Towards the New Frontiers of CSP. SolarPaces 2014.

[27] Burkholder F, Kutscher C. Heat loss testing of Schott's 2008 PTR70 parabolic trough receiver. NREL/TP-550-45633. May 2009.

[28] Solar Novatec. SAM lineal Fresnel solar boiler model, SAM Webinar. NREL SAM Conference 2013.

[29] Archimede solarenergy. Receiver Tubes.http://www.archimedesolarenergy.it

[30] Dyreby, J. (2014) Modeling the Supercritical Carbon Dioxide Brayton Cycle with Recompression.University of Wisconsin-Madison.

[31] T. Rowan, Functional Stability Analysis of Numerical Algorithms, Ph.D. thesis, Department of Computer Sciences, University of Texas at Austin, 1990.

[32] Powell, M. J. D. (2002). UOBYQA: unconstrained optimization by quadratic approximation. Mathematical Programming, Series B (Springer) 92: 555-582. doi: 10.1007/s101070100290.

[33] Powell, M. J. D. (November 2004). The NEWUOA software for unconstrained optimization without derivatives (PDF) (Report). Department of Applied Mathematics and Theoretical Physics, Cambridge University. DAMTP 2004/NA05. Retrieved 2014-01-14.

[34] T.Neises, C. Turchi. A comparison of Supercritical Carbon Dioxide Power Cycle Configurations with an Emphasis on CSP Applications. Energy Procedia of SolarPACES 2013, Volume 49, 2014, Pages 1187-1196.

[35] Gavic, D. (2012). Investigation Of Water, Air, and Hybrid Cooling For Supercritical Carbon Dioxide Brayton Cycles. University of Wisconsin-Madison.

[36] Thermoflow Inc. http://www.thermoflow.com/

[37] M. Biencinto, L.González, L.Valenzuela. A quasi-dynamic simulation model for direct steam generation in parabolic troughs using TRNSYS. Applied Energy 161 (2016) 133-142.

[38] J.B.Feldhoff, T.Hirsch, R.Pitz-Paal, L.Valenzuela. Analysis and potential of once-through steam generators in line focus systems - Final results of the DUKE project. SolarPaces 2015. AIP Conf. Proc. 1734, 100006 (2016).

[39] Klaus-J. Riffelmann, Timo Richert, Thomas Kuckelkorn. Optimized molten salt receivers for ultímate trough solar fields. SolarPace 2015. AIP Conf. Proc. 1734, 020021 (2016).

[40] Augusto Maccari, Sandro Donnola, Francesca Matino, Shiro Tamano. Archimede solar energy molten salt parabolic trough demo plant: Improvements and secondary year of operation. SolarPaces 2015. AIP Conf. Proc. 1734, 100007 (2016). 
9. Plantas termosolares con Colectores Lineales, configuración del campo solar Dual Loop acopladas a ciclos de potencia s- $\mathrm{CO}_{2}$ Brayton 
Capítulo 10

\title{
Plantas Termosolares de Colectores Lineales con Sales Fundidas o DSG como fluido caloportador acopladas a ciclos de potencia supercríticos Brayton con $\mathrm{C}_{2} \mathrm{H}_{6}$ como fluido de trabajo
}

\author{
Resumen \\ Debido a la similitud de las propiedades físicas en condiciones termodinámicas cercanas a \\ criticidad, en este capítulo se han estudiado dos fluidos de trabajo en los ciclos de potencia \\ Brayton en centrales termosolares: el Etano $\left(\mathrm{s}-\mathrm{C}_{2} \mathrm{H}_{6}\right)$ y el Dióxido de Carbono $\left(\mathrm{s}-\mathrm{CO}_{2}\right)$, ambos \\ fluidos en estado supercrítico.
}

Dos tipologías de colectores solares lineales han sido modelizados PTC y LF. Respecto al fluido caloportador en el campo solar se han analizado dos alternativas: las sales fundidas o la generación directa de vapor. Respecto a la configuración del ciclo de potencia Brayton, se han considerado las cuatro disposiciones ya definidas en capítulos anteriores [1]: SB, RC, PCRC, RCMCl.

Como principal resultado, se ha confirmado que los ciclos de potencia s- $\mathrm{C}_{2} \mathrm{H}_{6}$ Brayton proporcionan mayor eficiencia que los ciclos $\mathrm{s}-\mathrm{CO}_{2}$ Brayton, operando bajo similares condiciones termodinámicas. Es decir, con TIT $=300^{\circ} \mathrm{C}-550^{\circ} \mathrm{C}$, y TIP $=250$ bar. Como ejemplo más significativo de los resultados obtenidos, cabe destacar la eficiencia neta de la planta con ciclo $\mathrm{s}-\mathrm{C}_{2} \mathrm{H}_{6}$ Brayton $\mathrm{RCMCl}$, es de $42.11 \%$ para una $\mathrm{TIT}=400^{\circ} \mathrm{C}$. Sin embargo, el rendimiento de la planta termosolar con ciclo de potencia s- $\mathrm{CO}_{2}$ Brayton $\mathrm{RCMCl}$ es del $40 \%$.

En este capítulo también se realiza una comparación entre las plantas termosolares acopladas a ciclos (s- $\mathrm{CO}_{2}$ ó s- $\mathrm{C}_{2} \mathrm{H}_{6}$ ) Brayton, con la tecnología DSG en los colectores lineales y ciclo de potencia Rankine con DRH. Esta segunda tecnología, considerada de referencia en este capítulo, proporciona unos rendimientos energéticos netos de planta entre $40-41 \%$, para una temperatura de entrada en la turbina principal de $550^{\circ} \mathrm{C}$.

\subsection{Introducción}

Los ciclos Rankine son los más comunes para generación de potencia en las plantas termosolares. La eficiencia de los citados ciclos con el estado actual de la tecnología es aproximadamente un $43 \%$ con enfriamiento con agua, y de un $41.2 \%$ con enfriamiento mediante condensadores de aire en convección forzada [2]. Con la tecnología de ciclo Rankine, la presión del vapor vivo está limitada a condiciones termodinámicas subcríticas, normalmente con $\mathrm{TIP}=120$ bar y $\mathrm{TIT}=550^{\circ} \mathrm{C}$. Una posibilidad ya estudiada en capítulos anteriores de esta tesis para incrementar la potencia bruta de la instalación termosolar, es su integración con un ciclo de potencia supercrítico Rankine. Como también se ha indicado la capacidad de las turbinas de vapor de agua supercrítico disponible comercialmente es como mínimo de 400 MWe o mayor. En la referencia [3], se ha desarrollado el diseño básico de una planta termosolar con torre central y campo de heliostatos, y una capacidad de $400 \mathrm{MWe}$, acoplada a un ciclo de potencia supercritico Rankine, con una eficiencia bruta de $48.78 \%$ para una TIT = 
10. Plantas Termosolares con Sales Fundidas o DSG como fluido caloportador acopladas a ciclos de potencia supercríticos Brayton con $\mathrm{C}_{2} \mathrm{H}_{6}$ como fluido de trabajo

$590^{\circ} \mathrm{C}$. Recientemente, el SNL, en colaboración con Siemens Energy, han publicado un informe analizando la viabilidad de la adaptación de las turbinas de vapor subcrítico, marca comercial SST-900, para su utilización con vapor supercrítico en las plantas termosolares con torres central [4]. En este estudio se han considerado una presión de vapor vivo a la entrada de la turbina supercrítica entre 230 y 260 bar, y un rango de potencia generada entre 140 y 200 MWe. Un análisis similar se ha desarrollado en el capítulo 4 [5], para una planta termosolar con colectores PTC o LF acoplada a un ciclo de potencia supercrítico Rankine, obteniendo una eficiencia neta del $44 \%$ para una $\mathrm{TIT}=550^{\circ} \mathrm{C}$.

Otra solución para incrementar la eficiencia energética de plantas termosolares con colectores lineales, es su acoplamiento con los ciclos de potencia $\mathrm{s}-\mathrm{CO}_{2}$ Brayton, tal y como se ha estudiado en los capítulos 4 al 9. Los ciclos $\mathrm{s}-\mathrm{CO}_{2}$ Brayton constituyen una alternativa a los ciclos tradicionales Rankine, para incrementar la potencia neta de la planta termosolar y reducir el coste final de la electricidad producida en esta tipología de instalaciones. Los principales beneficios de la tecnología de ciclos s- $\mathrm{CO}_{2}$ Brayton, se sustenta en la utilización de un fluido de trabajo en estado supercrítico, con una alta densidad: la densidad en el punto crítico del $\mathrm{CO}_{2}$ es de $467.6 \mathrm{~kg} / \mathrm{m}^{3}$, y la densidad del $\mathrm{C}_{2} \mathrm{H}_{6}$ es $206.2 \mathrm{~kg} / \mathrm{m}^{3}$. Pero la principal razón para la selección del $\mathrm{CO}_{2}$ como principal fluido para desarrollo industrial de los equipos de los ciclos de potencia Brayton, es su punto de criticidad ( $31^{\circ} \mathrm{C}$ y 73.8 bar). El $\mathrm{C}_{2} \mathrm{H}_{6}$ se encuentra en estado supercrítico en condiciones ambientales, con alta densidad a la entrada del compresor para optimizar la eficiencia y reducir el trabajo de compresión. El punto crítico del $\mathrm{C}_{2} \mathrm{H}_{6}$ es de $32.2^{\circ} \mathrm{C}$ y 48.7 bar, muy similar al punto crítico del $\mathrm{CO}_{2}$ en cuanto a temperatura crítica.

Los ciclos de potencia s- $\mathrm{C}_{2} \mathrm{H}_{6}$ Brayton, fueron propuestos por Jeffrey A. Perez et al. [6], como un potencial sustitutivo del s- $\mathrm{CO}_{2}$ en los sistemas de conversión de potencia en los reactores nucleares Liquid Metal cooled Fast Breeder Reactor (LMFBR). En este estudio son analizados los potenciales riesgos y desventajas del uso del s- $\mathrm{C}_{2} \mathrm{H}_{6}$ como fluido de trabajo en los ciclos de potencia: descomposición química, inflamabilidad, auto-ignición. Así mismo, en los trabajos [7-8] el s- $\mathrm{C}_{2} \mathrm{H}_{6}$ es propuesto como posible alternativa al $\mathrm{s}-\mathrm{CO}_{2}$ para los ciclos de potencia Brayton, estudiando diferentes rangos del factor de Carnot (CN), definido como la relación entre la temperatura máxima y la temperatura mínima del ciclo Brayton. Con CN entre 1.5 y 2.5 , la temperatura mayor de foco caliente estudiada es inferior a $550^{\circ} \mathrm{C}$. Otro parámetro termodinámico decisivo para la selección del s- $\mathrm{C}_{2} \mathrm{H}_{6}$, es la relación entre el calor específico a presión constante $C_{p}$, y la constante de los gases $R$, medidos ambos en condiciones de gas ideal, $\left(C_{p} / R=4.5\right.$ para el $s-C_{2}$, y $C_{p} / R=6.418$ para el $\left.s-C_{2} H_{6}\right)$. Como se indica en la referencia [7-8], fluidos con valores altos del parámetro $C_{p} / R$ dan lugar a un mejor rendimiento energético del ciclo de potencia Brayton, que con fluidos con valores de $C_{p} / R$ bajos.

Como se ha tratado en los capítulos anteriores de esta tesis, se han publicado numerosos estudios sobre la adaptación de los ciclos s- $\mathrm{CO}_{2}$ Brayton a las plantas termosolares [9-13], como primera referencia se destaca la patente US 2012/0216536 A1, Aug. 30, 2012. En este capítulo se han considerado las mismas configuraciones de ciclos s- $\mathrm{C}_{2} \mathrm{H}_{6}$ Brayton definidas por NREL [1] en Estados Unidos y detalladas anteriormente: el ciclo simple con recuperación de calor Simple Brayton (SB), el ciclo con recompresión Recompression (RC), el ciclo con 
10. Plantas Termosolares con Sales Fundidas o DSG como fluido caloportador acopladas a ciclos de potencia supercríticos Brayton con $\mathrm{C}_{2} \mathrm{H}_{6}$ como fluido de trabajo

enfriamiento parcial y recompressión Partial Cooling with Recompresion (PCRC) y el ciclo con recompresión y enfriamiento intermedia en el compresor principal Recompression with Main Compression Intercooling ( $\mathrm{RCMCl})$. Las principales ventajas del ciclo simple SB es su reducido número de equipos y por tanto presumiblemente su bajo coste de inversión, figura 10.1, sin embargo, es importante resaltar el gran tamaño térmico (UA) requerido en su recuperador de calor, en relación con el resto de configuraciones de ciclos mencionados.

El objetivo fundamental del ciclo con recompresión (RC), figura 10.2, es reducir el pinch point debido a la utilización de un solo recuperador. Con dos recuperadores, uno de baja temperatura (LTR) y otro de alta temperatura (HTR), se obtienen menores diferencias de temperatura en el intercambiador de calor entre el campo solar y el ciclo de potencia, es decir, en el intercambiador de calor primario (PHX). Las necesidades de compresión del ciclo también se dividen entre el compresor principal y el secundario (también llamado recompressor). Esta configuración RC es la forma más simple de introducir enfriamiento intermedio antes de compresión y aprovechar los altos valores de densidad del fluido supercrítico. La configuración PCRC es muy similar a la RC, pero con dos etapas de enfriamiento intermedio y enfriamiento parcial antes de la separación de flujos a la entrada del compresor principal, figura 10.3. La última configuración $\mathrm{RCMCl}$, con dos enfriadores para minimizar el trabajo de compresión, figura 10.4. En este estudio se han simulado y comparado el comportamiento energético de los ciclos Brayton con dos fluidos supercríticos el $\mathrm{CO}_{2}$ y el $\mathrm{C}_{2} \mathrm{H}_{6}$, y dos tipologías diferentes de campos solares con colectores lineales PTC y con colectores LF.

\subsection{Estabilidad del Etano en los ciclos de potencia supercríticos Brayton}

La descomposición química del $\mathrm{C}_{2} \mathrm{H}_{6}$ es uno de sus principales inconvenientes cuando se utiliza en ciclos de potencia Brayton. La principal consecuencia directa es la reducción del inventario de HTF en el ciclo y de alteración de sus propiedades físicas, particularmente en la zona de entrada a los compresores, y a las turbinas, impactando directamente en la eficiencia de la instalación. Como regla general, la alta temperatura de operación, impulsan la generación de subproductos del $\mathrm{C}_{2} \mathrm{H}_{6}$, y las altas presiones favorecen la recombinación de estos subproductos, reduciendo la cinética de descomposición química.

Bajo los parámetros termodinámicos que operan los ciclos Brayton, la reacción química más probable es la descomposición del Etano $\left(\mathrm{C}_{2} \mathrm{H}_{6}\right)$ en Etileno $\left(\mathrm{C}_{2} \mathrm{H}_{4}\right)$, tal y como se ha demostrado a escala industrial en el proceso de producción de Etileno [6]. El $\mathrm{C}_{2} \mathrm{H}_{6}$ se descompone en $\mathrm{C}_{2} \mathrm{H}_{4}$ generando a su vez $\mathrm{H}_{2}$ y $\mathrm{CH}_{4}$, elemento muy perjudicial para el rendimiento del ciclo, dada su baja densidad y por tanto baja eficiencia de compresión [14], ver ecuación (10-1).

$$
\mathrm{C}_{2} \mathrm{H}_{6} \rightarrow \mathrm{C}_{2} \mathrm{H}_{4}+\mathrm{H}_{2}
$$

El $\mathrm{C}_{2} \mathrm{H}_{4}$ se produce a escala industrial en un proceso petroquímico mediante el cracking con vapor de agua, en este proceso se calientan los hidrocarburos hasta temperaturas entre $750^{\circ} \mathrm{C}$ $950^{\circ} \mathrm{C}$ [15], temperaturas muy superiores a los $400^{\circ} \mathrm{C}$ fijados en este estudio como temperatura máxima de operación de los ciclos Brayton y a una presión del ciclo entre 50 bar y 250 bar, también muy superiores a las de producción del $\mathrm{C}_{2} \mathrm{H}_{4}$. 
10. Plantas Termosolares con Sales Fundidas o DSG como fluido caloportador acopladas a ciclos de potencia supercríticos Brayton con $\mathrm{C}_{2} \mathrm{H}_{6}$ como fluido de trabajo

Algunos autores [6] han trabajado la reacción química de disociación del $\mathrm{C}_{2} \mathrm{H}_{6}$ con el programa informático HSC Chemistry 6.0 [16-17], obteniendo una cantidad de $\mathrm{C}_{2} \mathrm{H}_{6}$ disociado del $0.6 \%$ con $550^{\circ} \mathrm{C}$ y 200 bar. Las ecuaciones (10-2 y 10-3) de disociación del $\mathrm{C}_{2} \mathrm{H}_{6}$ dan como resultados dos ecuaciones indicadas a continuación, que definen la cantidad de $\mathrm{C}_{2} \mathrm{H}_{6}$ disociado a temperatura y presión constantes.

$$
X=191 \cdot e^{\frac{-8511}{T}}
$$

"X" Fracción disociada como función de la temperatura T (K), a 200 bar de presión constante.

$$
X=\frac{0.0876}{P^{0.5}}
$$

"X" Fracción disociada como función de la presión $\mathrm{P}$ (bar), a $550^{\circ} \mathrm{C}$ de temperatura constante.

El HSC Chemistry está enfocado a cálculos de minimización de la energía, y todos los resultados están dados en condiciones de equilibrio, únicamente considerando la descomposición del $\mathrm{C}_{2} \mathrm{H}_{6}$ en $\mathrm{C}_{2} \mathrm{H}_{4}$, ver ecuación (10-1), sin embargo, en la referencia [6] también se tienen en cuenta los mecanismos de generación de radicales libres y se predice la producción del $\mathrm{CH}_{4}, \mathrm{C}_{3} \mathrm{H}_{8}, \mathrm{C}_{4} \mathrm{H}_{10}, \mathrm{C}_{2} \mathrm{H}_{4}$ como se establece [17]. El $\mathrm{C}_{2} \mathrm{H}_{6}$ experimenta de forma predominante una degradación en $\mathrm{CH}_{4}$, y también produce más $\mathrm{C}_{2} \mathrm{H}_{4}$ que el previsto en la ecuación (10-1) listada anteriormente. Es probable que la reacción del $\mathrm{C}_{2} \mathrm{H}_{6}$ en Etileno ocurra más rápido que la del $\mathrm{C}_{2} \mathrm{H}_{6}$ en $\mathrm{CH}_{4}$. En este caso es posible producir $\mathrm{C}_{2} \mathrm{H}_{4} \sin$ producir $\mathrm{CH}_{4}$ simultáneamente si este proceso se realiza con celeridad, antes de que la mezcla llegue a su equilibrio. $\mathrm{Si}$ es éste el caso, un ciclo de potencia $\mathrm{s}-\mathrm{C}_{2} \mathrm{H}_{6}$ Brayton requeriría una mayor inversión de investigación para optimizar el lazo de potencia, y la disposición física y dimensiones de los equipos que lo integran con objeto de reducir el tiempo del $\mathrm{C}_{2} \mathrm{H}_{6}$ a altas temperaturas y evitar así su descomposición química.

Otros fenómenos físicos que pueden impactar negativamente en el desarrollo industrial de los ciclos $s-\mathrm{C}_{2} \mathrm{H}_{6}$ Brayton son su autoignición por combinación con oxígeno o aire y su inflamabilidad. Sin embargo, la presencia de aire u oxígeno es necesaria para la combustión o explosión del $\mathrm{C}_{2} \mathrm{H}_{6}$. Sería un requisito muy importante evitar las infiltraciones y fugas de estos gases en los sistemas de potencia con $\mathrm{C}_{2} \mathrm{H}_{6}$, de forma similar se comportan los sistemas con refrigeración con $\mathrm{H}_{2}$, ampliamente difundidos en las instalaciones nucleares.

\subsection{Planta termosolar de colectores lineales con DSG y ciclo subcrítico de potencia Rankine (configuración de referencia)}

Como se ha tratado en numerosas ocasiones a lo largo de este trabajo, la solución tecnológica de central termosolar de colectores lineales, con DSG en modo recirculación, acoplada a ciclo de potencia Rankine con DRH [18-19], es una de las tecnologías que integran el estado de la técnica actual en las instalaciones solares de concentración y generación de potencia. Esta configuración de planta termosolar ha sido considerada como la referencia para su comparación con las plantas termosolares con ciclos $s-\mathrm{C}_{2} \mathrm{H}_{6}$ Brayton propuestas en este capítulo. 
10. Plantas Termosolares con Sales Fundidas o DSG como fluido caloportador acopladas a ciclos de potencia supercríticos Brayton con $\mathrm{C}_{2} \mathrm{H}_{6}$ como fluido de trabajo

El desarrollo de la tecnología DSG en los colectores lineales, se consideró prioritaria en los centros de investigación a nivel mundial desde principios de 1990 para sustituir aceites térmicos, y como alternativa a las sales fundidas en los campos solares. Una recapitulación sobre la historia y estado de la técnica de DSG en colectores lineales se incluye en los capítulos 1 y 8.

Las principales ventajas de la DSG se pueden resumir en bajo impacto ambiental, alto grado de madurez tecnológica de los equipos del ciclo de potencia Rankine, bajo nivel de corrosión del material de los tubos absorbedores, acoplamiento directo con los ciclos Rankine, etc.

En la figura 10.1 se ha representado gráficamente la disposición de los equipos del campo solar con DSG en modo de recirculación de la fase líquida. Cabe destacar que la presión de operación del campo solar es cercana a los 100 bar, que se considera como un nivel de presión compatible para optimizar el material y espesor de los tubos absorbedores de los colectores solares. En la figura 10.2 se muestra el ciclo de potencia Rankine, integrado por cinco calentadores de agua de recirculación y un desaireador, obteniéndose una temperatura del agua de alimentación a la entrada del campo solar $239^{\circ} \mathrm{C}$ y una TIT $=550^{\circ} \mathrm{C}$. Ambos valores de temperatura son los principales responsables de la eficiencia neta del ciclo fijada entre 40 $41 \%$.

En fase de desarrollo industrial se encuentra otra tecnología, la generación directa de vapor en modo OT en los colectores lineales. Esta técnica para generación directa de vapor sin recirculación de fase líquida, incluye la inyección de agua líquida para evitar gradientes térmicos en las secciones de ebullición y de recalentamiento del agua. La DSG mediante OT es también potencialmente acoplable a ciclos de potencia Rankine dando valores similares de eficiencia energética $40-41 \%$ de la planta termosolar con DRH.

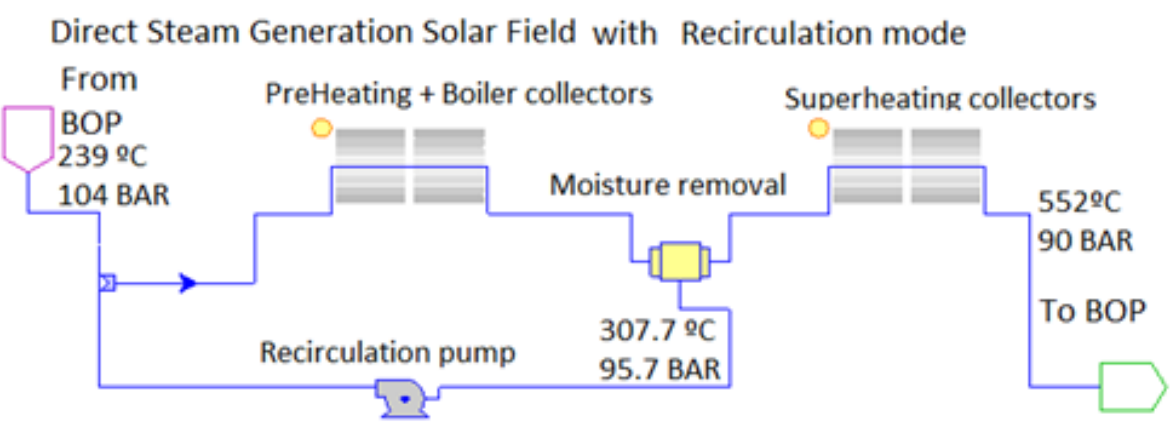

Figura 10.1. Planta termosolar integrada por colectores lineales con DSG en modo de recirculación. 
10. Plantas Termosolares con Sales Fundidas o DSG como fluido caloportador acopladas a ciclos de potencia supercríticos Brayton con $\mathrm{C}_{2} \mathrm{H}_{6}$ como fluido de trabajo

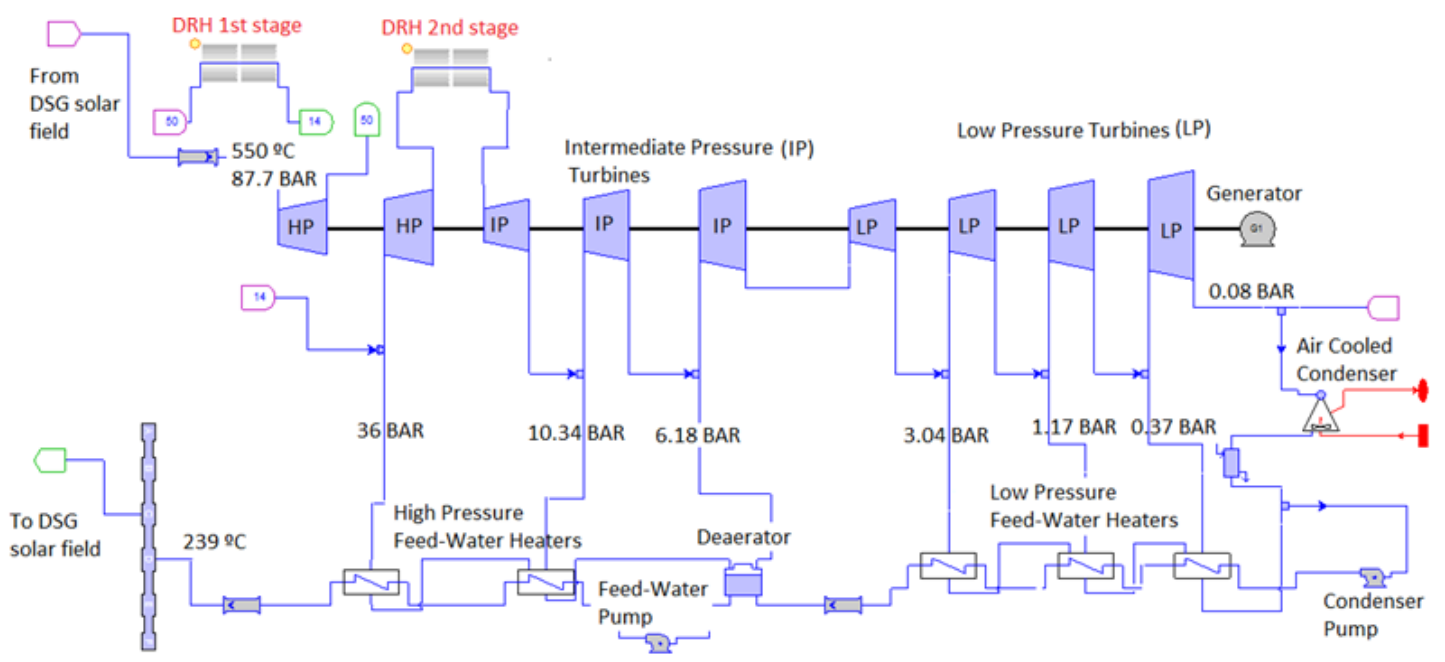

Figura 10.2. Ciclo de potencia Rankine y dos etapas de DRH.

Es importante hacer hincapié en la tecnología DRH en el ciclo Rankine, recientemente propuesto en otros trabajos [18-19]. Gracias a esta tecnología todavía no validada en ninguna instalación industrial, se incrementa la eficiencia neta del ciclo de potencia, ver la disposición de los equipos en la figura 10.2 y los resultados de eficiencia energética en las figuras 10.7 a 10.12. El éxito de su implantación está íntimamente relacionado con el diseño de detalle de los campos solares para recalentamiento del vapor. El principal objetivo es evitar pérdidas de presión excesivas en los tubos absorbedores limitando el flujo másico $\left(\mathrm{kg} / \mathrm{m}^{2} \cdot \mathrm{s}\right)$ y la longitud de los colectores y de las tuberías de distribución del vapor en el campo solar.

\subsection{El Campo Solar con DSG o Sales Fundidas}

Como se explicó en apartados anteriores de esta tesis, las configuraciones innovadoras de plantas termosolares con colectores con DSG o MS como HTF acopladas a ciclos de potencia Brayton con tipología SB, RC, PCRC y RCMCl están ilustradas en las figuras 10.3 a 10.6.

La alternativa de las sales fundidas MS como HTF en los campos solares con colectores lineales para sustituir a los aceites térmicos (como el VP1, Dowtherm A, etc.), está siendo ampliamente estudiada e implantada en plantas piloto [20-22]. El principal inconveniente de esta tecnología es la necesidad de traceado térmico para evitar la solidificación de las sales. Un objetivo sería ir adaptando los nuevos desarrollos de sales fundidas para reactores nucleares de nueva generación que soportan mayores temperaturas, para su implantación en las plantas termosolares.

La sal fundida comercial HITEC XL es un fluido que como HTF cada vez se usa más en plantas termosolares. La temperatura de solidificación del HITEC XL es menor que la Solar Salt $\mathrm{KNO}_{3}+\mathrm{NaNO}_{3}(60 \%-40 \%)$, y su máxima temperatura de operación es $540^{\circ} \mathrm{C}$, algo menor que la de la Solar Salt $\mathrm{KNO}_{3}+\mathrm{NaNO}_{3}$. A título informativo se citan los dos estudios de investigación más significativos sobre el desarrollo de sales fundidas para almacenamiento térmico [23], o como HTF [24] en plantas termosolares, y cuyos objetivos principales son: reducir el punto de fusión de las sales, una alta densidad de almacenamiento energético, una alta temperatura de operación, y un reducido coste. En el primer estudio [23] se ha definido una composición de sales incluyendo los siguientes componentes: $\left(\mathrm{NaNO}_{3}+\mathrm{LiNO}_{3}+\mathrm{Ca}\left(\mathrm{NO}_{3}\right)_{2}+\mathrm{KNO}_{3}\right)$ obteniendo 
10. Plantas Termosolares con Sales Fundidas o DSG como fluido caloportador acopladas a ciclos de potencia supercríticos Brayton con $\mathrm{C}_{2} \mathrm{H}_{6}$ como fluido de trabajo

una alta densidad de almacenamiento térmico y un bajo coste de las sales. En el segundo estudio [24] se ha desarrollado un sal fundida compuesta por: $\left(\mathrm{NaNO}_{3}+\mathrm{LiNO}_{3}+\mathrm{KNO}_{3}\right)$ y con un punto de fusión menor que la Solar Salt, para su utilización como HTF en los colectores lineales parabólicos.

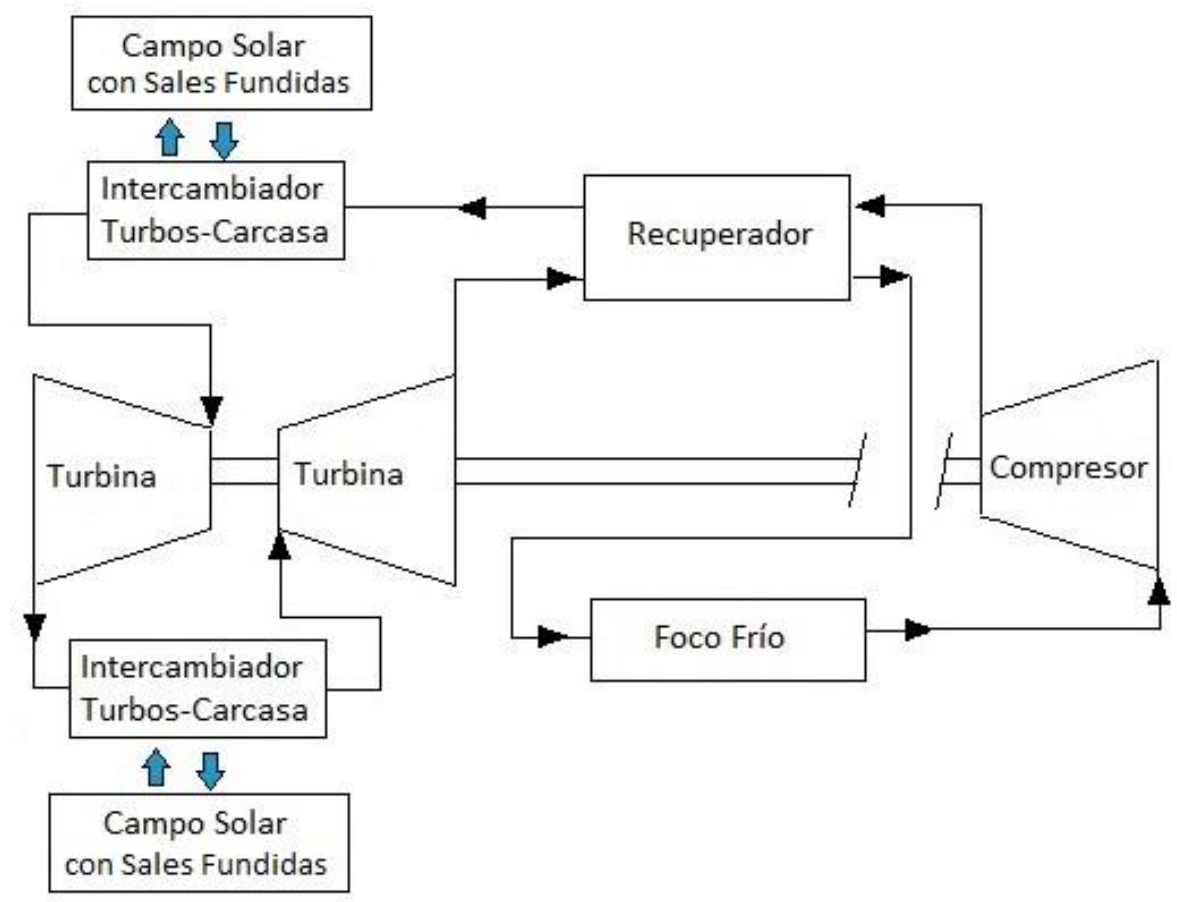

Figura 10.3. Ciclo de potencia Brayton SB con recalentamiento en turbinas.

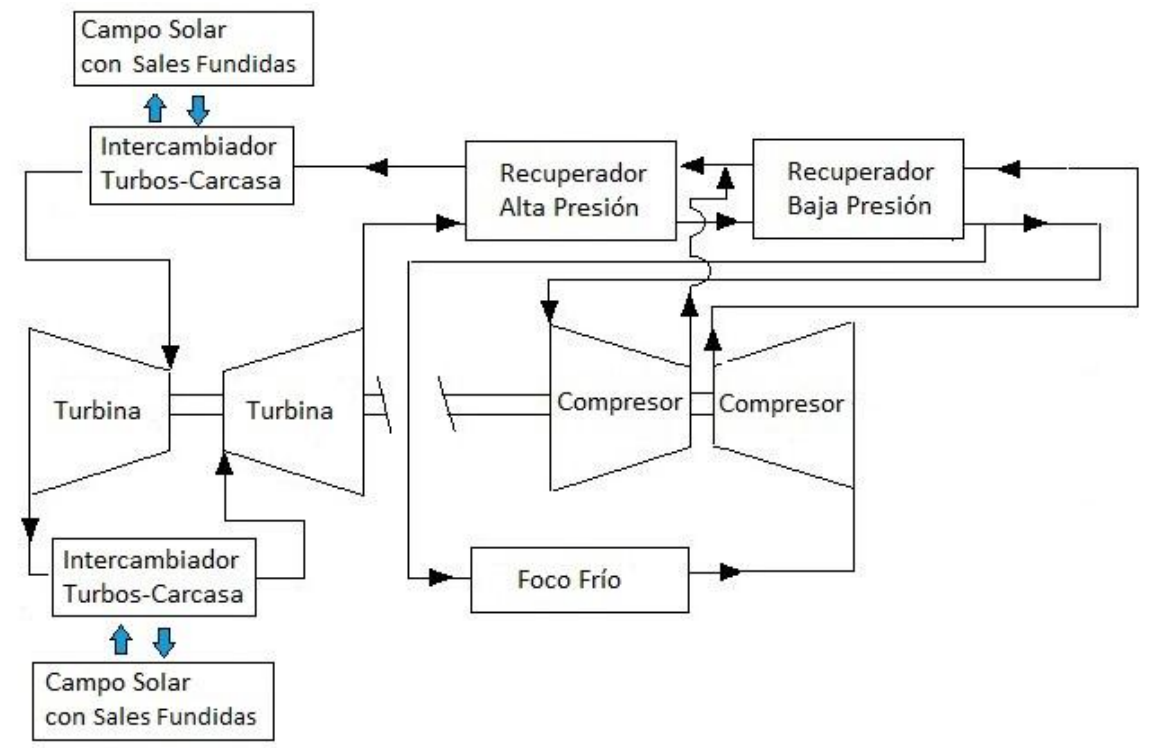

Figura 10.4. Ciclo de potencia Brayton RC con recalentamiento en turbinas.

Respecto a la presión de operación con sales fundidas como HTF, es menor que la del aceite térmico, debido a su baja viscosidad y a las menores pérdidas de presión en el campo solar. El valor de presión fijado es de 15 bar para las sales, y entre 20 y 30 bar para los aceites térmicos, con mayores viscosidades produciendo mayores pérdidas de presión. En relación a la máxima velocidad de las sales en los tubos absorbedores se limita a 3 o $4 \mathrm{~m} / \mathrm{s}$ para reducir la posible corrosión y erosión del material de los tubos, y reducir las pérdidas de presión en el campo 
10. Plantas Termosolares con Sales Fundidas o DSG como fluido caloportador acopladas a ciclos de potencia supercríticos Brayton con $\mathrm{C}_{2} \mathrm{H}_{6}$ como fluido de trabajo

solar. La principal desventaja de las sales fundidas en su solidificación y la necesidad de traceado térmico, así mismo presentan un alto grado de corrosión. Otra posible alternativa de integración de los colectores solares lineales con ciclos de potencia Brayton ha sido presentada en otros capítulos, utilizando las sales fundidas como HTF en el campo solar (capítulo 5 y 6) y en [25] DSG como HTF (capítulo 8), aceites térmicos como HTF (capítulo 7), o incluso la combinación en el mismo campo solar entre los diferentes HTFs indicados mediante la solución Dual-Loop (capítulo 9).

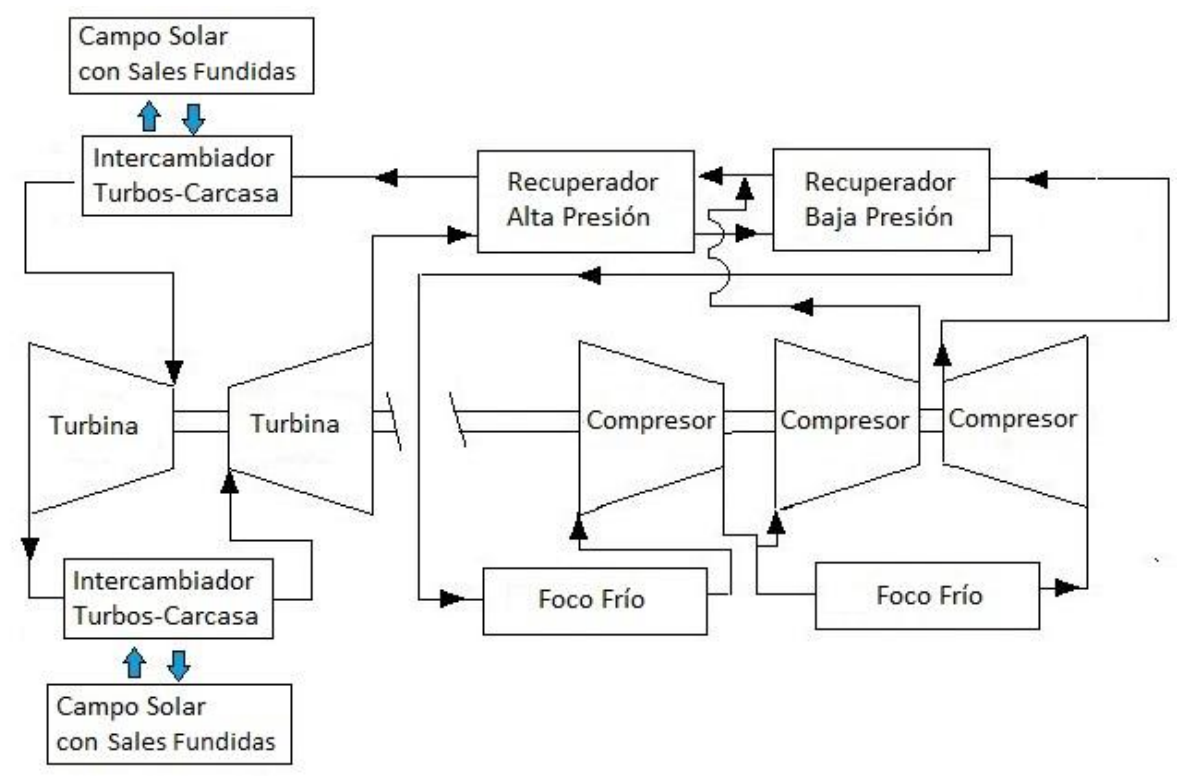

Figura 10.5. Ciclo de potencia Brayton PCRC con recalentamiento en turbinas.

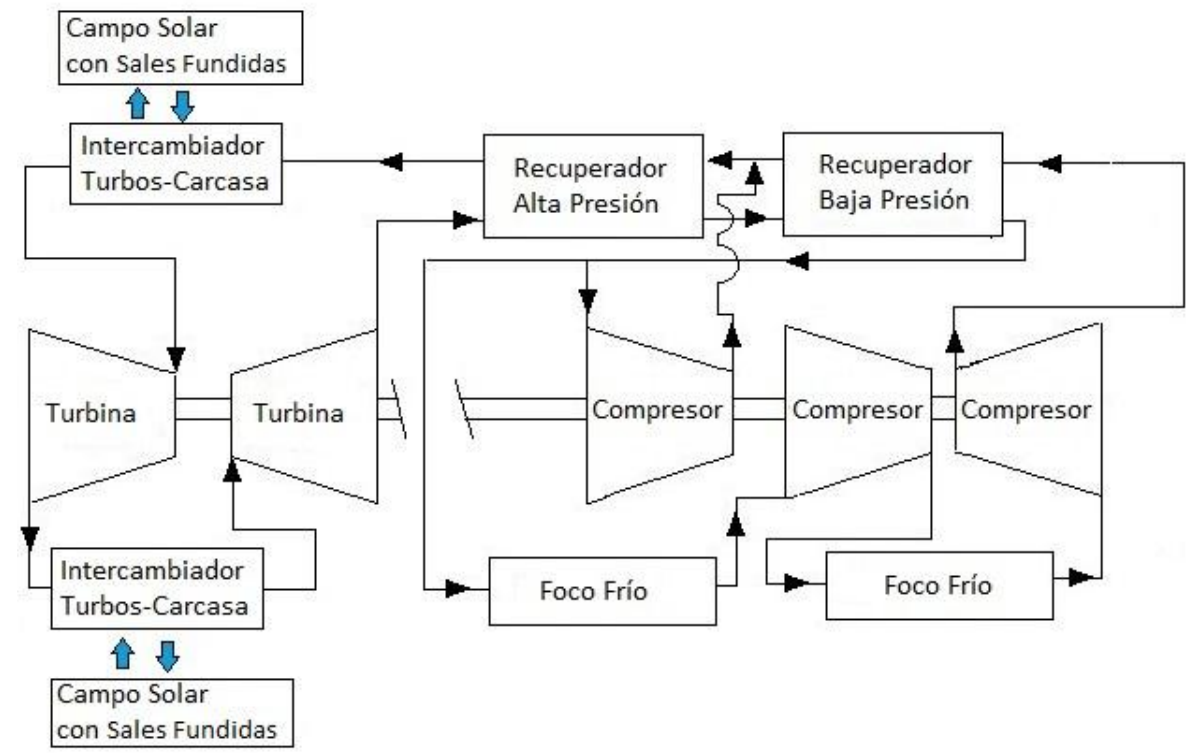

Figura 10.6. Ciclo de potencia Brayton $\mathrm{RCMCl}$ con recalentamiento en turbinas.

Los ciclos de potencia s- $\mathrm{CO}_{2}$ Brayton han sido tratados a lo largo de este trabajo. Cabe destacar la publicación [26], donde se realiza una recopilación del proyecto de fabricación de una planta de generación de energía con un ciclo s- $\mathrm{CO}_{2}$ Brayton de 10 MWe de potencia. 
10. Plantas Termosolares con Sales Fundidas o DSG como fluido caloportador acopladas a ciclos de potencia supercríticos Brayton con $\mathrm{C}_{2} \mathrm{H}_{6}$ como fluido de trabajo

Los ciclos de potencia s- $\mathrm{C}_{2} \mathrm{H}_{6}$ Brayton requieren una $\mathrm{TIT}<400^{\circ} \mathrm{C}$, para evitar su descomposición química. A la hora de diseñar los ciclos de potencia con $\mathrm{s}-\mathrm{C}_{2} \mathrm{H}_{6}$ es importante reducir el tiempo de permanencia del $\mathrm{C}_{2} \mathrm{H}_{6}$ a altas temperaturas y por tanto garantizar su estabilidad química [6]. La presión, la temperatura y el tiempo de reacción son las variables que mayor importancia presentan en la descomposición química del s- $\mathrm{C}_{2} \mathrm{H}_{6}$.

Debido a la limitación de $\mathrm{TIT}<400^{\circ} \mathrm{C}$ indicada del $\mathrm{s}-\mathrm{C}_{2} \mathrm{H}_{6}$, se ha estudiado también en este capítulo el acoplamiento del ciclo $\mathrm{s}-\mathrm{C}_{2} \mathrm{H}_{6}$ Brayton con colectores lineales con DSG. La tecnología DSG en los colectores lineales ha sido validada recientemente en plantas piloto, y se ha explicado en detalle en otros capítulos 1 y 8 de esta tesis.

\subsection{Hipótesis de cálculo}

En esta sección se detallan las hipótesis de cálculo consideradas en las simulaciones de las centrales termosolares con Thermoflow. En la tabla 10.1 se detallan las condiciones ambientales y de localización de las instalaciones analizadas. Estos datos son una referencia muy difundida en los estudios de plantas termosolares sirviendo como base para realizar estudios comparativos. En relación a los colectores lineales parabólicos se ha elegido el Eurotrough II, y para colectores LF los modelos sumistrados por Novatec, sus parámetros detallados están resumidos en las tablas 10.3 y 10.4. Respecto a los tubos absorbedores sus características principales se detallan en la tabla 10.2, hay principalmente dos marcas comerciales que los suministran Archimede Solar Energy y SCHOTT. Respecto a los ciclos de potencia, en este primer estudio se han considerado como parámetros de operación de los ciclos los incluidos en las tablas 10.5 y 10.6. Más adelante estos parámetros termodinámicos son modificados para optimizar la eficiencia energética.

Tabla 10.1. Localización y condiciones ambientales.

\begin{tabular}{lc}
\hline Localización: & Dagget,CA, USA. \\
Latitud: & $34.86^{\circ}$ \\
Longitud: & $-116.8^{\circ}$ \\
Zona Horaria: & -8 \\
Hora: & $11: 30 \mathrm{hr}$ \\
DNI: & $986 \mathrm{~W} / \mathrm{m}^{2}$ \\
Temperatura: & $25^{\circ} \mathrm{C}$ \\
Altitud: & $588 \mathrm{~m}$ \\
\hline
\end{tabular}

Tabla 10.2. Parámetros del tubo absorbedor.

\begin{tabular}{|c|c|}
\hline Diámetro Exterior: & $70 \mathrm{~mm}$ \\
\hline Espesor de pared: & $4.191 \mathrm{~mm}$ \\
\hline \multirow[t]{2}{*}{ Material: } & Acero Inoxidable (MS) \\
\hline & Steel (DSG) \\
\hline \multicolumn{2}{|c|}{$\begin{array}{l}\text { Vacio entre el tubo de acero y el tubo de cristal } \\
\text { and internal steel pipe }\end{array}$} \\
\hline Rugosidad: & $0.0457 \mathrm{~mm}$ \\
\hline HTF: HITEC XL (MS) & agua (DSG) \\
\hline
\end{tabular}


10. Plantas Termosolares con Sales Fundidas o DSG como fluido caloportador acopladas a ciclos de potencia supercríticos Brayton con $\mathrm{C}_{2} \mathrm{H}_{6}$ como fluido de trabajo

Tabla 10.3. Parámetros de colectores PTC.

\begin{tabular}{ll}
\hline Tipo colector: & EuroTrough II \\
Anchura apertura: & $5.77 \mathrm{~m}$ \\
Longitud focal: & $1.71 \mathrm{~m}$ \\
Factor limpieza: & 0.96 \\
Eficiencia óptica: & 0.75 \\
& $0.141 \Delta \mathrm{T}+6.48 \mathrm{e}-9 \Delta \mathrm{T}^{4}$ [27] \\
Pérdidas Térmicas: & (MS or DSG HTF) [41] \\
\hline
\end{tabular}

Tabla 10.4. Parámetros de colectores LF.

\begin{tabular}{ll}
\hline Tipo de colector: & SuperNova 1 (Novatec) \\
Dimensiones: & $16.56 \mathrm{~m} \times 44.8 \mathrm{~m}$ \\
Área de apertura: & $513.6 \mathrm{~m}^{2} /$ módulo \\
Eficiencia óptica: & $0.65 / 0.647$ (superheating) \\
Pérdidas Térmicas: & $1.06 \Delta \mathrm{T}+1.2 \cdot 10^{-8} \Delta \mathrm{T}^{4}$ [28] (DSG boiling) Novatec, [42] \\
Pérdidas Térmicas: & $0.15 \Delta \mathrm{T}+7.1510^{-9} \Delta \mathrm{T}^{4}$ [28] (MS or DSG HTF) [42] \\
\hline
\end{tabular}

Tabla 10.5. Parámetros del ciclo de potencia Brayton.

\begin{tabular}{ll}
\hline HP Turbina: & $\begin{array}{l}2 \text { etapas (87.7 bar; 36 bar) } \\
\text { IP Turbina: }\end{array}$ \\
& $\begin{array}{l}\text { (16.5 bar; } 10.34 \text { bar; 6.18 bar) } \\
5.17 \text { bar;3.04bar;1.17bar;0.37bar }\end{array}$ \\
LP Turbina: & $85 \%$ \\
Eficiencia Turbina: & 0.08 bar \\
Presión & \\
Condensador: & $98.23 \%$ (Punto de Diseño) \\
Eficiencia & \\
Generador: & $1 \%$ (Potencia Bruta) \\
Perdidas BOP: & $5^{\circ} \mathrm{C}$ \\
TTD: & $5^{\circ} \mathrm{C}$ \\
DCA: & 6.17 bar \\
Presión desaireador: &
\end{tabular}

Tabla 10.6. Parámetros del ciclo de potencia Rankine.

\begin{tabular}{lc}
\hline Eficiencia Turbina: & $93 \%$ \\
Eficiencia Compresor: & $89 \%$ \\
Eficiencia Intercambiadores: & $95 \%$ \\
No hay perdidas de presión en Intercambiadores \\
Temperatura entrada Turbina (TIT): & $550^{\circ} \mathrm{C}$ \\
Presión entrada Turbina: & $250 \mathrm{bar}$ \\
Presión Recalentamiento $\left(\mathrm{s}-\mathrm{CO}_{2}\right):$ & $173 \mathrm{bar}$ \\
Presión Recalentamiento $\left(\mathrm{s}-\mathrm{C}_{2} \mathrm{H}_{6}\right):$ & $116.9 \mathrm{bar}$ \\
Temperatura entrada Compresor $(\mathrm{CIT}):$ & $32^{\circ} \mathrm{C}$ \\
Presión entrada Compresor $\left(\mathrm{s}-\mathrm{CO}_{2}\right):$ & $74 \mathrm{bar}$ \\
Presión entrada Compresor $\left(\mathrm{s}-\mathrm{C}_{2} \mathrm{H}_{6}\right):$ & $50 \mathrm{bar}$ \\
Fracción de Flujo: & $71 \%, 29 \%$ \\
Perdidas BOP (\% Potencia Bruta): & $1 \%$ \\
Eficiencia Generador (Punto de Diseño): & $98.23 \%$ \\
\hline
\end{tabular}


10. Plantas Termosolares con Sales Fundidas o DSG como fluido caloportador acopladas a ciclos de potencia supercríticos Brayton con $\mathrm{C}_{2} \mathrm{H}_{6}$ como fluido de trabajo

\subsection{Eficiencia Neta en el Punto de Diseño}

Como primera aproximación para la simulación de los ciclos de potencia s- $\mathrm{CO}_{2}$ Brayton y s$\mathrm{C}_{2} \mathrm{H}_{6}$, se consideran las relaciones de presión a la entrada y salida de las turbinas resumidas en la tabla 10.7 (Opción A). Esta primera hipótesis de cálculo considera una TIP = 250 bar y una presión de entrada a la turbina de recalentamiento de 173 bar. Los resultados de las correspondientes simulaciones se resumen en las figuras 10.7 y 10.8 para diferentes temperaturas de entrada en turbinas.

Si se compara la eficiencia de las plantas termosolares propuestas en este trabajo con configuración (MS+Brayton), con la configuración de Referencia (DSG+Rankine), se concluye que las instalaciones de referencia presentan unos valores de eficiencia energéticas menores que los valores con ciclos de potencia Brayton tanto con s- $\mathrm{CO}_{2}$ como con s- $\mathrm{C}_{2} \mathrm{H}_{6}$, los resultados detallados se encuentran en las figuras 10.7 y 10.8. Y si se comparan los resultados de eficiencia neta de las plantas con ciclos Brayton, se concluye que la eficiencia de las plantas con $\mathrm{s}-\mathrm{C}_{2} \mathrm{H}_{6}$ es mayor que las instalaciones con s- $\mathrm{CO}_{2}$. Esta diferencia es aún mayor para valores medios de $\mathrm{TIT}$, entre $300^{\circ} \mathrm{C}$ y $400^{\circ} \mathrm{C}$, ver los resultados en las figuras 10.7 a 10.10). Otro hecho importante a destacar es la limitación de la temperatura máxima de operación del $\mathrm{C}_{2} \mathrm{H}_{6}$. Para evitar su descomposición química es conveniente limitar su temperatura de entrada en turbina a $400^{\circ} \mathrm{C}$.

Actualmente no se dispone de datos experimentales del comportamiento del $\mathrm{C}_{2} \mathrm{H}_{6}$ bajo las condiciones termodinámicas de presión y temperatura previstas en los ciclos considerados. Para $\mathrm{TIT} \leq 400^{\circ} \mathrm{C}$ la eficiencia neta del s- $\mathrm{C}_{2} \mathrm{H}_{6}$ es alrededor del $2 \%$ superior a la del s- $\mathrm{CO}_{2}$ en todas las configuraciones estudiadas.

Una primera optimización para incrementar la eficiencia neta de los ciclos Brayton se materializa en la modificación de las relaciones de presión a la entrada y salida de turbinas. $\mathrm{Si}$ se adaptan las relaciones de presión en turbina y compresores a los valores propuestos en la tabla 10.8 (Opción B), se puede incrementar la eficiencia neta de los ciclos de potencia en punto de diseño, los resultados se resumen en las figuras 10.9 y 10.10 .

Tabla 10.7. Relación de Presiones Opción A.

\begin{tabular}{lllll}
\hline Ciclo Potencia & SB & RC & PCRC & RCMCI \\
\hline $\mathrm{P}_{\text {ratio }} 1$ & 1.44 & 1.44 & 1.44 & 1.44 \\
$\mathrm{P}_{\text {ratio }}$ 2 & 2.33 & 2.33 & 2.33 & 2.33 \\
$\mathrm{P}_{\text {ratio }} 3$ & 3.37 & 3.37 & 1.39 & 3.37 \\
$\mathrm{P}_{\text {ratio }} 4$ & - & 3.37 & 2.42 & 1.39 \\
$\mathrm{P}_{\text {ratio }} 5$ & - & - & 2.42 & 2.42 \\
\hline
\end{tabular}

Tabla 10.8. Relación de Presiones Opción B.

\begin{tabular}{lllll}
\hline Ciclo Potencia & SB & RC & PCRC & RCMCI \\
\hline $\mathrm{P}_{\text {ratio }}$ 1 & 2.13 & 2.13 & 2.13 & 2.13 \\
$\mathrm{P}_{\text {ratio }}$ 2 & 2.33 & 2.33 & 2.33 & 2.33 \\
$\mathrm{P}_{\text {ratio }}$ 3 & 5 & 5 & 1.39 & 5 \\
$\mathrm{P}_{\text {ratio }} 4$ & - & 5 & 3.59 & 1.39 \\
$\mathrm{P}_{\text {ratio }} 5$ & - & - & 3.59 & 3.59 \\
\hline
\end{tabular}


10. Plantas Termosolares con Sales Fundidas o DSG como fluido caloportador acopladas a ciclos de potencia supercríticos Brayton con $\mathrm{C}_{2} \mathrm{H}_{6}$ como fluido de trabajo

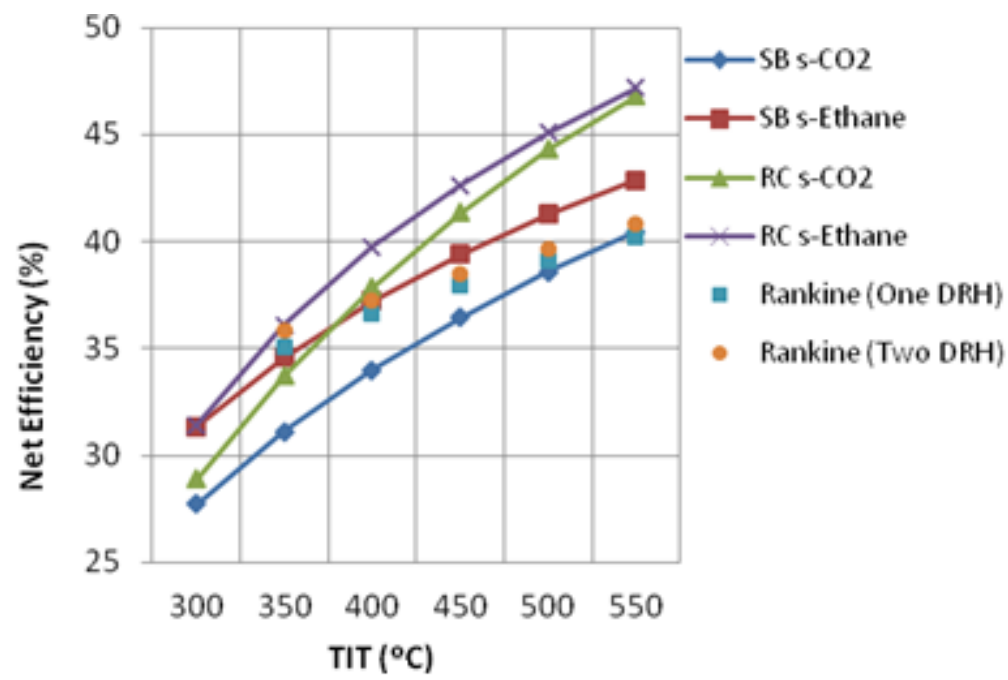

Figura 10.7. Eficiencia Neta \& $\mathrm{TIT}^{(1)}$.

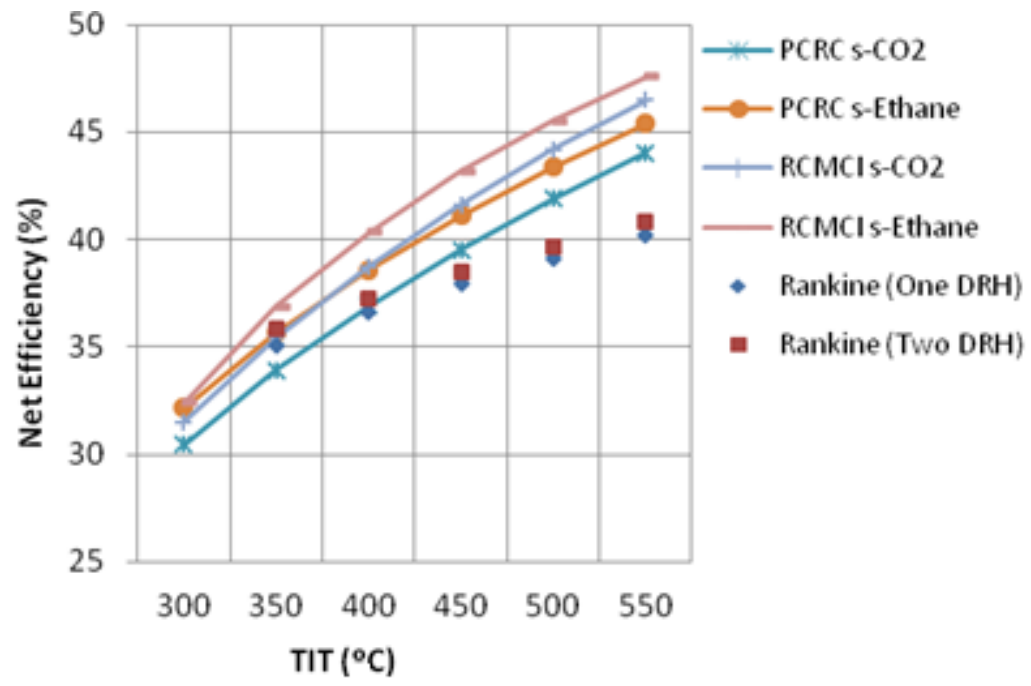

Figura 10.8. Eficiencia Neta \& $\mathrm{TIT}^{(1)}$.

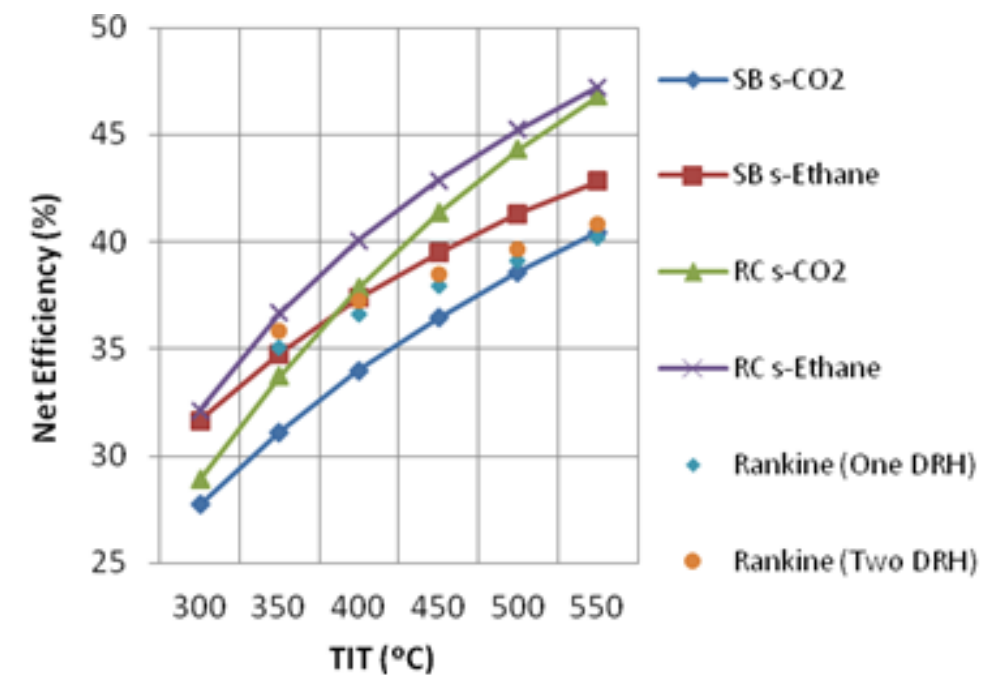

Figura 10.9. Eficiencia Neta \& $\mathrm{TIT}^{(2)}$.

${ }^{(1)}$ Relación de Presiones de la tabla 10.7 (Opción A) para los ciclos de potencia s- $\mathrm{CO}_{2}$ y s- $\mathrm{C}_{2} \mathrm{H}_{6}$ Brayton.

${ }^{(2)}$ Relación de Presiones de la tabla 10.7 (Opción A) para el ciclo de potencia s- $\mathrm{CO}_{2}$ Brayton. Relación de Presiones de la tabla 10.8 (Opción B) para el ciclo de potencia s- $\mathrm{C}_{2} \mathrm{H}_{6}$ Brayton. 
10. Plantas Termosolares con Sales Fundidas o DSG como fluido caloportador acopladas a ciclos de potencia supercríticos Brayton con $\mathrm{C}_{2} \mathrm{H}_{6}$ como fluido de trabajo

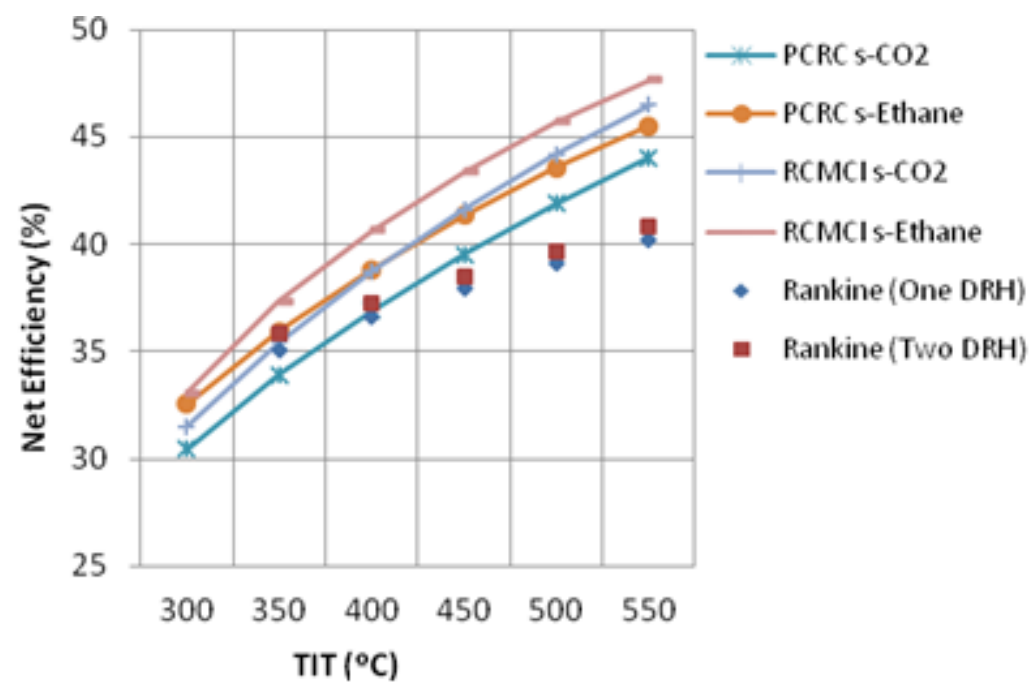

Figura 10.10. Eficiencia Neta \& $\mathrm{TIT}^{(2)}$.

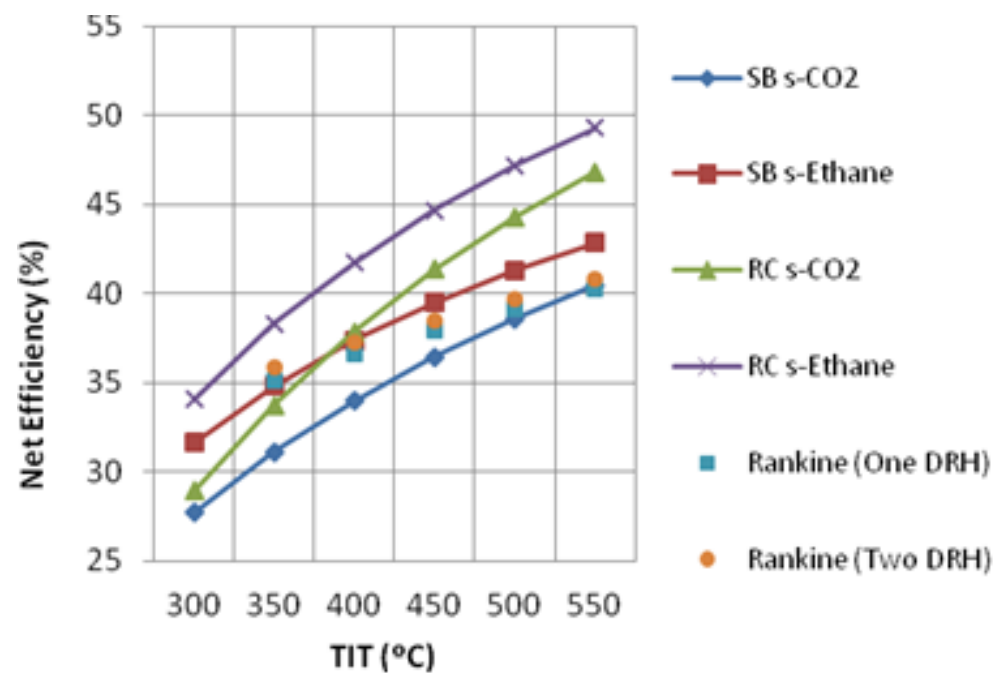

Figura 10.11. Eficiencia Neta \& $\mathrm{TIT}^{(2)}$.

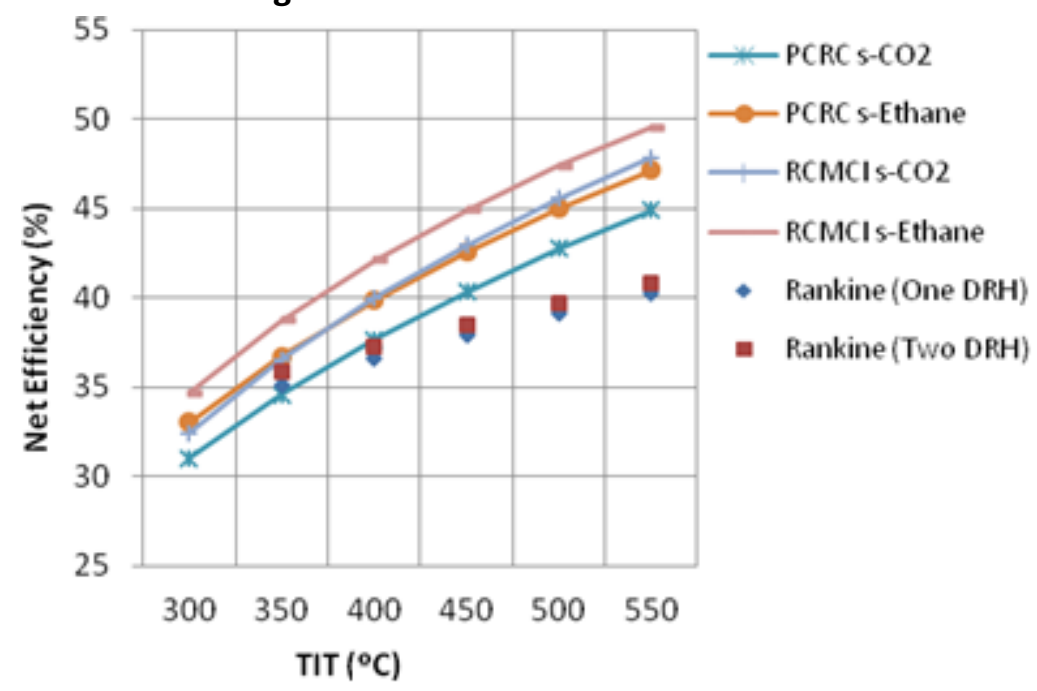

Figura 10.12. Eficiencia Neta \& TIT $^{(2)}$.

${ }^{(2)}$ Relación de Presiones de la tabla 10.7 (Opción A) para el ciclo de potencia s- $\mathrm{CO}_{2}$ Brayton. Relación de Presiones de la tabla 10.8 (Opción B) para el ciclo de potencia s- $\mathrm{C}_{2} \mathrm{H}_{6}$ Brayton. 
10. Plantas Termosolares con Sales Fundidas o DSG como fluido caloportador acopladas a ciclos de potencia supercríticos Brayton con $\mathrm{C}_{2} \mathrm{H}_{6}$ como fluido de trabajo

\subsection{Fracción de Caudal Óptimo}

En las configuraciones más complejas del ciclo de potencia Brayton, se realiza una división del caudal ante de su entrada en las etapas de compresión. Parte del caudal es comprimido sin enfriamiento previo alguno y otra parte del caudal es enfriado antes de su entrada a las etapas de compresión. De esta forma se consigue optimizar la eficiencia energética de los ciclos de potencia. En este capítulo de la tesis para las hipótesis de cálculo consideradas, se han identificado los valores óptimos de fracción de caudal antes de su compresión: para el s- $\mathrm{CO}_{2}$, RC (71\% - 29\%), PCRC (59\% - 41\%), RCMCI (64\% - 36\%); para el s- $\mathrm{C}_{2} \mathrm{H}_{6}: \mathrm{RC}(80 \%-20 \%), \mathrm{PCRC}$ $(85 \%-15 \%), \mathrm{RCMCl}(80 \%-20 \%)$. Con estos valores de fracción de caudal se obtienen los resultados ilustrados en las figuras 10.11 y 10.12 .

\subsection{Análisis de Sensibilidad del tamaño térmico (UA) en los Recuperadores}

El UA de los recuperadores, tanto de baja presión LTR como de alta presión HTR, es un parámetro que incluye una cuantificación tanto del área de intercambio de calor del intercambiador (A) como de su coeficiente general de transferencia de calor (U). Los recuperadores con una de las piezas fundamentales de los ciclos Brayton ya que su función es la de realizar el intercambio calorífico entre los dos focos de temperatura del ciclo termodinámico. Si se incrementa la eficiencia de los recuperadores, su área de intercambio y su coeficiente de transferencia de calor se consigue un mayor aprovechamiento energético del ciclo Brayton.

El UA también está íntimamente relacionado con la diferencia de temperatura de los fluidos a la salida de los recuperadores, es la conocida como pinch-point. Un incremento de la UA en los recuperadores disminuye los valores de pinch-point de los mismos. Sin embargo, existe un valor límite de UA en cada ciclo a partir del cual la diferencia de temperatura de los fluidos a la salida del cambiador es prácticamente nula, es decir, el pinch-point es casi cero. Por tanto, en la práctica habitual, el pinch-point de los cambiadores es de $3^{\circ} \mathrm{C} \circ 4^{\circ} \mathrm{C}$. Para fijar este valor sería necesario realizar un estudio económico evaluando si un incremento de las dimensiones de los recuperadores permite obtener una mejora sustancial de la eficiencia neta del ciclo de potencia. En este apartado, se han incrementado los valores del tamaño térmico (1.25.UA, 1.5.UA, y 1.75.UA) en los recuperadores (LTR y HTR) [29-30] para mejorar la eficiencia energética del ciclo. Los resultados de este análisis de sensibilidad frente al incremento de tamaño térmico en los recuperadores están resumidos en la tabla 10.9. Como contrapartida un incremento de las dimensiones de los recuperadores, y por tanto de su UA, trae consigo un incremento de las pérdidas de presión del fluido que lo atraviesa, impactando negativamente en la eficiencia del ciclo. Los resultados de este análisis frente al UA de los recuperadores se resumen en las figuras 10.9 y 10.10, tomando como hipótesis de cálculo las relaciones de presión Opción B listadas en la tabla 10.8.

Tabla 10.9. Planta Termosolar $\left(M S+s-C_{2} H_{6}\right)$. Eficiencia \& Tamaño Térmico en recuperadores ${ }^{(3)}$.

\begin{tabular}{lllll}
\hline Ciclo Potencia & SB & RC & PCRC & RCMCI \\
\hline TIT $=300^{\circ} \mathrm{C}$ (Referencia UA) & 31.64 & 32.12 & 32.54 & 33.06 \\
TIT $=300^{\circ} \mathrm{C}(1.25 \mathrm{UA})$ & 32.22 & 32.67 & 33.03 & 33.58 \\
TIT $=300^{\circ} \mathrm{C}(1.5 \mathrm{UA})$ & 32.59 & 33.03 & 33.34 & 33.91 \\
TIT $=300^{\circ} \mathrm{C}(1.75 \mathrm{UA})$ & 32.83 & 33.27 & 33.54 & 34.07
\end{tabular}


10. Plantas Termosolares con Sales Fundidas o DSG como fluido caloportador acopladas a ciclos de potencia supercríticos Brayton con $\mathrm{C}_{2} \mathrm{H}_{6}$ como fluido de trabajo

\begin{tabular}{lllll}
\hline Ciclo Potencia & SB & RC & PCRC & RCMCI \\
\hline TIT $=350^{\circ} \mathrm{C}$ (Referencia UA) & 34.79 & 36.67 & 35.95 & 37.34 \\
TIT $=350^{\circ} \mathrm{C}(1.25 \cup A)$ & 35.58 & 37.26 & 36.55 & 37.9 \\
TIT $=350^{\circ} \mathrm{C}(1.5 \cup A)$ & 36.1 & 37.62 & 36.93 & 38.25 \\
TIT $=350^{\circ} \mathrm{C}(1.75 \cup A)$ & 36.43 & 37.87 & 37.17 & 38.49 \\
TIT $=400^{\circ} \mathrm{C}$ (Referencia UA) & 37.36 & 40.07 & 38.83 & 40.64 \\
TIT $=400^{\circ} \mathrm{C}$ (1.25UA) & 38.38 & 40.78 & 39.54 & 41.34 \\
TIT $=400^{\circ} \mathrm{C}(1.5 \cup A)$ & 39.03 & 41.03 & 39.97 & 41.77 \\
TIT $=400^{\circ} \mathrm{C}(1.75 \cup A)$ & 39.46 & 41.54 & 40.26 & 42.06 \\
\hline
\end{tabular}

(3) Basado en los resultados obtenidos en las figuras 10.9 y 10.10, y las relaciones de presión de los ciclos $\mathrm{s}-\mathrm{C}_{2} \mathrm{H}_{6}$ Brayton Opción B listado en la tabla 10.8.

\subsection{Análisis de Sensibilidad de las pérdidas de presión en los Intercambiadores de Calor}

Los valores de eficiencia energética en el punto de diseño obtenidos en las figuras 10.7 a 10.12 son estimados y podrían considerarse como valores de referencia, pero en la realidad el comportamiento de la planta termosolar no puede nunca reproducir esos niveles de eficiencia energética porque existen unas pérdidas de presión en los intercambiadores de calor que impactan negativamente sobre el rendimiento de la instalación.

El desafío actual en los intercambiadores de tipología PCHE es la reducción de las pérdidas de presión, a pesar de las reducidas dimensiones de los canales, y de las turbulencias en los cabezales de entrada y salida al intercambiador. Debido a estos inconvenientes, hoy en día se continúa desarrollando la tecnología de los intercambiadores de calor para minimizar sus pérdidas de carga, maximizar su área de intercambio de calor, y minimizar el volumen que ocupan. En el caso de los intercambiadores PCHE, se están diseñando diferentes alternativas para el diseño de la sección transversal de los canales de flujo para reducir las pérdidas de carga.

Se dispone de varias alternativas, los canales rectangulares, los semicirculares, los canales con perfil de ala de avión o airfoil-fin, los que tienen geometría de s-fin, ver las referencias [31-32]. Para cuantificar el impacto real de las pérdidas de presión de los intercambiadores de calor en la eficiencia neta del ciclo de potencia, en este capítulo se han considerado dos escenarios, la caída de presión del $1 \%$ y del $2 \%$. Los resultados de este análisis están resumidos en las figuras 10.13 a 10.16. Estos resultados fueron obtenidos tomando como hipótesis de cálculo los valores representados gráficamente en las figuras 10.9 y 10.10 para condiciones ideales sin pérdidas de carga alguna.

Los estudios de sensibilidad frente a las pérdidas de presión en los intercambiadores de calor tienen como referencia los valores ideales incluidos en las figuras 10.9 y 10.10 , y las hipótesis de ratios de presión Opción B incluidos en la tabla 10.8. 
10. Plantas Termosolares con Sales Fundidas o DSG como fluido caloportador acopladas a ciclos de potencia supercríticos Brayton con $\mathrm{C}_{2} \mathrm{H}_{6}$ como fluido de trabajo

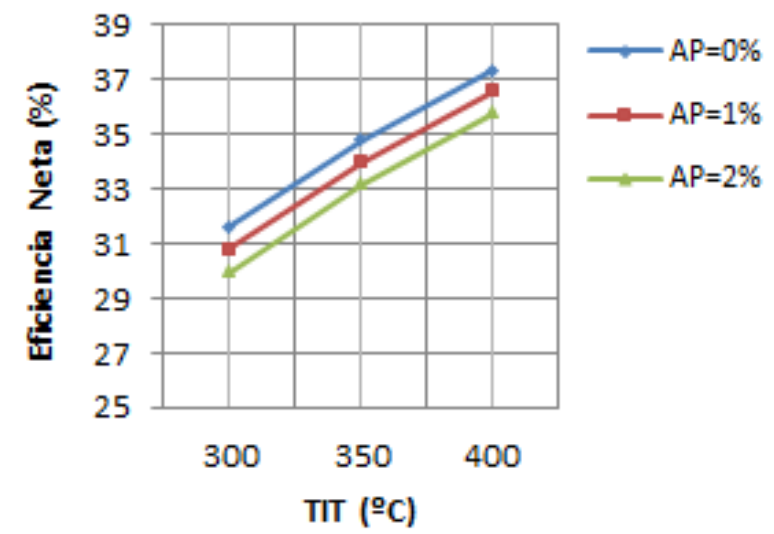

Figura 10.13. SB s- $\mathrm{C}_{2} \mathrm{H}_{6}$ Eficiencia Neta \& Pérdidas de Presión en $\mathrm{HX}^{(2)}$.

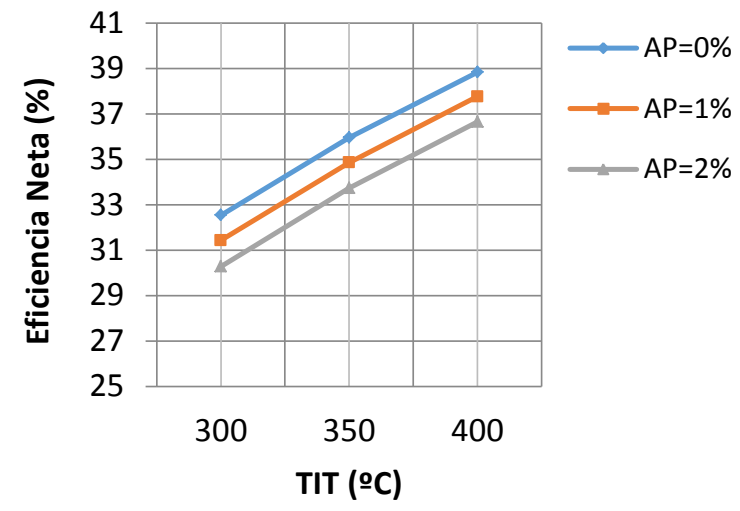

Figura 10.14. $\mathrm{PCRC} s-\mathrm{C}_{2} \mathrm{H}_{6}$ Eficiencia Neta \&Pérdidas de Presión en $\mathrm{HX}{ }^{(2)}$.

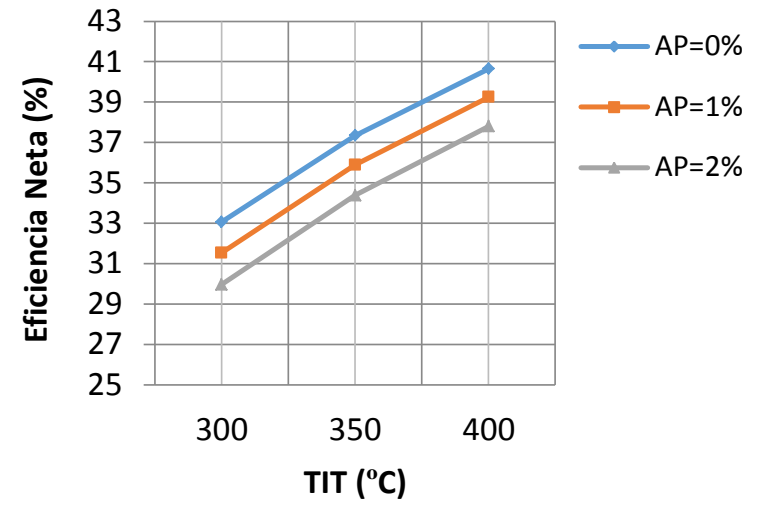

Figura 10.15. $\mathrm{RC} \mathrm{s}-\mathrm{C}_{2} \mathrm{H}_{6}$ Eficiencia Neta \& Pérdidas de Presión en $\mathrm{HX}{ }^{(2)}$.

${ }^{(2)}$ Relación de Presiones de la tabla 10.7 (Opción A) para el ciclo de potencia s- $\mathrm{CO}_{2}$ Brayton. Relación de Presiones de la tabla 10.8 (Opción B) para el ciclo de potencia s- $\mathrm{C}_{2} \mathrm{H}_{6}$ Brayton. 
10. Plantas Termosolares con Sales Fundidas o DSG como fluido caloportador acopladas a ciclos de potencia supercríticos Brayton con $\mathrm{C}_{2} \mathrm{H}_{6}$ como fluido de trabajo

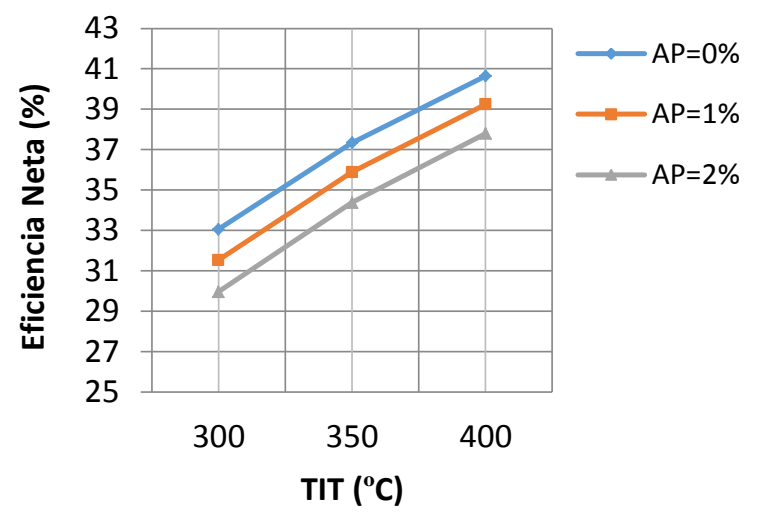

Figura 10.16. $\mathrm{RCMCl} \mathrm{s}-\mathrm{C}_{2} \mathrm{H}_{6}$ Eficiencia Neta \&Pérdidas de Presión en $\mathrm{HX}^{(2)}$.

\subsection{Análisis de Sensibilidad de la Temperatura de Entrada en el Compresor (CIT)}

La temperatura ambiental externa no es una variable controlada por el diseñador. El sistema de generación de potencia debería adaptarse a las condiciones meteorológicas que impactan directamente sobre la temperatura ambiente, la humedad relativa y como resultado sobre la temperatura de entrada al compresor Compressor Inlet Temperature (CIT). En este estudio se analizan dos condiciones de operación en el punto de diseño, $\mathrm{CIT}=25^{\circ} \mathrm{C}$ y $40^{\circ} \mathrm{C}$. El resultado de este análisis queda ilustrado en las figuras 10.17 a 10.20 , y tiene como base las mismas hipótesis de cálculo que las condiciones de $\mathrm{CIT}=32^{\circ} \mathrm{C}$ indicadas en las figuras 10.9 y 10.10 , y por tanto los ratios de presión Opción B listados en la tabla 10.8 .

Bajo condiciones climáticas adversas, la temperatura de operación a entrada del compresor puede ser superior a las del punto crítico del $\mathrm{CO}_{2}$ y del $\mathrm{C}_{2} \mathrm{H}_{6}$. Para contrarrestar este hecho, se incrementa la presión de entrada al compresor (CIP), tal y como se propone en [29-30]. El impacto es mayor en los ciclos de potencia Brayton con tipología SB y RC, las configuraciones $P C R C$ y $R C M C l$ incluyen más etapas de enfriamiento intermedio, limitando la fracción de caudal enfriado a bajas temperaturas. Para $\mathrm{TIT}>400^{\circ} \mathrm{C}$ la variación de la temperatura de entrada al compresor CIT tiene un impacto significativamente menor en la eficiencia neta que con $\mathrm{TIT}<300^{\circ} \mathrm{C}$; la razón principal es que las líneas isobáricas de las propiedades del gas están más separadas a altas temperaturas que a bajas temperaturas.

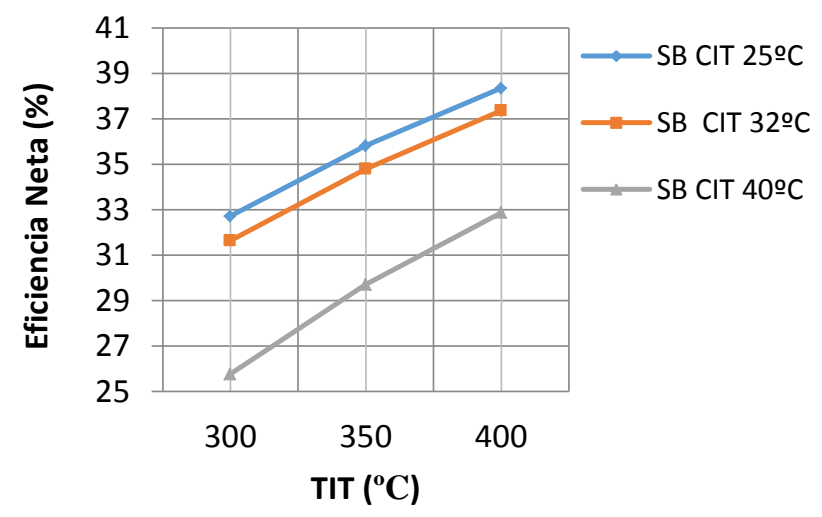

Figura 10.17. SB s- $\mathrm{C}_{2} \mathrm{H}_{6}$ Eficiencia Neta \& $\mathrm{CIT}^{(2)}$.

${ }^{(2)}$ Relación de Presiones de la tabla 10.7 (Opción A) para el ciclo de potencia s- $\mathrm{CO}_{2}$ Brayton. Relación de Presiones de la tabla 10.8 (Opción B) para el ciclo de potencia s $-\mathrm{C}_{2} \mathrm{H}_{6}$ Brayton. 
10. Plantas Termosolares con Sales Fundidas o DSG como fluido caloportador acopladas a ciclos de potencia supercríticos Brayton con $\mathrm{C}_{2} \mathrm{H}_{6}$ como fluido de trabajo

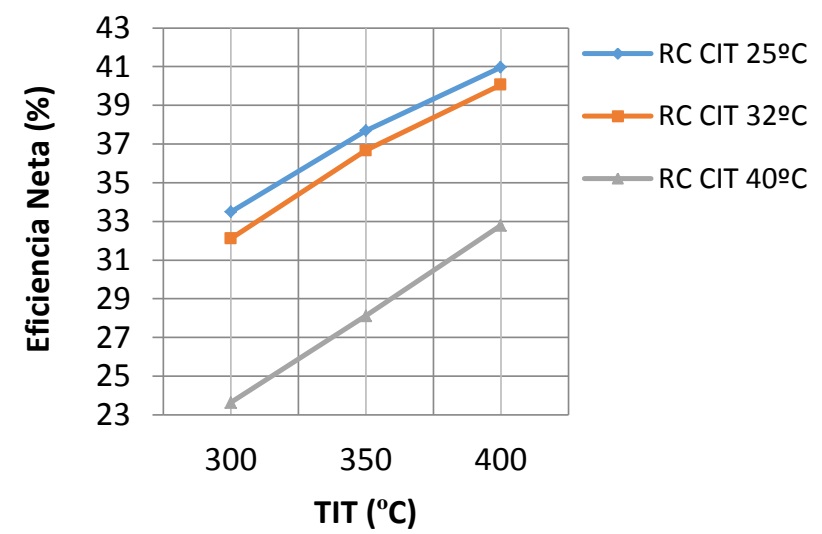

Figura 10.18. $\mathrm{RC} s-\mathrm{C}_{2} \mathrm{H}_{6}$ Eficiencia Neta \& $\mathrm{CIT}^{(2)}$.

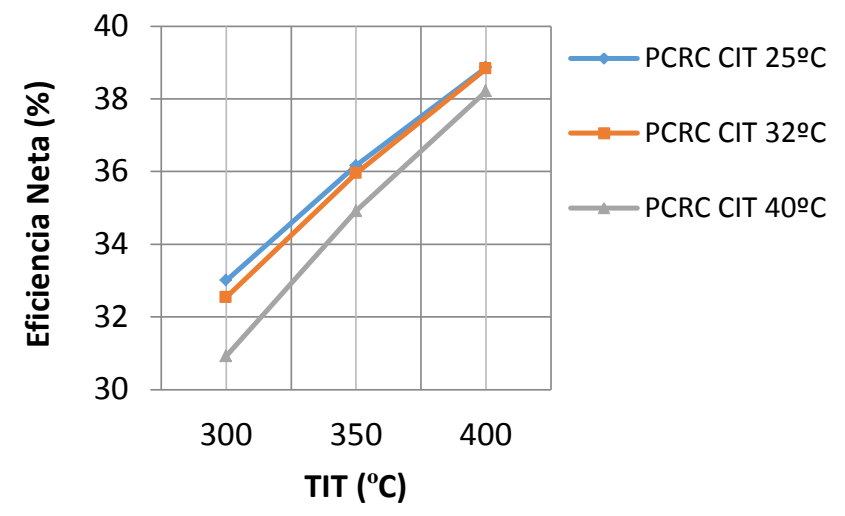

Figura 10.19. $\mathrm{PCRC} s-\mathrm{C}_{2} \mathrm{H}_{6}$ Eficiencia Neta \& $\mathrm{CIT}^{(2)}$.

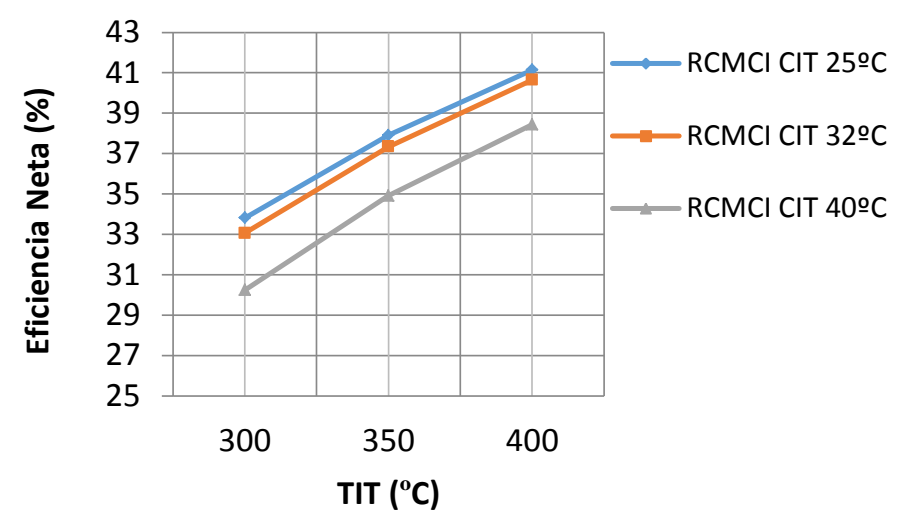

Figura 10.20. $\mathrm{RCMCl} \mathrm{s}-\mathrm{C}_{2} \mathrm{H}_{6}$ Eficiencia Neta \& $\mathrm{CIT}^{(2)}$.

${ }^{(2)}$ Relación de Presiones de la tabla 10.7 (Opción A) para el ciclo de potencia s- $\mathrm{CO}_{2}$ Brayton. Relación de Presiones de la tabla 10.8 (Opción B) para el ciclo de potencia s- $\mathrm{C}_{2} \mathrm{H}_{6}$ Brayton. 
10. Plantas Termosolares con Sales Fundidas o DSG como fluido caloportador acopladas a ciclos de potencia supercríticos Brayton con $\mathrm{C}_{2} \mathrm{H}_{6}$ como fluido de trabajo

Tabla 10.10. Eficiencia neta y temperatura entrada al compresor y pérdidas de presión en los intercambiadores de calor ${ }^{(3)}$.

\begin{tabular}{|c|c|c|c|c|}
\hline $\begin{array}{l}\text { Ciclo Potencia } \\
\text { Fluido de Trabajo }\end{array}$ & $\begin{array}{l}\mathrm{SB} \\
\mathrm{s}-\mathrm{C}_{2} \mathrm{H}_{6}\end{array}$ & $\begin{array}{l}\mathrm{RC} \\
\mathrm{s}-\mathrm{C}_{2} \mathrm{H}_{6}\end{array}$ & $\begin{array}{l}\text { PCRC } \\
\mathrm{s}-\mathrm{C}_{2} \mathrm{H}_{6}\end{array}$ & $\begin{array}{l}\mathrm{RCMCl} \\
\mathrm{s}-\mathrm{C}_{2} \mathrm{H}_{6}\end{array}$ \\
\hline $\left.\mathrm{TIT}=300^{\circ} \mathrm{C} \mathrm{CIT} 25^{\circ} \mathrm{C}, \mathrm{AP} 1 \%\right)$ & 31.93 & 32.06 & 31.99 & 32.37 \\
\hline TIT $=300^{\circ} \mathrm{C}\left(\mathrm{CIT} 25^{\circ} \mathrm{C}\right.$, AP $\left.2 \%\right)$ & 31.15 & 30.59 & 30.96 & 30.89 \\
\hline TIT $=300^{\circ} \mathrm{C}\left(\mathrm{CIT} 40^{\circ} \mathrm{C}\right.$, AP $\left.1 \%\right)$ & 24.68 & 21.65 & 29.56 & 28.61 \\
\hline TIT $=300^{\circ} \mathrm{C}\left(\mathrm{CIT} 40^{\circ} \mathrm{C}, \mathrm{AP} 2 \%\right)$ & 23.53 & 19.52 & 28.11 & 26.89 \\
\hline $\mathrm{TIT}=350^{\circ} \mathrm{C}\left(\mathrm{CIT} 25^{\circ} \mathrm{C}\right.$, AP $\left.1 \%\right)$ & 35.05 & 36.32 & 35.16 & 36.53 \\
\hline TIT $=350^{\circ} \mathrm{C}\left(\mathrm{CIT} 25^{\circ} \mathrm{C}, \mathrm{AP} 2 \%\right)$ & 34.28 & 34.91 & 34.14 & 35.11 \\
\hline TIT $=350^{\circ} \mathrm{C}\left(\mathrm{CIT} 40^{\circ} \mathrm{C}\right.$, AP $\left.1 \%\right)$ & 28.66 & 26.25 & 33.58 & 33.37 \\
\hline $\mathrm{TIT}=350^{\circ} \mathrm{C}\left(\mathrm{CIT} 40^{\circ} \mathrm{C}, \mathrm{AP} 2 \%\right)$ & 27.56 & 24.25 & 32.18 & 31.74 \\
\hline TIT $=400^{\circ} \mathrm{C}\left(\mathrm{CIT} 25^{\circ} \mathrm{C}, \mathrm{AP} 1 \%\right)$ & 37.62 & 39.63 & 37.87 & 39.8 \\
\hline $\mathrm{TIT}=400^{\circ} \mathrm{C}\left(\mathrm{CIT} 25^{\circ} \mathrm{C}, \mathrm{AP} 2 \%\right)$ & 36.87 & 38.28 & 36.85 & 38.44 \\
\hline TIT $=400^{\circ} \mathrm{C}\left(\mathrm{CIT} 40^{\circ} \mathrm{C}\right.$, AP $\left.1 \%\right)$ & 31.87 & 31.04 & 36.92 & 36.95 \\
\hline TIT $=400^{\circ} \mathrm{C}\left(\mathrm{CIT} 40^{\circ} \mathrm{C}\right.$, AP $\left.2 \%\right)$ & 30.81 & 29.13 & 35.56 & 35.39 \\
\hline
\end{tabular}

10.11. Efecto combinado de pérdida de presión en los cambiadores de calor y temperatura de entrada al compresor

El diseño del sumidero final de calor o Ultimate Heat Sink (UHS) es uno de los equipos claves durante el diseño de las plantas solares. Estas instalaciones están normalmente localizadas en zonas secas donde el agua es un recurso escaso. El estado del arte de los sistemas de enfriamiento del UHS está dominado por dos tecnologías: el enfriamiento mediante aire en convección forzada o Air Cooler Heat Exchangers (ACHE) y en el enfriamiento mediante agua en torres de refrigeración natural o de convección forzada. Un estudio detallado de estas dos alternativas se ha realizado en [11]. Con el sistema de enfriamiento húmedo (con agua de refrigeración) la densidad del $\mathrm{CO}_{2}$ a la entrada del compresor es muy alta, reduciendo el trabajo de la turbomáquina y mejorando la eficiencia del ciclo de potencia. Sin embargo, en la mayor parte de las plantas termosolares, la solución de diseño adoptada para el sumidero final de calor, es la solución seca (con aire).

El principal inconveniente de la eliminación de calor mediante aire en convección forzada es debido a la potencia eléctrica consumida por los ventiladores; como consecuencia directa la temperatura a la entrada del compresor es alta y se requiere un mayor trabajo de compresión del $\mathrm{CO}_{2}$.

(3) Basado en los resultados obtenidos en las figuras 10.9 y 10.10, y las relaciones de presión de los ciclos $\mathrm{s}-\mathrm{C}_{2} \mathrm{H}_{6}$ Brayton Opción B listado en la tabla 10.8 .

Así mismo, las pérdidas de carga en los cambiadores de calor influyen negativamente en la eficiencia neta de la planta termosolar. En la tabla 10.10 se han resumido los resultados obtenidos de un estudio de sensibilidad de los dos efectos citados: las pérdidas de presión en cambiadores y las altas temperaturas a la entrada del compresor.

Tabla 10.11. Costes Unitarios de Inversión en el campo solar $\left(\$ / \mathrm{m}^{2}\right)$.

\begin{tabular}{lllll}
\hline Colector solar tipo: & PTC & PTC & LF & LF \\
HTF: & MS & DSG & MS & DSG \\
Obra civil $\left(\$ / \mathrm{m}^{2}\right):$ & 25 & 25 & 20 & 20 \\
\hline
\end{tabular}


10. Plantas Termosolares con Sales Fundidas o DSG como fluido caloportador acopladas a ciclos de potencia supercríticos Brayton con $\mathrm{C}_{2} \mathrm{H}_{6}$ como fluido de trabajo

\begin{tabular}{lllll}
\hline Colector solar $\left(\$ / \mathrm{m}^{2}\right):$ & 317 & 270 & 245 & 181 \\
HTF sistema $\left(\$ / \mathrm{m}^{2}\right):$ & 90 & 90 & 35 & 35 \\
Coste Total Campo Solar $\left(\$ / \mathrm{m}^{2}\right):$ & 432 & 385 & 300 & 236 \\
\hline
\end{tabular}

Tabla 10.12. Costes de inversión en planta termosolar con colectores LF con MS como HTF (M\$). Potencia neta fija en todas las configuraciones $50 \mathrm{MWe}^{(3)}$.

\begin{tabular}{|c|c|c|c|c|c|c|c|c|c|c|}
\hline $\begin{array}{l}\text { Ciclo } \\
\text { Potencia }\end{array}$ & $\begin{array}{l}\text { Rankine } \\
\text { (Un } \\
\text { DRH) }\end{array}$ & $\begin{array}{l}\text { Rankine } \\
\text { (Dos } \\
\text { DRH) }\end{array}$ & $\begin{array}{l}\mathrm{SB} \\
\mathrm{s}-\mathrm{CO}_{2}\end{array}$ & $\begin{array}{l}\mathrm{SB} \\
\mathrm{S}-\mathrm{C}_{2} \mathrm{H}_{6}\end{array}$ & $\begin{array}{l}\mathrm{RC} \\
\mathrm{s}-\mathrm{CO}_{2}\end{array}$ & $\begin{array}{l}\mathrm{RC} \\
\mathrm{s}-\mathrm{C}_{2} \mathrm{H}_{6}\end{array}$ & $\begin{array}{l}\text { PCRC } \\
\text { s-CO }\end{array}$ & $\begin{array}{l}\text { PCRC } \\
\text { s- } \mathrm{C}_{2} \mathrm{H}_{6}\end{array}$ & $\begin{array}{l}\mathrm{RCMCl} \\
\mathrm{sCO}_{2}\end{array}$ & $\begin{array}{l}\mathrm{RCMCl} \\
\mathrm{s}-\mathrm{C}_{2} \mathrm{H}_{6}\end{array}$ \\
\hline Fluido & DSG & DSG & MS & MS & MS & MS & MS & MS & MS & MS \\
\hline $\mathrm{TIT}=300^{\circ} \mathrm{C}$ & - & - & 109.1 & 96.1 & 104.9 & 94.7 & 99.2 & 93.4 & 95.9 & 91.9 \\
\hline $\mathrm{TIT}=350^{\circ} \mathrm{C}$ & 60.1 & 59.7 & 97.4 & 88.1 & 90.5 & 83.8 & 89.5 & 85.2 & 85.8 & 82.2 \\
\hline $\mathrm{TIT}=400^{\circ} \mathrm{C}$ & 57.8 & 57.7 & 90.1 & 82.9 & 81.5 & 77.5 & 83.1 & 79.7 & 79.5 & 76.4 \\
\hline
\end{tabular}

Tabla 10.13. Costes de inversión en planta termosolar con colectores PTC con MS como HTF (M\$). Potencia neta fija en todas las configuraciones $50 \mathrm{MWe}^{(3)}$.

\begin{tabular}{|c|c|c|c|c|c|c|c|c|c|c|}
\hline $\begin{array}{l}\text { Ciclo } \\
\text { Potencia }\end{array}$ & $\begin{array}{l}\text { Rankine } \\
\text { (Un DRH) }\end{array}$ & $\begin{array}{l}\text { Rankine } \\
\text { (Dos } \\
\text { DRH) }\end{array}$ & $\begin{array}{l}\mathrm{SB} \\
\mathrm{s}-\mathrm{CO}_{2}\end{array}$ & $\begin{array}{l}\mathrm{SB} \\
\mathrm{s}-\mathrm{C}_{2} \mathrm{H}_{6}\end{array}$ & $\begin{array}{l}\mathrm{RC} \\
\mathrm{s}-\mathrm{CO}_{2}\end{array}$ & $\begin{array}{l}\mathrm{RC} \\
\mathrm{s}-\mathrm{C}_{2} \mathrm{H}_{6}\end{array}$ & $\begin{array}{l}\text { PCRC } \\
\mathrm{s}-\mathrm{CO}_{2}\end{array}$ & $\begin{array}{l}\text { PCRC } \\
s-\mathrm{C}_{2} \mathrm{H}_{6}\end{array}$ & $\begin{array}{l}\mathrm{RCMCl} \\
\mathrm{sCO}_{2}\end{array}$ & $\begin{array}{l}\mathrm{RCMCl} \\
\mathrm{s}-\mathrm{C}_{2} \mathrm{H}_{6}\end{array}$ \\
\hline Working Fluid & DSG & DSG & MS & MS & MS & MS & MS & MS & MS & MS \\
\hline $\mathrm{TIT}=300^{\circ} \mathrm{C}$ & - & - & 133.6 & 117.4 & 128.4 & 115.7 & 121.5 & 114.1 & 117.5 & 112.3 \\
\hline $\mathrm{TIT}=350^{\circ} \mathrm{C}$ & 79.6 & 77.9 & 119.7 & 107.8 & 111.1 & 102.5 & 109.9 & 104.4 & 105.4 & 100.6 \\
\hline $\mathrm{TIT}=400^{\circ} \mathrm{C}$ & 76.6 & 75.4 & 111.1 & 102.1 & 100.4 & 95.2 & 102.5 & 98.1 & 98.1 & 93.9 \\
\hline
\end{tabular}

Tabla 10.14. Costes de inversión en planta termosolar con colectores LF con DSG (M\$\$). Potencia neta fija en todas las configuraciones $50 \mathrm{MWe}^{(3)}$.

\begin{tabular}{|c|c|c|c|c|c|c|c|c|c|c|}
\hline $\begin{array}{l}\text { Ciclo } \\
\text { Potencia }\end{array}$ & $\begin{array}{l}\text { Rankine } \\
\text { (Un } \\
\text { DRH) }\end{array}$ & $\begin{array}{l}\text { Rankine } \\
\text { (Dos } \\
\text { DRH) }\end{array}$ & $\begin{array}{l}\mathrm{SB} \\
\mathrm{s}-\mathrm{CO}_{2}\end{array}$ & $\begin{array}{l}\text { SB } \\
s-\mathrm{C}_{2} \mathrm{H}_{6}\end{array}$ & $\begin{array}{l}\mathrm{RC} \\
\mathrm{s}-\mathrm{CO}_{2}\end{array}$ & $\begin{array}{l}\mathrm{RC} \\
\mathrm{s}-\mathrm{C}_{2} \mathrm{H}_{6}\end{array}$ & $\begin{array}{l}\text { PCRC } \\
\mathrm{s}-\mathrm{CO}_{2}\end{array}$ & $\begin{array}{l}\text { PCRC } \\
s-\mathrm{C}_{2} \mathrm{H}_{6}\end{array}$ & $\begin{array}{l}\mathrm{RCMCl} \\
\mathrm{sCO}_{2}\end{array}$ & $\begin{array}{l}\mathrm{RCMCl} \\
\mathrm{s}-\mathrm{C}_{2} \mathrm{H}_{6}\end{array}$ \\
\hline $\mathrm{TIT}=300^{\circ} \mathrm{C}$ & - & - & 73.9 & 65.1 & 71.2 & 64.23 & 67.3 & 63.3 & 65.1 & 62.4 \\
\hline $\mathrm{TIT}=350^{\circ} \mathrm{C}$ & 60.1 & 59.7 & 66.1 & 59.7 & 61.3 & 56.8 & 60.7 & 60.3 & 58.2 & 55.7 \\
\hline $\mathrm{TIT}=400^{\circ} \mathrm{C}$ & 57.8 & 57.7 & 61.1 & 56.3 & 55.3 & 52.6 & 56.4 & 54.1 & 53.9 & 51.8 \\
\hline
\end{tabular}

TABLA 10.15. Costes de inversión en planta termosolar con colectores PTC DSG (M\$\$). Potencia neta fija en todas las configuraciones $50 \mathrm{MWe}^{(3)}$.

\begin{tabular}{|c|c|c|c|c|c|c|c|c|c|c|}
\hline $\begin{array}{l}\text { Ciclo } \\
\text { Potencia }\end{array}$ & $\begin{array}{l}\text { Rankine } \\
\text { (Un } \\
\text { DRH) }\end{array}$ & $\begin{array}{l}\text { Rankine } \\
\text { (Dos } \\
\text { DRH) }\end{array}$ & $\begin{array}{l}\mathrm{SB} \\
\mathrm{s}-\mathrm{CO}_{2}\end{array}$ & $\begin{array}{l}\text { SB } \\
s-\mathrm{C}_{2} \mathrm{H}_{6}\end{array}$ & $\begin{array}{l}\mathrm{RC} \\
\mathrm{s}-\mathrm{CO}_{2}\end{array}$ & $\begin{array}{l}\mathrm{RC} \\
\mathrm{s}-\mathrm{C}_{2} \mathrm{H}_{6}\end{array}$ & $\begin{array}{l}\text { PCRC } \\
\mathrm{s}-\mathrm{CO}_{2}\end{array}$ & $\begin{array}{l}\text { PCRC } \\
s-\mathrm{C}_{2} \mathrm{H}_{6}\end{array}$ & $\begin{array}{l}\mathrm{RCMCl} \\
\mathrm{sCO}_{2}\end{array}$ & $\begin{array}{l}\mathrm{RCMCl} \\
\mathrm{s}-\mathrm{C}_{2} \mathrm{H}_{6}\end{array}$ \\
\hline HTF & DSG & DSG & DSG & DSG & DSG & DSG & DSG & DSG & DSG & DSG \\
\hline $\mathrm{TIT}=300^{\circ} \mathrm{C}$ & - & - & 102.7 & 90.2 & 98.6 & 88.8 & 93.4 & 87.7 & 90.3 & 86.3 \\
\hline $\mathrm{TIT}=350^{\circ} \mathrm{C}$ & 79.6 & 77.9 & 91.9 & 82.8 & 85.3 & 78.7 & 84.5 & 80.2 & 80.9 & 77.3 \\
\hline $\mathrm{TIT}=400^{\circ} \mathrm{C}$ & 76.6 & 75.4 & 85.3 & 78.4 & 77.2 & 73.2 & 78.7 & 75.3 & 75.3 & 72.1 \\
\hline
\end{tabular}

(3) Basado en los resultados obtenidos en las figuras 10.9 y 10.10, y las relaciones de presión de los ciclos s- $\mathrm{C}_{2} \mathrm{H}_{6}$ Brayton Opción B listado en la tabla 10.8.

\subsection{2. Área de Apertura y Coste de Inversión del Campo Solar}

Para traducir las mejoras de eficiencia neta del ciclo de potencia en ahorro de inversión en el campo solar, se ha calculado el área de apertura de los colectores en las diferentes configuraciones estudiadas. Los resultados obtenidos quedan resumidos en las figuras $10.21 \mathrm{y}$ 10.22 . 
10. Plantas Termosolares con Sales Fundidas o DSG como fluido caloportador acopladas a ciclos de potencia supercríticos Brayton con $\mathrm{C}_{2} \mathrm{H}_{6}$ como fluido de trabajo

En base a los costes unitarios indicados en la tabla 10.11, obtenidos del programa informático SAM desarrollado por NREL, se ha realizado una estimación de los costes de inversión de las diferentes configuraciones, tablas 10.12 a 10.15. En la tabla 10.12 y 10.13 , se ha asumido sales fundidas como HTF del campo solar, por este motivo se ha seleccionado acero inoxidable en los tubos absorbedores. En las tablas 10.14 y 10.15 se ha considerado que el HTF es agua, ya que se dispone de colectores con DSG. En este caso se ha elegido como material de comparación el acero al carbono u otro acero inoxidable de bajo coste para realizar la comparativa de inversión.

En la tabla 10.11 se puede apreciar como el coste de inversión en colectores LF es menor que en los colectores PTC. El principal motivo es la reducción del coste de fabricación de espejos rectos y de una estructura de suportación más liviana. Sin embargo, hablando desde el punto de vista de la producción de energía eléctrica de la planta termosolar a lo largo del año, los colectores PTC proporcionan una mayor potencia generada anual.

Comparando la inversión de capital en el campo solar en plantas acopladas a ciclos de potencia Rankine o ciclos Brayton, se concluye que las instalaciones con ciclo de potencia s- $\mathrm{C}_{2} \mathrm{H}_{6}$ Brayton $\mathrm{RCMCl}$ y DSG como HTF, son la solución de diseño optima desde el punto de vista de reducción del coste de inversión. Los resultados de esta comparativa están tabulados en las tablas 10.14 y 10.15 .

\subsection{Conclusiones}

El trabajo desarrollado en el presente capítulo demuestra como las centrales termosolares con colectores lineales acopladas a ciclos de potencia $s-\mathrm{C}_{2} \mathrm{H}_{6}$ Brayton tienen mayor eficiencia energética que las plantas con ciclos de potencia $s-\mathrm{CO}_{2}$ Brayton, y también superior que las centrales termosolares con ciclos de potencia Rankine.

La configuración $\mathrm{RCMCl}$ es la que proporciona mayor rendimiento para $\mathrm{TIT}<400^{\circ} \mathrm{C}$. La eficiencia neta de la planta termosolar acopladas a ciclos de potencia s- $\mathrm{C}_{2} \mathrm{H}_{6}$ Brayton es superior en 1-2 \% a la proporcionada por las plantas con fluido de trabajo s- $\mathrm{CO}_{2}$. Los resultados detallados están resumidos e ilustrados en las figuras 10.7 a 10.12 .

En este estudio también se han comparado las plantas con ciclos de potencia s- $\mathrm{CO}_{2}$ Brayton con la tecnología DSG en colectores lineales acoplada a ciclos de potencia Rankine. A pesar de la menor eficiencia de las plantas con ciclos Rankine en comparación con las plantas con ciclos Brayton, resultados detallados en las figuras 10.7 a 10.12; el coste del material de los equipos y componentes constituye una variable decisiva a tener en consideración a la hora de tomar decisiones de inversión en ambas tecnologías. En las tablas 10.12 a 10.15 se ha comparado el coste de capital en los campos solares. Como principal conclusión se hace hincapié en los mayores requisitos de corrosión de los materiales que están en contacto con las sales fundidas en comparación con la utilización de agua como HTF. Como caso extremo se ha considerado en los tubos receptores con sales fundidas acero inoxidable austenítico (AISI 304 o AISI 316), y para los tubos receptores con DSG acero al carbono. 
10. Plantas Termosolares con Sales Fundidas o DSG como fluido caloportador acopladas a ciclos de potencia supercríticos Brayton con $\mathrm{C}_{2} \mathrm{H}_{6}$ como fluido de trabajo

Los equipos integrantes del BOP son otro parámetro decisivo a tener en cuenta a la hora de realizar la comparativa tecnológica. En los ciclos de potencia Brayton es necesario utilizar aceros austeníticos muy aleados con Niobio (Nb) o Titanio (Ti) como elementos aleantes, es el caso del acero AISI 347. Sin embargo, los equipos del BOP de los ciclos de potencia Rankine pueden estar constituidos por aceros de baja aleación, optimizando por tanto su coste. El diseño y coste de fabricación de equipos sofisticados es otro punto muy importante a evaluar. Los ciclos de potencia Brayton requieren intercambiadores de calor con una alta resistencia mecánicas, operando a presiones del orden de 250 bar. Así mismo, las tasas de intercambio de calor son muy grandes, requiriendo grandes áreas de intercambio de calor en pequeños volúmenes, es decir, se requieren equipos muy compactos. Estos requisitos hacen necesaria la utilización de intercambiadores de calor de circuito impreso (PCHE), cuya tecnología de fabricación está en poder de unas pocas empresas en el mundo, constituyendo un monopolio a la hora de fijar su precio comercial.

En el caso de los ciclos de potencia Rankine, los equipos han sido ampliamente evaluados experimentalmente, y su nivel de tecnología de fabricación está muy difundido, existiendo un gran mercado de proveedores que facilita a obtener un precio competitivo, y una garantía de operación sólida. Lo mismo ocurre con las turbomáquinas, en proceso de desarrollo industrial para los ciclos de potencia Brayton.

Tabla 10.16. Coste de Capital en plantas con colectores LF (M\$). En todas las plantas termosolares se ha fijado la potencia neta $50 \mathrm{MWe}^{(3)}$.

\begin{tabular}{|c|c|c|c|c|c|c|c|c|c|c|}
\hline $\begin{array}{l}\text { Ciclo } \\
\text { Fluido }\end{array}$ & $\begin{array}{l}\text { Rankine } \\
\text { DSG }\end{array}$ & $\begin{array}{l}\text { Rankine } \\
\text { DSG }\end{array}$ & $\begin{array}{l}\mathrm{SB} \\
\mathrm{s}-\mathrm{CO}_{2}\end{array}$ & $\begin{array}{l}S B \\
s-C_{2} H_{6}\end{array}$ & $\begin{array}{l}\mathrm{RC} \\
\mathrm{s}-\mathrm{CO}_{2}\end{array}$ & $\begin{array}{l}\mathrm{RC} \\
\mathrm{s}-\mathrm{C}_{2} \mathrm{H}_{6}\end{array}$ & $\begin{array}{l}\mathrm{PCRC} \\
\mathrm{s}-\mathrm{CO}_{2}\end{array}$ & $\begin{array}{l}\text { PCRC } \\
s-\mathrm{C}_{2} \mathrm{H}_{6}\end{array}$ & $\begin{array}{l}\mathrm{RCMCl} \\
\mathrm{s}-\mathrm{CO}_{2}\end{array}$ & $\begin{array}{l}\mathrm{RCMCl} \\
\mathrm{s}-\mathrm{C}_{2} \mathrm{H}_{6}\end{array}$ \\
\hline $\mathrm{TIT}=300^{\circ} \mathrm{C}$ & - & - & 109.1 & 96.1 & 104.9 & 94.7 & 99.2 & 93.4 & 95.9 & 91.9 \\
\hline $\mathrm{TIT}=350^{\circ} \mathrm{C}$ & 60.1 & 59.7 & 97.4 & 88.1 & 90.5 & 83.8 & 89.5 & 85.2 & 85.8 & 82.2 \\
\hline $\mathrm{TIT}=400^{\circ} \mathrm{C}$ & 57.8 & 57.7 & 90.1 & 82.9 & 81.5 & 77.5 & 83.1 & 79.7 & 79.5 & 76.4 \\
\hline $\mathrm{TIT}=450^{\circ} \mathrm{C}$ & 55.9 & 56.1 & 85.1 & 79.7 & 75.8 & 73.7 & 78.9 & 76.1 & 75.1 & 72.7 \\
\hline $\mathrm{TIT}=500^{\circ} \mathrm{C}$ & 54.4 & 54.6 & 81.9 & 77.8 & 72.3 & 71.4 & 76.0 & 73.8 & 72.3 & 70.6 \\
\hline $\mathrm{TIT}=550^{\circ} \mathrm{C}$ & 53.2 & 53.5 & 80.2 & 77.3 & 70.4 & 70.5 & 74.4 & 72.7 & 70.7 & 69.7 \\
\hline
\end{tabular}

(3) Basado en los resultados obtenidos en las figuras 10.9 y 10.10, y las relaciones de presión de los ciclos $\mathrm{s}-\mathrm{C}_{2} \mathrm{H}_{6}$ Brayton Opción B listado en la tabla 10.8 .

Tabla 10.17. Coste de Capital en plantas con colectores PTC (M\$̦). En todas las plantas termosolares se ha fijado la potencia neta $50 \mathrm{MWe}{ }^{(3)}$.

\begin{tabular}{|c|c|c|c|c|c|c|c|c|c|c|}
\hline $\begin{array}{l}\text { Ciclo } \\
\text { Fluido }\end{array}$ & $\begin{array}{l}\text { Rankine } \\
\text { DSG }\end{array}$ & $\begin{array}{l}\text { Rankine } \\
\text { DSG }\end{array}$ & $\begin{array}{l}\mathrm{SB} \\
\mathrm{s}-\mathrm{CO}_{2}\end{array}$ & $\begin{array}{l}\mathrm{SB} \\
\mathrm{s}-\mathrm{C}_{2} \mathrm{H}_{6}\end{array}$ & $\begin{array}{l}\mathrm{RC} \\
\mathrm{s}-\mathrm{CO}_{2}\end{array}$ & $\begin{array}{l}\mathrm{RC} \\
\mathrm{s}-\mathrm{C}_{2} \mathrm{H}_{6}\end{array}$ & $\begin{array}{l}\text { PCRC } \\
\mathrm{s}-\mathrm{CO}_{2}\end{array}$ & $\begin{array}{l}\text { PCRC } \\
s-\mathrm{C}_{2} \mathrm{H}_{6}\end{array}$ & $\begin{array}{l}\mathrm{RCMCl} \\
\mathrm{s}-\mathrm{CO}_{2}\end{array}$ & $\begin{array}{l}\mathrm{RCMCl} \\
\mathrm{s}-\mathrm{C}_{2} \mathrm{H}_{6}\end{array}$ \\
\hline $\mathrm{TIT}=300^{\circ} \mathrm{C}$ & - & - & 133.6 & 117.3 & 128.4 & 115.7 & 121.5 & 114.1 & 117.5 & 112.3 \\
\hline $\mathrm{TIT}=350^{\circ} \mathrm{C}$ & 79.6 & 77.9 & 119.7 & 107.8 & 111.1 & 102.5 & 109.9 & 104.4 & 105.4 & 100.6 \\
\hline $\mathrm{TIT}=400^{\circ} \mathrm{C}$ & 76.6 & 75.4 & 111.1 & 102.1 & 100.4 & 95.2 & 102.5 & 98.1 & 98.1 & 93.9 \\
\hline $\mathrm{TIT}=450^{\circ} \mathrm{C}$ & 74.3 & 73.5 & 105.6 & 98.7 & 94.1 & 91.2 & 97.9 & 94.3 & 93.2 & 90.1 \\
\hline $\mathrm{TIT}=500^{\circ} \mathrm{C}$ & 72.4 & 71.7 & 102.7 & 97.5 & 90.6 & 89.3 & 95.3 & 92.7 & 90.6 & 88.9 \\
\hline $\mathrm{TIT}=550^{\circ} \mathrm{C}$ & 71.1 & 70.6 & 101.9 & 98.2 & 89.6 & 89.7 & 94.7 & 92.7 & 90.1 & 88.8 \\
\hline
\end{tabular}


10. Plantas Termosolares con Sales Fundidas o DSG como fluido caloportador acopladas a ciclos de potencia supercríticos Brayton con $\mathrm{C}_{2} \mathrm{H}_{6}$ como fluido de trabajo

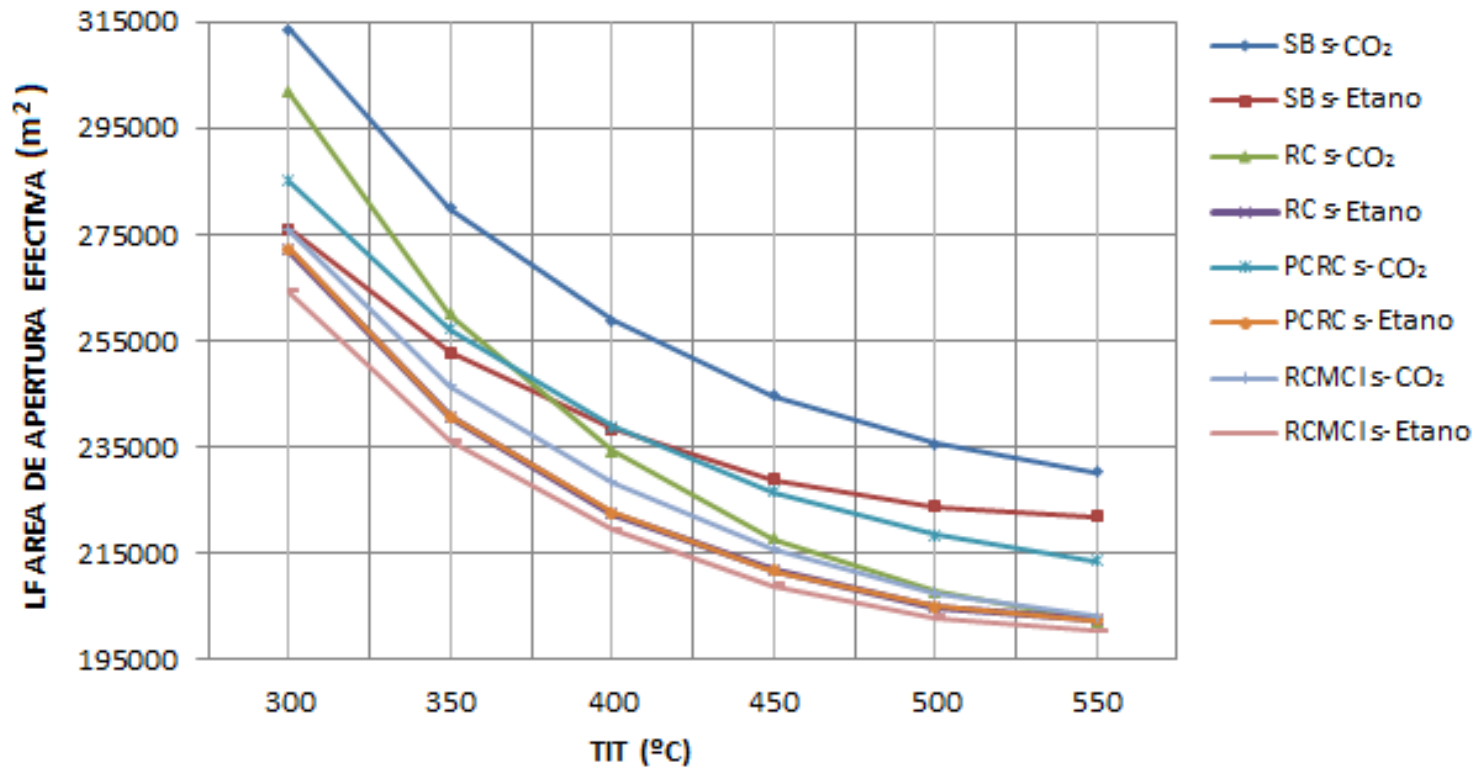

Figura 10.21. Área de Apertura Efectiva con colectores $\mathrm{LF}\left(\mathrm{m}^{2}\right)$ versus TIT. Potencia neta generada fija $50 \mathrm{MWe}$ netos ${ }^{(3)}$.

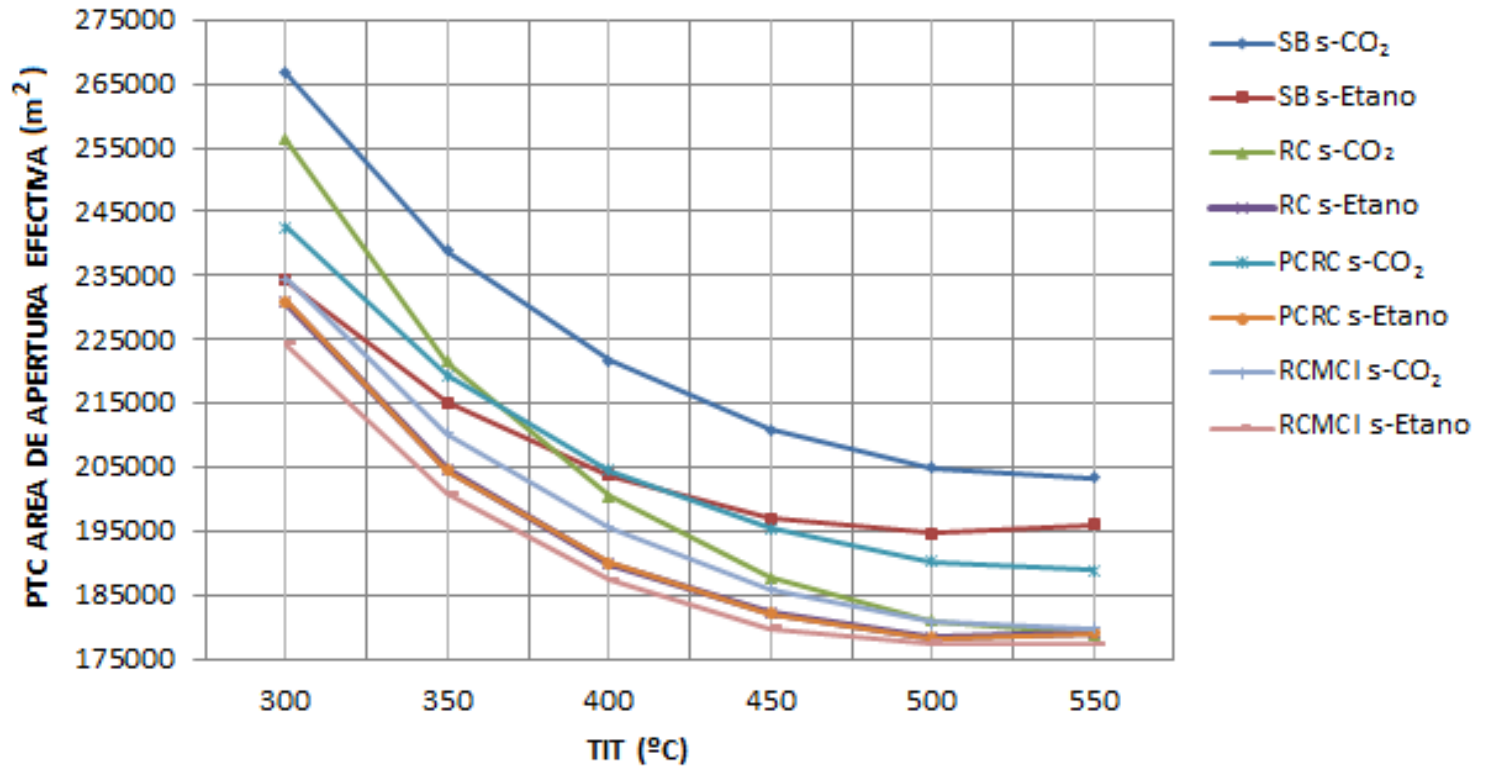

Figura 10.22. PTC Área de Apertura Efectiva $\left(\mathrm{m}^{2}\right)$ versus TIT. Potencia neta generada fija 50MWe netos ${ }^{(3)}$.

(3) Basado en los resultados obtenidos en las figuras 10.9 y 10.10, y las relaciones de presión de los ciclos $\mathrm{s}-\mathrm{C}_{2} \mathrm{H}_{6}$ Brayton Opción B listado en la tabla 10.8 .

Este estudio es conceptual y basado en simulaciones termodinámicas, calculando los flujos energéticos con las propiedades del fluido en estado supercrítico en los ciclos de potencia Brayton. No se ha evaluado la posible descomposición química u otras reacciones químicas. La literatura consultada [6-17] confirma el riesgo de disociación química del $\mathrm{C}_{2} \mathrm{H}_{6}$ debido a las altas temperaturas de operación de los ciclos $\mathrm{s}-\mathrm{C}_{2} \mathrm{H}_{6}$ Brayton, sin embargo, no se ha experimentado todavía sobre el posible efecto beneficioso de los altos valores de presión de operación (entre 50 y 250 bar). Una posible solución para evitar la descomposición del $\mathrm{C}_{2} \mathrm{H}_{6}$ en subproductos, es optimizar el diseño de disposición de los equipos que integran el ciclo de 
10. Plantas Termosolares con Sales Fundidas o DSG como fluido caloportador acopladas a ciclos de potencia supercríticos Brayton con $\mathrm{C}_{2} \mathrm{H}_{6}$ como fluido de trabajo

potencia para minimizar el tiempo a altas temperaturas. Para ello se debe maximizar la velocidad del fluido, teniendo en cuenta que un incremento de velocidad también puede dar lugar a un incremento de pérdidas de presión. Las reacciones de descomposición del $\mathrm{C}_{2} \mathrm{H}_{6}$ están muy influenciadas por la cinética del proceso.

Otro gran peligro de este tipo de ciclos de potencia son las infiltraciones de aire que puede provocar auto ignición y explosión del $\mathrm{C}_{2} \mathrm{H}_{6}$. Sin embargo, en este estudio se ha caracterizado la potencialidad de incrementar la eficiencia energética de las instalaciones termosolares gracias a los ciclos con $\mathrm{C}_{2} \mathrm{H}_{6}$.

\subsection{Referencias}

[1] Zhiwen Ma, Craig S. Turchi. Advanced Supercritical Carbon Dioxide Power Cycle Configurations for use in Concentrating Solar Power Systems. National Renewable Energy Laboratory.

[2] G.J. Kolb. An Evaluation of Possible Next-Generation High Temperature Molten-Salt Power Towers, SAND2011-9320, Sandia National Laboratories, Albuquerque, NM, 2011.

[3] Bruce Kelly. Advanced Thermal Storage for Central Receivers with Supercritical Coolants.

[4] James E. Pacheco, Thorsten Wolf, Nishant Muley. Incorporatin Supercritical steam Turbines into Advanced Molten-Salt Power Tower Plants: Feasibility and Performance. Sandia Report, SAN2013-1960, March 2013.

[5] L.Coco-Enríquez, J.Muñoz-Antón, J.M. Martínez-Val. Supercritical Steam power cycle for Line-Focus Solar Power Plants. 4th International Conference on Nuclear and Renewable Energy Resources NURER 2014, Turkey.

[6] Jeffrey A. Perez, Dr. M. Driscoll, Dr. P. Hejzlar. Evaluation of Ethane as a Power Conversion System Working Fluid for Fast Reactors. Massachusetts Institute of Technology MIT, June 2008. [7] A.Rovira, Javier Muñoz-Anton, Maria Jose Montes, Jose Maria Martinez-Val. Optimization of Brayton cycles for low to-moderate grade thermal energy sources. Enery 55, 15 June 2013.

[8] A.Rovira, C.Rubbia, M.Valdés, J.M.Martínez-Val. Thermodynamic cycles optimised for medium enthalpy units of concentrating solar power. Energy 67 (2014) 176-185.

[9] Craig S. Turchi, John Dyreby. Supercritical Carbon Dioxide power cycle configurations for use in Concentrating solar power systems. ASME Turbo Expo 2012, June 11-15 2012, Copenhagen, Denmark.

[10] Seidel, W. (2010). Model Development and Annual Simulation of the Supercritical Carbon Dioxide Brayton Cycle for Concentrating Solar Power Applications. University of WisconsinMadison.

[11] Gavic D. Investigation Of Water, Air, and Hybrid Cooling For Supercritical Carbon Dioxide Brayton Cycles. University of Wisconsin-Madison.

[12] Craig S. Turchi, Zhiwen Ma, Ty W. Neises, Michael J.Wagner. Thermodynamic Study of Advanced Supercritical Carbon Dioxide Power Cycles for Concentrating Solar Power Systems. ASME, Journal of Solar Energy Engineering, November 2013, Vol. 135.

[13] Ty W. Neises, Craig S. Turchi. Supercritical $\mathrm{CO}_{2}$ Power Cycles: Design Considerations for Concentrating Solar Power. The 4th International Symposium - Supercritical $\mathrm{CO}_{2}$ Power Cycles September 9-10, 2014, Pittsburgh, Pennsylvania.

[14] Alexander R. Ludington. Tools for Supercritical Carbon Dioxide Cycle Analysis and the Cycle's Applicability to Sodium Fast Reactors. Massachusetts Institute of Technology MIT, June 2009. 
10. Plantas Termosolares con Sales Fundidas o DSG como fluido caloportador acopladas a ciclos de potencia supercríticos Brayton con $\mathrm{C}_{2} \mathrm{H}_{6}$ como fluido de trabajo

[15] Database of Hazardous Materials. National Oceanic and Atmospheric Administration (NOAA), USA. https://cameochemicals.noaa.gov/chemical/8655

[16] A. Roine. Outokumpu HSC Chemistry for Windows: Chemical Reactions an Equilibrium Software with Extensive Thermodynamical Database, Version 5.1. User's Guide. 02103-ORC-T, Outokumpu Research Oy, Finlan, 2002.

[17] M.C. Lin and M.H Black. The Thermal Decomposition of Ethane. Dept. of Chemistry Thesis, University of Ottawa, 1996.

[18] T.Hirsch, A.Khenissi. A systematic comparison on power block efficiencies for CSP plants with direct steam generation. SolarPaces 2013, Las Vegas, U.S.

[19] L. Coco-Enríquez, J. Muñoz-Antón, J.M. Martínez-Val. Innovations on direct steam generation in linear Fresnel collectors. SolarPaces 2013, Las Vegas, U.S.

[20] A.Maccari, Archimede Solar Energy. Archimede Solar Energy Molten Salt Parabolic Trough Demo Plant: A Step Ahead Towards the New Frontiers of CSP. SolarPACES 2014.

[21] F. Matino, Archimede Solar Energy. Molten Salt Receivers Operated on Parabolic Trough Demo Plant and in Laboratory Conditions. SolarPACES 2014.

[22] G. Morin, Novatec Solar GmbH. Molten Salt as Heat Transfer Fluid in a Linear Fresnel Collector Comercial Application Backed by Demonstration. SolarPACES 2014.

[23] C.Parrado, A.Marzo, E.Fuentealba, A.G. Fernández. 2050 LCOE improvement using new molten salts for thermal energy storage in CSP plants. Renewable and Sustainable Energy Reviews, Volume 57, May 2016, Pages 505-514.

[24] S.Sau, N.Corsaro, T.Crescenzi, C. D'Ottavi, R.Liberatore, S.Licoccia, V.Russo, P.Tarquini, A.C. Tizzoni. Techno-economic comparison between CSP plants presenting two different heat transfer fluids. Applied Energy, Volume 168, 15 April 2016, Pages 96-109.

[25] L. Coco-Enríquez, J. Muñoz-Antón, J.M. Martínez-Val. Integration between DSG in linear solar collectors and Supercritical Carbon Dioxide Brayton Power cycles. 4th International Conference on Nuclear and Renewable Energy Resources NURER 2014, Turkey.

[26] Craig Turchi et al. $10 \mathrm{MW}$ Supercritical $\mathrm{CO}_{2}$ Turbine Test. NREL, DE-EE0001589, 01/27/2014.

[27] F.Burkholder, C.Kutscher. Heat Loss Testing of Schott's 2008 PTR70 Parabolic Trough Receiver. Report NREL/TP-550-45633, May 2009.

[28] Novatec Solar. SAM Linear Fresnel solar boiler model, SAM Webinar. NREL SAM Conference 2013.

[29] Dyreby J., Sanford Klein, Gregory Nellis, Douglas Reindl. Design Considerations for Supercritical Carbon Dioxide Brayton Cycles With Recompression. J. Eng. Gas Turbines Power 136(10), 101701 (Jul 22, 2014).

[30] Dyreby J. Thesis: Modeling the Supercritical Carbon Dioxide Brayton Cycle with Recompression. University of Wisconsin-Madison. September 2014.

[31] Jin Gyu Kwon, Hyun Sun Park, Tae Ho Kim, Moo Hwan Kim. Numerical Analysis of a fin arrangement for an optimal design of airfoil fin PCHE. The 4th International SymposiumSupercritical $\mathrm{CO}_{2}$ Power Cycles September 9-10, 2014, Pittsburgh, Pennsylvania.

[32] Carlson M. D., Kruizenga A. K., Schalansky C., Fleming D. F. Sandia Progress on advanced heat exchangers for $\mathrm{s}-\mathrm{CO}_{2}$ Brayton cycles. The 4 th International Symposium-Supercritical $\mathrm{CO}_{2}$ Power Cycles September 9-10, 2014, Pittsburgh, Pennsylvania. 
10. Plantas Termosolares con Sales Fundidas o DSG como fluido caloportador acopladas a ciclos de potencia supercríticos Brayton con $\mathrm{C}_{2} \mathrm{H}_{6}$ como fluido de trabajo 


\title{
Capítulo 11 \\ Comparación entre el $\mathrm{s}-\mathrm{CO}_{2}$ y otros fluidos de trabajo $\left(\mathrm{s}-\mathrm{C}_{2} \mathrm{H}_{6}, \mathrm{~s}\right.$ - $\mathrm{SF}_{6}, \mathrm{~s}-\mathrm{Xe}, \mathrm{s}-\mathrm{CH}_{4}, \mathrm{~s}-\mathrm{N}_{2}$ ) en las plantas termosolares acopladas a ciclos supercríticos Brayton
}

\begin{abstract}
Resumen
Las plantas termosolares con colectores lineales acopladas a ciclos de potencia Rankine o Brayton están madurando e implantándose a nivel industrial. En un futuro pueden ser una alternativa tecnológica a las plantas de generación de energía eléctrica con combustibles fósiles o nucleares. Las centrales termosolares proporcionan una fuente de energía renovables que permite reducir las emisiones de gases perjudiciales para el efecto invernadero y para la contaminación atmosférica. En este contexto, los gobiernos de las principales potencias mundiales, EEUU y UE, están realizando un esfuerzo inversor para desarrollo de los ciclos s- $\mathrm{CO}_{2}$ Brayton [1], para incrementar la eficiencia energética de las plantas termosolares con ciclos Rankine y el coste unitario de la electricidad generada, permitiendo que las plantas termosolares sean competitivas con las centrales de generación con combustibles fósiles o nucleares.
\end{abstract}

Poniendo especial consideración en los ciclos de potencia Brayton, hay fluidos caloportadores que presentan propiedades termodinámicas similares al $\mathrm{CO}_{2}$ en estado supercrítico, permitiendo optimizar el trabajo producido en las turbinas y el consumido por los compresores. En este estudio se considera la posibilidad de adoptar otros fluidos de trabajo en los ciclos de potencia supercríticos Brayton: el Etano $\left(\mathrm{C}_{2} \mathrm{H}_{6}\right)$, el Hexafluoruro de Azufre $\left(\mathrm{SF}_{6}\right)$, el Xenón $(\mathrm{Xe})$, el Metano $\left(\mathrm{CH}_{4}\right)$ y el Nitrógeno $\left(\mathrm{N}_{2}\right)$, línea de investigación que está siendo trabajada también por otros autores [2-6]. La principal novedad de este trabajo respecto a las referencias indicadas es la integración de los campos solares con colectores lineales trabajando con sales fundidas como HTF acoplados a ciclos de potencia supercríticos Brayton con recuperación de calor y recalentamiento.

Al igual que en capítulos anteriores, la principal innovación de este estudio la constituye la optimización de los parámetros termodinámicos de operación de los ciclos Brayton, para minimizar el área de apertura efectiva, y con ello, el coste de inversión de los campos solares de las plantas consideradas. Para ello se adoptará como principal referencia de trabajo el algoritmo de optimización SUBPLEX [7] propuesto por Dyreby [8]. En este trabajo se ha desarrollado un programa informático en con los lenguajes C\# y Fortran, para integrar los algoritmos matemáticos de optimización SUBPLEX [7], UOBYQA [9-11] y NEWUOA [12-13] para el cálculo de los ciclos de potencia supercríticos Brayton. Una vez obtenidos los parámetros termodinámicos óptimos de operación de los ciclos con el programa desarrollado ad hoc en esta tesis, se han introducido dichos parámetros en Thermoflow [14] para obtener el diseño optimizado de los campos solares con colectores lineales.

Como principal conclusión, se deduce la importancia que juega el tamaño térmico (UA) de los recuperadores en la eficiencia energética de la planta termosolar. Este incremento de eficiencia lleva aparejado una reducción del área de apertura efectiva de los colectores solares, 
11. Comparación entre el s- $\mathrm{CO}_{2}$ y otros fluidos de trabajo $\left(\mathrm{s}-\mathrm{C}_{2} \mathrm{H}_{6}, \mathrm{~s}-\mathrm{SF} \mathrm{F}_{6}, \mathrm{~s}-\mathrm{Xe}, \mathrm{s}-\mathrm{CH}_{4}, \mathrm{~s}-\mathrm{N}_{2}\right)$ en las plantas termosolares acopladas a ciclos supercríticos Brayton

y por tanto de su coste de inversión. El mínimo pinch-point en el recuperador es la principal restricción para un incremento de la eficiencia energética en el ciclo s- $\mathrm{CO}_{2}$ Brayton $\mathrm{SB}$, estableciéndose un tamaño térmico límite de $U A=10000 \mathrm{~kW} / \mathrm{K}$. Los fluidos caloportadores: $\mathrm{C}_{2} \mathrm{H}_{6}$, Xe y $\mathrm{SF}_{6}$ proporcionan valores de eficiencia energética del ciclo de potencia superiores a los del $\mathrm{CO}_{2}$ para un tamaño térmico del recuperador $\mathrm{UA}=15000-30000 \mathrm{~kW} / \mathrm{K}$. Los resultados detallados están resumidos en las figuras 11.9 a 11.14 .

En trabajos futuros, el objetivo será optimizar el diseño de los intercambiadores para maximizar su tamaño térmico, minimizando su volumen, su coste de fabricación y las pérdidas de presión a través de estos equipos. Para ello se están estudiando diferentes configuraciones de canales y procesos de fabricación [1]. La selección de los materiales para fabricación de los intercambiadores de calor, constituye otra pieza clave de investigación para permitir reducir la corrosión de los fluidos supercríticos a altas temperaturas y presiones. A la vez de proporcionar unos niveles aceptables de resistencia mecánica con un bajo coste de inversión.

\subsection{Introducción}

En este capítulo se estudian otros fluidos de trabajo en los ciclos supercríticos Brayton como alternativa al $\mathrm{s}-\mathrm{CO}_{2}$ para generación de potencia en centrales termosolares de colectores lineales. Los fluidos de trabajo estudiados y comparados con el $\mathrm{CO}_{2}$ son: $\mathrm{Xe}, \mathrm{C}_{2} \mathrm{H}_{6}, \mathrm{SF}_{6}, \mathrm{CH}_{4}$ y $\mathrm{N}_{2}$. La integración entre los ciclos de potencia supercríticos Brayton y los colectores lineales supone un esfuerzo importante para hacer más competitiva la energía solar de origen renovable, y optimizar el coste de la electricidad producida en plantas termosolares.

Los ciclos supercríticos Brayton tienen una historia dilatada, una de las primeras referencias al respecto se remonta a 1948, cuando Sulzer Bros patentó un ciclo Brayton con condensación parcial [15]. Actualmente se están desarrollando a escala industrial los equipos y componentes que integran los ciclos s- $\mathrm{CO}_{2}$ Brayton. Recientemente ha tenido lugar el Supercritical $\mathrm{CO}_{2}$ Power Cycles Syimposium [1] para promover las tecnologías relacionadas con los ciclos $\mathrm{s}-\mathrm{CO}_{2}$ Brayton. Estos simposios bianuales están celebrándose desde el año 2007 para implantar y comercializar los ciclos de potencia Brayton. $\mathrm{El} \mathrm{CO}_{2}$ como fluido de trabajo inicialmente investigado en Italia por Angelino 1968 [16-18]. E.G. Feher [19-20] lo seleccionó como fluido de trabajo más adecuado debido a las siguientes propiedades:

- la presión crítica del $\mathrm{CO}_{2}$ es un tercio de la del agua, permitiendo bajas presiones de operación.

- es un fluido estable e inerte para diferentes rangos de temperatura de operación del ciclo de potencia.

- existe documentación abundante de las propiedades del $\mathrm{CO}_{2}$, por tanto, el análisis y predicción de funcionamiento del ciclo termodinámico está basado en datos fehacientes.

- es un fluido abundante en la naturaleza, de bajo coste, no tóxico y sin impacto ambiental significativo en bajas cantidades.

En 2004 Dostal [21], publicó una tesis considerada una referencia en esta materia, constituyendo el renacimiento de la tecnología del $\mathrm{s}-\mathrm{CO}_{2}$. Recientemente, una importante innovación en esta materia ha sido publicada en [2-6], proponiendo otros fluidos de trabajo 
11. Comparación entre el s- $\mathrm{CO}_{2}$ y otros fluidos de trabajo $\left(\mathrm{s}-\mathrm{C}_{2} \mathrm{H}_{6}, \mathrm{~s}-\mathrm{SF} \mathrm{F}_{6}, \mathrm{~s}-\mathrm{Xe}, \mathrm{s}-\mathrm{CH}_{4}, \mathrm{~s}-\mathrm{N}_{2}\right)$ en las plantas termosolares acopladas a ciclos supercríticos Brayton

como alternativa al $\mathrm{CO}_{2}$ en los ciclos de potencia Brayton de propiedades termodinámicas similares en su estado supercrítico. En este capítulo se caracterizan los ciclos de potencia Brayton con diferentes fluidos de trabajo: $\mathrm{Xe}, \mathrm{C}_{2} \mathrm{H}_{6}, \mathrm{CH}_{4}, \mathrm{SF}_{6}, \mathrm{~N}_{2}$ y $\mathrm{CO}_{2}$.

En la tabla 11.1 se resumen las principales propiedades de los fluidos elegidos en este capítulo como fluidos de trabajo en los ciclos de potencia Brayton, para su acoplamiento a las plantas termosolares con colectores lineales. Uno de los aspectos más importantes considerados en la selección de los fluidos de trabajo es la temperatura del foco frío en el ciclo de potencia. El objetivo es que el fluido se encuentre en estado supercrítico a la entrada de los compresores para minimizar el trabajo en éstos.

Otro factor importante es la diferencia entre la temperatura crítica del fluido de trabajo y la temperatura ambiental donde se localiza la planta termosolar. Los equipos que integran el sumidero final de calor Ultimate Heat Sink (UHS) del ciclo tienen que trabajar con pinch-point entre $5^{\circ} \mathrm{C}$ y $10^{\circ} \mathrm{C}$, debido a las mínimas diferencias de temperatura en los intercambiadores de calor que lo integran.

Se ha confirmado experimentalmente que existe una excesiva sensibilidad a la temperatura de las propiedades a la entrada de los compresores [8], lo cual impacta significativamente en el rendimiento del ciclo de potencia, (ver figura 11.1). Para contrarrestar este fenómeno, cuando se incrementa la temperatura del UHS se aumenta de forma simultanea la presión a la entrada del compresor para intentar mantener una alta densidad del $\mathrm{s}-\mathrm{CO}_{2}$ que optimice el rendimiento energético de la turbomáquina.

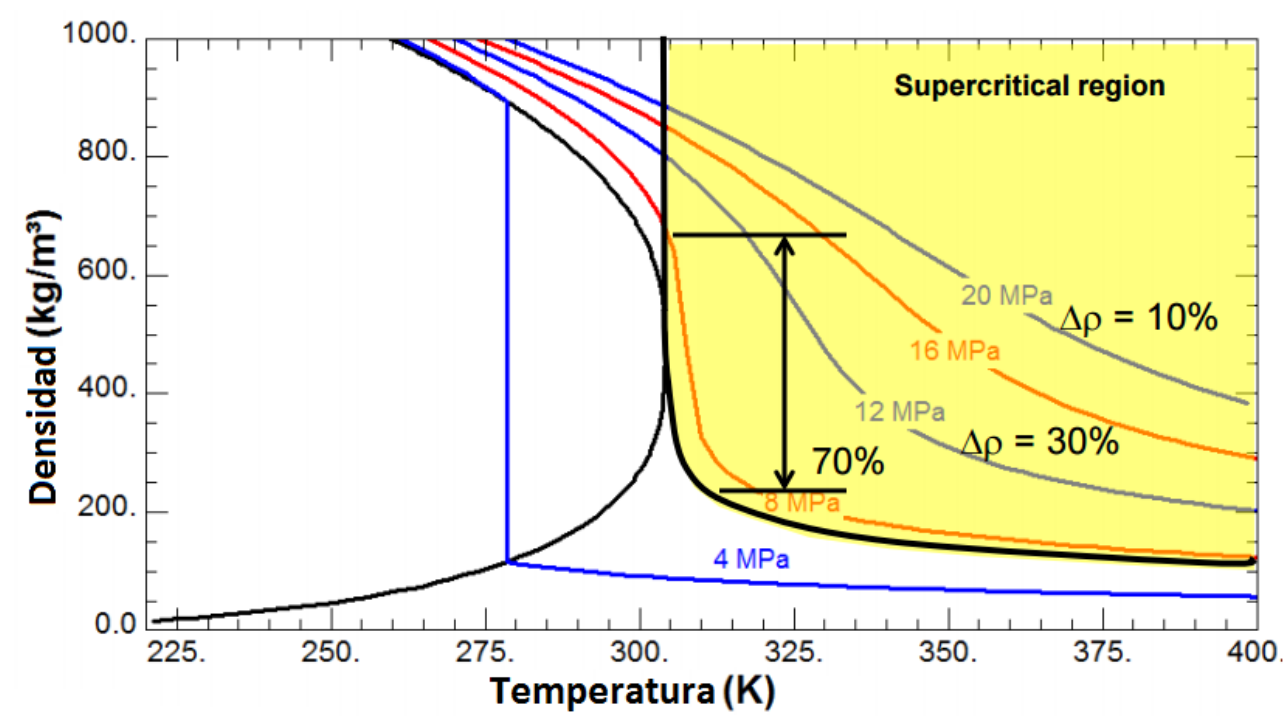

Figura 11.1. Representación gráfica del fenómeno físico de caída considerable de la densidad del s-CO2 en las cercanías del punto crítico debido a los cambios de presión y temperatura.

Recientes trabajos del SNL [22], se estudian dos casos en el diseño del UHS del ciclo Brayton:

- El sistema de enfriamiento seco o dry-cooling mediante convección forzada de aire [23]. Para este diseño del UHS se considera una temperatura de entrada al compresor $\mathrm{CIT}=47.85^{\circ} \mathrm{C}$, redondeando se puede considerar $\mathrm{CIT}=50^{\circ} \mathrm{C}$. La presión óptima para esta temperatura es alrededor de $\mathrm{CIP}=95$ bar. En este caso el pinch point, diferencia mínima 
11. Comparación entre el s- $\mathrm{CO}_{2}$ y otros fluidos de trabajo $\left(\mathrm{s}-\mathrm{C}_{2} \mathrm{H}_{6}, \mathrm{~s}-\mathrm{SF} \mathrm{F}_{6}, \mathrm{~s}-\mathrm{Xe}, \mathrm{s}-\mathrm{CH}_{4}, \mathrm{~s}-\mathrm{N}_{2}\right)$ en las plantas termosolares acopladas a ciclos supercríticos Brayton

entre la temperatura ambiente y la $\mathrm{CIT}$, es de $5^{\circ} \mathrm{C}$. Por tanto, para este diseño del UHS la máxima temperatura ambiente de operación podría estar entre $43-45^{\circ} \mathrm{C}$. Como ejemplo en España los valores máximos de temperatura normalmente rondan los $42^{\circ} \mathrm{C}$ en la estación de verano.

- El sistema de enfriamiento húmedo o wet-cooling con torre de refrigeración con agua [24-25]. Este diseño considera $\mathrm{CIT}=31.85^{\circ} \mathrm{C}$, y $\mathrm{CIP}=76$ bar. Para esta solución técnica es necesario un pinch point mínimo de $10^{\circ} \mathrm{C}$ entre la temperatura ambiente y $\mathrm{CIT}$. Por tanto, la temperatura ambiente máxima de operación sería de $21.8^{\circ} \mathrm{C}$. Para mayores temperaturas ambiente de operación es necesario incrementar CIP.

Otro punto muy importante con gran impacto en la eficiencia energética del ciclo de potencia, lo constituye el consumo eléctrico de los ventiladores del UHS. Este consumo ronda el $4 \%$ de la potencia del ciclo según las estimaciones realizadas [14].

El $\mathrm{SF}_{6}$ tiene una temperatura crítica de $45^{\circ} \mathrm{C}$, por tanto, en climas cálidos con temperaturas ambientales altas, este fluido de trabajo garantiza un buen desempeño del ciclo de potencia. $\mathrm{El}$ Xenón tiene una temperatura crítica de $16.5^{\circ} \mathrm{C}$, por esta razón este fluido de trabajo es apropiado para climas más fríos.

Otro parámetro de referencia a la hora de seleccionar el fluido de trabajo es la presión crítica, tabla 11.1. La presión crítica debería ser lo menor posible para no requerir umbrales de presión excesivos para maximizar la eficiencia y potencia generada en el ciclo. Para el caso del agua 22.06 MPa, lo que permite unas relaciones de expansión en turbina muy bajas, ya que la máxima presión de operación con los materiales actuales es del orden de $25 \mathrm{MPa}$.

Valores reducidos de presión y temperatura crítica permiten garantizar que el fluido de trabajo en la totalidad del ciclo Brayton se encuentra en estado supercrítico. No existe un estado bifásico que repercuta negativamente tanto en la transferencia de calor, como en el rendimiento con posible erosión y cavitación de las turbomáquinas.

Tabla 11.1. Propiedades de los fluidos de trabajo en estado de criticidad [26].

\begin{tabular}{lllll}
\hline Nombre & $\begin{array}{l}\text { Composición } \\
\text { Química }\end{array}$ & $\begin{array}{l}\text { Temperatura } \\
\text { Crítica }\left({ }^{\circ} \mathrm{C}\right)\end{array}$ & $\begin{array}{l}\text { Presión } \\
\text { Crítica }(\mathrm{MPa})\end{array}$ & $\begin{array}{l}\text { Densidad } \\
\text { Crítica }\left(\mathrm{kg} / \mathrm{m}^{3}\right)\end{array}$ \\
\hline Agua & $\mathrm{H}_{2} \mathrm{O}$ & 373.9 & 22.06 & 322.0 \\
Dióxido de Carbono & $\mathrm{CO}_{2}$ & 30.9 & 7.38 & 467.6 \\
Etano & $\mathrm{C}_{2} \mathrm{H}_{6}$ & 32.2 & 4.87 & 206.2 \\
Metano & $\mathrm{CH}_{4}$ & -82.6 & 4.59 & 162.6 \\
Hexafluoruro de Azufre & $\mathrm{SF}_{6}$ & 45.6 & 3.75 & 742.3 \\
Nitrógeno & $\mathrm{N}_{2}$ & -146.9 & 3.39 & 313.3 \\
Xenón & $\mathrm{Xe}$ & 16.6 & 5.84 & 1102.9 \\
\hline
\end{tabular}

La TIT es una variable termodinámica que tiene gran influencia en la eficiencia del ciclo Brayton, como ya se ha explicado previamente, debido al principio termodinámico de Carnot. El valor máximo de este parámetro está limitado por la estabilidad química de los fluidos de trabajo a altas presiones y temperaturas. También existe el riesgo de explosión, auto-ignición, 
11. Comparación entre el s- $\mathrm{CO}_{2}$ y otros fluidos de trabajo $\left(\mathrm{s}-\mathrm{C}_{2} \mathrm{H}_{6}, \mathrm{~s}-\mathrm{SF}_{6}, \mathrm{~s}-\mathrm{Xe}, \mathrm{s}-\mathrm{CH}_{4}, \mathrm{~s}-\mathrm{N}_{2}\right)$ en las plantas termosolares acopladas a ciclos supercríticos Brayton

y corrosión excesiva a altas presiones y temperaturas. En la tabla 11.2 se recomiendan unas presiones y temperaturas máximas de operación para los diferentes fluidos de trabajo.

Tabla 11.2. TIT máximas para diferentes fluidos de trabajo en ciclos de potencia Brayton.

\begin{tabular}{|c|c|c|}
\hline Fluido & TIT $\left({ }^{\circ} \mathrm{C}\right)$ & Razones para limitar la TIT \\
\hline $\mathrm{s}-\mathrm{CO}_{2}$ & 650 & Descomposición química. Corrosión de metales [27]. \\
\hline $\mathrm{s}-\mathrm{C}_{2} \mathrm{H}_{6}$ & 401.85 & $\begin{array}{l}\text { Límite de temperatura en base de datos de propiedades REFPROP [28]. } \\
\text { Descomposición química. Corrosión de metales. Riesgo de explosión. }\end{array}$ \\
\hline $\mathrm{s}-\mathrm{SF}_{6}$ & 351.85 & Descomposición química [29]. \\
\hline $\mathrm{s}-\mathrm{CH}_{4}$ & 351.85 & $\begin{array}{l}\text { Límite de temperatura en base de datos de propiedades REFPROP [30]. } \\
\text { Descomposición química. Corrosión de metales. Riesgo de explosión. }\end{array}$ \\
\hline $\mathrm{s}-\mathrm{N}_{2}$ & 550 & Corrosión de metales. Corrosión de metales [31]. \\
\hline$s-\mathrm{Xe}$ & 476.85 & Límite de temperatura en base de datos de propiedades REFPROP [32] \\
\hline
\end{tabular}

\subsection{Hipótesis de cálculo}

Los parámetros de diseño y condiciones de contorno consideradas en las simulaciones en el presente estudio, han sido resumidos en las tablas 11.3 a 11.7. En todas las simulaciones se ha fijado como potencia bruta $50 \mathrm{MWe}$.

Tabla 11.3. Localización y temperatura ambiental.

\begin{tabular}{ll}
\hline Localización: & Dagget,CA, USA. \\
Latitud: & $34.86^{\circ}$ \\
Longitud: & $-116.8^{\circ}$ \\
Zona horaria: & -8 \\
Hora: & $11: 30 \mathrm{hr}$ \\
DNI: & $986 \mathrm{~W} / \mathrm{m}^{2}$ \\
Ta ambiental: & $25^{\circ} \mathrm{C}$ \\
Altitud: & $588 \mathrm{~m}$ \\
\hline
\end{tabular}

Tabla 11.4. Parámetros del tubo absorbedor.

\begin{tabular}{ll}
\hline Diámetro exterior: & $70 \mathrm{~mm}$ \\
Espesor pared: & $4.191 \mathrm{~mm}$ \\
Material: & Stainless steel (MS) \\
Vacío entre tubo absorbedor & \\
y tubo vidrio & \\
Rugosidad: & $0.0457 \mathrm{~mm}$ \\
HTF: HITEC XL (MS) o & DSG \\
\hline
\end{tabular}

Tabla 11.5. Parámetros colectores PTC.

\begin{tabular}{ll}
\hline Tipo colector: & EuroTrough II \\
Anchura apertura: & $5.77 \mathrm{~m}$ \\
Longitud focal: & $1.71 \mathrm{~m}$ \\
Factor limpieza: & 0.96 \\
Eficiencia óptica: & 0.75 \\
Pérdidas térmicas: & $0.141 \Delta \mathrm{T}+6.48 \mathrm{e}-9 \Delta \mathrm{T} 4 \quad[33]$ \\
\hline
\end{tabular}


11. Comparación entre el s- $\mathrm{CO}_{2}$ y otros fluidos de trabajo $\left(\mathrm{s}-\mathrm{C}_{2} \mathrm{H}_{6}, \mathrm{~s}-\mathrm{SF}_{6}, \mathrm{~s}-\mathrm{Xe}, \mathrm{s}-\mathrm{CH}_{4}, \mathrm{~s}-\mathrm{N}_{2}\right)$ en las plantas termosolares acopladas a ciclos supercríticos Brayton

Tabla 11.6. Parámetros colectores LF.

\begin{tabular}{ll}
\hline Tipo colector: & SuperNova 1 (Novatec) \\
Dimensiones módulo: & $16.56 \mathrm{~m} \times 44.8 \mathrm{~m}$ \\
Área apertura: & $513.6 \mathrm{~m}^{2} /$ per Modulo \\
Eficiencia óptica: & 0.647 \\
Pérdidas térmicas: & $0.15 \Delta \mathrm{T}+7.1510^{-9} \Delta \mathrm{T}^{4}[34]$ \\
\hline
\end{tabular}

Tabla 11.7. Parámetros del ciclo Brayton.

\begin{tabular}{|c|c|}
\hline Eficiencia turbina: & $93 \%$ \\
\hline Eficiencia compresor: & $89 \%$ \\
\hline HX Efectividad: & $95 \%$ \\
\hline \multicolumn{2}{|c|}{ No se considera pérdidas de presión en HXs } \\
\hline Temperatura entrada Turbina (TIT): & $550^{\circ} \mathrm{C}$ \\
\hline Presión entrada en Turbina: & 250 bar \\
\hline Presión de recalentamiento: & Optimizada \\
\hline \multicolumn{2}{|c|}{ Temperatura entrada al compressor: $32^{\circ} \mathrm{C}$} \\
\hline Presión entrada a compresor: & Optimizada \\
\hline Fracción de recompresión: & Optimizada \\
\hline \multicolumn{2}{|c|}{ Consumos auxiliares (\% Potencia Bruta): $\quad 1 \%$} \\
\hline Eficiencia Generador (punto de diseñ & io): $98.23 \%$ \\
\hline
\end{tabular}

\subsection{Configuraciones ciclos de potencia Brayton}

En la figura 11.2 se ilustra la disposición de los equipos y componentes en el ciclo Brayton analizado en este capítulo. Su principal ventaja es su simplicidad y bajo número de equipos, reduciendo al máximo el coste de inversión. Incluye un recuperador de calor, y una etapa de recalentamiento en turbinas, para optimizar al máximo la eficiencia del ciclo.

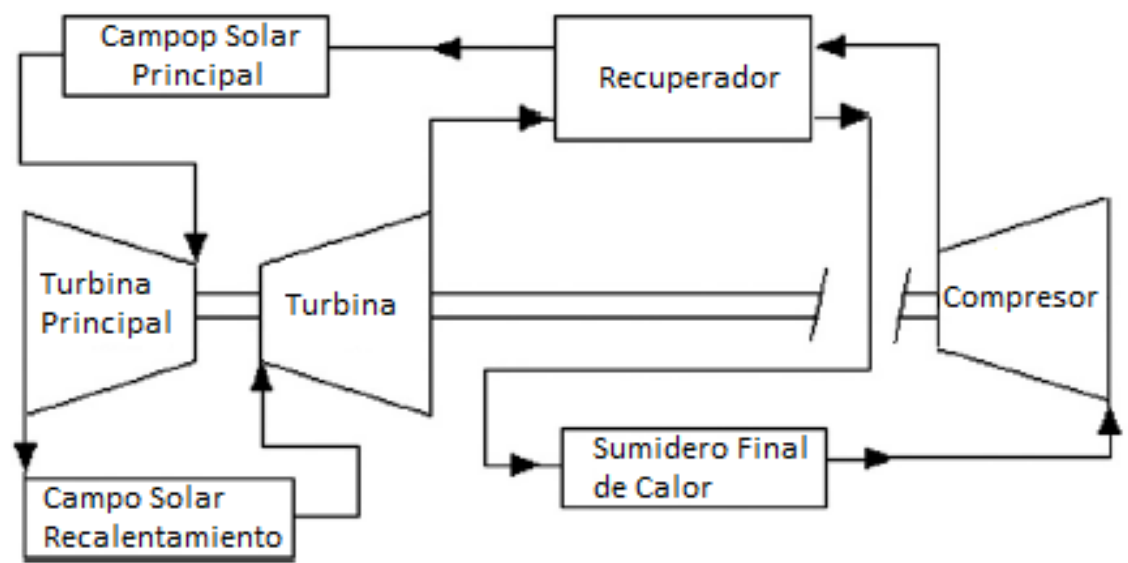

Figura 11.2. Ciclo de potencia Brayton $S B$ con fluido de trabajo supercrítico $\left(\mathrm{CO}_{2}, \mathrm{C}_{2} \mathrm{H}_{6}, \mathrm{SF}_{6}\right.$, $\mathrm{CH}_{4}, \mathrm{~N}_{2}$ y Xe). Incluye un recuperador y una etapa de recalentamiento en turbinas. Las fuentes de calor son dos campos solares con colectores lineales. 
11. Comparación entre el s- $\mathrm{CO}_{2}$ y otros fluidos de trabajo $\left(\mathrm{s}-\mathrm{C}_{2} \mathrm{H}_{6}, \mathrm{~s}-\mathrm{SF} \mathrm{F}_{6}, \mathrm{~s}-\mathrm{Xe}, \mathrm{s}-\mathrm{CH}_{4}, \mathrm{~s}-\mathrm{N}_{2}\right)$ en las plantas termosolares acopladas a ciclos supercríticos Brayton

Las fuentes térmicas provienen del calor aportado al fluido de trabajo en dos intercambiadores de calor ( $\mathrm{PHX}$ y RHX) tipo tubos-carcasa, que reciben la energía térmica del HTF de dos campos solares de concentración con colectores solares lineales. Un elemento clave para maximizar la eficiencia energética del ciclo es el enfriador Pre-Cooler, cuyo diseño debe ser optimizado para minimizar el consumo eléctrico de los equipos que lo integran (bombas, ventiladores, etc.) [2224]. Así mismo, en el Pre-Cooler, es donde mayor energía se pierde, por lo que es aconsejable utilizar la energía térmica del sumidero de calor para fines de cogeneración [35-37]: calentar agua sanitaria, generar vapor a baja presión, u otros fines de cogeneración de calor que sean compatible con las temperaturas del fluido de trabajo.

\subsection{Caracterización del ciclo de potencia Brayton}

Como se ha explicado en la introducción del este capítulo, el tamaño térmico de los recuperadores es una de las variables que más impacta en la eficiencia y producción de los ciclos de potencia supercríticos Brayton. En este apartado para caracterizar el ciclo Brayton de la figura 11.2, se ha seguido la metodología establecida por Dyreby [8], fijando el tamaño térmico (UA) de los recuperadores para diferentes TIT (desde $300^{\circ} \mathrm{C}$ hasta $550^{\circ} \mathrm{C}$ ). Los resultados obtenidos para los diferentes fluidos de trabajo estudiados $\left(\mathrm{CO}_{2}, \mathrm{C}_{2} \mathrm{H}_{6}, \mathrm{SF}_{6}, \mathrm{CH}_{4}, \mathrm{~N}_{2} \mathrm{y}\right.$ $\mathrm{Xe)}$, están incluidos en las figuras 11.3 a 11.8 y 11.9 a 11.14. Como resultado a remarcar, si el fluido de trabajo es $\mathrm{s}-\mathrm{CO}_{2}$, existe un valor máximo de tamaño térmico de los intercambiadores UA, a partir del cual, no es posible continuar incrementando la eficiencia del ciclo. Esto se debe a que la diferencia mínima de temperatura pinch-point en los recuperadores se reduce a un valor mínimo cercano a $1^{\circ} \mathrm{C}$. Los valores máximos del tamaño térmico (UA) de los recuperadores limitados por el pinch point mínimo en el recuperador son: para el $\mathrm{CO}_{2}$ $\mathrm{UA}=10000 \mathrm{~kW} / \mathrm{K}$, para el $\mathrm{C}_{2} \mathrm{H}_{6} \mathrm{UA}=15000 \mathrm{~kW} / \mathrm{K}$, para el $\mathrm{SF}_{6}, \mathrm{Xe}, \mathrm{CH}_{4}$ y el $\mathrm{N}_{2} \mathrm{UA}=30000 \mathrm{~kW} / \mathrm{K}$.

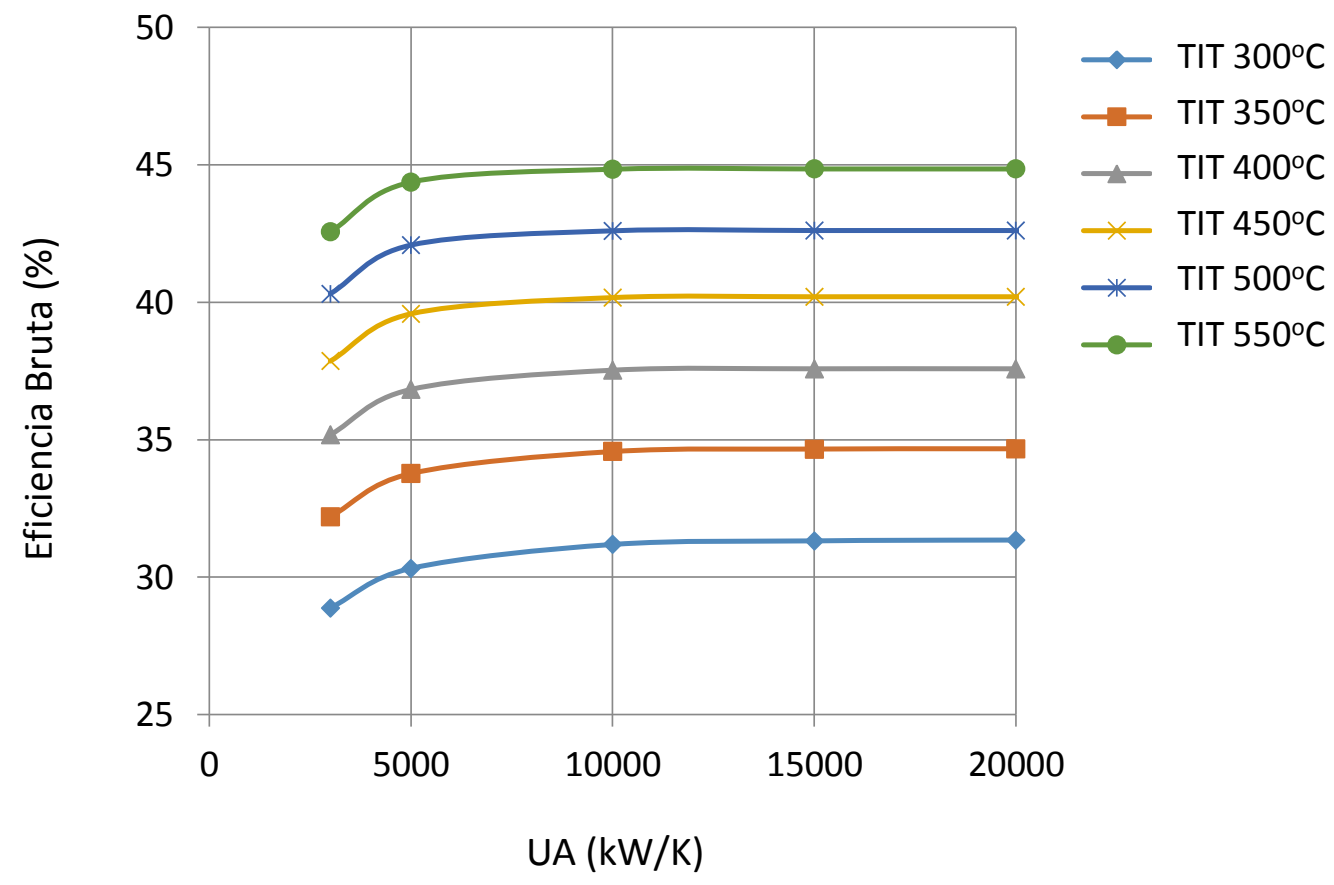

Figura 11.3. Eficiencia bruta versus tamaño térmico de los recuperadores. Ciclo Brayton SB con recuperación de calor. Fluido de trabajo $\mathrm{s}-\mathrm{CO}_{2}$. 
11. Comparación entre el s- $\mathrm{CO}_{2}$ y otros fluidos de trabajo $\left(\mathrm{s}-\mathrm{C}_{2} \mathrm{H}_{6}, \mathrm{~s}-\mathrm{SF} 6, \mathrm{~s}-\mathrm{Xe}, \mathrm{s}-\mathrm{CH}_{4}, \mathrm{~s}-\mathrm{N}_{2}\right)$ en las plantas termosolares acopladas a ciclos supercríticos Brayton

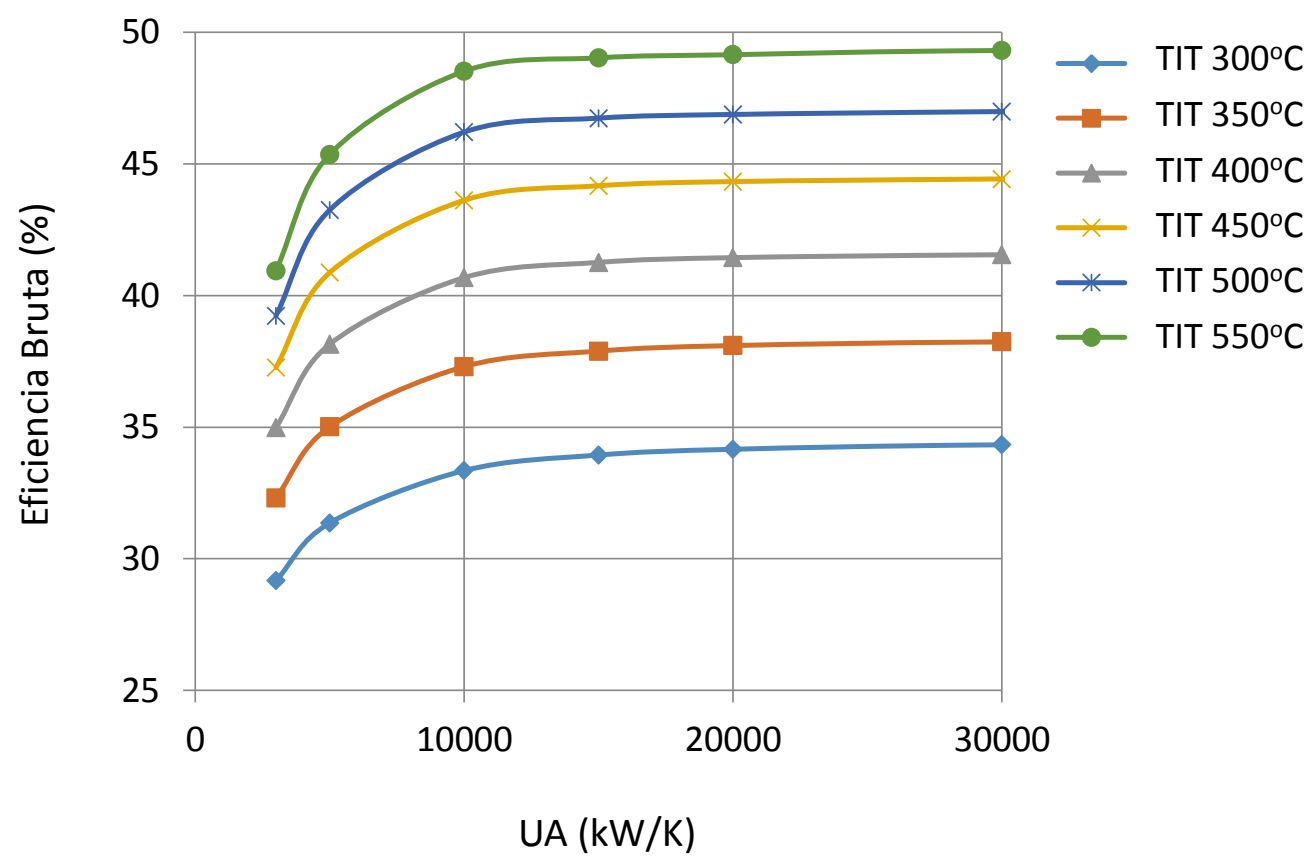

Figura 11.4. Eficiencia bruta versus tamaño térmico de los recuperadores. Ciclo Brayton SB con recuperación de calor. Fluido de trabajo $s-\mathrm{C}_{2} \mathrm{H}_{6}$.

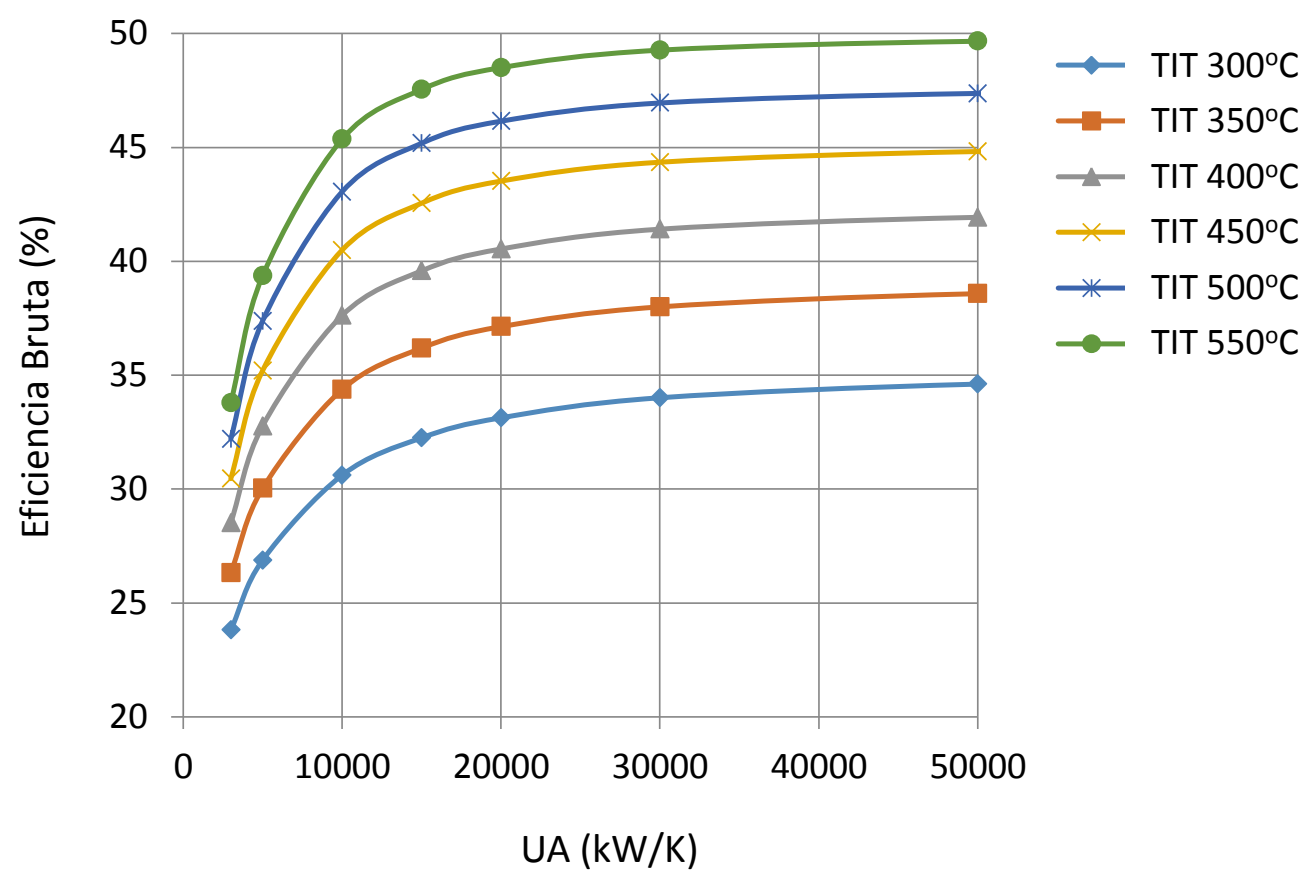

Figura 11.5. Eficiencia bruta versus tamaño térmico de los recuperadores. Ciclo Brayton SB con recuperación de calor. Fluido de trabajo s-SF 6 . 
11. Comparación entre el s- $\mathrm{CO}_{2}$ y otros fluidos de trabajo $\left(\mathrm{s}-\mathrm{C}_{2} \mathrm{H}_{6}, \mathrm{~s}-\mathrm{SF}_{6}, \mathrm{~s}-\mathrm{Xe}, \mathrm{s}-\mathrm{CH}_{4}, \mathrm{~s}-\mathrm{N}_{2}\right)$ en las plantas termosolares acopladas a ciclos supercríticos Brayton

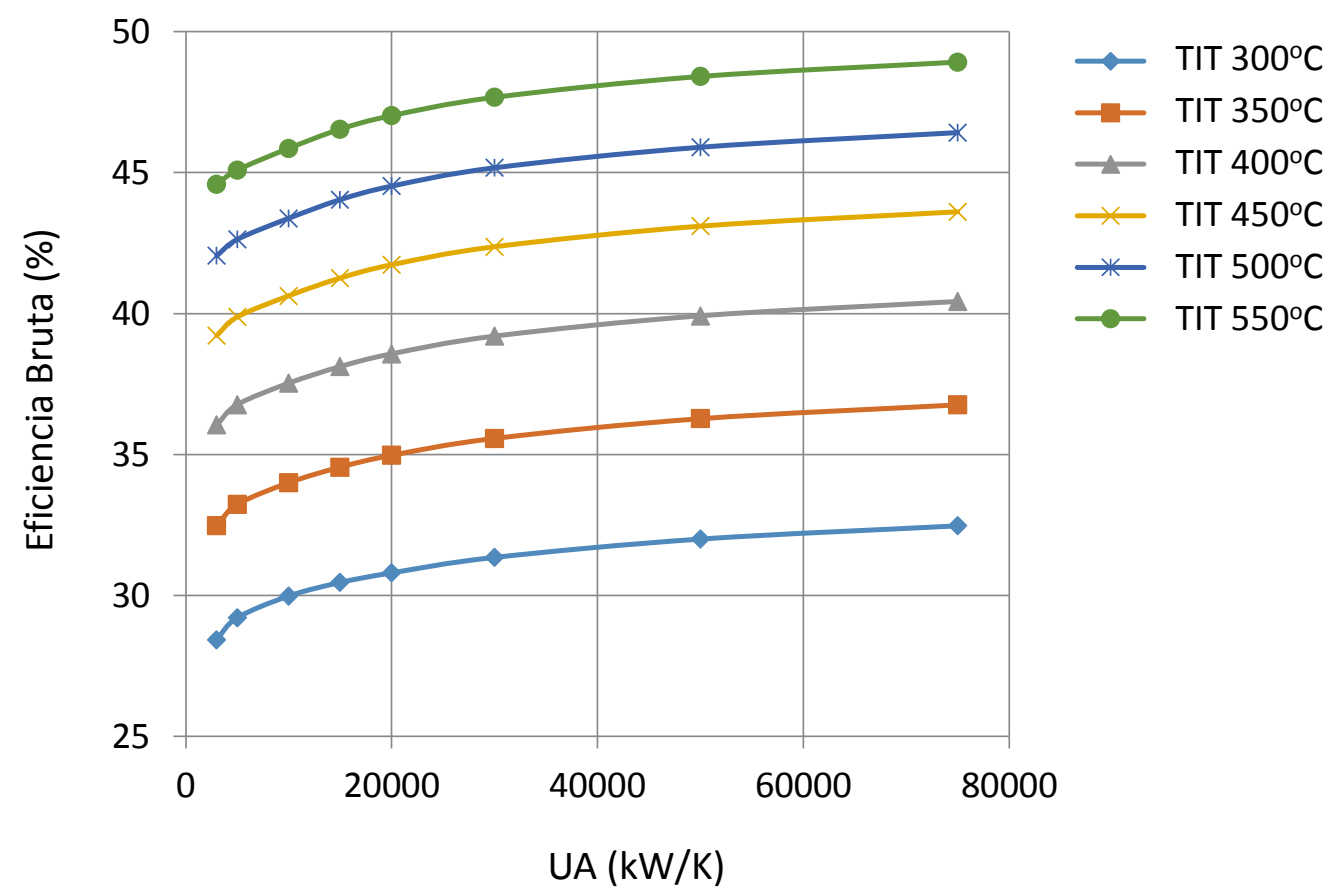

Figura 11.6. Eficiencia bruta versus tamaño térmico de los recuperadores. Ciclo Brayton SB con recuperación de calor. Fluido de trabajo s-Xe.

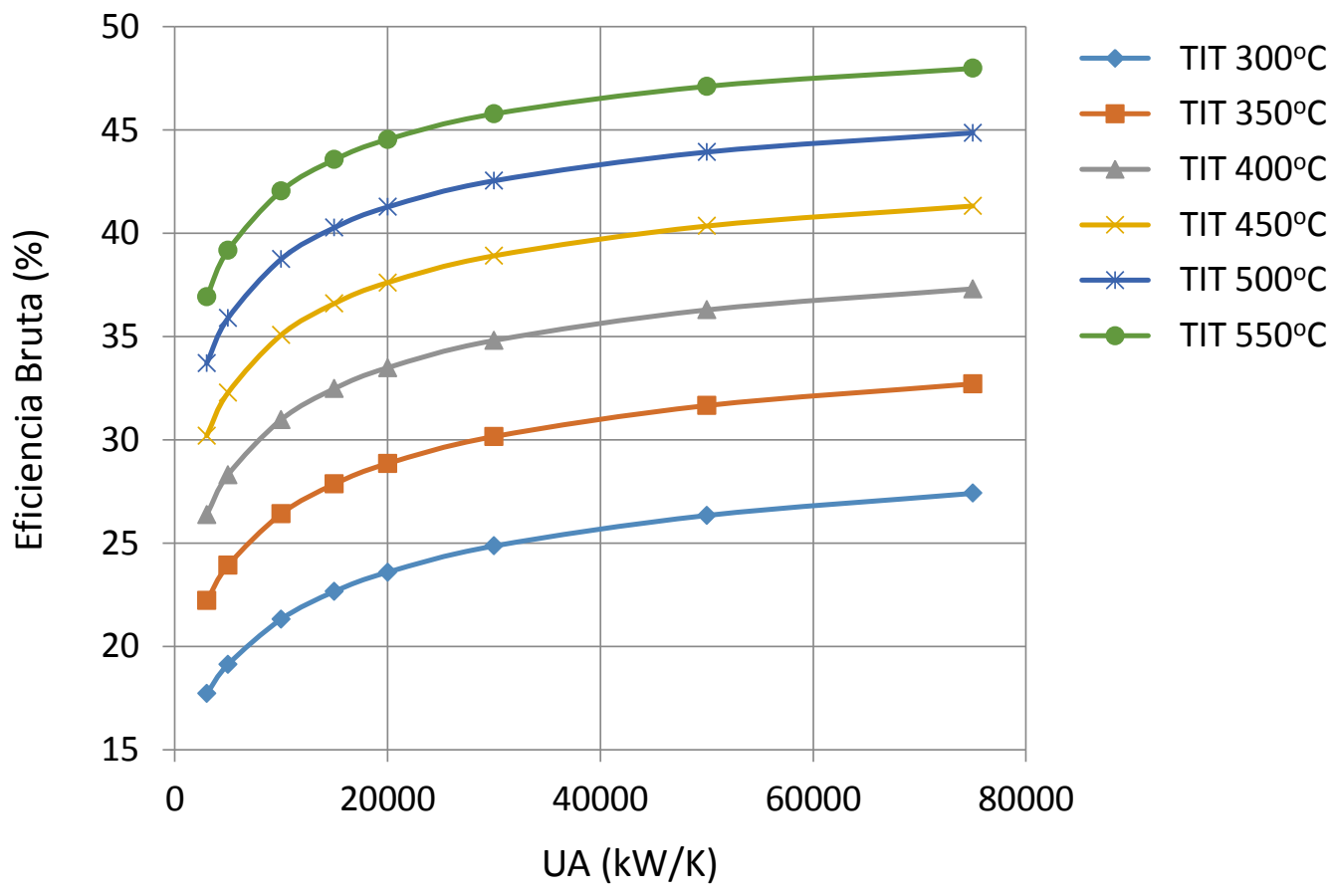

Figura 11.7. Eficiencia bruta versus tamaño térmico de los recuperadores. Ciclo Brayton SB con recuperación de calor. Fluido de trabajo s $-\mathrm{N}_{2}$. 
11. Comparación entre el s- $\mathrm{CO}_{2}$ y otros fluidos de trabajo $\left(\mathrm{s}-\mathrm{C}_{2} \mathrm{H}_{6}, \mathrm{~s}-\mathrm{SF}_{6}, \mathrm{~s}-\mathrm{Xe}, \mathrm{s}-\mathrm{CH}_{4}, \mathrm{~s}-\mathrm{N}_{2}\right)$ en las plantas termosolares acopladas a ciclos supercríticos Brayton

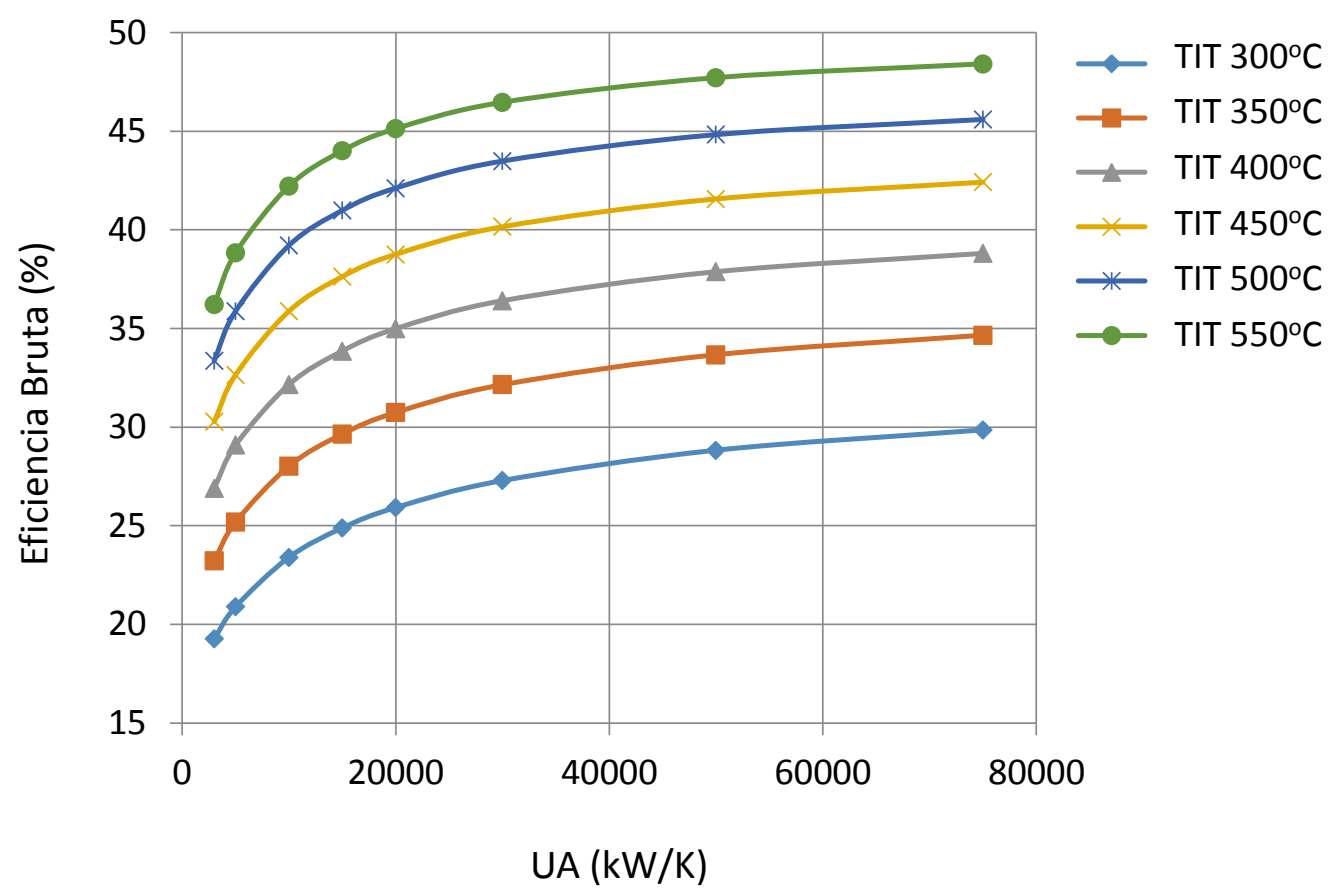

Figura 11.8. Eficiencia bruta versus tamaño térmico de los recuperadores. Ciclo Brayton SB con recuperación de calor. Fluido de trabajo $\mathrm{s}-\mathrm{CH}_{4}$.

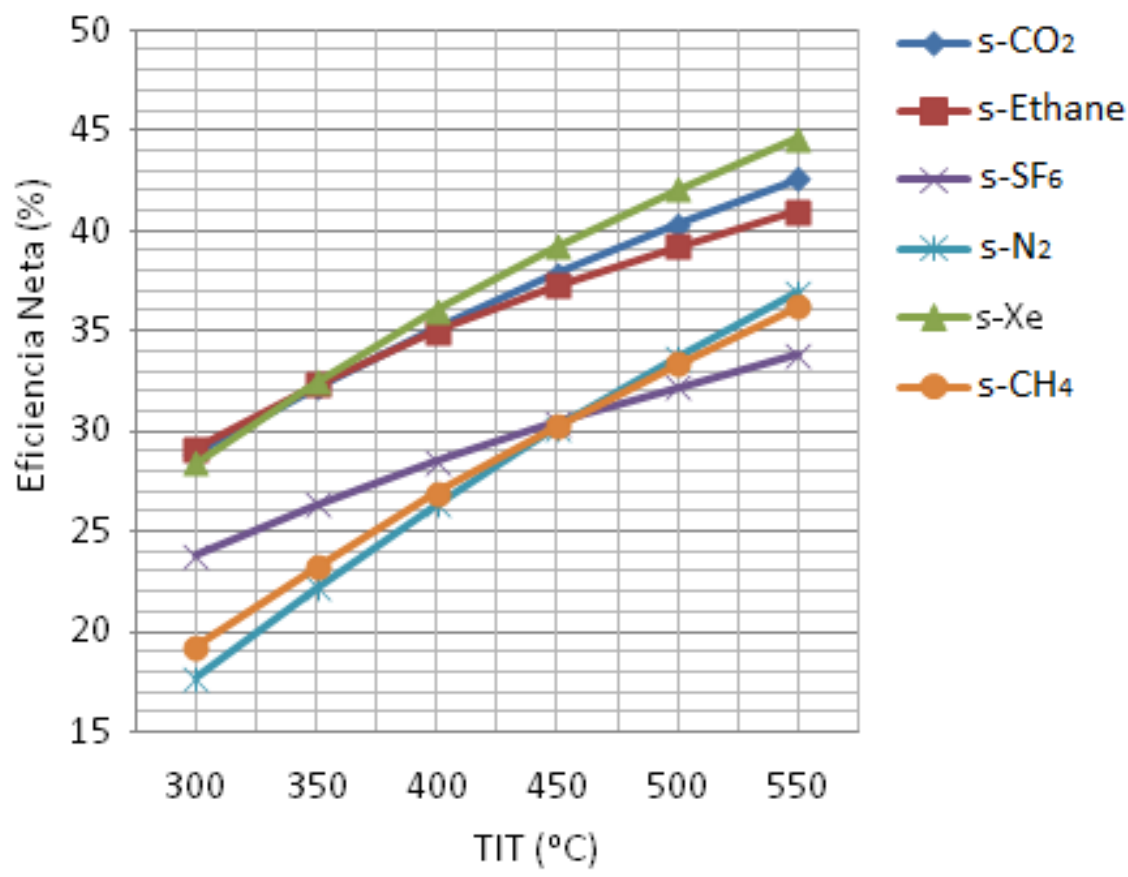

Figura 11.9. Eficiencia bruta versus TIT, fijado el $U A=3000 \mathrm{~kW} / \mathrm{K}$, para diferentes fluidos de trabajo: $\mathrm{CO}_{2}, \mathrm{C}_{2} \mathrm{H}_{6}, \mathrm{CH}_{4}, \mathrm{~N}_{2}$, Xe y $\mathrm{N}_{2}$. Ciclo Brayton $\mathrm{SB}$ con recuperación de calor. 
11. Comparación entre el s- $\mathrm{CO}_{2}$ y otros fluidos de trabajo $\left(\mathrm{s}-\mathrm{C}_{2} \mathrm{H}_{6}, \mathrm{~s}-\mathrm{SF} \mathrm{F}_{6}, \mathrm{~s}-\mathrm{Xe}, \mathrm{s}-\mathrm{CH}_{4}, \mathrm{~s}-\mathrm{N}_{2}\right)$ en las plantas termosolares acopladas a ciclos supercríticos Brayton

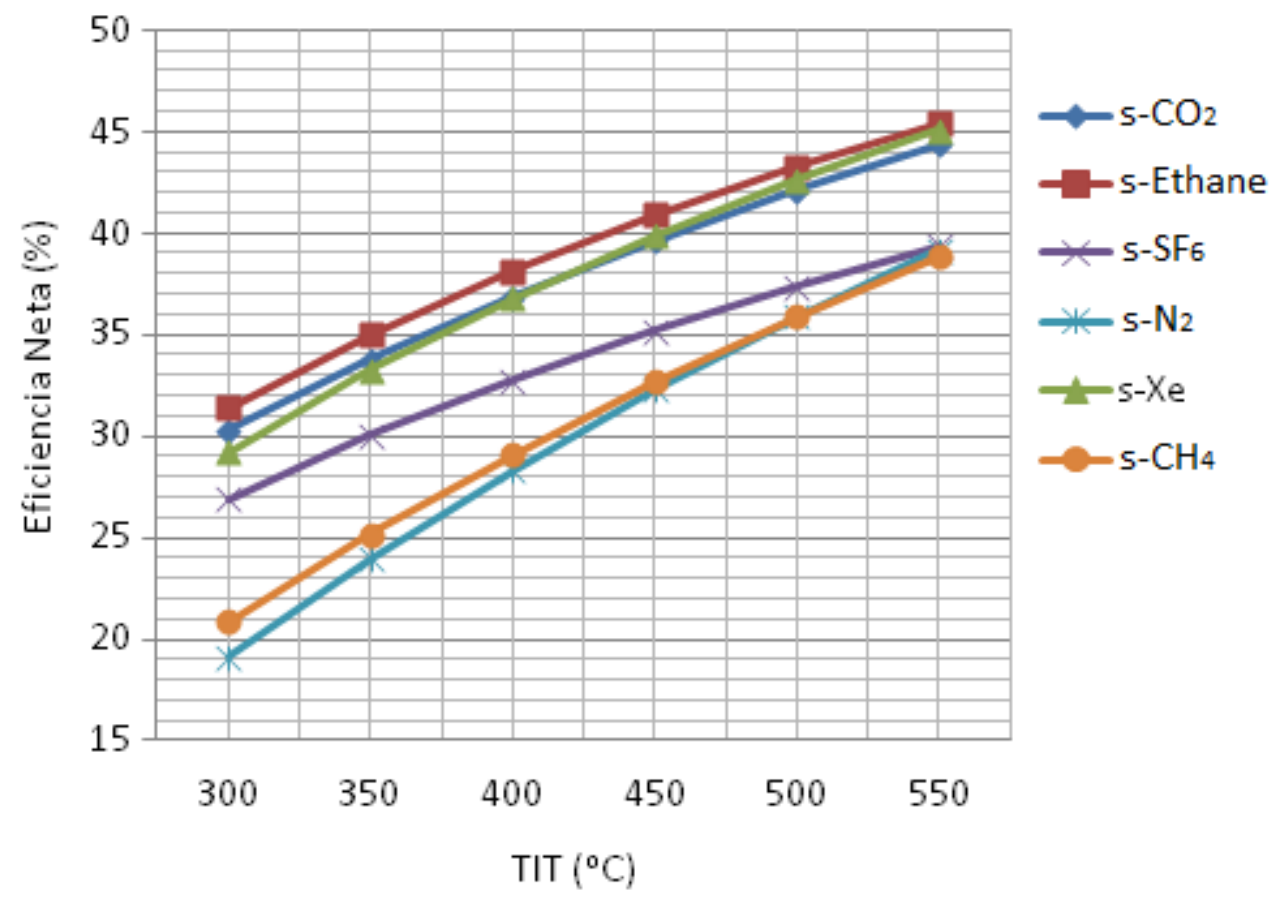

Figura 11.10. Eficiencia bruta versus TIT, fijado $U A=5000 \mathrm{~kW} / \mathrm{K}$ para diferentes fluidos de trabajo: $\mathrm{CO}_{2}, \mathrm{C}_{2} \mathrm{H}_{6}, \mathrm{CH}_{4}, \mathrm{~N}_{2}$, Xe y $\mathrm{N}_{2}$. Ciclo Brayton $\mathrm{SB}$ con recuperación de calor.

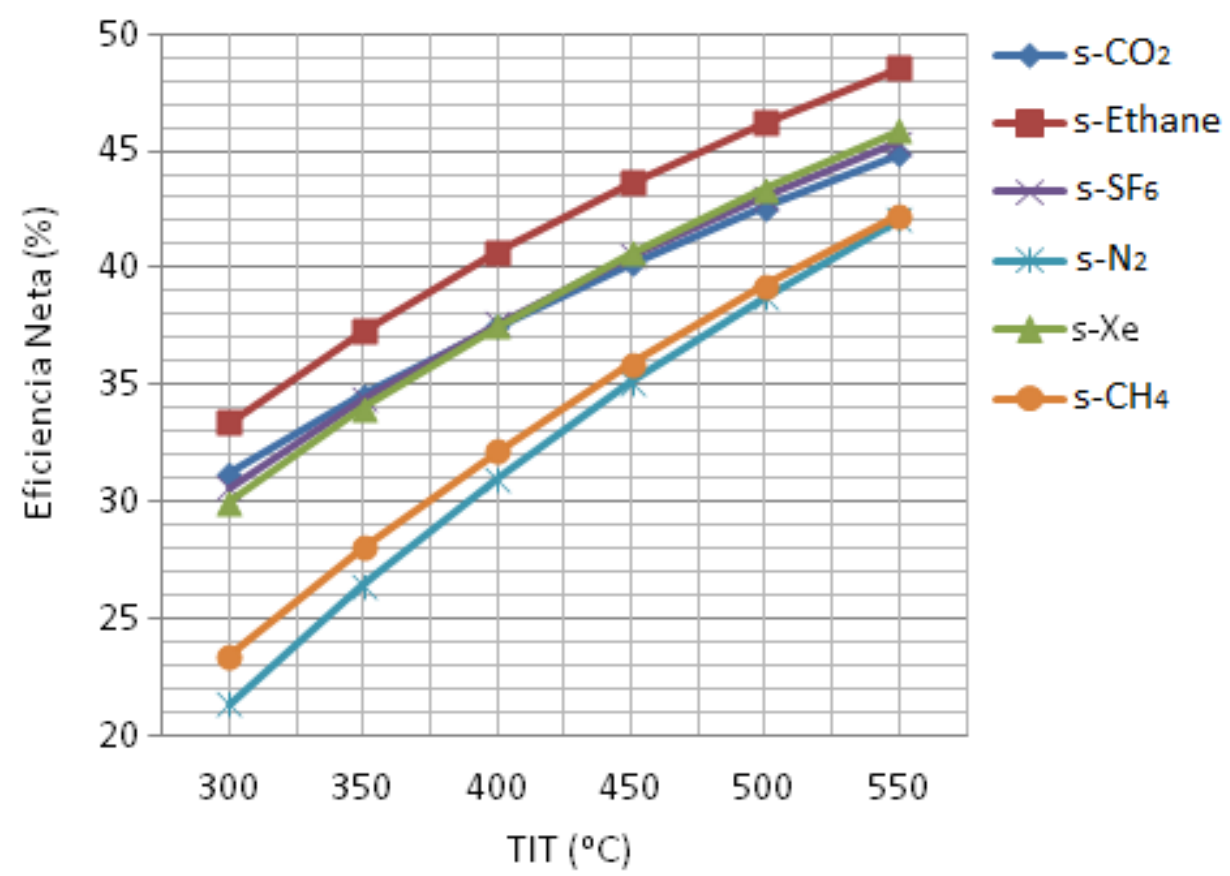

Figura 11.11. Eficiencia bruta versus TIT, fijado $U A=10000 \mathrm{~kW} / \mathrm{K}$ para diferentes fluidos de trabajo: $\mathrm{CO}_{2}, \mathrm{C}_{2} \mathrm{H}_{6}, \mathrm{CH}_{4}, \mathrm{~N}_{2}$, Xe y $\mathrm{N}_{2}$. Ciclo Brayton $\mathrm{SB}$ con recuperación de calor. 
11. Comparación entre el s- $\mathrm{CO}_{2}$ y otros fluidos de trabajo $\left(\mathrm{s}-\mathrm{C}_{2} \mathrm{H}_{6}, \mathrm{~s}-\mathrm{SF} \mathrm{F}_{6}, \mathrm{~s}-\mathrm{Xe}, \mathrm{s}-\mathrm{CH}_{4}, \mathrm{~s}-\mathrm{N}_{2}\right)$ en las plantas termosolares acopladas a ciclos supercríticos Brayton

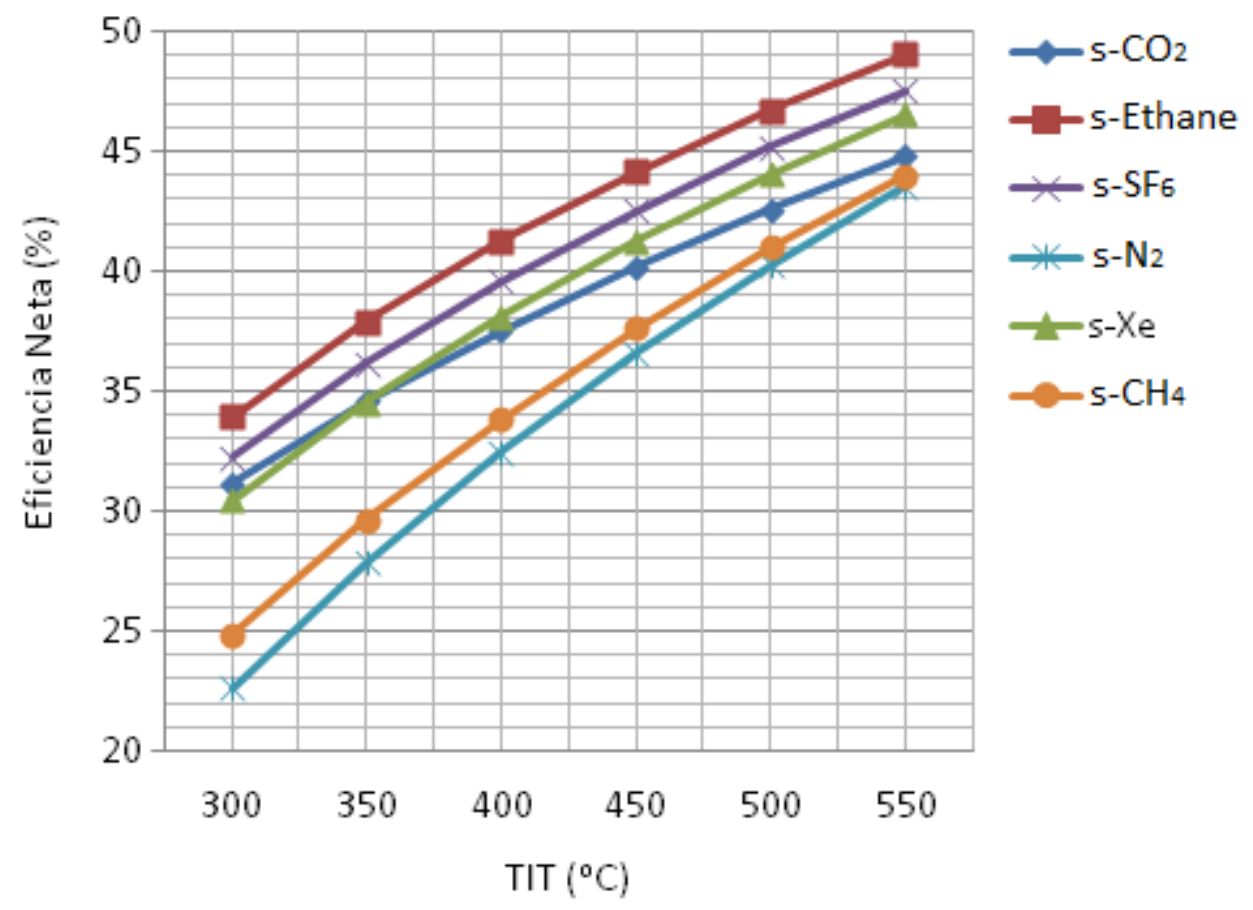

Figura 11.12. Eficiencia bruta versus TIT, fijado UA $=15000 \mathrm{~kW} / \mathrm{K}$ para diferentes fluidos de trabajo: $\mathrm{CO}_{2}, \mathrm{C}_{2} \mathrm{H}_{6}, \mathrm{CH}_{4}, \mathrm{~N}_{2}$, Xe y $\mathrm{N}_{2}$. Ciclo Brayton $\mathrm{SB}$ con recuperación de calor.

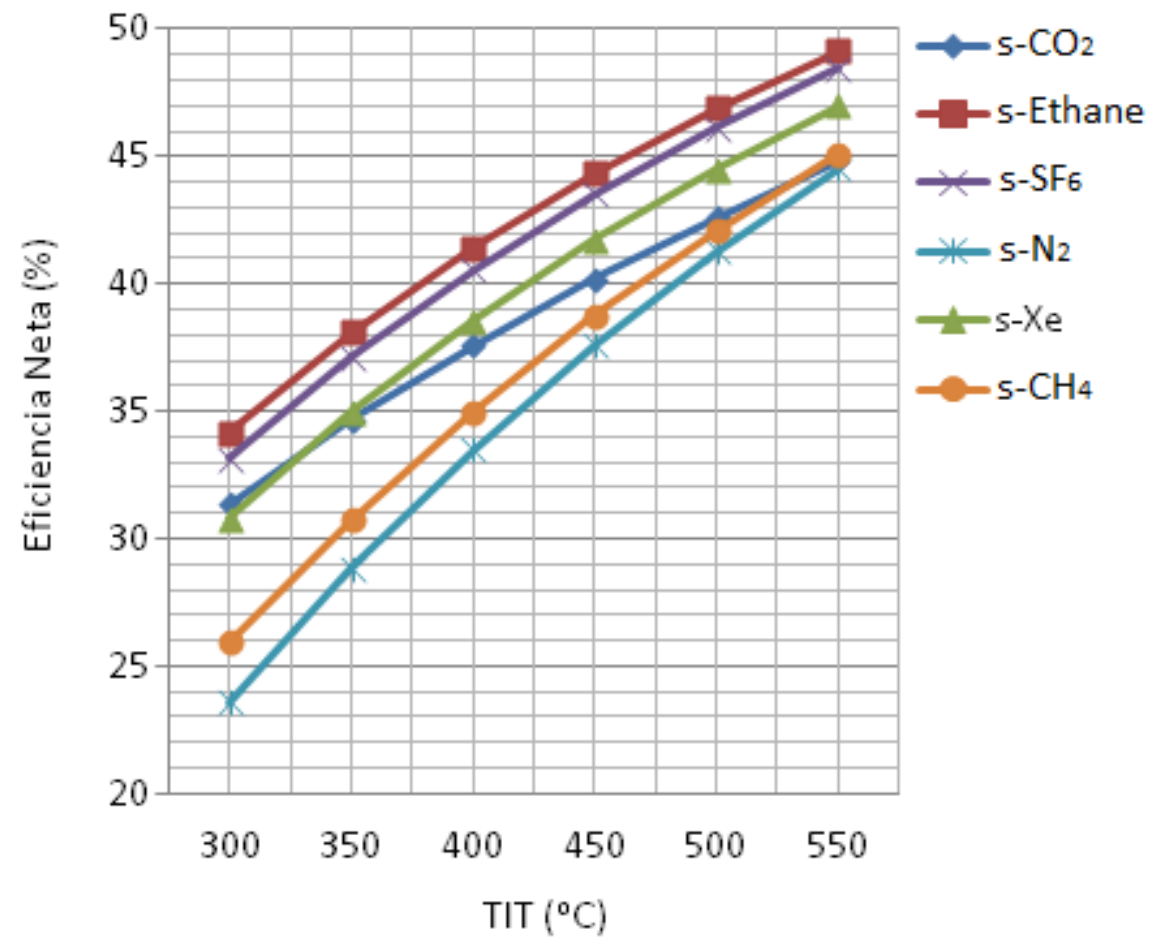

Figura 11.13. Eficiencia bruta \& TIT, fijado $U A=20000 \mathrm{~kW} / \mathrm{K}$ para diferentes fluidos de trabajo: $\mathrm{CO}_{2}, \mathrm{C}_{2} \mathrm{H}_{6}, \mathrm{CH}_{4}, \mathrm{~N}_{2}$, Xe y $\mathrm{N}_{2}$. Ciclo Brayton $\mathrm{SB}$ con recuperación de calor. 
11. Comparación entre el s- $\mathrm{CO}_{2}$ y otros fluidos de trabajo $\left(s-\mathrm{C}_{2} \mathrm{H}_{6}, \mathrm{~s}-\mathrm{SF}_{6}, \mathrm{~s}-\mathrm{Xe}, \mathrm{s}-\mathrm{CH}_{4}, \mathrm{~s}-\mathrm{N}_{2}\right)$ en las plantas termosolares acopladas a ciclos supercríticos Brayton

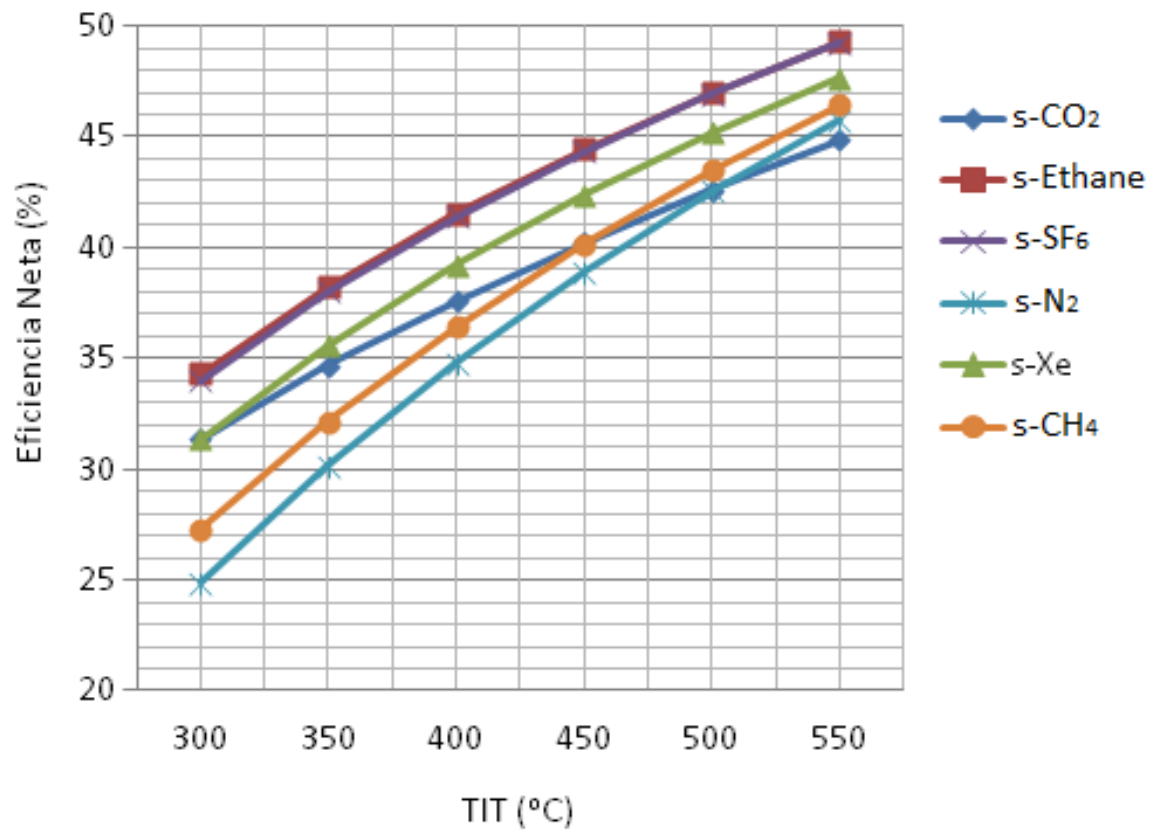

Figura 11.14. Eficiencia bruta versus TIT, fijado $U A=30000 \mathrm{~kW} / \mathrm{K}$ para diferentes fluidos de trabajo: $\mathrm{CO}_{2}, \mathrm{C}_{2} \mathrm{H}_{6}, \mathrm{CH}_{4}, \mathrm{~N}_{2}$, Xe y $\mathrm{N}_{2}$. Ciclo Brayton $\mathrm{SB}$ con recuperación de calor.

\section{5. Área de Apertura Efectiva del Campo Solar}

En este apartado se ha cuantificado tanto para colectores PTC como para colectores LF el área de apertura necesaria para cada uno de los fluidos de trabajo estudiados, figuras 11.15 a 11.24, obteniendo los siguientes resultados:

- En el caso de la comparación con el $\mathrm{C}_{2} \mathrm{H}_{6}$, se confirma como para el tamaño térmico de los recuperadores $\mathrm{UA}=10000 \mathrm{~kW} / \mathrm{K}$ el ahorro de superficie de captación solar es significativo $8 \%$, figuras 11.15 y 11.20 .

- En el caso de la comparativa con el $\mathrm{SF}_{6}$, como quedaba patente en el apartado anterior, donde se ha comparado la eficiencia bruta de la instalación solar, los ciclos con este gas de alta densidad requieren un mayor tamaño térmico de los recuperadores 3 veces mayores $\mathrm{UA}=15000 \mathrm{~kW} / \mathrm{K}$, para proporcionar valores de eficiencia y área de apertura superiores en un $5 \%$ a los del $\mathrm{CO}_{2}$, figuras 11.16 y 11.21. Xe, para un tamaño térmico $U A=5000 \mathrm{~kW} / \mathrm{K}$, requiere áreas similares de apertura en sus colectores a las solicitadas por el $\mathrm{CO}_{2}$, figuras 11.17 y 11.22 .

- Xe es un gas inerte, no corrosivo con los metales, y garantiza un bajo costes de los materiales de los equipos y componentes del ciclo de potencia, sin embargo, el tamaño de las turbomáquinas es un poco mayor que con $\mathrm{CO}_{2}$.

- Tanto el $\mathrm{N}_{2}$ como el $\mathrm{CH}_{4}$ requieren tamaños térmicos de los recuperadores mayores que los del $\mathrm{CO}_{2}$, para similares áreas de apertura del campo solar. Tanto para el $\mathrm{N}_{2}$ como para el $\mathrm{CH}_{4}$, su UA sería casi 4 veces mayor, entre 20000 a $30000 \mathrm{~kW} / \mathrm{K}$, para un UA del $\mathrm{CO}_{2}$ de $5000 \mathrm{~kW} / \mathrm{K}$, figuras $11.18,11.19,11.23$ y 11.24 . 
11. Comparación entre el s- $\mathrm{CO}_{2}$ y otros fluidos de trabajo $\left(\mathrm{s}-\mathrm{C}_{2} \mathrm{H}_{6}, \mathrm{~s}-\mathrm{SF} 6, \mathrm{~s}-\mathrm{Xe}, \mathrm{s}-\mathrm{CH}_{4}, \mathrm{~s}-\mathrm{N}_{2}\right)$ en las plantas termosolares acopladas a ciclos supercríticos Brayton

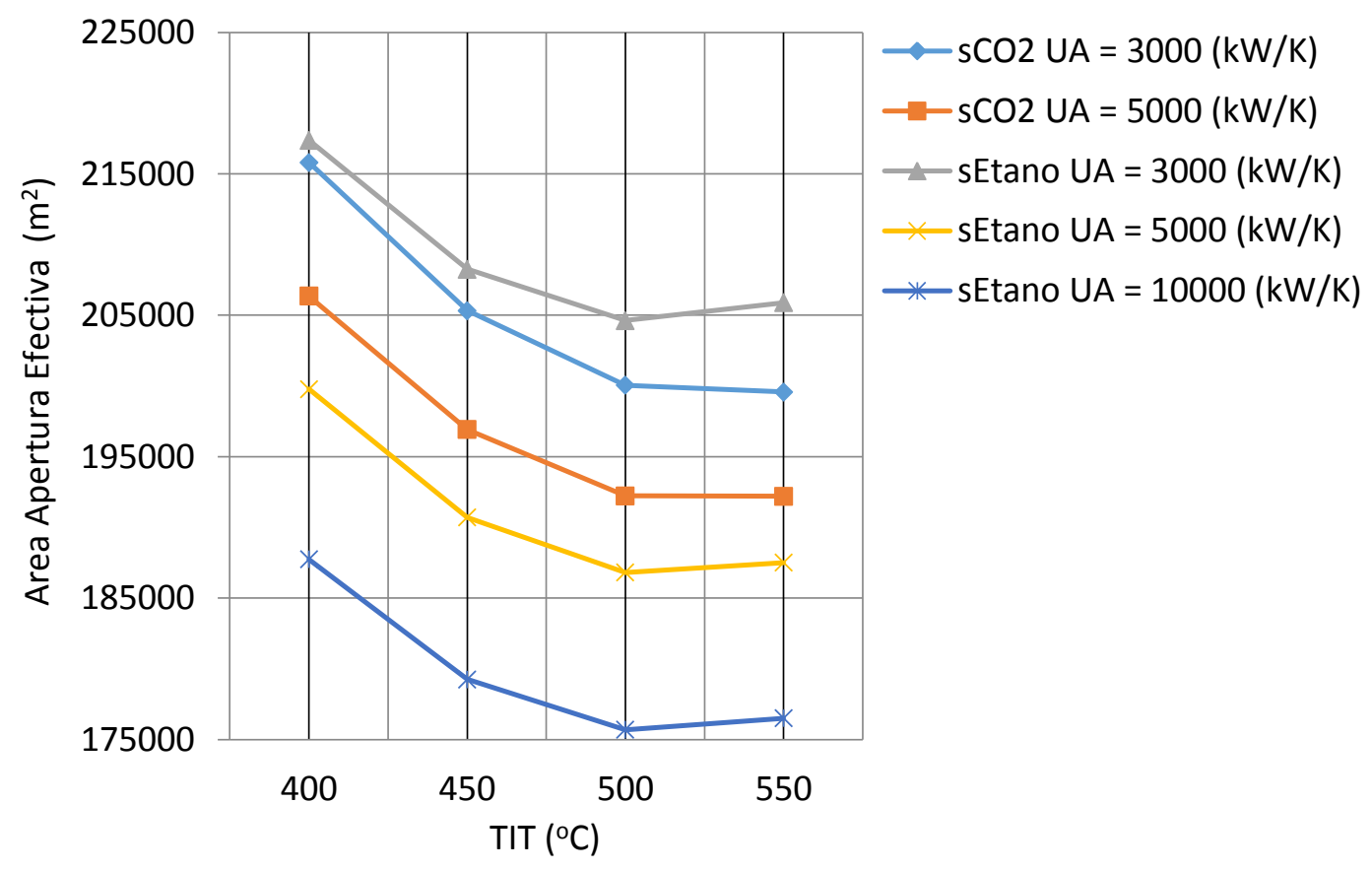

Figura 11.15. Comparación del área apertura efectiva versus TIT, en planta termosolar con colectores PTC acoplada a ciclo Brayton SB con s- $\mathrm{CO}_{2}$ o s- $\mathrm{C}_{2} \mathrm{H}_{6}$ como fluidos de trabajo.

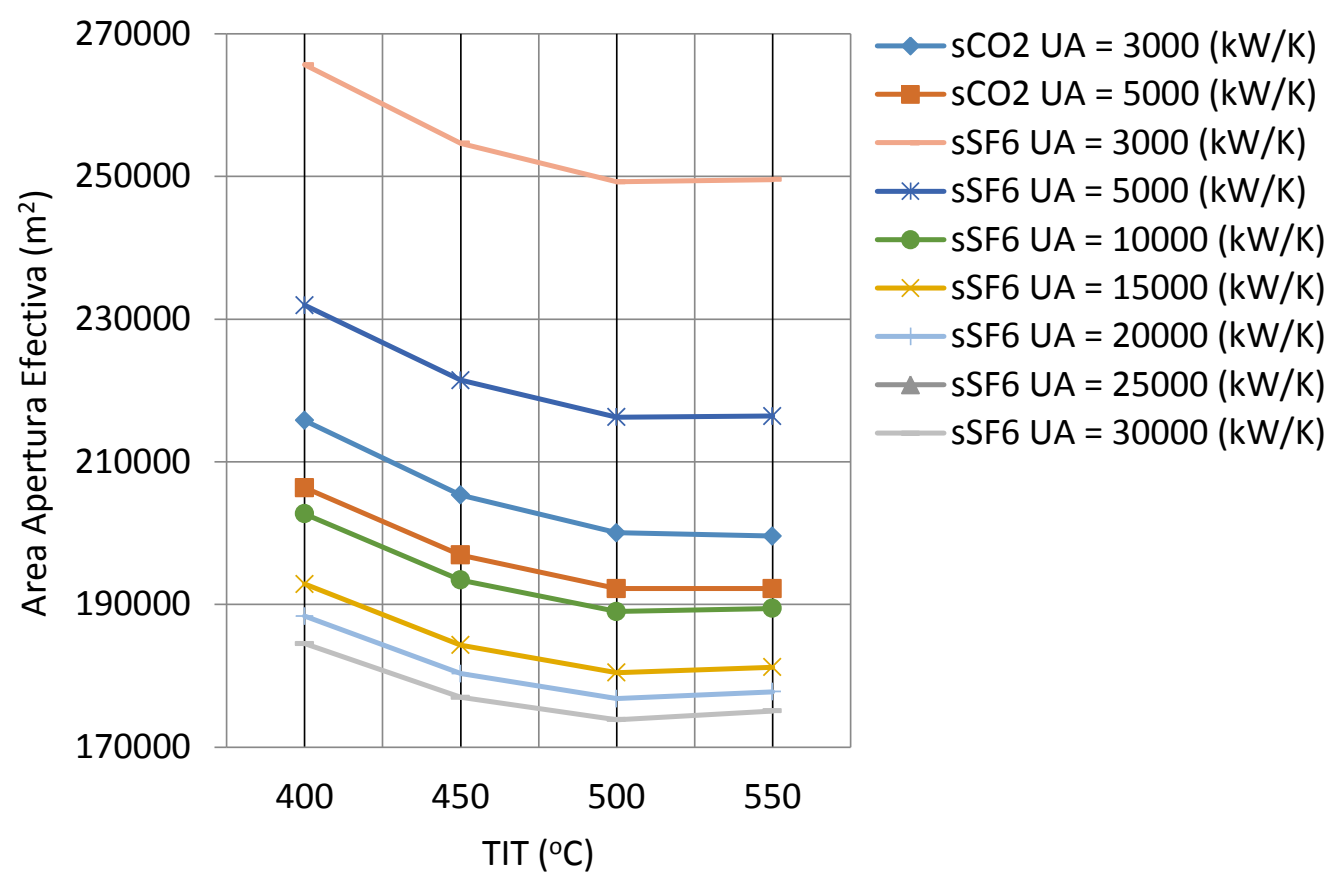

Figura 11.16. Comparación del área apertura efectiva versus TIT, en planta termosolar con colectores PTC acoplada a ciclo Brayton SB con s- $\mathrm{CO}_{2}$ o s-SF 6 como fluidos de trabajo. 
11. Comparación entre el s- $\mathrm{CO}_{2}$ y otros fluidos de trabajo $\left(s-\mathrm{C}_{2} \mathrm{H}_{6}, \mathrm{~s}-\mathrm{SF}_{6}, \mathrm{~s}-\mathrm{Xe}, \mathrm{s}-\mathrm{CH}_{4}, \mathrm{~s}-\mathrm{N}_{2}\right)$ en las plantas termosolares acopladas a ciclos supercríticos Brayton

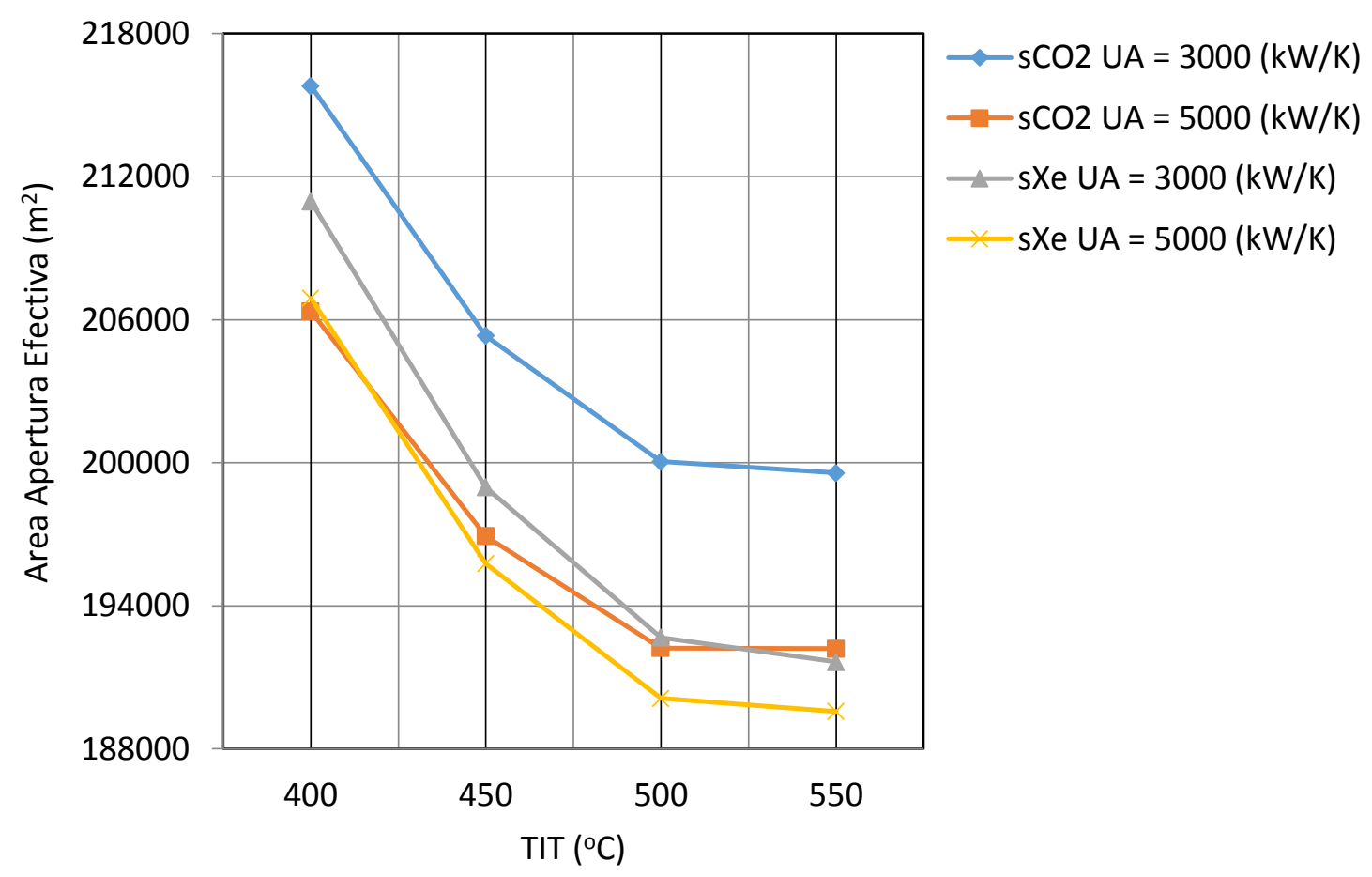

Figura 11.17. Comparación del área apertura efectiva versus TIT, en plantas termosolares con colectores PTC acoplada a ciclo Brayton SB con s- $\mathrm{CO}_{2}$ o s-Xe como fluidos de trabajo.

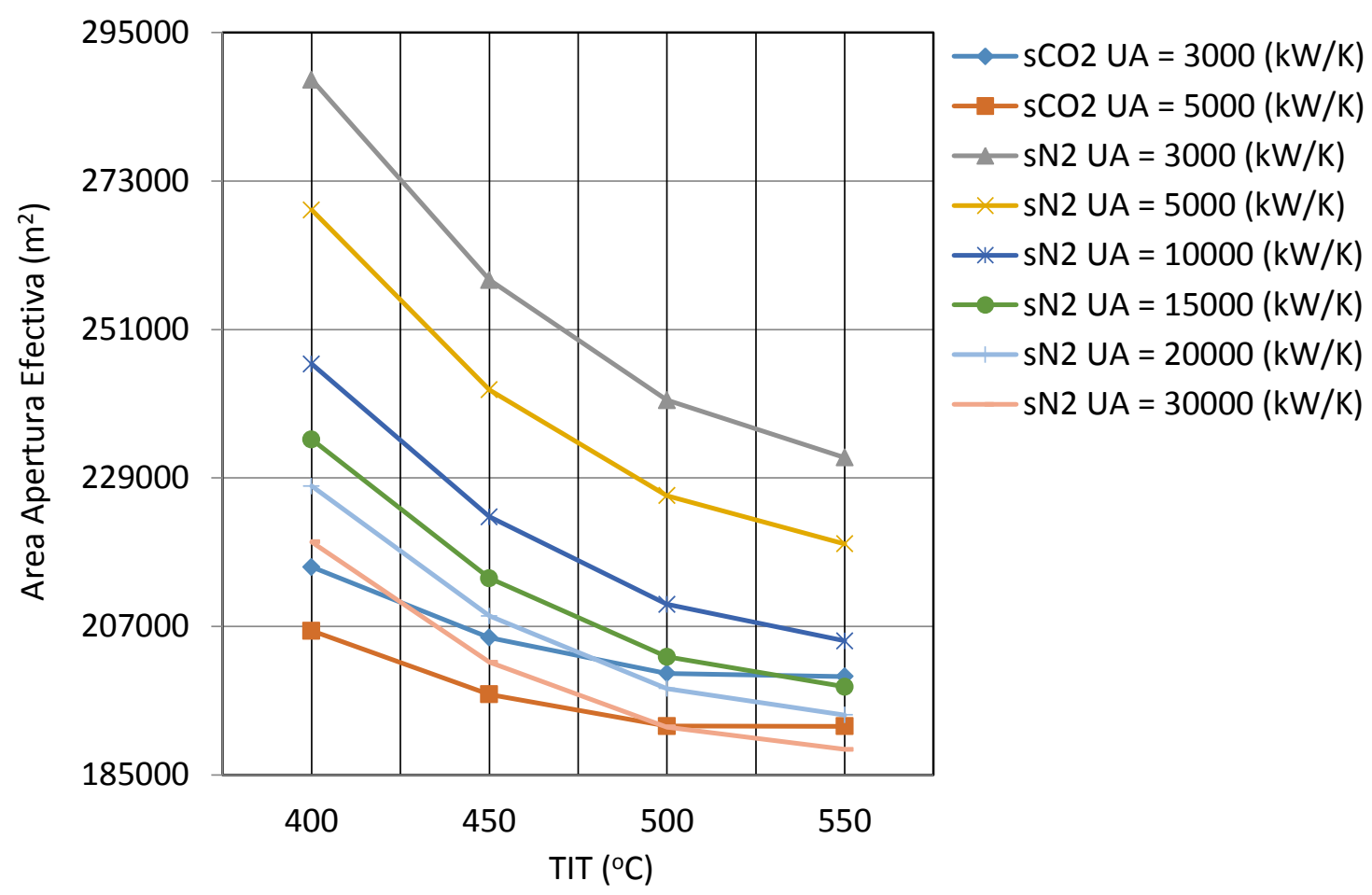

Figura 11.18. PT Comparación del área apertura efectiva versus TIT, en planta termosolar con colectores PTC acoplada a ciclo Brayton SB y s- $\mathrm{CO}_{2}$ o s- $\mathrm{N}_{2}$ como fluidos de trabajo. 
11. Comparación entre el s- $\mathrm{CO}_{2}$ y otros fluidos de trabajo $\left(\mathrm{s}-\mathrm{C}_{2} \mathrm{H}_{6}, \mathrm{~s}-\mathrm{SF} 6, \mathrm{~s}-\mathrm{Xe}, \mathrm{s}-\mathrm{CH}_{4}, \mathrm{~s}-\mathrm{N}_{2}\right)$ en las plantas termosolares acopladas a ciclos supercríticos Brayton

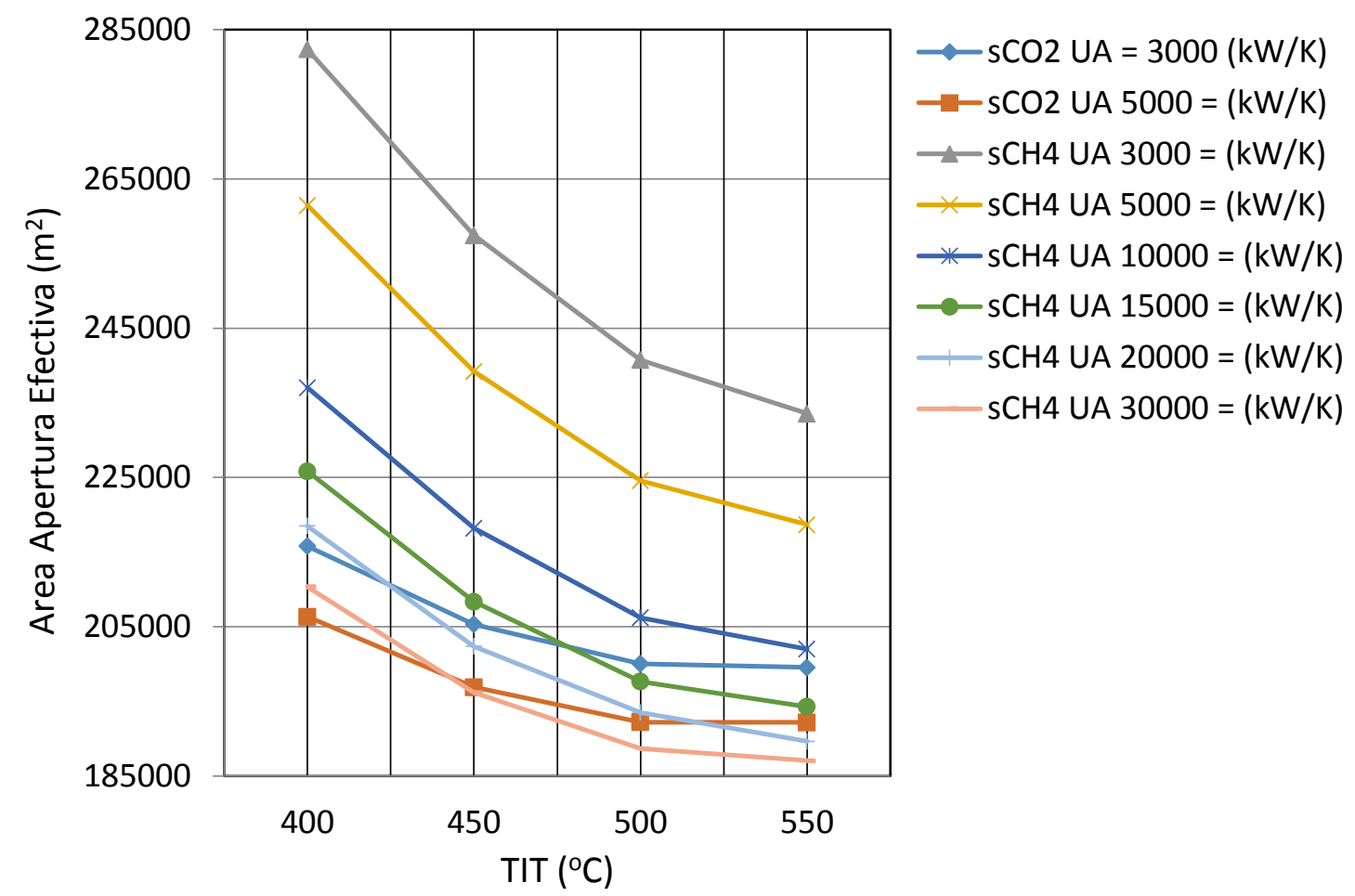

Figura 11.19. Comparación del área apertura efectiva versus TIT, en planta termosolar con colectores PTC acoplada a ciclo Brayton SB con s- $\mathrm{CO}_{2}$ o s- $\mathrm{CH}_{4}$ como fluidos de trabajo.

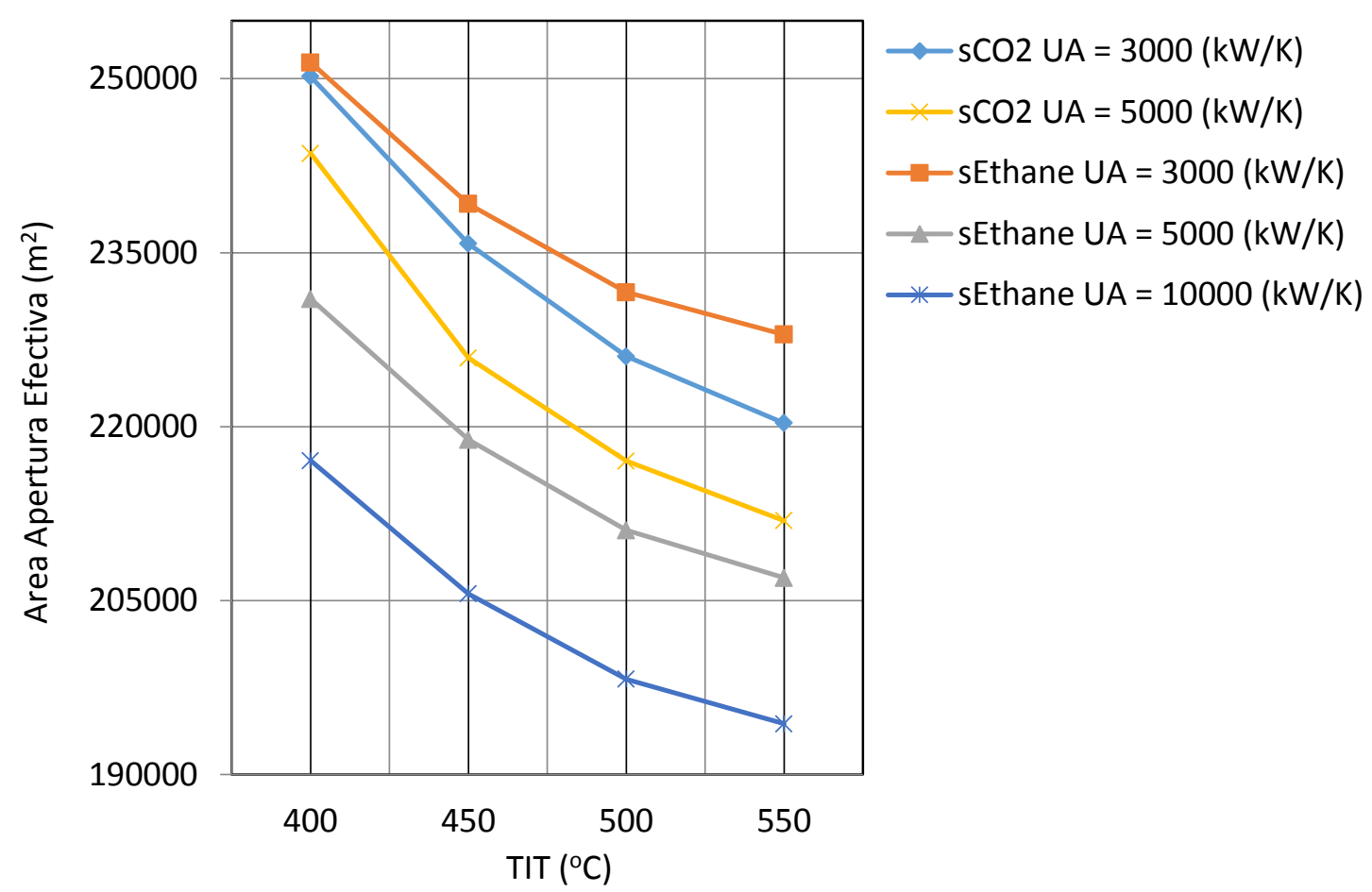

Figura 11.20. Comparación del área apertura efectiva versus TIT, en planta termosolar con LF acoplada a ciclo Brayton $\mathrm{SB}$ con s- $\mathrm{CO}_{2}$ o s- $\mathrm{C}_{2} \mathrm{H}_{6}$ como fluidos de trabajo. 
11. Comparación entre el s- $\mathrm{CO}_{2}$ y otros fluidos de trabajo $\left(\mathrm{s}-\mathrm{C}_{2} \mathrm{H}_{6}, \mathrm{~s}-\mathrm{SF} 6, \mathrm{~s}-\mathrm{Xe}, \mathrm{s}-\mathrm{CH}_{4}, \mathrm{~s}-\mathrm{N}_{2}\right)$ en las plantas termosolares acopladas a ciclos supercríticos Brayton

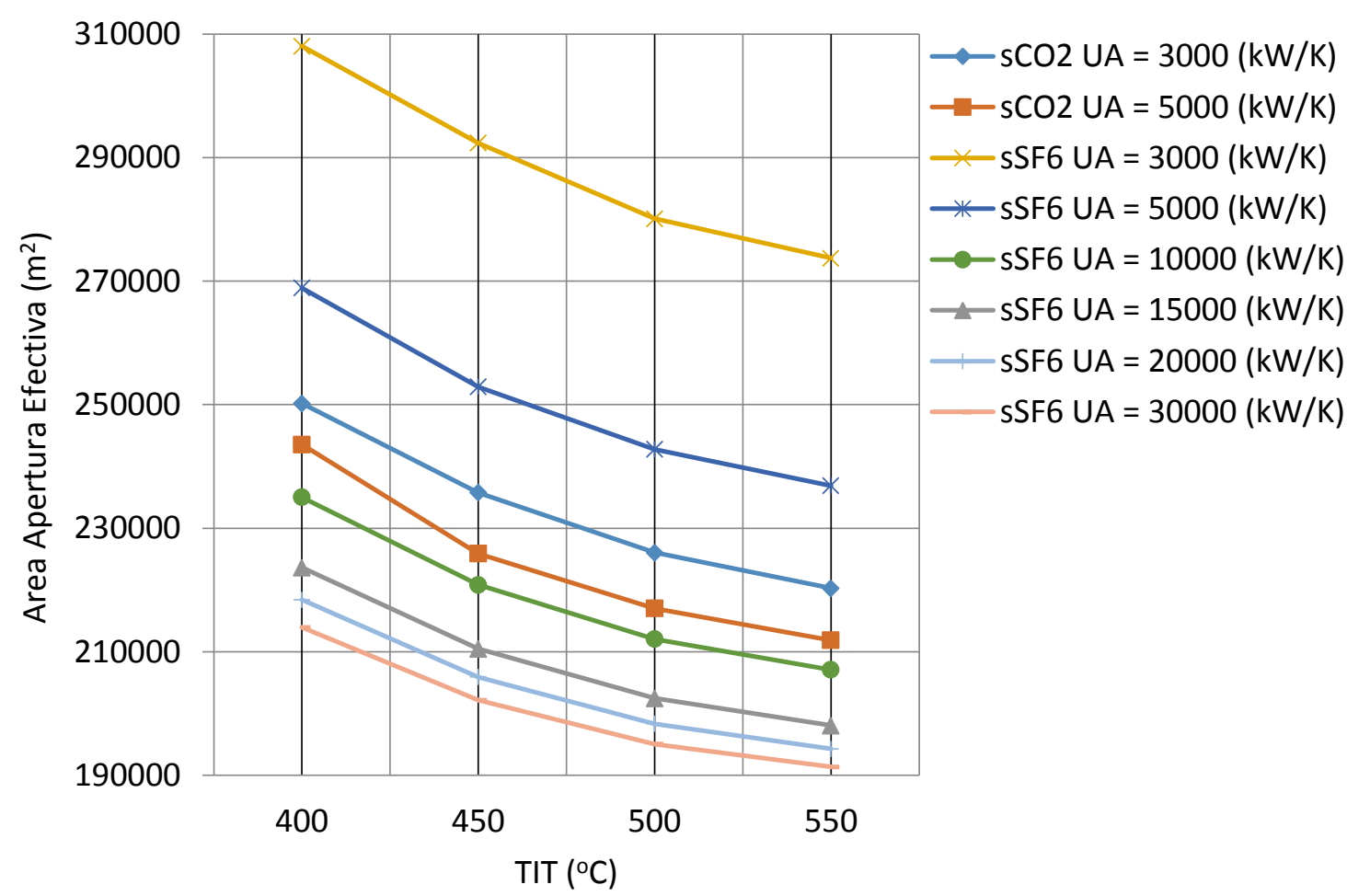

Figura 11.21. Comparación del área apertura efectiva versus TIT, en las planta termosolar con colectores LF acoplada a ciclo Brayton $\mathrm{SB}$ con s- $\mathrm{CO}_{2}$ o s-SF 6 como fluidos de trabajo.

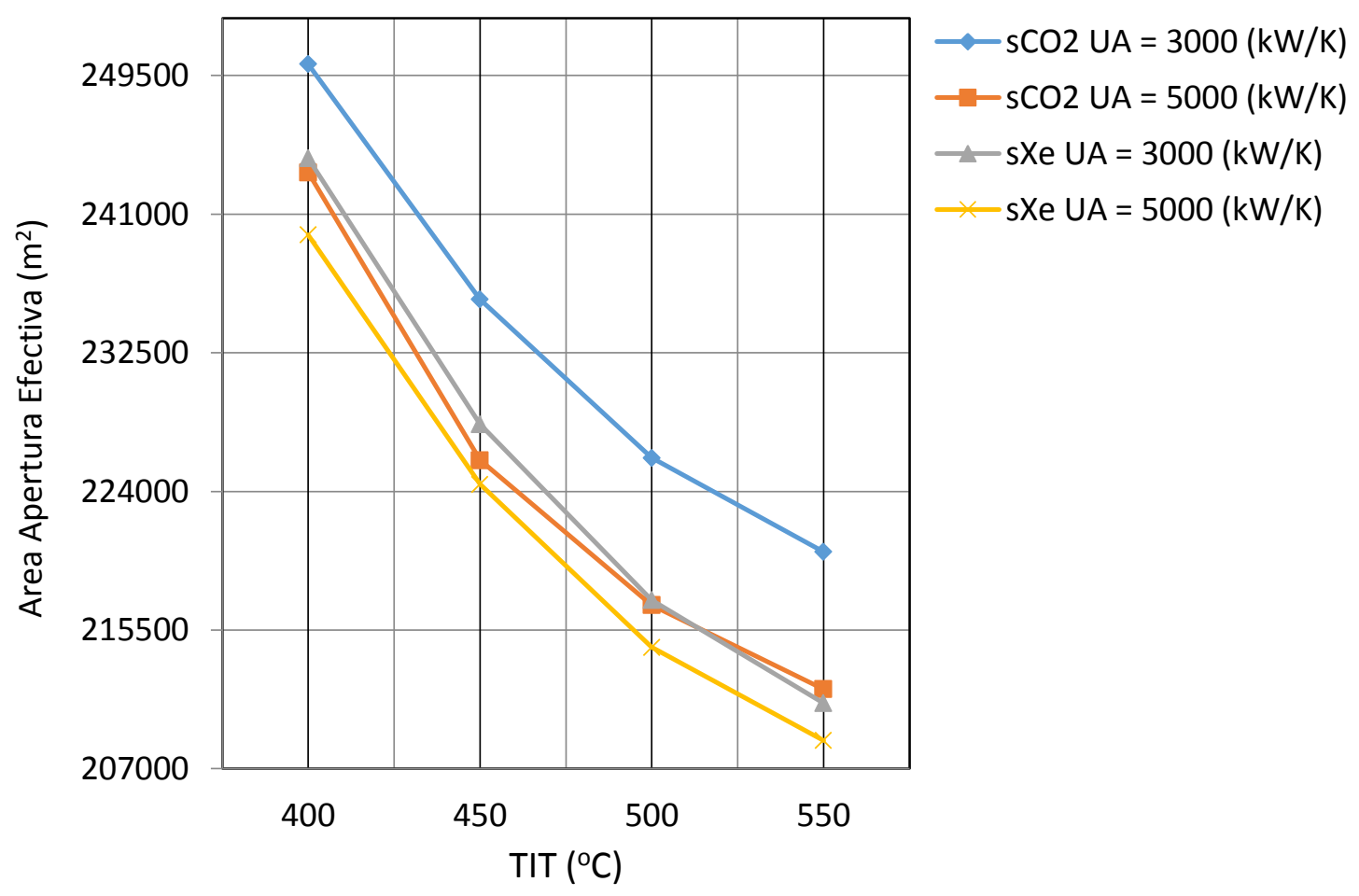

Figura 11.22. Comparación del área apertura efectiva versus TIT, en planta termosolar con colectores LF acoplada a ciclo Brayton SB con s- $\mathrm{CO}_{2}$ o s-Xe como fluidos de trabajo. 
11. Comparación entre el s- $\mathrm{CO}_{2}$ y otros fluidos de trabajo $\left(s-\mathrm{C}_{2} \mathrm{H}_{6}, \mathrm{~s}-\mathrm{SF}_{6}, \mathrm{~s}-\mathrm{Xe}, \mathrm{s}-\mathrm{CH}_{4}, \mathrm{~s}-\mathrm{N}_{2}\right)$ en las plantas termosolares acopladas a ciclos supercríticos Brayton

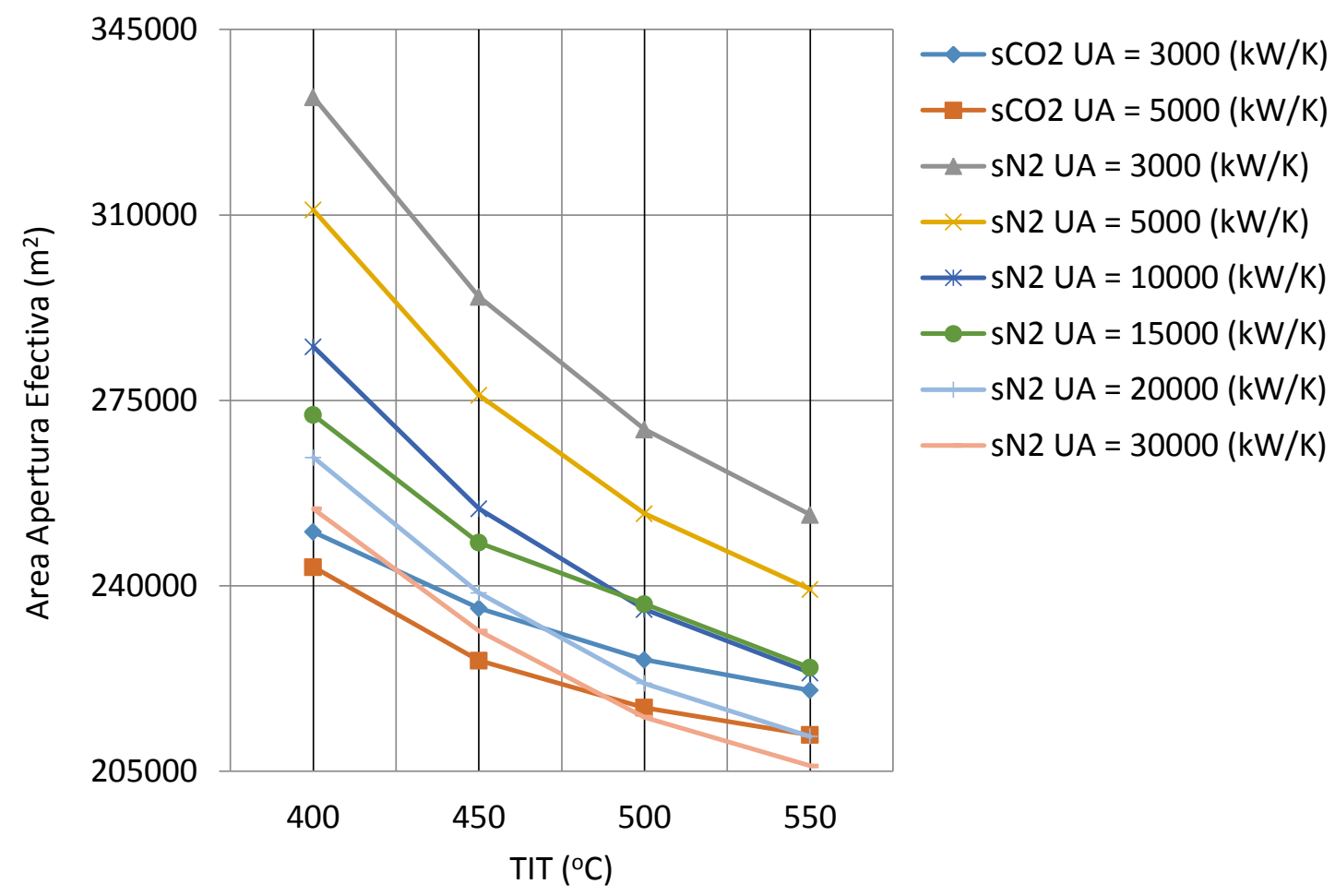

Figura 11.23. Comparación del área apertura efectiva versus TIT, en planta termosolar con colectores LF acoplada a ciclo Brayton SB con s- $\mathrm{CO}_{2}$ o s- $\mathrm{N}_{2}$ como fluidos de trabajo.

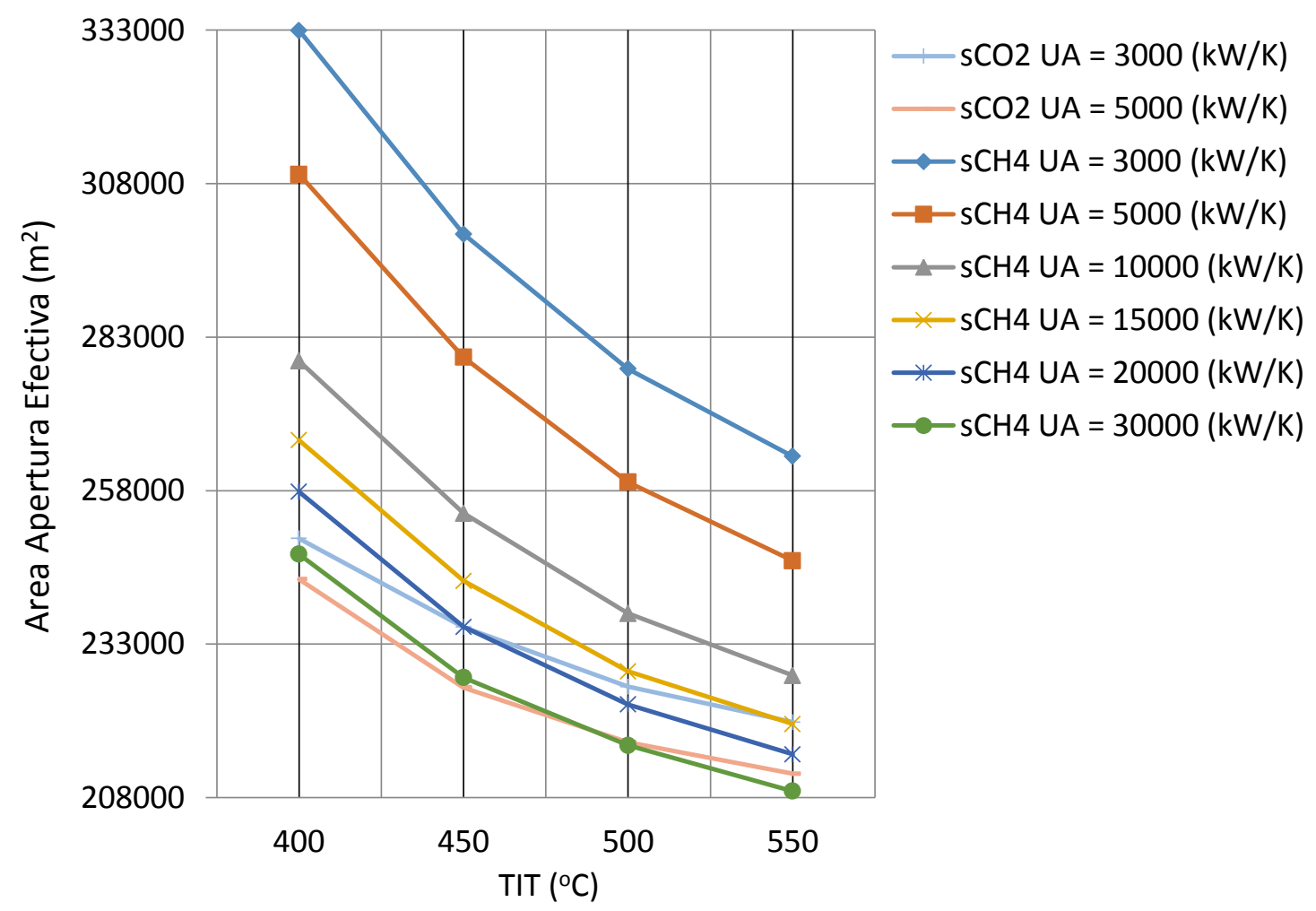

Figura 11.24. Comparación del área apertura efectiva versus $\mathrm{TIT}$, en planta termosolar con colectores LF acoplada a ciclo Brayton SB con s- $\mathrm{CO}_{2}$ o s- $\mathrm{CH}_{4}$ como fluidos de trabajo. 
11. Comparación entre el s- $\mathrm{CO}_{2}$ y otros fluidos de trabajo $\left(\mathrm{s}-\mathrm{C}_{2} \mathrm{H}_{6}, \mathrm{~s}-\mathrm{SF} \mathrm{F}_{6}, \mathrm{~s}-\mathrm{Xe}, \mathrm{s}-\mathrm{CH}_{4}, \mathrm{~s}-\mathrm{N}_{2}\right)$ en las plantas termosolares acopladas a ciclos supercríticos Brayton

11.6. Caracterización del coeficiente de transferencia de calor (HTC) de los fluidos de trabajo

Tomando como punto de partida la correlación de Dittus-Boelter [38] se ha realizado una comparativa para diferentes niveles de presión y temperatura, entre los coeficientes de transferencia de calor HTC de los diferentes fluidos de trabajo considerados en este capítulo $\left(\mathrm{CO}_{2}, \mathrm{SF}_{6}, \mathrm{C}_{2} \mathrm{H}_{6}, \mathrm{Xe}, \mathrm{N}_{2}\right.$ y $\left.\mathrm{CH}_{4}\right)$. Los resultados obtenidos están resumidos en las figuras 11.25 a 11.29. El objetivo es predecir el tamaño comparativo de los intercambiadores de calor con los diferentes fluidos. Para realizar esta comparativa se ha considerado un canal de $3 \mathrm{~mm}$ de diámetro, y se han fijado diferentes valores de velocidades del fluido (desde $3 \mathrm{~m} / \mathrm{s}$ hasta 30 $\mathrm{m} / \mathrm{s})$.

La correlación de Dittus-Boelter [38] viene definida por la ecuación (11-1):

$N u_{D}=0.023 \cdot R_{D}^{\frac{4}{5}} \cdot \operatorname{Pr}^{n}$

Siendo $\mathrm{n}=0.4$ para calentamiento del fluido, y $\mathrm{n}=0.3$ para enfriamiento del fluido.

Esta ecuación es válida para las siguientes condiciones (11-2) a (11-4) [38]:

$0.6<=\operatorname{Pr}<=160$

$\operatorname{Re}>=10000$

$L / D>=10$

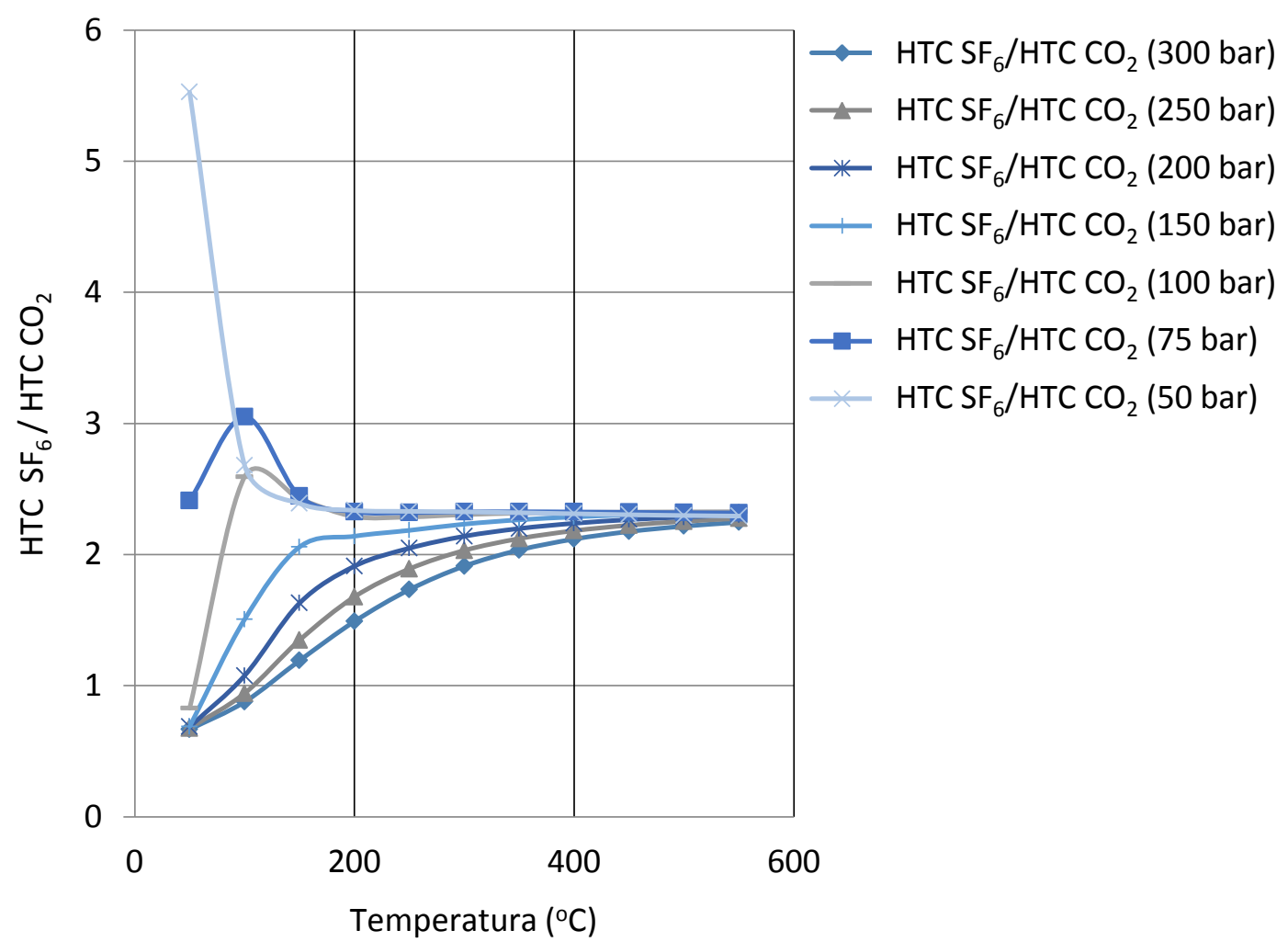

Figura 11.25. Comparación relativa de $\mathrm{HTC}$ en ciclos Brayton: $\mathrm{CO}_{2} \mathrm{Vs}_{\text {. }} \mathrm{SF}_{6}$.

El SF 6 presenta unos valores del $\mathrm{HTC}$ dos veces superiores al $\mathrm{CO}_{2}$ para temperaturas superiores a $250^{\circ} \mathrm{C}$ a $300^{\circ} \mathrm{C}$, como queda representado en la figura 11.25. En relación al $\mathrm{C}_{2} \mathrm{H}_{6}$, también presenta mayores valores de $\mathrm{HTC}$ en relación al $\mathrm{CO}_{2}$, su $\mathrm{HTC}$ se incrementa linealmente a partir 
11. Comparación entre el s- $\mathrm{CO}_{2}$ y otros fluidos de trabajo $\left(\mathrm{s}-\mathrm{C}_{2} \mathrm{H}_{6}, \mathrm{~s}-\mathrm{SF} \mathrm{F}_{6}, \mathrm{~s}-\mathrm{Xe}, \mathrm{s}-\mathrm{CH}_{4}, \mathrm{~s}-\mathrm{N}_{2}\right)$ en las plantas termosolares acopladas a ciclos supercríticos Brayton

de $200^{\circ} \mathrm{C}$ en relación al $\mathrm{CO}_{2}$. Los resultados detallados se muestran en la figura 11.26. También es interesante comparar las figuras 11.25 y 11.26, deducimos que el $\mathrm{SF}_{6}$ presenta mayores valores de $\mathrm{HTC}$ que el $\mathrm{C}_{2} \mathrm{H}_{6}$ y el $\mathrm{CO}_{2}$. Como se representa en la figura 11.27, el Nitrógeno proporciona valores de coeficiente de transferencia de calor tan buenos como el $\mathrm{CO}_{2}$, son entre un $50-70 \%$ menores.

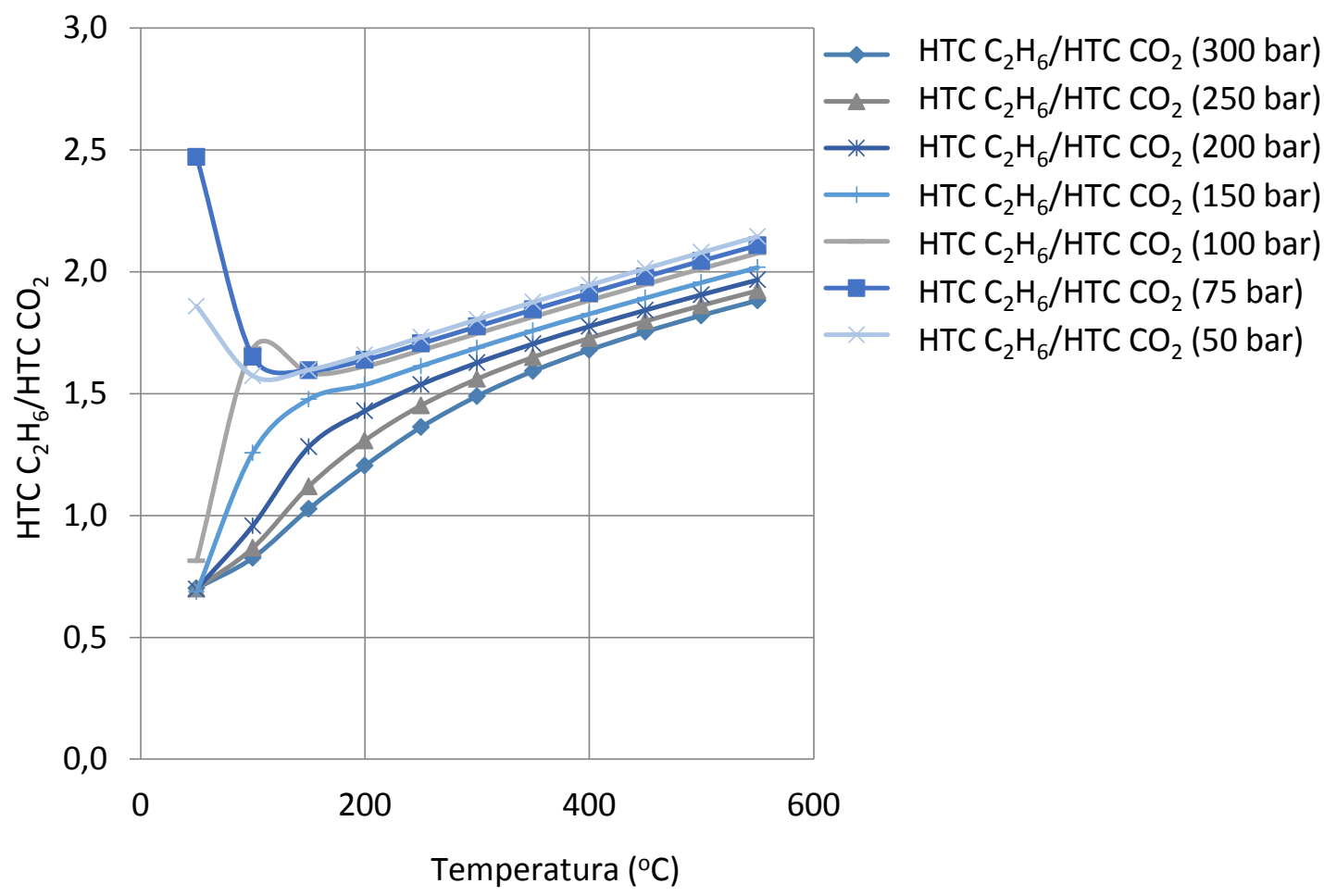

Figura 11.26. Comparación relativa de $\mathrm{HTC}$ de en ciclo Brayton: $\mathrm{CO}_{2} \mathrm{Vs} . \mathrm{C}_{2} \mathrm{H}_{6}$.

Los coeficientes de transferencia de calor del Xe, como se ilustra en la figura 11.28, también son inferiores a los del $\mathrm{CO}_{2}$, entre un $60 \%$ a un $40 \%$. Se concluye que el coeficiente de transferencia del Xe es el menor de todos los fluidos de trabajo que se han comparando. En relación al $\mathrm{CH}_{4}$, su HTC es similar al $\mathrm{CO}_{2}$ para bajas temperaturas y alrededor de un $15 \%$ a $55 \%$ superior al del $\mathrm{CO}_{2}$ para temperaturas superiores a los $300^{\circ} \mathrm{C}$, figura 11.29 . 
11. Comparación entre el s- $\mathrm{CO}_{2}$ y otros fluidos de trabajo $\left(\mathrm{s}-\mathrm{C}_{2} \mathrm{H}_{6}, \mathrm{~s}-\mathrm{SF} \mathrm{F}_{6}, \mathrm{~s}-\mathrm{Xe}, \mathrm{s}-\mathrm{CH}_{4}, \mathrm{~s}-\mathrm{N}_{2}\right)$ en las plantas termosolares acopladas a ciclos supercríticos Brayton

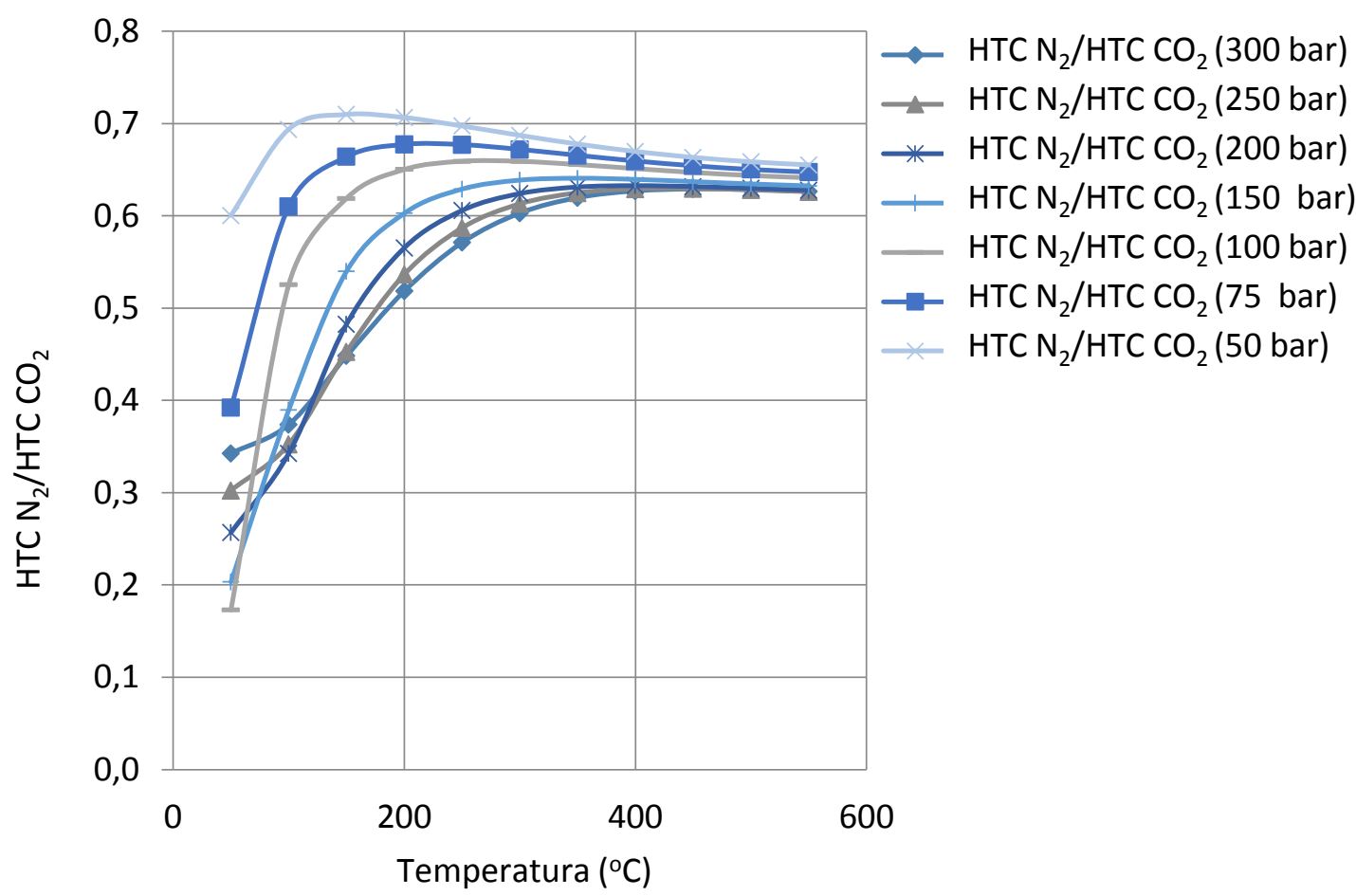

Figura 11.27. Comparación relativa de $\mathrm{HTC}$ en ciclo Brayton: $\mathrm{CO}_{2}$ Vs. $\mathrm{N}_{2}$.

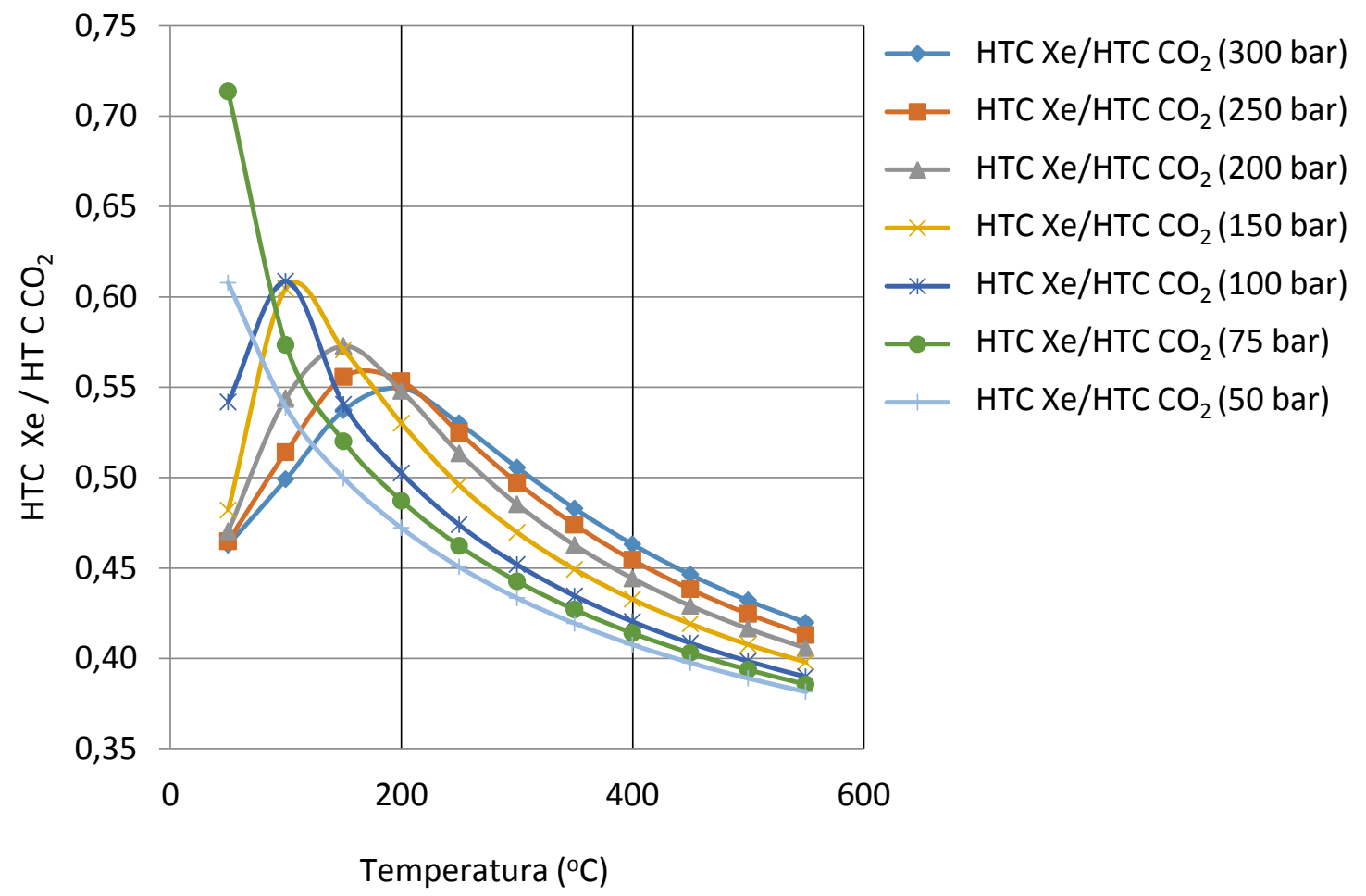

Figura 11.28. Comparación relativa de $\mathrm{HTC}$ en ciclo Brayton: $\mathrm{CO}_{2}$ Vs. Xe. 
11. Comparación entre el s- $\mathrm{CO}_{2}$ y otros fluidos de trabajo $\left(\mathrm{s}-\mathrm{C}_{2} \mathrm{H}_{6}, \mathrm{~s}-\mathrm{SF}_{6}, \mathrm{~s}-\mathrm{Xe}, \mathrm{s}-\mathrm{CH}_{4}, \mathrm{~s}-\mathrm{N}_{2}\right)$ en las plantas termosolares acopladas a ciclos supercríticos Brayton

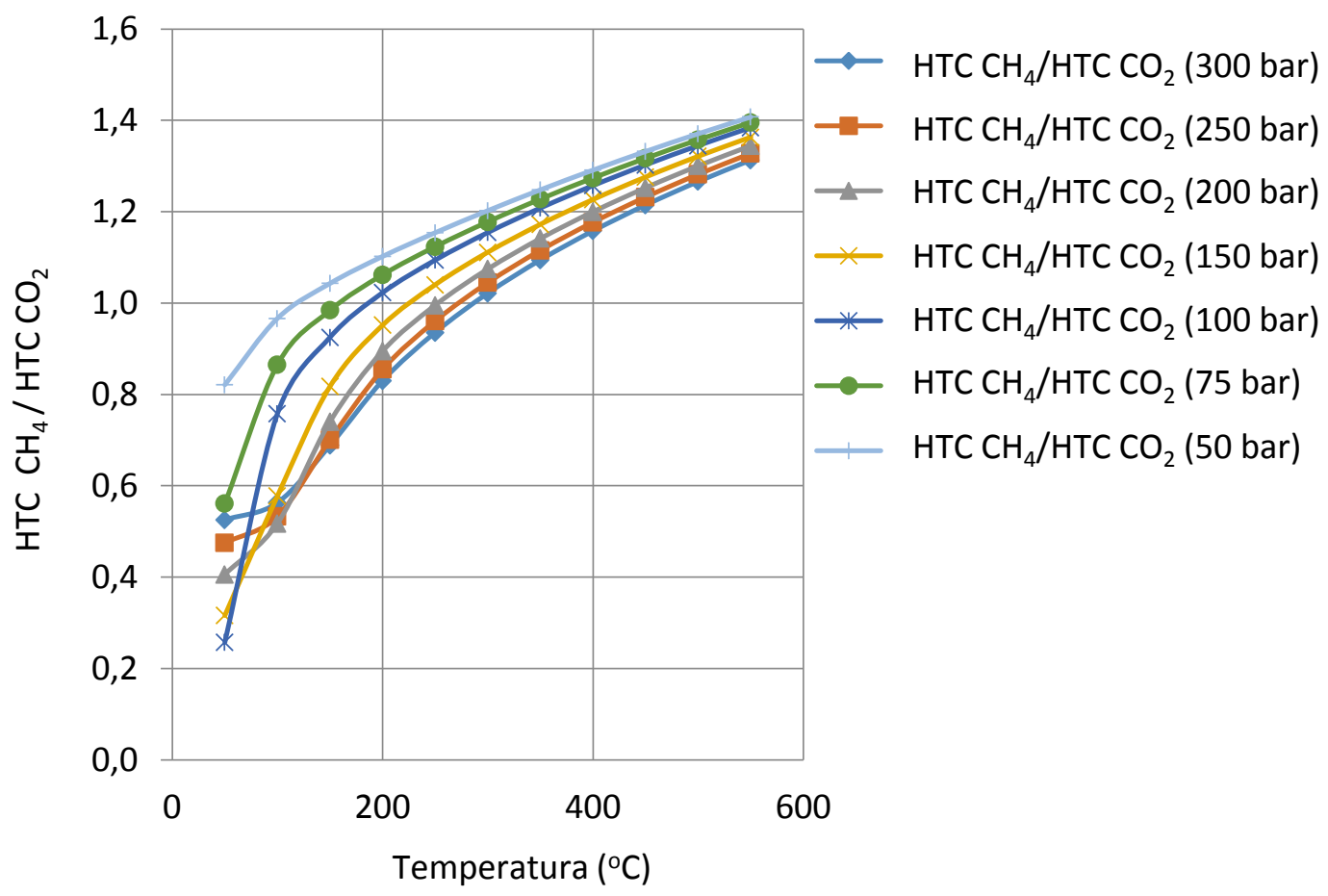

Figura 11.29. Comparación relativa de HTC en ciclo Brayton: $\mathrm{CO}_{2}$ Vs. $\mathrm{CH}_{4}$.

\subsection{Caracterización de las pérdidas de presión de los fluidos de trabajo}

En este apartado se han comparado las pérdidas de carga del $\mathrm{CO}_{2}$ con el resto de fluidos de trabajos analizados en este trabajo $\left(\mathrm{C}_{2} \mathrm{H}_{6}, \mathrm{SF}_{6}, \mathrm{Xe}, \mathrm{N}_{2}\right.$ y $\left.\mathrm{CH}_{4}\right)$. Para realizar esta comparativa se han fijado los diámetros de los canales, $3 \mathrm{~mm}$, y sus longitudes, $1.5 \mathrm{~m}$. Se ha realizado la comparativa para diferentes valores de velocidades de los fluidos $(3-30 \mathrm{~m} / \mathrm{s})$. El rango de velocidades elegido se debe a la similitud entre el estado supercrítico, y la fase líquida y vapor del fluido. En fase líquida la velocidad recomendable del fluido es de $3 \mathrm{~m} / \mathrm{s}$, porque con velocidades superiores se producen vibraciones y una excesiva erosión de los tubos. Para la fase vapor del fluido se recomiendan velocidades de hasta $30 \mathrm{~m} / \mathrm{s}$ ya que la densidad del fluido es menor.

Ecuación (11-5) para cálculo de las pérdidas de carga Darcy Weisbach [39]:

$\frac{\Delta P}{L}=f_{D} \cdot \frac{\rho}{2} \cdot \frac{V^{2}}{D}$

Ecuación (11-6) para cálculo del coeficiente de pérdidas de carga, Idelchik [40]:

$f_{c}=\left(\frac{1}{1.8 \cdot \log R e-1.5}\right)^{2}$

Ecuación (11-7) para cálculo del coeficiente de pérdidas de carga, Petukov [41]:

$f=\left(0.79 \cdot \ln \left(R e_{D}\right) \cdot 1.64\right)^{-2}$

Los resultados obtenidos se resumen en las figuras 11.30 a 11.34. Especial impacto en el cálculo de la pérdida de presión tienen la viscosidad y la densidad de los fluidos. De hecho las 
11. Comparación entre el s- $\mathrm{CO}_{2}$ y otros fluidos de trabajo $\left(s-\mathrm{C}_{2} \mathrm{H}_{6}, \mathrm{~s}-\mathrm{SF}_{6}, \mathrm{~s}-\mathrm{Xe}, \mathrm{s}-\mathrm{CH}_{4}, \mathrm{~s}-\mathrm{N}_{2}\right)$ en las plantas termosolares acopladas a ciclos supercríticos Brayton

pérdidas de presión con el $\mathrm{SF}_{6}$ son del orden de 2-3 veces superiores al $\mathrm{CO}_{2}$. En este caso la densidad del $\mathrm{SF}_{6}$ es superior al $\mathrm{CO}_{2}$, y también su viscosidad es del orden de 2 o 3 veces superior al $\mathrm{CO}_{2}$.

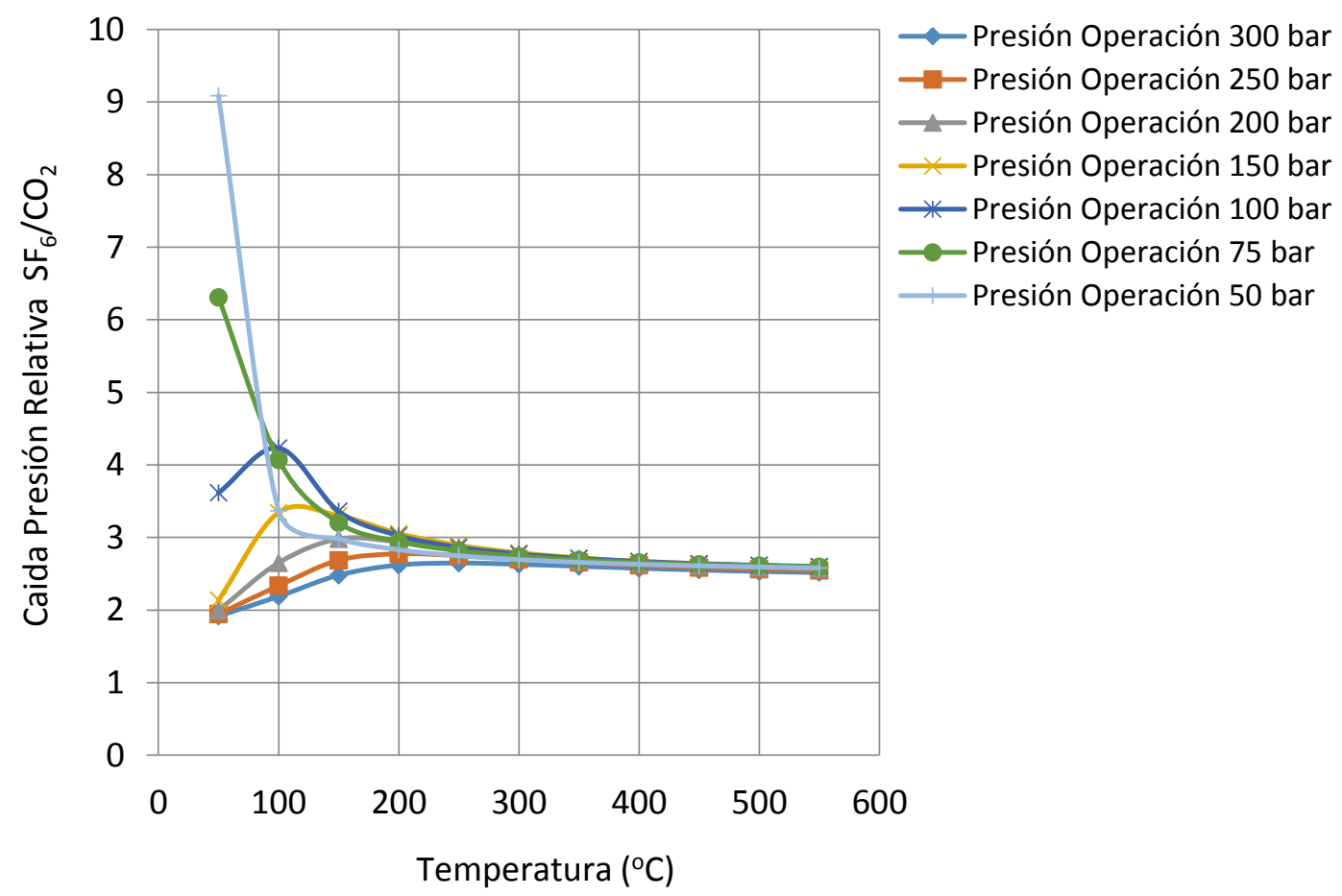

Figura 11.30. Comparación de la caída de presión relativa. $\mathrm{CO}_{2} \mathrm{Vs}$. $\mathrm{SF}_{6}$.

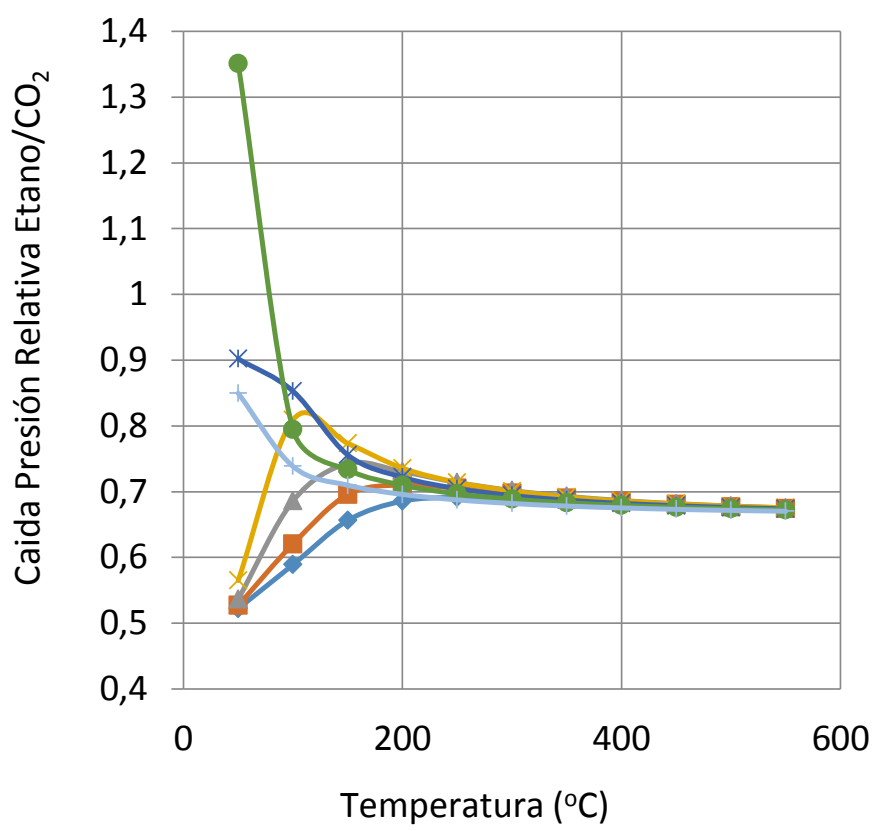

—Presión Operación 300 bar - Presión Operación 250 bar —-Presión Operación 200 bar * Presión Operación 150 bar * Presión Operación 100 bar - Presión Operación 75 bar - Presión Operación 50 bar

Figura 11.31. Comparación de la caída de presión relativa. $\mathrm{CO}_{2}$ Vs. $\mathrm{C}_{2} \mathrm{H}_{6}$.

Respecto al $\mathrm{C}_{2} \mathrm{H}_{6}$ presenta unas pérdidas de presión del $70 \%$ menor en comparación con el $\mathrm{CO}_{2}$, figura 11.31. La densidad del $\mathrm{C}_{2} \mathrm{H}_{6}$ es menor que la del $\mathrm{CO}_{2}$, pero la viscosidad del $\mathrm{CO}_{2}$ es mayor que la del $\mathrm{C}_{2} \mathrm{H}_{6}$. El $\mathrm{N}_{2}$, como se muestra la figura 11.32 presenta valores de pérdidas de carga 
11. Comparación entre el s- $\mathrm{CO}_{2}$ y otros fluidos de trabajo $\left(s-\mathrm{C}_{2} \mathrm{H}_{6}, \mathrm{~s}-\mathrm{SF}_{6}, \mathrm{~s}-\mathrm{Xe}, \mathrm{s}-\mathrm{CH}_{4}, \mathrm{~s}-\mathrm{N}_{2}\right)$ en las plantas termosolares acopladas a ciclos supercríticos Brayton

también entre un $60 \%$ y $70 \%$ inferiores a los del $\mathrm{CO}_{2}$. En este caso las viscosidades de ambos fluidos son similares, pero la densidad del $\mathrm{N}_{2}$ es muy inferior que la del $\mathrm{CO}_{2}$.

En el caso del Xenón, su densidad y su viscosidad son mayores que las del $\mathrm{CO}_{2}$ por esta razón sus pérdidas de carga son muy superiores a la de $\mathrm{CO}_{2}$, ver los resultados detallados en la figura 11.33. El $\mathrm{CH}_{4}$ presenta una gráfica de pérdida de presión muy similar a la del $\mathrm{N}_{2}$, en ambos casos sus densidades son bastante menores que la del $\mathrm{CO}_{2}$, y en el caso del $\mathrm{CH}_{4}$ su viscosidad es también inferior a la del $\mathrm{CO}_{2}$, y por tanto sus valores de pérdidas de presión son inferiores que el $\mathrm{CO}_{2}$, figura 11.34 .

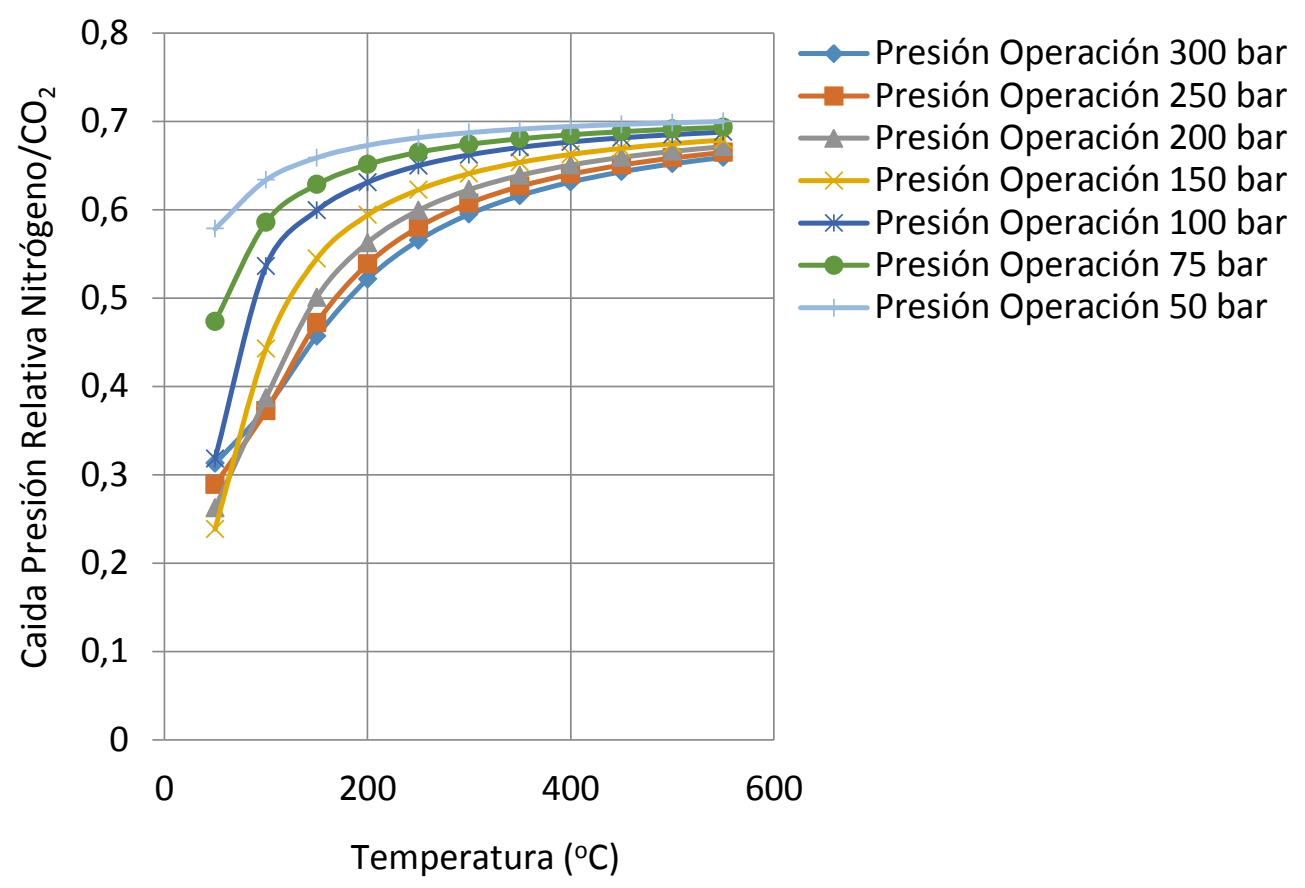

Figura 11.32. Comparación de la caída de presión relativa. $\mathrm{CO}_{2} \mathrm{Vs} . \mathrm{N}_{2}$.

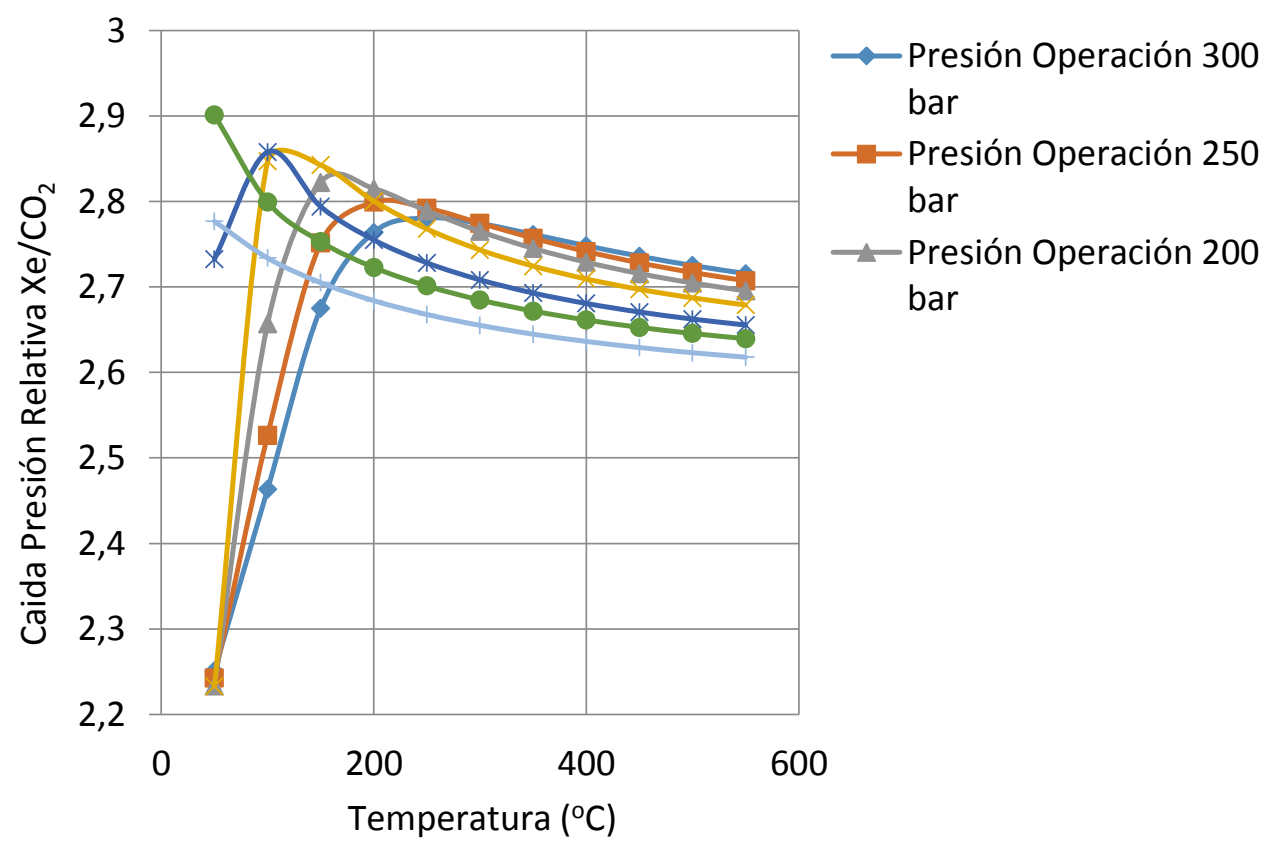

Figura 11.33. Comparación de la caída de presión relativa. $\mathrm{CO}_{2}$ Vs. Xe. 
11. Comparación entre el s- $\mathrm{CO}_{2}$ y otros fluidos de trabajo $\left(\mathrm{s}-\mathrm{C}_{2} \mathrm{H}_{6}, \mathrm{~s}-\mathrm{SF}_{6}, \mathrm{~s}-\mathrm{Xe}, \mathrm{s}-\mathrm{CH}_{4}, \mathrm{~s}-\mathrm{N}_{2}\right)$ en las plantas termosolares acopladas a ciclos supercríticos Brayton

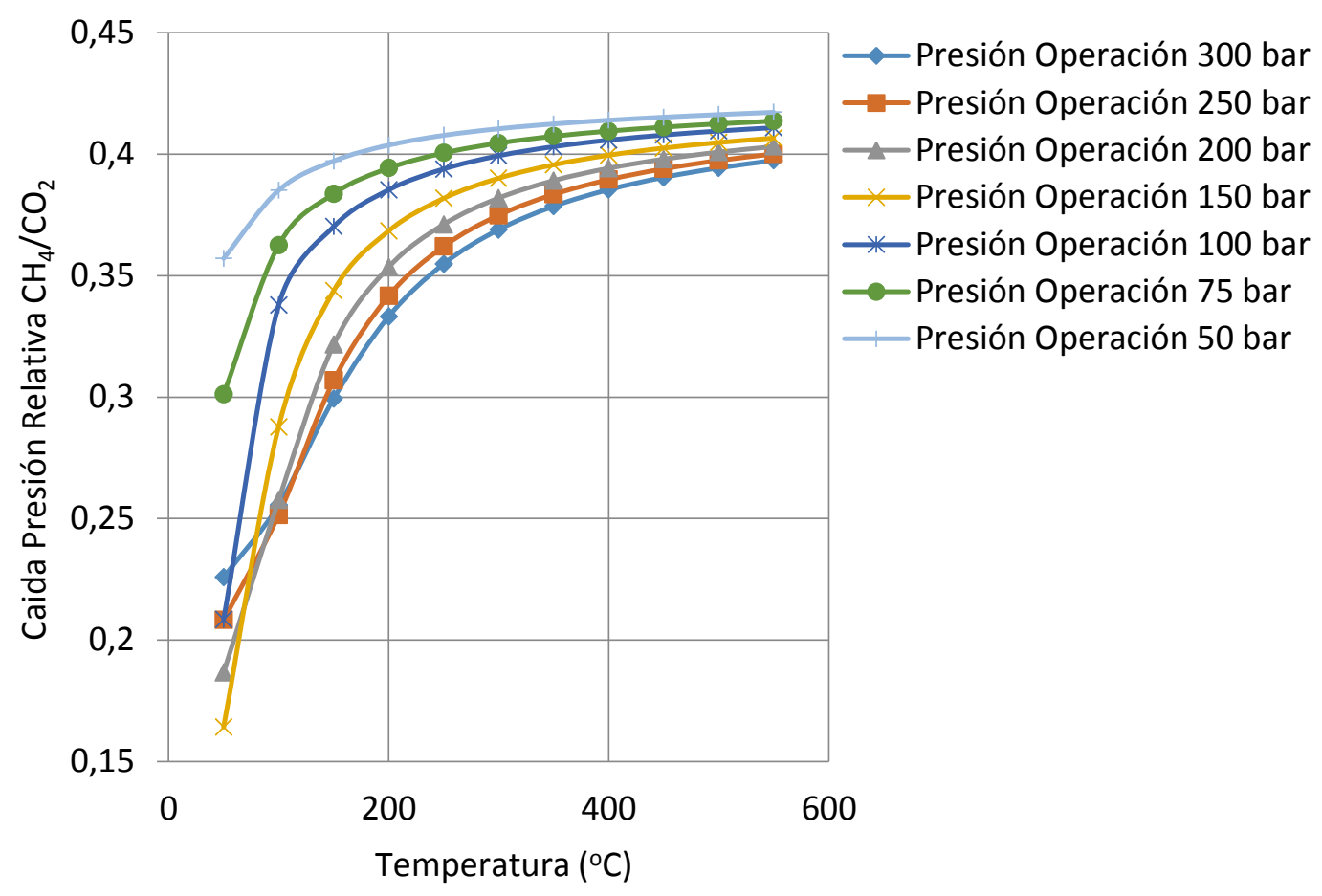

Figura 11.34. Comparación de la caída de presión relativa. $\mathrm{CO}_{2}$ Vs. $\mathrm{CH}_{4}$.

\subsection{Conclusiones}

Como primera tarea en este capítulo se ha caracterizado la eficiencia energética bruta del ciclo Brayton simple con recuperación de calor, representado en la figura 11.2, para diferentes TIT y tamaños térmicos (UA) de los recuperadores. Los resultados obtenidos están resumidos en las figuras 11.3 a 11.14. Los valores de eficiencia del ciclo representados en dichas gráficas han sido obtenidos de un estudio de optimización multivariable con los algoritmos matemáticos de cálculo SUBPLEX [7], UOBYQA [9-11] y NEWUOA [12-13]. En base a estos valores de eficiencia bruta se ha simulado en Thermoflow [14] la configuración de central termosolar propuesta en la figura 11.2, y se ha realizado un dimensionamiento del área de apertura efectiva de los campos solares con colectores PTC y LF. Los resultados obtenidos se resumen en las figuras 11.15 a 11.24 .

Se confirma que el $\mathrm{C}_{2} \mathrm{H}_{6}$ proporciona valores de eficiencia bruta del ciclo Brayton superiores al $\mathrm{CO}_{2}$, tal y como se había indicado en la referencia [4]. Sin embargo, la principal desventaja del $\mathrm{C}_{2} \mathrm{H}_{6}$ es su potencial descomposición a altas temperaturas, y su potencial riesgo de explosión (autoignición) por infiltraciones de aire en el ciclo [5-6].

El $\mathrm{SF}_{6}$ no presenta un alto grado de corrosión de los materiales a las temperaturas de operación del ciclo, pero su impacto ambiental negativo hace que tenga que ponerse especial interés en el desarrollo de sistemas para evitar posible fugas a la atmósfera. Este fluido presenta un mayor recorrido de optimización de la eficiencia en comparación con el $\mathrm{CO}_{2}$ para un aumento del tamaño térmico de los recuperadores de UA=20000 kW/K. 
11. Comparación entre el s- $\mathrm{CO}_{2}$ y otros fluidos de trabajo $\left(\mathrm{s}-\mathrm{C}_{2} \mathrm{H}_{6}, \mathrm{~s}-\mathrm{SF}_{6}, \mathrm{~s}-\mathrm{Xe}, \mathrm{s}-\mathrm{CH}_{4}, \mathrm{~s}-\mathrm{N}_{2}\right)$ en las plantas termosolares acopladas a ciclos supercríticos Brayton

El Xe es un fluido inerte, no provoca la corrosión de los metales de los equipos del ciclo de potencia Brayton. Presenta un alto potencial de ahorro en el coste del ciclo. El desarrollo de tubomáquinas para este fluido tendría unas dimensiones mayores que las del $\mathrm{CO}_{2}$. Habría que realizar un estudio de inversión para confirmar que el incremento de coste compensa el ahorro en materiales aleados, y las dimensiones de los intercambiadores de calor.

El $\mathrm{N}_{2}$ presenta un potencial de corrosión del acero. Este fluido ha sido experimentado en la industria nuclear en el CEA (Centre Energie Atomique) en Francia, y no se ha garantizado experimentalmente que sea una alternativa mejor que el $\mathrm{CO}_{2}$.

Finalmente, se ha estudiado el $\mathrm{CH}_{4}$ como un fluido que está siendo protagonista de la reducción del consumo de petróleo al ser el mayor componente del gas natural. Por tanto, es un fluido que se utiliza como combustible fósil en las centrales de generación de ciclo combinado y como alternativa al resto de subproductos del petróleo. En este estudio se ha caracterizado como fluido de trabajo en los ciclos Brayton y presenta menores valores de eficiencia bruta que el $\mathrm{CO}_{2}$.

\subsection{Referencias}

[1] Supercritical CO2 power cycles symposium. http://www.swri.org/4org/d18/sco2/

[2] A.Rovira, J.Muñoz-Antón, M.J.Montes, J.M.Martínez-Val. Optimization of Brayton cycles for low-to-moderate grade thermal energy sources. Elsevier Energy 55 (2013) 403-416.

[3] A.Rovira, C.Rubbia, M.Valdés, J.M. Martínez-Val. Thermodynamic cycles optimized for medium enthalpy units of concentrating solar power. Elsevier Energy 67 (2014) 176-185.

[4] L.Coco Enríquez, J.Muñoz-Antón, J.M.Martínez-Val Peñalosa. s-Ethane Brayton power conversion systems for Concentrated SolarPower plants. ASME Journal of Solar Energy Engineering, Accepted Manuscript.

[5] J.A. Perez, M. Driscoll, P. Hejzlar. Evaluation of Ethane as a Power Conversion System Working Fluid for Fast Reactors. Massachusetts Institute of Technology MIT, June 2008.

[6] M.C. Lin and M.H Black. The Thermal Decomposition of Ethane. Dept. of Chemistry Thesis, University of Ottawa, 1996.

[7] T. Rowan, Functional Stability Analysis of Numerical Algorithms. Ph.D. thesis, Department of Computer Sciences, University of Texas at Austin, 1990.

[8] John Dyreby. The University of Wisconsin, Madison. Thesis: Modeling the Supercritical CO2 Brayton Cycle with Recompression..

[9] Powell, M. J. D. (December 2000). UOBYQA: unconstrained optimization by quadratic approximation (Report). Department of Applied Mathematics and Theoretical Physics, Cambridge University. DAMTP 2000/NA14. Retrieved 2015-04-06.

[10] Powell, M. J. D. (2002). UOBYQA: Unconstrained Optimization By Quadratic Approximation. Mathematical Programming, Series B (Springer) 92: 555-582.

[11] Source code of UOBYQA software. Retrieved 2015-04-06.

[12] Source code of NEWUOA software. Retrieved 2014-01-14.

[13] Powell, M. J. D. (November 2004). The NEWUOA software for unconstrained optimization without derivatives (PDF) (Report). Department of Applied Mathematics and Theoretical Physics, Cambridge University. DAMTP 2004/NA05. Retrieved 2014-01-14.

[14] Thermoflow Inc. Sofware, http://www.thermoflow.com

[15] Sulzer Patent Verfahren zur Erzeugung von Arbeit aus Warme, Swiss Patent 269 599, (1948).

[16] Angelino G., Perspectives for the Liquid Phase Compression Gas Turbine, Journal of Engineering for Power, Trans. ASME, Vol. 89, No. 2, pp. 229-237, April, (1967). 
11. Comparación entre el s- $\mathrm{CO}_{2}$ y otros fluidos de trabajo $\left(\mathrm{s}-\mathrm{C}_{2} \mathrm{H}_{6}, \mathrm{~s}-\mathrm{SF}_{6}, \mathrm{~s}-\mathrm{Xe}, \mathrm{s}-\mathrm{CH}_{4}, \mathrm{~s}-\mathrm{N}_{2}\right)$ en las plantas termosolares acopladas a ciclos supercríticos Brayton

[17] Angelino G., Carbon Dioxide Condensation Cycles for Power Production, ASME Paper No. 68-GT-23, (1968).

[18] Angelino G., Real Gas Effects in Carbon Dioxide Cycles, ASME Paper No. 69-GT- 103, (1969).

[19] Feher E. G., Supercritical Thermodynamic Cycles for External and Internal Combustion Engines, Astropower, Inc. Engineering Report, May, (1962).

[20] Feher E. G., The Supercritical Thermodynamic Power Cycle, Douglas Paper No. 4348, presented to the IECEC, Miami Beach, Florida, August 13-17, (1967).

[21] V.Dostal, M.J. Driscoll, P.Hejzlar. A Supercritical Carbon Dioxide Cycle for Next Generation Nuclear Reactors. Advanced Nuclear Power Technology Program. MIT-ANP-TR-100, March 10, 2004. http://web.mit.edu/22.33/www/dostal.pdf

[22] Jim Pasch. Pressure-Enthalpy Diagram for Recompression Closed Brayton Cycle using s$\mathrm{CO}_{2}$. Sandia National Laboratories. SAND2016-4779 TR. http://energy.sandia.gov

[23] Sandeep R Pidaparti, Patrick J. Hruska, Anton Moisseytsev, James J. Sienicki, Devesh Ranjan. Technical and Economic feasibility of Dry Air Cooling for the Supercritical $\mathrm{CO}_{2}$ Brayton cycle using existing technology. The $5^{\text {th }}$ International Symposium- Supercritical $\mathrm{CO}_{2}$ Power Cycles, March 28-31, 2016, San Antonio, Texas, USA.

[24] Gavic D. Investigation Of Water, Air, and Hybrid Cooling For Supercritical Carbon Dioxide Brayton Cycles. University of Wisconsin-Madison.

[25] Timothy J. Held, Jason Miller, David J. Buckmaster. A comparative Study of Heat Rejection Systems for $\mathrm{sCO}_{2}$ Power cycles. The $5^{\text {th }}$ International Symposium- Supercritical $\mathrm{CO}_{2}$ Power Cycles, March 28-31, 2016, San Antonio, Texas, USA.

[26] E.W. Lemmon, M.L. Huber, M.O. McLinden. REFPROP database. Reference Fluid Thermodynamic and Transport Properties. NIST Standard Reference Database 23, Version 9.0.

Copyright 2010 by the U.S. Secretary of Commerce on behalf of the United States of America.

[27] Span, R. and Wagner, W.,A New Equation of State for Carbon Dioxide Covering the Fluid Region from the Triple-Point Temperature to $1100 \mathrm{~K}$ at Pressures up to $800 \mathrm{MPa}$. J. Phys. Chem. Ref. Data, 25(6):1509-1596, 1996.

[28] Buecker, D. and Wagner, W. A Reference Equation of State for the Thermodynamic Properties of Ethane for Temperatures from the Melting Line to $675 \mathrm{~K}$ and Pressures up to 900 MPa. J. Phys. Chem. Ref. Data, 35(1):205-266, 2006.

[29] Guder, C. and Wagner, W. A Reference Equation of State for the Thermodynamic Properties of Sulfur Hexafluoride (SF6) for Temperatures from the Melting Line to $625 \mathrm{~K}$ andPressures up to $150 \mathrm{MPa}$. J. Phys. Chem. Ref. Data, 38(1):33-94, 2009.

[30] Setzmann, U. and Wagner, W. A New Equation of State and Tables of Thermodynamic Properties for Methane Covering the Range from the Melting Line to $625 \mathrm{~K}$ at Pressures up to 1000 MPa. J. Phys. Chem. Ref. Data, 20(6):1061-1151, 1991.

[31] Span, R., Lemmon, E.W., Jacobsen, R.T, Wagner, W., and Yokozeki, A. A Reference Equation of State for the Thermodynamic Properties of Nitrogen for Temperatures from 63.151 to $1000 \mathrm{~K}$ and Pressures to $2200 \mathrm{MPa}$. J. Phys. Chem. Ref. Data, 29(6):1361-1433, 2000. [32] Xenon. Lemmon, E.W. and Span, R., Short Fundamental Equations of State for 20 Industrial Fluids. J. Chem. Eng. Data, 51:785-850, 2006.

[33] F.Burkholder, C.Kutscher. Heat Loss Testing of Schott's 2008 PTR70 Parabolic Trough Receiver. Report NREL/TP-550-45633, May 2009.

[34] Novatec Solar. SAM Linear Fresnel solar boiler model, SAM Webinar. NREL SAM Conference 2013.

[35] Leonid Moroz, Maksym Burlaka, Oleksii Rudenko. SoftInWay Inc. Study of a Supercritical $\mathrm{CO} 2$ Power Cycle Application in a Cogeneration Power Plant. Supercritical $\mathrm{CO}_{2}$ Power Cycle Symposium, September 9-10, 2014, Pittsburg, Pennsylvania, USA.

[36] Leonid Moroz, Boris Frolov, Maksym Burlaka. A New Concept to Designing a Combined Cycle Cogeneration Power Plant. SoftInWay Inc.

http://www.softinway.com 
11. Comparación entre el s- $\mathrm{CO}_{2}$ y otros fluidos de trabajo $\left(\mathrm{s}-\mathrm{C}_{2} \mathrm{H}_{6}, \mathrm{~s}-\mathrm{SF}_{6}, \mathrm{~s}-\mathrm{Xe}, \mathrm{s}-\mathrm{CH}_{4}, \mathrm{~s}-\mathrm{N}_{2}\right)$ en las plantas termosolares acopladas a ciclos supercríticos Brayton

[37] Dyreby J. Integration of a $10 \mathrm{MWe}$ Supercritical Carbon Dioxide Power Cycle into an Existing Cogeneration Power Plant. University of Wisconsin-Madision. $5^{\text {th }}$ International $\mathrm{s}-\mathrm{CO}_{2}$ Power Cycles Symposium, March 29-31, 2016, San Antonio, Texas, USA.

[38] Dittus Boelter. F.W. Dittus, L.M.K. Boelter. Heat transfer in automobile radiator of the tubular type. University of California at Berkley Publ. Eng., 2 (1930), pp. 443-461.

[39] Brown, Glenn. The Darcy-Weisbach Equation. Oklahoma State University-Stillwater.

[40] Idelchik, I.E., 1986, Handbook of Hydraulic Resistance, Second Edition, Hemisphere Publishing Corp.

[41] Petukov. Incropera, Frank P.; DeWitt, David P. (2007). Fundamentals of Heat and Mass Transfer (6th ed.). Hoboken: Wiley. pp. 490, 515.ISBN 978-0-471-45728-2. 


\title{
Capítulo 12 \\ Programa Informático para diseño y optimización de los ciclos Brayton en plantas termosolares con colectores lineales y diferentes fluidos caloportadores: Supercritical_CSP (SCSP)
}

\author{
Resumen \\ Se ha desarrollado software ad hoc para el diseño de las centrales termosolares con colectores \\ lineales acopladas a ciclos supercríticos Brayton. El programa informático se ha denominado: \\ Supercritical_CSP (SCSP).
}

Se ha partido del código en Fortran desarrollado por Dyreby [1]. Originalmente este código fue concebido para la simulación del ciclo de potencia s- $\mathrm{CO}_{2}$ Brayton $\mathrm{RC}$ sin recalentamiento en turbinas. Como principales aportaciones del SCSP se han añadido diversas configuraciones Brayton, todas ellas con opción de incluir una etapa de recalentamiento en las etapas de expansión en turbina. Así mismo, se han acoplado las configuraciones de ciclos Brayton citadas con campos solares con colectores lineales y diferentes fluidos caloportadores.

El código del SCSP se ha desarrollado en Fortran para cada una de las configuraciones citadas y se ha creado una interfaz en C\# para llamada de las librerías DLL, y representación del frontend, interfaz gráfico Graphical User Interface (GUI). Este desarrollo en C\# se ha llevado a cabo con Integrated Development Environment (IDE) de Microsoft Visual Studio 2010, creando una aplicación con ventanas tipo Windows.

El código fuente de Dyreby [1] fue concebido para el diseño de diferentes escenarios de operación: cálculo en el punto de diseño, cálculo de los parámetros de operación del ciclo Brayton para optimizar la eficiencia energética del ciclo mediante la integración del algoritmo matemático SUBPLEX [2], y finalmente cálculo en condiciones de carga parcial. Se ha incluido la utilización de algoritmos de cálculo alternativos para la optimización del cálculo del punto de diseño: UOBYQA [3] y NEWUOA [4]. Hay que destacar que los dos algoritmos citados proporcionan idénticos resultados que el SUBPLEX [2] para las configuraciones sin etapa de recalentamiento en turbina con que han sido comparados.

Otras características de SCSP son:

- Diseño detallado de equipos: recuperadores, intercambiador de acoplamiento con el foco frío, turbinas y compresores, campos solares con colectores lineales.

- Cálculo de consumos de equipos auxiliares de la planta: eficiencia del generador, consumo de las bombas auxiliares de recirculación de HTF en el campo solar, estimación del consumo ventiladores del foco frío, etc.

- Estimación del coste de los equipos del campo solar y del ciclo de potencia.

- Validación de resultados con Thermoflow [5]. 
12. Programa Informático para diseño y optimización de ciclos Brayton en plantas termosolares con diferentes fluidos caloportadores: Supercritical_CSP (SCSP)

\subsection{Introducción}

El diseño de la nueva generación de centrales termosolares acopladas a ciclos de potencia supercrítios Brayton, crea la necesidad de desarrollar una herramienta informática para la simulación y diseño de los equipos que integran estas nuevas instalaciones. Por ello se ha desarrollado ad hoc la herramienta Supercritical_CSP (SCSP). Si se realiza una recopilación de los programas informáticos comerciales aplicables a la solución del citado problema (Thermoflow [5], SAM [6], TRNSYS [7], IPSEpro [8], ASPEN/HSYS [9], Modelica [10], ECOSIM/PRO y PROOSIS [11], SolarTrace [12], SolarPILOT [13], TONATIUH [14], DELSOL3 [15]), se confirma que no existe en el mercado un programa que integre la optimización termodinámica de los ciclos supercríticos Brayton acoplados a los campos solares con colectores lineales.

System Advisor Model (SAM) [6] es una herramienta desarrollada por NREL, que en su versión actual (2016.3.14 para Windows) no dispone aún de la opción de simulación de los ciclos s- $\mathrm{CO}_{2}$ Brayton acoplados a campos solares. En la versión anterior (2015.6.30) fue incluido el acoplamiento de las plantas termosolares con torre central y campo de heliostatos a los ciclos de potencia Brayton. Sin embargo, posteriormente esta opción fue eliminada por el desarrollo detallado del sistema de control estas instalaciones termosolares. Según lo indicado por Turchi $[16,17]$ la tendencia principal de investigación en las plantas termosolares debería estar alineada con el acoplamiento de estas instalaciones con los ciclos s- $\mathrm{CO}_{2}$ Brayton. En [17] describe los objetivos en los próximos tres años (2016 a 2018) del NREL en el desarrollo del proyecto SUNLaMP Award NREL-1651. La tarea 2 de este proyecto tiene por objetivos:

- incrementar la velocidad de simulación y exactitud para hacer compatible los modelos de los ciclos $\mathrm{s}-\mathrm{CO}_{2}$ con el entorno de simulación SAM

- comparar la producción anual de las configuraciones RC y PCRC acopladas a CSP

- validar las estimaciones de las plantas solares acopladas a ciclos s-CO2 con los datos experimentales del programa STEP [19]

- y hacer recomendaciones a los diseñadores y fabricantes a escala industrial, para selección y operación con los ciclos $\mathrm{s}-\mathrm{CO}_{2}$ Brayton para aplicaciones de CSP. El desarrollo de la librería SDK y la exportación de datos a Excel, permiten la integración futura del SAM con la herramienta SCSP.

Thermoflow [5]: este programa informático en su última versión 26 (Septiembre 2016), permite la simulación de ciclos Brayton mediante la integración de la base de datos REFPROP [20] para el cálculo de las propiedades de los fluidos en su estado supercrítico. Así mismo, es posible el diseño y acoplamiento de los ciclos Brayton con campos solares con colectores lineales [21-23] y configuración de torre central con heliostatos. Sin embargo, este programa no integra algoritmos de optimización. Integración con Excel permite el intercambio de datos con SCSP.

TRaNsient SYstems Simulation Program (TRNSYS) [7]: desarrollado por la Universidad de Wisconsin. TRNSYS dispone de numerosas librerías de equipos, pero en la aplicación requerida es necesaria la programación de los equipos de los ciclos $\mathrm{s}-\mathrm{CO}_{2}$, como realizó Seidel [24]. 
12. Programa Informático para diseño y optimización de ciclos Brayton en plantas termosolares con diferentes fluidos caloportadores: Supercritical_CSP (SCSP)

IPSEpro [8]: es un programa muy similar Thermoflow. Dispone de numerosas librerías ya programadas (APP_Lib: Advanced Power Plant Library, CSP_Lib: Concentrating Solar Power Library, etc) para simulación de equipos y procesos [25, 26]. Destaca el estudio de Turchi [27] para el desarrollo y experimentación de una turbina de $10 \mathrm{MW}$ en un ciclo de potencia s- $\mathrm{CO}_{2}$ Brayton. Al igual que Thermoflow no dispone de algoritmos matémáticos integrados para cálculos de optimización.

ASPEN/HSYS [9]: tradicionalmente desarrollada para el sector petroquímico. Recientemente el National Energy Technology Laboratory (NETL) [28] y otros autores [29] han publicado trabajos de simulación de ciclos $\mathrm{s}-\mathrm{CO}_{2}$ con utilizando este software. Su principal inconveniente es que no dispone de librerías para simulación de equipos del campo solar. Como principal ventaja se destaca que integra numerosos programas para simulación detallada y diseño de los equipos que integran el ciclo Brayton, principalmente intercambiadores de calor y de ACHE. Así mismo, con el HSYS es posible realizar el diseño de separadores para analizar mezclas de sustancias como fluido de trabajo en el ciclo Brayton y su integración con procesos químicos.

MODELICA [10]: dispone de su propio lenguaje de programación para simulación de sistemas. Es más complejo que IPSEpro y Thermoflow, pero es más flexible y da acceso al código fuente los equipos. Al igual que el IPSEpro y el Thermoflow no integra algoritmos para la optimización de los parámetros de proceso en los ciclos Brayton. Existen trabajos [30] sobre modelización dinámica de los colectores PTC, simulación de la tecnología DSG en colectores lineales [31], y la la integración de ciclos Brayton en plantas termosolares [32].

ECOSIM/PRO y PROOSIS [11]: similar a Modélica, permite una gran flexibilidad de programación de componentes y equipos y simulaciones de transitorios y de punto de diseño. Integra diferentes algoritmos de resolución de sistemas de ecuaciones no lineales y de sistemas de ecuaciones de derivadas parciales. Para la simulación de plantas termosolares convencionales acopladas a ciclos Rankine dispone de una librería específica: Solar Thermal Plant Library. La librería: Heat Transfer Fluid System también puede ser utilizada para simulación de plantas termosolares, aprovechadas por algunos autores [33-35]

Herramientas para diseño óptico de los colectores solares como: SolarTrace [12], SolarPILOT [13], TONATIUH [14], DELSOL3 [15] no incluyen la integración de los campos solares con los ciclos de potencia Brayton, únicamente diseñan los colectores solares de forma aislada. Como trabajo futuro se propone el acoplamiento de estos programas con SCSP. 
12. Programa Informático para diseño y optimización de ciclos Brayton en plantas termosolares con diferentes fluidos caloportadores: Supercritical_CSP (SCSP)

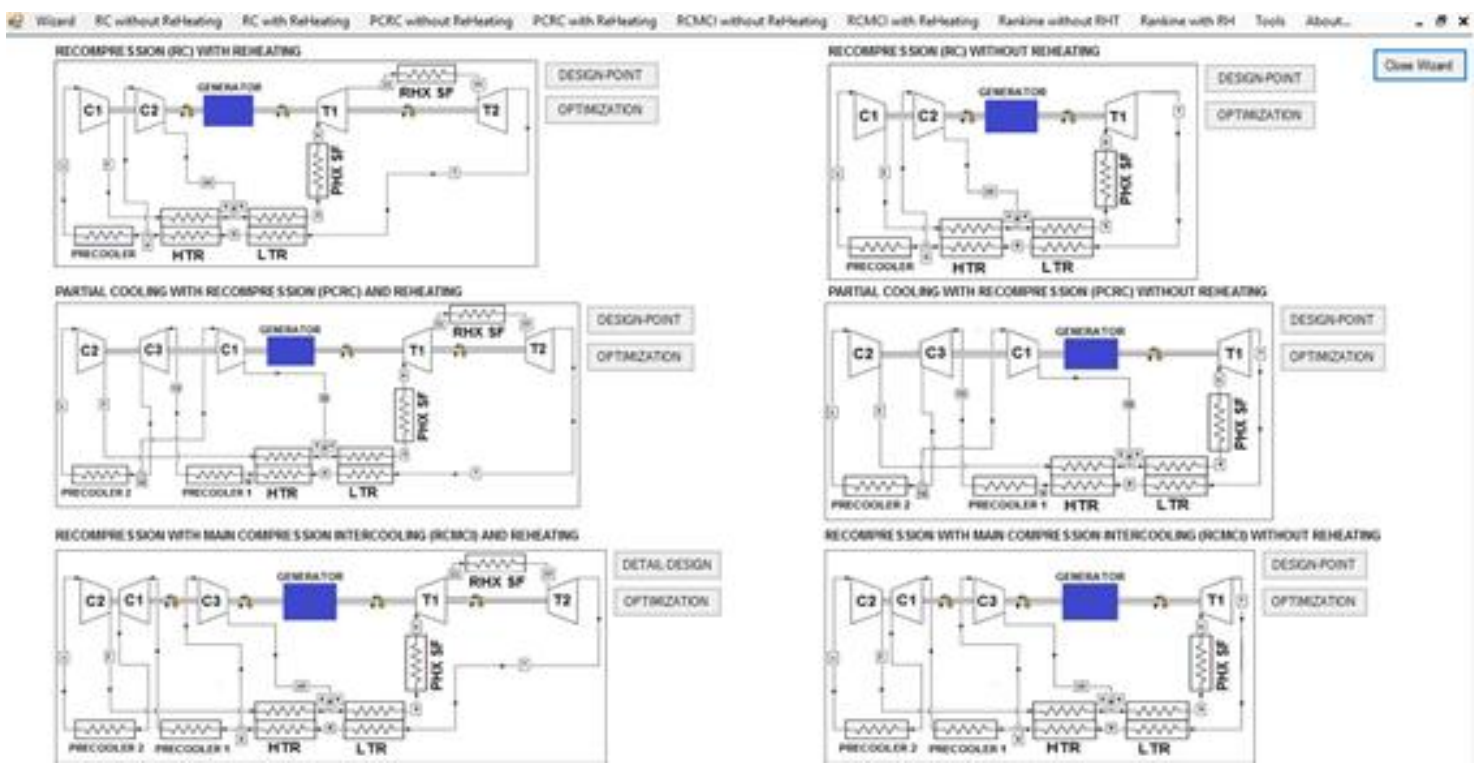

Figura 12.1. Pantalla de inicio de la herramienta SCSP.

En la figura 12.1 se muestra el cuadro de diálogo principal del programa SCSP. En esta pantalla de inicio es posible elegir la configuración de ciclo Brayton a analizar. La principal ventaja de este programa es que no hace falta programar código alguno para realizar la simulación de las plantas termosolares. Como principal inconveniente no se permite simular configuraciones diferentes a las programadas por el desarrollador. Los resultados de diseño de todos los equipos de la planta termosolar y su estimación de costes permite realizar una primera ingeniería básica de la instalación.

En futuras versiones se espera desarrollar las capacidades adicionales indicadas en el apartado de conclusiones. La figura 12.2 ilustra las opciones de análisis disponibles para cada configuración. En los siguientes apartados de este capítulo se detallan resultados obtenidos con cada una de las opciones citadas. Cabe destacar que el programa permite el ciclo Brayton con varios fluidos: $\mathrm{CO}_{2}, \mathrm{C}_{2} \mathrm{H}_{6}, \mathrm{Xe}, \mathrm{SF}_{6}, \mathrm{~N}_{2}$ y $\mathrm{CH}_{4}$. Los análisis termodinámicos citados han considerado las propiedades de los fluidos de trabajo en estado supercrítico de acuerdo a la información contenida en la base de datos REFPROP [20].

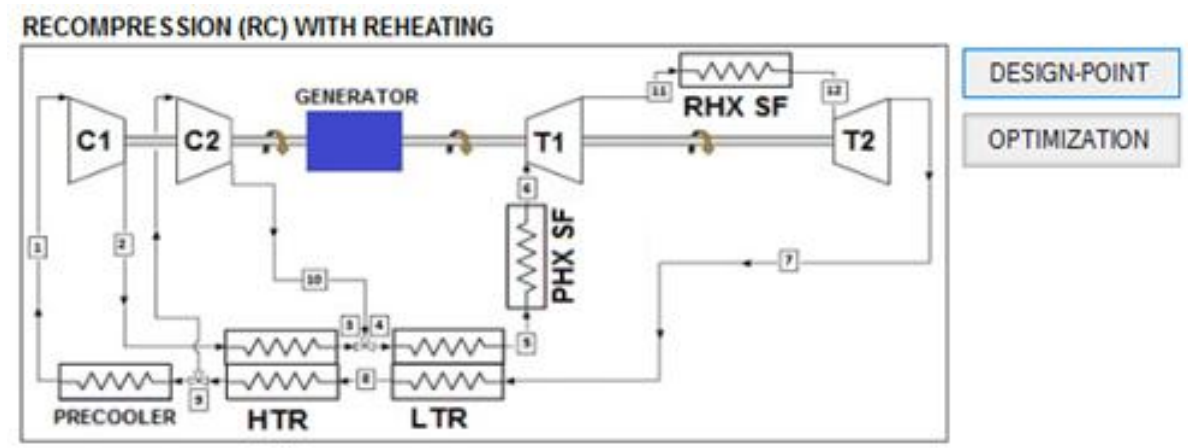

Figura 12.2. Ejemplo del GUI de la configuración de ciclo Brayton RC con recalentamiento con las opciones de cálculo en cada configuración: punto de diseño, optimización del diseño. 
12. Programa Informático para diseño y optimización de ciclos Brayton en plantas termosolares con diferentes fluidos caloportadores: Supercritical_CSP (SCSP)

\subsection{Configuraciones de las plantas CSP acoplada a ciclo Brayton}

En las figuras 12.3 y 12.4 representan dos ejemplos de la disposición de los equipos en las configuraciones de ciclos Brayton analizadas. Como puede apreciarse cada uno de los equipos incluye botón para el diseño de cada uno de los equipos. Las tuberías que unen los diferentes equipos quedan simuladas mediantes líneas de corrientes numeradas. La información de los parámetros termodinámicos de cada una de las corrientes: caudal, presión, temperatura y entalpía, quedan representados con una etiqueta cuando se desplaza el puntero del ratón encima del número de la corriente.

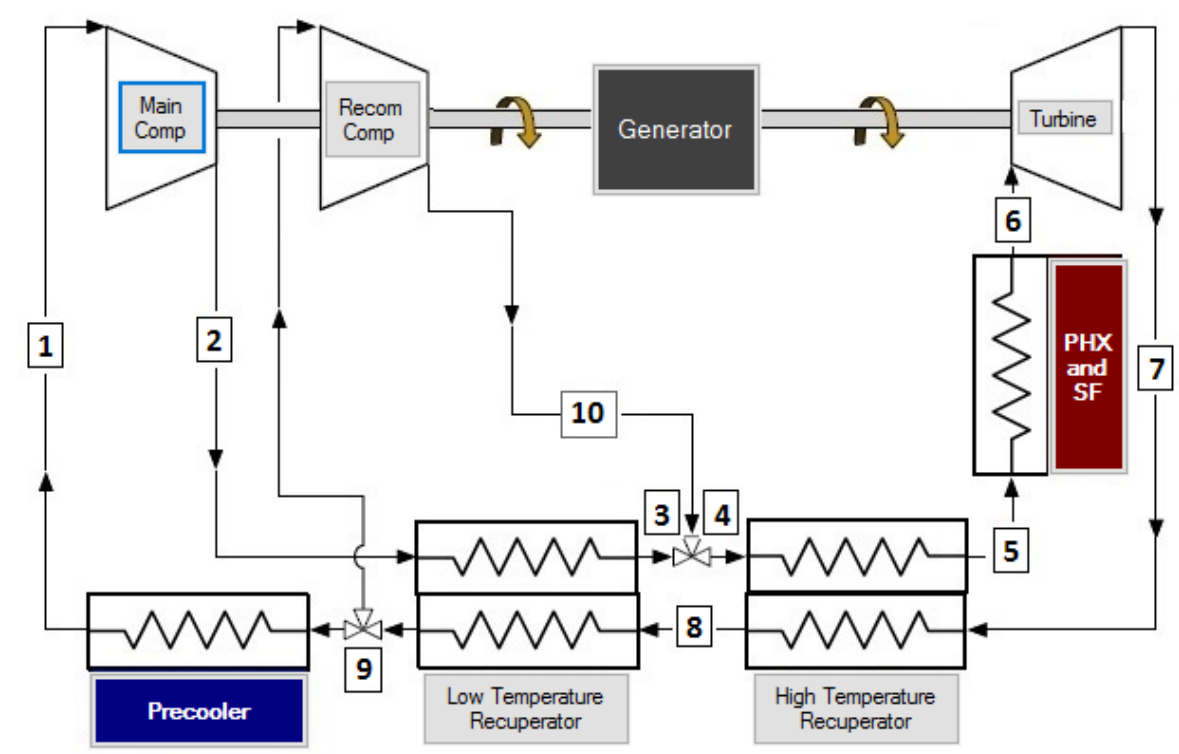

Figura 12.3. Ejemplo del GUI de la configuración de ciclo Brayton SB ó RC sin recalentamiento.

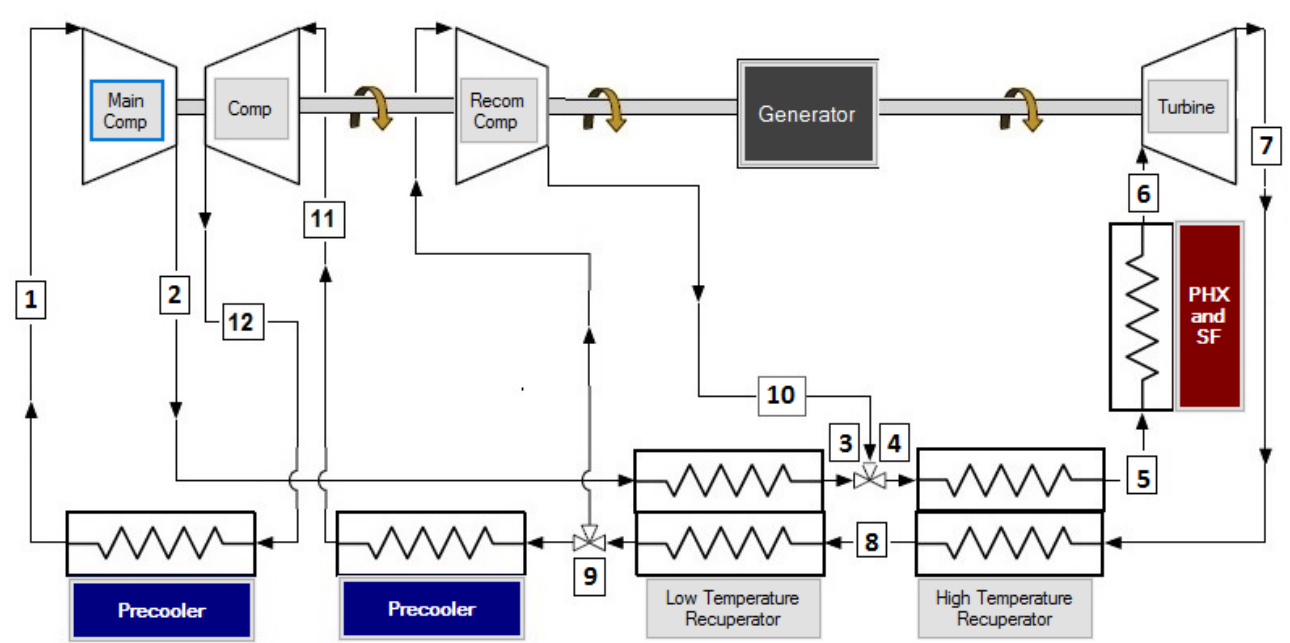

Figura 12.4. Ejemplo del GUI de la configuración de ciclo Brayton $\mathrm{RCMCl}$ sin recalentamiento.

\subsection{Cálculo del punto de diseño (Design-Point)}

Para este tipo de cálculo, el primer dato que debe introducir/seleccionar el usuario es el fluido de trabajo del ciclo Brayton entre los indicados anteriormente. 
12. Programa Informático para diseño y optimización de ciclos Brayton en plantas termosolares con diferentes fluidos caloportadores: Supercritical_CSP (SCSP)

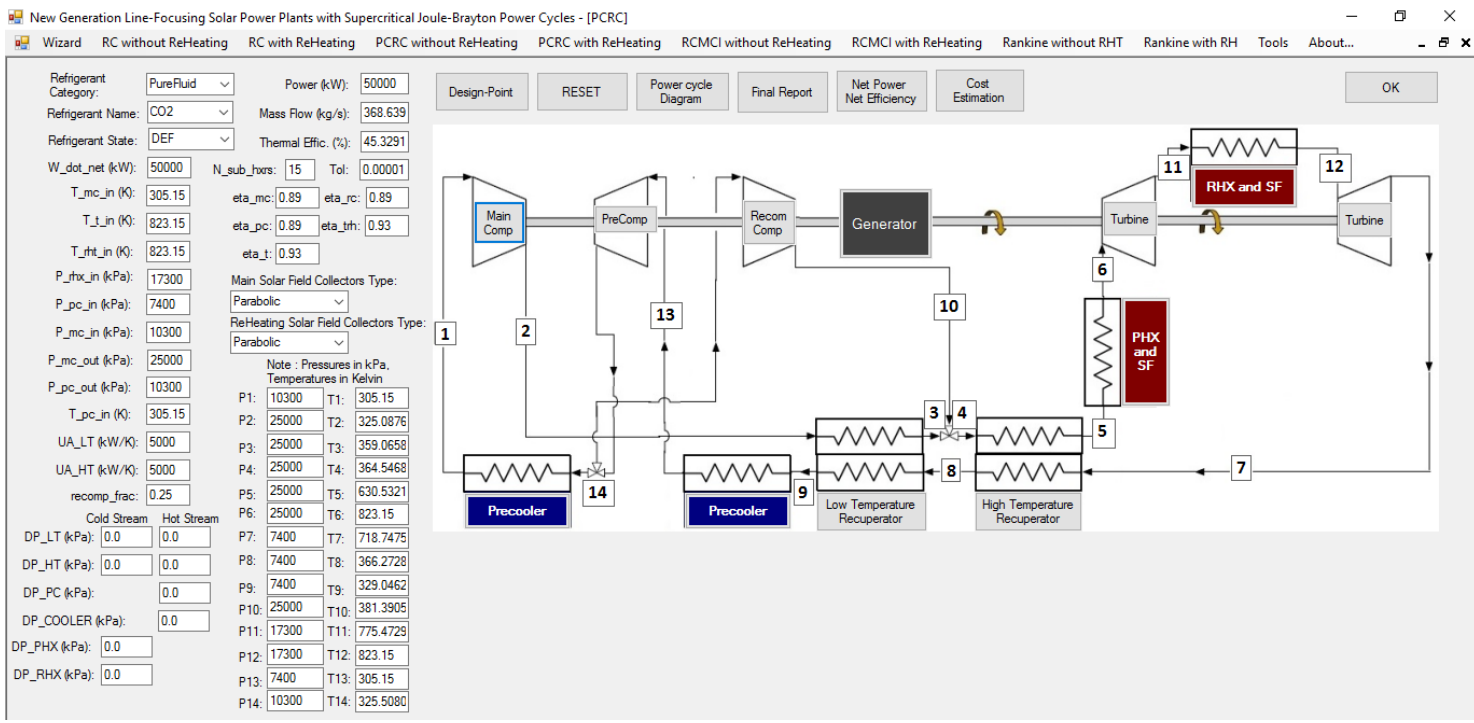

Figura 12.5. Cuadro de diálogo para cálculo del punto de diseño en la configuración PCRC con Recalentamiento.

En cada una de las configuraciones de ciclo Brayton estudiada es necesario introducir los datos de partida para poder realizar el cálculo en el punto de diseño, p.e. configuración PCRC con recalentamiento (figura 12.5). Algunos de los datos de partida introducidos por el usuario se ven en la figura 12.6 .

Main Solar Field
Collectors Type:
\begin{tabular}{|l|l|}
\hline Parabolic & $\checkmark$ \\
\hline Parabolic & \\
\hline $\begin{array}{l}\text { Fresnel } \\
\text { Dual-Loop }\end{array}$ \\
\hline
\end{tabular}
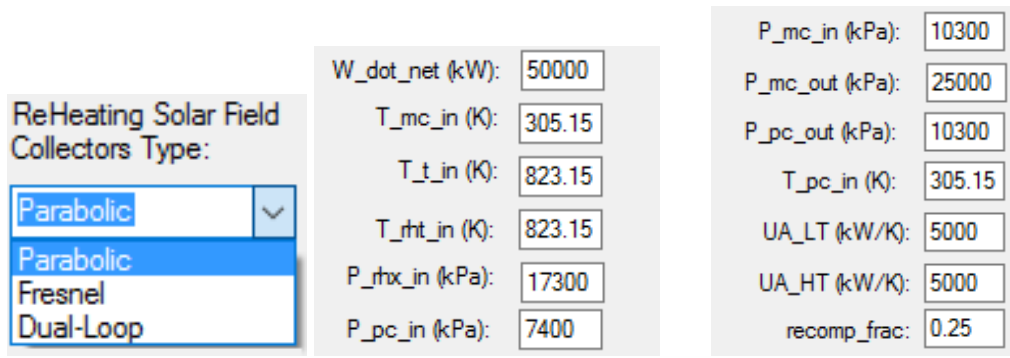

Figura 12.6. El usuario puede elegir entre diferentes tipologías de colectores lineales y DualLoop, para los campos solares considerados en la simulación, además de incluir otros datos de entrada (en este caso para cálculo de la configuración PCRC con recalentamiento)

\begin{tabular}{|c|c|c|c|c|c|c|c|}
\hline P1: & 10300 & T1: & 305.15 & P8: & 7400 & T8: & 366.2728 \\
\hline P2: & 25000 & T2: & 325.0876 & P9: & 7400 & TT & 329.0462 \\
\hline$P$ & 25000 & T3: & 359.0658 & P10: & 25000 & T10: & 381.3905 \\
\hline P4: & 25000 & T4: & 364.5468 & P11: & 17300 & T11: & 775.4729 \\
\hline$P$ & 25000 & T5: & 630.5321 & P12: & 17300 & T12: & 823.15 \\
\hline $\mathrm{P}$ & 25000 & T6: & 823.15 & & 7400 & T13: & 305.15 \\
\hline P7. & 7400 & T7: & 718.7475 & P14: & 10300 & T14: & 325.5080 \\
\hline
\end{tabular}

Figura 12.7. Ejemplo de resultados de presiones $(\mathrm{kPa})$ y temperaturas $(\mathrm{K})$ en cada una de las corrientes.

Entre los resultados obtenidos del cálculo en el punto de diseño, se destacan los valores de presión y temperatura en cada una de las corrientes, figura 12.7. El resto de resultados: representación de diagramas del ciclo, informes de cálculo de equipos, cálculo de parámetros de la planta, etc., están explicados en los siguientes apartados de este capítulo, figura 12.10. 
12. Programa Informático para diseño y optimización de ciclos Brayton en plantas termosolares con diferentes fluidos caloportadores: Supercritical_CSP (SCSP)

\subsection{Cálculo de las condiciones óptimas de operación}

La opción de optimización de los parámetros de operación del ciclo Brayton permite maximizar la eficiencia energética del ciclo para unas condiciones definidas por el usuario y una potencia generada fija. Como se ha explicado anteriormente, se han integrado tres algoritmos matemáticos de optimización multivariable: SUBPLEX [2], NEWUOA [3] y UOBYQA [4]. Cada uno de ellos puede ser ejecutado pulsando uno de los botones de la figura 12.8.

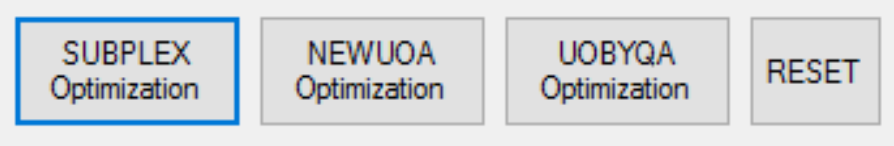

Figura 12.8. Botones para selección del algoritmo de optimización multivariable.

En este apartado se ha tomado como ejemplo la configuración PCRC con Recalentamiento, figura 12.9, para explicar tanto los datos de partida de la opción de optimización, como los resultados obtenidos en este proceso de cálculo.

Al igual que en la opción de cálculo en el punto de diseño, como primer paso se tiene que seleccionar el fluido de trabajo y la tipología de los colectores solares. Los parámetros a optimizar pueden ser considerados como fijos o variables (figura 12.9).

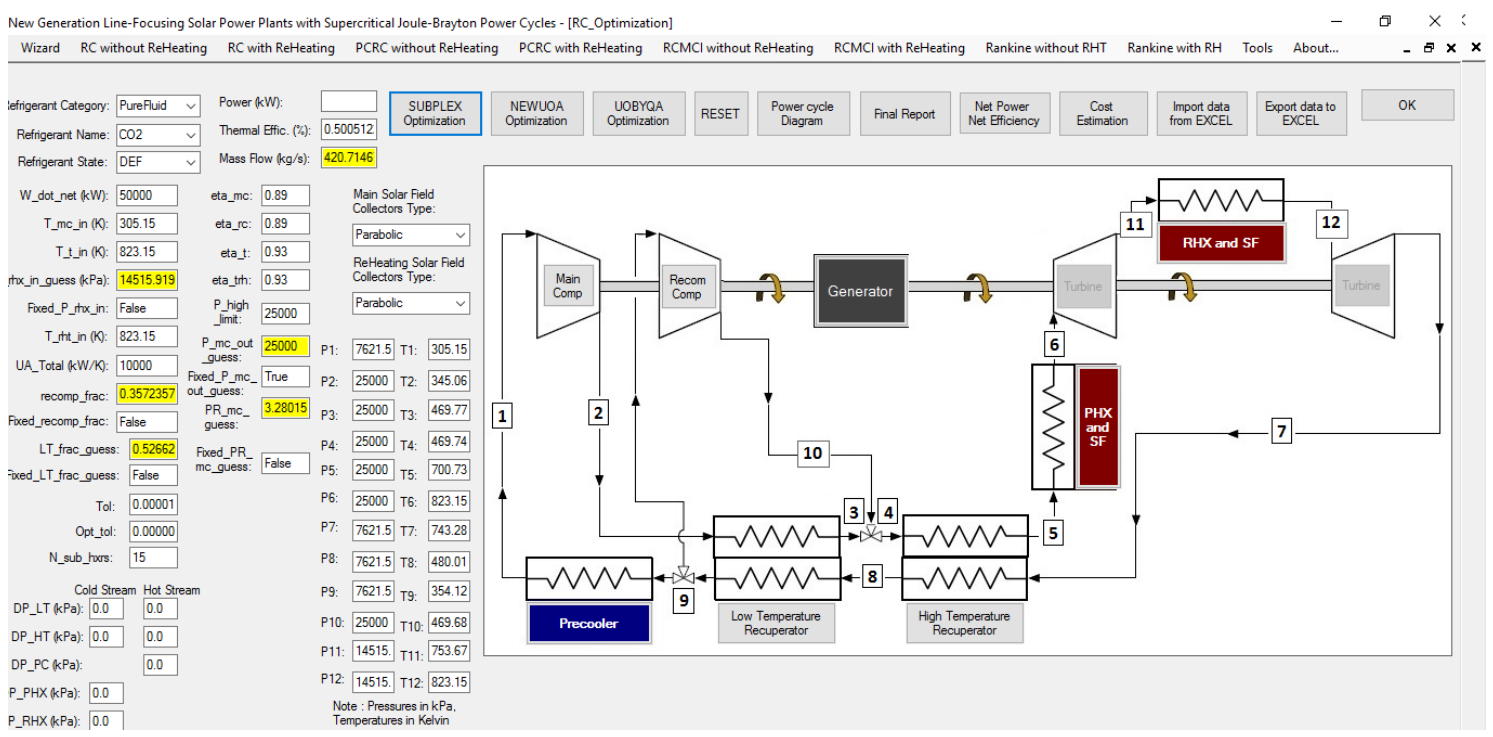

Figura 12.9. Cuadro de diálogo para optimización de las condiciones de operación en la configuración PCRC con Recalentamiento.

Se han definido cuatro botones para elegir los resultados del cálculo en el punto de diseño y de optimización multivariable, figura 12.10.

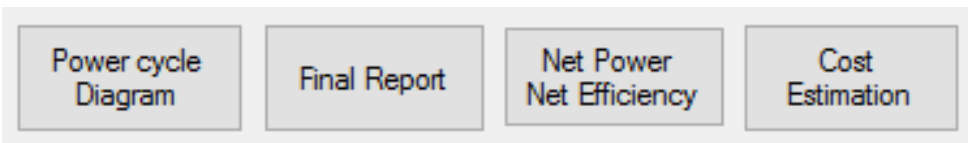

Figura 12.10. Botones para selección de los resultados del cálculo tanto en el punto de diseño como de optimización multivariable. 
12. Programa Informático para diseño y optimización de ciclos Brayton en plantas termosolares con diferentes fluidos caloportadores: Supercritical_CSP (SCSP)

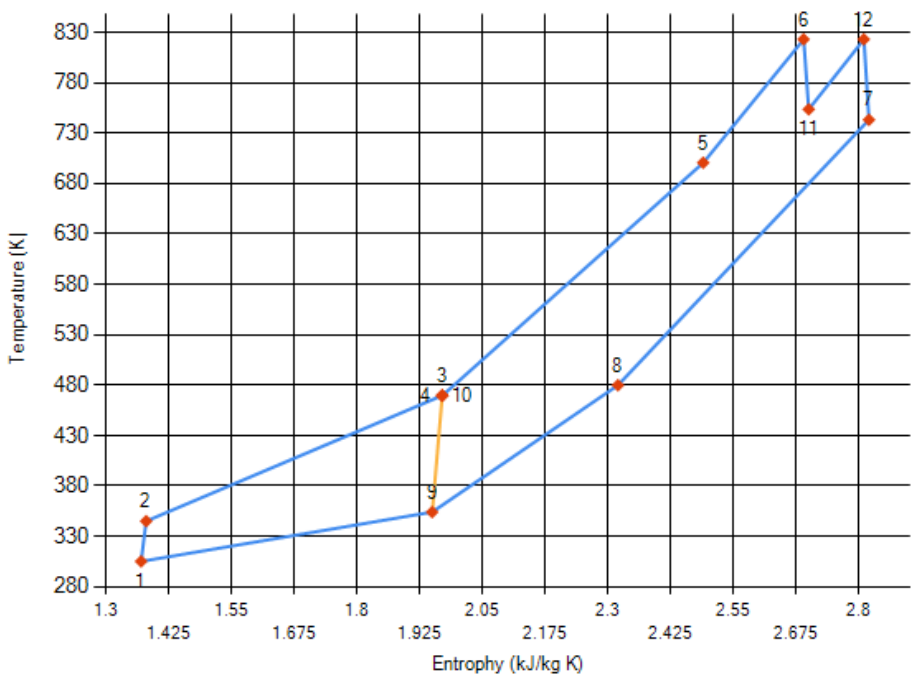

Figura 12.11. Diagrama Temperatura (K) Vs. Entropía (kJ/kg) de la configuración PCRC con recalentamiento.

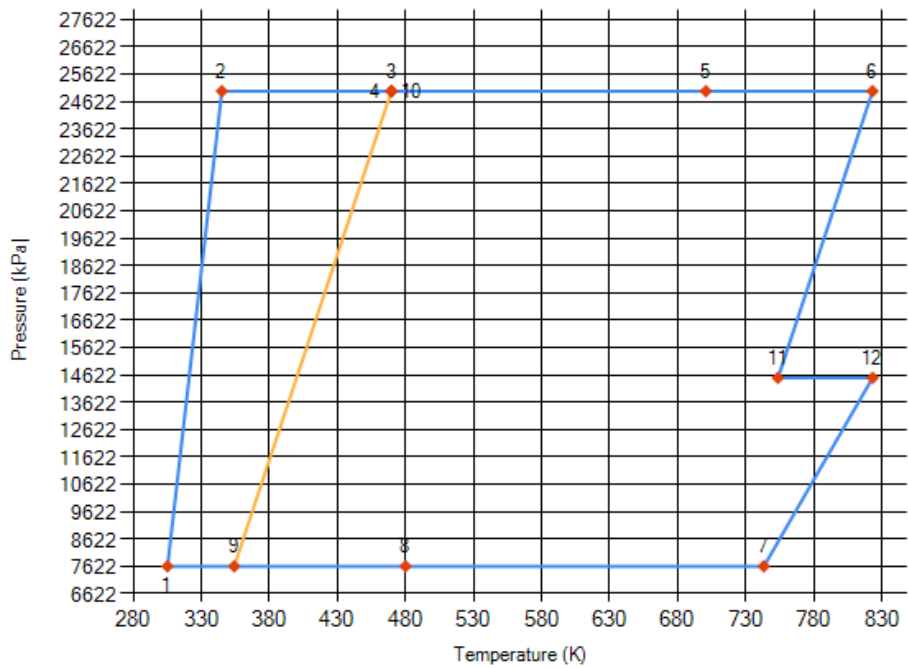

$$
\begin{aligned}
& \text { - Main_Flow } \\
& \text { - Recomp_Flow } \\
& \text { - Points_Values }
\end{aligned}
$$$$
\text { Vs. }
$$

Points_Values

Figura 12.12. Diagrama Presión ( $\mathrm{kPa}$ ) Vs. Temperatura (K) de la configuración PCRC con recalentamiento.

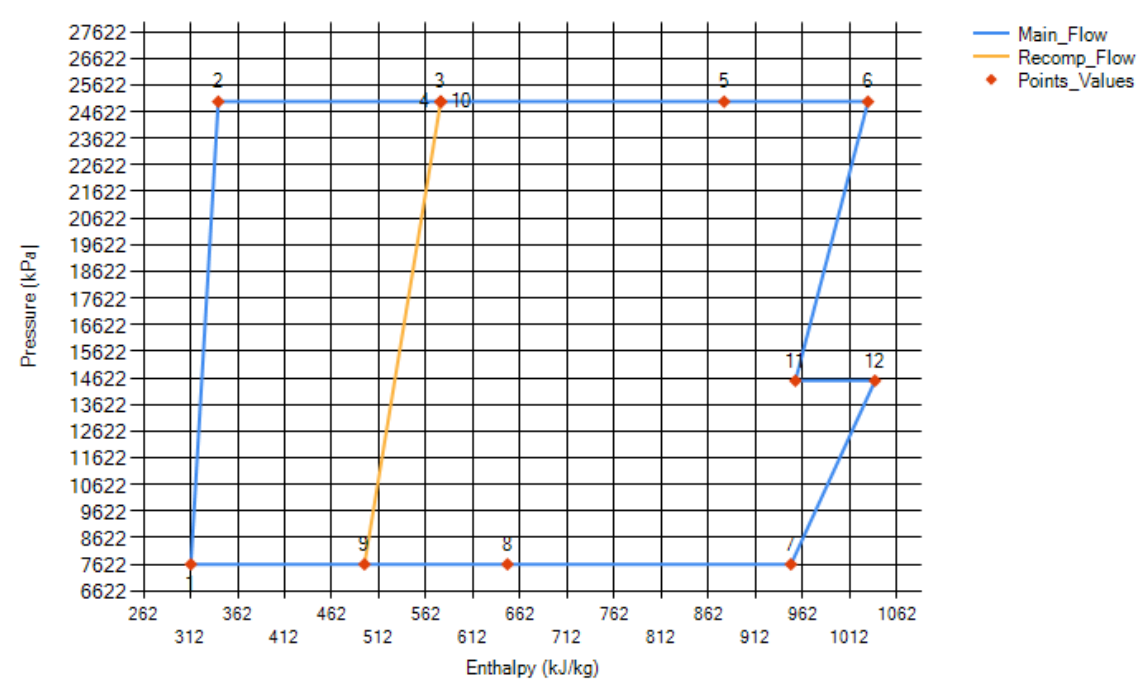

Figura 12.13. Diagrama P-s de la configuración PCRC con recalentamiento. 
12. Programa Informático para diseño y optimización de ciclos Brayton en plantas termosolares con diferentes fluidos caloportadores: Supercritical_CSP (SCSP)

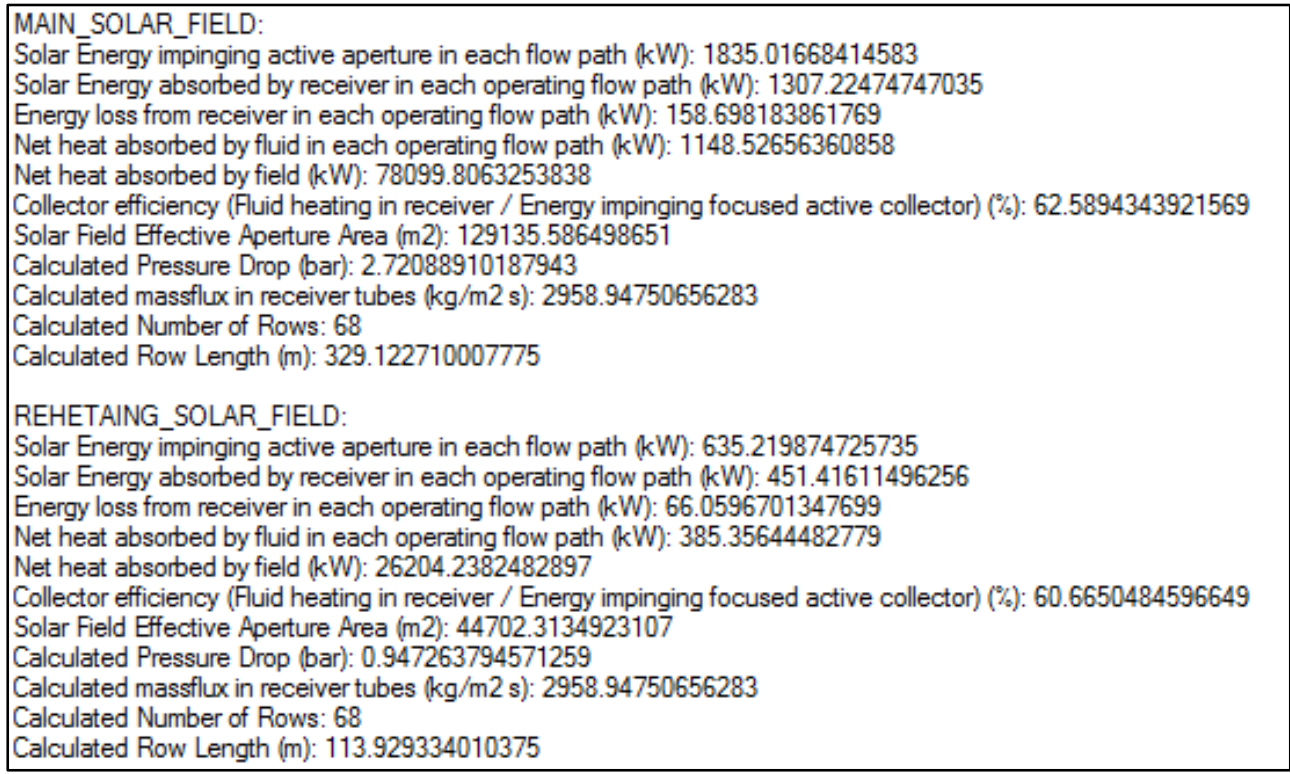

Figura 12.14. Resultados del cálculo de diseño de los campos solares principal y de recalentamiento.

LOW TEMPERATURE HEAT EXCHANGER:

Cold side inlet Pressure (bar): 25000

Cold side inlet Temperature (K): 381.301614279037

Hot side inlet Pressure (bar): 7400

Hot side inlet Temperature (K): 499.812097047058

Cold side outlet Pressure (bar): 25000

Hot side outlet Pressure (bar): 7400

Cold side mass flow $(\mathrm{kg} / \mathrm{s}): 335.514989537696$

Hot side mass flow $(\mathrm{kg} / \mathrm{s})$ : 447.353319383595

Heat Exchanged (kWth): 58750.488990068

Number of Sub-Heat Exchanger (Uds.): 15

Conductance (kW/K): 4999.97866830613

Number of Thermal Units: 9.60868798063722

Capacity Ratio: 0

Effectiveness: 0.961539343294878

Pinch-Point Temperature (K): 4.55795464149776

Number of Modules in paralell: 20

Heat Exchanged per module (kWth): 2937.5244495034

Cold side mass flow per module $(\mathrm{kg} / \mathrm{s}): 16.7757494768848$

Hot side mass flow per module (kg/s): 22.3676659691798

Conductance per module (kW/K): 249.998933415307

Number of Thermal Units per module: 9.60868798063722

Capacity Ratio per module: 0

Effectiveness per module: 0.96153964994106

Pinch-Point Tempertuature per module (K): 4.55795464149776 Pinch-Point Tempertuature per module (K): 9.43911355198782

HIGH TEMPERATURE HEAT EXCHANGER:

Cold side inlet Pressure (bar): 25000

Cold side inlet Temperature (K): 490.373107262744

Hot side inlet Pressure (bar): 7400

Hot side inlet Temperature (K): 718.747580010956

Cold side outlet Pressure (bar): 25000

Hot side outlet Pressure (bar): 7400

Cold side mass flow $(\mathrm{kg} / \mathrm{s}): 447.353319383595$

Hot side mass flow (kg/s): 447.353319383595

Heat Exchanged (kWth): 111636.383129119

Number of Sub-Heat Exchanger (Uds.): 15

Conductance (kW/K): 4999.96782002491

Number of Thermal Units: 9.8490439530552

Capacity Ratio: 0

Effectiveness: 0.958668805358466

Pinch-Point Temperature (K): 9.43911355198799

Number of Modules in paralell: 20

Heat Exchanged per module (kWth): 5581.81915645595

Cold side mass flow per module $(\mathrm{kg} / \mathrm{s}): 22.3676659691798$

Hot side mass flow per module (kg/s): 22.3676659691798

Conductance per module (kW/K): 249.998391001247

Number of Thermal Units per module: 9.84904395305527

Capacity Ratio per module: 0

Effectiveness per module: 0.958668263413656

Figura 12.15. Resultados del cálculo de diseño de los campos solares principal y de recalentamiento.

PRIMARY HEAT EXCHANGER:

Cold side inlet Pressure (bar): 25000

Cold side inlet Temperature (K): 683.32975428052

Hot side inlet Pressure (bar): 1500

Hot side inlet Temperature $(K): 833.15$

Cold side outlet Pressure (bar): 25000

Hot side outlet Pressure (bar): 1257.3

Cold side mass flow (kg/s): 447.353319383595

Hot side mass flow $(\mathrm{kg} / \mathrm{s}): 600$

Heat Exchanged (kWth): 78099.922532355

Number of Sub-Heat Exchanger (Uds.): 15

Conductance (kW/K): 2672.01535395299

Number of Thermal Units: 4.77854207445689

Capacity Ratio: 0.607752006728779

Effectiveness: 0.933252867317506

Pinch-Point Temperature (K): 10.0000719357091

Number of Modules in paralell: 20

Heat Exchanged per module (kW/h): 3904.99612661775
REHEATING HEAT EXCHANGER:

Cold side inlet Pressure (bar): 17300

Cold side inlet Temperature (K): 775.472945084985

Hot side inlet Pressure (bar): 1500

Hot side inlet Temperature $(K): 833.15$

Cold side outlet Pressure (bar): 17300

Hot side outlet Pressure (bar): 1257.3

Cold side mass flow (kg/s): 447.353319383595

Hot side mass flow $(\mathrm{kg} / \mathrm{s}): 600$

Heat Exchanged (kWth): 26204.0189854658

Number of Sub-Heat Exchanger (Uds.): 15

Conductance (kW/K): 1466.80006657826

Number of Thermal Units: 2.66708104504632

Capacity Ratio: 0.596705761438915

Effectiveness: 0.826619913260548

Pinch-Point Temperature (K): 10.0000527840775

Number of Modules in paralell: 20

Heat Exchanged per module (kWth): 1310.20094927329

Figura 12.16. Resultados del cálculo de diseño de los intercambiadores de acoplamiento entre los campos solares principal y de recalentamiento, y el ciclo de potencia Brayton. 
12. Programa Informático para diseño y optimización de ciclos Brayton en plantas termosolares con diferentes fluidos caloportadores: Supercritical_CSP (SCSP)

喝 Net_Power

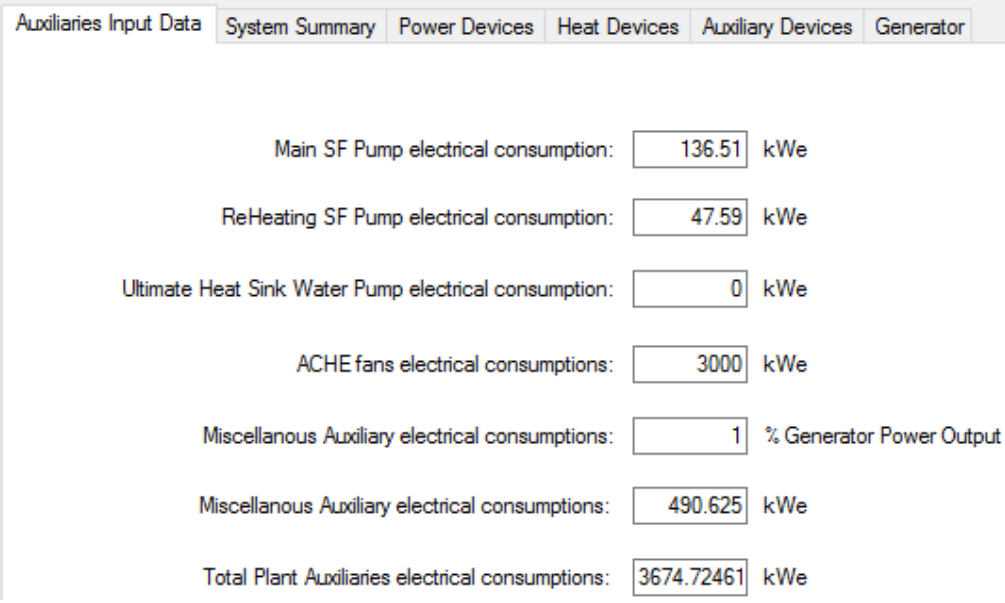

Figura 12.17. Definición de los consumos de los equipos auxiliares de los campos solares y del ciclo Brayton. El consumo de las bombas de recirculación de HTF es calculado automáticamente. El usuario debe estimar el consumo energético de los ventiladores del foco frío y de las pérdidas auxiliares del BOP.

唱 Net_Power

Auxiliaries Input Data System Summary Power Devices Heat Devices Auxilary Devices Generator

\begin{tabular}{|l|l|l|l|}
\hline & Parameter & Value & Unit \\
\hline & Net Total Heat Input: & 104303.9415 & $\mathrm{kWth}$ \\
\hline & Gross Power : & 49062.5 & $\mathrm{kWth}$ \\
\hline & Gross Efficiency : & 47.0380 & $\%$ \\
\hline & Net Power Output : & 45387.7775 & $\mathrm{~kW}$ \\
\hline & Net Efficiency : & 43.5149 & $\%$ \\
\hline & & & \\
\hline
\end{tabular}

Figura 12.18. Balance energético de la planta CSP.

Auxiliaries Input Data System Summary Power Devices Heat Devices Auxiliary Devices Generator

\begin{tabular}{|l|l|l|l|}
\hline & Component & Value & Unit \\
\hline \multirow{*}{*}{} & Main Turbine : & 23839.2820 & $\mathrm{~kW}$ \\
\hline & ReHeating Turbine : & 51786.8001 & $\mathrm{~kW}$ \\
\hline & Main Compressor : & -14595.0901 & $\mathrm{~kW}$ \\
\hline & ReCompressor : & -11030.9899 & $\mathrm{~kW}$ \\
\hline & Total TurboMachines Power : & 50000.0021 & $\mathrm{~kW}$ \\
\hline & Generator Efficiency : & 98.125 & $\%$ \\
\hline \multirow{2}{*}{ * } & Generator Output Power : & 49062.5021 & $\mathrm{~kW}$ \\
\hline & Net Power Output : & 45387.7775 & $\mathrm{~kW}$ \\
\hline & & & \\
\hline
\end{tabular}

Figura 12.19. Balance energético del ciclo de potencia. 
12. Programa Informático para diseño y optimización de ciclos Brayton en plantas termosolares con diferentes fluidos caloportadores: Supercritical_CSP (SCSP)

\begin{tabular}{|l|l|l|l|l|l}
\hline Auxiliaries Input Data System Summary & Power Devices Heat Devices Auxiliary Devices Generator
\end{tabular}

\begin{tabular}{|l|l|l|l|}
\hline & Component & Value & Unit \\
\hline * & Main Solar Field: & 78099.9225 & kWth \\
\hline & ReHeating Solar Field: & 26204.0190 & kWth \\
\hline \multirow{2}{*}{} & Pre-Cooler: & 54303.9415 & kWth \\
\hline
\end{tabular}

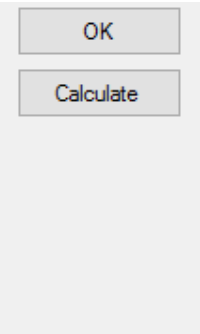

Figura 12.20. Cuantificación de la aportación energética en los campos solares (Main Solar Field, ReHeating Solar Field) y del consumo energético del foco frío (pre-cooler).

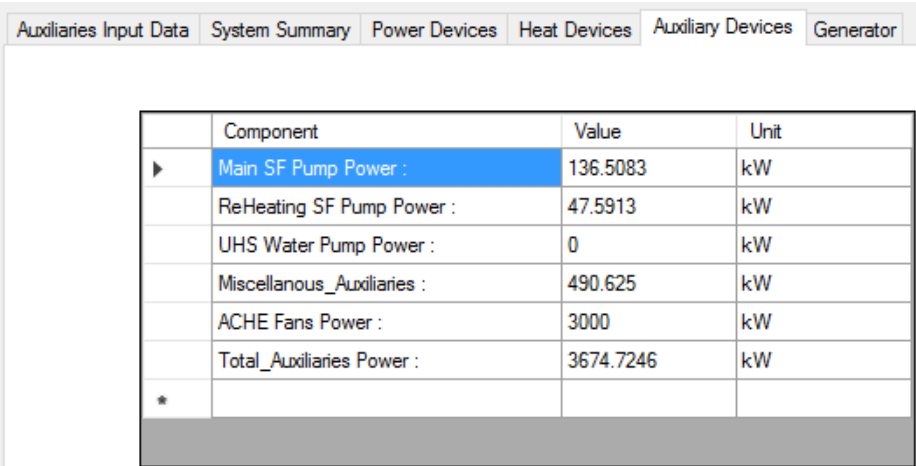

Figura 12.21. Detalle de las pérdidas energéticas de la planta CSP debidas al consumo de los equipos auxiliares del campo solar y del ciclo Brayton de potencia.

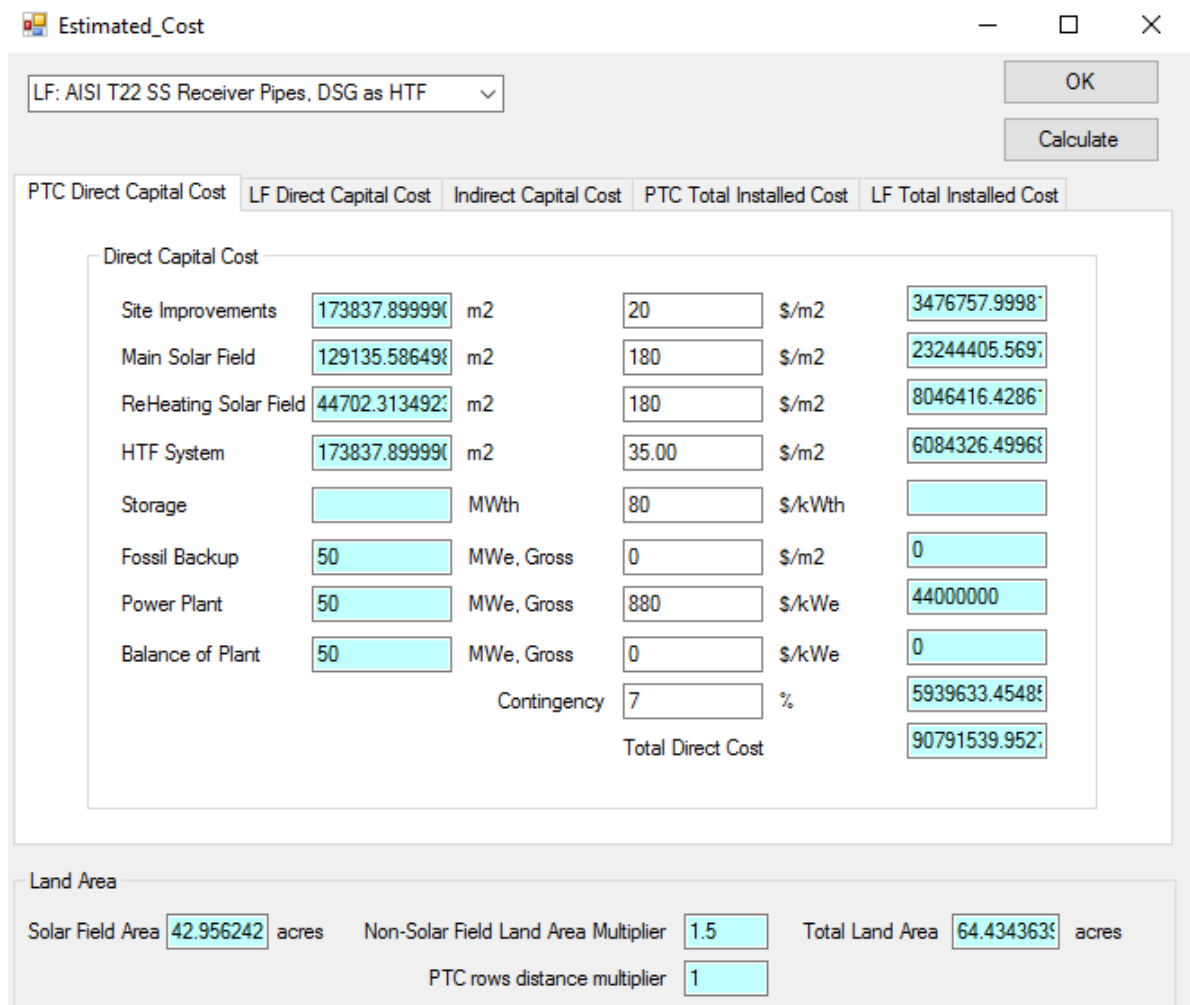

Figura 12.22. Resultados de la estimación de costes de los equipos y componentes de los campos solares principal y de recalentamiento, para colectores de tipología PTC y LF. 
12. Programa Informático para diseño y optimización de ciclos Brayton en plantas termosolares con diferentes fluidos caloportadores: Supercritical_CSP (SCSP)

\subsection{Diseño de los equipos de la planta CSP acoplada al ciclo supercrítico Brayton}

\subsubsection{Diseño de los Compresores}

Se ha considerado que el compresor principal es un compresor radial de una etapa, cuyo coeficiente de flujo $\phi=0.02971$. La metodología de cálculo se explica en la figura 2.18 del capítulo 2. Los polinomios aproximativos de la altura manométrica y eficiencia no dimensionales para el compresor de lazo de pruebas en SNL [12] están definidos en la figura 2.17, que son el resultado de la condensación de los datos experimentales representados en el mapa de operación del compresor, figuras 2.14 y 2.15. Respecto al recompresor, se ha considerado la opción de diseño de un compresor radial con dos etapas, ver figuras 2.19-2.21, para optimizar el diámetro de cada una de las etapas. Los datos de entrada y resultados de diseño de los compresores son los indicados en la figura 12.23.
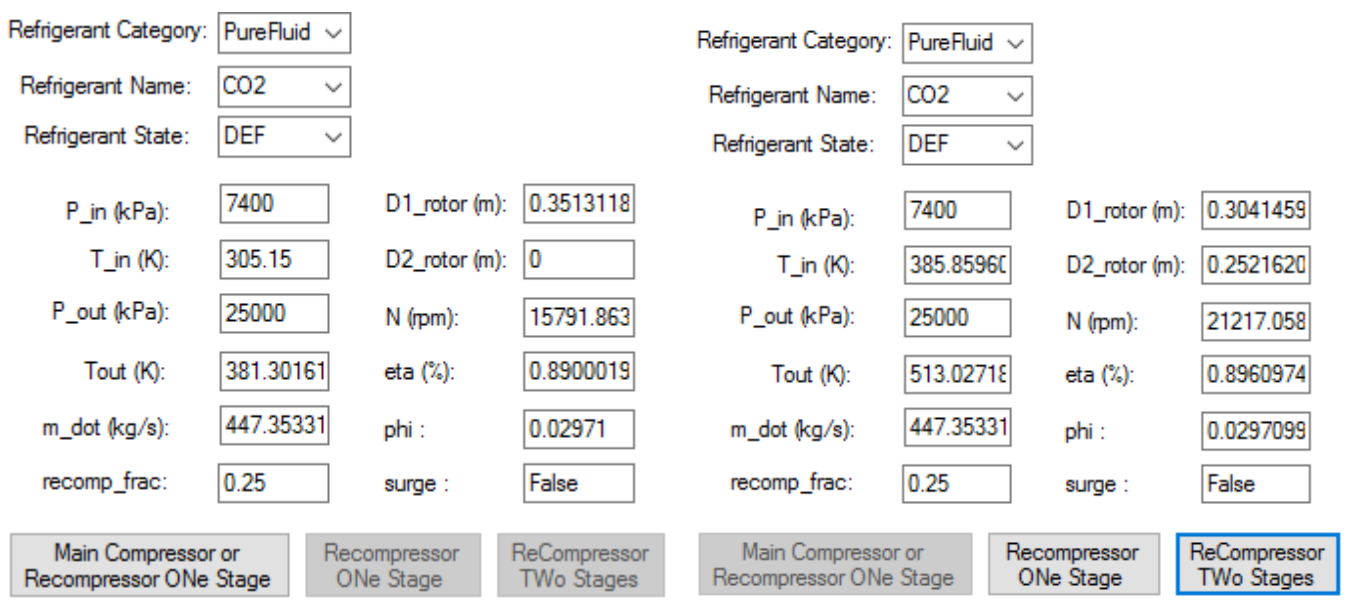

Figura 12.23. Cuadro de diálogo para diseño del compresor principal de una etapa de compresión, y del recompresor radial de dos etapas de compresión.

\subsubsection{Diseño de las Turbinas}

Respecto al diseño de la turbina principal de expansión y a la turbina de recalentamiento, la metodología para su diseño se explica en la figura 2.11 del capítulo 2. Los parámetros de operación considerados son los indicados en la figura 12.24:
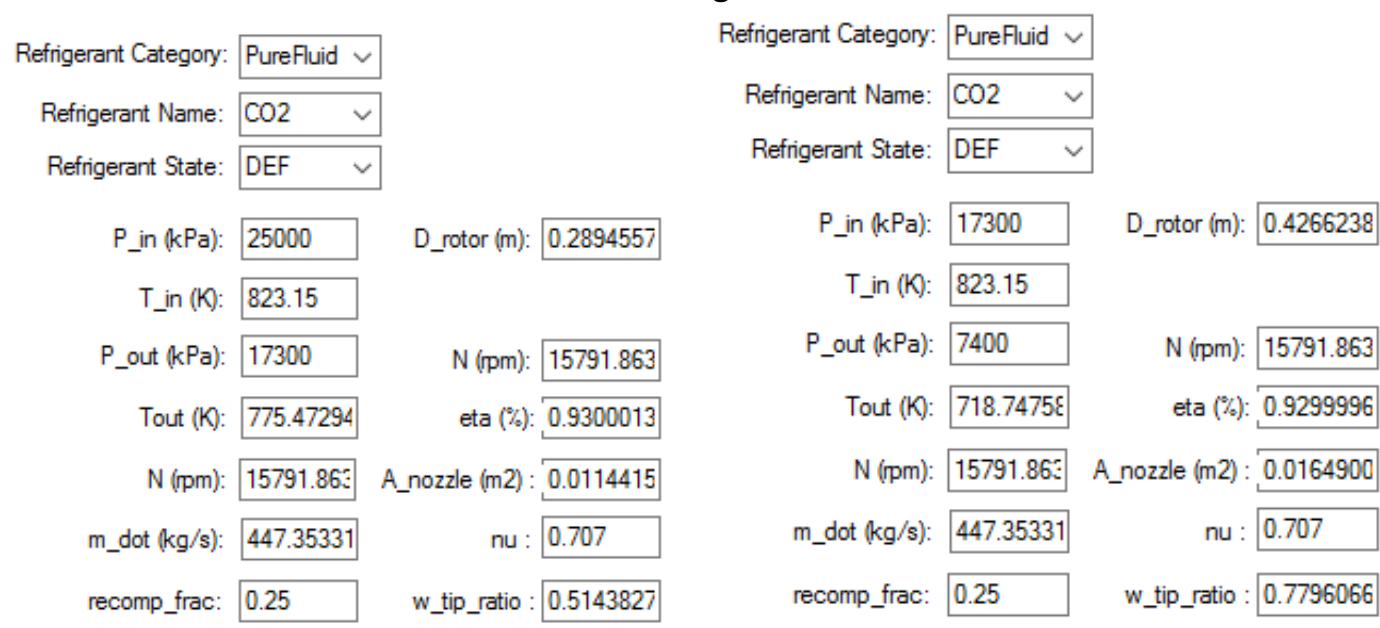

Figura 12.24. Cuadro de diálogo para diseño de las turbinas radiales, principal y de recalentamiento radial. 
12. Programa Informático para diseño y optimización de ciclos Brayton en plantas termosolares con diferentes fluidos caloportadores: Supercritical_CSP (SCSP)

\subsubsection{Diseño de intercambiadores del ciclo Brayton}

La metodología de cálculo de los cambiadores cuando uno de los fluidos que lo atraviesa el s$\mathrm{CO}_{2}$, viene especialmente detallada en el apartado 2.6 del capítulo 2, y se basa en la subdivisión en diferencias finitas del cambiador para realizar un cálculo lo más aproximado posible, poniendo especial consideración en que las propiedades de los fluidos supercríticos no presenta una variación lineal con la temperatura. La metodología de cálculo fue definida por por Klein and Nellis [36]. Los principales resultados obtenidos del diseño básico de cada intercambiador se indican en la figura 12.25 .
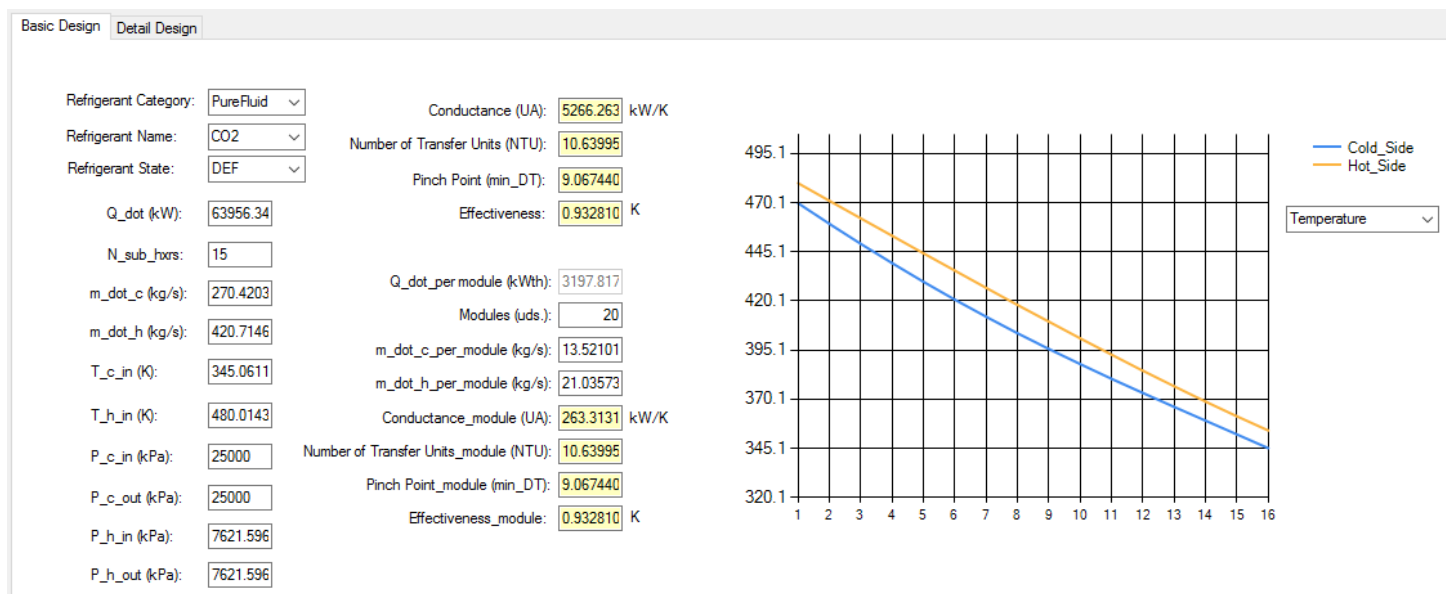

Figura 12.25. Cuadro de diálogo para diseño básico de los intercambiadores de calor (LRT, HTR, PHX, RHX, UHS) de la planta CSP acoplada a ciclo Brayton.

Los resultados gráficos muestran la distribución de las principales variables de operación en los diferentes tramos en los que se ha dividido el intercambiador de calor, figuras 12.25-12.28.

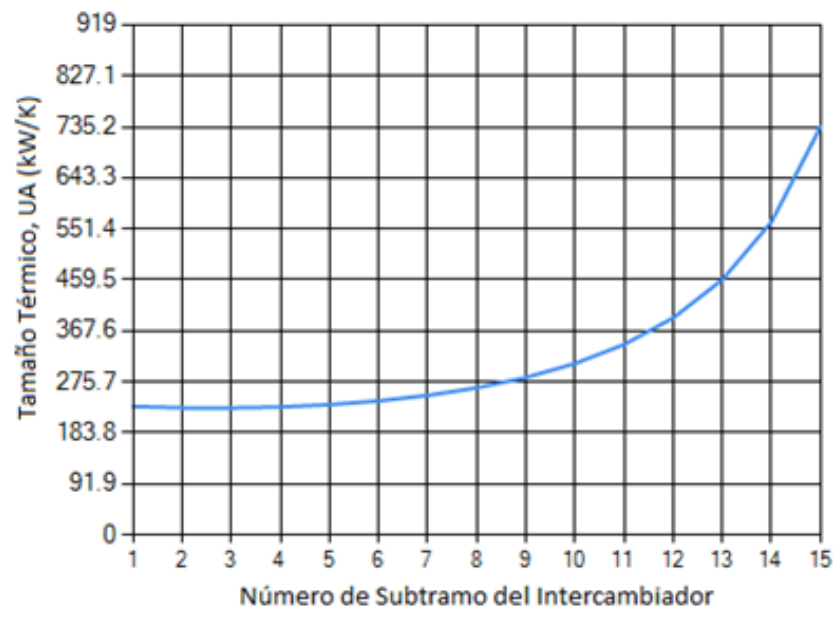

Figura 12.26. Variación de UA $(\mathrm{kW} / \mathrm{K})$ a lo largo del intercambiador de calor. 
12. Programa Informático para diseño y optimización de ciclos Brayton en plantas termosolares con diferentes fluidos caloportadores: Supercritical_CSP (SCSP)

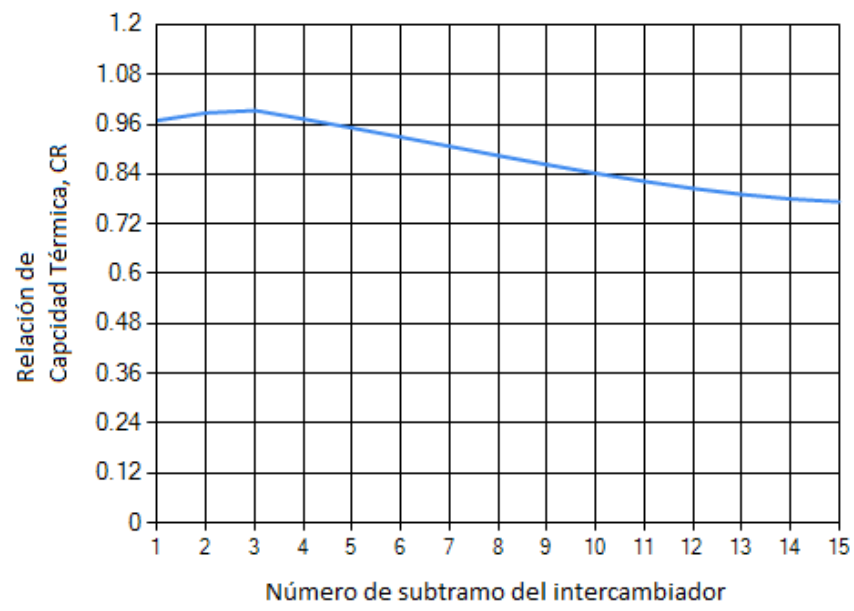

Figura 12.27. Variación de la relación de $C_{R}$ a lo largo del intercambiador de calor.

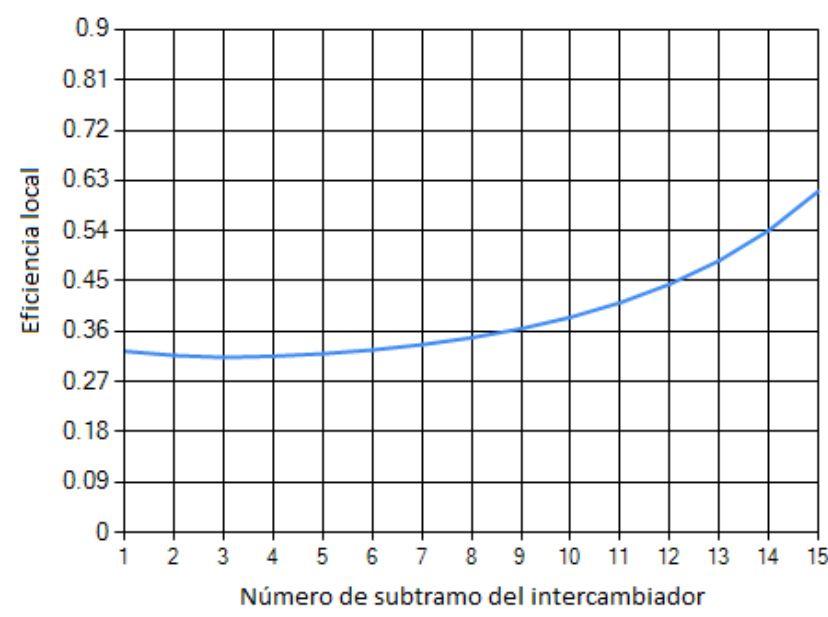

Figura 12.28. Variación de la eficiencia térmica a lo largo del intercambiador de calor.

El diseño de detalle de los intercambiadores, figura 12.31, tiene como principal objetivo definir las dimensiones del equipo para poder realizar una estimación económica del coste de su fabricación. Las correlaciones utilizadas para el cálculo de la distribución del HTC son las de Gnielinsky [37] y Dittus-Boelter [38]. La geometría estudiada se indica en la figura 12.29.

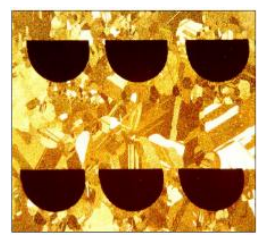

Figura 12.29. Sección transversal de los canales en los intercambiadores de calor de tipología circuito impreso (PCHE).

En trabajos futuros está prevista la modelización de otras tipologías de intercambiadores de calor compactos, pero con menor coste que los PCHE, son la tipología de intercambiadores Formed Plate Heat Exchanger (FPHE), figura 12.30. 
12. Programa Informático para diseño y optimización de ciclos Brayton en plantas termosolares con diferentes fluidos caloportadores: Supercritical_CSP (SCSP)
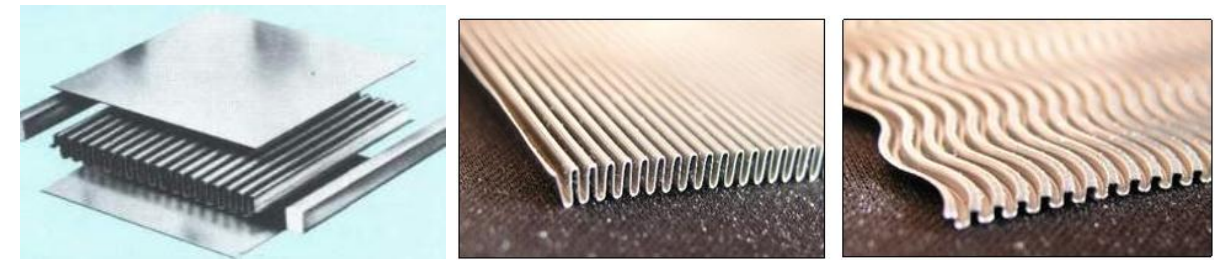

Figura 12.30. Sección transversal de los intercambiadores compactos de tipología (FPHE).
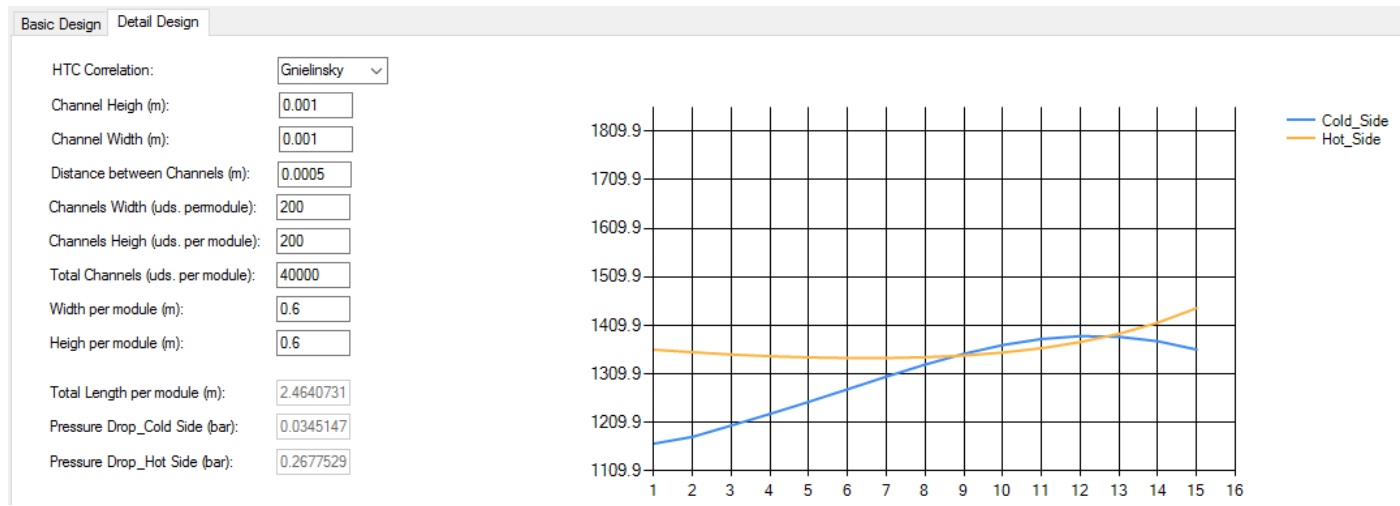

Figura 12.31. Cuadro de diálogo para diseño de detalle de los intercambiadores de calor de la planta CSP acoplada a ciclo Brayton.

\begin{tabular}{lllll} 
HTC Correlation: & Gnielinsky & \\
Channel Heigh $(\mathrm{m}):$ & 0.001 & & Channels Heigh (uds. per module): & 200 \\
Channel Width $(\mathrm{m}):$ & 0.001 & & Total Channels (uds. per module): & 40000 \\
Distance between Channels $(\mathrm{m}):$ & 0.0005 & Width per module (m): & 0.6 \\
Channels Width (uds. permodule): & 200 & Heigh per module (m): & 0.6 \\
\hline
\end{tabular}

Figura 12.32. Datos de partida para el diseño de detalle de los intercambiadores de calor.

Los principales resultados obtenidos del diseño de detalle de los intercambiadores tipo PCHE son:

- Total Length per module $(\mathrm{m})$ : longitud de cada módulo. Valor de referencia $1.5 \mathrm{~m}$.

- Pressure Drop_Cold Side (bar): pérdida de presión a través de cada módulo de la corriente fría. Valor de referencia 0.25 bar.

- Pressure Drop_Hot Side (bar): pérdida de presión a través de cada módulo de la corriente caliente. Valor de referencia 0.25 bar.

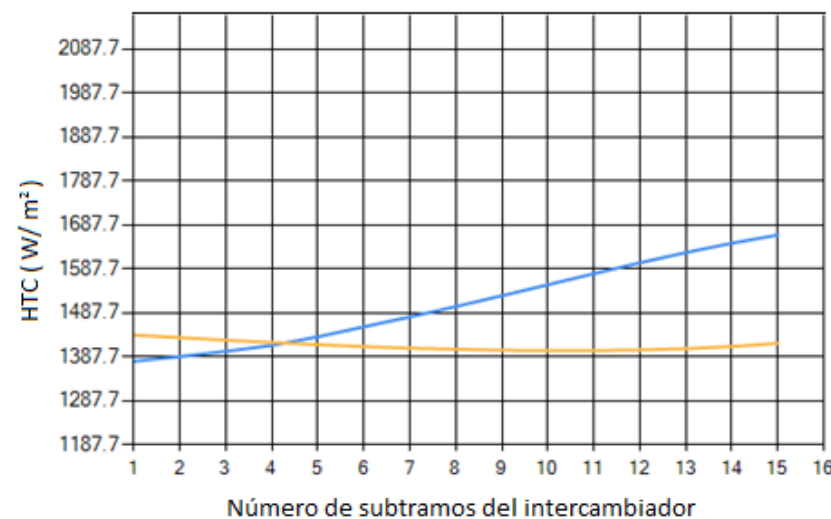

- Corriente fría

- Corriente caliente

Figura 12.33. Distribución de HTC a lo largo del intercambiador. 
12. Programa Informático para diseño y optimización de ciclos Brayton en plantas termosolares con diferentes fluidos caloportadores: Supercritical_CSP (SCSP)

1. Number of IAM data points
2. Line collector type: $0=$ parabolic trough, 1 =linear fresnel
3. Receiver tube outside diameter
4. Receiver tube wall thickness
5. Aperture width
6. Aperture width / Collector unit width (Fresnel only)
7. Collector unit width (for information Fresnel only)
8. Collector focal length
9. Number collector rows per flow path
10. Number collector row banks
11. Active reflector length as percent of total length
12. Collector unit row pitch / collector unit width
13. Reflector cleanliness factor
14. Row (tracking axis) rotation from due North
15. Row (tracking axis) tilt from horizontal
16. Coefficient A1 in heat loss per unit length equation
17. Coefficient $\mathrm{A} 2$ in heat loss per unit length equation
18. Overall heat loss correction factor
19. Number of computational segments along receiver

Figura 12.34. Ejemplo datos de partida para diseño del campo solar.

\subsubsection{Diseño del campo solar}

SCSP incluye la opción de diseño de dos tipologías receptor, realizándose su selección en el cuadro de diálogo principal de cada una de las configuraciones de ciclo Brayton, apartado 12.3. La metodología de diseño del campo solar ha sido explicada en el apartado 2.1.

En la figura 12.34 se muestra un ejemplo de los datos requeridos para diseño del campo solar y en la 12.35 se detallan resultados obtenidos del diseño detallado de los campos solares.

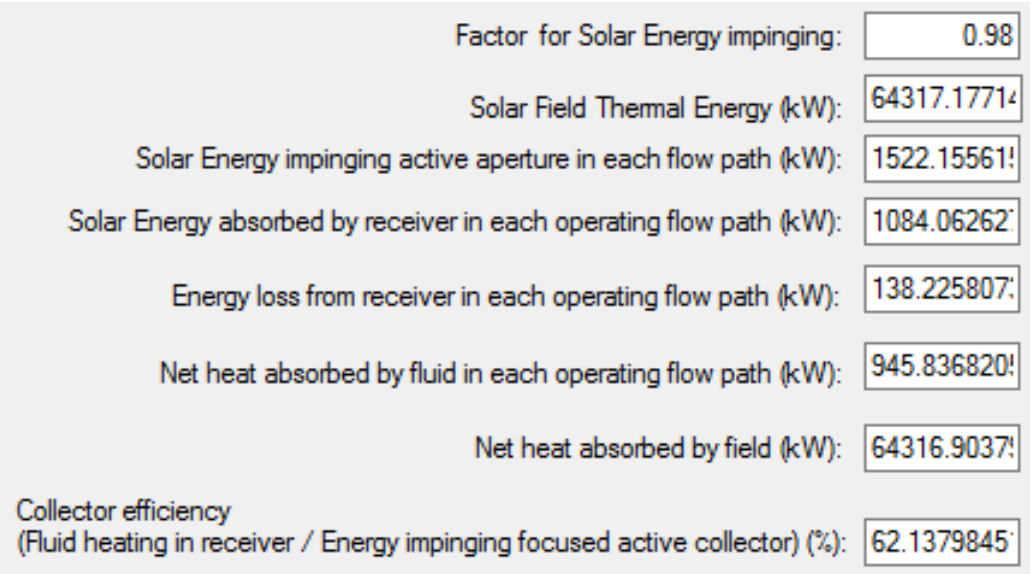

Figura 12.35. Ejemplo resultados del diseño de detalle del campo solar.

\subsubsection{Diseño del Generador}

El generador es un equipo cuyo rendimiento energético tiene un impacto directo sobre la potencia neta final producida en la planta termosolar. Como Valor de referencia se considera que el generador tiene una eficiencia energética alrededor del 98\%. Los datos de partida para 
12. Programa Informático para diseño y optimización de ciclos Brayton en plantas termosolares con diferentes fluidos caloportadores: Supercritical_CSP (SCSP)

diseño del generador están representados en la figura 12.36 y un ejemplo de resultados en la 12.37 .

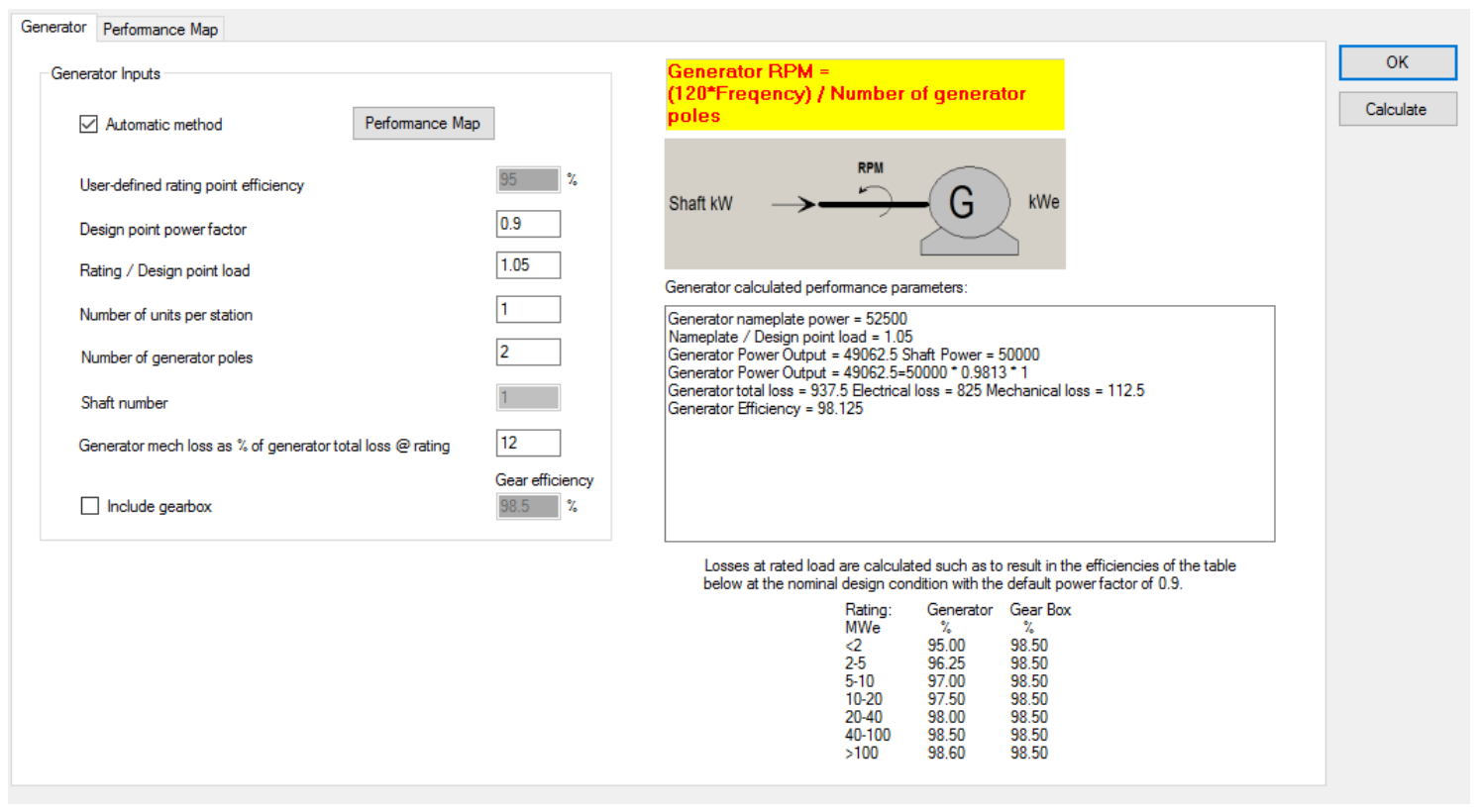

Figura 12.36. Cuadro de diálogo de diseño del Generador.

\begin{tabular}{|l|} 
Generator nameplate power $=52500$ \\
Nameplate $/$ Design point load $=1.05$ \\
Generator Power Output $=49062.5$ Shaft Power $=50000$ \\
Generator Power Output $=49062.5=50000 * 0.9813^{* 1}$ \\
Generator total loss $=937.5$ Electrical loss $=825$ Mechanical loss $=112.5$ \\
Generator Efficiency $=98.125$
\end{tabular}

Figura 12.37. Resultados del diseño del Generador.

\subsection{Validación de resultados con Thermoflow}

El objetivo de este apartado es realizar la validación y verificación de los procesos de cálculo que integran el programa informático SCSP. Para ello se han realizado tres validaciones.

En la primera validación se ha comparado el cálculo de los parámetros termodinámicos del ciclo s-CO2 Brayton RC sin recalentamiento con el programa SCSP con los valores obtenidos en un informe reciente del SNL [39] para el acoplamiento de un reactor nuclear refrigerado por He a un ciclo Brayton. Esta primera validación confirma que los valores termodinámicos de caudal, presión y temperatura de cada una de las corrientes del ciclo, así como los principales parámetros de operación en el punto de diseño (potencia neta generada, eficiencia energética neta y pérdidas de presión en cada equipo), son muy similares en ambos casos. En la figura 12.38 se representan los valores de referencia de la primera validación. En la figura 12.39. se recopilan los datos de partida en el SCSP para simulación del ciclo s- $\mathrm{CO}_{2}$ Brayton RC sin recalentamiento. En la figura 12.40 incluyen los resultados obtenidos con el programa SCSP, y se puede comprobar que se ajustan a los valores incluidos en la figura 12.38. Cabe destacar que el esquema de la figura 12.38 presenta un error en el caudal total del ciclo y ha sido corregido, ya que la suma de los caudales a través de cada compresor: $1456 \mathrm{~kg} / \mathrm{s}$ y $674.1 \mathrm{~kg} / \mathrm{s}$ da como resultado un caudal total en el ciclo de $2130.1 \mathrm{~kg} / \mathrm{s}$, sin embargo, en la figura original de la referencia [39] el valor es de $2267.24 \mathrm{~kg} / \mathrm{s}$. 
12. Programa Informático para diseño y optimización de ciclos Brayton en plantas termosolares con diferentes fluidos caloportadores: Supercritical_CSP (SCSP)

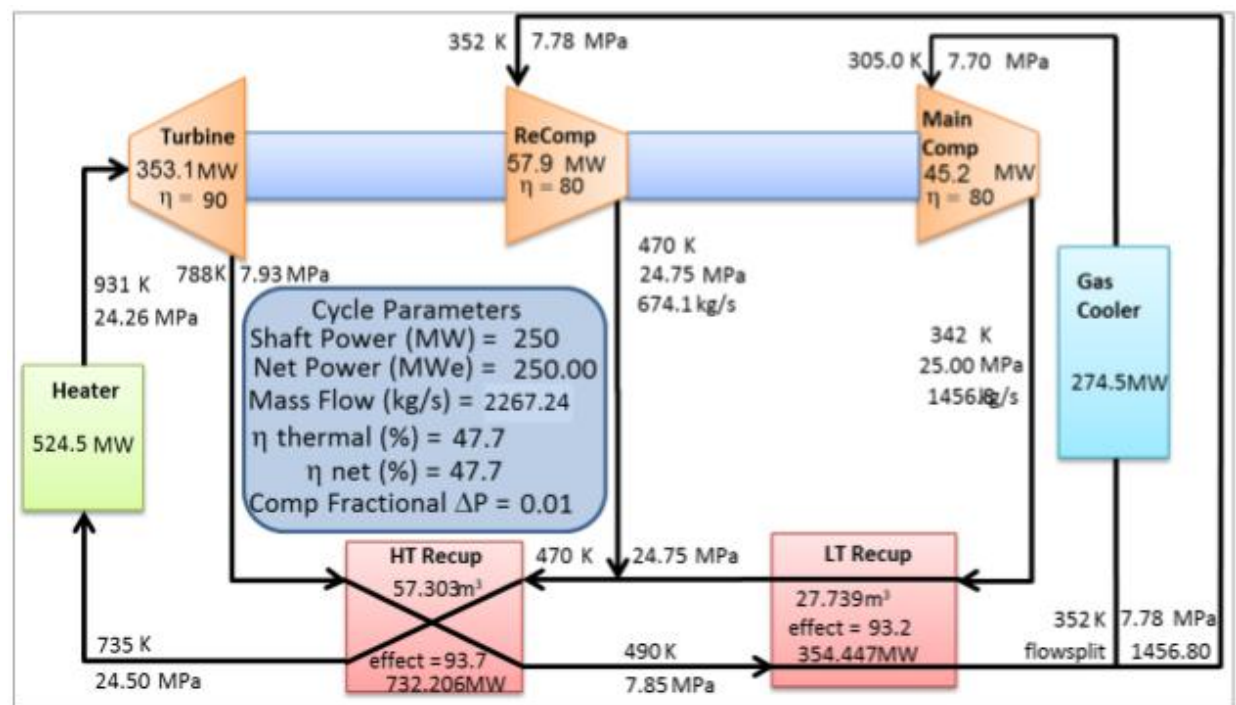

Figura 12.38. Esquema de referencia de la primera validación del programa informático SCSP. Ciclo de potencia s- $\mathrm{CO}_{2}$ Brayton acoplado a un reactor nuclear refrigerado por $\mathrm{He}$ [39].

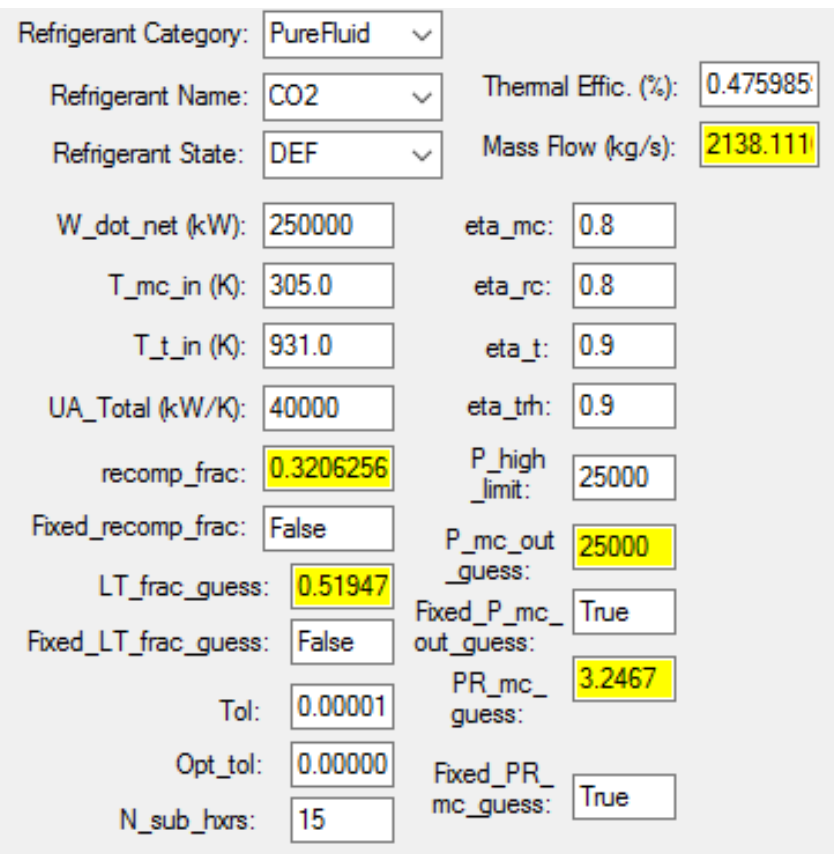

Figura 12.39. Datos de entrada en el SCSP para la primera validación.

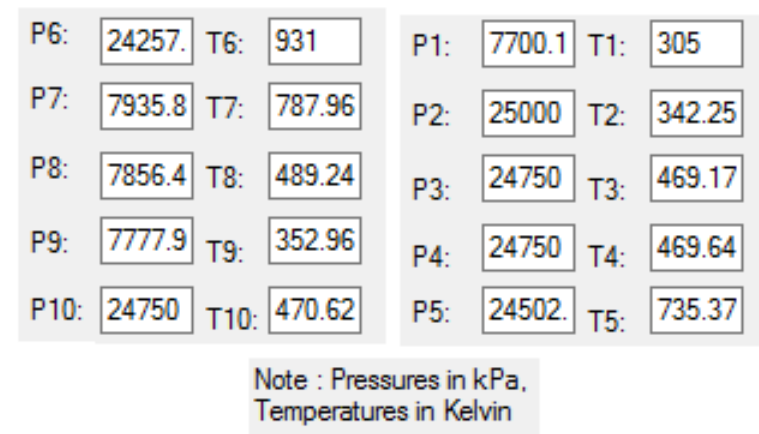

Figura 12.40. Resultados del SCSP de la primera validación. 
12. Programa Informático para diseño y optimización de ciclos Brayton en plantas termosolares con diferentes fluidos caloportadores: Supercritical_CSP (SCSP)

En la segunda validación se realiza una comparativa entre el diseño de tres ciclos Brayton definidos por Dyreby [1], figura 12.41, y las simulaciones correspondientes con SCSP. EI objetivo principal de esta verificación es comprobar que los parámetros de diseño básico de los equipos son similares en ambos casos.

En los tres casos estudiados en la segunda validación, se ha considerado una pérdida de presión del $1 \%$ en los intercambiadores de calor del ciclo Brayton, en su lado de fluido de trabajo s- $\mathrm{CO}_{2}$, figura 12.65 .

En todos los casos estudiados en la validación segunda, tablas 12.1-12.3, la turbina elegida para la validación con Dyreby [1] de la optimización de la configuración RC sin recalentamiento, es la turbina tipo SNL_Turbine radial del SNL. El programa incluye la turbina Radial_Turbine más general para el resto de configuraciones de ciclo Brayton.

\begin{tabular}{|c|c|c|c|}
\hline Power Output & \multicolumn{3}{|c|}{$10 \mathrm{MW}$} \\
\hline Turbine Inlet Temperature & \multicolumn{3}{|c|}{$550^{\circ} \mathrm{C}$} \\
\hline Compressor Outlet Pressure & \multicolumn{3}{|c|}{$25 \mathrm{MPa}$} \\
\hline Compressor Isentropic Efficiency & \multicolumn{3}{|c|}{0.89} \\
\hline Turbine Isentropic Efficiency & \multicolumn{3}{|c|}{0.93} \\
\hline Heat Exchanger Pressure Drops & \multicolumn{3}{|c|}{$1 \%$} \\
\hline Compressor Inlet Temperature & $32^{\circ} \mathrm{C}$ & $40^{\circ} \mathrm{C}$ & $50^{\circ} \mathrm{C}$ \\
\hline Compressor Inlet Pressure & $7.7 \mathrm{MPa}$ & $9 \mathrm{MPa}$ & $10 \mathrm{MPa}$ \\
\hline LT Recuperator Conductance & $1.74 \mathrm{MW} / \mathrm{K}$ & $1.59 \mathrm{MW} / \mathrm{K}$ & $1.52 \mathrm{MW} / \mathrm{K}$ \\
\hline LT Recuperator Minimum $\Delta \mathrm{T}$ & $5.3^{\circ} \mathrm{C}$ & $7.2^{\circ} \mathrm{C}$ & $7.2^{\circ} \mathrm{C}$ \\
\hline LT Recuperator Approx. Volume & $80 \mathrm{~m}^{3}$ & $50 \mathrm{~m}^{3}$ & $40 \mathrm{~m}^{3}$ \\
\hline HT Recuperator Conductance & $1.26 \mathrm{MW} / \mathrm{K}$ & $1.41 \mathrm{MW} / \mathrm{K}$ & $1.48 \mathrm{MW} / \mathrm{K}$ \\
\hline HT Recuperator Minimum $\Delta \mathrm{T}$ & $5.1^{\circ} \mathrm{C}$ & $7.7^{\circ} \mathrm{C}$ & $11.4^{\circ} \mathrm{C}$ \\
\hline HT Recuperator Approx. Volume & $40 \mathrm{~m}^{3}$ & $35 \mathrm{~m}^{3}$ & $35 \mathrm{~m}^{3}$ \\
\hline Compressor Rotor Diameter & $0.120 \mathrm{~m}$ & $0.148 \mathrm{~m}$ & $0.183 \mathrm{~m}$ \\
\hline RC First Stage Rotor Diameter & $0.162 \mathrm{~m}$ & $0.162 \mathrm{~m}$ & $0.157 \mathrm{~m}$ \\
\hline RC Second Stage Rotor Diameter & $0.137 \mathrm{~m}$ & $0.141 \mathrm{~m}$ & $0.139 \mathrm{~m}$ \\
\hline Turbine Rotor Diameter & $0.218 \mathrm{~m}$ & $0.241 \mathrm{~m}$ & $0.265 \mathrm{~m}$ \\
\hline Turbine Effective Nozzle Area & $1,140 \mathrm{~mm}^{2}$ & $1,450 \mathrm{~mm}^{2}$ & $1,790 \mathrm{~mm}^{2}$ \\
\hline Main Shaft Speed & $37,080 \mathrm{rpm}$ & $31,410 \mathrm{rpm}$ & $27,030 \mathrm{rpm}$ \\
\hline Recompressor Shaft Speed & $34,620 \mathrm{rpm}$ & $32,570 \mathrm{rpm}$ & $32,790 \mathrm{rpm}$ \\
\hline Recompression Fraction & 0.3752 & 0.3266 & 0.2578 \\
\hline Turbine Mass Flow Rate & $96.8 \mathrm{~kg} / \mathrm{s}$ & $114.5 \mathrm{~kg} / \mathrm{s}$ & $134.2 \mathrm{~kg} / \mathrm{s}$ \\
\hline Thermal Efficiency & $47.7 \%$ & $45.0 \%$ & $41.8 \%$ \\
\hline
\end{tabular}

Figura 12.41. Valores de referencia de la segunda validación. Obtenidos por Dyreby [1].

Un ejemplo de los datos de partida en SCSP para la segunda validación se resume en la figura 12.42 . 
12. Programa Informático para diseño y optimización de ciclos Brayton en plantas termosolares con diferentes fluidos caloportadores: Supercritical_CSP (SCSP)
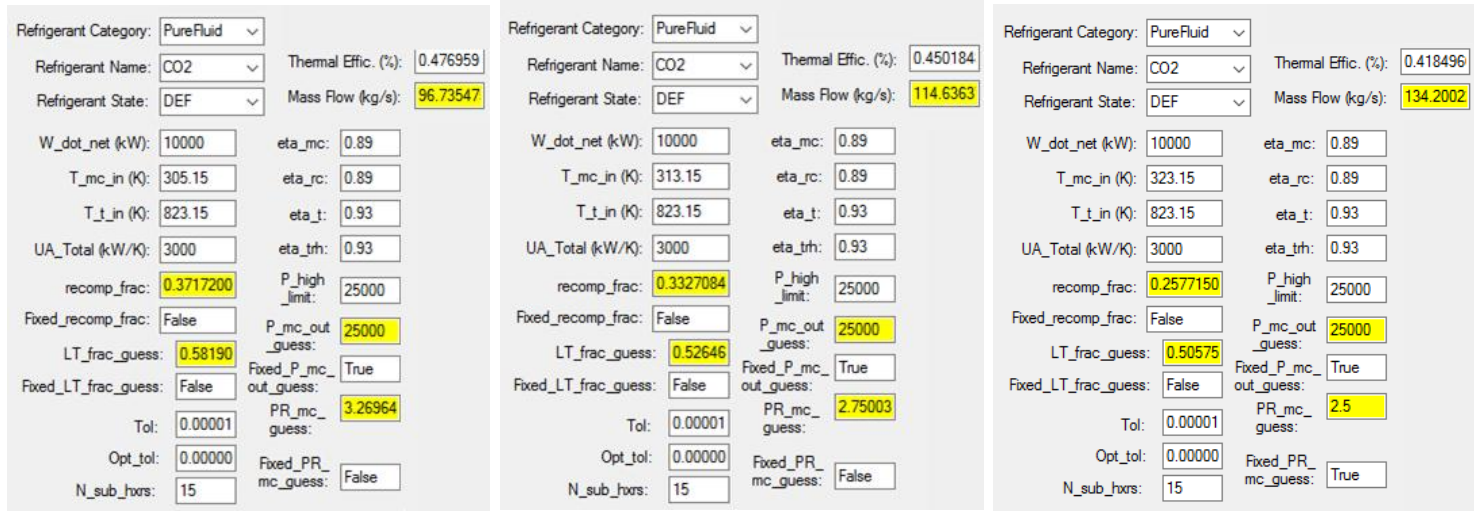

Figura 12.42. Datos de entrada en el SCSP para la segunda validación (caso $\mathrm{CIT}=32^{\circ} \mathrm{C}-$ izquierda, $40^{\circ} \mathrm{C}$ - centro, $50^{\circ} \mathrm{C}$ - derecha).

Los resultados de esta segunda validación con el SCSP se resumen en la tabla 12.1-12.3.

Tabla 12.1. Segunda validación caso $\mathrm{CIT}=32^{\circ} \mathrm{C}$.

\begin{tabular}{lcc}
\hline & Dyreby[1] & SCSP \\
Tamaño térmico recuperador LTR, UA $(\mathrm{kW} / \mathrm{K})$ & 1.74 & 1.74 \\
Tamaño térmico recuperador HTR, UA $(\mathrm{kW} / \mathrm{K})$ & 1.26 & 1.25 \\
Diámetro rotor Compresor principal $(\mathrm{m})$ & 0.120 & 0.122 \\
Diámetro primera etapa del recompresor $(\mathrm{m})$ & 0162 & 0.162 \\
Diámetro segunda etapa del recompresor $(\mathrm{m})$ & 0.137 & 0.136 \\
Diámetro de la turbina $(\mathrm{m})$ & 0.218 & 0.22 \\
\hline
\end{tabular}

Tabla 12.2. Segunda validación caso $\mathrm{CIT}=40^{\circ} \mathrm{C}$.

\begin{tabular}{lcc}
\hline & Dyreby[1] & SCSP \\
Tamaño térmico recuperador LTR, UA $(\mathrm{kW} / \mathrm{K})$ & 1.59 & 1.58 \\
Tamaño térmico recuperador HTR, UA $(\mathrm{kW} / \mathrm{K})$ & 1.41 & 1.42 \\
Diámetro rotor Compresor principal $(\mathrm{m})$ & 0.148 & 0.145 \\
Diámetro primera etapa del recompresor $(\mathrm{m})$ & 0.162 & 0.162 \\
Diámetro segunda etapa del recompresor $(\mathrm{m})$ & 0.141 & 0.142 \\
Diámetro de la turbina $(\mathrm{m})$ & 0.241 & 0.24 \\
\hline
\end{tabular}

Tabla 12.3. Segunda validación caso $\mathrm{CIT}=50^{\circ} \mathrm{C}$.

\begin{tabular}{lcc}
\hline & Dyreby[1] & SCSP \\
Tamaño térmico recuperador LTR, UA $(\mathrm{kW} / \mathrm{K})$ & 1.52 & 1.51 \\
Tamaño térmico recuperador HTR, UA $(\mathrm{kW} / \mathrm{K})$ & 1.48 & 1.48 \\
Diámetro rotor Compresor principal $(\mathrm{m})$ & 0.183 & 0.183 \\
Diámetro primera etapa del recompresor $(\mathrm{m})$ & 0.157 & 0.157 \\
Diámetro segunda etapa del recompresor $(\mathrm{m})$ & 0.139 & 0.138 \\
Diámetro de la turbina $(\mathrm{m})$ & 0.265 & 0.26 \\
\hline
\end{tabular}

\subsection{Conclusiones}

En este capítulo se han explicado las capacidades del programa SCSP, desarrollado ad hoc en este trabajo, para el diseño y dimensionamiento de los equipos en las nuevas centrales termosolares con colectores lineales acopladas a ciclos de potencia supercríticos Brayton. En la introducción se ha comparado la herramienta SCSP con el resto de programas informáticos 
12. Programa Informático para diseño y optimización de ciclos Brayton en plantas termosolares con diferentes fluidos caloportadores: Supercritical_CSP (SCSP)

comerciales, resaltando que actualmente no existe en el mercado una herramienta similar que integre el cálculo en condiciones de operación óptimas en los ciclos Brayton (integrando los algoritmos de cálculo SUBPELX [2], UOBYQA [3] y NEWUOA [4]) acoplados a un campo solar con colectores lineales.

Tomando como punto de partida el trabajo de Dyreby [1] se han programado configuraciones de ciclo Brayton adicionales: PCRC y $\mathrm{RCMCl}$, ver apartado 12.2. Así mismo se ha desarrollado la opción de incluir una etapa de recalentamiento en las turbinas de expansión. Las opciones de cálculo incluidas hasta ahora en la herramienta SCSP son: cálculo del punto operación en el punto de diseño y cálculo de las condiciones óptimas de operación en el punto de diseño. Se han incluido diferentes apartados explicando los parámetros de diseño para el cálculo y dimensionamiento de los equipos que integran estas plantas termosolares: campos solares, compresores, turbinas, intercambiadores.

Finalmente, se han realizado dos validaciones, comparando los resultados con las referencias [1] y [39], confirmando que los resultados obtenidos con el SCSP son fiables.

Se están desarrollando los análisis de sensibilidad ante variaciones de diferentes variables externas al ciclo: sensibilidad ante cambios de la CIT, sensibilidad ante variaciones de la fracción de recompresión y sensibilidad ante variaciones del TIP. Otros trabajos futuros que se proponen son:

- Condiciones de operación parcial, en el punto de diseño y en condiciones de optimización del punto de diseño, para las nuevas configuraciones con la opción de recalentamiento en turbina.

- Estimación de cálculo de la producción anual de electricidad.

- Interacción con Thermoflow mediante Excel para validación de resultados y ganar las sinergias de la combinación entre ambas herramientas.

- Validación de resultados con las versiones futuras de SAM.

- Incluir nuevos fluidos de trabajo en los ciclos Brayton y permitir la elaboración de mezcla de sustancias para optimizar la eficiencia energética de la planta.

- Acoplamiento con herramientas de cálculo de elementos finitos (ANSYS) y CFD (ANSYS CFX, Fluent) para diseño detallado de los equipos (turbomáquinas, intercambiadores, tubos absorbedores,etc.).

- Integración con campos solares con DSG en sus diferentes modos de operación (recirculación y un solo-paso).

- Integración con campos solares de tipología Dual-Loop.

- Incluir HTF innovadores en los campos solares (metales líquidos, nanofluidos, etc).

- Incluir la configuración de campo solar con torre central.

- Integración con sistema de almacenamiento térmico en foco caliente y foco frío.

- Diseño de detalle del foco frío.

- Acoplamiento con SAM mediante la librería SDK en C\#.

- Cálculo detallado de las pérdidas térmicas en los tubos absorbedores de los campos solares mediante correlaciones para cálculos de los HTC.

- Integración de innovadoras tipologías de colectores solares, Ultimate Trough, y absorbedores, Norwich. 
12. Programa Informático para diseño y optimización de ciclos Brayton en plantas termosolares con diferentes fluidos caloportadores: Supercritical_CSP (SCSP)

- Desarrollo de módulos para acoplamiento de la planta solar a instalaciones auxiliares: desaladoras, cogeneración de calor, producción de hidrógeno, acoplamiento a turbina de gas o caldera CFB.

- Módulo para simulación de los ciclos de subcríticos y supercríticos de Rankine.

\subsection{Referencias}

[1] John Dyreby. The University of Wisconsin, Madison. Thesis: Modeling the Supercritical CO2 Brayton Cycle with Recompression.

[2] T. Rowan, Functional Stability Analysis of Numerical Algorithms. Ph.D. thesis, Department of Computer Sciences, University of Texas at Austin, 1990.

[3] Powell, M. J. D. (2002). UOBYQA: Unconstrained Optimization By Quadratic Approximation. Mathematical Programming, Series B (Springer) 92: 555-582. doi:10.1007/s101070100290

[4] Powell, M. J. D. (November 2004). The NEWUOA software for unconstrained optimization without derivatives (PDF) (Report). Department of Applied Mathematics and Theoretical Physics, Cambridge University. DAMTP 2004/NA05. Retrieved 2014-01-14.

[5] Thermoflow Inc. Sofware, http://www.thermoflow.com.

[6] System Advisor Model (SAM). https://sam.nrel.gov/

[7] TRaNsient SYstems Simulation Program (TRNSYS). http://www.trnsys.com/

[8] SimTech, IPSEpro. http://www.simtechnology.com/CMS/index.php/ipsepro

[9] Aspentech/ Aspen HSYS. http://www.aspentech.com/products/aspen-hysys/

[10] 12th International Modelica Conference. https://www.modelica.org/

[11] ECOSIMPRO/PROOSIS. http://www.ecosimpro.com/

[12] SolTrace (NREL Software). http://www.nrel.gov/csp/soltrace.html

[13] Integrated Layout and Optimization Tool for Solar Power Towers, SolarPILOT (NREL Software). http://www.nrel.gov/csp/solarpilot.html

[14] TONATIUH (CENER-CIEMAT Software). http://www.cener.com

[15] A computer code for calculating the optical performance and optimal system design for solar thermal central receiver plants, DELSOL3

[16] Craig Turchi. Supercritical $\mathrm{CO}_{2}$ Power Cycles: Next-Gen Power for CSP?. SunShot, CSP Program Summit 2016.

[17] Craig Turchi. CSP Systems Analysis. SunShot, CSP Program Summit 2016.

[18] 2016 SunShot CSP Summit and Integration Workshop.

[19] Supercritical Transformational Electric Power (STEP) Program.

http://www.energy.gov/ne/articles/energy-department-announces-new-investments-

supercritical-transformational-electric

[20] NIST Reference Fluid Thermodynamic and Transport Properties Database (REFPROP): Version 9.1. https://www.nist.gov/srd/refprop

[21] L. Coco-Enríquez, J. Muñoz-Antón, J.M. Martínez-Val. Integration between direct steam generation in linear solar collectors and supercritical carbon dioxide Brayton power cycles. International Journal of Hydrogen Energy 40 (2015) 15284-15300.

[22] Luis Coco-Enríquez, Javier Muñoz-Antón, José María Martínez-Val Peñalosa. Dual Loop Line-Focusing Solar Power Plants with Supercritical Brayton Power cycles. $4^{\text {th }}$ European Conference on Renewables Energy Systems ECRES 2016.

[23] Luis Coco-Enríquez, Javier Muñoz-Antón, José María Martínez-Val Peñalosa. S-Ethane Brayton Power Conversion Systems for Concentrated Solar Power Plant. ASME Journal of Solar Energy Engineering, February 2016, Vol. 138 / 011012-1.

[24] Seidel W. Model Development and Annual Simulation of the Supercritical Carbon Dioxide Brayton Cycle for Concentrating Solar Power Applications. University of Wisconsin, Madison, USA, 2010. 
12. Programa Informático para diseño y optimización de ciclos Brayton en plantas termosolares con diferentes fluidos caloportadores: Supercritical_CSP (SCSP)

[25] R. Z. Mathkor, B. Agnew, M. A. Al-Weshahi and N. Eshoul. Exergy Modelling of an Organic Rankine Cycle Energized by Heat from Parabolic Trough Collector with Thermal Storage. Journal of Clean Energy Technologies, Vol. 4, No. 2, March 2016.

[26] C. Turchi, G. Zhu, M.I Wagner, T. Williams, D. Wendt. Geothermal / Solar Hybrid Designs: Use of Geothermal Energy for CSP Feedwater Heating. Geothermal Resources Council 2014 Annual Meeting, October 2014.

[27] C. Turchi, Christine Bing, Mark Lausten. $10 \mathrm{MW}$ Supercritical CO2 Turbine Test. NREL Nonpropietary Final Report DE-EE0001589.

[28] Klaus Brun, Aaron McClung, John Davis. Novel Supercritical Carbon Dioxide Power Cycle Utilizing Pressurized Oxy-Combustion in Conjuction with Cryogenic Compression. U.S. Department of Energy, NETL, Morgantown, WV. DOE Project DE-FE0009395.SwRI Project No. 17899. April 9, 2014.

[29] A.S.M. Arifur Chowdhury, Luz Bugarin, Antara Badhan, Ahsan Choudhuri, Norman Love. Thermodynamic analysis of a directly heated oxyfuel supercritical power system. Applied Energy, Volume 179, 1 October 2016, Pages 261-271.

[30] Robert Österholma, Jens Pålssonb. Dynamic modelling of a parabolic trough solar power plant. Proceedings of the 10th International Modelica Conference March 10-12, 2014, Lund, Sweden.

[31] Antoine Aurousseau, Valéry Vuillerme, Jean-Jacques Bezian. Modeling of Linear Concentrating Solar Power using Direct Steam Generation with Parabolic-Trough. Proceedings of the 11th International Modelica Conference September 21-23, 2015, Versailles, France.

[32] Casella Francesco, Colonna Piero. Development of a Modelica dynamic model of solar supercritical CO2 Brayton cycle power plants for control studies. Supercritical CO2 Power Cycle Symposium May 24-25, 2011, Boulder, Colorado.

[33] C. Kalathakis, N. Aretakis, I. Roumeliotis, A. Alexiou, K. Mathioudakis. ASME paper GT2016-57272: Assesment of Solar Steam Injection in Gas Turbines.

[34] C. Kalathakis, N. Aretakis, I. Roumeliotis, A. Alexiou, K. Mathioudakis. ASME paper GT2016-57700: Investigation of Different Solar Hybrid Gas Turbines and Exploitation of Rejected Sun Power.

[35] J. M. Torres, A. J. Gallego, J. M. Escaño, C. Bordons. Modelo en EcosimPro de Captador Solar Fresnel (Fresnel Lens Solar Concentrator).

[36] Klein, Nellis. 2009. Heat Transfer. Cambridge University Press.

[37] Incropera, Frank P.; DeWitt, David P. (2007). Fundamentals of Heat and Mass Transfer (6th ed.). Hoboken: Wiley. pp. 490, 515. ISBN 978-0-471-45728-2.

[38] Dittus Boelter. F.W. Dittus, L.M.K. Boelter. Heat transfer in automobile radiator of the tubular type. University of California at Berkley Publ. Eng., 2 (1930), pp. 443-461.

[39] Bobby D. Middleton, James R. Pasch, Alan Kruizenga, Matthew Walker. Coupling a Supercritical Carbon Dioxide Brayton Cycle to a Helium-Cooled Reactor. Sandia National Laboratories Report SAND2016-0696, Unlimited Release, January 2016. 
12. Programa Informático para diseño y optimización de ciclos Brayton en plantas termosolares con diferentes fluidos caloportadores: Supercritical_CSP (SCSP) 


\section{Capítulo 13 \\ Conclusiones y Trabajos Futuros}

\subsection{Conclusiones}

El objetivo principal de esta tesis es proponer diferentes diseños de plantas termosolares con colectores solares lineales, para su integración con las nuevas tecnologías de ciclos de potencia supercríticos tanto de tipología Rankine como Brayton.

En el primer capítulo de la tesis se realiza una recopilación de la eficiencia energética de las plantas termosolares, de tipología "SEGS", actualmente en operación, con colectores solares PTC, aceite térmico como HTF y ciclo de potencia Rankine, ver tabla 13.1.

Tabla 13.1. Resumen del rendimiento energético de las diferentes tecnologías analizadas en el capítulo primero.

\begin{tabular}{lllll}
\hline HTF & $\begin{array}{l}\text { Colectores } \\
\text { Solares }\end{array}$ & Ciclo de Potencia & $\begin{array}{c}\text { TIT } \\
\left({ }^{\circ} \mathrm{C}\right)\end{array}$ & $\begin{array}{l}\text { Eficiencia } \\
\text { Neta (\%) }\end{array}$ \\
\hline Therminol VP-1 & PTC / LF & $\begin{array}{l}\text { Rankine subcrítico } \\
\text { sin recalentamiento }\end{array}$ & 390 & 34.7 \\
\hline Dowtherm A & PTC / LF & $\begin{array}{l}\text { Rankine subcrítico } \\
\text { sin recalentamiento }\end{array}$ & 415 & 35.4 \\
\hline Therminol VP-1 & PTC / LF & $\begin{array}{l}\text { Rankine subcrítico } \\
\text { con recalentamiento }\end{array}$ & 390 & 36.3 \\
\hline Dowtherm A & PTC / LF & $\begin{array}{l}\text { Rankine subcrítico } \\
\text { con recalentamiento }\end{array}$ & 415 & 37 \\
\hline Solar Salt & PTC / LF & $\begin{array}{l}\text { Rankine subcrítico } \\
\text { sin recalentamiento }\end{array}$ & 550 & 38.5 \\
\hline Hitec XL & PTC / LF & $\begin{array}{l}\text { Rankine subcrítico } \\
\text { sin recalentamiento }\end{array}$ & 530 & 38 \\
\hline Solar Salt & PTC / LF & $\begin{array}{l}\text { Rankine subcrítico } \\
\text { con recalentamiento }\end{array}$ & 550 & 40.5 \\
\hline Hitec XL & PTC / LF & $\begin{array}{l}\text { Rankine subcrítico } \\
\text { con recalentamiento }\end{array}$ & 530 & 40 \\
\hline
\end{tabular}

Para realizar la comparativa entre las diferentes tecnologías se ha considerado en todos los casos 5 calentadores y un desaireador en el ciclo subcrítico Rankine [1]. En los proyectos más recientes de las centrales termosolares con colectores PTC, similares a los proyectos Solana y Mojave de la empresa Abengoa Solar [2], se podrían considerar 7 calentadores en el ciclo de subcrítico de Rankine con recalentamiento, incrementándose el rendimiento energético neto hasta un $37.7 \%$ con una TIT de $380^{\circ} \mathrm{C}$. Se concluye que el incremento de rendimiento energético que supone el incremento del número de calentadores desde 5 hasta 7 es $\approx 1.5 \%$.

En todos los casos estudiados se ha fijado la potencia bruta generada en 50 MWe. Los consumos parásitos del ciclo de potencia Rankine son del orden de $3 \mathrm{MWe}$, y se pueden desglosar en: 1.5 MWe los ventiladores del condensador de ciclo de potencia, 0.5 a $1 \mathrm{MWe}$ el consumo de las bombas de agua de alimentación del BOP, y $0.5 \mathrm{MWe}$ el consumo de las 
bombas de recirculación en el caso de aceite como HTF. En el caso de sales fundidas como HTF, este consumo se ve reducido debido a la menor viscosidad de las sales en comparación con los aceites. Sin embargo, las sales fundidas requieren un sistema de calefacción para evitar su solidificación a bajas temperaturas, ya mencionado en esta tesis Heat Tracing, lo cual supone un consumo adicional de energía. Así mismo no se ha tenido en cuenta el consumo eléctrico del sistema de renovación de los HTF, que necesitan un tratamiento para evitar su degradación con el tiempo.

Cabe destacar, como principal tendencia de diseño a medio plazo, la planta con colectores PTC y sales fundidas como HTF acoplada a ciclo Rankine, con un rendimiento del $38.4 \%$ sin recalentamiento, y del $40.5 \%$ con recalentamiento, tecnología desarrollada por Archimede Solar [3], compañía italiana pionera en este tipo de instalaciones.

En el primer capítulo, también se introduce otra tecnología que se está comenzando a implantar a escala industrial, es la DSG en colectores lineales acoplado directamente con ciclos de potencia de Rankine. La primera planta con DSG entró en operación en Tahilandia TSE1 [4]. Potencialmente esta tecnología proporciona rendimientos similares a los de las sales fundidas como HTF, con temperaturas de entrada a turbina y de recalentamiento de $550^{\circ} \mathrm{C}$. En este caso, la limitación de TIT se debe a la máxima temperatura de operación del material selectivo de los tubos absorbedores. En este sentido se están desarrollando nuevos tubos absorbedores para minimizar las pérdidas térmicas, sirvan como ejemplo los productos de las empresas Rioglass [5], Norwich Technologies [6-8], Schott Solar [9] y Archimede Solar [10]. Actualmente los tubos absorbedores soportan temperaturas de hasta $550^{\circ} \mathrm{C}$.

El capítulo segundo explica la metodología seguida a lo largo del desarrollo de la tesis: se han propuesto diferentes soluciones tecnológicas para el acoplamiento de los colectores solares lineales con los ciclos de potencia supercíticos de Rankine o Brayton. En relación con los ciclos de potencia supercríticos Brayton se ha introducido la optimización multiparámetro de las variables de operación de los ciclos de potencia Brayton, mediante la integración en los programas informáticos de cálculo con los algoritmos matemáticos de optimización: SUBPLEX [11], UOBYQA [12], y NEWOUA [13]. Esta optimización ha permitido minimizar la superficie de apertura efectiva y coste de los campo solares para las diferentes tecnologías de HTF estudiadas: Aceites caloportadores (capítulo 7), sales fundidas (capítulos 5 y 6), generación directa de vapor (capítulo 8), y sistema Dual-Loop (capítulo 9). Dentro del capítulo de metodología, se incluye una explicación detallada del código y algoritmos de diseño de equipos que integran el ciclo Brayton. Se ha desarrollado un programa informático en C\# ad hoc para esta tesis. Este software se ha utilizado para validar los resultados obtenidos con Thermoflow [14], para el dimensionamiento de los campo solares y resto de equipos, que integran las plantas termosolares de nueva generación acopladas a los ciclos de potencia supercríticos. Se ha desarrollado la integración en el programa informático de la librería de propiedades de los fluidos supercríticos caloportadores REFPROP [15], desarrollada por NIST, y programada originalmente en Fortran.

La primera tecnología propuesta, el Recalentamiento Directo (DRH), se define en el tercer capítulo. Esta solución tecnológica se propuso para plantas con colectores Fresnel en la 
Conferencia SolarPaces 2013 [16] y simultáneamente fue esbozada por Tobias Hirsch [17], en la misma conferencia. Mediante esta tecnología es posible evitar el intercambiador de calor entre el campo solar y el ciclo de potencia en la etapa o etapas de recalentemiento del vapor en las turbinas del BOP. Para ello la principal variable a considerar es la longitud de los colectores de recalentamiento, para minimizar las pérdidas de presión en el campo solar de recalentamiento, lo cual impacta negativamente en la eficiencia neta de la planta termosolar. Los resultados de la tecnología DRH en plantas LF con DSG acoplada a ciclos subcríticos Rankine, están resumidos en la tabla 13.2.

Tabla 13.2. Resumen del rendimiento energético de las diferentes soluciones tecnológicas analizadas en el capítulo 3.

\begin{tabular}{llllllll}
\hline HTF & Colector & Ciclo de Potencia & Fluido BOP & $\begin{array}{l}\text { TIT } \\
\left({ }^{\circ} \mathrm{C}\right)\end{array}$ & $\begin{array}{l}\text { Eficiencia } \\
\text { Neta }(\%)\end{array}$ & $\begin{array}{l}\text { Capítulo } \\
\text { Tesis }\end{array}$ & Ref. \\
\hline DSG & LF & Rankine con DRH & Agua & 373 & 38.3 & 3 & {$[16]$} \\
\hline DSG & LF & Rankine con DRH & Agua & 400 & 39.02 & 3 & {$[16]$} \\
\hline DSG & LF & Rankine con DRH & Agua & 450 & 40.17 & 3 & {$[16]$} \\
\hline DSG & LF & Rankine con DRH & Agua & 500 & 40.63 & 3 & {$[16]$} \\
\hline DSG & LF & Rankine con DRH & Agua & 517 & 40.82 & 3 & {$[16]$} \\
\hline
\end{tabular}

En el capítulo cuarto se lleva a cabo el diseño conceptual y balance energético de una planta termosolar con colectores lineales y ciclo de potencia de Rankine, con entrada de agua en estado supercrítico a la turbina principal. Los niveles de presión de agua supercrítica a entrada de turbina principal 260 bar, permiten la utilización de 7 calentadores de agua de alimentación, más un desaireador, y hasta tres etapas de recalentamiento.

Tabla 13.3. Resumen del rendimiento energético de las diferentes soluciones tecnológicas analizadas en el capítulo 4.

\begin{tabular}{|c|c|c|c|c|c|c|c|}
\hline HTF & Colector & Ciclo de Potencia & $\begin{array}{l}\text { Fluido } \\
\text { BOP }\end{array}$ & $\begin{array}{l}\text { TIT } \\
\left({ }^{\circ} \mathrm{C}\right)\end{array}$ & $\begin{array}{l}\text { Eficiencia } \\
\text { Neta (\%) }\end{array}$ & $\begin{array}{l}\text { Capítulo } \\
\text { Tesis }\end{array}$ & Ref. \\
\hline DSG & PTC & $\begin{array}{l}\text { Subcrítico Rankine } \\
\text { (2a etapa DRH) }\end{array}$ & Agua & 550 & 40.41 & 4 & {$[19,20]$} \\
\hline DSG & PTC & $\begin{array}{l}\text { Subcrítico Rankine } \\
\text { (1ª etapa DRH) }\end{array}$ & Agua & 550 & 40.09 & 4 & {$[19,20]$} \\
\hline DSG & PTC & $\begin{array}{l}\text { Supercrítico Rankine } \\
\text { (2⿺ etapa DRH) }\end{array}$ & Agua & 550 & 42.9 & 4 & {$[19,20]$} \\
\hline DSG & PTC & $\begin{array}{l}\text { Supercrítico Rankine } \\
\left(1^{\mathrm{a}}, 2^{\mathrm{a}}, 3^{\mathrm{a}} \text { etapa } \mathrm{DRH}\right)\end{array}$ & Agua & 550 & 44.52 & 4 & {$[19,20]$} \\
\hline DSG & PTC & $\begin{array}{l}\text { Supercrítico Rankine } \\
\left(1 \stackrel{a}{2} 2^{2} \text { etapa DRH) }\right.\end{array}$ & Agua & 550 & 43.81 & 4 & {$[19,20]$} \\
\hline DSG & PTC & $\begin{array}{l}\text { Supercrítico Rankine } \\
\text { (2⿺ } 3 \text { 3ㄹ etapa DRH) }\end{array}$ & Agua & 550 & 43.78 & 4 & {$[19,20]$} \\
\hline DSG & LF & $\begin{array}{l}\text { Subcrítico Rankine } \\
\text { (2ª etapa DRH) }\end{array}$ & Agua & 550 & 40.44 & 4 & {$[19,20]$} \\
\hline DSG & LF & $\begin{array}{l}\text { Subcrítico Rankine } \\
\text { (1ª etapa DRH) }\end{array}$ & Agua & 550 & 40.16 & 4 & {$[19,20]$} \\
\hline
\end{tabular}




\begin{tabular}{|c|c|c|c|c|c|c|c|}
\hline HTF & Colector & Ciclo de Potencia & $\begin{array}{l}\text { Fluido } \\
\text { BOP }\end{array}$ & $\begin{array}{l}\text { TIT } \\
\left({ }^{\circ} \mathrm{C}\right)\end{array}$ & $\begin{array}{l}\text { Eficiencia } \\
\text { Neta (\%) }\end{array}$ & $\begin{array}{l}\text { Capítulo } \\
\text { Tesis }\end{array}$ & Ref. \\
\hline DSG & $\mathrm{LF}$ & $\begin{array}{l}\text { Supercrítico Rankine } \\
\text { (2a etapa DRH) }\end{array}$ & Agua & 550 & 42.66 & 4 & {$[19,20]$} \\
\hline DSG & LF & $\begin{array}{l}\text { Supercrítico Rankine } \\
\left(1 \stackrel{a}{2} 2^{a}, 3^{\text {a }} \text { etapa DRH) }\right.\end{array}$ & Agua & 550 & 44.32 & 4 & {$[19,20]$} \\
\hline DSG & LF & $\begin{array}{l}\text { Supercrítico Rankine } \\
(1 \text { a }, 2 \text { a etapa } D R H)\end{array}$ & Agua & 550 & 43.9 & 4 & {$[19,20]$} \\
\hline DSG & LF & $\begin{array}{l}\text { Supercrítico Rankine } \\
(2 \text { a }, 3 \text { a etapa } \mathrm{DRH})\end{array}$ & Agua & 550 & 43.56 & 4 & {$[19,20]$} \\
\hline
\end{tabular}

La eficiencia de planta resultante estaría entre $43 \%$ y $45 \%$, ver los resultados detallados en las tablas 13.3 y 13.4, dependiendo de la tecnología de campo solar elegida, colectores lineales con DSG o MS. Correspondiendo el mayor rendimiento a la tecnología de sales fundidas como fluido caloportador, y el menor rendimiento a la solución de generación de vapor en los colectores lineales, ver el capítulo 4 para más información al respecto. El desarrollo de turbinas de vapor supercríticas continúa en fase de desarrollo industrial. Un primer esfuerzo de colaboración entre SNL y Siemens se indica en [18]. Este trabajo fue presentada en la Conferencia Internacional NURER 2014 [19], y posteriormente seleccionada para su publicación en la revista Journal of Polytechnics de la Universidad de Gazi en Turquía [20].

Tabla 13.4. Resumen del rendimiento energético de las diferentes soluciones tecnológicas analizadas en el capítulo 4.

\begin{tabular}{|c|c|c|c|c|c|c|c|}
\hline HTF & Colector & Ciclo de Potencia & $\begin{array}{l}\text { Fluido } \\
\text { BOP }\end{array}$ & $\begin{array}{l}\text { TIT } \\
\left({ }^{\circ} \mathrm{C}\right)\end{array}$ & $\begin{array}{l}\text { Eficiencia } \\
\text { Neta (\%) }\end{array}$ & $\begin{array}{l}\text { Capítulo } \\
\text { Tesis }\end{array}$ & Ref. \\
\hline MS & PTC & $\begin{array}{l}\text { Subcrítico Rankine } \\
\text { (2 etapa DRH) }\end{array}$ & Agua & 550 & 40.41 & 4 & {$[19,20]$} \\
\hline MS & PTC & $\begin{array}{l}\text { Subcrítico Rankine } \\
\text { (1 }{ }^{a} \text { etapa DRH) }\end{array}$ & Agua & 550 & 40.09 & 4 & {$[19,20]$} \\
\hline MS & PTC & $\begin{array}{l}\text { Supercrítico Rankine } \\
\text { (2a etapa DRH) }\end{array}$ & Agua & 550 & 43.82 & 4 & {$[19,20]$} \\
\hline MS & PTC & $\begin{array}{l}\text { Supercrítico Rankine } \\
(1 \underline{a}, 2 \underline{a}, 3 \underline{a} \text { etapa } D R H)\end{array}$ & Agua & 550 & 44.72 & 4 & {$[19,20]$} \\
\hline MS & PTC & $\begin{array}{l}\text { Supercrítico Rankine } \\
(1 \text { a }, 2 \text { a etapa } D R H)\end{array}$ & Agua & 550 & 44.39 & 4 & {$[19,20]$} \\
\hline MS & PTC & $\begin{array}{l}\text { Supercrítico Rankine } \\
(2 \text { a }, 3 \text { a etapa } \mathrm{DRH})\end{array}$ & Agua & 550 & 43.82 & 4 & {$[19,20]$} \\
\hline MS & LF & $\begin{array}{l}\text { Subcrítico Rankine } \\
\text { (2a etapa DRH) }\end{array}$ & Agua & 550 & 40.44 & 4 & {$[19,20]$} \\
\hline MS & LF & $\begin{array}{l}\text { Subcrítico Rankine } \\
\text { (1 } 1 \text { a etapa DRH) }\end{array}$ & Agua & 550 & 40.16 & 4 & {$[19,20]$} \\
\hline MS & LF & $\begin{array}{l}\text { Supercrítico Rankine } \\
\text { (2a etapa DRH) }\end{array}$ & Agua & 550 & 43.77 & 4 & {$[19,20]$} \\
\hline MS & LF & $\begin{array}{l}\text { Supercrítico Rankine } \\
(1 \text { a }, 2 \text { a }, 3 \text { a etapa DRH) }\end{array}$ & Agua & 550 & 45.06 & 4 & {$[19,20]$} \\
\hline MS & LF & $\begin{array}{l}\text { Supercrítico Rankine } \\
(1 \text { a }, 2 \text { a etapa } D R H)\end{array}$ & Agua & 550 & 44.62 & 4 & {$[19,20]$} \\
\hline
\end{tabular}




\begin{tabular}{llllllll}
\hline HTF & Colector & Ciclo de Potencia & $\begin{array}{l}\text { Fluido } \\
\text { BOP }\end{array}$ & $\begin{array}{l}\text { TIT } \\
\left({ }^{\circ} \mathrm{C}\right)\end{array}$ & $\begin{array}{l}\text { Eficiencia } \\
\text { Neta (\%) }\end{array}$ & $\begin{array}{l}\text { Capítulo } \\
\text { Tesis }\end{array}$ & Ref. \\
\hline MS & LF & $\begin{array}{l}\text { Supercrítico Rankine } \\
(2 \mathrm{a}, 3 \text { a etapa DRH) }\end{array}$ & Agua & 550 & 44.27 & 4 & {$[19,20]$} \\
\hline
\end{tabular}

En el capítulo quinto se realiza una primera aproximación de diseño del acoplamiento de los campos solares con colectores lineales PTC o LF, y los ciclos de potencia s- $\mathrm{CO}_{2}$ Brayton [21]. Como primera conclusión se deduce que la utilización de intercambiadores de calor de tipología tubos carcasa entre los campos solares y el ciclo de potencia, constituye una alternativa tecnológica viable desde el punto de vista económico y operacional frente a los intercambiadores de tipología de circuito impreso, con tecnología no desclasificada, y solo en poder pocas empresas a nivel mundial, como Heatric [22].

En el capítulo sexto ha continuado con el trabajo iniciado por Dyreby [53] y se han modelizado otras dos configuraciones adicionales al ciclo simple con recuperación (SB) y al ciclo con recompresión (RC). Las nuevas configuraciones analizadas son: configuración con enfriamiento parcial antes de la recompresión (PCRC), y configuración con recompresión y enfriamiento intermedio en el compresor principal ( $\mathrm{RCMCl}$ ). Así mismo, se ha integrado como opcional, una etapa de recalentamiento en las turbinas de todas configuaciones citadas: SB, RC, PCRC, $\mathrm{RCMCl}$. Se realiza la optimización multiparámetro de las variables de operación de los ciclos Brayton con los algoritmos de cálculo SUBPLEX [11], UOBYQA [12] y NEWUOA [13], fijando diferentes valores de tamaño térmico, UA, en los recuperadores del ciclo, y como resultado se obtienen valores de eficiencia neta del 50\%, optimizando por tanto, el área de apertura efectiva y el coste de inversión de los colectores solares. En la figura 13.1 se ilustran los resultados más siginificativos del capítulo 6 para la configuración de ciclo de potencia s- $\mathrm{CO}_{2} \mathrm{RC}$.

Así mismo se ha procedido al diseño de detalle de los recuperadores de alta (LTR) y baja presión (HTR), de los intercambiadores de acoplamiento del ciclo de potencia con los campos solares principal (PHX) y de recalentamiento (RHX), y finalmente se ha diseñado el intercambiador de acoplamiento del ciclo con el foco frío. En todos los casos se ha elegido como patrón de diseño la tipología de intercambiadores de circuito impreso (PCHE). Este diseño de detalle de los cambiadores permite un cálculo detallado de las pérdidas de presión a través de estos equipos, factor que tiene un impacto directo en la eficiencia energética del ciclo.

Se ha particularizado la aplicación de los ciclos Brayton mediante su acoplamiento a las plantas termosolares con colectores lineales PTC o LF. Se ha realizado el diseño detallado de los campos solares estimando el área de apertura efectiva para una potencia fija. Se han calculado las pérdidas de presión en el campo solar y se ha realizado una estimación económica del coste de los colectores solares.

Fijando diferentes valores del tamaño térmico (UA) de los recuperadores se ha comprobado que se mejora la eficiencia energética de los ciclos Brayton. Esta solución de diseño se puede llevar a la práctica incrementando la longitud de los cambiadores, o su sección transversal, así como optimizando el diseño de los canales para garantizar una mejora transferencia de calor. 
En este trabajo se ha fijado la presión de entrada en turbina a 250 bar. En la Dyreby [53] se confirma que un incremento de la presión de diseño a la entrada de turbina principal, no siempre se corresponde con una mejora de la eficiencia energética del ciclo. Existe una presión óptima a la salida del compresor que depende la máxima temperatura de operación, y está normalmente en el rango entre 300-350 bar, cuando el tamaño térmico de los recuperadores alcanzan su mínimo.

Los resultados también indican que el ciclo RC no es siempre inherentemente más eficiente que el ciclo SB con recuperación de calor. Para ciclos con recuperadores pequeños, la penalización asociada a desequilibrio en los recuperadores no justifica el trabajo adicional requerido por el recompresor.

La operación a temperaturas mayores en el foco frío (UHS) requiere un incremento de la presión a la entrada del compresor (CIP) para maximizar la eficiencia energética del ciclo. Las temperaturas mayore en el UHS también reducen la fracción de caudal de recompresión porque se opera lejos de la temperatura crítica del $\mathrm{CO}_{2}$, resultando un mayor equilibrio natural de la recuperación del proceso de transferencia de calor.

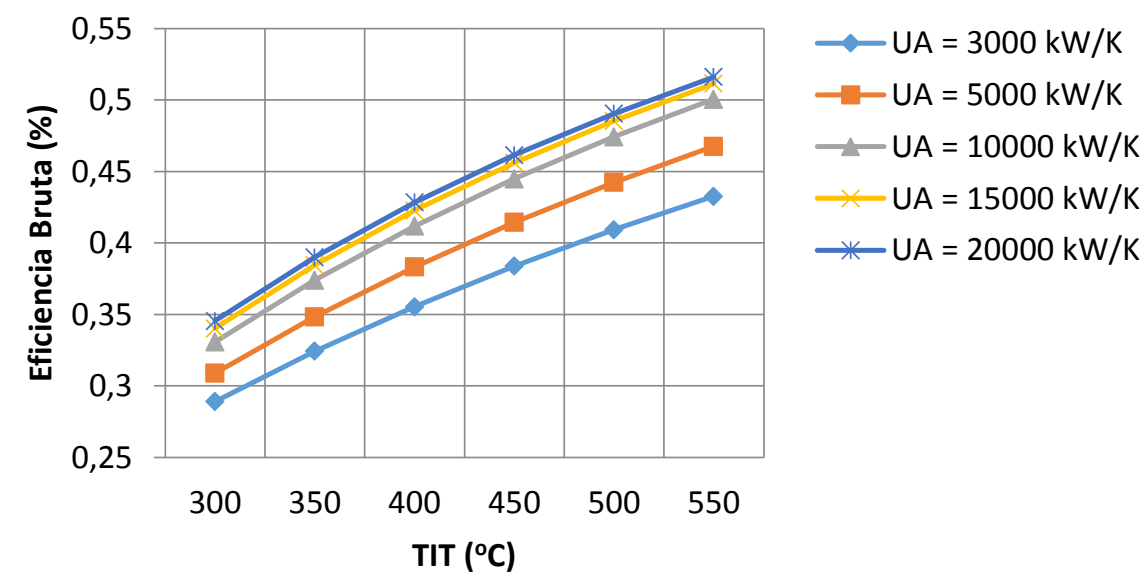

Figura 13.1. Resumen del rendimiento energético de las diferentes soluciones tecnológicas analizadas en el capítulo 6. Eficiencia Bruta Vs. TIT, ciclo $\mathrm{RC} \mathrm{s-} \mathrm{CO}_{2}$ con recalentamiento.

En este trabajo también se proponen otras soluciones tecnológicas para acoplar los ciclos s$\mathrm{CO}_{2}$ Brayton con colectores lineales. Las soluciones presentadas están alineadas con la utilización de diferentes HTF: aceites térmicos en los colectores lineales en el capítulo 7 [23] (tabla 13.5), la generación directa de vapor en los tubos absorbedores en el capítulo 8 [24] (tabla 13.6), y en los campos solares híbridos Dual-Loop, combinando diferentes HTFs en una misma planta CSP en el capítulo 9 [23] (tabla 13.7). Estas tres tecnologías permiten como principal ventaja elegir el coste y grado de aleación del material de los tubos absorbedores, ya que la corrosión provocada sobre los metales por los diferentes fluidos caloportadores es muy diferente. Así mismo, la temperatura de entrada en turbina ( 400 ㄷ) limita la opción de aceites como fluidos caloportadores a unos rendimientos energéticos al 42.5\%. Esta limitación es 
superada por la opción de generación directa de vapor y campos solares híbridos Dual-Loop con TIT de $550 \circ \mathrm{C}$, con rendimientos de hasta el $50 \%$.

En las tres configuraciones de planta indicadas, se concluye que la solución óptima de acoplamiento entre el ciclo de potencia y el campo solar lo constituirían los intercambiadores de calor tubos-carcasa, con $\mathrm{CO}_{2}$ por el interior de los tubos y el aceite térmico o vapor de agua, por el lado carcasa. Estos intercambiadores presentan costes inferiores a los cambiadores PCHE y permiten seleccionar un material con baja aleación para la carcasa ya que el aceite y el agua no son tan corrosivos como el $\mathrm{CO}_{2}$ a altas presiones y temperaturas, y a la vez presentan buenos coeficientes de transferencia de calor HTC, tal y como ha quedado experimentalmente comprobado por Bechtel [25].

Tabla 13.5. Resumen del rendimiento energético de las diferentes soluciones analizadas en el capítulo 7. Eficiencia Neta de planta termosolar con colectores lineales y aceite como HTF, acoplada a ciclo de potencia s- $\mathrm{CO}_{2}$ Brayton RC con Recalentamiento, UA=15000 kW/K.

\begin{tabular}{cccccc}
\hline $\begin{array}{c}\text { TIT } \\
\left({ }^{\circ} \mathrm{C}\right)\end{array}$ & $\begin{array}{c}\text { SF } \\
\text { Configuración }\end{array}$ & $\begin{array}{c}\text { Eficiencia } \\
\text { Bruta }(\%)\end{array}$ & $\begin{array}{c}\text { Eficiencia } \\
\text { Neta }(\%)\end{array}$ & $\begin{array}{c}\text { LTR Pinch } \\
\left({ }^{\circ} \mathrm{C}\right)\end{array}$ & $\begin{array}{c}\text { HTR Pinch } \\
\left({ }^{\circ} \mathrm{C}\right)\end{array}$ \\
\hline 550 & Solar Salt & 51.24 & 49.61 & 4.3 & 4.3 \\
520 & Hitec XL & 49.71 & 48.15 & 4.7 & 4.8 \\
415 & Dowtherm A & 43.42 & 41.96 & 6.2 & 7.1 \\
390 & Syltherm 800 & 41.66 & 40.17 & 6.2 & 7.8 \\
390 & Therminol VP1 & 41.63 & 40.22 & 6.2 & 7.8 \\
375 & Therminol 75 & 40.50 & 39.14 & 5.8 & 8.7 \\
\hline
\end{tabular}

Tabla 13.6. Resumen del rendimiento energético de las diferentes soluciones analizadas en el capítulo 8. Eficiencia neta de planta termosolar con colectores lineales y DSG como HTF, ciclo de potencia s- $\mathrm{CO}_{2}$ Brayton $\mathrm{RC}$, y diferencia mínimas de temperaturas en los recuperadores pinch-point.

\begin{tabular}{lllllll}
\hline $\begin{array}{l}\text { TIT } \\
\left({ }^{\circ} \mathrm{C}\right)\end{array}$ & $\begin{array}{l}\text { UA } \\
(\mathrm{kW} / \mathrm{K})\end{array}$ & $\begin{array}{l}\text { SF } \\
\text { Config. }\end{array}$ & $\begin{array}{l}\text { Eficiencia } \\
\text { Bruta }(\%)\end{array}$ & $\begin{array}{l}\text { Eficiencia } \\
\text { Neta }(\%)\end{array}$ & $\begin{array}{l}\text { LTR Pinch } \\
\left({ }^{\circ} \mathrm{C}\right)\end{array}$ & $\begin{array}{l}\text { HTR Pinch } \\
\left({ }^{\circ} \mathrm{C}\right)\end{array}$ \\
\hline 400 & 3000 & Configuración 1 & 33.39 & 32.34 & 12.2 & 17.9 \\
550 & 3000 & Configuración 2 & 42.94 & 38.91 & 24.1 & 58.7 \\
400 & 5000 & Configuración 1 & 36.32 & 35.19 & 14.8 & 33.6 \\
550 & 5000 & Configuración 2 & 46.26 & 42.16 & 15.8 & 30.9 \\
400 & 10000 & Configuración 1 & 38.84 & 37.63 & 8.7 & 14.2 \\
550 & 10000 & Configuración 2 & 49.45 & 45.25 & 9.1 & 10.2 \\
400 & 15000 & Configuración 1 & 39.84 & 38.61 & 6.1 & 7.8 \\
550 & 15000 & Configuración 2 & 50.54 & 46.28 & 4.5 & 4.5 \\
400 & 20000 & Configuración 1 & 40.36 & 39.11 & 4.6 & 4.9 \\
550 & 20000 & Configuración 2 & 51.01 & 46.75 & 2.4 & 2.4 \\
\hline
\end{tabular}


Tabla 13.7. Resumen del rendimiento energético de las diferentes soluciones analizadas en el capítulo 9. Planta termosolar con colectores lineales acoplada a ciclo de potencia s- $\mathrm{CO}_{2}$ Brayton SB con recalentamiento. Potencia Bruta fija 50MWe. Tamaño térmico de los recuperadores $U A=5000 \mathrm{~kW} / \mathrm{K}$.

\begin{tabular}{|c|c|c|c|c|c|c|c|}
\hline \multirow[b]{2}{*}{$\begin{array}{l}\text { Config. } \\
\text { SF }\end{array}$} & \multirow[b]{2}{*}{$\begin{array}{l}\text { TIT } \\
\left({ }^{\circ} \mathrm{C}\right)\end{array}$} & \multirow[b]{2}{*}{$\begin{array}{l}\text { SF principal } \\
\text { HTF }\end{array}$} & \multirow[b]{2}{*}{$\begin{array}{l}\text { SF Recalentamiento } \\
\text { HTF }\end{array}$} & \multicolumn{2}{|c|}{ Sin $\Delta \mathrm{P}$ en los $\mathrm{HX}$} & \multicolumn{2}{|c|}{ Con $\Delta \mathrm{P}$ en los $\mathrm{HX}$} \\
\hline & & & & $\begin{array}{l}\text { Effic. } \\
\text { Neta } \\
(\%)\end{array}$ & $\begin{array}{l}\text { Recuperador } \\
\text { pinch point } \\
\left({ }^{\circ} \mathrm{C}\right)\end{array}$ & $\begin{array}{l}\text { Effic. } \\
\text { Neta } \\
(\%)\end{array}$ & $\begin{array}{l}\text { Recuperador } \\
\text { pinch point } \\
\left({ }^{\circ} \mathrm{C}\right)\end{array}$ \\
\hline 1 & 400 & Solar Salt & Solar Salt & 36.05 & 3.7 & 35.48 & 4.1 \\
\hline 1 & 400 & DowTherm A & DowTherm A & 36.05 & 3.7 & 35.35 & 4.1 \\
\hline 3 (ver Nota) & 450 & DowTherm A & DowTherm A & 38.67 & 3.1 & 37.94 & 3.6 \\
\hline 2 & 450 & DowTherm A & Solar Salt & 38.70 & 3.1 & 38.15 & 3.4 \\
\hline 1 & 450 & Solar Salt & Solar Salt & 38.75 & 3.1 & 38.22 & 3.4 \\
\hline 2 & 500 & DowTherm A & Solar Salt & 41.17 & 2.6 & 40.59 & 3.1 \\
\hline 1 & 500 & Solar Salt & Solar Salt & 41.20 & 2.6 & 40.67 & 2.9 \\
\hline 2 & 550 & DowTherm A & Solar Salt & 43.42 & 2.3 & 42.84 & 2.7 \\
\hline 1 & 550 & Solar Salt & Solar Salt & 43.44 & 2.3 & 42.93 & 2.6 \\
\hline
\end{tabular}

Nota: La configuración 3 incluye campo solar Dual-Loop, tanto en el campo solar principal como en el campo solar de recalentamiento, figura 9.4. El campo con aceite térmico Dowtherm A, caliente al fluido de trabajo s- $\mathrm{CO}_{2}$ hasta $380^{\circ} \mathrm{C}$, y el campo solar con Solar Salt, sobrecalienta el fluido de trabajo s-CO $\mathrm{CO}_{2}$ hasta $450^{\circ} \mathrm{C}$.

En los dos últimos capítulos se estudian diferentes fluidos de trabajo en los ciclos de potencia supercríticos Brayton como alternativa al $\mathrm{CO}_{2}$. En el capítulo decimo se desarrolla un primer estudio de utilización del Etano como fluido de trabajo en el BOP, los resultados quedan documentados en [26], figura 13.2. Como principal conclusión se confirma que el rendimiento de los ciclos de etano es superior que la de los ciclos con $\mathrm{CO}_{2}$, del orden al $2 \%$. Sin embargo, hace falta realizar un esfuerzo inversor en investigación que garantice sistemas para evitar la descomposición química y posible riesgo de explosión del etano a altas presiones y temperaturas.

Otros fluidos de trabajo ( $\mathrm{Xe}, \mathrm{CH}_{4}, \mathrm{~N}_{2}$ y $\mathrm{SF}_{6}$ ) estudiados en el capítulo 11, permite disponer en cada caso de diferentes ventajas: en el caso del $\mathrm{Xe}$, es un fluido inerte no corrosivo pero la dimensión de las turbomáquinas sería algo mayor que con el $\mathrm{CO}_{2}$. El SF 6 presenta una alta densidad, lo cual garantiza un buen rendimiento de las turbomáquinas, pero es muy perjudicial para la atmósfera y requiere un control exhaustivo de las fugas, así mismo el tamaño térmico de los intercambiadores requeridos resulta muy superior al del $\mathrm{CO}_{2}$. Finalmente se ha caracterizado también el $\mathrm{CH}_{4}$ y el $\mathrm{N}_{2}$ como fluidos de trabajo en los ciclos. Los resultados de eficiencia energética neta para diferentes tamaños térmicos de los recuperadores de calor es la principal conclusión derivada de este trabajo [27] (figura 13.3).

El programa informático desarrollado en C\# y Fortran, ad hoc para este trabajo permite optimizar los parámetros termodinámicos para estos fluidos con los algoritmos citados SUBPLEX[11], UOBYQA[12], NEWOA[13], y obtener las eficiencias óptimas del ciclo de potencia para los diferentes tamaños térmicos UA de los recuperadores que integran el ciclo Brayton. Así mismo, permite cuantificar el área de apertura efectiva y coste de inversión de los campos solares con colectores lineales PTC o LF para cada configuración del ciclo de potencia Brayton. 
13. Conclusiones y trabajos futuros

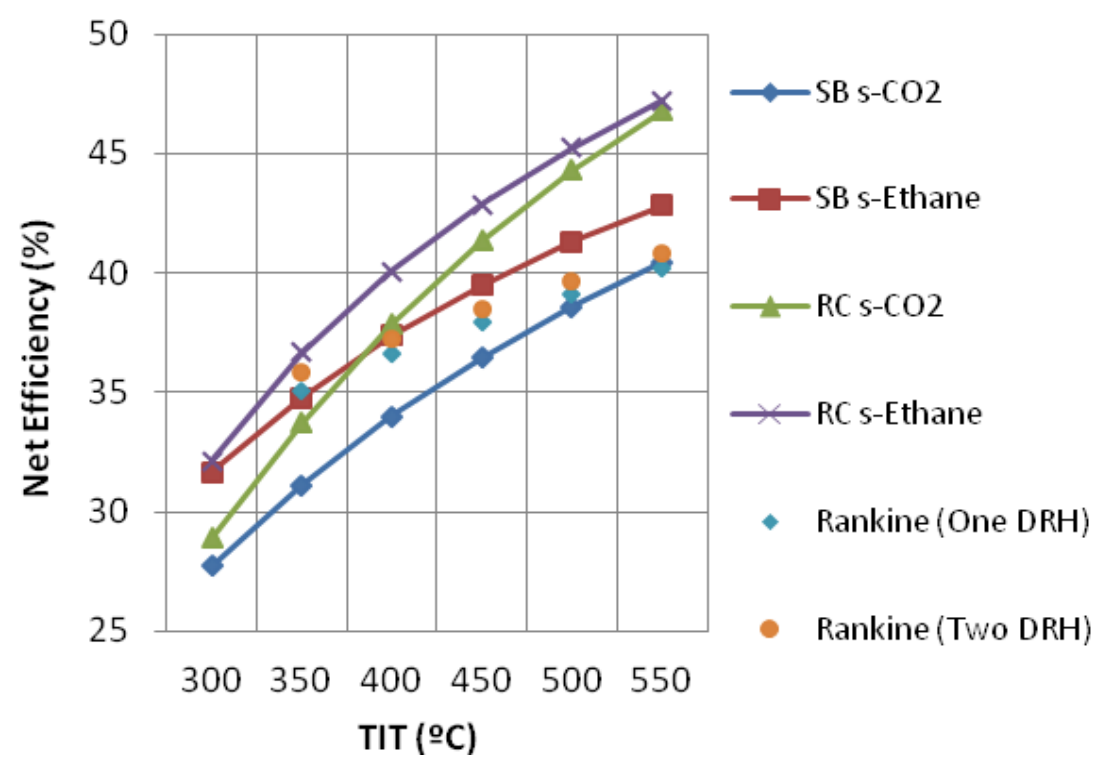

Figura 13.2. Resumen del rendimiento energético de las diferentes soluciones analizadas en el capítulo 10. Eficiencia Neta Vs. TIT ${ }^{(2)}$.

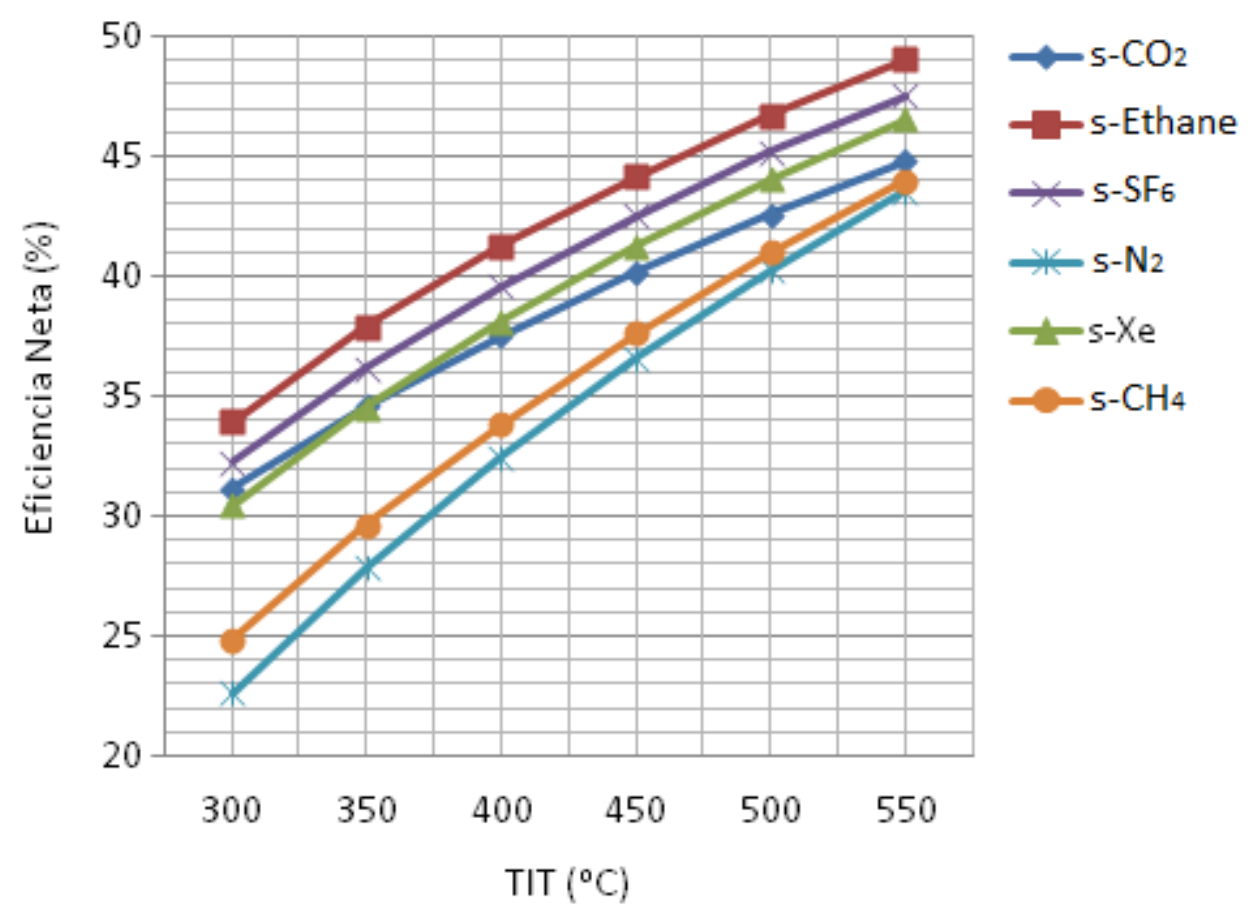

Figura 13.3. Resumen del rendimiento energético de las diferentes soluciones analizadas en el capítulo 11. Eficiencia bruta Vs. TIT, con UA $=15000 \mathrm{~kW} / \mathrm{K}$ para $\mathrm{CO}_{2}, \mathrm{C}_{2} \mathrm{H}_{6}, \mathrm{CH}_{4}, \mathrm{~N}_{2}$, Xe y $\mathrm{N}_{2}$. Ciclo Brayton SB con recuperación de calor.

\subsection{Trabajos Futuros}

En este apartado se tratan por separado los avances tecnológicos previsibles en las tecnologías que integran el campo solar, y las tecnologías que integran los ciclos de potencia.

Los colectores solares lineales continúan en fase de desarrollo. El principal objetivo es reducir su coste unitario manteniendo óptimas prestaciones de concentración de la radiación solar. Sirvan como ejemplo los trabajos para PTC [28-31] y para LF [32-37]. El futuro del desarrollo 
tecnológico también estará alineado con la búsqueda de procesos de fabricación con menor coste y en la utilización de materiales avanzados, como estructuras de materiales compuestos reforzados, similares a las de aviación, con sistemas de producción en serie y construcciones modulares prefabricadas.

En relación a la nueva generación de ciclos de potencia s- $\mathrm{CO}_{2}$ Brayton, bianualmente tiene lugar un simposio internacional [38] para materializar la implantación industrial de esta nueva tipología de BOP. Así mismo, en la cumbre anual de seguimiento de los avances de investigación en energía solar de la iniciativa SunShot [39], se estudian con mayor grado de detalle las tecnologías enfocadas a la nueva generación de plantas termosolares con ciclos de potencia s- $\mathrm{CO}_{2}$ Brayton. Cabe destacar las presentaciones de los proyectos de investigación en esta materia [40-49] principalmente al desarrollo a escala industrial de los equipos que integran los ciclos supercríticos y de los materiales para soportar sus altas temperaturas de operación.

En especial se destaca como trabajo futuro la caracterización del comportamiento de los ciclos Brayton bajo condiciones de irradiación solar fuera del punto de diseño, Part-Load. En este sentido se han publicado recientemente dos artículos [50-52] para calcular el tamaño térmico, UA, y eficiencia energética de los intercambiadores de calor que integran el ciclo Brayton. Dyreby [53], incluye unos primeros modelos del desempeño bajo condiciones Part-Load de las turbinas y compresores en base a los datos obtenidos del lazo de experimentación en SNL [54]. El objetivo sería calcular y predecir la producción anual de electricidad de una planta termosolar con colectores lineales y ciclo de potencia s- $\mathrm{CO}_{2}$ Brayton.

Las curvas características de operación en Part-Load sugieren que para aplicaciones donde es posible operar bajo condiciones fuera del punto de diseño, sería conveniente considerar como referencia de diseño una temperatura de operación del foco frío (UHS) superior a la entrada del compresor a la nominal. Si se diseña para temperaturas más bajas en el UHS permite una mayor eficiencia energética del ciclo en condiciones nominales, sin embargo, la operación de estos diseños a temperaturas mayores en el UHS, daría como resultado una limitación de la potencia y una menor eficiencia energética. La degradación energética en las condiciones Offdesign, cuando se incrementa la temperatura a la entrada del compresor, es principalmente debida al incremento de la relación entre la velocidad tip y la velocidad de chorro en la turbina, Vtip/Vspouting, reduciendo la eficiencia energética de este equipo. Para turbinas radiales lowreaction, la eficiencia bajo condiciones Off-design se ve reducida significativamente cuando el valor de la relación, Vtip/Vspouting, se aproxima a la unidad. Como valores de referencia se citan los siguientes (figura 13.4):

- Vtip/Vspouting=0.7 Eficiencia Turbina=1

- Vtip/Vspouting=0.8 Eficiencia Turbina $=0.95$

- Vtip/Vspouting=0.9 Eficiencia Turbina $=0.78$

- Vtip/Vspouting< 0.9 Caida exponencial de la Eficiencia de la Turbina

Como se puede apreciar este fenómeno de caída de la eficiencia de la turbina conforme se incrementa la relación, Vtip/Vspouting, no es tan acentuado en la turbina del lazo 
experimental en el SNL, tal y como destaca Dyreby [53]. Este hecho se debe a que la turbina de SNL tiene un grado de reacción no despreciable.

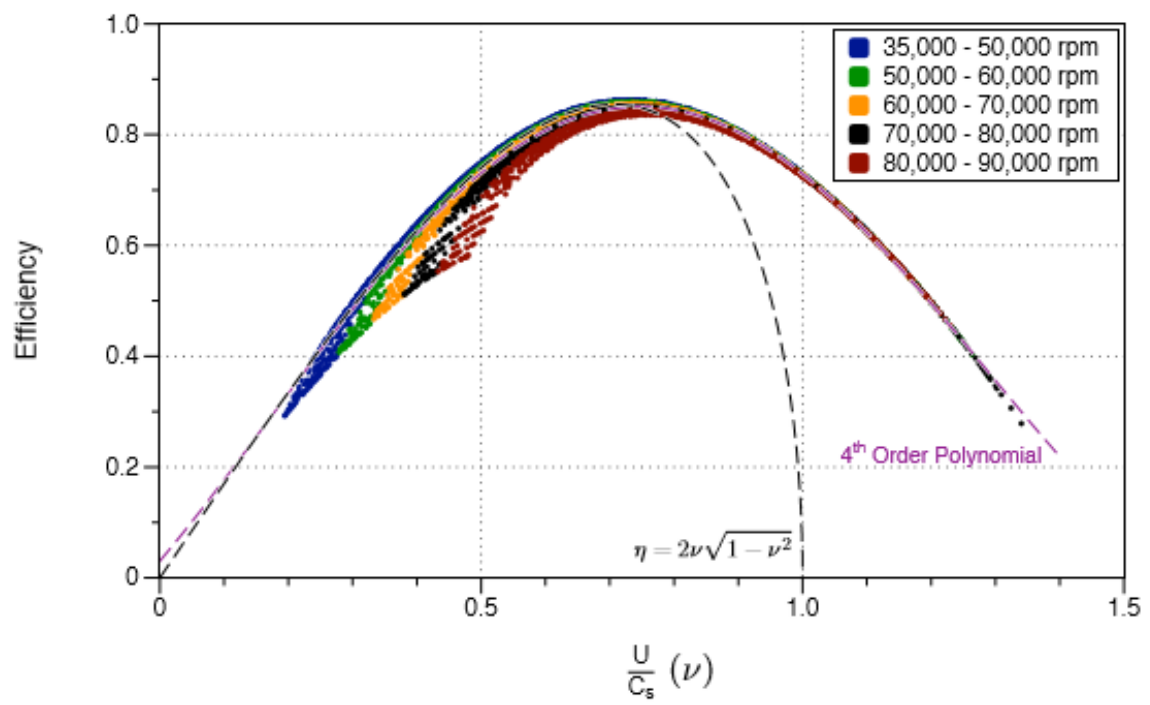

Figura 13.4. Predicción de la eficiencia de la turbina frente a la relación entre la velocidad del tip y la velocidad de chorro [53].

$\mathrm{U}_{\text {tip }}$ es la velocidad del alabe inducido.

La velocidad de chorro spouting velocity $\mathrm{C}_{\mathrm{s}}$ es la velocidad que se alcanzaría si el fluido fuera expandido en condiciones isoentrópicas:

$C_{s}=\sqrt{2 \cdot \Delta h_{i}} \quad$ Velocidad de Chorro, Spouting velocity.

Las observaciones confirman que los diseños a temperaturas menores a la nominal presentan una limitación de operación sustancial bajo condiciones de operación Off-design. Estos diseños enfocados a maximizar las condiciones de operación lo más cercanas posible al punto crítico del $\mathrm{CO}_{2}$ son ventajosas para plantas de potencia diseñadas para operar raramente fuera de las condiciones de diseño de la temperatura del foco frío (UHS). Estas plantas suelen estar enfriadas por agua cuya temperatura presenta variaciones tan significativas como el aire. Es el caso de plantas termosolares con enfriamiento seco, donde presumiblemente es muy posible que las condiciones ambientales de operación del foco frío varíen a lo largo del año considerablemente de las condiciones nominales. En este caso se aconseja considerar como temperatura de diseño del foco frío una temperatura mayor que la nominal de operación. Así mismo, el dimensionamiento del sistema de enfriamiento del foco frío será sobredimensionado a tal efecto.

El margen de sobredimensionamiento de diseño del foco frío, propuesto anteriormente para plantas termosolares acopladas a ciclos Brayton, está limitado por la operación a la entrada del compresor en condiciones termodinámicas por debajo de la temperatura crítica del $\mathrm{CO}_{2}$. Hay que poner especial cuidado y consideración a que operar a presiones y temperaturas inferiores a las del punto crítico puede dar lugar a condiciones bifásicas en el interior de los compresores. Lo cual podría generar el fenómeno de cavitación y daño de los álabes de las turbomáquinas. Una posibilidad para las condiciones bifásicas (asumiendo que el sistemas y las turbomáquinas 


\section{Conclusiones y trabajos futuros}

no están diseñados para condiciones transcríticas) es limitar la presión de entrada al compresor a presiones por encima de la presión crítica, pero estas condiciones pueden dar lugar que las turbomáquinas operen fuera de su rango de operación. Otra opción es introducir un bypass de control en el ciclo de potencia para prevenir que la potencia generada sea superior a la de diseño, aunque el efecto de este bypass sería negativo en el rendimiento energético de la instalación.

Otra opción particular para plantas termosolares, sería desviar el exceso de caudal del bypass hacia el sistema de almacenamiento térmico TES. Otra opción pasaría por sobredimensionar las turbomáquinas para aprovechar el exceso de potencia producida para alimentar a los equipos auxiliares (ventiladores del ACHE, bombas del SF, etc) y considerar las pérdidas parásitas del generador y resto de sistemas de apoyo.

Para evaluar el grado de sobredimensionamiento de diseño requerido, se propone realizar un estudio del incremento de coste de la planta termosolar considerando diferentes temperaturas del UHS por encima de las condiciones nominales de operación. También debería cuantificar la potencia, eficiencia energética y condiciones de operación cuando la temperatura del foco fría sea inferior a la de diseño.

La operación del sistemas bajos condiciones inferiores a la temperatura crítica, manteniendo la presión por encima del punto crítico para evitar condiciones bifásicas, también debería ser evaluadas.

El sistema de almacenamiento térmico TES de la planta termosolar proporciona un sistema de compensación de las fluctuaciones inherentes a las variaciones de temperatura en el UHS. Se propone estudiar la posibilidad de un almacenamiento térmico en el foco frío, aprovechando la noche para refrigeración de un fluido refrigerante, similar al propuesto en la referencia [68].

La utilización de otros fluidos de trabajo en el ciclo de potencia Brayton permite el estudio más detallado de su comportamiento para condiciones de operación fuera de la temperatura de diseño del foco frío (UHS).

Hay que hacer especial mención de los sistemas de control de los ciclos de potencias supercríticos acoplados a plantas solares. El Sol es una fuente de energía de naturaleza variable con el tiempo. La temperatura ambiente del foco frío también es variable a lo largo de los días. Y finalmente la demanda energética y el precio de la electricidad es también variable a lo largo del tiempo. Estas son los principales variables a las que se tienen que acomodar las tecnologías de los equipos que integran las plantas termosolares. Para ello se disponen de sistemas de almacenamiento térmico tanto en el foco caliente como en el foco frío. El almacenamiento térmico (TES) mediante tanques de sales fundidas [55] u otras alternativas tecnológicas [56-58], tendría también que ser integrado en las tipologías de plantas propuestas. Como alternativa a la generación de electricidad para optimizar el precio de venta de la producción energética, se puede continuar con el estudio de la producción de combustibles, como el Hidrógeno [59-60]. 
El estudio de detalle del sumidero de calor (UHS), para minimizar su consumo energético [6163], así como su integración con diferentes aplicaciones industriales [64] donde se requiera cogeneración de calor [65], desalación de agua del mar [66-67], procesos petroquímicos, refino de petróleo, permitiría incrementar sustancialmente la eficiencia neta de la planta termosolar. Hay que hacer especial mención al consumo eléctrico de los ventiladores del sistema de enfriamiento del sumidero de calor mediante aire en convección forzada (ACHE). En este estudio se ha estimado que su consumo para un sistema híbrido con agua sería del $4 \%$ de la potencia bruta generada. El objetivo de trabajos futuros estaría enfocado a reducir estos consumos auxiliares. Así mismo, se ha confirmado que existe una gran sensibilidad de la temperatura de entrada al compresor, muy relacionada con un difícil control y diseño del ACHE u otros sistemas de refrigeración. Se concluye que la optimización del diseño del UHS es pieza clave para garantizar la viabilidad industrial de los ciclos Brayton en las plantas termosolares.

Como solución innovadora recientemente propuesta se propone el almacenamiento térmico en el foco frío. Este concepto ha sido recientemente propuesto por Franklin Keith Miller de la Universidad de Wisconsin-Madison en [68]. Las sinergias con otras tecnologías de diseño del UHS, como el enfriamiento criogénico, tienen que ser estudiadas para intentar optimizar el rendimiento y operación de los compresores del ciclo Brayton. El diseño de detalle y desarrollo de procesos de fabricación con menores costes [22, 25, 42, 48], que permitan incrementar el tamaño térmico y la compacidad de los intercambiadores de calor y recuperadores del ciclo de potencia supercrítico, se considera otra pieza fundamental para incrementar el rendimiento energético de los ciclos Brayton teniendo como principal limitación la mínima diferencia de temperatura pinch-point entre el fluido caliente y el frío en los intercambiadores.

Respecto a las tubomáquinas (compresores y turbinas) de los ciclos supercríticos Rankine y Brayton para plantas termosolares, están en fase de desarrollo industrial para garantizar su escalabilidad, para potencias desde $10 \mathrm{MWe}$ hasta $300 \mathrm{MWe}$. Sirvan como ejemplos los las referencias [42, 45, 46, 57].

Finalmente, la hibridación con otras tecnologías de generación solar, como la fotovoltaica y termofotovoltaica [69-71] pueden proporcionar vías para incrementar el rendimiento energéticos de las plantas termosolares con ciclos de potencia supercríticos.

\subsection{Referencias}

[1] B. Kelly, D. Kearney. Thermal Storage Commercial Plant Design Study for a 2-Tank Indirect Molten Salt System. Final Report, May 13, 2002-December 31, 2004. National Renewable Energy Laboratory NREL, Subcontract Report NREL/SR-550-40166 July 2006.

[2] Abengoa solar power plants: Solana and Mojave. http://www.abengoasolar.com

[3] A.Maccari, S.Donnola, F.Matino, S.Tamano. Archimede solar energy molten salt parabolic trough demo plant: Improvements and secondary year of operation. SolarPaces 2015. AlP Conf. Proc. 1734, 100007 (2016).

[4] TSE-1. http://solarlite-csp.com/en/reference/tse-1-kanchanaburi-provinz-thailand/

[5] Rioglass. http://www.rioglass.com/

[6] Norwich Technologies, Inc. Cavity Receivers for Parabolic Solar Troughs. US Patent №: 20130220307 A1. 
[7] Joel Stettenheim. Norwich Technologies. Advanced low cost receiver for parabolic trough solar power. Design for manufacturing. 2016 SunShot CSP Summit and Integration Workshop. U.S. Department of Energy.

[8] S.Obrey, et al. High Temperature Heat Pipe Receiver for Parabolic Trough Collectors. 2016 SunShot CSP Summit and Integration Workshop. U.S. Department of Energy.

[9] Schott Solar. http://www.schott.com

[10] Archimede Solar. http://www.archimedesolarenergy.it

[11] T. Rowan. Functional Stability Analysis of Numerical Algorithms. Ph.D. thesis, Department of Computer Sciences, University of Texas at Austin, 1990.

[12] Powell M.J.D. (2002). UOBYQA: Unconstrained Optimization BY Quadratic Approximation. Mathematical Programming, Series B (Springer) 92: 555-582.

[13] Powell M.J.D. (November 2004). The NEWUOA software for unconstrained optimization without derivatives. Department of Applied Mathematics and Theoretical Physics, Cambridge University. DAMTP 2004/NA05. Retrieved 2014-01-14.

[14] Thermoflow, Inc. https://www.thermoflow.com/

[15] Eric W. Lemmon, Marcia L. Huber. Reference Fluid Thermodynamic and Transport Properties Database (REFPROP) Version 9.1. NIST, U.S. Department of Commerce.

[16] L.Coco-Enríquez, J.Muñoz-Antón, J.M. Martínez-Val. Innovations on direct steam generation in linear Fresnel collectors. SolarPaces 2013, Las Vegas, U.S.

[17] T.Hirsch, A.Khenissi. A systematic comparison on power block efficiencies for CSP plants with direct steam generation. SolarPaces 2013, Las Vegas, U.S.

[18] J.E. Pacheco, T.Wolf, N.Muley. Incorporating Supercritical Steam Turbines into Advanced Molten-Salt Power Tower Plants: Feasibility and Performance. SAND2013-1960, March 2013.

[19] L.Coco-Enríquez, J.Muñoz-Antón, J.M. Martínez-Val. Supercritical Steam power cycle for Line-Focus Solar Power Plants. 4th International Conference on Nuclear and Renewable Energy Resources NURER 2014, Turkey.

[20] L.Coco-Enriquez, J.Muñoz-Anton, J.M.Martinez-Val. Supercritical Steam Power Cycle For Line-Focusing Solar Power Plants. Journal of Polytechnic, 2015; 18 (4): 219-225.

[21] L.Coco-Enríquez, J.Muñoz-Antón, J.M. Martínez-Val. New Generation Line-Focusing Solar Power Plants with Molten Salts and Supercritical Brayton power cycles. 4th International Conference on Nuclear and Renewable Resources NURER 214, Turkey.

[22] D.Shiferaw, J.Montero Carrero, R. Le Pierres. Economic analysis of SCO2 cycles with PCHE Recuperator design optimisation. The $5^{\text {th }}$ International Symposium - Supercritical CO2 Power Cycles, March 28-31, 2016, San Antonio, Texas, USA.

[23] L.Coco-Enríquez, J.Muñoz-Antón, J.M.Martínez-Val. Dual Loop Line-Focusing Solar Power Plants with Supercritical Brayton Power cycles. $4^{\text {th }}$ European Conference on Renewables Energy Systems ECRES 2016.

[24] L. Coco-Enríquez, J. Muñoz-Antón, J.M. Martínez-Val. Integration between direct steam generation in linear solar collectors and supercritical carbon dioxide Brayton power cycles. International Journal of Hydrogen Energy 40 (2015) 15284-15300.

[25] T.L. Cox, P.M. Fourspring. Comparison of Measured and Analytical Performance of Shelland-Tube Heat Exchanger cooling and Heating supercritical Carbon Dioxide. The $4^{\text {th }}$ International Symposium - Supercritical CO2 Power Cycles, September 9-10, 2014, Pittsburgh, Pennsylvania, USA.

[26] L.Coco-Enríquez, J.Muñoz-Antón, J.M.Martínez-Val Peñalosa. S-Ethane Brayton Power Conversion Systems for Concentrated Solar Power Plant. ASME Journal of Solar Energy Engineering, February 2016, Vol. 138 / 011012-1.

[27] L.Coco-Enríquez, J.Muñoz-Antón, J.M.Martínez-Val Peñalosa. Comparison between s-CO2 and other supercritical working Fluids (s-Ethane, s-SF6, s-Xe, s-CH4, s-N2) in Line-Focusing Solar Power Plants with supercritical Brayton power cycles. $4^{\text {th }}$ ECRES 2016.

[28] K.C.Toussaint, L.J. Guo, G.Zhu. Development of a plannar focusing collector for CSP. 2016 SunShot CSP Summit and Integration Workshop. U.S. Department of Energy. 
[29] G.Mungas. Hyperlight Energy. Low Cost Concentrated Solar Power (CSP) Collector. 2016 SunShot CSP Summit and Integration Workshop. U.S. Department of Energy.

[30] P.Gleckman, Sunvapor Inc. Green Parabolic Trough Collector (GPTC). 2016 SunShot CSP Summit and Integration Workshop. U.S. Department of Energy.

[31] G.Mungas, Hyperlight Energy. Low Cost Concentrated Solar Power (CSP) Collector. 2016 SunShot CSP Summit and Integration Workshop. U.S. Department of Energy.

[32] G.Zhu, T.Wendelin, M.J. Wagner, C.Kutscher. History, current state, and future of linear Fresnel concentrating solar collectors. Solar Energy, Vol.103, 2014, pp 639-652.

[33] A.Rovira, R.Barbero, M.J.Montes, R.Abbas, F.Varela. A comparative analysis of configurations of linear Fresnel collectors for concentrating solar power. En. 73, 2014, 192-203. [34] Molten Salt as a Heat Transfer Fluid in a Linear Fresnel Collector - Commercial Application Backed by Demonstration. Energy Procedia, Volume 69, May 2015, Pages 689-698.

[35] A comprehensive optical characterization of linear Fresnel collectors by means of an analytic study. Applied Energy, 3 February 2016.

[36] M.J. Montes et al. Performance model and thermal comparison of different alternatives for the Fresnel single-tube receiver. App Th En, 104, 2016, pp 162-175.

[37] Paola Boito, Roberto Grena. Optimization of the geometry of Fresnel linear collectors. Solar Energy, Volume 135, October 2016, Pages 479-486.

[38] Supercritical $\mathrm{CO}_{2}$ Power Cycles Symposium. http://www.swri.org

[39] SunShot CSP Summit and Integration Workshop 2016. http://energy.gov

[40] Craig Turchi. Supercritical CO2 Power Cycles: Next-Gen Power for CSP? 2016 SunShot CSP Summit and Integration Workshop. U.S. Department of Energy.

[41] S.Sullivan, R.Zidan. Brayton Energy and Savannah River National Lab. High-Efficiency lowcost solar receiver for use in a supercritical $\mathrm{CO} 2$ recompression cycle. Low-cost metal hydride thermal energy storage system for CSP systems. Solar receiver with integrated thermal storage for a supercritical CO2 power cycle. 2016 SunShot CSP Summit and Integration Workshop. U.S. Department of Energy.

[42] J.Jeffrey Moore. Development of a High Efficiency Hot Gas Turbo-Expander and Low Cost Heat Exchanger for Optimized CSP s-CO2 Operation. 2016 SunShot CSP Summit and Integration Workshop. U.S. Department of Energy.

[43] Mark Anderson. University of Wisconsin Madison. Advanced s-CO2 cycles. 2016 SunShot CSP Summit and Integration Workshop. U.S. Department of Energy.

[44] C.Turchi. CSP Systems Analysis. 2016 SunShot CSP Summit and Integration Workshop. U.S. Department of Energy.

[45] A.Thatte. General Electric. An Integrated Coupled-Physics Framework for Performance and Life Prediction of Supercritical CO2 Turbomachines. 2016 SunShot CSP Summit and Integration Workshop. U.S. Department of Energy.

[46] J.Wilkes et al. Application of an Integrally Geared Compander (integrally-geared compressor-expander) to an s-CO2 Recompression Brayton Cycle. 2016 SunShot CSP Summit and Integration Workshop. U.S. Department of Energy.

[47] J.Moore, A.Thatte. Apollo High-Efficiency s-CO2 Centrifugal Compressor Development. 2016 SunShot CSP Summit and Integration Workshop. U.S. Department of Energy.

[48] K.H. Sandhage. Robust, Cost-Effective Heat Exchangers for $800^{\circ} \mathrm{C}$ Operation with supercritical CO2. 2016 SunShot CSP Summit and Integration Workshop. U.S. DOE.

[49] B.Pint. Lifetime Model Development for Supercritical CO2, CSP Systems. . 2016 SunShot CSP Summit and Integration Workshop. U.S. Department of Energy.

[50] K.Hoopes, David Sánchez, Francesco Crespi. A new method for modeling off-design performance of sCO2 Heat Exchangers without specifying detailed geometry..The 5th International Symposium - Supercritical CO2 Power Cycles March 29-31, 2016, San Antonio.

[51] L.A. Tse, T.Neises. Analysis and Optimization for Off-Design performance of the recompression sCO2 cycles for high temperature CSP. The 5th International Symposium Supercritical CO2 Power Cycles March 29-31, 2016, San Antonio, Texas. 
[52] J.D. Osorio, R.Hovsapian, J.C. Ordoñez. Dynamic analysis of concentrated solar supercritical $\mathrm{CO}_{2}$-based power generation clored-loop cycle. Applied Thermal Engineering, Volume 93, 25 January 2016, Pages 920-934.

[53] Dyreby, J. (2014) Thesis: Modeling the Supercritical Carbon Dioxide Brayton Cycle with Recompression. University of Wisconsin-Madison.

[54] Wright, S.A., Radel, R. F., Vernon, M. E., Rochau, G. E., \& Pickard, P. S. Operation and Analysis of a Supercritical CO2 Brayton Cycle. Sandia Report SAND2010-0171, (2010).

[55] C.Prieto, R.Osuna, A.I.Fernández, L.F. Cabeza. Thermal storage in a MW scale. Molten salt solar termal pilot facility: Plant description and commissioning experiences. Renewable Energy, Volume 99, December 2016, Pages 852-866.

[56] A.Lavine. Thermochemical Storage with Anhydrous Ammonia. 2016 SunShot CSP Summit and Integration Workshop. U.S. Department of Energy.

[57] A.Ambrosini. High Performance Reduction/Oxidation Metal Oxides for Thermochemical Energy Storage (PROMOTES). 2016 SunShot CSP Summit and Integration Workshop. U.S. Department of Energy.

[58] S.Gangwal, Andrew Muto. Demonstration of High-Temperature Calcium-Based Thermochemical Energy Storage System for Use with Concentrating Solar Power Facilities. 2016 SunShot CSP Summit and Integration Workshop. U.S. Department of Energy.

[59] J.Sanz-Bermejo, J.Muñoz-Antón, J.Gonzalez-Aguilar, M.Romero. Optimal integration of a solid-oxide electrolyser cell into a direct steam generation solar tower plant for zero-emission hydrogen production. Applied Energy, Volume 131, 15 October 2014, Pages 238-247.

[60] J.Sanz-Bermejo, J.Muñoz-Anton, J.Gonzalez-Aguilar, M.Romero. Part Load operation of a solid oxide electrolysis system for integration with renewable energy source. International Journal of Hydrogen Energy, Volume 40, Issue 26, 13 July 2015, Pages 8291-8303.

[61] P.J. Hruska, G.F. Nellis, S.A.Klein. Methodology of Modeling and Comparing the Use of Direct Air-Cooling for a Supercritical CO2 Brayton Cycle and a Steam Rankine Cycle. The $5^{\text {th }}$ International Symposium-Supercritical CO2 Power Cycles, March 28-31, 2016, San Antonio.

[62] S.R. Pidaparti, P.J. Hruska, A.Moisseytsev, J.J. Sienicki. Technical and Economic feasibility of dry air cooling for the supercritical $\mathrm{CO} 2$ Brayton cycle using existing technology. The $5^{\text {th }}$ International Symposium-Supercritical CO2 Power Cycles, March 28-31, 2016, San Antonio.

[63] Timothy J. Held, Jason Miller and David J. Buckmaster. A comparative Study of Heat Rejection Systems for $\mathrm{sCO}_{2}$ Power Cycles. The $5^{\text {th }}$ International Symposium-Supercritical $\mathrm{CO} 2$ Power Cycles, March 28-31, 2016, San Antonio, Texas.

[64] Y.Ahn, S.Jun Bae, M.Kim, S.Kuk Cho, Seungjoon Baik, Jeong Ik Lee, Jae Eun Cha. Review of supercritical $\mathrm{CO} 2$ power cycle technology and current status of research and development. Nuclear Engineering and Technology, Volume 47, Issue 6, 2015, pp.647-661.

[65] Parthiv Kurup and Craig Turchi. Initial Investigation into the Potential of CSP Industrial Process Heat for the Southwest United States. NREL, technical report NREL/TP-6A20-64709.

[66] A.Kouta, F.Al-Sulaiman, M.Atif, S.B.Marshad. Entropy, exergy, and cost analyses of solar driven cogenetion systems using supercritical $\mathrm{CO} 2$ Brayton cycles and MEE-TVC desalination system. Energy Conversion and Management, Vol. 115, 2016, pp 253-264.

[67] Osman Ahmed Hamed et al. Concentrating solar power for seawater thermal desalination. Desalination, Volume 396, 17 October 2016, Pages 70-78.

[68] F.K.Miller. Night sky cooling for concentrating solar plants. App. En 180:276-286, 2016.

[69] G.Segev, Y.Rosenwaks, A.Kribus. Sol. En. Mat. and Solar Cells, Vol.140, 2015, pp.464-476.

[70] A.Kribus, G.Segev. Solar energy conversion with photon-enhanced thermoionic emission. IOPScience, Journal of Optics.

[71] S.Balagopal, S.Yee. Ceramatec. Sodium Ion Expansion Power Block for Distributed CSP. 2016 SunShot CSP Summit and Integration Workshop. U.S. Department of Energy. 
ANEXO

\section{Resultados simulaciones capítulo 9. Plantas termosolares con colectores lineales, configuración del campo solar "Dual-Loop" acopladas a ciclos de potencia s- $\mathrm{CO}_{2}$ Brayton}

En las siguientes tablas se hace referencia a las configuraciones siguientes:

1. DSG (TIT=400C). Ver figura 8.8. capítulo 8.

2. Dual (DSG+Hitec $\mathrm{XL}$ ). Ver figuras 9.6 a 9.8 en capítulo 9.

3. Dual (DSG+Thermal Oil). Ver figuras 9.6 a 9.8 en capítulo 9.

4. Dual (DSG+Solar Salt). Ver figuras 9.6 a 9.8 en capítulo 9.

5. DSG (TIT=550C). Ver figura 8.9. capítulo 8 .

6. Solar Salt. Ver figuras 5.5 a 5.7 en capítulo 5 .

7. Hitec XL. Ver figuras 5.5 a 5.7 en capítulo 5.

8. Dual (MS+Thermal Oil). Ver figuras figuras 9.6 a 9.8 en capítulo 9.

\section{Caracterización del ciclo de potencia}

\subsection{Ciclo de potencia Brayton con Recompresión (RC)}

Tabla 1. Centrales Termosolares con campo "Dual-Loop" acopladas a ciclo de potencia RC s$\mathrm{CO}_{2}$ Brayton. Eficiencia y producción de energía.

\begin{tabular}{|c|c|c|c|c|c|c|}
\hline $\begin{array}{l}\text { TIT } \\
(\stackrel{\circ}{ })\end{array}$ & $\begin{array}{l}\text { SF } \\
\text { Config. }\end{array}$ & $\begin{array}{l}\text { UA } \\
(\mathrm{kW} / \mathrm{K})\end{array}$ & $\begin{array}{l}\text { Gross } \\
\text { Efficiency (\%) }\end{array}$ & $\begin{array}{l}\text { Gross Power } \\
\text { (kwe) }\end{array}$ & $\begin{array}{l}\text { Net Efficiency } \\
(\%)\end{array}$ & $\begin{array}{l}\text { Net Power } \\
(\mathrm{kWe})\end{array}$ \\
\hline 400 & $1: D S G$ & 3000 & 33.39 & 50004.2 & 32.34 & 48434 \\
\hline 400 & 2:Dual (DSG+Hitec XL) & 3000 & 35.57 & 50001.7 & 34.45 & 48428 \\
\hline 400 & 3:Dual (DSG+Thermal Oil) & 3000 & 35.47 & 50001.7 & 34.34 & 48195 \\
\hline 550 & 4:Dual (DSG+Solar Salt) & 3000 & 44.17 & 50020.4 & 40.79 & 46198 \\
\hline 550 & 5:DSG & 3000 & 42.94 & 50001.4 & 38.91 & 45306 \\
\hline 400 & $1: D S G$ & 5000 & 36.32 & 50002.3 & 35.19 & 48446 \\
\hline 400 & 2:Dual (DSG+Hitec XL) & 5000 & 38.37 & 50003.5 & 37.17 & 48437 \\
\hline 400 & 3:Dual (DSG+Thermal Oil) & 5000 & 38.44 & 50003.7 & 37.04 & 48181 \\
\hline 550 & 4:Dual (DSG+Solar Salt) & 5000 & 47.64 & 50032.4 & 44.33 & 46553 \\
\hline 550 & $5: D S G$ & 5000 & 46.26 & 50005.2 & 42.16 & 45572 \\
\hline 400 & 1:DSG & 10000 & 38.84 & 50003.2 & 37.63 & 48451 \\
\hline 400 & 2:Dual (DSG+Hitec XL) & 10000 & 41.22 & 50003.2 & 39.93 & 48444 \\
\hline 400 & 3:Dual (DSG+Thermal Oil) & 10000 & 41.30 & 50003.8 & 39.78 & 48165 \\
\hline 550 & 4:Dual (DSG+Solar Salt) & 10000 & 50.70 & 50019.1 & 47.64 & 47000 \\
\hline 550 & $5: D S G$ & 10000 & 49.45 & 49999.7 & 45.25 & 45750 \\
\hline 400 & 1:DSG & 15000 & 39.84 & 50004.8 & 38.61 & 48457 \\
\hline 400 & 2:Dual (DSG+Hitec XL) & 15000 & 42.32 & 50003.9 & 41.01 & 48451 \\
\hline 400 & 3:Dual (DSG+Thermal Oil) & 15000 & 42.41 & 50004.3 & 40.85 & 48164 \\
\hline 550 & 4:Dual (DSG+Solar Salt) & 15000 & 51.88 & 50025 & 48.76 & 47014 \\
\hline 550 & $5: D S G$ & 15000 & 50.54 & 50002.6 & 46.28 & 45795 \\
\hline 400 & $1: D S G$ & 20000 & 40.36 & 50004.4 & 39.11 & 48458 \\
\hline
\end{tabular}




\begin{tabular}{|c|c|c|c|c|c|c|}
\hline $\begin{array}{l}\text { TIT } \\
\text { (oC) }\end{array}$ & $\begin{array}{l}\text { SF } \\
\text { Config. }\end{array}$ & $\begin{array}{l}\text { UA } \\
(\mathrm{kW} / \mathrm{K})\end{array}$ & $\begin{array}{l}\text { Gross } \\
\text { Efficiency (\%) }\end{array}$ & $\begin{array}{l}\text { Gross Power } \\
\text { (kwe) }\end{array}$ & $\begin{array}{l}\text { Net Efficiency } \\
\text { (\%) }\end{array}$ & $\begin{array}{l}\text { Net Power } \\
(\mathrm{kWe})\end{array}$ \\
\hline 400 & 2:Dual (DSG+Hitec XL) & 20000 & 42.98 & 50004.1 & 41.39 & 48160 \\
\hline 400 & 3:Dual (DSG+Thermal Oil) & 20000 & 42.98 & 50002.4 & 41.39 & 48158 \\
\hline 550 & 4:Dual (DSG+Solar Salt) & 20000 & 52.39 & 50032.4 & 49.23 & 47014 \\
\hline 550 & $5: D S G$ & 20000 & 51.01 & 50003.2 & 46.75 & 45830 \\
\hline
\end{tabular}

Tabla 2. Centrales Termosolares con campo "Dual-Loop" acopladas a ciclo de potencia RC s$\mathrm{CO}_{2}$ Brayton. Eficiencia y producción de energía.

\begin{tabular}{|c|c|c|c|c|c|c|}
\hline$\overline{\mathrm{TIT}}\left({ }^{\circ} \mathrm{C}\right)$ & $\begin{array}{l}\text { SF } \\
\text { Config. }\end{array}$ & $\begin{array}{l}\mathrm{UA} \\
(\mathrm{kW} / \mathrm{K})\end{array}$ & $\begin{array}{l}\text { Gross Efficiency } \\
\text { (\%) }\end{array}$ & $\begin{array}{l}\text { Gross Power } \\
\text { (kwe) }\end{array}$ & $\begin{array}{l}\text { Net Efficiency } \\
\text { (\%) }\end{array}$ & $\begin{array}{l}\text { Net Power } \\
\text { (kWe) }\end{array}$ \\
\hline 400 & 6:Solar Salt & 3000 & 35.59 & 50003.9 & 34.43 & 48378 \\
\hline 400 & 7:Hitec XL & 3000 & 35.58 & 50025.7 & 34.43 & 48479 \\
\hline 450 & 6:Solar Salt & 3000 & 38.46 & 50083.2 & 37.22 & 48464 \\
\hline 450 & 7:Hitec XL & 3000 & 38.46 & 50083.1 & 37.22 & 48474 \\
\hline 450 & 8:Dual (MS+ Oil) & 3000 & 38.43 & 50002.5 & 37.17 & 48361 \\
\hline 500 & 6:Solar Salt & 3000 & 41.04 & 50040 & 39.71 & 48424 \\
\hline 500 & 7:Hitec XL & 3000 & 41.03 & 50040.1 & 39.72 & 48438 \\
\hline 500 & 8:Dual (MS+ Oil) & 3000 & 40.98 & 50000.8 & 39.65 & 48371 \\
\hline 520 & 6:Hitec XL & 3000 & 41.93 & 50018.8 & 40.59 & 48419 \\
\hline 550 & 7:Solar Salt & 3000 & 43.37 & 50080 & 41.97 & 48460 \\
\hline 400 & 6:Solar Salt & 5000 & 38.43 & 50002.1 & 37.2 & 48392 \\
\hline 400 & 7:Hitec XL & 5000 & 38.43 & 50037.6 & 37.2 & 48433 \\
\hline 450 & 6:Solar Salt & 5000 & 41.54 & 50003.9 & 40.21 & 48402 \\
\hline 450 & 7:Hitec XL & 5000 & 41.54 & 50003.8 & 40.22 & 48412 \\
\hline 450 & 8:Dual (MS+Oil) & 5000 & 41.49 & 50005.4 & 40.15 & 48389 \\
\hline 500 & 6:Solar Salt & 5000 & 44.34 & 50062.8 & 42.92 & 48461 \\
\hline 500 & 7:Hitec XL & 5000 & 44.34 & 50062.9 & 42.93 & 48474 \\
\hline 500 & 8:Dual (MS+Oil) & 5000 & 44.29 & 50001.7 & 42.86 & 48389 \\
\hline 520 & 6:Hitec XL & 5000 & 45.32 & 50002.1 & 43.88 & 48416 \\
\hline 550 & 7:Solar Salt & 5000 & 46.87 & 50024 & 45.37 & 48420 \\
\hline 400 & 6:Solar Salt & 10000 & 41.25 & 50003 & 39.93 & 48407 \\
\hline 400 & 7:Hitec XL & 10000 & 41.25 & 50041.5 & 39.94 & 48451 \\
\hline 450 & 6:Solar Salt & 10000 & 44.54 & 50000.9 & 43.13 & 48412 \\
\hline 450 & 7:Hitec XL & 10000 & 44.54 & 50001.2 & 43.13 & 48422 \\
\hline 450 & 8:Dual (MS+Oil) & 10000 & 44.54 & 50006 & 43.11 & 48402 \\
\hline 500 & 6:Solar Salt & 10000 & 47.50 & 50022.1 & 45.99 & 48434 \\
\hline 500 & 7:Hitec XL & 10000 & 47.49 & 50022.5 & 46 & 48446 \\
\hline 500 & 8:Dual (MS+Oil) & 10000 & 47.50 & 50004.6 & 45.98 & 48405 \\
\hline 520 & 6:Hitec XL & 10000 & 48.58 & 50003 & 47.05 & 48428 \\
\hline 550 & 7:Solar Salt & 10000 & 50.15 & 49999.1 & 48.55 & 48408 \\
\hline 400 & 6:Solar Salt & 15000 & 42.32 & 50002.2 & 40.98 & 48411 \\
\hline 400 & 7:Hitec XL & 15000 & 42.32 & 50002.3 & 40.98 & 48417 \\
\hline 450 & 6:Solar Salt & 15000 & 45.66 & 50040.6 & 44.22 & 48455 \\
\hline 450 & 7:Hitec XL & 15000 & 45.65 & 50041 & 44.22 & 48465 \\
\hline 450 & 8:Dual (MS+Oil) & 15000 & 45.70 & 50000.8 & 44.24 & 48401 \\
\hline 500 & 6:Solar Salt & 15000 & 48.60 & 50005 & 47.06 & 48421 \\
\hline 500 & 7:Hitec XL & 15000 & 48.60 & 50004.7 & 47.07 & 48433 \\
\hline 500 & 8:Dual (MS+Oil) & 15000 & 48.67 & 50004.3 & 47.05 & 48339 \\
\hline
\end{tabular}




\begin{tabular}{lllllll}
\hline TIT $\left({ }^{\circ} \mathrm{C}\right)$ & $\begin{array}{l}\text { SF } \\
\text { Config. }\end{array}$ & $\begin{array}{l}\text { UA } \\
(\mathrm{kW} / \mathrm{K})\end{array}$ & $\begin{array}{l}\text { Gross Efficiency } \\
(\%)\end{array}$ & $\begin{array}{l}\text { Gross Power } \\
(\mathrm{kwe})\end{array}$ & $\begin{array}{l}\text { Net Efficiency } \\
(\%)\end{array}$ & $\begin{array}{l}\text { Net Power } \\
(\mathrm{kWe})\end{array}$ \\
\hline 520 & 6:Hitec XL & 15000 & 49.71 & 50003.5 & 48.15 & 48432 \\
550 & 7:Solar Salt & 15000 & 51.24 & 50003.3 & 49.61 & 48416 \\
400 & 6:Solar Salt & 20000 & 42.88 & 50009.1 & 41.52 & 48420 \\
400 & 7:Hitec XL & 20000 & 42.88 & 50021.9 & 41.52 & 48439 \\
450 & 6:Solar Salt & 20000 & 46.20 & 50021.7 & 44.74 & 48439 \\
450 & 7:Hitec XL & 20000 & 46.20 & 50021.9 & 44.74 & 48448 \\
450 & 8:Dual (MS+Oil) & 20000 & 46.22 & 50002.5 & 44.75 & 48405 \\
500 & 6:Solar Salt & 20000 & 49.08 & 50044.2 & 47.53 & 48461 \\
500 & 7:Hitec XL & 20000 & 49.08 & 50044.2 & 47.54 & 48473 \\
500 & 8:Dual (MS+Oil) & 20000 & 49.21 & 50004.9 & 47.5 & 48271 \\
520 & 6:Hitec XL & 20000 & 50.21 & 50001.6 & 48.63 & 48432 \\
550 & 7:Solar Salt & 20000 & 51.73 & 50001.2 & 50.09 & 48416 \\
\hline
\end{tabular}

\subsection{Ciclo de potencia Brayton con Enfrimiento Parcial previo y posterior Recompresión} (PCRC).

Tabla 3. Centrales Termosolares con campo "Dual-Loop" acopladas a ciclo de potencia PCRC s$\mathrm{CO}_{2}$ Brayton. Eficiencia y producción de energía.

\begin{tabular}{|c|c|c|c|c|c|c|}
\hline $\begin{array}{l}\text { TIT } \\
\text { (oC) }\end{array}$ & $\begin{array}{l}\text { SF } \\
\text { Config. }\end{array}$ & $\begin{array}{l}\text { UA } \\
(\mathrm{kW} / \mathrm{K})\end{array}$ & $\begin{array}{l}\text { Gross } \\
\text { Efficiency(\%) }\end{array}$ & $\begin{array}{l}\text { Gross Power } \\
(\mathrm{kWe})\end{array}$ & $\begin{array}{l}\text { Net Efficiency } \\
\text { (\%) }\end{array}$ & $\begin{array}{l}\text { Net Power } \\
(\mathrm{kWe})\end{array}$ \\
\hline 400 & $1: D S G$ & 3000 & 33.94 & 50007.7 & 32.87 & 48430 \\
\hline 400 & 2:Dual (DSG+Hitec XL) & 3000 & 35.88 & 50004.6 & 34.75 & 48428 \\
\hline 400 & 3:Dual (DSG+Thermal Oil) & 3000 & 35.94 & 50004.6 & 34.63 & 48173 \\
\hline 550 & 4:Dual (DSG+Solar Salt) & 3000 & 44.15 & 50001.8 & 40.85 & 46261 \\
\hline 550 & $5: D S G$ & 3000 & 42.74 & 50019.7 & 38.87 & 45490 \\
\hline 400 & $1: D S G$ & 5000 & 35.86 & 50011.1 & 34.73 & 48438 \\
\hline 400 & 2:Dual (DSG+Hitec XL) & 5000 & 38.04 & 50004.9 & 36.85 & 48438 \\
\hline 400 & 3:Dual (DSG+Thermal Oil) & 5000 & 38.11 & 50004.9 & 36.71 & 48165 \\
\hline 550 & 4:Dual (DSG+Solar Salt) & 5000 & 46.53 & 50003.8 & 43.3 & 46533 \\
\hline 550 & $5: D S G$ & 5000 & 45.15 & 50006.7 & 41.19 & 45614 \\
\hline 400 & 1:DSG & 10000 & 37.38 & 50011.6 & 36.21 & 48444 \\
\hline 400 & 2:Dual (DSG+Hitec XL) & 10000 & 39.68 & 50011.3 & 38.44 & 48447 \\
\hline 400 & 3:Dual (DSG+Thermal Oil) & 10000 & 39.76 & 50011.3 & 38.29 & 48163 \\
\hline 550 & 4:Dual (DSG+Solar Salt) & 10000 & 48.16 & 50004.2 & 44.91 & 46623 \\
\hline 550 & $5: D S G$ & 10000 & 46.57 & 50028.1 & 42.57 & 45727 \\
\hline 400 & 1:DSG & 15000 & 37.83 & 50012.1 & 36.65 & 48451 \\
\hline 400 & 2:Dual (DSG+Hitec XL) & 15000 & 40.14 & 50005.1 & 38.88 & 48443 \\
\hline 400 & 3:Dual (DSG+Thermal Oil) & 15000 & 40.22 & 50005.1 & 38.73 & 48157 \\
\hline 550 & 4:Dual (DSG+Solar Salt) & 15000 & 48.51 & 50003.2 & 45.26 & 46653 \\
\hline 550 & $5: D S G$ & 15000 & 46.94 & 50024.8 & 42.92 & 45744 \\
\hline 400 & 1:DSG & 20000 & 38.04 & 50011.8 & 36.85 & 48450 \\
\hline 400 & 2:Dual (DSG+Hitec XL) & 20000 & 40.34 & 50010.4 & 39.07 & 48447 \\
\hline 400 & 3:Dual (DSG+Thermal Oil) & 20000 & 40.42 & 50010.4 & 38.93 & 48160 \\
\hline 550 & 4:Dual (DSG+Solar Salt) & 20000 & 48.65 & 50002.3 & 45.42 & 46688 \\
\hline 550 & $5: D S G$ & 20000 & 47.08 & 50023 & 43.09 & 45778 \\
\hline
\end{tabular}


Tabla 4. Centrales Termosolares con campo "Dual-Loop" acopladas a ciclo de potencia PCRC s$\mathrm{CO}_{2}$ Brayton. Eficiencia y producción de energía.

\begin{tabular}{|c|c|c|c|c|c|c|}
\hline 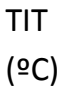 & $\begin{array}{l}\text { SF } \\
\text { Config. }\end{array}$ & $\begin{array}{l}\text { UA } \\
(\mathrm{kW} / \mathrm{K})\end{array}$ & $\begin{array}{l}\text { Gross } \\
\text { Efficiency(\%) }\end{array}$ & $\begin{array}{l}\text { Gross Power } \\
\text { (kWe) }\end{array}$ & $\begin{array}{l}\text { Net Efficiency } \\
(\%)\end{array}$ & $\begin{array}{l}\text { Net Power } \\
(\mathrm{kWe})\end{array}$ \\
\hline 400 & 6:Solar Salt & 3000 & 35.81 & 50003.9 & 34.64 & 48379 \\
\hline 400 & 7:Hitec XL & 3000 & 35.81 & 50003.5 & 34.65 & 48384 \\
\hline 450 & 6:Solar Salt & 3000 & 38.57 & 50003.8 & 37.32 & 48387 \\
\hline 450 & 7:Hitec XL & 3000 & 38.57 & 50003.8 & 37.33 & 48398 \\
\hline 450 & 8:Dual (MS+Oil) & 3000 & 38.57 & 50003.8 & 37.32 & 48372 \\
\hline 500 & 6:Solar Salt & 3000 & 41.08 & 50003.4 & 39.75 & 48389 \\
\hline 500 & 7:Hitec XL & 3000 & 41.07 & 50003.4 & 39.76 & 48403 \\
\hline 500 & 8:Dual (MS+Oil) & 3000 & 41.08 & 50003.4 & 39.74 & 48375 \\
\hline 520 & 6:Hitec XL & 3000 & 42.01 & 50002.6 & 40.67 & 48402 \\
\hline 550 & 7:Solar Salt & 3000 & 43.37 & 50001.8 & 41.97 & 48384 \\
\hline 400 & 6:Solar Salt & 5000 & 37.99 & 50008.1 & 36.77 & 48395 \\
\hline 400 & 7:Hitec XL & 5000 & 37.99 & 50008.1 & 36.77 & 48401 \\
\hline 450 & 6:Solar Salt & 5000 & 40.89 & 50007.1 & 39.58 & 48402 \\
\hline 450 & 7:Hitec XL & 5000 & 40.88 & 50007.1 & 39.58 & 48412 \\
\hline 450 & 8:Dual (MS+Oil) & 5000 & 40.89 & 50007.1 & 39.57 & 48389 \\
\hline 500 & 6:Solar Salt & 5000 & 43.50 & 50005.7 & 42.1 & 48402 \\
\hline 500 & 7:Hitec XL & 5000 & 43.49 & 50005.7 & 42.1 & 48415 \\
\hline 500 & 8:Dual (MS+Oil) & 5000 & 43.50 & 50005.7 & 42.1 & 48390 \\
\hline 520 & 6:Hitec XL & 5000 & 44.47 & 50005.6 & 43.05 & 48415 \\
\hline 550 & 7:Solar Salt & 5000 & 45.86 & 50004.3 & 44.39 & 48397 \\
\hline 400 & 6:Solar Salt & 10000 & 39.65 & 50009.1 & 38.37 & 48405 \\
\hline 400 & 7:Hitec XL & 10000 & 39.64 & 50009.1 & 38.38 & 48411 \\
\hline 450 & 6:Solar Salt & 10000 & 42.52 & 50007.7 & 41.16 & 48410 \\
\hline 450 & 7:Hitec XL & 10000 & 42.50 & 50007.6 & 41.15 & 48420 \\
\hline 450 & 8:Dual (MS+Oil) & 10000 & 42.53 & 50007.7 & 41.16 & 48396 \\
\hline 500 & 6:Solar Salt & 10000 & 45.07 & 50004.4 & 43.63 & 48407 \\
\hline 500 & 7:Hitec XL & 10000 & 45.06 & 50004.4 & 43.64 & 48420 \\
\hline 500 & 8:Dual (MS+Oil) & 10000 & 45.09 & 50004.4 & 43.64 & 48395 \\
\hline 520 & 6:Hitec XL & 10000 & 46.04 & 50004.7 & 44.58 & 48420 \\
\hline 550 & 7:Solar Salt & 10000 & 47.40 & 50004.2 & 45.88 & 48403 \\
\hline 400 & 6:Solar Salt & 15000 & 40.11 & 50008.1 & 38.82 & 48406 \\
\hline 400 & 7:Hitec XL & 15000 & 40.11 & 50008.1 & 38.83 & 48412 \\
\hline 450 & 6:Solar Salt & 15000 & 42.95 & 50006.4 & 41.58 & 48410 \\
\hline 450 & 7:Hitec XL & 15000 & 42.93 & 50006.4 & 41.56 & 48420 \\
\hline 450 & 8:Dual (MS+Oil) & 15000 & 42.96 & 50006.4 & 41.57 & 48396 \\
\hline 500 & 6:Solar Salt & 15000 & 45.48 & 50004.6 & 44.03 & 48409 \\
\hline 500 & 7:Hitec XL & 15000 & 45.45 & 50004.6 & 44.01 & 48422 \\
\hline 500 & 8:Dual (MS+Oil) & 15000 & 45.48 & 50004.6 & 44.02 & 48397 \\
\hline 520 & 6:Hitec XL & 15000 & 46.41 & 50004 & 44.94 & 48421 \\
\hline 550 & 7:Solar Salt & 15000 & 47.75 & 50003.2 & 46.22 & 48403 \\
\hline 400 & 6:Solar Salt & 20000 & 40.31 & 50008 & 39.02 & 48407 \\
\hline 400 & 7:Hitec XL & 20000 & 40.31 & 50008 & 39.02 & 48413 \\
\hline 450 & 6:Solar Salt & 20000 & 43.13 & 50005.8 & 41.75 & 48411 \\
\hline 450 & 7:Hitec XL & 20000 & 43.10 & 50005.8 & 41.74 & 48420 \\
\hline 450 & 8:Dual (MS+Oil) & 20000 & 43.13 & 50005.8 & 41.74 & 48397 \\
\hline
\end{tabular}




\begin{tabular}{|c|c|c|c|c|c|c|}
\hline $\begin{array}{l}\text { TIT } \\
(\stackrel{o}{C})\end{array}$ & $\begin{array}{l}\text { SF } \\
\text { Config. }\end{array}$ & $\begin{array}{l}\text { UA } \\
(\mathrm{kW} / \mathrm{K})\end{array}$ & $\begin{array}{l}\text { Gross } \\
\text { Efficiency(\%) }\end{array}$ & $\begin{array}{l}\text { Gross Power } \\
\text { (kWe) }\end{array}$ & $\begin{array}{l}\text { Net Efficiency } \\
\text { (\%) }\end{array}$ & $\begin{array}{l}\text { Net Power } \\
\text { (kWe) }\end{array}$ \\
\hline 500 & 6:Solar Salt & 20000 & 45.62 & 50003.9 & 44.16 & 48409 \\
\hline 500 & 7:Hitec XL & 20000 & 45.61 & 50003.9 & 44.17 & 48421 \\
\hline 500 & 8:Dual (MS+Oil) & 20000 & 45.64 & 50003.9 & 44.17 & 48397 \\
\hline 520 & 6:Hitec XL & 20000 & 46.56 & 50004 & 45.09 & 48421 \\
\hline 550 & 7:Solar Salt & 20000 & 47.90 & 50002.4 & 46.37 & 48403 \\
\hline
\end{tabular}

\subsection{Ciclo de potencia Brayton con Recompresión y Enfriamiento Intermedio en el Compresor Principal (RCMCl).}

Tabla 5. Centrales Termosolares con campo "Dual-Loop" acopladas a ciclo de potencia RCMCI $\mathrm{s}-\mathrm{CO}_{2}$ Brayton. Eficiencia y producción de energía.

\begin{tabular}{|c|c|c|c|c|c|c|}
\hline $\begin{array}{l}\text { TIT } \\
(\stackrel{o}{C})\end{array}$ & $\begin{array}{l}\text { SF } \\
\text { Config. }\end{array}$ & $\begin{array}{l}\text { UA } \\
(\mathrm{kW} / \mathrm{K})\end{array}$ & $\begin{array}{l}\text { Gross } \\
\text { Efficiency (\%) }\end{array}$ & $\begin{array}{l}\text { Gross Power } \\
\text { (kWe) }\end{array}$ & $\begin{array}{l}\text { Net Efficiency } \\
(\%)\end{array}$ & $\begin{array}{l}\text { Net Power } \\
(\mathrm{kWe})\end{array}$ \\
\hline 400 & $1: D S G$ & 3000 & 34.20 & 50010.3 & 33.13 & 48443 \\
\hline 400 & 2:Dual (DSG+Hitec XL) & 3000 & 36.03 & 50053.9 & 34.89 & 48478 \\
\hline 400 & 3:Dual (DSG+Thermal Oil) & 3000 & 36.09 & 50053.9 & 34.77 & 48220 \\
\hline 550 & 4:Dual (DSG+Solar Salt) & 3000 & 44.62 & 50001.6 & 41.2 & 46175 \\
\hline 550 & 5:DSG & 3000 & 43.38 & 50012.5 & 39.28 & 45280 \\
\hline 400 & $1: D S G$ & 5000 & 36.63 & 50017.5 & 35.49 & 48457 \\
\hline 400 & 2:Dual (DSG+Hitec XL) & 5000 & 38.63 & 50013.7 & 37.42 & 48447 \\
\hline 400 & 3:Dual (DSG+Thermal Oil) & 5000 & 38.70 & 50014 & 37.28 & 48180 \\
\hline 550 & 4:Dual (DSG+Solar Salt) & 5000 & 48.10 & 50007.2 & 44.72 & 46499 \\
\hline 550 & 5:DSG & 5000 & 46.68 & 50026.8 & 42.51 & 45548 \\
\hline 400 & 1:DSG & 10000 & 39.16 & 50008.5 & 37.95 & 48460 \\
\hline 400 & 2:Dual (DSG+Hitec XL) & 10000 & 41.56 & 50037.4 & 40.27 & 48475 \\
\hline 400 & 3:Dual (DSG+Thermal Oil) & 10000 & 41.65 & 50038 & 40.1 & 48177 \\
\hline 550 & 4:Dual (DSG+Solar Salt) & 10000 & 51.19 & 50007.3 & 47.87 & 46766 \\
\hline 550 & $5: D S G$ & 10000 & 49.70 & 50002.1 & 45.46 & 45737 \\
\hline 400 & 1:DSG & 15000 & 40.17 & 50004 & 38.93 & 48460 \\
\hline 400 & 2:Dual (DSG+Hitec XL) & 15000 & 42.67 & 50031.6 & 41.34 & 48470 \\
\hline 400 & 3:Dual (DSG+Thermal Oil) & 15000 & 42.77 & 50032 & 41.17 & 48164 \\
\hline 550 & 4:Dual (DSG+Solar Salt) & 15000 & 51.82 & 50014.8 & 49.18 & 47460 \\
\hline 550 & $5: D S G$ & 15000 & 50.75 & 50002 & 46.47 & 45782 \\
\hline 400 & 1:DSG & 20000 & 40.69 & 50005.7 & 39.43 & 48460 \\
\hline 400 & 2:Dual (DSG+Hitec XL) & 20000 & 43.21 & 50026.9 & 41.86 & 48467 \\
\hline 400 & 3:Dual (DSG+Thermal Oil) & 20000 & 43.32 & 50027.1 & 41.7 & 48161 \\
\hline 550 & 4:Dual (DSG+Solar Salt) & 20000 & 52.29 & 50000.2 & 49.7 & 47519 \\
\hline 550 & 5:DSG & 20000 & 51.21 & 50000.7 & 46.92 & 45814 \\
\hline
\end{tabular}

Tabla 6. Centrales Termosolares con campo "Dual-Loop" acopladas a ciclo de potencia RCMCI s- $\mathrm{CO}_{2}$ Brayton. Eficiencia y producción de energía.

\begin{tabular}{lllllll}
\hline $\begin{array}{l}\text { TIT } \\
\text { (o) })\end{array}$ & $\begin{array}{l}\text { SF } \\
\text { Config. }\end{array}$ & $\begin{array}{l}\text { UA } \\
(\mathrm{kW} / \mathrm{K})\end{array}$ & $\begin{array}{l}\text { Gross Efficiency } \\
(\%)\end{array}$ & $\begin{array}{l}\text { Gross Power } \\
(\mathrm{kWe})\end{array}$ & $\begin{array}{l}\text { Net Efficiency } \\
(\%)\end{array}$ & $\begin{array}{l}\text { Net Power } \\
(\mathrm{kWe})\end{array}$ \\
\hline 400 & 6:Solar Salt & 3000 & 36.01 & 50000.7 & 34.83 & 48370 \\
400 & 7:Hitec XL & 3000 & 36.01 & 50000.7 & 34.84 & 48374 \\
\hline
\end{tabular}




\begin{tabular}{|c|c|c|c|c|c|c|}
\hline $\begin{array}{l}\text { TIT } \\
(\stackrel{\circ}{ })\end{array}$ & $\begin{array}{l}\text { SF } \\
\text { Config. }\end{array}$ & $\begin{array}{l}\text { UA } \\
(\mathrm{kW} / \mathrm{K})\end{array}$ & $\begin{array}{l}\text { Gross Efficiency } \\
(\%)\end{array}$ & $\begin{array}{l}\text { Gross Power } \\
\text { (kWe) }\end{array}$ & $\begin{array}{l}\text { Net Efficiency } \\
\text { (\%) }\end{array}$ & $\begin{array}{l}\text { Net Power } \\
(\mathrm{kWe})\end{array}$ \\
\hline 450 & 6:Solar Salt & 3000 & 38.86 & 50004.1 & 37.6 & 48383 \\
\hline 450 & 7:Hitec XL & 3000 & 38.86 & 50004.1 & 37.6 & 48391 \\
\hline 450 & 8:Dual (MS+Oil) & 3000 & 38.86 & 50004.1 & 37.58 & 48357 \\
\hline 500 & 6:Solar Salt & 3000 & 41.43 & 50007.5 & 40.09 & 48389 \\
\hline 500 & 7:Hitec XL & 3000 & 41.43 & 50007.4 & 40.1 & 48401 \\
\hline 500 & 8:Dual (MS+Oil) & 3000 & 41.44 & 50000.1 & 40.09 & 48365 \\
\hline 520 & 6:Hitec XL & 3000 & 42.39 & 50012.8 & 41.03 & 48406 \\
\hline 550 & 7:Solar Salt & 3000 & 43.78 & 50009.2 & 42.36 & 48387 \\
\hline 400 & 6:Solar Salt & 5000 & 38.61 & 50003.3 & 37.37 & 48388 \\
\hline 400 & 7:Hitec XL & 5000 & 38.60 & 50004.2 & 37.35 & 48393 \\
\hline 450 & 6:Solar Salt & 5000 & 41.72 & 50007.4 & 40.38 & 48401 \\
\hline 450 & 7:Hitec XL & 5000 & 41.72 & 50007.3 & 40.39 & 48409 \\
\hline 450 & 8:Dual (MS+Oil) & 5000 & 41.73 & 50000.2 & 40.37 & 48374 \\
\hline 500 & 6:Solar Salt & 5000 & 44.50 & 50006.2 & 43.07 & 48402 \\
\hline 500 & 7:Hitec XL & 5000 & 44.51 & 50004.8 & 43.09 & 48412 \\
\hline 500 & 8:Dual (MS+Oil) & 5000 & 44.50 & 50006.2 & 43.06 & 48389 \\
\hline 520 & 6:Hitec XL & 5000 & 45.74 & 50009.5 & 44.28 & 48418 \\
\hline 550 & 7:Solar Salt & 5000 & 47.26 & 50002.8 & 45.74 & 48396 \\
\hline 400 & 6:Solar Salt & 10000 & 41.56 & 50004 & 40.23 & 48405 \\
\hline 400 & 7:Hitec XL & 10000 & 41.54 & 50005.2 & 40.22 & 48410 \\
\hline 450 & 6:Solar Salt & 10000 & 44.83 & 50004.2 & 43.4 & 48412 \\
\hline 450 & 7:Hitec XL & 10000 & 44.84 & 50003.3 & 43.42 & 48419 \\
\hline 450 & 8:Dual (MS+Oil) & 10000 & 44.84 & 50004.2 & 43.39 & 48393 \\
\hline 500 & 6:Solar Salt & 10000 & 47.77 & 50004.3 & 46.25 & 48413 \\
\hline 500 & 7:Hitec XL & 10000 & 47.75 & 50006.1 & 46.25 & 48426 \\
\hline 500 & 8:Dual (MS+Oil) & 10000 & 47.78 & 50004.3 & 46.25 & 48402 \\
\hline 520 & 6:Hitec XL & 10000 & 48.82 & 50028.3 & 47.27 & 48448 \\
\hline 550 & 7:Solar Salt & 10000 & 50.38 & 50005 & 48.78 & 48411 \\
\hline 400 & 6:Solar Salt & 15000 & 42.64 & 50004.4 & 41.35 & 48490 \\
\hline 400 & 7:Hitec XL & 15000 & 42.65 & 50004 & 41.36 & 48492 \\
\hline 450 & 6:Solar Salt & 15000 & 45.97 & 50000 & 44.51 & 48412 \\
\hline 450 & 7:Hitec XL & 15000 & 45.95 & 50001.3 & 44.5 & 48422 \\
\hline 450 & 8:Dual (MS+Oil) & 15000 & 45.97 & 50000 & 44.5 & 48400 \\
\hline 500 & 6:Solar Salt & 15000 & 48.87 & 50004.5 & 47.31 & 48418 \\
\hline 500 & 7:Hitec XL & 15000 & 48.84 & 50006.1 & 47.3 & 48430 \\
\hline 500 & 8:Dual (MS+Oil) & 15000 & 48.91 & 50004.5 & 47.26 & 48326 \\
\hline 520 & 6:Hitec XL & 15000 & 49.90 & 50027.3 & 48.32 & 48451 \\
\hline 550 & 7:Solar Salt & 15000 & 51.43 & 50001.2 & 49.79 & 48411 \\
\hline 400 & 6:Solar Salt & 20000 & 43.19 & 50002.9 & 41.88 & 48489 \\
\hline 400 & 7:Hitec XL & 20000 & 43.20 & 50001.8 & 41.89 & 48490 \\
\hline 450 & 6:Solar Salt & 20000 & 46.49 & 50001.4 & 45.01 & 48416 \\
\hline 450 & 7:Hitec XL & 20000 & 46.47 & 50002.7 & 45 & 48425 \\
\hline 450 & 8:Dual (MS+Oil) & 20000 & 46.49 & 50001.4 & 45 & 48403 \\
\hline 500 & 6:Solar Salt & 20000 & 49.35 & 50001.6 & 47.78 & 48417 \\
\hline 500 & 7:Hitec XL & 20000 & 49.33 & 50003.1 & 47.77 & 48429 \\
\hline 500 & 8:Dual (MS+Oil) & 20000 & 49.40 & 50001.6 & 47.72 & 48292 \\
\hline 520 & 6:Hitec XL & 20000 & 50.44 & 50042.5 & 48.85 & 48467 \\
\hline
\end{tabular}




\begin{tabular}{|c|c|c|c|c|c|c|}
\hline $\begin{array}{l}\text { TIT } \\
(\stackrel{o}{ } \text { C) }\end{array}$ & $\begin{array}{l}\text { SF } \\
\text { Config. }\end{array}$ & $\begin{array}{l}\text { UA } \\
(\mathrm{kW} / \mathrm{K})\end{array}$ & $\begin{array}{l}\text { Gross Efficiency } \\
(\%)\end{array}$ & $\begin{array}{l}\text { Gross Power } \\
\text { (kWe) }\end{array}$ & $\begin{array}{l}\text { Net Efficiency } \\
\text { (\%) }\end{array}$ & $\begin{array}{l}\text { Net Power } \\
\text { (kWe) }\end{array}$ \\
\hline 550 & 7:Solar Salt & 20000 & 51.89 & 50000.3 & 50.24 & 48412 \\
\hline
\end{tabular}

\section{2. Área de apertura efectiva y estimación de costes del campo solar}

\subsection{Ciclo Brayton con Recompresión (RC)}

Tabla 7. Centrales Termosolares con campo "Dual-Loop" acopladas a ciclo de potencia RC s$\mathrm{CO}_{2}$ Brayton. Área de apertura efectiva y estimación de costes de los colectores PTC.

\begin{tabular}{|c|c|c|c|c|c|c|c|}
\hline $\begin{array}{l}\text { TIT } \\
\left({ }^{\circ} \mathrm{C}\right)\end{array}$ & $\begin{array}{l}\text { SF } \\
\text { Config. }\end{array}$ & $\begin{array}{l}\text { UA } \\
(\mathrm{kW} / \mathrm{K})\end{array}$ & $\begin{array}{l}\text { PTC SF (m2) } \\
\text { stainless } \\
\text { steel }\end{array}$ & $\begin{array}{l}\text { PTC SF } \\
\left(\mathrm{m}^{2}\right) \\
\text { steel }\end{array}$ & $\begin{array}{l}\text { PTC SF } \\
\text { Cost } \\
\text { (M\$) }\end{array}$ & $\begin{array}{l}\text { Net } \\
\text { Power } \\
(\mathrm{kWe})\end{array}$ & $\begin{array}{l}\text { SF Unitary } \\
\text { Cost } \\
\text { (M\$/MWe) }\end{array}$ \\
\hline 400 & $1: D S G$ & 3000 & 225468 & 0 & 112.99 & 48434 & 2.33 \\
\hline 400 & 2:Dual (DSG+Hitec XL) & 3000 & 212379 & 0 & 106.43 & 48428 & 2.20 \\
\hline 400 & 3:Dual (DSG+Thermal Oil) & 3000 & 160142 & 52061 & 99.82 & 48195 & 2.07 \\
\hline 550 & 4:Dual (DSG+Solar Salt) & 3000 & 186420 & 0 & 93.42 & 46198 & 2.02 \\
\hline 550 & $5: D S G$ & 3000 & 189930 & 0 & 95.18 & 45306 & 2.10 \\
\hline 400 & $1: D S G$ & 5000 & 207272 & 0 & 103.87 & 48446 & 2.14 \\
\hline 400 & 2:Dual (DSG+Hitec XL) & 5000 & 197308 & 0 & 98.87 & 48437 & 2.04 \\
\hline 400 & 3:Dual (DSG+Thermal Oil) & 5000 & 138722 & 58435 & 91.48 & 48181 & 1.90 \\
\hline 550 & 4:Dual (DSG+Solar Salt) & 5000 & 173025 & 0 & 86.71 & 46553 & 1.86 \\
\hline 550 & $5: D S G$ & 5000 & 176294 & 0 & 88.34 & 45572 & 1.94 \\
\hline 400 & $1: D S G$ & 10000 & 194021 & 0 & 97.23 & 48451 & 2.01 \\
\hline 400 & 2:Dual (DSG+Hitec XL) & 10000 & 184754 & 0 & 92.58 & 48444 & 1.91 \\
\hline 400 & 3:Dual (DSG+Thermal Oil) & 10000 & 120116 & 64522 & 84.44 & 48165 & 1.75 \\
\hline 550 & 4:Dual (DSG+Solar Salt) & 10000 & 162835 & 0 & 81.60 & 47000 & 1.74 \\
\hline 550 & $5: D S G$ & 10000 & 164912 & 0 & 82.64 & 45750 & 1.81 \\
\hline 400 & $1: D S G$ & 15000 & 189150 & 0 & 94.79 & 48457 & 1.96 \\
\hline 400 & 2:Dual (DSG+Hitec XL) & 15000 & 180152 & 0 & 90.28 & 48451 & 1.86 \\
\hline 400 & 3:Dual (DSG+Thermal Oil) & 15000 & 113201 & 66826 & 81.84 & 48164 & 1.70 \\
\hline 550 & 4:Dual (DSG+Solar Salt) & 15000 & 159476 & 0 & 79.92 & 47014 & 1.70 \\
\hline 550 & $5: D S G$ & 15000 & 161374 & 0 & 80.87 & 45795 & 1.77 \\
\hline 400 & 1:DSG & 20000 & 186708 & 0 & 93.56 & 48458 & 1.93 \\
\hline 400 & 2:Dual (DSG+Hitec XL) & 20000 & 177595 & 0 & 89.00 & 48160 & 1.85 \\
\hline 400 & 3:Dual (DSG+Thermal Oil) & 20000 & 109803 & 67789 & 80.50 & 48158 & 1.67 \\
\hline 550 & 4:Dual (DSG+Solar Salt) & 20000 & 158057 & 0 & 79.21 & 47014 & 1.68 \\
\hline 550 & $5: D S G$ & 20000 & 159896 & 0 & 80.13 & 45830 & 1.75 \\
\hline
\end{tabular}

Tabla 8. Centrales Termosolares con campo "Dual-Loop" acopladas a ciclo de potencia RC s$\mathrm{CO}_{2}$ Brayton. Área de apertura efectiva y estimación de costes de los colectores PTC.

\begin{tabular}{lllllll}
\hline TIT $\left({ }^{\circ} \mathrm{C}\right)$ & $\begin{array}{l}\text { SF } \\
\text { Config. }\end{array}$ & $\begin{array}{l}\text { UA } \\
(\mathrm{kW} / \mathrm{K})\end{array}$ & $\begin{array}{l}\text { PTC SF }\left(\mathrm{m}^{2}\right) \\
\text { stainless } \\
\text { steel }\end{array}$ & $\begin{array}{l}\text { PTC SF } \\
\left(\mathrm{m}^{2}\right) \text { steel }\end{array}$ & PTC SF Cost (M\$) & $\begin{array}{l}\text { SF Unitary Cost } \\
(\mathrm{M} \$ / \mathrm{MWe})\end{array}$ \\
\hline 400 & 6:Solar Salt & 3000 & 211856 & 0 & 106.16 & 2.19 \\
400 & 7:Hitec XL & 3000 & 211872 & 0 & 106.17 & 2.19 \\
450 & 6:Solar Salt & 3000 & 200674 & 0 & 100.56 & 2.07 \\
450 & 7:Hitec XL & 3000 & 200455 & 0 & 100.45 & 2.07 \\
\hline
\end{tabular}




\begin{tabular}{|c|c|c|c|c|c|c|}
\hline $\operatorname{TIT}\left({ }^{\circ} \mathrm{C}\right)$ & $\begin{array}{l}\text { SF } \\
\text { Config. }\end{array}$ & $\begin{array}{l}\text { UA } \\
\text { (kW/K) }\end{array}$ & $\begin{array}{l}\text { PTC SF }\left(\mathrm{m}^{2}\right) \\
\text { stainless } \\
\text { steel }\end{array}$ & $\begin{array}{l}\text { PTC SF } \\
\left(\mathrm{m}^{2}\right) \text { steel }\end{array}$ & PTC SF Cost (M\$) & $\begin{array}{l}\text { SF Unitary Cost } \\
\text { (M\$/MWe) }\end{array}$ \\
\hline 450 & 8:Dual (MS+Oil) & 3000 & 97661 & 102528 & 87.47 & 1.81 \\
\hline 500 & 6:Solar Salt & 3000 & 193773 & 0 & 97.10 & 2.01 \\
\hline 500 & 7:Hitec XL & 3000 & 193328 & 0 & 96.88 & 2.00 \\
\hline 500 & 8:Dual (MS+Oil) & 3000 & 136428 & 56628 & 89.65 & 1.85 \\
\hline 520 & 6:Hitec XL & 3000 & 191256 & 0 & 95.84 & 1.98 \\
\hline 550 & 7:Solar Salt & 3000 & 191665 & 0 & 96.04 & 1.98 \\
\hline 400 & 6:Solar Salt & 5000 & 196537 & 0 & 98.48 & 2.04 \\
\hline 400 & 7:Hitec XL & 5000 & 196612 & 0 & 98.52 & 2.03 \\
\hline 450 & 6:Solar Salt & 5000 & 185991 & 0 & 93.20 & 1.93 \\
\hline 450 & 7:Hitec XL & 5000 & 185793 & 0 & 93.10 & 1.92 \\
\hline 450 & 8:Dual (MS+Oil) & 5000 & 104769 & 81226 & 83.03 & 1.72 \\
\hline 500 & 6:Solar Salt & 5000 & 180064 & 0 & 90.23 & 1.86 \\
\hline 500 & 7:Hitec XL & 5000 & 179689 & 0 & 90.04 & 1.86 \\
\hline 500 & 8:Dual (MS+Oil) & 5000 & 144630 & 34970 & 85.62 & 1.77 \\
\hline 520 & 6:Hitec XL & 5000 & 177611 & 0 & 89.00 & 1.84 \\
\hline 550 & 7:Solar Salt & 5000 & 178030 & 0 & 89.21 & 1.84 \\
\hline 400 & 6:Solar Salt & 10000 & 183417 & 0 & 91.91 & 1.90 \\
\hline 400 & 7:Hitec XL & 10000 & 183507 & 0 & 91.95 & 1.90 \\
\hline 450 & 6:Solar Salt & 10000 & 173822 & 0 & 87.10 & 1.80 \\
\hline 450 & 7:Hitec XL & 10000 & 173674 & 0 & 87.03 & 1.80 \\
\hline 450 & 8:Dual (MS+Oil) & 10000 & 111783 & 61956 & 79.30 & 1.64 \\
\hline 500 & 6:Solar Salt & 10000 & 168462 & 0 & 84.42 & 1.74 \\
\hline 500 & 7:Hitec XL & 10000 & 168143 & 0 & 84.26 & 1.74 \\
\hline 500 & 8:Dual (MS+Oil) & 10000 & 152239 & 16028 & 82.31 & 1.70 \\
\hline 520 & 6:Hitec XL & 10000 & 166298 & 0 & 83.33 & 1.72 \\
\hline 550 & 7:Solar Salt & 10000 & 166993 & 0 & 83.68 & 1.73 \\
\hline 400 & 6:Solar Salt & 15000 & 178851 & 0 & 89.62 & 1.85 \\
\hline 400 & 7:Hitec XL & 15000 & 178801 & 0 & 89.60 & 1.85 \\
\hline 450 & 6:Solar Salt & 15000 & 169830 & 0 & 85.10 & 1.76 \\
\hline 450 & 7:Hitec XL & 15000 & 169695 & 0 & 85.03 & 1.75 \\
\hline 450 & 8:Dual (MS+Oil) & 15000 & 114128 & 55323 & 77.98 & 1.61 \\
\hline 500 & 6:Solar Salt & 15000 & 164751 & 0 & 82.56 & 1.71 \\
\hline 500 & 7:Hitec XL & 15000 & 164419 & 0 & 82.39 & 1.70 \\
\hline 500 & 8:Dual (MS+Oil) & 15000 & 154664 & 9756 & 81.17 & 1.68 \\
\hline 520 & 6:Hitec XL & 15000 & 162675 & 0 & 81.52 & 1.68 \\
\hline 550 & 7:Solar Salt & 15000 & 163652 & 0 & 82.00 & 1.69 \\
\hline 400 & 6:Solar Salt & 20000 & 176610 & 0 & 88.50 & 1.83 \\
\hline 400 & 7:Hitec XL & 20000 & 176584 & 0 & 88.49 & 1.83 \\
\hline 450 & 6:Solar Salt & 20000 & 167841 & 0 & 84.10 & 1.74 \\
\hline 450 & 7:Hitec XL & 20000 & 167697 & 0 & 84.03 & 1.73 \\
\hline 450 & 8:Dual (MS+Oil) & 20000 & 114970 & 52622 & 77.39 & 1.60 \\
\hline 500 & 6:Solar Salt & 20000 & 163315 & 0 & 81.84 & 1.69 \\
\hline 500 & 7:Hitec XL & 20000 & 163004 & 0 & 81.68 & 1.69 \\
\hline 500 & 8:Dual (MS+Oil) & 20000 & 155312 & 7381 & 80.60 & 1.67 \\
\hline 520 & 6:Hitec XL & 20000 & 161127 & 0 & 80.74 & 1.67 \\
\hline 550 & 7:Solar Salt & 20000 & 162161 & 0 & 81.26 & 1.68 \\
\hline
\end{tabular}




\subsection{Ciclo Brayton con Enfriamiento previo y Recompresión (PCRC)}

Tabla 9. Centrales Termosolares con campo "Dual-Loop" acopladas a ciclo de potencia PCRC s$\mathrm{CO}_{2}$ Brayton. Área de apertura efectiva y estimación de costes de los colectores PTC.

\begin{tabular}{|c|c|c|c|c|c|c|c|}
\hline $\begin{array}{l}\text { TIT } \\
\left({ }^{\circ} \mathrm{C}\right)\end{array}$ & $\begin{array}{l}\text { SF } \\
\text { Config. }\end{array}$ & $\begin{array}{l}\text { UA } \\
(\mathrm{kW} / \mathrm{K})\end{array}$ & $\begin{array}{l}\text { PTC SF } \\
\left(\mathrm{m}^{2}\right) \\
\text { stainless } \\
\text { steel }\end{array}$ & $\begin{array}{l}\text { PTC SF } \\
\left(\mathrm{m}^{2}\right) \\
\text { steel }\end{array}$ & $\begin{array}{l}\text { PTC SF } \\
\text { Cost (M\$) }\end{array}$ & $\begin{array}{l}\text { Net } \\
\text { Power } \\
\text { (MWe) }\end{array}$ & $\begin{array}{l}\text { SF Unitary } \\
\text { Cost } \\
\text { (M\$/MWe) }\end{array}$ \\
\hline 400 & $1: D S G$ & 3000 & 221313 & 0 & 110.90 & 48430 & 2.29 \\
\hline 400 & 2:Dual (DSG+Hitec XL) & 3000 & 210321 & 0 & 105.40 & 48428 & 2.18 \\
\hline 400 & 3:Dual (DSG+Thermal Oil) & 3000 & 157894 & 52228 & 98.75 & 48173 & 2.05 \\
\hline 550 & 4:Dual (DSG+Solar Salt) & 3000 & 186241 & 0 & 93.33 & 46261 & 2.02 \\
\hline 550 & 5:DSG & 3000 & 190053 & 0 & 95.24 & 45490 & 2.09 \\
\hline 400 & 1:DSG & 5000 & 209713 & 0 & 105.09 & 48438 & 2.17 \\
\hline 400 & 2:Dual (DSG+Hitec XL) & 5000 & 198587 & 0 & 99.52 & 48438 & 2.05 \\
\hline 400 & 3:Dual (DSG+Thermal Oil) & 5000 & 141652 & 56743 & 92.31 & 48165 & 1.92 \\
\hline 550 & 4:Dual (DSG+Solar Salt) & 5000 & 176855 & 0 & 88.63 & 46533 & 1.90 \\
\hline 550 & $5: D S G$ & 5000 & 179872 & 0 & 90.14 & 45614 & 1.98 \\
\hline 400 & 1:DSG & 10000 & 201167 & 0 & 100.81 & 48444 & 2.08 \\
\hline 400 & 2:Dual (DSG+Hitec XL) & 10000 & 190722 & 0 & 95.57 & 48447 & 1.97 \\
\hline 400 & 3:Dual (DSG+Thermal Oil) & 10000 & 130792 & 59739 & 87.99 & 48163 & 1.83 \\
\hline 550 & 4:Dual (DSG+Solar Salt) & 10000 & 171154 & 0 & 85.77 & 46623 & 1.84 \\
\hline 550 & 5:DSG & 10000 & 174457 & 0 & 87.42 & 45727 & 1.91 \\
\hline 400 & 1:DSG & 15000 & 198755 & 0 & 99.60 & 48451 & 2.06 \\
\hline 400 & 2:Dual (DSG+Hitec XL) & 15000 & 188529 & 0 & 94.48 & 48443 & 1.95 \\
\hline 400 & 3:Dual (DSG+Thermal Oil) & 15000 & 128090 & 60254 & 86.83 & 48157 & 1.80 \\
\hline 550 & 4:Dual (DSG+Solar Salt) & 15000 & 169938 & 0 & 85.16 & 46653 & 1.83 \\
\hline 550 & 5:DSG & 15000 & 173084 & 0 & 86.74 & 45744 & 1.90 \\
\hline 400 & 1:DSG & 20000 & 197677 & 0 & 99.06 & 48450 & 2.04 \\
\hline 400 & 2:Dual (DSG+Hitec XL) & 20000 & 187620 & 0 & 94.02 & 48447 & 1.94 \\
\hline 400 & 3:Dual (DSG+Thermal Oil) & 20000 & 127038 & 60396 & 86.36 & 48160 & 1.79 \\
\hline 550 & 4:Dual (DSG+Solar Salt) & 20000 & 169482 & 0 & 84.93 & 46688 & 1.82 \\
\hline 550 & 5:DSG & 20000 & 172544 & 0 & 86.47 & 45778 & 1.89 \\
\hline
\end{tabular}

Tabla 10. Centrales Termosolares con campo "Dual-Loop" acopladas a ciclo de potencia PCRC $\mathrm{s}-\mathrm{CO}_{2}$ Brayton. Área de apertura efectiva y estimación de costes de los colectores PTC.

\begin{tabular}{|c|c|c|c|c|c|c|}
\hline $\operatorname{TIT}\left({ }^{\circ} \mathrm{C}\right)$ & $\begin{array}{l}\text { SF } \\
\text { Config. }\end{array}$ & $\begin{array}{l}\text { UA } \\
(\mathrm{kW} / \mathrm{K})\end{array}$ & $\begin{array}{l}\text { PTC SF }\left(\mathrm{m}^{2}\right) \\
\text { stainless } \\
\text { steel }\end{array}$ & $\begin{array}{l}\text { PTC SF }\left(\mathrm{m}^{2}\right) \\
\text { steel }\end{array}$ & PTC SF Cost (M\$) & $\begin{array}{l}\text { SF Unitary } \\
\text { Cost } \\
\text { (M\$̧/MWe) }\end{array}$ \\
\hline 400 & 6:Solar Salt & 3000 & 210717 & 0 & 105.59 & 2.18 \\
\hline 400 & 7:Hitec XL & 3000 & 210635 & 0 & 105.55 & 2.18 \\
\hline 450 & 6:Solar Salt & 3000 & 199807 & 0 & 100.12 & 2.07 \\
\hline 450 & 7:Hitec XL & 3000 & 199603 & 0 & 100.02 & 2.07 \\
\hline 450 & 8:Dual (MS+Oil) & 3000 & 93918 & 105424 & 86.68 & 1.79 \\
\hline 500 & 6:Solar Salt & 3000 & 193474 & 0 & 96.95 & 2.00 \\
\hline 500 & 7:Hitec XL & 3000 & 193047 & 0 & 96.74 & 2.00 \\
\hline 500 & 8:Dual (MS+Oil) & 3000 & 130308 & 62092 & 88.63 & 1.83 \\
\hline 520 & 6:Hitec XL & 3000 & 191577 & 0 & 96.00 & 1.98 \\
\hline 550 & 7:Solar Salt & 3000 & 191444 & 0 & 95.93 & 1.98 \\
\hline 400 & 6:Solar Salt & 5000 & 198912 & 0 & 99.67 & 2.06 \\
\hline 400 & 7:Hitec XL & 5000 & 198859 & 0 & 99.65 & 2.06 \\
\hline 450 & 6:Solar Salt & 5000 & 188872 & 0 & 94.64 & 1.96 \\
\hline 450 & 7:Hitec XL & 5000 & 188704 & 0 & 94.56 & 1.95 \\
\hline 450 & 8:Dual (MS+Oil) & 5000 & 98582 & 89888 & 83.18 & 1.72 \\
\hline
\end{tabular}




\begin{tabular}{|c|c|c|c|c|c|c|}
\hline $\operatorname{TIT}\left({ }^{\circ} \mathrm{C}\right)$ & $\begin{array}{l}\text { SF } \\
\text { Config. }\end{array}$ & $\begin{array}{l}\text { UA } \\
(\mathrm{kW} / \mathrm{K})\end{array}$ & $\begin{array}{l}\text { PTC SF }\left(\mathrm{m}^{2}\right) \\
\text { stainless } \\
\text { steel }\end{array}$ & $\begin{array}{l}\text { PTC SF }\left(\mathrm{m}^{2}\right) \\
\text { steel }\end{array}$ & PTC SF Cost (M\$) & $\begin{array}{l}\text { SF Unitary } \\
\text { Cost } \\
\text { (M\$/MWe) }\end{array}$ \\
\hline 500 & 6:Solar Salt & 5000 & 183233 & 0 & 91.82 & 1.90 \\
\hline 500 & 7:Hitec XL & 5000 & 182864 & 0 & 91.63 & 1.89 \\
\hline 500 & 8:Dual (MS+Oil) & 5000 & 135162 & 47248 & 85.49 & 1.77 \\
\hline 520 & 6:Hitec XL & 5000 & 181626 & 0 & 91.01 & 1.88 \\
\hline 550 & 7:Solar Salt & 5000 & 181727 & 0 & 91.06 & 1.88 \\
\hline 400 & 6:Solar Salt & 10000 & 190812 & 0 & 95.62 & 1.98 \\
\hline 400 & 7:Hitec XL & 10000 & 190749 & 0 & 95.58 & 1.97 \\
\hline 450 & 6:Solar Salt & 10000 & 181858 & 0 & 91.13 & 1.88 \\
\hline 450 & 7:Hitec XL & 10000 & 181744 & 0 & 91.07 & 1.88 \\
\hline 450 & 8:Dual (MS+Oil) & 10000 & 101246 & 80241 & 80.89 & 1.67 \\
\hline 500 & 6:Solar Salt & 10000 & 177114 & 0 & 88.75 & 1.83 \\
\hline 500 & 7:Hitec XL & 10000 & 176759 & 0 & 88.57 & 1.83 \\
\hline 500 & 8:Dual (MS+Oil) & 10000 & 137544 & 38824 & 83.51 & 1.73 \\
\hline 520 & 6:Hitec XL & 10000 & 175754 & 0 & 88.07 & 1.82 \\
\hline 550 & 7:Solar Salt & 10000 & 176187 & 0 & 88.29 & 1.82 \\
\hline 400 & 6:Solar Salt & 15000 & 188648 & 0 & 94.53 & 1.95 \\
\hline 400 & 7:Hitec XL & 15000 & 188586 & 0 & 94.50 & 1.95 \\
\hline 450 & 6:Solar Salt & 15000 & 180087 & 0 & 90.24 & 1.86 \\
\hline 450 & 7:Hitec XL & 15000 & 180006 & 0 & 90.20 & 1.86 \\
\hline 450 & 8:Dual (MS+Oil) & 15000 & 101572 & 78150 & 80.27 & 1.66 \\
\hline 500 & 6:Solar Salt & 15000 & 175595 & 0 & 87.99 & 1.82 \\
\hline 500 & 7:Hitec XL & 15000 & 175319 & 0 & 87.85 & 1.81 \\
\hline 500 & 8:Dual (MS+Oil) & 15000 & 137731 & 37217 & 83.00 & 1.72 \\
\hline 520 & 6:Hitec XL & 15000 & 174401 & 0 & 87.39 & 1.80 \\
\hline 550 & 7:Solar Salt & 15000 & 174954 & 0 & 87.67 & 1.81 \\
\hline 400 & 6:Solar Salt & 20000 & 187728 & 0 & 94.07 & 1.94 \\
\hline 400 & 7:Hitec XL & 20000 & 187667 & 0 & 94.04 & 1.94 \\
\hline 450 & 6:Solar Salt & 20000 & 179373 & 0 & 89.88 & 1.86 \\
\hline 450 & 7:Hitec XL & 20000 & 179282 & 0 & 89.84 & 1.86 \\
\hline 450 & 8:Dual (MS+Oil) & 20000 & 101626 & 77379 & 80.00 & 1.65 \\
\hline 500 & 6:Solar Salt & 20000 & 175068 & 0 & 87.73 & 1.81 \\
\hline 500 & 7:Hitec XL & 20000 & 174728 & 0 & 87.56 & 1.81 \\
\hline 500 & 8:Dual (MS+Oil) & 20000 & 137727 & 36633 & 82.78 & 1.71 \\
\hline 520 & 6:Hitec XL & 20000 & 173852 & 0 & 87.12 & 1.80 \\
\hline 550 & 7:Solar Salt & 20000 & 174445 & 0 & 87.41 & 1.81 \\
\hline
\end{tabular}

\subsection{Ciclo Brayton con Recompresión y Enfriamiento Intermedio en el Compresor Principal (RCMCl)}

Tabla 11. Centrales Termosolares con campo "Dual-Loop" acopladas a ciclo de potencia RCMCI s- $\mathrm{CO}_{2}$ Brayton. Área de apertura efectiva y estimación de costes de los colectores PTC.

\begin{tabular}{llllllll}
\hline $\begin{array}{l}\text { TIT } \\
\left({ }^{\circ} \mathrm{C}\right)\end{array}$ & $\begin{array}{l}\text { SF } \\
\text { Config. }\end{array}$ & $\begin{array}{l}\text { UA } \\
(\mathrm{kW} / \mathrm{K})\end{array}$ & $\begin{array}{l}\text { PTC SF }\left(\mathrm{m}^{2}\right) \\
\text { stainless } \\
\text { steel }\end{array}$ & $\begin{array}{l}\text { PTC SF }\left(\mathrm{m}^{2}\right) \\
\text { Steel }\end{array}$ & $\begin{array}{l}\text { PTC SF } \\
\text { Cost (M\$) }\end{array}$ & $\begin{array}{l}\text { Net } \\
\text { Power } \\
(\mathrm{MWe})\end{array}$ & $\begin{array}{l}\text { SF Unitary } \\
\text { Cost } \\
\mathrm{MW}\end{array}$ \\
\hline 400 & 1:DSG & 3000 & 220124 & 0 & 110.31 & 48443 & 2.28 \\
400 & 2:Dual (DSG+Hitec XL) & 3000 & 209982 & 0 & 105.23 & 48478 & 2.17 \\
400 & 3:Dual (DSG+Thermal Oil) & 3000 & 156747 & 53038 & 98.48 & 48220 & 2.04 \\
550 & 4:Dual (DSG+Solar Salt) & 3000 & 185728 & 0 & 93.07 & 46175 & 2.02 \\
550 & 5:DSG & 3000 & 188247 & 0 & 94.33 & 45280 & 2.08 \\
400 & 1:DSG & 5000 & 205791 & 0 & 103.13 & 48457 & 2.13 \\
400 & 2:Dual (DSG+Hitec XL) & 5000 & 196031 & 0 & 98.24 & 48447 & 2.03
\end{tabular}


Anexo

\begin{tabular}{|c|c|c|c|c|c|c|c|}
\hline 400 & 3:Dual (DSG+Thermal Oil) & 5000 & 140581 & 55275 & 91.22 & 48180 & 1.89 \\
\hline 550 & 4:Dual (DSG+Solar Salt) & 5000 & 172683 & 0 & 86.53 & 46499 & 1.86 \\
\hline 550 & 5:DSG & 5000 & 174993 & 0 & 87.69 & 45548 & 1.93 \\
\hline 400 & 1:DSG & 10000 & 192447 & 0 & 96.44 & 48460 & 1.99 \\
\hline 400 & 2:Dual (DSG+Hitec XL) & 10000 & 182995 & 0 & 91.70 & 48475 & 1.89 \\
\hline 400 & 3:Dual (DSG+Thermal Oil) & 10000 & 119378 & 63460 & 83.67 & 48177 & 1.74 \\
\hline 550 & 4:Dual (DSG+Solar Salt) & 10000 & 162846 & 0 & 81.61 & 46766 & 1.74 \\
\hline 550 & 5:DSG & 10000 & 164311 & 0 & 82.34 & 45737 & 1.80 \\
\hline 400 & 1:DSG & 15000 & 187591 & 0 & 94.01 & 48460 & 1.94 \\
\hline 400 & 2:Dual (DSG+Hitec XL) & 15000 & 179175 & 0 & 89.79 & 48470 & 1.85 \\
\hline 400 & 3:Dual (DSG+Thermal Oil) & 15000 & 113431 & 65579 & 81.49 & 48164 & 1.69 \\
\hline 550 & 4:Dual (DSG+Solar Salt) & 15000 & 161097 & 0 & 80.73 & 47460 & 1.70 \\
\hline 550 & 5:DSG & 15000 & 160885 & 0 & 80.62 & 45782 & 1.76 \\
\hline 400 & 1:DSG & 20000 & 185222 & 0 & 92.82 & 48460 & 1.92 \\
\hline 400 & 2:Dual (DSG+Hitec XL) & 20000 & 176912 & 0 & 88.65 & 48467 & 1.83 \\
\hline 400 & 3:Dual (DSG+Thermal Oil) & 20000 & 111229 & 65514 & 80.36 & 48161 & 1.67 \\
\hline 550 & 4:Dual (DSG+Solar Salt) & 20000 & 159938 & 0 & 80.15 & 47519 & 1.69 \\
\hline 550 & 5:DSG & 20000 & 159449 & 0 & 79.90 & 45814 & 1.74 \\
\hline
\end{tabular}

Tabla 12. Centrales Termosolares con campo "Dual-Loop" acopladas a ciclo de potencia PCRC $\mathrm{s}-\mathrm{CO}_{2}$ Brayton. Área de apertura efectiva y estimación de costes de los colectores PTC.

\begin{tabular}{|c|c|c|c|c|c|c|}
\hline TIT $\left({ }^{\circ} \mathrm{C}\right)$ & $\begin{array}{l}\text { SF } \\
\text { Config. }\end{array}$ & $\begin{array}{l}\text { UA } \\
(\mathrm{kW} / \mathrm{K})\end{array}$ & $\begin{array}{l}\text { PTC SF }\left(\mathrm{m}^{2}\right) \\
\text { stainless steel }\end{array}$ & $\begin{array}{l}\text { PTC SF }\left(\mathrm{m}^{2}\right) \\
\text { steel }\end{array}$ & PTC SF Cost (M\$) & $\begin{array}{l}\text { SF Unitary Cost } \\
\text { (M\$/MWe) }\end{array}$ \\
\hline 400 & 6:Solar Salt & 3000 & 209565 & 0 & 105.01 & 2.17 \\
\hline 400 & 7:Hitec XL & 3000 & 209486 & 0 & 104.97 & 2.17 \\
\hline 450 & 6:Solar Salt & 3000 & 198373 & 0 & 99.40 & 2.05 \\
\hline 450 & 7:Hitec XL & 3000 & 198157 & 0 & 99.30 & 2.05 \\
\hline 450 & 8:Dual (MS+Oil) & 3000 & 97133 & 100863 & 86.58 & 1.79 \\
\hline 500 & 6:Solar Salt & 3000 & 191878 & 0 & 96.15 & 1.99 \\
\hline 500 & 7:Hitec XL & 3000 & 191424 & 0 & 95.92 & 1.98 \\
\hline 500 & 8:Dual (MS+Oil) & 3000 & 135190 & 55778 & 88.71 & 1.83 \\
\hline 520 & 6:Hitec XL & 3000 & 189942 & 0 & 95.18 & 1.97 \\
\hline 550 & 7:Solar Salt & 3000 & 189705 & 0 & 95.06 & 1.96 \\
\hline 400 & 6:Solar Salt & 5000 & 195769 & 0 & 98.10 & 2.03 \\
\hline 400 & 7:Hitec XL & 5000 & 195780 & 0 & 98.10 & 2.03 \\
\hline 450 & 6:Solar Salt & 5000 & 185215 & 0 & 92.81 & 1.92 \\
\hline 450 & 7:Hitec XL & 5000 & 185023 & 0 & 92.71 & 1.92 \\
\hline 450 & 8:Dual (MS+Oil) & 5000 & 103473 & 81443 & 82.46 & 1.70 \\
\hline 500 & 6:Solar Salt & 5000 & 179242 & 0 & 89.82 & 1.86 \\
\hline 500 & 7:Hitec XL & 5000 & 178792 & 0 & 89.59 & 1.85 \\
\hline 500 & 8:Dual (MS+Oil) & 5000 & 143064 & 35678 & 85.10 & 1.76 \\
\hline 520 & 6:Hitec XL & 5000 & 176778 & 0 & 88.58 & 1.83 \\
\hline 550 & 7:Solar Salt & 5000 & 176587 & 0 & 88.49 & 1.83 \\
\hline 400 & 6:Solar Salt & 10000 & 182206 & 0 & 91.30 & 1.89 \\
\hline 400 & 7:Hitec XL & 10000 & 182211 & 0 & 91.31 & 1.89 \\
\hline 450 & 6:Solar Salt & 10000 & 172749 & 0 & 86.56 & 1.79 \\
\hline 450 & 7:Hitec XL & 10000 & 172550 & 0 & 86.46 & 1.79 \\
\hline 450 & 8:Dual (MS+Oil) & 10000 & 109813 & 62808 & 78.63 & 1.62 \\
\hline 500 & 6:Solar Salt & 10000 & 167459 & 0 & 83.91 & 1.73 \\
\hline 500 & 7:Hitec XL & 10000 & 167186 & 0 & 83.78 & 1.73 \\
\hline 500 & 8:Dual (MS+Oil) & 10000 & 149809 & 17468 & 81.63 & 1.69 \\
\hline 520 & 6:Hitec XL & 10000 & 166250 & 0 & 83.31 & 1.72 \\
\hline 550 & 7:Solar Salt & 10000 & 166252 & 0 & 83.31 & 1.72 \\
\hline 400 & 6:Solar Salt & 15000 & 177805 & 0 & 89.10 & 1.84 \\
\hline 400 & 7:Hitec XL & 15000 & 177746 & 0 & 89.07 & 1.84 \\
\hline
\end{tabular}


Anexo

\begin{tabular}{lllllll}
\hline TIT $\left({ }^{\circ} \mathrm{C}\right)$ & $\begin{array}{l}\text { SF } \\
\text { Config. }\end{array}$ & $\begin{array}{l}\text { UA } \\
(\mathrm{kW} / \mathrm{K})\end{array}$ & $\begin{array}{l}\text { PTC SF }\left(\mathrm{m}^{2}\right) \\
\text { stainless steel }\end{array}$ & $\begin{array}{l}\text { PTC SF }\left(\mathrm{m}^{2}\right) \\
\text { steel }\end{array}$ & PTC SF Cost (M\$) & $\begin{array}{l}\text { SF Unitary Cost } \\
(\mathrm{M} \$ \mathrm{MWe})\end{array}$ \\
\hline 450 & 6:Solar Salt & 15000 & 168573 & 0 & 84.47 & 1.74 \\
450 & 7:Hitec XL & 15000 & 168479 & 0 & 84.42 & 1.74 \\
450 & 8:Dual (MS+Oil) & 15000 & 111865 & 56543 & 77.30 & 1.60 \\
500 & 6:Solar Salt & 15000 & 163868 & 0 & 82.11 & 1.70 \\
500 & 7:Hitec XL & 15000 & 163609 & 0 & 81.98 & 1.69 \\
500 & 8:Dual (MS+Oil) & 15000 & 151799 & 11785 & 80.49 & 1.67 \\
520 & 6:Hitec XL & 15000 & 162815 & 0 & 81.59 & 1.68 \\
550 & 7:Solar Salt & 15000 & 163040 & 0 & 81.70 & 1.69 \\
400 & 6:Solar Salt & 20000 & 175602 & 0 & 87.99 & 1.81 \\
400 & 7:Hitec XL & 20000 & 175505 & 0 & 87.94 & 1.81 \\
450 & 6:Solar Salt & 20000 & 166757 & 0 & 83.56 & 1.73 \\
450 & 7:Hitec XL & 20000 & 166670 & 0 & 83.52 & 1.72 \\
450 & 8:Dual (MS+Oil) & 20000 & 112554 & 54046 & 76.71 & 1.58 \\
500 & 6:Solar Salt & 20000 & 162313 & 0 & 81.33 & 1.68 \\
500 & 7:Hitec XL & 20000 & 162060 & 0 & 81.21 & 1.68 \\
500 & 8:Dual (MS+Oil) & 20000 & 152338 & 9671 & 79.97 & 1.66 \\
520 & 6:Hitec XL & 20000 & 161200 & 0 & 80.78 & 1.67 \\
550 & 7:Solar Salt & 20000 & 161648 & 0 & 81.00 & 1.67 \\
\hline
\end{tabular}




\section{LISTA DE ACRÓNIMOS}

ACHE: "Air Cooled Heat Exchanger". Intercambiador mediante aire en convección forzada. BOP: "Balance Of Plant". Ciclo de potencia.

CIT: "Compressor Inlet Temperature". Temperatura de entrada en el compresor.

CSP: "Concentrated Solar Power". Energía de concentración solar.

DISS: "DIrect Solar Steam". Proyecto de investigación para la generación directa de vapor en los colectores solares cilindro-parabólicos en España.

DNI: "Direct Normal Irradiance". Irradiación solar directa.

DRH: "Direct ReHeating". Recalentamiento directo.

DSG: "Direct Steam Generation". Generación directa de vapor.

DSG+s-CO2: planta termosolar con colectores lineales con generación directa de vapor, y ciclo de potencia Brayton con Dióxido de Carbono en estado supercrítico.

DSG+s-Ethane: planta termosolar con colectores lineales con generación directa de vapor, y ciclo de potencia Brayton con Etano en estado supercrítico.

DSG+s-Water Rankine: planta termosolar con generación directa de calor en los colectores lineales, y ciclo de potencia supercritico de Rankine.

DSG+Water Rankine: planta termosolar con generación directa de calor en los colectores lineales, y ciclo de potencia de Rankine.

Dual-Loop+s-CO2: planta termosolar con combinación de dos fluidos caloportadores en sus campos solares, y ciclo de potencia Brayton con Dióxido de Carbono en estado supercrítico. Dual-Loop+Water Rankine: planta termosolar con combinación de dos fluido caloportadores en sus campos solares, y ciclo de potencia de Rankine.

ENEA: “Agenzia nazionale per le nouve tecnologie, l'energia e lo sviluppo económico sostenible". Agencia nacional para las Nuevas Tecnologías, Energía y desarrollo Económico Sostenible en Italia.

HTF: "Heat Transfer Fluid". Fluido caloportador.

HTR: "High Temperature Recuperator". Recuperador de calor de alta presión.

HX: "Heat Exchanger". Intercambiador de calor.

INDITEP: "Integration of dsg technology for electricity production". Integración de la generación directa de vapor para la producción de electricidad.

LF: "Linear Fresnel". Colector lineal Fresnel.

LTR: "Low Temperatura Recuperator". Recuperador de calor de baja presión.

MS: "Molten Salt". Sales fundidas.

MS+s-CO2: planta termosolar con colectores lineales con sales fundidas como fluido caloportador, y ciclo de potencia Brayton con Dióxido de Carbono en estado supercrítico.

MS+s-Ethane: planta termosolar con colectores lineales con sales fundidas como fluido caloportador, y ciclo de potencia Brayton con Etano en estado supercrítico.

MS+s-Water Rankine: planta termosolar con sales fundidas como fluido caloportador, y ciclo de potencia supercritico de Rankine.

MS+Water Rankine: planta termosolar con sales fundidas como fluido caloportador, y ciclo de potencia de Rankine.

NIST: "National Institute of Standards and Technology". Instituto Nacional de Normas y Tecnología en Estados Unidos.

NREL: “National Renewable Laboratory". Laboratorio central de energías renovables en Estados Unidos.

Oil+s-CO2: planta termosolar con colectores lineales con aceite fundido como fluido caloportador, y ciclo de potencia Brayton con Dióxido de Carbono en estado supercrítico. Oil+Water Rankine: planta termosolar con aceite térmico como fluido caloportador, y ciclo de potencia de Rankine. 
PCRC: "Partial Cooling with Recompression Brayton power cycle". Ciclo Brayton con enfríamiento previo a la recompresion.

PHX: "Primary Heat Exchanger". Intercambiador de calor para intercambio energético con campo solar principal.

PSA: Plataforma Solar de Almería.

PTC: "Parabolic Trough Collector". Colector cilindro-parabólico.

RC: "Recompression Brayton power cycle". Ciclo Brayton con recompression.

$\mathrm{RCMCl}$ : "Recompression with Main Compression Intercooling Brayton power cycle". Ciclo

Brayton con recompresion y enfriamiento intermedio en el compresor principal.

RHX: "ReHeating Heat Exchanger". Intercambiador de calor para intercambio energético con campo solar de recalentamiento.

SAM: "System Advisor Model"

SB: "Simple Brayton power cycle". Ciclo Brayton con recuperación de calor.

$\mathrm{s}-\mathrm{CH} 4$ : "supercritical Methane". Metano en estado supercrítico.

s-CO2: "supercritical Carbon Dioxide". Dióxido de Carbono en estado supercrítico.

s-Etano: "supercritical Ethane". Etano en estado supercrítico.

SF: "Solar Field". Campo solar.

s-N2: "supercritical Nitrogen". Nitrógeno en estado supercrítico.

STE: "Solar Thermal Energy". Energía termosolar.

s-Water: "supercritical water". Agua en estado supercritico.

s-Xe: "supercritical Xenon". Xenón en estado supercrítico.

TES: "Thermal Energy Storage". Almacenamiento térmico de energía.

TIP: "Turbine Inlet Pressure". Presión de entrada en la turbina.

TIT: "Turbine Inlet Temperature". Temperatura de entrada en turbina.

UA: Tamaño térmico del intercambiador de calor. 


\section{LISTA DE FIGURAS}

1. Estimación de la aportación de las Energías Renovables al Consumo Global de Energía en 2014 [3].

2. Potencia instalada en el mundo de energías renovables 2014 [3].

3. Mapa mundial de la distribución de Irradiación solar directa (DNI) anual kWh/m2 /yr, y previsiones de Producción y Consumo de energía termosolar en el año 2050 en TWh [6]. Las flechas indican la transferencia de electricidad generadas por CSP desde las zonas con mayor irradiación solar hacia las zonas con mayor consumo eléctrico.

4. Previsión de reducción del coste unitario e incremento de la producción en CSP [4]

5. Representación esquemática de las diferentes tecnologías de concentración solar en las plantas termosolares de generación de energía eléctrica [4].

6. Eficiencia en función de la temperatura de trabajo para varias relaciones de concentración solar [47].

7. Planta termosolar de tipología SEGS-I a SEGS-IX, construida por la compañía Luz en Estados Unidos, entre 1985 y 1991. Ciclo de potencia Rankine con recalentamiento. Eficiencia del ciclo $37.6 \%$. Potencia nominal eléctrica $80 \mathrm{MWe}$. Eficiencia eléctrica anual $13.6 \%$.

8. Planta termosolar con colectores lineales (PTC), sales fundidas (MS) como fluido caloportador (HTF), sistema de almacenamiento térmico (TES) con sales fundidas, y caldera de fuel de apoyo [53].

9. Planta termosolar con colectores lineales PTC, DSG como HTF, y caldera de biomasa/biogas de apoyo [53].

10. Capacidad Global de potencia termosolar por países en el Mundo (GW) [3].

11. Vista aérea de las plantas termosolares Andasol 1 y 2, con colectores PTC [50]

12. Representación gráfica del principal objetivo de la iniciativa SunShot, reducir el coste unitario de le energía generada en la planta termosolar a $6 \mathrm{c} \$ / \mathrm{kWh}$ [67].

13. Desglose de los objetivos de coste en las plantas termosolares, SunShot [67].

1.1. Ilustración del cálculo de la constante solar.

1.2. Representación del espectro de la radiación solar a nivel extraterrestres y a nivel de la superficie terrestre. También se compara dichos espectros con el espectro de emisión de un cuerpo negro a una temperatura de 5250oC (temperatura del Sol) [2]. 1.3. Balance Energético de la Tierra. Se representa gráficamente los flujos energéticos, tanto de la energía incidente sobre la Tierra procedente del Sol, la energía absorbida por la Tierra, y la energía que la Tierra emite al Espacio [3].

1.4. Mapa de la distribución de Irradiación Solar Directa DNI (kWh/m2) [3].

1.5. Sección transversal de un colector solar tipo cilindro parabólico (PTC) [5].

1.6. Relaciones geométricas que definen una parábola. Esta forma geométrica ha sido utilizada para el diseño de los espejos de los colectores solares cilindro-parabólicos [6].

1.7. Representación gráfica de los ángulos solares y orientación de un colector PTC [7].

1.8. Definición de la dirección normal al área de apertura del colector solar (PTC) [7]. 25

1.9. Sistema de seguimiento solar, mediante la rotación del colector PTC [6]. 26

1.10. Ilustración de un captador solar PTC [1]. 27

1.11. Balance energético de un colector solar PTC [6]. 27 
1.12. Sección transversal de un colector cilindro-parabólico representando los parámetros ópticos principales de los componentes que lo integran: transmitancia, absortancia y reflectancia [6].

1.13. Representación del valor de los factores de modificación del ángulo de incidencia IAM para diferentes valores del ángulo de incidencia [6].

1.14. En esta figura se ilustran los parámetros: área de apertura, ángulo de incidencia en un colector PTC [6].

1.15. Dos filas de colectores adyacentes pueden darse sombra una fila sobre la otra si el ángulo de seguimiento solar llega a unos valores extremos. La sombra depende de la anchura de apertura de los colectores, de la distancia entre las filas (entre los ejes centrales de las dos filas), y del ángulo de seguimiento solar de los colectores [20]

1.16. Sombra entre dos filas adyacentes de los colectores solares PTC [6]

1.17. Pérdidas geométricas del extremo de un colector CCP (izq.) [6]. Sección transversal del colector parabólico para definición de su la longitud focal media Lf,ave (der.) [20].

1.18. Sección longitudinal de un tubo absorbedor de un colector solar PTC [6].

1.19. Balance energético de una sección transversal de tubo absorbedor [23].

1.20. Representación gráfica de las resistencias térmicas correspondientes al modelo unidimensional del tubo absorbedor representado en la figura 1.19 [23].

1.21. Principales componentes que integran un colector solar PTC [6].

1.22. Sistema de seguimiento solar mediante actuadores de dos pistones [6].

1.23. Sistema de seguimiento solar. Sensor de seguimiento de la luz [6].

1.24. Manguera flexibe y junta de bola, dos elementos que permiten el acoplamiento entre los tubos absorbedores y las tuberías principales de distribución del campo solar [6].

1.25. Sección transversal de un colector solar tipo LF [2].

1.26. Vista de los colectores solares tipo LF en la Central Térmica de Liddell en Australia [27].

1.27. Las lentes patentadas por Augustin Fresnel permiten reducir el espesor de las lentes utilizadas hasta entonces para focalizar la luz en luminarias doméstricas [8].

1.28. Sección transversal de un colector solar lineal tipo LF [5].

1.29. Vista general de un colector solar tipo LF.

1.30. Vista detallada de los elementos que integran el colector LF con un solo tubo absorbedor y su reflector secundario.

1.31. Esquema de funcionamiento de un colector solar tipo LF multitubos, 1) el agua circula a través del interior del tubo absorbedor, 2) los espejos reflectores enfocan los rayos del Sol hacia la superficie del tubo absorbedor y hacia el reflector secundario, 3) el calor de la superficie del tubo absorbedor, debido a la incidencia de los rayos solares, calienta el agua del interior del tubo aborbedor y la convierte en vapor sobrecalentado [17].

1.32. Sección transversal del diseño de dos receptores (cavidades trapezoidales) en los colectores LF, figura izquierda [18], figura derecha [19].

1.33. Sistema de seguimiento solar de un colector LF [7].

1.34. Definición de la sección transversal de un módulo de los colectores LF de la marca comercial Novatec Solar [18]. 
1.35. Ilustración de los ángulos de incidencia $(\theta \mathrm{i})$, ángulo de incidencia longitudinal (Өlong) y transversal ( $\theta$ trans) en un colector LF de la empresa Novatec [18].

1.36. Modificadores del ángulo de incidencia en función del ángulo de incidencia solar. En línea continua los valores del IAM transversales y en línea discontinua los valores del IAM longitudinales [7].

1.37. Representación gráfica de los ángulos solares sobre un espejo plano de un colector lineal de tipología LF [7].

1.38. Definición de los parámetro trigonométricos que definen el ángulo de incidencia tanto longitudinal como transversal dependiendo de la orientación del colector LF en relación al Sol (orientación arbitraria, orientación Norte-Sur, orientación Este-Oeste) [7].

1.39. Explicación de los tres efectos físicos que disminuyen la radiación solar reflejada por los espejos de los colectores LF cuando el Sol está en posición bajas [7].

1.40. Estudio de la variación de la eficiencia óptica, de los colectores solares PTC y LF con tubos absorbedores al vacío, en relación al ángulo de incidencia de la radiación solar [7].

1.41. Se ilustra la radiación solar $(\mathrm{W} / \mathrm{m} 2)$ sobre los colectores lineales, LF y PTC, corregidas por el IAM, y su comparación con la radiación solar directa incidente DNI [7].

1.42. La potencia por metro cuadrado en LF muestra un pico en verano, mientras que en PTC muestra una meseta más amplia. Localización considerada Daggett USA [7].

1.43. Comparación de la variación de la Eficiencia Térmica de PTC y LF respecto a la temperatura de los colectores. Se han estudiado dos escenarios radiación solar incidente de $850 \mathrm{~W} / \mathrm{m} 2$ y de $500 \mathrm{~W} / \mathrm{m} 2$ [7].

1.44. Planta Termosolar con tecnología de receptor central y campo de heliostatos [1].

1.45. Vista de los colectores solares tipo Heliostatos en una Central Termosolar con tecnología de Torre Central [1].

1.46. Captador Solar Tipo Disco de Stirling [36].

1.47. Captadores/concentradores solares tipo discos Stirling [1].

1.48. Esquema de planta solar con campo solar con aceite térmico o sales fundidas como HTF, y ciclo de potencia Rankine sin Recalentamiento.

1.49. Esquema de planta solar con campo solar con aceite térmico o sales fundidas como HTF, y ciclo de potencia Rankine con Recalentamiento.

2.1. Algoritmo de cálculo del área de apertura efectiva y pérdidas de presión en los campos solares con colectores lineales.

2.2. Representación gráfica de las longitudes focales para el cálculo de la longitud focal media [2].

2.3. Sombra entre dos filas paralelas de colectores parabólicos. La geometría de la sombra depende de la distancia entre las dos filas de colectores (Lspacing), de su ángulo de seguimiento solar (Wcol), y de la longitud de apertura de los colectores (W) [2].

2.4. Representación gráfica 3D de los principales componentes y equipos que integran el lazo de pruebas de ciclo supercritical Carbon Dioxide (s-CO2) Brayton en SNL [11].

2.5. Esquema del ciclo de potencia s-CO2 Brayton del lazo de pruebas de SNL [11]. 
2.6. Tecnología principal o key technology, Turbo-Alternador-Compresor diseñado con rodamientos tipología gas foil bearings. Longitud 24 pulgadas y diámetro 12 pulgadas [11].

2.7. Fotografía de las dos turbinas radiales fabricadas por la empresa Baber -Nichols [13], que integran el ciclo de pruebas de ciclo de potencia s-CO2 Brayton en SNL [11].

2.8. Eficiencia de una turbina radial ideal en función de la relación de velocidades ( $v=U$ tip/Cs). El valor normalmente elegido es de $v=0.707$ para obtener una eficiencia aerodinámica máxima (Japikse y Baines [15]).

2.9. Mapa de funcionamiento de la turbina acoplada al compresor principal [11].

2.10. Mapa de funcionamiento de la turbina acoplada al recompresor [11].

2.11. Algoritmo para el diseño Design-Point de las turbinas radiales del ciclo de potencia s-CO2 Brayton [12].

2.12. Algoritmo de cálculo para determinar las condiciones de operación Off-design de la turbina [12].

2.13. Representación 3D y fotografía de los compresores radiales de la marca comercial Baber-Nichols [13] que integran el lazo experimental de ciclo de potencia sCO2 en SNL [11].

2.14. El mapa de operación del compresor radial es representado para un rango de velocidades de 35,40 y $45 \mathrm{krpm}$ [11].

2.15. Gráfica de detalle del mapa de operación del compresor radial, en lazo de pruebas del ciclo s-CO2 Brayton, en SNL [11].

2.16. Curvas adimensionales que relacionan el coeficiente de flujo $(\phi)$, con la eficiencia $(\eta)$ y el coeficiente de altura manométrica $(\Psi)$, para el compresor del lazo de pruebas de SNL [12].

2.17. Polinomios aproximativos de la altura manométrica y eficiencia no dimensionales para el compresor de lazo de pruebas en SNL [12].

2.18. Algoritmo de diseño de los compresores radiales del ciclo Brayton con una etapa de compresión [12].

2.19. Algoritmo de diseño del Recompresor radial de dos etapas de compresión del ciclo Brayton [12].

2.20. Algoritmo de diseño del Recompresor radial de dos etapas de compresión del ciclo Brayton [12]

2.21. Algoritmo de diseño del Recompresor radial de dos etapas de compresión del ciclo Brayton [12].

2.22. Algoritmo de cálculo para simulación de un Compresor radial de una sola etapa de compresión fuera de sus condiciones del punto de diseño Off-Design [12].

2.23. Subdivisión del intercambiador de calor con fluidos supercriticos $[17,18]$.

2.24. Algoritmo para el cálculo de los intercambiadores de calor en un ciclo de potencia Brayton con fluido de trabajo en estado supercrítico [17-18].

2.25. Algoritmo de cálculo para cálculo de la longitud total y pérdidas de presiones de un cambiador de calor en un ciclo Brayton con fluido de trabajo en estado supercrítico [17].

2.26. Movimientos básicos del método Nelder-Mead para buscar la solución [22] 86

2.27. Definición del algoritmo NEWUOA [26-27].

3.1. Lazo integrado por colectores solares LF y DSG en modo recirculación. 
3.2. Configuración de referencia integrada por lazos de colectores solares LF con DSG en modo RC conectados en paralelo [26].

3.3. (a) Modelo del campo solar con colectores LF y DSG con modo recirculación RC. Realizado con el programa Informático Thermoflow [26].

3.3. (b) Modelo Thermoflow del BOP con DRH con colectores LF.

3.4. Planta termosolar de $50 \mathrm{MWe}$ con dos grupos de 13 lazos conectados en paralelo. Cada lazo dispone de 12 módulos para ebullición del agua y 5 módulos para sobrecalentamiento del vapor. Todos los módulos están integrados por colectores LF tipo Novatec [7]. El bloque de potencia BOP está localizado en el centro del campo solar.

3.5. Configuración propuesta [27].

4.1. DSG en los colectores lineales PTC o LF en modo Recirculación de la fase líquida.

4.2. Ciclo subcrítico Rankine con 5 calentadores del agua de alimentación y un desaireador.

4.3. Planta Termosolar con colectores lineales (PTC o LF) con generación directa de vapor como HTF acoplado con ciclo de potencia supercrítico Rankine.

4.4. Planta Termosolar con colectores lineales con sales fundidas como HTF acoplado a ciclo de potencia supercrítico Rankine.

5.1. Adaptación de los ciclos supercriticos s-CO2 Brayton a las plantas termosolares con colectores lineales.

5.2. Planta Termosolar con colectores lineales y aceite como HTF [22].

5.3. Planta Termosolar con colectores lineales (PTC o LF) y sales fundidas como HTF [22]

5.4. Planta termosolar con colectores lineales y ciclo de potencia Brayton con s-CO2 y configuración básica con recuperación SB.

5.5. Planta termosolar con colectores lineales y ciclo de potencia Brayton con s-CO2 y configuración con recompresión RC.

5.6. Planta termosolar con colectores lineales y ciclo de potencia Brayton con s-CO2 y configuración con enfriamiento parcial y recompresión PCRC.

5.7. Planta termosolar con colectores lineales y ciclo de potencia Brayton con s-CO2 y configuración con recompresión y enfriamiento intermedia en el compresor principal RCMCl.

5.8. Eficiencia Neta frente a TIT. Ciclos Brayton sin Recalentamiento.

5.9. Eficiencia Neta frente a TIT. Ciclos Brayton con Recalentamiento.

5.10. Potencia Unitaria (Potencia Neta / Área Apertura Efectiva SF) frente a TIT, Sin Recalentamiento.

5.11. Potencia Unitaria (Potencia Neta / Área Apertura Efectiva SF) frente a TIT, Con Recalentamiento.

6.1. Eficiencia Bruta \& TIT, ciclo RC s-CO2 sin recalentamiento.

6.2. Eficiencia Bruta \& TIT, ciclo RC s-CO2 con recalentamiento.

6.3. Eficiencia Bruta \& TIT, ciclo PCRC s-CO2 sin recalentamiento.

6.4. Eficiencia Bruta \& TIT, ciclo PCRC s-CO2 con recalentamiento.

6.5. Eficiencia Bruta \& TIT, ciclo RCMCl s- $\mathrm{CO} 2$ sin recalentamiento.

6.6. Eficiencia Bruta \& $\mathrm{TIT}$, ciclo $\mathrm{RCMCl}$ s- $\mathrm{CO} 2$ con recalentamiento. 
6.7. Tamaño térmico óptimo del Recuperador de Baja Presión (LTR), frente a TIT, ciclo termodinámico (RC) s-CO2 sin Recalentamiento.

6.8. Tamaño térmico óptimo del Recuperador de Alta Presión (HTR), frente a TIT. Ciclo termodinámico (RC) s-CO2 sin Recalentamiento.

6.9. Tamaño térmico óptimo del Recuperador de Baja Presión (LTR), frente a TIT. Ciclo termodinámico (RC) s-CO2 con Recalentamiento.

6.10. Tamaño térmico óptimo del Recuperador de Baja Presión (HTR), frente a TIT. Ciclo termodinámico (RC) s-CO2 con Recalentamiento.

6.11. Tamaño térmico óptimo del Recuperador de Baja Presión (LTR), frente a TIT. Ciclo termodinámico (PCRC) s-CO2 sin Recalentamiento.

6.12. Tamaño térmico óptimo del Recuperador de Baja Presión (HTR), frente a la TIT. Ciclo termodinámico (PCRC) s-CO2 sin Recalentamiento.

6.13. Tamaño térmico óptimo del Recuperador de Baja Presión (LTR), frente a TIT. Ciclo termodinámico (PCRC) s-CO2 con Recalentamiento.

6.14. Tamaño térmico óptimo del Recuperador de Baja Presión (HTR), frente a TIT. Ciclo termodinámico (PCRC) s-CO2 con Recalentamiento.

6.15. Tamaño térmico óptimo del Recuperador de Baja Presión (LTR), frente a TIT. Ciclo termodinámico ( $\mathrm{RCMCl}) \mathrm{s}-\mathrm{CO} 2$ sin Recalentamiento.

6.16. Tamaño térmico óptimo del Recuperador de Baja Presión (HTR), frente a TIT. Ciclo termodinámico ( $\mathrm{RCMCl}$ ) s-CO2 sin Recalentamiento.

6.17. Tamaño térmico óptimo del Recuperador de Baja Presión (LTR), frente a TIT. Ciclo termodinámico ( $\mathrm{RCMCl}$ ) s-CO2 con Recalentamiento.

6.18. Tamaño térmico óptimo del Recuperador de Baja Presión (HTR), frente a TIT. Ciclo termodinámico ( $\mathrm{RCMCl}$ ) s-CO2 con Recalentamiento.

6.19. Área de Apertura Efectiva con colectores solares MS PTC y ciclo de potencia RC s-CO2 con recalentamiento.

6.20. Área de Apertura Efectiva con colectores solares MS LF y ciclo de potencia RC s$\mathrm{CO} 2$ con recalentamiento.

6.21. Área de Apertura Efectiva con colectores solares MS PTC y ciclo de potencia PCRC $\mathrm{s}-\mathrm{CO} 2$ con recalentamiento.

6.22. Área de Apertura Efectiva con colectores solares MS LF y ciclo de potencia PCRC $\mathrm{s}-\mathrm{CO} 2$ con recalentamiento.

6.23. Área de Apertura Efectiva con colectores solares MS PTC y ciclo de potencia $\mathrm{RCMCl}$ s- $\mathrm{CO} 2$ con recalentamiento.

6.24. Área de Apertura Efectiva con colectores solares MS LF y ciclo de potencia RCMCI $\mathrm{s}$ - $\mathrm{CO} 2$ con recalentamiento.

6.25. Estimación de Costes de los colectores solares PTC y ciclo de potencia RC s-CO2 con recalentamiento.

6.26. Estimación de Costes de los colectores solares LF y ciclo de potencia RC s-CO2 con recalentamiento.

6.27. Estimación de Costes de los colectores solares PTC y ciclo de potencia PCRC s$\mathrm{CO} 2$ con recalentamiento.

6.28. Estimación de Costes de los colectores solares LF y ciclo de potencia PCRC s-CO2 con recalentamiento. 
6.29. Estimación de Costes de los colectores solares PTC y ciclo de potencia RCMCl s$\mathrm{CO} 2$ con recalentamiento.

6.30. Estimación de Costes de los colectores solares LF y ciclo de potencia RCMCl s$\mathrm{CO} 2$ con recalentamiento.

7.1. Planta termosolar de colectores lineales con aceite térmico como HTF acoplada a ciclo de potencia s-CO2 Brayton RC.

7.2. Planta termosolar con colectores lineales con aceite térmico como HTF acoplada a ciclo de potencia s-CO2 Brayton PCRC.

7.3. Planta termosolar con colectores lineales con aceite térmico como HTF acoplada a ciclo de potencia s-CO2 Brayton $\mathrm{RCMCl}$.

7.4. Área de Apertura Efectiva con colectores solares lineales PTC acoplada a ciclo de potencia s-CO2 Brayton RC con recalentamiento.

7.5. Área de Apertura Efectiva con colectores solares lineales LF acoplada a ciclo de potencia s-CO2 Brayton RC con recalentamiento.

7.6. Área de Apertura Efectiva con colectores solares lineales PTC acoplada a ciclo de potencia s-CO2 Brayton PCRC con recalentamiento.

7.7. Área de Apertura Efectiva con colectores solares lineales LF acoplada a ciclo de potencia s-CO2 Brayton PCRC con recalentamiento.

7.8. Área de Apertura Efectiva con colectores solares lineales PTC acoplada a ciclo de potencia s- $\mathrm{CO} 2$ Brayton $\mathrm{RCMCl}$ con recalentamiento.

7.9. Área de Apertura Efectiva con colectores solares lineales LF acoplada a ciclo de potencia s- $\mathrm{CO} 2$ Brayton $\mathrm{RCMCl}$ con recalentamiento.

150000

7.10. Estimación de Costes de campo solar con colectores lineales PTC acoplada a ciclo de potencia s- $\mathrm{CO} 2$ Brayton RC con recalentamiento.

7.11. Estimación de Costes de campo solar con colectores lineales LF acoplada a ciclo de potencia s-CO2 Brayton RC con recalentamiento.

7.12. Estimación de Costes de campo solar con colectores lineales PTC acoplada a ciclo de potencia s-CO2 Brayton PCRC con recalentamiento.

7.13. Estimación de Costes de campo solar con colectores lineales LF acoplada a ciclo de potencia s-CO2 Brayton PCRC con recalentamiento.

7.14. Estimación de Costes de campo solar con colectores lineales PTC acoplada a ciclo de potencia s-CO2 Brayton $\mathrm{RCMCl}$ con recalentamiento.

7.15. Estimación de Costes de campo solar con colectores lineales LF acoplada a ciclo de potencia s-CO2 Brayton $\mathrm{RCMCl}$ con recalentamiento.

55

8.1. Configuración de central termosolar con colectores lineales acoplada a ciclo de potencia s-CO2 Brayton SB.

8.2. Configuración de central termosolar con colectores lineales acoplada a ciclo de potencia s-CO2 Brayton RC.

8.3. Configuración de central termosolar con colectores lineales acoplada a ciclo de potencia s-CO2 Brayton PCRC.

8.4. Configuración de central termosolar con colectores lineales acoplada a ciclo de potencia s-CO2 Brayton RCMCl. 
8.5. Campo solar integrado por colectores lineales con generación directa de vapor en modo recirculación (configuración de referencia).

8.6. Ciclo de potencia Rankine sin recalentamiento en turbinas (configuración de referencia).

8.7. Integración de las Configuraciones 1 y 2 en la misma planta termosolar.

8.8. Planta termosolar con colectores lineales con generación directa de vapor acoplada a ciclo de potencia s-CO2 Brayton sin recalentamiento (configuración 1).

8.9. Planta termosolar con colectores lineales para sobrecalentamineto del vapor acoplada a ciclo de potencia s-CO2 Brayton sin recalentamiento (configuración 2).

8.10. Plano de planta del Campo Solar diseñado para la configuración 2.

8.11. Planta Termosolar con colectores lineales con DSG, acoplada a dos ciclos de potencia s- $\mathrm{CO} 2$ Bratyon $\mathrm{RCMCl}$ en cascada con recalentamiento en el ciclo inferior. (configuración 3).

8.12. Planta termosolar con colectores lineales con DSG en campo solar principal, y con sales fundidas en el campo solar para recalentamiento, acoplada a ciclo de potencia s-CO2 Brayton (configuración 4).

8.13. Área de apertura efectiva del campo solar con colectores PTC Vs. UA de los recuperadores del ciclo s-CO2 Brayton $\mathrm{RC}$ sin recalentamiento.

8.14. Área de apertura efectiva del campo solar con colectores LF Vs. UA de los recuperadores del ciclo s-CO2 Brayton RC sin recalentamiento.

8.15. Área de apertura efectiva del campo solar con colectores PTC Vs. UA de los recuperadores del ciclo s-CO2 Brayton PCRC sin recalentamiento.

8.16. Área de apertura efectiva del campo solar con colectores LF Vs. el UA de los recuperadores del ciclo s-CO2 Brayton PCRC sin recalentamiento.

8.17. Área de apertura efectiva del campo solar con colectores PTC Vs. UA de los recuperadores del ciclo s-CO2 Brayton $\mathrm{RCMCl}$ sin recalentamiento.

8.18. Área de apertura efectiva del campo solar con colectores LF Vs. UA de los recuperadores del ciclo s-CO2 Brayton $\mathrm{RCMCl}$ sin recalentamiento.

9.1. Diagrama de flujo del sistema Dual-Loop propuesto por Vogel 2014 [10].

9.2. Configuración 1. Planta termosolar con campos solares con aceites térmicos 0 sales fundidas como HTF acopladas a ciclo de potencia s-CO2 Brayton SB con recuperación de calor y recalentamiento.

9.3. Configuración 2. Planta termosolar con campos sola principal Dual-Loop, y campo solar de recalentamiento Single-Loop, acoplada a ciclo de potencia s-CO2 Brayton SB con recuperación de calor y recalentamiento.

9.4. Configuración 3. Planta termosolar con campo solare principal y de recalentamiento de tipo Dual-Loop, acoplada a ciclo de potencia s-CO2 Brayton SB con recuperación de calor y recalentamiento.

9.5. Eficiencia Neta de los ciclos de potencia s-CO2 Brayton SB con recuperación de calor y recalentamiento en función del UA de los recuperadores de calor y de TIT.

9.6 Planta CSP con colectores solares lineales con dos campos solares: el campo principal de tipología 'Dual Loop' y el campo de recalentamiento acoplados a ciclo s$\mathrm{CO} 2$ Brayton RC con recalentamiento 
9.7. Planta CSP con colectores solares lineales, con dos campos solares: el campo principal de tipología 'Dual Loop' y el campo de recalentamiento acoplados a ciclo s$\mathrm{CO} 2$ Brayton PCRC con recalentamiento

9.8. Planta CSP con colectores solares lineales, con dos campos solares: el campo principal de tipología 'Dual-Loop' y el campo de recalentamiento acoplados a ciclo s$\mathrm{CO} 2$ Brayton $\mathrm{RCMCl}$ con recalentamiento

9.9. Eficiencia Neta de los ciclos de potencia s- $\mathrm{CO} 2$ Brayton RC con recuperación de calor y recalentamiento en función del UA de los recuperadores de calor y de TIT.

9.10. Eficiencia Neta de los ciclos de potencia s-CO2 Brayton PCRC con recuperación de calor y recalentamiento en función del UA de los recuperadores de calor y de TIT.

9.11. Eficiencia Neta de los ciclos de potencia s- $\mathrm{CO} 2$ Brayton $\mathrm{RCMCl}$ con recuperación de calor y recalentamiento en función del UA de los recuperadores de calor y de TIT.

10.1. Planta termosolar integrada por colectores lineales con DSG en modo de recirculación.

10.2. Ciclo de potencia Rankine y dos etapas de DRH.

10.3. Ciclo de potencia Brayton SB con recalentamiento en turbinas.

10.4. Ciclo de potencia Brayton RC con recalentamiento en turbinas.

10.5. Ciclo de potencia Brayton PCRC con recalentamiento en turbinas.

10.6. Ciclo de potencia Brayton $\mathrm{RCMCl}$ con recalentamiento en turbinas.

10.7. Eficiencia Neta \& TIT (1).

10.8. Eficiencia Neta \& TIT (1).

10.9. Eficiencia Neta \& TIT (2)

10.10. Eficiencia Neta \& TIT (2).

10.11. Eficiencia Neta \& TIT (2)

10.12. Eficiencia Neta \& TIT (2).

10.13. SB s-C2H6 Eficiencia Neta \& Pérdidas de Presión en HX (2).

10.14. PCRC s-C2H6Eficiencia Neta \&Pérdidas de Presión en HX (2).

10.15. RC s-C2H6 Eficiencia Neta \& Pérdidas de Presión en HX (2).

10.16. $\mathrm{RCMCl}$ s-C2H6 Eficiencia Neta \&Pérdidas de Presión en HX (2).

10.17. SB s-C2H6 Eficiencia Neta \& CIT (2).

10.18. RC s-C2H6 Eficiencia Neta \& CIT (2).

10.19. PCRC s-C2H6 Eficiencia Neta \& CIT (2).

10.20. RCMCl s-C2H6 Eficiencia Neta \& CIT (2).

10.21. Área de Apertura Efectiva con colectores LF (m2) versus TIT. Potencia neta generada fija $50 \mathrm{MWe}$ netos (3).

10.22. PTC Área de Apertura Efectiva (m2) versus TIT. Potencia neta generada fija $50 \mathrm{MWe}$ netos (3).

11.1. Representación gráfica del fenómeno físico de caída considerable de la densidad del s-CO2 en las cercanías del punto crítico debido a los cambios de presión y temperatura.

11.2. Ciclo de potencia Brayton SB con fluido de trabajo supercrítico (CO2, C2H6, SF6, $\mathrm{CH} 4, \mathrm{~N} 2$ y $\mathrm{Xe}$ ). Incluye un recuperador y una etapa de recalentamiento en turbinas. Las fuentes de calor son dos campos solares con colectores lineales.

11.3. Eficiencia bruta versus tamaño térmico de los recuperadores. Ciclo Brayton SB con recuperación de calor. Fluido de trabajo s-CO2. 
11.4. Eficiencia bruta versus tamaño térmico de los recuperadores. Ciclo Brayton SB con recuperación de calor. Fluido de trabajo s-C2H6.

11.5. Eficiencia bruta versus tamaño térmico de los recuperadores. Ciclo Brayton SB con recuperación de calor. Fluido de trabajo s-SF6.

11.6. Eficiencia bruta versus tamaño térmico de los recuperadores. Ciclo Brayton SB con recuperación de calor. Fluido de trabajo s-Xe.

11.7. Eficiencia bruta versus tamaño térmico de los recuperadores. Ciclo Brayton SB con recuperación de calor. Fluido de trabajo s-N2.

11.8. Eficiencia bruta versus tamaño térmico de los recuperadores. Ciclo Brayton SB con recuperación de calor. Fluido de trabajo s-CH4.

11.9. Eficiencia bruta versus TIT, fijado el UA $=3000 \mathrm{~kW} / \mathrm{K}$, para diferentes fluidos de trabajo: $\mathrm{CO} 2, \mathrm{C} 2 \mathrm{H} 6, \mathrm{CH} 4, \mathrm{~N} 2$, Xe y N2. Ciclo Brayton SB con recuperación de calor.

11.10. Eficiencia bruta versus TIT, fijado $U A=5000 \mathrm{~kW} / \mathrm{K}$ para diferentes fluidos de trabajo: $\mathrm{CO} 2, \mathrm{C} 2 \mathrm{H} 6, \mathrm{CH} 4, \mathrm{~N} 2$, Xe y N2. Ciclo Brayton SB con recuperación de calor.

11.11. Eficiencia bruta versus TIT, fijado $U A=10000 \mathrm{~kW} / \mathrm{K}$ para diferentes fluidos de trabajo: $\mathrm{CO} 2, \mathrm{C} 2 \mathrm{H} 6, \mathrm{CH} 4, \mathrm{~N} 2$, Xe y N2. Ciclo Brayton SB con recuperación de calor.

11.12. Eficiencia bruta versus TIT, fijado UA $=15000 \mathrm{~kW} / \mathrm{K}$ para diferentes fluidos de trabajo: $\mathrm{CO} 2, \mathrm{C} 2 \mathrm{H} 6, \mathrm{CH} 4, \mathrm{~N} 2, \mathrm{Xe}$ y N2. Ciclo Brayton SB con recuperación de calor.

11.13. Eficiencia bruta \& TIT, fijado $U A=20000 \mathrm{~kW} / \mathrm{K}$ para diferentes fluidos de trabajo: $\mathrm{CO} 2, \mathrm{C} 2 \mathrm{H} 6, \mathrm{CH} 4, \mathrm{~N} 2, \mathrm{Xe}$ y N2. Ciclo Brayton SB con recuperación de calor.

11.14. Eficiencia bruta versus TIT, fijado $U A=30000 \mathrm{~kW} / \mathrm{K}$ para diferentes fluidos de trabajo: $\mathrm{CO} 2, \mathrm{C} 2 \mathrm{H} 6, \mathrm{CH} 4, \mathrm{~N} 2$, Xe y N2. Ciclo Brayton SB con recuperación de calor.

11.15. Comparación del área apertura efectiva versus TIT, en planta termosolar con colectores PTC acoplada a ciclo Brayton SB con s-CO2 o s-C2H6 como fluidos de trabajo.

11.16. Comparación del área apertura efectiva versus TIT, en planta termosolar con colectores PTC acoplada a ciclo Brayton SB con s-CO2 o s-SF6 como fluidos de trabajo.

11.17. Comparación del área apertura efectiva versus TIT, en plantas termosolares con colectores PTC acoplada a ciclo Brayton SB con s-CO2 o s-Xe como fluidos de trabajo.

11.18. PT Comparación del área apertura efectiva versus TIT, en planta termosolar con colectores PTC acoplada a ciclo Brayton SB y s-CO2 o s-N2 como fluidos de trabajo.

11.19. Comparación del área apertura efectiva versus TIT, en planta termosolar con colectores PTC acoplada a ciclo Brayton SB con s- $\mathrm{CO} 2$ o s-CH4 como fluidos de trabajo.

11.20. Comparación del área apertura efectiva versus TIT, en planta termosolar con LF acoplada a ciclo Brayton SB con s-CO2 o s- $\mathrm{C} 2 \mathrm{H} 6$ como fluidos de trabajo.

11.21. Comparación del área apertura efectiva versus TIT, en las planta termosolar con colectores LF acoplada a ciclo Brayton SB con s-CO2 o s-SF6 como fluidos de trabajo.

11.22. Comparación del área apertura efectiva versus TIT, en planta termosolar con colectores LF acoplada a ciclo Brayton SB con s-CO2 o s-Xe como fluidos de trabajo.

11.23. Comparación del área apertura efectiva versus TIT, en planta termosolar con colectores LF acoplada a ciclo Brayton SB con s-CO2 o s-N2 como fluidos de trabajo.

11.24. Comparación del área apertura efectiva versus $\mathrm{TIT}$, en planta termosolar con colectores LF acoplada a ciclo Brayton SB con s- $\mathrm{CO} 2$ o s-CH4 como fluidos de trabajo.

11.25. Comparación relativa de HTC en ciclos Brayton: CO2 Vs. SF6. 
11.27. Comparación relativa de HTC en ciclo Brayton: CO2 Vs. N2. 297

11.28. Comparación relativa de HTC en ciclo Brayton: CO2 Vs. Xe. 297

11.29. Comparación relativa de HTC en ciclo Brayton: $\mathrm{CO} 2$ Vs. CH4. 298

11.30. Comparación de la caída de presión relativa. CO2 Vs. SF6. 299

11.31. Comparación de la caída de presión relativa. CO2 Vs. C2H6. 299

11.32. Comparación de la caída de presión relativa. CO2 Vs. N2. 300

11.33. Comparación de la caída de presión relativa. CO2 Vs. Xe. 300

11.34. Comparación de la caída de presión relativa. CO2 Vs. CH4. 301

12.1. Pantalla de inicio de la herramienta SCSP.

12.2. Ejemplo del GUI de la configuración de ciclo Brayton RC con recalentamiento con las opciones de cálculo en cada configuración: punto de diseño, optimización del 308 diseño.

12.3. Ejemplo del GUI de la configuración de ciclo Brayton SB ó RC sin recalentamiento.

12.4. Ejemplo del GUI de la configuración de ciclo Brayton $\mathrm{RCMCl}$ sin recalentamiento.

12.5. Cuadro de diálogo para cálculo del punto de diseño en la configuración PCRC con Recalentamiento.

12.6. El usuario puede elegir entre diferentes tipologías de colectores lineales y DualLoop, para los campos solares considerados en la simulación, además de incluir otros datos de entrada (en este caso para cálculo de la configuración PCRC con recalentamiento).

12.7. Ejemplo de resultados de presiones $(\mathrm{kPa})$ y temperaturas $(\mathrm{K})$ en cada una de las corrientes.

12.8. Botones para selección del algoritmo de optimización multivariable.

12.9. Cuadro de diálogo para optimización de las condiciones de operación en la configuración PCRC con Recalentamiento.

12.10. Botones para selección de los resultados del cálculo tanto en el punto de diseño como de optimización multivariable.

12.11. Diagrama Temperatura (K) Vs. Entropía $(\mathrm{kJ} / \mathrm{kg}$ ) de la configuración PCRC con recalentamiento.

12.12. Diagrama Presión ( $\mathrm{kPa})$ Vs. Temperatura $(\mathrm{K})$ de la configuración PCRC con recalentamiento.

12.13. Diagrama P-s de la configuración PCRC con recalentamiento.

12.14. Resultados del cálculo de diseño de los campos solares principal y de recalentamiento.

12.15. Resultados del cálculo de diseño de los campos solares principal y de recalentamiento.

12.16. Resultados del cálculo de diseño de los intercambiadores de acoplamiento entre los campos solares principal y de recalentamiento, y el ciclo de potencia Brayton.

12.17. Definición de los consumos de los equipos auxiliares de los campos solares y del ciclo Brayton. El consumo de las bombas de recirculación de HTF es calculado automáticamente. El usuario debe estimar el consumo energético de los ventiladores del foco frío y de las pérdidas auxiliares del BOP.

12.18. Balance energético de la planta CSP.

12.19. Balance energético del ciclo de potencia. 
12.20. Cuantificación de la aportación energética en los campos solares (Main Solar Field, ReHeating Solar Field) y del consumo energético del foco frío (pre-cooler).

12.21. Detalle de las pérdidas energéticas de la planta CSP debidas al consumo de los equipos auxiliares del campo solar y del ciclo Brayton de potencia.

12.22. Resultados de la estimación de costes de los equipos y componentes de los campos solares principal y de recalentamiento, para colectores de tipología PTC y LF.

12.23. Cuadro de diálogo para diseño del compresor principal de una etapa de compresión, y del recompresor radial de dos etapas de compresión.

12.24. Cuadro de diálogo para diseño de las turbinas radiales, principal y de recalentamiento radial.

12.25. Cuadro de diálogo para diseño básico de los intercambiadores de calor (LRT, HTR, PHX, RHX, UHS) de la planta CSP acoplada a ciclo Brayton.

12.26. Variación de UA ( $\mathrm{kW} / \mathrm{K})$ a lo largo del intercambiador de calor.

317

12.27. Variación de la relación de CR a lo largo del intercambiador de calor.

318

12.28. Variación de la eficiencia térmica a lo largo del intercambiador de calor.

12.29. Sección transversal de los canales en los intercambiadores de calor de tipología circuito impreso (PCHE).

12.30. Sección transversal de los intercambiadores compactos de tipología (FPHE).

12.31. Cuadro de diálogo para diseño de detalle de los intercambiadores de calor de la planta CSP acoplada a ciclo Brayton.

12.32. Datos de partida para el diseño de detalle de los intercambiadores de calor. $\quad 319$

12.33. Distribución de HTC a lo largo del intercambiador. 319

12.34. Ejemplo datos de partida para diseño del campo solar. 320

12.35. Ejemplo resultados del diseño de detalle del campo solar. 320

12.36. Cuadro de diálogo de diseño del Generador. 321

12.37. Resultados del diseño del Generador. 321

12.38. Esquema de referencia de la primera validación del programa informático SCSP.

Ciclo de potencia s-CO2 Brayton acoplado a un reactor nuclear refrigerado por He [39].

12.39. Datos de entrada en el SCSP para la primera validación.

12.40. Resultados del SCSP de la primera validación.

12.41. Valores de referencia de la segunda validación. Obtenidos por Dyreby [1].

12.42. Datos de entrada en el SCSP para la segunda validación (caso $\mathrm{CIT}=32^{\circ} \mathrm{C}-$ izquierda, $40^{\circ} \mathrm{C}$ - centro, $50^{\circ} \mathrm{C}$ - derecha).

13.1. Resumen del rendimiento energético de las diferentes soluciones tecnológicas analizadas en el capítulo 6. Eficiencia Bruta Vs. TIT, ciclo RC s-CO2 con recalentamiento.

13.2. Resumen del rendimiento energético de las diferentes soluciones analizadas en el capítulo 10. Eficiencia Neta Vs. TIT (2).

13.3. Resumen del rendimiento energético de las diferentes soluciones analizadas en el capítulo 11. Eficiencia bruta Vs. TIT, con UA $=15000 \mathrm{~kW} / \mathrm{K}$ para CO2, C2H6, CH4, N2, Xe y N2. Ciclo Brayton SB con recuperación de calor.

13.4. Predicción de la eficiencia de la turbina frente a la relación entre la velocidad del tip y la velocidad de chorro [53]. 


\section{LISTA DE TABLAS}

1. Principales características técnicas de las plantas termosolares SEGS [54].

2. Producción Anual de las plantas termosolares SEGS [54].

3. Potencia eléctrica Neta producida (MWh) en las plantas termosolares SEGS [54].

4. Plantas termosolares con colectores lineales PTC o LF y ciclo de potencia Rankine.

1.1. Definición de los ángulos solares y de orientación de un colector PTC.

1.2. Coeficientes de pérdidas térmicas y eficiencia óptica de los colectores LF marca comercial Novatec Solar [18].

1.3. Principales parámetros de diseño del colector LF de la marca comercial Novatec [18].

1.4. Definición de los ángulos solares de un colector LF [7].

1.5. Ecuaciones de ángulos de incidencia longitudinal $(\theta I)$ y transversal ( $\theta$ trans) en LF [6].

1.6. Eficiencia y potencia de las centrales termosolares con colectores lineales PTC y ciclo de potencia Rankine sin Recalentamiento. Valores en punto de diseño.

1.7. Eficiencia y potencia de las centrales termosolares con colectores lineales LF y ciclo de potencia Rankine sin Recalentamiento. Valores en punto de diseño.

1.8. Eficiencia y potencia de las centrales termosolares con colectores lineales PTC y ciclo de potencia Rankine con Recalentamiento. Valores en punto de diseño.

1.9. Eficiencia y potencia de las centrales termosolares con colectores lineales LF y ciclo de potencia Rankine con Recalentamiento. Valores en punto de diseño.

1.10. Eficiencia y potencia de las centrales termosolares con colectores lineales PTC y ciclo de potencia Rankine sin Recalentamiento. Valores en punto de diseño.

1.11. Eficiencia y potencia de las centrales termosolares con colectores lineales LF y ciclo de potencia Rankine sin Recalentamiento. Valores en punto de diseño.

1.12. Eficiencia y potencia de las centrales termosolares con colectores lineales PTC y ciclo de potencia Rankine con Recalentamiento. Valores en punto de diseño.

1.13. Eficiencia y potencia de las centrales termosolares con colectores lineales LF y ciclo de potencia Rankine con Recalentamiento. Valores en punto de diseño.

3.1. Comparación entre las capacidades de los programas informáticos Thermoflow y SAM para el diseño de plantas termosolares con colectores LF y DSG.

3.2. Parámetros de localización de la planta termosolar con colectores LF y DSG.

3.3a. Parámetros de los colectores LF con DSG y del campo solar.

3.3b. Parámetros de los colectores LF con DSG y del campo solar.

3.4. BOP principales parámetros termodinámicos.

3.5. Datos de entrada en el punto de Diseño 21 Junio.

3.6. Comparación parámetros del campo solar LF (punto de diseño 21 Junio).

3.7. Comparación parámetros del campo solar LF (punto de diseño 21 Junio).

3.8. Parámetros óptimos del modulo de recalentamiento (punto de diseño 21 Junio).

3.9. Comparación parámetros termodinámicos SF LF (punto de Diseño 21 Junio). 
3.11. Principales propiedades termodinámicas de las Corrientes energéticas entre equipos en la configuración de Referencia (punto de Diseño 21 Junio).

3.12. Configuración de Referencia. Potencia energética anual (resultados mensuales).

3.13. Potencia y eficiencia de la configuración de Referencia con Recalentamiento Directa DRH (punto de diseño 21 Junio).

3.14. Configuración de Referencia, potencia anual (mensual).

3.15. Parámetros termodinámicos de operación de la configuración propuesta [27] (Punto de diseño 21 de Junio).

3.16. Configuración propuesta [27], potencia anual (mensual).

3.17. Configuración propuesta [27], potencia anual (mensual) comparada para el cálculo con SAM y Thermoflow

4.1. Localización y condiciones ambientales.

105

4.2. Parámetros tubo absorbedor.

4.3. Parámetros colector PTC.

4.4. Parámetros colector LF.

4.5. Ciclo Rankine (supercritico).

4.6. Ciclo Rankine (supercrítico).

4.7. Planta Termosolar con colectores LF con DSG y ciclos de potencia Rankine. Comparación de la eficiencia neta de los ciclos subcríticos y los supercríticos $\left(\mathrm{TIT}=550^{\circ} \mathrm{C}\right)$.

4.8. Planta Termosolar con colectores PTC con DSG, y ciclos de potencia Rankine. Comparación de la eficiencia neta de los ciclos subcríticos y los supercríticos (TIT $\left.=550^{\circ} \mathrm{C}\right)$.

4.9. Planta Termosolar con colectores LF, con sales fundidas como HTF, y ciclos de potencia de Rankine. Comparación de la eficiencia neta de los ciclos subcríticos y los supercríticos $\left(\mathrm{TIT}=550^{\circ} \mathrm{C}\right)$.

4.10. Planta Termosolar con colectores PTC, con sales fundidas como HTF, y ciclos de potencia de Rankine. Comparación de la eficiencia neta de los ciclos subcríticos y los supercríticos $\left(\mathrm{TIT}=550^{\circ} \mathrm{C}\right)$.

5.1. Localización y condiciones ambientales. 130

5.2. Parámetros tubo absorbedor.

5.3 Parámetros colectores PTC.

5.4. Parámetros colectores LF.

5.5. Ciclo de potencia Rankine.

5.6. Ciclo de potencia s-CO2 Brayton.

5.7. Planta Termosolar con colectores lineales con sales fundidas como fluido caloportador y ciclos de potencia Brayton. Principales parámetros del diseño básico de los intercambiadores de calor (PHX y RHX) entre los campos solars y el ciclo de potencia s-CO2 Brayton. Para la comparativa de resultados se ha fijado la potencia neta generada $55 \mathrm{MWe}$ y la $\mathrm{TIT}=550^{\circ} \mathrm{C}$.

5.8. Planta Termosolar con colectores lineales con sales fundidas como fluido caloportador y ciclos de potencia Brayton. Diseño de detalle de los intercambiadores de calor (PHX y RHX) entre los campos solares y el ciclo de potencia s-CO2 Brayton. Para la comparativa de resultados se ha fijado la potencia neta generada $55 \mathrm{MWe}$ y la $\mathrm{TIT}=550^{\circ} \mathrm{C}$. 
6.1. Propiedades físicas de las sales fundidas como HTF.

6.2. Localización, condiciones ambientales.

6.3. Parámetros tubo absorbedor.

6.4. Parámetros colectores PTC.

6.5. Parámetros colectores Fresnel.

6.6. Ciclo potencia Rankine.

6.7. Ciclo potencia s-CO2 Brayton.

6.8. Variables termodinámicas para optimizar el rendimiento energético del ciclo de potencia Brayton ( $R C$ sin recalentamiento) en centrales termosolares con colectores lineales.

6.9. Variables termodinámicas para optimizar el rendimiento energético del ciclo de potencia Brayton (RC sin Recalentamiento) en centrales termosolares con colectores lineales.

6.10. Variables termodinámicas para optimizar el rendimiento energético del ciclo de potencia Brayton (RC sin Recalentamiento) en centrales termosolares con colectores lineales.

6.11. Variables termodinámicas para optimizar el rendimiento energético del ciclo de potencia Brayton (RC con Recalentamiento) en centrales termosolares con colectores lineales.

6.12. Variables termodinámicas para optimizar el rendimiento energético del ciclo de potencia Brayton ( $\mathrm{RC}$ con Recalentamiento) en centrales termosolares con colectores lineales lineales.

6.13. Variables termodinámicas para optimizar el rendimiento energético del ciclo de potencia Brayton ( $\mathrm{RC}$ con Recalentamiento) en centrales termosolares con colectores lineales.

6.14. Variables termodinámicas para optimizar el rendimiento energético del ciclo de potencia Brayton (PCRC sin Recalentamiento) en centrales termosolares con colectores lineales.

6.15. Variables termodinámicas para optimizar el rendimiento energético del ciclo de potencia Brayton (PCRC sin Recalentamiento) en centrales termosolares con colectores lineales.

6.16. Variables termodinámicas para optimizar el rendimiento energético del ciclo de potencia Brayton (PCRC sin Recalentamiento) en centrales termosolares con colectores lineales.

6.17. Variables termodinámicas para optimizar el rendimiento energético del ciclo de potencia Brayton (PCRC con Recalentamiento) en centrales termosolares con colectores lineales.

6.18. Variables termodinámicas para optimizar el rendimiento energético del ciclo de potencia Brayton (PCRC con Recalentamiento) en centrales termosolares con colectores lineales.

6.19. Variables termodinámicas para optimizar el rendimiento energético del ciclo de potencia Brayton (PCRC con Recalentamiento) en centrales termosolares con colectores lineales. 
6.20. Variables termodinámicas para optimizar el rendimiento energético del ciclo de potencia Brayton ( $\mathrm{RCMCl}$ sin Recalentamiento) en centrales termosolares con colectores lineales.

6.21. Variables termodinámicas para optimizar el rendimiento energético del ciclo de potencia Brayton ( $\mathrm{RCMCl}$ sin Recalentamiento) en centrales termosolares con colectores lineales.

6.22. Variables termodinámicas para optimizar el rendimiento energético del ciclo de potencia Brayton ( $\mathrm{RCMCl}$ sin Recalentamiento) en centrales termosolares con colectores lineales.

6.23. Variables termodinámicas para optimizar el rendimiento energético del ciclo de potencia Brayton ( $\mathrm{RCMCl}$ con Recalentamiento) en centrales termosolares con colectores lineales.

6.24. Variables termodinámicas para optimizar el rendimiento energético del ciclo de potencia Brayton ( $\mathrm{RCMCl}$ con Recalentamiento) en centrales termosolares con colectores lineales.

6.25. Variables termodinámicas para optimizar el rendimiento energético del ciclo de potencia Brayton ( $\mathrm{RCMCl}$ con Recalentamiento) en centrales termosolares con colectores lineales (PTC o LF).

7.1. Principales aceites térmicos como HTF en las plantas termosolaresy sus principales propiedades físicas.

7.2. Principales sales fundidas como HTF en las plantas termosolares y sus principales propiedades físicas.

7.3. Localización, condiciones ambientales.

7.4. Parámetros tubo absorbedor.

7.5. Parámetros colectores PTC.

7.6. Parámetros colector LF.

7.7. Ciclo potencia Rankine.

7.8. Ciclo potencia s-CO2 Brayton.

7.9. Eficiencia Neta de planta termosolar con colectores lineales acoplada a ciclo de potencia Rankine sin Recalentamiento.

7.10. Eficiencia Neta de planta termosolar con colectores lineales acoplada a ciclo de potencia Rankine con Recalentamiento.

7.11. Eficiencia Neta de planta termosolar con colectores lineales acoplada a ciclo de potencia s-CO2 Brayton RC con recalentamiento, UA=3000 kW/K.

7.12. Eficiencia Neta de planta termosolar con colectores lineales acoplada a ciclo de potencia s-CO2 Brayton RC con recalentamiento, UA=5000 kW/K.

7.13. Eficiencia Neta de planta termosolar con colectores lineales acoplada a ciclo de potencia s-CO2 Brayton RC con recalentamiento, UA=10000 kW/K.

7.14. Eficiencia Neta de planta termosolar con colectores lineales acoplada a ciclo de potencia s-CO2 Brayton RC con recalentamiento, UA=15000 kW/K.

7.15. Eficiencia Neta de planta termosolar con colectores lineales acoplada a ciclo de potencia s-CO2 Brayton RC con recalentamiento, UA=20000 kW/K.

7.16. Eficiencia Neta de planta termosolar con colectores lineales acoplada a ciclo de potencia s-CO2 Brayton PCRC con recalentamiento, UA=3000 kW/K. 
7.17. Eficiencia Neta de planta termosolar con colectores lineales acoplada a ciclo de potencia s-CO2 Brayton PCRC con recalentamiento, UA=5000 kW/K.

7.18. Eficiencia Neta de planta termosolar con colectores lineales acoplada a ciclo de potencia s-CO2 Brayton PCRC con recalentamiento, UA=10000 kW/K.

7.19. Eficiencia Neta de planta termosolar con colectores lineales acopladas a ciclo de potencia s-CO2 Brayton PCRC con recalentamiento, UA=15000 kW/K.

7.20. Eficiencia Neta de planta termosolar con colectores lineales acoplada a ciclo de potencia s-CO2 Brayton PCRC con Recalentamiento, UA=20000 kW/K.

7.21. Eficiencia Neta de planta termosolar con colectores lineales acoplada a ciclo de potencia s-CO2 Brayton $\mathrm{RCMCl}$ con Recalentamiento, $\mathrm{UA}=3000 \mathrm{~kW} / \mathrm{K}$.

7.22. Eficiencia Neta de planta termosolar con colectores lineales acoplada a ciclo de potencia s-CO2 Brayton $\mathrm{RCMCl}$ con Recalentamiento, UA=5000 kW/K.

7.23. Eficiencia Neta de planta termosolar con colectores lineales acoplada a ciclo de potencia s-CO2 Brayton $\mathrm{RCMCl}$ con Recalentamiento), UA=10000 kW/K.

7.24. Eficiencia Neta de planta termosolar con colectores lineales acoplada a ciclo de potencia s-CO2 Brayton $\mathrm{RCMCl}$ con Recalentamiento, UA=15000 kW/K.

7.25. Eficiencia Neta de planta termosolar con colectores lineales acoplada a ciclo de potencia s-CO2 Brayton $\mathrm{RCMCl}$ con Recalentamiento, $U A=20000 \mathrm{~kW} / \mathrm{K}$.

7.26. Variables en la optimización del rendimiento energético de planta termosolar con colectores lineales, Dowtherm A como HTF, acopladas a ciclos de potencia s-CO2 Brayton RC con Recalentamiento.

7.27. Variables en la optimización del rendimiento energético de planta termosolar con colectores lineales, Therminol VP/Syltherm 800 como HTF, acoplada a ciclos de potencia s-CO2 Brayton RC con Recalentamiento.

7.28. Variables en la optimización del rendimiento energético de planta termosolar con colectores lineales, Therminol 75 como HTF, acopladas a ciclos de potencia s-CO2 Brayton RC con Recalentamiento.

7.29. Variables en la optimización del rendimiento energético de planta termosolar con colectores lineales, Dowtherm A como HTF, acopladas a ciclos de potencia s-CO2 Brayton PCRC con Recalentamiento.

7.30. Variables en la optimización del rendimiento energético de planta termosolar con colectores lineales, Therminol VP/Syltherm 800 como HTF, acopladas a ciclos de potencia sCO2 Brayton PCRC con Recalentamiento).

7.31. Variables en la optimización del rendimiento energético de planta termosolar con colectores lineales, Therminol 75 como HTF, acopladas a ciclos de potencia s-CO2 Brayton PCRC con Recalentamiento.

7.32. Variables en la optimización del rendimiento energético de planta termosolar con colectores lineales, Dowtherm A como HTF, acopladas a ciclos de potencia s-CO2 Brayton $\mathrm{RCMCl}$ con Recalentamiento.

7.33. Variables en la optimización del rendimiento energético de planta termosolar con colectores lineales, Therminol VP/Syltherm 800 como HTF, acopladas a ciclos de potencia sCO2 Brayton $\mathrm{RCMCl}$ con Recalentamiento.

7.34. Variables en la optimización del rendimiento energético de planta termosolar con colectores lineales, Therminol 75 como HTF, acopladas a ciclos de potencias-CO2 Brayton $\mathrm{RCMCl}$ con Recalentamiento. 
7.35. Diseño y parámetros de operación de los recuperadores. Tamaño térmico total de los recuperadores UA=3000 kW/K. Ciclo de potencia s-CO2 Brayton RC.

7.36. Diseño y parámetros de operación de los recuperadores. Tamaño térmico total de los recuperadores UA=5000 kW/K. Ciclo de potencia s-CO2 Brayton RC.

7.37. Diseño y parámetros de operación de los recuperadores. Tamaño térmico total de los recuperadores UA=10000 kW/K. Ciclo de potencia s-CO2 Brayton RC.

7.38. Diseño y parámetros de operación de los recuperadores. Tamaño térmico total de los recuperadores UA=15000 kW/K. Ciclo de potencia s-CO2 Brayton RC.

7.39. Diseño y parámetros de operación de los recuperadores. Tamaño térmico total de los recuperadores UA=20000 kW/K. Ciclo de potencia s-CO2 Brayton RC.

7.40. Diseño y parámetros de operación de los recuperadores. Tamaño térmico total de los recuperadores UA=3000 kW/K. Ciclo de potencia s-CO2 Brayton PCRC.

7.41. Diseño y parámetros de operación de los recuperadores. Tamaño térmico total de los recuperadores UA $=5000 \mathrm{~kW} / \mathrm{K}$. Ciclo de potencia s-CO2 Brayton PCRC.

7.42. Diseño y parámetros de operación de los recuperadores. Tamaño térmico total de los recuperadores UA=10000 kW/K. Ciclo de potencia s-CO2 Brayton PCRC.

7.43. Diseño y parámetros de operación de los recuperadores. Tamaño térmico total de los recuperadores UA $=15000 \mathrm{~kW} / \mathrm{K}$. Ciclo de potencia s-CO2 Brayton PCRC.

7.44. Diseño y parámetros de operación de los recuperadores. Tamaño térmico total de los recuperadores UA=20000 kW/K. Ciclo de potencia s-CO2 Brayton PCRC.

7.45. Diseño y parámetros de operación de los recuperadores. Tamaño térmico total de los recuperadores UA=3000 kW/K. Ciclo de potencia s-CO2 Brayton RCMCI.

7.46. Diseño y parámetros de operación de los recuperadores. Tamaño térmico total de los recuperadores UA=5000 kW/K. Ciclo de potencia s-CO2 Brayton RCMCl.

7.47. Diseño y parámetros de operación de los recuperadores. Tamaño térmico total de los recuperadores UA=10000 kW/K. Ciclo de potencia s-CO2 Brayton RCMCI.

7.48. Diseño y parámetros de operación de los recuperadores. Tamaño térmico total de los recuperadores UA=15000 kW/K. Ciclo de potencia s-CO2 Brayton RCMCI.

7.49. Diseño y parámetros de operación de los recuperadores. Tamaño térmico total de los recuperadores UA=20000 kW/K. Ciclo de potencia s-CO2 Brayton RCMCI.

8.1. Eficiencia energética de las plantas termosolares con colectores lineales con DSG acopladas a ciclos de potencia s-CO2 Brayton.

8.2. Listado de proyectos de investigación más significativos para el desarrollo de la DSG en los colectores lineales.

8.3. Localización y condiciones ambientales. 202

8.4. Parámetros tubos receptores. 202

8.5. Parámetros de los colectores PTC. 202

8.6. Parámetros de los colectores LF. 202

8.7. Parámetros del ciclo s-CO2 Brayton. 202

8.8. Hipótesis y resultados del Balance Hidráulico del campo solar de la configuración 2. 207

8.9. Eficiencia neta de plantas termosolares con colectores LF. Comparación de 201 resultados entre la configuración 1 y la de referencia.

8.10. Eficiencia neta de plantas termosolares con colectores PTC. Comparación resultados entre la configuración 1 y la de referencia. 
8.11. Eficiencia neta de plantas termosolares con colectores LF. Comparación de resultados entre la configuración 2 y la de referencia.

8.12. Eficiencia neta de plantas termosolares con colectores PTC. Comparación de resultados entre la configuración 2 y la de referencia.

8.13. Eficiencia neta de plantas termosolares con colectores LF. Comparación de resultados entre la configuración 3 y la de referencia.

8.14. Eficiencia neta de plantas termosolares con colectores lineales. Comparación de resultados entre la configuración 4 y diferentes tipologías de ciclos s-CO2 Brayton.

8.15. Potencia Unitaria de plantas termosolares con colectores LF. Comparación de resultados entre la configuración 1 y la de referencia, $\mathrm{TIT}=400^{\circ} \mathrm{C}$.

8.16. Potencia Unitaria de plantas termosolares con colectores LF. Comparación de resultados entre la configuración 4 y la de referencia, $\mathrm{T}=550^{\circ} \mathrm{C}$.

8.17. Área de apertura y coste del campo solar en plantas con LF. Comparación de resultados entre la configuración 1 y la de referencia. Potencia neta $40 \mathrm{MWe}$, $\mathrm{TIT}=400^{\circ} \mathrm{C}$.

8.18. Área de apertura y coste del campo solar en plantas con PTC. Comparación de resultados entre la configuración 1 y la de referencia. Potencia neta $40 \mathrm{MWe}$, $\mathrm{TIT}=400^{\circ} \mathrm{C}$.

8.19. Área de apertura y coste del campo solar en plantas con LF. Comparación de resultados entre la configuración 2 y la de referencia. Potencia neta $55 \mathrm{MWe}$, $\mathrm{TIT}=550^{\circ} \mathrm{C}$.

8.20. Área de apertura y coste del campo solar en plantas con PTC. Comparación de resultados entre la configuración 2 y la de referencia. Potencia neta $55 \mathrm{MWe}, 214$ $\mathrm{TIT}=550^{\circ} \mathrm{C}$.

8.21. Área de apertura y coste del campo solar en plantas con LF. Comparación de resultados entre la configuración 4 y la de referencia. Potencia neta $55 \mathrm{MWe}, 214$ $\mathrm{TIT}=550^{\circ} \mathrm{C}$.

8.22. Área de apertura y coste del campo solar en plantas con PTC. Comparación de resultados entre la configuración 4 y la de referencia. Potencia neta $55 \mathrm{MWe}, 214$ $\mathrm{TIT}=550^{\circ} \mathrm{C}$.

8.23. Tamaño térmico de los intercambiadores en plantas termosolares con LF. Comparación entre la configuración 1 y la referencia. Potencia neta 40 MWe, 215 $\mathrm{TIT}=400^{\circ} \mathrm{C}$.

8.24. Tamaño térmico de los intercambiadores en plantas termosolares con PTC. Comparación entre la configuración 1 y la referencia. Potencia neta 40 MWe, 216 $\mathrm{TIT}=400^{\circ} \mathrm{C}$.

8.25. Tamaño térmico de los intercambiadores en plantas termosolares con LF. Comparación entre la configuración 2 y la de referencia. Potencia neta $55 \mathrm{MWe}, 216$ $\mathrm{TIT}=550^{\circ} \mathrm{C}$.

8.26. Tamaño térmico de los intercambiadores en plantas termosolares con PTC. Comparación entre la configuración 2 y la de referencia. Potencia neta $55 \mathrm{MWe}, 216$ $\mathrm{TIT}=550^{\circ} \mathrm{C}$.

8.27. Caracterización de los materiales del ciclo de potencia s-CO2 Brayton [39]. 
8.28. Coste de inversión de los intercambiadores en plantas termosolares con LF. Comparación entre la configuración 1 y la de referencia. Potencia Neta 40 MWe, 217 $\mathrm{TIT}=400^{\circ} \mathrm{C}$.

8.29. Coste de inversión de los intercambiadores del ciclo de potencia Rankine. Planta termosolar con LF. Potencia Neta $40 \mathrm{MWe}, \mathrm{TIT}=400^{\circ} \mathrm{C}$.

8.30. Coste de inversión de los intercambiadores en plantas termosolares con PTC. Comparación entre la configuración 1 y la de referencia. Potencia Neta $40 \mathrm{MWe}$, $\mathrm{TIT}=400^{\circ} \mathrm{C}$.

8.31. Coste de inversión de los intercambiadores del ciclo de potencia Rankine. Planta termosolar con PTC. Potencia Neta $40 \mathrm{MWe}, \mathrm{TIT}=400^{\circ} \mathrm{C}$.

8.32. Coste de inversión de los intercambiadores en plantas termosolares con LF. Comparación entre la configuración 2 y la de referencia. Potencia Neta $55 \mathrm{MWe}, 218$ $\mathrm{TIT}=550^{\circ} \mathrm{C}$.

8.33. Coste de inversión de los intercambiadores del ciclo de potencia Rankine. Planta termosolar con LF. Potencia Neta $55 \mathrm{MWe}, \mathrm{TIT}=550^{\circ} \mathrm{C}$.

8.34. Coste de inversión de los intercambiadores en plantas termosolares con PTC. Comparación entre la configuración 2 y la de referencia. Potencia Neta $55 \mathrm{MWe}$, $\mathrm{TIT}=550^{\circ} \mathrm{C}$

8.35. Coste de inversión de los intercambiadores del ciclo de potencia Rankine. Planta termosolar con PTC. Potencia Neta $55 \mathrm{MWe}, \mathrm{TIT}=550^{\circ} \mathrm{C}$.

8.36. Coste de inversión de los intercambiadores en plantas termosolares con LF. Comparación entre la configuración 4 y la de referencia. Potencia Neta $55 \mathrm{MWe}$, $\mathrm{TIT}=550^{\circ} \mathrm{C}$.

8.37. Coste de inversión de los intercambiadores en plantas termosolares con PTC. Comparación entre la configuración 4 y la de referencia. Potencia Neta $55 \mathrm{MWe}$, $\mathrm{TIT}=550^{\circ} \mathrm{C}$.

8.38. Coste de inversión total. Planta termosolar con LF. Suma del coste de los colectores solares y de los intercambiadores de calor del ciclo de potencia s-CO2 Brayton. Comparación entre la configuración 1 y la de referencia. Potencia Neta 40 $\mathrm{MWe}, \mathrm{TIT}=400^{\circ} \mathrm{C}$.

8.39. Coste de inversión total. Planta termosolar con PTC. Suma del coste de los colectores solares y de los intercambiadores de calor del ciclo de potencia s-CO2 Brayton. Comparación entre la configuración 1 y la de referencia. Potencia Neta 40 $\mathrm{MWe}, \mathrm{TIT}=400^{\circ} \mathrm{C}$.

8.40. Coste de inversión total. Planta termosolar con LF. Suma del coste de los colectores solares y de los intercambiadores de calor del ciclo de potencia s-CO2 Brayton. Comparación entre la configuración 2 y la de referencia. Potencia Neta 55 $\mathrm{MWe}, \mathrm{TIT}=550^{\circ} \mathrm{C}$.

8.41. Coste de inversión total. Planta termosolar con PTC. Suma del coste de los colectores solares y de los intercambiadores de calor del ciclo de potencia s-CO2 Brayton. Comparación entre la configuración 2 y la de referencia. Potencia Neta 55 $\mathrm{MWe}, \mathrm{TIT}=550^{\circ} \mathrm{C}$.

8.42. Eficiencia neta del ciclo de potencia s-CO2 Brayton RC, y diferencia mínimas de temperaturas en los recuperadores pinch-point. 
8.43. Eficiencia neta del ciclo de potencia s-CO2 Brayton PCRC, y diferencia mínimas de temperaturas en los recuperadores pinch-point.

8.44. Eficiencia neta del ciclo de potencia s- $\mathrm{CO} 2$ Brayton $\mathrm{RCMCl}$, y diferencia mínimas de temperaturas en los recuperadores pinch-point.

8.45. Tamaño térmico UA y estimación del coste de los intercambiadores de plantas termosolares con colectores lineales con DSG, acopladas a ciclos s-CO2 Brayton RC.

8.46. Tamaño térmico UA y estimación del coste de los intercambiadores de plantas termosolares con colectores lineales con DSG, acopladas a ciclos s-CO2 Brayton PCRC.

8.47. Tamaño térmico UA y estimación del coste de los intercambiadores de plantas termosolares con colectores lineales con DSG, acopladas a ciclos s-CO2 Brayton RCMCI.

9.1. Comparación del contenido de materiales (en Toneladas) de tres plantas termosolares con diferente tecnología. Potencia 103 MWe y 6 horas de almacenamiento térmico [8].

9.2. Comparación resultados configuraciones acopladas a ciclo de potencia Rankine [15].

9.3. Localización y condiciones ambientales.

9.4. Parámetros colectores PTC y LF.

9.5. Parámetros tubo absorbedor [29]

9.6. Parámetros ciclo s-CO2 Brayton.

9.7. Planta termosolar con colectores lineales acoplada a ciclo de potencia s-CO2 Brayton SB con recalentamiento. Potencia Bruta fija 50MWe. Tamaño térmico de los recuperadores $\mathrm{UA}=3000 \mathrm{~kW} / \mathrm{K}$.

9.8. Planta termosolar con colectores lineales acoplada a ciclo de potencia s-CO2 Brayton SB con recalentamiento. Potencia Bruta fija 50MWe. Tamaño térmico de los recuperadores $U A=5000 \mathrm{~kW} / \mathrm{K}$.

9.9. Estimación central termosolar con colectores PTC acoplada a ciclo Potencia s-CO2 Brayton SB. Potencia bruta fija $50 \mathrm{MWe}$.

9.10. Estimación central termosolar con colectores LF acoplada a ciclo Potencia s-CO2 Brayton SB. Potencia bruta fija 50 MWe.

9.11. Centrales Termosolares con campo Dual-Loop acopladas a ciclo de potencia sCO2 Brayton RC. Eficiencia y producción de energía.

9.12. Centrales Termosolares con campo Dual-Loop acopladas a ciclo de potencia sCO2 Brayton PCRC. Eficiencia y producción de energía.

9.13. Centrales Termosolares con campo Dual-Loop acopladas a ciclo de potencia s$\mathrm{CO} 2$ Brayton RCMCl. Eficiencia y producción de energía.

9.14. Centrales Termosolares con campo Dual-Loop (oil+sal) acopladas a ciclo de potencia s-CO2 Brayton RC. Área de apertura efectiva y estimación de costes de los colectores PTC.

9.15. Centrales Termosolares con campo Dual-Loop acopladas a ciclo de potencia PCRC s-CO2 Brayton. Área de apertura efectiva y estimación de costes de los colectores PTC.

9.16. Centrales Termosolares con campo Dual-Loop acopladas a ciclo de potencia s$\mathrm{CO} 2$ Brayton RCMCI. Área de apertura efectiva y estimación de costes de los colectores PTC.

10.1. Localización y condiciones ambientales. 
10.3. Parámetros de colectores PTC.

10.4. Parámetros de colectores LF.

10.5. Parámetros del ciclo de potencia Brayton. 260

10.6. Parámetros del ciclo de potencia Rankine. 260

10.7. Relación de Presiones Opción A. 261

10.8. Relación de Presiones Opción B 261

10.9. Planta Termosolar (MS+s-C2H6). Eficiencia \& Tamaño Térmico en recuperadores (3).

10.10. Eficiencia neta y temperatura entrada al compresor y pérdidas de presión en los intercambiadores de calor (3).

10.11. Costes Unitarios de Inversión en el campo solar $(\$ / \mathrm{m} 2)$.

10.12. Costes de inversión en planta termosolar con colectores LF con MS como HTF (M\$). Potencia neta fija en todas las configuraciones 50MWe (3).

10.13. Costes de inversión en planta termosolar con colectores PTC con MS como HTF (M\$). Potencia neta fija en todas las configuraciones 50MWe (3).

10.14. Costes de inversión en planta termosolar con colectores LF con DSG (M\$). Potencia neta fija en todas las configuraciones 50MWe (3).

TABLA 10.15. Costes de inversión en planta termosolar con colectores PTC DSG (M\$). Potencia neta fija en todas las configuraciones 50MWe (3).

10.16. Coste de Capital en plantas con colectores LF (M\$). En todas las plantas termosolares se ha fijado la potencia neta 50MWe (3).

10.17. Coste de Capital en plantas con colectores PTC (M\$়). En todas las plantas termosolares se ha fijado la potencia neta $50 \mathrm{MWe}(3)$.

11.1. Propiedades de los fluidos de trabajo en estado de criticidad [26].

11.2. TIT máximas para diferentes fluidos de trabajo en ciclos de potencia Brayton. $\quad 281$

11.3. Localización y temperatura ambiental. 281

11.4. Parámetros del tubo absorbedor. 281

11.5. Parámetros colectores PTC. $\quad 281$

11.6. Parámetros colectores LF. 282

11.7. Parámetros del ciclo Brayton. $\quad 282$

12.1. Segunda validación caso $\mathrm{CIT}=32^{\circ} \mathrm{C}$. 324

12.2. Segunda validación caso $\mathrm{CIT}=40^{\circ} \mathrm{C}$. 324

12.3. Segunda validación caso $\mathrm{CIT}=50^{\circ} \mathrm{C}$. 325

13.1. Resumen del rendimiento energético de las diferentes tecnologías analizadas en el capítulo primero.

13.2. Resumen del rendimiento energético de las diferentes soluciones tecnológicas analizadas en el capítulo 3.

13.3. Resumen del rendimiento energético de las diferentes soluciones tecnológicas analizadas en el capítulo 4.

13.4. Resumen del rendimiento energético de las diferentes soluciones tecnológicas analizadas en el capítulo 4.

13.5. Resumen del rendimiento energético de las diferentes soluciones analizadas en el capítulo 7. Eficiencia Neta de planta termosolar con colectores lineales y aceite como HTF, acoplada a ciclo de potencia s-CO2 Brayton RC con Recalentamiento, UA=15000 $\mathrm{kW} / \mathrm{K}$. 
13.6. Resumen del rendimiento energético de las diferentes soluciones analizadas en el capítulo 8. Eficiencia neta de planta termosolar con colectores lineales y DSG como HTF, ciclo de potencia s-CO2 Brayton RC, y diferencia mínimas de temperaturas en los recuperadores pinch-point.

13.7. Resumen del rendimiento energético de las diferentes soluciones analizadas en el capítulo 9. Planta termosolar con colectores lineales acoplada a ciclo de potencia s-CO2 Brayton SB con recalentamiento. Potencia Bruta fija 50MWe. Tamaño térmico de los recuperadores UA=5000 kW/K.

1. Centrales Termosolares con campo "Dual-Loop" acopladas a ciclo de potencia RC sCO2 Brayton. Eficiencia y producción de energía.

2. Centrales Termosolares con campo "Dual-Loop" acopladas a ciclo de potencia RC sCO2 Brayton. Eficiencia y producción de energía.

3. Centrales Termosolares con campo "Dual-Loop" acopladas a ciclo de potencia PCRC sCO2 Brayton. Eficiencia y producción de energía.

4. Centrales Termosolares con campo "Dual-Loop" acopladas a ciclo de potencia PCRC sCO2 Brayton. Eficiencia y producción de energía.

5. Centrales Termosolares con campo "Dual-Loop" acopladas a ciclo de potencia RCMCl s-CO2 Brayton. Eficiencia y producción de energía.

6. Centrales Termosolares con campo "Dual-Loop" acopladas a ciclo de potencia RCMCl s-CO2 Brayton. Eficiencia y producción de energía.

7. Centrales Termosolares con campo "Dual-Loop" acopladas a ciclo de potencia RC sCO2 Brayton. Área de apertura efectiva y estimación de costes de los colectores PTC.

8. Centrales Termosolares con campo "Dual-Loop" acopladas a ciclo de potencia RC sCO2 Brayton. Área de apertura efectiva y estimación de costes de los colectores PTC.

9. Centrales Termosolares con campo "Dual-Loop" acopladas a ciclo de potencia PCRC sCO2 Brayton. Área de apertura efectiva y estimación de costes de los colectores PTC.

10. Centrales Termosolares con campo "Dual-Loop" acopladas a ciclo de potencia PCRC s-CO2 Brayton. Área de apertura efectiva y estimación de costes de los colectores PTC.

11. Centrales Termosolares con campo "Dual-Loop" acopladas a ciclo de potencia $\mathrm{RCMCl}$ s-CO2 Brayton. Área de apertura efectiva y estimación de costes de los colectores PTC.

12. Centrales Termosolares con campo "Dual-Loop" acopladas a ciclo de potencia PCRC s-CO2 Brayton. Área de apertura efectiva y estimación de costes de los colectores PTC. 